WSRC-TR-93-059

FOURTH QUARTER 1992

AND 1992 SUMMARY

\title{
H-AREA SEEPAGE BASINS \\ GROUNDWATER \\ MONITORING REPORT (U)
}

PUBLICATION DATE: MARCH 1993
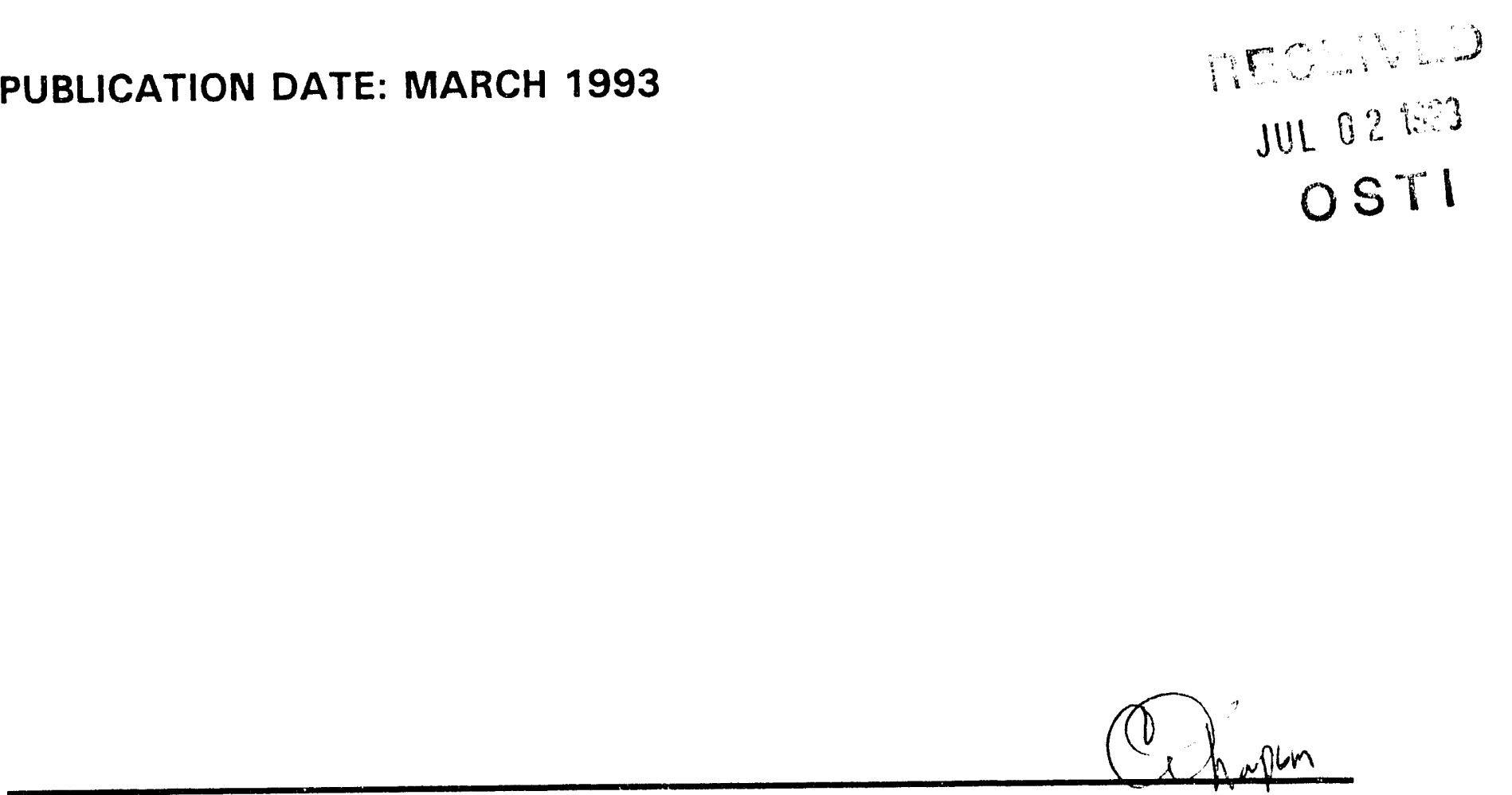


\section{DISCLAIMER}

This report was prepared by Westinghouse Savannah River Company (WSRC) for the United States Department of Energy under Contract No. DE-AC09-89SR18035 and is an account of work performed under that contract. Reference herein to any specific commercial product, process, or service by trademark, name, manufacturer, or otherwise does not necessarily constitute or imply endorsement, recommendation, or favoring of same by WSRC or by the United States Government or any agency thereof. The views and opinions of the authors expressed herein do not necessarily state or reflect those of the United States Government or any agency thereof. 
WSRC-TR-93-059

\section{Abstract}

During fourth quarter 1992, the groundwater at the H-Area Seepage Basins (HASB) was monitored in compliance with South Carolina Hazardous Waste Management Regulations, R61-79.265, Subpart F. Samples were collected from 130 wells that monitor the three separate hydrostratigraphic units that make up the uppermost aquifer beneath the HASB. A detailed description of the uppermost aquifer is included in the Resource Conservation and Recovery Act Part B Post-Closure Care Permit Application for the H-Area Hazardous Waste Management Facility submitted to the South Carolina Department of Health and Environmental Control in December 1990.

Historically, as well as currently, tritium, nitrate, total alpha-emitting radium, gross alpha, and mercury have been the primary constituents observed above final Primary Drinking Water Standards (PDWS) in groundwater at the HASB. Nonvolatile beta has consistently exceeded its drinking water screening level. Other radionuclides and hazardous constituents also have exceeded the final PDWS in the groundwater at the HASB. These constituents are found primarily in Aquifer Zone $\mathrm{IIB}_{2}$ (Water Table) and in the upper portion of Aquifer Zone $\mathrm{IIB}_{1}$ (Barnwell/McBean). However, constituents exceeding standards also occur in several wells in the lower portion of Aquifer Zone $\mathrm{IIB}_{1}$ (Barnwell/McBean) and Aquifer Unit IIA (Congaree).

Isoconcentration/isoactivity maps included in this report indicate both the concentration/ activity and extent of the primary contaminants in each of the three hydrostratigraphic units during first and fourth quarters 1992 . Water-level maps indicate that the groundwater flow rates and directions at the HASB have remained relatively constant since the basins ceased to be active in 1988 . 
WSRC-TR-93-059 


\section{Contents}

Page

Abstract $\ldots \ldots \ldots \ldots \ldots \ldots \ldots \ldots \ldots \ldots \ldots \ldots \ldots \ldots \ldots \ldots \ldots \ldots \ldots$

List of Figures $\ldots \ldots \ldots \ldots \ldots \ldots \ldots \ldots \ldots \ldots \ldots \ldots \ldots \ldots$

List of Tables $\ldots \ldots \ldots \ldots \ldots \ldots \ldots \ldots \ldots \ldots \ldots \ldots \ldots \ldots \ldots \ldots \ldots$

Executive Summary $\ldots \ldots \ldots \ldots \ldots \ldots \ldots \ldots \ldots \ldots \ldots \ldots \ldots \ldots$

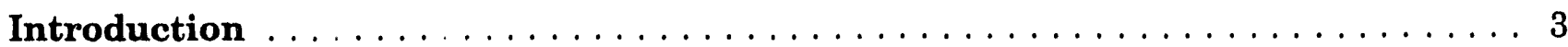

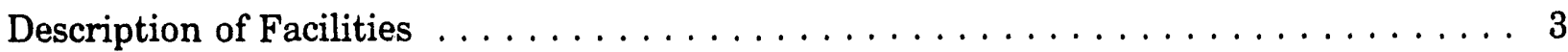

Hydrostratigraphic Units . . . . . . . . . . . . . . . . . . . . . 4

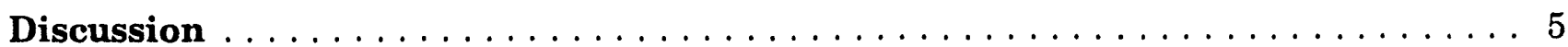

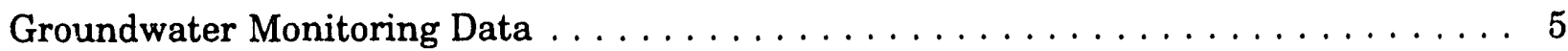

Integrity of the Monitoring Well Network $\ldots \ldots \ldots \ldots \ldots \ldots \ldots \ldots$

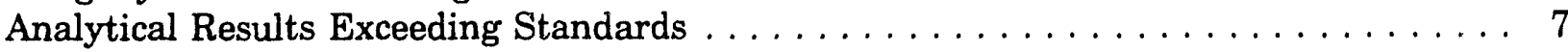

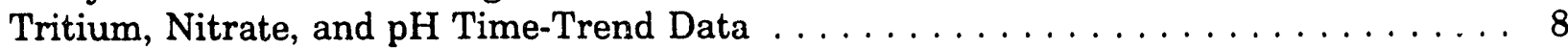

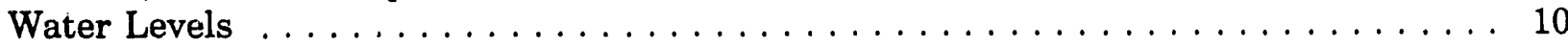

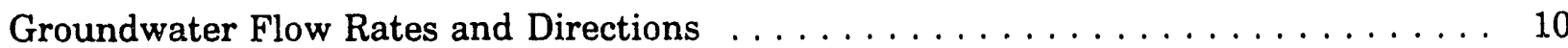

Upgradient Versus Downgradient Results $\ldots \ldots \ldots \ldots \ldots \ldots \ldots \ldots$

Conclusions $\ldots \ldots \ldots \ldots \ldots \ldots \ldots \ldots \ldots \ldots \ldots \ldots \ldots \ldots \ldots \ldots$

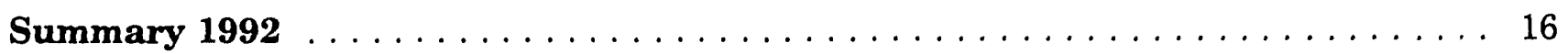

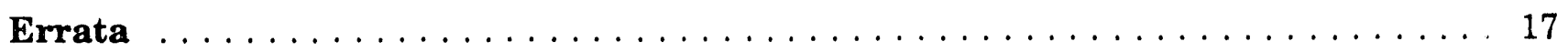

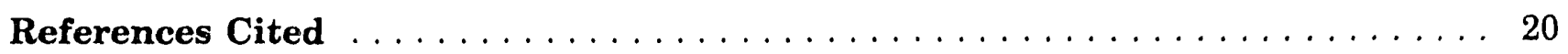

Appendix A-Final Primary Drinking Water Standards $\ldots \ldots \ldots \ldots \ldots$

Appendix B-Flagging Criteria $\ldots \ldots \ldots \ldots \ldots \ldots \ldots \ldots \ldots \ldots \ldots \ldots$

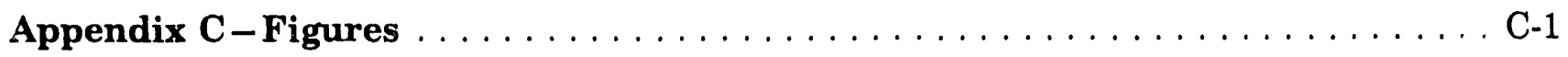

Appendix D-Groundwater Monitoring Results Tables $\ldots \ldots \ldots \ldots \ldots \ldots$ 
Appendix E-Data Quality/Useability Assessment $\ldots \ldots \ldots \ldots \ldots \ldots \ldots$

Appendix F-Time Series Plots $\ldots \ldots \ldots \ldots \ldots \ldots \ldots \ldots \ldots \ldots \ldots \ldots \ldots \ldots$

Appendix G-Hydrographs $\ldots \ldots \ldots \ldots \ldots \ldots \ldots \ldots \ldots \ldots \ldots \ldots$ 


\section{List of Figures}

Page

1. Hydrostratigraphic Nomenclature $\ldots \ldots \ldots \ldots \ldots \ldots \ldots \ldots \ldots \ldots \ldots$. . . . . .

2. Regional Correlation of Hydrostratigraphic and Lithostratigraphic Nomenclatures $\ldots \ldots \ldots \ldots \ldots \ldots \ldots \ldots \ldots \ldots \ldots \ldots \ldots$

3. Location of the H-Area Seepage Basins at the Savannah River Site . . . . . . . . . C-4

4. Location of the Groundwater Monitoring Wells at the H-Area Seepage Basins . . . C-5

5. Location of Aquifer Zone $\mathrm{IIB}_{2}$ (Water Table) Groundwater Monitoring Wells at the H-Area Seepage Basins . . . . . . . . . . . . . . . . . . C-6

6. Location of Upper Portion Aquifer Zone IIB 1 (Barnwell/McBean) Groundwater Monitoring Wells at the H-Area Seepage Basins $\ldots \ldots \ldots \ldots \ldots \ldots$

7. Location of Lower Portion Aquifer Zone IIB 1 (Barnwell/McBean) Groundwater Monitoring Wells at the H-Area Seepage Basins $\ldots \ldots \ldots \ldots \ldots \ldots$

8. Location of Aquifer Unit IIA (Congaree) Groundwater Monitoring Welis at the H-Area Seepage Basins . . . . . . . . . . . . . . . . . . . C-9

9. Tritium Activities in Aquifer Zone $\mathrm{IIB}_{2}$ (Water Table) at the H-Area Seepage Basins, First Quarter $1992 \ldots \ldots \ldots \ldots \ldots \ldots \ldots \ldots \ldots \ldots$. . . . . . . . . . . . . .

10. Tritium Activities in Aquifer Zone $\mathrm{IIB}_{2}$ (Water Table) at the H-Area Seepage Basins, Fourth Quarter $1992 \ldots \ldots \ldots \ldots \ldots \ldots \ldots \ldots \ldots$. . . . . . . . . . . . . .

11. Nitrate Concentrations in Aquifer Zone $\mathrm{IIB}_{2}$ (Water Table) at the H-Area Seepage Basins, First Quarter $1992 \ldots \ldots \ldots \ldots \ldots \ldots \ldots \ldots \ldots \ldots$. . . . . . . . . . . . .

12. Nitrate Concentrations in Aquifer Zone $\mathrm{IIB}_{2}$ (Water Table) at the H-Area Seepage Basins, Fourth Quarter $1992 \ldots \ldots \ldots \ldots \ldots \ldots \ldots \ldots \ldots \ldots$. . . . . . . . . . . . . .

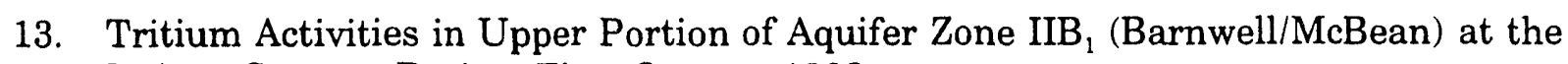
H-Area Seepage Basins, First Quarter $1992 \ldots \ldots \ldots \ldots \ldots \ldots \ldots \ldots$. . . . . . . . . .

14. Tritium Activities in Upper Portion of Aquifer Zone IIB $_{1}$ (Barnwell/McBean) at the H-Area Seepage Basins, Fourth Quarter $1992 \ldots \ldots \ldots \ldots \ldots \ldots \ldots$. . . . . . . . . . . . 
15. Nitrate Concentrations in Upper Portion of Aquifer Zone $\mathrm{IIB}_{1}$ (Barnwell/McBean) at the H-Area Seepage Basins, First Quarter $1992 \ldots \ldots \ldots \ldots \ldots \ldots$

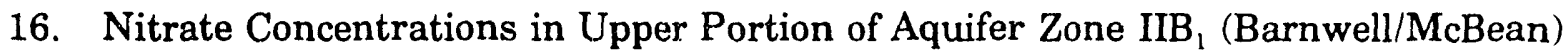
at the H-Area Seepage Basins, Fourth Quarter $1992 \ldots \ldots \ldots \ldots \ldots \ldots$. . . . . . . . .

17. Tritium Activities in Lower Portion of Aquifer Zone IIB $_{1}$ (Barnwell/McBean) at the H-Area Seepage Basins, First Quarter $1992 \ldots \ldots \ldots \ldots \ldots \ldots \ldots \ldots$. . . . . . . . . .

18. Tritium Activities in Lower Portion of Aquifer Zone $\mathrm{IIB}_{1}$ (Barnwell/McBean) at the H-Area Seepage Basins, Fourth Quarter $1992 \ldots \ldots \ldots \ldots \ldots \ldots \ldots$

19. Nitrate Concentrations in Lower Portion of Aquifer Zone $\mathrm{IIB}_{1}$ (Barnwell/McBean) at the H-Area Seepage Basins, First Quarter $1992 \ldots \ldots \ldots \ldots \ldots \ldots \ldots$

20. Nitrate Concentrations in Lower Portion of Aquifer Zone $\mathrm{IIB}_{1}$ (Barnwell/McBean) at the H-Area Seepage Basins, Fourth Quarter $1992 \ldots \ldots \ldots \ldots \ldots \ldots$. . . . . .

21. Tritium Activities in Aquifer Unit IIA (Congaree) at the H-Area Seepage Basins,

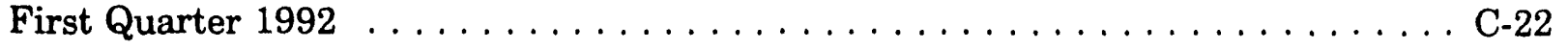

22. Tritium Activities in Aquifer Unit IIA (Congaree) at the H-Area Seepage Basins, Fourth Quarter $1992 \ldots \ldots \ldots \ldots \ldots \ldots \ldots \ldots \ldots \ldots \ldots \ldots \ldots$. . . . . . . . . . . . . . . .

23. Nitrate Concentrations in Aquifer Unit IIA (Congaree) at the H-Area Seepage Basins, First Quarter 1992

24. Nitrate Concentrations in Aquifer Unit IIA (Congaree) at the H-Area Seepage Basins, Fourth Quarter $1992 \ldots \ldots \ldots \ldots \ldots \ldots \ldots \ldots \ldots \ldots \ldots$

25. Gross Alpha Activities in Aquifer Zone $\mathrm{IIB}_{2}$ (Water Table) at the H-Area Seepage Basins, First Quarter 1992

26. Gross Alpha Activities in Aquifer Zone $\mathrm{IIB}_{2}$ (Water Table) at the H-Area Seepage Basins, Fourth Quarter 1992

27. Nonvolatile Beta Activities in Aquifer Zone $\mathrm{IIB}_{2}$ (Water Table) at the H-Area Seepage Basins, First Quarter 1992

28. Nonvolatile Beta Activities in Aquifer Zone $\mathrm{IIB}_{2}$ (Water Table) at the H-Area

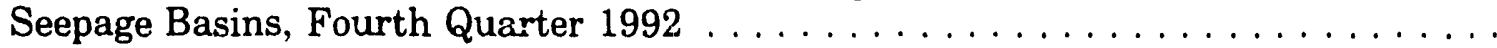

29. Gross Alpha Activities in Upper Portion of Aquifer Zone IIB (Barnwell/McBean) at the H-Area Seepage Basins, First Quarter $1992 \ldots \ldots \ldots \ldots \ldots \ldots \ldots \ldots$. . . . . . . . .

30. Gross Alpha Activities in Upper Portion of Aquifer Zone IIB (Barnwell/McBean) at the H-Area Seepage Basins, Fourth Quarter 1992 
31. Nonvolatile Beta Activities in Upper Portion of Aquifer Zone $\mathrm{IIB}_{1}$ (Barnwell/McBean) at the H-Area Seepage Basins, First Quarter 1992

32. Nonvolatile Beta Activities in Upper Portion of Aquifer Zone IIB $_{1}$ (Barnwell/McBean) at the H-Area Seepage Basins, Fourth Quarter $1992 \ldots \ldots$. . .

33. Gross Alpha Activities in Lower Portion of Aquifer Zone IIB 1 (Barnwell/McBean) at the H-Area Seepage Basins, First Quarter 1992

34. Gross Alpha Activities in Lower Portion of Aquifer Zone IIB $_{1}$ (Barnwell/McBean) at the H-Area Seepage Basins, Fourth Quarter $1992 \ldots \ldots \ldots \ldots \ldots \ldots \ldots$

35. Nonvolatile Beta Activities in Lower Portion of Aquifer Zone IIB (Barnwell/McBean) at the H-Area Seepage Basins, First Quarter 1992

36. Nonvolatile Beta Activities in Lower Portion of Aquifer Zone IIB $_{1}$ (Barnwell/McBean) at the H-Area Seepage Basins, Fourth Quarter 1992 . . . . . . .

37. Gross Alpha Activities in Aquifer Unit IIA (Congaree) at the H-Area Seepage Basins, First Quarter 1992

38. Gross Alpha Activities in Aquifer Unit IIA (Congaree) at the H-Area Seepage Basins, Fourth Quarter $1992 \ldots \ldots \ldots \ldots \ldots \ldots$. . . . . . . . . . . . . . . . . .

39. Nonvolatile Beta Activities in Aquifer Unit IIA (Congaree) at the H-Area Seepage

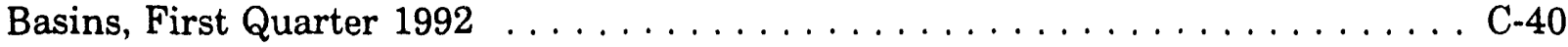

40. Nonvolatile Beta Activities in Aquifer Unit IIA (Congaree) at the H-Area Seepage Basins, Fourth Quarter $1992 \ldots \ldots \ldots \ldots$. . . . . . . . . . . . . . . . . . . . . .

41. Mercury Concentrations in Aquifer Zone $\mathrm{IIB}_{2}$ (Water Table) at the H-Area Seepage Basins, First Quarter 1992

42. Mercury Concentrations in Aquifer Zone $\mathrm{IIB}_{2}$ (Water Table) at the H-Area Seepage Basins, Fourth Quarter $1992 \ldots \ldots \ldots \ldots \ldots \ldots \ldots \ldots \ldots$

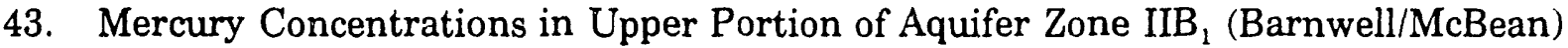
at the H-Area Seepage Basins, First Quarter 1992

44. Mercury Concentrations in Upper Portion of Aquifer Zone IIB $_{1}$ (Barnwell/McBean) at the H-Area Seepage Basins, Fourth Quarter $1992 \ldots \ldots \ldots \ldots$. . . . . . . . . . . . .

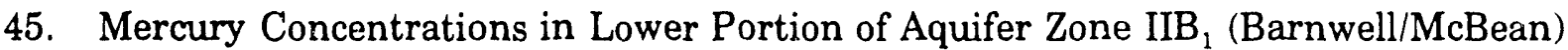
at the H-Area Seepage Basins, First Quarter $1992 \ldots \ldots \ldots \ldots \ldots$. . . . . . . . . .

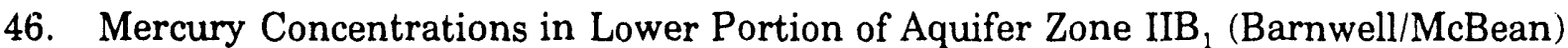
at the H-Area Seepage Basins, Fourth Quarter 1992 
47. Mercury Concentrations in Aquifer Unit IIA (Congaree) at the H-Area Seepage

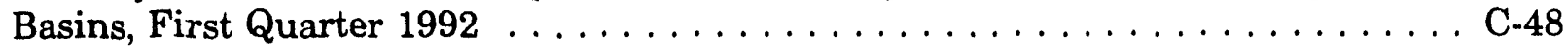

48. Mercury Concentrations in Aquifer Unit IIA (Congaree) at the H-Area Seepage Basins, Fourth Quarter $1992 \ldots \ldots \ldots \ldots \ldots \ldots \ldots \ldots \ldots$. . . . . . . . . . . . . . . . .

49. $\mathrm{pH}$ Levels in Aquifer Zone $\mathrm{IIB}_{2}$ (Water Table) at the H-Area Seepage Basins, Fourth Quarter $1992 \ldots \ldots \ldots \ldots \ldots \ldots \ldots \ldots \ldots \ldots \ldots \ldots$

50. Specific Conductance in Aquifer Zone $\mathrm{IIB}_{2}$ (Water Table) at the H-Area Seepage Basins, Fourth Quarter 1992

51. $\mathrm{pH}$ Levels in Upper Portion of Aquifer Zone IIB $_{1}$ (Barnwell/McBean) at the H-Area Seepage Basins, Fourth Quarter $1992 \ldots \ldots \ldots \ldots \ldots \ldots \ldots \ldots \ldots \ldots$

52. Specific Conductance in Upper Portion of Aquifer Zone IIB $_{1}$ (Barnwell/McBean) at the H-Area Seepage Basins, Fourth Quarter $1992 \ldots \ldots \ldots \ldots \ldots \ldots \ldots \ldots$. . . . . C-53

53. $\mathrm{pH}$ Levels in Lower Portion of Aquifer Zone IIB $_{1}$ (Barnwell/McBean) at the H-Area Seepage Basins, Fourth Quarter $1992 \ldots \ldots \ldots \ldots \ldots \ldots \ldots \ldots$. . . . . . . . . . . .

54. Specific Conductance in Lower Portion of Aquifer Zone IIB $_{1}$ (Barnwell/McBean) at the H-Area Seepage Basins, Fourth Quarter $1992 \ldots \ldots \ldots \ldots \ldots \ldots \ldots$. . . . . . C-55

55. pH Levels in Aquifer Unit IIA (Congaree) at the H-Area Seepage Basins, Fourth

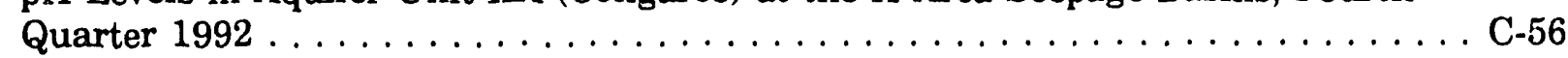

56. Specific Conductance in Aquifer Unit IIA (Congaree) at the H-Area Seepage Basins, Fourth Quarter 1992

57. Piezometric Surface Map of Aquifer Zone $\mathrm{IIB}_{2}$ (Water Table) at the H-Area

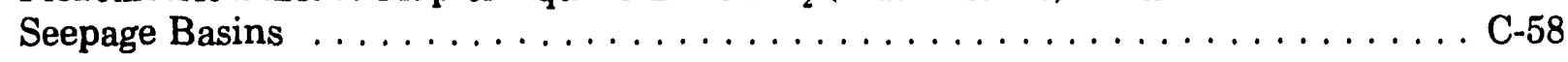

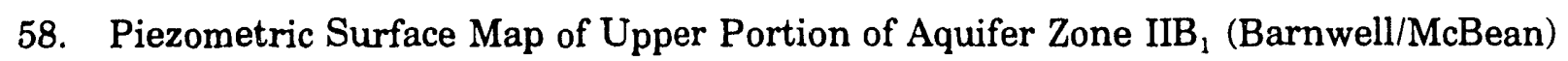

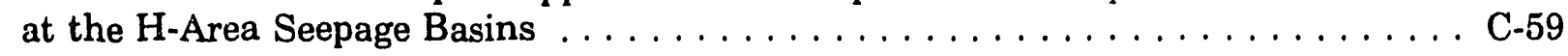

59. Piezometric Surface Map of Lower Portio. of Aquifer Zone IIB (Barnwell/McBean) $^{-}$

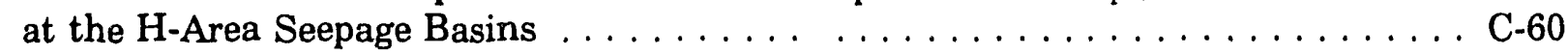

60. Piezometric Surface Map of Aquifer Unit IIA (Congaree) at the H-Area Seepage Basins 
WSRC-TR-93-059

\section{List of Tables}

Page

1. Constituents Exceeding the Final Primary Drinking Water Standards . . . . . . D-5

2. Constituents Exceeding Half the Final Primary Drinking Water Standards

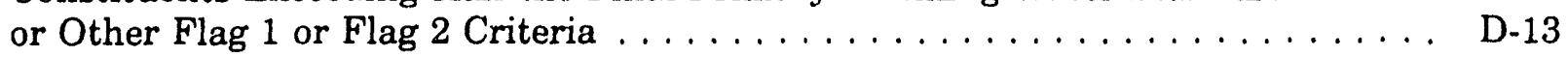

3. Groundwater Monitoring Results for Individual Wells . . . . . . . . . . . . . D-22 
WSRC-TR-93-059 


\section{Executive Summary}

Currently, 130 wells of the HSB series monitor the groundwater in the uppermost aquifer beneath the H-Area Seepage Basins (HASB) at the Savannah River Site (SRS) as required by the South Carolina Hazardous Waste Management Regulations. The wells are sampled and analyzed quarterly for certain indicator parameters, heavy metals, radionuclides, and other constituents. This grourdwater quality assessment report describes the monitoring results that exceeded final Primary Drinking Water Standards (PDWS), drinking water screening levels, or SRS flagging criteria for fourth quarter 1982.

Reports for first, second, and third quarters are submitted to the South Carolina Department of Health and Environmental Control (SCDHEC) 90 days after the end of each quarter as required by Administrative Consent Order 85-70-SW (as amended in 1988). The fourth quarter report, which includes the annual report, is submitted to SCDHEC 90 days after the end of the quarter as negotiated by SCDHEC with the U.S. Environmental Protection Agency. Under the approved HASB Hazardous Waste Permit issued November 2, 1992 (SCDHEC, 1992), semiannual reports will be required during 1993. The report for first and second quarters 1993 will be submitted September 30,1993, and the report for third and fourth quarters 1993 will be submitted March 31, 1994.

During fourth quarter 1992, 10 constituents exceeded the final PDWS at one or more wells at the HASB. As in previous quarters, tritium and nitrate were the primary constituents found in the groundwater at the HASB. Of the 130 groundwater monitoring wells, $84(64 \%)$ exhibited elevated tritium activities and $44(34 \%)$ exhibited elevated nitrate activities. These two constituents were found primarily in Aquifer Zone $\mathrm{IIB}_{2}$ (Water Tabls) and in the upper portion of Aquifer Zone IIB $_{1}$ (Barnwell/McBean). However, tritium activities exceeding the final PDWS also occurred in several wells in the lower portion of Aquifer Zone IIB $_{1}$ (Barnwell/McBean) and Aquifer Unit IIA (Congaree).

Mercury, lead, and cadmium historically have been the primary heavy metals exhibiting elevated cc ncentrations at the HASB. During fourth quarter 1992, mercury exceeded its final PDWS in 11 wells in Aquifer Zcne $\mathrm{IIB}_{2}$ (Water Table) (HSB 68, 101D, 102D, 103D, 104D, 105D, 108D, 125D, 126D, 127D, and 145D). Lead was elevated in 2 wells in Aquifer Zone $\mathrm{IB}_{2}$ (Water Table) (HSB115D and 152D). Mercury and lead did not exceed standards in any of the lower water-bearing units. Cadmium did not exceed its final PDWS in any well at the HASB during the quarter.

Tetrachloroethylene was elevated in wells HSB106C, 139C, and 145C, and trichloroethylene was elevated in well HSB $145 \mathrm{C}$ during fourth quarter 1.32. These wells are located in the upper portion of Aquifer Zone $\mathrm{IIB}_{1}$ (Barnwell/McBean).

No constituents exceeded the final PDWS in upgradient Aquifer Zone IIB $_{2}$ (Water Table) wells HSB 66 or $85 \mathrm{C}$, ungradient wells in the lower portion of Aquifer Zone IIB $_{1}$ 
(Barnwell/McBean), or upgradient Aquifer Unit IIA (Congaree) wells. Upgradient Aquifer Zone $\mathrm{IIB}_{2}$ (Water Table) well HSB 65C exhibited elevated tritium levels. One or more of the downgradient wells at the HASB contained elevated levels of tritium, nitrate, nonvolatile beta, total alpha-emitting radium, gross alpha, mercury, tetrachloroethylene, lead, arsenic, or trichloroethylene.

Using SRS grid coordinates, groundwater flow in Aquifer Zone $\mathrm{IIB}_{2}$ (Water Table) is to the southwest toward Fourmile Branch; flows in the upper and lower portions of Aquifer Zone IIB $_{1}$ (Barnwell/McBean) are south-southwest. Flow in Aquifer Unit IIA (Congaree) is northwest toward Upper Three Runs Creek. Flow rates during fourth quarter 1992 were estimated to range between approximately $120 \mathrm{ft} / \mathrm{yr}$ and $470 \mathrm{ft} / \mathrm{yr}$ in Aquifer Zone $\mathrm{IIB}_{2}$ (Water Table), between $200 \mathrm{ft} / \mathrm{yr}$ and $550 \mathrm{ft} / \mathrm{yr}$ in the upper portion of Aquifer Zone $\mathrm{IIB}_{1}(\mathrm{~B} \varepsilon \mathrm{nwell} /$ McBean), between $55 \mathrm{ft} / \mathrm{yr}$ and $110 \mathrm{ft} / \mathrm{yr}$ in the lower portion of Aquifer Zone IIB $_{1}$ (Barnwell/ McBean), and at about $330 \mathrm{ft} / \mathrm{yr}$ in Aquifer Unit ILA (Congaree). 
WSRC-TR-93-059

\section{Introduction}

\section{Description of Facilities}

In 1955, three H-Area Seepage Basins (HASB) (H-1, H-2, and H-3) were constructed at the Savannah River Site (SRS) to contain waste water from the H-Area Separations Facility at SRS. In 1962, another basin (H-4) was constructed to replace Basin $\mathrm{H}-3$ due to the slow seepage of waste water from Basin H-3. These unlined, hydraulically connected basins received waste water containing elevated amounts of nitrate, tritium, and other constituents that aiso included low-level radionuclides and other constituents. Primary sources for this waste water included the nitric acid recovery overheads, the general-purpose evaporator overheads. overheads from the two waste tank farm evaporators, cooling water from the tritium facilities, and retention basin transfers (Heffner and Exploration Resources, 1991).

The basins allowed the waste water either to evaporate or to percolate into the underlying soils where some of the waste constituents were removed from the water by interaction with the soils. However, tritium does not interact with the soils but instead continues to migrate downward into the groundwater. Some of the tritium in groundwater decays before reaching surface water. The half-life of tritium is 12.26 years.

The groundivater at the HASB contains elevated levels of low-level radionuclides and chemicals from 30 years of operation. Tritium and nitrate are the primary constituents and are mainly exhibited in Aquifer Zones $\mathrm{IIB}_{2}$ (Water Table) and IIB 1 (Barnwell/McBean). Since use of the HASB ceased and the basins were closed, overall tritium and nitrate levels have declined.

Discharge of waste water to the HASB was discontinued on November 7, 1988, and the basins' closure certification was effective in November 31,1991, according to the South Carolina Hazardous Waste Management Regulations (SCDHEC, 1990). Low permeability closure caps have been constructed over the basins to prevent infiltration of rainwater through basin sediments and to minimize migration of contaminants into the groundwater.

On December 3, 1990, SRS submitted a Resource Conservation and Recovery Act (RCRA) Part B Post-Closure Care Permit Application that includes an implementable plan for groundwater remediation to SCDHEC (WSRC, 1990). SCDHEC issued a RCRA Part B permit with conditions in response to this application on November 2, 1992. The permit requires that a detailed corrective action plan for groundwater remediation at the HASB be submitted. 


\section{Hydrostratigraphic Units}

Historically, groundwater quality assessment reports for the HASB have used the nomenclature Water Table, Barnwell, McBean, and Congaree to identify hydrostratigraphic units. However, an interim alphanumeric system developed by Aadland and Bledsoe (1990)

(Figure 1, Appendix C) defines the aquifer and aquitard units at SRS using hydrostratigraphic rather than lithostratigraphic designations. Figure 2 (Appendix C) shows a correlation of these designations. For clarity, this report uses the newer nomenclature and also includes the older names used in earlier repcrts. The HASB RCRA Part B Post-Closure Care Permit Application, submitted December 1990 (WSRC, 1990), includes an in-depth explanation of the new nomenclature.

The HASB well network monitors three distinct hydrostratigraphic units in the uppermost aquifer beneath the facility: Aquifer Zone $\mathrm{IIB}_{2}$ (Water Table), which is supported by Confining Zone $\mathrm{IIB}_{1}-\mathrm{IIB}_{2}$ (Tan Clay); the poorly confined Aquifer Zone $\mathrm{IIB}_{1}$ (Barnwell/McBean); and the semi-confined Aquifer Unit IIA (Congaree), which is separated from the overlying Aquifer Zone IIB $_{1}$ (Barnwell/McBean) by Confining Unit IIA-IIB (Green Clay). The base of the uppermost aquifer is defined as the uppermost bed of Confining System I-II (Ellenton Formation), which lies approximately $300 \mathrm{ft}$ below the surface of the area. The HASB RCRA Part B Post-Closure Care Permit Application includes a detailed description of the geologic and hydrogeologic systems at the HASB. 
WSRC-TR-93-059

\section{Discussion}

\section{Groundwater Monitoring Data}

The groundwater sampling procedure was modified beginning fourth quarter 1992 in response to regulatory guidance and advances in sampling equipment design (WSRC, 1992). The modified procedure requires evacuation of a minimum of two well volumes and stabilization of $\mathrm{pH}$, specific conductance, and turbidity prior to sample collection. Stability is achieved over a minimum of three successive measurements taken within a given time period. If a well pumps dry before two well volumes are purged or before stabilization is achieved, it must be revisited within 24 hours for the data to be considered from a single sampling event. On the second visit within 24 hours, samples are taken without purging or stability measurements; thus, these samples may not be representative of the groundwater quality.

A further modification in the procedure is that samples collected for metals analyses are not filtered. Thus, the analyses are for total metals rather than dissolved metals. Variable-speed pumps have been installed in some wells that have had a history of elevated metals. Samples from these wells are collected at a slower rate to minimize turbidity, which has been associated with elevated metal levels. Decreased aluminum and iron concentrations as well as lower turbidity values have been observed for samples from wells with variable-speed pumps. At present, wells HSB 68, 69, 84A, 84D, 86C, 86D, 101D, 102D, 103D, 104D, 105D, 106D, $107 \mathrm{D}, 108 \mathrm{D}, 110 \mathrm{D}, 111 \mathrm{D}, 111 \mathrm{E}, 112 \mathrm{D}, 112 \mathrm{E}, 113 \mathrm{D}, 114 \mathrm{D}, 116 \mathrm{C}, 116 \mathrm{D}, 125 \mathrm{D}$, and 136D have variable-speed pumps.

During fourth quarter 1992, samples from wells at the HASB were analyzed for certain indicator parameters, heavy metals, radionuclides, and cther constituents as part of the SRS Environmental Protection Department/Environmental Monitoring Section (EPD/EMS) Groundwater Monitoring Program. Monitoring results that exceeded the Safe Drinking Water Act final Primary Drinking Water Standards (PDWS) or screening levels, established by the U.S. Environmental Protection Agency (EPA) (see Appendix A), the South Carolina final Primary Drinking Water Standard for lead (see Appendix A), or other SRS flagging criteria (see Appendix B) are discussed in this report.

The drinking water standard for lead was changed to the South Carolina Primary Drinking Water Standard of $50 \mu \mathrm{g} / \mathrm{L}$ fourth quarter 1992. Lead data for the earlier quarters of 1992 were made consistent with the $50 \mu \mathrm{g} / \mathrm{L}$ standard for this annual report. The SRS flagging criteria are based on final and proposed PDWS, Secondary Drinking Water Standards, and method detection limits For simplicity, results that either equal or exceed standards are described only as exceeding or above standards. Constituent levels that exceed the final PDWS, screening levels, or Flag 2 criteria are described as elevated, and constituent levels that exceed Flag 1 criteria are described as slightly elevated. 
The final PDWS for individual analytes provided in Appendix A may not always match the SRS flagging criteria provided in Appendix B. The final PDWS are used as guidelines in this compliance report to meet regulatory requirements; the flagging criteria are used by EPD/EMS to identify relative levels of constituents in the groundwater and as guides for scheduling groundwater sampling.

Appendix $\mathrm{C}$ contains illustrations of the hydrostratigraphic units beneath the HASB at SRS (Figures 1 and 2); the monitored waste management unit (Figure 3); the individual monitoring wells (Figure 4); the monitoring wells in the different hydrostratigraphic zones and units (Figures 5 through 8); the lateral distributions of tritium, nitrate, gross alpha, nonvolatile beta, mercury, specific conductance, and $\mathrm{pH}$ (Figures 9 through 56); and the water-elevation contours of the different hydrostratigraphic zones and units and the groundwater flow directions (Figures 57 through 60). The monitoring results tables as well as analyses that exceeded the holding times, final PDWS, and other flagging criteria are in Appendix D; data quality/useability assessment information is in Appendix E; time series plots for tritium, nitrate, and pH for selected wells are in Appendix F; and hydrographs are in Appendix G.

\section{Integrity of the Monitoring Well Network}

The HASB groundwater monitoring well network (Figure 4, Appendix C) provides groundwater samples from the three hydrostratigraphic units that make up the uppermost aquifer below the facility. A program is in place to rehabilitate and replace wells that do not produce representative samples from the units being monitored.

The 58 Aquifer Zone $\mathrm{IIB}_{2}$ (Water Table) wells are HSB 65, 65C, 66, 67, 68, 69, 70, 71, 83D, 84D, 85C, 86D, 100D, 101D, 102D, 103D, 104D, 105D, 106D, 107D, 108D, 109D, 110D, 111D, $111 \mathrm{E}, 112 \mathrm{D}, 112 \mathrm{E}, 113 \mathrm{D}, 114 \mathrm{D}, 115 \mathrm{D}, 116 \mathrm{D}, 117 \mathrm{D}, 125 \mathrm{D}, 126 \mathrm{D}, 127 \mathrm{D}, 129 \mathrm{D}, 130 \mathrm{D}, 131 \mathrm{D}$, 132D, 133D, 134D, 135D, 136D, 137D, 138D, 139D, 140D, 141D, 142D, 143D, 145D, 146D, 147D, 148D, 149D, 150D, 151D, and 152D (Figure 5, Appendix C).

The 46 wells screened in the upper portion of Aquifer Zone $\mathrm{IIB}_{1}$ (Barnwell/McBean) are HSB $68 \mathrm{C}, 70 \mathrm{C}, 71 \mathrm{C}, 83 \mathrm{C}, 84 \mathrm{C}, 86 \mathrm{C}, 100 \mathrm{C}, 101 \mathrm{C}, 102 \mathrm{C}, 103 \mathrm{C}, 104 \mathrm{C}, 105 \mathrm{C}, 106 \mathrm{C}, 107 \mathrm{C}$, $108 \mathrm{C}, 109 \mathrm{C}, 110 \mathrm{C}, 111 \mathrm{C}, 112 \mathrm{C}, 113 \mathrm{C}, 114 \mathrm{C}, 115 \mathrm{C}, 116 \mathrm{C}, 117 \mathrm{C}, 125 \mathrm{C}, 126 \mathrm{C}, 127 \mathrm{C}, 129 \mathrm{C}$, $130 \mathrm{C}, 131 \mathrm{C}, 132 \mathrm{C}, 133 \mathrm{C}, 134 \mathrm{C}, 135 \mathrm{C}, 136 \mathrm{C}, 137 \mathrm{C}, 139 \mathrm{C}, 140 \mathrm{C}, 141 \mathrm{C}, 142 \mathrm{C}, 143 \mathrm{C}, 145 \mathrm{C}$, 146C, 148C, 151C, and 152C (Figure 6, Appendix C).

The 6 wells screened in the lower portion of Aquifer Zone IIB $($ Barnwell/McBean) are HSB 65B, 68B, 83B, 84B, 85B, and 86B (Figure 7, Appendix C).

Twenty wells monitor Aquifer Unit IIA (Congaree). Thirteen of these wells are screened in the upper portion (HSB 85A, 118A, 119A, 120A, 121A, 122A, 123A, 124AR, 139A, 140A, $141 \mathrm{~A}, 144 \mathrm{~A}$, and $146 \mathrm{~A}$ ), and four are screened in the lower portion (HSB 65A, 68A, 84A, and 86A) (Figure 8, Appendix C). Wells HSB 69A, 83A, and 117A are screened in the middle portion of the Congaree.

Table 3 (Appendix D) lists the number of well volumes purged from each of the HSB wells during fourth quarter 1992. Where the casing diameter is not known, the well diameter was assumed to be $4 \mathrm{in}$. to calculate the number of well volumes purged. Aquifer $\mathrm{Z}_{\text {one }} \mathrm{IIB}_{2}$ 
(Water Table) wells HSB102D, 112E, 115D, 126D, 132D, 141D, 147D, 148D, and 150D pumped dry during purging. Aquifer Zone $\mathrm{IIB}_{2}$ (Water Table) well HSB106D could not be sampled because the pump would not start. Aquifer Zone $\mathrm{IIB}_{2}$ (Water Table) well HSB131D could not be sampled because the pump was removed for repairs.

Wells HSB 68C, 70C, 71C, 84C, 129C, 132C, 136C, 137C, 139C, 141C, and 148C, located in the upper portion of Aquifer Zone $\mathrm{IIB}_{1}$ (Barnwell/McBean), pumped dry during purging. Well HSB117C, also in the upper portion of Aquifer Zone IIB 1 (Barnwell/McBean), was not sampled because the pump has been removed. Wells HSB $68 \mathrm{~B}$ and $85 \mathrm{~B}$, located in the lower

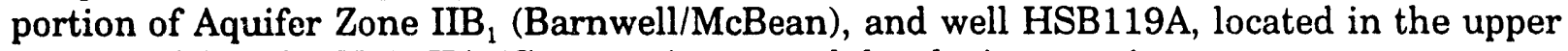
portion of Aquifer Unit IIA (Congaree), pumped áry during purging.

Wells HSB 67, 68, 69, 86D, 102D, 104D, 105D, 109D, 113D, 114D, 115D, 116D, and 136D had field $\mathrm{pH}$ values equal to or less than 4; wells $\mathrm{HSB} 70 \mathrm{C}, 84 \mathrm{~B}, 85 \mathrm{~B}, 101 \mathrm{D}, 104 \mathrm{C}, 123 \mathrm{~A}, 135 \mathrm{C}$, $136 \mathrm{C}, 141 \mathrm{~A}, 141 \mathrm{C}, 148 \mathrm{C}$, and $148 \mathrm{D}$ had field $\mathrm{pH}$ values equal to or greaier than 8.

\section{Analytical Results Exceeding Standards}

Results for analytes that exceeded the final PDWS (see Appendix A) during fourth quarter 1992 are summarized in Table 1 (Appendix D).

Forty-seven of the 58 Aquifer Zone $\mathrm{IIB}_{2}$ (Water Table) wells contained tritium, nitrate, nonvolatile beta, total alpha-emitting radium (radium-223, radium-224, and radium-226), gross alpha, mercury, lead, or arsenic levels that exceeded standards Tritium was elevated in 46 wells, with activities up to $1.5 \mathrm{E}+04 \mathrm{pCi} / \mathrm{mL}$ in well HSB112D; nitrate was elevated in 26 wells, with concentrations up to $57,500 \mu \mathrm{g} / \mathrm{L}$ in well HSB111D; nonvolatile beta exceeded its drinking water screening level in 28 wells, with activities up to $7.3 \mathrm{E}+03 \mathrm{pCi} / \mathrm{L}$ in well HSB116D; total alpha-emitting radium was elevated in 22 wells, with activities up to $8.9 \mathrm{E}+01$ pCi/L in wells HSB 68 and 116D; and gross alpha was elevated in 17 wells, with a maximum activity of $1.7 \mathrm{E}+02$ in well HSB102D. Mercury was elevated in 11 wells, lead in 2 wells, and arsenic in 1 well during the quarter.

Thirty-two of the 46 wells that monitor the upper portion of Aquifer Zone IIB $_{1}$ (Barnwell/ McBean) contained levels of tritium, nitrate, nonvolatile beta, total alpha-emitting radium, tetrachloroethylene, gross alpha, or trichloroethylene that exceeded standards. Tritium was elevated in 32 wells, with activities up to $1.6 \mathrm{E}+04 \mathrm{pCi} / \mathrm{mL}$ in well $\mathrm{HSB} 86 \mathrm{C}$; nitrate was elevated in 17 wells, with concentrations up to $60,000 \mu \mathrm{g} / \mathrm{L}$ in well $\mathrm{HSB} 137 \mathrm{C}$; and nonvolatile beta exceeded its drinking water screening level in 9 wells, with activities up to $5.5 \mathrm{E}+02$ $\mathrm{pCi} / \mathrm{L}$ in well HSB $86 \mathrm{C}$. Total alpha-emitting radium was elevated in 4 wells, tetrachloroethylene in 3 wells, gross alpha in 2 wells, and trichloroethylene in 1 well.

Two of the 6 wells that monitor the lower portion of Aquifer Zone IIB $_{1}$ (Barnwell/McBean) contained levels of tritium that exceeded its final PDWS. Wells HSB 68B and 84B exhibited $3.8 \mathrm{E}+01 \mathrm{pCi} / \mathrm{mL}$ and $8.4 \mathrm{E}+01 \mathrm{pCi} / \mathrm{mL}$ of tritium activity, respectively.

Four of the 20 wells in Aquifer Unit IIA (Congaree) contained elevated levels of tritium, with activities up to $5.0 \mathrm{E}+03 \mathrm{pCi} / \mathrm{mL}$ in well HSB118A (in the upper portion of this hydrostrati- 
graphic unit). Well HSB 84A contained elevated nonvolatile beta, and well HSB118A contained elevated nitrate.

During fourth quarter 1992, mercury exceeded its final PDWS in 11 wells in Aquifer Zone $\mathrm{IIB}_{2}$ (Water Table) (HSB 68, 101D, 102D, 103D, 104D, 105D, 108D, 125D, 126D, 127D, and $145 \mathrm{D})$, with the highest concentration at $7.9 \mu \mathrm{g} / \mathrm{L}$ in well HSB126D. Lead was elevated in 2 wells in Aquifer Zone $\mathrm{IIB}_{2}$ (Water Table) (HSB115D and 152D), with the highest concentration at $71 \mu \mathrm{g} / \mathrm{L}$ in well HSB152D. Mercury and lead did not exceed standards in any of the lower water-bearing units. Cadmium did not exceed its final PDWS in any well at the HASB during the quarter.

Tetrachloroethylene was elevated in wells HSB106C, 139C, and 145C, and trichloroethylene was elevated in well HSB145C during fourth quarter 1992. These wells are located in the upper portion of Aquifer Zone $\mathrm{IIB}_{1}$ (Barnwell/McBean).

Results for analytes that exceeded other SRS flagging criteria (see Appendix B) during fourth quarter 1992 are summarized in Table 2 (Appendix D). Table 3 (Appendix D) shows the results for all of the constituents and identifies the results that received modifiers or that exceeded the EPA-approved holding time, the final PDWS, or other standards during fourth quarter 1992.

Constituent results are compared with the PDWS in the database of values reported by the laboratory. Many constituents are reported to more significant digits in the database than in these reports. Thus, some constituent results in Table 3 that appear to equal the PDWS are not marked in the $D$ column. Those results are below the PDWS in the database.

During 1992, nitrate results were obtained by two different methods and reported as nitrate as nitrogen and nitrate-nitrite as nitrogen. In this text, these results are treated as a single evaluation of nitrate frequency in the HASB, and the terms nitrate and nitrate-nitrite are used interchangeably.

The lateral distributions of tritium, nitrate, gross alpha, nonvolatile beta, mercury, specific conductance, and $\mathrm{pH}$ in Aquifer Zone $\mathrm{IIB}_{2}$ (Water Table), the upper portion of Aquifer

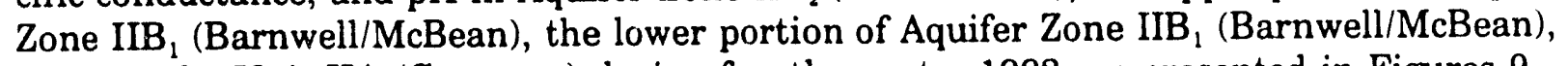
and Aquifer Unit IIA (Congaree) during fourth quarter 1992 are presented in Figures 9 through 56 (Appendix C).

\section{Tritium, Nitrate, and pH Time-Trend Data}

Time series plots for tritium, nitrate, and $\mathrm{pH}$ through fourth quarter 1992 for certain wells and well clusters at the HASB appear in Appendix F. The designations $U$. Congaree, $M$. Congaree, and L. Congaree used in Appendix F represent the upper, middle, and lower portions, respectively, of Aquifer Unit IIA (Congaree).

Tritium activities in wells HSB $66,83 \mathrm{~B}, 85 \mathrm{~B}, 85 \mathrm{C}, 86 \mathrm{~A}, 100 \mathrm{C}, 125 \mathrm{C}, 130 \mathrm{C}, 130 \mathrm{D}$, and 131D have been consistently below the final PDWS when sampled for at least the past 3 years. Most of the other wells shown in Appendix F exhibit tritium in levels that have exceeded the 
final PDWS since at least early 1990. The time series plots for wells HSB 68, 68A, 69, 70, 83D, 84A, 84D, 101D, 102D, 104D, 105D, 106D, 107C, 107D, 108D, 109D, 110D, 113D, 114D, 116D, 117D, 125D, 127D, 129D, 134D, 135D, 136D, 137D, and 138D show tritium activities declining with time. The time series plots for wells HSB1.19A and 137C indicate that tritium activities are increasing with time. Data from wells HSB 83C, 118A, 140A, 140C, 140D, 141A, 141C, 141D, 142D, 143C, 143D, 144A, 145C, 145D, 146A, 146C, 146D, 147D, 148C, $148 \mathrm{D}, 149 \mathrm{D}, 150 \mathrm{D}, 151 \mathrm{C}, 151 \mathrm{D}, 152 \mathrm{C}$, and 152D are insufficient to detect general trends in tritium levels. However, tritium levels in wells HSB 83C, 140A, and 140C have been near or below the final PDWS since sampling began. The remaining HSB wells presented in Appendix $\mathrm{F}$ exhibit no detectable long-term patterns.

Well HSB131C, located on the south side of Fourmile Branch, has continuously exhibited elevated levels of tritium. It is believed that the upper portion of Aquifer Zone IIB $_{1}$ (Barnwell/McBean) discharges into Fourmile Branch from the south and north sides of the creek. The hypothesis that constituents can discharge into Fourmile Branch from the south will be tested by upper Aquifer Zone IIB (Barnwell/McBean) wells HSB140C and 148C, installed during late 1990 south of Fourmile Branch. Tritium has been detected in these wells at levels below the final PDWS, except during first quarter 1992 when elevated levels were detected in well HSB148C. It is possible that Fourmile Branch incises upper Aquifer Zone IIB $_{1}$ (Barnwell/McBean), creating a discharge area for that water-bearing unit so that tritium cannot migrate further within the groundwater system.

Nitrate concentrations in wells HSB $65 \mathrm{~A}, 65 \mathrm{~B}, 65 \mathrm{C}, 65 \mathrm{D}, 66,68 \mathrm{~A}, 68 \mathrm{~B}, 68 \mathrm{C}, 70,71,83 \mathrm{~A}, 83 \mathrm{~B}$, $83 \mathrm{C}, 84 \mathrm{~A}, 84 \mathrm{~B}, 85 \mathrm{~B}, 85 \mathrm{C}, 100 \mathrm{C}, 100 \mathrm{D}, 101 \mathrm{C}, 104 \mathrm{C}, 105 \mathrm{C}, 106 \mathrm{C}, 107 \mathrm{C}, 109 \mathrm{C}, 109 \mathrm{D}, 110 \mathrm{C}$, 117A, 117D, 125C, 130D, 131D, 132C, 132D, 133D, 134C, 135C, 135D, 139A, and 139D have been consistently below the final PDWS for the past 3 years. Most of the remaining wells contain nitrate concentrations that have exceeded the final PDWS since at least early 1990. The time series plots for wells HSB 69, 71C, 83D, 101D, 103D, 104D, 105D, 106D, 107D, 108D, 117C, 126D, 134D, 135D, and 138D indicate nitrate concentrations are declining with time. The plots for wells $\mathrm{HSB} 68,70 \mathrm{C}$, and $118 \mathrm{~A}$ show increasing nitrate concentrations. Wells HSB 84C, 110D, 112C, 112D, 113D, 114C, 115C, 115D, 116C, 116D, 119A, 127C, 129D, and $136 \mathrm{C}$ have what appear to be anomalously high results for fourth quarter 1991 . Wells HSB 69A, 121A, 122A, 123A, 124AR, 133C, 140C, 140D, 141C, 141D, 142C, 142D, 143C, $143 \mathrm{D}, 144 \mathrm{~A}, 145 \mathrm{C}, 145 \mathrm{D}, 146 \mathrm{~A}, 146 \mathrm{C}, 146 \mathrm{D}, 147 \mathrm{D}, 148 \mathrm{C}, 148 \mathrm{D}, 149 \mathrm{D}, 150 \mathrm{D}, 151 \mathrm{C}, 151 \mathrm{D}$, $152 \mathrm{C}$, and $152 \mathrm{D}$ have insufficient data to determine long-term trends. However, nitrate concentrations in wells HSB 69A, 140C, 140D, 141C, 141D, 142C, 142D, 143C, 143D, 144A, $146 \mathrm{~A}, 146 \mathrm{C}, 146 \mathrm{D}, 147 \mathrm{D}, 148 \mathrm{D}, 149 \mathrm{D}, 150 \mathrm{D}, 151 \mathrm{C}, 151 \mathrm{D}, 152 \mathrm{C}$, and 152D have been near or below final PDWS since sampling began. The remaining HSB wells presented in Appendix F exhibit no detectable long-term patterns.

Aquifer Zone $\mathrm{IIB}_{2}$ (Water Table) well HSB 66 and well clusters HSB 69, 84, 104, 111, 116, 126 , and 131 were used to characterize the $\mathrm{pH}$ levels for certain locations within the HASB. Well HSB 66, the only well that represents $\mathrm{pH}$ conditions upgradient of the HASB, is somewhat acidic. Aquifer Zone $\mathrm{IIB}_{2}$ (Water Table) wells HSB 69 and $84 \mathrm{D}$, located midway between Basin H-4 and Fourmile Branch, usually exhibit $\mathrm{pH}$ levels between 3 and 4 . Aquifer Unit IIA (Congaree) well HSB 69A has exhibited a pH range between 6 and 10, but the $\mathrm{pH}$ level is usually near 7. The wells in the HSB 84 well cluster have $\mathrm{pH}$ levels that have ranged from approximately 4 to 10 . The pH levels in well cluster HSB 84 have become more consistent 
since first quarter 1990, with the range since that time between 6 and 8 in wells HSB 84A and $84 \mathrm{C}$. HSB $84 \mathrm{D}$ remains consistently acidic, and HSB $84 \mathrm{~B}$ remains basic. Well cluster HSB104, located between Basin H-3 and Fourmile Branch, exhibits a pH range from

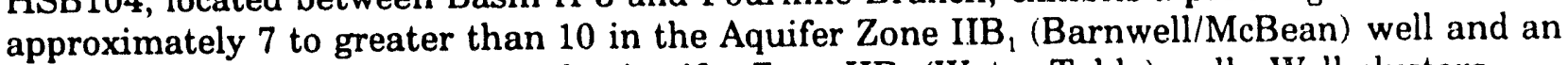
acidic $\mathrm{pH}$ of approximately 4 in the Aquifer Zone IIB $_{2}$ (Water Table) well. Well clusters HSB111 and HSB116, located directly downgradient of the edge of Basin H-4, exhibit acidic conditions generally between 4 and 6 . Well clusters HSB126 and HSB131, located near Fourmile Branch, have pH levels fluctuating around neutral in the Aquifer Zone IIB $_{1}$ (Barnwell/McBean) wells and somewhat acidic $\mathrm{pH}$ levels in the Aquifer Zone IIB $_{2}$ (Water Table) wells.

\section{Water Levels}

Hydrographs showing the water elevations through time for well clusters at the HASB are provided in Appendix F. The average water elevation in Aquifer Zone IIB $_{2}$ (Water Table) during third quarter 1992 was $222.37 \mathrm{ft} \mathrm{msl}$. The average water elevation in this zone during fourth quarter 1992 was $222.17 \mathrm{ft} \mathrm{msl}$, a decrease of $0.2 \mathrm{ft}$ since the previous quarter. The average water elevation in the upper portion of Aquifer Zone IIB $_{1}$ (Barnwell/McBean) during third quarter 1992 was $216.92 \mathrm{ft} \mathrm{msl}$. The average water elevation in this zone during fourth quarter 1992 was $216.87 \mathrm{ft} \mathrm{msl}$, a decrease of $0.05 \mathrm{ft}$ since the previous quarter. The average water elevation in the lower portion of Aquifer Zone IIB $_{1}$ (Barnwell/McBean) during third quarter 1992 was $221.80 \mathrm{ft} \mathrm{msl}$. The average water elevation in the lower portion of Aquifer Zone $\mathrm{IIB}_{1}$ (Barnwell/McBean) during fourth quarter 1992 was $221.90 \mathrm{ft} \mathrm{msl}$, an increase of 0.1 $\mathrm{ft} \mathrm{msl}$. The average water elevation in Aquifer Unit IIA (Congaree) during third quarter 1992 was $171.15 \mathrm{ft}$ msl. The average water elevation in Aquifer Unit IIA (Congaree) during fourth quarter 1992 was $171.33 \mathrm{ft} \mathrm{msl}$, an increase of $0.18 \mathrm{ft}$ since the previous quarter.

A consistent downward vertical head relationship exists between the hydrostratigraphic zones and units monitored at the HASB. Flow potential is downward from Aquifer Zone $\mathrm{IIB}_{2}$ (Water Table) to Aquifer Zone IIB, (Barnwell/McBean) (although at several well clusters, the head difference is very small) and downward from Aquifer Zone IIB $_{1}$ (Barnwell/McBean) to Aquifer Unit IIA (Congaree). These hydrostratigraphic zones have exhibited consistent head relationships since 1988. There are no wells screened in the lower portion of Aquifer Unit IIA (Congaree) to evaluate the head relationship in this unit.

\section{Groundwater Flow Rates and Directions}

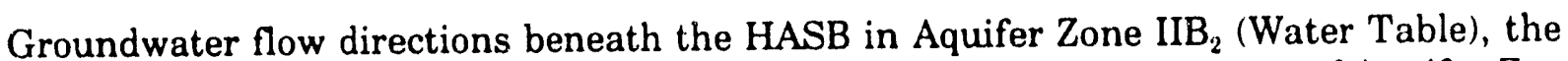

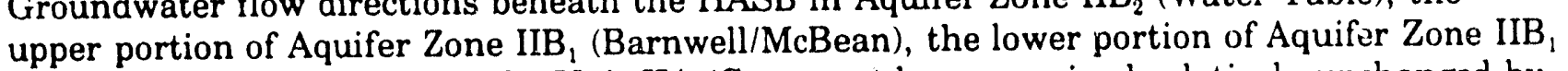
(Barnwell/McBean), and Aquifer Unit IIA (Congaree) have remained relatively unchanged by closure activities. Water-level maps (Figures 57 through 60, Appendix C) for the monitored water-bearing units illustrate groundwater flow patterns. Using SRS grid coordinates, flow in Aquifer Zone $\mathrm{IIB}_{2}$ (Water Table) is to the southwest toward Fourmile Branch; flows in the upper and lower portions of Aquifer Zone IIB $_{1}$ (Barnwell/McBean) are south-southwest. Flow in Aquifer Unit IIA (Congaree) is northwest toward Upper Three Runs Creek. 
To estimate the transport rate of any constituents originating from the HASB, the horizontal flow rate of groundwater is estimated for each hydrostratigraphic unit. Estimated horizontal flow rates in Aquifer Zone $\mathrm{IIB}_{2}$ (Water Table) and the upper and lower portions of Aquifer Zone IIB ${ }_{1}$ (Barnwell/McBean) are calculated along two paths (designated flow paths A and B) for each unit. These flow paths characterize the approximate maximum and minimum groundwater flow rates within these units in areas downgradient from the basins. The estimated horizontal flow rate is calculated along a single flow path for Aquifer Unit IIA (Congaree).

Flow rate calculations are approximations based on inferred or estimated parameters. For this reason, estimations of flow rates should be considered accurate to an order of magnitude only. The groundwater flow rate estimates for Aquifer Zone $\mathrm{IIB}_{2}$ (Water Table), the upper portion of Aquifer Zone IIB $_{1}$ (Barnwell/McBean), the lower portion of Aquifer Zone IIB (Barnwell/McBean), and Aquifer Unit IIA (Congaree) beneath the HASB are based on the following one-dimensional flow equation:

$$
\text { Flow }(\mathrm{ft} / \text { day })=\frac{\text { Hydraulic Conductivity }(\mathrm{ft} / \text { day) }}{\text { Porosity (unitless) }} \times \frac{d h(\mathrm{ft})}{d l(\mathrm{ft})}
$$

The hydraulic conductivity $(\mathrm{Kh})$ constants are $10 \mathrm{ft} /$ day for Aquifer Zone $\mathrm{IIB}_{2}$ (Water Table), $10 \mathrm{ft} /$ day for the upper portion of Aquifer Zone $\mathrm{IIB}_{1}$ (Barnwell/McBean), $5.3 \mathrm{ft} /$ day for the

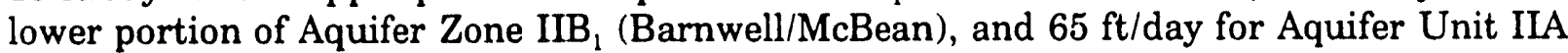
(Congaree) (Geraghty \& Miller, 1990). An effective porosity value of $20 \%$ is used for each hydrostratigraphic unit (Jaegge et al., 1987). This value is based on field and laboratory measurements of porosity and is assumed to be a conservative estimate. The gradient in each calculation represents the change in head $(d h)$ divided by the horizontal distance $(d l)$ along each flow direction arrow. Flow rate estimates vary depending upon the vertical gradient between wells, the size of the area under consideration, and the number of data points.

Flow rate estimates are calculated as follows: flow path length is calculated to the nearest $50 \mathrm{ft}$ for path B of Aquifer Zone $\mathrm{IIB}_{2}$ (Water Table) and to the nearest $100 \mathrm{ft}$ for the remaining flow paths. Flow rate per day is calculated to two significant figures using the above equation. This value is then multiplied by 365 and rounded to two significant figures for the flow rate per year.

The approximate range of groundwater flow rates in Aquifer Zone $\mathrm{IIB}_{2}$ (Water Table) is estimated as follows (Figure 57, Appendix C):

Flow path A

$$
\begin{aligned}
& \frac{10}{0.20} \times \frac{18}{700} \approx 1.3 \mathrm{ft} / \text { day } \\
& 1.3 \mathrm{ft} / \text { day } \times 365 \text { days } \approx 470 \mathrm{ft} / \mathrm{yr}
\end{aligned}
$$


Flow path B

$$
\begin{aligned}
& \frac{10}{0.20} \times \frac{10}{1,550} \approx 0.32 \mathrm{ft} / \text { day } \\
& 0.32 \mathrm{ft} / \text { day } \times 365 \text { days } \approx 120 \mathrm{ft} / \mathrm{yr}
\end{aligned}
$$

The approximate range of groundwater flow rates in the upper portion of Aquifer Zone IIB $_{1}$ (Barnwell/McBean) is estimated as follows (Figure 58, Appendix C):

Flow path $\mathrm{A}$

$$
\begin{aligned}
& \frac{10}{0.20} \times \frac{12}{400} \approx 1.5 \mathrm{ft} / \text { day } \\
& 1.5 \mathrm{ft} / \text { day } \times 365 \text { days } \approx 550 \mathrm{ft} / \mathrm{yr}
\end{aligned}
$$

Flow path B

$$
\begin{aligned}
& \frac{10}{0.20} \times \frac{10}{900} \approx 0.56 \mathrm{ft} / \text { day } \\
& 0.56 \mathrm{ft} / \text { day } \times 365 \mathrm{davs} \approx 200 \mathrm{ft} / \mathrm{yr}
\end{aligned}
$$

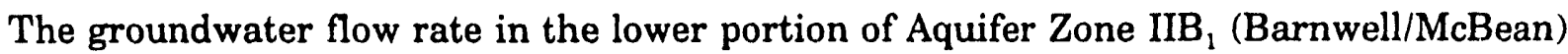
is estimated as follows (Figure 59, Appendix C):

Flow path $\mathrm{A}$

$$
\begin{aligned}
& \frac{5.3}{0.20} \times \frac{10}{900} \approx 0.29 \mathrm{ft} / \text { day } \\
& 0.29 \mathrm{ft} / \text { day } \times 365 \text { days } \approx 110 \mathrm{ft} / \mathrm{yr}
\end{aligned}
$$

Flow path B

$$
\begin{aligned}
& \frac{5.3}{0.20} \times \frac{10}{1,800} \approx 0.15 \mathrm{ft} / \text { day } \\
& 0.15 \mathrm{ft} / \text { day } \times 365 \text { days } \approx 55 \mathrm{ft} / \mathrm{yr}
\end{aligned}
$$

The groundwater flow rate in Aquifer Unit IIA (Congaree) is estimated as follows (Figure 60, Appendix C):

$$
\begin{aligned}
& \frac{65}{0.20} \times \frac{8}{2,900} \approx 0.9 \mathrm{ft} / \text { day } \\
& 0.9 \mathrm{ft} / \text { day } \times 365 \text { days } \approx 330 \mathrm{ft} / \mathrm{yr}
\end{aligned}
$$




\section{Upgradient Versus Downgradient Results}

Groundwater flow in Aquifer Zone $\mathrm{IIB}_{2}$ (Water Table) is to the southwest toward Fourmile Branch (Figure 57, Appendix C), using SRS grid coordinates. Wells HSB 65C, 66, and 85C are upgradient wells for Aquifer Zone $\mathrm{IIB}_{2}$ (Water Table). All remaining Aquifer Zone $\mathrm{IIB}_{2}$ (Water Table) wells monitor downgradient water quality (Figure 5, Appendix C).

Flows in the upper and lower portions of Aquifer Zone $\mathrm{IIB}_{1}$ (Barnwell/McBean) are to the south-southwest toward Fourmile Branch (Figures 58 and 59, Appendix C). No wells monitor upgradient water quality in the upper portion of Aquifer Zone $\mathrm{IIB}_{1}$ (Barnwell/McBean) (Figure 6, Appendix C). The upgradient wells in the lower portion of Aquifer Zone IIB $_{1}$ (Barnwell/McBean) are HSB 65B and 85B (Figure 7, Appendix C).

Flow in the Aquifer Unit IIA (Congaree) is to the northwest toward Upper Three Runs Creek. Unlike the shallower units, flow directions in Aquifer Unit IIA (Congaree) are affected by Upper Three Runs Creek (Figure 60, Appendix C). The upgradient wells in Aquifer Unit ILA (Congaree) wells are HSB140.A, 141A, and 146A.

No constituents exceeded the final PDWS in upgradient wells HSB 66 or 85C, while upgradient well HSB 65C exhibited elevated tritium activities. Downgradient wells screened in Aquifer Zone $\mathrm{IIB}_{2}$ (Water Table) and the upper portion of Aquifer Zone IIB (Barnwell/ McBean) contained elevated levels of tritium, nitrate, nonvolatile beta, total alpha-emitting radium, gross alpha, mercury, tetrachloroethylene, lead, arsenic, or trichloroethylene during fourth quarter 1992 .

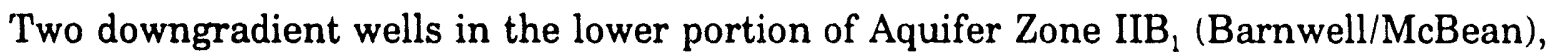
HSB $68 \mathrm{~B}$ and $84 \mathrm{~B}$, contained elevated tritium activities; upgradient wells HSB $65 \mathrm{~B}$ and $85 \mathrm{~B}$ did not contained elevated constituents.

Six downgradient wells in Aquifer Unit IIA (Congaree) contained elevated levels of tritium, nonvolatile beta, or nitrate. Upgradient wells HSB140A, 141A, and 146A contained no elevated constituents.

Wells HSB 66, 83A, and 85B are the designated background wells for the HASB. During fourth quarter 1992, none of these wells contained elevated constituents. 


\section{Conclusions}

The groundwater at the HASB contains elevated levels of low-level radionuclides and chemicais, resulting from 30 years of receiving waste water from the H-Area Separations Facility. During fourth quarter 1992, 10 constituents exceeded the final PDWS at one or more wells at the HASB.

As in previous quarters, tritium and nitrate were the primary constituents found in the groundwater at the HASB. Of the 130 groundwater monitoring wells, $84(64 \%)$ exhibited elevated tritium activities and $44(34 \%)$ exhibited elevated nitrate concentrations. These two constituents were found primarily in Aquifer Zone $\mathrm{IIB}_{2}$ (Water Table) and in the upper portion of Aquifer Zone IIB $_{1}$ (Barnwell/McBean). However, tritium activities exceeding the final PDWS also occurred in several wells in the lower portion of Aquifer Zone IIB $_{1}$ (Barnwell/McBean) and Aquifer Unit IIA (Congaree). The highest tritium activity was found in the upper portion of Aquifer Zone IIB 1 (Barnwell/McBean) well HSB 86C $(1.6 \mathrm{E}+04$ $\mathrm{pCi} / \mathrm{mL}$ ). The highest nitrate concentration occurred in Aquifer Zone IIB $_{1}$ (Barnwell/McBean) well HSB137C $(60,000 \mu \mathrm{g} / \mathrm{L})$. Tritium and nitrate levels appear to be decreasing over time since closure of the HASB, at least in Aquifer Zone $\mathrm{IIB}_{2}$ (Water Table).

The presence of tritium and other constituents in the lower hydrostratigraphic units and the head difference indicate that vertical pathways into the deeper water-bearing units exist at the HASB. A current groundwater flow model for the General Separations Area indicates that the vertical component beneath this area is important (GeoTrans, Inc., 1992).

Mercury, lead, and cadmium historically have been the primary heavy metals exhibiting elevated concentrations at the HASB. During fourth quarter 1992, mercury exceeded its final PDWS in 11 wells in Aquifer Zone IIB $_{2}$ (Water Table) (HSB 68, 101D, 102D, 103D, 104D, $105 \mathrm{D}, 108 \mathrm{D}, 125 \mathrm{D}, 126 \mathrm{D}, 127 \mathrm{D}$, and 145D), with the highest concentration at $7.9 \mu \mathrm{g} / \mathrm{L}$ in well HSB126D. Lead was elevated in 2 wells in Aquifer Zone IIB $_{2}$ (Water Table) (HSB115D and 152D), with the highest concentration at $71 \mu \mathrm{g} / \mathrm{L}$ in well HSB152D. Mercury and lead did not exceed standards in any of the lower water-bearing units. Cadmium did not exceed its final PDWS in any well at the HASB during the quarter. Wells with elevated mercury and lead lie south of the seepage basins.

Tetrachloroethylene was elevated in wells HSB106C, 139C, and 145C, and trichloroethylene was elevated in well HSB145C during fourth quarter 1992. These wells are located in the upper portion of Aquifer Zone IIB $_{1}$ (Barnwell/McBean). No other volatile organic compounds exceeded the final PDWS at the HASB during this quarter. Historically, volatile organic compounds were not placed in the HASB; however, elevated levels of these constituents have occurred sporadically over the years.

No constituents exceeded the final PDWS in upgradient Aquifer Zone $\mathrm{IIB}_{2}$ (Water Table) wells HSB 66 or $85 \mathrm{C}$, while upgradient Aquifer Zone IIB $_{2}$ (Water Table) well HSB 65C 
exhibited elevated tritium levels. Downgradient wells screened in Aquifer Zone IIB $_{2}$ (Water Table) and the upper portion of Aquifer Zone IIB (Barnwell/McBean) contained elevated levels of tritium, nitrate, nonvciatile beta, total alpha-emitting radium, gross alpha, mercury, tetrachloroethylene, lead, arsenic, or trichloroethylene.

Upgradient wells in the lower portion of Aquifer Zone IIB $_{1}$ (Barnwell/McBean) and in Aquifer Unit IIA (Congaree) did not contain elevated constituents during the quarter. However, several dowrigradient wells in the $\epsilon_{-}$units contained elevated levels of tritium, nonvolatile beta, or nitrate. Generally, elevated levels of constituents found in downgradient wells but not in upgradient wells at a waste management unit are considered products of the waste management unit.

Flow rates during fourth quarter 1992 were estimated to range between approximately 120 $\mathrm{ft} / \mathrm{yr}$ and $470 \mathrm{ft} / \mathrm{yr}$ in Aquifer Zone $\mathrm{IIB}_{\mathrm{z}}$ (Water Table), between $200 \mathrm{ft} / \mathrm{yr}$ and $550 \mathrm{ft} / \mathrm{yr}$ in the upper portion of Aquifer Zone $\mathrm{IH}_{1}$ (Barnwell/McBean), between $55 \mathrm{ft} / \mathrm{yr}$ and $110 \mathrm{ft} / \mathrm{yr}$ in the lower portior: of Aquifer Zone $\mathrm{IIB}_{1}$ (Barnwell/McBean), and at about $330 \mathrm{ft} / \mathrm{yr}$ in Aquifer Unit IIA (Congaree). 
WSRC-TR-93-059

\section{Summary 1992}

During 1992, samples from 93 of the 130 groundwater monitoring wells (73\%) at the HASB contained elevated levels of tritium, nitrate, nonvolatile beta, total alpha-emitting radium, gross alpha, mercury, tetrachloroethylene, arsenic, lead, trichloroethylene, or cadmium (Table 1, Appendix D).

As in 1991, tritium and nitrate exceeded their final PDWS more frequently and more consistently than did other constituents. Tritium exceeded its final PDWS in 90 wells during 1992 , with the maximum tritium activity $(1.8 \mathrm{E}+04 \mathrm{pCi} / \mathrm{mL})$ found in Aquifer Zone $\mathrm{IIB}_{2}$ (Water Table) well HSB112D during first and second quarters. Nitrate was elevated in 50 wells during 1992 , with a maximum concentration of $90,000 \mu \mathrm{g} / \mathrm{L}$ in well HSB137C, located in the upper portion of Aquifer Zone $\mathrm{IIB}_{1}$ (Barnwell/McBean), during first quarter. In 1991, tritium and nitrate occurred in 96 and 53 wells, respectively; the maximum tritium activity $\left(2.2 \mathrm{E}+04 \mathrm{pCi} / \mathrm{mL}\right.$ ) occurred in Aquifer Zone $\mathrm{IIB}_{2}$ (Water Table) well HSB101D, and the maximum nitrate concentration $\left(695,000 \mu \mathrm{g} / \mathrm{L}\right.$ ) occurred in Aquifer Zone IIB $_{2}$ (Water Table) well HSB115D.

Nonvolatile beta exceeded its drinking water screening level in 48 wells during 1992 , with a maximum activity of $1.3 \mathrm{E}+04 \mathrm{pCi} / \mathrm{L}$ in Aquifer Zone $\mathrm{IIB}_{2}$ (Water Table) well HSB116D during second quarter. Gross alpha was elevated in 24 wells during the year, with activities up to $2.2 \mathrm{E}+02 \mathrm{pCi} / \mathrm{L}$ in Aquifer Zone $\mathrm{IIB}_{2}$ (Water Table) well HSB102D during second quarter. Total alpha-emitting radium exceeded its final PDWS in 37 wells, with maximum activities up to $1.7 \mathrm{E}+02 \mathrm{pCi} / \mathrm{L}$ in Aquifer Zone $\mathrm{IIB}_{2}$ (Water Table) well HSB116D. Mercury exceeded its final PDWS in 15 wells; tetrachloroethylene in 5 wells; arsenic, lead, and trichloroethylene in 2 wells each; and cadmium in 1 well. Radionuclides and volatile organics occurred with less frequency in 1992 than in 1991; heavy metals occurred with greater frequency.

As in the preceding year, elevated constituents were found primarily in Aquifer Zone $\mathrm{IIB}_{2}$ (Water Table) and Aquifer Zone IIB $_{1}$ (Barnwell/McBean) wells. However, several Aquifer Unit IIA (Congaree) wells contained elevated levels of some constituents, primarily tritium, during both years.

Using SRS grid coordinates, groundwater flow directions beneath the HASB have remained relatively unchanged by closure activities, which began in the late $1980 \mathrm{~s}$. Flow in Aquifer Zone $\mathrm{IIB}_{2}$ (Water Table) is to the southwest toward Fourmile Branch; tlows in the upper and lower portions of Aquifer Zone IIB (Barnwell/McBean) are south-southwest. Flow in Aquifer Unit IIA (Congaree) is northwest toward Upper Three Runs Creek. Groundwater flow rate estimates during the year ranged from approximately $110 \mathrm{ft} / \mathrm{yr}$ to $470 \mathrm{ft} / \mathrm{yr}$ in Aquifer Zone IIB $_{2}$ (Water Table), from $150 \mathrm{ft} / \mathrm{yr}$ to $550 \mathrm{ft} / \mathrm{yr}$ in the upper portion of Aquifer Zone IIB (Barnwell/McBean), from $37 \mathrm{ft} / \mathrm{yr}$ to $120 \mathrm{ft} / \mathrm{yr}$ in the lower portion of Aquifer Zone $\mathrm{IIB}_{1}$ (Barnwell/McBean), and from $330 \mathrm{ft} / \mathrm{yr}$ to $690 \mathrm{ft} / \mathrm{yr}$ in Aquifer Unit IIA (Congaree). 


\section{Errata}

Some of the values for earlier quarters presented in the results tables of this report may differ from the values for those same quarters presented in previous reports, and reported values may not match reported sample dates. These differences result from the following: (1) the computer program that creates the analytical results tables was revised beginning second quarter 1992 to present the highest value for analytes with more than one result (previously, the program presented the first value encountered in the database); (2) a new computer program, which rounds numbers differently from the former computer program, was first used during third quarter 1992; and (3) some reanalyses may have been performed by the laboratories after the quarterly reports had gone to press. The sample dates in the tables are the dates when the field data were collected. These dates may differ from the dates of the laboratory analyses if the highest results were obtained for samples collected on different dates.

First Quarter 1992:

- Page 1, paragraph 1, line 8; page 3, paragraph 3, line 8: The December 1990 Part B PostClosure Care Permit Application is no longer the active document. This statement should identify the revised permit application submitted March 13, 1992.

- Page 1, paragraph 2, line 7: The statement referring to Administrative Consent Order 85-70-SW should be corrected to indicate that the first, second, and third quarters are submitted to SCDHEC 90 days after the end of each quarter as required by Administrative Consent Order 85-70-SW (as amended in 1988). The fourth quarter report, which includes the annual report, is submitted to SCDHEC 90 days after the end of the quarter as negotiated by SCDHEC with EPA.

- Page 8, paragraph 1, line 1: The well identified as HSB 85D should be identified as HSB 85A.

- Page 8, paragraph 1, line 4: The well identified as HSB 69A should be identified as HSB 69.

- Page 9, Water Levels, paragraph 1, line 2: "Aquifer Zones IIB $_{2}$ (Water Table)" should read "Aquifer Zone IIB $_{2}$ (Water Table)."

- Page E-66: The time series plot is incorrectly labeled as HSB 69A. The correct label is HSB 69 . 


\section{Second Quarter 1992:}

- Page 11, Upgradient Versus Downgradient Results, paragraph 1, line 2: The statement "Wells HSB 65, 66, and 85C are designated upgradient wells..." should read "Wells HSB 65C, 66, and 85C are designated upgradient wells...." The discussion of upgradient versus downgradient results should be revised accordingly.

- Page D-11: The second result for well HSB143C (23 $\mu \mathrm{g} / \mathrm{L})$ is for trichloroethylene, not tetrachloroethylene.

\section{Third Quarter 1992:}

- Prior to third quarter 1992, the results for certain analyses for nitrate-nitrite as nitrogen were reported incorrectly by the General Engineering laboratory as nitrate as nitrogen results. The analyses in the results tables for reports beginning third quarter 1992 are reported correctly (i.e., nitrate-nitrite results have been distinguished from true nitrate results).

- Page D-65, Well HSB 84D: The result for cobalt-60 of $5.4 \mathrm{E}+02 \pm 1.3 \mathrm{E}+02 \mathrm{pCi} / \mathrm{L}$ is incorrect. The correct result is $5.4 \mathrm{E}+01 \pm 1.3 \mathrm{E}+01 \mathrm{pCi} / \mathrm{L}$. The corrected result does not exceed the standard for cobalt-60 presented in Appendix A.

- Page D-79, Well HSB 86D: The result for cobalt-60 of $7.8 \mathrm{E}+02 \pm 1.5 \mathrm{E}+02 \mathrm{pCi} / \mathrm{L}$ is incorrect. The correct result is $7.8 \mathrm{E}+01 \pm 1.5 \mathrm{E}+01 \mathrm{pCi} / \mathrm{L}$. The corrected result does not exceed the standard for cobalt-60 presented in Appendix A.

- Page D-106, Well HSB108D: The result for cobalt-60 of $1.4 \mathrm{E}+02 \pm 1.4 \mathrm{E}+03 \mathrm{pCi} / \mathrm{L}$ is incorrect. The correct result is $1.4 \mathrm{E}+02 \pm 2.1 \mathrm{E}+01 \mathrm{pCi} / \mathrm{L}$.

- Page D-108, Well HSB109D: The result for cobalt-60 of $2.6 \mathrm{E}+02 \pm 5.1 \mathrm{E}+03 \mathrm{pCi} / \mathrm{L}$ is incorrect. The correct result is $2.6 \mathrm{E}+01 \pm 1.4 \mathrm{E}+01 \mathrm{pCi} / \mathrm{L}$. The corrected result does not exceed the standard for cobalt-60 presented in Appendix A.

- Page D-109, Well HSB109D: The result for niobium-95 should be deleted.

- Page D-121, Well HSB112E: The result for cobalt-60 of $1.8 \mathrm{E}+02 \pm 8.3 \mathrm{E}+01 \mathrm{pCi} / \mathrm{L}$ is not correct. The correct result is $1.8 \mathrm{E}+01 \pm 8.3 \mathrm{E}+00 \mathrm{pCi} / \mathrm{L}$. The corrected result does not exceed the standard for cobalt-60 presented in Appendix A. 


\section{References Cited}

Aadland, R. K., and H. W. Bledsoe, 1990. Classification of Hydrostratigraphic Units at the Savannah River Site, South Carolina, WSRC-RP-90-987. Westinghouse Savannah River Company, Savannah River Site, Aiken, SC.

GeoTrans, Inc., 1992. Groundwater Flow Model for the General Separations Area, Savannah River Site. Prepared for Westinghouse Savannah River Company, Environmental Restoration Department, Savannah River Site, Aiken, SC.

Geraghty \& Miller, Inc., 1990. Evaluation of Integrated Waste Facility Closure Capping on Ground-Water Flow and Solute Transport in General Separations Area, Savannah River Site: Flow Model and Particle-Tracking Analysis, Final Report. Prepared by Geraghty \& Miller Modeling Group for Westinghouse Savannah River Company, Waste Management Technology, Savannah River Site, Aiken, SC.

Heffner, J. D., and Exploration Resources, Inc., 1991. Technical Summary of Groundwater Quality Protection Program at the Savannah River Site (1952-1986), Volume I-Site Geohydrology and Waste Sites, DPSP-88-1002. Westinghouse Savannah River Company, Aiken, SC.

Jaegge, W. J., N. L. Kolb, B. B. Looney, I. W. Marine, O. A. Towler, and J. R. Cook, 1987. Radioactive Waste Burial Grounds, DPST-85-694. Savannah River Laboratory, E. I. du Pont de Nemours \& Company, Aiken, SC.

SCDHEC (South Carolina Department of Health and Environmental Control), 1990. South Carolina Hazardous Waste Management Regulations; R.61-79.124, .260 through .266, .268 , and .270; November 1990. Columbia, SC.

SCDHEC (South Carolina Department of Health and Environmental Control), 1992. Hazardous Waste Permit SC1-890-008-989. Office of Environmental Quality Control, Bureau of Solid and Hazardous Waste Management, Columbia, SC.

WSRC (Westinghouse Savannah River Company), 1990. Application for a Hazardous Waste Part B Post-Closure Care Permit. Volume IV. F-Area Hazardous Waste Management Facility. Revision 2, December 3, 1990. Savannah River Site, Aiken, SC.

WSRC (Westinghouse Savannah River Company), 1992. Hydrogeologic Data Collection Procedures and Specifications: Sampling Groundwater Monitoring Wells, Manual 3Q5, Chapter 14, Revision 0. Environmental Protection Department, Environmental Monitoring Section, Savannah River Site, Aiken, SC. 
WSRC-TR-93-059 
WSRC-TR-93-059

Appendix A - Final Primary Drinking Water Standards 
Final Primary Drinking Water Standards

\begin{tabular}{|c|c|c|c|c|}
\hline Analyte & Unit & Level & Status & Reference \\
\hline Arsenic & $\mu g / L$ & 50 & Final & CFR, 1991 \\
\hline Barium & $\mu g / L$ & 2,000 & Final & CFR, 1991 \\
\hline Benzene & $\mu g / L$ & 5 & Final & CFR, 1991 \\
\hline Bromodichloromethane & $\mu g / L$ & $100^{a}$ & Final & CFR, 1991 \\
\hline Bromoform & $\mu \mathrm{g} / \mathrm{L}$ & $100^{a}$ & Final & CFR, 1991 \\
\hline Cadmium & $\mu g / L$ & 5 & Final & CFR, 1991 \\
\hline Carbon tetrachloride & $\mu g / L$ & 5 & Final & CFR, 1991 \\
\hline Chlordane & $\mu \mathrm{g} / \mathrm{L}$ & 2 & Final & CFR, 1991 \\
\hline Chloroethene (Vinyl chloride) & $\mu g / L$ & 2 & Final & CFR, 1991 \\
\hline Chloroform & $\mu g / L$ & $100^{a}$ & Final & CFR, 1991 \\
\hline Chromium & $\mu g / L$ & 100 & Final & CFR, 1991 \\
\hline Copper & $\mu g / L$ & 1,300 & Final & CFR, 1991 \\
\hline Dibromochloromethane & $\mu g / L$ & $100^{a}$ & Final & CFR, 1991 \\
\hline Dibromochloropropane & $\mu \mathrm{g} / \mathrm{L}$ & 0.2 & Final & CFR, 1991 \\
\hline 1,2-Dichlorobenzene & $\mu g / L$ & 600 & Final & CFR, 1991 \\
\hline 1.4-Dichlorobenzene & $\mu g / L$ & 75 & Final & CFR, 1991 \\
\hline 1.2-Dichloroethane & $\mu g / L$ & 5 & Final & CFR, 1991 \\
\hline 1.1-Dichloroethylene & $\mu g / L$ & 7 & Final & CFR, 1991 \\
\hline cis-1,2-Dichloroethylene & $\mu g / L$ & 70 & Final & CFR, 1991 \\
\hline trans-1,2-Dichloroethylene & $\mu g / L$ & 100 & Final & CFR, 1991 \\
\hline 2,4-Dichlorophenoxyacetic acid & $\mu g / L$ & 70 & Final & CFR, 1991 \\
\hline 1,2-Dichloropropane & $\mu g / L$ & 5 & Fin?al & CFR, 1991 \\
\hline Endrin & $\mu g / L$ & 0.2 & Final & CFR, 1991 \\
\hline Ethylbenzene & $\mu g / L$ & 700 & Final & CFR, 1991 \\
\hline Fluoride & $\mu g / L$ & 4,000 & Final & CFR, 1991 \\
\hline Gross alpha ${ }^{b}$ & $\mathrm{pCi} / \mathrm{L}$ & $1.5 E+01$ & Final & CFR, 1991 \\
\hline Heptachlor & $\mu g / L$ & 0.4 & Final & CFR, 1991 \\
\hline Heptachlor epoxide & $\mu g / L$ & 0.2 & Final & CFR, 1991 \\
\hline Lead & $\mu g / L$ & 50 & Final & SCDHEC, 1981 \\
\hline Lindane & $\mu g / L$ & 0.2 & Final & CFR, 1991 \\
\hline Mercury & $\mu g / L$ & 2 & Final & CFR, 1991 \\
\hline Methoxychlor & $\mu g / L$ & 40 & Final & CFR, 1991 \\
\hline Nitrate as nitrogen & $\mu g / L$ & 10,000 & Final & CFR, 1991 \\
\hline Nitrate-nitrite as nitrogen & $\mu g / L$ & 10,000 & Final & CFR, 1991 \\
\hline Nitrite as nitrogen & $\mu g / L$ & 1,000 & Final & CFR, 1991 \\
\hline Nonvolatile beta ${ }^{c}$ & $\mathrm{pCi} / \mathrm{L}$ & $5 E+01$ & Final & EPA, 1977 \\
\hline $\mathrm{PCBs}^{d}$ & $\mu g / L$ & 0.5 & Final & CFR, 1991 \\
\hline Pentachlorophenol & $\mu g / L$ & 1 & Final & CFR, 1991 \\
\hline Selenium & $\mu g / L$ & 50 & Final & CFR, 1991 \\
\hline Strontium- $89 / 90^{\circ}$ & $\mathrm{pCi} / \mathrm{L}$ & $8 E+00$ & Final & CFR, 1991 \\
\hline Strontium-90 & $\mathrm{pCi} / \mathrm{L}$ & $8 E+00$ & Final & CFR, 1991 \\
\hline Styrene & $\mu g / L$ & 100 & Final & CFR, 1991 \\
\hline Tetrachloroethylene & $\mu g / L$ & 5 & Final & CFR, 1991 \\
\hline Toluene & $\mu q / L$ & 1.000 & Final & CFR, 1991 \\
\hline Total radium (Radium-226 and -228 ) & $\mathrm{pCi} / \mathrm{L}$ & $5 E+00$ & Final & CFR, 1991 \\
\hline Total trihalomethanes & $\mu g / L$ & 100 & Final & CFR, 1991 \\
\hline Toxaphene & $\mu g / L$ & 3 & Final & CFR, 1991 \\
\hline $2,4,5-T P$ (Silvex) & $\mu g / L$ & 50 & Final & CFR, 1991 \\
\hline 1,1,1-Trichloroethane & $\mu g / L$ & 200 & Final & CFR, 1991 \\
\hline
\end{tabular}


Analyte

Trichloroethylene

Tritium

Xylenes

$\begin{array}{llll}\text { Unit } & \text { Level } & \text { Status } & \text { Reference } \\ \mu \mathrm{g} / \mathrm{L} & 5 & \text { Final } & \text { CFR, 1991 } \\ \mathrm{pCi} / \mathrm{mL} & 2 \mathrm{E}+01 & \text { Final } & \text { CFR, 1991 } \\ \mu \mathrm{g} / \mathrm{L} & 10,000 & \text { Final } & \text { CFR, 1991 }\end{array}$

Note: The drinking water standard for lead was changed to the South Carolina Primary Drinking Water Standard of $50 \mu \mathrm{g} / \mathrm{L}$ fourth quarter 1992.

a This value is the drinking water standard for total trihalomethanes (the sum of bromoform, bromodichloromethane, chloroform, and dibromochloromethane).

b The standard given is for gross alpha including radium-226 but excluding radon and uranium.

c This is the screening level above which providers of public drinking water should perform analyses for specific man-made radionuclides. The standard for the total dose equivalent from all such radionuclides is 4 mrem per year.

d Analyses were conducted in 1992 for the following: РCB 1016, РCB 1221, РCB 1232, РCB 1242, PCB 1248, PCB 1254, and PCB 1260.

- For double radionuclide analyses where each separate radionuclide has its own standard, the more stringent standard is used.

\section{References}

CFR (Code of Federal Regulations), 1991. National Primary Drinking Water Regulations, 40 CFR, Part 141, pp. 578-715. Washington, DC.

EPA (U.S. Environmental Protection Agency), 1977. National Interim Primary Drinking Water Regulations, EPA-570/9-76-003. Washington, DC.

SCDHEC (South Carolina Department of Health and Environmental Control), 1981. State Primary Drinking Water Regulations, R.61-58.5. Columbia, SC. 
WSRC-TR-93.059 
WSRC-TR-93-059

\section{Appendix B - Flagging Criteria}




\section{Flagging Criteria}

Beginning in 1991, the Savannah River Site Environmental Protection Department/ Environmental Monitoring Section modified its guidelines for flagging constituents in the Groundwater Monitoring Program. These flagging criteria are as follows:

- Flag 2 criteria for constituents equal the Safe Drinking Water Act (SDWA) final Primary Drinking Water Standard (PDWS), the SDWA proposed PDWS, or the SDWA Secondary Drinking Water Standard (SDWS). If a constituent does not have a drinking water standard, the Flag 2 criterion equals 10 times the method detection limit (MDL) calculated as the 90 th percentile detection limit obtained recently by one of the primary analytical laboratories.

- Flag 1 criteria for constituents equal one-half of the final PDWS, one-half the proposed PDWS, or one-half the SDWS. If a constituent does not have an drinking water standard, the Flag 1 criterion equals 5 times the MDL calculated as the 90th percentile detection limit obtained recently by one of the primary analytical laboratories.

- Flag 0 criteria are assigned to constituent levels below Flag 1 criteria, constituent levels below the sample detection limits, or constituents having no flagging criteria.

The following parameters are not assigned flagging criteria: alkalinity, calcium, carbonate, color, corrosivity, magnesium, odor, potassium, Eh, silica, sodium, total dissolved solids, total phosphorus, total phosphates (as P), and turbidity. In addition, common laboratory contaminants and cleaners including phthalates, methylene chloride, ketones, and toluene are not assigned flagging criteria.

Analyte
Acenaphthene
Acenaphthylene
Acetone
Acetonitrile (Methyl cyanide)
Acetophenone
2-Acetylaminofluorene
Acrolein
Acrylonitrile
Aldrin
Alkalinity (as $\mathrm{CaCO}_{3}$ )
Allyl chloride
Aluminum
Americium-241
Americium-243
4 -Aminobiphenyl
Ammonia
Ammonia nitrogen
Aniline
Anthracene
Antimony
Antimony-125
Aramite

H-Area Seepage Basins

\begin{tabular}{|c|c|c|c|}
\hline Unit & Flag 1 & Flag 2 & Source \\
\hline$\mu g / L$ & 50 & 100 & EPA Method 8270 \\
\hline$\mu g / L$ & 50 & 100 & EPA Method 8270 \\
\hline$\mu g / L$ & 50 & 100 & EPA Method 8240 \\
\hline$\mu g / L$ & 500 & 1.000 & EPA Method 8240 \\
\hline$\mu g / L$ & 50 & 100 & EPA Method 8270 \\
\hline$\mu g / L$ & 50 & 100 & EPA Method 8270 \\
\hline$\mu g / L$ & 100 & 200 & EPA Method 8240 \\
\hline$\mu g / L$ & 100 & 200 & EPA Method 8240 \\
\hline$\mu g / L$ & 2.5 & 5 & EPA Method 8080 \\
\hline & No flag & No flag & Set by EPD/EMS \\
\hline$\mu \mathrm{g} / \mathrm{L}$ & 250 & 500 & EPA Method 8240 \\
\hline$\mu g / L$ & 25 & 50 & Secondary DWS (CFR, 1991b) \\
\hline $\mathrm{pCi} / \mathrm{L}$ & $3.17 E+00$ & $6.34 E+00$ & Proposed DWS (EPA, 1991) \\
\hline $\mathrm{pCi} / \mathrm{L}$ & $3.19 E+00$ & $6.37 E+00$ & Proposed DWS (EPA, 1991) \\
\hline$\mu g / L$ & 50 & 100 & EPA Method 8270 \\
\hline$\mu g / L$ & 500 & 1,000 & APHA Method 417B \\
\hline$\mu g / L$ & 50 & 100 & EPA Method 350.1 \\
\hline$\mu g / L$ & 50 & 100 & EPA Method 8270 \\
\hline$\mu g / L$ & 50 & 100 & EPA Method 8270 \\
\hline$\mu g / L$ & 2.5 & 5 & Proposed DWS (EPA, 1990) \\
\hline $\mathrm{pCi} / \mathrm{L}$ & $1.5 E+02$ & $3 E+02$ & Final DWS (EPA, 1977) \\
\hline$\mu g / L$ & 50 & 100 & EPA Method 8270 \\
\hline
\end{tabular}




\begin{tabular}{|c|c|c|c|c|}
\hline Analyte & $\underline{\text { Unit }}$ & Flag 1 & Flag 2 & Source \\
\hline Arsenic & $\mu g / L$ & 25 & 50 & Final DWS (CFR, 1991a) \\
\hline Barium & $\mu g / L$ & 1,000 & 2,000 & Final DWS (CFR, 1991a) \\
\hline Barium-140 & $\mathrm{pCi} / \mathrm{L}$ & $4.5 E+01$ & $9 E+01$ & Final DWS (EPA, 1977) \\
\hline Benzene & $\mu g / L$ & 2.5 & 5 & Final DWS (CFR, 1991a) \\
\hline alpha-Benzene hexachloride & $\mu g / L$ & 2.5 & 5 & EPA Method 8080 \\
\hline beta-Benzene hexachloride & $\mu g / L$ & 2.5 & 5 & EPA Method 8080 \\
\hline delta-Benzene hexachloride & $\mu g / L$ & 2.5 & 5 & EPA Method 8080 \\
\hline Benzidine & $\mu g / L$ & 250 & 500 & EPA Method 8270 \\
\hline Benzolalanthracene & $\mu g / L$ & 0.05 & 0.1 & Proposed DWS (EPA, 1990 ) \\
\hline Benzolblfluoranthene & $\mu g / L$ & 0.1 & 0.2 & Proposed DWS (EPA, 1990) \\
\hline Benzolk]fluoranthene & $\mu g / L$ & 0.1 & 0.2 & Proposed DWS (EPA, 1990) \\
\hline Benzolg,h,i]perylene & $\mu g / L$ & 50 & 100 & EPA Method 8270 \\
\hline Benzolalpyrene & $\mu g / L$ & 0.1 & 0.2 & Proposed DWS (EPA, 1990) \\
\hline Benzoic acid & $\mu g / L$ & 250 & 500 & EPA Method 8270 \\
\hline 1,4-Benzoquinone & $\mu g / L$ & 50 & 100 & EPA Method 8270 \\
\hline Benzyl alcohol & $\mu g / L$ & 100 & 200 & EPA Method 8270 \\
\hline Beryllium & $\mu g / L$ & 0.5 & 1 & Proposed DWS (EPA, 1990) \\
\hline Beryllium-7 & $\mathrm{pCi} / \mathrm{L}$ & $3 E+03$ & $6 E+03$ & Final DWS (EPA, 1977) \\
\hline Bis(2-chloroethoxy) methane & $\mu g / L$ & 50 & 100 & EPA Method 8270 \\
\hline Bis (2-chloroethyl) ether & $\mu g / L$ & 50 & 100 & EPA Method 8270 \\
\hline Bis (2-chloroisopropyl) ether & $\mu g / L$ & 50 & 100 & EPA Method 8270 \\
\hline Bis(chloromethyl) ether & $\mu g / L$ & 50 & 100 & EPA Method 8270 \\
\hline Bis(chloromethyl-ethyl) ether & $\mu g / L$ & 50 & 100 & EPA Method 8270 \\
\hline Bis(2-ethylhexyl) phthalate & & No flag & No flag & Set by EPD/EMS \\
\hline Bromide & $\mu g / L$ & 5,000 & 10,000 & EPA Method 300.0 \\
\hline Bromodichloromethane & $\mu g / L$ & 50 & 100 & Final DWS (CFR, 1991a) \\
\hline Bromoform & $\mu g / L$ & 50 & 100 & Final DWS (CFR, 1991a) \\
\hline Bromomethane (Methyl bromide) & $\mu \mathrm{g} / \mathrm{L}$ & 5 & 10 & EPA Method 8240 \\
\hline 4-Bromophenyl phenyl ether & $\mu g / L$ & 50 & 100 & EPA Method 8270 \\
\hline 2-sec-Butyl-4,6-dinitrophenol & $\mu g / L$ & 3.5 & 7 & Proposed DWS (EPA, 1990) \\
\hline Butyibenzyl phthalate & & No flag & No flag & Set by EPD/EMS \\
\hline Cadmium & $\mu g / L$ & 2.5 & 5 & Final DWS (CFR, 1991a) \\
\hline Calcium & & No flag & No flag & Set by EPD/EMS \\
\hline Carbon disulfide & $\mu g / L$ & 5 & 10 & EPA Method 8240 \\
\hline Carbon tetrachloride & $\mu g / L$ & 2.5 & 5 & Final DWS (CFR, 1991a) \\
\hline Carbon-14 & $\mathrm{pCi} / \mathrm{L}$ & $1 E+03$ & $2 E+03$ & Final DWS (EPA, 1977) \\
\hline Carbonate & $\mu g / L$ & 500 & 1,000 & EPA Method 310.1 \\
\hline Cerium-141 & $\mathrm{pCi} / \mathrm{L}$ & $1.5 E+02$ & $3 E+02$ & Final DWS (EPA, 1977) \\
\hline Cerium-144 & $\mathrm{pCi} / \mathrm{L}$ & $1.31 E+02$ & $2.61 E+02$ & Proposed DWS (EPA, 1991) \\
\hline Cesium-134 & $\mathrm{pCi} / \mathrm{L}$ & $4.07 E+01$ & $8.13 E+01$ & Proposed DWS (EPA, 1991 ) \\
\hline Cesium-137 & $\mathrm{pCi} / \mathrm{L}$ & $1 E+02$ & $2 E+02$ & Final DWS (EPA, 1977) \\
\hline Chlordane & $\mu g / L$ & 1 & 2 & Final DWS (CFR, 1991a) \\
\hline Chloride & $\mu g / L$ & 125,000 & 250,000 & Secondary DWS (CFR, 1991b) \\
\hline 4-Chloroaniline & $\mu g / L$ & 50 & 100 & EPA Method 8270 \\
\hline Chlorobenzene & $\mu \mathrm{g} / \mathrm{L}$ & 5 & 10 & EPA Method 8240 \\
\hline Chlorobenzilate & $\mu \mathrm{g} / \mathrm{L}$ & 50 & 100 & EPA Method 8270 \\
\hline Chloroethane & $\mu g / L$ & 5 & 10 & EPA Method 8240 \\
\hline Chloroethene (Vinyl chloride) & $\mu g / L$ & 1 & 2 & Final DWS (CFR, 1991a) \\
\hline Chloroethyl vinyl ether & $\mu g / L$ & 5 & 10 & EPA Method 8240 \\
\hline 2-Chloroethyl vinyl ether & $\mu g / L$ & 5 & 10 & EPA Method 8240 \\
\hline Chloroform & $\mu g / L$ & 50 & 100 & Final DWS (CFR, 1991a) \\
\hline para-Chloro-meta-cresol & $\mu g / L$ & 50 & 100 & EPA Method 8270 \\
\hline Chloromethane (Methyl chloride) & $\mu g / L$ & 5 & 10 & EPA Method 8240 \\
\hline 2.Chloronaphthalene & $\mu g / L$ & 50 & 100 & EPA Method 8240 \\
\hline 2-Chlorophenol & $\mu g / L$ & 50 & 100 & EPA Method 8270 \\
\hline
\end{tabular}




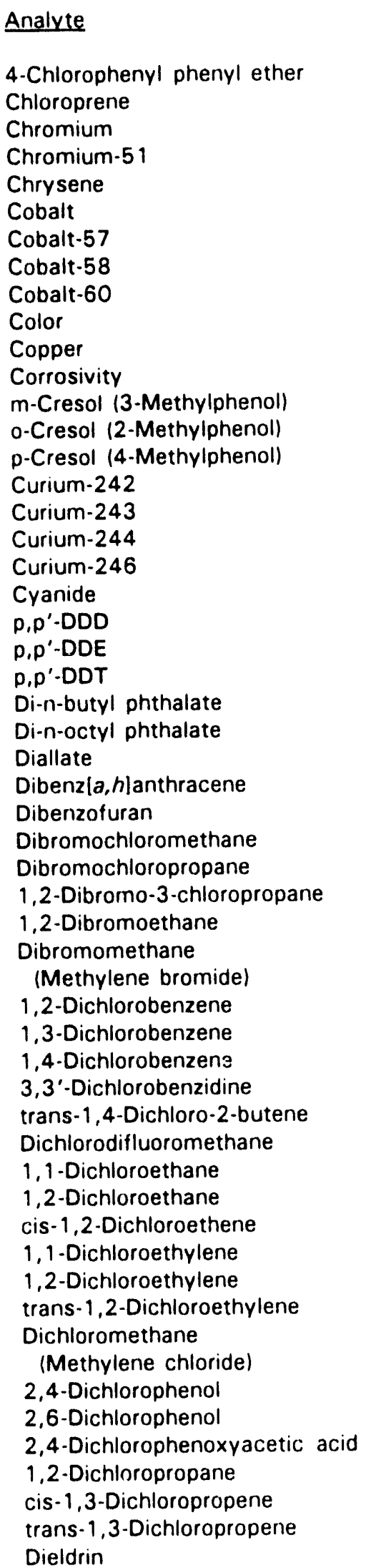

\begin{tabular}{|c|c|c|}
\hline Unit & Flag 1 & Flag 2 \\
\hline$\mu g / L$ & 50 & 100 \\
\hline$\mu g / L$ & 1,000 & 2,000 \\
\hline$\mu \mathrm{g} / \mathrm{L}$ & 50 & 100 \\
\hline pCi/L & $3 E+03$ & $6 E+03$ \\
\hline$\mu g / L$ & 0.1 & 0.2 \\
\hline$\mu g / L$ & 20 & 40 \\
\hline $\mathrm{pCi} / \mathrm{L}$ & $5 E+02$ & $1 E+03$ \\
\hline $\mathrm{pCi} / \mathrm{L}$ & $4.5 E+03$ & $9 E+03$ \\
\hline $\mathrm{pCi} / \mathrm{L}$ & $\begin{array}{l}5 E+01 \\
\text { No flag }\end{array}$ & $\begin{array}{l}1 E+02 \\
\text { No flag }\end{array}$ \\
\hline$\mu g / L$ & $\begin{array}{l}650 \\
\text { No flag }\end{array}$ & $\begin{array}{l}1,300 \\
\text { No flag }\end{array}$ \\
\hline$\mu \mathrm{g} / \mathrm{L}$ & 50 & 100 \\
\hline$\mu g / L$ & 50 & 100 \\
\hline$\mu g / L$ & 50 & 100 \\
\hline $\mathrm{pCi} / \mathrm{L}$ & $6.65 E+01$ & $1.33 E+02$ \\
\hline $\mathrm{pCi} / \mathrm{L}$ & $4.15 E+00$ & $8.3 E+00$ \\
\hline $\mathrm{pCi} / \mathrm{L}$ & $4.92 E+00$ & $9.84 E+00$ \\
\hline $\mathrm{pCi} / \mathrm{L}$ & $3.14 E+00$ & $6.27 E+00$ \\
\hline$\mu g / L$ & 100 & 200 \\
\hline$\mu g / L$ & 2.5 & 5 \\
\hline$\mu g / L$ & 2.5 & 5 \\
\hline$\mu g / L$ & 2.5 & 5 \\
\hline & No flag & No flag \\
\hline & No flag & No flag \\
\hline$\mu g / L$ & 50 & 100 \\
\hline$\mu g / L$ & 0.15 & 0.3 \\
\hline$\mu g / L$ & 50 & 100 \\
\hline$\mu g / L$ & 50 & 100 \\
\hline$\mu g / L$ & 0.1 & 0.2 \\
\hline$\mu y / L$ & 250 & 500 \\
\hline$\mu g / L$ & 100 & 200 \\
\hline$\mu \mathrm{g} / \mathrm{L}$ & 5 & 10 \\
\hline$\mu g / L$ & 300 & 600 \\
\hline$\mu g / L$ & 50 & 100 \\
\hline$\mu \mathrm{g} / \mathrm{L}$ & 37.5 & 75 \\
\hline$\mu g / L$ & 50 & 100 \\
\hline$\mu g / L$ & 150 & 300 \\
\hline$\mu \mathrm{g} / \mathrm{L}$ & 5 & 10 \\
\hline$\mu \mathrm{g} / \mathrm{L}$ & 5 & 10 \\
\hline$\mu g / L$ & 2.5 & 5 \\
\hline$\mu g / L$ & 35 & 70 \\
\hline$\mu g / L$ & 3.5 & 7 \\
\hline$\mu g / L$ & 25 & 50 \\
\hline$\mu g / L$ & 50 & 100 \\
\hline & No flag & No flag \\
\hline$\mu g / L$ & 50 & 100 \\
\hline$\mu g / L$ & 50 & 100 \\
\hline$\mu \mathrm{g} / \mathrm{L}$ & 35 & 70 \\
\hline$\mu g / L$ & 2.5 & 5 \\
\hline$\mu \mathrm{g} / \mathrm{L}$ & 5 & 10 \\
\hline$\mu g / L$ & 5 & 10 \\
\hline$\mu g / L$ & 2.5 & 5 \\
\hline
\end{tabular}

\section{Source}

EPA Method 8270

EPA Method 8240

Final DWS (CFR, 1991a)

Final DWS (EPA, 1977)

Proposed DWS (EPA, 1990)

EPA Method 6010

Final DWS (EPA, 1977)

Final DWS (EPA, 1977)

Final DWS (EPA, 1977)

Set by EPD/EMS

Final DWS (CFR, 1991a)

Set by EPD/EMS

EPA Method 8270

EPA Method 8270

EPA Method 8270

Proposed DWS (EPA, 1991)

Proposed DWS (EPA, 1991)

Proposed DWS (EPA, 1991)

Proposed DWS (EPA, 1991)

Proposed DWS (EPA, 1990)

EPA Method 8080

EPA Method 8080

EPA Method 8080

Set by EPD/EMS

Set by EPD/EMS

EPA Method 8270

Proposed DWS (EPA, 1990)

EPA Method 8270

Final DWS (CFR, 1991a)

Final DWS (CFR, 1991a)

EPA Method 8240

EPA Method 8240

EPA Method 8240

Final DWS (CFR, 1991a)

EPA Method 8270

Final DWS (CFR, 1991a)

EPA Method 8270

EPA Method 8240

EPA Method 8240

EPA Method 8240

Final DWS (CFR, 1991a)

Final DWS (CFR, 1991a)

Final DWS (CFR, 1991a)

EPA Method 8240

Final DWS (CFR, 1991a)

Set by EPD/EMS

EPA Method 8270

EPA Method 8270

Final DWS (CFR, 1991a)

Final DWS (CFR, 1991a)

EPA Method 8240

EPA Method 8240

EPA Method 8080 
Analyte

Diethyl phthalate

Dimethoate

p-Dimethylaminoazobenzene

p-\{Dimethylamino)ethylbenzene

7,12-Dimethylbenzla]anthracene

3,3'-Dimethylbenzidine

a,a-Dimethylphenethylamine

2,4-Dimethyl phenol

Dimethyl phthalate

1,3-Dinitrobenzene

4,6-Dinitro-ortho-cresol

2,4-Dinitrophenol

2,4-Dinitrotoluene

2,6-Dinitrotoluene

1,4-Dioxane

Diphenylamine

1,2-Diphenylhydrazine

Dissolved organic carbon

Disulfoton

Eh

alpha-Endosulfan

beta-Endosulfan

Endosulfan I

Endosulfan II

Endosulfan sulfate

Endrin

Endrin aldehyde

Endrin ketone

Ethyl methacrylate

Ethyl methanesulfonate

Ethylbenzene

Europium-154

Europium-155

Famphur

Fluoranthene

Fluorene

Fluoride

Gross alpha

Heptachlor

Heptachlor epoxide

Heptachlorodibenzo-p-dioxin isomers

$1,2,3,4,6,7,8$-Heptachlorodibenzop-dioxin

Heptachlorodibenzo- $\mathrm{p}$-furan

isomers

$1,2,3,4,6,7,8$-Heptachlorodibenzop-furan

Hexachlorobenzene

Hexachlorobutadiene

Hexachlorocyclopentadiene

Hexachlorodibenzu-p-dioxin isomers

$1,2,3,4,7,8$-Hexachlorodibenzop-dioxin

Hexachlorodibenzo-p-furan isomers
Unit

$\mu g / L$

$\mu g / L$

$\mu g / L$

$\mu \mathrm{g} / \mathrm{L}$

$\mu \mathrm{g} / \mathrm{L}$

$\mu g / L$

$\mu \mathrm{g} / \mathrm{L}$

$\mu \mathrm{g} / \mathrm{L}$

$\mu \mathrm{g} / \mathrm{L}$

$\mu \mathrm{g} / \mathrm{L}$

$\mu \mathrm{g} / \mathrm{L}$

$\mu g / L$

$\mu \mathrm{g} / \mathrm{L}$

$\mu g / L$

$\mu \mathrm{g} / \mathrm{L}$

$\mu \mathrm{g} / \mathrm{L}$

$\mu \mathrm{g} / \mathrm{L}$

$\mu \mathrm{g} / \mathrm{L}$

$\mu g / L$

$\mu g / L$

$\mu g / L$

$\mu \mathrm{g} / \mathrm{L}$

$\mu \mathrm{g} / \mathrm{L}$

$\mu \mathrm{g} / \mathrm{L}$

$\mu g / L$

$\mu \mathrm{g} / \mathrm{L}$

$\mu \mathrm{g} / \mathrm{L}$

pCi/L

pCi/L.

$\mu g / h$

$\mu g / l$

$\mu \mathrm{g} / \mathrm{L}$

$\mu \mathrm{g} / \mathrm{L}$

$\mathrm{pCi} / \mathrm{L}$

$\mu g / L$

$\mu g / L$

$\mu g / L$

\section{Flag 1}

No flag

50

50

50

50

50

50

50

No flag

50

250

250

50

50

50

50

50

5,000

50

No flag

50

50

2.5

2.5

2.5

0.1

2.5

No flag

50

50

350

$1 E+02$

$3 E+02$

50

50

50

2,000

$7.5 E+00$

0.2

0.1

0.00325

$\mu \mathrm{g} / \mathrm{L}$

0.00325

$\mu g / L$

0.00225

$\mu \mathrm{g} / \mathrm{L}$

$\mu g / L$

$\mu \mathrm{g} / \mathrm{L}$

$\mu g / L$

$\mu \mathrm{g} / \mathrm{L}$

$\mu \mathrm{g} / \mathrm{L}$

$\mu g / L$
Fligg $2 \quad$ Source

No flag Set by EPD/EMS

100 EPA Method 8270

100 EPA Method 8270

$100 \quad$ EPA Methoif 8270

100 EPA Methoid 8270

$100 \quad$ EPA Methoid 8270

$100 \quad$ EP,A Methoci 8270

100 EPA Method 8270

No flag Set by EPD/EMS

$100 \quad$ EPA Method $\Sigma 270$

$500 \quad$ EPA Method 8270

$500 \quad$ EPA Method 8270

100 EPA Method 8270

$100 \quad$ EPA Method 8270

100 EPA Method 8270

100 EPA Method 8270

$100 \quad$ EPA Method 8270

10,000 EPA Method SŪ60

$100 \quad$ EFA Method 8270

No flag Set by EPD/EMS

$100 \quad$ EPA Method 8270

100 EPA Method 8270

5 EPA Method 8080

5 EPA Method 8080

5 EPA Method 8080

0.2 Final DWS (CFR, 1991a)

5 EPA Method 8080

No flag Set by EPD/EMS

$100 \quad$ EPA Method 8270

100 EPA Method 8270

700

$2 \mathrm{E}+02$

Final DWS (CFR, 1991a)

Final DWS (EPA, 1977)

Final DWS (EPA, 1977)

EPA Method 8270

EPA Method 8270

EPA Method 8270

100

4,000

$1.5 E+01$

0.4

0.2

Final DWS (CFR, 1991a)

Final DWS (CFR, 1991a)

Final DWS (Cl:R, 1991a)

Final DWS (CFR, 1991a)

0.0065

EPA Method 8280

0.0065

EPA Method 8280

0.0045

EPA Method 8280

0.0045

1

100

50

0.0045

$0.00 \%$

0.004
EPA Method 8280

Proposed DWS (EPA, 1990)

EPA Method 8270

Proposed DWS (EPA, 1990)

EPA Method 8280

EPA Method 8280

EPA Method 8280 
Analyte

$1,2,3,4,7,8$-Hexachlorodibenzop-furan

Hexachloroethane

Hexachlorophene

Hexachloropropene

2-Hexanone

Indeno[1,2,3-c, 0]pyrene

lodine

lodine-129

lodine-131

lodomethane (Methyl iodide)

Iron

Iron-55

Iron-59

Isobutyl alcohol

Isodrin

Isophorone

Isosafrole

Kepone

Lanthanum $=140$

Lead

Lindane

Lithium

Magnesium

Manganese

Manganese-54

Mercury

Methacrylonitrile

Methapyrilene

Methoxychlor

3-Metnylcholanthrene

2-Methyl-4,6-dinitrophenol

Methyl ethyl ketone

Methyl isobutyl ketone

Methyl methacrylate

Methyl methanesulfonate

2-Methyinaphthalene

Molybdenurn

Naphthalene

1,4-Naphthoquinone

1-Naphthylamine

2-Naphthylamine

Neptunium-237

Nickel

Nickel-59

Nickel-63

Niobium-95

Nitrate as nitrogen

Nitrate-nitrite as nitrogen

Nitrite as nitrogen

2-Nitroaniline

3-Nitroaniline

4-Nitroaniline

Nitrobenzene

Nitrogen by Kjeldahl method
Unit

Flag 1

Flag 2

Source

$\mu$ $\mu \mathrm{g} / \mathrm{L}$

$\mu \mathrm{g} / \mathrm{L}$

$\mathrm{pCi} / \mathrm{L}$

pCi/L

$\mu \mathrm{g} / \mathrm{L}$

$\mu \mathrm{g} / \mathrm{L}$

$\mu \mathrm{g} / \mathrm{L}$

$\mu \mathrm{g} / \mathrm{L}$

$\mu g / L$

pCi/L

$\mu \mathrm{g} / \mathrm{L}$

$\mu \mathrm{g} / \mathrm{L}$

$\mu g / L$

$\mu \mathrm{g} / \mathrm{L}$

$\mathrm{pCi} / \mathrm{L}$

$\mu \mathrm{g} / \mathrm{L}$

$\mu \mathrm{g} / \mathrm{L}$

$\mu g / L$

$\mu \mathrm{g} / \mathrm{L}$

$\mu g / L$

$\mu \mathrm{g} / \mathrm{L}$

$\mu \mathrm{g} / \mathrm{L}$

$\mu \mathrm{g} / \mathrm{L}$

$\mu \mathrm{g} / \mathrm{L}$

$\mu g / L$

$\mu g / L$

$\mu \mathrm{g} / \mathrm{L}$

$\mu \mathrm{g} / \mathrm{L}$

$\mu \mathrm{g} / \mathrm{L}$

$\mathrm{pCi} / \mathrm{L}$

$\mu \mathrm{g} / \mathrm{L}$

pCi/L

$\mathrm{pCi} / \mathrm{L}$

$\mathrm{pCi/L}$

$\mu \mathrm{g} / \mathrm{L}$

$\mu \mathrm{g} / \mathrm{L}$

$\mu g / L$

$\mu \mathrm{g} / \mathrm{L}$

$\mu \mathrm{g} / \mathrm{L}$

$\mu g / \mathrm{L}$

$\mu g / L$

$\mu \mathrm{g} / \mathrm{L}$

$\begin{array}{lll}0.002 & 0.004 & \text { EPA Method } 8280 \\ 50 & 100 & \text { EPA Method } 8270 \\ 250 & 500 & \text { EPA Method } 8270 \\ 50 & 100 & \text { EPA Method } 8270 \\ 100 & 200 & \text { EPA Method } 8240 \\ 50 & 100 & \text { EPA Method } 8270 \\ 500 & 1,000 & \text { EPA Method } 415\end{array}$

$5 E-01 \quad 1 E+00$

$1.5 E+00 \quad 3 E+00$

$75 \quad 150$

$150 \quad 300$

$1 E+03 \quad 2 E+03$

$1 E+02 \quad 2 E+02$

500

50

1,000

100

100

$\begin{array}{ll}50 & 100 \\ 50 & 100\end{array}$

$3 E+01 \quad 6 E+01$

$7.5 \quad 15$

$\begin{array}{ll}0.1 & 0.2\end{array}$

$25 \quad 50$

No flag No flag

25

$1.5 \mathrm{E}+02$

50

$3 E+02$

$\begin{array}{ll}1 & 2 \\ 250 & 500\end{array}$

250

50

20

50

250

No flag

No flag

100

40

100

500

No flag

No flag

100

100

100

500

100

100

100

100

$7.06 E+O C$

100

$3.53 E+00$

50

$\begin{array}{ll}1.5 E+02 & 3 E+02 \\ 2.5 E+01 & 5 E+01\end{array}$

$1.5 \mathrm{E}+02 \quad 3 \mathrm{E}+02$

$5,000 \quad 10,000$

$5,000 \quad 10,000$

$500 \quad 1,000$

$50 \quad 100$

$50 \quad 100$

$50 \quad 100$

$50 \quad 100$

500

EPA Method 8270
Final DWS (EPA, 1977)

Final DWS (EPA, 1977)

EPA Method 8240

Secondary DWS (CFR, 1991b)

Final DWS (EPA, 1977)

Final DWS (EPA, 1977)

EPA Method 8240

EPA Method 8270

EPA Method 8270

EPA Method 8270

Final DWS (EPA, 1977)

Final DWS (CFR, 1991a)

Final DWS (CFR, 1991a)

EPA Method 6010

Set by EPD/EMS

Secondary DWS (CFR, 1991b)

Final DWS (EPA, 1977)

Final DWS (CFR, 1991a)

EPA Method 8240

EPA Method 8270

Final DWS (CFR, 1991a)

EPA Method 8270

EPA Method 8270

Set by EPD/EMS

Set by EPD/EMS

EPA Method 8270

EPA Method 8270

EPA Method 8270

EPA Method 6010

EPA Method 8270

EPA Method 8270

EPA Method 8270

EPA Method 8270

Proposed DWS (EPA, 1991)

Proposed DWS (EPA, 1990)

Final DWS (EPA, 1977)

Final DWS (EPA, 1977)

Final DWS (EPA, 1977)

Final DWS (CFR, 1991a)

Final DWS (CFR, 1991a)

Final DWS (CFR, 1991a)

EPA Method 8270

EPA Method 8270

EPA Method 8270

EPA Method 8270

EPA Method 351.2 


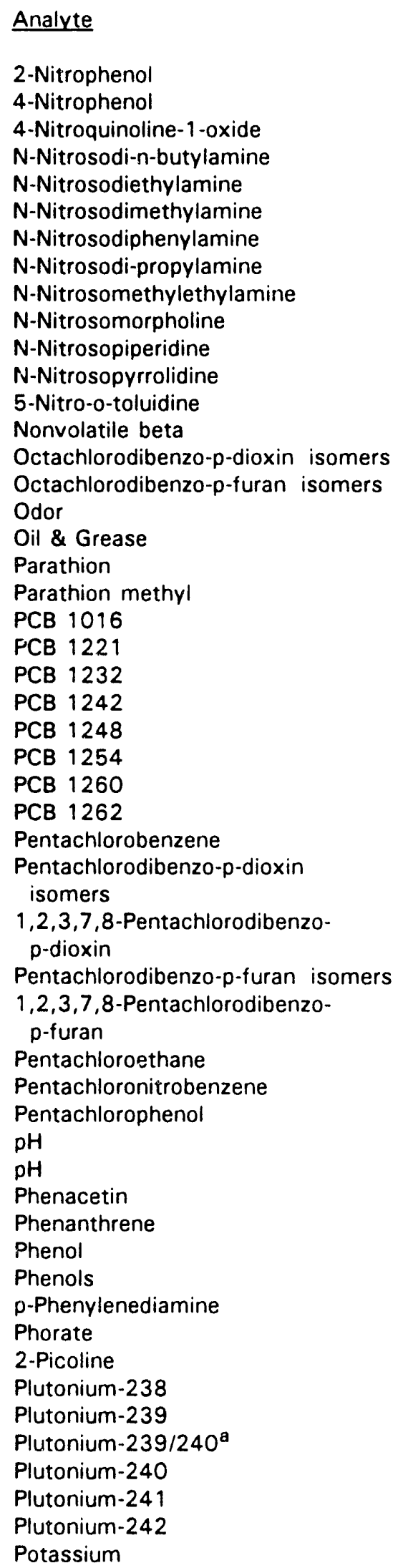

\begin{tabular}{|c|c|c|c|}
\hline$\underline{\text { Unit }}$ & Flag 1 & Flag 2 & Source \\
\hline$\mu g / L$ & 50 & 100 & EPA Method 8270 \\
\hline$\mu g / L$ & 50 & 100 & EPA Method 8270 \\
\hline$\mu g / L$ & 50 & 100 & EPA Method 8270 \\
\hline$\mu \mathrm{g} / \mathrm{L}$ & 50 & 100 & EPA Method 8270 \\
\hline$\mu \mathrm{g} / \mathrm{L}$ & 50 & 100 & EPA Method 8270 \\
\hline$\mu g / L$ & 50 & 100 & EPA Method 8270 \\
\hline$\mu \mathrm{g} / \mathrm{L}$ & 50 & 100 & EPA Method 8270 \\
\hline$\mu \mathrm{g} / \mathrm{L}$ & 50 & 100 & EPA Method 8270 \\
\hline$\mu g / L$ & 50 & 100 & EPA Method 8270 \\
\hline$\mu g / L$ & 50 & 100 & EPA Method 8270 \\
\hline$\mu g / L$ & 50 & 100 & EPA Method 8270 \\
\hline$\mu g / L$ & 50 & 100 & EPA Method 8270 \\
\hline$\mu \mathrm{g} / \mathrm{L}$ & 50 & 100 & EPA Method 8270 \\
\hline $\mathrm{pCi} / \mathrm{L}$ & $2.5 E+01$ & $5 E+01$ & Proposed DWS (EPA, 1986) \\
\hline$\mu g / L$ & 0.005 & 0.01 & EPA Method 8280 \\
\hline$\mu g / L$ & 0.005 & 0.01 & EPA Method 8280 \\
\hline & No flag & No flag & Set by EPD/EMS \\
\hline$\mu g / L$ & 5,000 & 10,000 & EPA Method 413.1 \\
\hline$\mu g / L$ & 2.5 & 5 & EPA Method 8080 \\
\hline$\mu g / L$ & 2.5 & 5 & EPA Method 8080 \\
\hline$\mu g / L$ & 0.25 & 0.5 & Final DWS (CFR, 1991a) \\
\hline$\mu \mathrm{g} / \mathrm{L}$ & 0.25 & 0.5 & Final DWS (CFR, 1991a) \\
\hline$\mu g / L$ & 0.25 & 0.5 & Final DWS (CFR, 1991a) \\
\hline$\mu \mathrm{g} / \mathrm{L}$ & 0.25 & 0.5 & Final DWS (CFR, 1991a) \\
\hline$\mu \mathrm{g} / \mathrm{L}$ & 0.25 & 0.5 & Final DWS (CFR, 1991a) \\
\hline$\mu \mathrm{g} / \mathrm{L}$ & 0.25 & 0.5 & Final DWS (CFR, 1991a) \\
\hline$\mu g / L$ & 0.25 & 0.5 & Final DWS (CFR, 1991a) \\
\hline$\mu g / L$ & 0.25 & 0.5 & Final DWS (CFR, 1991a) \\
\hline$\mu g / L$ & 50 & 100 & EPA Method 8270 \\
\hline$\mu g / L$ & 0.00275 & 0.0055 & EPA Method 8280 \\
\hline$\mu g / L$ & 0.00275 & 0.0055 & EPA Method 8280 \\
\hline$\mu g / L$ & 0.00275 & 0.0055 & EPA Method 8280 \\
\hline$\mu \mathrm{g} / \mathrm{L}$ & 0.00275 & 0.0055 & EPA Method 8280 \\
\hline$\mu \mathrm{g} / \mathrm{L}$ & 50 & 100 & EPA Method 8270 \\
\hline$\mu g / L$ & 50 & 100 & EPA Method 8270 \\
\hline$\mu \mathrm{g} / \mathrm{L}$ & 0.5 & 1 & Final DWS (CFR, 1991a) \\
\hline $\mathrm{pH}$ & 8 & 10 & Set by EPD/EMS \\
\hline $\mathrm{pH}$ & 4 & 3 & Set by EPDiEMS \\
\hline$\mu \mathrm{g} / \mathrm{L}$ & 50 & 100 & EPA Method 8270 \\
\hline$\mu g / L$ & 50 & 100 & EPA Method 8270 \\
\hline$\mu g / L$ & 50 & 100 & EPA Method 8270 \\
\hline$\mu \mathrm{g} / \mathrm{L}$ & 25 & 50 & EPA Method 420.1 \\
\hline$\mu \mathrm{g} / \mathrm{L}$ & 50 & 100 & EPA Method 8270 \\
\hline$\mu \mathrm{g} / \mathrm{L}$ & 2.5 & 5 & EPA Method 8080 \\
\hline$\mu \mathrm{g} / \mathrm{L}$ & 50 & 100 & EPA Method 8270 \\
\hline $\mathrm{pCi} / \mathrm{L}$ & $3.51 E+00$ & $7.02 E+00$ & Proposed DWS (EPA, 1991) \\
\hline $\mathrm{pCi} / \mathrm{L}$ & $3.11 E+01$ & $6.21 E+01$ & Proposed DWS (EPA, 1991) \\
\hline $\mathrm{pCi} / \mathrm{L}$ & $3.11 E+01$ & $6.21 E+01$ & Proposed DWS (EPA, 1991) \\
\hline $\mathrm{pCi} / \mathrm{L}$ & $3.11 E+01$ & $6.22 E+01$ & Proposed DWS (EPA, 1991) \\
\hline $\mathrm{pCi} / \mathrm{L}$ & $3.13 E+01$ & $6.26 E+01$ & Proposed DWS (EPA, 1991) \\
\hline \multirow[t]{2}{*}{$\mathrm{pCi} / \mathrm{L}$} & $3.27 E+01$ & $6.54 E+01$ & Proposed DWS (EPA, 1991) \\
\hline & No flag & No flag & Set by EPD/EMS \\
\hline
\end{tabular}




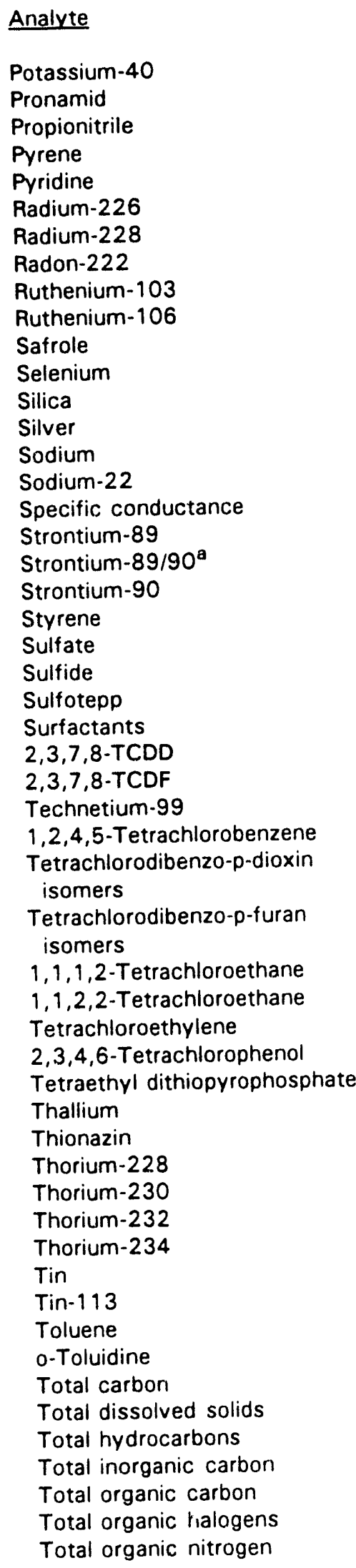

H-Area Seepage Rasins

\begin{tabular}{|c|c|c|c|}
\hline Unit & Flag 1 & Flag 2 & Source \\
\hline $\begin{array}{l}\mathrm{pCi} / \mathrm{L} \\
\mu g / L\end{array}$ & $\begin{array}{l}1.5 E+02 \\
50\end{array}$ & $\begin{array}{l}3 E+02 \\
100\end{array}$ & $\begin{array}{l}\text { Proposed DWS (EPA, 1986) } \\
\text { EPA Method } 8270\end{array}$ \\
\hline$\mu g / L$ & 1,000 & 2,000 & $\begin{array}{l}\text { EPA Method } 8270 \\
\text { EPA Method } 8240\end{array}$ \\
\hline$\mu g / L$ & 50 & 100 & EPA Method 8270 \\
\hline$\mu g / L$ & 50 & 100 & EPA Method 8270 \\
\hline $\mathrm{pCi} / \mathrm{L}$ & $7.85 E+00$ & $1.57 E+01$ & Proposed DWS (EPA, 1991) \\
\hline $\mathrm{pCi} / \mathrm{L}$ & $3.93 E+00$ & $7.85 E+00$ & Proposed DWS (EPA, 1991) \\
\hline $\mathrm{pCi} / \mathrm{L}$ & $1.5 E+02$ & $3 E+02$ & Proposed DWS (EPA, 1991) \\
\hline $\mathrm{pCi} / \mathrm{L}$ & $1 E+02$ & $2 E+02$ & Final DWS (EPA, 1977) \\
\hline $\mathrm{pCi} / \mathrm{L}$ & $1.5 E+01$ & $3 E+01$ & Final DWS (EPA, 1977) \\
\hline$\mu g / L$ & 50 & 100 & EPA Method 8270 \\
\hline$\mu g / L$ & 25 & 50 & Final DWS (CFR, 1991a) \\
\hline$\mu g / L$ & $\begin{array}{l}\text { No flag } \\
25\end{array}$ & $\begin{array}{l}\text { No flag } \\
50\end{array}$ & $\begin{array}{l}\text { Set by EPD/EMS } \\
\text { Final DWS (CFR, 1991a) }\end{array}$ \\
\hline & No flag & No flag & $\begin{array}{l}\text { Final DWS (CFR, 1991a) } \\
\text { Set by EPD/EMS }\end{array}$ \\
\hline $\mathrm{pCi} / \mathrm{L}$ & $2.33 E+02$ & $4.66 E+02$ & Proposed DWS (EPA, 1991) \\
\hline $\begin{array}{l}\mu \mathrm{S} / \mathrm{cm} \\
\mathrm{pCi} / \mathrm{L}\end{array}$ & 250 & 500 & Set by EPD/EMS \\
\hline $\begin{array}{l}\mathrm{pCi} / \mathrm{L} \\
\mathrm{pCi} / \mathrm{L}\end{array}$ & $1 E+01$ & $2 E+01$ & Final DWS (EPA, 1977) \\
\hline $\begin{array}{l}\mathrm{pCi} / \mathrm{L} \\
\mathrm{pCi} / \mathrm{L}\end{array}$ & $4 E+00$ & $8 E+00$ & Final DWS (CFR, 1991a) \\
\hline $\begin{array}{l}\mathrm{pCi} / \mathrm{L} \\
\mu \mathrm{g} / \mathrm{L}\end{array}$ & $4 E+00$ & $8 E+00$ & Final DWS (CFR, 1991a) \\
\hline $\begin{array}{l}\mu g / L \\
\mu g / L\end{array}$ & 50 & 100 & Final DWS (CFR, 1991a) \\
\hline $\begin{array}{l}\mu g / L \\
\mu g / L\end{array}$ & 200,000 & 400,000 & Proposed DWS (EPA, 1990) \\
\hline $\begin{array}{l}\mu g / L \\
\mu g / L\end{array}$ & 5,000 & 10,000 & EPA Method 9030 \\
\hline$\mu g / L$ & 50 & 100 & EPA Method 8270 \\
\hline$\mu g / L$ & $\begin{array}{l}\text { No flag } \\
0.00225\end{array}$ & $\begin{array}{l}\text { No flag } \\
0.0045\end{array}$ & $\begin{array}{l}\text { Set by EPD/EMS } \\
\text { EPA Method } 8280\end{array}$ \\
\hline$\mu g / L$ & 0.002 & $\begin{array}{l}0.0045 \\
0.004\end{array}$ & \\
\hline $\mathrm{pCi} / \mathrm{L}$ & $4.5 E+02$ & $9 E+02$ & $\begin{array}{l}\text { EPA Method } 8280 \\
\text { Final DWS (EPA, 1977) }\end{array}$ \\
\hline$\mu \mathrm{g} / \mathrm{L}$ & 50 & 100 & \\
\hline & & & EPA Method 8280 \\
\hline $\begin{array}{l}\mu g / L \\
\mu g / L\end{array}$ & 0.002 & 0.004 & EPA Method 8280 \\
\hline $\begin{array}{l}\mu g / L \\
\mu g / L\end{array}$ & 5 & 10 & EPA Method 8240 \\
\hline $\begin{array}{l}\mu g / L \\
\mu g / L\end{array}$ & 5 & 10 & EPA Method 8240 \\
\hline $\begin{array}{l}\mu g / L \\
\mu g / L\end{array}$ & 2.5 & 5 & Final DWS (CFR, 1991a) \\
\hline $\begin{array}{l}\mu g / L \\
\mu g / L\end{array}$ & 50 & 100 & EPA Method 8270 \\
\hline$\mu g / L$ & 50 & $\begin{array}{l}100 \\
1\end{array}$ & $\begin{array}{l}\text { EPA Method } 8270 \\
\text { Proposed DWS (EPA, 1990) }\end{array}$ \\
\hline$\mu g / L$ & $\begin{array}{l}0.5 \\
50\end{array}$ & $\begin{array}{l}1 \\
100\end{array}$ & $\begin{array}{l}\text { Proposed DWS (EPA, } 1990 \text { ) } \\
\text { EPA Method } 8270\end{array}$ \\
\hline $\mathrm{pCi} / \mathrm{L}$ & $\begin{array}{l}50 \\
6.25 E+01\end{array}$ & $\begin{array}{l}100 \\
1.25 E+02\end{array}$ & $\begin{array}{l}\text { EPA Method } 8270 \\
\text { Proposed DWS (EPA, 1991) }\end{array}$ \\
\hline $\mathrm{pCi} / \mathrm{L}$ & $\begin{array}{l}6.25 E+01 \\
3.96 E+01\end{array}$ & $\begin{array}{l}1.25 E+02 \\
7.92 E+01\end{array}$ & \\
\hline $\mathrm{pCi} / \mathrm{L}$ & $4.4 E+01$ & $8.8 E+01$ & $\begin{array}{l}\text { Proposed DWS (EPA, 1991) } \\
\text { Proposed DWS (EPA, 1991) }\end{array}$ \\
\hline $\mathrm{pCi} / \mathrm{L}$ & $2 E+02$ & $4.01 E+02$ & \\
\hline$\mu g / L$ & 10 & 20 & $\begin{array}{l}\text { Proposed DWS (EPA, 1991) } \\
\text { EPA Method } 282.2\end{array}$ \\
\hline $\mathrm{pCi} / \mathrm{L}$ & $1.5 E+02$ & $3 E+02$ & $\begin{array}{l}\text { EPA Method } 282.2 \\
\text { Final DWS (EPA, 1977) }\end{array}$ \\
\hline$\mu g / L$ & 500 & 1,000 & $\begin{array}{l}\text { Final DWS (EPA, 1977) } \\
\text { Final DWS (CFR, 1991a) }\end{array}$ \\
\hline$\mu g / L$ & 50 & 100 & $\begin{array}{l}\text { Final DWS (CFR, 1991a) } \\
\text { EPA Method } 8270\end{array}$ \\
\hline$\mu g / L$ & 5,000 & 10,000 & $\begin{array}{l}\text { EPA Method } 8270 \\
\text { EPA Method } 9060\end{array}$ \\
\hline & No flag & No flag & $\begin{array}{l}\text { EPA Method } 9060 \\
\text { Set by EPD/EMS }\end{array}$ \\
\hline$\mu g / L$ & 5,000 & 10,000 & $\begin{array}{l}\text { Set by EPD/EMS } \\
\text { EPA Method } 418.1\end{array}$ \\
\hline$\mu \mathrm{g} / \mathrm{L}$ & 5,000 & 10,000 & $\begin{array}{l}\text { EPA Method } 418.1 \\
\text { EPA Method } 9060\end{array}$ \\
\hline$\mu g / L$ & 5,000 & 10,000 & $\begin{array}{l}\text { EPA Method } 9060 \\
\text { EPA Method } 9060\end{array}$ \\
\hline$\mu \mathrm{g} / \mathrm{L}$ & 25 & 50 & $\begin{array}{l}\text { EPA Method } 9060 \\
\text { EPA Method } 9020\end{array}$ \\
\hline$\mu$ & 500 & & $\begin{array}{l}\text { EPA Method } 9020 \\
\text { EPA Method } 420\end{array}$ \\
\hline & & & \\
\hline
\end{tabular}

Fourth Quarter 1992 


\section{Analyte}

Total petroleum hydrocarbons

Total phosphates (as P)

Total phosphorus

Total radium

Total silica

Total trihalomethanes

Toxaphene

2,4,5-TP (Silvex)

Tributyl phosphate

1,2,4-Trichlorobenzene

$1,1,1$-Trichloroethane

1,1,2-Trichloroethane

Trichloroethylene

Trichlorofluoromethane

2,4,5-Trichlorophenol

2,4,6-Trichlorophenol

2,4,5-Trichlorophenoxyacetic acid

1,2,3-Trichloropropane

$0,0,0$-Triethyl phosphorothioate

1,3,5-Trinitrobenzene

Tritium

Turbidity

Uranium

Uranium alpha activity

Uranium-233/234 ${ }^{\text {a }}$

Uranium-234

Uranium-235

Uranium-238

Vanadium

Vinyl acetate

Xylenes

Zinc

Zinc-65

Zirconium-95

Zirconium/Niobium-95

\begin{tabular}{|c|c|c|}
\hline Unit & Flag 1 & Flag 2 \\
\hline \multirow[t]{3}{*}{$\mu \mathrm{g} / \mathrm{L}$} & 5,000 & 10,000 \\
\hline & No flag & No fiag \\
\hline & No flag & No flag \\
\hline $\mathrm{pCi} / \mathrm{L}$ & $2.5 E+00$ & $5 E+00$ \\
\hline$\mu g / L$ & 500 & 1,000 \\
\hline$\mu g / L$ & 50 & 100 \\
\hline$\mu g / L$ & 1.5 & 3 \\
\hline$\mu \mathrm{g} / \mathrm{L}$ & 25 & 50 \\
\hline$\mu g / L$ & 50 & 100 \\
\hline$\mu g / L$ & 4.5 & 9 \\
\hline$\mu g / L$ & 100 & 200 \\
\hline$\mu g / L$ & 2.5 & 5 \\
\hline$\mu g / L$ & 2.5 & 5 \\
\hline$\mu g / L$ & 5 & 10 \\
\hline$\mu g / L$ & 50 & 100 \\
\hline$\mu \mathrm{g} / \mathrm{L}$ & 50 & 100 \\
\hline$\mu \mathrm{g} / \mathrm{L}$ & 2.5 & 5 \\
\hline$\mu \mathrm{g} / \mathrm{L}$ & 5 & 10 \\
\hline$\mu g / L$ & 50 & 100 \\
\hline$\mu g / L$ & 50 & 100 \\
\hline $\mathrm{pCi} / \mathrm{mL}$ & $1 E+01$ & $2 E+01$ \\
\hline & No flag & No flag \\
\hline$\mu g / L$ & 10 & 20 \\
\hline $\mathrm{pCi} / \mathrm{L}$ & $1.5 E+01$ & $3 E+01$ \\
\hline $\mathrm{pCi} / \mathrm{L}$ & 6.9E-00 & $1.38 E+01$ \\
\hline $\mathrm{pCi} / \mathrm{L}$ & $6.95 E+00$ & $1.39 E+01$ \\
\hline $\mathrm{pCi} / \mathrm{L}$ & $7.25 E+00$ & $1.45 E+01$ \\
\hline $\mathrm{pCi} / \mathrm{L}$ & $7.3 E+00$ & $1.46 \mathrm{E}+01$ \\
\hline$\mu \mathrm{g} / \mathrm{L}$ & 50 & 100 \\
\hline$\mu g / L$ & 5 & 10 \\
\hline$\mu g / L$ & 5,000 & 10,000 \\
\hline$\mu g / L$ & 2,500 & 5,000 \\
\hline $\mathrm{pCi} / \mathrm{L}$ & $1.5 E+02$ & $3 E+02$ \\
\hline $\mathrm{pCi} / \mathrm{L}$ & $1 E+02$ & $2 E+02$ \\
\hline $\mathrm{pCi} / \mathrm{L}$ & $1 E+02$ & $2 E+02$ \\
\hline
\end{tabular}

$\underline{\text { Source }}$

EPA Method 418.1

Set by EPD/EMS

Set by EPD/EMS

Final DWS (CFR, 1991a)

EPA Method 6010

Final DWS (CFR, 1991a)

Final DWS (CFR, 1991a)

Final DWS (CFR, 1991a)

EPA Method 8270

Proposed DWS (EPA, 1990)

Final DWS (CFR, 1991a)

Proposed DWS (EPA, 1990)

Final DWS (CFR, 1991a)

EPA Method 8240

EPA Method 8270

EPA Method 8270

EPA Method 8150

EPA Method 8240

EPA Method 8270

EPA Method 8270

Final DWS (CFR, 1991a)

Set by EPD/EMS

Proposed DWS (EPA, 1991)

Proposed DWS (EPA, 1991)

Proposed DWS (EPA, 1991)

Proposed DWS (EPA, 1991)

Proposed DWS (EPA, 1991)

Proposed DWS (EPA, 1991)

EPA Method 6010

EPA Method 8240

Final DWS (CFR, 1991a)

Secondary DWS (CFR, 1991b)

Final DWS (EPA, 1977)

Final DWS (EPA, 1977)

Final DWS (EPA, 1977)

a For double radionuclide analyses where each separate radionuclide has its own standard, the more stringent standard is used.

\section{References}

APHA (American Public Health Association, American Water Works Association, and Water Pollution Control Federation), 1985. Standard Methods for the Examination of Water and Wastewater, 16th edition. Washington, DC.

CFR (Code of Federal Regulations), 1991a. National Primary Drinking Water Regulations, 40 CFR, Part 141, pp. 578-715. Washington, DC.

CFR (Code of Federal Regulations), 1991b. National Secondary Drinking Water Regulations, 40 CFR, Part 143, pp. 758-762. Washington, DC. 
EPA (U.S. Environmental Protection Agency), 1977. National Interim Primary Drinking Water Regulations, EPA-570/9-76-003. Washington, DC.

EPA (U.S. Environmental Protection Agency), 1986. Water Pollution Control; National Primary Drinking Water Regulations, Radionuclides (Proposed). Federal Register, September 30, 1986, pp. 34836-34862. Washington, DC.

EPA (U.S. Environmental Protection Agency), 1990. National Primary and Secondary Drinking Water Regulations; Synthetic Organic Chemicals and Inorganic Chemicals (Proposed Rule). Federal Register, July 25, 1990, pp. 30369-30448. Washington, DC.

EPA (U.S. Environmental Protection Agency), 1991. National Primary Drinking Water Regulations; Radionuclides; Proposed Rule. Federal Register, July 18, 1991, pp. 33052-33127. Washington, DC. 
WSRC-TR-93-059

\section{Appendix C - Figures}




\begin{tabular}{|c|c|c|c|c|c|c|}
\hline$\frac{.0}{\frac{0}{0}} \frac{0}{\frac{0}{0}}$ & \multicolumn{3}{|c|}{$\begin{array}{c}\text { Lithostratigraphic } \\
\text { Units }\end{array}$} & \multicolumn{3}{|c|}{$\begin{array}{c}\text { Hydrostatigraphic } \\
\text { Units }\end{array}$} \\
\hline \multirow{9}{*}{$\underset{\frac{Z}{\pi}}{\stackrel{\frac{\pi}{\sigma}}{0}}$} & \multicolumn{2}{|c|}{ "Upland Unit" } & \multirow{7}{*}{ 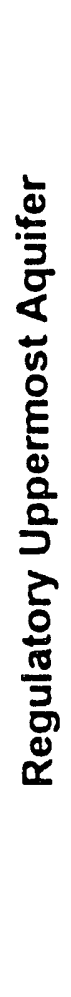 } & \multirow{5}{*}{ 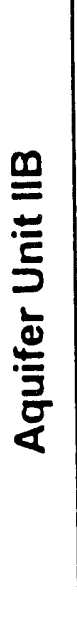 } & \multirow{2}{*}{$\begin{array}{l}\text { Aquifer } \\
\text { Zone } \| B_{2}\end{array}$} & \multirow{9}{*}{ 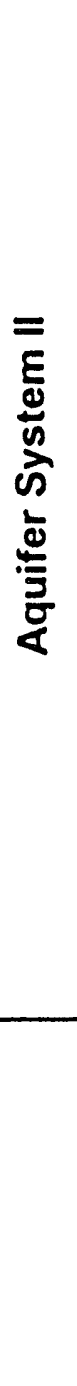 } \\
\hline & \multirow{3}{*}{ 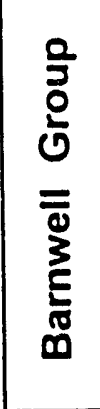 } & $\left|\begin{array}{c}\text { Tobacco Rd } \\
\text { Formation }\end{array}\right|$ & & & & \\
\hline & & $\begin{array}{l}\text { Dry Branch } \\
\text { Formation }\end{array}$ & & & $\begin{array}{c}\text { Confining } \\
\text { Zone }\left\|B_{1}-\right\| B_{2}\end{array}$ & \\
\hline & & $\begin{array}{l}\text { Clinchfield } \\
\text { Formation }\end{array}$ & & & \multirow{2}{*}{$\begin{array}{l}\text { Aquifer } \\
\text { Zone IIB, }\end{array}$} & \\
\hline & \multicolumn{2}{|c|}{$\begin{array}{c}\text { Santee Limestone } \\
\text { Formation }\end{array}$} & & & & \\
\hline & \multicolumn{2}{|c|}{$\begin{array}{l}\text { Warley Hill } \\
\text { Formation }\end{array}$} & & \multicolumn{2}{|c|}{$\begin{array}{c}\text { Confining Unit } \\
\text { IAA-IB }\end{array}$} & \\
\hline & \multicolumn{2}{|c|}{$\begin{array}{l}\text { Congareel } \\
\text { Fishburne } \\
\text { Formations }\end{array}$} & & \multicolumn{2}{|c|}{ Aquifer Unit IIA } & \\
\hline & \multicolumn{2}{|c|}{$\begin{array}{c}\text { Williamsburg } \\
\text { Formation }\end{array}$} & & \multirow{2}{*}{\multicolumn{2}{|c|}{$\begin{array}{l}\text { Confining } \\
\text { System I-II }\end{array}$}} & \\
\hline & \multicolumn{3}{|c|}{$\begin{array}{l}\text { Ellenton } \\
\text { Formation }\end{array}$} & & & \\
\hline
\end{tabular}

Figure 1. Hydrostratigraphic Nomenclature 


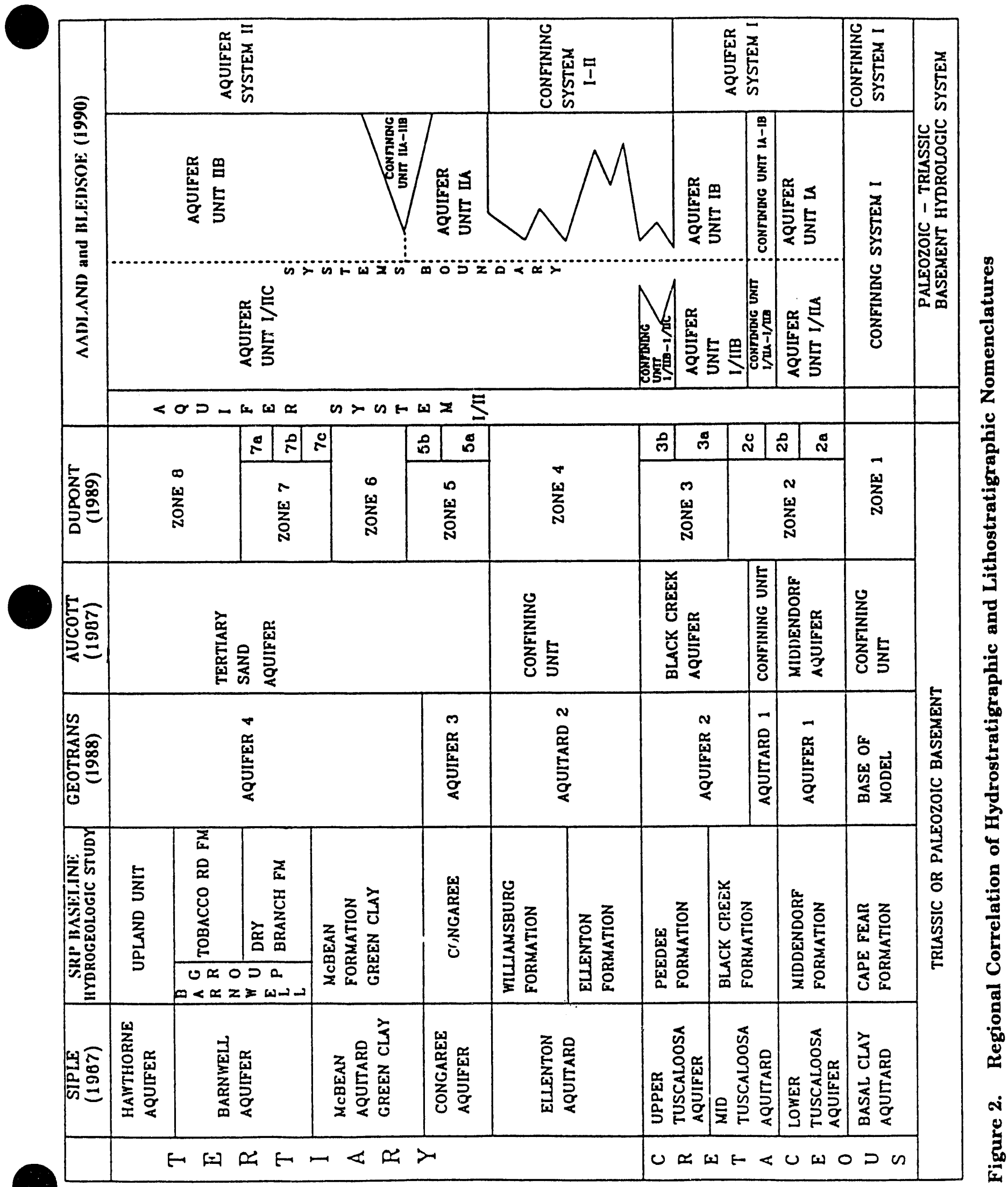




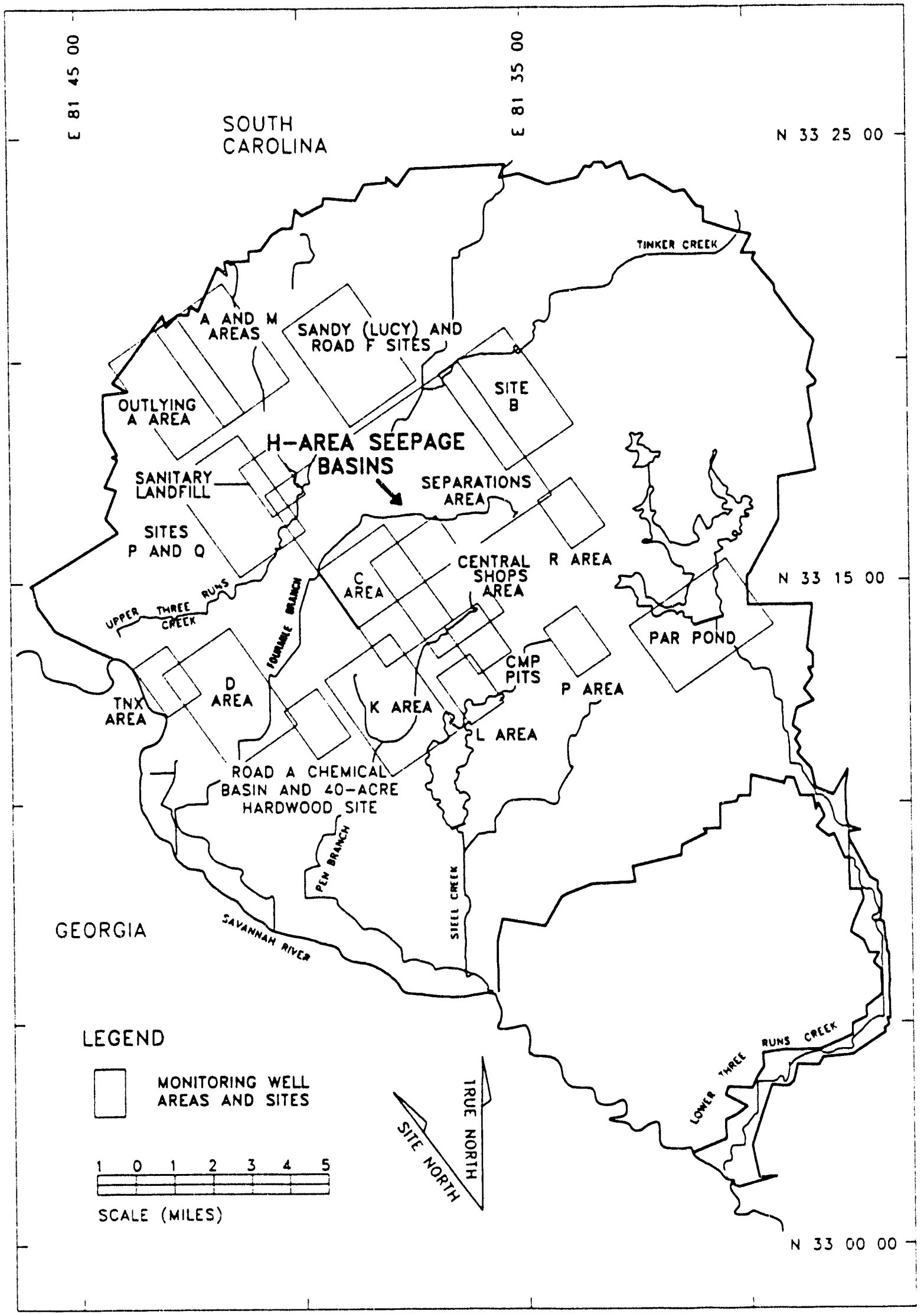

Figure 3. Location of the H-Area Seepage Basing at the Savannah River Site 


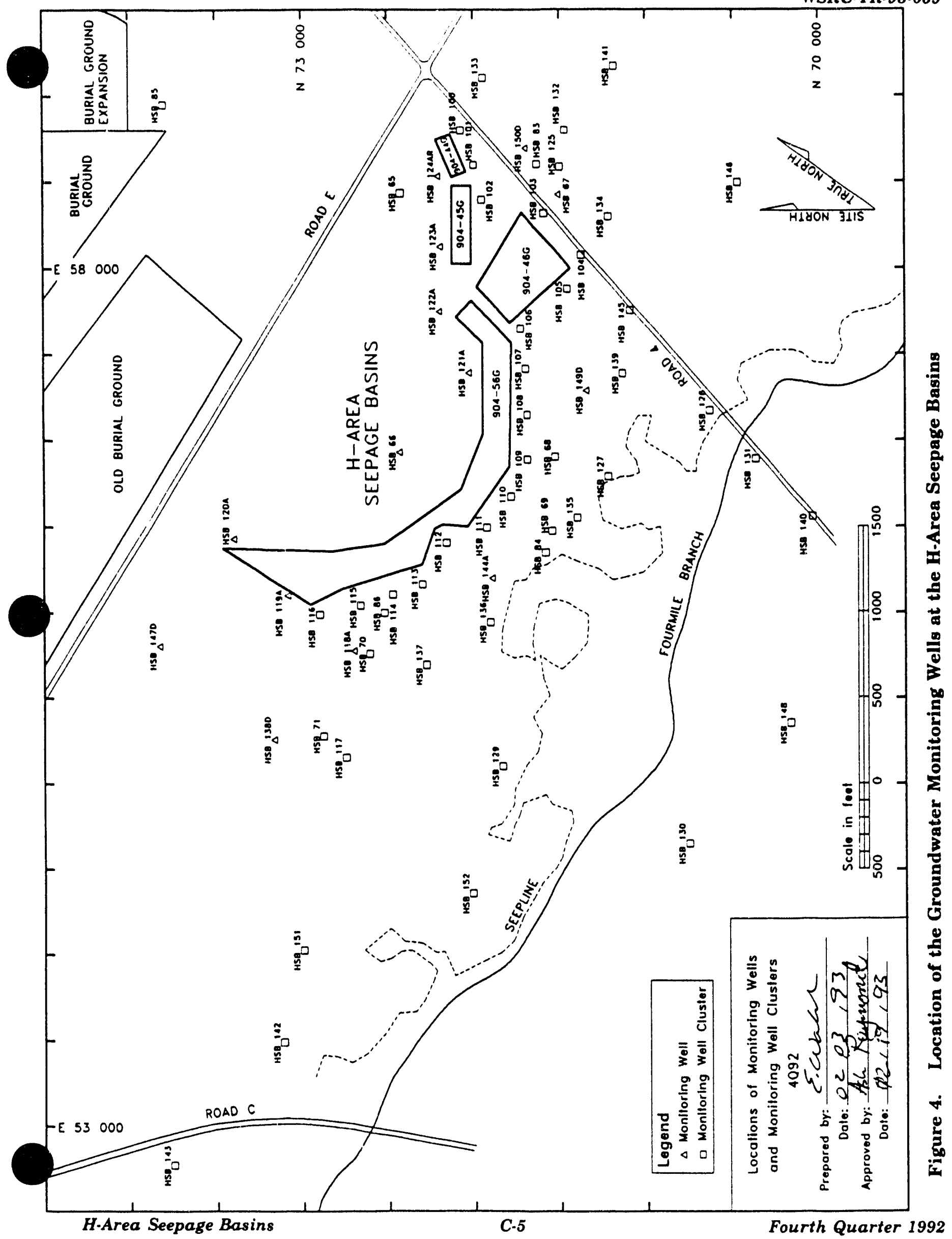


WSRC-TR.93.059

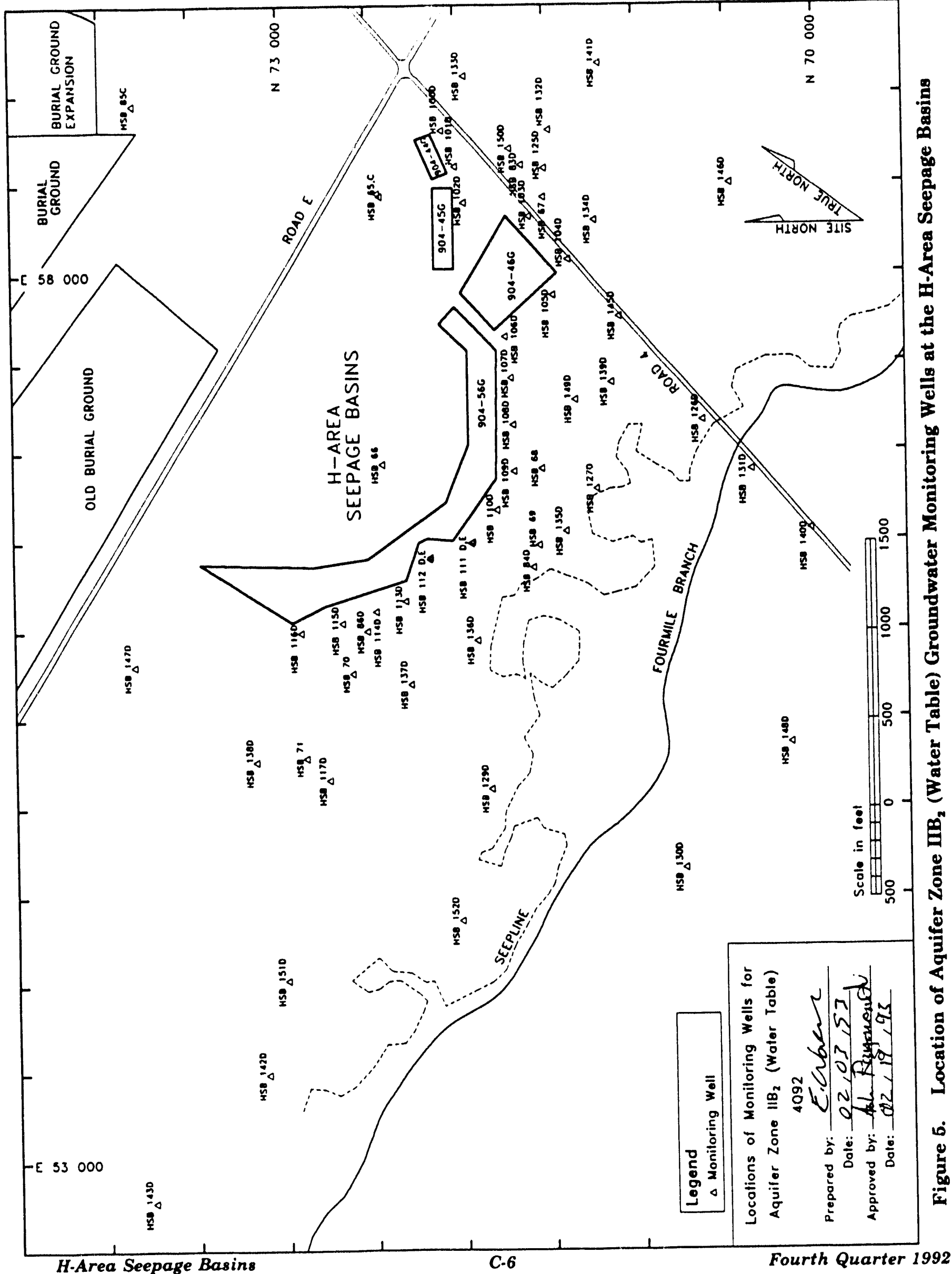




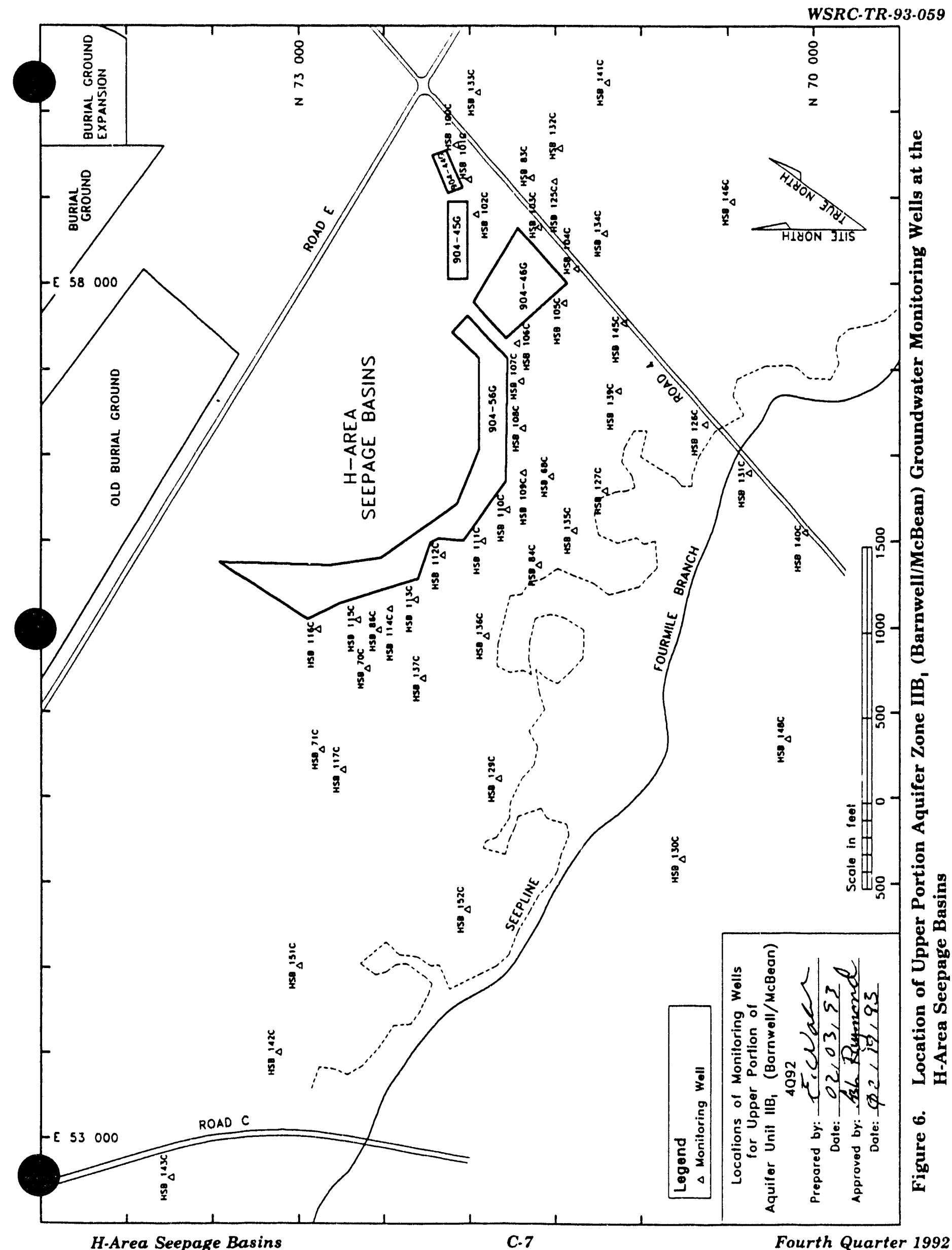




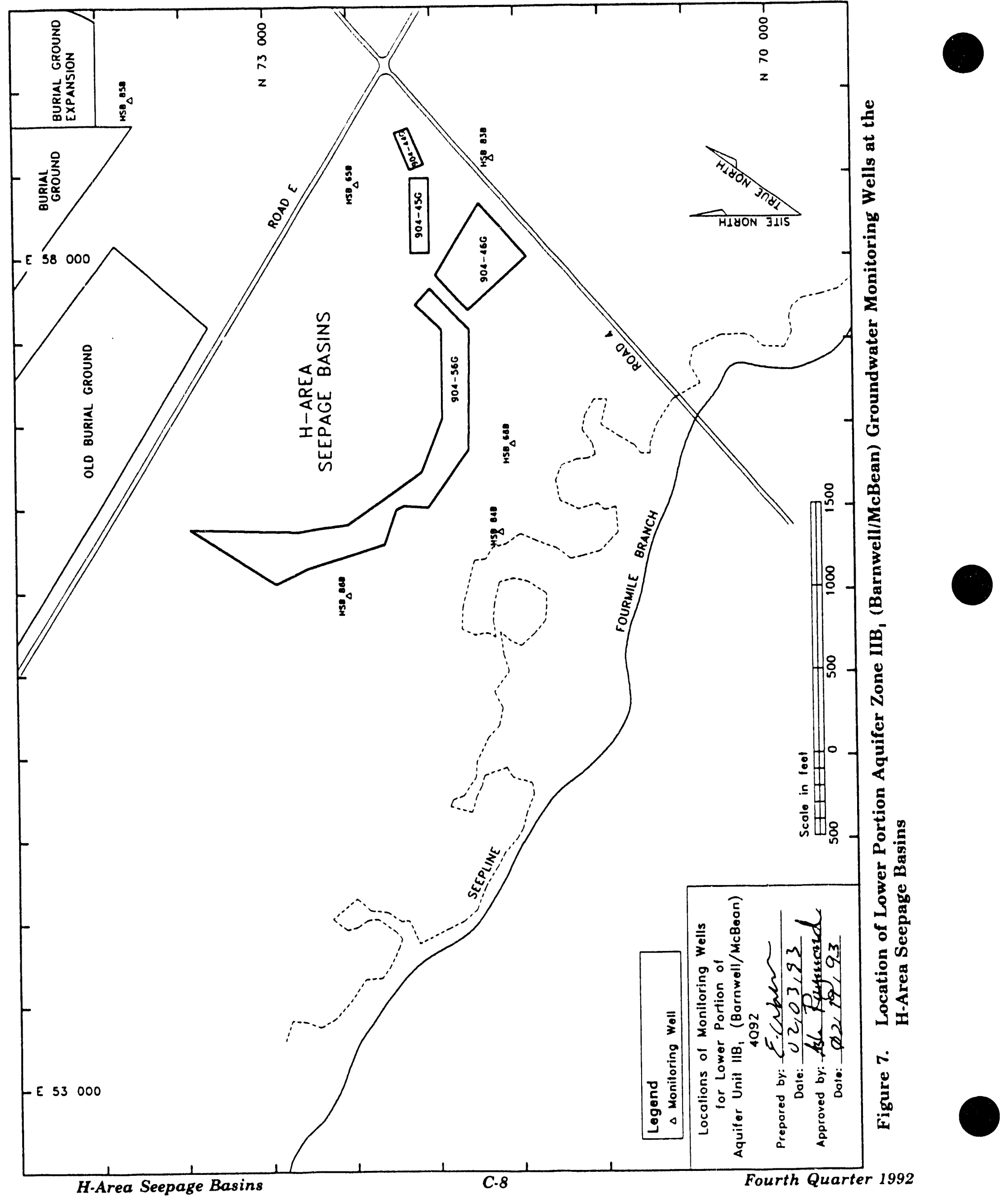




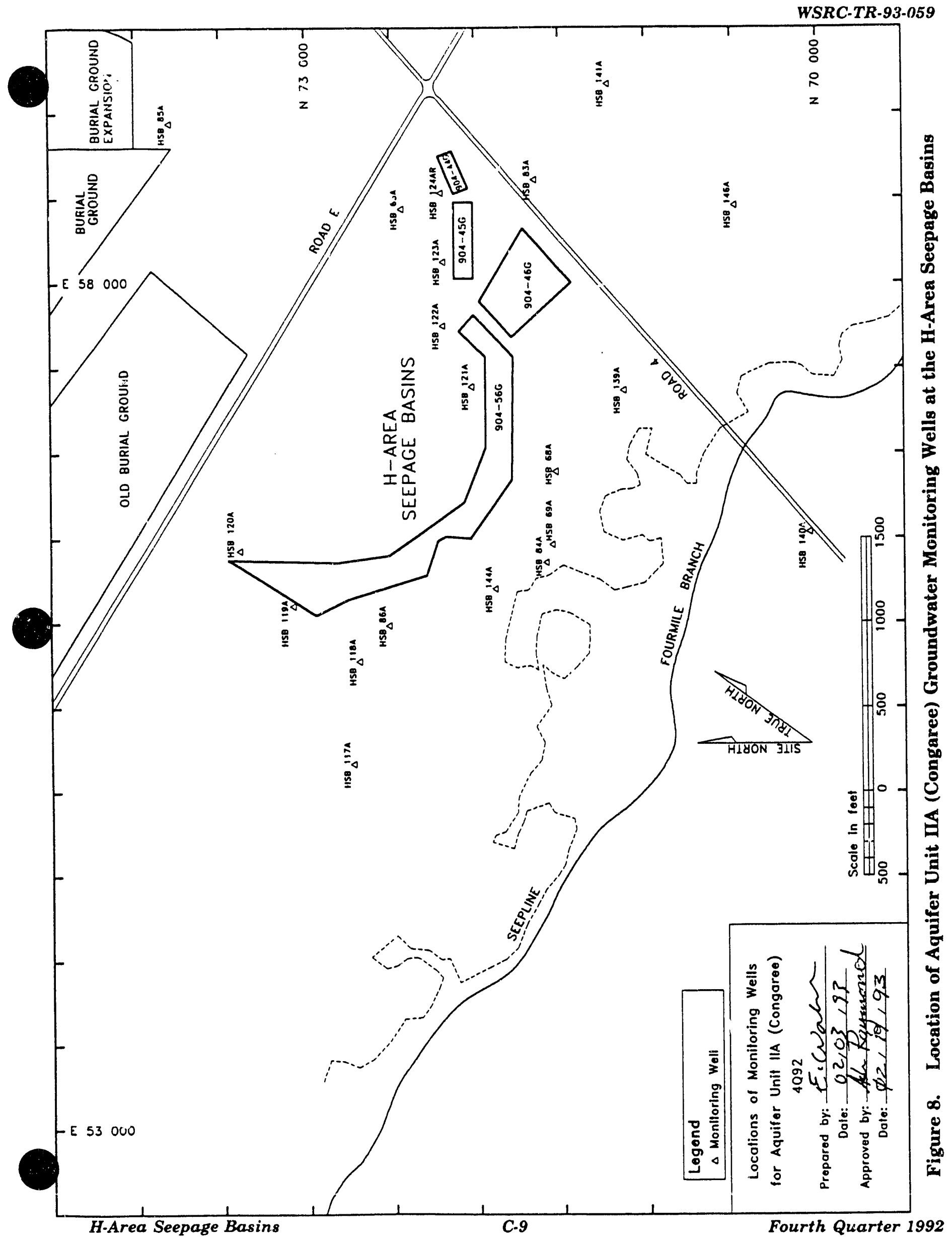


$\ddot{n} 4$

$\bar{i} \triangleleft$
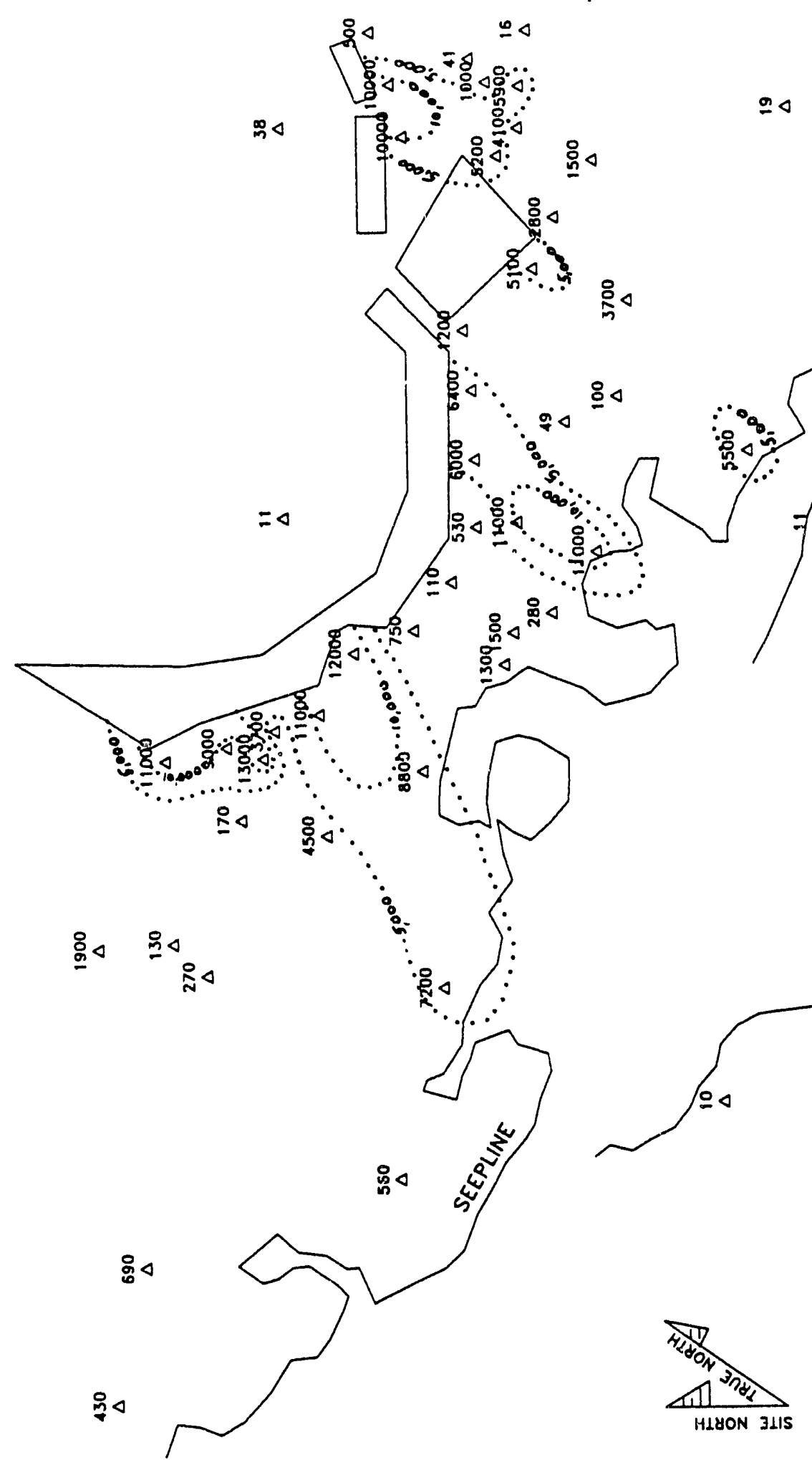


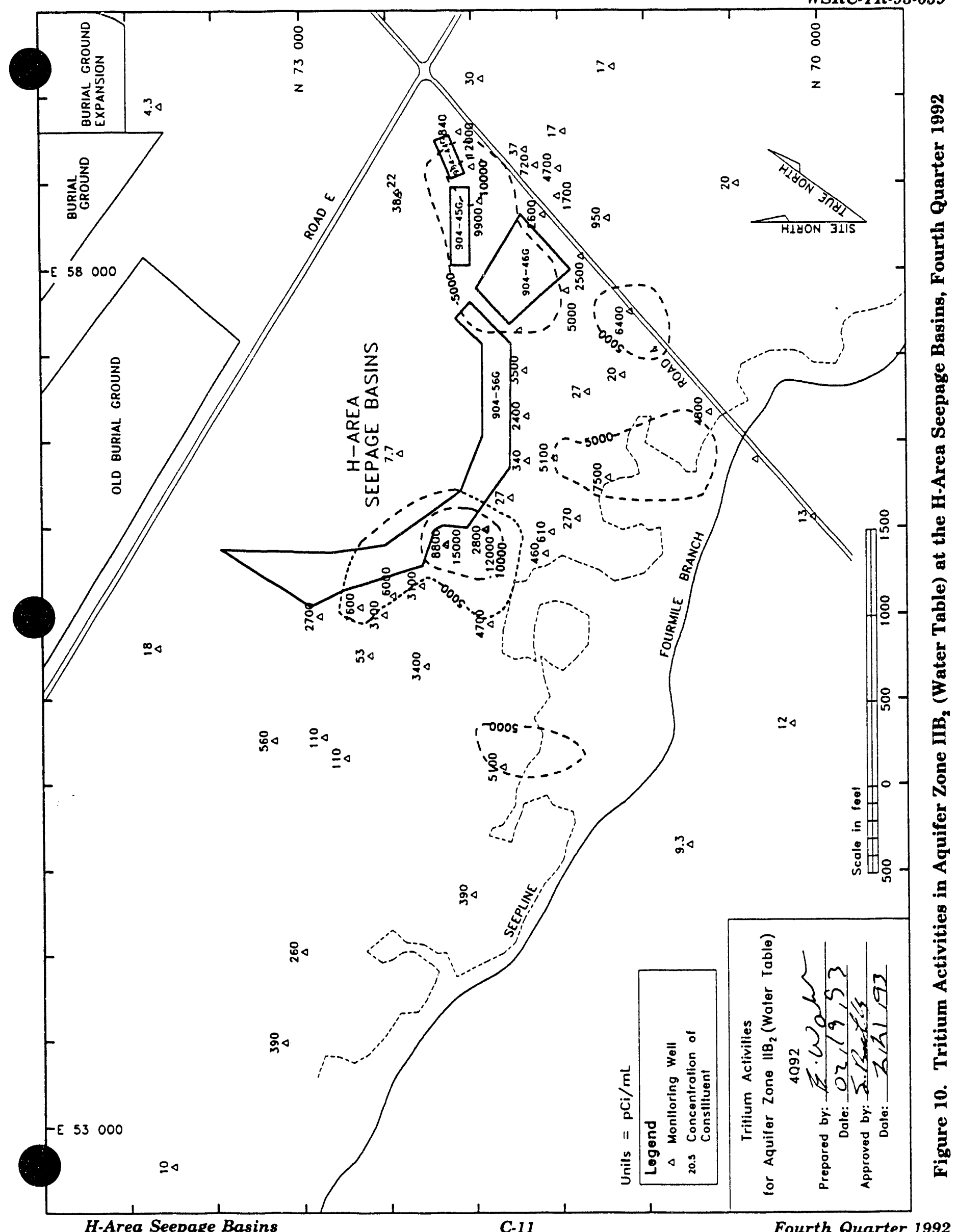


온

ㅇำ

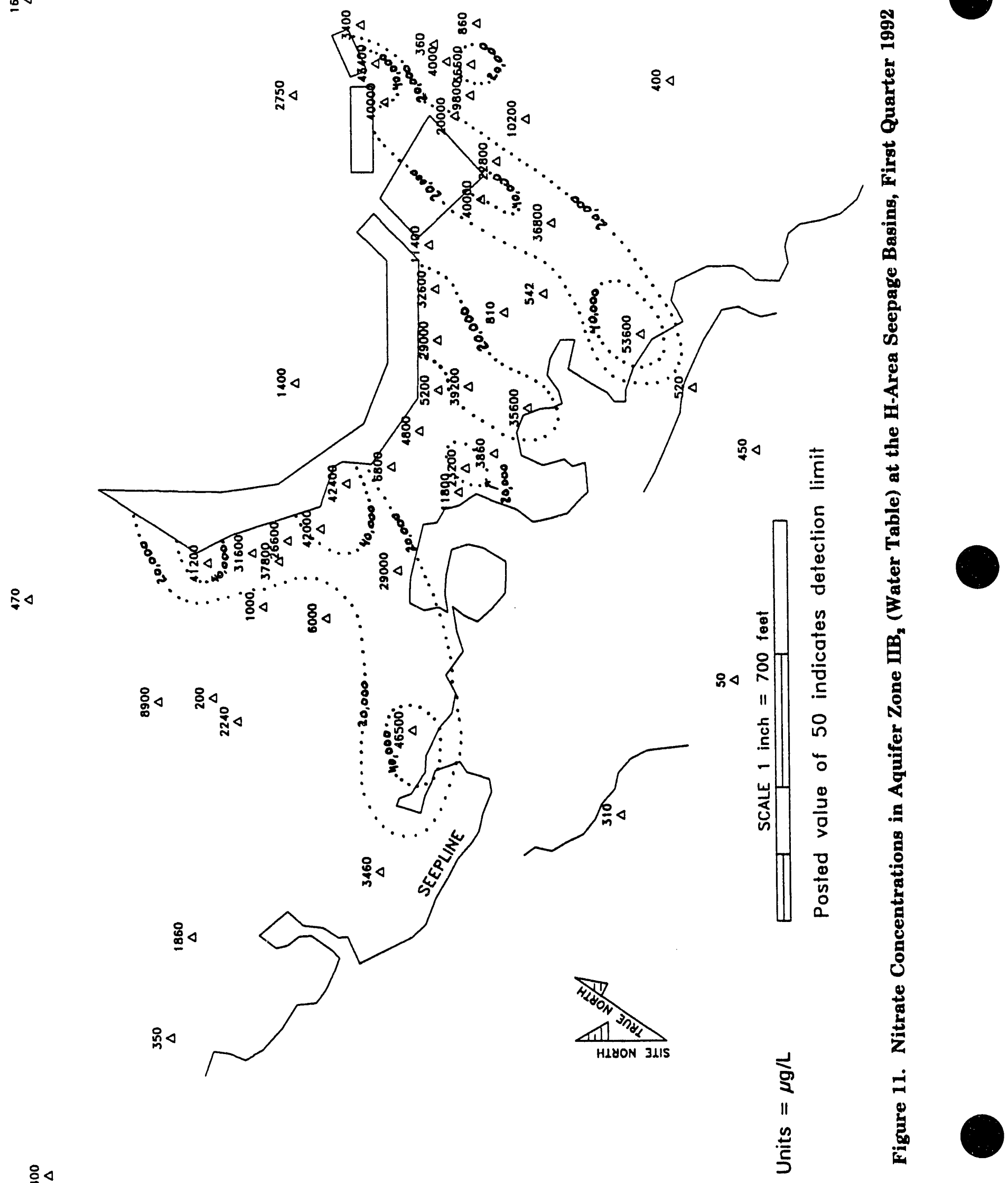




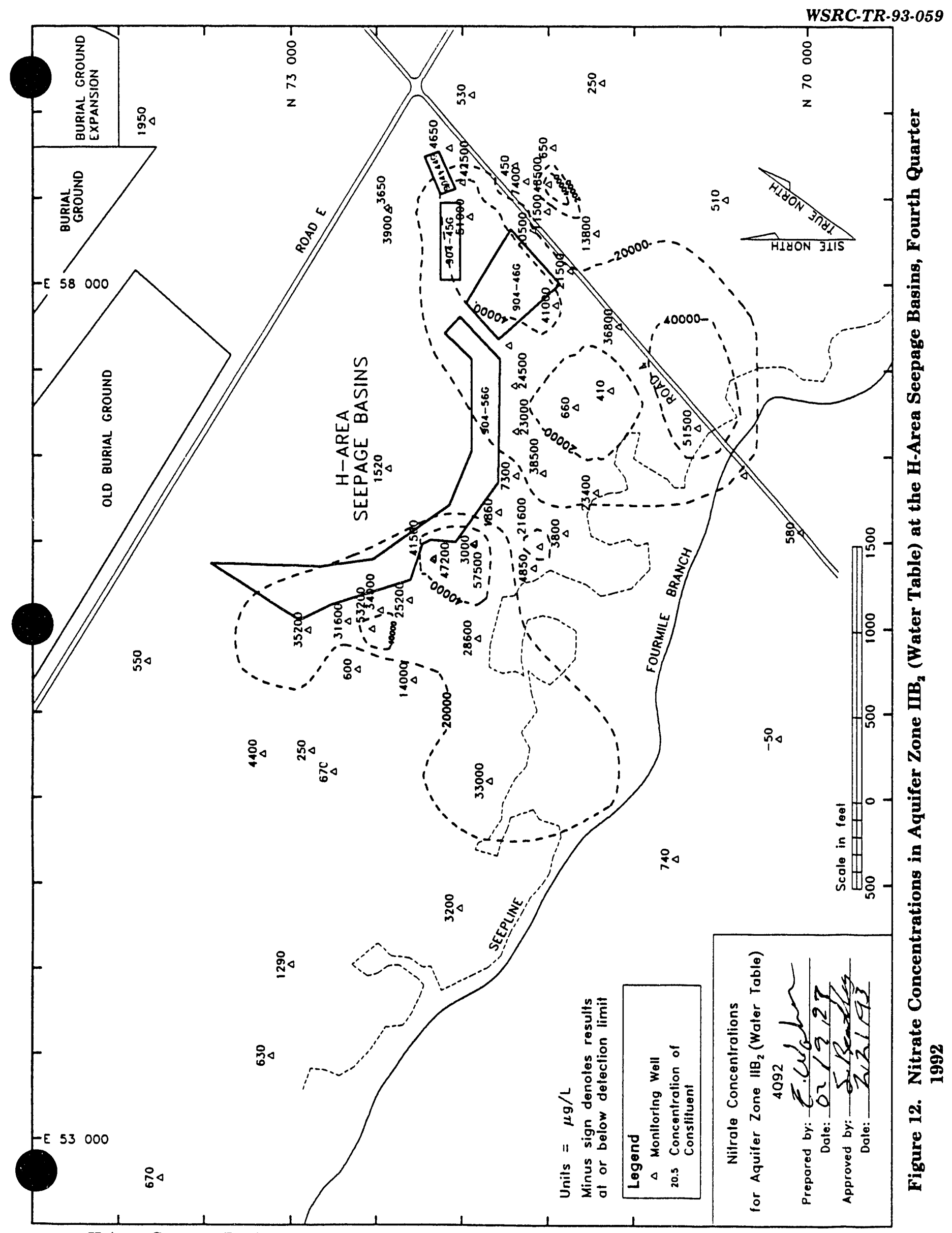




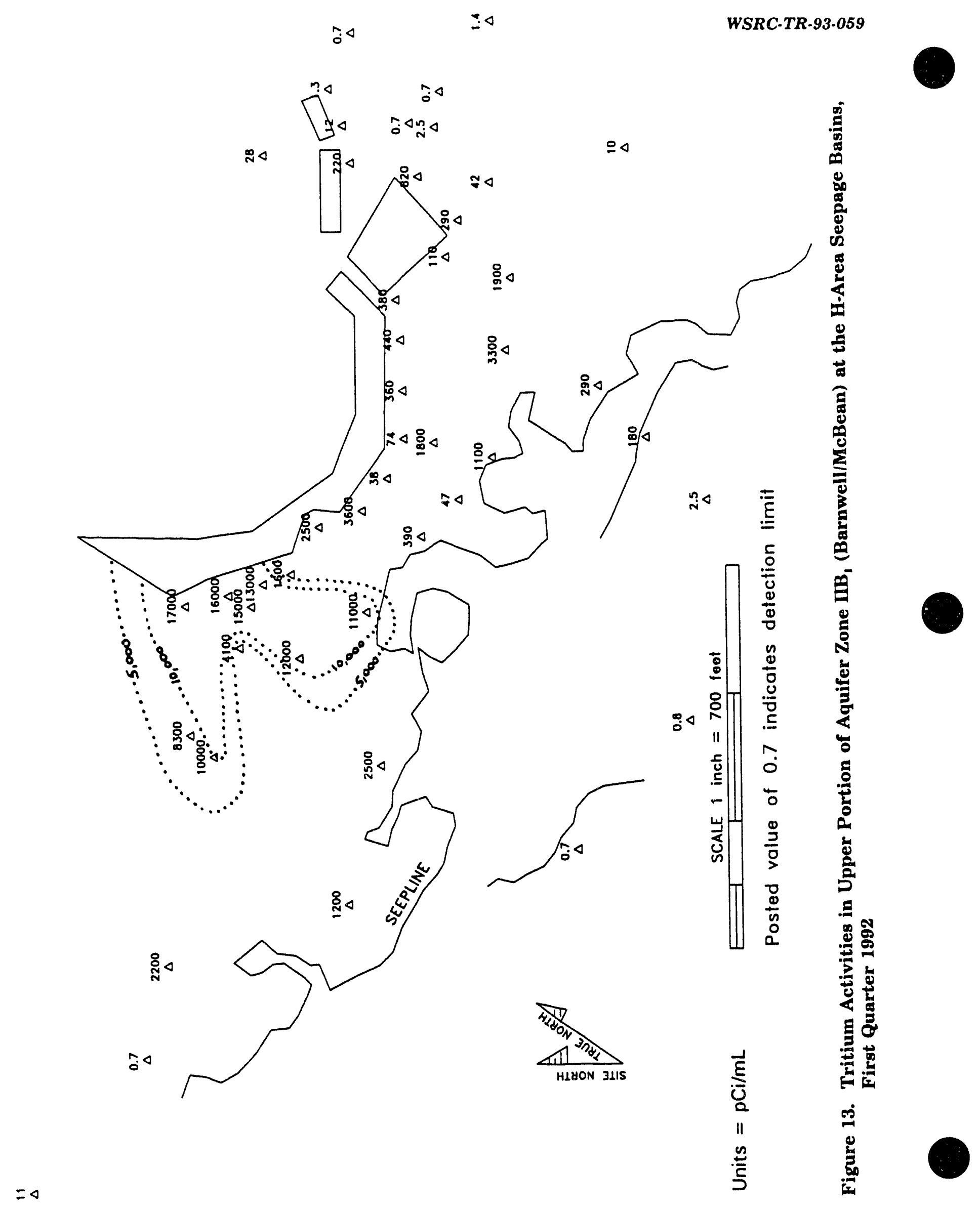




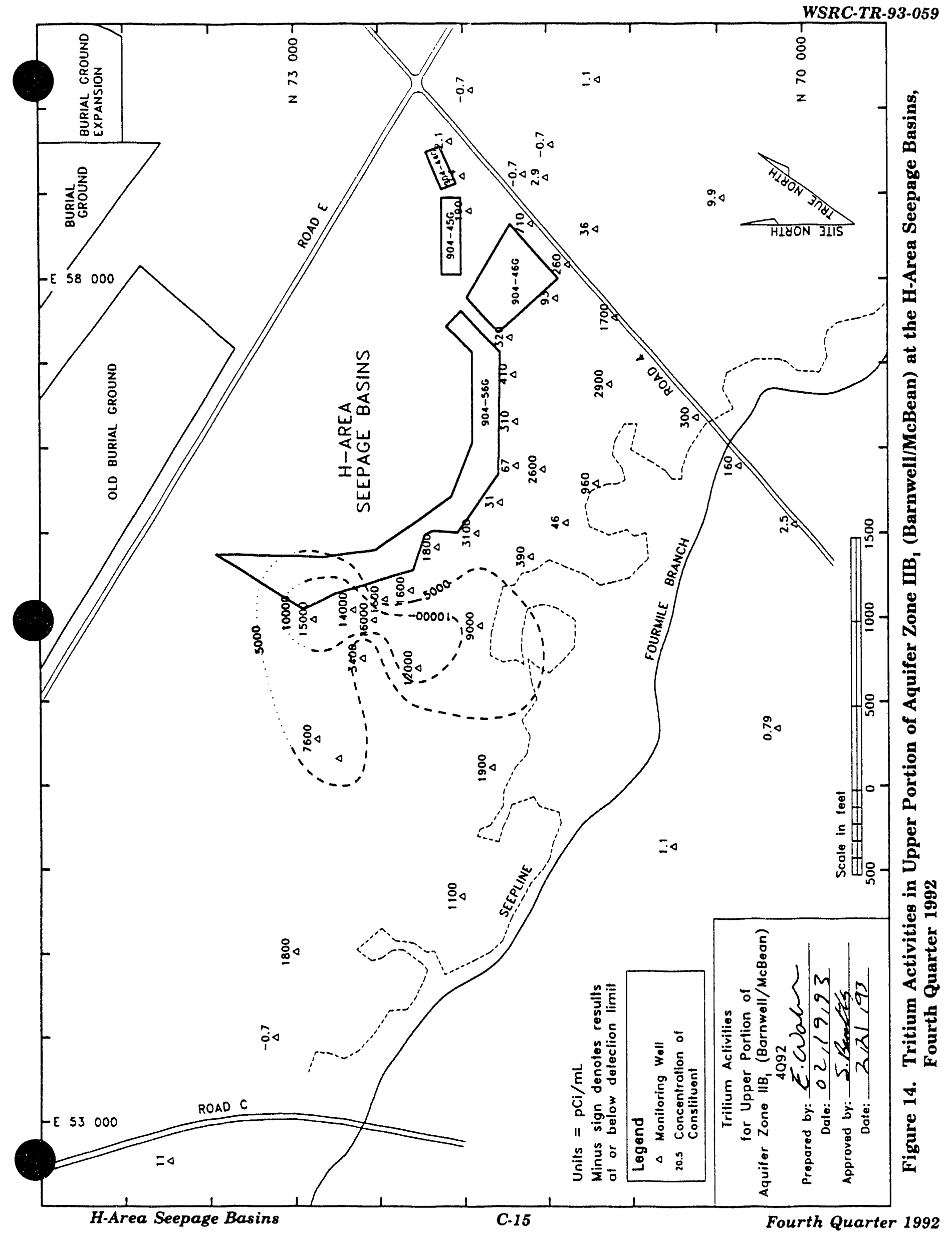




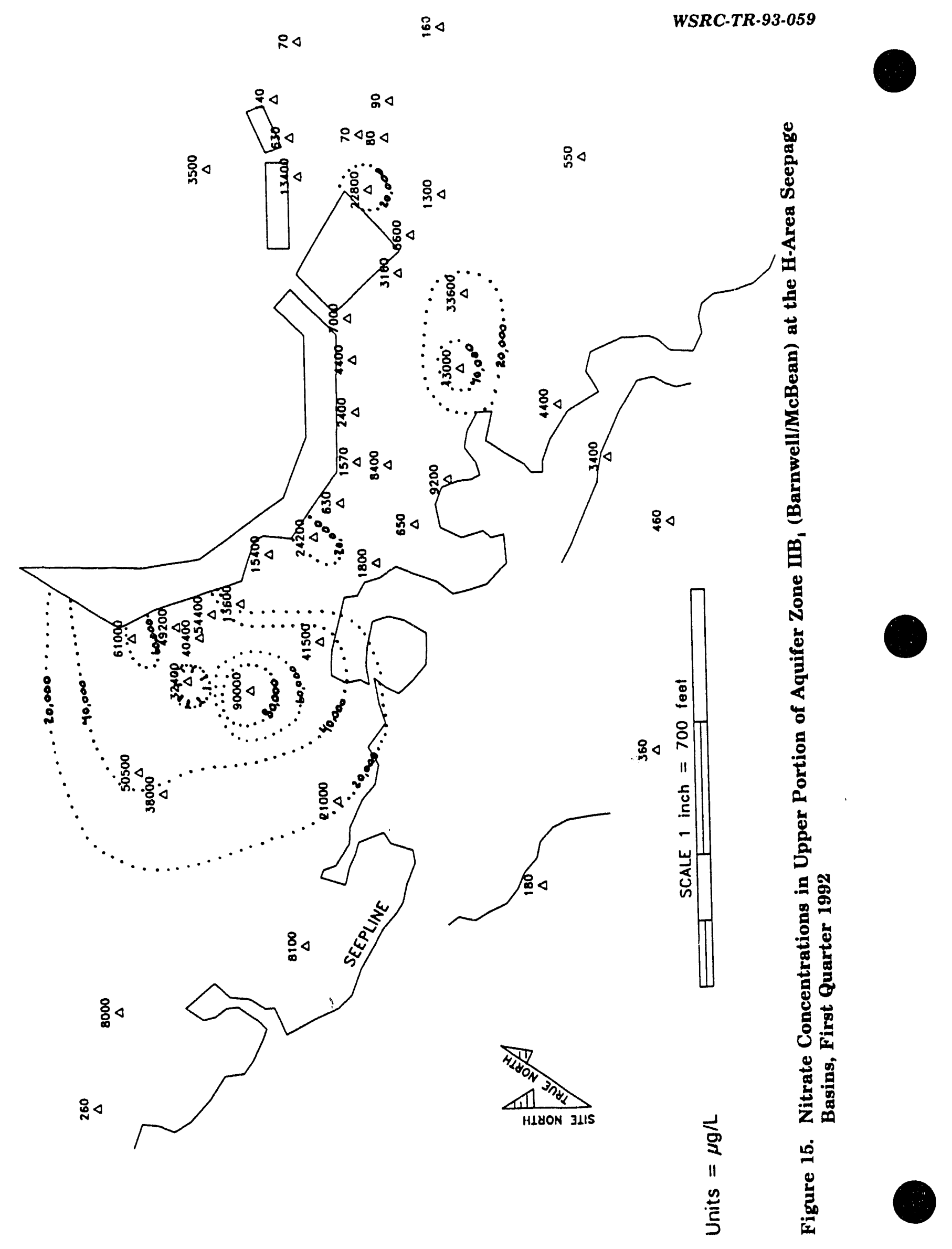

$\stackrel{\circ}{\circ} \triangleleft$ 


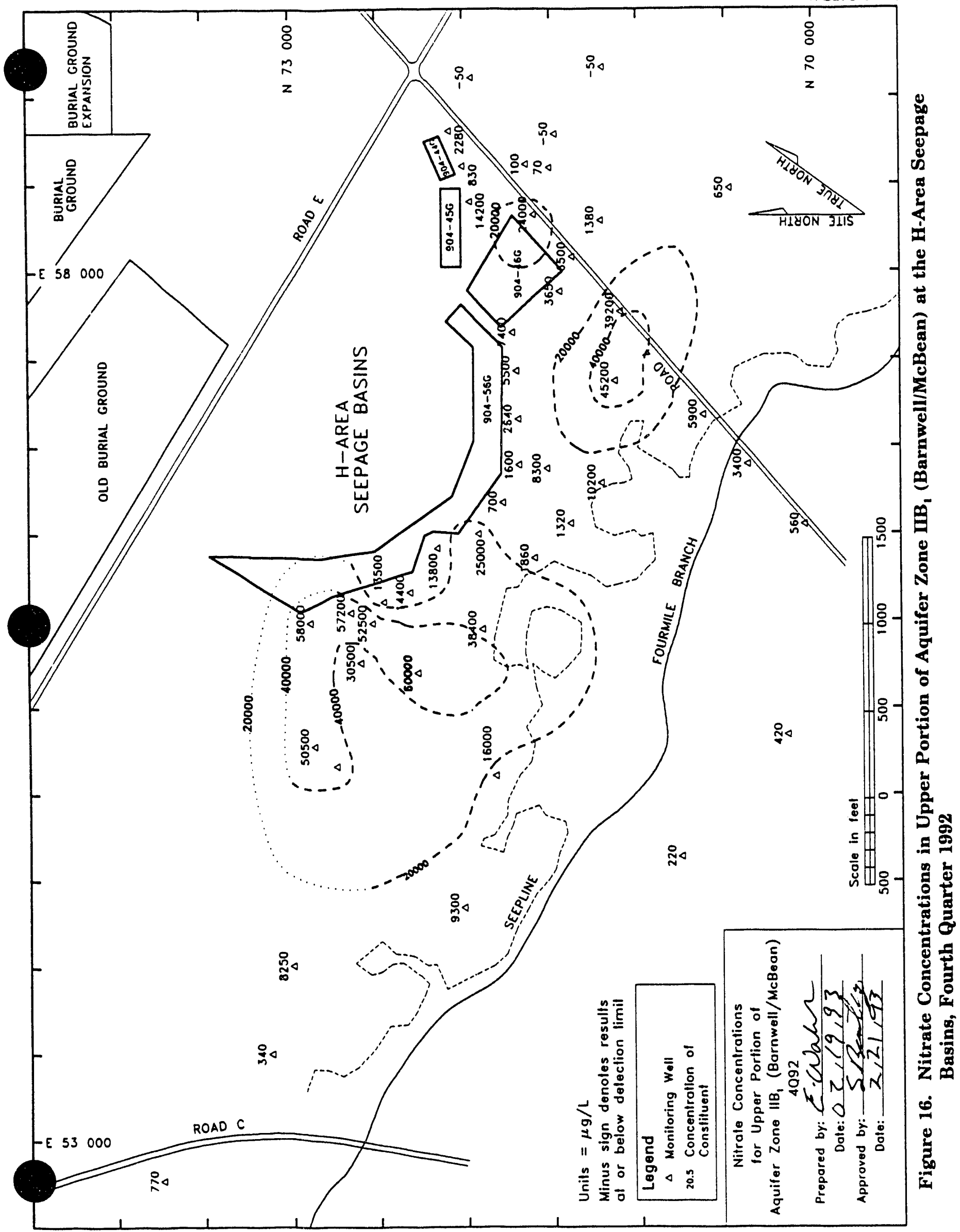


.

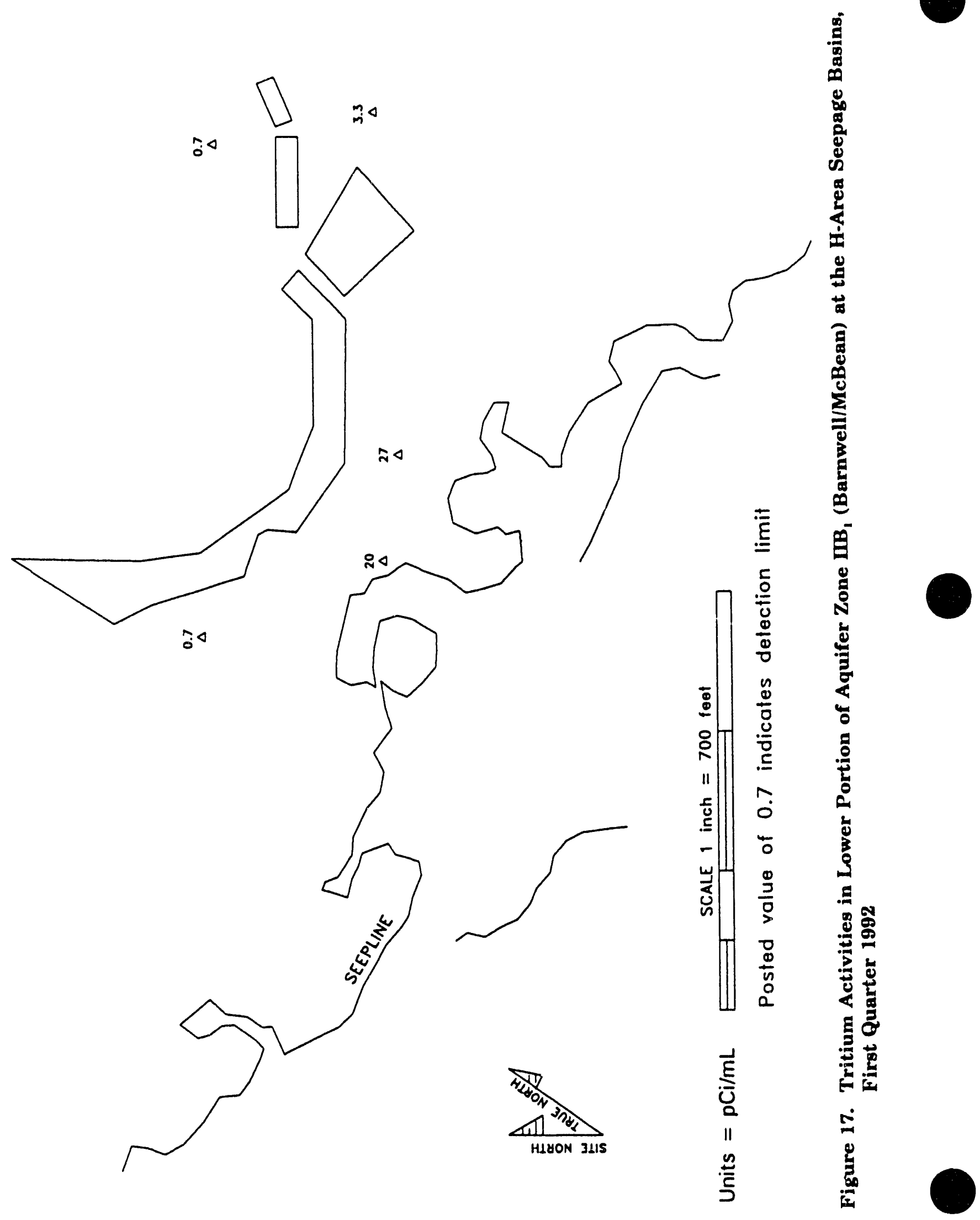




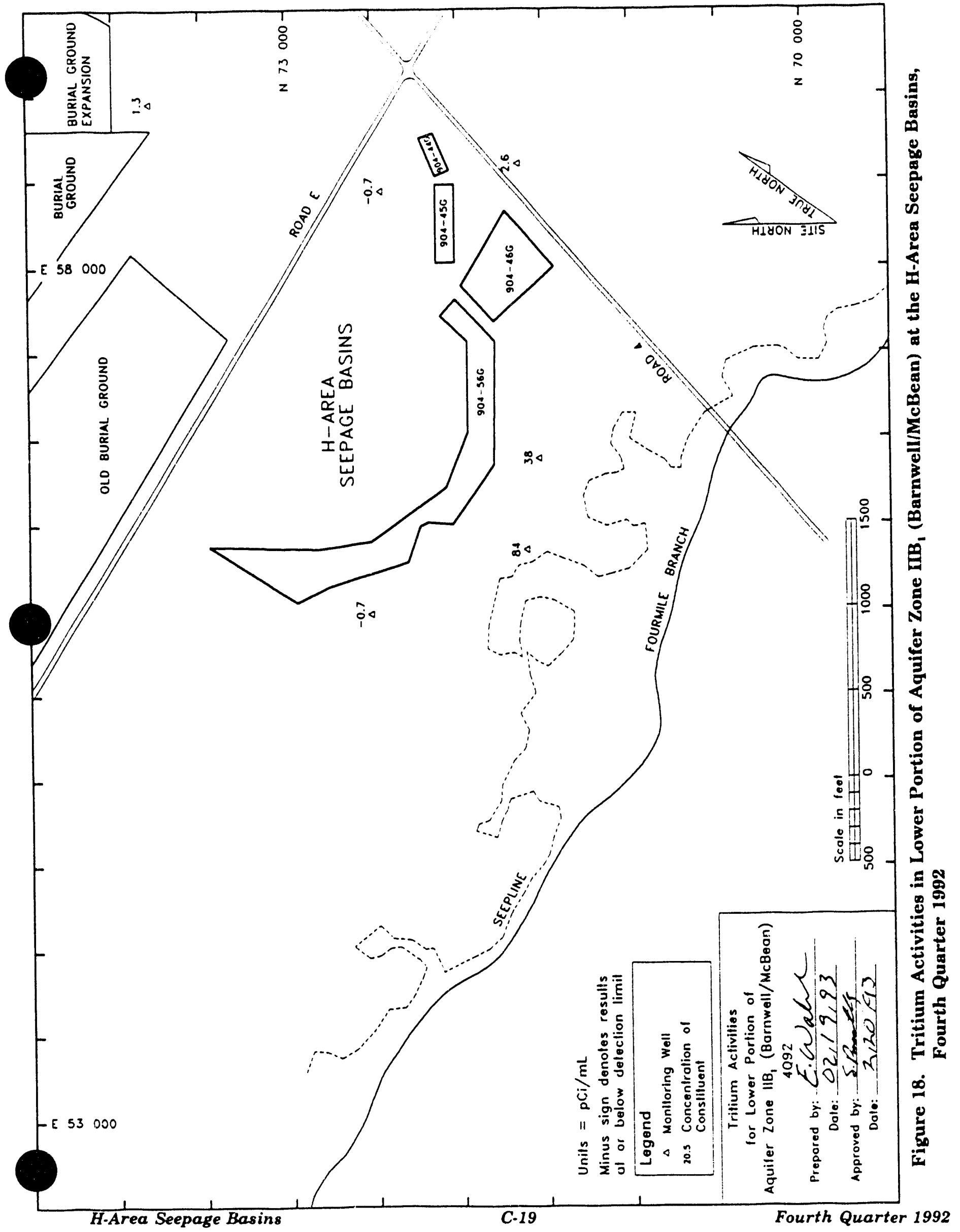


$\underline{-}$

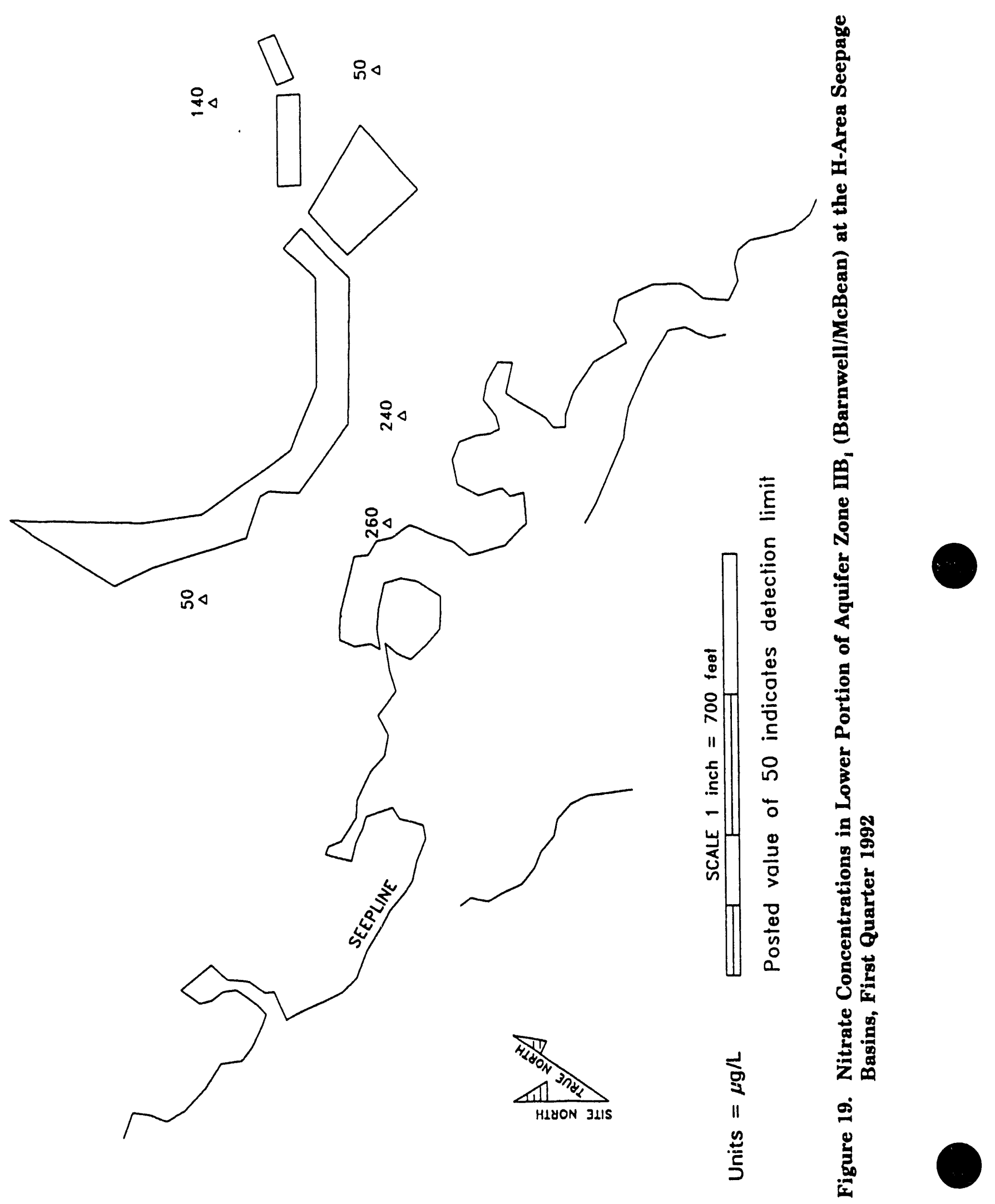




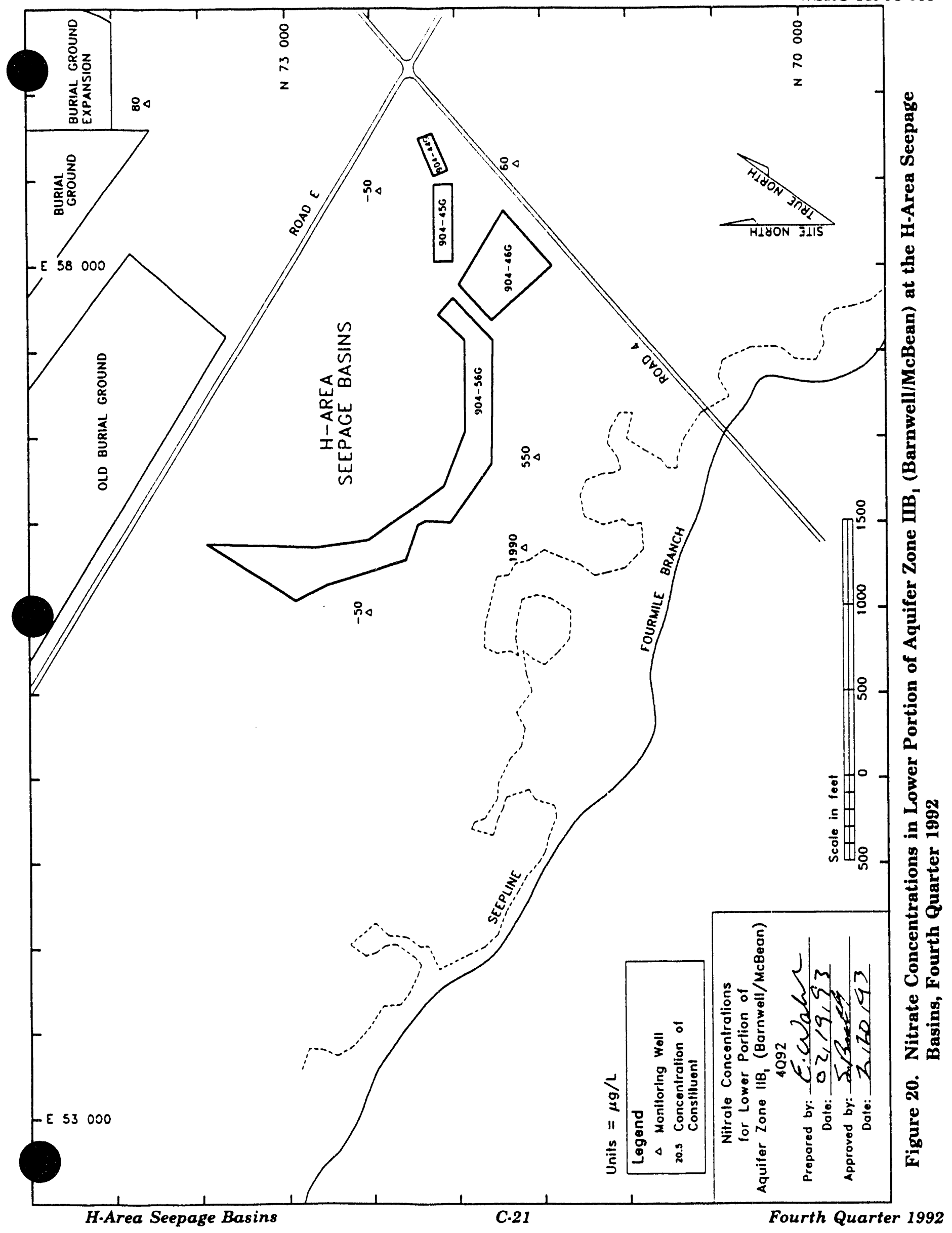


ก.

م.

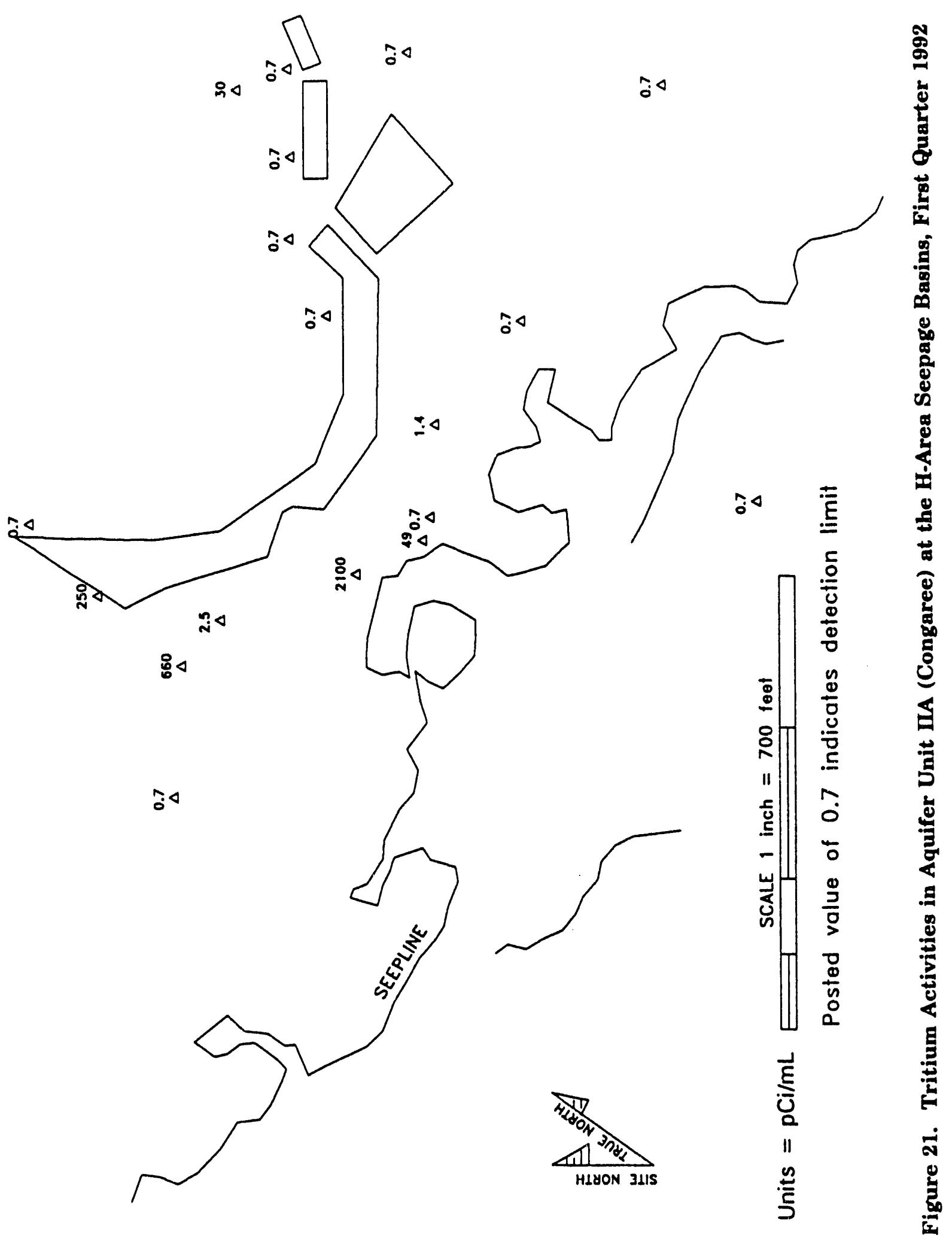




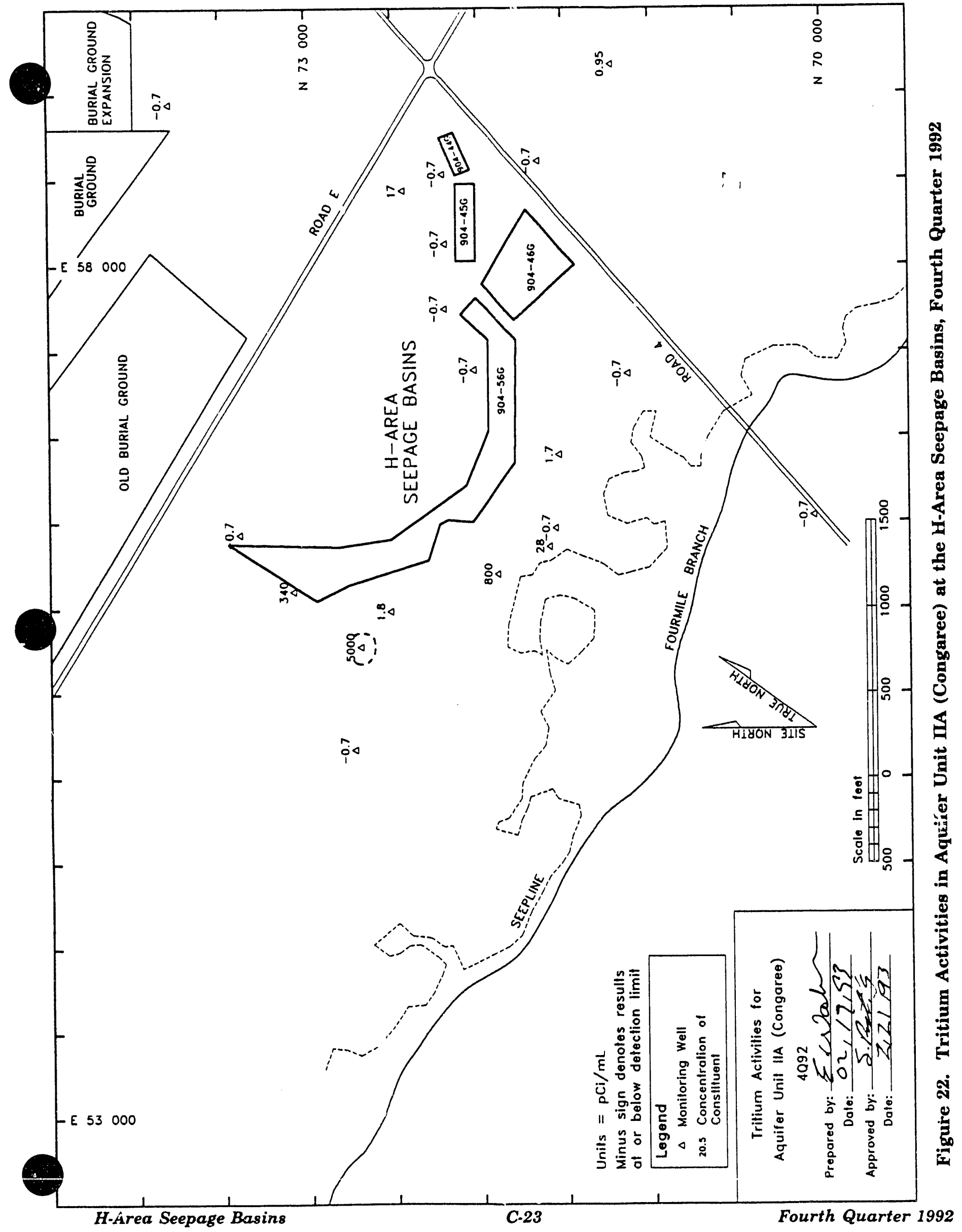


은

은

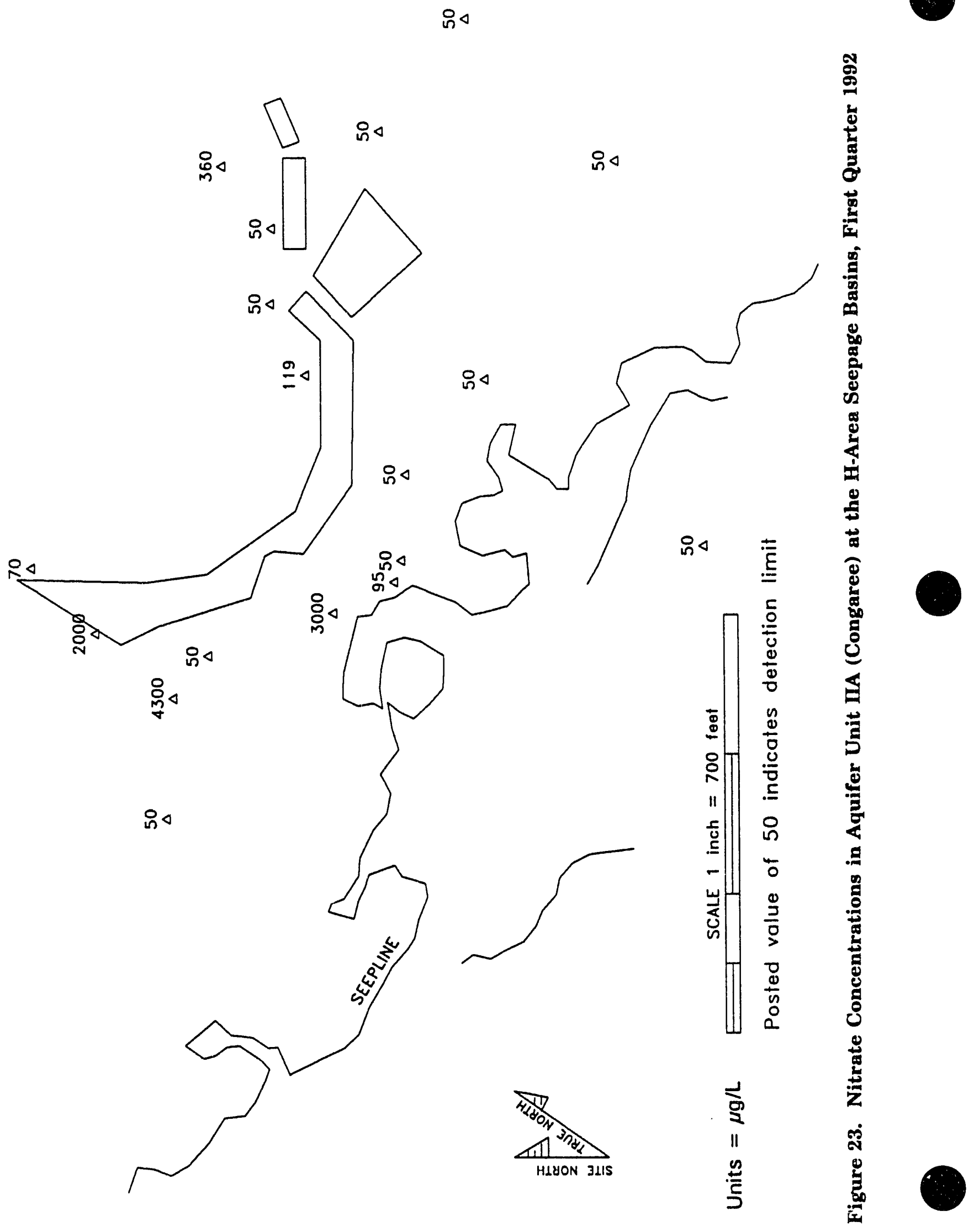




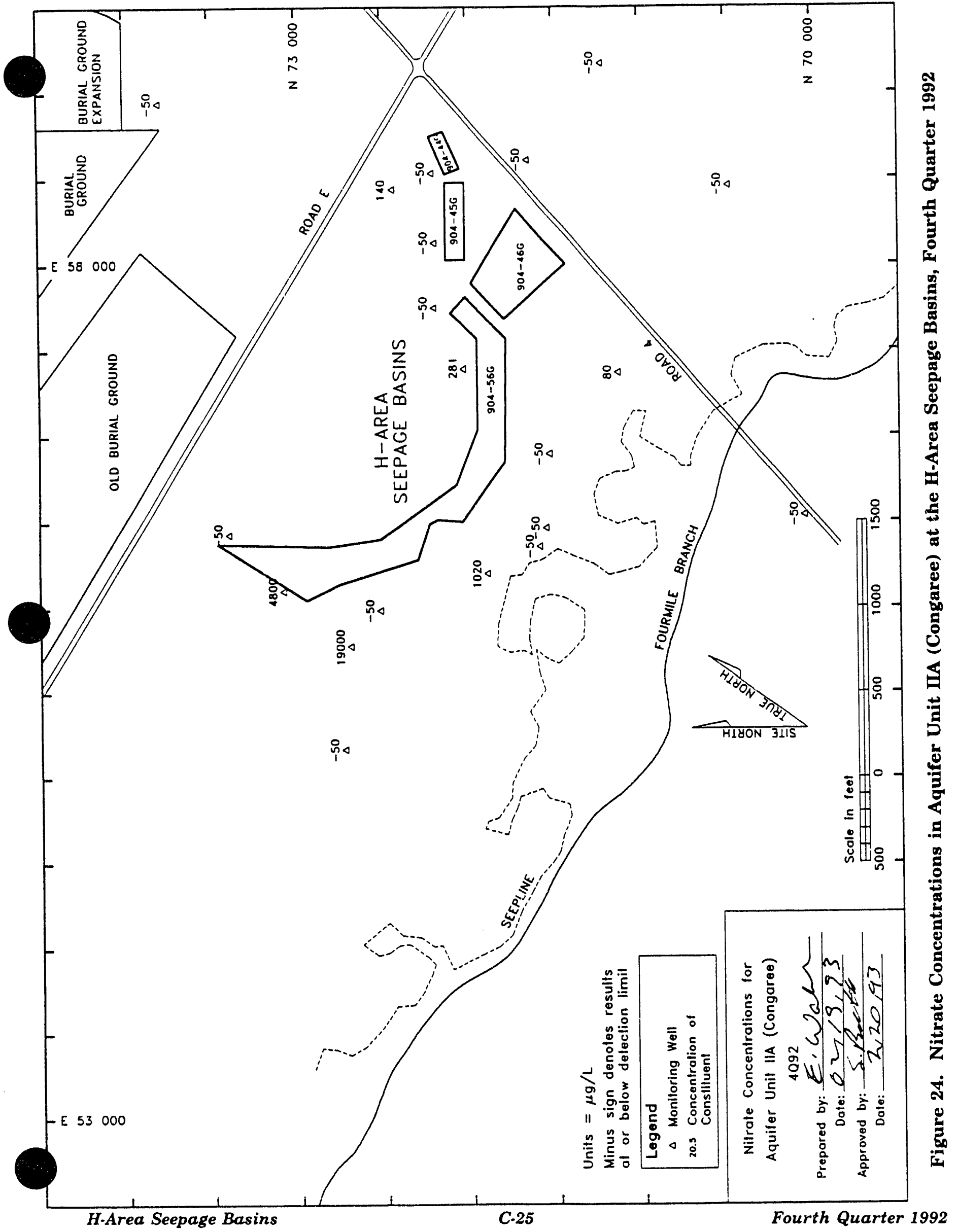


$N \triangle$

$N S$

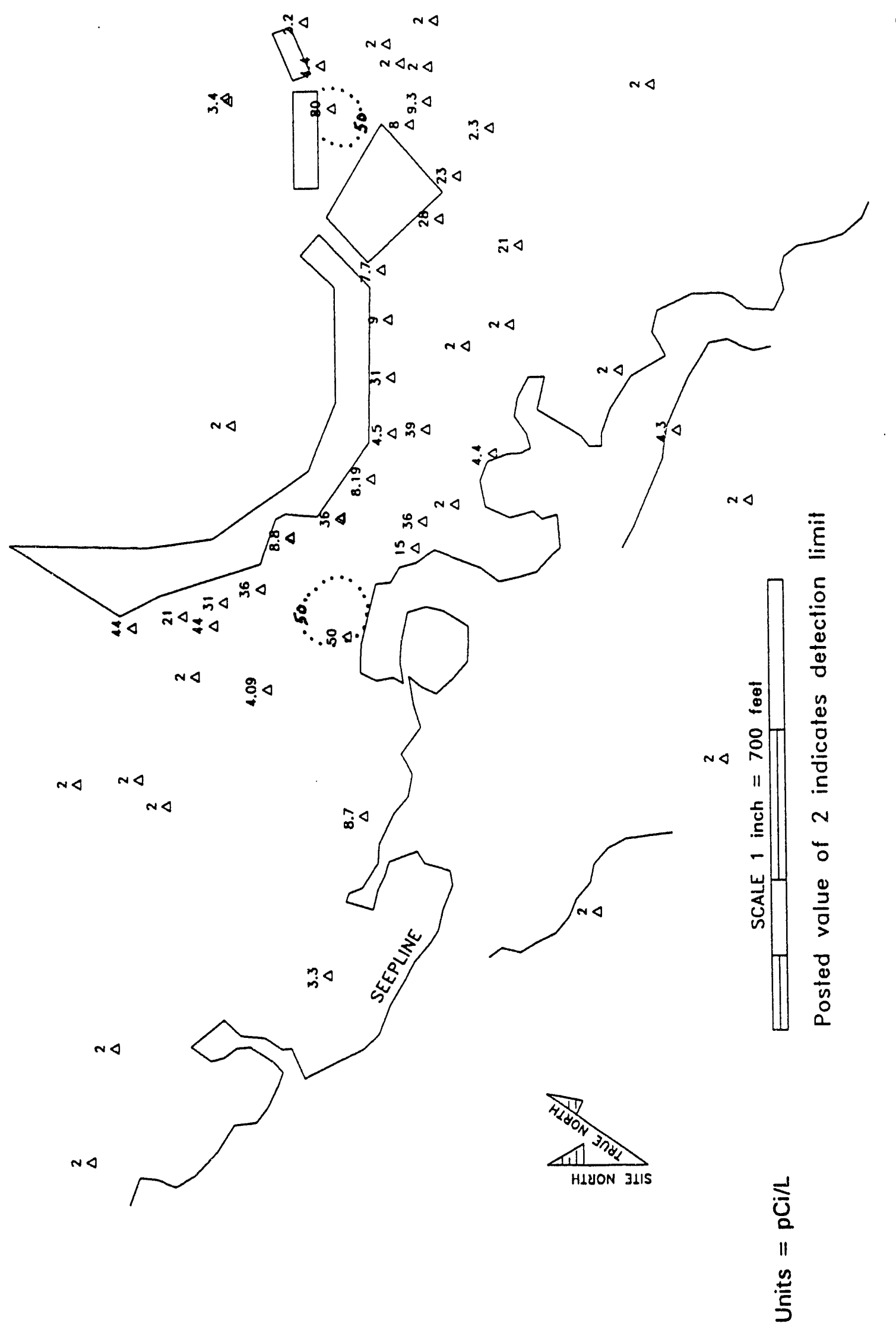

总

命 


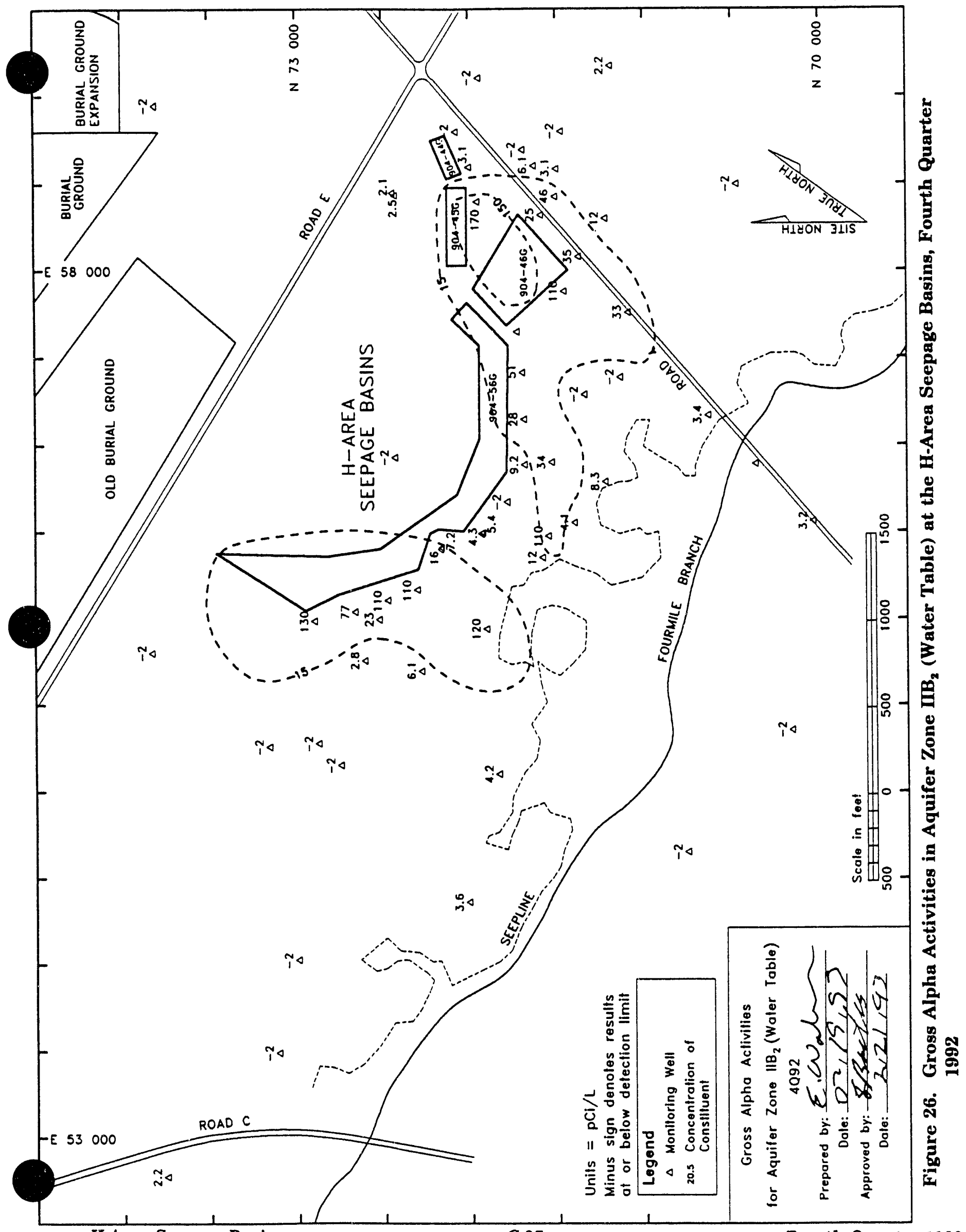




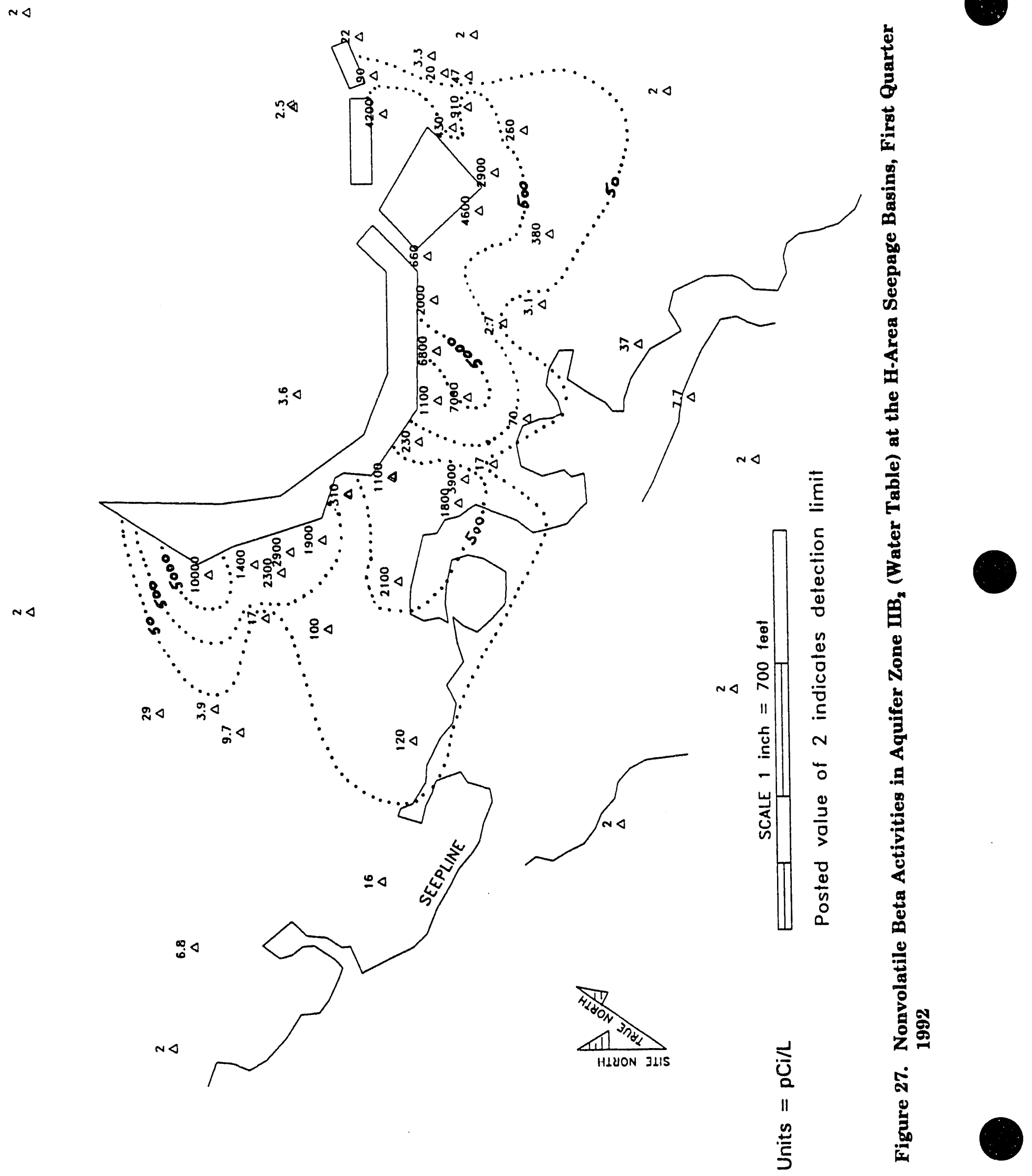

$N \triangleleft$ 


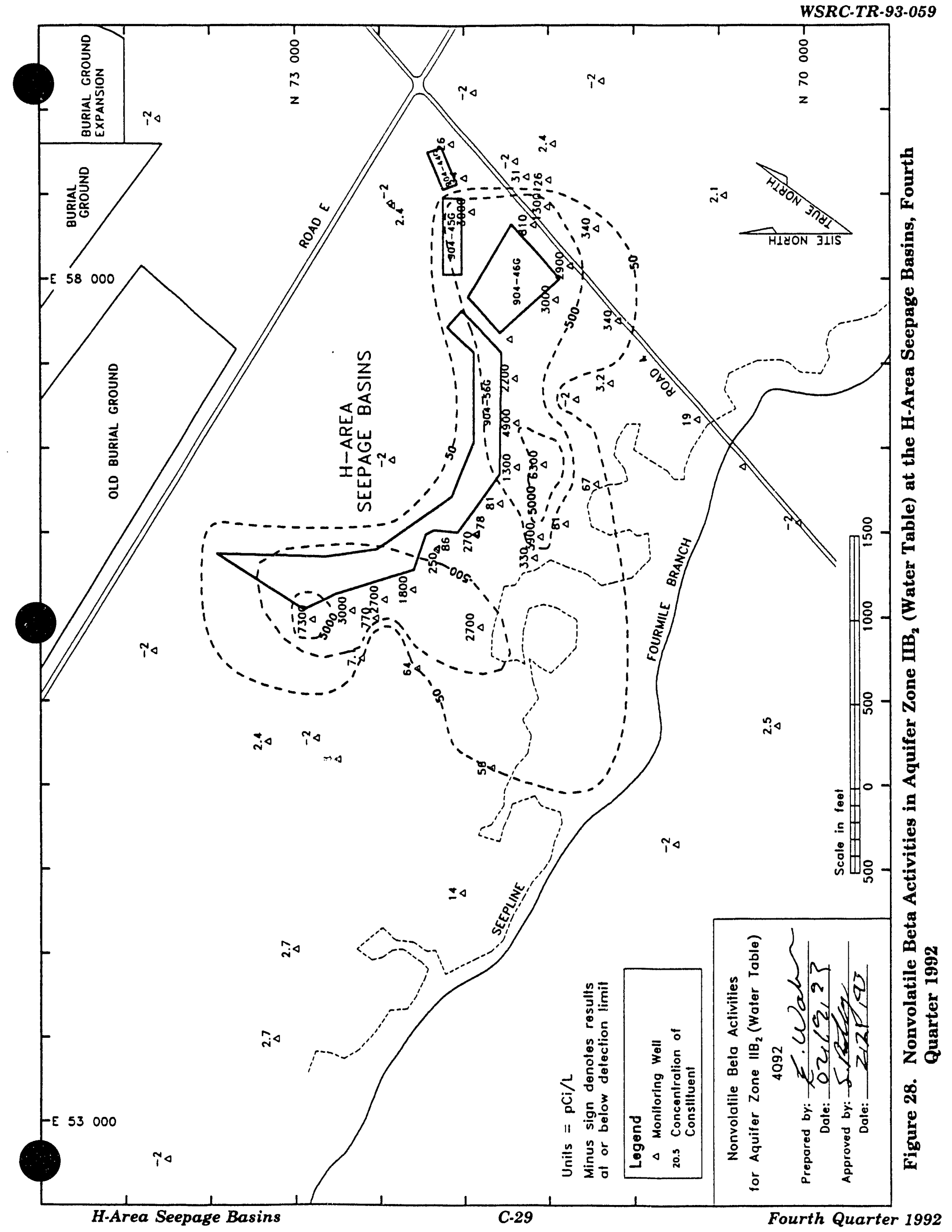




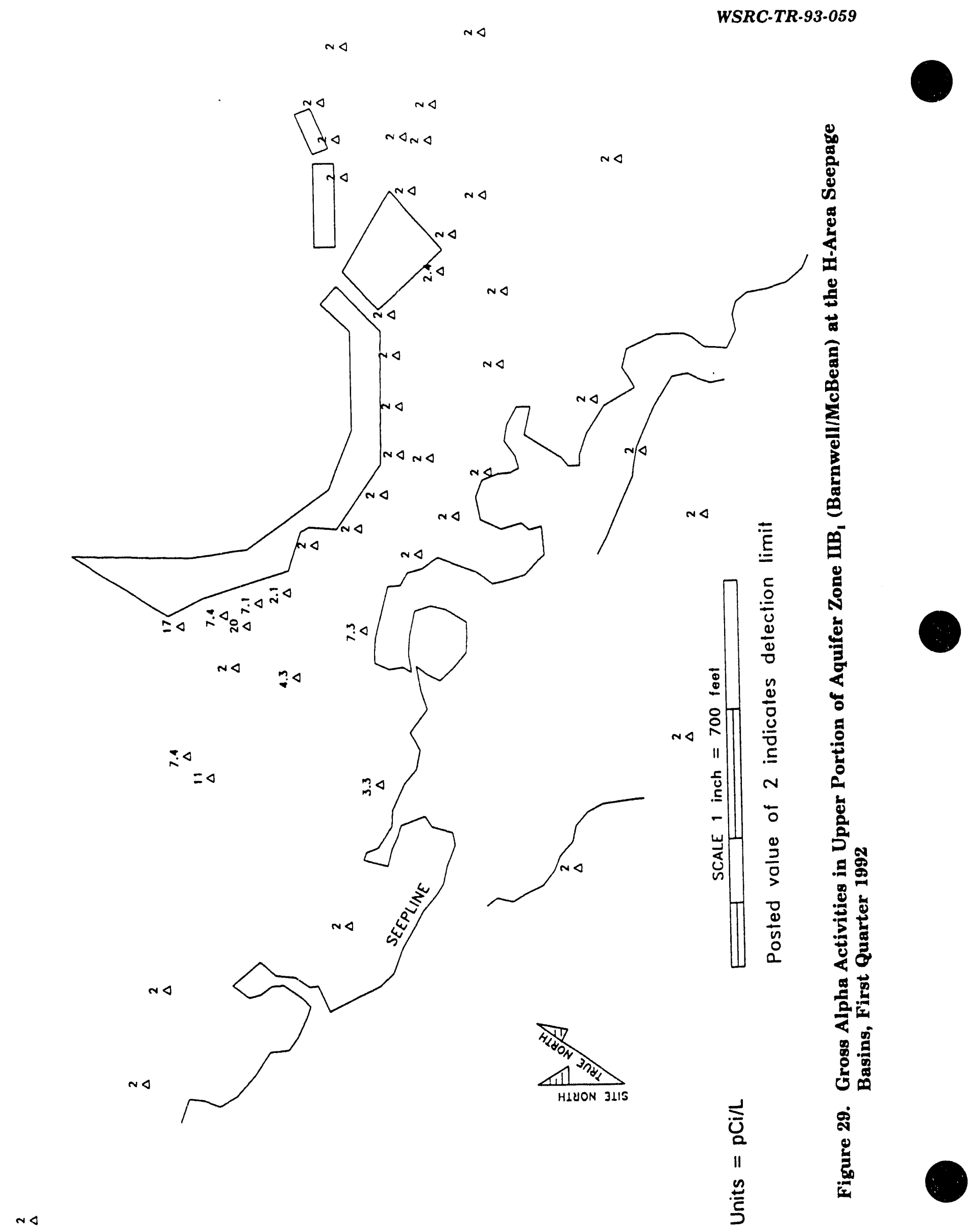




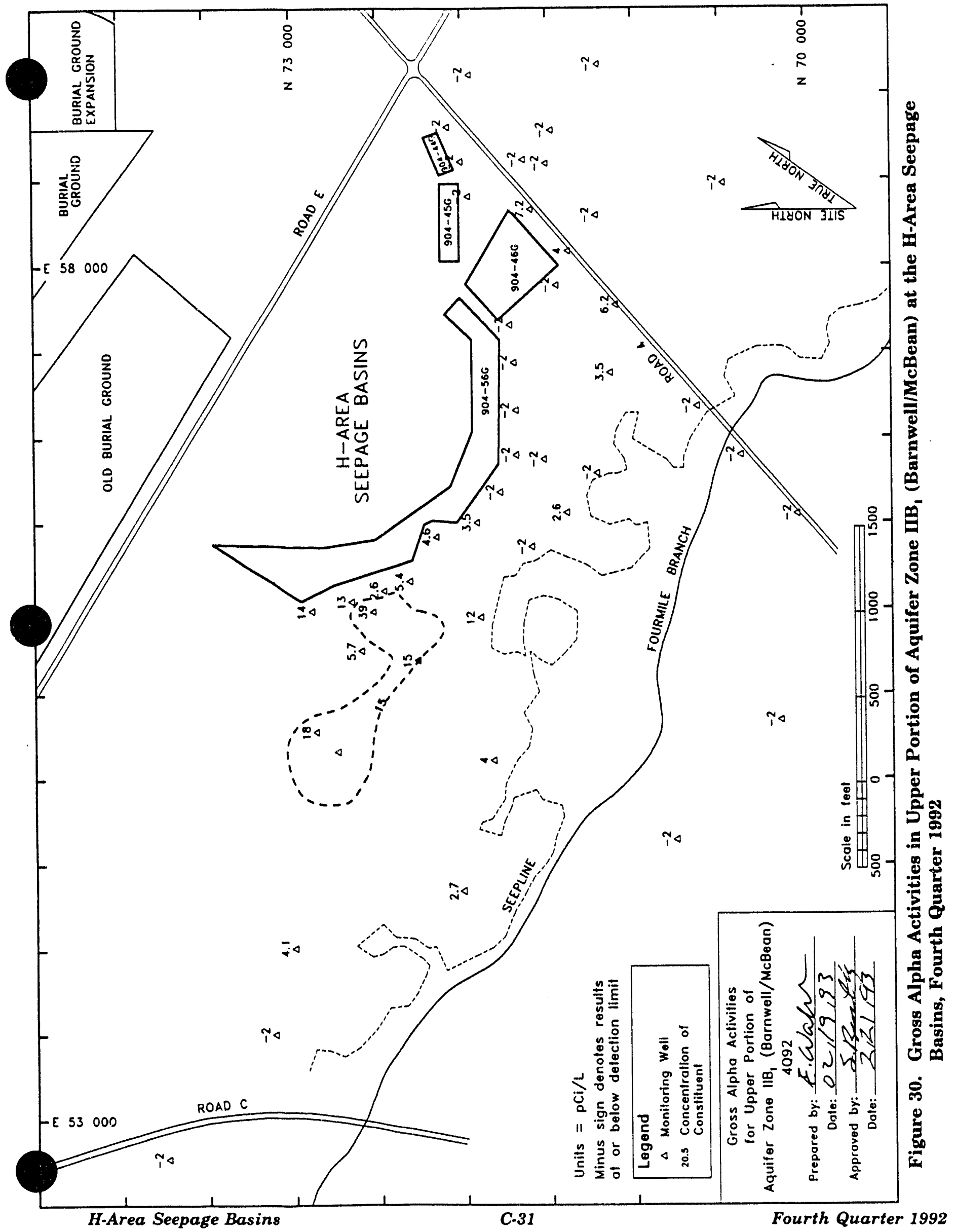




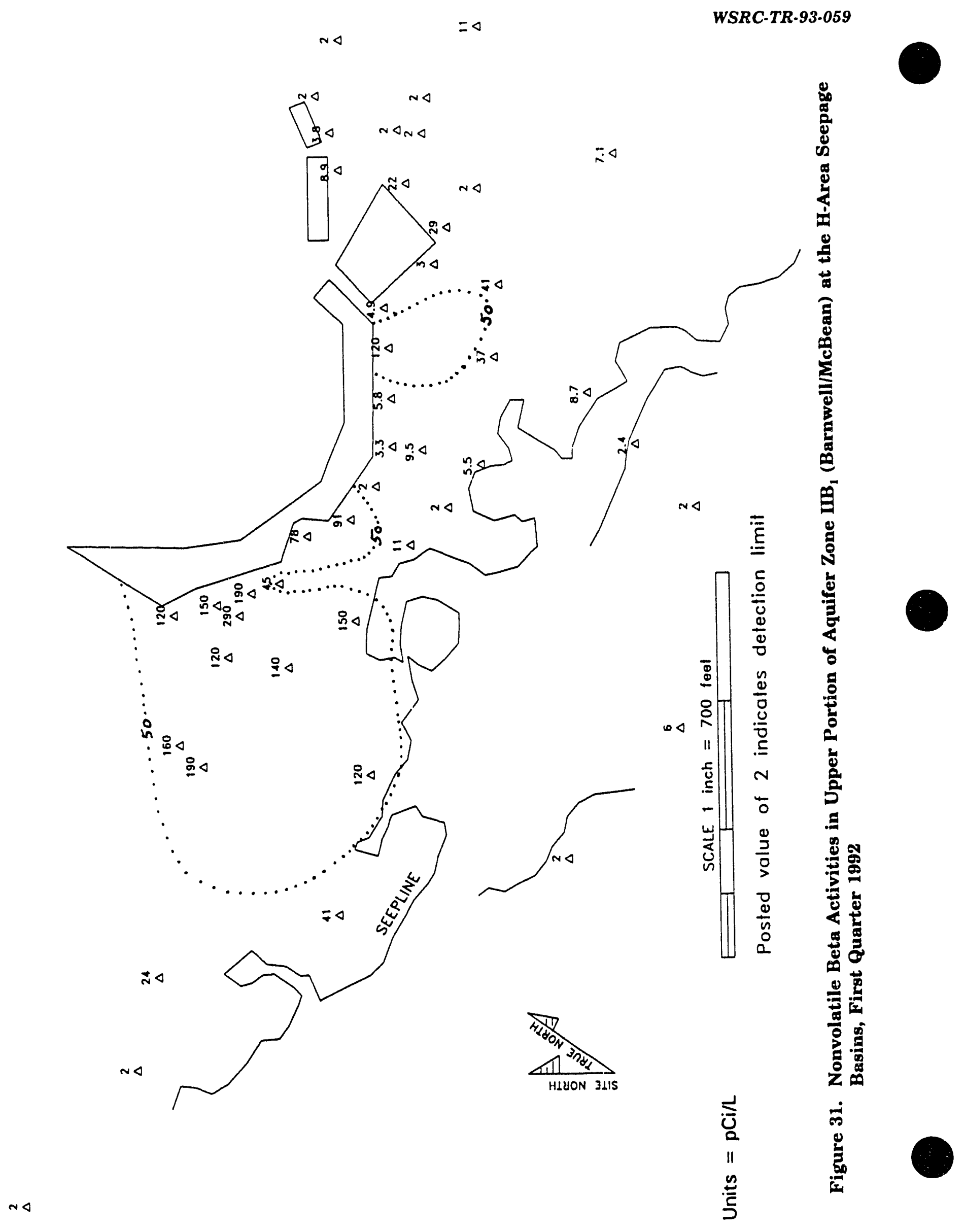




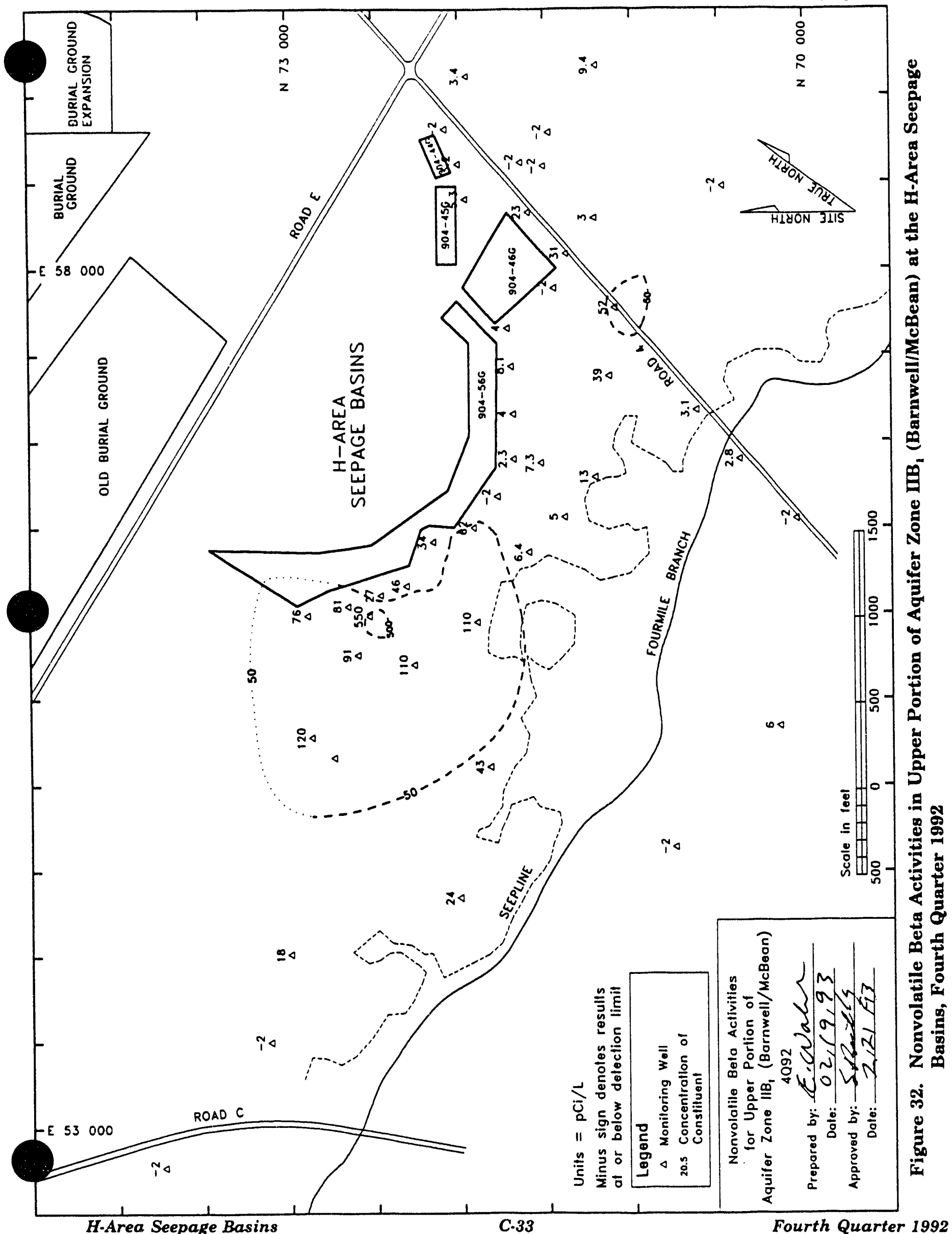




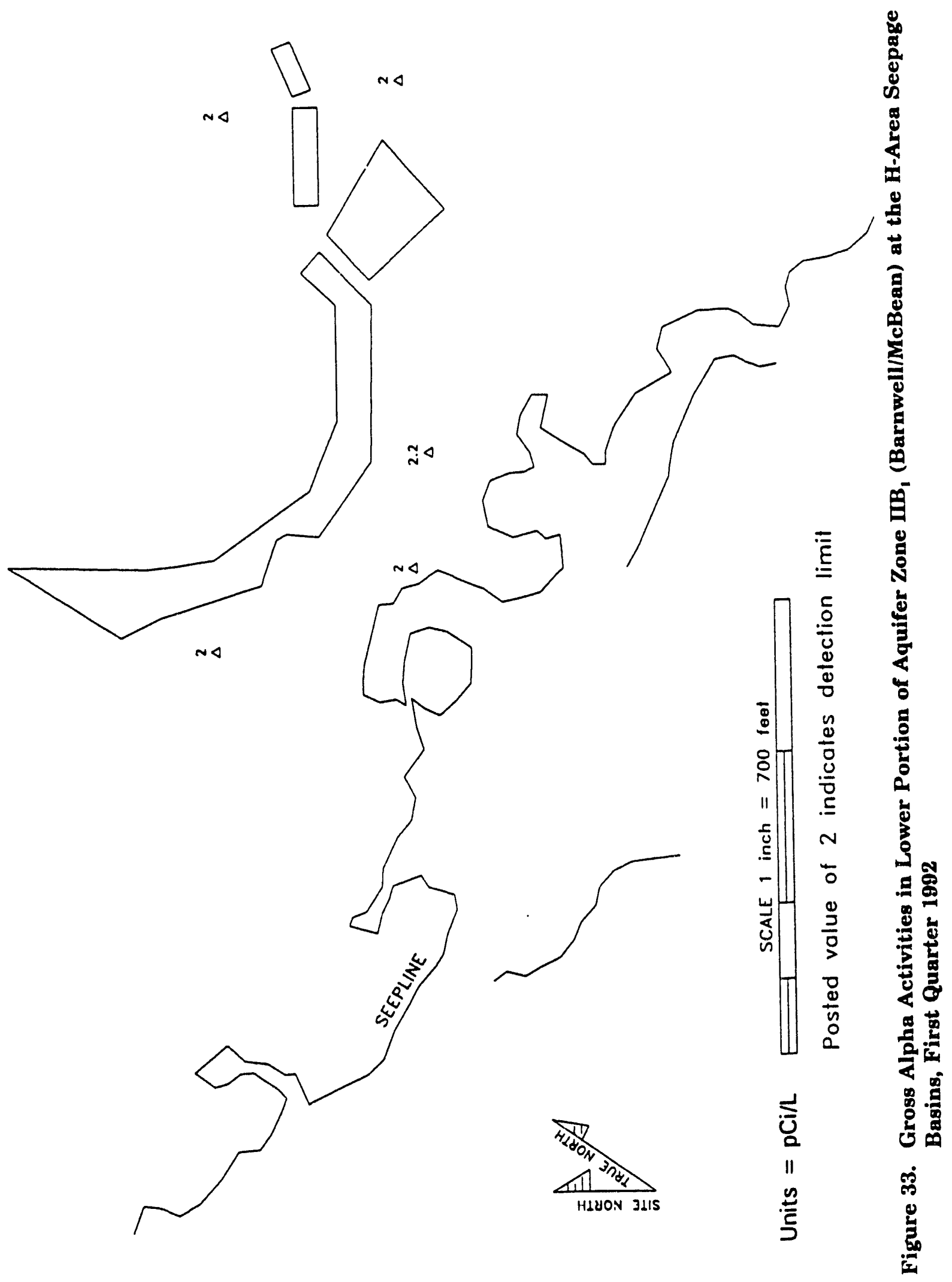




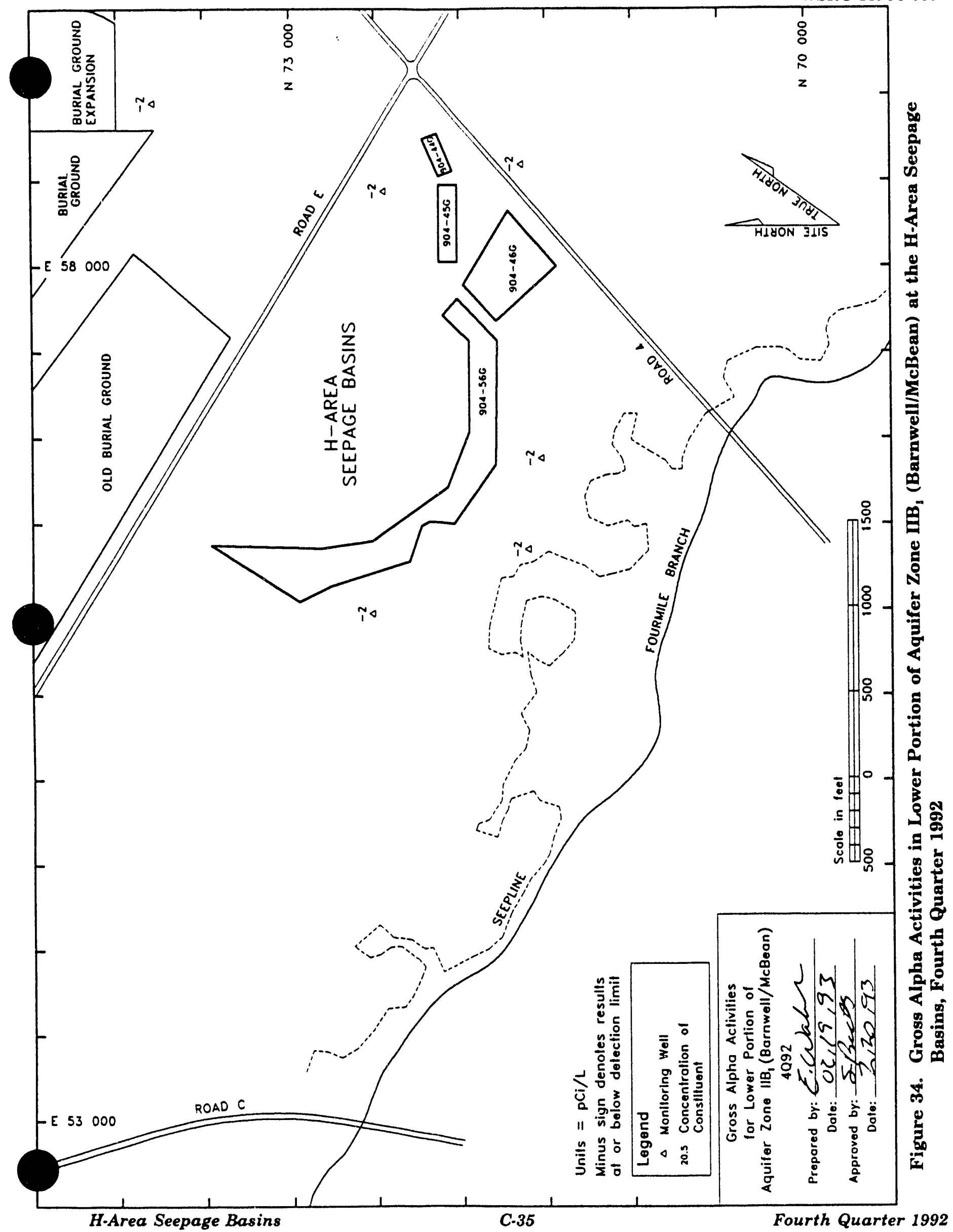




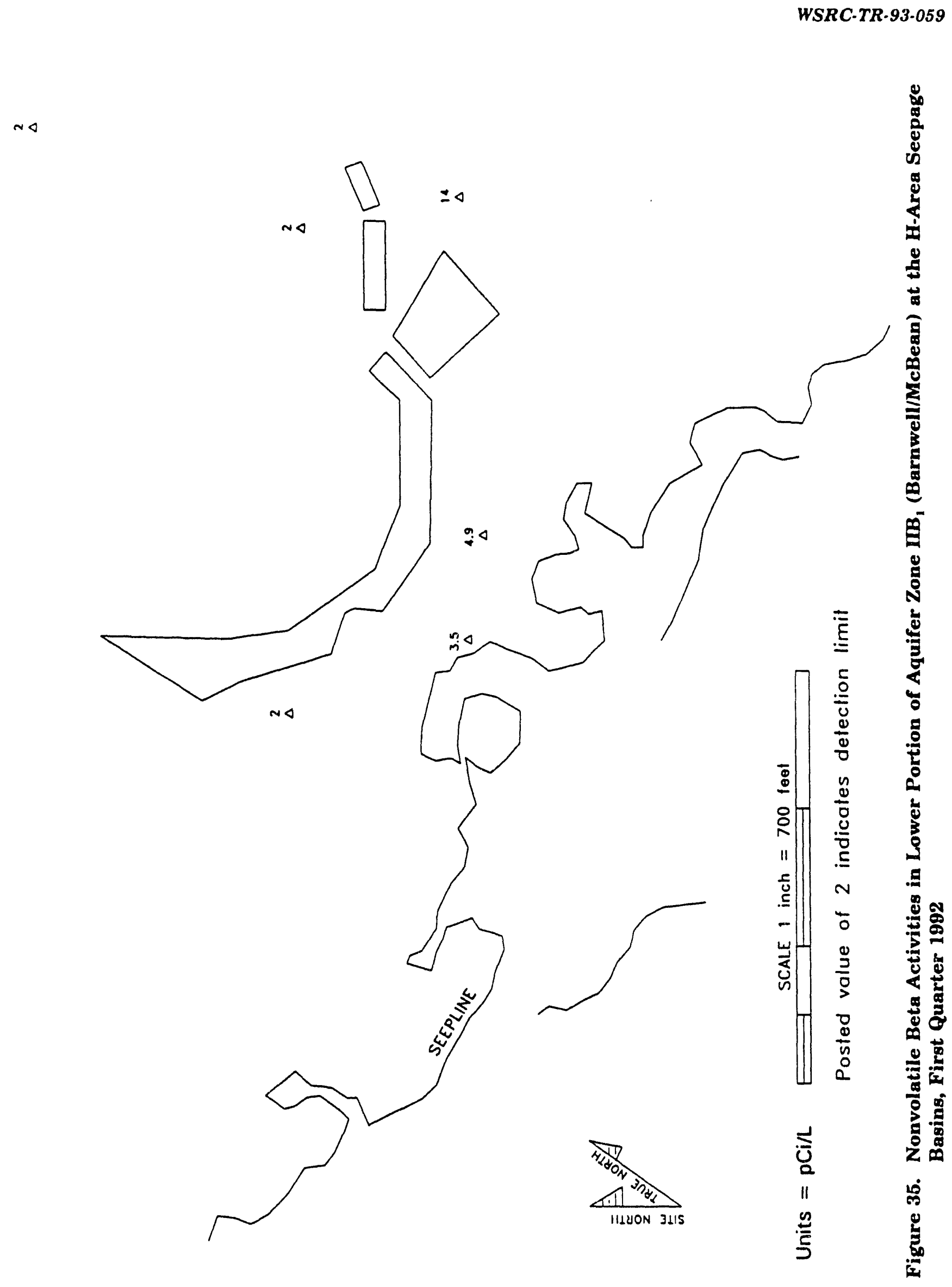




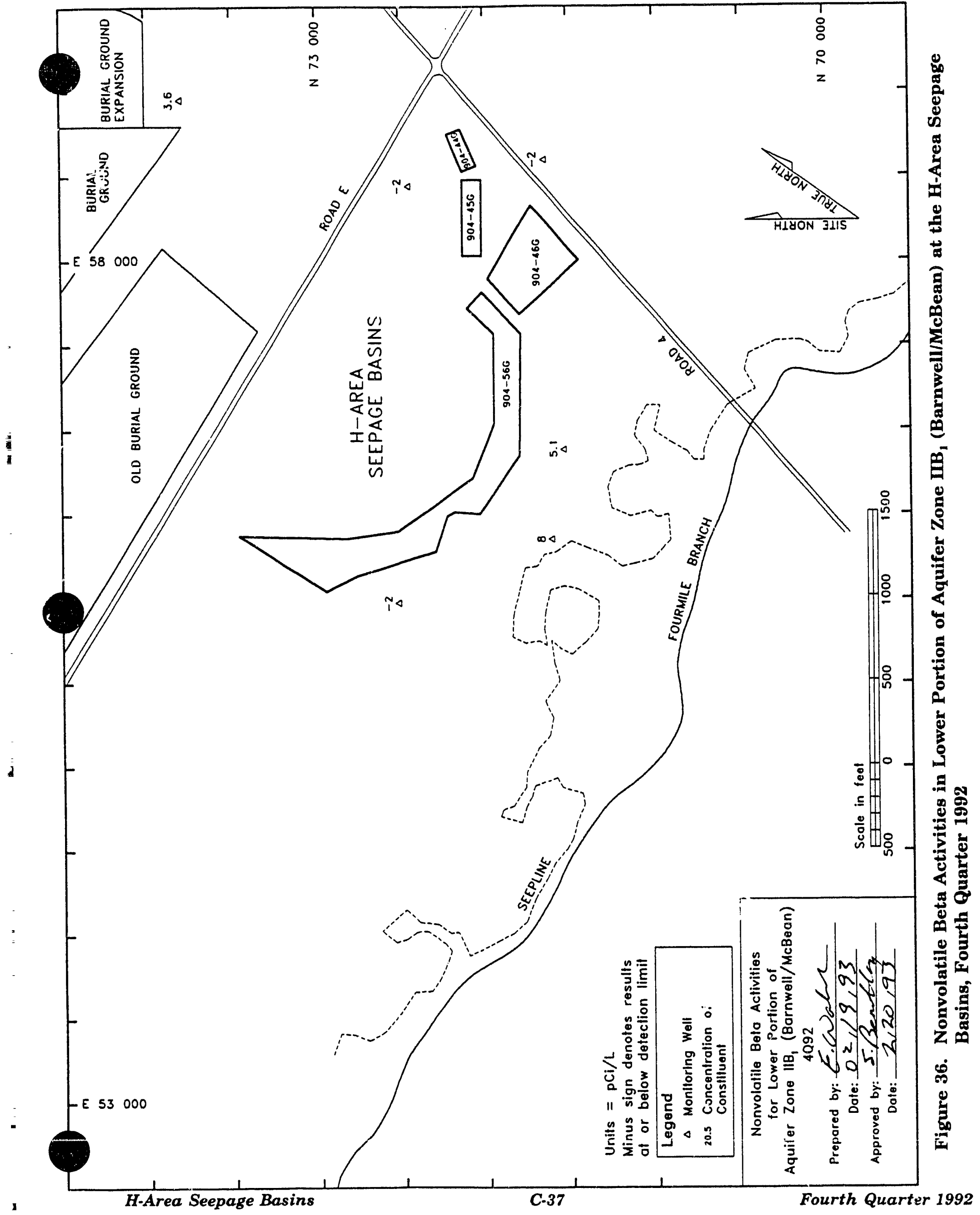


$N \triangleleft$

$m \triangleleft$

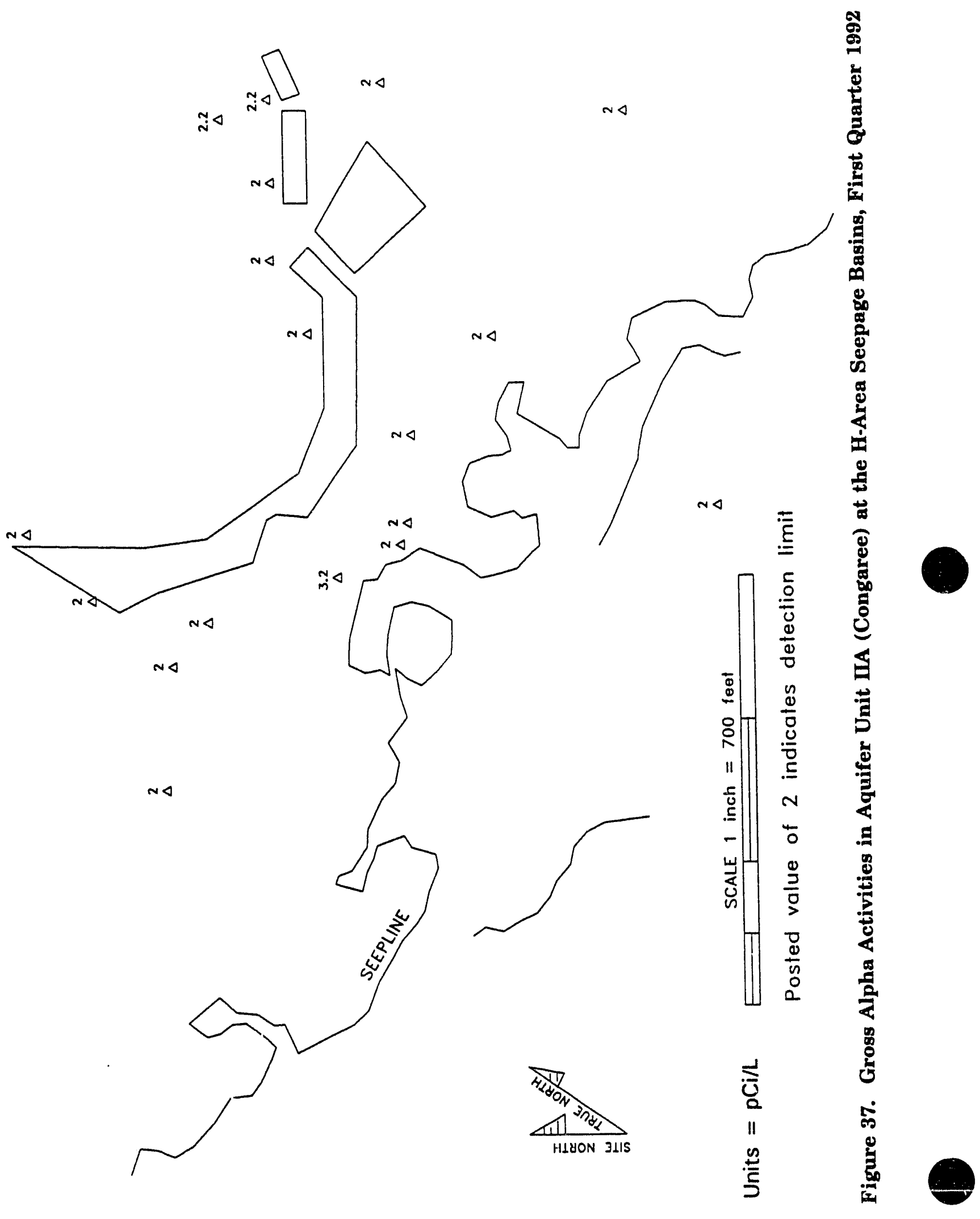




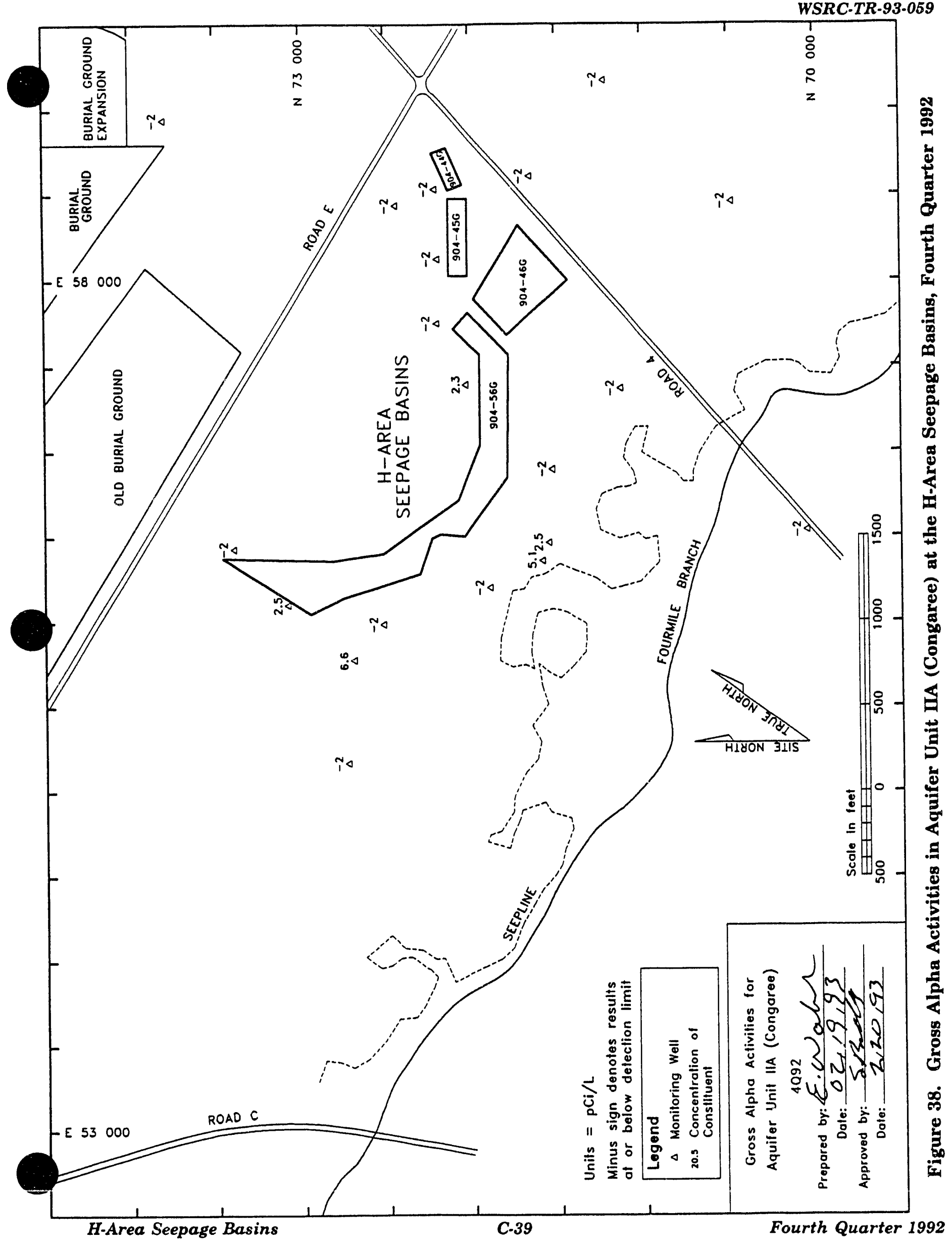


in

$n \triangleleft$

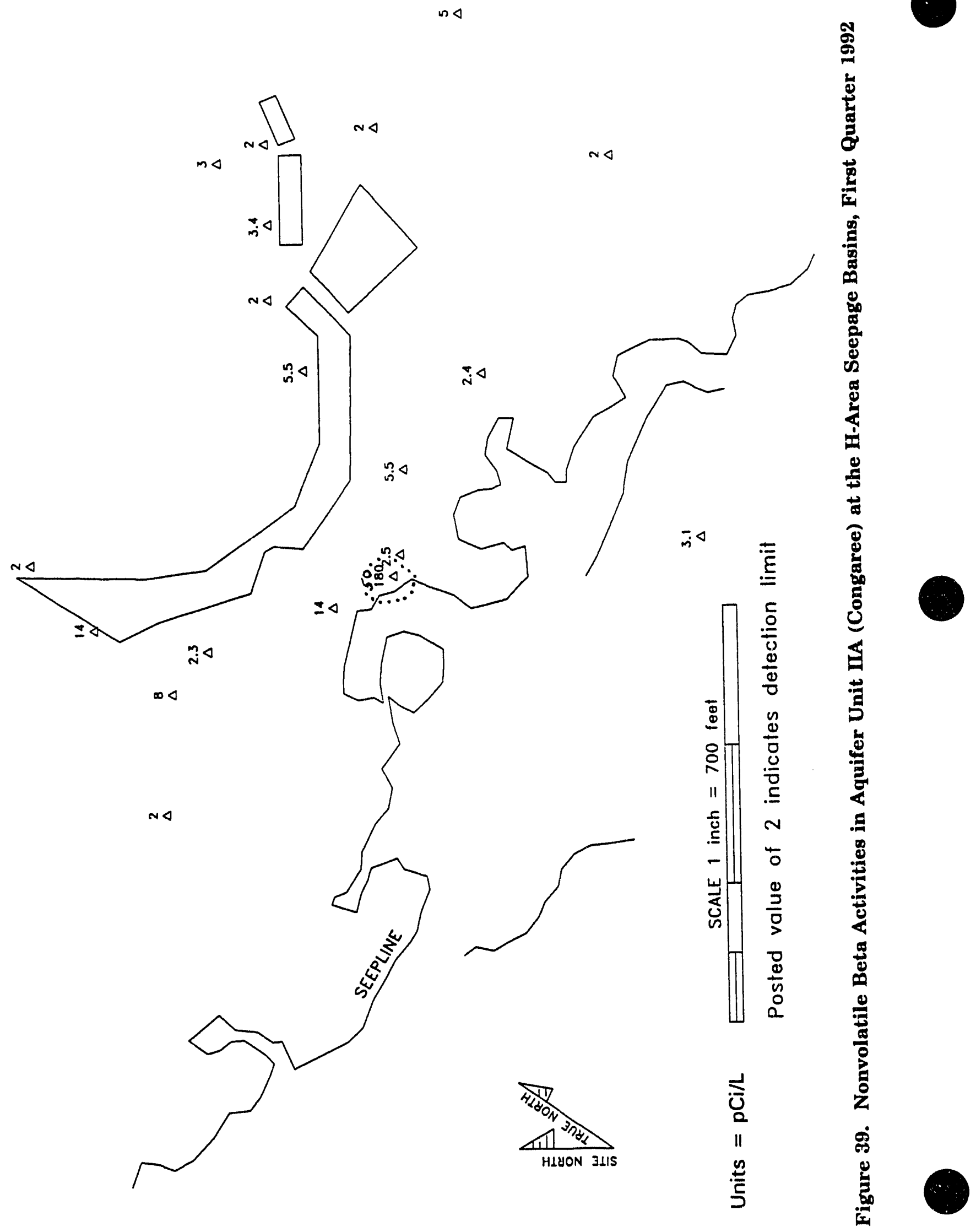




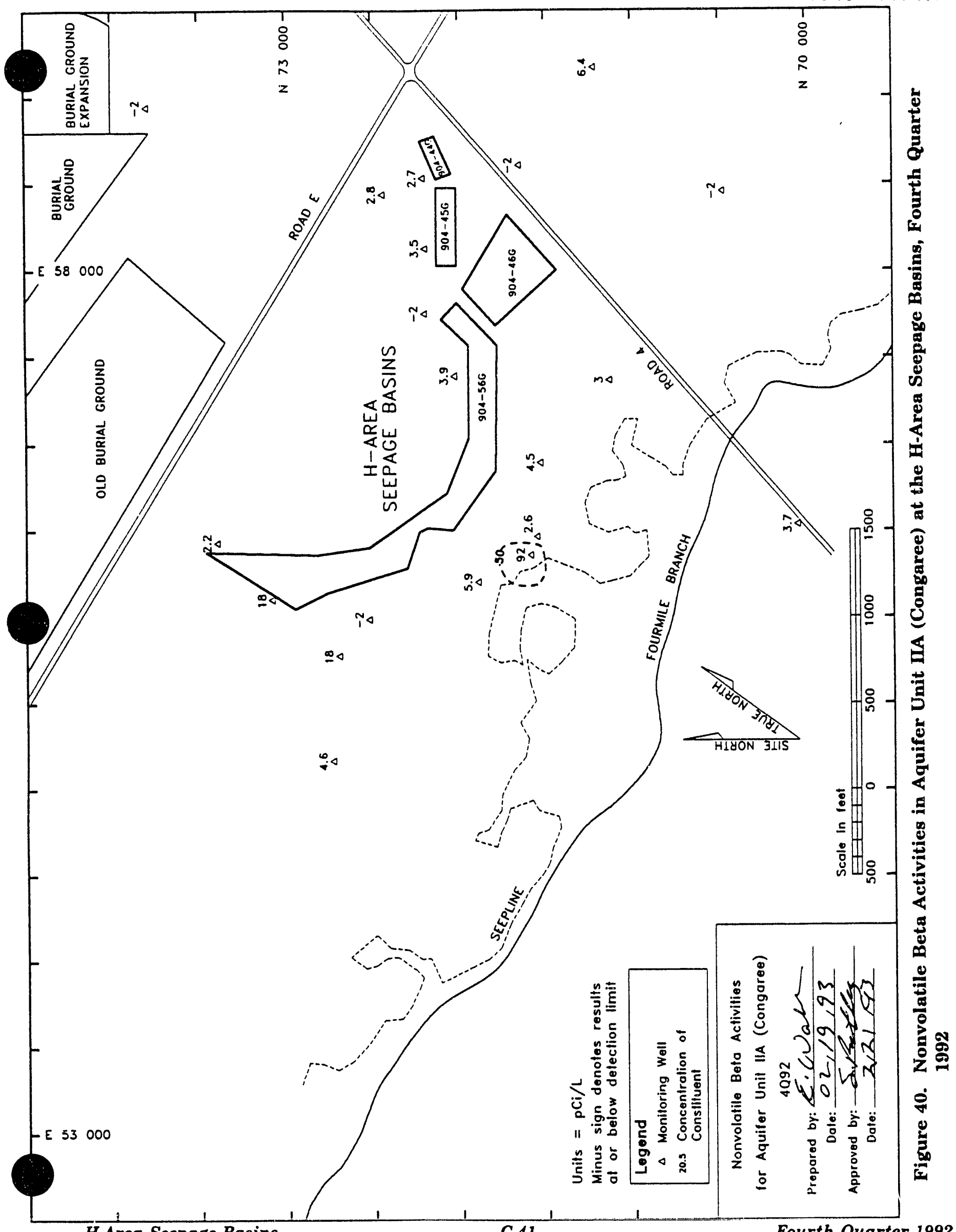




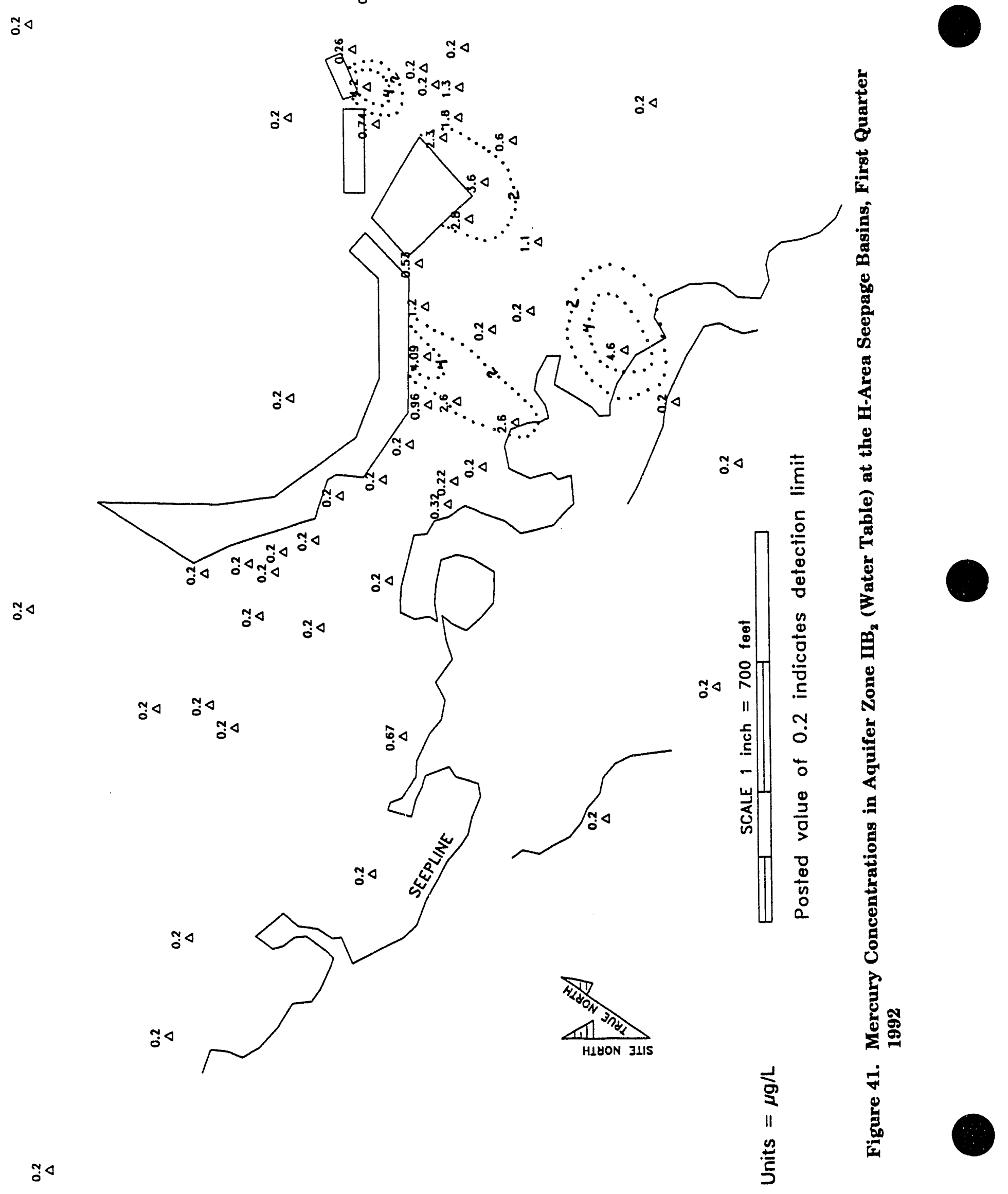




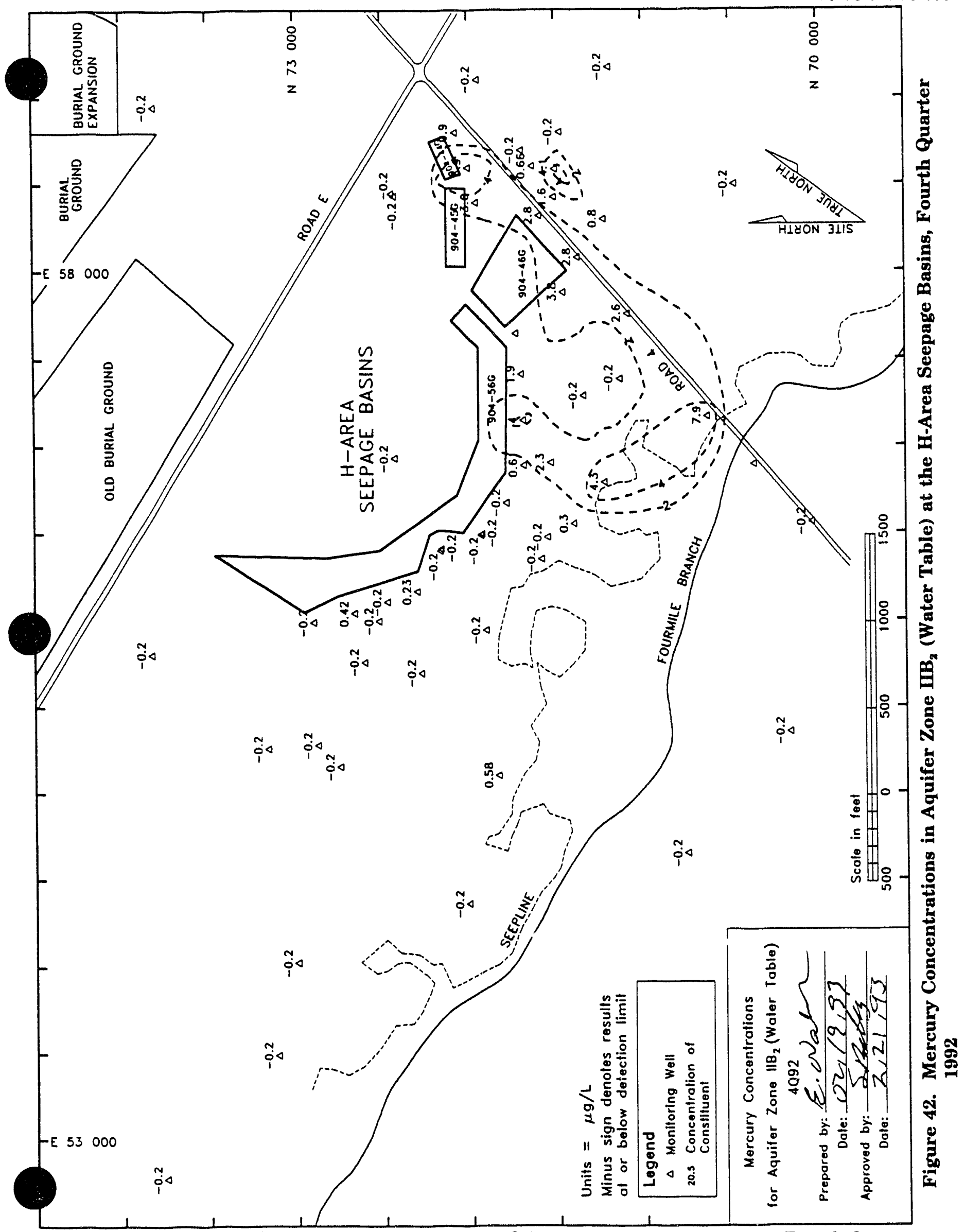




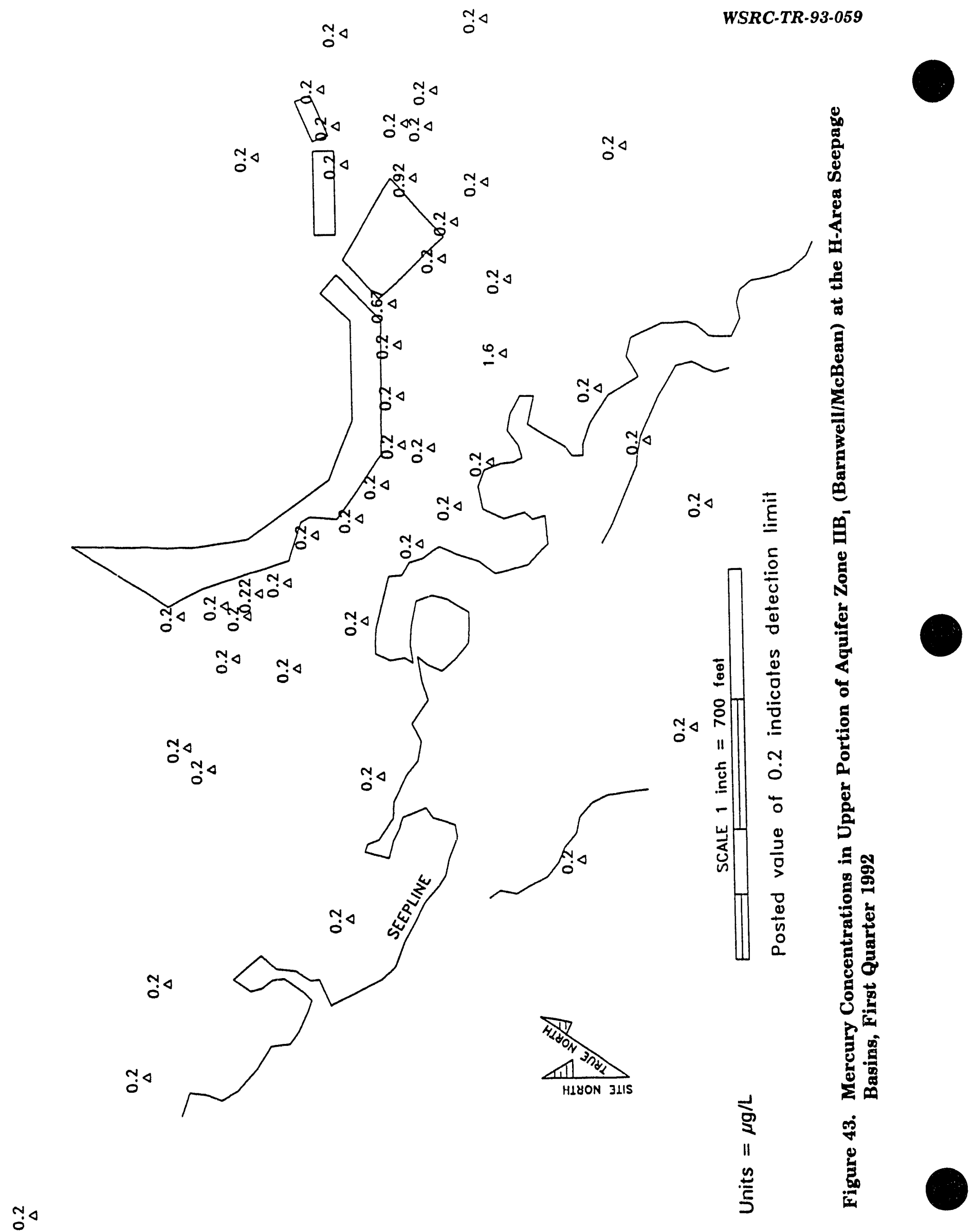




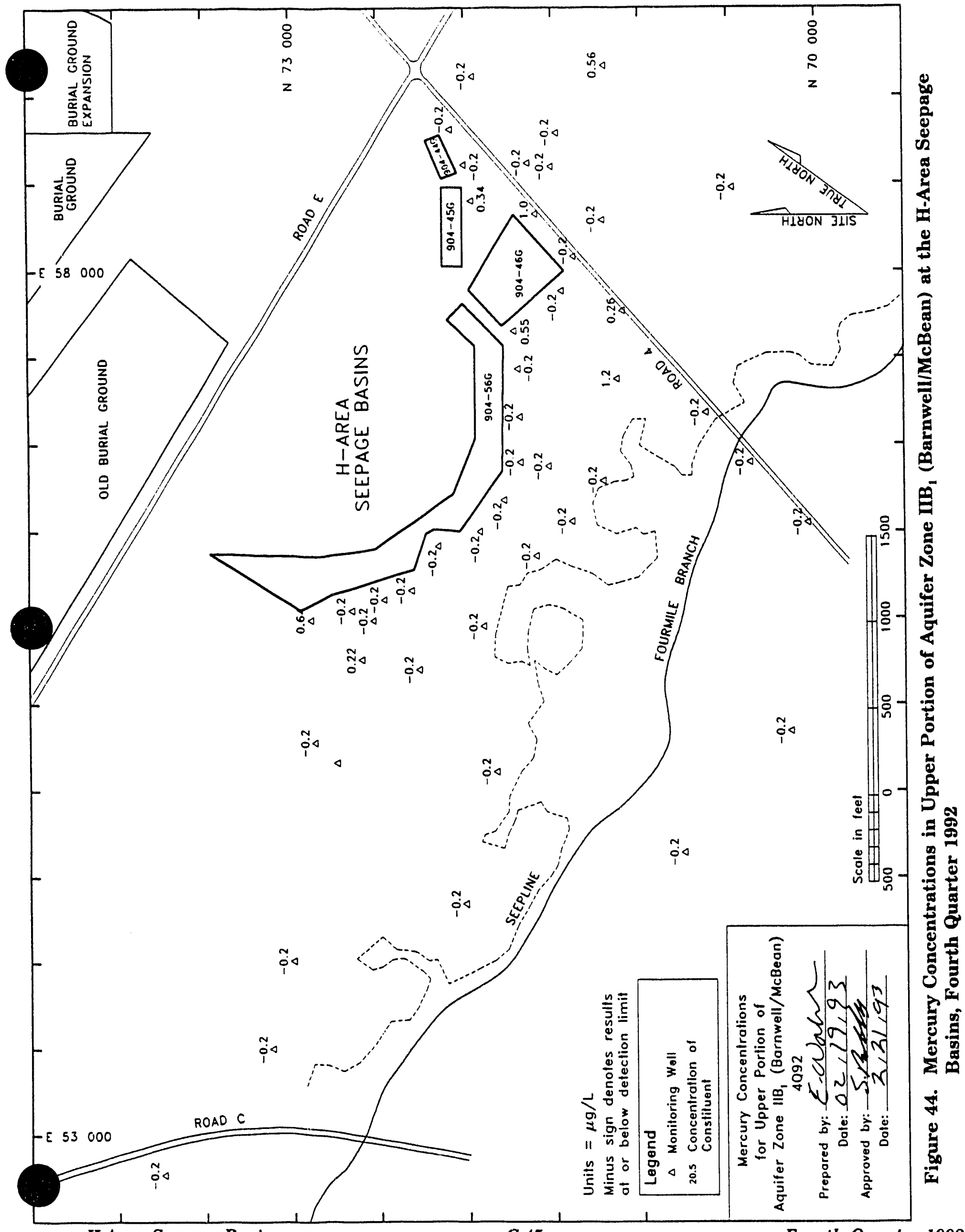


WSRC-TR-93-059

กิธ

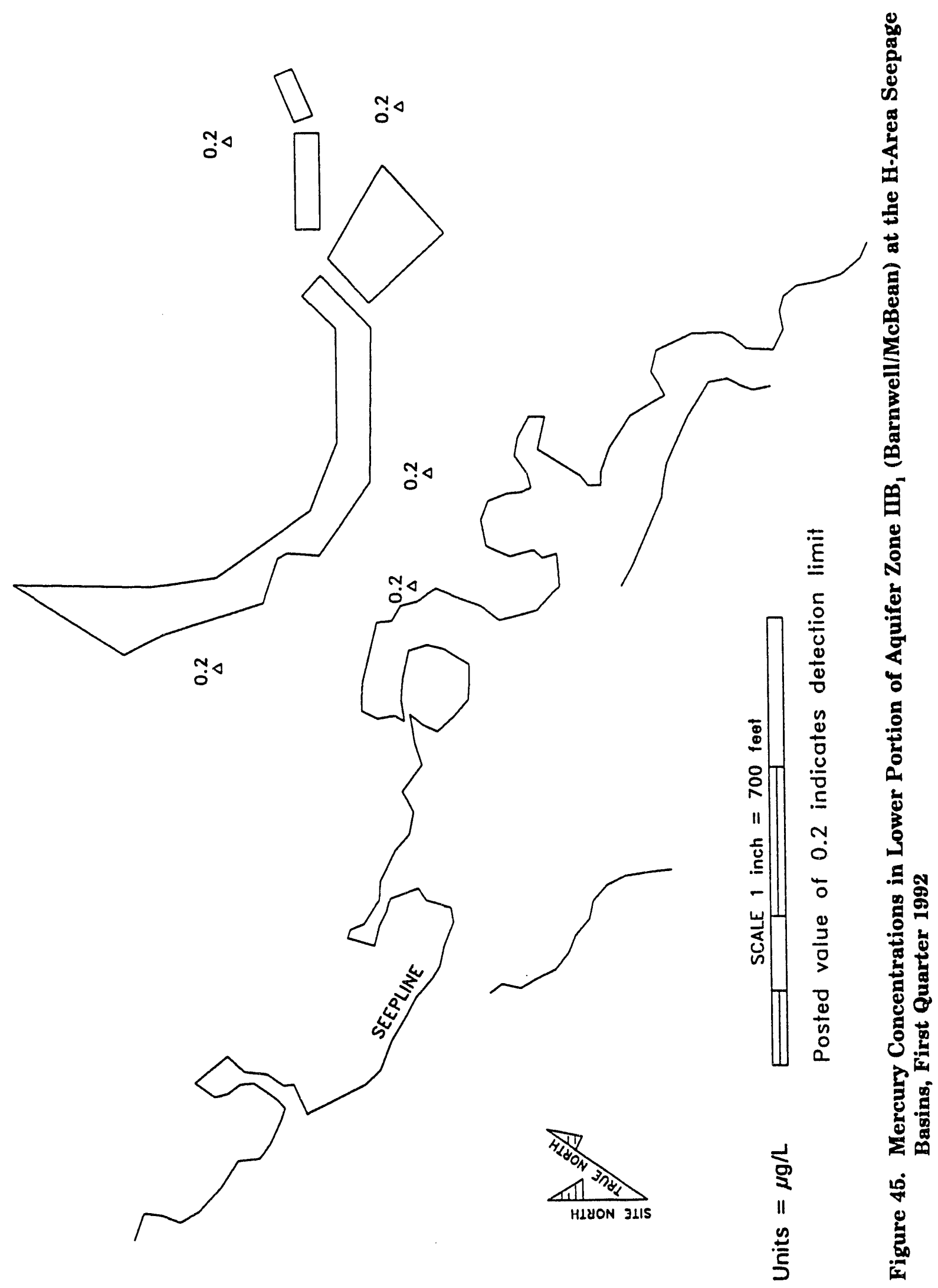


WSRC-TR-93-059

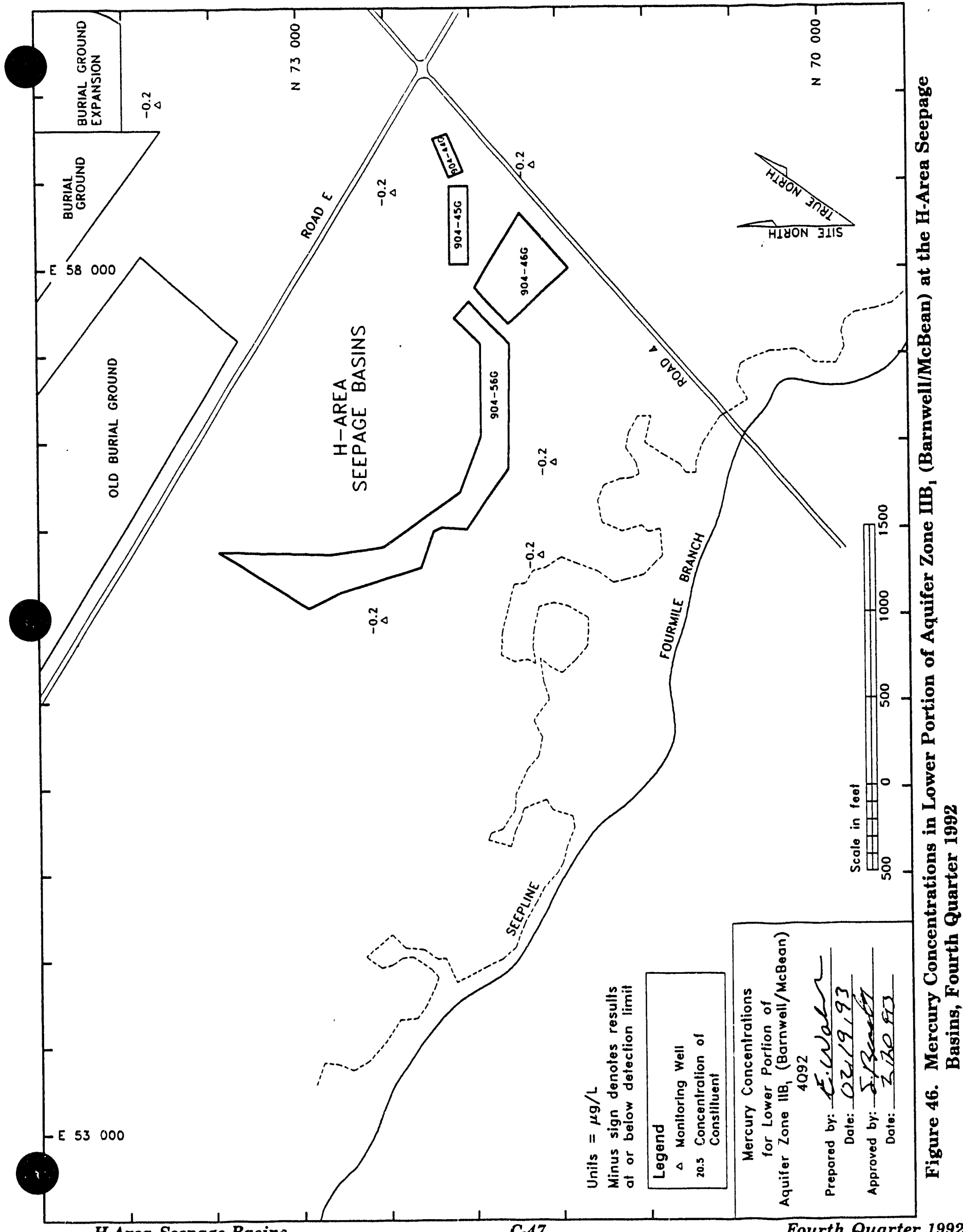


WSRC.TR-93-059

$\dddot{n}$

$\overbrace{}^{3} \triangleleft$

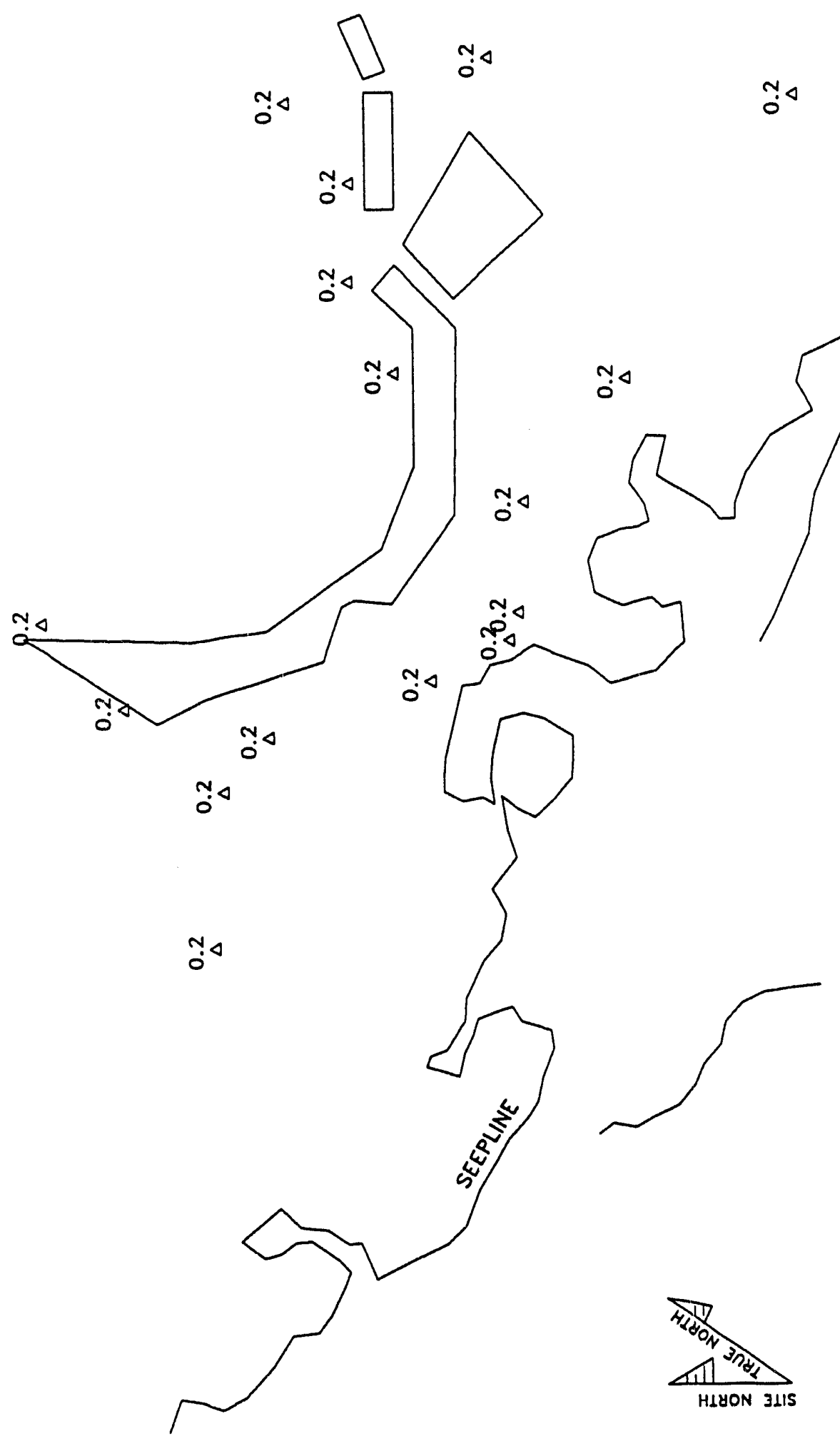

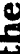

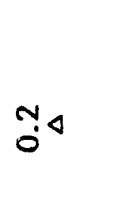

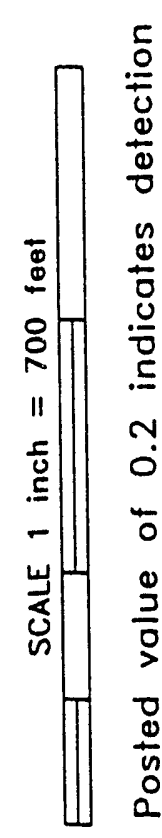

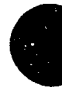


WSRC-TR-93.059

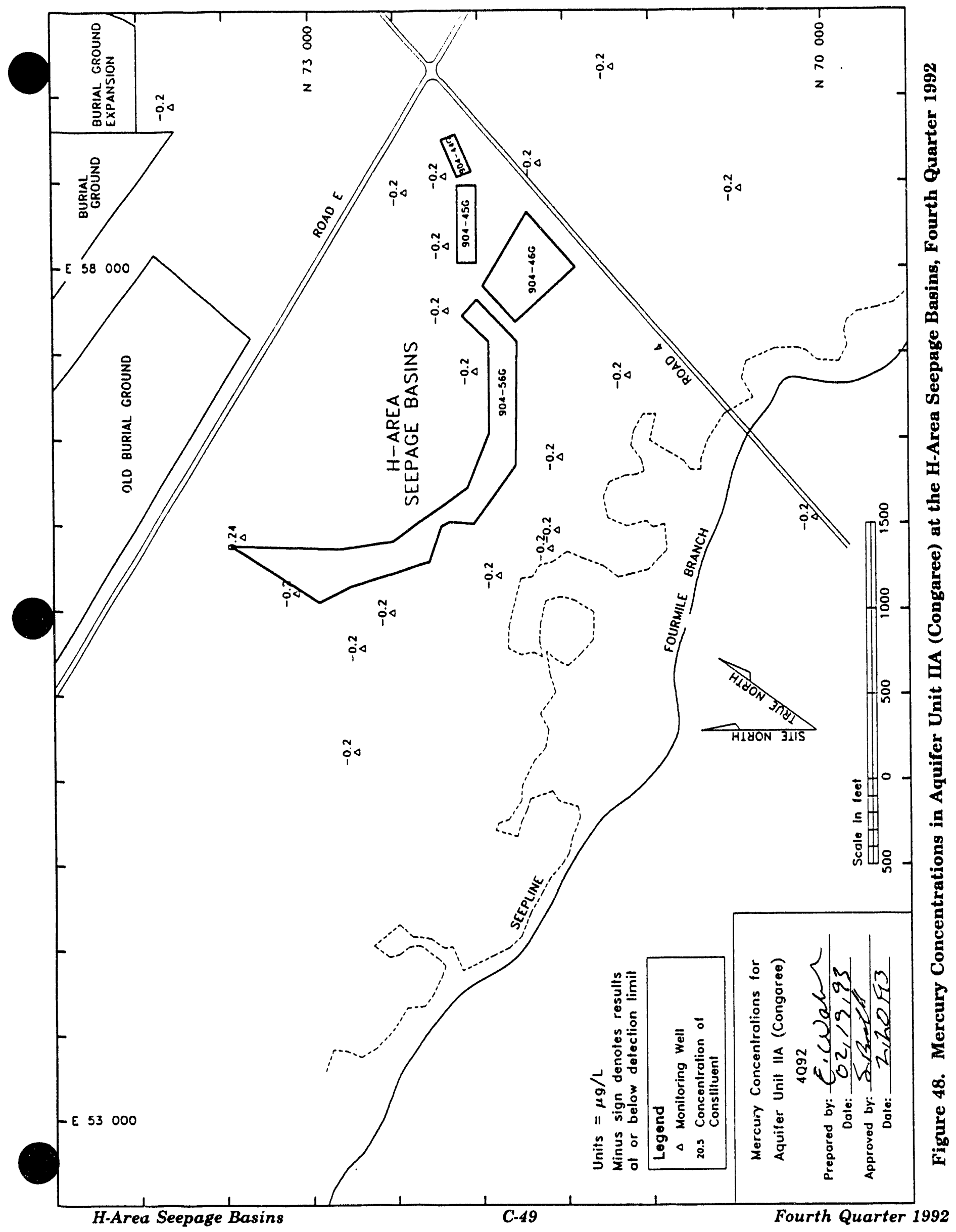


WSRC-TR.93-059

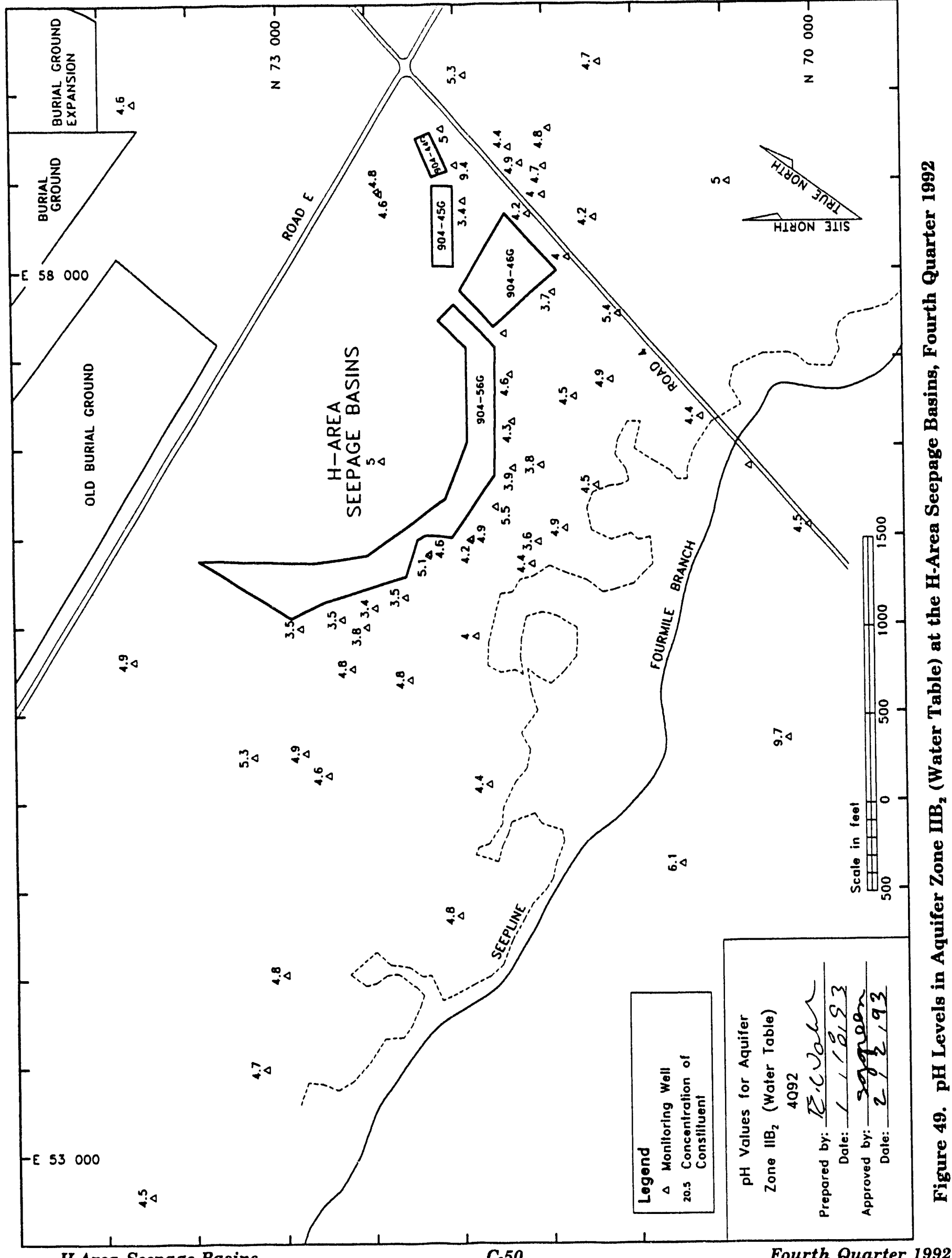


WSRC-TR-93-059

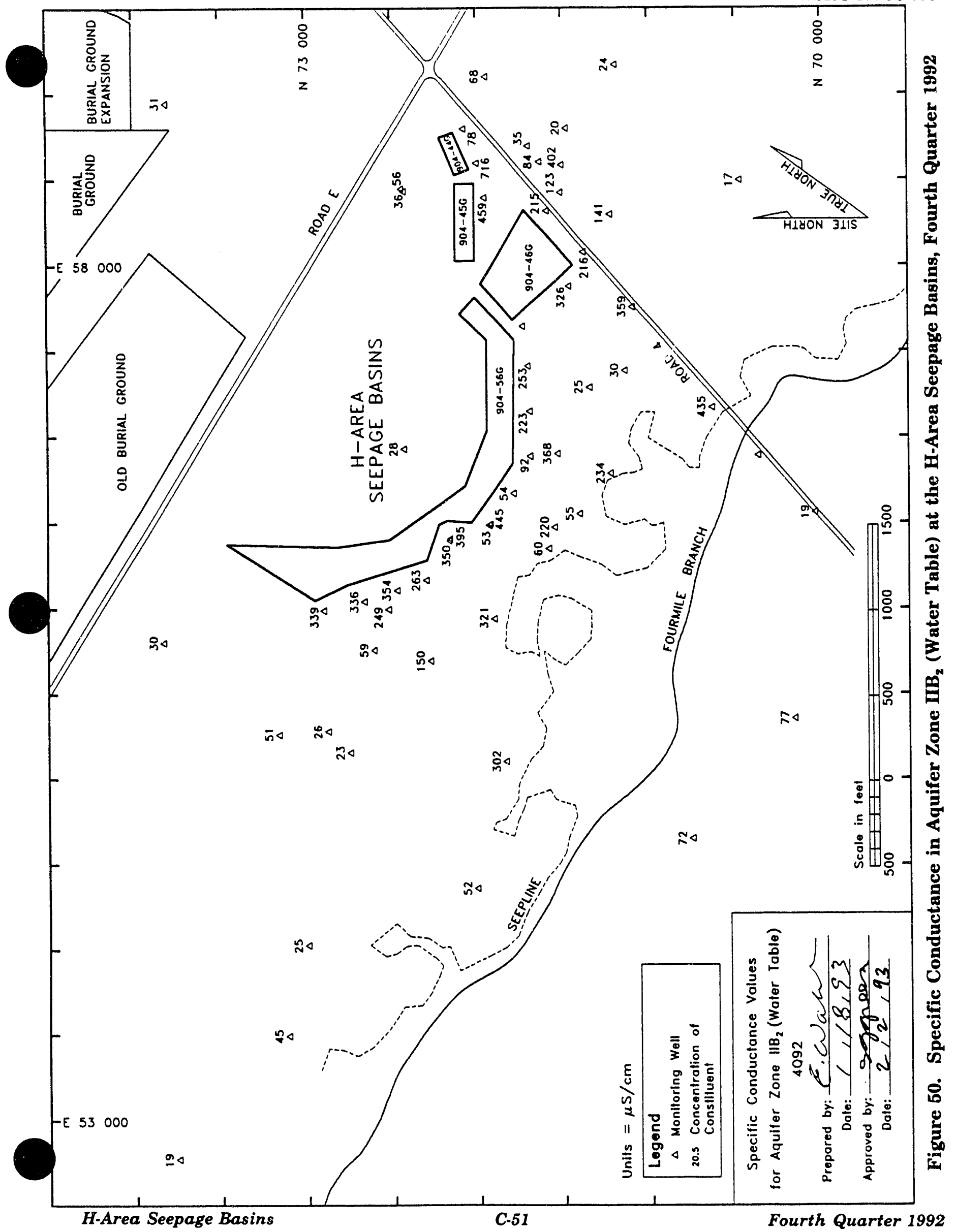




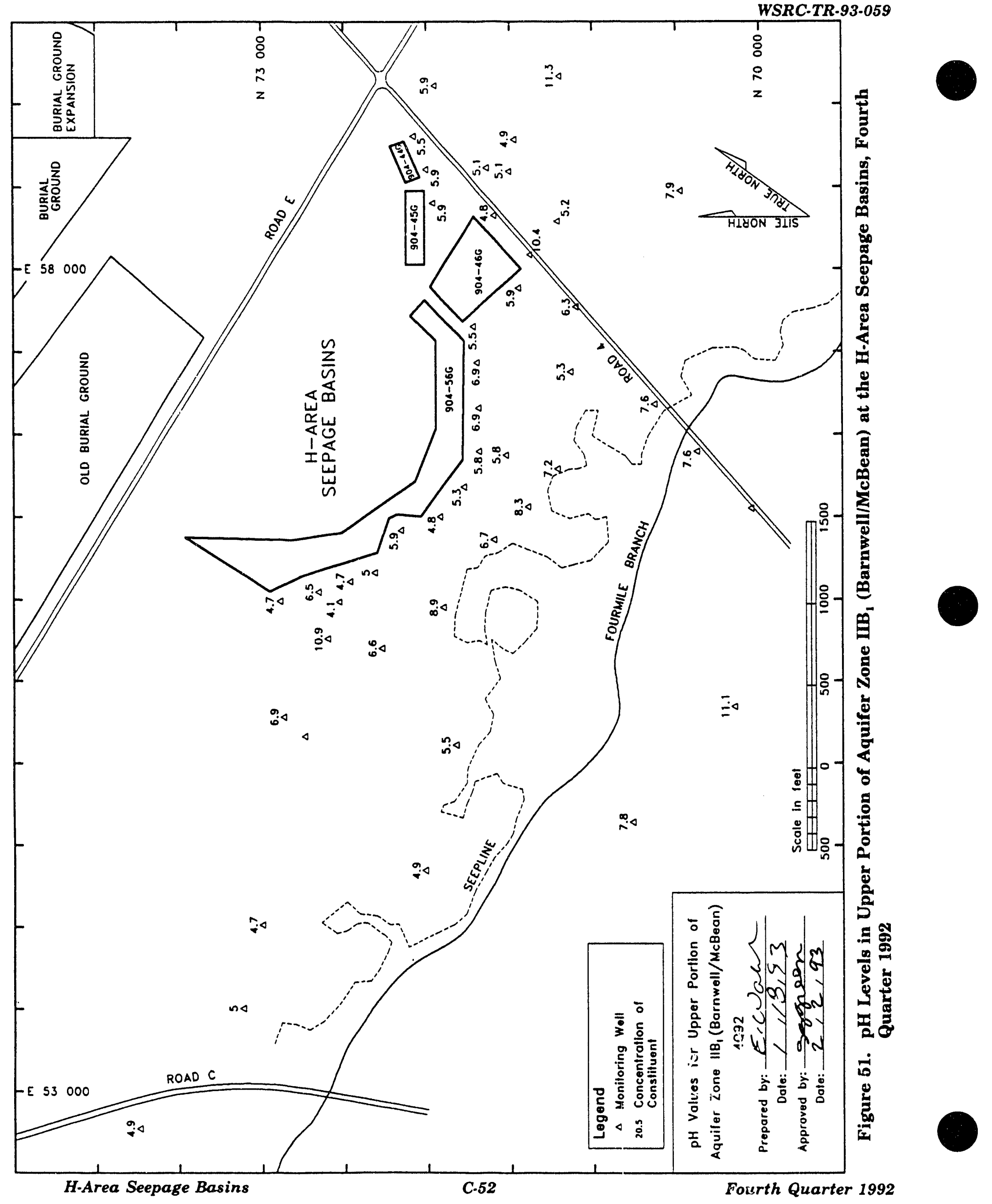




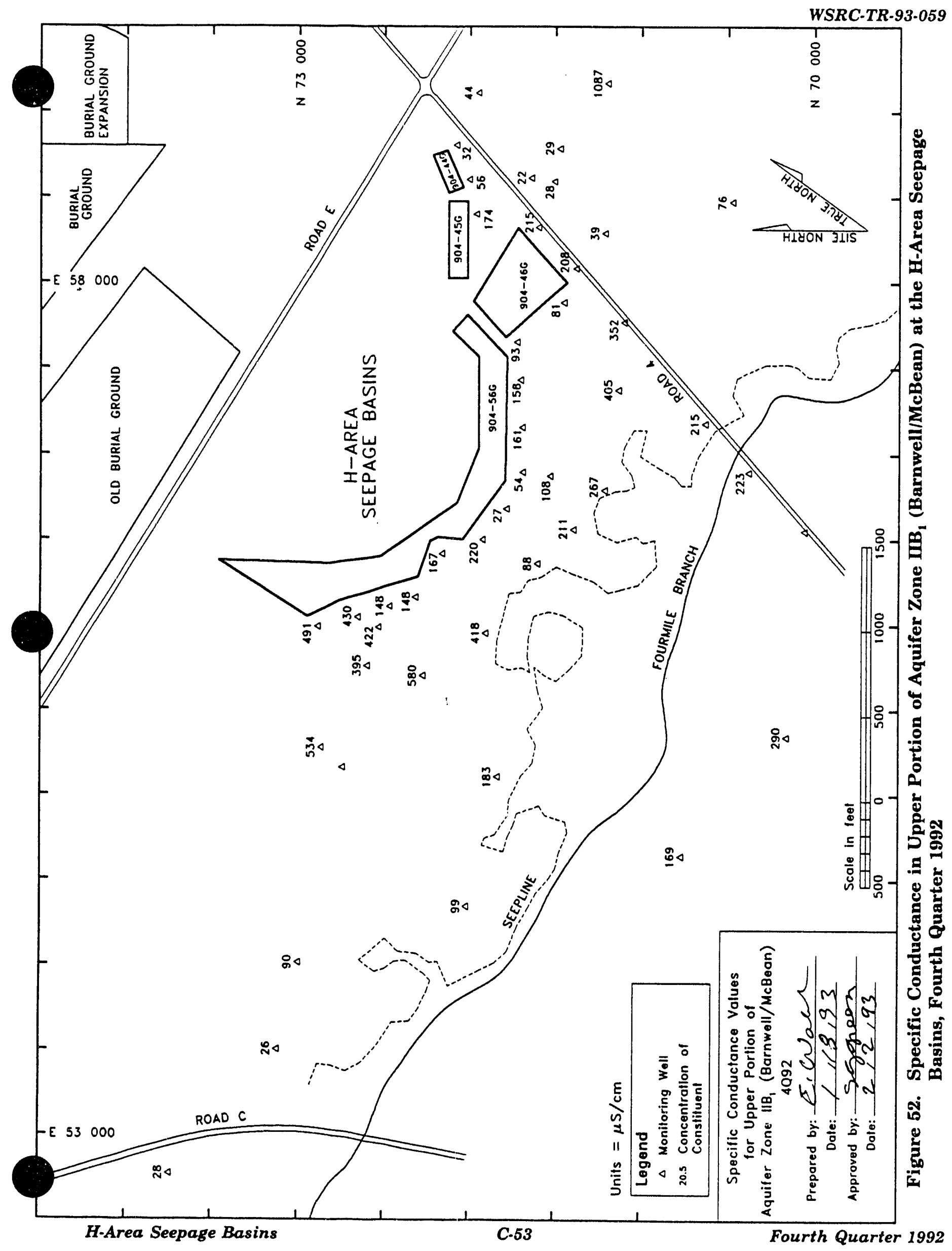




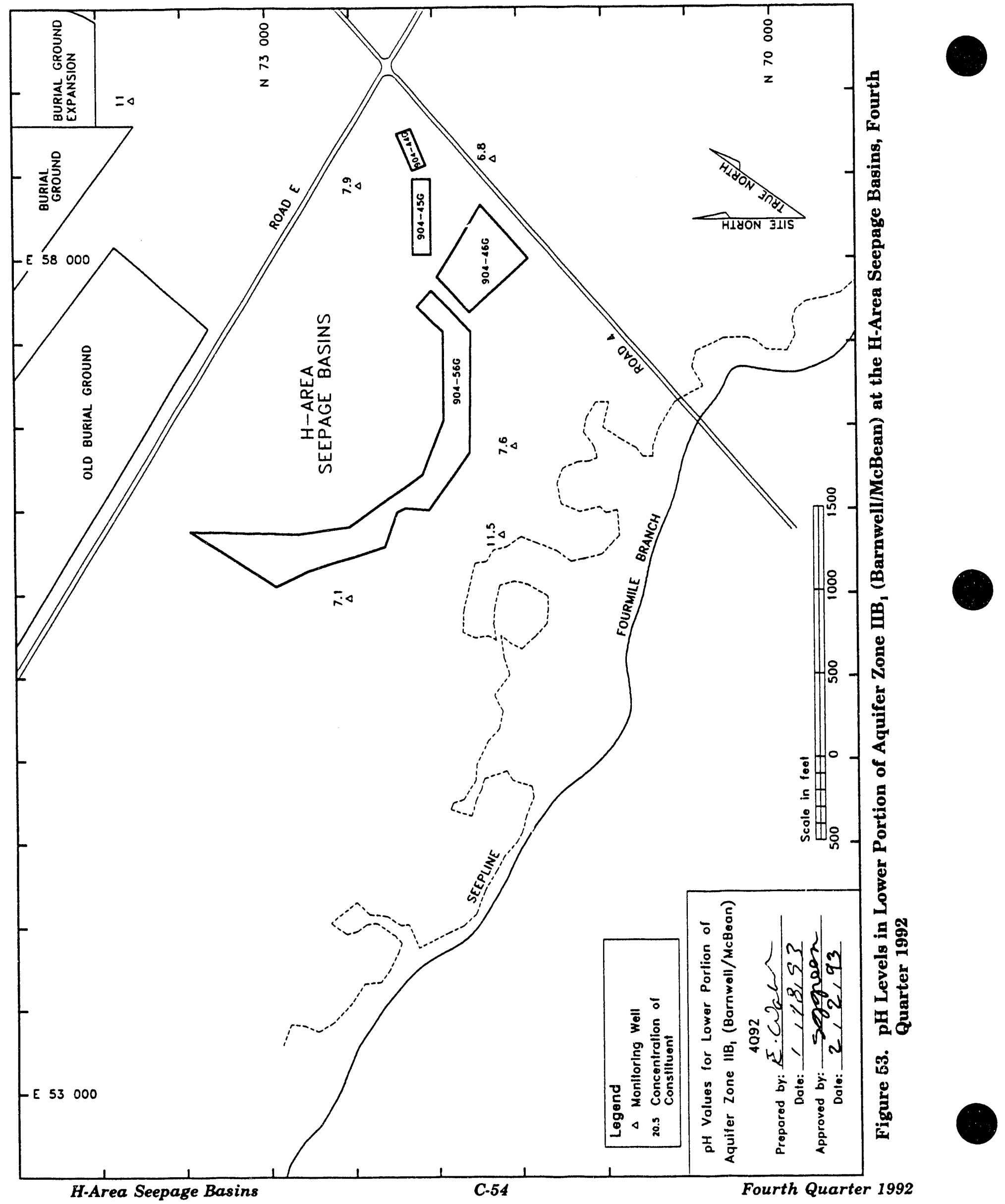




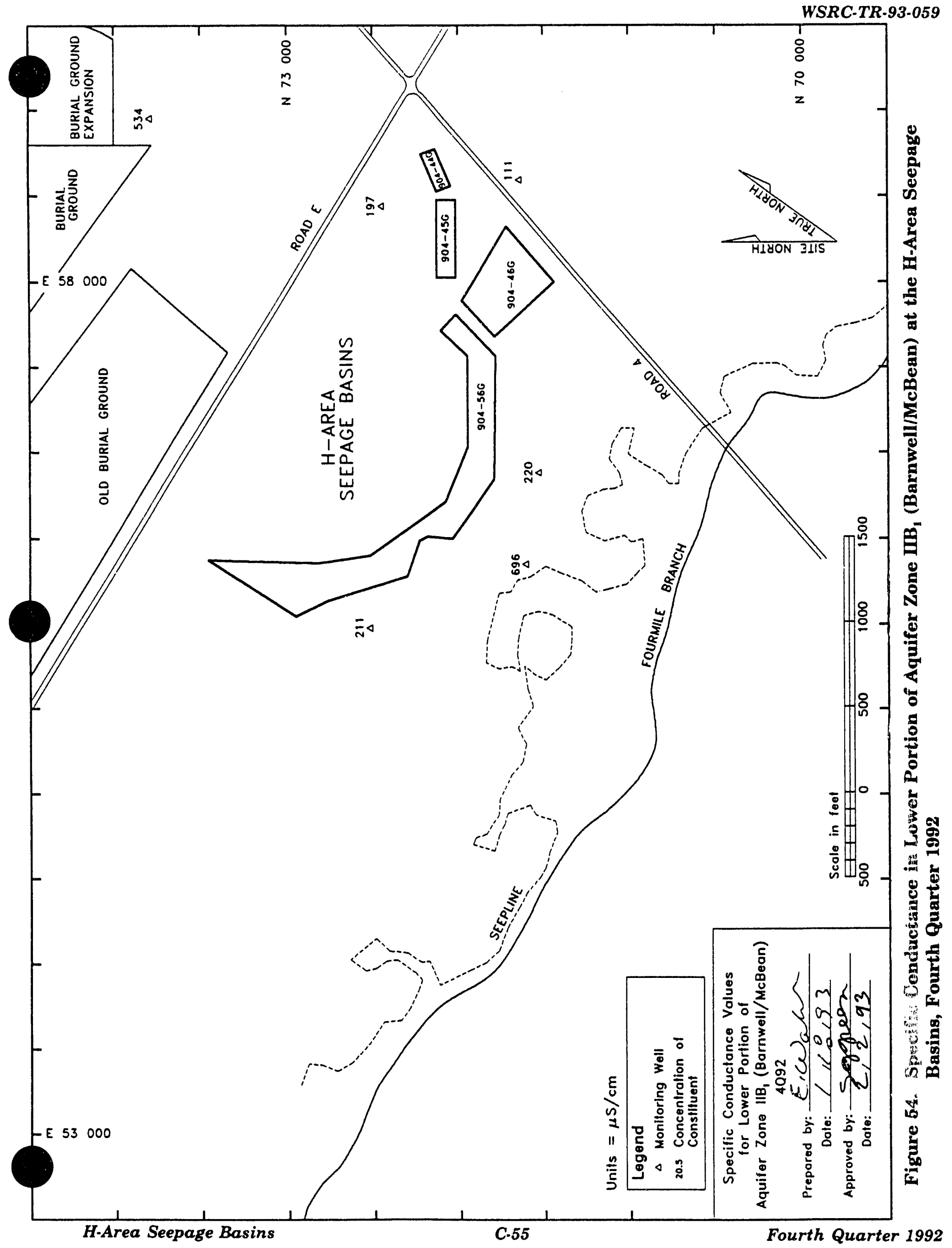




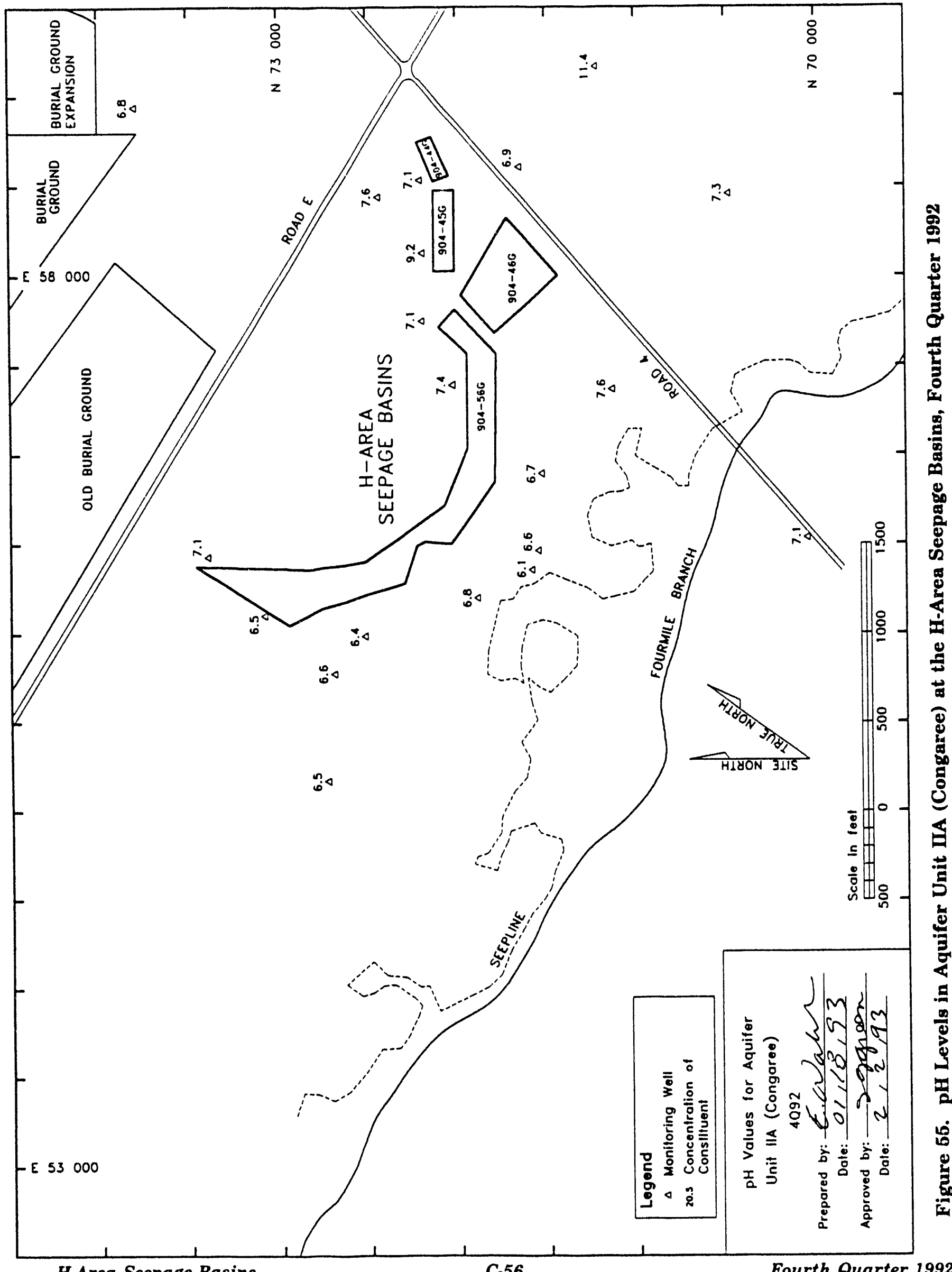




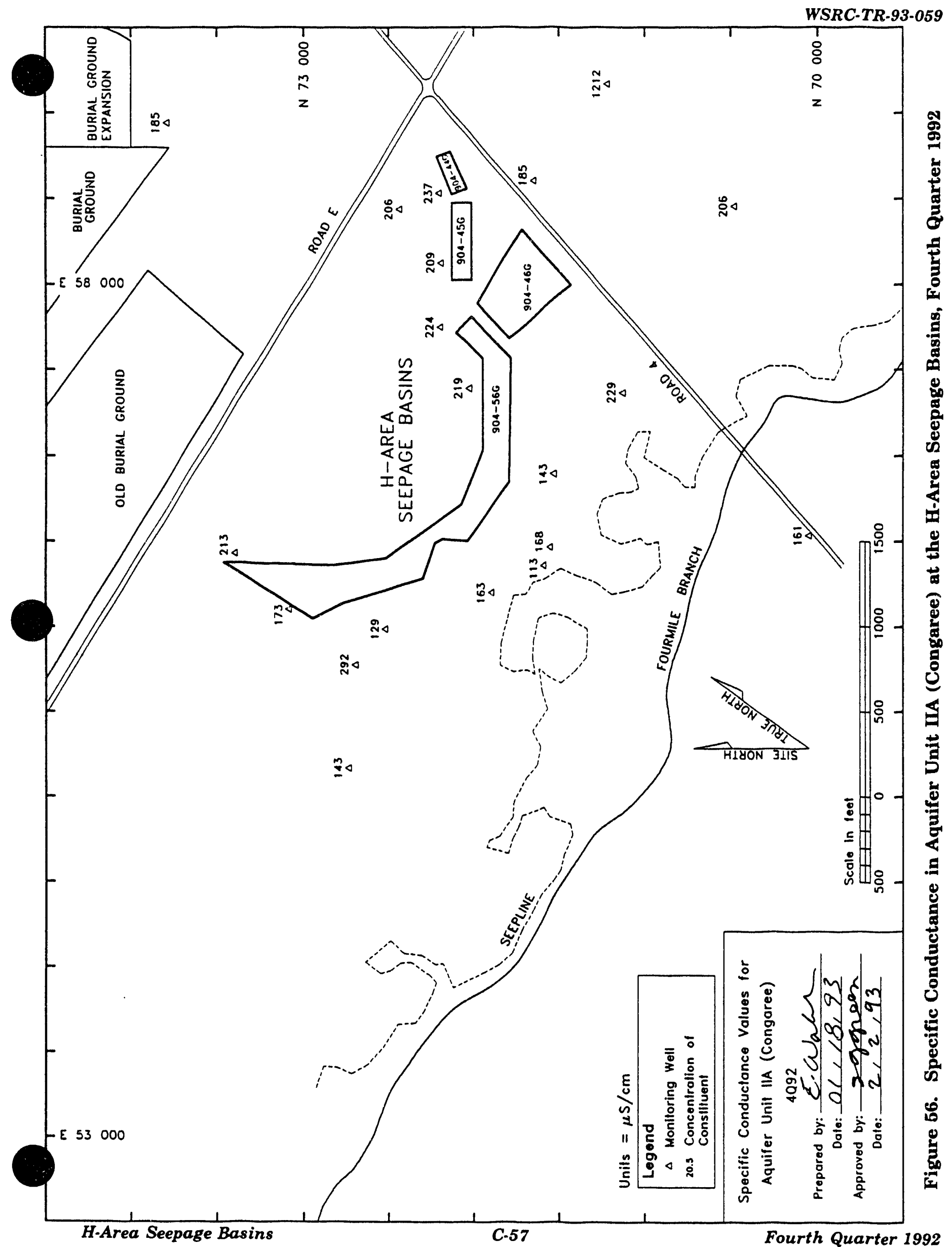




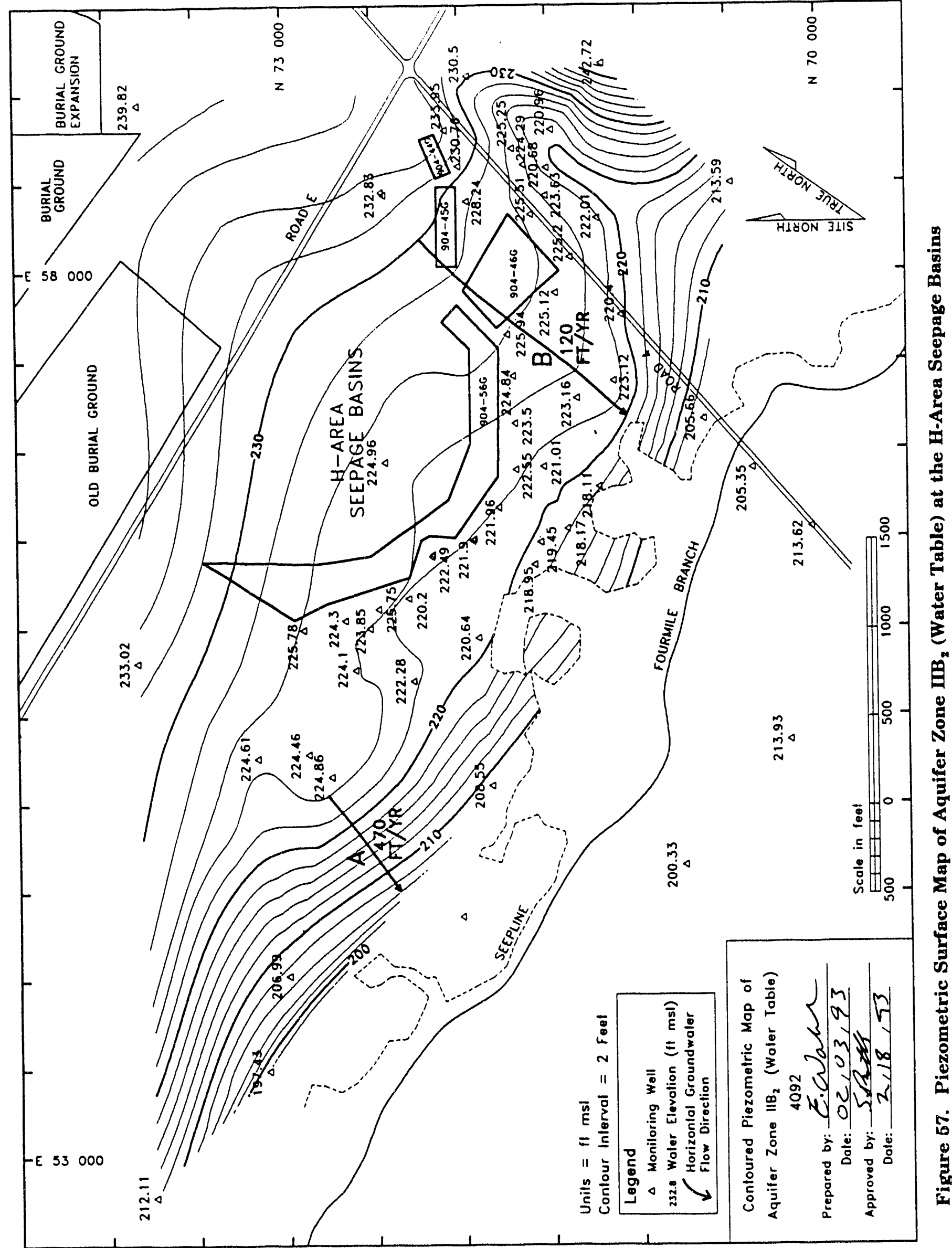




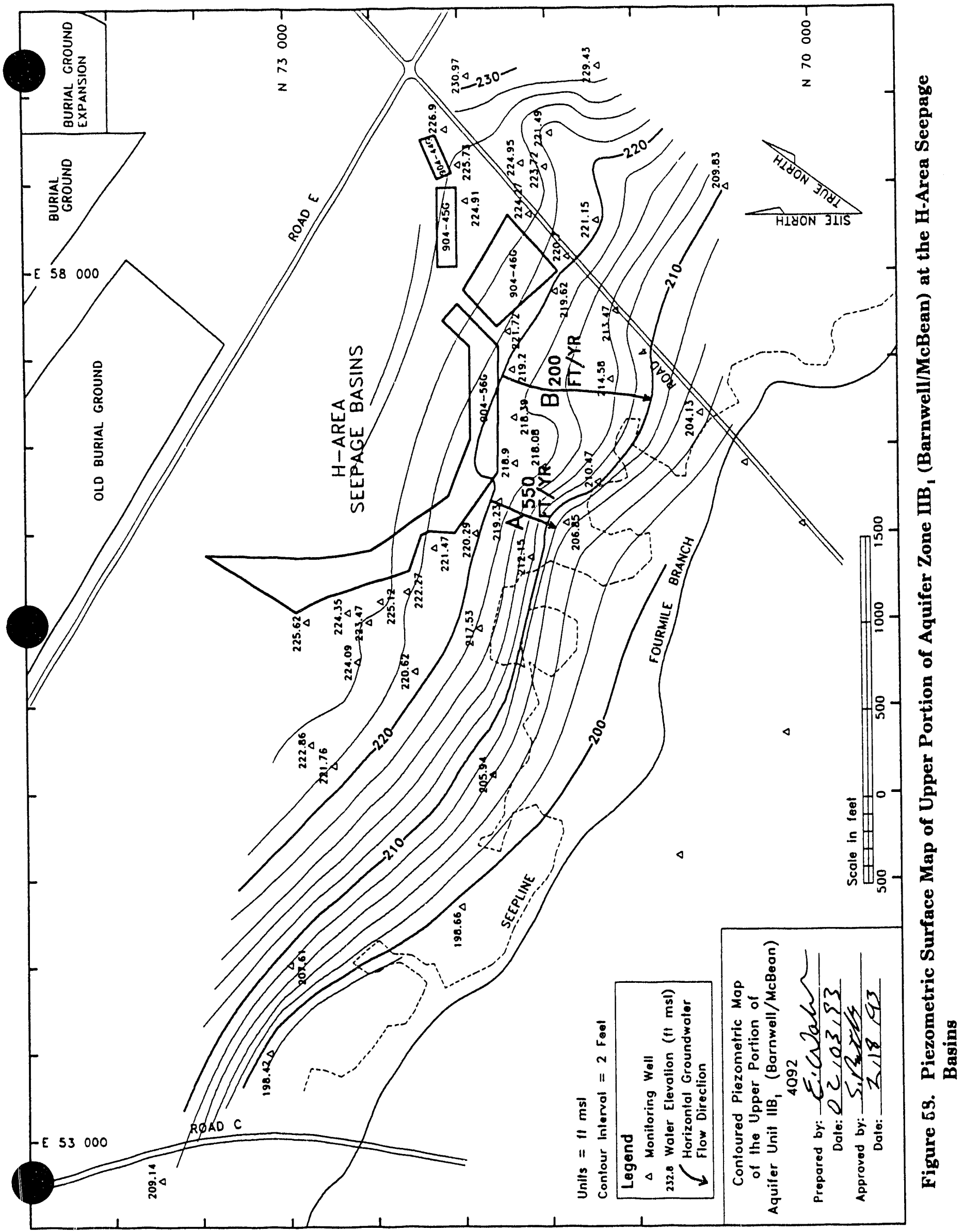


WSRC-TR-93-059

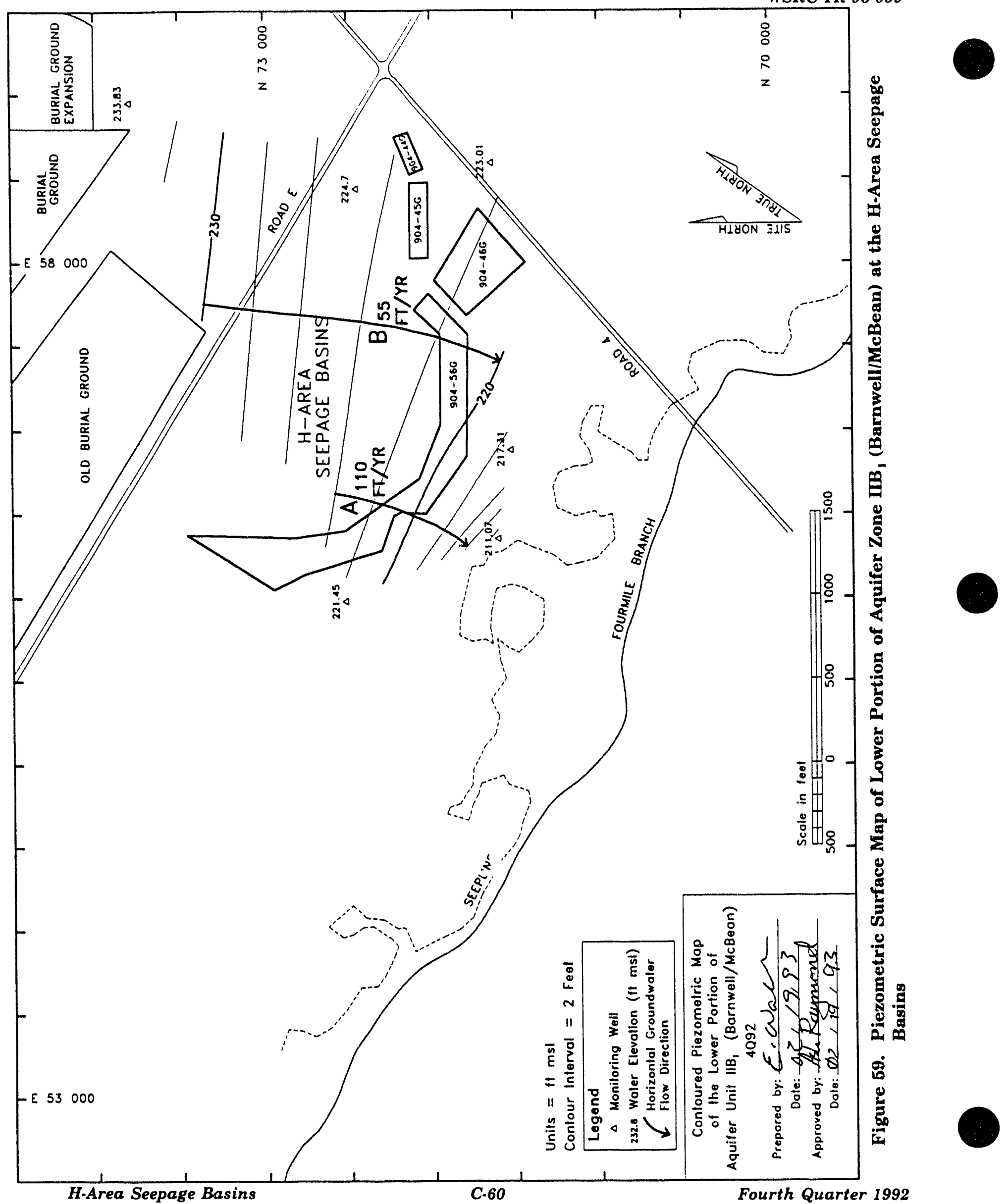




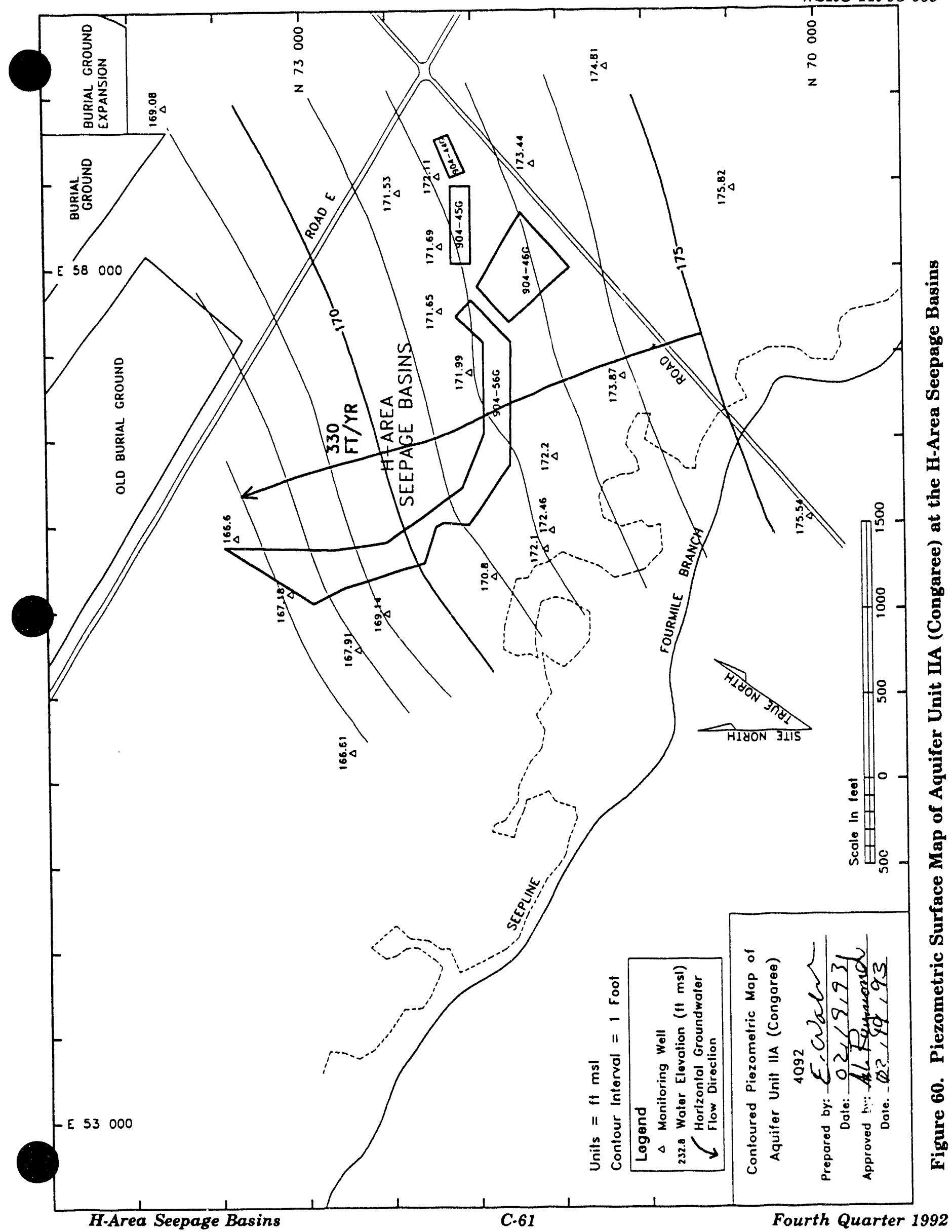




\section{References}

Aadiand, R. K., and H. W. Bledsoe, 1990. Classification of Hydrostratigraphic Units at the Savannah River Site, South Carolina, WSRC-RP-90-987. Westinghouse Savannah River Company, Savannah River Site, Aiken, SC.

Aucott, W. R., M. E. Davis, and G. K. Speiran, 1987. Geohydrologic Framework of the Coastal Plain Aquifers of South Carolina, U.S. Geological Survey Water-Resources Investigations Report 85-4271.

DuPont (E. I. du Pont de Nemours \& Company), 1989. Final Safety Analysis Report of the Defense Waste Processing Facility, DPSTSA-200-10. Savannah River Plant, Aiken, SC.

GeoTrans, Inc., 1988. A Numerical Model of the Hydrogeological System Underlying the Savannah River Plant, Final report submitted to Savannah River Laboratory. GeoTrans, Inc., Sterling, VA.

Siple, G. E., 1967. Geology and Ground Water of the Savannah River Plant and Vicinity, South Carolina. Geological Survey Water-Supply Paper 1841, Reston, VA. 
WSRC-TR-93-059

\section{Appendix D - Groundwater Monitoring Results Tables}




\section{Key to Reading the Tables}

The following abbreviations may appear in the tabular data:

B = sample collected from well using an open bucket bailer

$\mathrm{BA}=$ Barringer Laboratories, Inc.

$\mathrm{CN}=$ Clemson Technical Center, Inc.

$\mathrm{CS}=$ carbon steel

$\mathrm{D}=$ primary drinking water standard (PDWS)

$\mathrm{E}=$ exponential notation (e.g., $1.1 \mathrm{E}-09=1.1 \times 10^{-9}=0.0000000011$ )

$\mathrm{EM}=$ Environmental Protection Department/Environmental Monitoring Section (EPD/EMS)

Laboratory

$\mathrm{GE}=$ General Engineering Laboratories

GP = Environmental Physics, Inc.

$\mathrm{H}=$ holding time

1,2,3,4,6,7,8-HPCDD $=1,2,3,4,6,7,8$-heptachlorodibenzo-p-dioxin

1,2,3,4,6,7,8-HPCDF $=1,2,3,4,6,7,8$-heptachlorodibenzo-p-furan

1,2,3,4,7,8-HXCDD = 1,2,3,4,7,8-hexachlorodibenzo-p-dioxin

$1,2,3,4,7,8$-HXCDF $=1,2,3,4,7,8$-hexachlorodibenzo-p-furan

Lindane = gamma-benzene hexachloride

$\mathrm{mg} / \mathrm{L}=$ milligrams per liter

Mod $=$ modifier

msl $=$ mean sea level

MSL $=$ million structures per liter

NTU = turbidity unit

$\mathrm{P}=$ sample collected from well using a bladder pump

$\mathrm{PCB}=$ polychlorinated biphenyl

1,2,3,7,8-PCDD $=1,2,3,7,8$-pentachlorodibenzo-p-dioxin

1,2,3,7,8-PCDF $=1,2,3,7,8$-pentachlorodibenzo-p-furan

$\mathrm{pCi} / \mathrm{L}=$ picocuries per liter

$\mathrm{pCi} / \mathrm{mL}=$ picocuries per milliliter

PDWS $=$ primary drinking water standard

$\mathrm{pH}=\mathrm{pH}$ unit

PVC = polyvinyl chloride

$\mathrm{S}=$ sample collected from well using a single-speed centrifugal downhole pump

Sp. conductance $=$ specific conductance

$\mathrm{SP}=$ Spencer Testing Services, Inc.

TCDD = tetrachlorodibenzo-p-dioxin

TCDF = tetrachlorodibenzo-p-furan

$\mathrm{TM}=\mathrm{TMA} /$ Eberline

TOC $=$ top of casing

$\mathrm{V}=$ sample collected from well using a variable-speed pump

$\mathrm{WA}=$ Roy $\mathrm{F}$. Weston, Inc.

$\mu \mathrm{g} / \mathrm{L}=$ micrograms per liter

$\mu \mathrm{S} / \mathrm{cm}=$ microsiemens per centimeter 


\section{Holding Times}

Standard analytical methods include a limit, called holding time, on the maximum elapsed time between sample collection and extraction or analysis by the laboratory. In the data tables, a large $\operatorname{dot}(\bullet)$ in the $\mathrm{H}$ (holding time) - olumn indicates that holding time was exceeded. Analyses performed beyond holding time may not yield valid results.

The South Carolina Department of Health and Environmental Control allows only 15 minutes to elapse between sampling and analysis for $\mathrm{pH}$. Thus, only field $\mathrm{pH}$ measurements can meet the holding time criterion; laboratory $\mathrm{pH}$ analyses will always exceed it.

Laboratory-initiated procedures for reducing the number of other analyses performed out of holding time include subcontracting analyses when difficulties with equipment, personnel, or work load would prevent timely analyses. Beginning fourth quarter 1991, SRS reduced the compensation to laboratories for analyses performed out of holding time.

\section{Data Qualification}

The contract laboratories continually assess their own accuracy and precision according to U.S. Environmental Protection Agency (EPA) guidelines. They submit sample- or batchspecific quality assurance/quality control information either at the same time as analytical results or in a quarterly summary. Properly defined and used result modifiers (also referred to as qualifiers) can be a key component in assessing data useability. Result modifiers designed by EPD/EMS and provided to the primary laboratories are defined below. These modifiers appear in the data tables under the column "Mod."

Result modifier

(Blank)

$A^{a}$

$J^{a}$

$L^{a}$

$M^{a}$

$\mathrm{R}^{\mathrm{a}}$

$\mathrm{T}^{\mathrm{a}}$

$v^{a}$

1

2
Definition

Data are not quanfied. Number should be interpreted exactly as reported.

Value reported is the mean of two or more determinations.

Value is estimated because quantitation in the sample or in associated quality control samples did not meet specifications.

Value is off-scale high. The actual value is not known but is known to be greater than the value shown.

Presence of the analyte is verified but not quantified.

Result was rejerted because performance requirements in the sample analysis or associated qualicy control analyses were not met.

Analyte was not detected; if present, it was below the criteria for detection.

Analyte was detected in the associated method blank.

Result may be an underestimation of the true value due to analytical bias.

Result may be an overestimation of the true value due to analytical bias. 
WSRC-TR-93-059

\section{Result modifier Definition}

3

The associated result may be of poor precision (high variability) due to analytical bias.

6

The associated result is from a reanalysis performed out of holding time due to problems with an earlier analysis.

a These codes are based on the STORET codes from EPA. 
WSRC-TR-93-059

Table 1. Constituents Exceeding the Final Primary Drinking Water Standards

Aquifer Zone $\mathrm{IBB}_{2}$ (Water Table)

\begin{tabular}{|c|c|c|c|c|c|c|}
\hline Well & Constituent & Unit & 1092 & $\underline{2092}$ & 3092 & 4092 \\
\hline HSB 65 & Tritium & $\mathrm{pCi} / \mathrm{mL}$ & $3.8 E+01$ & $3.3 E+01$ & $\therefore 6 \mathrm{E}+01$ & $3.8 E+01$ \\
\hline HSB 65C & Tritium & $\mathrm{pCi} / \mathrm{mL}$ & $2.8 \mathrm{E}+01$ & $2.7 E+01$ & $2.4 E+01$ & $2.2 E+01$ \\
\hline HSB 67 & $\begin{array}{l}\text { Gross alpha } \\
\text { Mercury } \\
\text { Nitrate-nitrite as nitrogen } \\
\text { Nonvolatile beta } \\
\text { Total alpha-emitting radium }{ }^{b} \\
\text { Tritium }\end{array}$ & $\begin{array}{l}\mathrm{pCi} / \mathrm{L} \\
\mu \mathrm{g} / \mathrm{L} \\
\mu \mathrm{g} / \mathrm{L} \\
\mathrm{pCi} / \mathrm{L} \\
\mathrm{pCi} / \mathrm{L} \\
\mathrm{pCi} / \mathrm{mL}\end{array}$ & $\begin{array}{l}-a \\
- \\
- \\
9.1 E+02 \\
2.2 E+01 \\
4.1 E+03\end{array}$ & $\begin{array}{l}2.3 E+01 \\
\overline{11} \\
2.600 \\
4.7 E+03 \\
4.3 E+03\end{array}$ & $\begin{array}{l}4.8 E+01 \\
2.0 \\
13,900 \\
1.7 E+03 \\
2.5 E+01 \\
3.8 E+03\end{array}$ & $\begin{array}{l}4.6 E+01 \\
- \\
11.500 \\
1.3 E+03 \\
3.5 E+01 \\
1.7 E+03\end{array}$ \\
\hline HSB 68 & $\begin{array}{l}\text { Gross alpha } \\
\text { Mercury } \\
\text { Nitrate-nitrite as nitrogen } \\
\text { Nonvolatile beta } \\
\text { Total alphe-emitting radium } \\
\text { Tritium }\end{array}$ & $\begin{array}{l}\mathrm{pCi} / \mathrm{L} \\
\mu \mathrm{g} / \mathrm{L} \\
\mu \mathrm{g} / \mathrm{L} \\
\mathrm{pCi} / \mathrm{L} \\
\mathrm{pCi} / \mathrm{L} \\
\mathrm{pCi} / \mathrm{mL}\end{array}$ & $\begin{array}{l}3.9 E+01 \\
2.6 \\
39,200 \\
7.0 E+03 \\
1.1 E+02 \\
1.1 E+04\end{array}$ & $\begin{array}{l}6.9 E+01 \\
2.6 \\
40.100 \\
9.4 E+03 \\
1.3 E+02 \\
9.4 E+03\end{array}$ & $\begin{array}{l}3.6 \mathrm{E}+01 \\
2.5 \\
39,000 \\
5.8 \mathrm{E}+03 \\
6.6 \mathrm{E}+01 \\
6.0 \mathrm{E}+03\end{array}$ & $\begin{array}{l}3.4 E+01 \\
2.3 \\
38.500 \\
6.3 E+03 \\
8.9 E+01 \\
5.1 E+03\end{array}$ \\
\hline HSB 69 & $\begin{array}{l}\text { Gross alpha } \\
\text { Nitrate-nitrite as nitrogen } \\
\text { Nonvolatile beta } \\
\text { Total alpha-emitting radium } \\
\text { Tritium }\end{array}$ & $\begin{array}{l}\mathrm{pCi} / \mathrm{L} \\
\mu \mathrm{g} / \mathrm{L} \\
\mathrm{pCi} / \mathrm{L} \\
\mathrm{pCi} / \mathrm{L} \\
\mathrm{pCi} / \mathrm{mL}\end{array}$ & $\begin{array}{l}3.6 E+01 \\
23,200 \\
3.9+03 \\
3.8 E+01 \\
1.5 E+03\end{array}$ & $\begin{array}{l}2.4 E+01 \\
21,000 \\
5.0+03 \\
9.7 E+01 \\
1.1 E+03\end{array}$ & $\begin{array}{l}4.3 E+01 \\
21,200 \\
4.1 E+03 \\
4.5 E+01 \\
7.3 E+02\end{array}$ & $\begin{array}{l}1.1 E+02 \\
21,600 \\
3.9 E+03 \\
8.8 E+01 \\
6.1 E+02\end{array}$ \\
\hline HSB 70 & $\begin{array}{l}\text { Total alpha-emitting radium } \\
\text { Tritium }\end{array}$ & $\begin{array}{l}\mathrm{pCi} / \mathrm{L} \\
\mathrm{pCi} / \mathrm{mL}\end{array}$ & $\overline{1.7 E+02}$ & $\begin{array}{l}7.0 E+00 \\
7.6 E+01\end{array}$ & $\overline{4} .6 E+01$ & $\overline{5.3 E}+01$ \\
\hline HSB 71 & Tritium & $\mathrm{pCi} / \mathrm{mL}$ & $1.3 E+02$ & $1.1 E+02$ & $5.9 E+01$ & $1.1 E+02$ \\
\hline HSB 83D & Tritium & $\mathrm{pCi} / \mathrm{mL}$ & $1.0 E+03$ & $1.0 E+03$ & $8.2 E+02$ & $7.2 E+02$ \\
\hline HSB 84D & $\begin{array}{l}\text { Gross alpha } \\
\text { Nitrate-nitrite as nitrogen } \\
\text { Nonvolatile beta } \\
\text { Total alpha-emitting radium } \\
\text { Tritium }\end{array}$ & $\begin{array}{l}\mathrm{pCi} / \mathrm{L} \\
\mu \mathrm{g} / \mathrm{L} \\
\mathrm{pCi} / \mathrm{L} \\
\mathrm{pCi} / \mathrm{L} \\
\mathrm{pCi} / \mathrm{mL}\end{array}$ & $\begin{array}{l}1.5 E+01 \\
11,800 \\
1.8 E+03 \\
2.0 E+01 \\
1.3 E+03\end{array}$ & $\begin{array}{l}- \\
10,200 \\
1.6 E+03 \\
2.9 E+01 \\
9.1 E+02\end{array}$ & $\begin{array}{l}- \\
- \\
\overline{1.7 E}+01 \\
7.0 E+02\end{array}$ & $\begin{array}{l}- \\
- \\
3.3 E+02 \\
1.1 E+01 \\
4.6 E+02\end{array}$ \\
\hline HSB 86D & $\begin{array}{l}\text { Gross alpha } \\
\text { Nitrate as nitrogen } \\
\text { Nitrate-nitrite as nitrogen } \\
\text { Nonvolatile beta } \\
\text { Total aipha-emitting radium } \\
\text { Tritium }\end{array}$ & $\begin{array}{l}\mathrm{pCi} / \mathrm{L} \\
\mu \mathrm{g} / \mathrm{L} \\
\mu \mathrm{g} / \mathrm{L} \\
\mathrm{pCi} / \mathrm{L} \\
\mathrm{pCi} / \mathrm{L} \\
\mathrm{pCi} / \mathrm{mL}\end{array}$ & $\begin{array}{l}4.4 E+01 \\
37.800 \\
N A \\
2.3 E+03 \\
7.2 E+01 \\
1.3 E+04\end{array}$ & $\begin{array}{l}6.9 E+01 \\
N A^{C} \\
34,200 \\
2.1 E+03 \\
3.5 E+01 \\
8.3 E+03\end{array}$ & $\begin{array}{l}- \\
N A \\
35,000 \\
1.3 E+03 \\
2.7 E+01 \\
5.9 E+03\end{array}$ & $\begin{array}{l}2.3 E+01 \\
N A \\
53,200 \\
7.7 E+02 \\
4.7 E+01 \\
3.1 E+03\end{array}$ \\
\hline HSB100D & Tritium & $\mathrm{pCi} / \mathrm{mL}$ & $5.0 E+02$ & $5.9 E+02$ & $8.2 E+02$ & $8.4 E+02$ \\
\hline HSB101D & $\begin{array}{l}\text { Arsenic } \\
\text { Mercury } \\
\text { Nitrate-nitrite as nitrogen } \\
\text { Nonvolatile beta } \\
\text { Tritium }\end{array}$ & $\begin{array}{l}\mu g / L \\
\mu g / L \\
\mu g / L \\
\mathrm{pCi} / \mathrm{L} \\
\mathrm{pCi} / \mathrm{mL}\end{array}$ & $\begin{array}{l}81 \\
4.2 \\
43,400 \\
9.0 E+01 \\
1.0 E+04\end{array}$ & $\begin{array}{l}83 \\
2.8 \\
72,000 \\
- \\
1.1 E+04\end{array}$ & $\begin{array}{l}\overline{2.6} \\
38,500 \\
\overline{8} .8 \mathrm{E}+03\end{array}$ & $\begin{array}{l}102 \\
5.9 \\
42,500 \\
- \\
1.2 E+04\end{array}$ \\
\hline
\end{tabular}


Aquifer Zone $\mathrm{IB}_{2}$ (Woter Table)

\begin{tabular}{|c|c|c|c|c|c|c|}
\hline Woll & Constituent & $\underline{\text { Unit }}$ & 1092 & $\underline{2092}$ & $\underline{3092}$ & 4092 \\
\hline \multirow[t]{6}{*}{ HSB102D } & Gross alpha & $\mathrm{pCi} / \mathrm{L}$ & $8.0 E+01$ & $2.2 E+02$ & - & $1.7 E+02$ \\
\hline & Mercury & $\mu g / L$ & - & 3.3 & 3.5 & 3.6 \\
\hline & Nitrate-nitrite as nitrogen & $\mu \mathrm{g} / \mathrm{L}$ & 40,000 & 42,000 & 46,000 & 51,000 \\
\hline & Nonvolatile beta & $\mathrm{pCi} / \mathrm{L}$ & $4.2 E+03$ & $7.5 E+03$ & $7.0 E+03$ & $3.0 E+03$ \\
\hline & Total alpha-emitting radium & $\mathrm{pCi} / \mathrm{L}$ & $7.3 E+01$ & $7.2 E+01$ & - & $4.3 E+01$ \\
\hline & Tritium & $\mathrm{pCi} / \mathrm{mL}$ & $1.0 E+04$ & $1.5 E+04$ & $1.1 E+04$ & $9.9 E+03$ \\
\hline \multirow[t]{7}{*}{ HSB103D } & Arsenic & $\mu g / L$ & - & - & 75 & - \\
\hline & Gross alpha & $\mathrm{pCi} / \mathrm{L}$ & - & $2.1 E+01$ & $1.5 E+01$ & $2.5 E+01$ \\
\hline & Mercury & $\mu g / L$ & 2.3 & - & - & 2.8 \\
\hline & Nitrato-nitrite as nitrogen & $\mu \mathrm{g} / \mathrm{L}$ & 20,000 & 20,000 & 21,000 & 20,500 \\
\hline & Nonvolatile beta & $\mathrm{pCi} / \mathrm{L}$ & $4.3 E+02$ & $4.3 E+02$ & $4.7 E+02$ & $8.1 E+02$ \\
\hline & Total alpho-emitting radium & $\mathrm{pCi} / \mathrm{L}$ & $1.9 E+01$ & $1.2 E+01$ & $1.2 E+01$ & $3.0 E+01$ \\
\hline & Tritium & $\mathrm{pCi} / \mathrm{mL}$ & $5.2 E+03$ & $4.3 E+03$ & $3.4 E+03$ & $2.6 E+03$ \\
\hline \multirow[t]{6}{*}{ HSB104D } & Gross aipha & $\mathrm{pCi} / \mathrm{L}$ & $2.3 E+01$ & $2.7 E+01$ & $3.5 E+01$ & $3.5 E+01$ \\
\hline & Mercury & $\mu g / L$ & 3.6 & 2.3 & - & 2.8 \\
\hline & Nitrate-nitrite as nitrogen & $\mu \mathrm{g} / \mathrm{L}$ & 22,800 & 23,000 & 17,600 & 21,500 \\
\hline & Nonvolatile beta & $\mathrm{pCi} / \mathrm{L}$ & $2.9 E+03$ & $2.5 E+03$ & $1.7 E+03$ & $2.9 E+03$ \\
\hline & Total alpha-emitting radium & $\mathrm{pCi} / \mathrm{L}$ & $3.8 E+01$ & $1.8 E+01$ & $1.1 E+01$ & $4.3 E+01$ \\
\hline & Tritium & $\mathrm{pCi} / \mathrm{mL}$ & $2.8 E+03$ & $2.6 E+03$ & $6.9 E+02$ & $2.5 E+03$ \\
\hline \multirow[t]{6}{*}{ HSB105D } & Gross alpha & $\mathrm{pCi} / \mathrm{L}$ & $2.8 E+01$ & $5.4 E+01$ & $5.3 E+01$ & $1.1 E+02$ \\
\hline & Mercury & $\mu g / L$ & 2.8 & 4.6 & 5.4 & 3.8 \\
\hline & Nitrate-nitrite as nitrogen & $\mu \mathrm{g} / \mathrm{L}$ & 40,000 & 58,000 & 36,500 & 41,000 \\
\hline & Nonvolatile beta & $\mathrm{pCi} / \mathrm{L}$ & $4.6 E+03$ & $4.9 E+03$ & $3.2 E+03$ & $3.0 E+03$ \\
\hline & Total alpha-emitting radium & $\mathrm{pCi} / \mathrm{L}$ & $5.4 E+01$ & $5.7 E+01$ & $2.6 E+01$ & $3.8 \mathrm{E}+01$ \\
\hline & Tritium & $\mathrm{pCi} / \mathrm{mL}$ & $5.1 E+03$ & $7.7 E+03$ & $5.5 E+03$ & $5.0 E+03$ \\
\hline \multirow[t]{4}{*}{ HSB106D } & Nitrate-nitrite as nitrogen & $\mu g / L$ & 11,400 & 24,000 & 11,500 & NA \\
\hline & Nonvolatile beta & $\mathrm{pCi} / \mathrm{L}$ & $6.6 E+02$ & $6.8 \mathrm{E}+02$ & $4.9 E+02$ & NA \\
\hline & Total alpha-emitting radium & $\mathrm{pCi} / \mathrm{L}$ & $9.7 E+00$ & $8.4 E+00$ & $7.9 E+00$ & NA \\
\hline & Tritium & $\mathrm{pCi} / \mathrm{mL}$ & $1.2 E+03$ & $1.1 E+03$ & $1.1 E+03$ & NA \\
\hline \multirow[t]{6}{*}{ HSB107D } & Gross alpha & $\mathrm{pCi} / \mathrm{L}$ & - & $1.7 E+01$ & $3.1 E+01$ & $5.1 E+01$ \\
\hline & Mercury & $\mu g / L$ & - & 3.5 & & \\
\hline & Nitrate-nitrite as nitrogen & $\mu g / L$ & 32,600 & 28,000 & 26,000 & 24,500 \\
\hline & Nonvolatile beta & $\mathrm{pCi} / \mathrm{L}$ & $2.0 E+03$ & $3.2 E+03$ & $3.2 E+03$ & $2.2 E+03$ \\
\hline & Total alpha-emitting radium & $\mathrm{pCi} / \mathrm{L}$ & $3.4 E+01$ & $8.1 E+01$ & $1.8 E+01$ & $2.8 E+01$ \\
\hline & Tritium & pCiimL & $6.5 E+03$ & $4.6 E+03$ & $3.3 E+03$ & $3.5 E+03$ \\
\hline \multirow[t]{5}{*}{ HSB108D } & Gross alpha & $\mathrm{pCi} / \mathrm{L}$ & $3.1 E+01$ & $3.3 E+01$ & $4.9 E+01$ & $2.8 E+01$ \\
\hline & Mercury & $\mu g / L$ & 4.1 & 2.8 & 5.0 & \\
\hline & Nitrate-nitrite as nitrogen & $\mu g / L$ & $\begin{array}{l}29,400 \\
6.8 E+03\end{array}$ & $\begin{array}{l}29,000 \\
6.3 E+03\end{array}$ & $\begin{array}{l}26,500 \\
5.9 E+03\end{array}$ & 23,000 \\
\hline & Nonvolatile beta & $\begin{array}{l}\mathrm{pCi} / \mathrm{L} \\
\mathrm{pCi} / \mathrm{L}\end{array}$ & $8.1 E+01$ & $\begin{array}{l}0.3 E+03 \\
1.4 E+02\end{array}$ & $\begin{array}{l}5.9 E+03 \\
3.6 E+01\end{array}$ & $\begin{array}{l}4.9 E+03 \\
6.9 E+01\end{array}$ \\
\hline & $\begin{array}{l}\text { Total alpha-emitting radium } \\
\text { Tritium }\end{array}$ & $\begin{array}{l}\mathrm{pCi} / \mathrm{L} \\
\mathrm{pCi} / \mathrm{mL}\end{array}$ & $6.0 E+03$ & $4.9 E+03$ & $2.6 E+03$ & $\begin{array}{l}6.9 E+01 \\
2.4 E+03\end{array}$ \\
\hline \multirow{4}{*}{ HSB109D } & & & & & & \\
\hline & Nonvolatile beta & $\mathrm{pCi} / \mathrm{L}$ & $1.1 E+03$ & $1.5 E+03$ & $1.4 E+03$ & $1.3 E+03$ \\
\hline & Total alpha-emitting radium & $\mathrm{pCi} / \mathrm{L}$ & $1.6 \mathrm{E}+01$ & $1.7 E+01$ & $7.8 E+00$ & $2.6 E+01$ \\
\hline & Tritium & $\mathrm{pCi} / \mathrm{mL}$ & $5.3 E+02$ & $3.4 E+02$ & $3.2 E+02$ & $3.4 E+02$ \\
\hline \multirow[t]{3}{*}{ HSB110D } & Nonvolatile beta & $\mathrm{pCi} / \mathrm{L}$ & $2.4 E+02$ & $1.1 E+02$ & $6.0 E+01$ & $8.1 E+01$ \\
\hline & Total alpha-emitting radium & $\mathrm{pCi} / \mathrm{L}$ & $5.0 E+00$ & - & - & - \\
\hline & Tritium & $\mathrm{pCi} / \mathrm{mL}$ & $1.2 \mathrm{E}+02$ & $6.4 E+01$ & $2.3 E+01$ & $2.7 E+01$ \\
\hline
\end{tabular}


WSRC-TR-93-059

Aquifer Zone $\mathrm{IB}_{2}$ (Water Table)

\begin{tabular}{|c|c|c|c|c|c|c|}
\hline Well & Constituent & Unit & 1092 & 2092 & 3092 & 4092 \\
\hline HSB111D & $\begin{array}{l}\text { Nitrate-nitrite as nitrogen } \\
\text { Nonvolatile beta } \\
\text { Total alpha-emitting radium } \\
\text { Tritium }\end{array}$ & $\begin{array}{l}\mu \mathrm{g} / \mathrm{L} \\
\mathrm{pCi} / \mathrm{L} \\
\mathrm{pCi} / \mathrm{L} \\
\mathrm{pCi} / \mathrm{mL}\end{array}$ & $\begin{array}{l}41,600 \\
9.9 E+01 \\
- \\
1.4 E+04\end{array}$ & $\begin{array}{l}64.000 \\
7.2 E+01 \\
7.5 E+C O \\
1.4 E+04\end{array}$ & $\begin{array}{l}58,000 \\
6.6 E+01 \\
6.5 E+00 \\
1.2 E+04\end{array}$ & $\begin{array}{l}57.500 \\
7.8 E+01 \\
6.6 E+00 \\
1.2 E+04\end{array}$ \\
\hline HSB111E & $\begin{array}{l}\text { Gross alpha } \\
\text { Nonvolatile beta } \\
\text { Total alpha-emitting radium } \\
\text { Tritium }\end{array}$ & $\begin{array}{l}\mathrm{pCi} / \mathrm{L} \\
\mathrm{pCi} / \mathrm{L} \\
\mathrm{pCi} / \mathrm{L} \\
\mathrm{pCi} / \mathrm{mL}\end{array}$ & $\begin{array}{l}3.6 E+01 \\
1.1 E+03 \\
1.5 E+01 \\
7.5 E+02\end{array}$ & $\begin{array}{l}- \\
7.6 E+02 \\
9.1 E+00 \\
3.3 E+02\end{array}$ & $\begin{array}{l}- \\
1.2 E+03 \\
7.9 E+00 \\
2.4 E+03\end{array}$ & $\begin{array}{l}-\overline{2.7 E}+02 \\
9.0 E+00 \\
2.8 E+03\end{array}$ \\
\hline HSB1 12D & $\begin{array}{l}\text { Nitrate-nitrite as nitrogen } \\
\text { Nonvolatile beta } \\
\text { Total alpha-emitting radium } \\
\text { Tritium }\end{array}$ & $\begin{array}{l}\mu \mathrm{g} / \mathrm{L} \\
\mathrm{pCi} / \mathrm{L} \\
\mathrm{pCi} / \mathrm{L} \\
\mathrm{pCi} / \mathrm{mL}\end{array}$ & $\begin{array}{l}48,800 \\
7.5 E+01 \\
- \\
1.8 E+04\end{array}$ & $\begin{array}{l}53,000 \\
1.1 E+02 \\
8.6 E+00 \\
1.8 E+04\end{array}$ & $\begin{array}{l}48,000 \\
6.2 E+01 \\
6.4 E+00 \\
1.5 E+04\end{array}$ & $\begin{array}{l}47,200 \\
8.6 E+01 \\
- \\
1.5 E+04\end{array}$ \\
\hline HSB112E & $\begin{array}{l}\text { Gross alpha } \\
\text { Nitrate as nitrogen } \\
\text { Nitrate-nitrite as nitrogen } \\
\text { Nonvolatile beta } \\
\text { Total alpha-emitting radium } \\
\text { Tritium }\end{array}$ & $\begin{array}{l}\mathrm{pCi} / \mathrm{L} \\
\mu \mathrm{g} / \mathrm{L} \\
\mu \mathrm{g} / \mathrm{L} \\
\mathrm{pCi} / \mathrm{L} \\
\mathrm{pCi} / \mathrm{L} \\
\mathrm{pCi} / \mathrm{mL}\end{array}$ & $\begin{array}{l}- \\
42,400 \\
N A \\
3.1 E+02 \\
1.2 E+01 \\
1.2 E+04\end{array}$ & $\begin{array}{l}- \\
\text { NA } \\
44,000 \\
3.2 E+02 \\
9.4 E+00 \\
1.0 E+04\end{array}$ & $\begin{array}{l}- \\
N A \\
42,000 \\
3.1 E+02 \\
2.2 E+01 \\
9.7 E+03\end{array}$ & $\begin{array}{l}1.6 E+01 \\
N A \\
41,500 \\
2.5 E+02 \\
6.6 E+00 \\
8.8 E+03\end{array}$ \\
\hline HSB1 13D & $\begin{array}{l}\text { Gross alpha } \\
\text { Nitrate as nitrogen } \\
\text { Nitrate-nitrite as nitrogen } \\
\text { Nonvolatile beta } \\
\text { Total alpha-emitting radium } \\
\text { Tritium }\end{array}$ & $\begin{array}{l}\mathrm{pCi} / \mathrm{L} \\
\mu g / L \\
\mu g / L \\
\mathrm{pCi} / \mathrm{L} \\
\mathrm{pCi} / \mathrm{L} \\
\mathrm{pCi} / \mathrm{mL}\end{array}$ & $\begin{array}{l}3.6 E+01 \\
42,000 \\
N A \\
1.9 E+03 \\
5.7 E+01 \\
1.1 E+04\end{array}$ & $\begin{array}{l}9.7 E+01 \\
N A \\
37.900 \\
2.8 E+03 \\
4.4 E+01 \\
1.0 E+04\end{array}$ & $\begin{array}{l}6.4 E+01 \\
N A \\
42.500 \\
2.3 E+03 \\
3.3 E+01 \\
9.6 E+03\end{array}$ & $\begin{array}{l}1.1 E+02 \\
N A \\
25,200 \\
1.8 E+03 \\
3.6 E+01 \\
3.1 E+03\end{array}$ \\
\hline HSB114D & $\begin{array}{l}\text { Gross alpha } \\
\text { Mercury } \\
\text { Nitrate as nitrogen } \\
\text { Nitrate-nitrite as nitrogen } \\
\text { Nonvolatile beta } \\
\text { Total alpha-emitting radium } \\
\text { Tritium }\end{array}$ & $\begin{array}{l}\mathrm{pCi} / \mathrm{L} \\
\mu g / \mathrm{L} \\
\mu \mathrm{g} / \mathrm{L} \\
\mu \mathrm{g} / \mathrm{L} \\
\mathrm{pCi} / \mathrm{L} \\
\mathrm{pCi} / \mathrm{L} \\
\mathrm{pCi} / \mathrm{mL}\end{array}$ & $\begin{array}{l}3.1 E+01 \\
- \\
26,600 \\
N A \\
3.0 E+03 \\
6.8 E+01 \\
3.7 E+03\end{array}$ & $\begin{array}{l}5.1 E+01 \\
- \\
N A \\
24,800 \\
3.4 E+03 \\
4.2 E+01 \\
3.5 E+03\end{array}$ & $\begin{array}{l}4.1 E+01 \\
2.6 \\
N A \\
25,000 \\
2.7 E+03 \\
2.8 E+01 \\
3.0 E+03\end{array}$ & $\begin{array}{l}1.1 E+02 \\
- \\
N A \\
34,000 \\
2.7 E+03 \\
5.6 E+01 \\
6.0 E+03\end{array}$ \\
\hline HSB115D & $\begin{array}{l}\text { Gross alpha } \\
\text { Lead } \\
\text { Mercury } \\
\text { Nitrate as nitrogen } \\
\text { Nitrate-nitrite as nitrogen } \\
\text { Nonvolatile beta } \\
\text { Total alpha-emitting radium } \\
\text { Tritium }\end{array}$ & $\begin{array}{l}\mathrm{pCi} / \mathrm{L} \\
\mu \mathrm{g} / \mathrm{L} \\
\mu \mathrm{g} / \mathrm{L} \\
\mu \mathrm{g} / \mathrm{L} \\
\mu \mathrm{g} / \mathrm{L} \\
\mathrm{pCi} / \mathrm{L} \\
\mathrm{pCi} / \mathrm{L} \\
\mathrm{pCi} / \mathrm{mL}\end{array}$ & $\begin{array}{l}2.1 E+01 \\
54 \\
- \\
31,600 \\
N A \\
1.4 E+03 \\
3.9 E+01 \\
9.0 E+03\end{array}$ & $\begin{array}{l}3.3 E+01 \\
71 \\
- \\
N A \\
36,000 \\
2.4 E+03 \\
3.8 E+01 \\
8.6 E+03\end{array}$ & $\begin{array}{l}2.5 E+01 \\
60 \\
3.6 \\
N A \\
36,000 \\
2.6 E+03 \\
2.8 E+01 \\
7.0 E+03\end{array}$ & $\begin{array}{l}7.7 E+01 \\
66 \\
- \\
\text { NA } \\
31,600 \\
3.0 E+03 \\
6.1 E+01 \\
7.6 E+03\end{array}$ \\
\hline HSB116D & $\begin{array}{l}\text { Gross alpha } \\
\text { Nitrate-nitrite as nitrogen } \\
\text { Nonvolatile beta } \\
\text { Total alpha-emitting radium } \\
\text { Tritium }\end{array}$ & $\begin{array}{l}\mathrm{pCi} / \mathrm{L} \\
\mu \mathrm{g} / \mathrm{L} \\
\mathrm{pCi} / \mathrm{L} \\
\mathrm{pCi} / \mathrm{L} \\
\mathrm{pCi} / \mathrm{mL}\end{array}$ & $\begin{array}{l}4.4 E+01 \\
41.200 \\
1.0 E+04 \\
1.2 E+02 \\
1.1 E+04\end{array}$ & $\begin{array}{l}7.3 E+01 \\
40,800 \\
1.3 E+04 \\
1.7 E+02 \\
8.1 E+03\end{array}$ & $\begin{array}{l}5.2 E+01 \\
44.000 \\
1.1 E+04 \\
5.8 E+01 \\
6.3 E+03\end{array}$ & $\begin{array}{l}1.3 E+02 \\
35,200 \\
7.3 E+03 \\
8.9 E+01 \\
2.7 E+03\end{array}$ \\
\hline HSB117D & Tritium & $\mathrm{pCi} / \mathrm{mL}$ & $2.7 E+02$ & $1.5 E+02$ & $1.2 E+02$ & $1.1 E+02$ \\
\hline HSB1 25D & $\begin{array}{l}\text { Mercury } \\
\text { Nitrate-nitrite as nitrogen } \\
\text { Nonvolatile beta } \\
\text { Tritium }\end{array}$ & $\begin{array}{l}\mu g / L \\
\mu g / L \\
\mathrm{pCi} / \mathrm{L} \\
\mathrm{pCi} / \mathrm{mL}\end{array}$ & $\begin{array}{l}- \\
36,600 \\
\overline{5} .9 E+03\end{array}$ & $\begin{array}{l}- \\
32,000 \\
5.1 E+01 \\
5.1 E+03\end{array}$ & $\begin{array}{l}2.7 \\
34,000 \\
- \\
3.8 E+03\end{array}$ & $\begin{array}{l}4.1 \\
48,500 \\
- \\
4.7 E+03\end{array}$ \\
\hline
\end{tabular}


WSRC-TR-93-059

Aquifer Zone $\mathrm{IIB}_{2}$ (Water Table)

\begin{tabular}{|c|c|c|c|c|c|c|}
\hline Well & Constituent & Unit & 1092 & 2092 & $\underline{3092}$ & 4092 \\
\hline HSB1 26D & $\begin{array}{l}\text { Mercury } \\
\text { Nitrate-nitrite as nitrogen } \\
\text { Tritium }\end{array}$ & $\begin{array}{l}\mu \mathrm{g} / \mathrm{L} \\
\mu \mathrm{g} / \mathrm{L} \\
\mathrm{pCi} / \mathrm{mL}\end{array}$ & $\begin{array}{l}4.6 \\
53,600 \\
5.5 E+03\end{array}$ & $\begin{array}{l}6.8 \\
59.000 \\
5.4 E+03\end{array}$ & $\begin{array}{l}6.0 \\
54,800 \\
4.8 E+03\end{array}$ & $\begin{array}{l}7.9 \\
51.500 \\
4.8 E+03\end{array}$ \\
\hline HSB1 27D & $\begin{array}{l}\text { Mercury } \\
\text { Nitrate-nitrite as nitrogen } \\
\text { Nonvolatile beta } \\
\text { Tritium }\end{array}$ & $\begin{array}{l}\mu g / L \\
\mu g / L \\
\mathrm{pCi} / \mathrm{L} \\
\mathrm{pCi} / \mathrm{mL}\end{array}$ & $\begin{array}{l}2.6 \\
35,600 \\
7.0 E+01 \\
1.1 E+04\end{array}$ & $\begin{array}{l}3.4 \\
26,000 \\
6.1 E+01 \\
8.0 E+03\end{array}$ & $\begin{array}{l}3.1 \\
25,500 \\
5.0 E+01 \\
7.4 E+03\end{array}$ & $\begin{array}{l}4.3 \\
23,400 \\
6.7 E+01 \\
7.5 E+03\end{array}$ \\
\hline HSB129D & $\begin{array}{l}\text { Nitrate-nitrite as nitrogen } \\
\text { Nonvolatile beta } \\
\text { Total alpha-emitting radium } \\
\text { Tritium }\end{array}$ & $\begin{array}{l}\mu \mathrm{g} / \mathrm{L} \\
\mathrm{pCi} / \mathrm{L} \\
\mathrm{pCi} / \mathrm{L} \\
\mathrm{pCi} / \mathrm{mL}\end{array}$ & $\begin{array}{l}46,500 \\
1.2 E+02 \\
9.0 E+00 \\
7.2 E+03\end{array}$ & $\begin{array}{l}40,000 \\
5.2 E+01 \\
- \\
6.9 E+C .3\end{array}$ & $\begin{array}{l}36,000 \\
7.1 E+01 \\
- \\
6.3 E+03\end{array}$ & $\begin{array}{l}33,000 \\
5.6 E+01 \\
- \\
5.1 E+03\end{array}$ \\
\hline HSB133D & Tritium & $\mathrm{pCi} / \mathrm{mL}$ & $3.5 E+01$ & $3.3 E+01$ & $2.9 E+01$ & $3.0 E+01$ \\
\hline HSB134D & $\begin{array}{l}\text { Nitrate-nitrite as nitrogen } \\
\text { Nonvolatile beta } \\
\text { Total alpha-emitting radium } \\
\text { Tritium }\end{array}$ & $\begin{array}{l}\mu \mathrm{g} / \mathrm{L} \\
\mathrm{pCi} / \mathrm{L} \\
\mathrm{pCi} / \mathrm{L} \\
\mathrm{pCi} / \mathrm{mL}\end{array}$ & $\begin{array}{l}10,200 \\
2.6 E+C 2 \\
- \\
1.5 E+03\end{array}$ & $\begin{array}{l}15,800 \\
3.2 E+02 \\
1.1 E+01 \\
1.6 E+03\end{array}$ & $\begin{array}{l}12,000 \\
4.6 E+02 \\
- \\
8.6 E+02\end{array}$ & $\begin{array}{l}13,800 \\
3.4 E+02 \\
8.4 E+00 \\
9.5 E+02\end{array}$ \\
\hline HSB135D & $\begin{array}{l}\text { Nonvolatile beta } \\
\text { Tritium }\end{array}$ & $\begin{array}{l}\mathrm{pCi} / \mathrm{L} \\
\mathrm{pCi} / \mathrm{mL}\end{array}$ & $\overline{2} .8 E+02$ & $\begin{array}{l}6.8 E+01 \\
3.4 E+02\end{array}$ & $\overline{1} .5 E+02$ & $\begin{array}{l}8.1 E+01 \\
2.7 E+02\end{array}$ \\
\hline HSB136D & $\begin{array}{l}\text { Gross alpha } \\
\text { Nitrate-nitrite as nitrogen } \\
\text { Nonvolatile beta } \\
\text { Total alpha-emitting radium } \\
\text { Tritium }\end{array}$ & $\begin{array}{l}\mathrm{pCi} / \mathrm{L} \\
\mu \mathrm{g} / \mathrm{L} \\
\mathrm{pCi} / \mathrm{L} \\
\mathrm{pCi} / \mathrm{L} \\
\mathrm{pCi} / \mathrm{mL}\end{array}$ & $\begin{array}{l}5.0 E+01 \\
29,000 \\
2.1 E+03 \\
7.4 E+01 \\
8.8 E+03\end{array}$ & $\begin{array}{l}9.7 E+01 \\
28400 \\
2.8 E+03 \\
5.5 E+01 \\
6.2 E+03\end{array}$ & $\begin{array}{l}6.0 E+01 \\
27.400 \\
2.0 E+03 \\
4.4 E+01 \\
4.5 E+03\end{array}$ & $\begin{array}{l}1.2 E+02 \\
28,600 \\
2.7 E+03 \\
6.5 E+01 \\
4.7 E+03\end{array}$ \\
\hline HSB137D & $\begin{array}{l}\text { Nitrate-nitrite as nitrogen } \\
\text { Nonvolatile beta } \\
\text { Tritium }\end{array}$ & $\begin{array}{l}\mu g / L \\
\mathrm{pCi} / \mathrm{L} \\
\mathrm{pCi} / \mathrm{mL}\end{array}$ & $\begin{array}{l}- \\
1.1 E+02 \\
4.6 E+03\end{array}$ & $\begin{array}{l}10,000 \\
8.4 E+01 \\
3.7 E+03\end{array}$ & $\begin{array}{l}18,200 \\
5.8 E+01 \\
3.0 E+03\end{array}$ & $\begin{array}{l}14,000 \\
6.4 E+01 \\
3.4 E+03\end{array}$ \\
\hline HSB138D & Tritium & $\mathrm{pCi} / \mathrm{mL}$ & $2.0 E+03$ & $9.4 E+02$ & $4.8 E+02$ & $5.6 E+02$ \\
\hline HSB141D & Tritium & $\mathrm{pCi} / \mathrm{mL}$ & $2.3 E+01$ & $2.1 E+01$ & - & - \\
\hline HSB142D & Tritium & $\mathrm{pCi} / \mathrm{mL}$ & $4.3 E+0.2$ & $6.7 E+02$ & $4.2 E+02$ & $3.9 E+02$ \\
\hline HSB143D & $\begin{array}{l}\text { Tetrachloroethylene } \\
\text { Total alpha-emitting radium }\end{array}$ & $\begin{array}{l}\mu g / L \\
\mathrm{pCi} / \mathrm{L}\end{array}$ & $\begin{array}{l}- \\
-\end{array}$ & $\begin{array}{l}8.7 \\
-\end{array}$ & $\overline{1.7 E}+01$ & - \\
\hline HSB145D & $\begin{array}{l}\text { Gross alpha } \\
\text { Mercury } \\
\text { Nitrate-nitrite as nitrogen } \\
\text { Nonvolatile beta } \\
\text { Total alpha-emitting radium } \\
\text { Tritium }\end{array}$ & $\begin{array}{l}\mathrm{pCi} / \mathrm{L} \\
\mu \mathrm{g} / \mathrm{L} \\
\mu \mathrm{g} / \mathrm{L} \\
\mathrm{pCi} / \mathrm{L} \\
\mathrm{pCi} / \mathrm{L} \\
\mathrm{pCi} / \mathrm{mL}\end{array}$ & $\begin{array}{l}2.1 E+01 \\
- \\
36,800 \\
3.8 E+02 \\
1.7 E+01 \\
3.7 E+03\end{array}$ & $\begin{array}{l}5.7 E+01 \\
- \\
39,000 \\
4.6 E+02 \\
3.3 E+01 \\
4.8 E+03\end{array}$ & $\begin{array}{l}5.6 E+01 \\
- \\
39,200 \\
4.4 E+02 \\
1.6 E+01 \\
5.4 E+03\end{array}$ & $\begin{array}{l}3.3 E+01 \\
2.6 \\
36,800 \\
3.4 E+02 \\
2.4 E+01 \\
6.4 E+03\end{array}$ \\
\hline HSB146D & Tritium & $\mathrm{pCi} / \mathrm{mL}$ & - & $2.0 E+01$ & $2.3 E+01$ & $2.0 E+01$ \\
\hline HSB147D & Tritium & $\mathrm{pCi} / \mathrm{mL}$ & $2.4 E+01$ & $2.2 E+01$ & $2.1 E+01$ & - \\
\hline HSB149D & Tritium & $\mathrm{pCi} / \mathrm{mL}$ & $5.0 E+01$ & $3.1 E+01$ & $2.8 E+01$ & 2.7E + 01 \\
\hline
\end{tabular}


WSRC-TR-93-059

Aquifer Zone $\mathrm{IIB}_{2}$ (Water Table)

\begin{tabular}{|c|c|c|c|c|c|c|}
\hline Well & Constituent & Unit & 1092 & $\underline{2092}$ & 3092 & 4092 \\
\hline HSB150D & Tritium & $\mathrm{pCi} / \mathrm{mL}$ & 4.1E + 01 & $3.3 E+01$ & - & $3.7 E+01$ \\
\hline HSB151D & Tritium & $\mathrm{pCi} / \mathrm{mL}$ & $6.9 E+02$ & $4.8 E+02$ & $2.6 E+02$ & $2.6 E+02$ \\
\hline HSB152D & $\begin{array}{l}\text { Lead } \\
\text { Tritium }\end{array}$ & $\begin{array}{l}\mu \mathrm{g} / \mathrm{L} \\
\mathrm{pCi} / \mathrm{mL}\end{array}$ & $\overline{5.6 E}+02$ & $\overline{4.7 E}+02$ & $\overline{2 . O E}+02$ & $\begin{array}{l}71 \\
3.9 E+02\end{array}$ \\
\hline
\end{tabular}

Aquifer Zone IIB, (Barnwell/McBean, upper portion)

\begin{tabular}{|c|c|c|c|c|c|c|}
\hline Well & Constituent & Unit & 1092 & $\underline{2092}$ & 3092 & 40.92 \\
\hline HSB $68 C$ & Tritium & $\mathrm{pCi} / \mathrm{mL}$ & $1.8 \mathrm{E}+03$ & $2.3 E+03$ & $2.4 E+03$ & $2.6 E+03$ \\
\hline HSB 7OC & $\begin{array}{l}\text { Nitrate as nitrogen } \\
\text { Nitrate-nitrite as nitrogen } \\
\text { Nonvolatile beta } \\
\text { Tritium }\end{array}$ & $\begin{array}{l}\mu g / L \\
\mu g / L \\
\mathrm{pCi} / \mathrm{L} \\
\mathrm{pCi} / \mathrm{mL}\end{array}$ & $\begin{array}{l}32,600 \\
N A \\
1.2 E+02 \\
4.1 E+03\end{array}$ & $\begin{array}{l}\text { NA } \\
27.000 \\
7.6 E+01 \\
3.1 E+03\end{array}$ & $\begin{array}{l}\text { NA } \\
27.500 \\
1.0 E+02 \\
3.1 E+03\end{array}$ & $\begin{array}{l}\text { NA } \\
30,500 \\
9.1 E+01 \\
3.4 E+03\end{array}$ \\
\hline HSB 71C & $\begin{array}{l}\text { Gross alpha } \\
\text { Nitrate-nitrite as nitrogen } \\
\text { Nonvolatile beta } \\
\text { Total alpha-emitting radium } \\
\text { Tritium }\end{array}$ & $\begin{array}{l}\mathrm{pCi} / \mathrm{L} \\
\mu \mathrm{g} / \mathrm{L} \\
\mathrm{pCi} / \mathrm{L} \\
\mathrm{pCi} / \mathrm{L} \\
\mathrm{pCi} / \mathrm{mL}\end{array}$ & $\begin{array}{l}- \\
50,500 \\
1.6 E+02 \\
1.2 E+01 \\
8.6 E+03\end{array}$ & $\begin{array}{l}- \\
51,000 \\
1.9 E+02 \\
5.9 E+00 \\
8.3 E+03\end{array}$ & $\begin{array}{l}2.6 E+01 \\
52,000 \\
1.4 E+02 \\
6.8 E+00 \\
7.8 E+03\end{array}$ & $\begin{array}{l}1.8 E+01 \\
50,500 \\
1.2 E+02 \\
8.7 E+00 \\
7.6 E+03\end{array}$ \\
\hline HSB 84C & Tritium & $\mathrm{pCi} / \mathrm{mL}$ & $3.9 E+02$ & $4.0 E+02$ & $3.8 E+02$ & $3.9 E+02$ \\
\hline HSB 86C & $\begin{array}{l}\text { Cadmium } \\
\text { Gross alpha } \\
\text { Nitrate as nitrogen } \\
\text { Nitrate-nitrite as nitrogen } \\
\text { Nonvolatile beta } \\
\text { Total alpha-emitting radium } \\
\text { Tritium }\end{array}$ & $\begin{array}{l}\mu g / L \\
\mathrm{pCi} / \mathrm{L} \\
\mu g / L \\
\mu g / L \\
\mathrm{pCi} / \mathrm{L} \\
\mathrm{pCi} / \mathrm{L} \\
\mathrm{pCi} / \mathrm{mL}\end{array}$ & $\begin{array}{l}9.8 \\
2.0 E+01 \\
40,400 \\
N A \\
2.9 E+02 \\
1.6 E+01 \\
1.5 E+04\end{array}$ & $\begin{array}{l}8.9 \\
5.8 E+01 \\
N A \\
39.700 \\
4.4 E+02 \\
2.1 E+01 \\
1.7 E+04\end{array}$ & $\begin{array}{l}8.3 \\
- \\
\text { NA } \\
45,000 \\
2.6 E+02 \\
1.7 E+01 \\
1.6 E+04\end{array}$ & $\begin{array}{l}- \\
3.9 E+01 \\
N A \\
52,500 \\
5.5 E+02 \\
1.7 E+01 \\
1.6 E+04\end{array}$ \\
\hline HSB101C & Tritium & $\mathrm{pCi} / \mathrm{mL}$ & - & $2.0 E+01$ & - & - \\
\hline HSB102C & $\begin{array}{l}\text { Nitrate-nitrite as nitrogen } \\
\text { Tritium }\end{array}$ & $\begin{array}{l}\mu g / L \\
\mathrm{pCi} / \mathrm{mL}\end{array}$ & $\begin{array}{l}13,400 \\
2.2 E+02\end{array}$ & $\begin{array}{l}14,400 \\
2.2 E+02\end{array}$ & $\begin{array}{l}15,200 \\
1.9 E+02\end{array}$ & $\begin{array}{l}14,200 \\
1.9 E+02\end{array}$ \\
\hline HSB $103 C$ & $\begin{array}{l}\text { Nitrate-nitrite as nitrogen } \\
\text { Tritium }\end{array}$ & $\begin{array}{l}\mu \mathrm{g} / \mathrm{L} \\
\mathrm{pCi} / \mathrm{mL}\end{array}$ & $\begin{array}{l}22,800 \\
8.2 E+02\end{array}$ & $\begin{array}{l}22,000 \\
8.1 E+02\end{array}$ & $\begin{array}{l}24,000 \\
7.3 E+02\end{array}$ & $\begin{array}{l}24,000 \\
7.1 E+02\end{array}$ \\
\hline HSB104C & Tritium & $\mathrm{pCi} / \mathrm{mL}$ & $3.0 E+02$ & $2.9 E+02$ & $2.6 E+02$ & $2.6 E+02$ \\
\hline HSB105C & Tritium & $\mathrm{pCi} / \mathrm{mL}$ & $1.1 E+02$ & $1.0 E+02$ & $1.0 E+02$ & $9.3 E+01$ \\
\hline HSB106C & $\begin{array}{l}\text { Tetrachloroethylene } \\
\text { Tritium }\end{array}$ & $\begin{array}{l}\mu \mathrm{g} / \mathrm{L} \\
\mathrm{pCi} / \mathrm{mL}\end{array}$ & $\begin{array}{l}5.2 \\
3.8 E+02\end{array}$ & $\begin{array}{l}5.7 \\
3.6 E+02\end{array}$ & $\overline{3.1 E}+02$ & $\begin{array}{l}6.6 \\
3.2 E+02\end{array}$ \\
\hline HSB107C & $\begin{array}{l}\text { Nonvolatile beta } \\
\text { Tritium }\end{array}$ & $\begin{array}{l}\mathrm{pCi} / \mathrm{L} \\
\mathrm{pCi} / \mathrm{mL}\end{array}$ & $\begin{array}{l}1.2 E+02 \\
4.4 E+02\end{array}$ & $\overline{4} .2 E+02$ & $\overline{3} .7 E+02$ & $\overline{4} .1 E+02$ \\
\hline
\end{tabular}


Aquifer Zone IIB, (Barnwell/McBean, upper portion)

\begin{tabular}{|c|c|c|c|c|c|c|}
\hline Well & Constituent & $\underline{\text { Unit }}$ & 1092 & $\underline{2092}$ & $\underline{3092}$ & 4092 \\
\hline HSB108C & $\begin{array}{l}\text { Nitrate-nitrite as nitrogen } \\
\text { Tritium }\end{array}$ & $\begin{array}{l}\mu \mathrm{g} / \mathrm{L} \\
\mathrm{pCi} / \mathrm{mL}\end{array}$ & $\overline{3} .6 \mathrm{E}+02$ & $\overline{3} .6 \mathrm{E}+02$ & $\begin{array}{l}32,000 \\
3.3 E+02\end{array}$ & $\overline{3.1 E}+02$ \\
\hline HSB109C & Tritium & $\mathrm{pCi} / \mathrm{mL}$ & $7.8 E+01$ & $7.8 E+01$ & $6.9 E+01$ & $6.7 E+01$ \\
\hline HSB110C & Tritium & $\mathrm{pCi} / \mathrm{mL}$ & $3.8 E+01$ & $3.6 \mathrm{E}+01$ & $3.0 E+01$ & $3.1 E+01$ \\
\hline HSB111C & $\begin{array}{l}\text { Nitrate-nitrite as nitrogen } \\
\text { Nonvolatile beta } \\
\text { Tritium }\end{array}$ & $\begin{array}{l}\mu \mathrm{g} / \mathrm{L} \\
\mathrm{pCi} / \mathrm{L} \\
\mathrm{pCi} / \mathrm{mL}\end{array}$ & $\begin{array}{l}24,200 \\
9.1 E+01 \\
3.6 E+03\end{array}$ & $\begin{array}{l}24,000 \\
\overline{3.4 E}+03\end{array}$ & $\begin{array}{l}24.000 \\
6.0 E+01 \\
3.2 E+03\end{array}$ & $\begin{array}{l}25,000 \\
6.2 E+01 \\
3.1 E+03\end{array}$ \\
\hline HSB112C & $\begin{array}{l}\text { Nitrate-nitrite as nitrogen } \\
\text { Nonvolatile beta } \\
\text { Tritium }\end{array}$ & $\begin{array}{l}\mu \mathrm{g} / \mathrm{L} \\
\mathrm{pCi} / \mathrm{L} \\
\mathrm{pCi} / \mathrm{mL}\end{array}$ & $\begin{array}{l}15,400 \\
7.8 E+01 \\
2.5 E+03\end{array}$ & $\begin{array}{l}14,000 \\
5.1 E+01 \\
2.3 E+03\end{array}$ & $\begin{array}{l}14,000 \\
- \\
2.0 E+03\end{array}$ & $\begin{array}{l}13,800 \\
- \\
1.8 E+03\end{array}$ \\
\hline HSB113C & $\begin{array}{l}\text { Nitrate-nitrite as nitrogen } \\
\text { Nonvolatile beta } \\
\text { Tritium }\end{array}$ & $\begin{array}{l}\mu \mathrm{g} / \mathrm{L} \\
\mu \mathrm{g} / \mathrm{L} \\
\mathrm{pCi} / \mathrm{mL}\end{array}$ & $\begin{array}{l}13,600 \\
- \\
1.6 E+03\end{array}$ & $\begin{array}{l}14,200 \\
5.4 E+01 \\
1.6 E+03\end{array}$ & $\begin{array}{l}14,800 \\
5.0 E+01 \\
1.6 E+03\end{array}$ & $\begin{array}{l}14,400 \\
- \\
1.6 E+03\end{array}$ \\
\hline HSB114C & $\begin{array}{l}\text { Gross alpha } \\
\text { Nitrate as nitrogen } \\
\text { Nitrate-nitrite as nitrogen } \\
\text { Nonvolatile beta } \\
\text { Total alpha-emitting radium } \\
\text { Tritium }\end{array}$ & $\begin{array}{l}\mathrm{pCi} / \mathrm{L} \\
\mu \mathrm{g} / \mathrm{L} \\
\mu \mathrm{g} / \mathrm{L} \\
\mathrm{pCi} / \mathrm{L} \\
\mathrm{pCi} / \mathrm{L} \\
\mathrm{pCi} / \mathrm{mL}\end{array}$ & $\begin{array}{l}- \\
54,400 \\
N A \\
1.9 E+02 \\
9.7 E+00 \\
1.3 E+04\end{array}$ & $\begin{array}{l}1.7 E+01 \\
N A \\
56,500 \\
1.8 E+02 \\
8.5 E+00 \\
1.4 E+04\end{array}$ & $\begin{array}{l}- \\
N A \\
56,500 \\
1.4 E+02 \\
1.0 E+01 \\
1.3 E+04\end{array}$ & $\begin{array}{l}\overline{N A} \\
13,500 \\
- \\
\overline{1} \\
\overline{1.6 E}+03\end{array}$ \\
\hline HSB115C & $\begin{array}{l}\text { Nitrate-nitrite as nitrogen } \\
\text { Nonvolatile beta } \\
\text { Total alpha-emitting radium } \\
\text { Tritium }\end{array}$ & $\begin{array}{l}\mu g / L \\
\mathrm{pCi} / \mathrm{L} \\
\mathrm{pCi} / \mathrm{L} \\
\mathrm{pCi} / \mathrm{mL}\end{array}$ & $\begin{array}{l}49.200 \\
1.5 E+02 \\
- \\
1.6 E+04\end{array}$ & $\begin{array}{l}53,000 \\
1.7 E+02 \\
5.4 E+00 \\
1.6 E+04\end{array}$ & $\begin{array}{l}51,500 \\
1.3 E+02 \\
- \\
1.4 E+04\end{array}$ & $\begin{array}{l}57.200 \\
8.1 E+01 \\
6.9 E+00 \\
1.4 E+04\end{array}$ \\
\hline HSB116C & $\begin{array}{l}\text { Gross alpha } \\
\text { Nitrate-nitrite as nitrogen } \\
\text { Nonvolatile beta } \\
\text { Total alpha-emitting radium } \\
\text { Tritium }\end{array}$ & $\begin{array}{l}\mathrm{pCi} / \mathrm{L} \\
\mu \mathrm{g} / \mathrm{L} \\
\mathrm{pCi} / \mathrm{L} \\
\mathrm{pCi} / \mathrm{L} \\
\mathrm{pCi} / \mathrm{mL}\end{array}$ & $\begin{array}{l}1.7 E+01 \\
61.000 \\
1.2 E+02 \\
6.1 E+00 \\
1.7 E+04\end{array}$ & $\begin{array}{l}2.4 E+01 \\
57.200 \\
5.8 E+01 \\
6.5 E+00 \\
1.7 E+04\end{array}$ & $\begin{array}{l}- \\
69,000 \\
7.8 E+01 \\
8.8 E+00 \\
1.5 E+04\end{array}$ & $\begin{array}{l}- \\
58,000 \\
7.6 E+01 \\
8.7 E+00 \\
1.5 E+04\end{array}$ \\
\hline HSB117C & $\begin{array}{l}\text { Gross alpha } \\
\text { Nitrate-nitrite as nitrogen } \\
\text { Nonvolatile beta } \\
\text { Total alpha-emitting radium } \\
\text { Tritium }\end{array}$ & $\begin{array}{l}\mathrm{pCi} / \mathrm{L} \\
\mu \mathrm{g} / \mathrm{L} \\
\mathrm{pCi} / \mathrm{L} \\
\mathrm{pCi} / \mathrm{L} \\
\mathrm{pCi} / \mathrm{mL}\end{array}$ & $\begin{array}{l}- \\
38,000 \\
1.9 E+02 \\
8.8 E+00 \\
1.0 E+04\end{array}$ & $\begin{array}{l}3.0 E+01 \\
25,700 \\
8.1 E+01 \\
1.4 E+01 \\
9.5 E+03\end{array}$ & $\begin{array}{l}2.3 E+01 \\
- \\
9.8 E+01 \\
6.2 E+00 \\
7.9 E+03\end{array}$ & $\begin{array}{l}\text { NA } \\
\text { NA } \\
\text { NA } \\
\text { NA } \\
\text { NA }\end{array}$ \\
\hline HSB126C & $\begin{array}{l}\text { Nitrate-nitrite as nitrogen } \\
\text { Total alpha-emitting radium } \\
\text { Tritium }\end{array}$ & $\begin{array}{l}\mu \mathrm{g} / \mathrm{L} \\
\mathrm{pCi} / \mathrm{L} \\
\mathrm{pCi} / \mathrm{mL}\end{array}$ & $\begin{array}{l}- \\
2.9 E+02\end{array}$ & $\begin{array}{l}52,000 \\
- \\
3.0 E+02\end{array}$ & $\begin{array}{l}- \\
5.9 E+00 \\
2.9 E+02\end{array}$ & $\begin{array}{l}- \\
3.0 E+02\end{array}$ \\
\hline HSB1 27C & $\begin{array}{l}\text { Nitrate-nitrite as nitrogen } \\
\text { Total alpha-emitting radium } \\
\text { Tritium }\end{array}$ & $\begin{array}{l}\mu \mathrm{g} / \mathrm{L} \\
\mathrm{pCi} / \mathrm{L} \\
\mathrm{pCi} / \mathrm{mL}\end{array}$ & 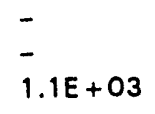 & $\overline{-}-\overline{O E}+03$ & $\begin{array}{l}11,000 \\
3.4 E+01 \\
9.5 E+02\end{array}$ & $\begin{array}{l}10,200 \\
- \\
9.6 \mathrm{E}+02\end{array}$ \\
\hline HSB1 29C & $\begin{array}{l}\text { Nitrate-nitrite as nitrogen } \\
\text { Nonvolatile beta } \\
\text { Tritium }\end{array}$ & $\begin{array}{l}\mu g / L \\
p C i / L \\
p C i / m L\end{array}$ & $\begin{array}{l}21,000 \\
1.2 E+02 \\
2.5 E+03\end{array}$ & $\begin{array}{l}21,000 \\
- \\
2.4 E+03\end{array}$ & $\begin{array}{l}13,500 \\
5.0 E+01 \\
1.8 E+03\end{array}$ & $\begin{array}{l}16,000 \\
- \\
1.9 E+03\end{array}$ \\
\hline HSB131C & Tritium & $\mathrm{pCi} / \mathrm{mL}$ & $1.8 E+02$ & $1.8 E+02$ & $1.6 E+02$ & $1.6 E+.02$ \\
\hline
\end{tabular}


Aquifer Zone IIB, (Barnwell/McBean, upper portion)

\begin{tabular}{|c|c|c|c|c|c|c|}
\hline Well & Constituent & Unit & 1092 & $\underline{2092}$ & 3092 & 4092 \\
\hline HSB134C & Tritium & $\mathrm{pCi} / \mathrm{mL}$ & $4.2 E+01$ & $4.3 E+01$ & $3.8 E+01$ & $3.6 E+01$ \\
\hline HSB135C & Tritium & $\mathrm{pCi} / \mathrm{mL}$ & $4.7 E+01$ & $5.1 E+01$ & $6.2 E+01$ & $4.6 E+01$ \\
\hline HSB136C & $\begin{array}{l}\text { Nitrate-nitrite as nitrogen } \\
\text { Nonvolatile beta } \\
\text { Tritium }\end{array}$ & $\begin{array}{l}\mu \mathrm{g} / \mathrm{L} \\
\mathrm{pCi} / \mathrm{L} \\
\mathrm{pCi} / \mathrm{mL}\end{array}$ & $\begin{array}{l}41,500 \\
1.5 E+02 \\
1.1 E+04\end{array}$ & $\begin{array}{l}44,200 \\
5.2 E+01 \\
1.0 E+04\end{array}$ & $\begin{array}{l}37.600 \\
8.7 E+01 \\
8.1 E+03\end{array}$ & $\begin{array}{l}38,400 \\
1.1 E+02 \\
9.0 E+03\end{array}$ \\
\hline HSB137C & $\begin{array}{l}\text { Nitrate-nitrite as nitrogen } \\
\text { Nonvolatile beta } \\
\text { Tritium }\end{array}$ & $\begin{array}{l}\mu \mathrm{g} / \mathrm{L} \\
\mathrm{pCi} / \mathrm{L} \\
\mathrm{pCi} / \mathrm{mL}\end{array}$ & $\begin{array}{l}90,000 \\
1.5 E+02 \\
1.2 E+04\end{array}$ & $\begin{array}{l}64,000 \\
1.6 E+02 \\
1.2 E+04\end{array}$ & $\begin{array}{l}60,800 \\
1.7 E+02 \\
1.2 E+04\end{array}$ & $\begin{array}{l}60,000 \\
1.1 E+02 \\
1.2 E+04\end{array}$ \\
\hline HSB139C & $\begin{array}{l}\text { Nitrate-nitrite as nitrogen } \\
\text { Tetrachloroethylene } \\
\text { Tritium }\end{array}$ & $\begin{array}{l}\mu g / L \\
\mu g \cdot L \\
\mathrm{pCi} / \mathrm{mL}\end{array}$ & $\begin{array}{l}43,000 \\
9.8 \\
3.4 E+03\end{array}$ & $\begin{array}{l}50,000 \\
8.8 \\
3.3 E+03\end{array}$ & $\begin{array}{l}48,800 \\
8.8 \\
3.0 E+03\end{array}$ & $\begin{array}{l}45,200 \\
12 \\
2.9 E+03\end{array}$ \\
\hline HSB143C & $\begin{array}{l}\text { Tetrachloroethylene } \\
\text { Total alpha-emitting radium } \\
\text { Trichloroethylene }\end{array}$ & $\begin{array}{l}\mu g / L \\
\mathrm{pCi} / \mathrm{L} \\
\mu g / L\end{array}$ & $\begin{array}{l}- \\
-\end{array}$ & $\begin{array}{l}11 \\
- \\
23\end{array}$ & $\overline{1.2 E}+01$ & $\begin{array}{l}- \\
- \\
-\end{array}$ \\
\hline HSB145C & $\begin{array}{l}\text { Nitrate-nitrite as nitrogen } \\
\text { Nonvolatile beta } \\
\text { Tetrachloroethylene } \\
\text { Trichloroethylene } \\
\text { Tritium }\end{array}$ & $\begin{array}{l}\mu \mathrm{g} / \mathrm{L} \\
\mathrm{pCi} / \mathrm{L} \\
\mu \mathrm{g} / \mathrm{L} \\
\mu \mathrm{g} / \mathrm{L} \\
\mathrm{pCi} / \mathrm{mL}\end{array}$ & $\begin{array}{l}33,600 \\
- \\
12 \\
\overline{2} \\
2.0 E+03\end{array}$ & $\begin{array}{l}33,200 \\
\overline{14} \\
\overline{1} \\
1.9 \mathrm{E}+03\end{array}$ & $\begin{array}{l}33,600 \\
5.0 E+01 \\
16 \\
- \\
1.7 E+03\end{array}$ & $\begin{array}{l}39,200 \\
5.2 E+01 \\
32 \\
35 \\
1.7 E+03\end{array}$ \\
\hline HSB151C & Tritium & $\mathrm{pCi} / \mathrm{mL}$ & $2.2 E+03$ & $2.2 E+03$ & $1.8 E+03$ & $1.8 E+03$ \\
\hline HSB152C & $\begin{array}{l}\text { Nitrate-nitrite as nitrogen } \\
\text { Tritium }\end{array}$ & $\begin{array}{l}\mu \mathrm{g} / \mathrm{L} \\
\mathrm{pCi} / \mathrm{mL}\end{array}$ & $\overline{1.2 \mathrm{E}+03}$ & $\overline{1.2 E}+03$ & $\begin{array}{l}10,500 \\
1.2 E+03\end{array}$ & $\overline{1} .1 E+03$ \\
\hline
\end{tabular}

Aquifer Zone IIB, (Barnwell/McBean, lower portion)

$\begin{array}{llllllll}\text { Well } & \text { Constituent } & \text { Unit } & \text { 1092 } & \text { 2092 } & \text { 3092 } & \underline{4092} & \text { Mod } \\ \text { HSB 68B } & \text { Tritium } & \text { pCi } / \mathrm{mL} & 2.7 \mathrm{E}+01 & 1.9 \mathrm{E}+02 & 2.5 \mathrm{E}+01 & 3.8 \mathrm{E}+01 \\ \mathrm{HSB} \mathrm{848} & \text { Tritium } & \mathrm{pCi} / \mathrm{mL} & 2.1 \mathrm{E}+01 & 8.6 \mathrm{E}+01 & 7.8 \mathrm{E}+01 & 8.4 \mathrm{E}+01\end{array}$

\begin{tabular}{|c|c|c|c|c|c|c|}
\hline Well & Constituent & Unit & 1092 & $\underline{2092}$ & 3092 & 4092 \\
\hline HSB 65A & Tritium & $\mathrm{pCi} / \mathrm{mL}$ & $3.0 E+01$ & $3.0 E+01$ & $2.2 E+01$ & - \\
\hline HSB 84A & $\begin{array}{l}\text { Nonvolatile beta } \\
\text { Tritium }\end{array}$ & $\begin{array}{l}\mathrm{pCi} / \mathrm{L} \\
\mathrm{pCi} / \mathrm{mL}\end{array}$ & $\begin{array}{l}1.8 E+02 \\
4.9 E+01\end{array}$ & $\begin{array}{l}1.7 E+02 \\
4.2 E+01\end{array}$ & $\begin{array}{l}1.5 E+02 \\
3.7 E+01\end{array}$ & $\begin{array}{l}9.2 E+01 \\
2.8 E+01\end{array}$ \\
\hline HSB1 18A & $\begin{array}{l}\text { Nitrate-nitrite as nitrogen } \\
\text { Tritium }\end{array}$ & $\begin{array}{l}\mu \mathrm{g} / \mathrm{L} \\
\mathrm{pCi} / \mathrm{mL}\end{array}$ & $\overline{6} .6 \mathrm{E}+02$ & $\overline{2} .2 E+03$ & $\begin{array}{l}13,500 \\
2.8 E+03\end{array}$ & $\begin{array}{l}18,600 \\
5.0 E+03\end{array}$ \\
\hline HSB1 19A & Tritium & $\mathrm{pCi} / \mathrm{mL}$ & $2.5 E+02$ & $2.5 E+02$ & $2.7 E+02$ & $3.4 E+02$ \\
\hline
\end{tabular}


WSRC-TR-93-059

Aquifor Unit IIA (Congaree)

$\begin{array}{llllllll}\text { Well } & \text { Constituent } & \text { Unit } & \text { 1092 } & \text { 2092 } & \underline{3092} & \underline{4092} & \text { Mod } \\ \text { HSB139A } & \text { Nonvolatile beta } & \text { pCi/L } & - & - & 6.0 E+01 & - \\ \text { HSB144A } & \text { Tritium } & \mathrm{pCi} / \mathrm{mL} & 2.1 E+03 & 1.5 E+03 & 1.1 E+03 & 8.0 E+02\end{array}$

Note: Table 1 presents the highest value for duplicate/replicate results. The drinking water standard for lead was changed to the South Carolina Primary Drinking Water Standard or $50 \mu \mathrm{g} / \mathrm{L}$ fourth quarter 1992.

- - = analyzed but not above final PDWS.

b The final POWS for total radium was applied to total alpha-emitting radium.

c $N A=$ not analyzed. 
Table 2. Constituents Exceeding Half the Final Primary Drinking Water Standards or Other Flag 1 or Flag 2 Criteria

Aquifer Zone $\|_{2}$ (Water Table)

\begin{tabular}{|c|c|c|c|c|c|}
\hline Well & Constituent & Unit & 4092 & Mod & Flag \\
\hline \multirow[t]{2}{*}{ HSB 65} & Aluminum & $\mu g / L$ & 78 & & 2 \\
\hline & Lead & $\mu \mathrm{g} / \mathrm{L}$ & 11 & & 1 \\
\hline HSB $65 \mathrm{C}$ & Aluminum & $\mu g / L$ & 35 & & 1 \\
\hline HSB 66 & Aluminum & $\mu g / L$ & 174 & & 2 \\
\hline \multirow[t]{4}{*}{ HSB 67} & Aluminum & $\mu g / L$ & 1,610 & & 2 \\
\hline & Manganese & $\mu g / L$ & 184 & & 2 \\
\hline & Mercury & $\mu \mathrm{g} / \mathrm{L}$ & 1.6 & & 1 \\
\hline & $\mathrm{pH}$ & $\mathrm{pH}$ & 3.7 & $\mathrm{~J}$ & 1 \\
\hline \multirow[t]{4}{*}{ HSB 68} & Aluminum & $\mu g / L$ & 4,550 & & 2 \\
\hline & Cobalt- 60 & $\mathrm{pCi} / \mathrm{L}$ & $1.1 E+02$ & & 2 \\
\hline & Manganese & $\mu g / L$ & 993 & & 2 \\
\hline & Specific conductance & $\mu S / c m$ & 365 & & 1 \\
\hline \multirow[t]{4}{*}{ HSB 69} & Aluminum & $\mu g / L$ & 4,810 & & 2 \\
\hline & Cobalt -60 & $\mathrm{pCi} / \mathrm{L}$ & $1.7 E+02$ & & 2 \\
\hline & Manganese & $\mu g / L$ & 728 & & 2 \\
\hline & $\mathrm{pH}$ & $\mathrm{pH}$ & 3.9 & $J$ & 1 \\
\hline \multirow[t]{2}{*}{ HSB 70} & Aluminum & $\mu g / L$ & 69 & & 2 \\
\hline & Antimony & $\mu g / L$ & 3.1 & & 1 \\
\hline HSB 71 & Aluminum & $\mu \mathrm{g} / \mathrm{L}$ & 77 & & 2 \\
\hline \multirow[t]{5}{*}{ HSB 83D } & Aluminum & $\mu g / L$ & 198 & & 2 \\
\hline & Manganese & $\mu g / L$ & 59 & & 2 \\
\hline & Nitrate-nitrite as nitrogen & $\mu g / L$ & 7,400 & & 1 \\
\hline & Nonvolatile beta & $\mathrm{pCi} / \mathrm{L}$ & $3.1 E+01$ & & 1 \\
\hline & Total alpha-emitting radium ${ }^{\mathrm{a}}$ & $\mathrm{pCi} / \mathrm{L}$ & $2.6 E+00$ & & 1 \\
\hline \multirow[t]{3}{*}{ HSB 84D } & Aluminum & $\mu g / L$ & 588 & & 2 \\
\hline & Gross alpha & $\mathrm{pCi} / \mathrm{L}$ & $1.2 E+01$ & & 1 \\
\hline & Manganese & $\mu g / L$ & 46 & & 1 \\
\hline HSB 85C & Aluminum & $\mu g / L$ & 80 & & 2 \\
\hline \multirow[t]{3}{*}{ HSB 86D } & Aluminum & $\mu g / L$ & 2,300 & & 2 \\
\hline & Cobalt- 60 & $\mathrm{pCi} / \mathrm{L}$ & $6.4 E+01$ & & 1 \\
\hline & Manganese & $\mu g / L$ & 234 & & 2 \\
\hline \multirow[t]{4}{*}{ HSB 10OD } & Aluminum & $\mu g / L$ & 48 & & 1 \\
\hline & Manganese & $\mu g / L$ & 36 & & 1 \\
\hline & Nonvolatile beta & $\mathrm{pCi} / \mathrm{L}$ & $2.6 \mathrm{E}+01$ & & 1 \\
\hline & Total alpha-emitting radium & $\mathrm{pCi} / \mathrm{L}$ & $3.3 E+00$ & & 1 \\
\hline
\end{tabular}


Aquifer Zone $\mathrm{IB}_{2}$ (Water Table)

\begin{tabular}{|c|c|c|c|c|c|}
\hline Well & Constituent & Unit & 4092 & Mod & Flag \\
\hline HSB101D & $\begin{array}{l}\text { Aluminum } \\
\mathrm{pH} \\
\text { Vanadium }\end{array}$ & $\begin{array}{l}\mu g / L \\
\mathrm{pH} \\
\mu g / L\end{array}$ & $\begin{array}{l}845 \\
9.7 \\
506\end{array}$ & $J$ & $\begin{array}{l}2 \\
1 \\
2\end{array}$ \\
\hline HSB 102D & $\begin{array}{l}\text { Aluminum } \\
\text { Cobalt } 60 \\
\text { Iron } \\
\text { Manganese } \\
\text { pH } \\
\text { Silica, total } \\
\text { Specific conductance }\end{array}$ & $\begin{array}{l}\mu \mathrm{g} / \mathrm{L} \\
\mathrm{pCi} / \mathrm{L} \\
\mu \mathrm{g} / \mathrm{L} \\
\mu \mathrm{g} / \mathrm{L} \\
\mathrm{pH} \\
\mu \mathrm{g} / \mathrm{L} \\
\mu \mathrm{S} / \mathrm{cm}\end{array}$ & $\begin{array}{l}16,300 \\
1.3 E+02 \\
255 \\
1,250 \\
3.7 \\
13,800 \\
430\end{array}$ & J & $\begin{array}{l}2 \\
2 \\
1 \\
2 \\
1 \\
2 \\
1\end{array}$ \\
\hline HSB103D & $\begin{array}{l}\text { Aluminum } \\
\text { Manganese }\end{array}$ & $\begin{array}{l}\mu g / L \\
\mu g / L\end{array}$ & $\begin{array}{l}1,670 \\
267\end{array}$ & & $\begin{array}{l}2 \\
2\end{array}$ \\
\hline HSB 104D & $\begin{array}{l}\text { Aluminum } \\
\text { Cobalt }-60 \\
\text { Manganese }\end{array}$ & $\begin{array}{l}\mu \mathrm{g} / \mathrm{L} \\
\mathrm{pCi} / \mathrm{L} \\
\mu g / \mathrm{L}\end{array}$ & $\begin{array}{l}6,400 \\
5.8 E+01 \\
384\end{array}$ & & $\begin{array}{l}2 \\
1 \\
2\end{array}$ \\
\hline HSB 105D & $\begin{array}{l}\text { Aluminum } \\
\text { Manganese } \\
\text { Specific conductance }\end{array}$ & $\begin{array}{l}\mu \mathrm{g} / \mathrm{L} \\
\mu \mathrm{g} / \mathrm{L} \\
\mu \mathrm{S} / \mathrm{cm}\end{array}$ & $\begin{array}{l}6,110 \\
392 \\
320\end{array}$ & & $\begin{array}{l}2 \\
2 \\
1\end{array}$ \\
\hline HSB 107D & $\begin{array}{l}\text { Aluminum } \\
\text { Manganese } \\
\text { Mercury } \\
\text { Silica, total }\end{array}$ & $\begin{array}{l}\mu g / L \\
\mu g / L \\
\mu g / L \\
\mu g / L\end{array}$ & $\begin{array}{l}103 \\
181 \\
1.9 \\
8,760\end{array}$ & & $\begin{array}{l}2 \\
2 \\
1 \\
2\end{array}$ \\
\hline HSB 108D & $\begin{array}{l}\text { Aluminum } \\
\text { Cobalt }-60 \\
\text { Manganese }\end{array}$ & $\begin{array}{l}\mu g / L \\
\mathrm{pCi} / \mathrm{L} \\
\mu g / L\end{array}$ & $\begin{array}{l}1,710 \\
1.1 E+02 \\
629\end{array}$ & & $\begin{array}{l}2 \\
2 \\
2\end{array}$ \\
\hline HSB 1090 & $\begin{array}{l}\text { Aluminum } \\
\text { Gross alpha } \\
\text { Manganese } \\
\text { Nitrate-nitrite as nitrogen }\end{array}$ & $\begin{array}{l}\mu g / L \\
\mathrm{pCi} / \mathrm{L} \\
\mu g / L \\
\mu g / L\end{array}$ & $\begin{array}{l}1,100 \\
9.2 E+00 \\
188 \\
7,300\end{array}$ & & $\begin{array}{l}2 \\
1 \\
2 \\
1\end{array}$ \\
\hline HSB $110 D$ & $\begin{array}{l}\text { Aluminum } \\
\text { Manganese } \\
\text { Total alpha-emitting radium }\end{array}$ & $\begin{array}{l}\mu g / L \\
\mu g / L \\
p C i / L\end{array}$ & $\begin{array}{l}264 \\
51 \\
3.9 E+00\end{array}$ & & $\begin{array}{l}2 \\
2 \\
1\end{array}$ \\
\hline HSB 1110 & $\begin{array}{l}\text { Aluminum } \\
\text { Manganese } \\
\text { Specific conductance }\end{array}$ & $\begin{array}{l}\mu \mathrm{g} / \mathrm{L} \\
\mu \mathrm{g} / \mathrm{L} \\
\mu \mathrm{S} / \mathrm{cm}\end{array}$ & $\begin{array}{l}220 \\
49 \\
450\end{array}$ & & $\begin{array}{l}2 \\
1 \\
1\end{array}$ \\
\hline HSB $111 E$ & Aluminum & $\mu g / L$ & 436 & & 2 \\
\hline HSB $112 D$ & $\begin{array}{l}\text { Aluminum } \\
\text { Manganese } \\
\text { Silica, total } \\
\text { Specific conductance } \\
\text { Total alphaemitting radium }\end{array}$ & $\begin{array}{l}\mu \mathrm{g} / \mathrm{L} \\
\mu \mathrm{g} / \mathrm{L} \\
\mu \mathrm{g} / \mathrm{L} \\
\mu \mathrm{S} / \mathrm{cm} \\
\mathrm{pCi} / \mathrm{L}\end{array}$ & $\begin{array}{l}115 \\
125 \\
7,220 \\
360 \\
3.4 E+00\end{array}$ & & $\begin{array}{l}2 \\
2 \\
2 \\
1 \\
1\end{array}$ \\
\hline
\end{tabular}


Aquifer Zone $\|_{2} B_{2}$ (Water Table)

\begin{tabular}{|c|c|c|c|c|}
\hline Well & Constituent & Unit & $\underline{4092}$ & Mod \\
\hline HSB112E & $\begin{array}{l}\text { Aluminum } \\
\text { Manganese } \\
\text { Specific conductance }\end{array}$ & $\begin{array}{l}\mu \mathrm{g} / \mathrm{L} \\
\mu \mathrm{g} / \mathrm{L} \\
\mu \mathrm{S} / \mathrm{cm}\end{array}$ & $\begin{array}{l}403 \\
543 \\
315\end{array}$ & \\
\hline HSB113D & $\begin{array}{l}\text { Aluminum } \\
\text { Cobalt }-60 \\
\text { Iron } \\
\text { Lead } \\
\text { Manganese } \\
\mathrm{pH}\end{array}$ & $\begin{array}{l}\mu \mathrm{g} / \mathrm{L} \\
\mathrm{pCi} / \mathrm{L} \\
\mu \mathrm{g} / \mathrm{L} \\
\mu \mathrm{g} / \mathrm{L} \\
\mu \mathrm{g} / \mathrm{L} \\
\mathrm{pH}\end{array}$ & $\begin{array}{l}6,470 \\
1.4 E+02 \\
154 \\
11 \\
566 \\
3.9\end{array}$ & $J$ \\
\hline HSB114D & $\begin{array}{l}\text { Aluminum } \\
\text { Cobalt }-60 \\
\text { Manganese } \\
\text { pH } \\
\text { Specific conductance }\end{array}$ & $\begin{array}{l}\mu \mathrm{g} / \mathrm{L} \\
\mathrm{pCi} / \mathrm{L} \\
\mu \mathrm{g} / \mathrm{L} \\
\mathrm{pH} \\
\mu S / \mathrm{cm}\end{array}$ & $\begin{array}{l}8,290 \\
1.6 \mathrm{E}+02 \\
510 \\
3.8 \\
310\end{array}$ & $J$ \\
\hline HSB $115 D$ & $\begin{array}{l}\text { Aluminum } \\
\text { Cadmium } \\
\text { Cobalt } \\
\text { Cobalt-60 } \\
\text { Iron } \\
\text { Manganese } \\
\text { Nickel } \\
\text { Specific conductance }\end{array}$ & $\begin{array}{l}\mu \mathrm{g} / \mathrm{L} \\
\mu \mathrm{g} / \mathrm{L} \\
\cdot \mathrm{g} / \mathrm{L} \\
\mathrm{pCC} / \mathrm{L} \\
\mu \mathrm{g} / \mathrm{L} \\
\mu \mathrm{g} / \mathrm{L} \\
\mu \mathrm{g} / \mathrm{L} \\
\mu \mathrm{S} / \mathrm{cm}\end{array}$ & $\begin{array}{l}8,360 \\
3.7 \\
50 \\
1.7 E+02 \\
1,690 \\
1,730 \\
55 \\
300\end{array}$ & \\
\hline HSB1 16D & $\begin{array}{l}\text { Aluminum } \\
\text { Cobalt-60 } \\
\text { Leac' } \\
\text { Manganese } \\
\text { Speciitic conductance }\end{array}$ & $\begin{array}{l}\mu \mathrm{g} / \mathrm{L} \\
\mathrm{pCi} / \mathrm{L} \\
\mu \mathrm{g} / \mathrm{L} \\
\mu \mathrm{g} / \mathrm{L} \\
\mu \mathrm{S} / \mathrm{cm}\end{array}$ & $\begin{array}{l}2.730 \\
2.6 E+02 \\
12 \\
941 \\
280\end{array}$ & \\
\hline HSB 1170 & Aluminum & $\mu g / L$ & 45 & \\
\hline HSB 125D & $\begin{array}{l}\text { Aluminum } \\
\text { Manganese } \\
\text { Nonvolatile beta } \\
\text { Specific conductance }\end{array}$ & $\begin{array}{l}\mu \mathrm{g} / \mathrm{L} \\
\mu \mathrm{g} / \mathrm{L} \\
\mathrm{pCi} / \mathrm{L} \\
\mu \mathrm{S} / \mathrm{cm}\end{array}$ & $\begin{array}{l}99 \\
71 \\
2.6 E+01 \\
400\end{array}$ & \\
\hline HSB 126D & $\begin{array}{l}\text { Aluminum } \\
\text { Antimony } \\
\text { Manganese } \\
\text { Specific conductance } \\
\text { Total alpha-emitting radium }\end{array}$ & $\begin{array}{l}\mu \mathrm{g} / \mathrm{L} \\
\mu \mathrm{g} / \mathrm{L} \\
\mu \mathrm{g} / \mathrm{L} \\
\mu \mathrm{S} / \mathrm{cm} \\
\mathrm{pCi} / \mathrm{L}\end{array}$ & $\begin{array}{l}472 \\
4.1 \\
45 \\
430 \\
4.9 E+00\end{array}$ & \\
\hline HISE 127D & $\begin{array}{l}\text { A.luminum } \\
\text { Gross alpha } \\
\text { Manganese } \\
\text { Total alpha-emitting radium }\end{array}$ & $\begin{array}{l}\mu \mathrm{g} / \mathrm{L} \\
\mathrm{pCi} / \mathrm{L} \\
\mu \mathrm{g} / \mathrm{L} \\
\mathrm{pCi} / \mathrm{L}\end{array}$ & $\begin{array}{l}175 \\
8.3 E+00 \\
308 \\
4.3 E+00\end{array}$ & \\
\hline
\end{tabular}

Flag

2

2

2

2$$
2
$$$$
1
$$

2$$
2
$$

1

$$
\text { (1) }
$$$$
1
$$$$
\text { (n) }
$$

$$
2
$$

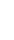$$
1
$$$$
1
$$$$
1
$$$$
1
$$

2

1

2
1 
Aquifer Zone $\|_{2}$ (Water Tahle)

\begin{tabular}{|c|c|c|c|c|c|}
\hline Well & Constituent & Unit & 4092 & Mod & Flag \\
\hline HSB 129D & $\begin{array}{l}\text { Aluminum } \\
\text { Specific conductance } \\
\text { Total alpha-emitting radium }\end{array}$ & $\begin{array}{l}\mu \mathrm{g} / \mathrm{L} \\
\mu \mathrm{S} / \mathrm{cm} \\
\mathrm{pCi} / \mathrm{L}\end{array}$ & $\begin{array}{l}146 \\
270 \\
4.9 E+00\end{array}$ & & $\begin{array}{l}2 \\
1 \\
1\end{array}$ \\
\hline HSB132D & $\begin{array}{l}\text { Aluminum } \\
\text { Iron } \\
\text { Tritium }\end{array}$ & $\begin{array}{l}\mu \mathrm{g} / \mathrm{L} \\
\mu \mathrm{g} / \mathrm{L} \\
\mathrm{pCi} / \mathrm{mL}\end{array}$ & $\begin{array}{l}685 \\
238 \\
1.7 E+01\end{array}$ & & $\begin{array}{l}2 \\
1 \\
1\end{array}$ \\
\hline HSB 133D & Aluminum & $\mu \mathrm{g} / \mathrm{L}$ & 156 & & 2 \\
\hline HSB 134D & $\begin{array}{l}\text { Aluminum } \\
\text { Gross alpha } \\
\text { Manganese }\end{array}$ & $\begin{array}{l}\mu \mathrm{g} / \mathrm{L} \\
\mathrm{pCi} / \mathrm{L} \\
\mu \mathrm{g} / \mathrm{L}\end{array}$ & $\begin{array}{l}473 \\
1.2 E+01 \\
113\end{array}$ & & $\begin{array}{l}2 \\
1 \\
2\end{array}$ \\
\hline HSB 1 35D & Aluminum & $\mu g / L$ & 176 & & 2 \\
\hline HSB136D & $\begin{array}{l}\text { Aluminum } \\
\text { Cobalt- } 60 \\
\text { Manganese } \\
\text { Specific conductance }\end{array}$ & $\begin{array}{l}\mu \mathrm{g} / \mathrm{L} \\
\mathrm{pCi} / \mathrm{L} \\
\mu \mathrm{g} / \mathrm{L} \\
\mu \mathrm{S} / \mathrm{cm}\end{array}$ & $\begin{array}{l}4,710 \\
1.7 E+02 \\
416 \\
260\end{array}$ & & $\begin{array}{l}2 \\
2 \\
2 \\
1\end{array}$ \\
\hline HSB 1 37D & $\begin{array}{l}\text { Aluminum } \\
\text { Manganese } \\
\text { Total alpha-emitting radium }\end{array}$ & $\begin{array}{l}\mu \mathrm{g} / \mathrm{L} \\
\mu \mathrm{g} / \mathrm{L} \\
\mathrm{pCi} / \mathrm{L}\end{array}$ & $\begin{array}{l}100 \\
65 \\
4.7 E+00\end{array}$ & & $\begin{array}{l}2 \\
2 \\
1\end{array}$ \\
\hline HSB $138 D$ & Lead & $\mu \mathrm{g} / \mathrm{L}$ & 8.1 & & 1 \\
\hline HSB139D & $\begin{array}{l}\text { Aluminum } \\
\text { Tritium }\end{array}$ & $\begin{array}{l}\mu \mathrm{g} / \mathrm{L} \\
\mathrm{pCi} / \mathrm{mL}\end{array}$ & $\begin{array}{l}51 \\
2.0 E+01\end{array}$ & & $\begin{array}{l}2 \\
1\end{array}$ \\
\hline HSB140D & $\begin{array}{l}\text { Aluminum } \\
\text { Tritium }\end{array}$ & $\begin{array}{l}\mu \mathrm{g} / \mathrm{L} \\
\mathrm{pCi} / \mathrm{mL}\end{array}$ & $\begin{array}{l}61 \\
1.3 E+01\end{array}$ & & $\begin{array}{l}2 \\
1\end{array}$ \\
\hline HSB 141D & $\begin{array}{l}\text { Aluminum } \\
\text { Iron } \\
\text { Manganese } \\
\text { Tritium }\end{array}$ & $\begin{array}{l}\mu \mathrm{g} / \mathrm{L} \\
\mu \mathrm{g} / \mathrm{L} \\
\mu \mathrm{g} / \mathrm{L} \\
\mathrm{pCi} / \mathrm{mL}\end{array}$ & $\begin{array}{l}539 \\
643 \\
42 \\
1.7 E+01\end{array}$ & & $\begin{array}{l}2 \\
2 \\
1 \\
1\end{array}$ \\
\hline HSB 142D & Aluminum & $\mu g / L$ & 171 & & 2 \\
\hline HSB 143D & $\begin{array}{l}\text { Aluminum } \\
\text { Tritium }\end{array}$ & $\begin{array}{l}\mu \mathrm{g} / \mathrm{L} \\
\mathrm{pCi} / \mathrm{mL}\end{array}$ & $\begin{array}{l}56 \\
1.0 E+01\end{array}$ & & $\begin{array}{l}2 \\
1\end{array}$ \\
\hline HSB 145D & $\begin{array}{l}\text { Aluminum } \\
\text { Manganese } \\
\text { Specific conductance } \\
\text { Trichloroethylene }\end{array}$ & $\begin{array}{l}\mu \mathrm{g} / \mathrm{L} \\
\mu \mathrm{g} / \mathrm{L} \\
\mu \mathrm{S} / \mathrm{cm} \\
\mu \mathrm{g} / \mathrm{L}\end{array}$ & $\begin{array}{l}1,140 \\
925 \\
350 \\
2.8\end{array}$ & & $\begin{array}{l}2 \\
2 \\
1 \\
1\end{array}$ \\
\hline HSB146D & Aluminum & $\mu g / L$ & 195 & & 2 \\
\hline
\end{tabular}


Aquifer Zone $\mathrm{IB}_{2}$ (Water Table)

\begin{tabular}{|c|c|c|c|c|c|}
\hline Well & Constituent & Unit & 4092 & Mod & Flag \\
\hline HSB 147D & $\begin{array}{l}\text { Aluminum } \\
\text { Lead } \\
\text { Tritium }\end{array}$ & $\begin{array}{l}\mu g / L \\
\mu g / L \\
p C i / m L\end{array}$ & $\begin{array}{l}245 \\
8.6 \\
1.8 E+01\end{array}$ & & $\begin{array}{l}2 \\
1 \\
1\end{array}$ \\
\hline HSB148D & $\begin{array}{l}\text { Aluminum } \\
\text { Iron } \\
\mathrm{pH} \\
\text { Tritium }\end{array}$ & $\begin{array}{l}\mu \mathrm{g} / \mathrm{L} \\
\mu \mathrm{g} / \mathrm{L} \\
\mathrm{pH} \\
\mathrm{pCi} / \mathrm{mL}\end{array}$ & $\begin{array}{l}695 \\
191 \\
9.8 \\
1.2 E+01\end{array}$ & $\begin{array}{l}\mathrm{J} 24 \\
\mathrm{~J}\end{array}$ & $\begin{array}{l}2 \\
1 \\
1 \\
1\end{array}$ \\
\hline HSB149D & Aluminum & $\mu g / L$ & 200 & & 2 \\
\hline HSB150D & $\begin{array}{l}\text { Aluminum } \\
\text { Iron }\end{array}$ & $\begin{array}{l}\mu g / L \\
\mu g / L\end{array}$ & $\begin{array}{l}1,770 \\
639\end{array}$ & & $\begin{array}{l}2 \\
2\end{array}$ \\
\hline HSB151D & Aluminum & $\mu g / L$ & 44 & & 1 \\
\hline HSB 152D & $\begin{array}{l}\text { Aluminum } \\
\text { Iron } \\
\text { Manganese }\end{array}$ & $\begin{array}{l}\mu g / L \\
\mu g / L \\
\mu g / L\end{array}$ & $\begin{array}{l}5,050 \\
2,930 \\
36\end{array}$ & $\mathrm{~J} 2$ & $\begin{array}{l}2 \\
2 \\
1\end{array}$ \\
\hline
\end{tabular}

Aquifer Zone $\| B_{1}$ (Barnwell/McBean, upper portion)

\begin{tabular}{|c|c|c|c|c|c|}
\hline Well & Constituent & Unit & 4092 & $\underline{\text { Mod }}$ & Flag \\
\hline \multirow[t]{2}{*}{ HSB 688} & $\begin{array}{l}\text { Aluminum } \\
\text { Iron }\end{array}$ & $\begin{array}{l}\mu g / L \\
\mu g / L\end{array}$ & $\begin{array}{l}310 \\
317\end{array}$ & & $\begin{array}{l}2 \\
2\end{array}$ \\
\hline & $\mathrm{pH}$ & $\mathrm{pH}$ & 8.1 & $J$ & 1 \\
\hline \multirow[t]{5}{*}{ HSB $68 \mathrm{C}$} & Aluminum & $\mu \mathrm{g} / \mathrm{L}$ & 117 & & 2 \\
\hline & Copper & $\mu g / L$ & 865 & & 1 \\
\hline & Iron & $\mu \mathrm{g} / \mathrm{L}$ & 326 & & 2 \\
\hline & Manganese & $\mu g / L$ & 48 & & 1 \\
\hline & Nitrate-nitrite as nitrogen & $\mu \mathrm{g} / \mathrm{L}$ & 8,300 & & 1 \\
\hline \multirow[t]{3}{*}{ HSB 70C } & Aluminum & $\mu g / L$ & 110 & & 2 \\
\hline & $\mathrm{pH}$ & $\mathrm{pH}$ & 11 & $J$ & 2 \\
\hline & Specific conductance & $\mu \mathrm{S} / \mathrm{cm}$ & 340 & & 1 \\
\hline \multirow[t]{3}{*}{ HSB 71C } & Aluminum & $\mu g / L$ & 1,000 & & 2 \\
\hline & Manganese & $\mu g / L$ & 100 & & 2 \\
\hline & Specific conductance & $\mu \mathrm{S} / \mathrm{cm}$ & 600 & & 2 \\
\hline HSB $83 C$ & Aluminum & $\mu g / L$ & 67 & & 2 \\
\hline HSB $84 C$ & Aluminum & $\mu \mathrm{g} / \mathrm{L}$ & 99 & & 2 \\
\hline \multirow[t]{2}{*}{ HSB $86 B$} & Aluminum & $\mu g / L$ & 85 & & 2 \\
\hline & Total organic halogens & $\mu g / L$ & 51 & & 2 \\
\hline
\end{tabular}


Aquifer Zone IIB, (Barnwell/McBean, upper portion)

Well

Constituent

Unit

4092

$\underline{\text { Mod }}$

Flag

HSB 86C

Aluminum
Cadmium
Cobalt
Manganese
Specific conductance

$\mu g / L$

806

$\mu g / L$

$\mu g / L$

$\mu \mathrm{g} / \mathrm{L}$

$\mu \mathrm{S} / \mathrm{cm}$

4.7

24

1,180

355

$\begin{array}{ll}\text { HSB100C } & \begin{array}{l}\text { Aluminum } \\ \text { Antimony }\end{array} \\ \text { HSB101C } & \text { Tritium } \\ \text { HSB102C } & \text { Manganese } \\ \text { HSB103C } & \begin{array}{l}\text { Aluminum } \\ \text { Manganese } \\ \text { Mercury } \\ \text { Total alpha-emitting radium }\end{array}\end{array}$

$\mu \mathrm{g} / \mathrm{L} . \quad 56$

$\mu g / L$

J3

$1.4 E+01$

$\mathrm{pCi} / \mathrm{mL}$

63

2

1

1

2

1

$\mu \mathrm{g} / \mathrm{L}$

197

$\mu \mathrm{g} / \mathrm{L}$

520

$\mu \mathrm{g} / \mathrm{L}$.

1.0

$\mathrm{pCi} / \mathrm{L}$

$3.2 \mathrm{E}+00$

2

HSB 104C

Aluminum

$\mu g / L$

425

Nitrate-nitrite as nitrogen

Nonvolatile beta

$\mathrm{pH}$

$\mu g / L$

pCi/L

$\mathrm{pH}$

Total alpha-emitting radium

$\mathrm{pCi} / \mathrm{L}$

6,500

$3.1 E+01$

9.8

$2.8 E+00$

$\mu \mathrm{g} / \mathrm{L}$

7,400

$\mu g / L$

$\mu \mathrm{g} / \mathrm{L}$

$\mu \mathrm{g} / \mathrm{L}$

$\mu \mathrm{g} / \mathrm{L}$

49

163

292

Manganese

Nitrate-nitrite as nitrogen

$\mu g / L$

47

$\mu g / L$

69

$\mu g / L$

$\mu \mathrm{g} / \mathrm{L}$

195

Aluminum

Manganese

Specific conductance

$\mu \mathrm{S} / \mathrm{cm}$

35

550

$\mu \mathrm{g} / \mathrm{L}$

86

$\mu \mathrm{g} / \mathrm{L}$

pCi/L

25

Manganese

Nonvolatile beta

$\mathrm{pCi} / \mathrm{L}$

$3.4 \mathrm{E}+01$

2.7E + 00

$\mu \mathrm{g} / \mathrm{L}$

$\mu \mathrm{g} / \mathrm{L}$

139

Manganese

Nonvolatile beta

$\mathrm{pCi} / \mathrm{L}$

Total alpha-emitting radium

$\mathrm{pCi} / \mathrm{L}$

47

$4.6 \mathrm{E}+01$

$2.5 E+00$

$\mu g / L$

$\mu \mathrm{g} / \mathrm{L}$

$\mathrm{pCi} / \mathrm{L}$

178

55

2.7E + 01

Manganese

Nonvolatile beta 
Aquifer Zone IIB, (Barnwell/McBean, upper portion)

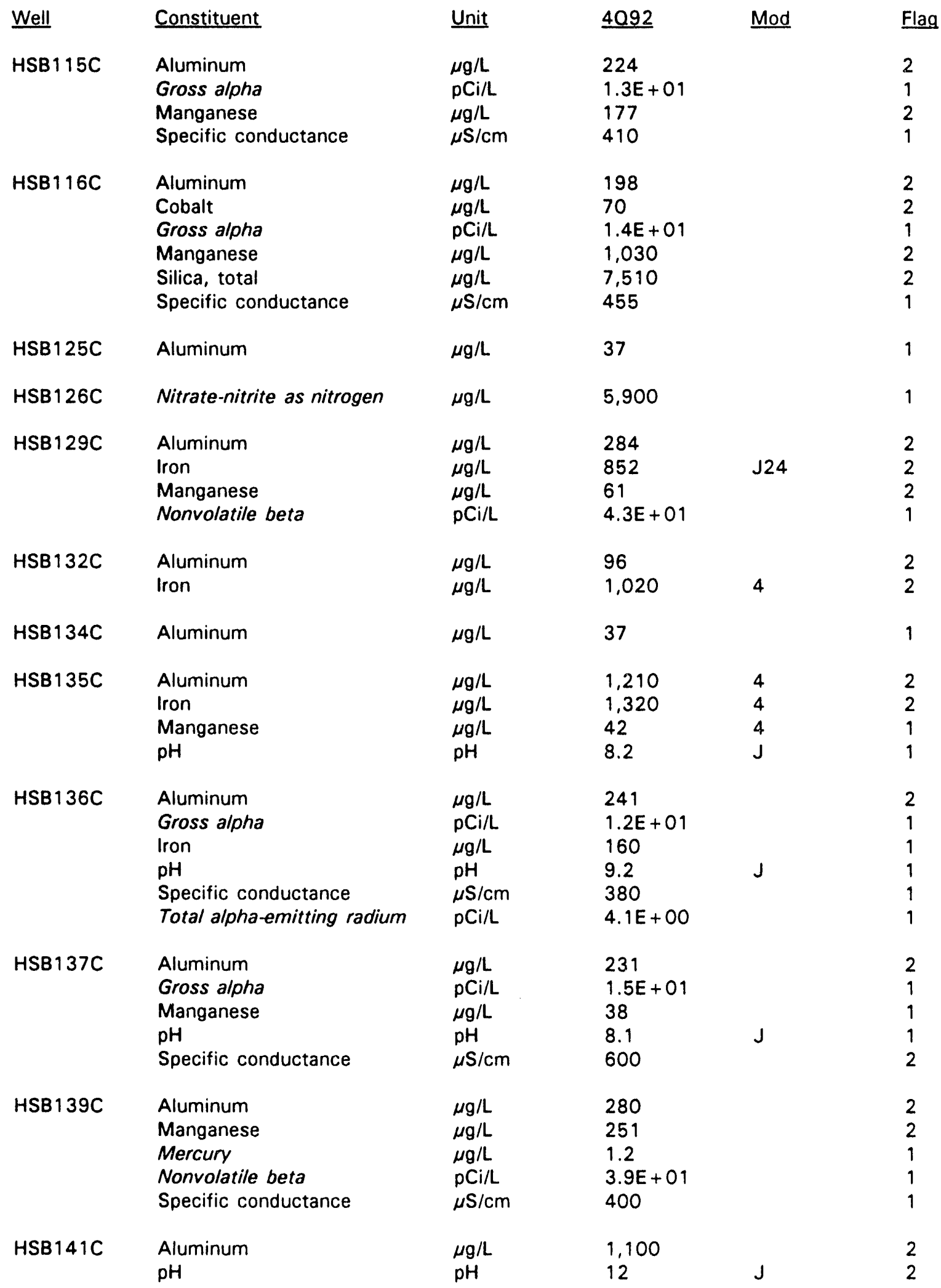


Aquifer Zone IIB, (Barnwell/McBean, upper portion)

\begin{tabular}{|c|c|c|c|c|c|}
\hline Well & Constituent & Unit & 4092 & Mod & Flag \\
\hline & Specific conductance & $\mu \mathrm{S} / \mathrm{cm}$ & 850 & & 2 \\
\hline HSB142C & Aluminum & $\mu g / L$ & 76 & & 2 \\
\hline HSB 143C & $\begin{array}{l}\text { Aluminum } \\
\text { Tritium }\end{array}$ & $\begin{array}{l}\mu \mathrm{g} / \mathrm{L} \\
\mathrm{pCi} / \mathrm{mL}\end{array}$ & $\begin{array}{l}33 \\
1.1 E+01\end{array}$ & & $\begin{array}{l}1 \\
1\end{array}$ \\
\hline HSB145C & $\begin{array}{l}\text { Aluminum } \\
\text { Manganese } \\
\text { Specific conductance } \\
\text { Total alpha-emitting radium }\end{array}$ & $\begin{array}{l}\mu g / L \\
\mu g / L \\
\mu S / c m \\
p C i / L\end{array}$ & $\begin{array}{l}218 \\
127 \\
310 \\
4.0 E+00\end{array}$ & & $\begin{array}{l}2 \\
2 \\
1 \\
1\end{array}$ \\
\hline HSB 146C & Aluminum & $\mu g / L$ & 165 & & 2 \\
\hline HSB148C & $\begin{array}{l}\text { Aluminum } \\
\mathrm{pH} \\
\text { Specific conductance }\end{array}$ & $\begin{array}{l}\mu \mathrm{g} / \mathrm{L} \\
\mathrm{pH} \\
\mu \mathrm{S} / \mathrm{cm}\end{array}$ & $\begin{array}{l}911 \\
11 \\
270\end{array}$ & $J$ & $\begin{array}{l}2 \\
2 \\
1\end{array}$ \\
\hline HSB151C & $\begin{array}{l}\text { Aluminum } \\
\text { Nitrate-nitrite as nitrogen }\end{array}$ & $\begin{array}{l}\mu \mathrm{g} / \mathrm{L} \\
\mu \mathrm{g} / \mathrm{L}\end{array}$ & $\begin{array}{l}125 \\
8,250\end{array}$ & & $\begin{array}{l}2 \\
1\end{array}$ \\
\hline HSB152C & $\begin{array}{l}\text { Aluminum } \\
\text { Manganese } \\
\text { Nitrate-nitrite as nitrogen }\end{array}$ & $\begin{array}{l}\mu g / L \\
\mu g / L \\
\mu g / L\end{array}$ & $\begin{array}{l}87 \\
37 \\
9,300\end{array}$ & & $\begin{array}{l}2 \\
1 \\
1\end{array}$ \\
\hline
\end{tabular}

Aquifer Zone IIB, (Barnwell/McBean, lower portion)

\begin{tabular}{|c|c|c|c|c|c|}
\hline Well & Constituent & Unit & 4092 & Mod & Flag \\
\hline HSB 83B & Aluminum & $\mu g / L$ & 48 & & 1 \\
\hline HSB 84B & $\begin{array}{l}\text { Aluminum } \\
\text { Iron } \\
\mathrm{pH}\end{array}$ & $\begin{array}{l}\mu g / L \\
\mu g / L \\
\mathrm{pH}\end{array}$ & $\begin{array}{l}762 \\
596 \\
10\end{array}$ & $\begin{array}{l}4 \\
J\end{array}$ & $\begin{array}{l}2 \\
2 \\
2\end{array}$ \\
\hline HSB 85B & $\begin{array}{l}\text { Aluminum } \\
\text { Chloroethene (Vinyl chloride) } \\
\text { pH } \\
\text { Specific conductance }\end{array}$ & $\begin{array}{l}\mu \mathrm{g} / \mathrm{L} \\
\mu \mathrm{g} / \mathrm{L} \\
\mathrm{pH} \\
\mu \mathrm{S} / \mathrm{cm}\end{array}$ & $\begin{array}{l}2,520 \\
1.3 \\
11 \\
440\end{array}$ & $J$ & $\begin{array}{l}2 \\
1 \\
2 \\
1\end{array}$ \\
\hline
\end{tabular}

Aquifer Unit IIA (Congaree)

\begin{tabular}{|c|c|c|c|c|}
\hline Well & Constituent & Unit & 4Q992 & Mod \\
\hline HSB 65A & Tritium & $\mathrm{pCi} / \mathrm{mL}$ & $1.7 E+01$ & \\
\hline HSB 69A & Aluminum & $\mu g / L$ & 60 & \\
\hline
\end{tabular}


WSRC-TR-93-059

Aquifer Unit IIA ICongareet

\begin{tabular}{|c|c|c|c|c|c|}
\hline Well & Constituent & $\underline{\text { Unit }}$ & 4092 & $\underline{\text { Mod }}$ & \\
\hline HSB 84A & Aluminum & $\mu g / L$ & 71 & & \\
\hline HSB 85A & $\begin{array}{l}\text { Aluminum } \\
\text { Total alphaemitting radium }\end{array}$ & $\begin{array}{l}\mu g / L \\
\mathrm{pCi} / \mathrm{L}\end{array}$ & $\begin{array}{l}43 \\
3.4 E+00\end{array}$ & & \\
\hline HSB 86A & Aluminum & $\mu g / L$ & 48 & & \\
\hline HSB117A & Manganese & $\mu g / L$ & 74 & & \\
\hline HSB118A & $\begin{array}{l}\text { Manganese } \\
\text { Specific conductance } \\
\text { Total alpha-emitting radium } \\
\text { Total organic carbon }\end{array}$ & $\begin{array}{l}\mu \mathrm{g} / \mathrm{L} \\
\mu \mathrm{S} / \mathrm{cm} \\
\mathrm{pCi} / \mathrm{L} \\
\mu \mathrm{g} / \mathrm{L}\end{array}$ & $\begin{array}{l}54 \\
270 \\
3.1 E+00 \\
12,200\end{array}$ & & \\
\hline HSB119A & $\begin{array}{l}\text { Aluminum } \\
\text { Iron } \\
\text { Lead } \\
\text { Silica, total }\end{array}$ & $\begin{array}{l}\mu g / L \\
\mu g / L \\
\mu g / L \\
\mu g / L\end{array}$ & $\begin{array}{l}1,160 \\
348 \\
12 \\
36,600\end{array}$ & $\sqrt{2}$ & \\
\hline HSB 121A & Total alpha-emitting radium & $\mathrm{pCi} / \mathrm{L}$ & $3.5 E+00$ & & \\
\hline HSB123A & $\begin{array}{l}\text { Aluminum } \\
\text { pH } \\
\text { Total alpha-emitting radium }\end{array}$ & $\begin{array}{l}\mu \mathrm{g} / \mathrm{L} \\
\mathrm{pH} \\
\mathrm{pCi} / \mathrm{L}\end{array}$ & $\begin{array}{l}25 \\
8.8 \\
2.6 E+00\end{array}$ & $J$ & \\
\hline HSB124AR & Manganese & $\mu g / L$ & 29 & & \\
\hline HSB140A & Aluminum & $\mu g / L$ & 33 & & \\
\hline HSB 141A & $\begin{array}{l}\text { Aluminum } \\
\text { pH } \\
\text { Specific conductance }\end{array}$ & $\begin{array}{l}\mu \mathrm{g} / \mathrm{L} \\
\mathrm{pH} \\
\mu \mathrm{S} / \mathrm{cm}\end{array}$ & $\begin{array}{l}2,960 \\
12 \\
1,000\end{array}$ & $J$ & \\
\hline HSB144A & Manganese & $\mu g / L$ & 43 & & \\
\hline HSB 146A & Aluminum & $\mu g / L$ & 119 & & \\
\hline
\end{tabular}

Note: Constituents exceeding half the final PDWS appear italicized. These results do not include field data results.

a Flagging criteria for total radium were applied to total alpha-emitting radium. 
Table 3. Groundwater Monitoring Results for Individual Wells

\section{WELL HSB 65}

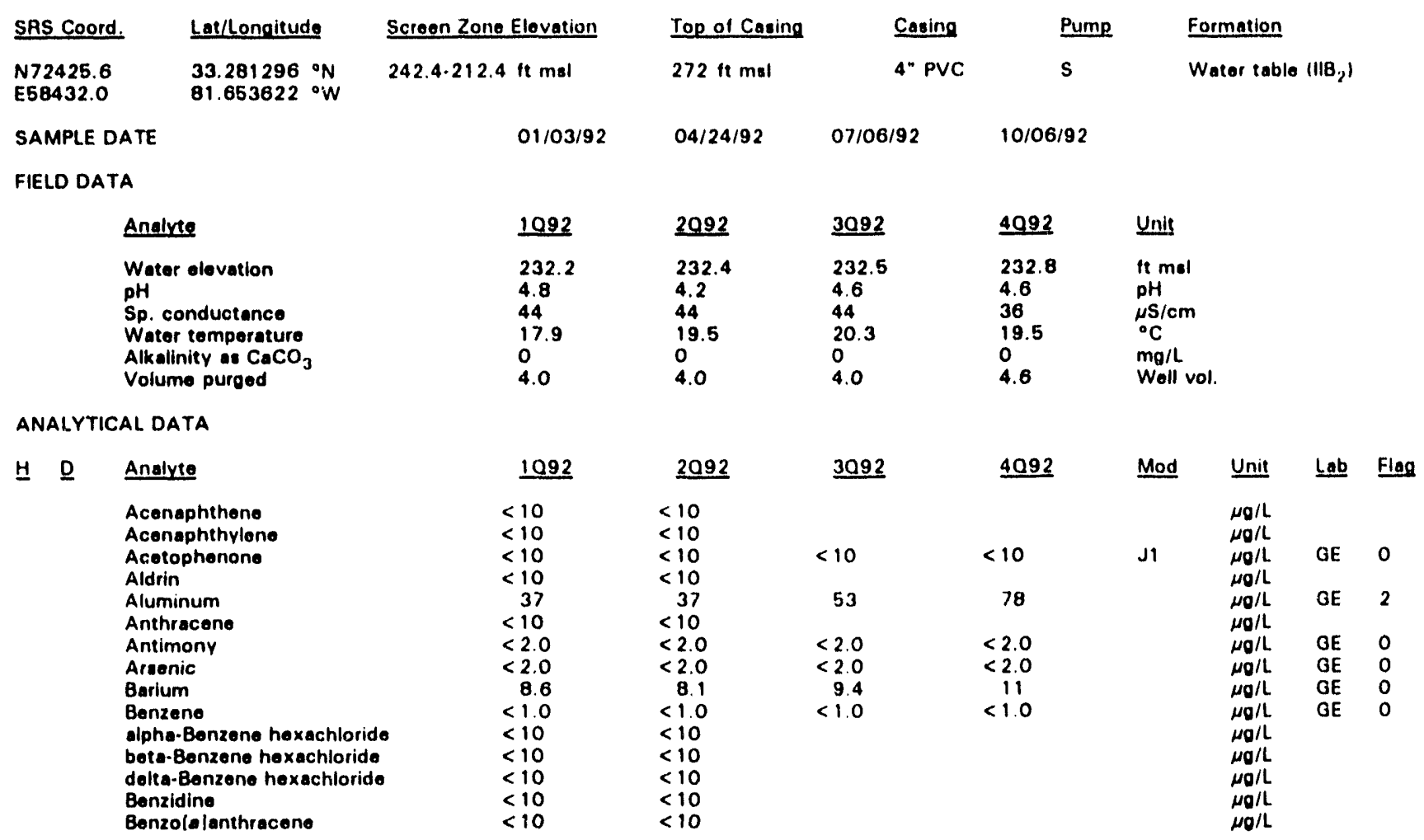

Note: Flagging levels, modifiers, and laboratories are for 4 th quarter 1992 data only. See Appendix $B$ for flagging criteria.

- exceeded holding time for 4th quarter 1992.

- exceeded final primary drinking water standard for 4th quartor 1992. 
WSRC-TR-93-059

Woll HSB 65 continued

ANALYTICAL DATA

H D Anolyte

Benzolblfluoranthene

Benzolklfluoranthene

Benzolg,h,ilperylene

Benzolelpyrene

Bis (2-chloroethoxy) methane

Bis (2-chloroethyl) other

Bis/2-chloroisopropyl) other

Bis/2-ethylhexyll phthalate

Bromodichloromethane

Bromotor

Bromomothane (Methyl bromide)

4-Bromophenyl phenyl other

Butylbenzyl phthalate

Cadmium

Calcium

Carbon tetrardane

Chloride

Chlorobenzene

para-Chloro-meta-cresol

Chloroethane

Chloroothene (Vinyl chloride)

2-Chloroethyl vinyl other

Chloroform

Chloromethane (Methyl chloride)

2.Chloronaphthalene

2-Chlorophonol

4-Chlorophenyl phenyl ether

Chromium

Chrysene

Cobalt

Copper

Cyanide

p.p'-DDD

p.P'-DDE

p.p'-DDT

Dibenzla,hlanthracene

Dibromochloromethan

Di-n-butyl phthalate

3. $3^{\prime}$-Dichlorobenzidine

1.1-Dichloroethane

1.2-Dichloroethane

1.1-Dichloroethylene

trans-1,2-Dichloroethylene

Dichloromethane (Methylene chloride)

2.4-Dichlorophenol

2,4-Dichlorophenoxyacetic acid

1,2-Dichloropropane

cie-1.3-Dichloropropene

trans-1,3-Dichloropropene

Dieldrin

Diothyl phthalate

2.4-Dimethyl pheno

Dimethyl phthalate

2.4-Dinitrophonol

2,4-Dinitrotoluene

2,6-Dinitrotoluene

Di-n-octyl phthalate

1.2-Diphenylhydrazine

Endosulfan I

Endosulfan II

Endosulfan sulfate

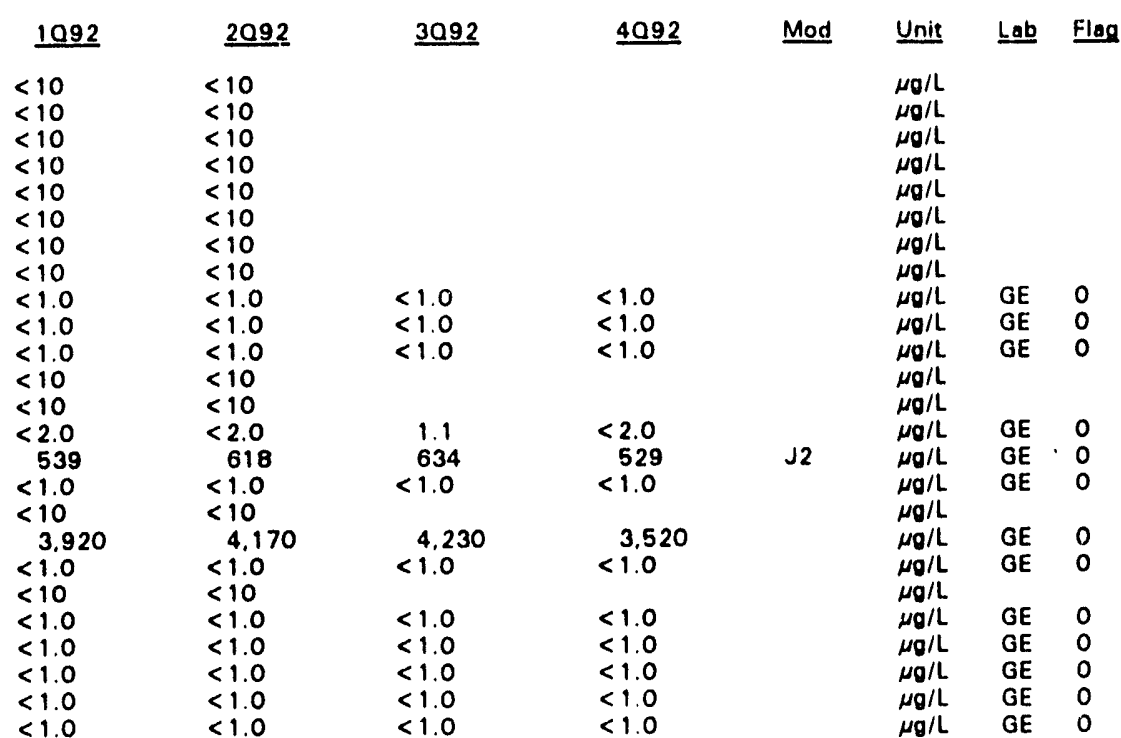

$\begin{array}{rr}61 & 56 \\ <5.0 & <5.0\end{array}$

$<10$

$<10$

$<10$

$<10$

$<1.0$

$<10$
$<10$

$<1.0$

$<1.0$

$<1.0$

$<1.0$

$<1.0$

$<10$

$<0.30$

$<1.0$

$<1.0$

$<1.0$

$<10$

$<10$

$<10$

$<10$

$<45$

$<10$

$<10$

$<10$

$<10$

$<10$

$<10$

$<10$
$<1.1<4.0$

$1.1<4.0$

$\begin{array}{cc}56 & 88 \\ <5.0 & <5.0\end{array}$

$\mu g / L$

$\mu \mathrm{g} / \mathrm{L}$

$\mu \mathrm{g} / \mathrm{L}$

$\mu \mathrm{g} / \mathrm{L}$

$\mu_{g} / L$

$\mu \mathrm{g} / \mathrm{L}$

$\mu \mathrm{g} / \mathrm{L}$

$\mu \mathrm{g} / \mathrm{L}$

$\mu \mathrm{g} / \mathrm{L}$

$\mu \mathrm{g} / \mathrm{L}$

$<1.0<1.0$

$<1.0<1.0$

$<1.0<1.0$

$<10<10$

$<1.0<1.0$

2.1

$<0.30$

$<0.30$

$<1.0$

$<1.0$

$<1.0$

$<1.0$

$<1.0$
GE 0

GE 0

GE 0

$$
\text { OE } 0
$$

GE $O$

GE 0

GE 0

GE 0

GE 0

GE 0

GE 0

GE $\quad 0$

Note: Flagging levels, modifiers, and laboratories are for 4 th quarter 1992 data only. See Appendix B for flagging criteria.

- =xceeded holding time for 4th quarter 1992.

- exceeded final primary drinking water standard for 4th quarter 1992.

H-Area Seepage Basins 
WSRC-TR.93.059

Well HSB 65 continued

ANALYTICAL DATA

H D Anclute

Endrin

Endrin aldehyde

Ethylbenzene

Fluoranthene

Fluorene

Fluoride

Grose alpha

Heptachlor

Heptechlor epoxide

Hexachlorobenzene

Hexechlorobutediene

Hexechlorocyclopentediene

Hexechloroethane

Indenol 1,2,3-c,dlpyrene

iron

leophorone

Leed

lind ane

Magnesium

Manganese

Mercury

Mothoxychlor

2-Methyl-4,6-dinitrophenol

Nephthalene

Nickel

Nitrate as nitrogen

Nitrate-nitrite as nitrogen

Nitrobenzene

2. Nitrophenol

4-Nitropheno

N-Nitrosodimethylamine

N.Nitrosodiphenylamine

N-Nitrosodipropylamine

Nonvolatile beta

PCB 1016

PCB 1221

PCB 1232

PCB 1242

PCB 1248

PCB 1254

PCB 1260

Pentechlorophenol

- pH

Phenenthrene

Phenol

Phonole

Potassium

Pyrene

Radium-226

Redium-228

Solenium

Silice

Silver

Sodium

Specific conductance

Sulfate

1,1,2,2. Tetrachloroethane

Tetrachloroethylene

Tolueno

Total alpha-emitting radium

Total alpha-emitting radium
Total diesolved solids

$\begin{aligned} & 1092 \\ < & 10 \\ < & 10 \\ < & 1.0 \\ < & 10 \\ < & 10 \\ < & 100 \\ 3.4 E+00 & <10 \\ < & 10\end{aligned}$

\begin{tabular}{|c|c|c|c|}
\hline 2092 & 3092 & 4092 & Mod \\
\hline $\begin{array}{l}<0.0060 \\
<10 \\
<1.0\end{array}$ & $\begin{array}{l}<0.0060 \\
<1.0\end{array}$ & $\begin{array}{l}<0.0060 \\
<1.0\end{array}$ & \\
\hline
\end{tabular}

$\begin{array}{lll}\text { Unit } & \text { Lab } & \text { Flag } \\ \mu g / L & \text { GE } & 0 \\ \mu \sigma / L & & \\ \mu \sigma / L & \text { GE } & 0 \\ \mu g / L & & \\ \mu g / L & & \\ \mu \sigma / L & \text { OE } & 0\end{array}$

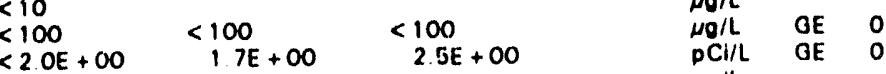

$<10<10$

$<10<10$

$<10<10$

$<10<10$

$<10<10$

$<10<10$

$11<4.0$

$<10$

14
$<10$

902

$<2.0$

$<0.20$

$<0.50$

$<10$

$<10$

$<4.0$

2.750

$<10$

$<10$

$<10$

$<10$

$<10$

$<10$

$2.5 E+00$

$<150$

$<150$

$<150$

$<150$

$<150$

$<150$

$<150$

$<10$

4.5

$<10$

$<10$

$<5.0$

$<500$

$<10$

$<4.0$
$<10$

$<10$
11
$<0.0050$

$<0.0050$
901

$<2.0$

$<0.20$

$<0.20$
$<0.50$

$<10$

$<10$

$<4.0$

$<2.0$

6.750

$<2.0$

4,850

40

2.010

$<1.0$

$<1.0$

$<2.0$

$<1.0$

1. $1 E+00$

33.000

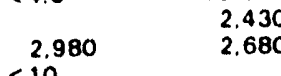

36

13

$<0.0050$

975

12

$<0.20$

$<0.50$

$<10$

2.680

No

$\mu \mathrm{g} / \mathrm{L}$

$\mu / L$

$\mu \sigma / L$

$\mu \sigma / L$

$\mu \mathrm{N} / \mathrm{L}$

$\mu \sigma / L$
$\mu \sigma / L$

$\mu \mathrm{mo} / \mathrm{L}$

$\mu \mathrm{g} / \mathrm{L}$ OE 1

GE 0

11

$<0.0050$

1.100

$<2.0$

$<0.20$

$<0.50$

$<10$

3.000

$<10$
$<10$

$<10$

$<10$

$<10$

$<10$

$<10$

$<2.0 E+00$

$<150$

$<150$

$<150$

$<150$

$<150$

$<150$

$<150$

$<10$
4.9

4.9
$<10$

10

50

$<5.0$
$<500$

$<500$
$<10$

$<2.0$

7.220

$<2.0$

40

$<1.000$

$<1.0$

$<1.0$

$<2.0$

$<1.0$

$1.1 E+00$

19.000

$2.9 E+00$



4.8
$<5.0$
282

$1.1 E+00$
$8.4 E \cdot 01$
$<2.0$
6.840
0.72
4.700
42
$<1.000$
$<1.0$
$<1.0$
2.3
$<1.0$
$<1.0 E+00$
60.000

3.900

$\begin{array}{ll}\text { GE } & 0 \\ \text { GE } & 0\end{array}$

GE 0

GE 0

GE $O$

OE 0

$\mu \sigma / L \quad O E$

$\mu \sigma / L$

$\mu \mathrm{g} / \mathrm{h}$

$\mu \mathrm{g} / \mathrm{L}$

$\mu \mathrm{g} / \mathrm{L}$

$\mu g / L$

$\mu \mathrm{g} / \mathrm{L}$

$\mu \mathrm{O} / \mathrm{L}$

$\mu \mathrm{g} / \mathrm{L}$

$\mu \mathrm{g} / \mathrm{l}$.

$\mu \mathrm{m} / \mathrm{L}$

Nol

no

$\mu \mathrm{g} / \mathrm{L}$ GE

$\mu \mathrm{g} / \mathrm{L}$

$\mu / L$ GE 0

$<5.0$

$<500$

$<2.0$

6.000

$<2.0$

2.960

$<1,000$

$<1.000$

$<1.0$

$<1.0$

$<2.0$

2.1E +00

20.000

$\mu \mathrm{g} / \mathrm{L}$

PCi/L

pCi/L

$\mu \sigma / L$ OE 0

$\mu / L$ OE $O$

$\mu / L$ GE 0

Ng/L OE

$\mu S / \mathrm{cm}$ OE

$\mu \mathrm{O} / \mathrm{L}$ GE $O$

HOL GE

NOL GE

$\begin{array}{lll}\mu \mathrm{g} / \mathrm{L} & \mathrm{GE} & 0 \\ \mu \mathrm{g} / \mathrm{L} & \mathrm{GE} & 0\end{array}$

$\mu \mathrm{N} / \mathrm{L}$ OE 0

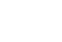

PCB 1242
PCB 1248
PCB 1254
PCB 1260
Pontachlorophenol
PH
Phenanthrone
Phenol
Phonols
Potassium
Pyrane
Radium-226
Redium-228
Selenium
Silica
Silver
Sodium
Specific conductance
Sulfate
$1.1 .2 .2 \cdot$ Totrachloroethane
Totrachloroethylene
Tln
Toluene
Total alpho-emitting radium
Total diesolved solid


WSRC.TR.93.059

Well HSB 65 continued

ANALYTICAL DATA

H 2 Analyte

Total organic carbon

Total orgenic halogens

Total phosphates las P)

Toxaphene

2.4.5-TP (Silvex)

1.2.4-Trichlorobenzene

1.1.1-Trichloroethene

1.1,2-Trichloroethene

Trichloroethylene

Trichlorolluoromethane

2,4,6. Trichlorophonol

- Tritium

Turbidity

Vanadium

Xrlenee

Zine

$\begin{aligned} & 1092 \\ &< 1.000 \\ &< 5.0 \\ &< 50 \\ &< 10 \\ &< 0.090 \\ &< 10 \\ &<1.0 \\ &<1.0 \\ &<1.0 \\ &<1.0 \\ &<10 \\ & 3.8 E+01 \\ & 1.9 \\ &< 8.0 \\ &< 2.0 \\ & 12\end{aligned}$

$\begin{aligned} & 2092 \\ &< 1.000 \\ &< 5.0 \\ &<50 \\ &<0.24 \\ &<0.090 \\ &<10 \\ &<1.0 \\ &<1.0 \\ &<1.0 \\ &<1.0 \\ &<10 \\ & 3.3 \mathrm{E}+01 \\ &<0.10 \\ &<8.0 \\ &<2.0 \\ & 12\end{aligned}$

$\quad 3092$
1.210
28
31
$<0.24$
$<0.090$
$<1.0$
$<1.0$
$<1.0$
$<1.0$

$3.6 E+01$
0.73
$<0.88$
$<2.0$
96

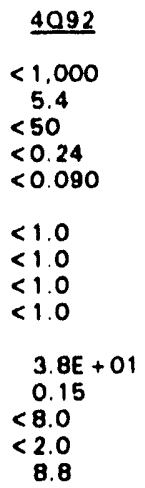

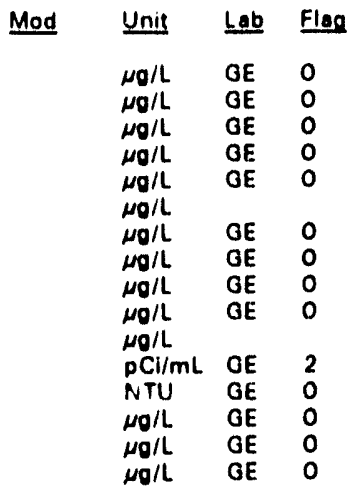

\section{WELL HSB 65A}

\begin{tabular}{|c|c|}
\hline SAS coord. & Lat/Longitude \\
\hline $\begin{array}{l}N 72436.2 \\
E 58436.0\end{array}$ & $\begin{array}{l}33.281326{ }^{\circ} \mathrm{N} \\
81.653633^{\circ} \mathrm{W}\end{array}$ \\
\hline
\end{tabular}

$\frac{\text { Screen Zone Elovation }}{73.2 \cdot 62.5 \mathrm{ft} \mathrm{msl}}$

$\frac{\text { Top of Casing }}{273.6 \mathrm{ft} \mathrm{msl}}$

\begin{tabular}{cc} 
Casing & Pump \\
\hline 4* PVC & $s$ \\
$6 / 92$ & $10 / 05 / 92$
\end{tabular}

$01 / 03 / 92$

$04 / 24 / 92$

$07 / 06 / 92$

10/05/92

FIELD DATA

\section{Analve}

Water elevation

pH

Sp. conductance

Water temperature

Alkalinity \& $\mathrm{CaCO}_{3}$

Volume purged

\section{ANALYTICAL DATA}

H $\underline{D}$

Analyte
Acenaphthene
Acenaphthylene
Acetophenone
Aldrin
Aluminum
Anthracene
Antimony
Arsenic
Berium
Benzene
alpha-Benzene hexachloride
beta-Benzene hexachloride
detta-Benzene hexachloride
Benzidine
Benzolelenthracene
Benzolblfluoranthene
Benzolkifluoranthene
Benzolo,h,iperylene
Benzolelpyrene
Bis (2-chlorvethoxy) methane
Bis (2-chloroethyll other
Bis (2-chloroisopropyl) ether

$\begin{array}{ll}1092 & \underline{2092} \\ 171.8 & 171.9 \\ 7.5 & 7.0 \\ 211 & 210 \\ 18.7 & 20.3 \\ 89 & 80 \\ 4.0 & 4.0\end{array}$

3092
171.5
7.0
211
20.4
76
4.0

4092
171.5
7.6
206
19.9
84
2.3

Unie
tt mel
$\mathrm{pH}$
$\mu \mathrm{S} / \mathrm{cm}$
${ }^{\circ} \mathrm{C}$
$\mathrm{mg} / \mathrm{L}$
Woll vol.

$<10$

$<10$

$<10$

$<10$

$<10$
24

$<10$

$<2.0$

$<2.0$

44
$<1.0$

$<1.0$

$<10$
$<10$

$<10$

$<10$

$<10$

$<10$

$<10$

$<10$

$<10$

$<10$
$<10$

$<10$
$<10$

$<10$

$\begin{aligned} & 2092 \\ &< 10 \\ &< 10 \\ &< 10 \\ &< 10 \\ & 27 \\ &< \\ &<10 \\ &<2.0 \\ &< \\ &<.0 \\ & 48 \\ &<1.0 \\ &<10 \\ &<10 \\ &<10 \\ &<10 \\ &<10 \\ &<10 \\ &<10 \\ &<10 \\ &<10 \\ &<10 \\ &<10 \\ &<10\end{aligned}$

\section{$\underline{3092}$}

$<10$

32

$<2.0$

$<2.0$

54

$<1.0$

$\quad \underline{4092}$
$<10$
24
$<2.0$
$<2.0$
39
$<1.0$

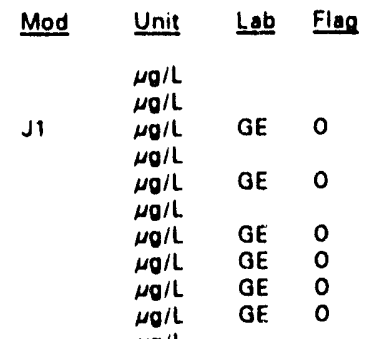

L. Congareo IIIAI

$\mu \mathrm{g} / \mathrm{L}$

$\mu \mathrm{o} / \mathrm{L}$

$\mu \mathrm{g} / \mathrm{L}$

$\mu \mathrm{g} / \mathrm{L}$

$\mu \sigma / L$

$\underset{\mu \sigma / L}{\mu g / L}$

$\mu \mathrm{g} / \mathrm{L}$

$\mu g / L$

$\mu \mathrm{g} / \mathrm{L}$

$\mu \mathrm{g} / \mathrm{L}$

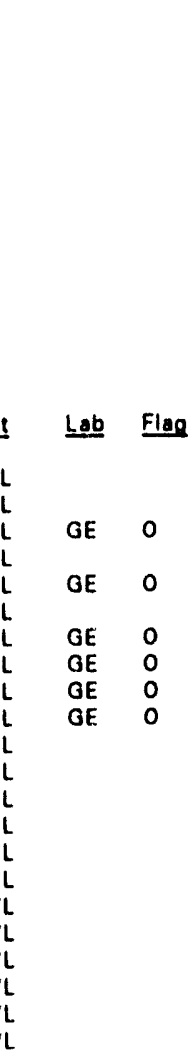

Note: Flagging levels, modifiers, and laboratories are for 4th quarter 1992 data only. See Appendix B for flagging criteria. - = exceeded holding time for 4th quarter 1992.

- =xceeded final primary drinking water standard for 4th quarter 1992. 


\section{ANALYTICAL DATA}

H D Anolvte

Bie12-ethylhexyll phthalate Bromodichloromothan.

Bromolorm

Bromomethane (Muthyl bromide)

4.Bromophenyl pheriyl other

Butylbenzyl phthalate

Cadmium

Cerbon tetrechloride

Cerbon terro

Chloride

Chlorobenzene

para-Chloro-meta-cresol

Chloroethano

Chloroethene IVinyl chloride)

2.Chloroethyl vinyl other

Chloroform

Chloromethane (Mathyl chloride)

2.Chloronaphthalene

2. Chlorophenol

4.Chlorophenyl phenyl ether

Chromium

Chrysene

Cobalt

Copper

Cranide

p.p'DDD

P.P'-DDE

P.p. hlanthracene

Dibromochloremethene

Di-n-butyl phthalate

3.3'-Dichlorobenzidine

1.1.Dichloroethane

1.2-Dichloroethane

1.1-Dichloroethylene

trans-1.2-Dichloroethylene

Dichloromethane (Methylene chloride)

2.4-Dichlorophenol

2,4-Dichlorophenoxyacetic acid

1.2-Dichloropropane

cis-1.3-Dichloropropene

trane-1.3-Dichloropropene

Dioldrin

Diethyl phthalate

2.4-Dimethyl phenol

Dimethyl phthalate

2,4-Dinitrophenol

2.4-Dinitrotoluene

2.6-Dinitrotoluene

Di-n-octyl phthalate

1.2-Diphenylhydrazine

Endosulfan I

Endosulfan II

Endosulfan sulfato

Endrin

Endrin aldehyde

Ethylbenzene

Fluoranthene

Fluorene

Fluoride

Groes alphe

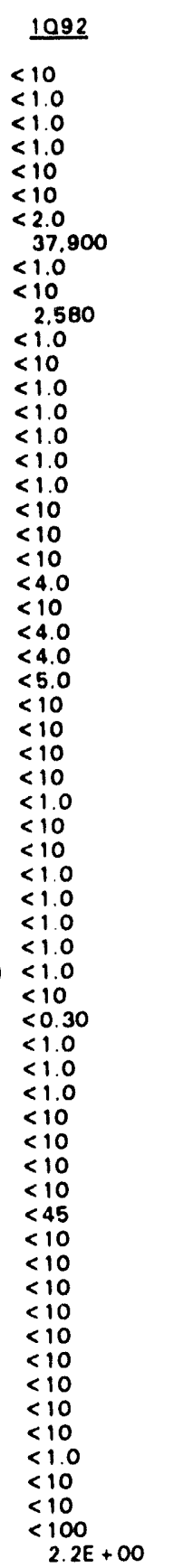

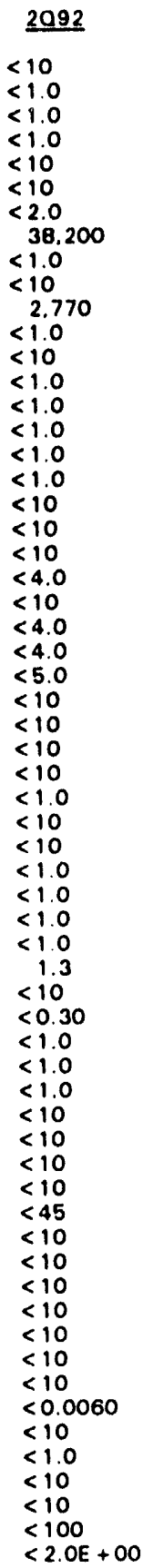

3992

4092

Mod

$<1.0$

$<1.0$
$<1.0$

$<1.0$

$<1.0$

$<2.0$

42.200

$<1.0$

2.560

$<1.0$

$<1.0$

$<10$

$<10$

$<1.0$

$<1.0$

$<4.0$

$<4.0$

$<4.0$
$<5.0$

$<1.0$

$<2.0$

34.900

$<1.0$

2.520

$<1.0$

$<1.0$

$<1.0$

$<1.0$

$<1.0$

$<1.0$

$<1.0$

$<4.0$

$<4.0$

$<4.0$

$<5.0$

$<1.0$

$<1.0$

$<1.0$

$<10$

$<1.0$

1.1

$<0.30$

$<1.0$

$<1.0$

$<1.0$

$<1.0$

$<1.0$

$<1.0$

2.5

$<0.30$

$<1.0$

$<1.0$

$<1.0$

\section{$<0.0060$}

$<0.0060$

$<1.0$

$<1.0$

$\begin{array}{ll}<100 & <100 \\ <2.0 E+00 & <2.0 E+00\end{array}$
OE 0

GE 0

GE $O$

GE 0

GE 0

GE

GE 0

GE 0

GE 0

GE 0

GE 0

GE 0

$\begin{array}{ll}\text { GE } & 0 \\ \text { GE } & 0\end{array}$

OE 0

GE 0

GE 0

GE 0

GE 0

GE 0

GE 0

$\mu g / L$

$\mu \mathrm{g} / \mathrm{h}$

$\mu \mathrm{g} / \mathrm{L}$

$\mu \mathrm{o} / \mathrm{L}$

$\mu \boldsymbol{g} / \mathrm{L}$

$\mu g / L$

$\mu \sigma / L$

$\mu \sigma / L$

$\mu \mathrm{d} / \mathrm{L}$

$\mu \mathrm{g} / \mathrm{L}$

$\mu \mathrm{g} / \mathrm{L}$

GE 0

GE 0

$\mu \mathrm{g} / \mathrm{L}$

$\mu \mathrm{g} / \mathrm{L}$

$\mu g / L$ GE $O$

Note: Flagging levels, modifiers, and laboratories are for 4 th quarter 1992 data only. See Appendix B for flagging criteria.

- = exceeded holding time for 4 th quarter 1992.

= exceeded final primary drinking water standard for 4th quarter 1992. 
WSRC.TR.93-059

Woll HSB 65A continued

ANALYTICAL DATA

H $\mathrm{D}$ Analyte

Heptachlor

Heptachlor epoxide

Hexachlorobenzene

Hexachlorobutadiene

Hexechlorocyclopentadiene

Hexechloroethane

Indenol 1,2,3-c.d)pyrene

Iron

leophorone

Lead

Lindane

Magnesium

Manganese

Mercury

Methoxychlor

2.Methyl-4.6-dinitrophenol

Naphthalene

Nickel

Nitrate-nitrite as nitrogen

Nitrobenzene

2. Nitropheno

4-Nitrophenol

N-Nitrosodimethyiamine

N-Nitrosodiphenylamine

N-Nitrosodipropylamine

Nonvolatile beta

PCB 1016

PCB 1221

PCB 1232

PCB 1242

PCB 1248

PCB 1254

PCB 1260

Pentachlorophenoi

pH

Phenanthrene

Phenol

Phenols

Potassium

Pyrono

Solenium

Silica

Silver

Sodium

Specific conductance

Sulfate

1,1,2,2-Tetrachloroethane

Tetrachloroethylene

Tin

Toluene

Total alpha-emitting radium

Total dissolved solids

Total organic carbon

Total organic halogens

Total phosphates (as P)

Toxaphene

2.4.5.TP (Silvex)

1.2.4-Trichlorobenzene

1.1.1-Trichloroethane

1.1.2. Trichlorosthene

Trichloroethylene

Trichlorofluoromethane

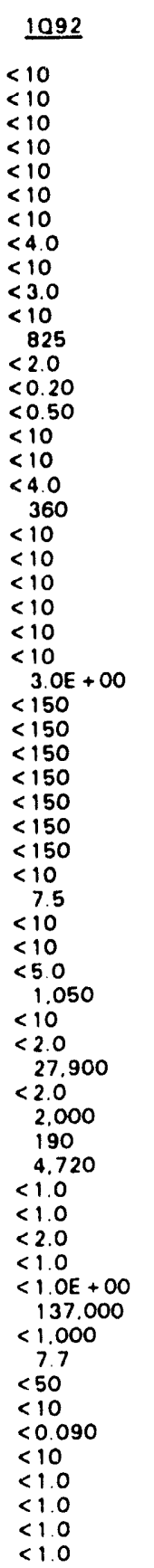

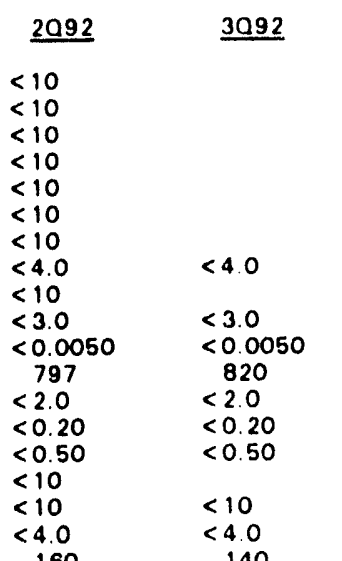

4092

Mod

$\underline{\text { Lab }}$

Flag

160

$<10$

$<10$

$<10$

$<10$

$<10$

$<10$

$<2.0 E+00<2.0 E+O O$

$<150$

$<150$

$<150$

$<150$

$<150$

$<150$

(10

$<.5$
$<10$
$<10$

$<10$

$<5.0$

$<10$

28.400

$<2.0$

1.850

170

5.400

$<1.0$

$<1.0$

$<2.0$

$<10$

$<1$. OE + OO

140

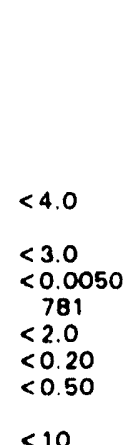

$<10$
$<4.0$

140

133.000

$<1.000$

11
$<50$

$<50$

$<0.24$

$<0.090$
$<10$

$<10$
$<1.0$

$<1.0$

$<1.0$

(

$2.8 E+00$

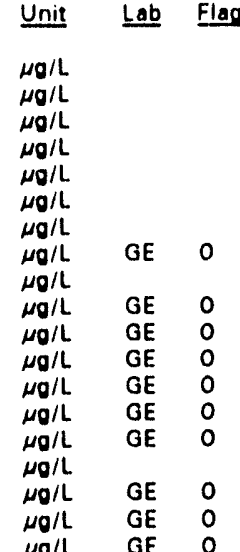

$<1.0$

$\begin{aligned} & 7.3 \\ &<5.0 \\ & 1.240 \\ &< 2.0 \\ & 26.500 \\ &< 2.0 \\ & 2.070 \\ & 198 \\ & 4.860 \\ &< 1.0 \\ &<1.0 \\ &< 2.0 \\ &<1.0 \\ &<1.0 E+00 \\ & 121.000 \\ &<1.000 \\ &<5.0 \\ &<50 \\ &<0.24 \\ &<0.090 \\ &<1.0 \\ &<10 \\ &<10 \\ &<1.0 \\ &<1.0\end{aligned}$

7.3

$<5.0$

1.120

$<2.0$

22.300

$<2.0$

1.900

198

5.390

$<1.0$

$<1.0$

$<2.0$

$<1.0$

$<1, O E+O O$

139.000

$<1.000$

$<5.0$

$<50$

$<0.24$

$<0.090$

$<1.0$

$<1.0$

$<1.0$

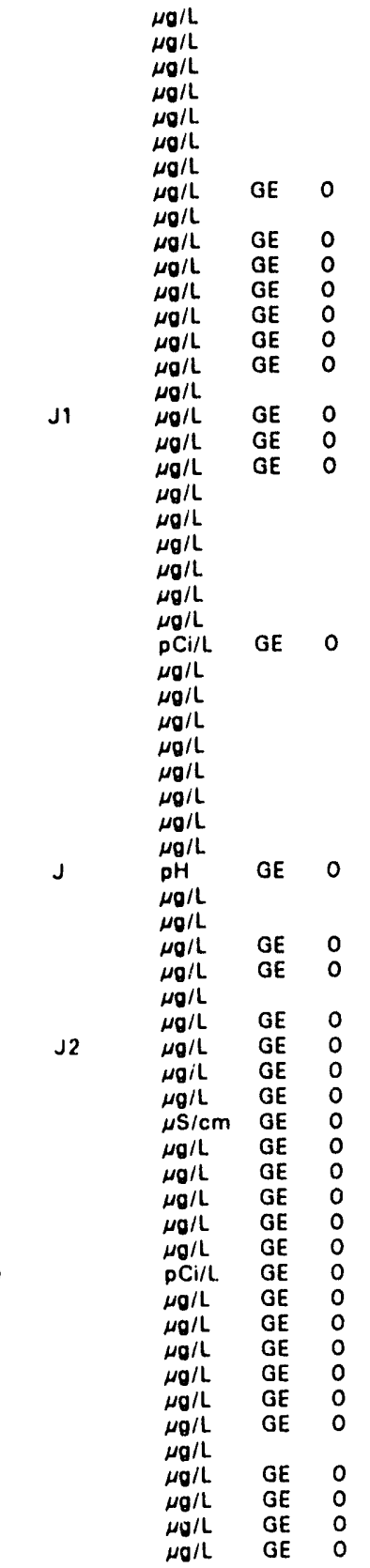

Note: Flagging levels, modifiers, and laboratories are for 4th quarter 1992 data only. See Appendix B for fiagging criteria.

- = exceeded holding time for 4 th quarter 1992.

- =xceeded final primary drinking water standard for 4 th quarter 1992.

H-Area Seepage Basins 
WSRC-TR-93-059

Well HSB 65A continued

ANALYTICAL DATA

H D Analyte

2,4,6-Trichlorophenol

Tritium

Turbidity

Vanadium

Xylones

Zinc

$\begin{array}{lll}1092 & \underline{2092} & \underline{3092} \\ <10 & <10 & \\ 3.0 \mathrm{E}+01 & 3.0 \mathrm{E}+01 & 2.2 \mathrm{E}+01 \\ 0.20 & <0.10 & <0.10 \\ <8.0 & <8.0 & <8.0 \\ <2.0 & <2.0 & <2.0 \\ <2.0 & <2.0 & <2.0\end{array}$

$4092 \quad$ Mod

$\begin{array}{lll}\text { Unit } & \text { Lab } & \text { Flag } \\ \mu \mathrm{g} / \mathrm{L} & & \\ \mathrm{pCi} / \mathrm{mL} & \mathrm{GE} & 1 \\ \mathrm{NTU} & \mathrm{GE} & 0 \\ \mu \mathrm{g} / \mathrm{L} & \mathrm{GE} & 0 \\ \mu \mathrm{g} / \mathrm{L} & \mathrm{GE} & 0 \\ \mu \mathrm{g} / \mathrm{L} & \mathrm{GE} & 0\end{array}$

1.7E+01

0.36

$<8.0$

$<2.0$

$<2.0$

\section{WELL HSB 65B}

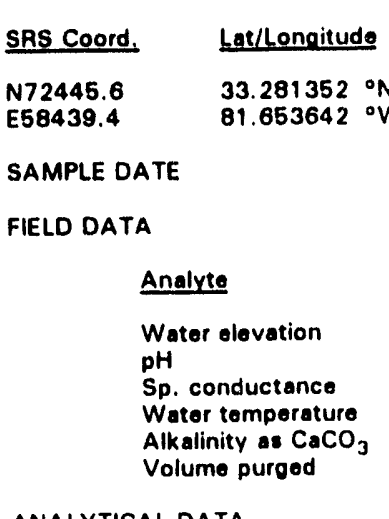

ANALYTICAL DATA

Acenaphthene
Acenaphthylene
Acetophenone
Aldrin
Aluminum
Anthracene
Antimony
Arsenic
Barium
Benzene
alpha-Benzene hexachloride
beta-Benzene hexachloride
delta-Benzene hexachloride
Benzidine
Benzolalenthracene
Benzolb)fluoranthene
Benzolk)fluoranthene
Benzolo,h,iperylene
Benzolalpyrene
Bie (2-chloroethoxy) methane
Bis (2-chloroethyl) ether
Bis (2-chloroisopropyl) ether
Bis (2-ethylhexyll phthalate
Bromodichloromethane
Bromoform
Bromomethane (Methyl bromide)
4-Bromophenyl phenyl ether
Butylbenzyl phthalate
Cadmium
Calcium
Carbon tetrachloride
Chlordane

\section{Screen Zone Elevation \\ 133.3.123.3 $\mathrm{ft} \mathrm{msl}$}

$01 / 03 / 92$
Top of Casing

$273.7 \mathrm{ft} \mathrm{ms}$

$04 / 24 / 92$

2092
224.5
7.4
205
19.6
95
4.0

1092

$<10$

$<10$

$<10$

$<10$

$<20$
$<10$

$<10$
$<2.0$

$<2.0$
$<2.0$

11

$<1.0$
$<10$

$<10$
$<10$
$<10$

$<10$

$<10$
$<10$
$<10$

$<10$

$<10$

$<10$

$<10$

$<10$

$<10$

$<1.0$

$<1.0$

$<1.0$

$<10$

$<10$

$<2.0$

38.500

$<1.0$

$<10$
2092

$<10$

$<10$

$<10$

$<10$

31
$<10$

$<2.0$

$<2.0$
$<2.0$

15
$<1.0$

$<1.0$

$<10$

$<10$

$<10$

$<10$

$<10$

$<10$

$<10$
$<10$

$<10$

$<10$

$<10$

$<10$

$<10$
$<1.0$

$<1.0$
$<1.0$

$<1.0$

$<10$

$<10$

$<2.0$
37.400

$<1.0$

$<10$

ag 
Well HSB 656 continued

\section{ANALYTICAL DATA}

H D

Analyte

Chloride

pera-Chloro-meta-cresol

Chloroethane

Chioroethene (Vinyl chloride)

2-Chloroestiyl vinyl other

Chloroform

Chloromethane (Methyt chloride)

2-Chloronaphthalene

2-Chlorophenol

4-Chlorophenyl phenyl other

Chromium

Chrysene

Cobatt

Copper

Cyanide

p.p'·DDD

p.p'-ODE

P.P'-DDT

Dibenzle, hlanthracene

Dibromochloromethane

Di-n-butyl phthalate

3, 3'-Dichlorobenzidine

1.1-Dichloroethane

1.2-Dichloroethane

1,1-Dichloroethylene

trans-1.2-Dichloroathylene

Dichloromethane (Methylene chloride)

2.4-Dichlorophenol

2.4-Dichlorophenoxyacetic acid

1,2-Dichloropropane

cis-1.3-Dichloropropene

trans-1.3-Dic hloropropene

Dieldrin

Diethyl phthalate

2.4-Dimethyl phenol

Dimethyl phthalate

2,4-Dinitrophenol

2.4-Dinitrotoluene

2.6-Dinitrotoluene

Di-n-octyl phehalate

1.2-Diphenylhydrazine

Endosulfan I

Endosulfan II

Endosulfan sulfate

Endrin

Endrin aldahyde

Ethylbenzene

Fluoranthene

Fluorene

Fluoride

Grose alpha

Heptachlor

Heptachlor epoxide

Hexachlorobenzene

Hexachlorobutadien

Hexachlorocyclopentadiene

Hexachloroethane

Indenol 1,2,3-c, dlpyrene

Iron

Isophorone

Lead

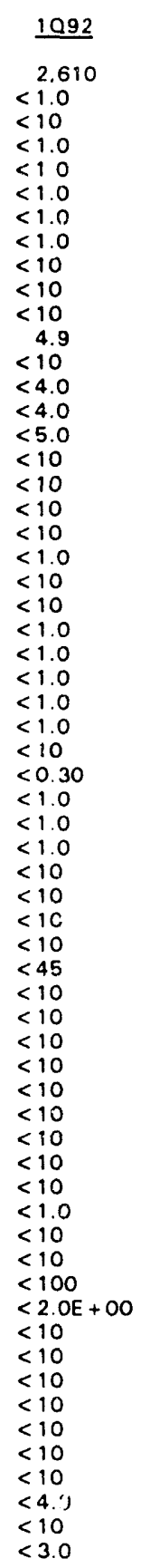

2092

2,620
$<1.0$

$<1.0$

$<10$
$\because 1.0$

$<1.0$

$<1.0$

$<1.0$

$<1.0$

$<10$

$<10$

$<10$

$<4.0$

$<10$

$<4.0$

$<4.0$

$<10$

$<10$

$<10$

$<10$

$<1.0$

$<10$

$<10$

$<1.0$

$<1.0$

$<1.0$

$<1.0$

2.6

$<10$

$<0.30$

$<1.0$

$<1.0$

$<1.0$

$<10$

$<10$

$<10$

$<10$

$<45$

$<10$

$<10$

$<10$

$<10$

$<10$

$<10$

$<10$

$<0.0060$

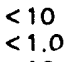

$<10$

$<10$

$<100$

$<2.0 E+00$

$<10$

$<10$

$<10$

$<10$

$<10$
$<10$

$<10$

$<10$

$<4.0$

$<10$
$<3.0$

$\begin{array}{ll}\underline{3092} & \underline{4092} \\ 2.590 & <1.570 \\ <1.0 & <1.0 \\ <1.0 & <1.0 \\ <1.0 & <0\end{array}$

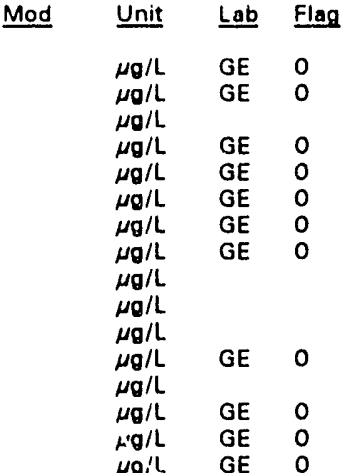

$<4.0$
$<5.0$

$<4.0$

$<4.0$
$<5.0$

$<1.0$

$<0$

$<1.0$

$<1.0$

$<1.0$

$<4.0$

$<1.0$

$\mu \mathrm{g}$

$\mu g / L$
$\mu g / L$
$\mu g / L$

$\mu g / L$

$\mu \mathrm{g} / \mathrm{L}$

$\mu_{g} / L$

$\mu \mathrm{g} / \mathrm{L}$

$<1.0$

$<1.0$

$<1.0$

$<1.0$

3.5

$<0.30$

$<1.0$

$<1.0$

$<1.0$

GE $O$

$<1.0$

$<1.0$

$<1.0$

$<1.0$

1.7

$<0.30$

$<1.0$

$<1.0$

$<1.0$

\begin{tabular}{|c|c|}
\hline$<0.0060$ & $<0.0060$ \\
\hline$<1.0$ & $<1.0$ \\
\hline $\begin{array}{l}<100 \\
<2.0 E+00\end{array}$ & $\begin{array}{l}<100 \\
<2.0 E+00\end{array}$ \\
\hline
\end{tabular}

GE 0

GE $O$

GE 0

GE $O$

$\mu g / L$

$\mu g / L$

$\mu g / L$

$\mu \mathrm{g} / \mathrm{L}$

$\mu \mathrm{g} / \mathrm{L}$

$\mu g / L$

$\mu g / L$

GE 0

GE $O$

Note: Flagging levels, modifiers, and laboratories are for 4 th quarter 1992 data only. See Appendix B for flagging criteria. - exceeded holding time for 4th quarter 1992.

- exceeded finai primary drinking water standard for 4th quarter 1992.

H-Area Seepage Basins 
WSRC-TR-93-059

Woll HSB 658 continued

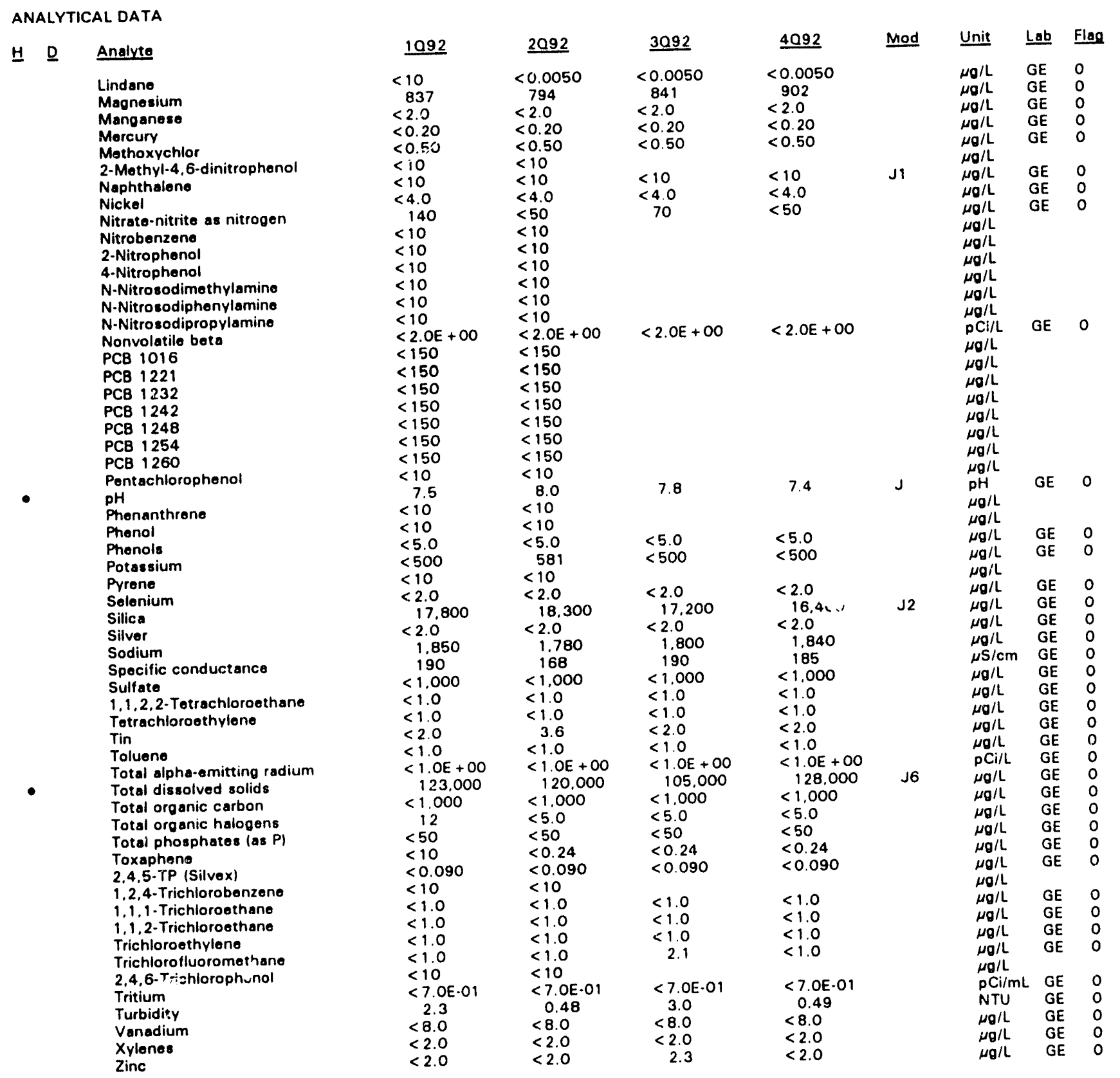

Note: Flagging levels, modifiers, and laboratories are for 4 th quarter 1992 data only. See Appendix B for flagging criteria. - = exceeded holding time for 4 th quarter 1992.

- exceeded final primary drinking water standard for 4 th quarter 1992.

H-Area Seepage Basins

D.30

Fourth Quarter 1992 
WSRC-TR-93-059

WELL HSB 65C

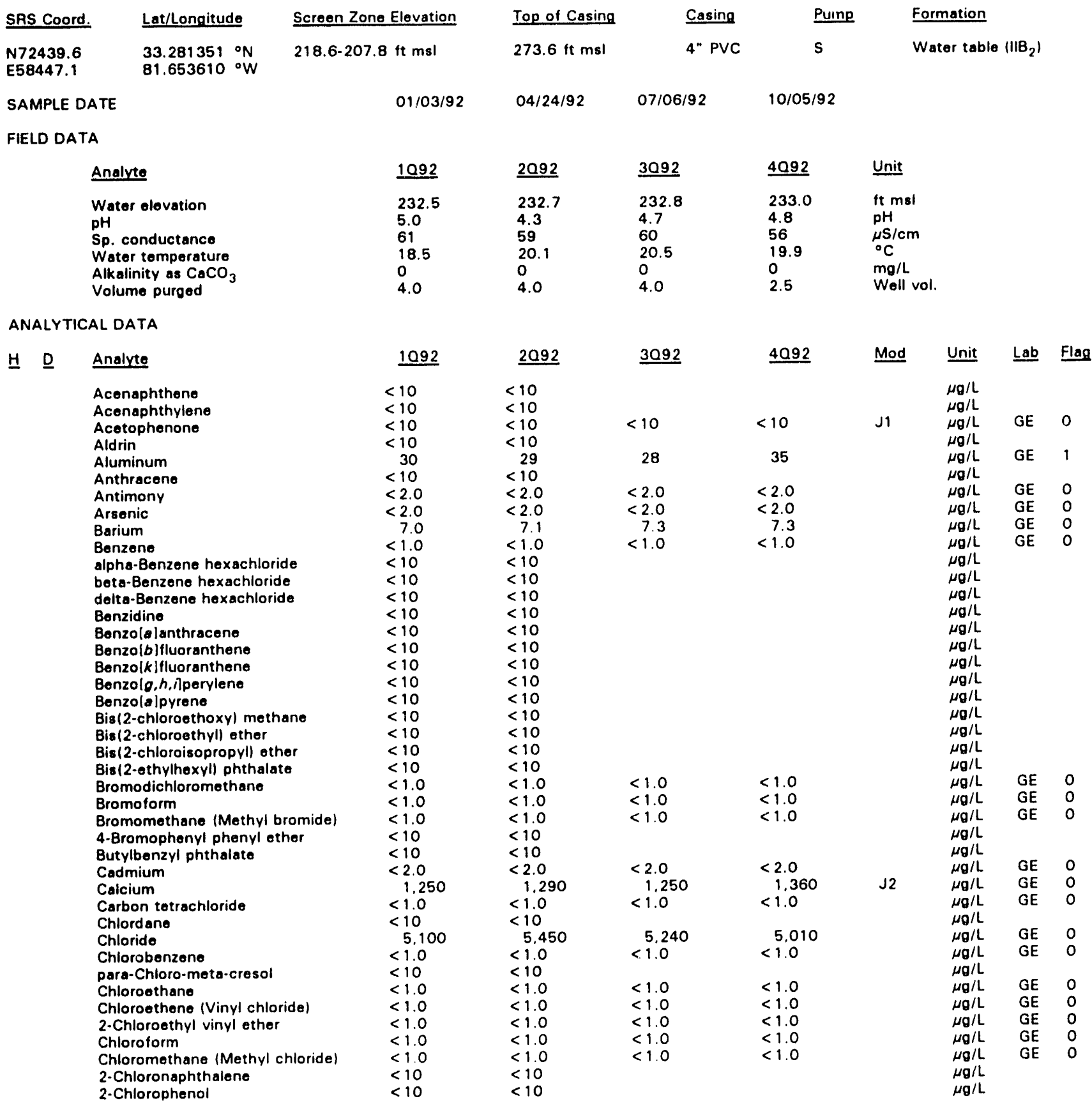

Note: Flagging levels, modifiers, and laboratories are for 4th quarter 1992 data only. See Appendix B for flagging criteria.

- exceeded holding time for 4th quarter 1992.

- =xceeded final primary drinking water standard for 4th quarter 1992.

H-Area Seepage Basins 
WSRC-TR-93-059

Well HSB 65C continued

ANALYTICAL DATA

H D Analyte

4-Chlorophenyl phenyl ether Chromium

Chrysene

Cobalt

Copper

Cyanide

p.p'-DDE

Dibenzle, hlanthracene

Dibromochloromethane

Di-n-butyl phthalate

3,3'-Dichlorobenzidine

1.1-Dichloroethane

1.2-Dichloroethane

1.1-Dichloroethylene

trans-1,2-Oichloroethylene

Dichloromethane (Methylene chloride)

2.4-Dichlorophenol

2.4-Dichlorophenoxyacetic acid

1,2-Dichloropropane

cis-1.3-Dichloropropene

trans-1,3-Dichloropropene

Dieldrin

Diethyl phthalate

2,4-Dimethyl phienol

Dimethyl phthalate

2.4-Dinitrophenol

2.4-Dinitrotoluene

2,6-Dinitrotoluene

Di-n-octyl phthalate

1,2-Diphenylhydrazine

Endosulfan I

Endosulfan II

Endosulfan sulfate

Endrin

Endrin aldehydo

Ethylbenzene

Fluoranthene

Fluorene

Fluoride

Gross alpha

Hoptachlor

Heptachlor epoxide

Hexachlorobenzene

Hexachlorobutadiene

Hexachlorocyclopentadiene

Hexachloroethane

Indenol 1,2,3-c,dlpyrene

Iron

Isophorone

Lead

Lind ane

Magnesium

Manganese

Mercury

Mothoxychlor

2-Methyl-4,6-dinitrophenol

Naphthalene

Nickel

Nitrate-nitrite as nitrogen

Nitrobenzene
1092

\section{$\underline{2092}$}

$<10$

$<4.0$

$<10$

$<4.0$

6.4

$<5.0$

$<10$

$<10$

$<10$

$<10$

$<1.0$

$<10$

$<10$

$<1.0$

$<1.0$

$<1.0$

$<1.0$

1.3

$<10$

$<0.30$

$<1.0$

$<1.0$

$<1.0$

$<10$

$<10$

$<10$

$<10$

$<10$
$<45$

$<10$

$<10$

$<10$

$<10$
$<10$

$<10$

$<10$
$<10$

$<10$

$<10$

$<10$
$<10$

$<10$
$<1.0$

$<1.0$
$<10$

$<10$

$<100$

$<2 . \mathrm{OE}+1) 0$

$<10$

$<10$

$<10$

$<10$

$<10$

$<10$
$<10$

$<10$
$<10$

56

$<10$

$<3.0$

$<10$

9.5

$<0.20$

$<0.50$

$<10$

$<10$

$<4.0$

3.500

$<10$
$<10$

$<4.0$

$<10$

$<4.0$

6.5
$<5.0$

$<5.0$
$<10$

$<10$

$<10$

$<10$

$<1.0$

$<10$

$<10$

$<1.0$

$<1.0$

$<1.0$

2.2

$<10$

$<0.30$

$<1.0$

$<1.0$

$<1.0$

$<10$

$<10$

$<10$

$<10$

$<45$

$<10$

$<10$

$<10$

$<10$

$<10$

$<10$
$<10$

$<10$

$<0.0060$

$<10$

$<1.0$

$<10$

$<10$

$<100$

$<2.0 E+00$

$<10$

$<10$

$<10$

$<10$

$<10$

$<10$

$<10$

16
$<10$

$<3.0$

$<0.0050$

730
9.4

$<.4$

$<0.50$

$<10$

$<10$

$<4.0$

5.700

$<10$

\begin{tabular}{|c|c|c|c|c|}
\hline 3092 & 4092 & Mod & Unit & $\underline{L a b}$ \\
\hline$<4.0$ & $<4.0$ & & $\begin{array}{l}\mu g / L \\
\mu g / L \\
\mu g / L\end{array}$ & $\mathrm{GE}$ \\
\hline $\begin{array}{r}<4.0 \\
7.8 \\
<5.0\end{array}$ & $\begin{array}{r}<4.0 \\
8.0 \\
<5.0\end{array}$ & & $\begin{array}{l}\mu_{g} / L \\
\mu_{0} / L \\
\mu_{g} / L\end{array}$ & $\begin{array}{l}\mathrm{GE} \\
\mathrm{GE} \\
\mathrm{GE}\end{array}$ \\
\hline
\end{tabular}

$<1.0$

$<1.0$

$<1.0$

$<1.0$

$<1.0$

$<1.0$

$<1.0$

$<0.30$

$<1.0$

$<1.0$

$<1.0$

$<5.0$

$<1.0$

$<1.0$

$<1.0$

$\begin{array}{r}2.5 \\ \hline\end{array}$

$<0.30$

$<1.0$

1.0

$<1.0$

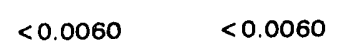

$<1.0$

$<1.0$

$<100$

$<2.0 E+00$

2.1E + 00

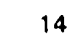

$<3.0$

$<0.0050$

732
8.6

$<0.20$

$<0.50$

$<10$

$<4.0$

3.600

$\begin{aligned} & 19 \\ & 3.0 \\ &< 0.0050 \\ & 849 \\ & 10 \\ &< 0.20 \\ &< 0.50 \\ &< 10 \\ &< 4.0 \\ & 3.650\end{aligned}$

GE

GE $O$

GE 0

GE O

J1

J1

$\mu \mathrm{g} / \mathrm{L}$

$\mu \mathrm{g} / \mathrm{L}$

$\mu g / L$ GE O

$\mu \mathrm{g} / \mathrm{L}$

Note: Flagging levels, modifiers, and laboratories are for 4 th quarter 1992 data only. See Appendix B for flagging criteria.

- =xceeded holding time for 4th quarter 1992 .

- exceeded final primary drinking water standard for 4 th quarter 1992.

H-Area Seepage Basins

D-32

Fourth Quarter 1992 
WSRC-TR-93-059

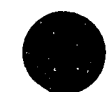

Well HSB 65C continued

\section{ANALYTICAL DATA}

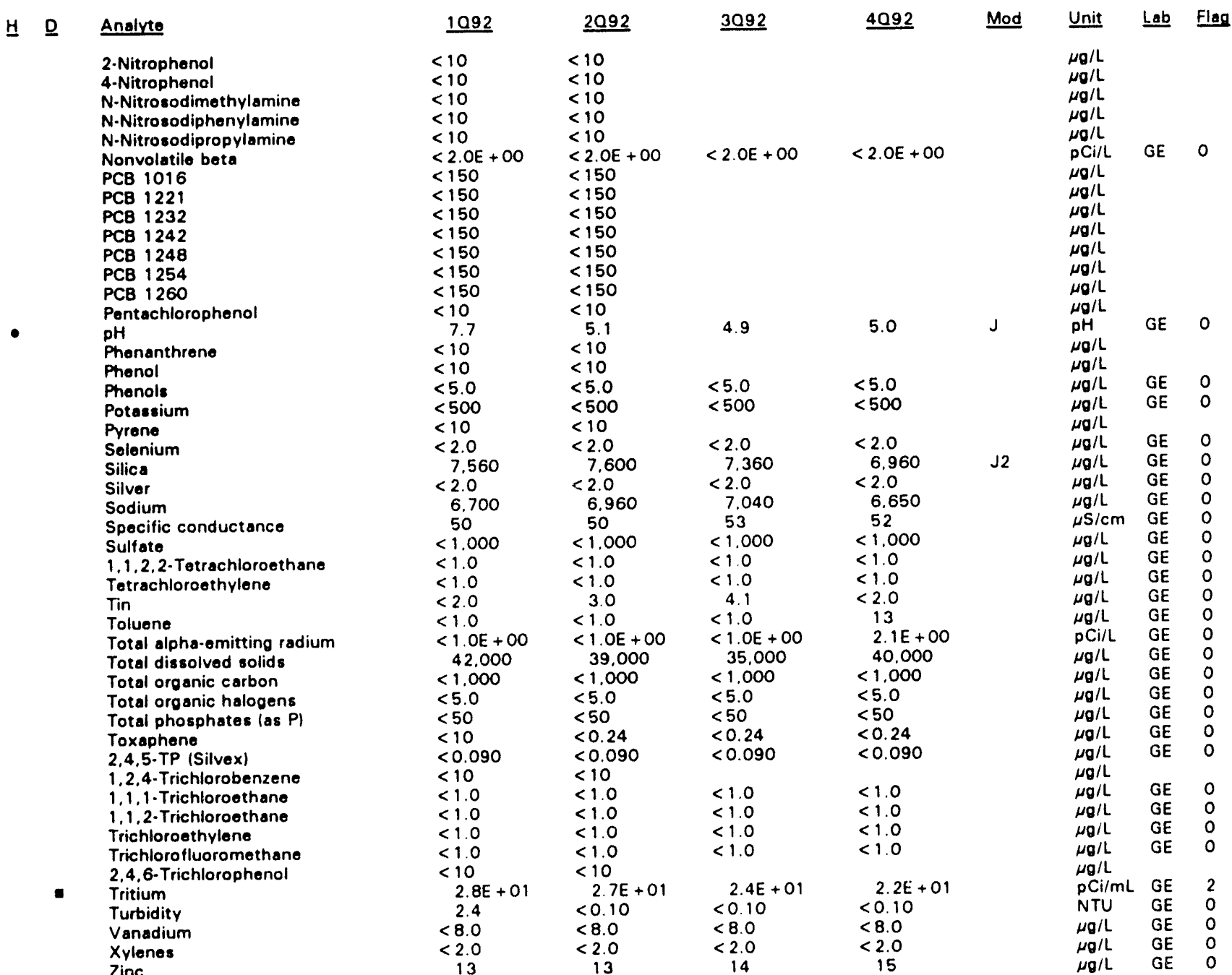

Note: Flagging levels, modifiers, and laboratories are for 4 th quarter 1992 data only. See Appendix B for flagging criteria. - =xceeded holding time for 4th quarter 1992.

- exceeded final primary drinking water standard for 4th quarter 1992. 
WSRC-TR-93-059

WELL HSB 66

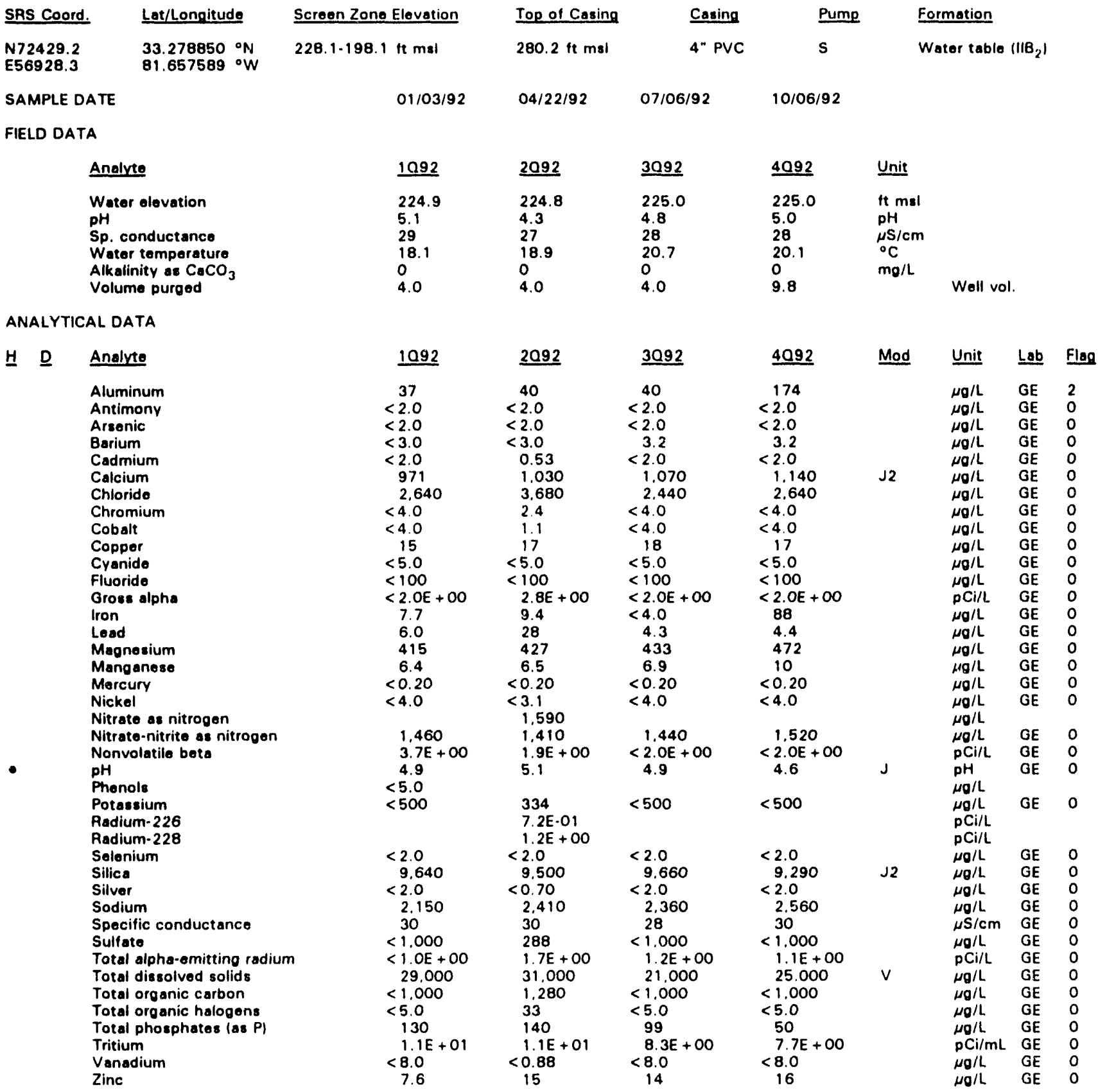

Note: Flagging levels, modifiers, and laboratories are for 4 th quarter 1992 data only. See Appendix $B$ for flagging criteria.

- = exceeded holding time for 4th quarter 1992.

- =xceeded final primary drinking water standard for 4th quarter 1992. 
WELL HSB 67

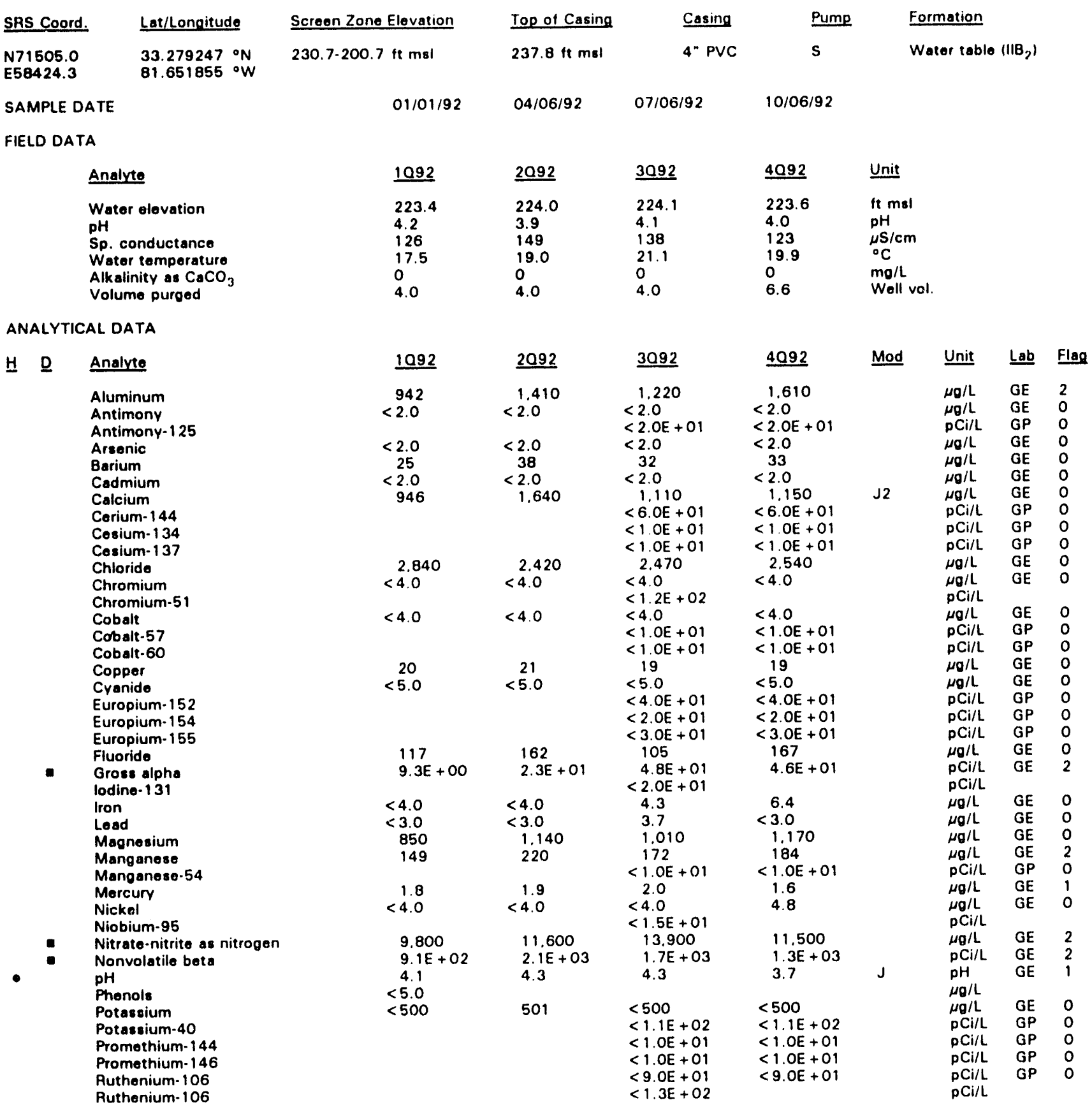

Note: Flagging levels, modifiers, and laboratories are for 4 th quarter 1992 data only. See Appendix B for flagging criteria. - exceeded holding time for 4th quarter 1992.

- exceeded final primary drinking water standard for 4th quarter 1992. 
ANALYTICAL DATA

H. $\quad$ Analyte
Solenium
Silica
Silver
Sodium
Sodium-22
Specific conductance
Sulfate
Total activity
Total alpha-emitting radium
Total diseolved solids
Total organic carbon
Total organic halogens
Total phosphates las P)
Tritium
Vanadium
Yttrium-88
Zinc
Zinc-65
Zirconium-95

\section{WELL HSB 68}

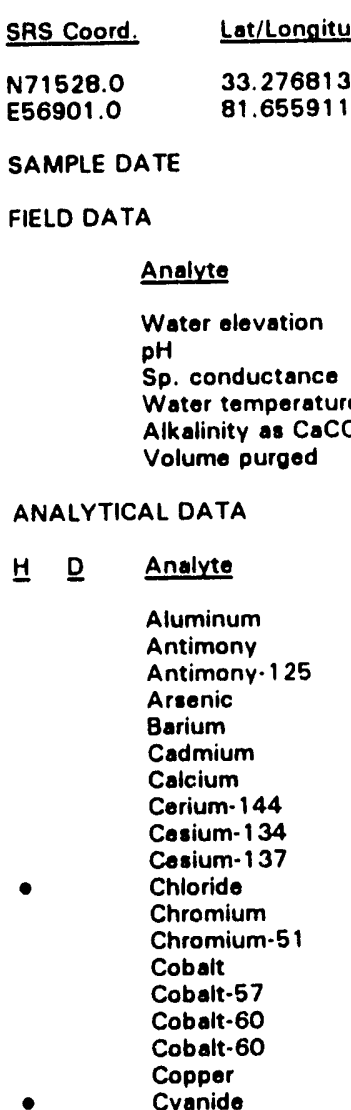

$\begin{array}{lll}1092 & \underline{2092} & \underline{3092} \\ <2.0 & <2.0 & <2.0 \\ 7.320 & 7.410 & 7.410 \\ <2.0 & <2.0 & <2.0 \\ 12.000 & 11.900 & 13.500 \\ 95 & 122 & <1.0 E+01 \\ 12.100 & 2.020 & 115 \\ 4.2 \mathrm{E}+06 & 4.7 \mathrm{E}+06 & 2.870 \\ 2.2 \mathrm{OE}+01 & 4.7 \mathrm{E}+01 & 2.5 \mathrm{E}+06 \\ 77.000 & 92.000 & 80.000 \\ <1.000 & <1.000 & <1.000 \\ <5.0 & <5.0 & <5.0 \\ <50 & <50 & <50 \\ 4.1 \mathrm{E}+03 & 4.3 \mathrm{E}+03 & 3.8 \mathrm{OE}+03 \\ <8.0 & <8.0 & <8.0 \\ & & <6.0 \mathrm{E}+01 \\ 7.4 & 11 & 8.3 \\ & & <2.0 \mathrm{E}+01 \\ & & <2.0 \mathrm{E}+01\end{array}$

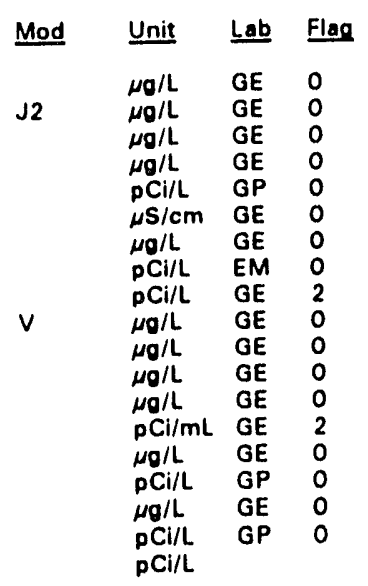


ANALYTICAL DATA

H D Analyte

Europium-152

Europium-154

Europium-155

Fluoride

- Groses alphe

lodine-131

Iron

Lead

Magnesium

Mangenese

Menganese.54

- Mercury

Nickel

Niobjum-95

- Nitrate-nitrite as nitrogen

- Nonvolatilo teto

pH

Phenols

Potassium

Potassium-40

Promethium-144

Promethium-146

Ruthenium-106

Ruthenium-106

Selenium

Silica

Silver

Sodium

Sodium

Sodium-22

Specific conductance

Sullate

- Total alpha-emitting radium

Total dissolved solids

Total organic carbon

Total organic halogens

Total phosphates las P

- Tritium

Vanadium

Yttrium-88

Zinc

Zinc-65

Zirconium-95

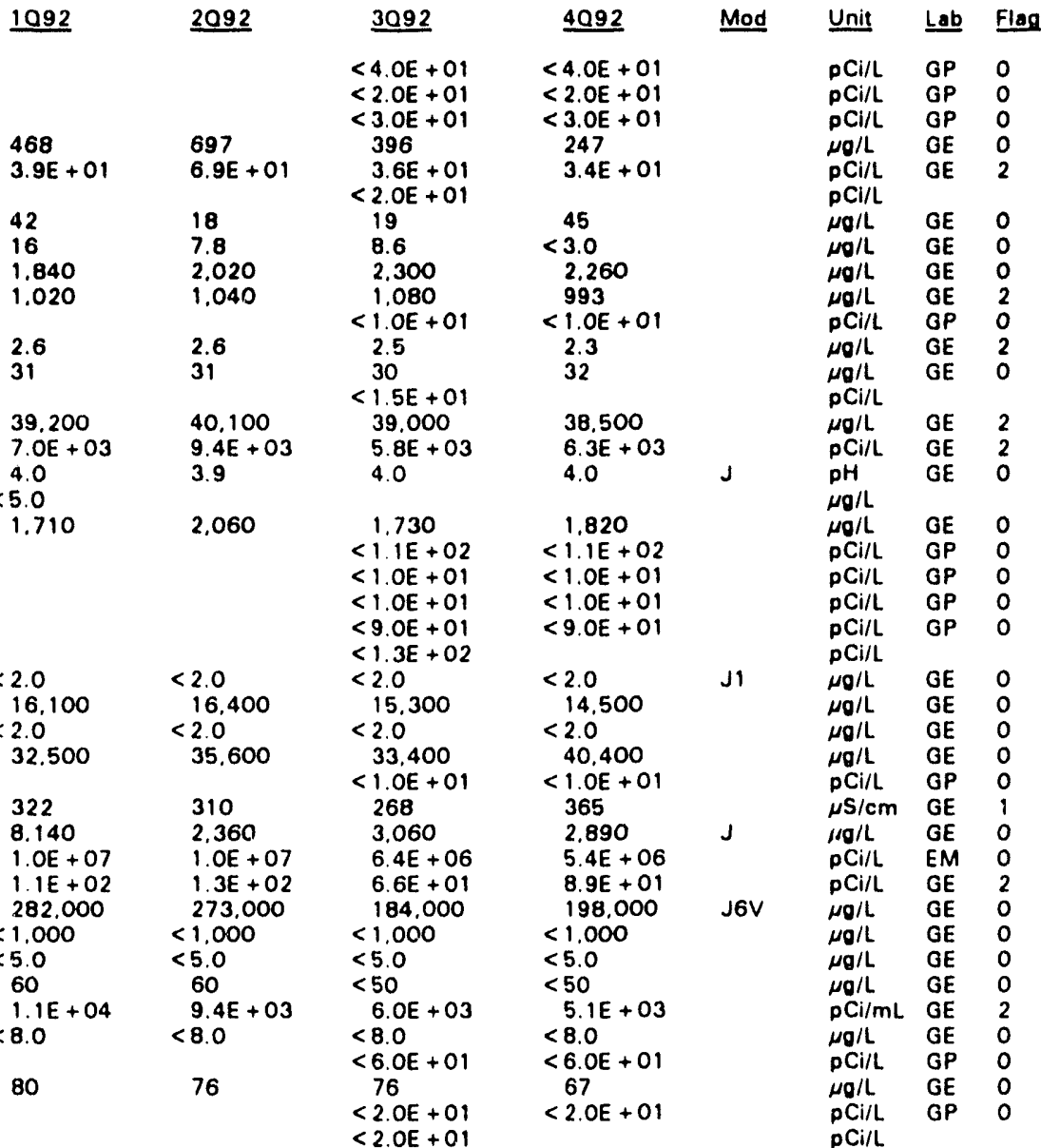

Note: Flagging levels, modifiers, and laboratories are for 4 th quarter 1992 data only. See Appendix B for flagging criteria.

- =xceeded holding time for 4th quarter 1992.

- =xceeded final primary drinking water standard for 4th quarter 1992. 
WSRC.TR.93.059

WELL HSB 68A

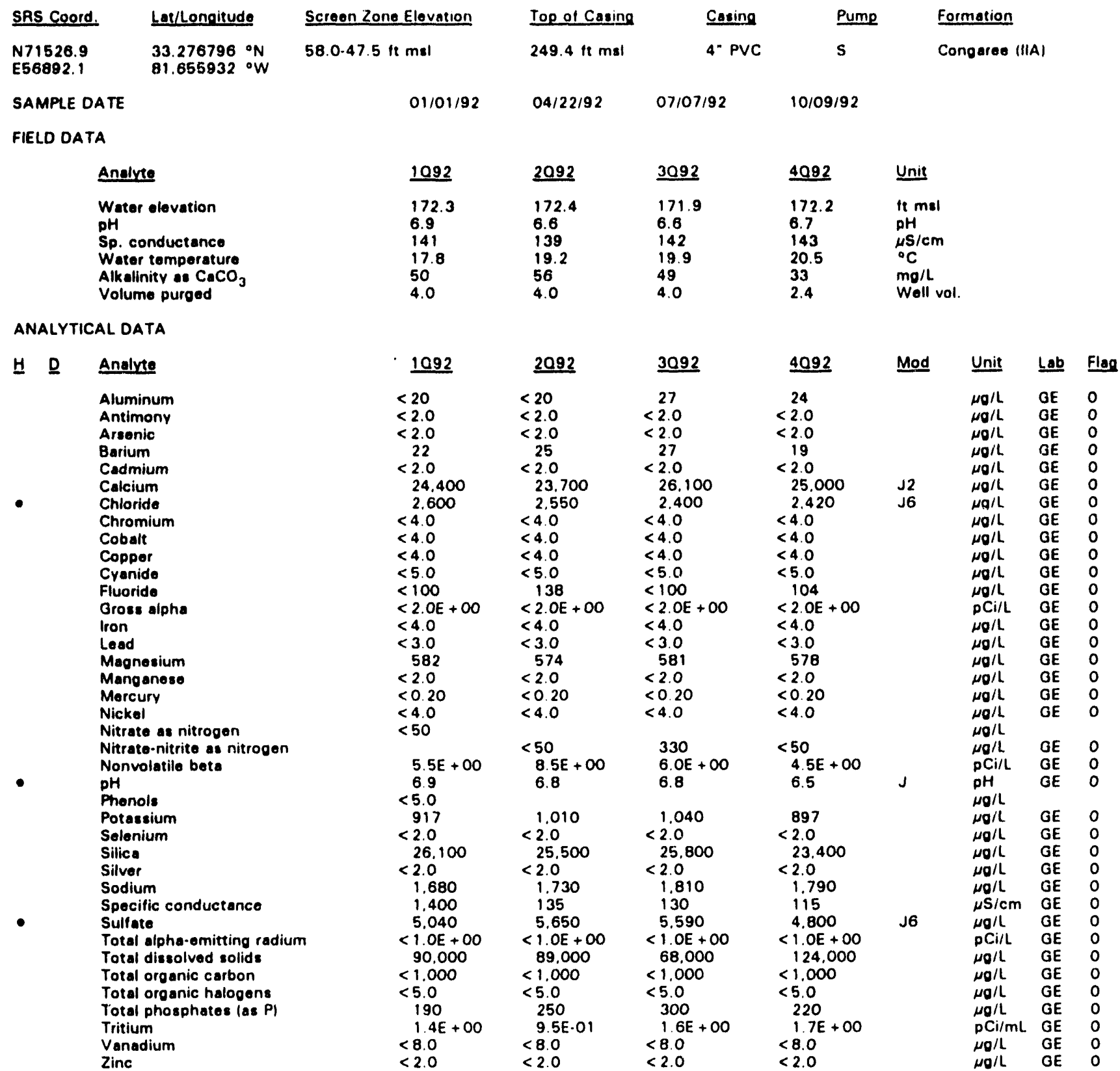

Note: Flagging levels, modifiers, and laboratories are for 4 th quarter 1992 data only. See Appendix B for flagging criteria.

- =xceeded holding time for 4th quarter 1992.

- =xceeded final primary drinking water standard for 4th quarter 1992. 
WSRC-TR-93.059

WELL HSB 68B

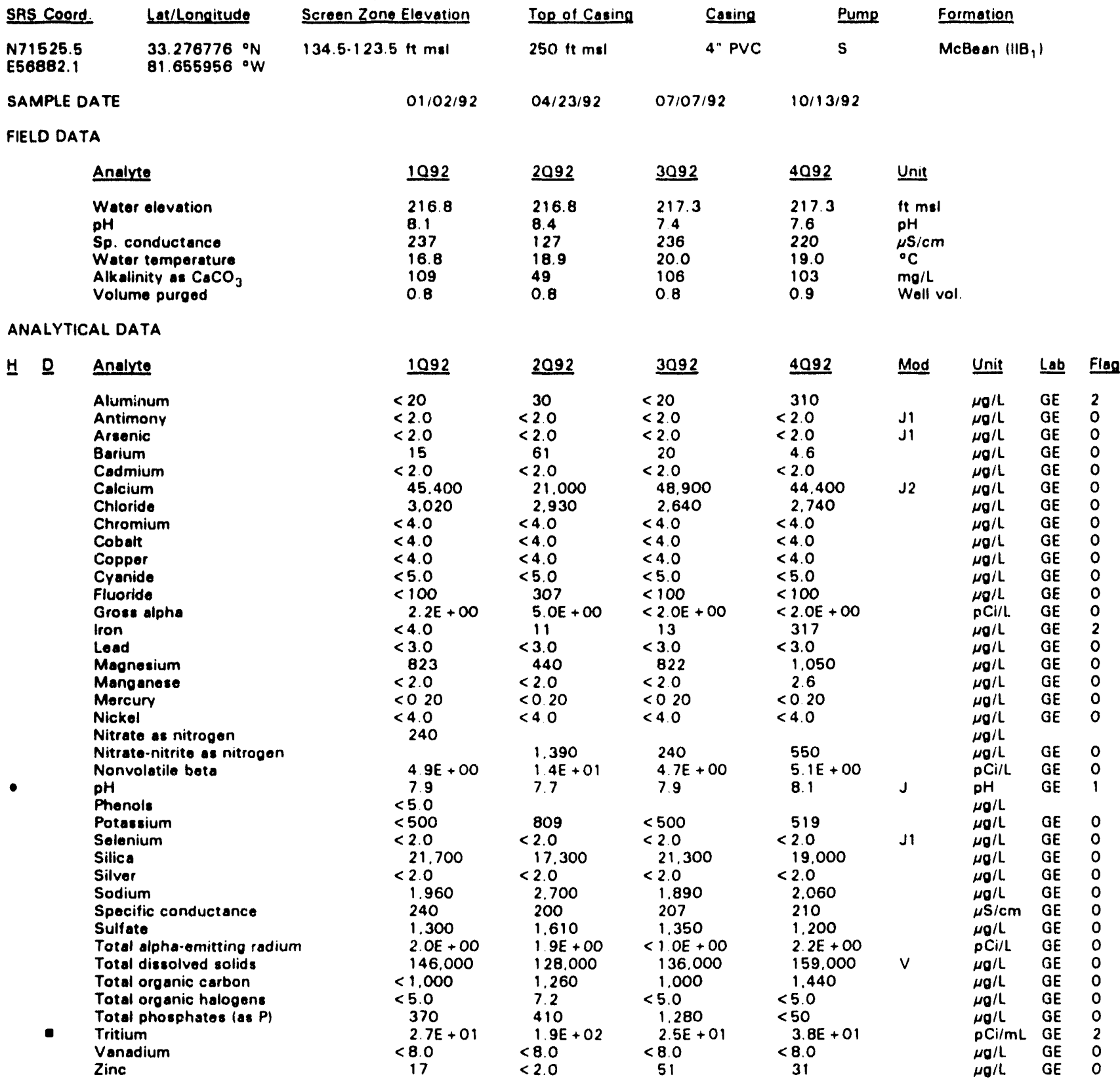

Note: Flagging levels, modifiers, and laboratories are for 4 th quarter 1992 data only. See Appendix B for tlagging criteria.

- =xceeded holding time for 4th quarter 1992.

- exceeded final primary drinking water standard for 4th quarter 1992.

H-Area Seepage Basins

D-39

Fourth Quarter 1992 
WSRC.TR.93-059

WELL HSB 68C

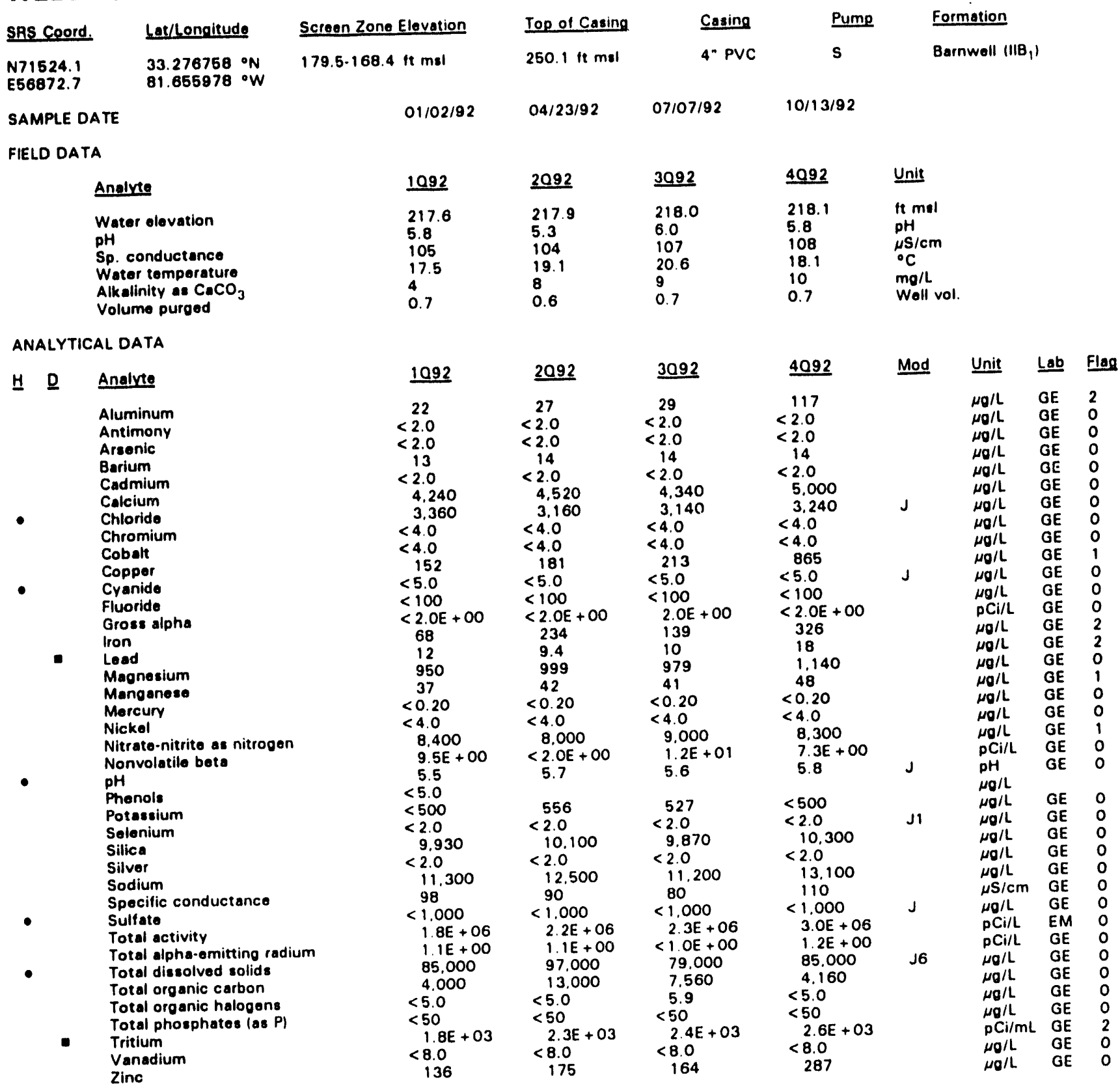

Note: Flagging levels, modifiers, and laboratories are for 4 th quarter 1992 data only. See Appendix B for flagging criteria.

- =xceeded holding time for 4th quarter 1992.

- =xceeded final primary drinking water standard for 4th quarter 1992.

H-Area Seepage Basins

$D .40$

Fourth Quarter 1992 
WSRC-TR-93-059

\section{WELL HSB 69}

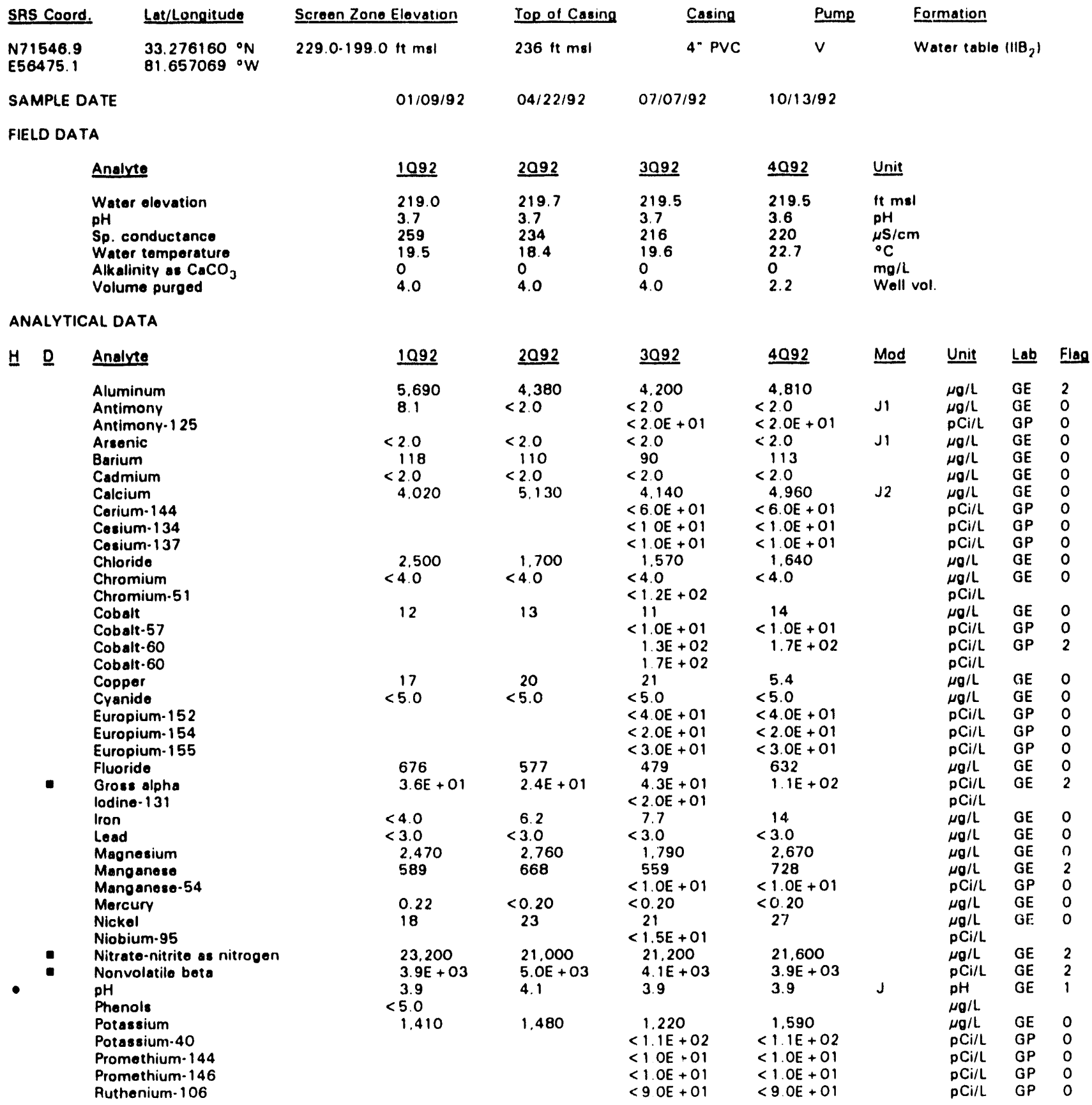

Note: Flagging levels, modifiers, and laboratories are for 4 th quarter 1992 data only. See Appendix B for flagging criteria. - exceeded holding time for 4 th quarter 1992.

- exceeded final primary drinking water standard for 4th quarter 1992. 
WSRC-TR-93.059

Well HSB 69 continued

ANALYTICAL DATA

H D Analyte

Ruthenium-106

Solenium

Silica

Silver

Sodium

Sodium.22

Specific conductance

Sulfate

Total activity

- Total alpha-emitting radium

Total dissolved solids

Total organic carbon

Total organic halogens

Total phosphates las P

Tritium

Vanadium

Yttrium. 88

Zine

Zine-65

Zirconium-95

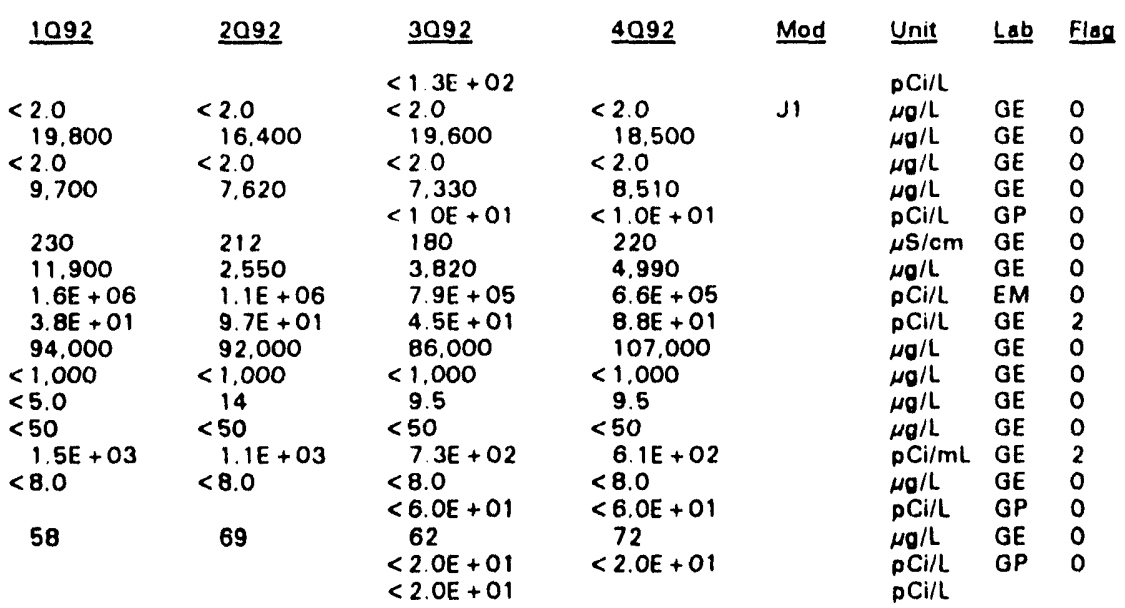

\section{WELL HSB 69A}

\begin{tabular}{|c|c|}
\hline SAS Coord. & Las/Longitude \\
\hline $\begin{array}{l}\text { N71549.4 } \\
\text { E56465.1 }\end{array}$ & $\begin{array}{l}33.2761490^{\circ} \mathrm{N} \\
81.657100^{\circ} \mathrm{W}\end{array}$ \\
\hline
\end{tabular}

$\frac{\text { Screen Zone Elevation }}{93.1 \cdot 83.1 \mathrm{ft} \mathrm{msi}}$

\section{Top of Casing \\ $236.6 \mathrm{ft} \mathrm{msl}$}

$01 / 09 / 92$

$04 / 22 / 92$

$07 / 07 / 92$

Casing

\section{Pump}

S

Formation

SAMPLE DATE

FIELD DATA

\section{Analyte}

Water olovation

pH

Water temperature

Alkalinity as $\mathrm{CaCO}_{3}$

Volume purged

ANALYTICAL DATA
Sp. conductance

$\begin{array}{ll}1092 & \underline{2092} \\ 172.5 & 172.7 \\ 6.9 & 6.7 \\ 170 & 165 \\ 18.6 & 19.0 \\ 55 & 56 \\ 4.0 & 4.0\end{array}$

\begin{tabular}{l}
3092 \\
\hline 172.3 \\
6.7 \\
166 \\
19.6 \\
59 \\
4.0
\end{tabular}

10/09/92

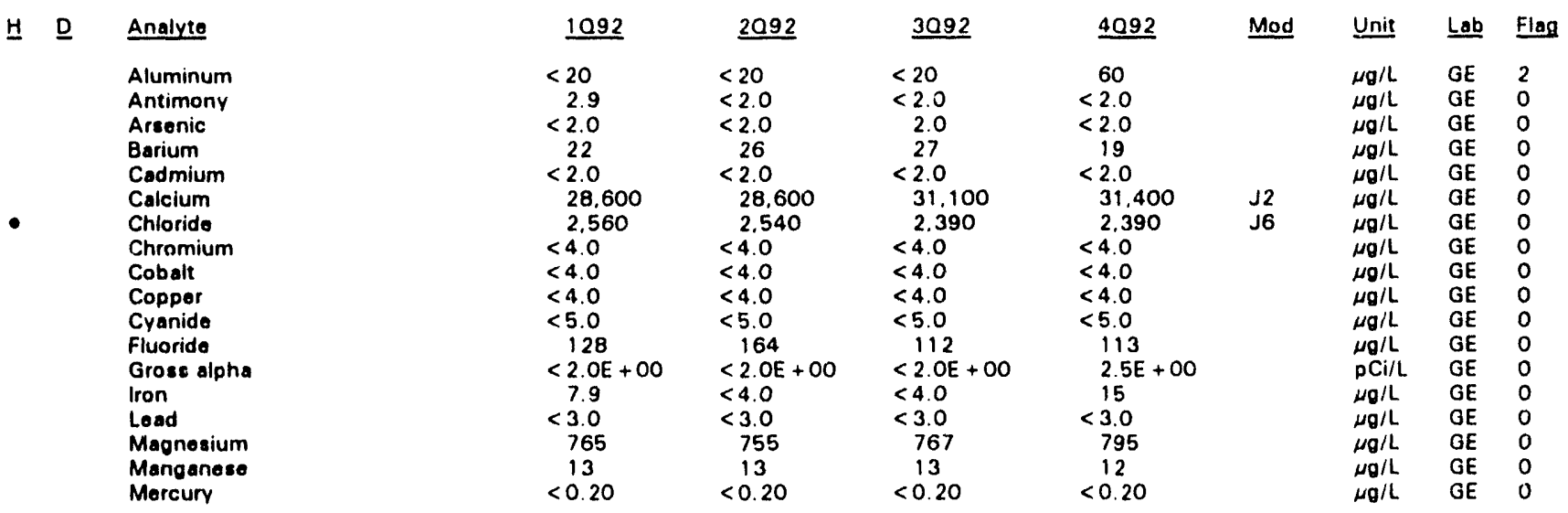

Note: Flagging levels, modifiers, and laboratories are for 4th quarter 1992 data only. See Appendix B for flagging criteria.

- exceeded holding time for 4 th quarter 1992.

- exceeded final primary drinking water standard for 4th quarter 1992.

H-Area Seepage Basins

$D \cdot 42$

Fourth Quarter 1992 
WSRC-TR-93-059

Well HSB 69A continued

ANALITICAL DATA

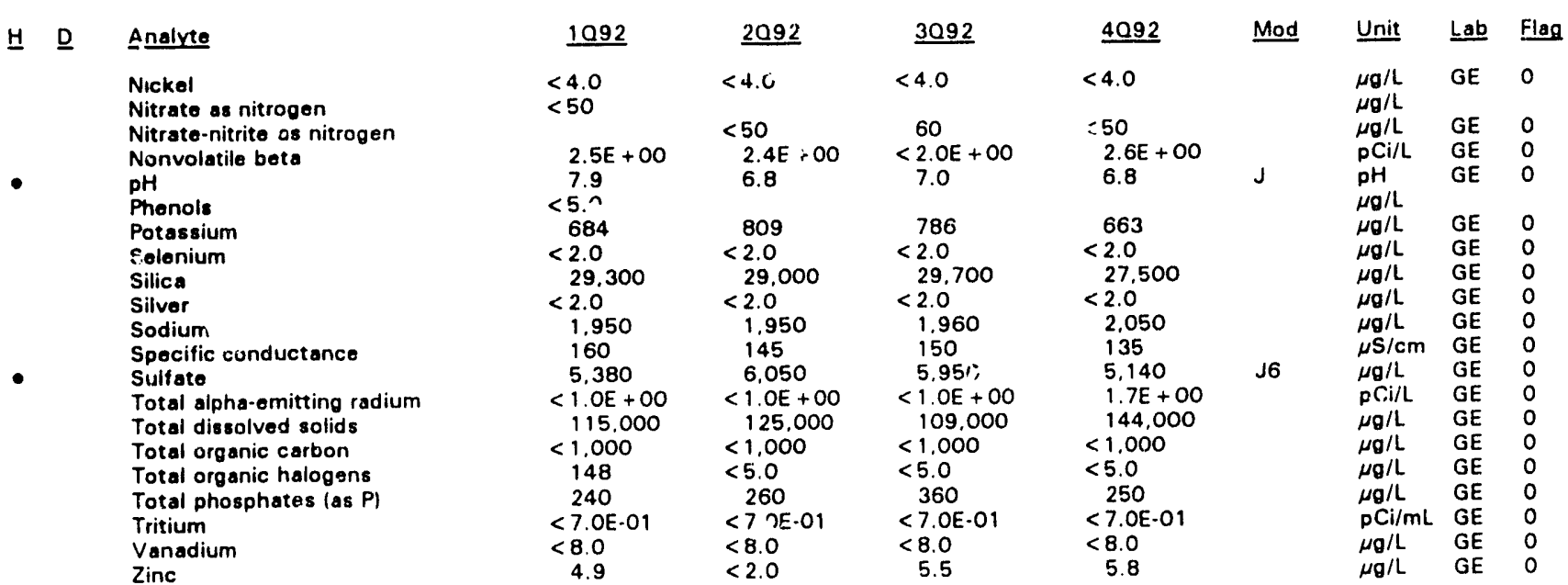

\section{WELL HSB 70}

\begin{tabular}{|c|c|}
\hline SAS Coord. & Lat/Longitude \\
\hline $\begin{array}{l}\text { N72606.9 } \\
\text { E55758.9 }\end{array}$ & $\begin{array}{l}33.277336{ }^{\circ} \mathrm{N} \\
81.661013^{\circ} \mathrm{V}\end{array}$ \\
\hline
\end{tabular}

\section{Screen Zone Elevation}

Top of Casing

\section{Casing}

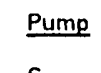

Formation

SAMPLE DATE

$01 / 07 / 92$

$242.8 \mathrm{ft} \mathrm{msl}$

$$
\text { 4" PVC }
$$

S

Water table $\left(11 \mathrm{~B}_{2}\right.$;

FIELD DATA

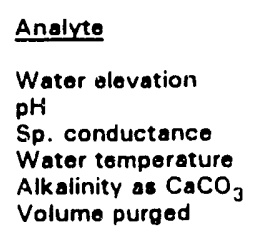

1092
223.4
5.2
68
19.4
0
4.0

2092
224.4
4.7
61
19.7
0
4.0

3092
225.3
5.1
58
20.4
0
4.0

\begin{tabular}{l}
4092 \\
\hline 224.1 \\
4.8 \\
59 \\
19.3 \\
0 \\
3.3
\end{tabular}

Unit

ANALYTICAL DATA

$H \quad \mathrm{Analyte}$
Aluminum
Antimony
Arsenic
Barium
Cadmium
Calcium
Chloride
Chromium
Cobalt
Copper
Cyanide
Fluoride
Gross alpho
Iron
Lead
Magnesium
Manganese

$\begin{array}{ll}\frac{109}{10} & \underline{2092} \\ <20 & <20 \\ <2.0 & <2.0 \\ <20 & <2.0 \\ 79 & 63 \\ <2.0 & <2.0 \\ 4.910 & 4.280 \\ 2.620 & 2.920 \\ <4.0 & <4.0 \\ <4.0 & <4.0 \\ 397 & 504 \\ <5.0 & <5.0 \\ <100 & <100 \\ <2.0 \mathrm{E}+00 & <2.0 \mathrm{O}+00 \\ 28 & 29 \\ 38 & 22 \\ 1.830 & 1.730 \\ 31 & 13\end{array}$

$\begin{aligned} & 3092 \\ & 25 \\ & 7.7 \\ < & 2.0 \\ & 61 \\ & 0.38 \\ & 4.390 \\ & 2.840 \\ < & 1.1 \\ < & 0.88 \\ & 815 \\ < & 5.0 \\ < & 100 \\ & 2.7 E+00 \\ & 26 \\ & 43 \\ & 1.600 \\ & 13\end{aligned}$

4092
69
3.1
3.9
61
$<2.0$
4.360
2.180
$<4.0$
$<4.0$
527
$<5.0$
$<100$
$2.8 \mathrm{E}+00$
16
30
1.690
11

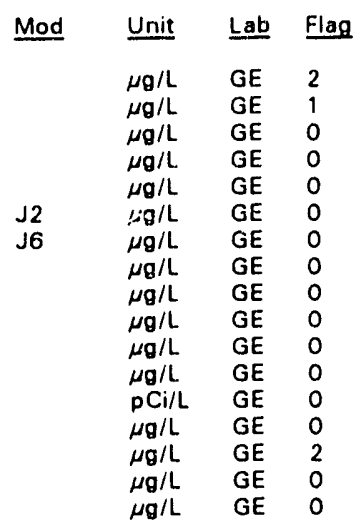

Note: Flagging levels, modifiers, and laboratories are for 4 th quarter 1992 data only. See Appendix $B$ for flagging criteria. - =xceeded holding time for 4th quarter 1992.

- exceeded final primary drinking water standar $\rfloor$ for 4 th quarter 1992. 
Well HSB 70 continued

ANALYTICAL DATA

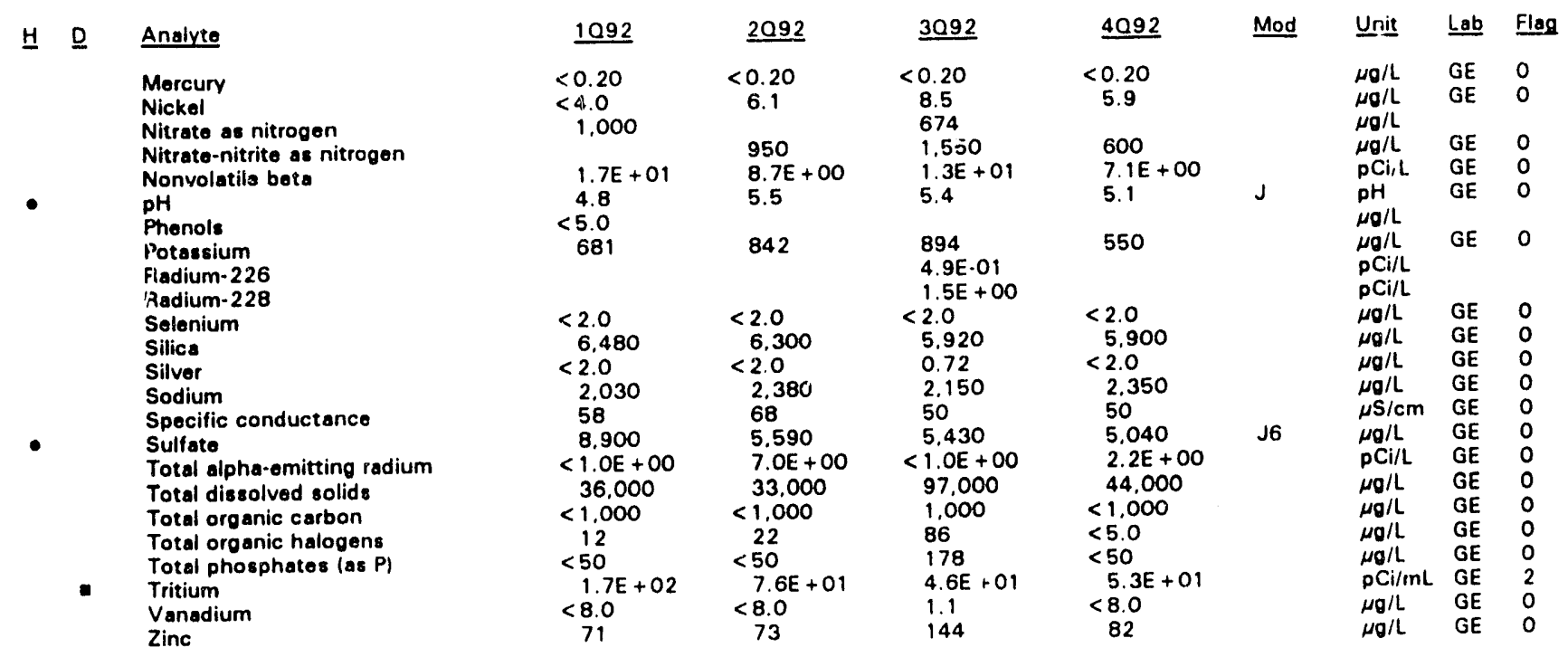

\section{WELL HSB 70C}

$\begin{array}{lll}\text { SRS Coord. } & \text { Lat/Longitude } & \text { S } \\ \text { N72597.3 } & 33.277311^{\circ} \mathrm{N} & 174 \\ \text { E55757.1 } & 81.660999{ }^{\circ} \mathrm{W}\end{array}$

\section{Screen Zone Elevation}

Top of Casing

$$
\text { Casing }
$$

$$
\text { Pump }
$$

Formation

SAMPLE DATE

$01 / 08 / 92$

$04 / 22 / 92$

$07 / 08 / 92$

$10 / 13 / 92$

FIELD DATA

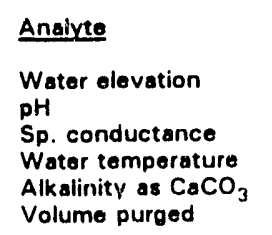

$\begin{array}{lll}1092 & \underline{2092} & \underline{3092} \\ 222.7 & 223.4 & 223.9 \\ 9.8 & 11.1 & 11.6 \\ 351 & 608 & 604 \\ 17.1 & 18.8 & 19.9 \\ 18 & 43 & 65 \\ 0.7 & 0.7 & 0.7\end{array}$

ANALYTICAL DATA

$H \quad$ Analyte
Aluminum
Antimony
Antimony-125
Arsenic
Barium
Cadmium
Calcium
Corium-144
Cesium-134
Cosium-137
Chloride
Chromium
Chromium-51
Cobalt

$\begin{array}{lll}1092 & \underline{2092} & \underline{3092} \\ 26 & 41 & 26 \\ 3.9 & <2.0 & <2.0 \\ <2.0 & <2.0 & <2.0 E+01 \\ 98 & 105 & <2.0 \\ <2.0 & <2.0 & 105 \\ 23.200 & 27.600 & <2.0 \\ & & 24.500 \\ & & <6.0 E+01 \\ 3.900 & 3.510 & <1.0 E+01 \\ <4.0 & <4.0 & 3.0 E+01 \\ & <4.00 \\ <4.0 & <4.0 & <4.0 \\ & & <1.2 E+02\end{array}$

$\begin{array}{ll}\text { 4092 } & \text { Unit } \\ 224.1 & \mathrm{ft} \mathrm{msl} \\ 10.9 & \mathrm{pH} \\ 395 & \mu \mathrm{S} / \mathrm{cm} \\ 18.8 & { }^{\circ} \mathrm{C} \\ 98 & \mathrm{mg} / \mathrm{L} \\ 0.7 & \text { Well vol. }\end{array}$

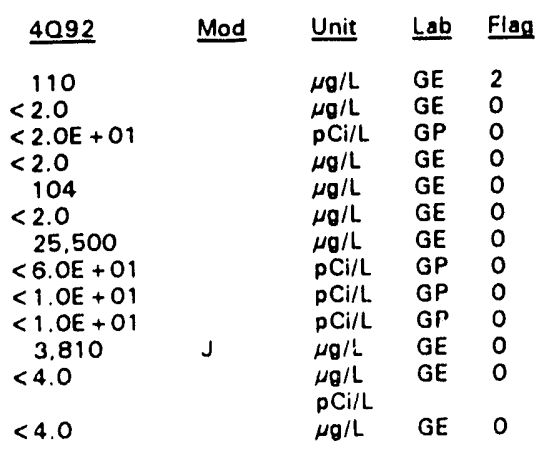

Note: Flagging levels, modifiers, and laboratories are for 4 th quarter 1992 data only. See Appendix B for flagging criteria. - =xceeded holding time for 4 th quarter 1992.

- =xceeded final primary drinking water standard for 4th quarter 1992. 
ANALYTICAL DATA

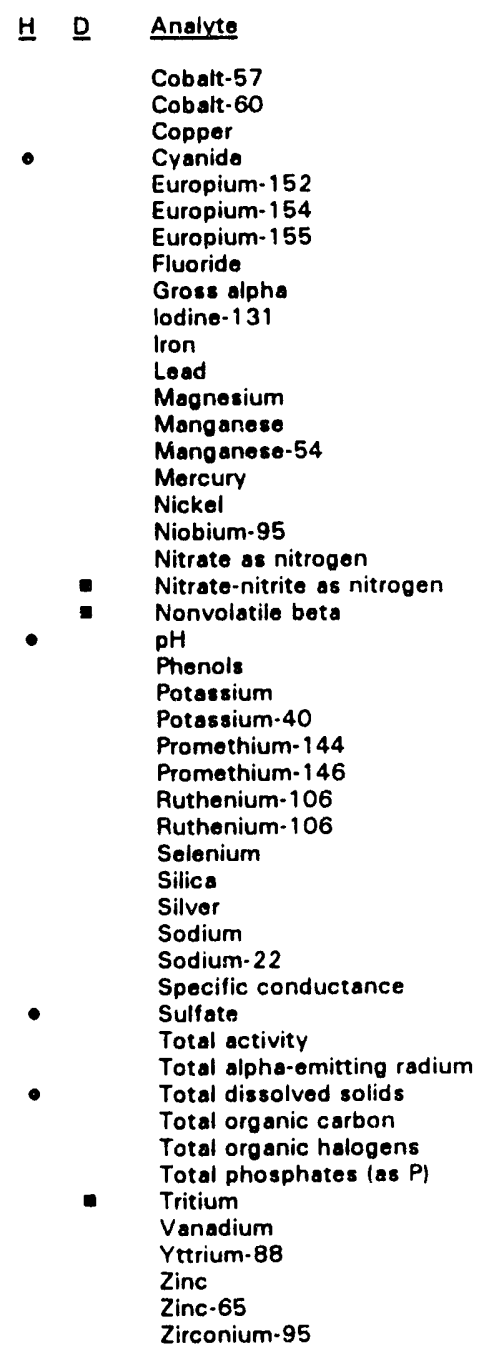

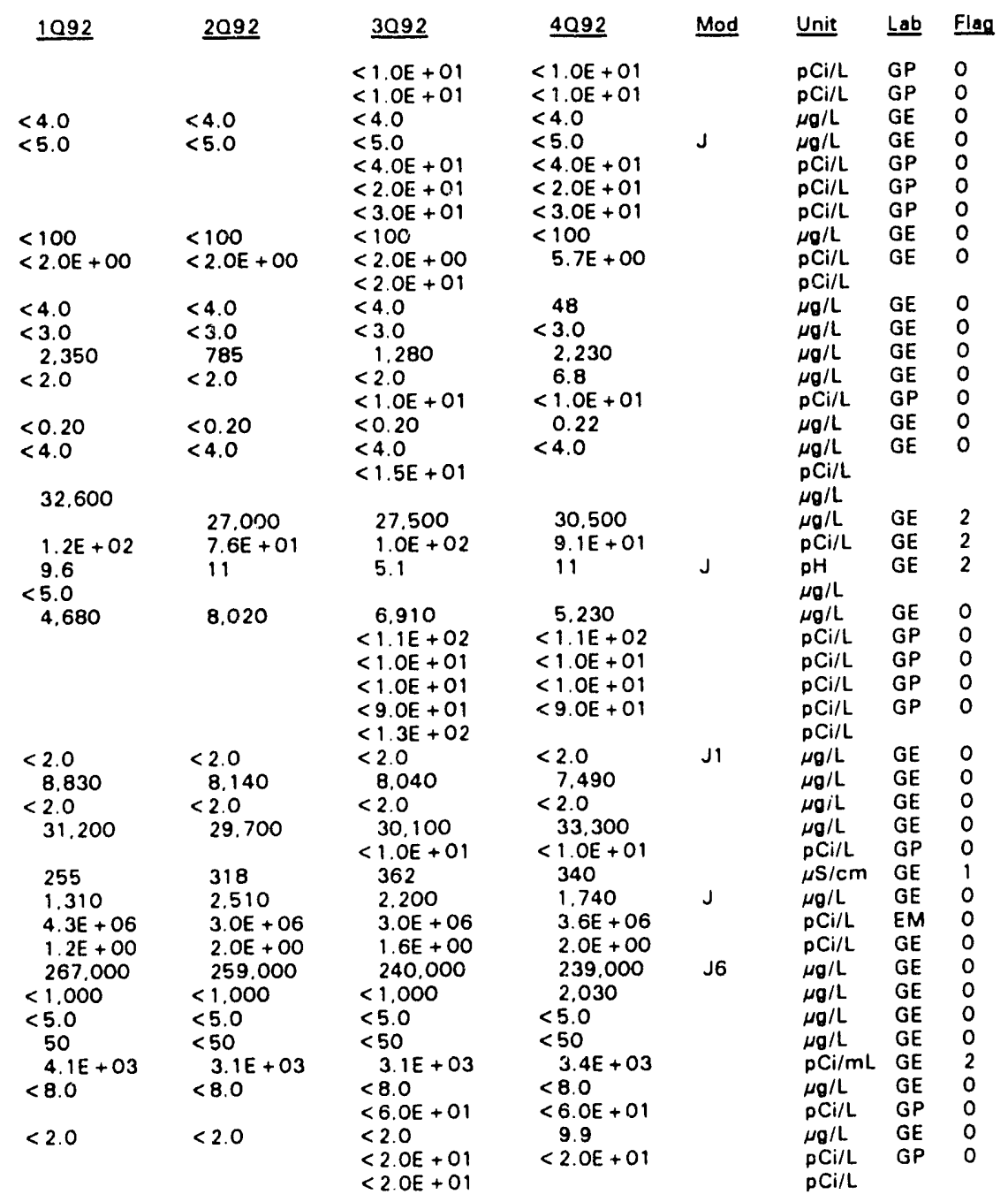

Note: Flagging levels, modifiers, and laboratories are for 4 th quarter 1992 data only. See Appendix $B$ for flagging criteria. - =xceeded holding time for 4 th quarter 1992.

- exceeded final primary drinking water standard for 4th quarter 1992. 
WELL HSB 71

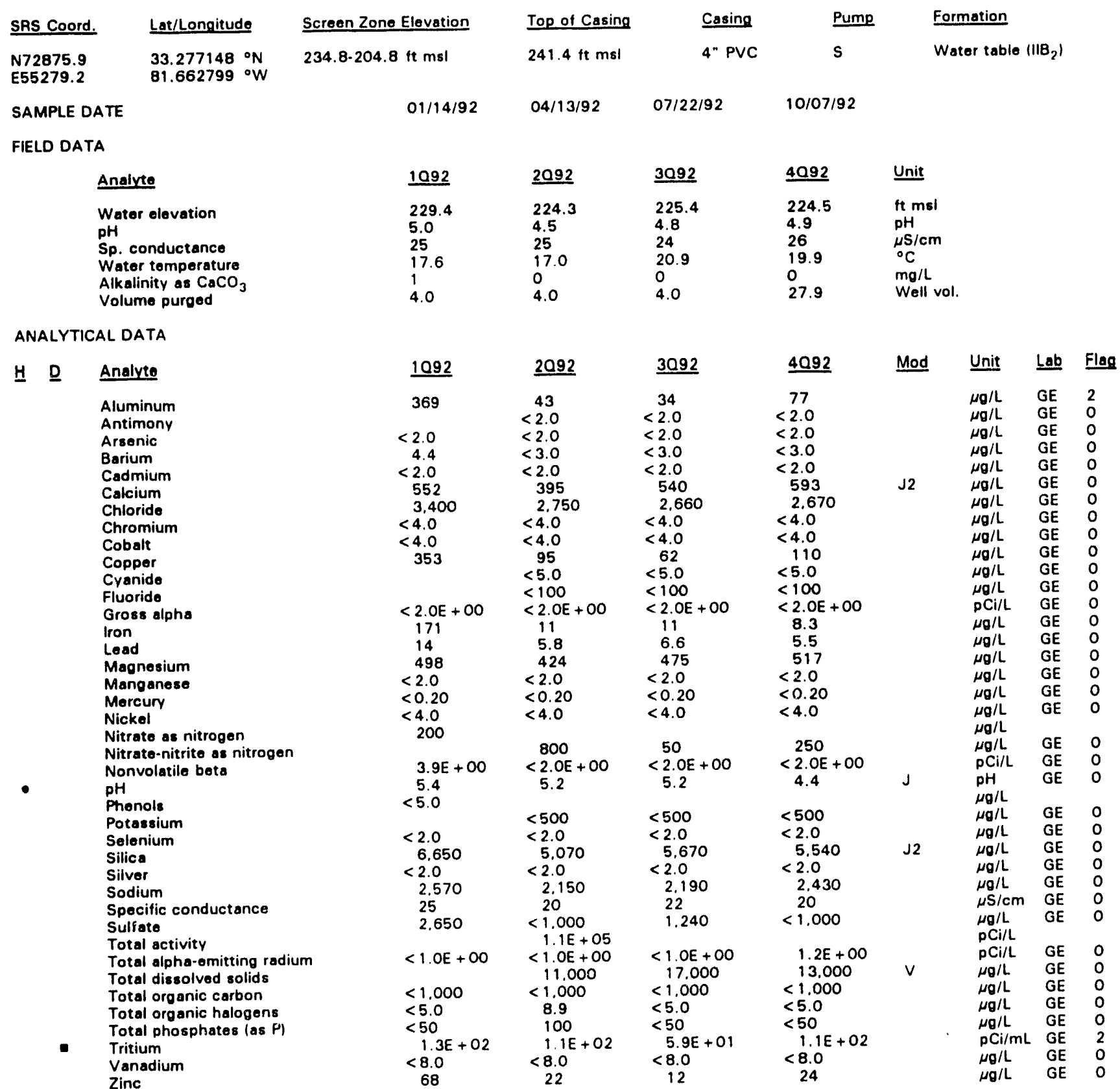

Note: Flagging levels, modifiers, and laboratories are for 4 th quarter 1992 data only. See Appendix B for flagging criteria. - = exceeded holding time for 4 th quarter 1992.

- =xceeded final primary drinking water standard for 4th quarter 1992. 
WELL HSB 71C

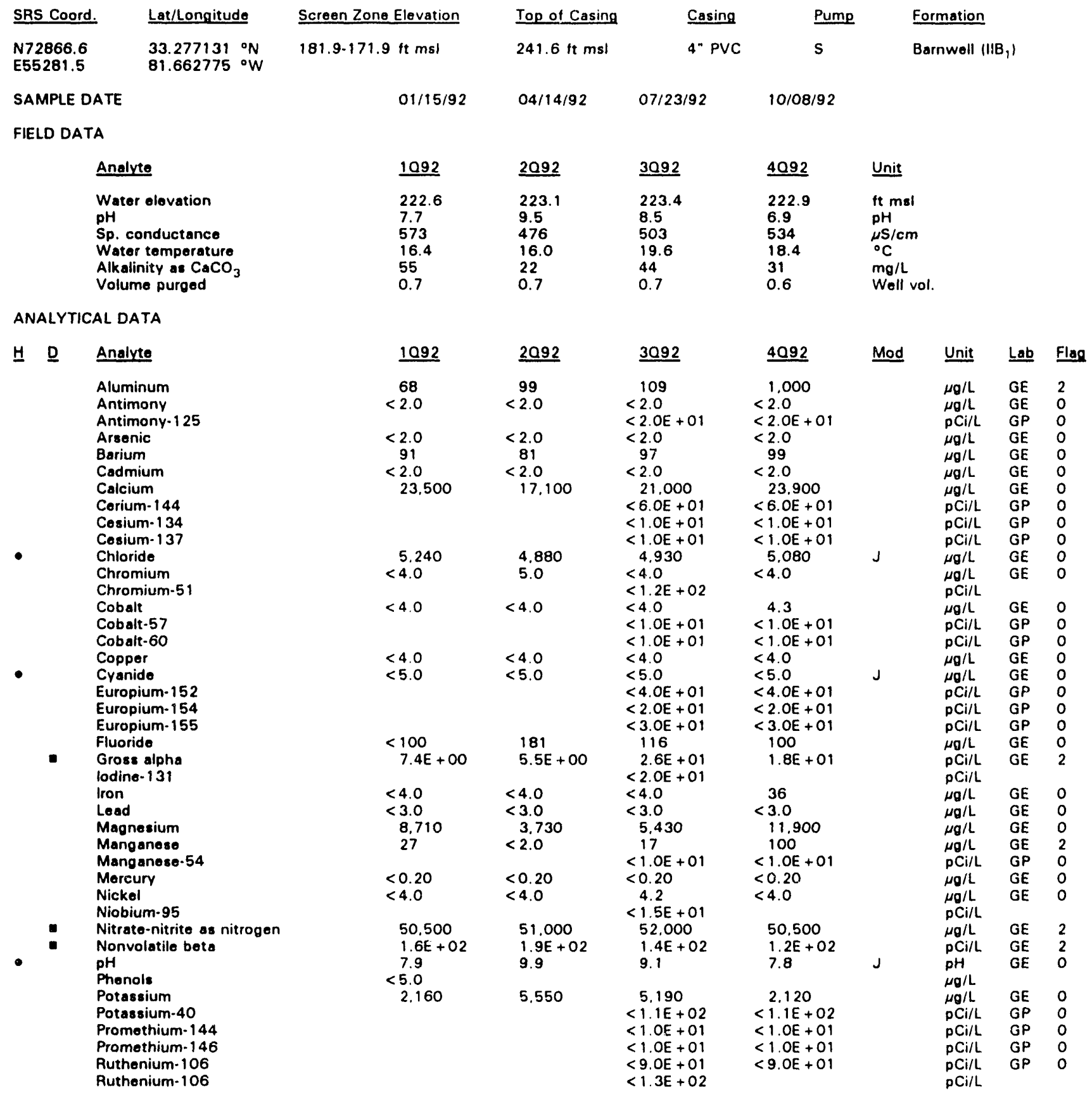

Note: Flagging levels, modifiers, and laboratories are for 4 th quarter 1992 data only. See Appendix B for flagging criteria. - =xceeded holding time for 4th quarter 1992.

- =xceeded final primary drinking water standard for 4th quarter 1992. 


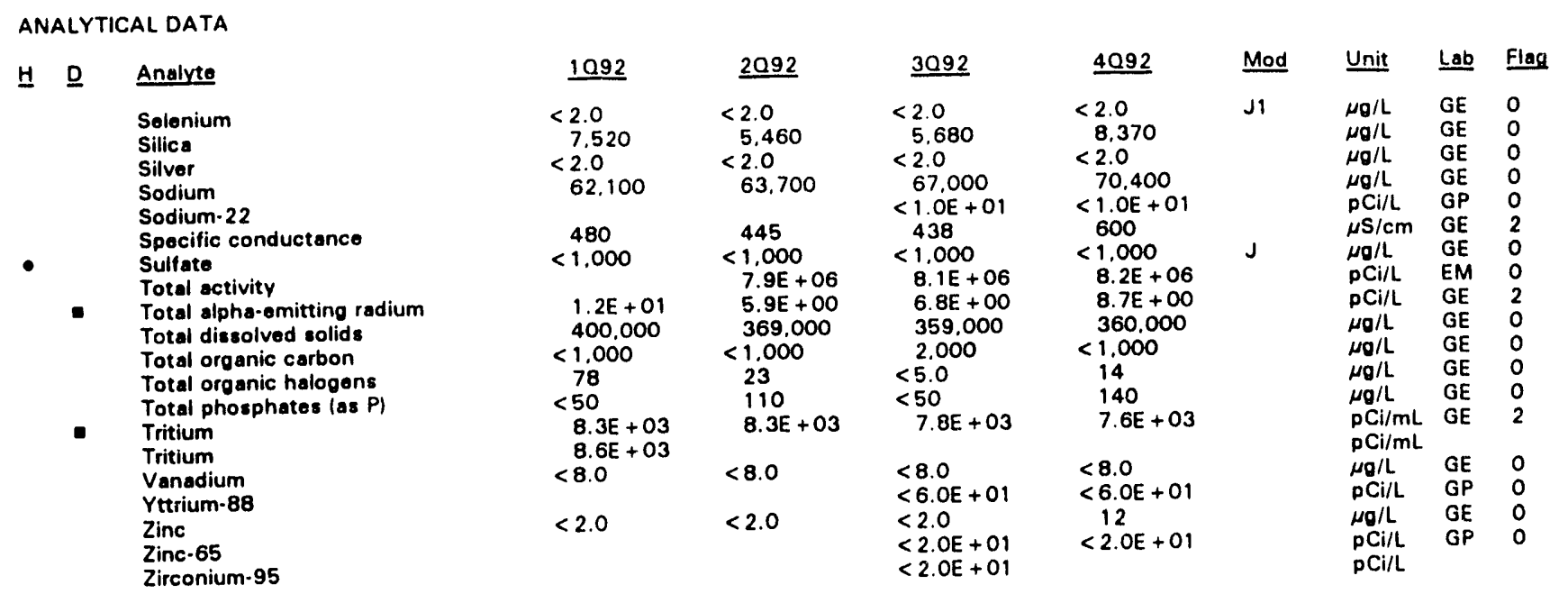

\section{WELL HSB 83A}

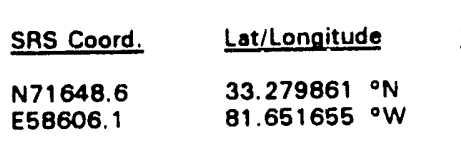

SAMPLE DATE

FIELD DATA

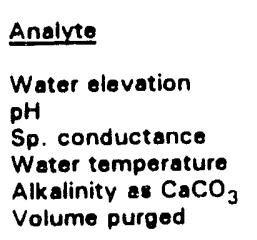

ANALYTICAL DATA

H. Analyte
Aluminum
Antimony
Arsenic
Barium
Cadmium
Calcium
Chloride
Chromium
Cobalt
Copper
Cyanide
Fluoride
Gross alpha
Iron
Lead
Magnesium
Manganese
Mercury

$\frac{\text { Screen Zone Elevation }}{76.0-65.2 \mathrm{ft} \mathrm{msl}}$

$01 / 07 / 92$

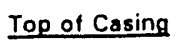

$237.3 \mathrm{ft} \mathrm{msl}$

1092
173.7
7.2
193
17.6
74
4.0

2092
173.7
6.4
189
19.0
77
4.0

$\begin{array}{lll} & 1092 & \underline{2092} \\ <20 & & <20 \\ <2.0 & <2.0 \\ <2.0 & <2.0 \\ 25 & 31 \\ <2.0 & <2.0 \\ 34.500 & 35.800 \\ 2.630 & 2.430 \\ <4.0 & <4.0 \\ <4.0 & <4.0 \\ <4.0 & <4.0 \\ <5.0 & <5.0 \\ <100 & <100 \\ <2.0 \mathrm{E}+00 & <2.0 \mathrm{E}+00 \\ <4.0 & <4.0 \\ <3.0 & <3.0 \\ 759 & & 783 \\ <2.0 & <2.0 \\ <0.20 & <0.20\end{array}$

$\begin{array}{ll}\text { Casing } & \text { Pump } \\ \text { 4" PVC } & \mathrm{S}\end{array}$

Formation

M. Congaree (IIA)

$04 / 27 / 92 \quad 07 / 07 / 92 \quad 10 / 07 / 92$ 
WSRC-TR-93-059

Well HSB 83A continued

ANALYTICAL DATA

H D Analyte
Nickel
Nitrate as nitrogen
Nitrate-nitrite as nitrogen
Nonvolatile bota
pH
Phenols
Potassium
Solenium
Silica
Silver
Sodium
Specific conductance
Sulfate
Total alpha-emitting radium
Total diesolved solids
Total organic carbon
Total organic halogens
Total phosphates las Pf
Tritium
Vanadium
Zinc

$\begin{array}{lll}1092 & \underline{2092} & \underline{3092} \\ <4.0 & <4.0 & <4.0 \\ <50 & & \\ & <50 & 190 \\ <2.0 \mathrm{E}+00 & <2.0 \mathrm{E}+00 & <2.0 \mathrm{E}+00 \\ 6.7 & 7.4 & 7.0 \\ <5.0 & & \\ 876 & 1.010 & 1.020 \\ <2.0 & <2.0 & <2.0 \\ 27.700 & 27.200 & 27.200 \\ <2.0 & <2.0 & <2.0 \\ 1.680 & 1.760 & 1.760 \\ 170 & 180 & 122 \\ 4.940 & 5.650 & 5.570 \\ 1.5 \mathrm{E}+00 & <1.0 \mathrm{E}+00 & <1.0 \mathrm{OE}+00 \\ 119.000 & 125.000 & 117.000 \\ <1.000 & <1.000 & <1.000 \\ <5.0 & <5.0 & <5.0 \\ <50 & <50 & 90 \\ <7.0 \mathrm{E} \cdot 01 & <7.0 \mathrm{O} \cdot 01 & <7.0 \mathrm{E}-01 \\ <8.0 & <8.0 & <8.0 \\ <2.0 & <2.0 & <2.0\end{array}$

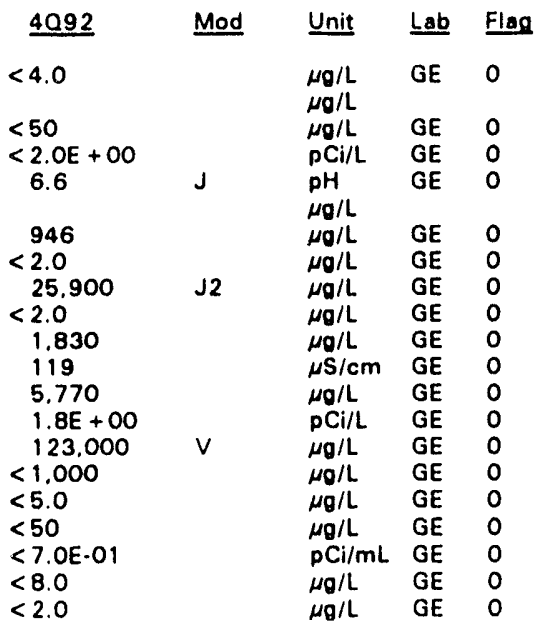

\section{WELL HSB 83B}

\begin{tabular}{|c|c|c|c|c|c|c|c|c|c|}
\hline \multicolumn{2}{|l|}{ SRS Coord. } & Screen Zone Elevation & Top of Casing & Casing & Pump & \multicolumn{3}{|c|}{ Formation } & \\
\hline $\begin{array}{l}\text { N71639.6 } \\
\text { E58594.9 }\end{array}$ & $\begin{array}{l}33.279823^{\circ} \mathrm{N} \\
81.651667{ }^{\circ} \mathrm{W}\end{array}$ & $132.1-121.2 \mathrm{ft} \mathrm{msl}$ & $237 \mathrm{ft} \mathrm{msl}$ & 4" PVC & s & \multicolumn{4}{|c|}{ McBean (IIB, ) } \\
\hline \multicolumn{2}{|c|}{ SAMPLE DATE } & $01 / 07 / 92$ & $04 / 27 / 92$ & $07 / 07 / 92$ & $10 / 07 / 92$ & & & & \\
\hline \multicolumn{10}{|c|}{ FIELD DATA } \\
\hline \multicolumn{2}{|r|}{ Analyte } & 1092 & $\underline{2092}$ & 3092 & 4092 & \multicolumn{4}{|l|}{ Unit } \\
\hline \multicolumn{2}{|r|}{$\begin{array}{l}\text { Water elevation } \\
\text { pH } \\
\text { Sp. conductance } \\
\text { Water temperature } \\
\text { Alkalinity as } \mathrm{CaCO}_{3} \\
\text { Volume purged }\end{array}$} & $\begin{array}{l}222.6 \\
7.0 \\
118 \\
17.6 \\
40 \\
4.0\end{array}$ & $\begin{array}{l}223.0 \\
6.1 \\
115 \\
18.6 \\
46 \\
4.0\end{array}$ & $\begin{array}{l}223.0 \\
6.5 \\
118 \\
20.0 \\
47 \\
4.0\end{array}$ & $\begin{array}{l}223.0 \\
6.8 \\
111 \\
19.1 \\
38 \\
2.9\end{array}$ & \multicolumn{3}{|c|}{$\begin{array}{l}\text { ft msl } \\
\mathrm{pH} \\
\mu \mathrm{S} / \mathrm{cm} \\
{ }^{\circ} \mathrm{C} \\
\mathrm{mg} / \mathrm{L} \\
\text { Well vol. }\end{array}$} & \\
\hline \multicolumn{10}{|c|}{ ANALYTICAL DATA } \\
\hline \multirow[t]{2}{*}{$\underline{H} \quad \underline{D}$} & Analyte & 1092 & $\underline{2092}$ & 3092 & 4092 & Mod & Unit & $\underline{\text { Lab }}$ & Flag \\
\hline & $\begin{array}{l}\text { Aluminum } \\
\text { Antimony } \\
\text { Arsenic } \\
\text { Barium } \\
\text { Cadmium } \\
\text { Calcium } \\
\text { Chloride } \\
\text { Chromium } \\
\text { Cobalt } \\
\text { Copper } \\
\text { Cyanide } \\
\text { Fluoride } \\
\text { Gross alpha } \\
\text { Iron } \\
\text { Lead } \\
\text { Magnesium } \\
\text { Manganese }\end{array}$ & $\begin{aligned} &< 20 \\
&< 2.0 \\
&< 2.0 \\
& 34 \\
&< 2.0 \\
& 18.400 \\
& 2.730 \\
& 4.3 \\
&< 4.0 \\
&< 4.0 \\
&< 5.0 \\
& 152 \\
&< 2.0 E+00 \\
&< 4.0 \\
&<3.0 \\
& 544 \\
&< 2.0\end{aligned}$ & $\begin{aligned} & 31 \\
< & 2.0 \\
< & 2.0 \\
& 37 \\
< & 2.0 \\
& 19.100 \\
& 2.510 \\
< & 4.0 \\
< & 4.0 \\
< & 4.0 \\
< & 5.0 \\
& 168 \\
< & 2.0 E+00 \\
< & 4.0 \\
< & 3.0 \\
& 569 \\
< & 2.0\end{aligned}$ & $\begin{aligned} & 34 \\
< & 2.0 \\
< & 2.0 \\
& 37 \\
< & 2.0 \\
& 19.500 \\
& 2.460 \\
< & 4.0 \\
< & 4.0 \\
< & 4.0 \\
< & 5.0 \\
& 140 \\
< & 2.0 E+00 \\
< & 4.0 \\
< & 3.0 \\
& 555 \\
< & 2.0\end{aligned}$ & $\begin{aligned} & 48 \\
< & 2.0 \\
< & 2.0 \\
& 33 \\
< & 2.0 \\
& 19.600 \\
& 2.550 \\
< & 4.0 \\
< & 4.0 \\
< & 4.0 \\
< & 5.0 \\
& 175 \\
< & 2.0 E+00 \\
& 15 \\
< & 3.0 \\
& 572 \\
< & 2.0\end{aligned}$ & J2 & $\begin{array}{l}\mu g / L \\
\mu g / L \\
\mu g / L \\
\mu g / L \\
\mu g / L \\
\mu g / L \\
\mu g / L \\
\mu g / L \\
\mu g / L \\
\mu g / L \\
\mu g / L \\
\mu g / L \\
\rho C i / L \\
\mu g / L \\
\mu g / L \\
\mu g / L \\
\mu g / L\end{array}$ & $\begin{array}{l}\mathrm{GE} \\
\mathrm{GE} \\
\mathrm{GE} \\
\mathrm{GE} \\
\mathrm{GE} \\
\mathrm{GE} \\
\mathrm{GE} \\
\mathrm{GE} \\
\mathrm{GE} \\
\mathrm{GE} \\
\mathrm{GE} \\
\mathrm{GE} \\
\mathrm{GE} \\
\mathrm{GE} \\
\mathrm{GE} \\
\mathrm{GE} \\
\mathrm{GE}\end{array}$ & $\begin{array}{l}1 \\
0 \\
0 \\
0 \\
0 \\
0 \\
0 \\
0 \\
0 \\
0 \\
0 \\
0 \\
0 \\
0 \\
0 \\
0 \\
0\end{array}$ \\
\hline
\end{tabular}

Note: Flagging levels, modifiers, and laboratories are for 4 th quarter 1992 data only. See Appendix $B$ for flagging criteria. - =xceeded holding time for 4th quarter 1992 .

- exceeded final primary drinking water standard for 4th quarter 1992. 
WSRC.TR-93-059

Woll HSB B3B continued

ANALYTICAL DATA

H. D Analyte
Morcury
Nickel
Nitrate as nitrogen
Nitrate-nitrite as nitrogen
Nonvolatile beta
pH
Phenols
Potaseium
Solenium
Silica
Silver
Sodium
Specific conductance
Sulfate
Total alpha-omitting radium
Total dissolved solids
Total organic carbon
Total organic halogens
Total phosphates las P)
Tritium
Vonadium
Zinc

$\begin{array}{lll}1092 & \underline{2092} & \underline{3092} \\ <0.20 & <0.20 & <0.20 \\ <4.0 & <4.0 & <4.0 \\ <50 & & \\ & <50 & 990 \\ 1.4 E+01 & <2.0 E+00 & <2.0 E+00 \\ 6.5 & 7.1 & 6.8 \\ <5.0 & & \\ 702 & 811 & 806 \\ <2.0 & <2.0 & <2.0 \\ 28.700 & 28.800 & 28.500 \\ <2.0 & <2.0 & <2.0 \\ 3.290 & 3.500 & 3.330 \\ 105 & 115 & 108 \\ 1.110 & 1.260 & 1.160 \\ <1.0 E+00 & <1.0 E+00 & <1.0 E+00 \\ 78.000 & 87.000 & 74,000 \\ <1.000 & <1.000 & <1.000 \\ 30 & <5.0 & <5.0 \\ 450 & 460 & 732 \\ 3.3 E+00 & 2.2 E+00 & 3.0 E+00 \\ <8.0 & <8.0 & <8.0 \\ 9.3 & 9.1 & 9.5\end{array}$

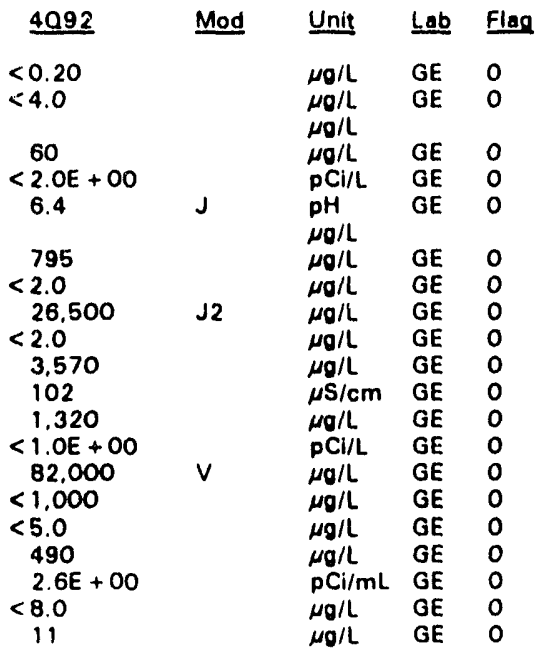

\section{WELL HSB 83C}

\begin{tabular}{|c|c|c|c|c|c|c|c|c|c|}
\hline SRS Coord. & Lat/Longitude & Screen Zone Elovation & Top of Casing & Casing & Pump & \multicolumn{4}{|c|}{ Formation } \\
\hline $\begin{array}{l}N 71636.9 \\
E 58614.8\end{array}$ & $\begin{array}{l}33.279849{ }^{\circ} \mathrm{N} \\
81.651609{ }^{\circ} \mathrm{W}\end{array}$ & $171.2 \cdot 160.2 \mathrm{ft} \mathrm{msl}$ & $237.1 \mathrm{ft} \mathrm{msl}$ & 4" PVC & $\mathbf{s}$ & \multicolumn{4}{|c|}{ Barnwell (IIB, } \\
\hline \multicolumn{2}{|c|}{ SAMPLE DATE } & $01 / 07 / 92$ & $04 / 27 / 92$ & $07 / 07 / 92$ & $10 / 07 / 92$ & & & & \\
\hline \multicolumn{10}{|c|}{ FIELD DATA } \\
\hline \multicolumn{2}{|r|}{ Analyte } & 1092 & $\underline{2092}$ & 3092 & 4092 & \multicolumn{4}{|l|}{ Unit } \\
\hline \multicolumn{2}{|r|}{$\begin{array}{l}\text { Water elevation } \\
\text { pH } \\
\text { Sp. conductance } \\
\text { Water temperature } \\
\text { Alkalinity as } \mathrm{CaCO}_{3} \\
\text { Volume purged }\end{array}$} & $\begin{array}{l}224.4 \\
5.4 \\
24 \\
18.1 \\
1 \\
4.0\end{array}$ & $\begin{array}{l}224.8 \\
4.4 \\
22 \\
18.4 \\
1 \\
4.0\end{array}$ & $\begin{array}{l}224.8 \\
5.1 \\
24 \\
19.6 \\
1 \\
4.0\end{array}$ & $\begin{array}{l}225.0 \\
5.1 \\
22 \\
18.9 \\
1 \\
3.2\end{array}$ & \multicolumn{3}{|c|}{$\begin{array}{l}\text { ft msl } \\
\text { pH } \\
\mu S / \mathrm{cm} \\
{ }^{\circ} \mathrm{C} \\
\mathrm{mg} / \mathrm{L} \\
\text { Well vol. }\end{array}$} & \\
\hline \multicolumn{10}{|c|}{ ANALYTICAL DATA } \\
\hline \multirow[t]{2}{*}{$\underline{H} \quad \underline{D}$} & Analyte & 1092 & $\underline{2092}$ & 3092 & $\underline{4092}$ & Mod & Unit & $\underline{\text { Lab }}$ & Flag \\
\hline & $\begin{array}{l}\text { Aluminum } \\
\text { Antimony } \\
\text { Arvenic } \\
\text { Barium } \\
\text { Cadmium } \\
\text { Calcium } \\
\text { Chloride } \\
\text { Chromium } \\
\text { Cobait } \\
\text { Copper } \\
\text { Cyanide } \\
\text { Fluoride } \\
\text { Gross alpha } \\
\text { lron } \\
\text { Lead } \\
\text { Magnesium }\end{array}$ & $\begin{aligned} &<20 \\
&<2.0 \\
&<2.0 \\
&<3.0 \\
&<2.0 \\
& \quad 1.080 \\
& 2.680 \\
&<4.0 \\
&<4.0 \\
& \quad 9.7 \\
&<5.0 \\
&<100 \\
&<2.0 E+00 \\
& 5.3 \\
&<3.0 \\
& 447\end{aligned}$ & $\begin{aligned}<20 \\
<2.0 \\
<2.0 \\
<3.0 \\
<2.0 \\
1.160 \\
2.510 \\
<4.0 \\
<4.0 \\
7.3 \\
<5.0 \\
<100 \\
<2.0 E+00 \\
4.3 \\
<3.0 \\
463\end{aligned}$ & $\begin{aligned} &< 20 \\
&<2.0 \\
&<2.0 \\
&<3.0 \\
&<2.0 \\
& 1.270 \\
& 2.510 \\
&<4.0 \\
&<4.0 \\
& 11 \\
&<5.0 \\
&<100 \\
&<2.0 \mathrm{E}+\mathrm{OO} \\
& 4.8 \\
&<3.0 \\
& 454\end{aligned}$ & $\begin{aligned} & 67 \\
&< 2.0 \\
&< 2.0 \\
&< 3.0 \\
&<2.0 \\
& 1.230 \\
& 2.480 \\
& 4.1 \\
&< 4.0 \\
& 12 \\
&< 5.0 \\
&< 100 \\
&< 2.0 E+00 \\
& 41 \\
&< 3.0 \\
& 493\end{aligned}$ & J2 & $\begin{array}{l}\mu g / L \\
\mu g / L \\
\mu g / L \\
\mu g / L \\
\mu g / L \\
\mu g / L \\
\mu g / L \\
\mu g / L \\
\mu g / L \\
\mu g / L \\
\mu g / L \\
\mu g / L \\
p C i / L \\
\mu g / L \\
\mu g / L \\
\mu g / L\end{array}$ & $\begin{array}{l}\mathrm{GE} \\
\mathrm{GE} \\
\mathrm{GE} \\
\mathrm{GE} \\
\mathrm{GE} \\
\mathrm{GE} \\
\mathrm{GE} \\
\mathrm{GE} \\
\mathrm{GE} \\
\mathrm{GE} \\
\mathrm{GE} \\
\mathrm{GE} \\
\mathrm{GE} \\
\mathrm{GE} \\
\mathrm{GE} \\
\mathrm{GE}\end{array}$ & $\begin{array}{l}2 \\
0 \\
0 \\
0 \\
0 \\
0 \\
0 \\
0 \\
0 \\
0 \\
0 \\
0 \\
0 \\
0 \\
0 \\
0\end{array}$ \\
\hline
\end{tabular}

Note: Flagging levels, modifiers, and laboratories are for 4th quarter 1992 data only. See Appendix B for flagging criteria. - =xceeded holding time for 4th quarter 1992.

a = exceeded final primary drinking water standard for 4th quarter 1992. 
WSRC.TR.93.059

Woll HSB 83C continued

ANALYTICAL DATA

$\underline{H}$

Analyte
Manganese
Mercury
Nickel
Nitrate as nitrogen
Nitrate-nitrite as nitrogen
Nonvolatile beta
pH
Phenols
Potassium
Solenium
Silica
Silver
Sodium
Specific conductance
Sulfate
Total alpha-omitting radium
Total dissolved solids
Total organic carbon
Total organic halogens
Total phosphates las PI
Tritium
Vanadium
Zinc

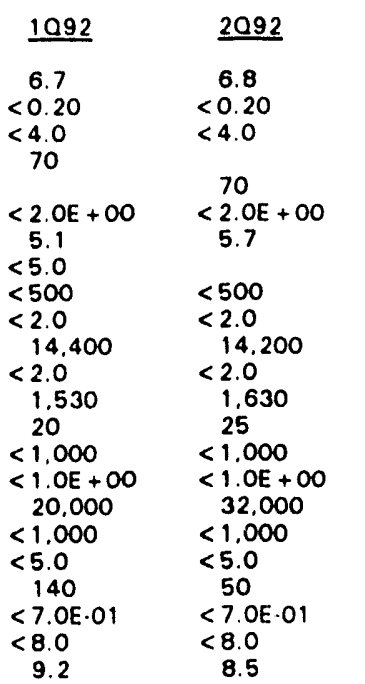

$\begin{array}{rl} & 3092 \\ 7 & 7.0 \\ < & 0.20 \\ < & 40 \\ & 80 \\ < & 2.0 E+O 0 \\ & 5.4 \\ < & 500 \\ < & 2.0 \\ & 14.300 \\ < & 2.0 \\ & 1.600 \\ & 20 \\ < & 1.000 \\ < & 1.0 E+00 \\ & 23.000 \\ < & 1.000 \\ <5.0 \\ & 101 \\ & 1.0 \mathrm{E}+00 \\ < & 8.0 \\ 11\end{array}$

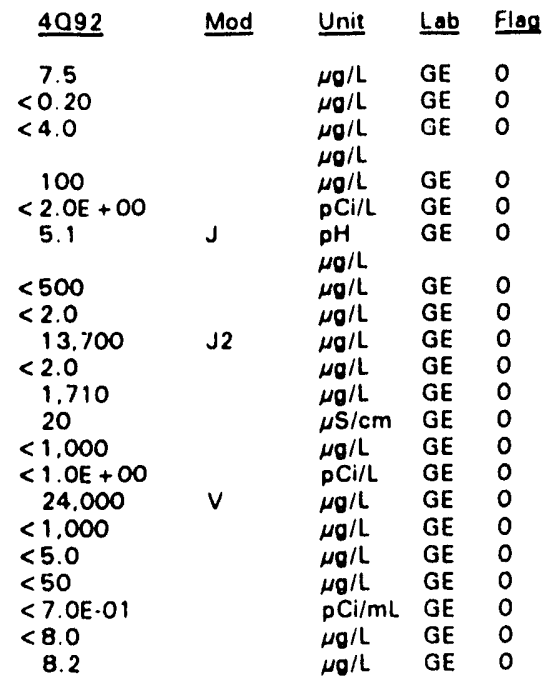

\section{WELL HSB 83D}

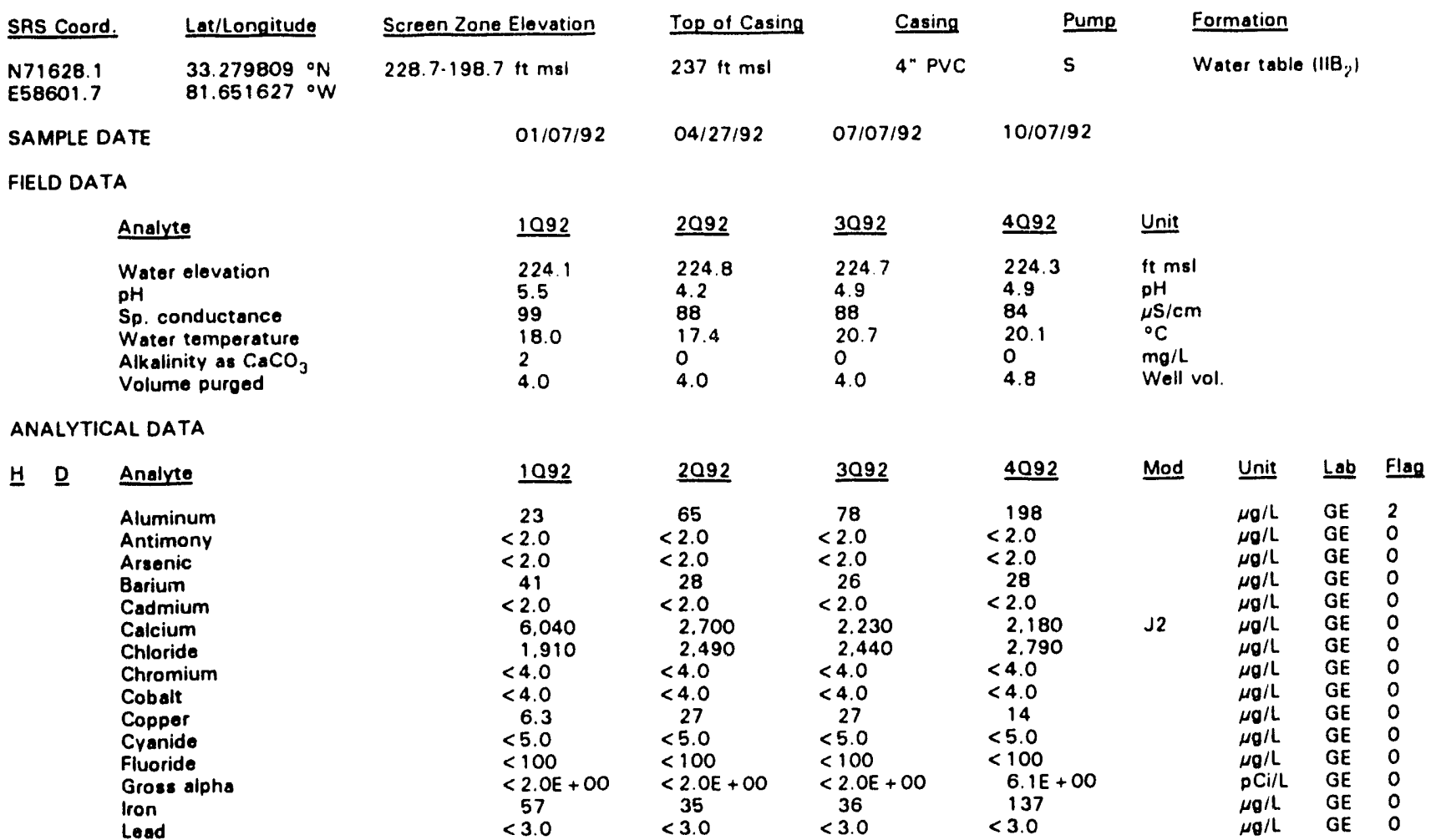

Note: Flagging levels, modifiers, and laboratories are for 4 th quarter 1992 data only. See Appendix B for flagging criteria.

- = exceeded holding time for 4th quarter 1992.

- =xceeded final primary drinking water standard for 4th quarter 1992 .

H-Area Seepage Basins 


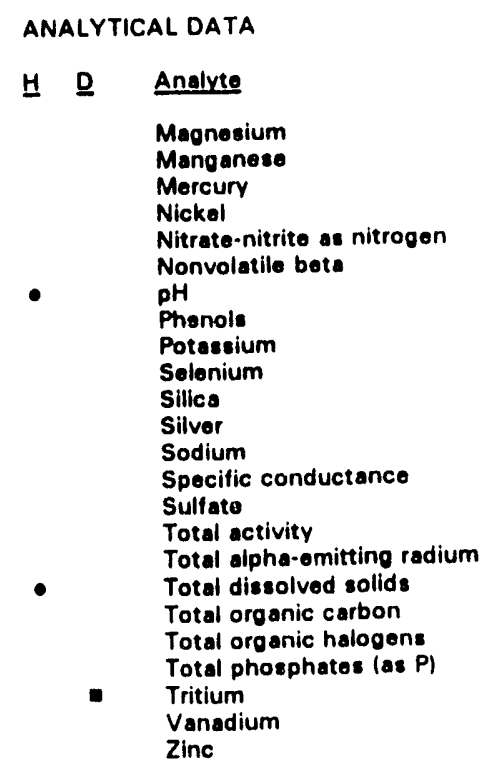

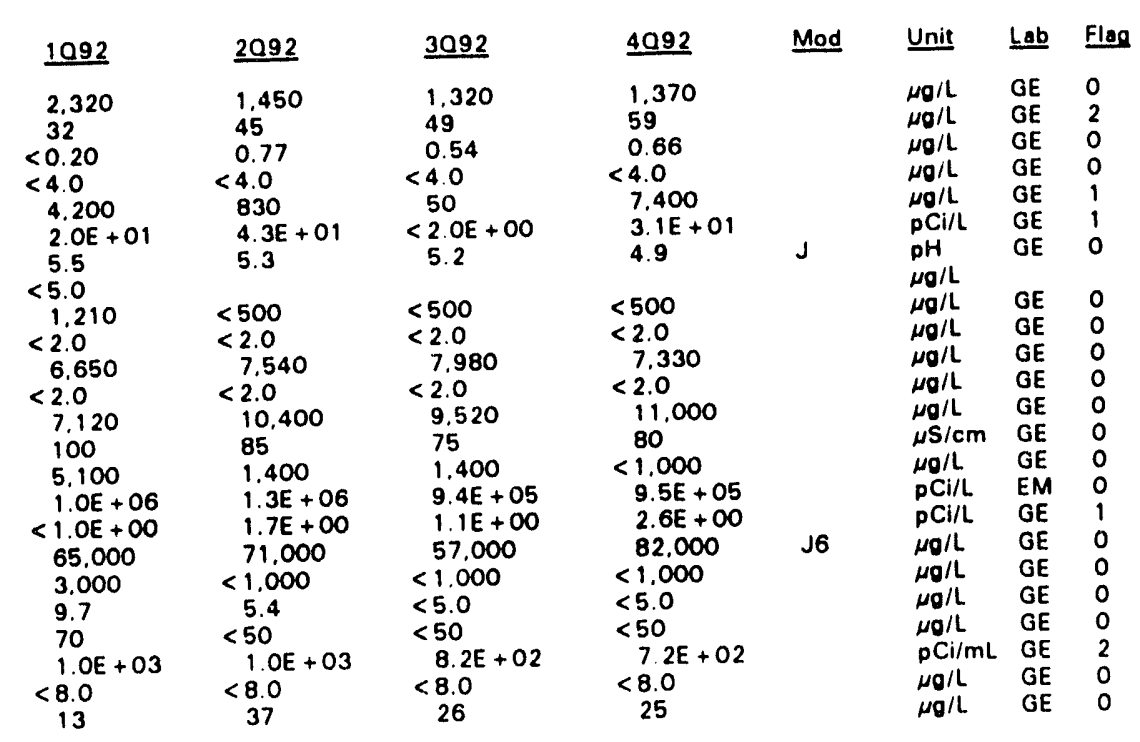

\section{WELL HSB 84A}

\begin{tabular}{|c|c|}
\hline SAS Coord. & Lat/Longitude \\
\hline $\begin{array}{l}\text { N71586.2 } \\
\text { E56359.1 }\end{array}$ & $\begin{array}{l}33.2760577^{\circ} \mathrm{N} \\
81.657450^{\circ} \mathrm{W}\end{array}$ \\
\hline
\end{tabular}
5.9-64.7 ft ms!

SAMPLE DATE

$01 / 09 / 92$

Top of Casing

$228.7 \mathrm{ft} \mathrm{msl}$

$04 / 21 / 92$

2092
172.3
6.3
114
19.9
34
4.0

ANALYTICAL DATA

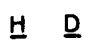

$$
\begin{aligned}
& \text { Analyte } \\
& \text { Aluminum } \\
& \text { Antimony } \\
& \text { Antimony-125 } \\
& \text { Arsenic } \\
& \text { Barium } \\
& \text { Cadmium } \\
& \text { Calcium } \\
& \text { Cerium-144 } \\
& \text { Cesium-134 } \\
& \text { Cosium-137 } \\
& \text { Chloride } \\
& \text { Chromium } \\
& \text { Chromium-51 }
\end{aligned}
$$

Cobalt

$\begin{array}{lll}\text { Casing } & \text { Pump } & \text { Formation } \\ \text { 4" PVC }^{n} & V & \text { L. Congaree (IIA) }\end{array}$

$07 / 08 / 92 \quad 10 / 27 / 92$

$\begin{array}{lll}3092 & \underline{4092} & \text { Unit } \\ 172.0 & 172.1 & \mathrm{ft} \mathrm{msl} \\ 6.4 & 6.1 & \mathrm{pH} \\ 114 & 113 & \mu \mathrm{S} / \mathrm{cm} \\ 20.0 & 20.3 & { }^{\circ} \mathrm{C} \\ 31 & 34 & \mathrm{mg} / \mathrm{L} \\ 4.0 & 2.1 & \text { Well vol. }\end{array}$

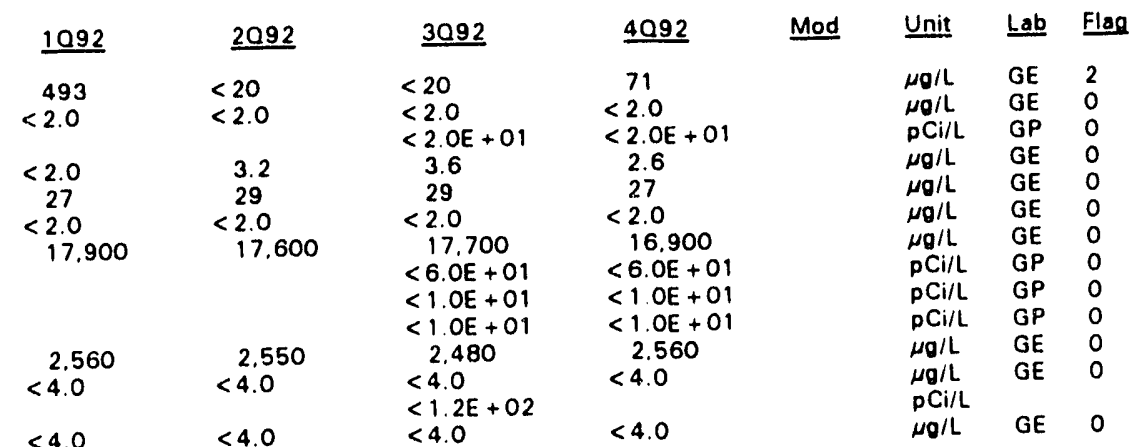

Note: Flagging levels, modifiers, and laboratories are for 4 th quarter 1992 data only. See Appendix B for flagging criteria.

- =xceeded holding time for 4th quarter 1992.

- =xceeded final primary drinking water standard for 4th quarter 1992. 
Well HSB 84A continued

\section{ANALYTICAL DATA}

D. Analyte
Cobalt-57
Cobalt-60
Coppor
Cyanida
Europium-152
Europium-154
Europium-155
Fluoride
Gross alpha
lodine-131
Iron
Lead
Magnesium
Manganese
Manganese-54
Mercury
Nickol
Niobium-95
Nitrate as nitrogen
Nitrato-nitrite as nitrogen
Nonvolatile beta
pH
Phenols
Potassium
Potassium-40
Promethium-144
Promethium-146
Ruthenium-106
Ruthenium-106
Selenium
Silica
Silver
Sodium
Sodium-22
Specific conductance
Sulfate
Total alpha-emitting radium
Total dissolved solids
Total organic carben
Total organic halogens
Total phosphates las P)
Tritium
Vanadium
Yttrium-88
Zinc
Zinc-65
Zirconium-95

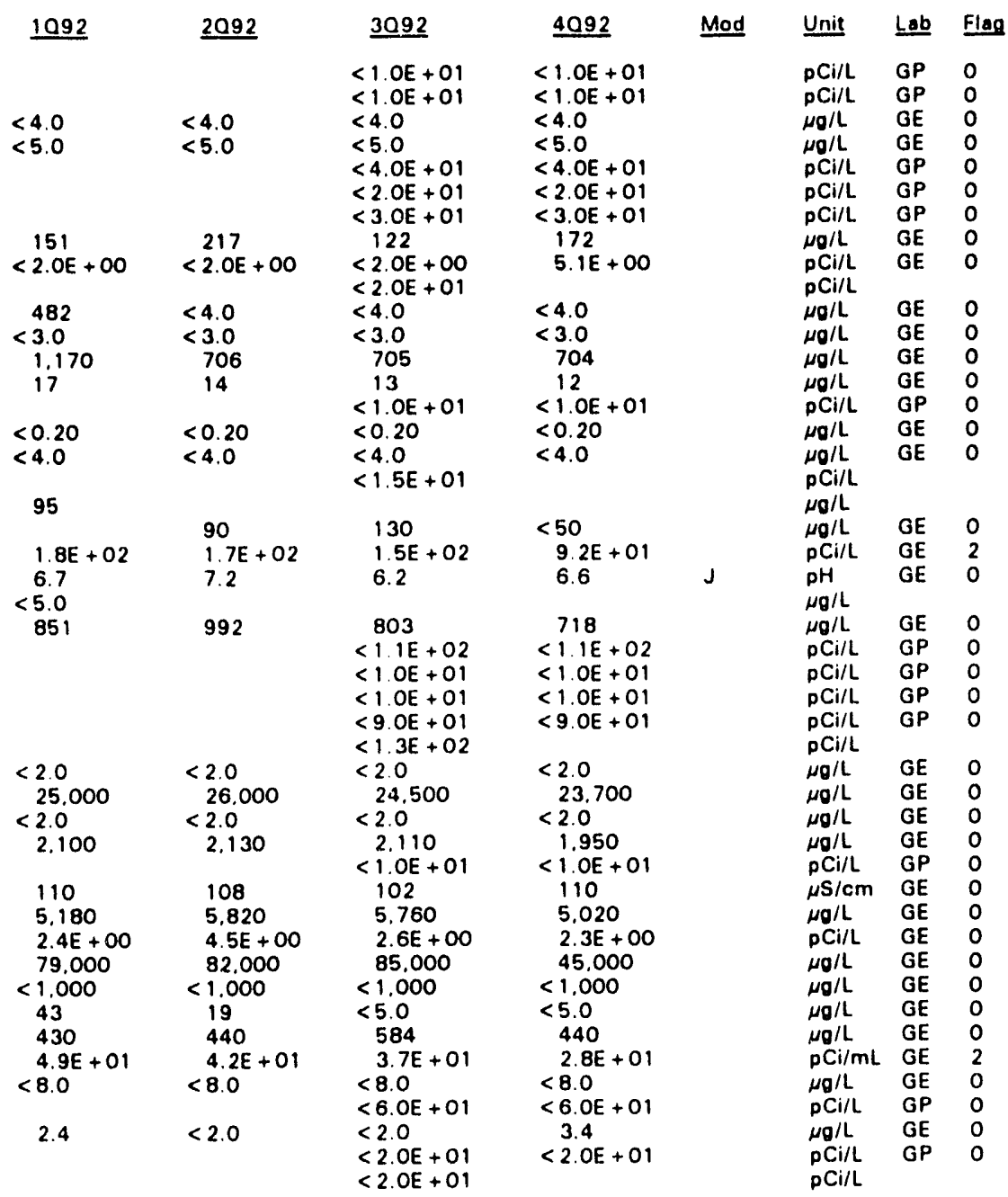

Note: Flagging levels, modifiers, and laboratories are for 4 th quarter 1992 data only. See Appendix B for flagging criteria. - = exceeded holding time for 4th quarter 1992.

- =xceeded final primary drinking water standard for 4th quarter 1992. 
WELL HSB 84B

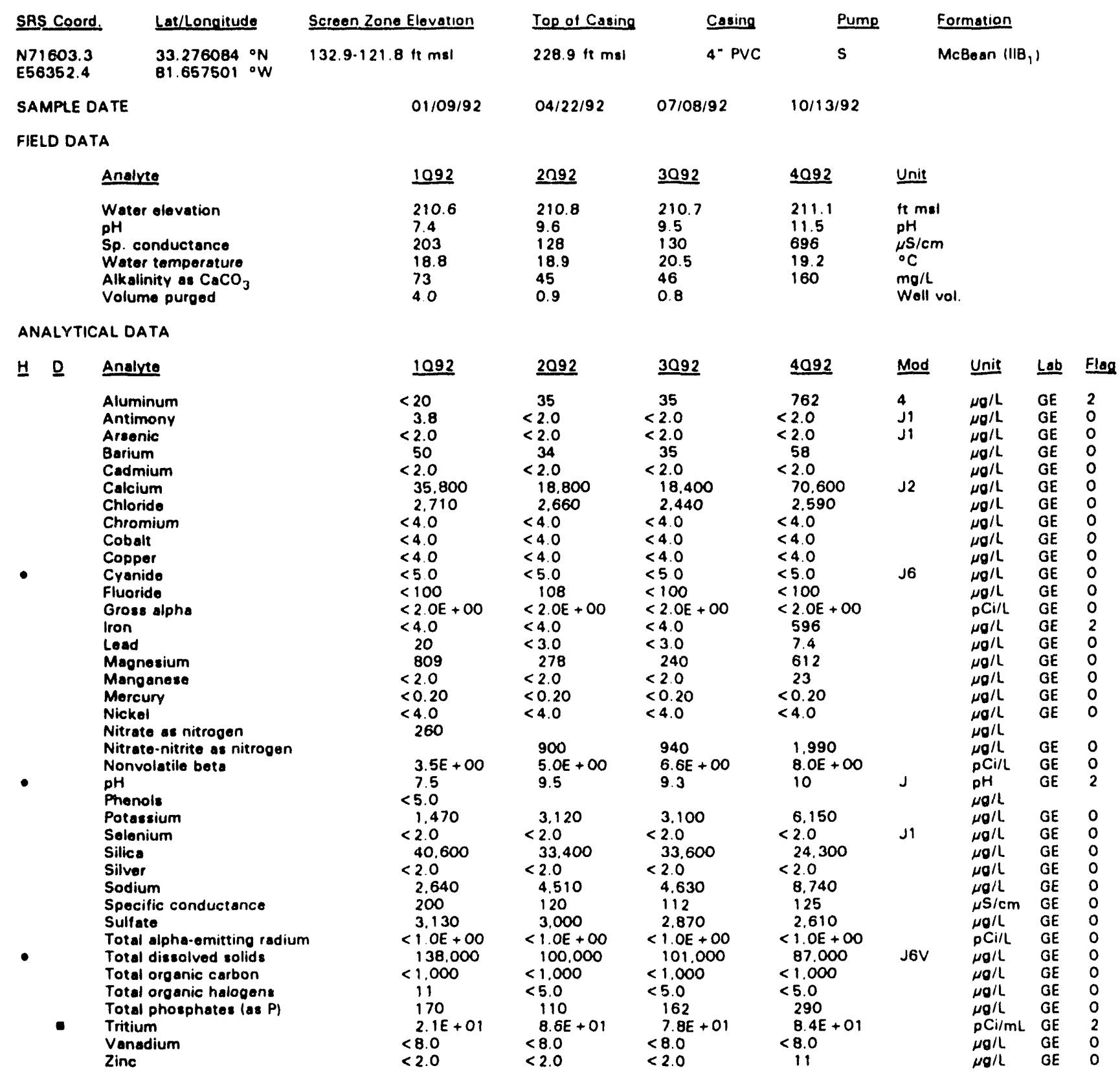

Note: Flagging levels, modifiers, and laboratories are for 4 th quarter 1992 data only. See Appendix B for flagging criteria.

- = exceeded holding time for 4th quarter 1992.

- exceeded final primary drinking water standard for 4th quarter 1992. 
WSRC-TR-93-059

WELL HSB 84C

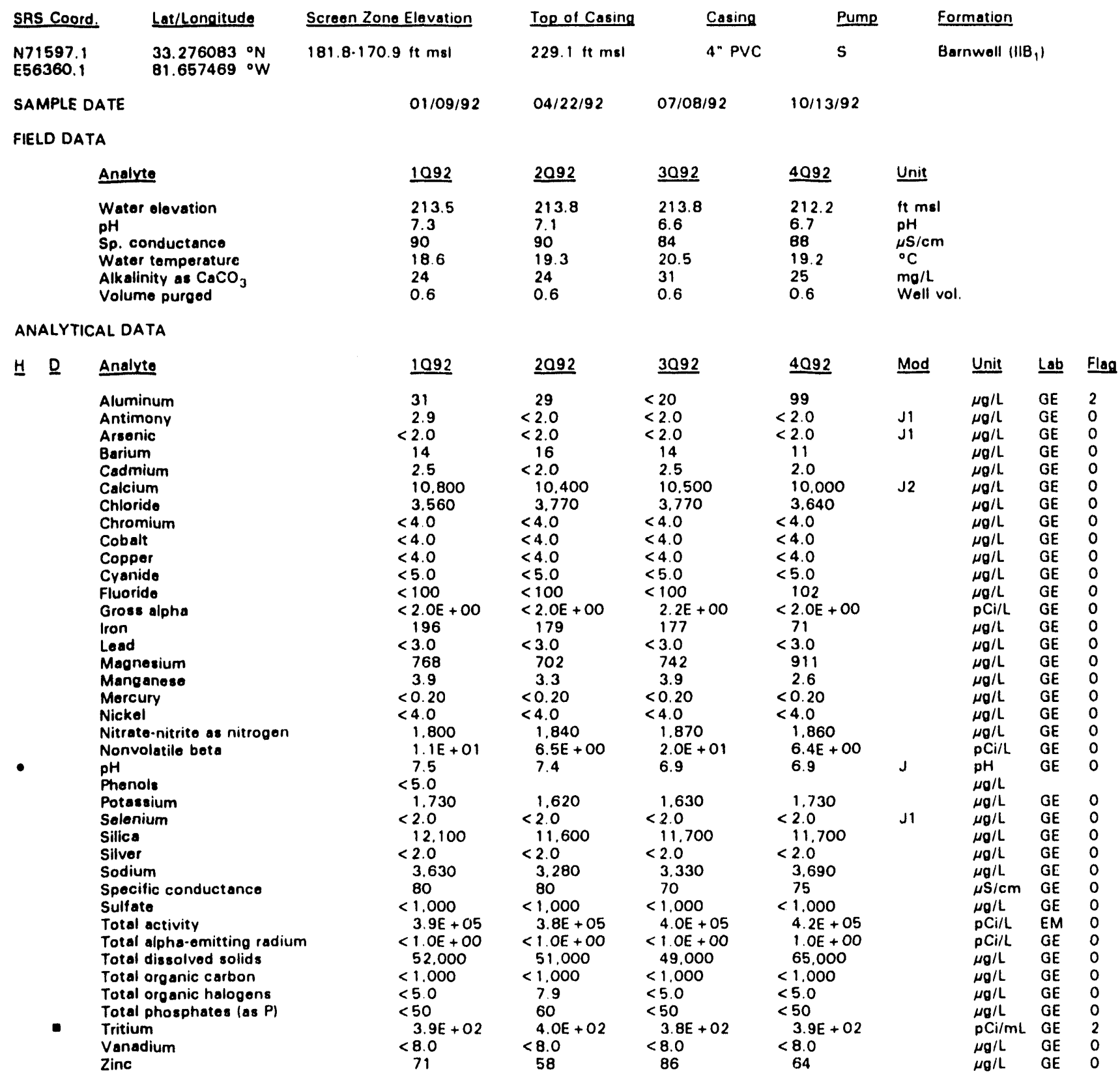

Note: Flagging levels, modifiers, and laboratories are for 4 th quarter 1992 data onlv. See Appendix B for flagging criteria. - = exceeded holding time for 4 th quarter 1992.

- exceeded final primary drinking water standard for 4th quarter 1992. 
WSRC.TR-93.059

WELL HSB 84D

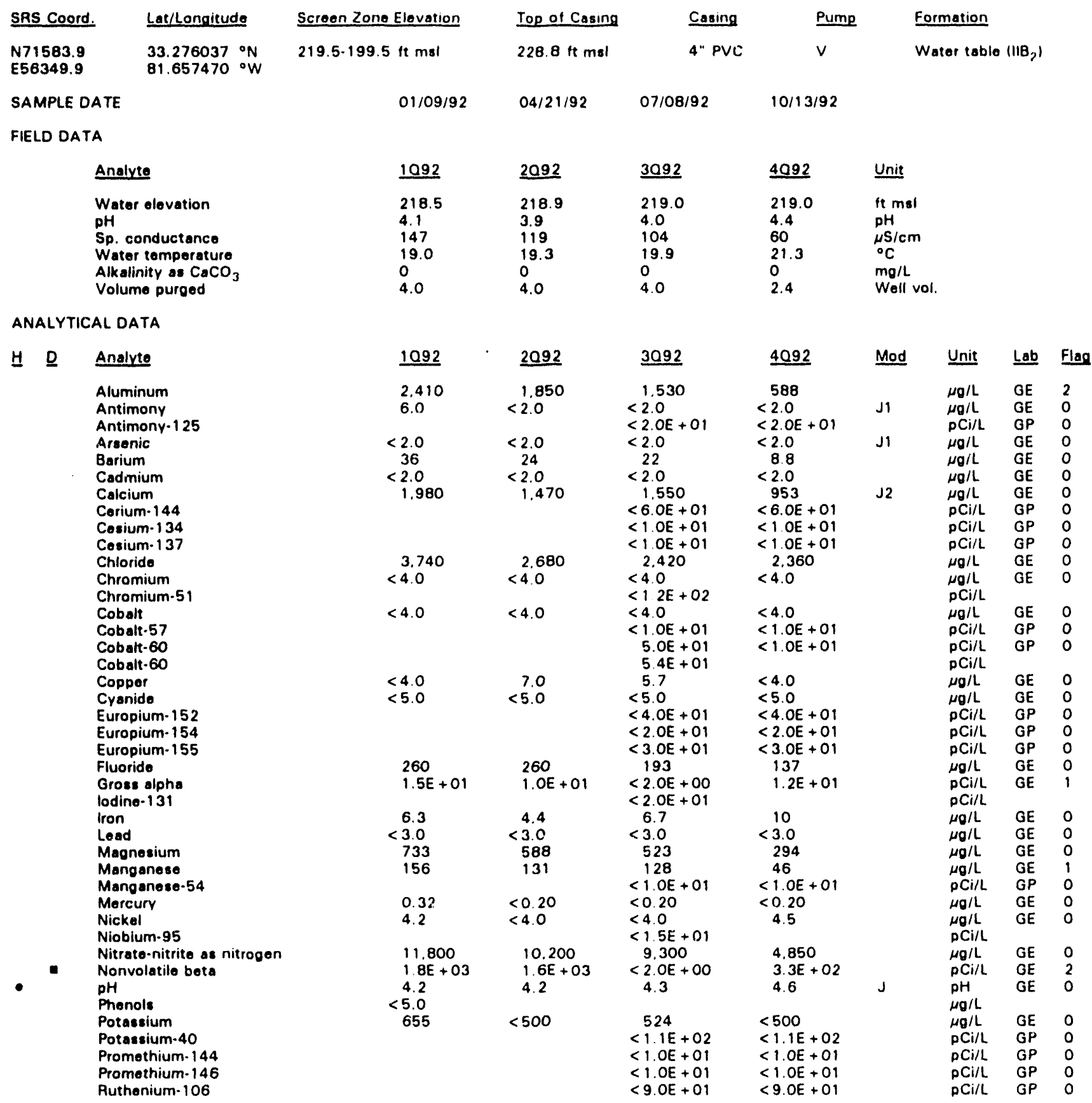

Note: Flagging levels, modifiers, and laboratories are for 4th quarter 1992 data only. See Appendix B for flagging criteria.

- = exceeded holding time for 4 th quarter 1992.

- exceeded final primary drinking water standard for 4th quarter 1992. 


H. Analyte
Ruthenium-106
Solenium
Silica
Silver
Sodium
Sodium-22
Specific conductance
Sulfate
Total activity
Toral alpha-omitting radium
Total dissolved solids
Total organic carbon
Total organic halogens
Total phosphates (as P)
Tritium
Vanadium
Yttrium-88
Zinc
Zinc-65
Zirconium-95

\section{WELL HSB 85A}

\begin{tabular}{|c|c|c|c|c|c|c|c|c|c|}
\hline SRS Coord. & Lat/Longitude & Screen Zone Elevation & Top of Casing & Casing & Pump & \multicolumn{3}{|c|}{ Formation } & \\
\hline $\begin{array}{l}N 73791.9 \\
\text { E58943.4 }\end{array}$ & $\begin{array}{l}33.285152{ }^{\circ} \mathrm{N} \\
81.6549300^{\circ} \mathrm{W}\end{array}$ & $71.1-61.1 \mathrm{ft} \mathrm{msl}$ & $294.4 \mathrm{ft} \mathrm{msl}$ & 4" PVC & s & \multicolumn{4}{|c|}{ U. Congaree (IIA) } \\
\hline \multicolumn{2}{|c|}{ SAMPLE DATE } & $01 / 15 / 92$ & $04 / 16 / 92$ & $07 / 08 / 92$ & $10 / 09 / 92$ & & & & \\
\hline \multicolumn{10}{|c|}{ FIELD DATA } \\
\hline \multicolumn{2}{|r|}{ Analyte } & 1092 & $\underline{2092}$ & 3092 & 4092 & \multicolumn{3}{|l|}{ Unit } & \\
\hline \multicolumn{2}{|r|}{$\begin{array}{l}\text { Water elevation } \\
\text { pH } \\
\text { Sp. conductance } \\
\text { Water temperature } \\
\text { Alkalinity as } \mathrm{CaCO}_{3} \\
\text { Volume purged }\end{array}$} & $\begin{array}{l}169.4 \\
7.0 \\
196 \\
18.5 \\
76 \\
4.0\end{array}$ & $\begin{array}{l}169.3 \\
6.9 \\
189 \\
20.5 \\
69 \\
4.0\end{array}$ & $\begin{array}{l}169.1 \\
6.6 \\
185 \\
20.3 \\
63 \\
4.0\end{array}$ & $\begin{array}{l}169.1 \\
6.8 \\
185 \\
20.1 \\
68 \\
2.5\end{array}$ & \multicolumn{3}{|c|}{$\begin{array}{l}\text { ft msi } \\
\mathrm{pH} \\
\mu \mathrm{S} / \mathrm{cm} \\
{ }^{\circ} \mathrm{C} \\
\mathrm{mg} / \mathrm{L} \\
\text { Well vol. }\end{array}$} & \\
\hline \multicolumn{10}{|c|}{ ANALYTICAL DATA } \\
\hline \multirow[t]{2}{*}{$\underline{H} \quad \underline{D}$} & Analyte & 1092 & $\underline{2092}$ & $\underline{3092}$ & 4092 & Mod & Unit & $\underline{\text { Lab }}$ & Flag \\
\hline & $\begin{array}{l}\text { Acenaphthene } \\
\text { Acenaphthylene } \\
\text { Acetophenone } \\
\text { Aldrin } \\
\text { Aluminum } \\
\text { Anthracene } \\
\text { Antimony } \\
\text { Arsenic } \\
\text { Barium } \\
\text { Benzene } \\
\text { alpha-Benzene hexachloride } \\
\text { beta-Benzene hexachloride } \\
\text { delta-Benzene hexachloride } \\
\text { Benzidine } \\
\text { Benzolalanthracene } \\
\text { Benzolblfluoranthene } \\
\text { Benzolklfluoranthene } \\
\text { Benzolg,h,iperylene }\end{array}$ & $\begin{array}{l}<10 \\
<10 \\
<10 \\
<10 \\
<20 \\
<10 \\
<2.0 \\
<2.0 \\
<11 \\
<1.0 \\
<10 \\
<10 \\
<10 \\
<10 \\
<10 \\
<10 \\
<10 \\
<10 \\
<10\end{array}$ & $\begin{array}{l}<10 \\
<10 \\
<10 \\
<10 \\
<20 \\
<10 \\
<2.0 \\
<2.0 \\
\quad 31 \\
<1.0 \\
<10 \\
<10 \\
<10 \\
<10 \\
<10 \\
<10 \\
<10 \\
<10\end{array}$ & $\begin{array}{l}<10 \\
<20 \\
<2.0 \\
<2.0 \\
31 \\
<\therefore 0\end{array}$ & $\begin{array}{r}<10 \\
\\
43 \\
<2.0 \\
<2.0 \\
23 \\
<1.0\end{array}$ & J1 & $\begin{array}{l}\mu g / L \\
\mu g / L \\
\mu g / L \\
\mu g / L \\
\mu g / L \\
\mu g / L \\
\mu g / L \\
\mu g / L \\
\mu g / L \\
\mu g / L \\
\mu g / L \\
\mu g / L \\
\mu g / L \\
\mu g / L \\
\mu g / L \\
\mu g / L \\
\mu g / L \\
\mu g / L\end{array}$ & $\begin{array}{l}\mathrm{GE} \\
\mathrm{GE} \\
\mathrm{GE} \\
\mathrm{GE} \\
\mathrm{GE} \\
\mathrm{GE}\end{array}$ & $\begin{array}{l}0 \\
1 \\
0 \\
0 \\
0 \\
0\end{array}$ \\
\hline
\end{tabular}

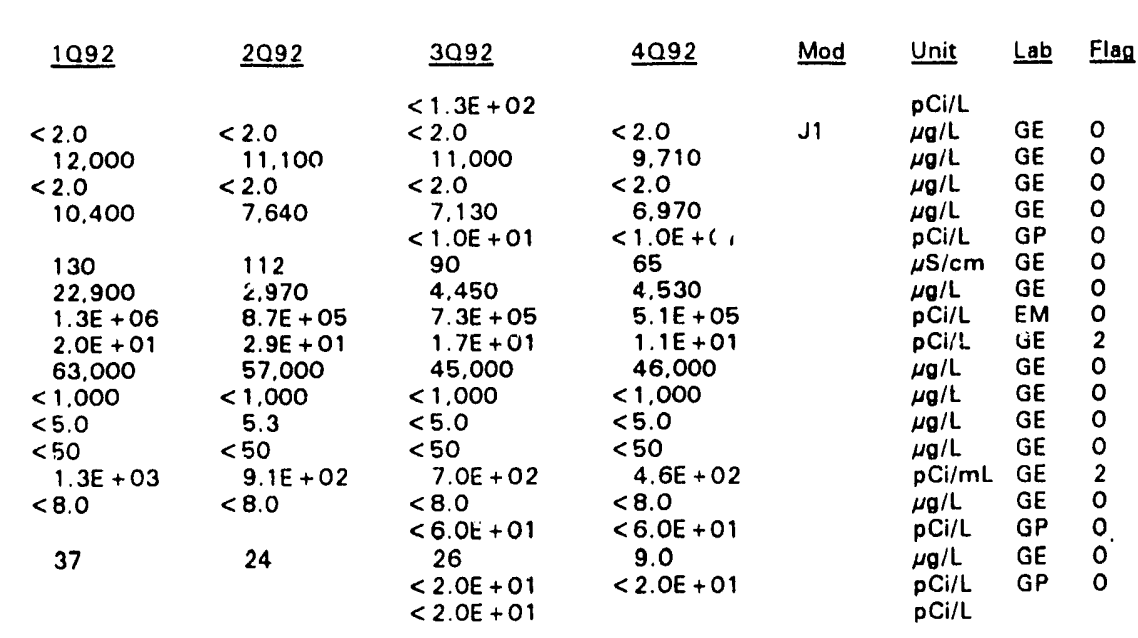

Analyt 


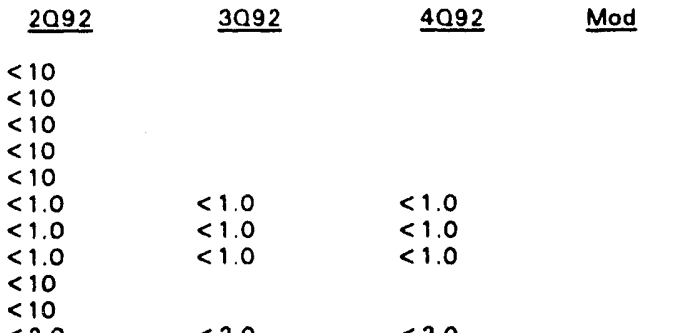

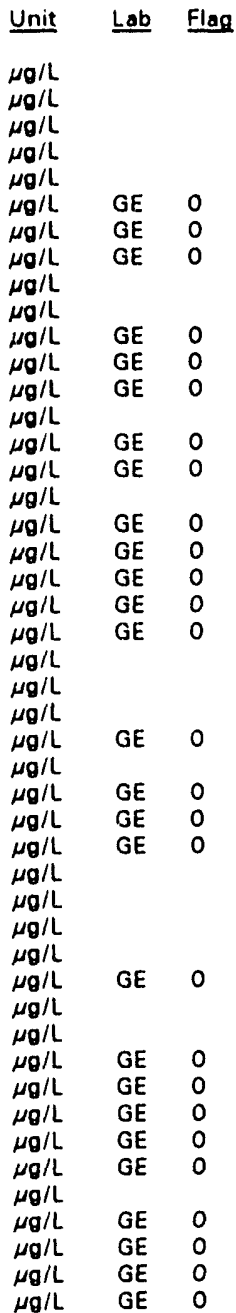

Benzolalpyrene

$<10$

$<10$

Bis(2-chloroethoxy) methan

Bis (2-chloroothyl) other

Bis (2-chloroisopropyl) other

Bis(2-othylhexyl) phthalate

Bromodichloromethane

Bromoform

4-Bromophenyl phenyl ether

Butylbenzyl phthalate

Cadmium

Calcium

Carbon tetrachloride

Chlord ane

- Chloride

Chlorobenzene

para-Chloro-meta-cresol

Chloroethane

Chloroethene (Vinyl chloride)

2-Chloroethyl vinyl ether

Chloroform

Chloromethane (Methyl chloride)

2. Chloronaphthalene

2-Chlorophenol

4-Chiorophenyl phenyl ethe

Chromium

Chrysene

Cobalt

Copper

Cyanido

p.p'-DDD

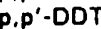

Dibenzla,hlanthracene

Dibromochloromethane

Di-n-butyl phthalate

3, $3^{\prime}$-Dichlorobenzidine

1.1-Dichloroethane

1.2-Dichloroethane

1,1-Dichloroethylene

trans-1,2-Dichloroethylene

Dichloromethane (Methylene chloride)

2,4-Dichloropheniul

2.4-Dichlorophenoxyacetic acid

1,2-Dichloropropane

cis-1.3-Dichloropropene

trans-1,3-Dichloropropene

Dieldrin

Diethyl phthalate

2,4-Dimethyl pheno

Oimethyl phthalate

2,4-Dinitrophenol

2,4-Dinitrotoluene

2,6-Dinitrotoluene

Di-n-octyl phthslate

1,2-Diphenylhydrazine

Endosulfan I

Endosulfan II

Endosulfan sulfate

Endrin

Endrin aldehyde

Ethylbenzene
$<1$

$<10$

$<10$

$<1.0$

$<1.0$

$<10$

$<10$

$<.0$
32.800

$<1.0$

$<10$

2.480

$<1.0$

$<10$

$<1.0$

$<1.0$

$<1.0$

$<0$

$<1.0$

$<10$

$<10$

$<10$

$<4.0$

$<10$

$<4.0$

$<4.0$

$<5.0$

$<10$

$<10$

$<10$

$<10$

$<1.0$

$<10$

$<10$

$<1.0$

$<1.0$

$<1.0$

$<1.0$

2.5

$<10$
$<0.30$

$<1.0$

$<1.0$

$<1.0$

$<10$

$<10$

$<10$

$<10$

$<45$

$<10$

$<10$

$<10$

$<10$

$<10$

$<10$

$<10$

$<10$

$<10$

$<1.0$
$<2.0$

33.500

$<1.0$

$<10$

2,490
$<1.0$

$<10$

$<1.0$

$<1.0$

$<1.0$

$<1.0$

$<1.0$

$<10$

$<10$

$<10$

$<4.0$

$<10$

$<4.0$

$<4.0$

$<5.0$

$<10$

$<10$

$<10$

$<10$

$<1.0$

$<10$

$<10$

$<1.0$

$<1.0$

$<1.0$

$<1.0$

$<0.30$

$<1.0$

$<1.0$

$<1.0$

$<10$

$<10$

$<10$

$<10$

$<45$

$<10$

$<10$

$<10$

$<10$

$<10$
$<10$
$<10$

$<10$

$<0.0060$

$<2.0$
$<3.200$
$<1.0$

$<2.0$

$<1.0$

2.350

2.410

$J 2$

$<1.0$

$<1.0$

$<1.0$

$<1.0$

$<1.0$

$<1.0$

$<4.0$

$<40$

$<4.0$

$<5.0$

$<1.0$

$<1.0$

$<1.0$

$<1.0$

$<1.0$

$<1.0$

$<0.30$

$<1.0$

$<1.0$

$<10$

$<1.0$

$<0.0060$

$<0.0060$

$<1.0$

$<1.0$

$<1.0$

$<1.0$

$<1.0$

$<4.0$

$<4.0$

$<4.0$

$<5.0$

(1.0

$<1.0$

$<1.0$

$<1.0$

$<1.0$

$<0.30$

$<1.0$

$\mu \mathrm{g} / \mathrm{L}$

$\mu g / L$

$\mu g / L$

$\mu \mathrm{g} / \mathrm{L}$

$\mu \mathrm{g} / \mathrm{L}$

ro

$\mu \mathrm{g} / \mathrm{L}$

$\mu g / L$

$\mu g / L$

$\mu \mathrm{g} / \mathrm{L}$

$\mu g /$

$\mu g / L$

$\mu \mathrm{g} / \mathrm{L}$

GE $O$

GE 0

Note: Flagging levels, modifiers, and laboratories are for 4 th quarter 1992 data only. See Appendix $B$ for flagging criteria.

- = exceeded holding time for 4th quarter 1992.

- =xceeded final primary drinking water standard for 4th quarter 1992.

H-Area Seepaga Dasins 


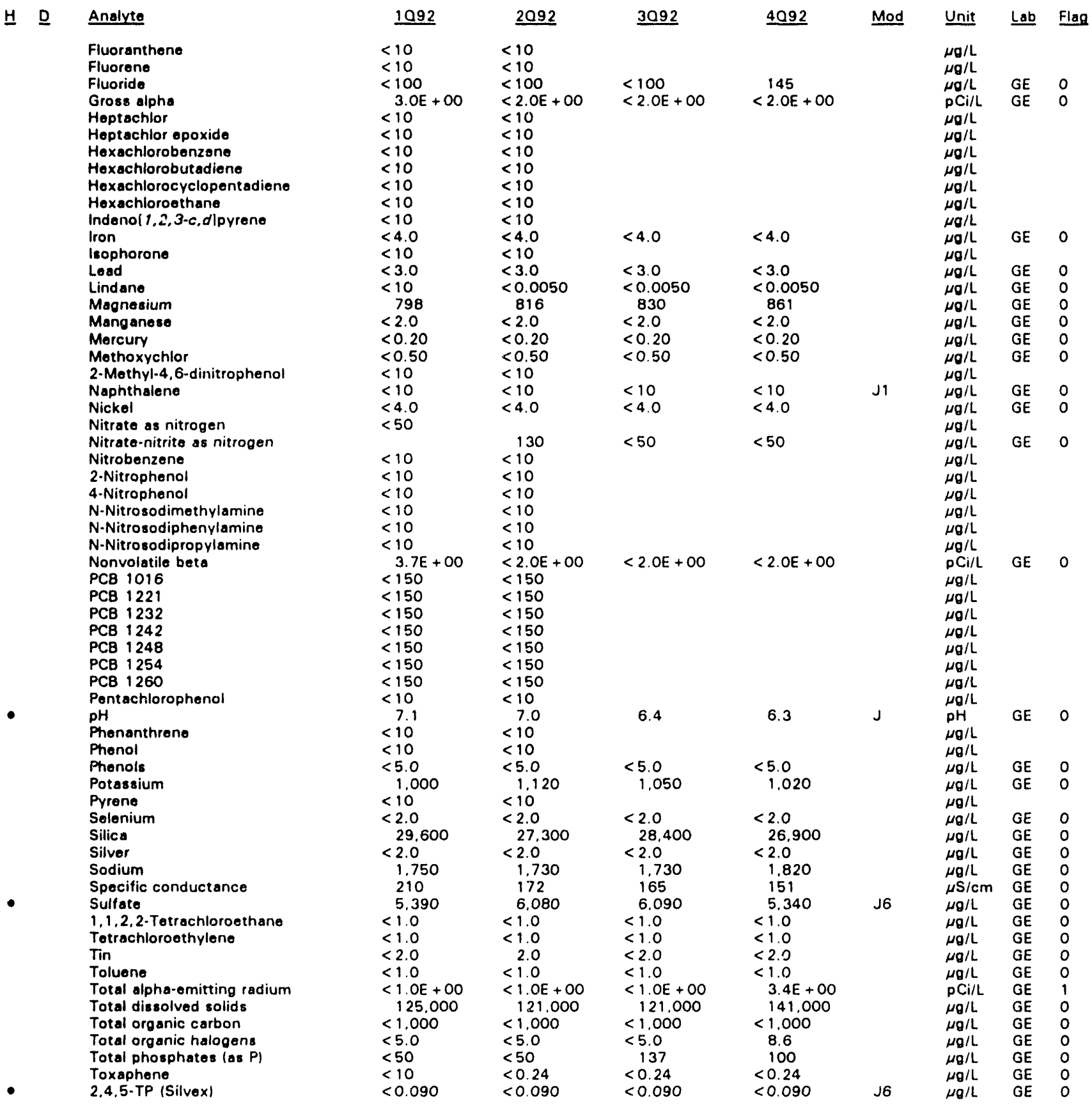

Note: Flagging levels, modifiers, and laboratories are for 4 th quarter 1992 data only. See Appendix B for flagging criteria. - = exceeded holding time for 4th quarter 1992 .

- =xceeded final primary drinking water standard for 4th quarter 1992. 
WSRC-TR-93-059

Well HSB 85A continued

ANALYTICAL DATA

\section{H D Analyte}

1,2.4-Trichlorobenzene

$1,1,1$-Trichloroethane

1,1,2-Trichloroethane

Trichloroethylene

Trichlorofluoromethane

2.4.6-Trichlorophenol

- Tritium

Turbidity

Xylones

Zinc

$\begin{aligned} & 1092 \\ &< 10 \\ &< 1.0 \\ &< 1.0 \\ &< 1.0 \\ &< 1.0 \\ &< 10 \\ &<7.0 E-01 \\ &<0.10 \\ &<8.0 \\ &<2.0 \\ &<2.0\end{aligned}$

$\begin{aligned} & 2092 \\ &< 10 \\ &< 1.0 \\ &< 1.0 \\ &< 1.0 \\ &<1.0 \\ &<10 \\ &<7.0 \mathrm{E}-01 \\ &<0.10 \\ &<8.0 \\ &<2.0 \\ &<2.0\end{aligned}$

3092

$<1.0$

$<1.0$

$<1.0$
6.5

$<7$. OE-01

$<0.10$

$<8.0$

$<2.0$

$<2.0$
4092

$<1.0$

$<1.0$

$<1.0$

$<1.0$

$<7.0 E \cdot 01$

0.44

0.44
$<8.0$

$<2.0$

$<2.0$
Mod Unit Lab Flag

$\mu g / L \quad G E \quad O$

$\mu g / L \quad$ GE $\quad 0$

$\mu g / L$ GE $O$

$\mu \mathrm{g} / \mathrm{L}$

$\mu \mathrm{g} / \mathrm{L}$

$\mathrm{pCi} / \mathrm{mL}$ GE 0

NTU GE 0

$\mu \mathrm{g} / \mathrm{L}$ GE 0

$\mu \mathrm{g} / \mathrm{L} \quad \mathrm{GE} \quad \mathrm{O}$

\section{WELL HSB 85B}

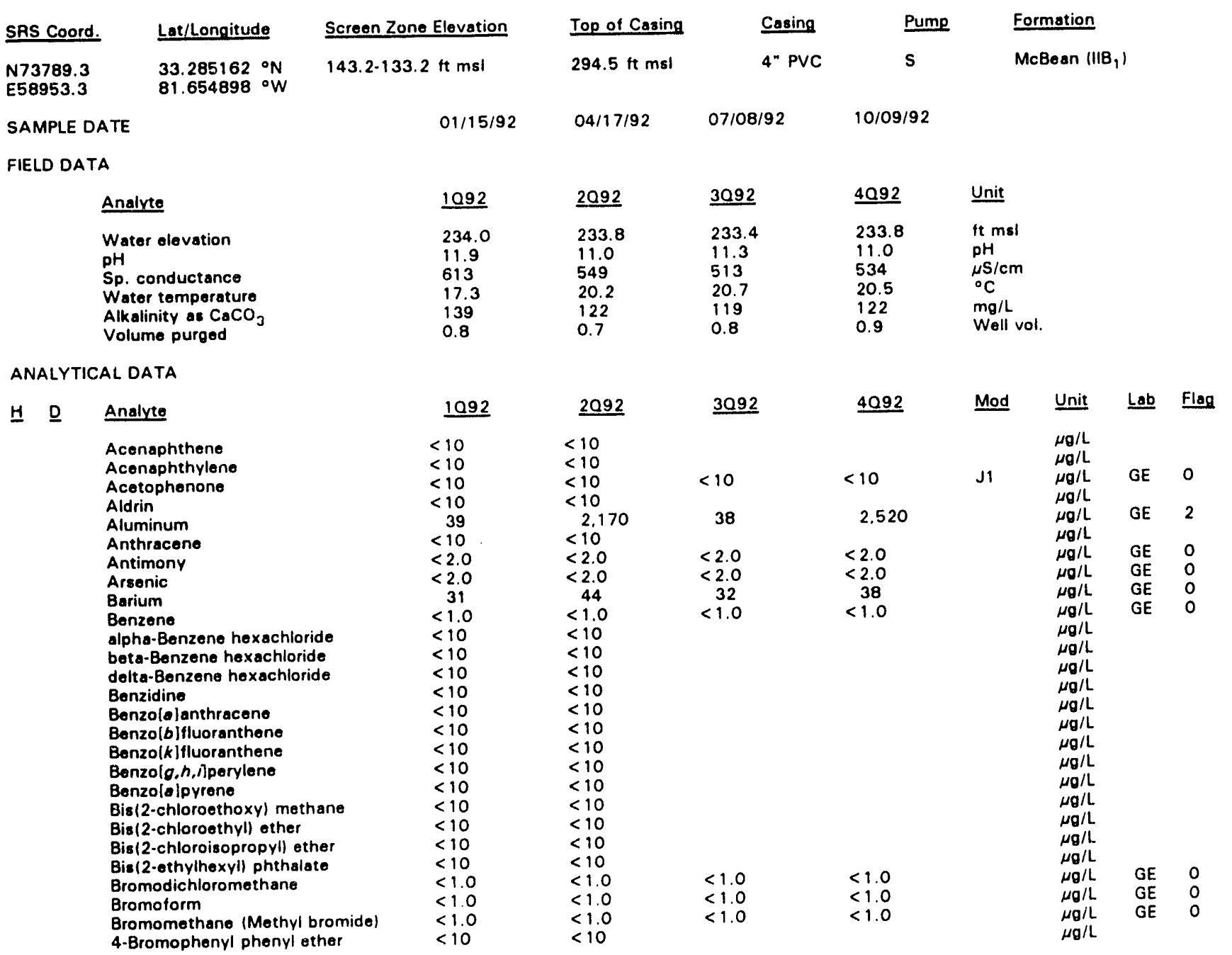

Note: Flagging levels, modifiers, and laboratories are for 4 th quarter 1992 data only. See Appendix $B$ for flagging criteria.

- = exceeded holding time for 4th quarter 1992.

- =xceeded final primary drinking water standard for 4 th quarter 1992. 
WSRC-TR-93-059

Woll HSB 858 continued

\section{ANALYTICAL DATA}

H D Analvte

Butylbenzyl phthaiate

Cadmium

Calcium

Carbon tetrachloride

Chlordane

Chloride

Chlorobenzene

para-Chloro-meta-cresol

Chloroethane

Chloroethene (Vinyl chloride)

2-Chloroethyl vinyl ether

Chloroform

Chloromethane (Methyl chloride)

2-Chloronaphthalene

2-Chlorophenol

4. Chlorophenyl phenyl ether $\quad<10$

Chromium $<4.0$

Chrysene $<10$

Cobalt

Copper

Cyanide

p.p'-DDD

p.p'-DDE

p.p'-DDT

Dibenzla,hlanthracene

Dibromochloromethane

Di-n-butyl phthalate

3. 3'-Dichlorobenzidine

1.1-Dichloroethane

1.2-Dichloroethane

1.1-Dichloroethylene

$<1.0$

Dichloromethane (Methylene chloride) 3.4

2,4-Dichlorophenol

2,4-Dichlorophenoxyacetic acid $<0.30$

1,2-Dichloropropane

cis-1,3-Dichloropropene

trans-1,3-Dichloropropene

Dieldrin

Diethyl phthalate

2.4-Dimethyl phenol

Dimothyl phthalate

2.4-Dinitrophenol

2,4-Dinitrotoluene

2.6-Dinitrotoluene

Di-n-octyl phthalate

1.2-Diphenylhydrazine

Endosulfan I

Endosulfan II

Endosulfan sulfate

Endrin

Endrin aldehyde

Ethylbenzene

Fluoranthene

Fluorene

Fluoride

Gross alpha

Heptachlor

Heptachlor epoxide

Hexachlorobenzene

Hexachlorobutadiene

Hexachlorocyclopent adiene

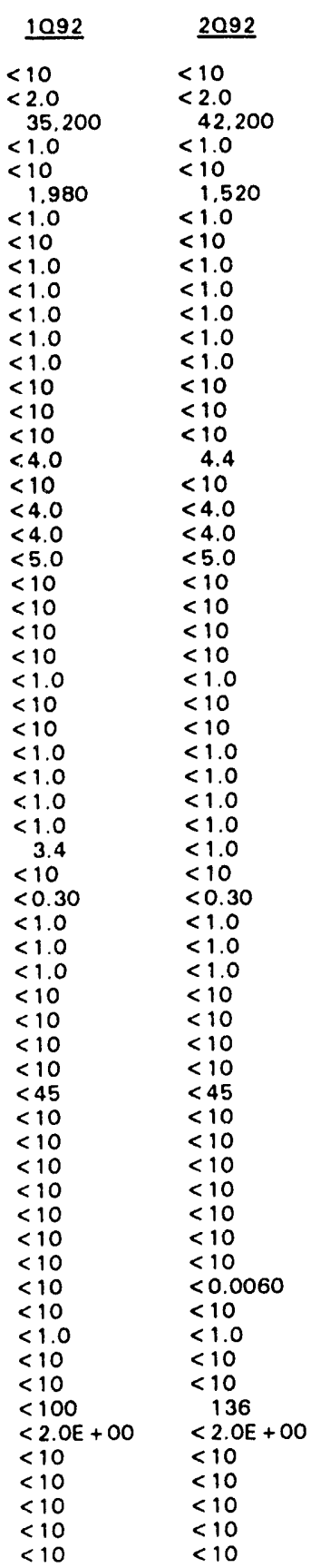

$\underline{3092} \underline{4092}$ Mod Unit Lab Flag

$<2.0$

36.300

$<1.0$

1.960

$<1.0$

$<1.0$

$<1.0$

$<1.0$

$<1.0$

$<1.0$

$<4.0$

$<4.0$

$<4.0$

$<5.0$

(2.0

(n)

$\begin{array}{lll}\mu \mathrm{g} / \mathrm{L} & & \\ \mu \mathrm{g} / \mathrm{L} & \mathrm{GE} & 0 \\ \mu \mathrm{g} / \mathrm{L} & \mathrm{GE} & 0 \\ \mu \mathrm{g} / \mathrm{L} & \mathrm{GE} & 0 \\ \mu \mathrm{g} / \mathrm{L} & \mathrm{GE} & 0 \\ \mu \mathrm{g} / \mathrm{L} & \mathrm{GE} & 0 \\ \mu \mathrm{g} / \mathrm{L} & \mathrm{GE} & 0 \\ \mu \mathrm{g} / \mathrm{L} & \mathrm{GE} & 0 \\ \mu \mathrm{g} / \mathrm{L} & \mathrm{GE} & 0 \\ \mu \mathrm{g} / \mathrm{L} & \mathrm{GE} & 1 \\ \mu \mathrm{g} / \mathrm{L} & \mathrm{GE} & 0 \\ \mu \mathrm{g} / \mathrm{L} & \mathrm{GE} & 0 \\ \mu \mathrm{g} / \mathrm{L} & \mathrm{GE} & 0\end{array}$

$<1.0<1.0$

45.800

$<1.0$

1.350
$<1.0$

$<1.0$

1.3

$<1.0$

$<1.0$

$<1.0$
$<1.0$

$<1.0$
$<1.0$

$<1.0$

$<1.0$

$<0.30$

$<1.0$

$<1.0$

$<1.0$

$$
4.5
$$

$<4.0$

$<4.0$

$<5.0$

$<1.0$

$<1.0$
$<1.0$

$<1.0$

$<1.0$

1.2

$<0.30$

$<1.0$

$<1.0$

$<0.0060$

$<0.0060$

$<1.0$

$<1.0$

$<100$

$<2 . \mathrm{OE}+00$

175

$<2 . \mathrm{OE}+00$

GE 0

GE 0

$\begin{array}{ll}\mathrm{GE} & 0 \\ \mathrm{GE} & 0\end{array}$

J 0

GE 0

GE $\quad 0$

GE 0

$\begin{array}{ll}\text { GE } & 0 \\ \text { GE } & 0\end{array}$

GE 0

GE $\quad 0$

GE 0

rg/

$\mu \mathrm{g} / \mathrm{L}$

$\mu g / L$

$\mu \mathrm{g} / \mathrm{L}$

$\mu \mathrm{g} / \mathrm{L}$

$\mu g / L$

$\mu g / L$

$\mu \mathrm{g} / \mathrm{L}$

$\mu \mathrm{g} / \mathrm{L}$

$\mu g / L$

$\mu \mathrm{g} / \mathrm{L}$

$\mu g / L$
$\mu g / L$

GE 0

GE $O$

$\mu g / L$

$\mu g / L$

$\mu \mathrm{g} / \mathrm{L}$ GE 0

PCi/L GE

$\mu_{g} / L$

$\mu g / L$

$\mu g / L$

$\mu g / L$

$\mu \mathrm{g} / \mathrm{L}$

Note: Flagging levels, modifiers, and laboratories are for 4 th quarter 1992 data only. See Appendix B for flagging criteria.

- =xceeded holding time for 4th quarter 1992.

- exceeded final primary drinking water standard for 4 th quarter 1992. 
WSRC-TR.93-059

Woll HSB 85B continued

ANALYTICAL DATA

H D Analyte

Hexachloroethane

Indenol 1,2,3-c,dipyrene

Iron

Isophorone

Load

Lindane

Magnesium

Manganose

Mercury

Methoxychlor

2-Methyl-4,6-dinitrophenol

Naphthalene

Nickel

Nitrate as nitrogen

Nitrate-nitrite as nitrogen

Nitrobenzene

2-Nitrophenol

4-Nitrophenol

$\mathrm{N}$-Nitrosodimethylamine

N-Nitrosodiphenylamine

N-Nitrosodipropylamine

Nonvolatile beta

PCB 1016

PCB 1221

PCB 1232

PCB 1242

PCB 1248

PCB 1254

PCB 1260

Pentachlorophenol

pH

Phenanthrene

Phenol

Phenols

Potassium

Pyreno

Selenium

Silica

Silver

Sodium

Specific conductance

- Sulfate

1,1,2,2-Tetrachloroethane

Tetrachloroethylene

Tin

Toluene

Total alpha-emitting radium

Total dissolved solids

Total organic carbon

Total organic halogens

Total phosphates (as P)

Toxaphene

2,4,5-TP (Silvex)

1,2,4-Trichlorobenzene

1 1. 1-Trichloroethane

1,1,2-Trichloroethone

Trichloroethylene

Trichlorofluoromethane

2.4,6-Trichlorophenol

Tritium

Turbidity

Vanadium

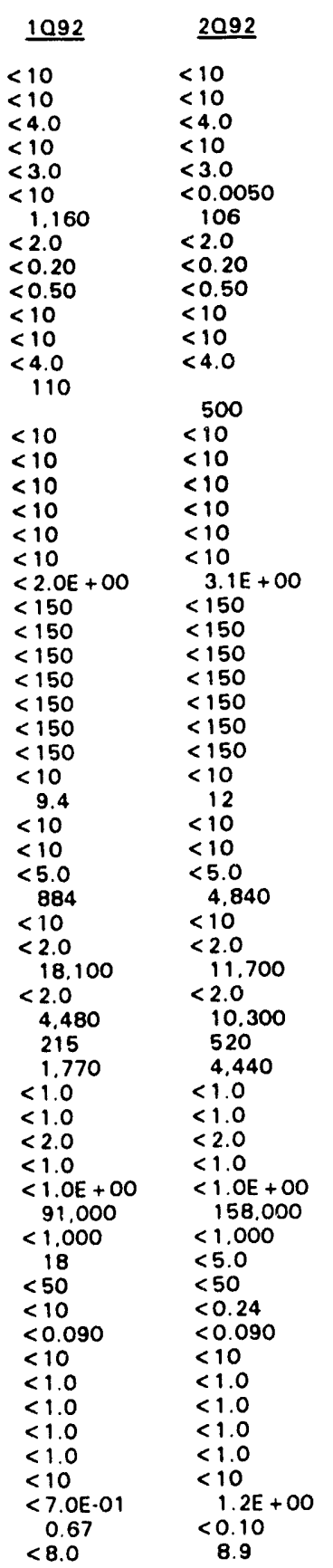

3092

4092

Mod Unit Lab Flag

$<4.0$

$<3.0$
$<0.0050$

1.270

$\begin{aligned} & 1.270 \\ &<2.0\end{aligned}$

$<0.20$

$<0.50$

$<10$

$<4.0$

520

9.5

$<3.0$

$<0.0050$

57

$<2.0$

$<0.20$

$<0.50$

$<10$

80

$<2.0 \mathrm{OE}+0 \mathrm{O}$

3. $6 E+00$

Unit
$\mu g / L$
$\mu g / L$

$\mu g / L$ GE $O$

$\mu g / L$

$\mu \mathrm{g} / \mathrm{L}$ GE 0

$\mu g / L$ GE

$\mu \mathrm{g} / \mathrm{L}$ GE 0

$\begin{array}{lll}\mu \mathrm{g} / \mathrm{L} & \mathrm{GE} & 0 \\ \mu \mathrm{g} / \mathrm{L} & \mathrm{GE} & 0\end{array}$

$\mu g / L$

GE 0

GE 0

GE 0

$\mu g / L$

$\mu \mathrm{g} / \mathrm{L}$

$\mu \mathrm{g} / \mathrm{L}$

$\mu g / L$

$\mu g / L$

$\mu g / L$
$\mathrm{PCi} / \mathrm{L}$
$\mu \mathrm{g} / \mathrm{L}$

GE 0

$\mu \mathrm{g} / \mathrm{L}$

$\mu \mathrm{g} / \mathrm{L}$

$\mu g / L$

$\mu \mathrm{g} / \mathrm{L}$

$\mu \mathrm{g} / \mathrm{L}$

8. 7

$<5.0$

901

$<2.0$

17.800

$<2.0$

4.280

175

1.580

$<1.0$

$<2.0$

$<1.0$

$<1 . \mathrm{OE}+00$

149.000

2.000

$<5.0$

$<50$

$<0.24$
$<0.090$

$<1.0$

$<1.0$

$<1.0$

$<1.0$

$1.4 E+00$

$<0.10$

$<8.0$
pH GE 2

$\mu g / L$

$<5.0$

5,200

$<2.0$

10.600

$<2.0$

11,200

440

3.740

$<1.0$

$<1.0$

$<2.0$

$<1.0 \mathrm{E}+00$

$1.0 E+00$
158.000

1.420

$<5.0$

$<50$

$<0.24$

$<0.090$

$<1.0$

$<1.0$

$<1.0$

$<1.0$

1. $3 E+00$

0.35

11 
WSRC-TR-93-059

Well HSB 858 continued

ANALYTICAL DATA

H $\underline{D}$ Analyte

Xylenes

$\begin{array}{ll}\frac{1092}{2092} & \underline{2092} \\ <2.0 & <2.0 \\ <2.0 & <2.0\end{array}$

$\begin{aligned} & 3092 \\ < & 2.0 \\ < & 2.0\end{aligned}$

4092
$<2.0$
7.2

$\begin{array}{lll}\text { Mod Unit } & \text { Lab } & \text { Flag } \\ \mu g / L & \text { GE } & 0 \\ \mu g / L & \text { GE } & 0\end{array}$

WELL HSB 85C

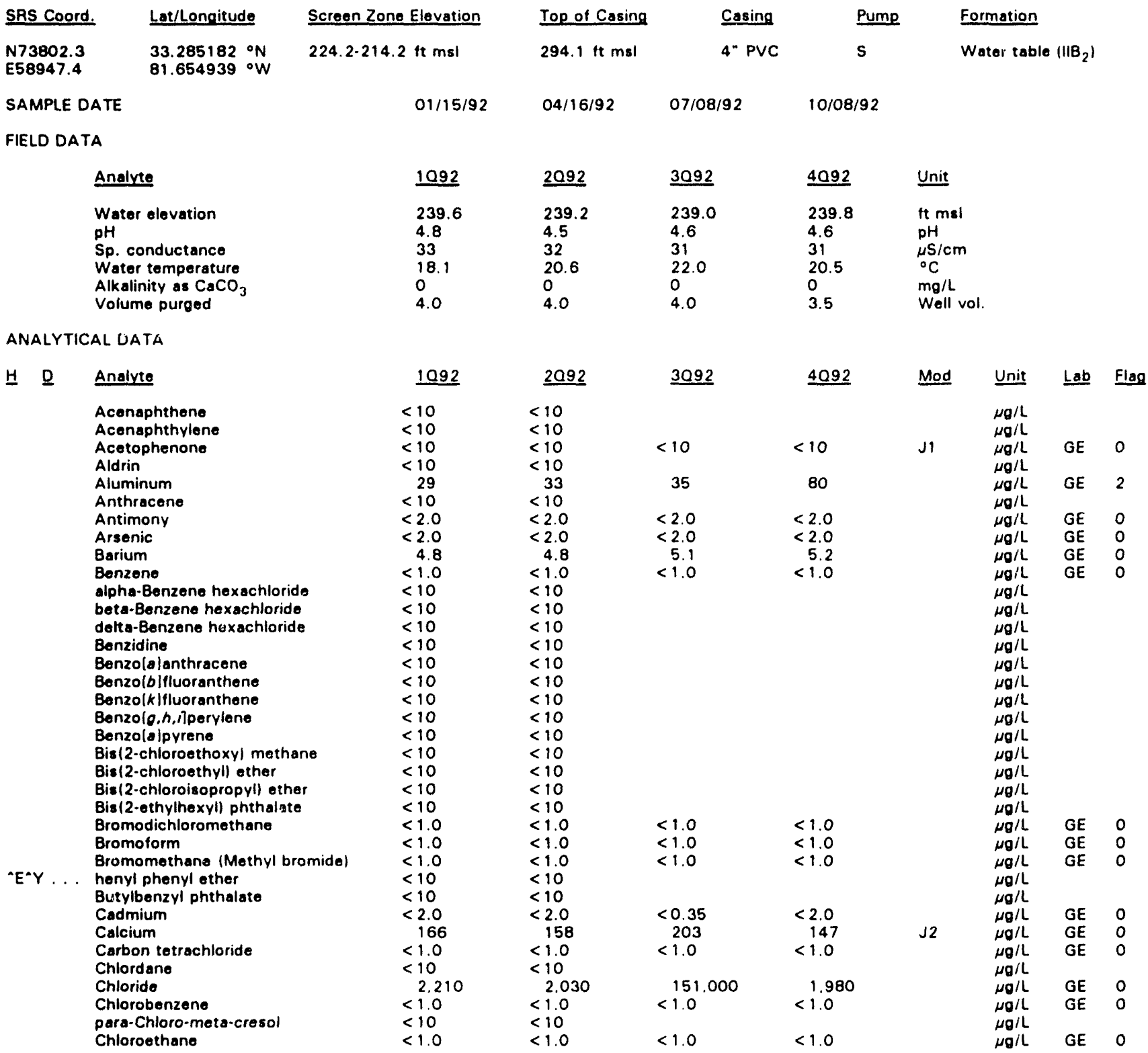

Note: Flagging levels, modifiers, and laboratories are for 4th quarter 1992 data only. See Appendix $B$ for flagging criteria.

- = exceeded holding time for 4th quarter 1992 .

- =xceeded final primary drinking water standard for 4th quarter 1992.

H-Area Seepage Basins 
H D Anaiyte

2.Chloroethyl vinyl other

Chloroform

Chloromethane (Methyl chloride)

2-Chloronaphthalene

2.Chlorophenol

4-Chlorophenyl phenyl ether

Chromium

Chrysene

Cobalt

Copper

Cyanide

p.p'-DDD

P.P'-DDE

p.p'-DDT

Dibenzle, hlanthracene

Dibromochloromethane

Di-n-butyl phthalate

3, 3'-Dichlorobenzidine

1,1-Dichloroothane

1.2-Dichloroethane

1.1-Dichloroethylene

trans-1,2-Dichloroethylene

Dichloromethane iMethytene chloridel

2.4-Dichlorophenol

2.4-Dichlorophenoxyacetic acid

1.2-Dichloropropane

cis-1,3-Dichloropropene

trans-1,3-Dichloropropene

Dieldrin

Diethyl phthalate

2.4-Dimethyl phenol

Dimethyl phthalate

2.4-Dinitrophenol

2,4-Dinitrotoluene

2,6-Dinitrotoluene

Di-n-octyl phthalate

1.2-Diphenylhydrazine

Endosulfan I

Endosulfan II

Endosulfan sulfate

Endrin

Endrin aldehyde

Ethylbenzene

Fluoranthene

Fluorene

Fluoride

Gross alpha

Heptachior

Heptachlor epoxide

Hexachlorobenzene

Hexachlorobutadiene

Hexachlorocyclopent adiene

Hexachloroethane

Indeno $[1,2,3-c, d]$ pyrene

Iron

Isophorone

Lead

Lindane

Magnesium

Manganese

Mercury

$\underline{2092}$

$<1.0$

$<1.0$

$<1.0$

$<1.0$

$<10$

$<10$

$<10$

$<4.0$

$<10$

$<4.0$

23
$<5.0$

$<10$

$<10$

$<10$

$<10$

$<1.0$

$<10$

$<10$

$<1.0$

$<1.0$

$<1.0$

$<1.0$

$<1.0$

$<10$

$<0.30$

$<1.0$
$<1.0$

$<1.0$

$<10$

$<10$

$<10$

$<10$

$<45$

$<10$

$<10$

$<10$

$<10$

$<10$

$<10$

$<10$

$<10$

$<10$

$<1.0$

$<10$

$<10$

$<100$

$<10$

$<10$
$<10$

$<10$
$<10$
$<10$

$<10$

$<10$

$<4.0$

$<10$

$<3.0$

$<10$

102

2.4

$<0.20$
$<1.0$

$<1.0$

$<1.0$

$<1.0$

$<10$
$<10$

$<10$
$<10$

$<4.0$

$<10$

$<4.0$

23
$<5.0$

$<10$

$<10$

$<10$

$<10$

$<10$
$<1.0$

$<10$

$<10$

$<10$

$<1.0$

$<1.0$

$<1.0$

1.7

$<10$

$<0.30$

$<1.0$

$<1.0$

$<1.0$

$<10$

$<10$

$<10$

$<10$

$<45$

$<10$

$<10$

$<10$

$<10$

$<10$

$<10$

$<10$
$<0.0060$

$<10$

$<1.0$

$<1.0$

$<10$

$<10$
$<100$

$<2.0 E+00$

$<10$

$<10$

$<10$

$<10$

$<10$

$<10$

$<10$
$<4.0$

$<4.0$
$<10$

$<3.0$

$<0.0050$

99

2.5
$<0.20$ $\underline{3092}$

31

$<1.0$

$<1.0$

$<1.0$

$<1.1$

$<0.88$

$\begin{aligned} & 26 \\ &<5.0\end{aligned}$

$<1.0$

$<1.0$

$<1.0$

$<1.0$

$<1.0$

5.1

$<0.30$

$<1.0$

$<1.0$

$<1.0$

.

$\begin{array}{ll}<0.0060 & <0.0060 \\ <1.0 & <1.0 \\ <100 & <100 \\ 2.4 E+00 & <2.0 E+00\end{array}$

Mod

$\begin{array}{lll}\text { Unit } & \text { Lab } & \text { Fla } \\ \mu g / L & \text { GE } & 0 \\ \mu g / L & \text { GE } & 0 \\ \mu g / L & \text { GE } & 0 \\ \mu g / L & \text { GE } & 0\end{array}$

$\mu \sigma / L$

$\mu \mathrm{\mu g} / \mathrm{L}$

$\mu \sigma / L$

$\mu g / L$

$\mu \mathrm{g} / \mathrm{L}$

$\mu \mathrm{g} / \mathrm{L}$

$\mu \mathrm{g} / \mathrm{L}$

$\mu \mathrm{g} / \mathrm{L}$

$\mu \mathrm{g} / \mathrm{L}$

$\mu g / L$

$\mu g / L$

$\mu \mathrm{g} / \mathrm{L}$

$\mu g / L$

$\mu \mathrm{g} / \mathrm{L}$

$\mu g / L$

$\mu g / L$
$\mu g / L$
$\mu g / L$

$\mu g / L$

$\mu \mathrm{g} / \mathrm{L}$

$\mu g / L$
$\mu g / L$
$\mu g / L$

$\mu \mathrm{g} / \mathrm{L}$

$\mu \mathrm{g} / \mathrm{L}$

$\mu \mathrm{g} / \mathrm{L}$

$\mu g / L$

$\mu \mathrm{g} / \mathrm{L}$

$\mu g / L$

$\mu \mathrm{g} / \mathrm{L}$

$\mu \mathrm{g} / \mathrm{L}$

$\mu_{g} / L$

$\mu g / L$

$\mu \mathrm{g} / \mathrm{L}$

$\mu_{0} / L$

$\mu g / L$

$\mu \mathrm{g} / \mathrm{L}$

$\mu \mathrm{g} / \mathrm{L}$

pCi/L

$\mu \mathrm{g} / \mathrm{L}$

$\mu \mathrm{g} / \mathrm{L}$

$\mu \mathrm{g} / \mathrm{L}$

$\mu \mathrm{g} / \mathrm{L}$

$\mu g / L$

$\mu g / L$

$\mu g / L$

$\mu \mathrm{g} /$

$\mu g /$

$\mu g / L$

GE 0

GE 0

$\begin{array}{ll}\text { GE } & 0 \\ \text { GE } & 0\end{array}$

GE $O$

GE 0

GE 0

GE 0

GE 0

GE 0

GE 0

GE $O$

GE 0

GE 0

$<0.0050<0.0050$

$103 \quad 111$

$<\begin{array}{cc}2.4 & <.4 \\ <0.20 & <0.20\end{array}$

ag

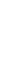


H D Analyte

Methoxychlor

2-Mothyl-4,6-dinitrophenol Naphthalene

Nickel

Nitrate as nitrogen

Nitrate-nitrite as nitrogen

Nitrobenzene

2-Nitrophenol

4-Nitrophenol

N-Nitrosodimethylamine

N-Nitrosodiphenylamine

$\mathrm{N}$-Nitrosodipropylamine

Nonvolatile beta

PCB 1016

PCB 1221

PB 1232

PCB 1242

PCB 1248

PCB 1254

PCB 1260

Pontachlorophenol

pH

Phenanthrene

Phenol

Phenols

Potassium

Pyrone

Radium-226

Radium-228

Selenium

Silica

Silver

Sodium

Specific conductance

Sulfate

1,1,2,2-Tetrachloroethane

Tetrachloroethylene

Tin

Toluene

Total alpha-emitting radium

Total dissolved solids

Total organic carbon

Total organic halogens

Total phosphates (as P)

Toxaphene

2,4,5.TP (Silvex)

1,2,4-Trichlorobenzene

1,1,1-Trichloroethane

1,1,2-Trichloroethane

Trichloroethylene

Trichlorofluoromethane

2,4,6-Trichlorophenol

Tritium

Turbidity

Vanadium

Xylenes

Zinc

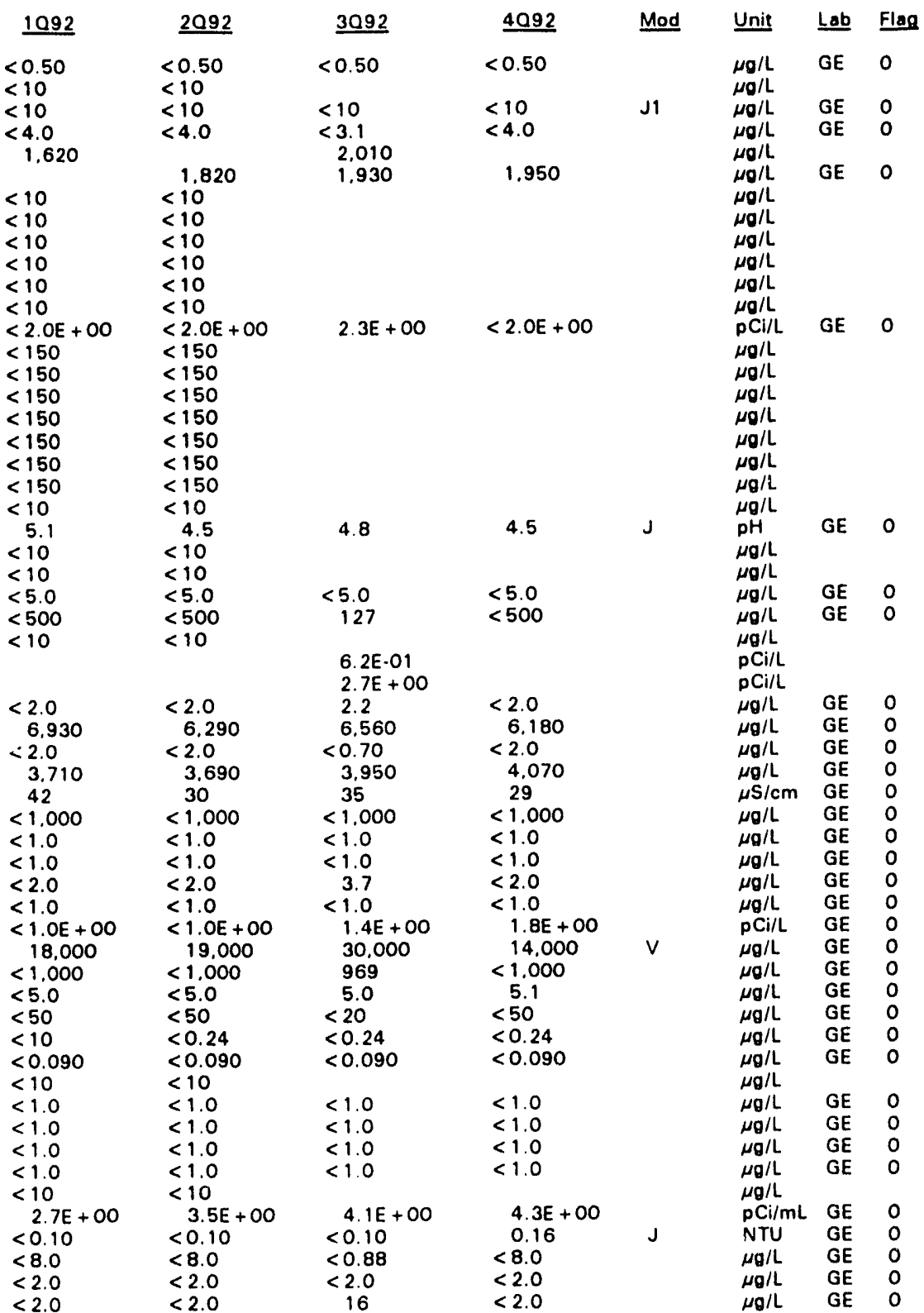

Note: Flagging levels, modifiers, and laboratories are for 4 th quarter 1992 data only. See Appendix B for flagging criteria.

- =xceeded holding time for 4th quarter 1992.

- =xceeded final primary drinking water standard for 4th quarter 1992. 
WSRC.TR.93.059

WELL HSB 86A

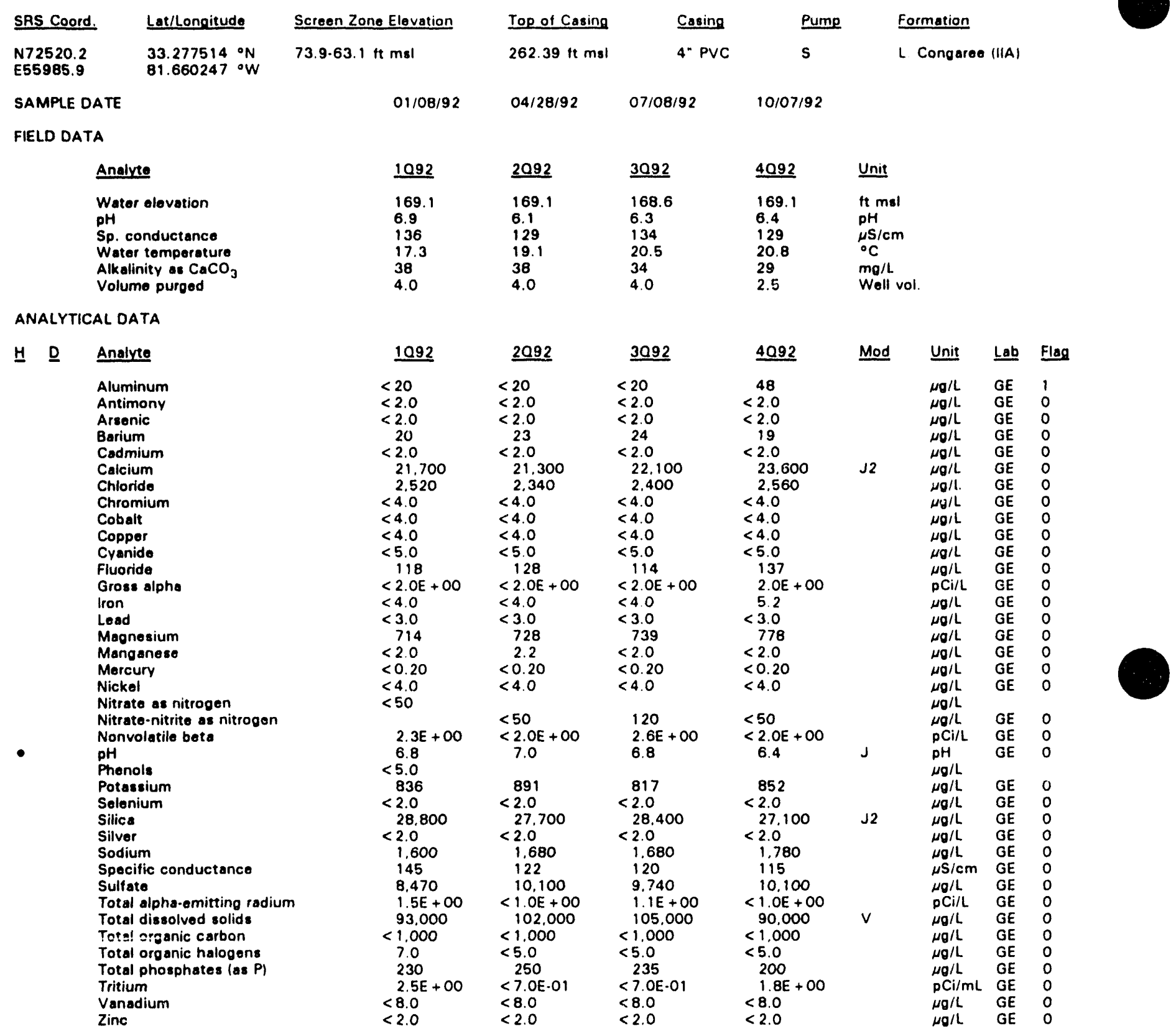

Note: Flagging levels, modifiers, and laboratories are for 4 th quarter 1992 data only. See Appendix B for flagging criteria. - = exceeded holding time for 4 th quarter 1992.

- =xceeded final primary drinking water standard for 4 th quarter 1992.

H-Area Seepage Basins 
WELL HSB 86B

\begin{tabular}{|c|c|c|c|c|c|c|}
\hline SRS Coord. & Lat/Longitude & Screen Zone Elevation & Top of Casing & Casing & Pump & Formation \\
\hline $\begin{array}{l}\text { N72519.0 } \\
\text { E55976.9 }\end{array}$ & $\begin{array}{l}33.277497{ }^{\circ} \mathrm{N} \\
81.660269{ }^{\circ} \mathrm{W}\end{array}$ & $124.0-113.8 \mathrm{ft} \mathrm{msl}$ & $261.89 \mathrm{ft} \mathrm{msl}$ & 4" PVC & $\mathbf{s}$ & McBoan (IIB, ) \\
\hline SAMPLE DA & & $01 / 08 / 92$ & $04 / 28 / 92$ & $07 / 08 / 92$ & $10 / 07 / 92$ & \\
\hline FIELD DATA & & & & & & \\
\hline & & 1092 & $\underline{2092}$ & 3092 & 4092 & Unit \\
\hline & $\begin{array}{l}\text { elevation } \\
\text { onductance } \\
\text { temperature } \\
\text { nity as } \mathrm{CaCO}_{3} \\
\text { le purged }\end{array}$ & $\begin{array}{l}221.0 \\
7.6 \\
224 \\
19.5 \\
80 \\
4.0\end{array}$ & $\begin{array}{l}221.8 \\
7.0 \\
214 \\
19.5 \\
95 \\
4.0\end{array}$ & $\begin{array}{l}221.9 \\
6.9 \\
220 \\
22.4 \\
87 \\
4.0\end{array}$ & $\begin{array}{l}221.4 \\
7.1 \\
211 \\
21.0 \\
87 \\
2.5\end{array}$ & $\begin{array}{l}\text { ft msl } \\
\mathrm{pH} \\
\mu \mathrm{S} / \mathrm{cm} \\
{ }^{\circ} \mathrm{C} \\
\mathrm{mg} / \mathrm{h} \\
\text { Well vol. }\end{array}$ \\
\hline
\end{tabular}

ANALYTICAL DATA

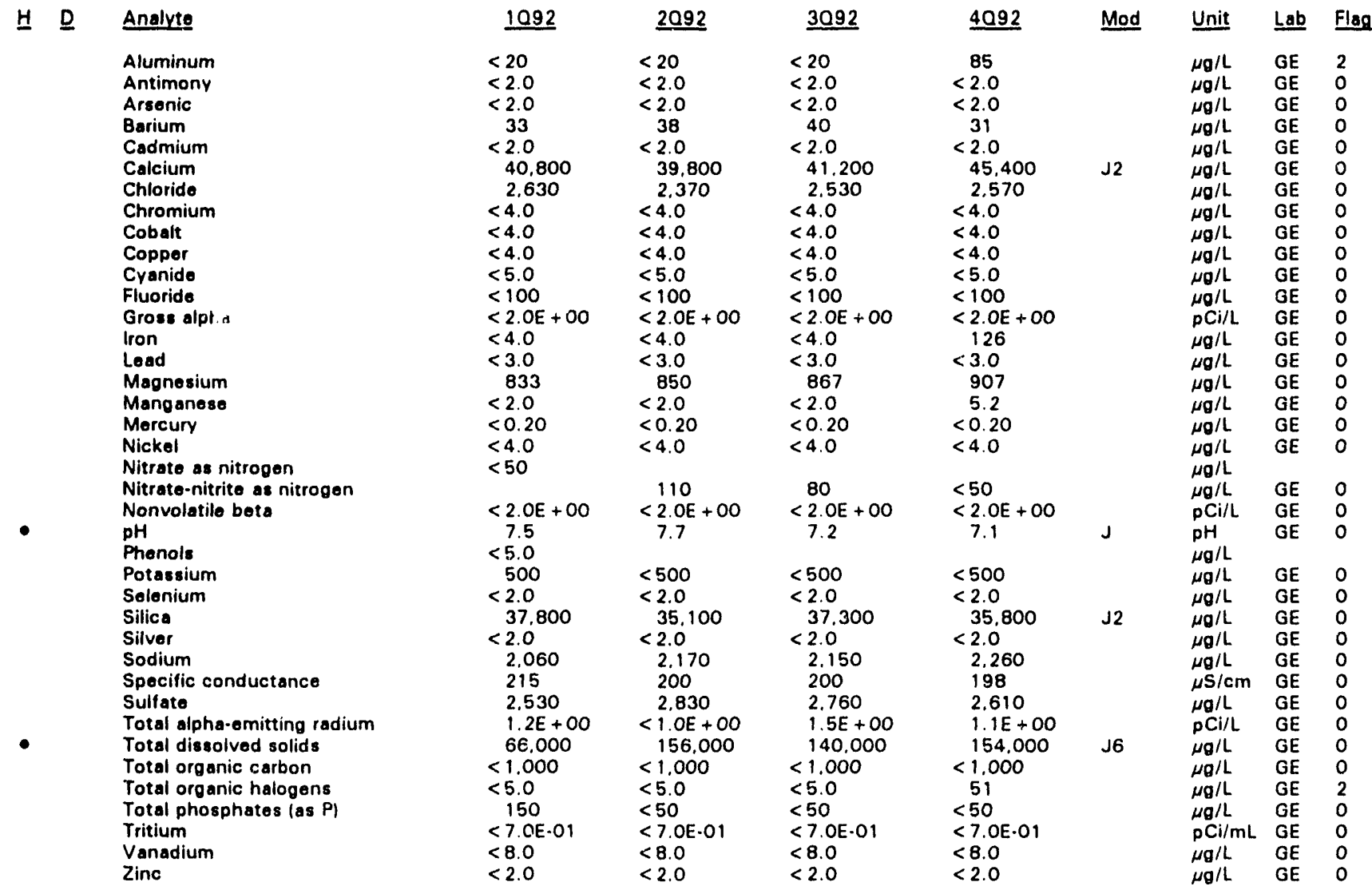

Note: Flagging levels, modifiers, and laboratories are for 4th quarter 1992 data only. See Appendix B for flagging criteria. - = exceeded holding time for 4th quarter 1992.

- =xceeded final primary drinking water standard for 4th quarter 1992. 
WSRC.TR-93.059

WELL HSB 86C

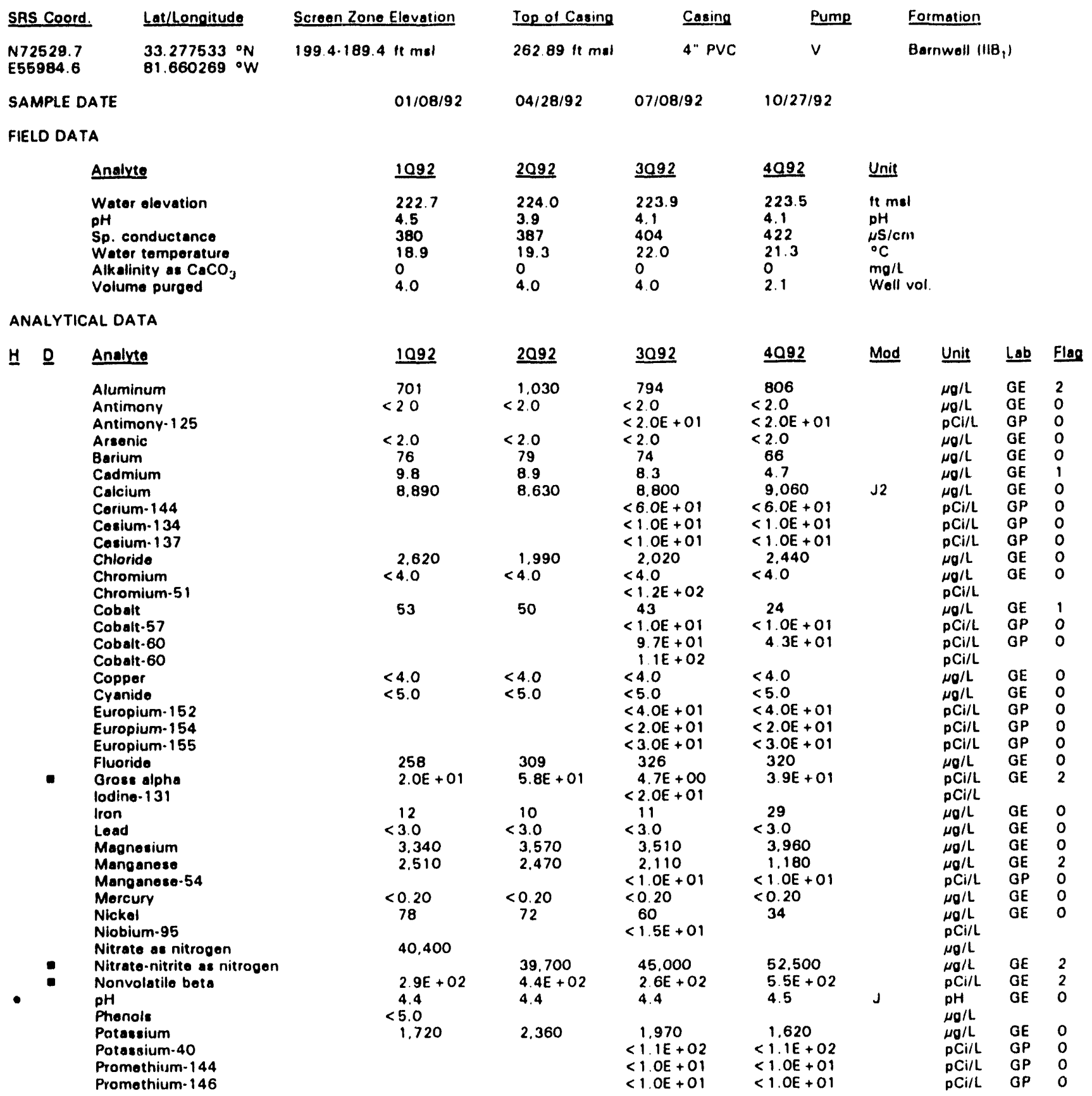

Note: Flagging levels, modifiers, and laboratories are for 4 th quarter 1992 data only. See Appendix 8 for flagging criteria. - =xceeded holding time for 4th quarter 1992.

- exceeded final primary drinking water standard for 4th quarter 1992. 
WSRC.TR-93.059

Well HSB 86C continued

ANALYTICAL DATA

H D Analyte

Ruthenium-106

Ruthenium-106

Solonium

Silica

Silver

Sodium

Sodium-22

Specific conductance

Sulfate

Total activity

- Total alpha-emitting radium

Total dissolved solids

Total organic carbon

Total orgenic halogens

Total phosphates (as P)

- Tritium

Vanadium

Yttrium-88

Zinc

Zinc-65

Zirconium-95

$\begin{array}{lll}1092 & \underline{2092} & \underline{3092} \\ & & <9.0 E+01 \\ & <1.3 E+02 \\ <2.0 & <2.0 & <2.0 \\ 18,900 & 17.700 & 17.900 \\ <2.0 & <2.0 & <2.0 \\ 38.600 & 43.100 & 45.800 \\ & & <1.0 E+01 \\ 282 & 340 & 400 \\ 24.500 & 2.490 & 4.060 \\ 1.5 E+07 & 1.7 E+07 & 1.7 E+07 \\ 1.6 E+01 & 2.1 E+01 & 1.7 E+01 \\ 246.000 & 281.000 & 263.000 \\ <1.000 & <1.000 & <1.000 \\ 15 & 60 & <5.0 \\ 50 & <50 & <50 \\ 1.5 E+04 & 1.7 E+04 & 1.6 E+04 \\ <8.0 & <8.0 & <8.0 \\ 70 & 66 & <6.0 E+01 \\ 70 & 65 & 55 \\ & & <2.0 E+01 \\ & & <2.0 E+01\end{array}$

$\begin{aligned} & 4092 \quad \text { Mod } \\ < & 9.0 E+01 \\ < & 2.0 \\ & 14.900 \\ < & 2.0 \\ & 54.000 \\ < & 1.0 E+01 \quad J 2 \\ & 355 \\ & 2.530 \\ & 1.7 E+07 \\ & 1.7 E+01 \\ < & 278.000 \\ < & 1.000 \\ < & 5.0 \\ < & 50 \\ & 1.6 E+04 \\ < & 8.0 \\ < & 6.0 E+01 \\ & 39 \\ < & 2.0 E+01\end{aligned}$

\section{WELL HSB 86D}

$\begin{array}{lll}\text { SRS Coord. } & \text { Lat/Longitude } & \text { Scr } \\ \text { N72522.1 } & 33.277536{ }^{\circ} \mathrm{N} & 236 \\ \text { E55996.5 } & 81.660223{ }^{\circ} \mathrm{W}\end{array}$

$\frac{\text { Screen Zone Elevation }}{236.6-206.6 \mathrm{ft} \mathrm{msl}}$

\section{Top of Casing}

$04 / 28 / 92$

$07 / 08 / 92$

Casing

Pump

Formation

SAMPLE DATE

$01 / 08 / 92$

v

Water table $\left(11 \mathrm{~B}_{2}\right)$

FIELD DATA

Analvte
Water elevation
pH
Sp. conductance
Water temperature
Alkalinity as $\mathrm{CaCO}_{3}$
Volume purged

ANALYTICAL DATA

$\begin{array}{ll}1092 & \underline{2092} \\ 222.7 & 224.0 \\ 3.9 & 3.5 \\ 410 & 351 \\ 19.2 & 19.3 \\ 0 & 0 \\ 4.0 & 4.0\end{array}$

4.0

$\begin{array}{cc}\frac{1092}{2092} \\ 5.860 & 3.390 \\ <2.0 & <2.0 \\ <2.0 & <2.0 \\ 77 & 55 \\ <2.0 & <2.0 \\ 2.930 & 2.080 \\ & \\ 2.600 & 1.930 \\ <4.0 & <4.0 \\ 12 & 8.3\end{array}$

3092
223.9
3.7
332
21.6
0
4.0

3092

3.090

$<2.0$

$<2.0 \mathrm{E}+01$

$<2.0$

52

$<2.0$

2.150
$<6.0 E+01$

$<6.0 E+01$
$<1.0 E+01$

$<1 . O E+01$

1.800

$<4.0$

$<1.2 \mathrm{E}+02$

8. 1

$<1.0 E+01$

6. $8 E+01$

7. $8 E+01$
$10 / 15 / 92$

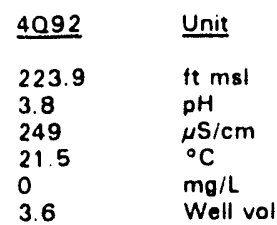

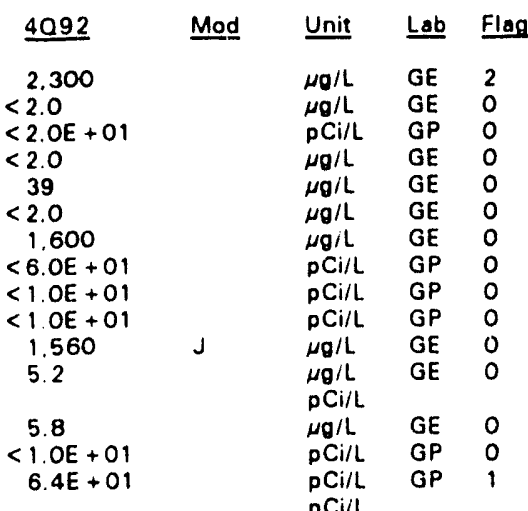

Note: Flagging levels, modifiers, and laboratories are for 4th quarter 1992 data only. See Appendix B for flagging criteria. - =xceeded holding time for 4 th quarter 1992.

- =xceeded final primary drinking water standard for 4th quarter 1992. 
WSRC.TR-93-059

Well HS8 860 continued

\section{ANALYTICAL DATA}

H

D. $\quad$ Analyte
Coppep
Cyanide
Europium-152
Europium-154
Europium-155
Fluoride
Grose alphs
lodine-131
Iron
Lead
Magnesium
Manganese
Manganese-54
Morcury
Nickel
Niobium-95
Nitrate as nitrogen
Nitrate-nitrite as nitrogen
Nonvolatile beta
pH
Phonols
Potassium
Potassium-40
Promethium-144
Promethium-146
Ruthenium-106
Ruthenium-106
Solenium
Silica
Silver
Sodium
Sodium-22
Specific conductance
Sulfate
Total activity
Total alpha-emitting radium
Total dissolved solids
Total organic carbon
Total organic halogens
Total phosphates las P)
Tritium
Vanadium
Yttrium-88
Zinc
Zinc-65
Zirconium-95

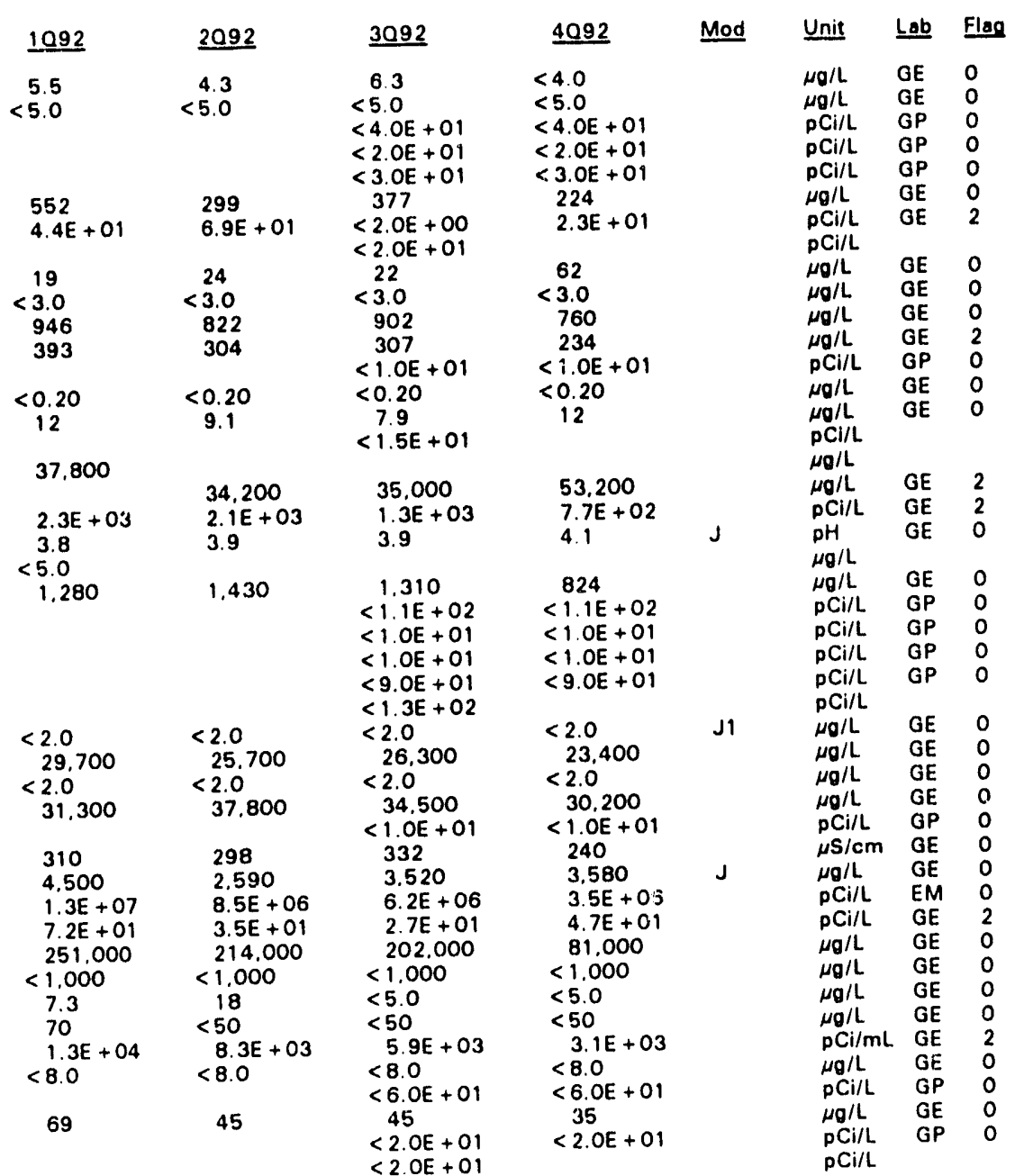

Note: Flagging levels, modifiers, and laboratories are for 4 th quarter 1992 data only. See Appendix $B$ for flagging criteria.

- = exceeded holding time for 4th quarter 1992.

- =xceeded final primary drinking water standard for 4th quarter 1992. 
WSRC-TR-93-059

WELL HSB100C

\begin{tabular}{|c|c|c|c|c|c|c|}
\hline SRS Coord. & Lat/Longitude & Screen Zone Elevation & Top of Casing & Casing & Pump & Formation \\
\hline $\begin{array}{l}\text { N72077.2 } \\
\text { E58806.5 }\end{array}$ & $\begin{array}{l}33.281136{ }^{\circ} \mathrm{N} \\
81.651960^{\circ} \mathrm{W}\end{array}$ & $163.0 .1-1.0 \mathrm{ft} \mathrm{msl}$ & $260.2 \mathrm{ft} \mathrm{msl}$ & 4" PVC & $\mathbf{s}$ & Barnwell (IIB, \\
\hline SAMPLE JA & & $01 / 01 / 92$ & $04 / 02 / 92$ & $07 / 08 / 92$ & $11 / 06 / 92$ & \\
\hline FIELD DATA & & & & & & \\
\hline & & 1092 & $\underline{2092}$ & $\underline{3092}$ & 4092 & Unit \\
\hline & $\begin{array}{l}\text { elevation } \\
\text { nductance } \\
\text { temperature } \\
\text { hity as } \mathrm{CaCO}_{3} \\
\text { e purged }\end{array}$ & $\begin{array}{l}226.4 \\
5.8 \\
34 \\
18.6 \\
4 \\
4.0\end{array}$ & $\begin{array}{l}226.7 \\
4.9 \\
36 \\
19.1 \\
2 \\
4.0\end{array}$ & $\begin{array}{l}226.6 \\
5.4 \\
34 \\
21.5 \\
3 \\
4.0\end{array}$ & $\begin{array}{l}226.9 \\
5.5 \\
32 \\
20.2 \\
4 \\
2.5\end{array}$ & $\begin{array}{l}\text { ft msi } \\
\mathrm{pH} \\
\mu \mathrm{S} / \mathrm{cm} \\
{ }^{\circ} \mathrm{C} \\
\mathrm{mg} / \mathrm{L} \\
\text { Well vol. }\end{array}$ \\
\hline
\end{tabular}

\section{ANALYTICAL DATA}

H D Analyte

Aluminum
Antimony
Arsenic
Barium
Cadmium
Calcium
Chloride
Chromium
Cobalt
Copper
Cyanide
Fluoride
Gross alpha
Iron
Lead
Magnesium
Manganese
Mercury
Nickel
Nitrate as nitrogen
Nitrate-nitrite as nitrogen
Nonvolatile beta
pH
Phenols
Potassium
Radium-226
Radium-228
Solenium
Silica
Silver
Sodium
Spocific conductance
Sulfate
Total alpha-emitting radium
Total dissolved solids
Total organic carbon
Total organic halogens
Total phosphates las Pl
Tritium
Vanadium
Zinc

1092

$<20$

$<2.0$

$<2.0$
$<2.0$

$<3.0$

$<2.0$

3.150

2.770

$<4.0$

$<4.0$

$<4.0$

$<5.0$

$<2.0 E+00$

$<2.0 E$
$<4.0$

$<4.0$
$<3.0$

412

2.6

$<0.20$

$<4.0$
140

$<2.0 E+00$

5.6

$<5.0$

$<500$

$<2.0$

14.500

$<2.0$

1.740

320

$<1,000$

$1.4 \mathrm{E}+00$

25,000

$<1,000$

57

440

$1.3 E$
$<8.0$
4.4

\section{$\underline{2092}$}

$<20$

$<2.0$

$<2.0$

$<3.0$

$<2.0$

3.160

2.650

$<4.0$

$<4.0$

$<4.0$

$<5.0$

157

$<2.0 E+O 0$

$<4.0$

$<3.0$

409

2.6

$<0.20$

$<4.0$

150

$<2.0 \mathrm{E}+00$

5.7

$<500$

$<2.0$

$<2.0$

$<2.0$

1.770

29

$<1,000$

$<1.0 E+00$

28,000

$<1,000$

8.1
510

510
$1.4 E+00$

$<8.0$

$<8.0$
$<2.0$
3092

$<20$

$<2.0$

$<2.0$

$<3.0$

$<2.0$

3.130

2.590

$<4.0$

$<4.0$

$<4.0$

$<5.0$

121

$<2.0 \mathrm{E}+00$

$<4.0$

$<3.0$

407

2.8

$<0.20$

$<4.0$

160

$<2.0 \mathrm{E}+0 \mathrm{OO}$

5.8

$<500$

$<2.0$

13.900

$<2.0$

1.750

32

$<1,000$

$<1.0 E+O 0$

39,000

$<1,000$

$<5.0$

481

1. $3 E+00$

$<8.0$

$<.0$
3.4
4092 Mod Unit Lab Flag

56

3.3

$<2.0$
$<3.0$

0.72

3.390
3.420

3.4

3.3
1.4

1.4
1.5

$<5.0$

152

9.0E-01

8.7
$<2.0$

$<2.0$
430

2.9

$<0.20$

$<3.1$

2.280

160

1. $6 E+00$

6.0

559

$<2.4 \mathrm{E} \cdot 01$

3.0E.01

$<2.0$

15.100

$<0.70$

1.850

35

310

$<1.0 E+00$
83.000

83,000
$<500$

$<5.0$

530

$2.1 E+00$

2.0
7.0

$\begin{array}{llll} & \mu g / L & \text { GE } & 2 \\ & 3 g & \text { WA } & 1 \\ & \mu g / L & \text { GE } & 0\end{array}$

$\begin{array}{llll} & \mu g / L & \text { GE } & 0 \\ \text { J3 } & \mu g / L & \text { GE } & 0 \\ \mu g / L & \text { WA } & 0\end{array}$

$\begin{array}{llll}J 2 & \mu g / L & G E & 0\end{array}$

$\mu g / L$ WA 0

$J 3 \mu g / L$ WA 0

J3 $\mu g / L$ WA 0

J3 $\mu \mathrm{g} / \mathrm{L}$ WA 0

$\begin{array}{lll}\mu \mathrm{g} / \mathrm{L} & \mathrm{GE} & 0 \\ \mu \mathrm{g} / \mathrm{L} & \mathrm{GE} & 0\end{array}$

$\mu g / L$ GE

J3 ${ }_{\mu \mathrm{g} / \mathrm{L}}^{\mathrm{PC} / \mathrm{L}}$ WA 0

$\mu g / L$ WA $O$

$\mu \mathrm{g} / \mathrm{L}$ WA

$\mu \mathrm{G} L \mathrm{GE}$

$\mu g / L$ WA 0

$\mu g / L \quad$ WA 0

$\begin{array}{lll}\mu \mathrm{g} / \mathrm{L} & W A & 0 \\ \mu \mathrm{g} / \mathrm{L} & \mathrm{GE} & 0\end{array}$

$\mathrm{PCi} / \mathrm{L}$ TM 0

$\begin{array}{lll}\mathrm{PH} & \text { WA } & 0 \\ \mu \mathrm{g} / L & & \end{array}$

$\mu \mathrm{g} / L$ WA 0

$P C \mathrm{C} / L$ TM $O$

$\mathrm{PCi} / \mathrm{L}$ TM 0

$\mu g / L \quad G E$

$\begin{array}{lll}\mu g / L & G E & 0 \\ \mu g / L & W A & O\end{array}$

$\begin{array}{lll}\mu g / L & \text { WA } & 0 \\ \mu g / L & W A & 0\end{array}$

$\begin{array}{lll}\mu \mathrm{g} / \mathrm{L} & \text { WA } & 0 \\ \mu \mathrm{S} / \mathrm{cm} & \mathrm{GE} & 0\end{array}$

$\mu g / L$ WA $O$

$\mathrm{pCi} / \mathrm{L}$ GE $O$

$\mu g / L$ WA $O$

$\begin{array}{lll}\mu g / L & W A & 0 \\ \mu g / L & W A & 0\end{array}$

$\mu g / L$ GE 0

$\mu g / L$ WA 0

$\mathrm{pCi} / \mathrm{mL}$ TM 0

$\begin{array}{llll}V & \mu g / L & W A & 0 \\ v & \mu g / L & W A & 0\end{array}$

Note: Flagging levels, modifiers, and laboratories are for 4th quarter 1992 data only. See Appendix B for íagging criteria. - = exceeded holding time for 4th quarter 1992.

- = exceeded final primary drinking water standard for 4th quarter 1992.

H-Area Seepage Basins 
WELL HSB100D

\begin{tabular}{|c|c|c|c|c|c|c|c|c|c|}
\hline \multicolumn{2}{|r|}{ Lat/Longitude } & \multirow{2}{*}{$\frac{\text { Screen Zone Elevation }}{236.9 \cdot 216.9 \mathrm{ft} \mathrm{msl}}$} & Top of Casing & Casing & \multicolumn{3}{|c|}{ Formation } & & \\
\hline $\begin{array}{l}\text { N72073.8 } \\
\text { E58796.9 }\end{array}$ & $\begin{array}{l}33.281113 \circ \mathrm{o} \\
81.651978 \text { o }\end{array}$ & & $260.1 \mathrm{ft} \mathrm{msl}$ & 4" PVC & $\mathbf{s}$ & $W_{a}$ & ter table & $\left(\| B_{2}\right)$ & \\
\hline SAMPLE DA & ATE & $01 / 01 / 92$ & $04 / 02 / 92$ & $07 / 08 / 92$ & $10 / 18 / 92$ & & & & \\
\hline FIELD DATA & & & & & & & & & \\
\hline & Analyte & 1092 & 2092 & $\underline{3092}$ & 4092 & Unit & & & \\
\hline & $\begin{array}{l}\text { Water elevation } \\
\text { pH } \\
\text { Sp. conductance } \\
\text { Water temperature } \\
\text { Alkalinity as } \mathrm{CaCO}_{3} \\
\text { Volume purged }\end{array}$ & $\begin{array}{l}233.1 \\
5.3 \\
68 \\
19.4 \\
1 \\
4.0\end{array}$ & $\begin{array}{l}233.7 \\
4.4 \\
68 \\
20.1 \\
0 \\
4.0\end{array}$ & $\begin{array}{l}233.8 \\
4.9 \\
79 \\
22.4 \\
0 \\
4.0\end{array}$ & $\begin{array}{l}234.0 \\
5.0 \\
78 \\
21.1 \\
0 \\
3.6\end{array}$ & $\begin{array}{l}\mathrm{ft} \mathrm{msl} \\
\mathrm{pH} \\
\mu \mathrm{S} / \mathrm{cm} \\
{ }^{\circ} \mathrm{C} \\
\mathrm{mg} / \mathrm{L} \\
\text { Well vol. }\end{array}$ & & & \\
\hline ANALYTICA & AL DATA & & & & & & & & \\
\hline$\underline{H} \quad \underline{D}$ & Analyte & 1092 & $\underline{2092}$ & 3092 & 4092 & Mod & Unit & $\underline{\text { Lab }}$ & Flag \\
\hline (1) & $\begin{array}{l}\text { Aluminum } \\
\text { Antimony } \\
\text { Arsenic } \\
\text { Barium } \\
\text { Cadmium } \\
\text { Calcium } \\
\text { Chloride } \\
\text { Chromium } \\
\text { Cobalt } \\
\text { Copper } \\
\text { Cyanide } \\
\text { Fluoride } \\
\text { Gross alpha } \\
\text { Iron } \\
\text { Lead } \\
\text { Magnesium } \\
\text { Manganese } \\
\text { Mercury } \\
\text { Nickel } \\
\text { Nitrate-nitrite as nitrogen } \\
\text { Nonvolatile beta } \\
\text { pH } \\
\text { Phenols } \\
\text { Potassium } \\
\text { Selenium } \\
\text { Silica } \\
\text { Silver } \\
\text { Sodium } \\
\text { Specific conductance } \\
\text { Sulfate } \\
\text { Total activity } \\
\text { Total alpha-emitting radium } \\
\text { Total dissolved solids } \\
\text { Total organic carbon } \\
\text { Total organic halogens } \\
\text { Total phosphates las P) } \\
\text { Tritium } \\
\text { Vanadium } \\
\text { Zinc } \\
\text { a }\end{array}$ & 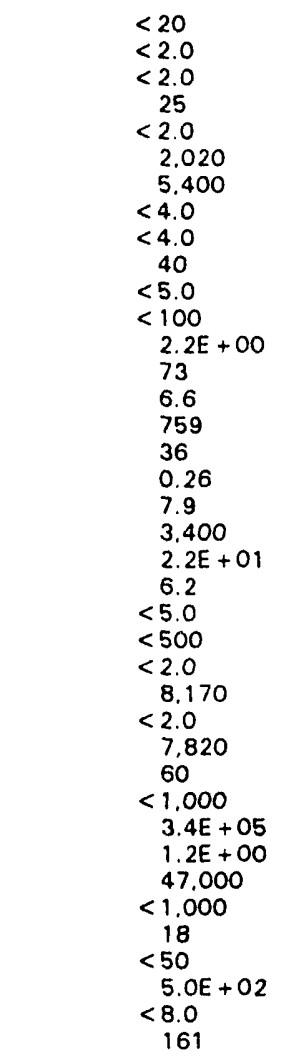 & $\begin{array}{l}<20 \\
<2.0 \\
<2.0 \\
20 \\
<2.0 \\
1.310 \\
5.090 \\
<4.0 \\
<4.0 \\
26 \\
<5.0 \\
<100 \\
<2.0 \mathrm{E}+00 \\
12 \\
4.4 \\
631 \\
29 \\
0.27 \\
<4.0 \\
3.120 \\
1.9 \mathrm{E}+01 \\
4.9 \\
<500 \\
<2.0 \\
8.330 \\
<2.0 \\
7.090 \\
58 \\
<1.000 \\
7.9 \mathrm{E}+05 \\
1.9 \mathrm{E}+00 \\
43.000 \\
<1.000 \\
6.6 \\
<50 \\
5.9 \mathrm{E}+02 \\
<8.0 \\
99\end{array}$ & $\begin{aligned} & 20 \\
&< 2.0 \\
&< 2.0 \\
& 22 \\
&<2.0 \\
& 1.370 \\
& 5.050 \\
&<4.0 \\
&<4.0 \\
& 28 \\
&<5.0 \\
&<100 \\
&<2.0 \mathrm{E}+00 \\
& 15 \\
& 4.7 \\
& 677 \\
& 33 \\
& 0.29 \\
& 4.8 \\
& 5.000 \\
& 3.4 \mathrm{E}+01 \\
& 5.2 \\
&<500 \\
&<500 \\
&<2.0 \\
& 7.730 \\
&<2.0 \\
& 10.300 \\
& 60 \\
& 1.230 \\
& 8.8 \mathrm{E}+05 \\
& 2.0 \mathrm{E}+00 \\
& 46.000 \\
&<1.000 \\
&<5.0 \\
&<50 \\
& 8.2 \mathrm{E}+02 \\
&<8.0 \\
& 79\end{aligned}$ & $\begin{aligned} & 48 \\
&< 2.0 \\
&< 2.0 \\
& 23 \\
&<2.0 \\
& 1.340 \\
& 5.020 \\
&<4.0 \\
&<4.0 \\
& 32 \\
&<5.0 \\
&<100 \\
&<2.0 \mathrm{E}+00 \\
& 47 \\
& 6.5 \\
& 743 \\
& 36 \\
& 0.90 \\
& 5.1 \\
& 4.650 \\
& 2.6 \mathrm{E}+01 \\
& 5.6 \\
&<500 \\
&<2.0 \\
&<2.01 \\
& 7.250 \\
&<2.0 \\
& 11.100 \\
& 70 \\
&<1.000 \\
& 8.8 \mathrm{E}+05 \\
& 3.3 \mathrm{E}+00 \\
& 44.000 \\
&<1.000 \\
&<5.0 \\
&<50 \\
& 8.4 \mathrm{E}+02 \\
&<8.0 \\
& 89\end{aligned}$ & $J$ & $\begin{array}{l}\mu g / L \\
\mu g / L \\
\mu g / L \\
\mu g / L \\
\mu g / L \\
\mu g / L \\
\mu g / L \\
\mu g / L \\
\mu g / L \\
\mu g / L \\
\mu g / L \\
\mu g / L \\
p C i / L \\
\mu g / L \\
\mu g / L \\
\mu g / L \\
\mu g / L \\
\mu g / L \\
\mu g / L \\
\mu g / L \\
p C i / L \\
p H \\
\mu g / L \\
\mu g / L \\
\mu g / L \\
\mu g / L \\
\mu g / L \\
\mu g / L \\
\mu S / c m \\
\mu g / L \\
p C i / L \\
p C i / L \\
\mu g / L \\
\mu g / L \\
\mu g / L \\
\mu g / L \\
p C i / m L \\
\mu g / L \\
\mu g / L\end{array}$ & $\begin{array}{l}\mathrm{GE} \\
\mathrm{GE} \\
\mathrm{GE} \\
\mathrm{GE} \\
\mathrm{GE} \\
\mathrm{GE} \\
\mathrm{GE} \\
\mathrm{GE} \\
\mathrm{GE} \\
\mathrm{GE} \\
\mathrm{GE} \\
\mathrm{GE} \\
\mathrm{GE} \\
\mathrm{GE} \\
\mathrm{GE} \\
\mathrm{GE} \\
\mathrm{GE} \\
\mathrm{GE} \\
\mathrm{GE} \\
\mathrm{GE} \\
\mathrm{GE} \\
\mathrm{GE} \\
\mathrm{GE} \\
\mathrm{GE} \\
\mathrm{GE} \\
\mathrm{GE} \\
\mathrm{GE} \\
\mathrm{GE} \\
\mathrm{GE} \\
\mathrm{EM} \\
\mathrm{GE} \\
\mathrm{GE} \\
\mathrm{GE} \\
\mathrm{GE} \\
\mathrm{GE} \\
\mathrm{GE} \\
\mathrm{GE} \\
\mathrm{GE}\end{array}$ & $\begin{array}{l}1 \\
0 \\
0 \\
0 \\
0 \\
0 \\
0 \\
0 \\
0 \\
0 \\
0 \\
0 \\
0 \\
0 \\
0 \\
0 \\
1 \\
0 \\
0 \\
0 \\
1 \\
0 \\
0 \\
0 \\
0 \\
0 \\
0 \\
0 \\
0 \\
0 \\
1 \\
0 \\
0 \\
0 \\
0 \\
2 \\
0 \\
0\end{array}$ \\
\hline
\end{tabular}

Note: Flagging levels, modifiers, and laboratcries are for 4 th quarter 1992 data only. See Appendix $B$ for flagging criteria.

- = exceeded holding time for 4th quarter 1992.

- =xceeded final primary drinking water standard for 4th quarter 1992. 
WELL HSB101C

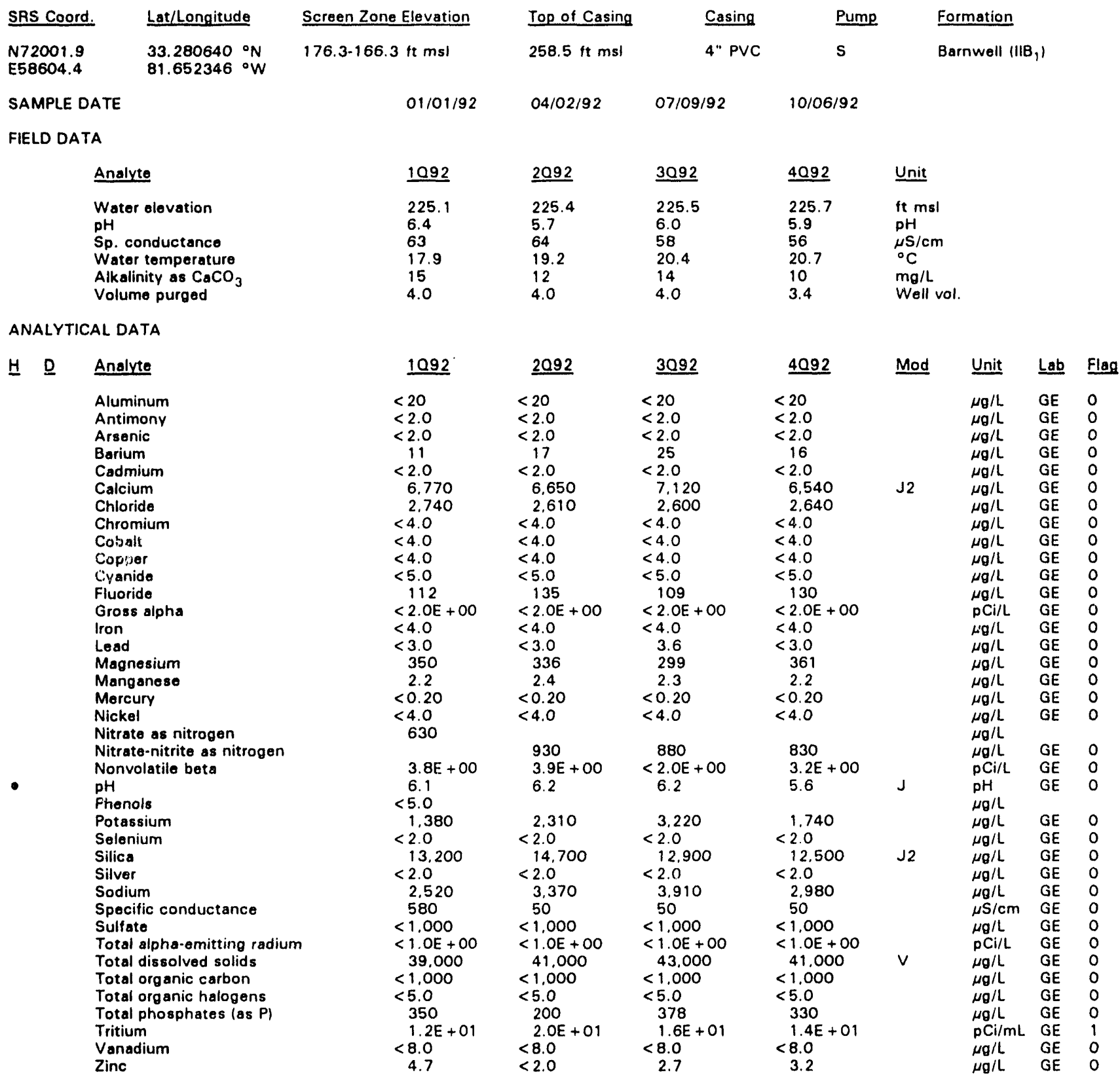

Note: Flagging levels, modifiers, and laboratories are for 4th quarter 1992 data only. See Appendix B for fiagging criteria. - = exceeded holding time for 4th quarter 1992.

- =xceeded final primary drinking water standard for 4th quarter 1992. 
WSRC-TR-93-059

WELL HSB101D

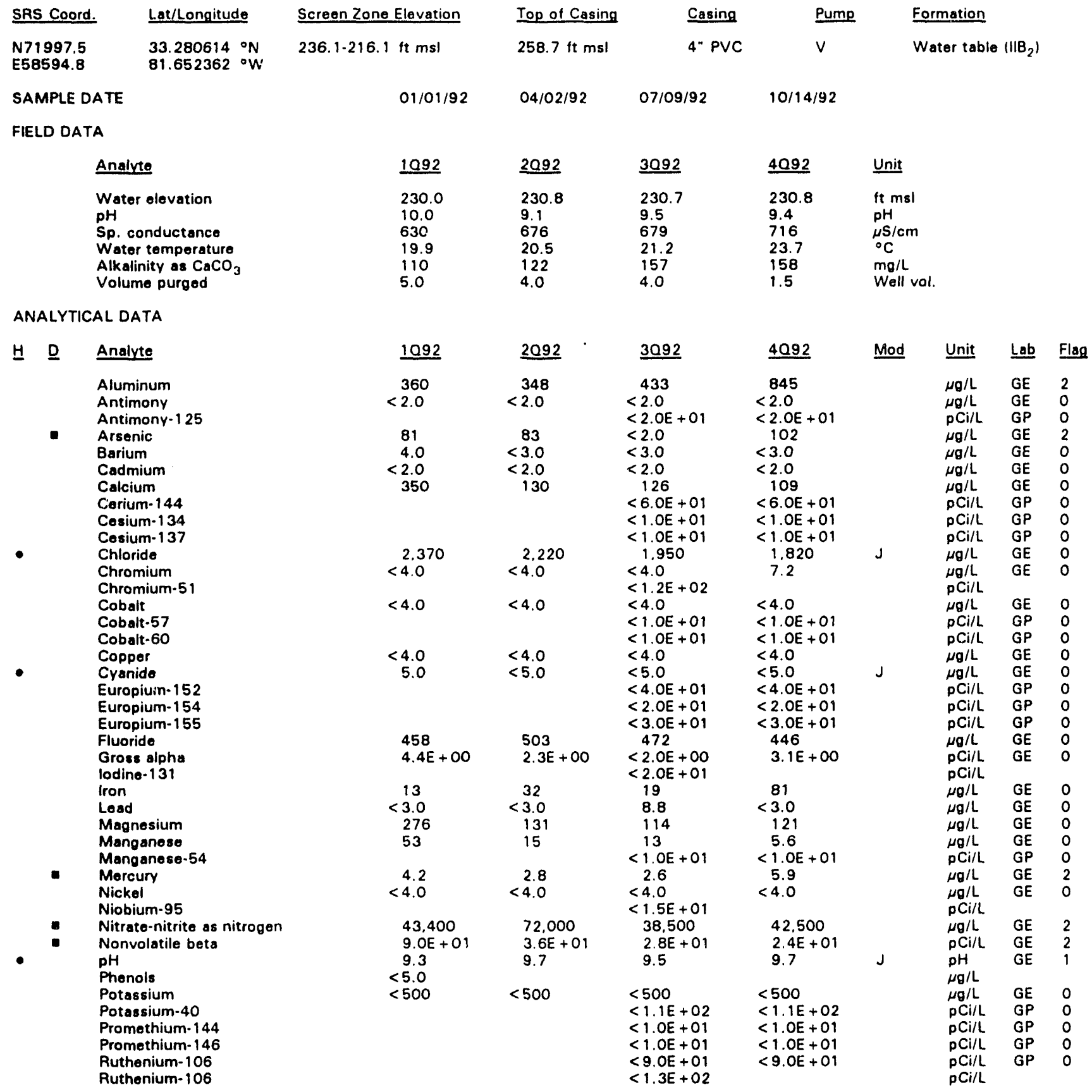

Note: Flagging levels, modifiers, and laboratories are for 4 th quarter 1992 data only. See Appendix B for flagging criteria.

- exceeded holding time for 4th quarter 1992

- =xceeded final primary drinking water standard for 4 th quarter 1932. 
Well HSB101D continued

ANALYTICAL DATA

H $\quad$ Analyte
Selenium
Silica
Silver
Sodium
Sodium-22
Specific conductance
Sulfate
Total activity
Total alpha-emitting radium
Total dissolved solids
Total organic carbon
Total organic halogens
Total phosphates las P)
Tritium
Vanadium
Yttrium-88
Zinc
Zinc-65
Zirconium-95

$\begin{array}{lll}1092 & \underline{2092} & \underline{3092} \\ <2.0 & <2.0 & <2.0 \\ 4.830 & 4.500 & 4.330 \\ <2.0 & <2.0 & <2.0 \\ 115.000 & 113.000 & 115.000 \\ & & <1.0 \mathrm{E}+01 \\ 610 & 700 & 600 \\ 3.420 & 5.260 & 5.010 \\ 9.6 \mathrm{E}+06 & 1.1 \mathrm{E}+07 & 9.6 \mathrm{E}+06 \\ 2.0 \mathrm{E}+00 & <1.0 \mathrm{E}+00 & <1.0 \mathrm{E}+00 \\ 430.000 & 409,000 & 435.000 \\ <1.000 & <1.000 & <1.000 \\ 8.0 & 18 & <5.0 \\ 2.280 & 2.520 & 2.960 \\ 1.0 \mathrm{E}+04 & 1.1 \mathrm{E}+04 & 8.8 \mathrm{E}+03 \\ 323 & 300 & 304 \\ <2.0 & <2.0 & <6.0 \mathrm{E}+01 \\ & & <2.0 \\ & & <2.0 \mathrm{E}+01 \\ & & <2.0 \mathrm{E}+01\end{array}$

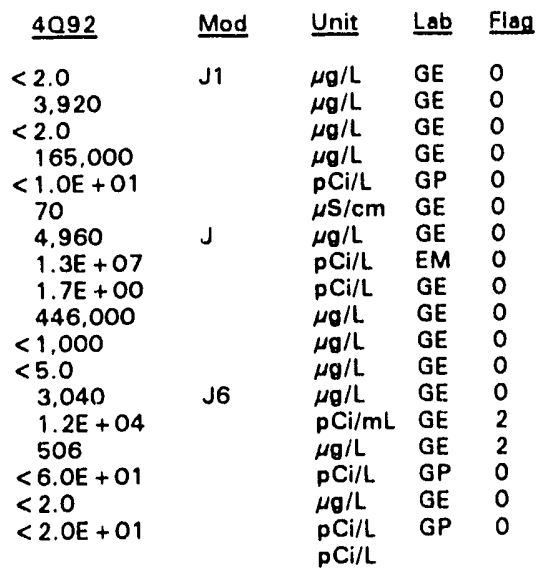

\section{WELL HSB102C}

\begin{tabular}{|c|c|c|c|c|c|c|c|c|c|}
\hline SRS Coord. & Lat/Longitude & Screen Zone Elevation & Top of Casing & Casing & Pump & \multicolumn{4}{|c|}{ Formation } \\
\hline $\begin{array}{l}\text { N71960.1 } \\
\text { E58399.7 }\end{array}$ & $\begin{array}{l}33.280213^{\circ} \mathrm{N} \\
81.652803^{\circ} \mathrm{W}\end{array}$ & $176.7-166.7 \mathrm{ft} \mathrm{ms}$ & $259 \mathrm{ft} \mathrm{msl}$ & 4" PVC & $\mathbf{s}$ & \multicolumn{4}{|c|}{ Barnwell (ItB, } \\
\hline \multicolumn{2}{|c|}{ SAMPLE DATE } & $01 / 01 / 92$ & $04 / 14 / 92$ & $07 / 09 / 92$ & $10 / 20 / 92$ & & & & \\
\hline \multicolumn{10}{|c|}{ FIELD DATA } \\
\hline \multicolumn{2}{|r|}{ Analyte } & 1092 & $\underline{2092}$ & 3092 & 4092 & \multicolumn{4}{|l|}{ Unit } \\
\hline & $\begin{array}{l}\text { Water elevation } \\
\text { pH } \\
\text { Sp. conductance } \\
\text { Water temperature } \\
\text { Alkalinity as } \mathrm{CaCO}_{3} \\
\text { Volume purged }\end{array}$ & $\begin{array}{l}224.3 \\
6.0 \\
178 \\
18.0 \\
10 \\
4.0\end{array}$ & $\begin{array}{l}224.6 \\
5.4 \\
184 \\
19.0 \\
11 \\
4.0\end{array}$ & $\begin{array}{l}224.5 \\
5.7 \\
194 \\
20.3 \\
12 \\
4.0\end{array}$ & $\begin{array}{l}224.9 \\
5.9 \\
174 \\
20.4 \\
8 \\
5.8\end{array}$ & \multicolumn{4}{|c|}{$\begin{array}{l}\mathrm{ft} \mathrm{msl} \\
\mathrm{pH} \\
\mu \mathrm{S} / \mathrm{cm} \\
{ }^{\circ} \mathrm{C} \\
\mathrm{mg} / \mathrm{L} \\
\text { Well vol. }\end{array}$} \\
\hline \multicolumn{10}{|c|}{ ANALYTICAL DATA } \\
\hline \multirow[t]{2}{*}{$\underline{H} \quad \underline{D}$} & Analyte & 1092 & $\underline{2092}$ & 3092 & 4092 & Mod & $\underline{\text { Unit }}$ & $\underline{\text { Lab }}$ & Flag \\
\hline & $\begin{array}{l}\text { Aluminum } \\
\text { Antimony } \\
\text { Arsenic } \\
\text { Barium } \\
\text { Cadmium } \\
\text { Calcium } \\
\text { Chloride } \\
\text { Chromium } \\
\text { Cobalt } \\
\text { Copper } \\
\text { Cyanide } \\
\text { Fluoride } \\
\text { Gross alpha } \\
\text { Iron } \\
\text { Lesd } \\
\text { Magnesium } \\
\text { Manganese } \\
\text { Mercury } \\
\text { Nickel }\end{array}$ & $\begin{aligned}< & 20 \\
< & 2.0 \\
< & 2.0 \\
& 22 \\
< & 2.0 \\
& 6.290 \\
& 5.000 \\
< & 4.0 \\
< & 4.0 \\
< & 4.0 \\
< & 5.0 \\
< & 100 \\
< & 2.0 E+00 \\
< & 4.0 \\
< & 3.0 \\
& 1.520 \\
& 57 \\
< & 0.20 \\
< & 4.0\end{aligned}$ & $\begin{aligned} &< 20 \\
&< 2.0 \\
&< 2.0 \\
& 26 \\
&< 2.0 \\
& 6.340 \\
& 5.310 \\
&< 4.0 \\
&< 4.0 \\
&<4.0 \\
&<5.0 \\
&<100 \\
&<2.0 E+00 \\
&<4.0 \\
&<3.0 \\
& 1.800 \\
& 64 \\
& 0.22 \\
&< 4.0\end{aligned}$ & $\begin{aligned}< & 20 \\
< & 2.0 \\
< & 2.0 \\
& 28 \\
< & 2.0 \\
& 7.080 \\
& 5.030 \\
< & 4.0 \\
< & 4.0 \\
< & 4.0 \\
< & 5.0 \\
< & 100 \\
< & 2.0 E+00 \\
< & 4.0 \\
< & 3.0 \\
& 1.890 \\
& 59 \\
< & 0.20 \\
& 4.4\end{aligned}$ & $\begin{array}{rl}< & 20 \\
< & 2.0 \\
<2 & 2.0 \\
& 28 \\
< & 2.0 \\
& 6.450 \\
& 5.110 \\
< & 4.0 \\
< & 4.0 \\
< & 4.0 \\
<5.0 \\
< & 100 \\
< & 2.0 \mathrm{E}+\mathrm{OO} \\
& 5.4 \\
< & 3.0 \\
& 2.030 \\
& 63 \\
& 0.34 \\
& 4.4\end{array}$ & $\mathrm{~J} 2$ & $\begin{array}{l}\mu g / L \\
\mu g / L \\
\mu g / L \\
\mu g / L \\
\mu g / L \\
\mu g / L \\
\mu g / L \\
\mu g / L \\
\mu g / L \\
\mu g / L \\
\mu g / L \\
\mu g / L \\
p C i / L \\
\mu g / L \\
\mu g / L \\
\mu g / L \\
\mu g / L \\
\mu g / L \\
\mu g / L\end{array}$ & $\begin{array}{l}\mathrm{GE} \\
\mathrm{GE} \\
\mathrm{GE} \\
\mathrm{GE} \\
\mathrm{GE} \\
\mathrm{GE} \\
\mathrm{GE} \\
\mathrm{GE} \\
\mathrm{GE} \\
\mathrm{GE} \\
\mathrm{GE} \\
\mathrm{GE} \\
\mathrm{GE} \\
\mathrm{GE} \\
\mathrm{GE} \\
\mathrm{GE} \\
\mathrm{GE} \\
\mathrm{GE} \\
\mathrm{GE}\end{array}$ & $\begin{array}{l}0 \\
0 \\
0 \\
0 \\
0 \\
0 \\
0 \\
0 \\
0 \\
0 \\
0 \\
0 \\
0 \\
0 \\
0 \\
0 \\
2 \\
0 \\
0\end{array}$ \\
\hline
\end{tabular}

Note: Flagging levels, modifiers, and laboratories are for 4 th quarter 1992 data only. See Appendix B for flagging criteria.

- =xceeded holding time for 4th quarter 1992.

- =xceeded final primary drinking water standard for 4th quarter 1992. 
Woll HSB102C continued

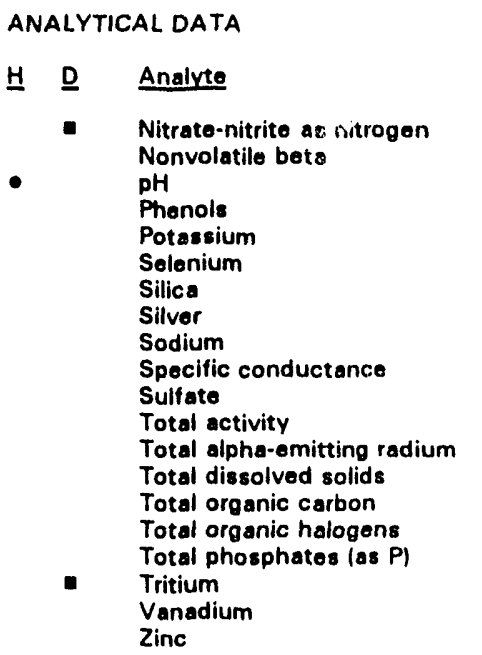

$\begin{array}{lll}1092 & \underline{2092} & \underline{3092} \\ 13.400 & 14.400 & 15.200 \\ 8.9 \mathrm{E}+00 & 6.1 \mathrm{E}+00 & 7.8 \mathrm{E}+00 \\ 5.9 & 5.6 & 6.1 \\ <5.0 & & \\ 2.950 & 3.120 & 2.820 \\ <2.0 & <2.0 & <2.0 \\ 10.300 & 10.100 & 9.800 \\ <2.0 & <2.0 & <2.0 \\ 17.900 & 20.700 & 21.800 \\ 160 & 170 & 152 \\ <1.000 & <1.000 & <1.000 \\ 2.3 \mathrm{E}+05 & & 1.9 \mathrm{E}+05 \\ <1.0 \mathrm{E}+00 & 1.7 \mathrm{E}+00 & <1.0 \mathrm{E}+00 \\ 130.000 & 132.000 & 135.000 \\ <1.000 & <1.000 & <1.000 \\ <5.0 & <5.0 & <5.0 \\ 80 & <50 & <50 \\ 2.2 \mathrm{E}+02 & 2.2 \mathrm{E}+02 & 1.9 \mathrm{E}+02 \\ <8.0 & <8.0 & <8.0 \\ 11 & 13 & 16\end{array}$

$\frac{4092}{}$
14.200
$5.3 \mathrm{E}+00$
5.9
2.380
$<2.0$
10.100
$<2.19$
23.200
150
$<1.000$
$2.0 \mathrm{E}+05$
$<1.0 \mathrm{E}+00$
122.000
$<1.000$
5.0
$<50$
$1.9 \mathrm{E}+02$
$<8.0$
17

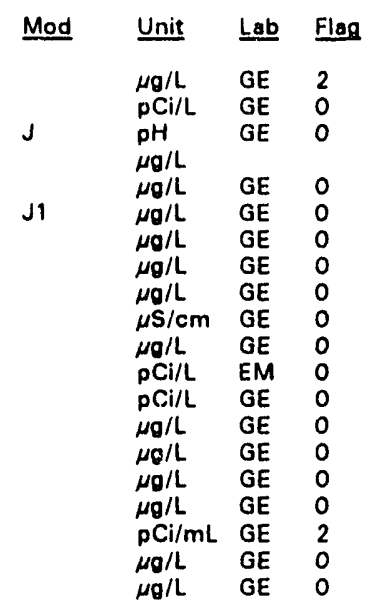

\section{WELL HSB102D}

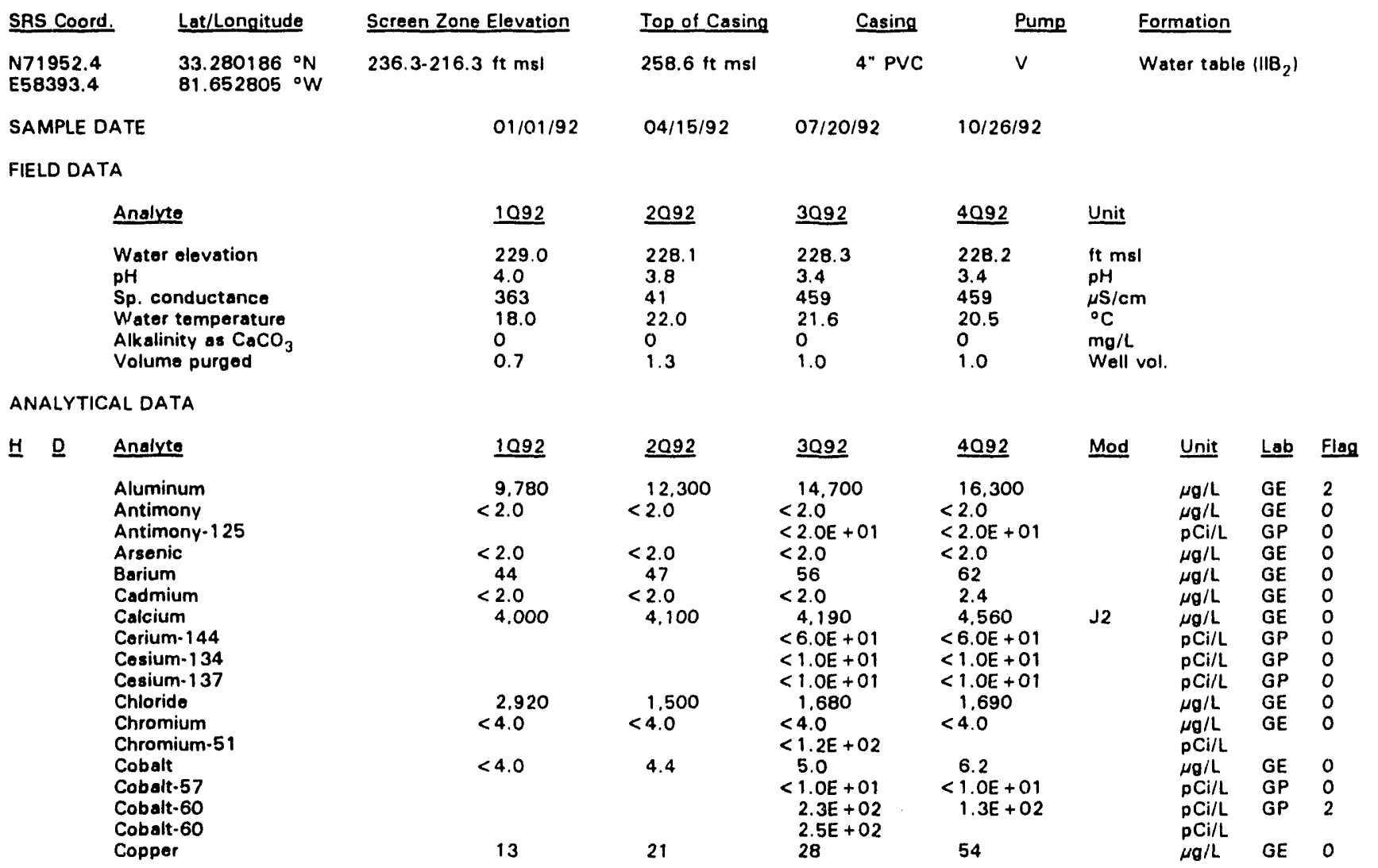

Note: Flagging levels, modifiers, and laboratories are for 4 th quarter 1992 data only. See Appendix 8 for flagging criteria.

- =xceeded holding time for 4 th quarter 1992.

- exceeded final primary drinking water standard for 4th quarter 1992. 
ANALYTICAL DATA

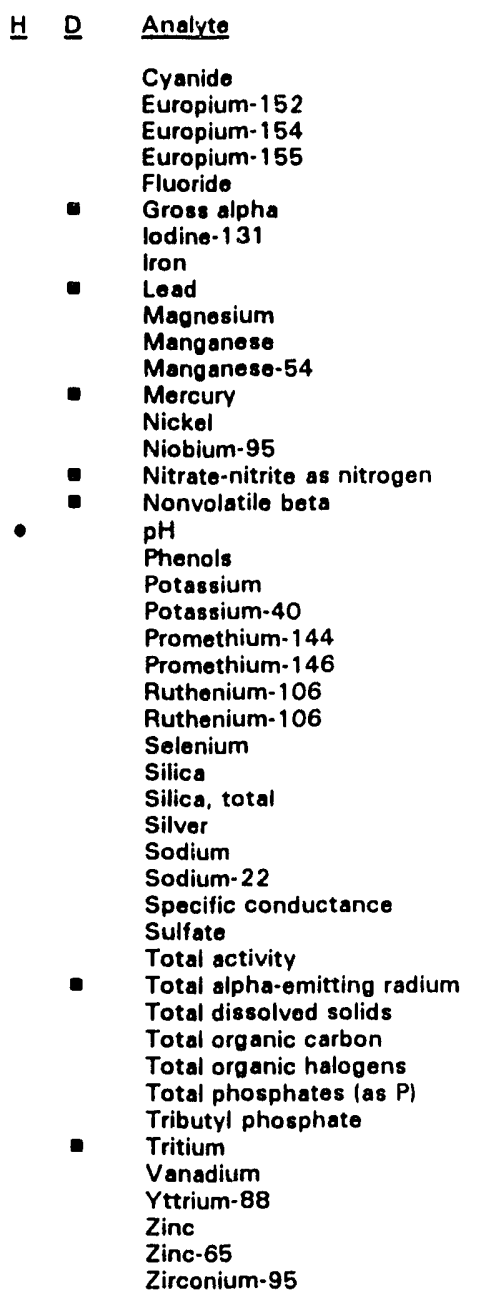

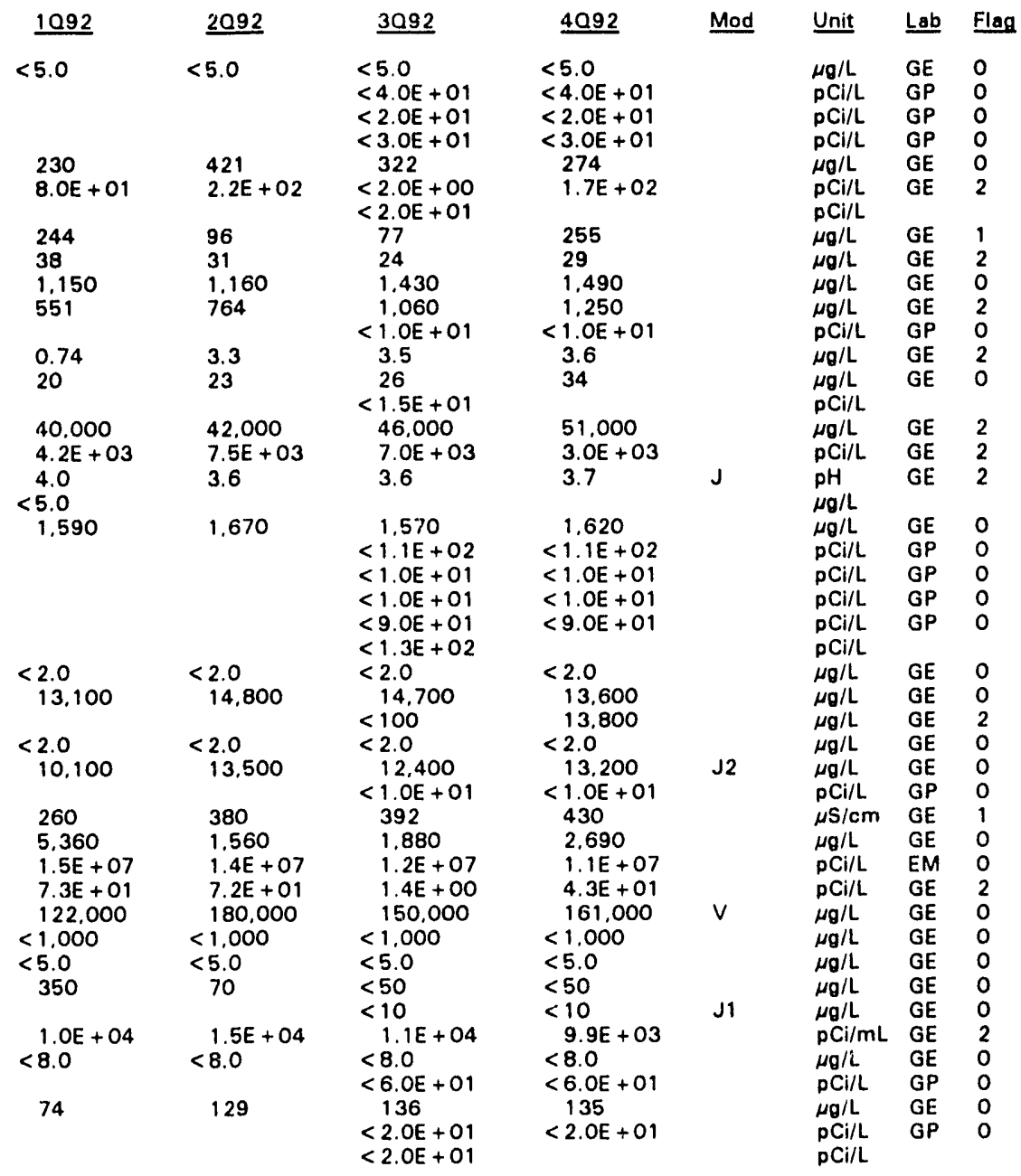

\footnotetext{
Note: Flagging levels, modifiers, and laboratories are for 4 th quarter 1992 data only. See Appendix B for flagging criteria. - = exceeded holding time for 4 th quarter 1992.
}

- =xceeded final primary drinking water standard for 4th quarter 1992. 
WELL HSB 103C

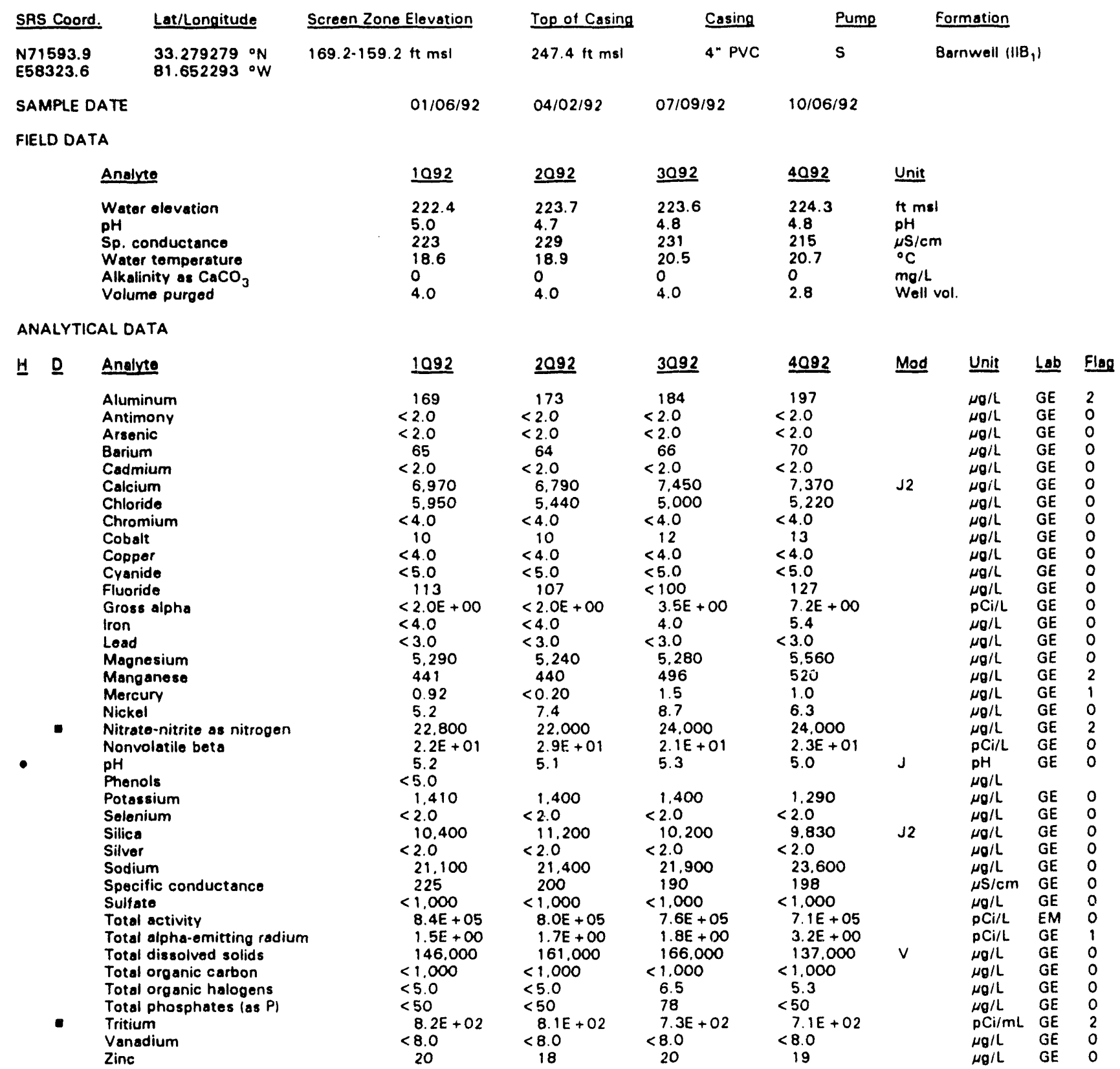

Note: Flagging levels, modifiers, and laboratories are for 4th quarter 1992 data only. See Appendix B for flagging criteria. - = exceeded holding time for 4th quarter 1992.

- =xceeded final primary drinking water standard for 4th quarter 1992. 
WELL HSB103D

\begin{tabular}{|c|c|c|c|c|c|c|}
\hline SRS Coord. & Lat/Longitude & Screen Zone Elevation & Top of Casing & Casing & Pump & Formation \\
\hline $\begin{array}{l}N 71588.1 \\
E 58315.6\end{array}$ & $\begin{array}{l}33.279253{ }^{\circ} \mathrm{N} \\
81.652302{ }^{\circ} \mathrm{W}\end{array}$ & $233.7 .213 .7 \mathrm{ft} \mathrm{ms}$ & $247.6 \mathrm{ft} \mathrm{msl}$ & 4" PVC & V & Water table $\left(11 B_{2}\right)$ \\
\hline SAMPLE DA & & $01 / 06 / 92$ & $04 / 02 / 92$ & $07 / 09 / 92$ & $10 / 14 / 92$ & \\
\hline FIELD DATA & & & & & & \\
\hline & & 1092 & $\underline{2092}$ & 3092 & 4092 & $\underline{\text { Unit }}$ \\
\hline & $\begin{array}{l}\text { elevation } \\
\text { onductance } \\
\text { temperature } \\
\text { nity as } \mathrm{CaCO}_{3} \\
\text { te purged }\end{array}$ & $\begin{array}{l}224.7 \\
4.3 \\
206 \\
20.7 \\
0 \\
4.0\end{array}$ & $\begin{array}{l}225.6 \\
4.0 \\
216 \\
19.9 \\
0 \\
4.0\end{array}$ & $\begin{array}{l}225.6 \\
4.0 \\
209 \\
21.4 \\
0 \\
4.0\end{array}$ & $\begin{array}{l}225.5 \\
4.2 \\
215 \\
21.6 \\
0 \\
4.1\end{array}$ & $\begin{array}{l}\text { ft msl } \\
\mathrm{pH} \\
\mu \mathrm{S} / \mathrm{cm} \\
{ }^{\circ} \mathrm{C} \\
\mathrm{mg} / \mathrm{L} \\
\text { Well vol. }\end{array}$ \\
\hline
\end{tabular}

ANALYTICAL DATA

H D Analyte

Aluminum

Antimony

Antimony-125

Arsenic

Barium

Cadmium

Calcium

Cerium-144

Cesium-134

Cesium-137

Chloride

Chromium

Chromium-5

Cobait

Cobalt-57

Cobalt-60

Copper

Cyanide

Europium-152

Europium-154

Europium-155

Fluoride

- Gross alpha

lodine-131

Iron

Lead

Magnesium

Manganese

Manganese-54

- Marcury

Nickel

Niobium-95

- Nitrate-nitrite as nitrogen

Nonvolatile beta

$\mathrm{pH}$

Phenols

Potassium

Potassium-40

Promethium-144

Promethium-146

Ruthenium.106

Ruthenium-106
1092

682

$<2.0$

$<2.0$

28
$<2.0$

924

4,920

$<4.0$

$<4.0$

$<4.0$

5.0

$<100$

$8.0 E+00$

11
$<3.0$

$<3.0$
1.320

151

2.3
$<4.0$

20.000

4. $3 E+02$

4.4

4.4
$<5.0$
863

$\begin{aligned} & 2092 \\ & 704 \\ &< 2.0 \\ &< 2.0 \\ & 27 \\ &<2.0 \\ & 859\end{aligned}$

859

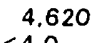

$<4.0$

$<4.0$

$<4.0$

$<5.0$

$<100$

2. $1 \mathrm{E}+0$

10
$<3.0$

1.310

1.8

$<4.0$

20.000

4. $3 E+02$

4.5

843
3092

784

$<2.0$

$<2.0 E+01$

75
32

$<2.0$

1.090

$<6.0 E+01$

$<1.0 E+01$

$<1.0 E+01$

4,180

$<4.0$

$<1.2 \mathrm{E}+02$

$<4.0$

$<1.0 E+01$

$<1.0 E+01$

$<4.0$

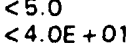

$<4.0 E+01$

$<2.0 E+01$
$<3.0 E+01$

107

$1.5 E+01$

$<2.0 E+01$

15

$<3.0$

1.490

215

$<1.0 E+01$

1.7

$<4.0$

$<1.5 \mathrm{E}+0$

21.000

4. $7 E+02$

4.5

1.000

$<1.1 E+02$

$<1.0 E+01$

$<1.0 E+01$

$<9.0 E+01$

$<1.3 E+02$
4092

1.670

$<2.0$

$<2.0 E+01$

$<2.0$

42
$<2.0$

$\begin{aligned} & 1.230 \\ < & 6.0 E+01\end{aligned}$

$<1.0 E+01$

$<1.0 E+01$

4.160

4.6

$<4.0$

$<1.0 E+01$

$<1.0 E+01$

$<4.0$

$<5.0$

$<4.0 E+01$

$<2.0 E+01$

$<3.0 E+01$

103

$2.5 \mathrm{E}+01$

111

$<3.0$

1.560

267

$<1.0 E+01$

2.8
7.3

20.500

8. $1 E+02$

4.4

1,000

$<1.1 E+02$

$<1$. OE + 01

$<1.0 E+01$

$<9.0 E+01$
Mod Unit Lab Flag

$\mu \mathrm{g} / \mathrm{L} \quad \mathrm{GE} \quad 2$

$\mu \mathrm{g} / \mathrm{L} \quad \mathrm{GE}$

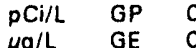

$\mu \mathrm{g} / \mathrm{L}$ GE

$\mu_{g} / L$ GE

$\mu_{g} / L \quad$ GE

$p C i / L \quad G P$

pCi/L GP

$\mu_{g} / L$ GE $O$

$\mu \mathrm{g} / \mathrm{L} \quad \mathrm{GE}$

pCi/L

$\mu \mathrm{g} / \mathrm{L} \quad \mathrm{GE} \quad \mathrm{O}$

PCi/L GP

$\mathrm{pCi} / \mathrm{L}$ GP

$\mu \mathrm{g} / \mathrm{L} \quad \mathrm{GE}$

$\mu g / L \quad G E$

$\mathrm{PCi} / \mathrm{L}$ GP

oCi/L GP

$\mu g / L \quad$ GE

$\mathrm{pCi} / \mathrm{L}$

$\mu g / L \quad G E$ O

$\mu g / L$ GE 0

$\mu g / L$ GE

$\mathrm{PCi} / \mathrm{L}$ GP

$\begin{array}{lll}\mu \mathrm{g} / \mathrm{L} & \mathrm{GE} & 2 \\ \mu \mathrm{g} / \mathrm{L} & \mathrm{GE} & 0\end{array}$

$\mathrm{pCi} / \mathrm{L}$

$\mu \mathrm{g} / \mathrm{L}$ GE 2

$\mathrm{PCi} / \mathrm{L}$ GE 2

$\mathrm{pH}$ GE

rou

$\mu g /$

pCi/L GE

$\mathrm{pCi} / \mathrm{L}$ GP

$\mathrm{pCi} / \mathrm{L}$ GP

PCil GP 0

$\mathrm{PCi} / \mathrm{L}$ GP

Note: Flagging levels, modifiers, and laboratories are for 4th quarter 1992 data only. See Appendix B for flagging criteria.

- =xceeded holding time for 4th quarter 1992.

- exceeded final primary drinking water standard for 4th quarter 1992. 
Well HSB103D continusd

ANALYTICAL DATA

$\begin{array}{ll}\text { H. } & \text { Analvte } \\ \text { Selenium } \\ \text { Silica } \\ \text { Silver } \\ \text { Sodium } \\ \text { Sodium-22 } \\ \text { Specific conductance } \\ \text { Sulfate } \\ \text { Total activity } \\ \text { Total alpha-emitting radium } \\ \text { Total dissolved solids } \\ \text { Total organic carbon } \\ \text { Total organic halogens } \\ \text { Total phosphates las P) } \\ \text { Tritium } \\ \text { Vanadium } \\ \text { Yttrium-88 } \\ \text { Zinc } \\ \text { Zinc-65 } \\ \text { Zirconium-95 }\end{array}$

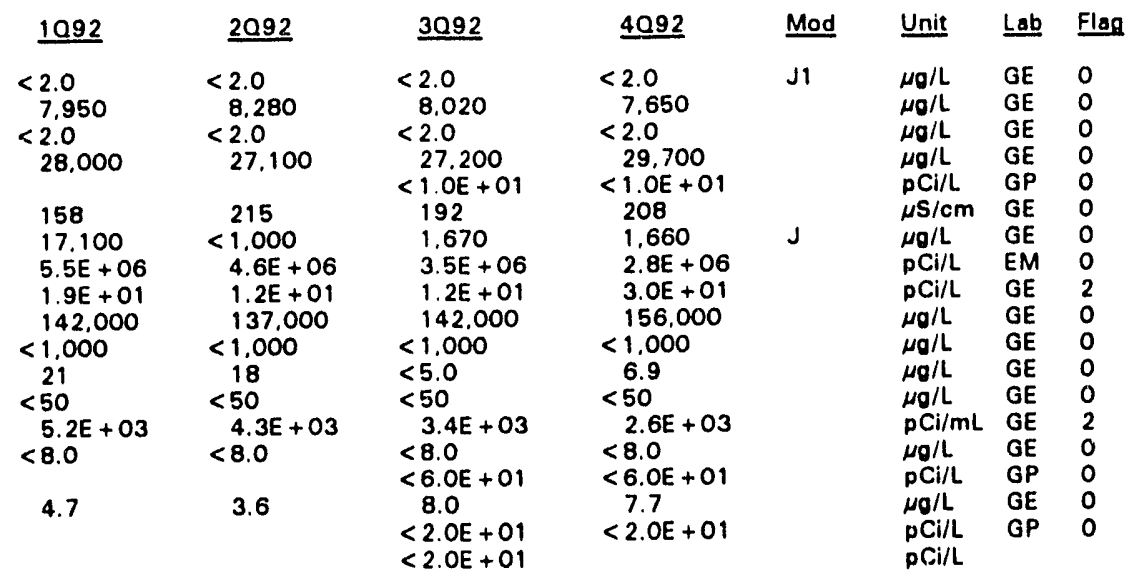

\section{WELL HSB104C}

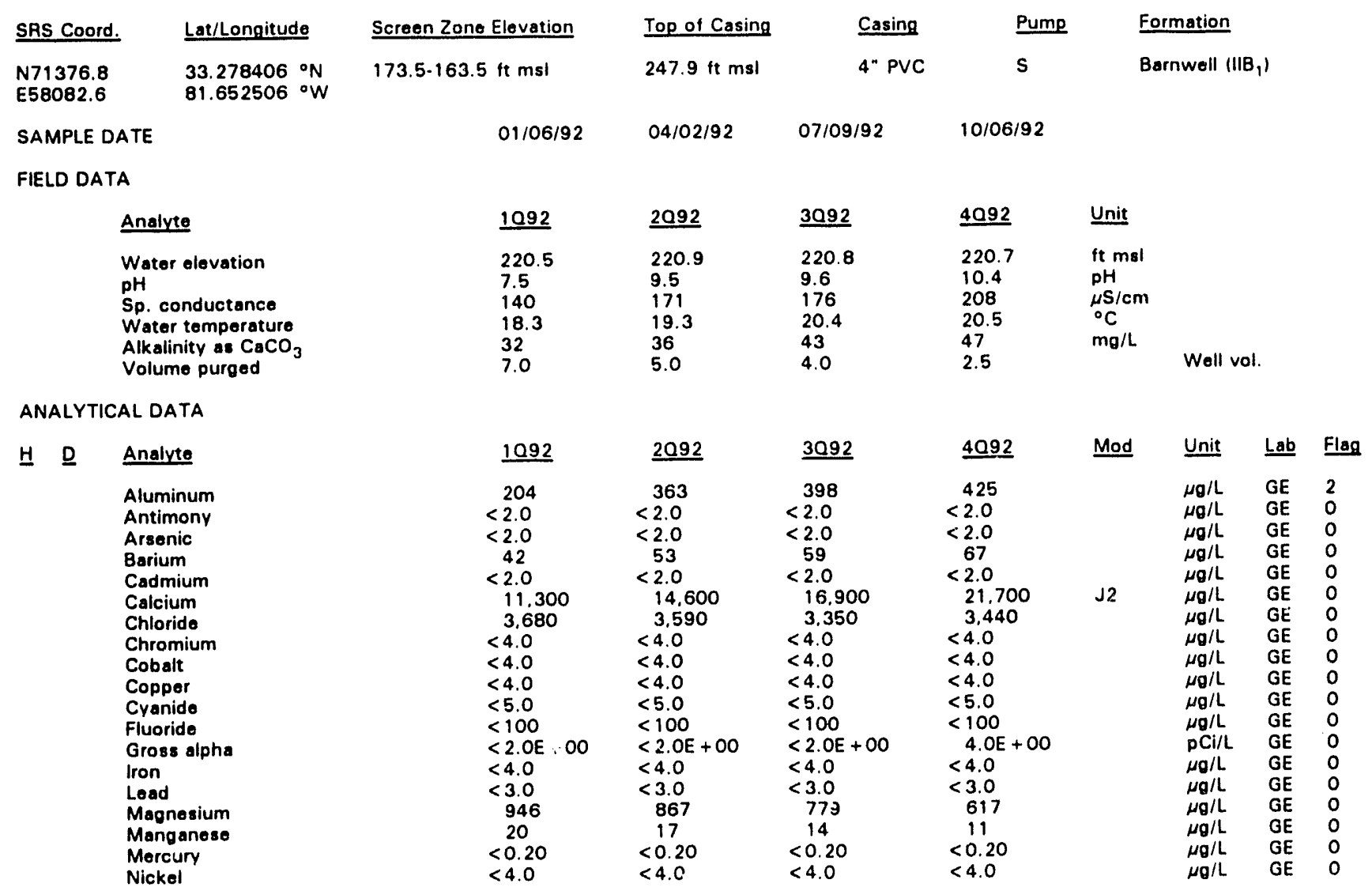

Note: Flagging levels, modifiers, and laboratories are for 4th quarter 1992 data only. See Appendix B for flagging criteria.

- = exceeded holding time for 4th quarter 1992.

- =xceeded final primary drinking water standard for 4th quarter 1992. 


D $\quad$ Analyte
- Nitrate-nitrite as nitrogen
Nonvolatile beta
pH
Phenols
Potassium
Selenium
Silica
Silver
Sodium
Specific conductance
Sulfate
Total activity
Total alpha-omitting radium
Total dissolved solids
Total organic carbon
Total organic halogens
Total phosphates (as P)
Tritium
Vonadium
Zinc

$\begin{array}{lll}1092 & \underline{2092} & \underline{3092} \\ 5.600 & 5.700 & 5.400 \\ 2.9 E+01 & 2.2 \mathrm{E}+01 & 2.4 \mathrm{E}+01 \\ 7.6 & 9.2 & 9.7 \\ <5.0 & & \\ 6.160 & 7.080 & 7.800 \\ <2.0 & <2.0 & <2.0 \\ 12.400 & 13.600 & 12.200 \\ <2.0 & <2.0 & <2.0 \\ 9.440 & 10.300 & 10.600 \\ 135 & 147 & 145 \\ <1.000 & <1.000 & <1.000 \\ 3.0 \mathrm{E}+05 & 2.8 \mathrm{E}+05 & 2.8 \mathrm{E}+05 \\ <1.0 \mathrm{E}+00 & 1.2 \mathrm{E}+00 & <1.0 \mathrm{E}+00 \\ 87.000 & 103,000 & 110.000 \\ <1.000 & <1.000 & <1.000 \\ <5.0 & 7.8 & <5.0 \\ <50 & <50 & <50 \\ 3.0 \mathrm{E}+02 & 2.9 \mathrm{E}+02 & 2.6 \mathrm{E}+02 \\ <8.0 & <8.0 & <8.0 \\ 2.9 & <2.0 & <2.0\end{array}$

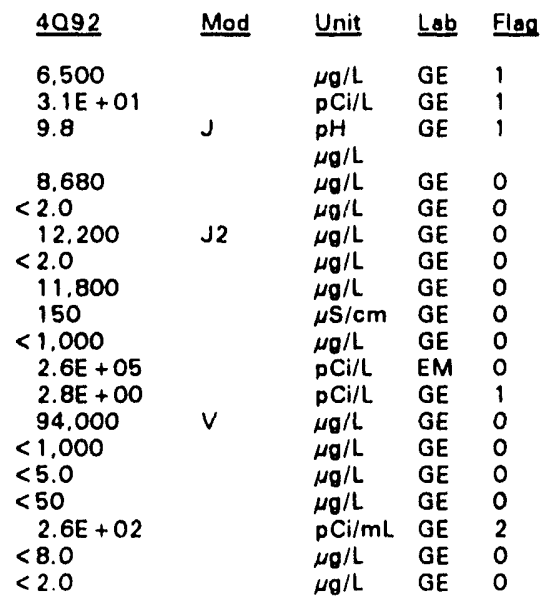

\section{WELL HSB104D}

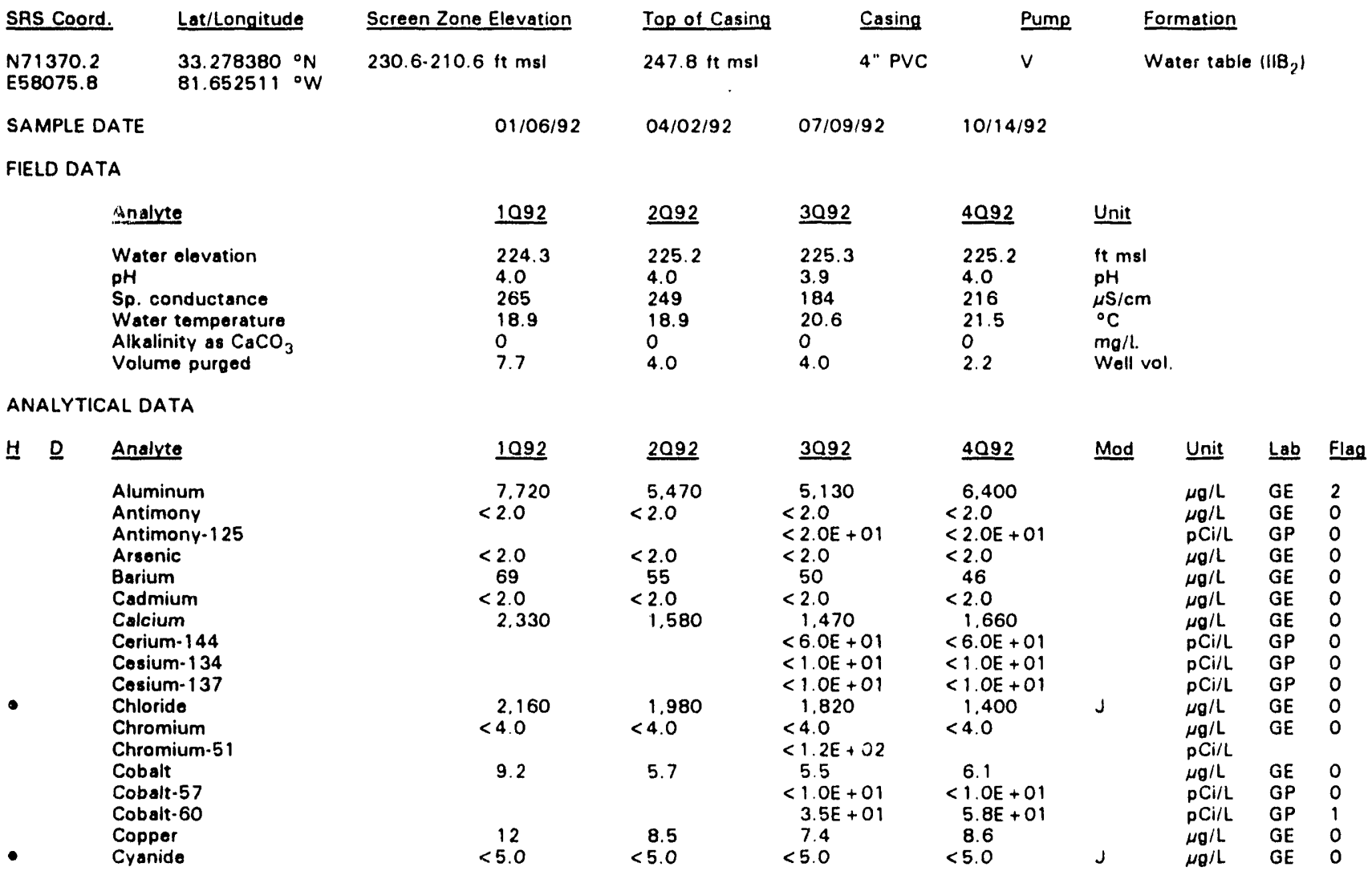

Note: Flagging levels, modifiers, and laboratories are for 4 th quarter 1992 data only. See Appendix B for flagging criteria. - exceeded holding time for 4 th quarter 1992.

- =xceeded final primary drinking water standard for 4th quarter 1992. 
ANALYTICAL DATA

D. $\quad$ Analyte
Europium-152
Europium-154
Europium-155
Fluorido
Gross alpha
lodino-131
Iron
Lead
Magnesium
Manganese
Manganeso-54
Mercury
Nickel
Niobium-95
Nitrate-nitrite as nitrogen
Nonvolatile bota
pH
Phenols
Potassium
Potassium-40
Promothium-144
Promethium-146
Ruthenium-106
Ruthenium-106
Selenium
Silica
Silver
Sodium
Sodium-22
Specific conductance
Sulfate
Total activity
Total alpha-emitting radium
Total dissolved solids
Total organic carbon
Total organic halogens
Total phosphates las PI
Tritium
Vanadium
Yttrium-88
Zinc
Zinc-65
Zirconium-95

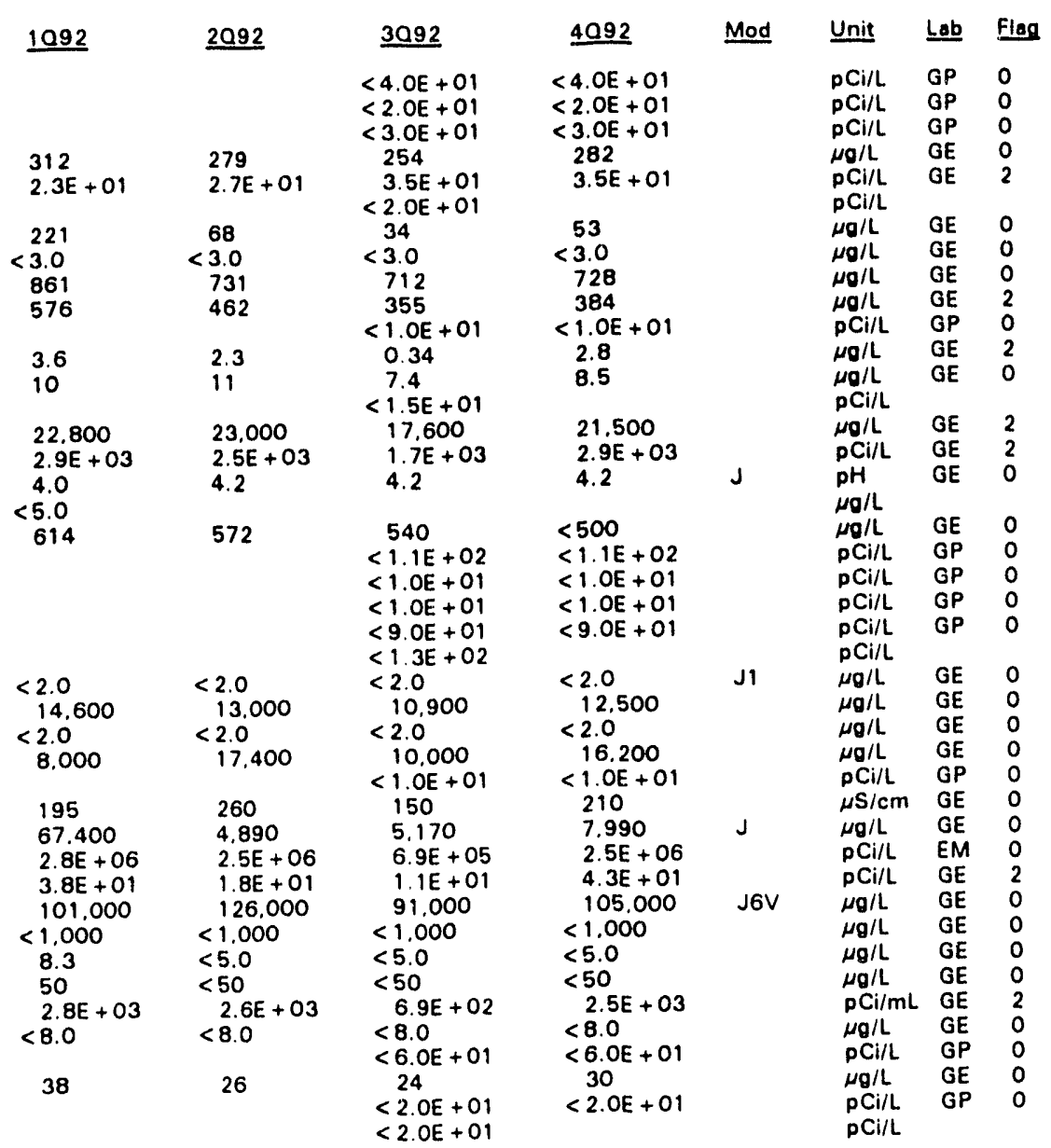

Note: Flagging levels, modifiers, and laboratories are for 4 th quarter 1992 data only. See Appendix B for flagging criteria.

- = exceeded holding time for 4th quarter 1992.

- =xceeded final primary drinking water standard for 4th quarter 1992.

\section{H-Area Seepage Basins}


WELL HSB105C

\begin{tabular}{|c|c|c|c|c|c|c|}
\hline SRS Coord. & Lot/Longitude & Screen Zone Elovation & Top of Casing & Casing & Pump & Formation \\
\hline $\begin{array}{l}N 71447.3 \\
E 57883.8\end{array}$ & $\begin{array}{l}33.278237{ }^{\circ} \mathrm{N} \\
81.653160^{\circ} \mathrm{W}\end{array}$ & $162.2 \cdot 152.2 \mathrm{ft} \mathrm{msl}$ & $249.5 \mathrm{ft} \mathrm{msl}$ & 4" PVC & $\mathbf{s}$ & Barriwell $\left(11 \theta_{1}\right)$ \\
\hline SAMPLE DA & & $01 / 01 / 92$ & $04 / 02 / 92$ & $07 / 10 / 92$ & $10 / 07 / 92$ & \\
\hline FIELD DATA & & & & & & \\
\hline & & 1092 & $\underline{2092}$ & 3092 & 4092 & Unit \\
\hline & $\begin{array}{l}\text {-levation } \\
\text { nductance } \\
\text { temperature } \\
\text { ity as } \mathrm{CaCO}_{3} \\
\text { - purged }\end{array}$ & $\begin{array}{l}219.5 \\
6.2 \\
82 \\
17.8 \\
14 \\
4.0\end{array}$ & $\begin{array}{l}219.9 \\
5.6 \\
87 \\
18.3 \\
11 \\
4.0\end{array}$ & $\begin{array}{l}219.7 \\
5.8 \\
84 \\
20.0 \\
14 \\
4.0\end{array}$ & $\begin{array}{l}219.6 \\
5.9 \\
81 \\
19.9 \\
14 \\
3.8\end{array}$ & $\begin{array}{l}\text { ft mal } \\
\mathrm{pH} \\
\mu \mathrm{S} / \mathrm{cm} \\
{ }^{\circ} \mathrm{C} \\
\mathrm{mg} / \mathrm{L} \\
\text { Well vol. }\end{array}$ \\
\hline
\end{tabular}

ANALYTICAL DATA

\begin{tabular}{|c|c|c|c|c|c|c|c|c|c|}
\hline$\underline{H} \quad \underline{D}$ & Analyte & 1092 & 2092 & 3092 & 4092 & Mod & Unit & Lab & Flag \\
\hline a & 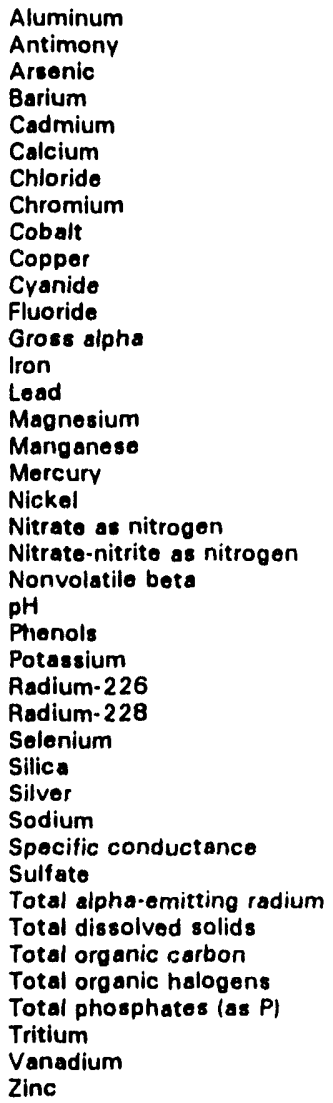 & $\begin{aligned} &< 20 \\
&< 2.0 \\
&< 2.0 \\
& 4.6 \\
&<2.0 \\
& 9.080 \\
& 3.680 \\
&< 4.0 \\
&< 4.0 \\
&< 4.0 \\
&< 5.0 \\
&< 100 \\
& 2.4 E+00 \\
&< 4.0 \\
&< 3.0 \\
& 833 \\
& 3.1 \\
&<0.20 \\
&<4.0 \\
& 3.160 \\
& 3.0 E+00 \\
& 5.9 \\
&< 5.0 \\
& 1.350\end{aligned}$ & $\begin{aligned}< & 20 \\
< & 2.0 \\
< & 2.0 \\
& 6.9 \\
< & 2.0 \\
& 8.550 \\
& 3.490 \\
< & 4.0 \\
< & 4.0 \\
< & 4.0 \\
< & 5.0 \\
< & 100 \\
< & 2.0 \mathrm{E}+00 \\
< & 4.0 \\
< & 3.0 \\
& 843 \\
& 3.1 \\
< & 0.20 \\
< & 4.0 \\
& 3.450 \\
& 1.4 \mathrm{E}+01 \\
& 5.8 \\
& 2.430\end{aligned}$ & 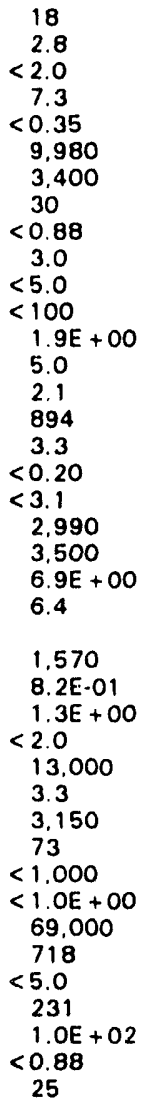 & $\begin{aligned} &< 20 \\
&<2.0 \\
&<2.0 \\
& 4.0 \\
&<2.0 \\
& 9.840 \\
& 3.370 \\
&<4.0 \\
&<4.0 \\
&<4.0 \\
&<5.0 \\
&<100 \\
&<2.0 E+00 \\
& 4.7 \\
&<3.0 \\
& \quad 895 \\
& 3.5 \\
&<0.20 \\
&<4.0 \\
& \\
& 3.650 \\
& 2.0 E+O O \\
& 6.1 \\
& \\
& 1.380\end{aligned}$ & $\mathrm{~J} 2$ & 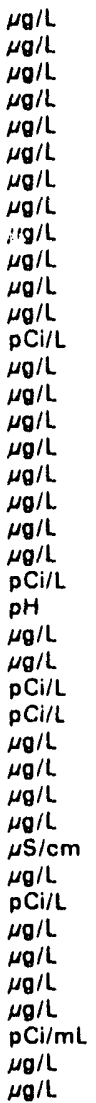 & $\begin{array}{l}G E \\
G E \\
G E \\
G E \\
G E \\
G E \\
G E \\
G E \\
G E \\
G E \\
G E \\
G E \\
G E \\
G E \\
G E \\
G E \\
G E \\
G E \\
G E \\
G E \\
G E \\
G E \\
G E\end{array}$ & $\begin{array}{l}0 \\
0 \\
0 \\
0 \\
0 \\
0 \\
0 \\
0 \\
0 \\
0 \\
0 \\
0 \\
0 \\
0 \\
0 \\
0 \\
0 \\
0 \\
0 \\
0 \\
0 \\
0 \\
0\end{array}$ \\
\hline
\end{tabular}

\footnotetext{
Note: Flagging levels, modifiers, and laboratories are for 4 th quarter 1992 data only. See Appendix $B$ for flagging criteria.

- = exceeded holding time for 4th quarter 1992.

- exceeded final primary drinking water standard for 4th quarter 1992.
} 
WSRC-TR-93.059

\section{WELL HSB105D}

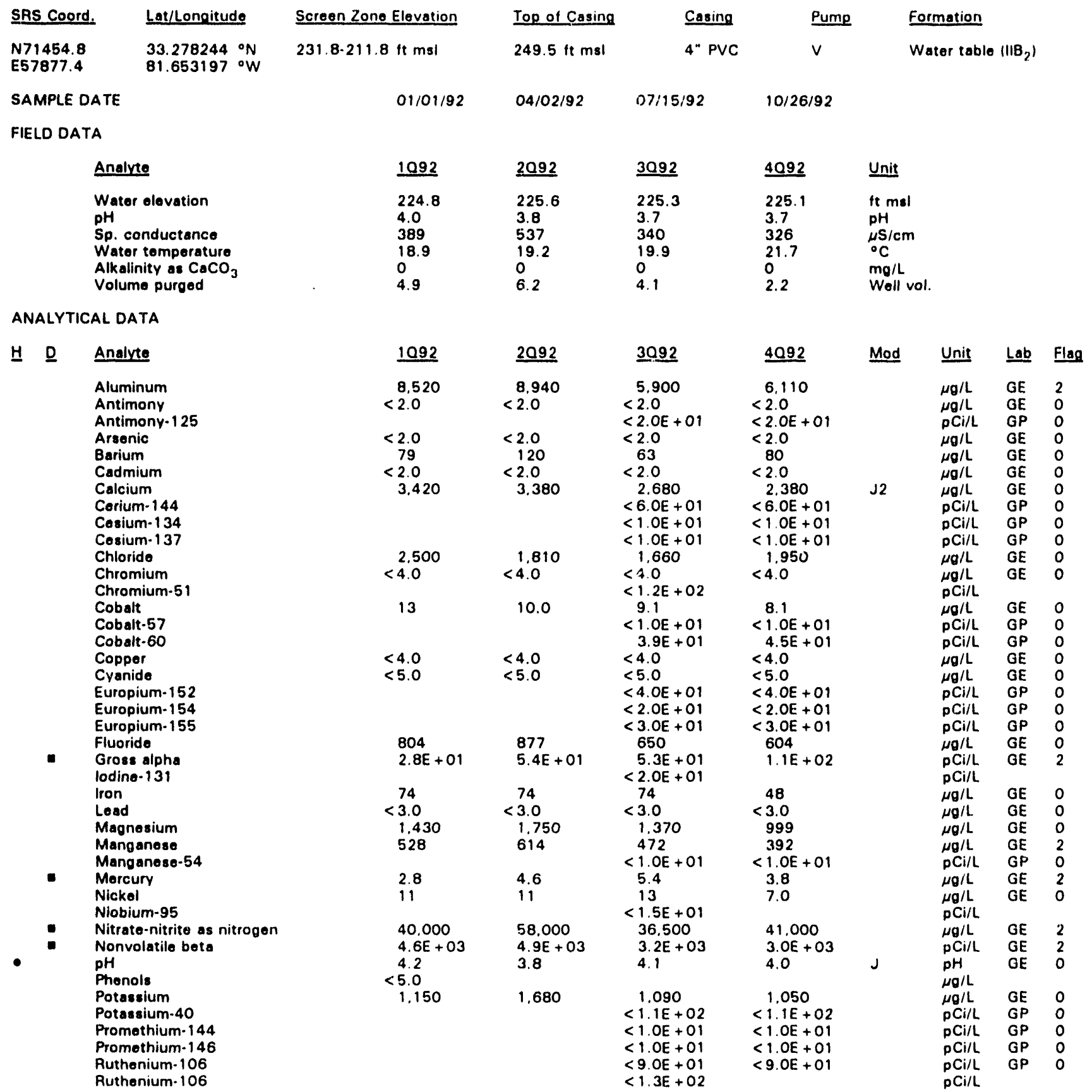

Note: Flagging levels, modifiers, and laboratories are for 4 th quarter 1992 data only. See Appendix B for flagging criteria. - = exceeded holding time for 4 th quarter 1992.

- =xceeded final primary drinking water standard for 4th quarter 1992. 
WSRC-TR-93-059

Well HSB105D continued

ANALYTICAL DATA

H. $\quad$ Analyte
Solenium
Silica
Silver
Sodium
Sodium-22
Specific conductance
Sulfate
Total activity
Total alpha-emitting radium
Total dissolved solids
Total organic carbon
Total organic halogens
Total phosphatos las P)
Tritium
Vanadium
Yttrium-88
Zinc
Zinc-65
Zirconium-95

$\begin{array}{lll}1092 & \underline{2092} & \underline{3092} \\ <2.0 & <2.0 & <2.0 \\ 7.520 & 8.190 & 7.310 \\ <2.0 & <2.0 & <2.0 \\ 22.600 & 40.100 & 28.000 \\ & & <1.0 E+01 \\ 315 & 600 & 280 \\ 20.600 & 2.830 & 3.810 \\ 5.2 E+06 & 8.0 E+06 & 6.2 E+06 \\ 5.4 E+01 & 5.7 E+01 & 2.6 E+01 \\ 157.000 & 287.000 & 163.000 \\ <1.000 & <1.000 & <1.000 \\ 6.1 & 5.7 & <5.0 \\ <50 & <50 & <50 \\ 5.1 E+03 & 7.7 E+03 & 5.5 E+03 \\ <8.0 & <8.0 & <8.0 \\ 77 & 55 & <6.0 E+01 \\ 77 & & 45 \\ & & <2.0 E+01 \\ & & <2.0 E+01\end{array}$

\begin{tabular}{|c|c|c|c|c|}
\hline 4092 & Mod & Unit & Lab & Flag \\
\hline$<2.0$ & & $\mu g / L$ & GE & 0 \\
\hline 7.370 & & $\mu g / L$ & $\mathrm{GE}$ & 0 \\
\hline$<2.0$ & & $\mu g / L$ & GE & 0 \\
\hline 35,600 & J2 & $\mu \mathbf{g} / \mathrm{L}$ & GE & 0 \\
\hline $\begin{array}{l}<1.0 E+01 \\
320\end{array}$ & & $\begin{array}{l}\mathrm{pCi} / \mathrm{L} \\
\mu \mathrm{S} / \mathrm{cm}\end{array}$ & $\begin{array}{l}\text { GP } \\
G E\end{array}$ & $\begin{array}{l}0 \\
1\end{array}$ \\
\hline 4,480 & & $\mu \mathrm{g} / \mathrm{L}$ & $\mathrm{GE}$ & 0 \\
\hline $5.6 E+06$ & & $\mathrm{pCi} / \mathrm{L}$ & $E M$ & 0 \\
\hline $3.8 E+01$ & & $\mathrm{pCi} / \mathrm{L}$ & GE & 2 \\
\hline 182,000 & V & $\mu g / L$ & GE & 0 \\
\hline $\begin{aligned} & 1.140 \\
< & 5.0\end{aligned}$ & & $\begin{array}{l}\mu \mathrm{g} / \mathrm{L} \\
\mu \mathrm{g} / \mathrm{L}\end{array}$ & $\begin{array}{l}\mathrm{GE} \\
\mathrm{GE}\end{array}$ & $\begin{array}{l}0 \\
0\end{array}$ \\
\hline $\begin{array}{l}<5.0 \\
<50\end{array}$ & & $\mu g / L$ & GE & 0 \\
\hline $5.0 E+03$ & & $\mathrm{pCi} / \mathrm{mL}$ & $\begin{array}{l}\text { GE } \\
\text { GE }\end{array}$ & $\begin{array}{l}2 \\
0\end{array}$ \\
\hline $\begin{array}{l}<8.0 \\
<6.0 E+01\end{array}$ & & $\begin{array}{l}\mu \mathrm{g} / \mathrm{L} \\
\mathrm{pCi} / \mathrm{L}\end{array}$ & $\begin{array}{l}\text { GE } \\
\text { GP }\end{array}$ & 0 \\
\hline 52 & & $\mu g / L$ & GE & 0 \\
\hline$<2 . \mathrm{OE}$ & & $\begin{array}{l}\mathrm{pCi} / \mathrm{L} \\
\mathrm{pCi} / \mathrm{L}\end{array}$ & GP & 0 \\
\hline
\end{tabular}

\section{WELL HSB106C}

\begin{tabular}{lll} 
SRS Coord. & Lat/Longitude & Scr \\
\cline { 3 - 3 } N71720.9 & $33.278464{ }^{\circ} \mathrm{N}$ & 1 \\
E57651.5 & $81.654309^{\circ} \mathrm{W}$ & \\
SAMPLE DATE & \\
FIELD DATA &
\end{tabular}
Screen Zone Elevation

Top of Casing

$$
01 / 01 / 92
$$

$04 / 14 / 92$

$07 / 15 / 92$

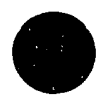

Analyte

Water elovation

$\mathrm{pH}$

Sp. conductance

Water tempeiature

Alkalinity as $\mathrm{CaCO}_{3}$

Volume purged

ANALYTICAL DATA

H D Analyte

Aluminum

Antimony

Arsenic

Barium

Benzene

Bromodichloromethane

Bromoform

Bromomethane (Methyl bromide)

Cadmium

Calcium

Carbon tetrachloride

Chloride

Chlorobenzene

Chloroethane

Chloroethene (Vinyl chloride)

2-Chloroethyl vinyl ether

Chloroform

Chloromethane (Methyl chloride)

Chromium

$\begin{array}{lll}1092 & \underline{2092} & \underline{3092} \\ 221.5 & 221.7 & 221.7 \\ 5.9 & 5.3 & 5.7 \\ 95 & 100 & 97 \\ 18.3 & 19.0 & 19.5 \\ 4 & 4 & 5 \\ 4.0 & 4.0 & 4.0\end{array}$

$10 / 08 / 92$

$\begin{array}{lll}\text { Casing } & \text { Pump } & \text { Formation } \\ \text { 4" PVC } & S & \text { Barnwell (IIB, ) }\end{array}$




\begin{tabular}{|c|c|c|c|c|c|c|c|c|c|c|}
\hline 브 & $\underline{\mathrm{D}}$ & Analyte & 1092 & 2092 & 3092 & 4092 & Mod & Unit & $\underline{\mathrm{Lab}}$ & Flag \\
\hline • & च & 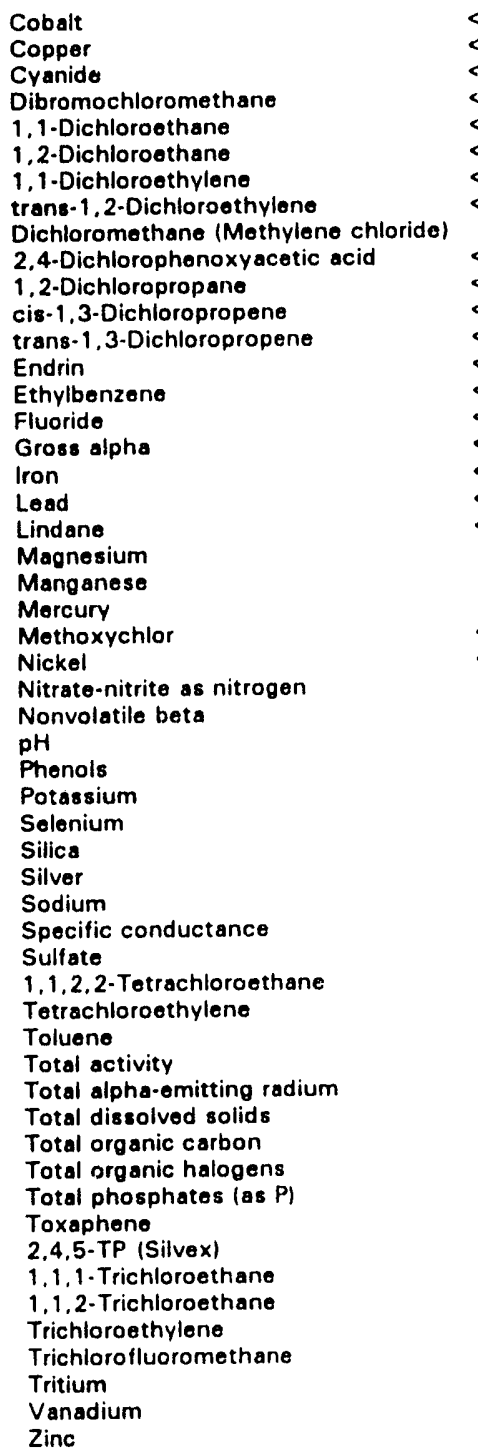 & 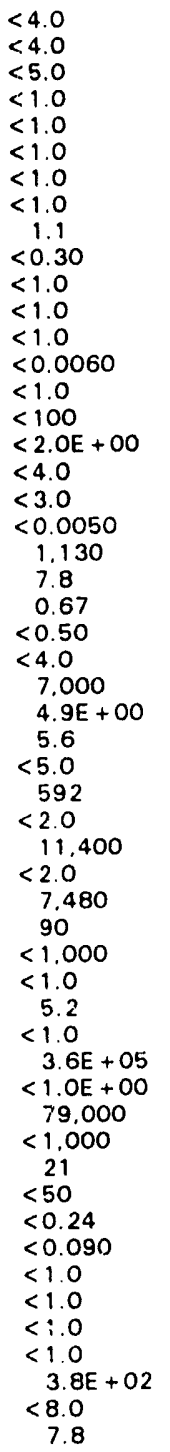 & 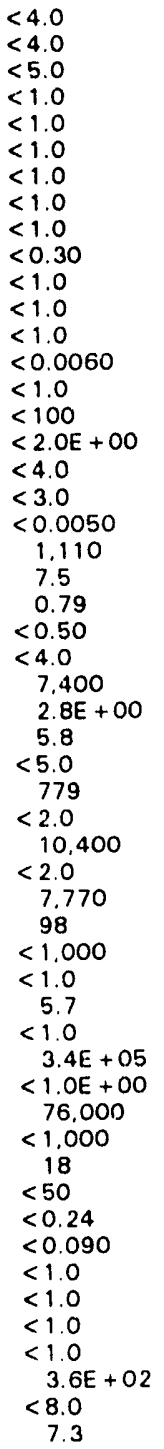 & 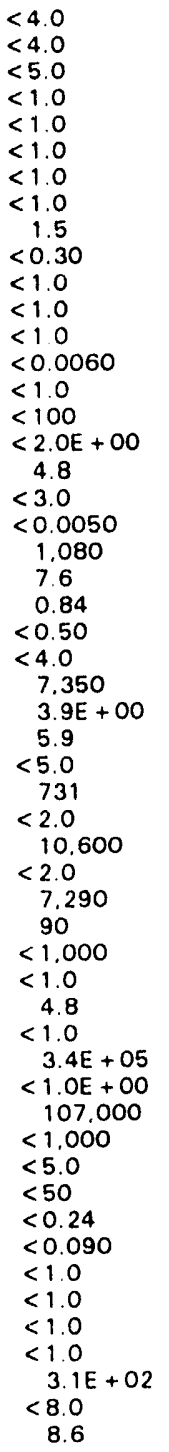 & 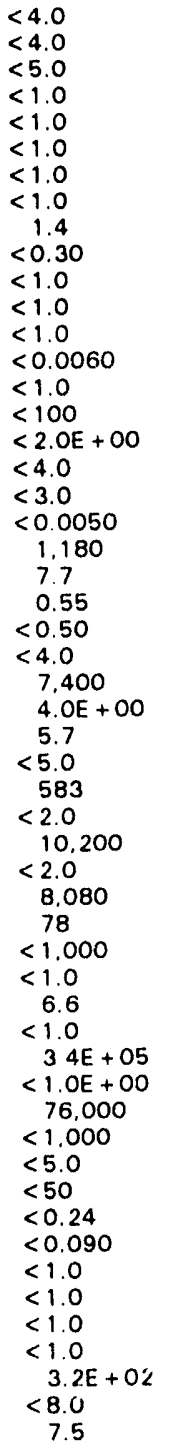 & J6 & $\begin{array}{l}\mu g / L \\
\mu g / L \\
\mu g / L \\
\mu g / L \\
\mu g / L \\
\mu g / L \\
\mu g / L \\
\mu g / L \\
\mu g / L \\
\mu g / L \\
\mu g / L \\
\mu g / L \\
\mu g / L \\
\mu g / L \\
\mu g / L \\
\mu g / L \\
p C i / L \\
\mu g / L \\
\mu g / L \\
\mu g / L \\
\mu g / L \\
\mu g / L \\
\mu g / L \\
\mu g / L \\
\mu g / L \\
\mu g / L \\
p C i / L \\
p H \\
\mu g / L \\
\mu g / L \\
\mu g / L \\
\mu g / L \\
\mu g / L \\
\mu g / L \\
\mu S / C m \\
\mu g / L \\
\mu g / L \\
\mu g / L \\
\mu g / L \\
\mathrm{p} C \mathrm{Ci} / \mathrm{L} \\
\mathrm{pCCi} / \mathrm{L} \\
\mu g / L \\
\mu g / L \\
\mu g / L \\
\mu g / L \\
\mu g / L \\
\mu g / L \\
\mu g / L \\
\mu g / L \\
\mu g / L \\
\mu g / L \\
p C i / m L \\
\mu g / L \\
\mu g / L \\
\mu\end{array}$ & $\begin{array}{l}\mathrm{GE} \\
\mathrm{GE} \\
\mathrm{GE} \\
\mathrm{GE} \\
\mathrm{GE} \\
\mathrm{GE} \\
\mathrm{GE} \\
\mathrm{GE} \\
\mathrm{GE} \\
\mathrm{GE} \\
\mathrm{GE} \\
\mathrm{GE} \\
\mathrm{GE} \\
\mathrm{GE} \\
\mathrm{GE} \\
\mathrm{GE} \\
\mathrm{GE} \\
\mathrm{GE} \\
\mathrm{GE} \\
\mathrm{GE} \\
\mathrm{GE} \\
\mathrm{GE} \\
\mathrm{GE} \\
\mathrm{GE} \\
\mathrm{GE} \\
\mathrm{GE} \\
\mathrm{GE} \\
\mathrm{GE} \\
\mathrm{GE} \\
\mathrm{GE} \\
\mathrm{GE} \\
\mathrm{GE} \\
\mathrm{GE} \\
\mathrm{GE} \\
\mathrm{GE} \\
\mathrm{GE} \\
\mathrm{GE} \\
\mathrm{GE} \\
\mathrm{GE} \\
\mathrm{GE} \\
\mathrm{GE} \\
\mathrm{GE} \\
\mathrm{GE} \\
\mathrm{GE} \\
\mathrm{GE} \\
\mathrm{GE} \\
\mathrm{GE} \\
\end{array}$ & $\begin{array}{l}0 \\
0 \\
0 \\
0 \\
0 \\
0 \\
0 \\
0 \\
0 \\
0 \\
0 \\
0 \\
0 \\
0 \\
0 \\
0 \\
0 \\
0 \\
0 \\
0 \\
0 \\
0 \\
0 \\
0 \\
0 \\
0 \\
0 \\
0 \\
0 \\
1 \\
0 \\
0 \\
0 \\
0 \\
0 \\
0 \\
0\end{array}$ \\
\hline
\end{tabular}

Note: Flagging levels, modifiers, and laboratories are for 4 th quarter 1992 data only. See Appendix 8 for flagging criteria.

- = exceeded holding time for 4th quarter 1992.

- =xceeded final primary drinking water standard for 4th quarter 1992. 
WSRC-TR-9.3-059

\section{WELL HSB 106D}

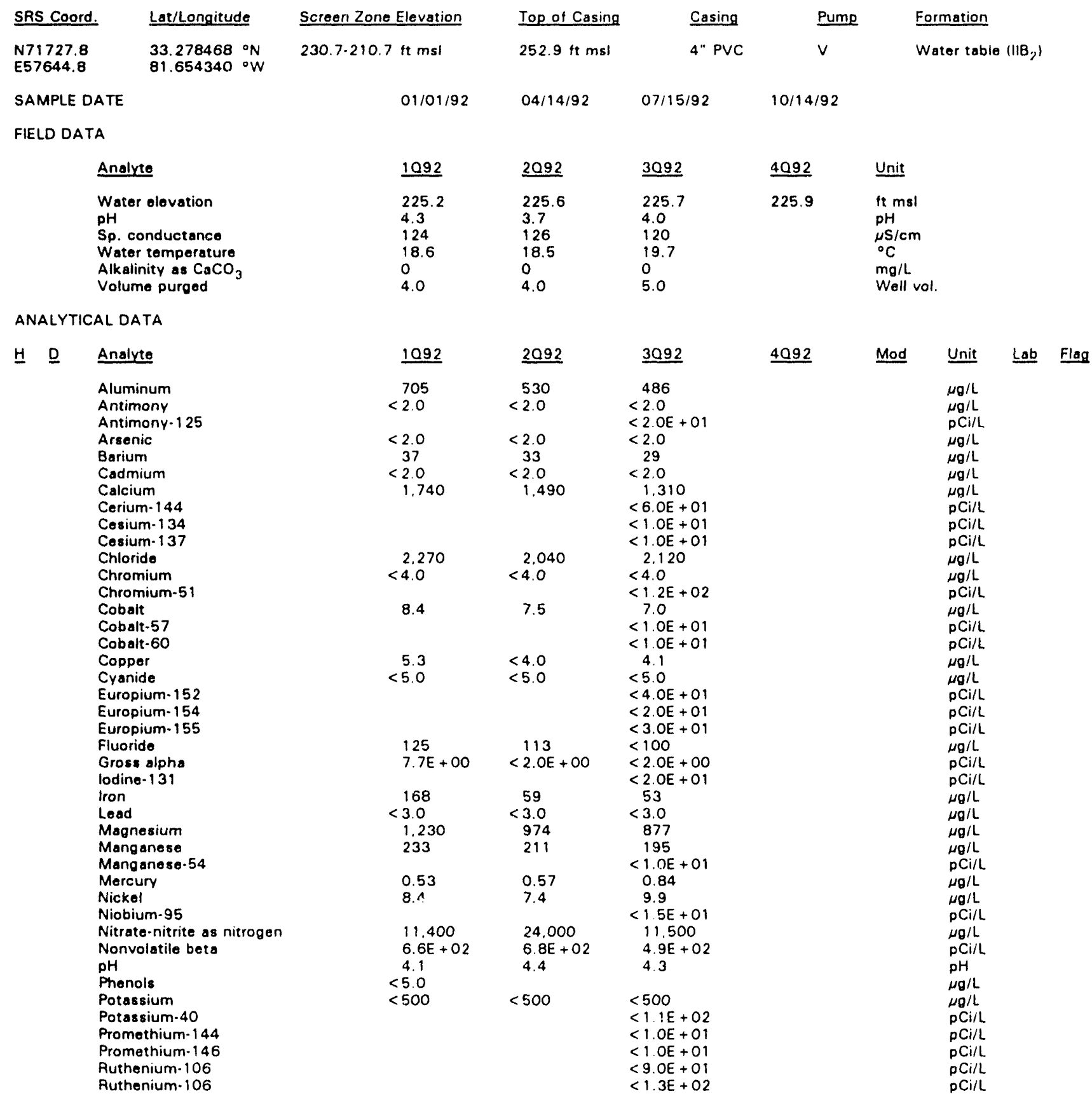

Note: Flagging levels, modifiers, and laboratories are for 4 th quarter 1992 data only. See Appendix B for flagging criteria. - = exceeded holding time for 4 th quarter 1992.

- exceeded final primary drinking water standard for 4ih quarter 1992. 
WSRC-TR-93-059

Well HSB106D continued

ANALYTICAL DATA

H. Analyte
Selerium
Silica
Silver
Sodium
Sodium-22
Specific conductance
Sulfate
Total activity
Total alpha-omitting radium
Total diseolved solids
Total organic carbon
Total organic halogens
Total phosphates las PI
Tritium
Vanadium
Yttrium-88
Zinc
Zinc-65
Zirconium-95

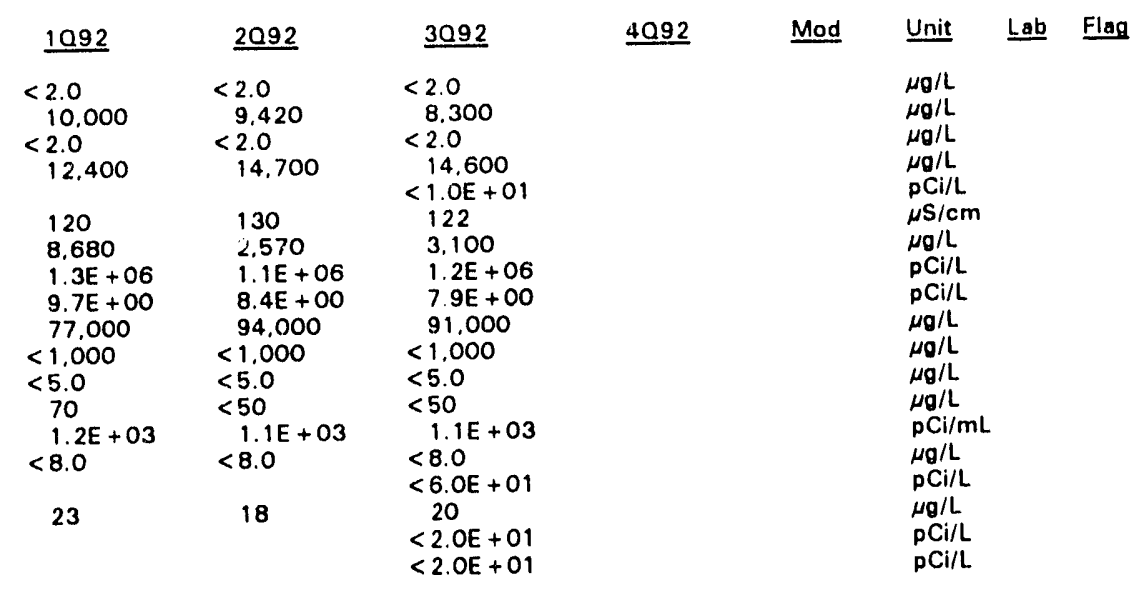

\section{WELL HSB107C}

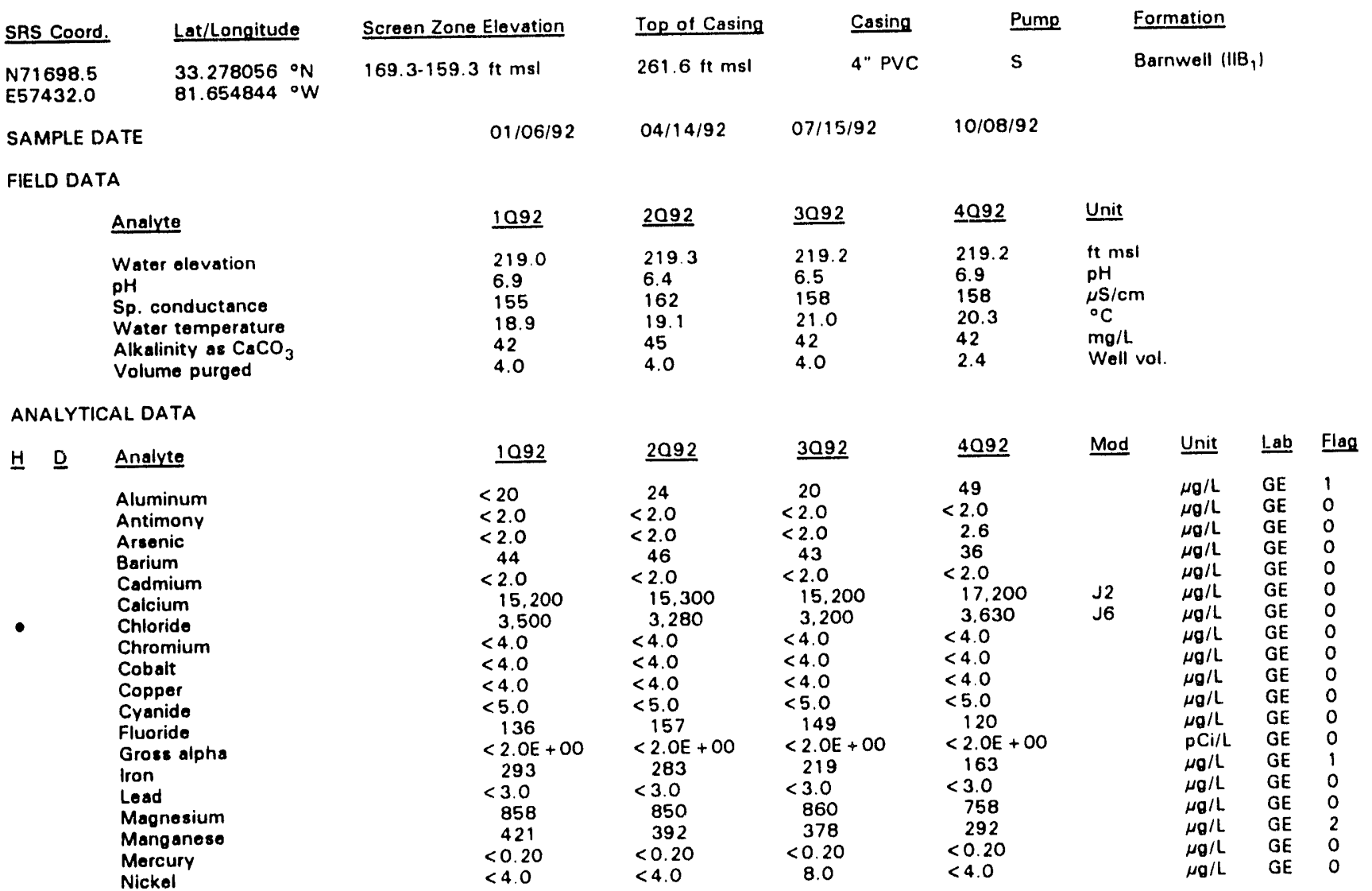

Note: Flagging levels, modifiers, and laboratories are for 4th quarter 1992 data only. See Appendix B for flagging criteria.

- exceeded holding time for 4th quarter 1992.

- =xceeded final primary drinking water standard for 4th quarter 1992.

H-Area Seepage Basins 
Well HSB107C continued

ANALYTICAL DATA

H D $\quad$ Analyte
Nitrate-nitrite as nitrogen
Nonvolatile beta
pH
Phenols
Potassium
Solenium
Silica
Silver
Sodium
Specific conductance
Sulfate
Total activity
Total alpha-emitting radium
Total dissolved solids
Total organic carbon
Total organic halogens
Total phosphates (as P)
Tritium
Vanadium
Zinc

\begin{tabular}{cc}
\hline 1092 & $\underline{2092}$ \\
4.400 & 4.900 \\
$1.2 \mathrm{E}+02$ & $1.3 \mathrm{E}+01$ \\
7.2 & 7.1 \\
$<5.0$ & \\
2.770 & 2.930 \\
$<2.0$ & $<2.0$ \\
10.900 & 11.300 \\
$<2.0$ & $<2.0$ \\
11.200 & 11.000 \\
150 & 155 \\
$<1.000$ & $<1.000$ \\
$4.4 \mathrm{E}+05$ & $4.0 \mathrm{E}+05$ \\
$<1.0 \mathrm{E}+00$ & $<1.0 \mathrm{E}+00$ \\
90.000 & 92.000 \\
$<1.000$ & $<1.000$ \\
$<5.0$ & $<5.0$ \\
200 & 190 \\
$4.4 \mathrm{E}+02$ & $4.2 \mathrm{E}+02$ \\
$<8.0$ & $<8.0$ \\
4.5 & 3.9
\end{tabular}

$\begin{array}{rl} & 3092 \\ & 5.050 \\ 1.1 E+01 & 7.0 \\ & 2.590 \\ < & 2.0 \\ & 10.500 \\ < & 2.0 \\ & 10.100 \\ & 150 \\ < & 1.000 \\ & 3.9 E+05 \\ & 1.2 E+00 \\ & 100.000 \\ < & 1.000 \\ < & 5.0 \\ & 220 \\ & 3.7 E+02 \\ < & 8.0 \\ & 8.5\end{array}$

$\begin{aligned} & 4092 \\ & \text { Mod } \\ & 5.500 \\ & 8.1 \mathrm{E}+00 \\ & 6.8 \\ & \mathrm{~J} \\ & 2.750 \\ &< 2.0 \\ & 9.900 \\ &< 2.0 \\ & 11.600 \\ & 140 \\ & 1.000 \\ & 4.2 \mathrm{E}+05 \\ & 1.5 \mathrm{E}+00 \\ & 116.000 \\ &< 1.000 \\ &<5.0 \\ & 190 \\ & 4.1 \mathrm{E}+02 \\ &< 8.0 \\ & 5.4 \\ &\end{aligned}$

\begin{tabular}{lll} 
Unit & Lab & Flag \\
\cline { 1 - 1 }$\mu \mathrm{g} / \mathrm{L}$ & $\mathrm{GE}$ & 1 \\
$\mathrm{pCi} / \mathrm{L}$ & $\mathrm{GE}$ & 0 \\
$\mathrm{pH}$ & $\mathrm{GE}$ & 0 \\
$\mu \mathrm{g} / \mathrm{L}$ & & \\
$\mu \mathrm{g} / \mathrm{L}$ & $\mathrm{GE}$ & 0 \\
$\mu \mathrm{g} / \mathrm{L}$ & $\mathrm{GE}$ & 0 \\
$\mu \mathrm{g} / \mathrm{L}$ & $\mathrm{GE}$ & 0 \\
$\mu \mathrm{g} / \mathrm{L}$ & $\mathrm{GE}$ & 0 \\
$\mu \mathrm{g} / \mathrm{L}$ & $\mathrm{GE}$ & 0 \\
$\mu \mathrm{S} / \mathrm{cm}$ & $\mathrm{GE}$ & 0 \\
$\mu \mathrm{g} / \mathrm{L}$ & $\mathrm{GE}$ & 0 \\
$\mathrm{pCi} / \mathrm{L}$ & $\mathrm{EM}$ & 0 \\
$\mathrm{pCi} / \mathrm{L}$ & $\mathrm{GE}$ & 0 \\
$\mu \mathrm{g} / \mathrm{L}$ & $\mathrm{GE}$ & 0 \\
$\mu \mathrm{g} / \mathrm{L}$ & $\mathrm{GE}$ & 0 \\
$\mu \mathrm{g} / \mathrm{L}$ & $\mathrm{GE}$ & 0 \\
$\mu \mathrm{g} / \mathrm{L}$ & $\mathrm{GE}$ & 0 \\
$\mathrm{pCi} / \mathrm{mL}$ & $\mathrm{GE}$ & 2 \\
$\mu \mathrm{g} / \mathrm{L}$ & $\mathrm{GE}$ & 0 \\
$\mu \mathrm{g} / \mathrm{L}$ & $\mathrm{GE}$ & 0
\end{tabular}

\section{WELL HSB107D}

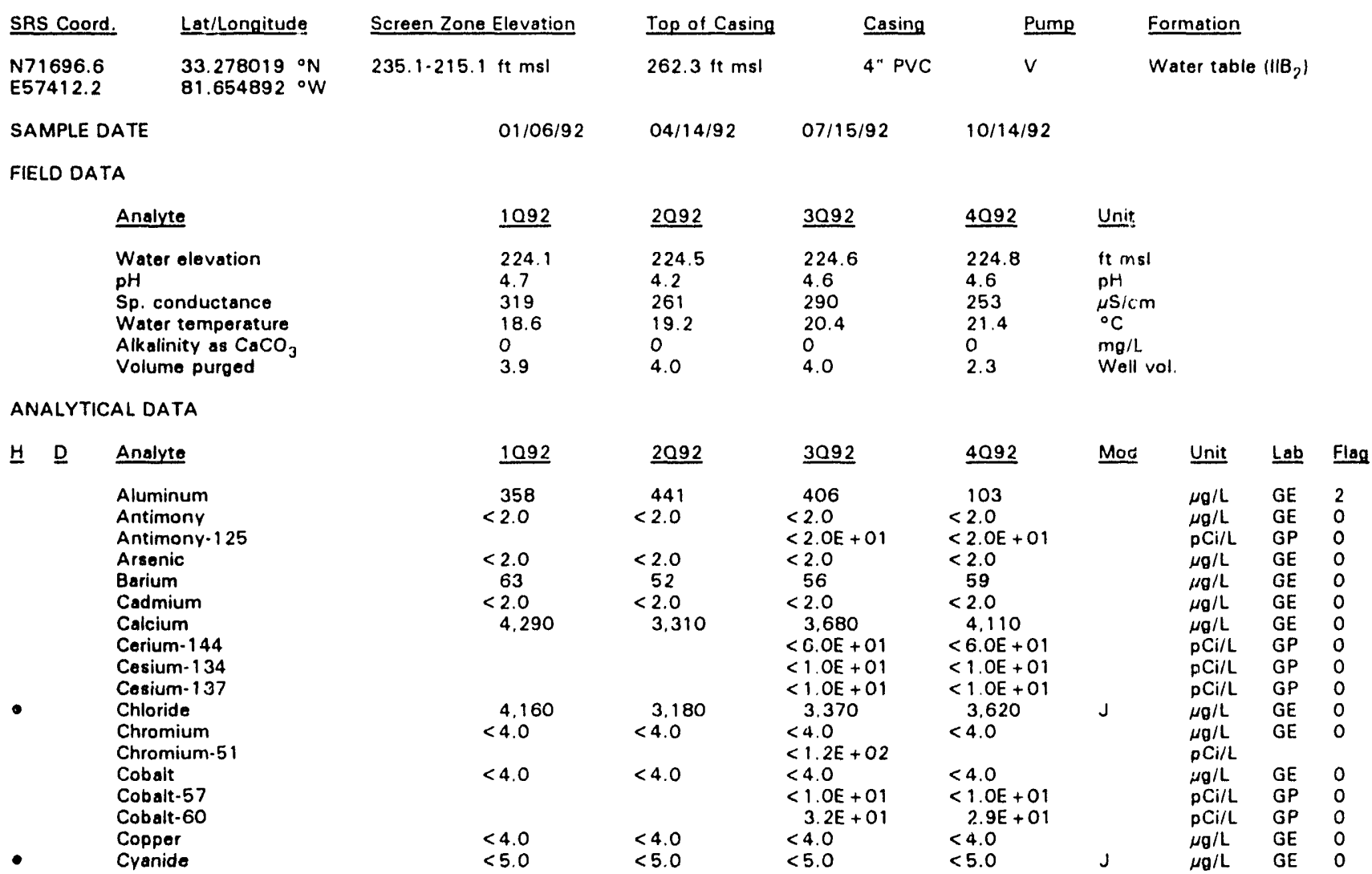

Note: Flagging levels, modifiers, and laboratories are for 4th quarter 1992 data only. See Appendix B for flagging criteria.

- = exceeded holding time for 4th quarter 1992.

- = exceeded final primary drinking water standard for 4th quarter 1992.

H-Area Seepage Basins 
WSRC-TR-93-059

Well HSB107D cuntinued

ANALYTICAL DATA

H D Analyte

Europium-152

Europium-154

Europium- 155

Fluoride

Grose alpha

lodine-131

Iron

Lead

Magnesium

Manganese

Manganese-54

Mercury

Nickol

Niobium-95

- Nitrate-nitrito as nitrogen

Nonvolatilo bots

pH

Phenols

Potassium

Potassium-40

Promethium-144

Promethium-146

Ruthonium-106

Ruthenium-106

Solonium

Silica

Silica, total

Silver

Sodium

Sodium-22

Specific conductance

Total activity

- Total alpha-omitting radium

Total dissolved solids

Total organic carbon

Total organic halogens

Total phosphates (as P)

Tributyl phosphate

Tritium

Vanadium

Yttrium-88

Zinc

Zinc. 65

Zirconium-95
1092

2092

3092

$<4.0 E+01$

$<2.0 E+01$

$<3 . \mathrm{OE}+01$

$<100$

168
$1.7 E+0$

138

$9.0 E+00$

14

$\begin{aligned} & 26 \\ < & 3.0\end{aligned}$

3.150

$<3.0$

2.430

180

1.2
5.0

32.600

$2.0 E+03$

$2.0 \mathrm{E}+$
5.0

$<5.0$

1.290

3.5

4.4

28.000

3. $2 \mathrm{E}+03$

$3.2 \mathrm{E}+$
4.7

1.400

$3.1 E+01$
$<2.0 E+01$

10

$<3.0$

2.930

183

$<1.0 E+01$

2.1

6.1

$<1.5 \mathrm{E}+01$

26.000

3. $2 \mathrm{E}+03$

4.9

1.280

$<1.1 E+02$

$<1, O E+01$

$<1.0 E+01$

$<9$ OE + 01

$<1.3 E+02$

$<2.0$

9.370

$<2.0$

37.900

$<2.0$

9.120

$<2.0$

31.100

225

$6.9 E+06$

730

$<1.000$

4. $3 E+06$

8.1E + 01

207,000

$3.4 E+01$
217.000

$<1,000$

$<5.0$
50

$<1,000$

$<5.0$
60

$6.5 E+03$

$<8.0$

$4.6 E+03$

$<8.0$

16

13
$<2.0$

8.920

9.130

$<2.0$

30.700

$<1.0 \mathrm{E}+01$

198
$<1.000$

3. $7 E+06$

$1.8 E+01$

173,000

$<1.000$

$\begin{aligned} & 9.6 \\ < & 50\end{aligned}$

$<10$

3. $3 E+03$

$<8.0$

$<6.0 E+01$

13

$<2.0 E+01$

$<2.0 E+01$

\section{Q92}

$<4.0 E+01$

$<2.0 E+01$

$<3.0 E+01$

$<100$

5.1E+01

17
$<3.0$

3.420

$<1.0 E+01$

$1.0 E+$
1.9

4.3

24,500

2. $2 \mathrm{E}+\mathrm{O} 3$

5.0

1.150

$<1.1 E+02$

$<1.0 E+01$

$<1.0 E+01$

$<9.0 E+01$

$<2.0$

8.680

8.760

$<2.0$

33,600

$<1.0 E+01$

235

$<1,000$

3. $7 \mathrm{E}+06$

$2.8 E+01$

172.000

$<1.000$

5.2

$<50$

$<10$

$3.5 \mathrm{E}+03$

$<8.0$

$<6 . \mathrm{OE}+01$

11

$<2.0 E+01$
Mod Unit Lab Flag

pCi/L GP 0

$\mathrm{PCi} / \mathrm{L}$ GP 0



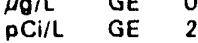

$\mathrm{pCi} / \mathrm{L}$

$\mu \mathrm{g} / \mathrm{L}$

$\mu \mathrm{g} / \mathrm{L}$

$\mu \mathrm{g} / \mathrm{L}$

$\mu \mathrm{pCi} / \mathrm{L}$

$\mu \mathrm{pCi} / \mathrm{L}$

$\mu \mathrm{pCi} / \mathrm{L}$

$\mu \mathrm{g} / \mathrm{L}$ GE 2

$\mathrm{pCi} / \mathrm{L}$ GE 2

$\mathrm{pH}$ GE

$\mu \mathrm{g} / \mathrm{L}$

$\mu \mathrm{g} /$

pCill GE 0

pCi/L GP

pCill GP

pCil GP

$\mathrm{pCi} / \mathrm{L}$

$\mathrm{pCi} / \mathrm{L}$

$\mu \mathrm{g} / \mathrm{L}$ GE

$\mu \mathrm{g} / \mathrm{L}$ GE

$\mu \mathrm{g} / \mathrm{L}$ GE

$\mu \mathrm{g} / \mathrm{L}$ GE 0

$\mu$ Gi/L GE

$\mathrm{PCi} / \mathrm{L}$ GP 0

$\mu S / \mathrm{cm}$ GE

$\mu \mathrm{g} / \mathrm{L}$ GE 0

$\mathrm{pCi} / \mathrm{L}$ GE

$\mu \mathrm{g} / \mathrm{L}$ GE

$\mu g / L \quad G E$

$\mu g / L \quad G \quad G$

$\mu \mathrm{g} / \mathrm{L} \quad \mathrm{GE}$

$\mathrm{pCi} / \mathrm{mL}$ GE 2

GE

$\mu g / L \quad G E$

$\begin{array}{ll}\mathrm{pCi} / \mathrm{L} & \mathrm{GP} \\ \mu \mathrm{g} / \mathrm{L} & \mathrm{GE}\end{array}$

$p \mathrm{Ci} / \mathrm{L}$ GP

Note: Flagging levels, modifiers, and laboratories are for 4th quarter 1992 data only. See Appendix B for flagging criteria. - = exceeded holding time for 4th quarter 1992.

- =xceeded final primary drinking water standard for 4th quarter 1992. 
WSRC-TR-93-059

\section{WELL HSB108C}

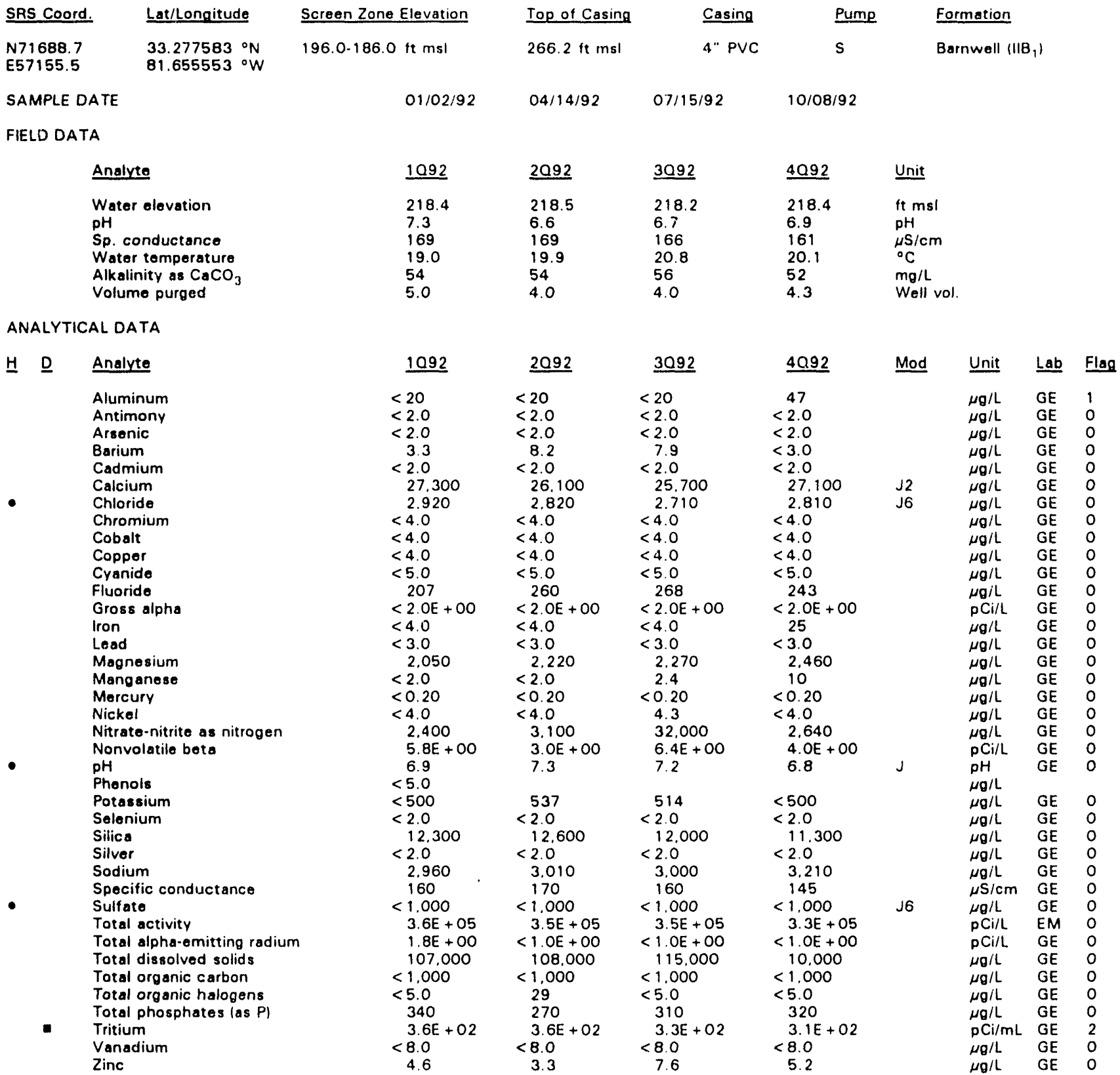

Note: Flagging levels, modifiers, and laboratories are for 4 th quarter 1992 data only. See Appendix $B$ for flagging criteria. - = exceeded holding time for 4 th quarter 1992.

- =xceeded final primary drinking water standard for 4th quarter 1992.

H-Area Seepage Basins

$D .91$

Fourth Quarter 1992 
WSRC-TR-93.059

WELL HSB 108D

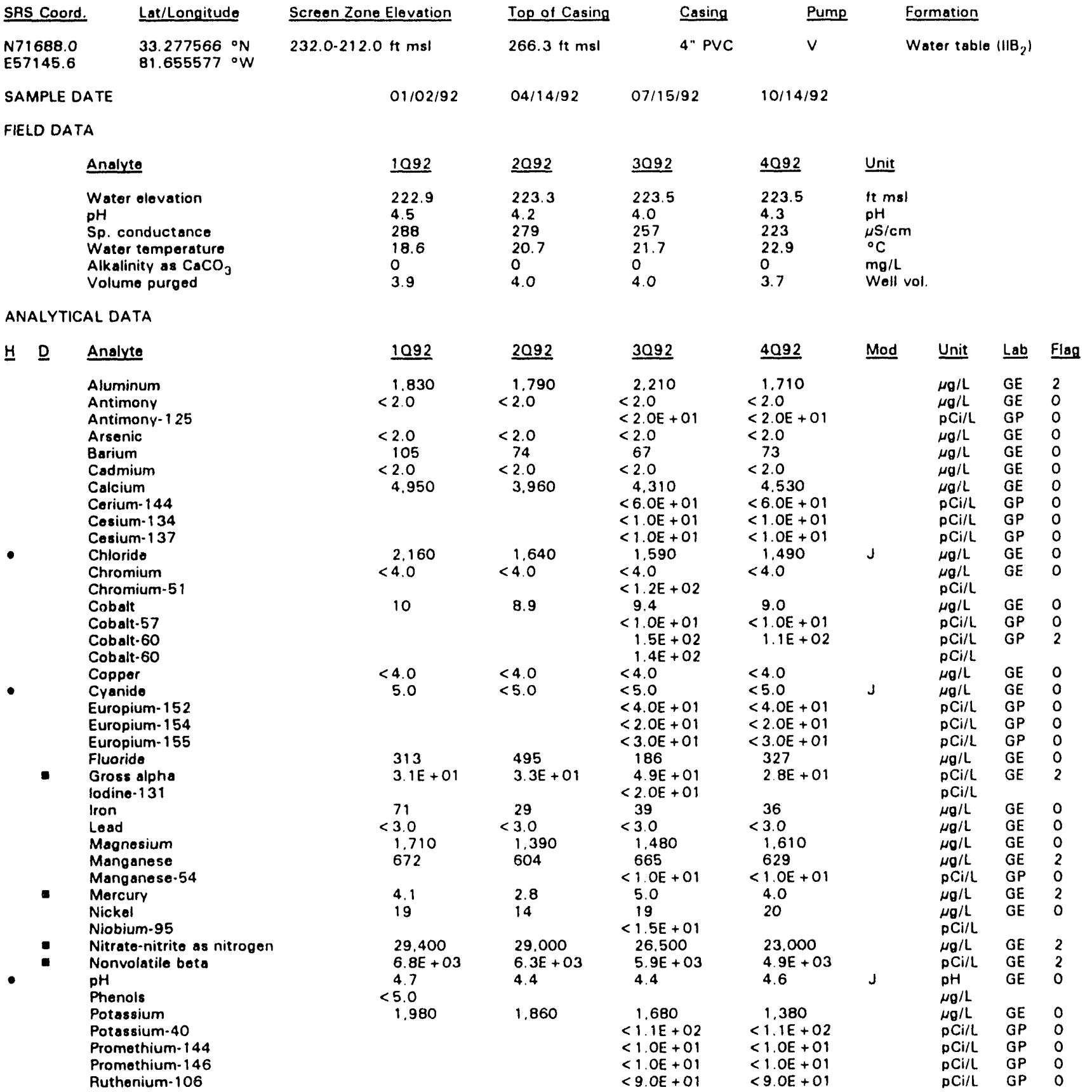

Note: Flagging levels, modifiers, and laboratories are for 4th quarter 1992 data only. See Appendix B for flagging criteria.

- =xceeded holding time for 4th quarter 1992.

- exceeded final primary drinking water standard for 4th quarter 1992.

H-Area Seepage Basins

$D .92$
Fourth Quarter 1992 
WSRC-TR-93-059

Woll HSB108D continued

ANALYTICAL DATA

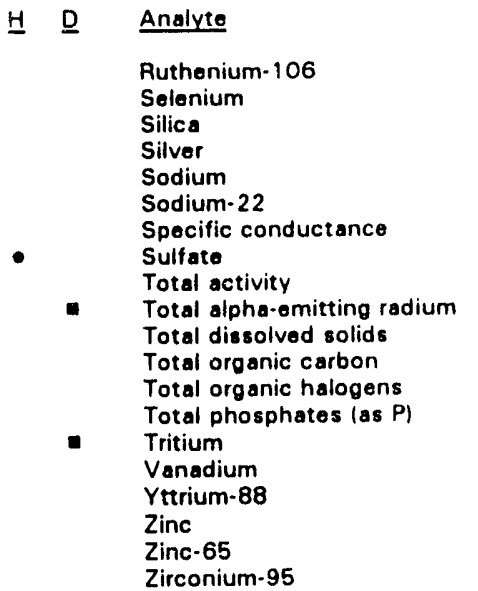

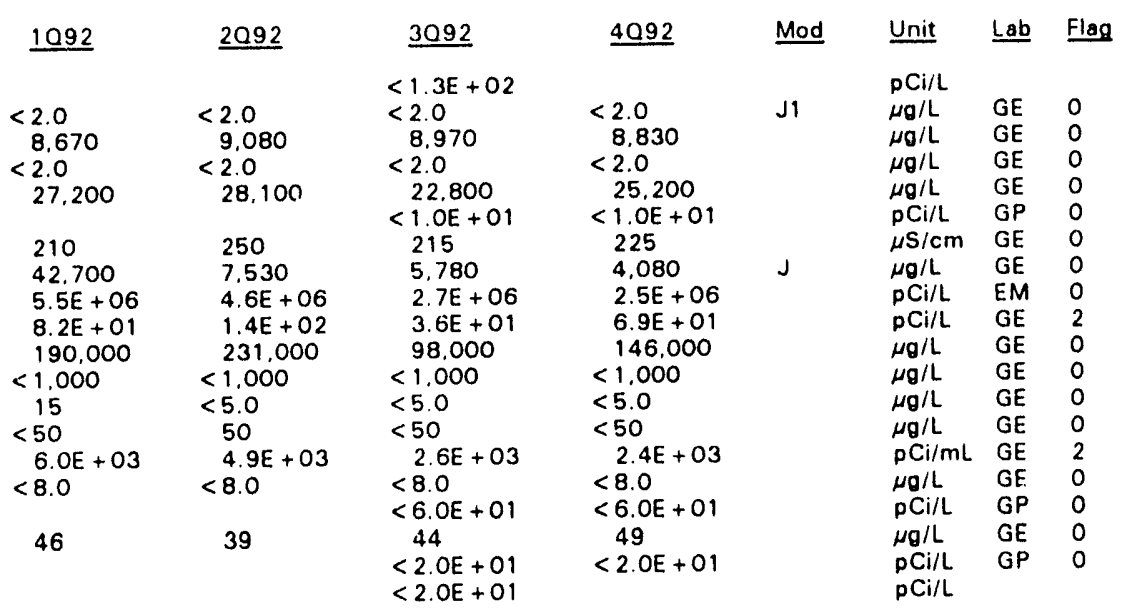

\section{WELL HSB109C}

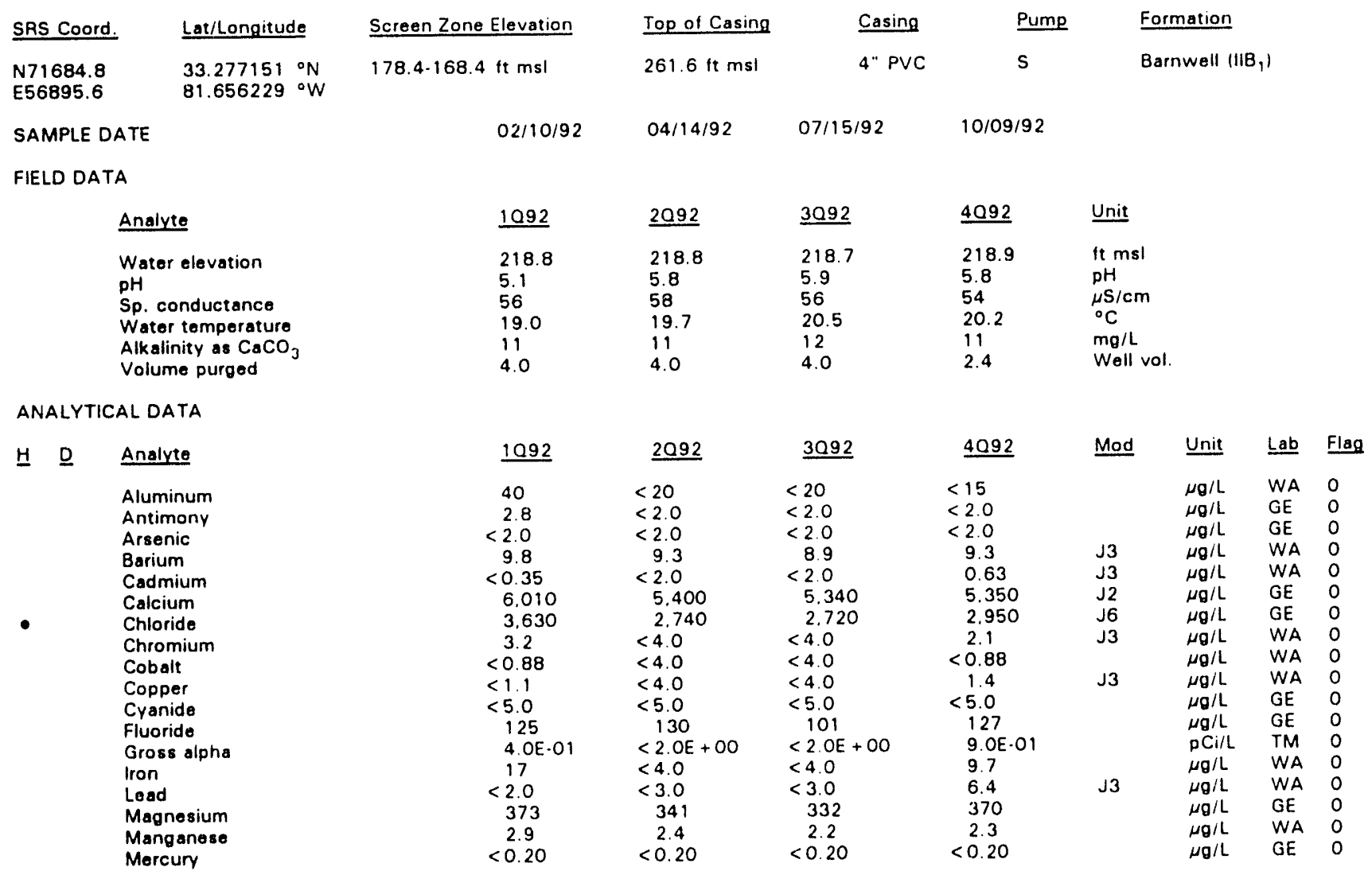

Note: Flagging levels, modifiers, and laboratories are for 4 th quarter 1992 data only. See Appendix B for flagging criteria.

- = exceeded holding time for 4th quarter 1992 .

- =xceeded final primary drinking water standard for 4th quarter 1992.

H-Area Seepage Basins 
WSRC-TR-93.059

Well HSB109C continued

ANALYTICAL DATA

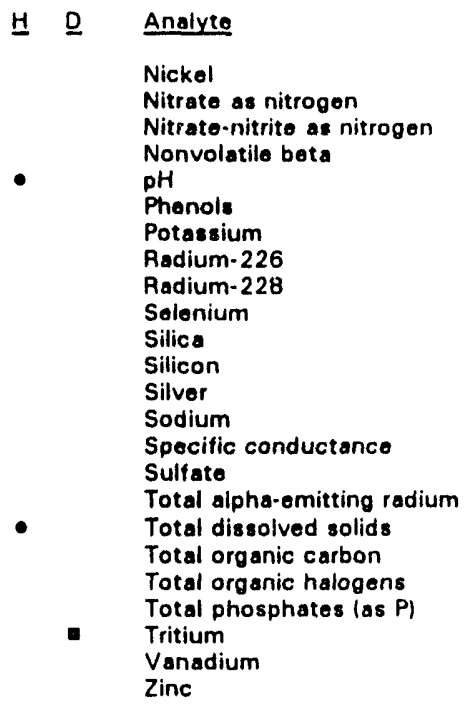

$\begin{array}{lll}1092 & \underline{2092} & \underline{3092} \\ <3.1 & <4.0 & <4.0 \\ 1.570 & & 1.400 \\ & 1.450 & <.0 E+00 \\ 4.4 \mathrm{E}+00 & 2.5 \mathrm{E}+00 & <2.0 \mathrm{l} \\ 6.4 & 6.5 & \\ 9.9 & & \\ 553 & 632 & <500 \\ 3.0 \mathrm{E} \cdot 01 & & \\ 9.0 \mathrm{E} \cdot 01 & & \\ <2.0 & <2.0 & <2.0 \\ 11.400 & 11.000 & 11.100 \\ 5.190 & & \\ <0.70 & <2.0 & <2.0 \\ 4.490 & 4.490 & 4.250 \\ 50 & 52 & 50 \\ <1.000 & <1.000 & <1.000 \\ <1.0 \mathrm{OE}+00 & <1.0 \mathrm{E}+00 & <1.0 \mathrm{E}+00 \\ 40.000 & 43.000 & 43.000 \\ 1.040 & <1.000 & <1.000 \\ 37 & <5.0 & <5.0 \\ 393 & 210 & 522 \\ 7.8 \mathrm{E}+01 & 7.8 \mathrm{E}+01 & 6.9 \mathrm{E}+01 \\ 1.2 & <8.0 & <8.0 \\ 30 & 5.4 & 6.0\end{array}$

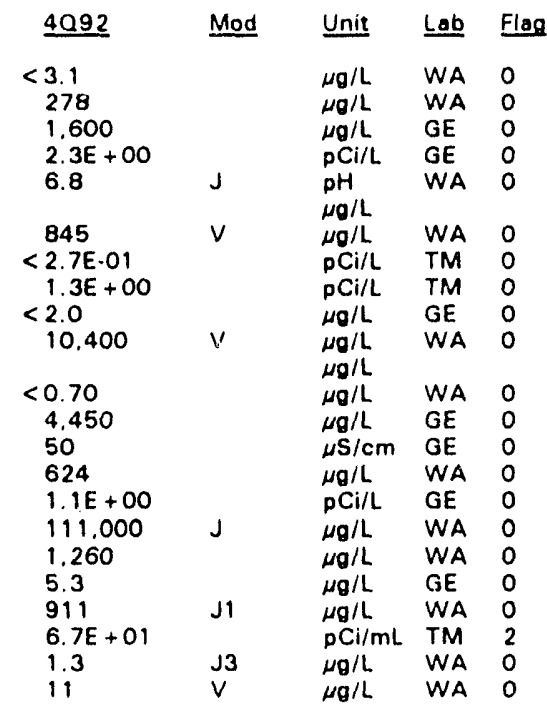

\section{WELL HSB109D}

\begin{tabular}{|c|c|c|c|c|c|c|c|c|c|}
\hline \multicolumn{2}{|r|}{ Lat/Lonnitude } & \multirow{2}{*}{$\frac{\text { Screen Zone Elevation }}{233.0 \cdot 213.0 \mathrm{ft} \mathrm{msl}}$} & Top of Casing & Casing & \multicolumn{3}{|c|}{ Formation } & & \\
\hline $\begin{array}{l}\text { N71685.6 } \\
\text { E56885.5 }\end{array}$ & $\begin{array}{l}33.277136{ }^{\circ} \mathrm{N} \\
81.656257{ }^{\circ} \mathrm{W}\end{array}$ & & $261.2 \mathrm{ft} \mathrm{msl}$ & 4" PVC & S & \multicolumn{4}{|c|}{ Water table $(1 \mid B$, , } \\
\hline \multicolumn{2}{|c|}{ SAMPLE DATE } & $02 / 10 / 92$ & $04 / 14 / 92$ & $07 / 15 / 92$ & $10 / 15 / 92$ & & & & \\
\hline \multicolumn{10}{|c|}{ FIELD DATA } \\
\hline \multicolumn{2}{|r|}{ Anaiyte } & 1092 & 2092 & 3092 & 4092 & \multicolumn{4}{|l|}{ Unit } \\
\hline \multicolumn{2}{|r|}{$\begin{array}{l}\text { Water elevation } \\
\text { pH } \\
\text { Sp. conductance } \\
\text { Water temperature } \\
\text { Alkalinity as } \mathrm{CaCO}_{3} \\
\text { Volume purged }\end{array}$} & $\begin{array}{l}222.3 \\
3.5 \\
76 \\
18.8 \\
0 \\
3.9\end{array}$ & $\begin{array}{l}222.6 \\
4.1 \\
80 \\
20.7 \\
0 \\
4.0\end{array}$ & $\begin{array}{l}222.5 \\
4.0 \\
86 \\
21.8 \\
0 \\
4.0\end{array}$ & $\begin{array}{l}222.6 \\
3.9 \\
92 \\
21.3 \\
0 \\
2.7\end{array}$ & \multicolumn{3}{|c|}{$\begin{array}{l}\mathrm{ft} \mathrm{msl} \\
\mathrm{pH} \\
\mu \mathrm{S} / \mathrm{cm} \\
{ }^{\circ} \mathrm{C} \\
\mathrm{mg} / \mathrm{L} \\
\text { Well vol. }\end{array}$} & \\
\hline \multicolumn{10}{|c|}{ ANALYTICAL DATA } \\
\hline \multirow[t]{2}{*}{$\underline{H} \quad \underline{D}$} & Analyte & 1092 & $\underline{2092}$ & $\underline{3092}$ & 4092 & Mod & Unit & $\underline{L a b}$ & Flag \\
\hline & $\begin{array}{l}\text { Aluminum } \\
\text { Antimony } \\
\text { Antimony-125 } \\
\text { Arsonic } \\
\text { Barium } \\
\text { Cadmium } \\
\text { Calcium } \\
\text { Cerium-144 } \\
\text { Cesium-134 } \\
\text { Cesium-137 } \\
\text { Chloride } \\
\text { Chromium } \\
\text { Chromium-51 } \\
\text { Cobalt }\end{array}$ & $\begin{aligned} & 603 \\
< & 2.0 \\
& <2.0 \\
& 37 \\
< & 2.0 \\
& 1.050\end{aligned}$ & $\begin{aligned} & 715 \\
< & 2.0 \\
& <2.0 \\
& 46 \\
< & 2.0 \\
& 1.230\end{aligned}$ & $\begin{aligned} & 882 \\
< & 2.0 \\
< & 2.0 E+01 \\
< & 2.0 \\
& 57 \\
< & 2.0 \\
& 1.200 \\
< & 6.0 E+01 \\
< & 1.0 E+01 \\
< & 1.0 E+01 \\
& 2.170 \\
< & 4.0 \\
< & 1.2 E+02 \\
& 4.1\end{aligned}$ & $\begin{aligned} & 1.100 \\
< & 2.0 \\
< & 2.0 E+01 \\
< & 2.0 \\
& 73 \\
< & 2.0 \\
& 1.100 \\
< & 6.0 E+01 \\
< & 1.0 E+01 \\
< & 1.0 E+01 \\
& 1.880 \\
< & 4.0 \\
< & 4.0\end{aligned}$ & $\mathrm{~J} 2$ & $\begin{array}{l}\mu g / \mathrm{L} \\
\mu \mathrm{g} / \mathrm{L} \\
\mathrm{pCi} / \mathrm{L} \\
\mu \mathrm{g} / \mathrm{L} \\
\mu \mathrm{g} / \mathrm{L} \\
\mu \mathrm{g} / \mathrm{L} \\
\mu \mathrm{g} / \mathrm{L} \\
\mathrm{pCi} / \mathrm{L} \\
\mathrm{pCi} / \mathrm{L} \\
\mathrm{pCi} / \mathrm{L} \\
\mu g / \mathrm{L} \\
\mu g / \mathrm{L} \\
\mathrm{pCi} / \mathrm{L} \\
\mu \mathrm{g} / \mathrm{L}\end{array}$ & $\begin{array}{l}G E \\
G E \\
G P \\
G E \\
G E \\
G E \\
G E \\
G P \\
G P \\
G P \\
G E \\
G E \\
\text { GE }\end{array}$ & $\begin{array}{l}2 \\
0 \\
0 \\
0 \\
0 \\
0 \\
0 \\
0 \\
0 \\
0 \\
0 \\
0 \\
0\end{array}$ \\
\hline
\end{tabular}

Note: Flagging levels, modifiers, and laboratories are for 4 th quarter 1992 data only. See Appendix $B$ for flagging criteria.

- =xceeded holding time for 4 th quarter 1992

- exceeded final primary drinking water standard for 4th quarter 1992. 
WSRC-TR-93.059

Well HS8109D continued

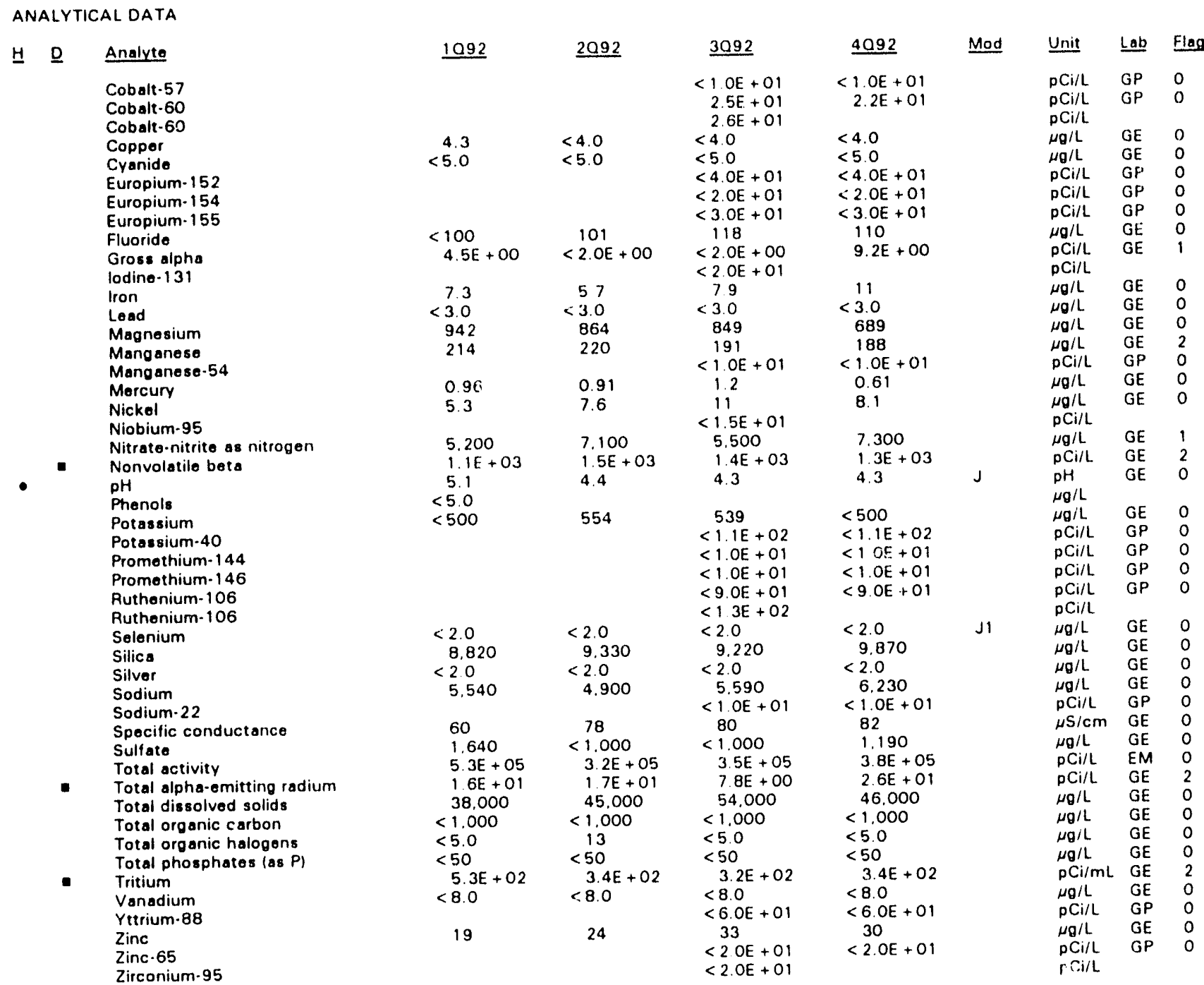

Note: Flagging levels, modifiers, and laboratories are for 4 ih quarter 1992 data only. See Appendix 'j for flagging criteria. $\checkmark=$ exceeded holding time for 4 th quarter 1992.

- exceeded final primary drinkıng water standard for 4th quarter 1992. 
WSRC.TR.93.059

\section{WELL HSB1 10C}

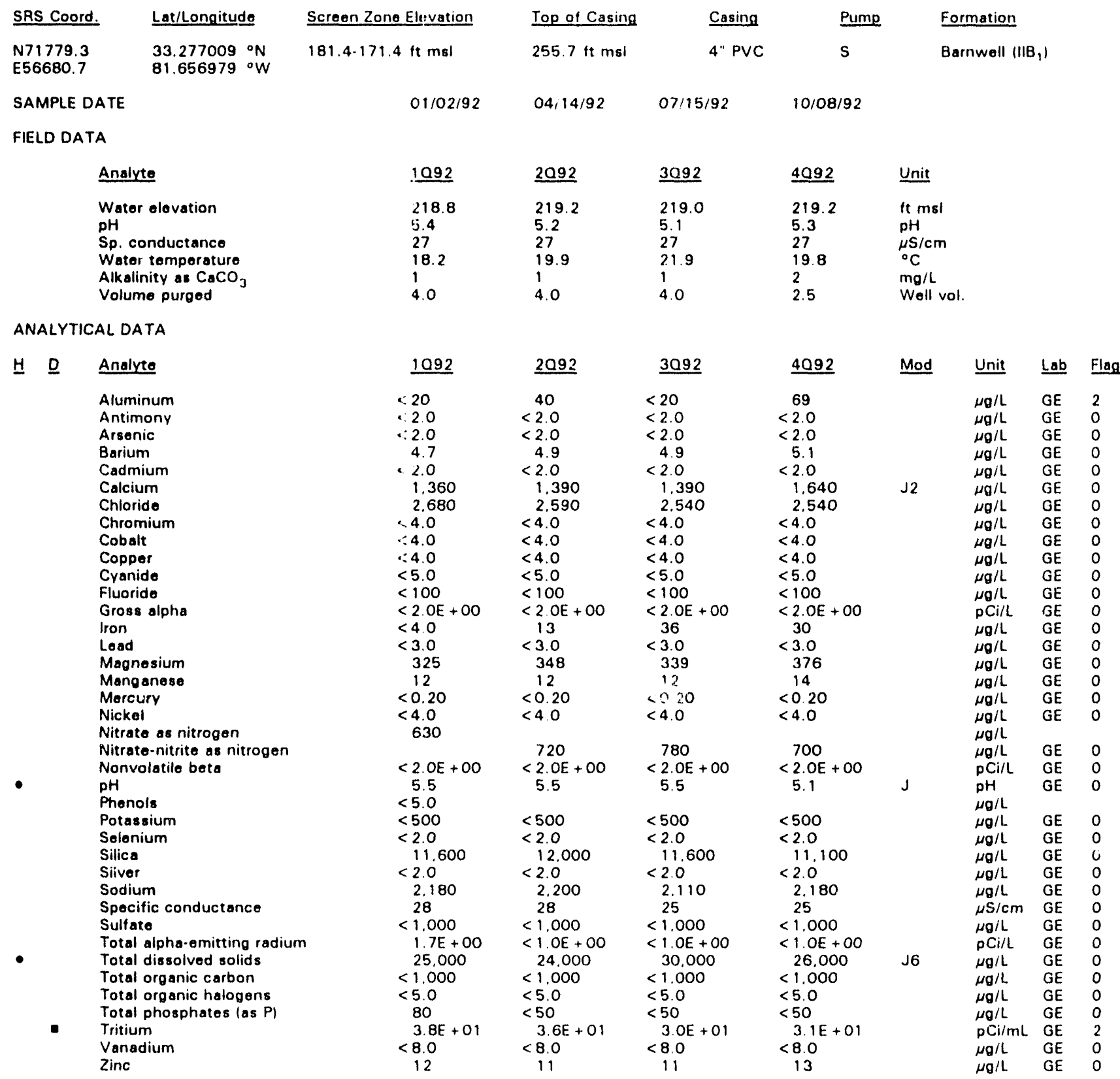

Note: Flagging levels, modifiers, and laboratories are for 4th quarter 1992 data only. See Appendix B for flagging criteria. - = exceeded holding time for 4th quarter 1992.

- exceeded final primary drinking water standard for 4 th quarter 1992. 


\section{WELL HSB110D}

\section{SRS Coord. \\ N71785.2 E56672.1}

SAMPLE DATE

Lat/Longitude

$33.277008^{\circ} \mathrm{N}$ $81.657013^{\circ} \mathrm{W}$

\section{Screen Zone Elevation \\ $231.4 \cdot 211.4 \mathrm{ft} \mathrm{msl}$}

$01 / 02 / 92$

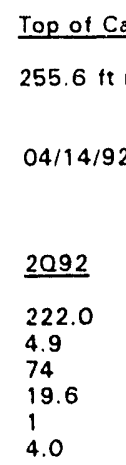

1092
221.6
4.3
97
18.5
0
4.0

ANALYTICAL DATA

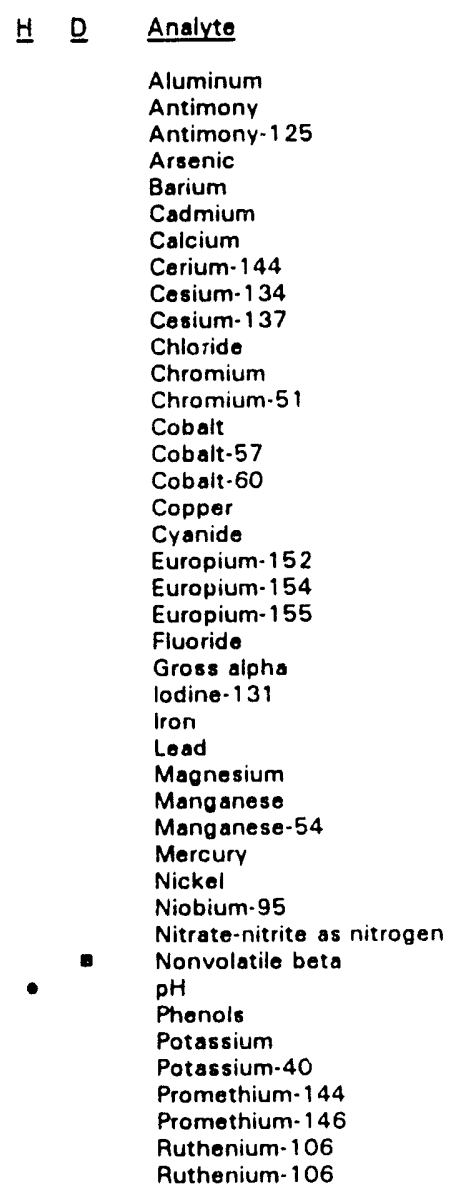

\section{Analyte}

Water elevation

$\mathrm{pH}$

Sp. conductance Water temperature

Alkalinity as $\mathrm{CaCO}_{3}$

Alkalinity as CaCC

$\begin{array}{rr}\frac{1092}{2092} \\ 912 & 176 \\ <2.0 & <2.0 \\ <2.0 & <2.0 \\ 9.3 & 4.8 \\ <2.0 & <2.0 \\ 617 & 440\end{array}$$$
309
$$$$
\begin{array}{r}
214 \\
<2.0
\end{array}
$$$$
<2.0 E+01
$$$$
<2.0
$$$$
\begin{array}{r}
<.3 \\
8
\end{array}
$$$$
\begin{array}{r}
8.3 \\
<2.0
\end{array}
$$$$
614
$$$$
<6.0 \mathrm{OE}+01
$$$$
<1.0 E+01
$$$$
<1.0 E+01
$$

$\begin{array}{ll}3.090 & 2.730 \\ <4.0 & <4.0\end{array}$$$
<4.0
$$$$
<4.0
$$$$
<4.0
$$$$
<5.0
$$

$$
103
$$$$
\text { 8. } 2 \mathrm{E}+00
$$$$
\begin{array}{r}
5.9 \\
<3.0
\end{array}
$$$$
\begin{array}{r}
<3.0 \\
140
\end{array}
$$$$
\begin{aligned}
& 140 \\
& 72
\end{aligned}
$$$$
<0.20
$$$$
\begin{gathered}
<0.20 \\
4.6
\end{gathered}
$$

4.800

$2.4 \mathrm{E}+02$

4.2

4.2
$<5.0$

1.010

$<4.0$

$<1.2 \mathrm{E}+02$

$<4.0$

$<1.0 E+01$

$<1.0 E+01$
$<1.0 E+01$

$<4.0$

$<5.0$

$<4.0 E+01$

$<2.0 E+01$

$<3.0 E+01$

$<100$

$<2.0 E+O O$

$<2.0 E+01$

14
$<3.0$

$<3.0$
198

198
48

$<1.0 E+01$

$<0.20$

$<4.0$

$<1.5 \mathrm{E}+01$

1,860

$6.0 \mathrm{E}+01$

1. $1 E+02$

5.6

2.620

$<1.1 \mathrm{E}+02$

$<1.0 E+01$

$<1.0 E+01$

$<9.0 E+01$

$<1.3 E+02$

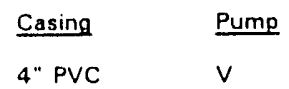

Formation

Water tsble $\left(11 \mathrm{~B}_{2}\right)$

$<1.0 E+01<1.0 E+01$

$2.700 \quad 2.700$

\begin{tabular}{|c|c|c|c|c|}
\hline 4092 & Mod & Unit & $\underline{\text { Lab }}$ & Fla \\
\hline 264 & & $\mu g / L$ & $\mathrm{GE}$ & 2 \\
\hline$<2.0$ & J1 & $\mu g / L$ & GE & 0 \\
\hline$<2.0 E+01$ & & $\mathrm{pCi} / \mathrm{L}$ & $G P$ & 0 \\
\hline$<2.0$ & J1 & $\mu g / L$ & $G E$ & 0 \\
\hline 4.6 & & $\mu \mathbf{g} / \mathrm{L}$ & $G E$ & 0 \\
\hline$<2.0$ & & $\mu g / L$ & GE & 0 \\
\hline 594 & J2 & $\mu g / L$ & GE & 0 \\
\hline$<6.0 E+01$ & & $\mathrm{pCi} / \mathrm{L}$ & $G P$ & 0 \\
\hline$<1.0 E+01$ & & $\mathrm{pCi} / \mathrm{L}$ & GP & 0 \\
\hline$<1.0 E+01$ & & $\mathrm{pCi} / \mathrm{L}$ & GP & 0 \\
\hline 2,700 & & $\mu g / L$ & GE & 0 \\
\hline$<4.0$ & & $\begin{array}{l}\mu_{g} / \mathrm{L} \\
\mathrm{pCi} / \mathrm{L}\end{array}$ & GE & 0 \\
\hline$<4.0$ & & $\mu g / L$ & GE & 0 \\
\hline$<1.0 E+01$ & & $\mathrm{pCi} / \mathrm{L}$ & GP & 0 \\
\hline$<1.0 E+01$ & & $\mathrm{pCi} / \mathrm{L}$ & GP & 0 \\
\hline$<4.0$ & & $\mu \mathrm{g} / \mathrm{L}$ & $\mathrm{GE}$ & 0 \\
\hline$<5.0$ & & $\mu g / L$ & GE & 0 \\
\hline$<4.0 E+01$ & & $\mathrm{pCi} / \mathrm{L}$ & GP & 0 \\
\hline$<2.0 E+01$ & & $\mathrm{pCi} / \mathrm{L}$ & GP & 0 \\
\hline$<3.0 \mathrm{E}+01$ & & $\mathrm{pCi} / \mathrm{L}$ & $G P$ & 0 \\
\hline$<100$ & & $\mu \mathbf{g} / \mathbf{L}$ & $G E$ & 0 \\
\hline$<2.0 E+00$ & & $\begin{array}{l}\mathrm{pCi} / \mathrm{L} \\
\mathrm{pCi} / \mathrm{L}\end{array}$ & GE & 0 \\
\hline 32 & & $\mu g / L$ & GE & 0 \\
\hline$<3.0$ & & $\mu \mathrm{g} / \mathrm{L}$ & GE & 0 \\
\hline 163 & & $\mu g / L$ & $\mathrm{GE}$ & 0 \\
\hline 51 & & $\mu_{g} / \mathrm{L}$ & $G E$ & 2 \\
\hline $\begin{array}{l}<1.0 E+01 \\
<0.20\end{array}$ & & $\begin{array}{l}\mathrm{PC} 1 / \mathrm{L} \\
\mu \mathrm{g} / \mathrm{L}\end{array}$ & $\begin{array}{l}\text { GP } \\
\text { GE }\end{array}$ & 0 \\
\hline 4.5 & & $\begin{array}{l}\mu \mathrm{g} / \mathrm{L} \\
\mathrm{pCi} / \mathrm{L}\end{array}$ & GE & 0 \\
\hline 1.860 & & $\mu_{g} / \mathrm{L}$ & GE & 0 \\
\hline 8.1E+01 & & $\mathrm{pCi} / \mathrm{L}$ & GE & 2 \\
\hline 5.7 & $J$ & $\begin{array}{l}\mathrm{pH} \\
\mu \mathrm{g} / \mathrm{L}\end{array}$ & GE & 0 \\
\hline 2.760 & & $\mu_{g} / \mathrm{L}$ & GE & 0 \\
\hline$<1.1 E+02$ & & $\begin{array}{l}\mathrm{pCi} / \mathrm{L} \\
\mathrm{pCi} / \mathrm{L}\end{array}$ & $\begin{array}{l}\text { GP } \\
\text { GP }\end{array}$ & \\
\hline $\begin{array}{l}<1.0 E+01 \\
<1.0 E+01\end{array}$ & & $\mathrm{pCi} / \mathrm{L}$ & GP & 0 \\
\hline $\begin{array}{l}<1.0 E+01 \\
<9.0 E+01\end{array}$ & & $\mathrm{pCi} / \mathrm{L}$ & GP & $\sigma$ \\
\hline & & $\mathrm{pCi} / \mathrm{L}$ & & \\
\hline
\end{tabular}

Note: Flagging levels, modifiers, and laboratories are for 4 th quarter 1992 data only. See Appendix $B$ for flagging criteria.

- =xceeded holdiny time for 4 th quarter 1992.

- =xceeded final primary drinking water standard for 4th quarter 1992. 
Well HSB1100 continued

ANALYTICAL DATA

H D Analyte

Solenium

Silica

Silver

Sodium

Sodium- 22

Specific conductance

Sulfate

Total activity

Total alpha-emitting radium

Total dissolved solid

Total organic carbon

Total organic halouens

Total phosphates las P

- Tritium

Vanadium

Yetrium-88

Zinc

Zinc.65

Zirconium-95

\begin{tabular}{|c|c|c|c|c|c|c|c|}
\hline 1092 & $\underline{2092}$ & 3092 & 4092 & Mod & Unit & $\underline{L a b}$ & Flag \\
\hline $\begin{aligned}< & 2.0 \\
& 12.900 \\
< & 2.0 \\
& 4.880\end{aligned}$ & $\begin{array}{l}<2.0 \\
10.800 \\
<2.0 \\
7.350\end{array}$ & $\begin{aligned}< & 2.0 \\
& 10.300 \\
< & 2.0 \\
& 12.900 \\
< & 1.0 E+01\end{aligned}$ & $\begin{aligned}< & 2.0 \\
& 10.100 \\
< & 2.0 \\
& 8.420 \\
< & 1.0 E+01\end{aligned}$ & J1 & $\begin{array}{l}\mu \mathrm{g} / \mathrm{L} \\
\mu \mathrm{g} / \mathrm{L} \\
\mu \mathrm{g} / \mathrm{L} \\
\mu \mathrm{g} / \mathrm{L} \\
\mathrm{pCi} / \mathrm{L}\end{array}$ & $\begin{array}{l}G E \\
G E \\
G E \\
G E \\
G P\end{array}$ & $\begin{array}{l}0 \\
0 \\
0 \\
0 \\
0\end{array}$ \\
\hline $\begin{array}{l}70 \\
41.900 \\
1.4 E+05\end{array}$ & $\begin{array}{l}60 \\
4.620 \\
5.5 E+04\end{array}$ & $\begin{array}{l}55 \\
4.380\end{array}$ & $\begin{array}{l}50 \\
4.180\end{array}$ & & $\begin{array}{l}\mu \mathrm{S} / \mathrm{cm} \\
\mu \mathrm{g} / \mathrm{L} \\
\mathrm{pCi} / \mathrm{L}\end{array}$ & $\begin{array}{l}G E \\
G E\end{array}$ & $\begin{array}{l}0 \\
0\end{array}$ \\
\hline $\begin{array}{l}5.0 E+00 \\
47.000\end{array}$ & $\begin{array}{l}2.3 E+00 \\
47.000\end{array}$ & $\begin{array}{l}1.4 E+00 \\
46.000\end{array}$ & $\begin{array}{l}3.9 E+00 \\
43.000\end{array}$ & & $\begin{array}{l}\mathrm{PCi} / \mathrm{L} \\
\mu \mathrm{g} / \mathrm{L}\end{array}$ & $\begin{array}{l}\text { GE } \\
\mathrm{GE}\end{array}$ & $\begin{array}{l}1 \\
0\end{array}$ \\
\hline$<\begin{array}{l}1.000 \\
13\end{array}$ & $<1,000$ & $<1,000$ & $<1,000$ & & $\mu \mathrm{g} / \mathrm{L}$ & $\mathrm{GE}$ & 0 \\
\hline $\begin{array}{r}13 \\
<50\end{array}$ & $\begin{array}{r}44 \\
<50\end{array}$ & $\begin{aligned} & 5.3 \\
< & 50\end{aligned}$ & $\begin{array}{l}<5.0 \\
<50\end{array}$ & & $\begin{array}{l}\mu g / L \\
\mu g / L\end{array}$ & $\begin{array}{l}\mathrm{GE} \\
\mathrm{GE}\end{array}$ & $\begin{array}{l}0 \\
0\end{array}$ \\
\hline $\begin{array}{rl}<50 & 1.2 E+02 \\
< & 8.0\end{array}$ & $\begin{array}{l}6.4 E+01 \\
<8.0\end{array}$ & $\begin{aligned} & 2.3 E+01 \\
< & 8.0 \\
< & 6.0 E+01\end{aligned}$ & $\begin{aligned} & 2.7 E+01 \\
< & 8.0 \\
< & 6.0 E+01\end{aligned}$ & & $\begin{array}{l}\mathrm{pCi} / \mathrm{mL} \\
\mu \mathrm{g} / \mathrm{L} \\
\mathrm{pCi} / \mathrm{L}\end{array}$ & $\begin{array}{l}\text { GE } \\
G E \\
G P\end{array}$ & $\begin{array}{l}2 \\
0 \\
0\end{array}$ \\
\hline 15 & 6.6 & $\begin{aligned} & 11 \\
< & 2.0 E+01 \\
< & 2.0 E+01\end{aligned}$ & $\begin{aligned} & 6.3 \\
< & 2.0 E+01\end{aligned}$ & & $\begin{array}{l}\mu \mathrm{g} / \mathrm{L} \\
\mathrm{pCi} / \mathrm{L} \\
\mathrm{pCi} / \mathrm{L}\end{array}$ & $\begin{array}{l}G E \\
G P\end{array}$ & $\begin{array}{l}0 \\
0\end{array}$ \\
\hline
\end{tabular}

\section{WELL HSB111C}

$\begin{array}{ll}\text { SAS Coord. } & \text { Lat/Longitude } \\ \text { N71919.4 } & 33.277027{ }^{\circ} \mathrm{N} \\ \text { E56501.9 } & 81.657722{ }^{\circ} \mathrm{W}\end{array}$

Screen Zone Elevation

150.7.140.7 ft msi

$01 / 02 / 92$

SAMPLE DATE

FIELD DATA

Analyte

Woter elevation

$\mathrm{pH}$

Sp. conductance

Water temperature

Alkalinity as $\mathrm{CaCO}_{3}$

Volume purged

ANALYTICAL DATA

H D Analyte

Aluminum

Antimony

Antimony-125

Arsonic

Barium

Cadmium

Calcium

Cerium- 144

Cesium-134

Cosium-137

Chloride

Chromium

Chromium.51

Cobalt

Cobalt -57

Cobalt- 60

\section{Copper \\ Copper
Cyanide
Europium-152 \\ Copper
Cyanide
Europium-152}

$\begin{array}{ll}1092 & \underline{2092} \\ 220.0 & 220.4 \\ 4.8 & 4.6 \\ 234 & 226 \\ 18.8 & 19.8 \\ 0 & 0 \\ 4.0 & 4.0\end{array}$

\section{Top of Casing \\ $256 \mathrm{ft} \mathrm{ms}$}

$04 / 23 / 92$

$07 / 15 / 92$

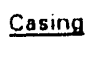

4" PVC

Pumn

s

Formation

Barnwell (IIB,

$\begin{array}{cc}\frac{1092}{20.3} & \underline{2092} \\ <2.0 & <2.0 \\ <2.0 & <2.0 \\ 21 & 23 \\ <2.0 & <2.0 \\ 16.000 & 15.900 \\ & \\ 3.860 & \\ <4.0 & 3.590 \\ <4.0 & <4.0 \\ & <4.0 \\ <4.0 & <4.0 \\ 5.0 & <5.0\end{array}$

3092
220.2
4.6
230
21.7
0
4.0

4092
220.3
4.8
220
20.1
0
2.7

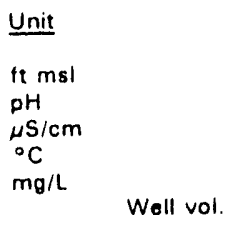

3092

4092

Mod Unit Lab Flag

149

$<2.0$

$<2.0 E+01$

$<2.0$

23

$<2.0$

14,300

$<6.0 E+01$

$<1.0 E+01$

$<1 \mathrm{DE}+01$

3.520

$<4.0$

$<1.2 \mathrm{E}+02$

$<4.0$

$<1.0 E+01$

$<1 . O E+01$

$<4.0$

$<5.0$
$<4.0 E+0$

195

$<2.0$

$<2.0 E+01$

$<2.0$

19

$<2.0$

13.900

$<6.0 E+01$

$<1.0 E+01$

$<1.0 E+01$

3.660

$<4.0$

$<4.0$

$<1.0 E+01$

$<1 . O E+01$

$<4.0$

$<5.0$

$<4.0 E+01$

$\begin{array}{lll}\mu g / L & G E & 2 \\ \mu g / L & G E & 0 \\ p C i / L & G P & 0 \\ \mu g / L & G E & 0 \\ \mu g / L & G E & 0 \\ \mu g / L & G E & 0 \\ \mu g / L & G E & 0 \\ p C i / L & G P & 0 \\ p C i / L & G P & 0 \\ \mathrm{pCi} / \mathrm{L} & G P & 0 \\ \mu g / L & G E & 0 \\ \mu g / L & G E & 0 \\ p C i / L & & \\ \mu g / L & G E & 0 \\ \mathrm{pCi} / L & G P & 0 \\ p C i / L & G P & 0 \\ \mu g / L & G E & 0 \\ \mu g / L & G E & 0 \\ \mathrm{pCi} / L & G P & 0\end{array}$

Note: Flagging levels, modifiers, and laboratories are for 4 th quarter 1992 data only. See Appendix B for flagging criteria.

- = exceeded holding time for 4th quarter 1992.

- =xceeded final primary drinkıng water standard for 4th quarter 1992. 
ANALYTICAL DATA

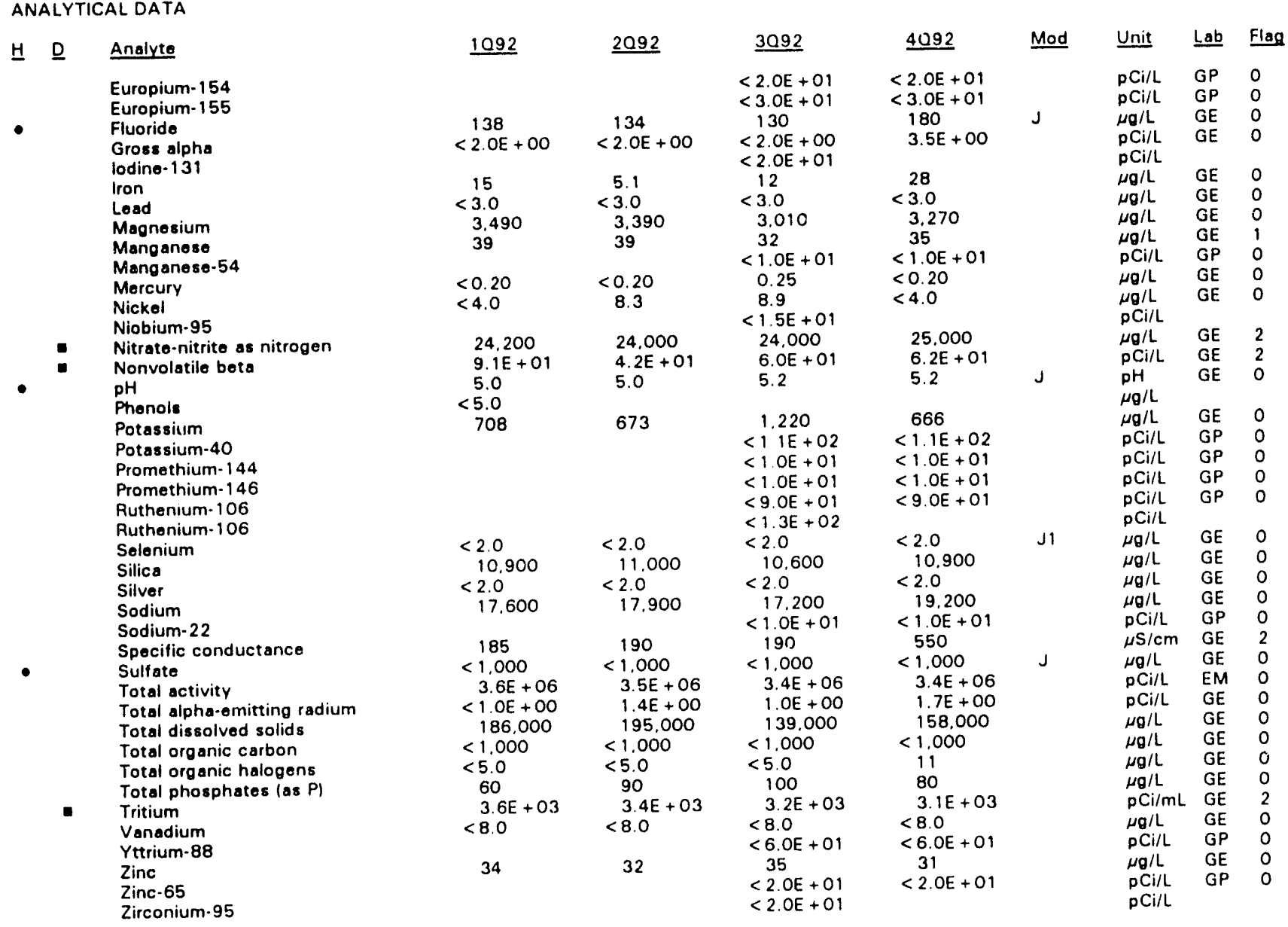

Note: Flagging levels, modifiers, and laboratories are for 4 th quarter 1992 data only. See Appendix B for flagging criteria.

- = exceeded holding time for 4th quarter 1992.

- =xceeded final primary drinking water standard for 4 th quarter 1992. 
WSRC-TR-93-059

WELL HSB111D

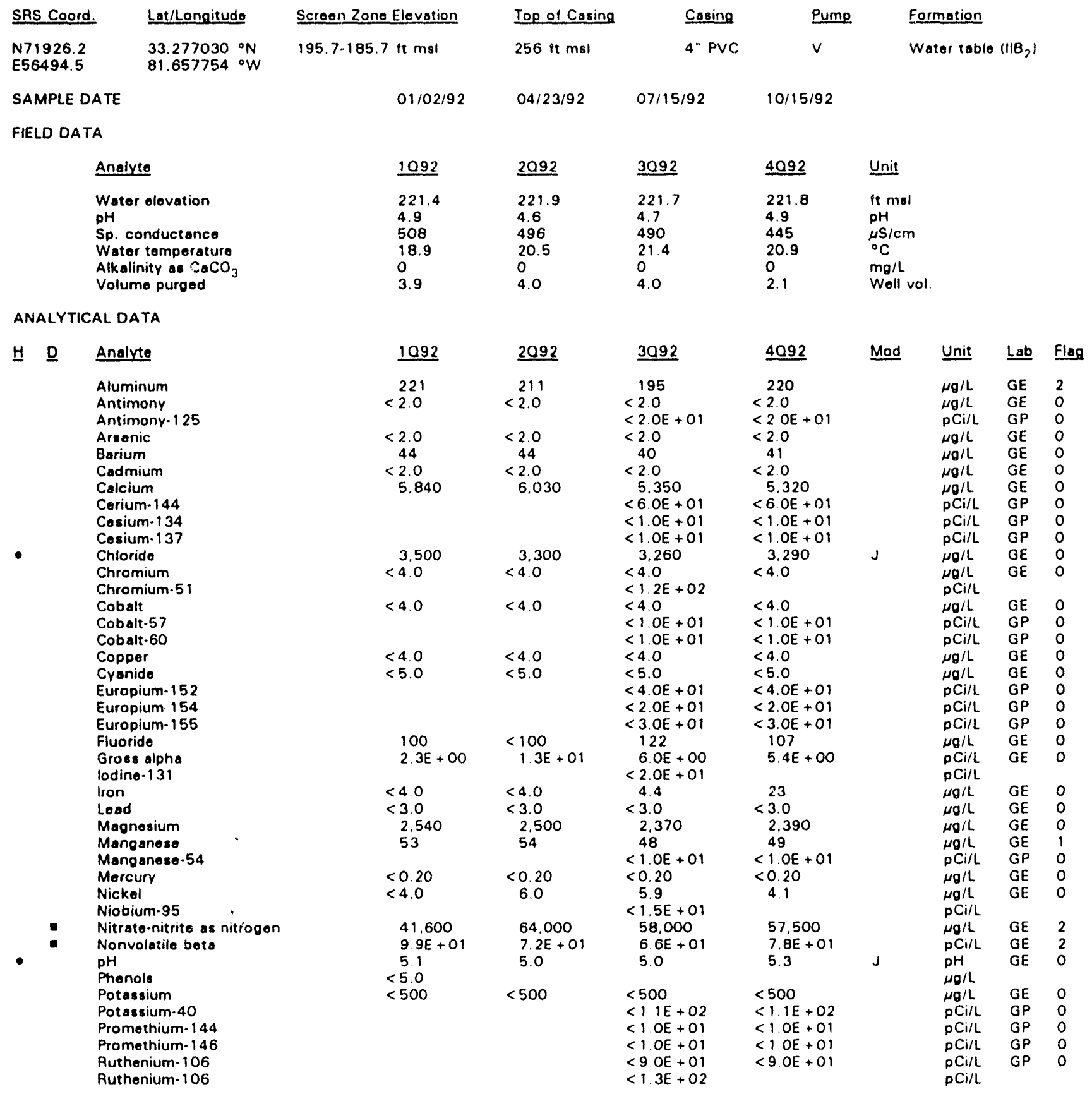

Note: Flagging levels, modifiers, and laboratories are for 4 th quarter 1992 data only. See Apperidix B for flagging criteria. - = exceeded holding time for 4 th quarter 1992.

- =xceeded final primary drinking water standard for 4th quarter 1992.

H-Area Seepage Basins

D. 100
Fourth Quarter 1992 
Well HSB1110 continued

\section{ANALYTICAL DATA}

$\begin{array}{ll}\text { H. } & \text { Anaiyte } \\ \text { Selenium } \\ \text { Silica } \\ \text { Silver } \\ \text { Sodium } \\ \text { Sodium-22 } \\ \text { Specific conductance } \\ \text { Sulfate } \\ \text { Total activity } \\ \text { Total alpha-emitting radium } \\ \text { Total dissolved solids } \\ \text { Total organic carbon } \\ \text { Total organic halogens } \\ \text { Total phosphates (as P) } \\ \text { Tritium } \\ \text { Vanadium } \\ \text { Yttrium-88 } \\ \text { Zinc } \\ \text { Zinc-65 } \\ \text { Zirconium-95 }\end{array}$

$\begin{array}{lll}1092 & \underline{2092} & \underline{3092} \\ <2.0 & <2.0 & <2.0 \\ 8.960 & 9.160 & 8.670 \\ <2.0 & <2.0 & <2.0 \\ 81.700 & 82.000 & 78,100 \\ & & <1.0 \mathrm{E}+01 \\ 405 & 450 & 412 \\ 4.820 & <1.000 & <1.000 \\ 1.3 \mathrm{E}+07 & 1.4 \mathrm{E}+07 & 1.2 \mathrm{E}+07 \\ 2.0 \mathrm{E}+00 & 7.5 \mathrm{E}+00 & 6.5 \mathrm{E}+00 \\ 378,000 & 385,000 & 364.000 \\ <1.000 & <1.000 & <1.000 \\ 25 & <5.0 & <5.0 \\ <50 & <50 & 50 \\ 1.4 \mathrm{E}+04 & 1.4 \mathrm{E}+04 & 1.2 \mathrm{E}+04 \\ <8.0 & <8.0 & <8.0 \\ & & <6.0 \mathrm{O}+01 \\ 14 & 12 & 13 \\ & & <2.0 \mathrm{O}+01 \\ & & <2 \mathrm{OE}+01\end{array}$

$\begin{aligned} & 4092 \\ &< 2.0 \\ & 8.400 \\ &< 2.0 \\ & 88.000 \\ &< 1.0 \mathrm{E}+01 \\ & 450 \\ &< 1.000 \\ & 1.2 \mathrm{E}+07 \\ & 6.6 \mathrm{E}+00 \\ & 334,000 \\ &< 1.000 \\ &< 5.0 \\ &< 50 \\ & 1.2 \mathrm{E}+04 \\ &< 8.0 \\ &< 6.0 \mathrm{E}+01 \\ & 13 \\ &< 2.0 \mathrm{OE}+01 \\ &\end{aligned}$

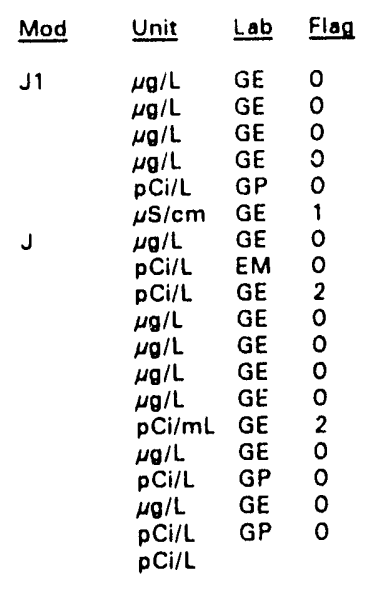

\section{WELL HSB111E}

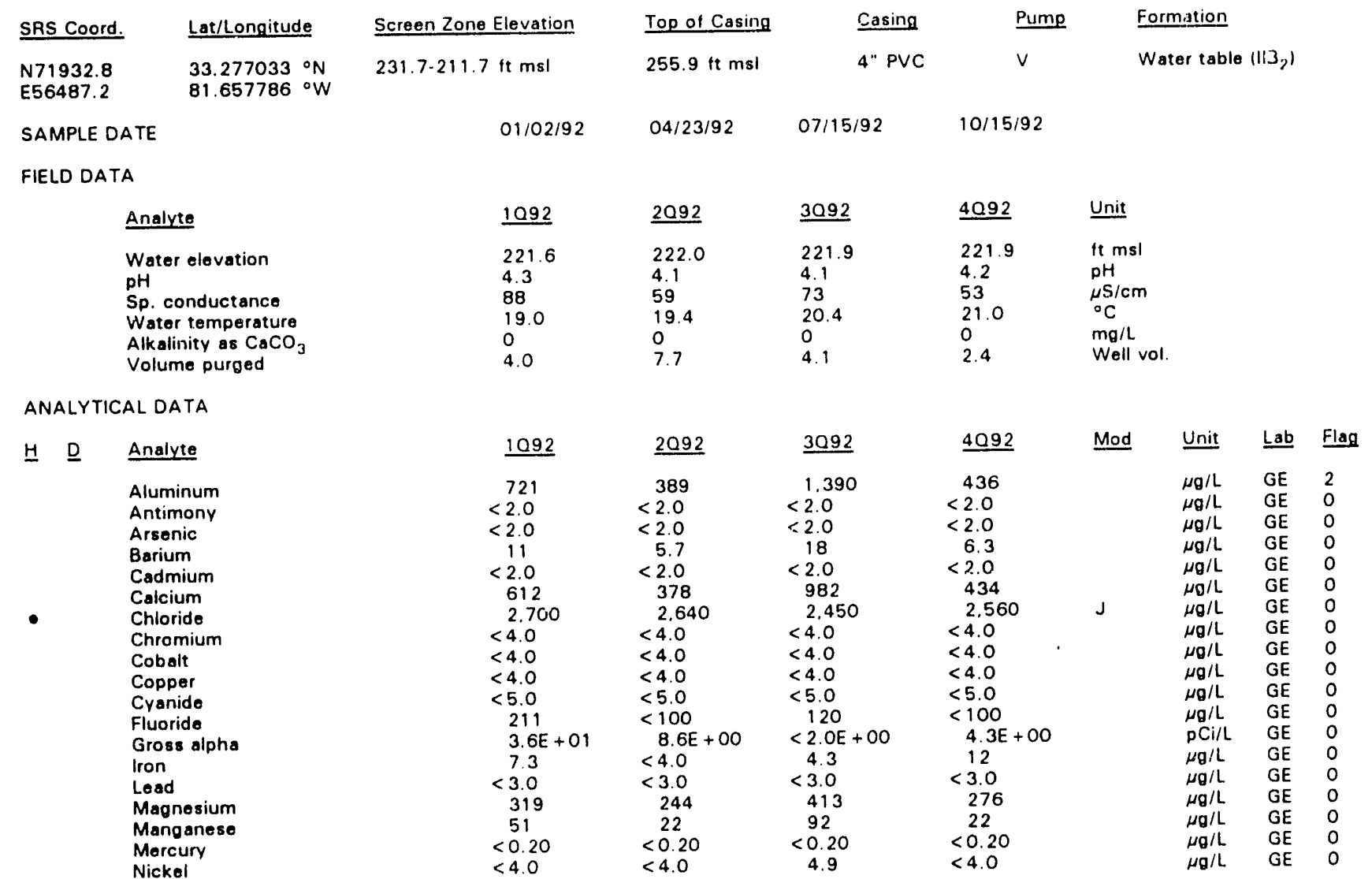

Note: Flagging levels, modifiers, and laboratories are for 4th quarter 1992 data only. See Appendix $B$ for flagging criteria. - exceeded holding time for 4 th quarter 1992.

- exceeded final primary drinking water standard for 4th quarter 1992. 


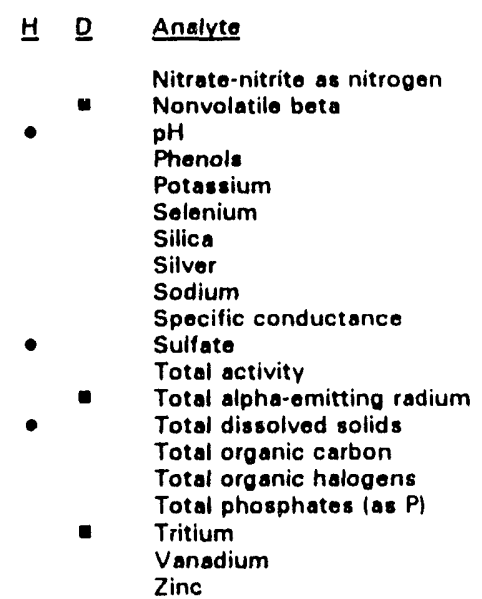

$\begin{array}{lll}\frac{2092}{1092} & \underline{3092} \\ 6.800 & 3.160 & 5.700 \\ 1.1 \mathrm{E}+03 & 7.6 \mathrm{E}+02 & 1.2 \mathrm{E}+03 \\ 4.1 & 4.5 & 4.4 \\ <5.0 & & \\ <500 & <500 & 550 \\ <20 & <2.0 & <2.0 \\ 7.360 & 7.440 & 8.040 \\ <2.0 & <2.0 & <2.0 \\ 5.740 & 4.140 & 8.640 \\ 110 & 48 & 68 \\ 18.300 & 2.250 & 3.270 \\ 4.3 \mathrm{E}+05 & 3.4 \mathrm{E}+05 & 2.7 \mathrm{E}+06 \\ 1.5 \mathrm{E}+01 & 9.1 \mathrm{E}+00 & 7.9 \mathrm{E}+00 \\ 56.000 & 23.000 & 60.000 \\ <1.000 & <1.000 & <1.000 \\ 9.6 & <5.0 & <5.0 \\ <50 & <50 & <50 \\ 7.5 \mathrm{E}+02 & 3.3 \mathrm{E}+02 & 2.4 \mathrm{E}+03 \\ <8.0 & <8.0 & <8.0 \\ 9.6 & 5.7 & 17\end{array}$

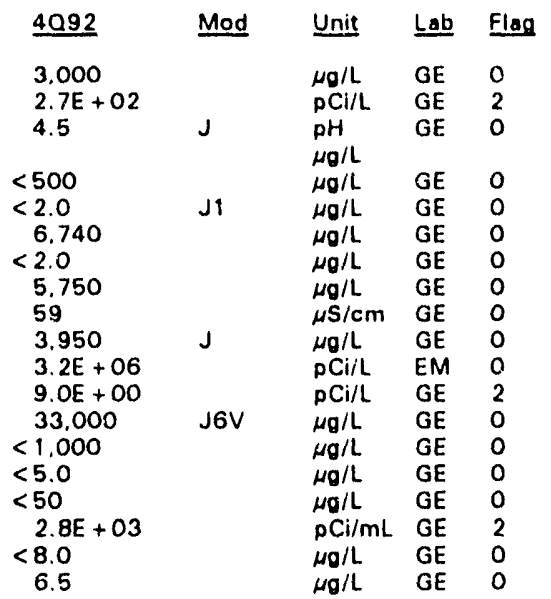

\section{WELL HSB112C}

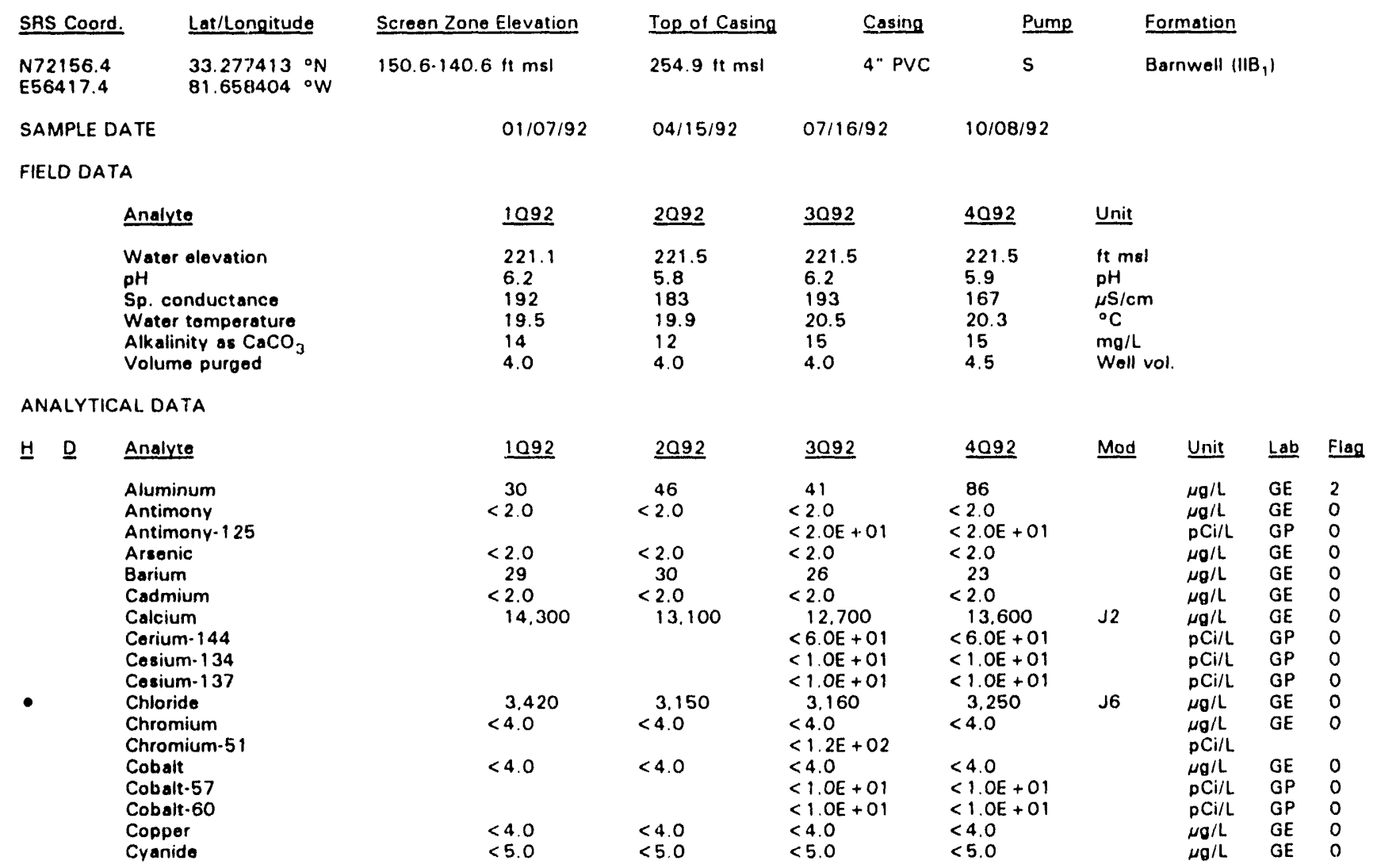

Note: Flagging levels, modifiers, and laboratories are for 4 th quarter 1992 data only. See Appendix B for flagging criteria.

- =xceeded holding time for 4 th quarter 1992.

- exceeded final primary drinking water standard for 4th quarter 1992.

H-Area Seepage Basins
Fourth Quarter 1992 
WSRC.TR.93.059

Well HSB112C continued

ANALYTICAL DATA

H D Analyte

Europium-152

Europium-154

Europium-155

Fluorido

Groses alphe

lodine-131

Iron

Load

Magnesium

Manganese

Manganese. 54

Mercury

Nickel

Niobium-95

- Nitrate-nierite as nitrogen

Nonvolatile beta

pH

Phenols

Potassium

Potassium-40

Promethium-144

Promethium-146

Ruthenium-106

Ruthenium-106

Selenium

Silica

Silver

Sodium

Sodium 22

Specific conductance

- Sulfate

Total activity

Total alpha-emitting radium

Total dissolved solids

Total organic carbon

Total organic halogens

Total phosphates (as P)

- Tritium

Vanadium

Yttrium-88

Zinc

Zirconium.95

\begin{tabular}{|c|c|}
\hline 1092 & 2092 \\
\hline $\begin{array}{l}175 \\
<2.0 E+00\end{array}$ & $\begin{array}{l}299 \\
2.5 E+00\end{array}$ \\
\hline $\begin{aligned}< & 4.0 \\
< & 3.0 \\
& 3.060 \\
& 29\end{aligned}$ & $\begin{aligned}< & 4.0 \\
< & 3.0 \\
& 2.920 \\
& 29\end{aligned}$ \\
\hline $\begin{array}{l}<0.20 \\
<4.0\end{array}$ & $\begin{array}{l}<0.20 \\
<4.0\end{array}$ \\
\hline $\begin{array}{l}15.400 \\
7.8 E+01 \\
6.3 \\
<5.0 \\
614\end{array}$ & $\begin{array}{l}14.000 \\
5.1 E+01 \\
6.2 \\
705\end{array}$ \\
\hline $\begin{aligned}< & 2.0 \\
& 12.000 \\
< & 2.0 \\
& 13,400\end{aligned}$ & $\begin{aligned}< & 2.0 \\
& 12.300 \\
< & 2.0 \\
& 13.000\end{aligned}$ \\
\hline $\begin{aligned} & 140 \\
< & 1.000 \\
& 2.6 E+06 \\
< & 10 E+00 \\
& 158.000 \\
< & 1.000 \\
< & 5.0 \\
& 430 \\
& 2.5 E+03 \\
< & 8.0\end{aligned}$ & $\begin{aligned} & 170 \\
< & 1.000 \\
& 2.3 E+06 \\
& 1.6 E+00 \\
& 166.000 \\
< & 1.000 \\
< & 5.0 \\
& 520 \\
& 2.3 E+03 \\
< & 8.0\end{aligned}$ \\
\hline
\end{tabular}

23 $\frac{3092}{<4.0 E+01}$

$<2.0 E+01$

$<3 . \mathrm{OE}+01$

203

$<2.0 E+O O$

$<2.0 E+01$

$<4.0$

$<3.0$

2.700

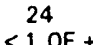

$<1.0 E+01$

0.36

5.2

< $1.5 E+01$

14.000

2. $7 E+01$

6.4

673

$<1.1 E+02$

$<1 . \mathrm{OE}+01$

$<1.0 E+01$

$<9.0 E+01$

$<1.3 E+02$

$<2.0$

11.600

$<2.0$

12.300

$<1 . \mathrm{OE}+01$

142

$<1.000$

2. $4 \mathrm{E}+06$

$<1$ OE + OO

137.000

$<1.000$

$<5.0$

630

$2.0 E+03$

$<8.0$

$<6.0 E+01$

22
4092 Mod

$<4.0 E+01$

$<2.0 E+01$

$<3 . \mathrm{OE}+01$

197

$4.6 E+00$

8.4
$<3.0$

2.650

2.65

$<1.0 E+01$

$<0.20$

4.8

13,800

3. $4 \mathrm{E}+01$

6.2

$<500$

$<1.1 \mathrm{E}+02$

$<1.0 E+01$

$<1.0 E+01$

$<9.0 E+01$

$<2.0$

11.000

$<2.0$

12.400

$<1.0 E+01$

145

$<1,000$

2. $0 E+06$

2. $7 E+00$

146.000

$<1.000$

$<5.0$

480

$1.8 E+03$

$<8.0$

$<6.0 E+01$

21

$<2 . \mathrm{OE}+01$
Mod Unit Lab Flag

$\mathrm{PCi} / \mathrm{L} \quad \mathrm{GP} \quad 0$

$\mathrm{PCi} / \mathrm{L}$ GP 0

$\mu \mathrm{g} / \mathrm{L}$ GE $O$

$\mathrm{pCi} / \mathrm{L} \quad \mathrm{GE} \quad 0$

$\mathrm{pCi} / \mathrm{L}$

$\mu \mathrm{g} / \mathrm{L}$ GE 0

$\mu \mathrm{g} / \mathrm{L} \quad \mathrm{GE} \quad 0$

GE

$\mathrm{pCi} / \mathrm{L}$ GP

$\mu g / L$ GE $O$

$\mu g / L$ GE 0

$\mathrm{PCi} / \mathrm{L}$ GE

$\mu \mathrm{g} / \mathrm{L} \quad \mathrm{GE} 2$

$\begin{array}{lll}\mathrm{pCi} / \mathrm{L} & \mathrm{GE} & 1 \\ \mathrm{pH} & \mathrm{GE} & 0\end{array}$

$\mu g / L \quad G E$ O

PCi/L GP

PCIL GP O

$\mathrm{PCi} / \mathrm{G}$ GP 0

$\begin{array}{lll}\mathrm{PCi} / \mathrm{L} & \mathrm{GP} & 0 \\ \mathrm{pCi} / \mathrm{L} & \mathrm{GP} & 0\end{array}$

$\mathrm{PCi} / \mathrm{L}$

$\mu_{g} / \mathrm{L}$ GE $O$

$\mu g / L$ GE 0

$\begin{array}{lll}\mu \mathrm{g} / \mathrm{L} & \mathrm{GE} & 0 \\ \mu \mathrm{g} / \mathrm{L} & \mathrm{GE} & 0\end{array}$

$\mathrm{PCi} / \mathrm{L}$ GP 0

S/Cm GE 0

SIL $O$

$\mu \mathrm{g} / \mathrm{L}$ GE 0

PCi $\quad$ GE 1

$\mu g / L$ GE 0

$\mu \mathrm{g} / \mathrm{L}$ GE 0

NG/ GE

$\mu \mathrm{g} / \mathrm{L}$ GE 0

$\begin{array}{lll}\mu \mathrm{g} / \mathrm{L} & \mathrm{GE} & 0 \\ \mathrm{pCi} / \mathrm{mL} & \mathrm{GE} & 2\end{array}$

$\mu \mathrm{g} / \mathrm{L}$ GE 0

$\mathrm{pCi} / \mathrm{L}$ GP 0

GE $/ \mathrm{L}$ GE

$\begin{array}{lll}\mu \mathrm{g} / \mathrm{L} & \mathrm{GE} & 0 \\ \mathrm{pCi} / \mathrm{L} & \mathrm{GP} & 0\end{array}$

$<2.0 E+01$

$<2.0 E+01$

Note: Flagging levels, modifiers, and laboratories are for 4 th quarter 1992 data only. See Appendix B for flagging criteria.

- =xceeded holding time for 4th quarter 1992.

- =xceeded final primary drinking water standard for 4th quarter 1992. 
WELL HSB112D

\begin{tabular}{|c|c|}
\hline SRS Coord & Lat/Longitude \\
\hline $\begin{array}{l}\text { N72161.6 } \\
\text { E56408.1 }\end{array}$ & $\begin{array}{l}33.277410^{\circ} \mathrm{N} \\
81.6584399^{\circ} \mathrm{W}\end{array}$ \\
\hline
\end{tabular}

FIELD DATA

\section{Analyte}

Water olevation

$\mathrm{pH}$

Sp. conductance

Water temperature

Alkalinity as $\mathrm{CaCO}_{3}$

Volume purged

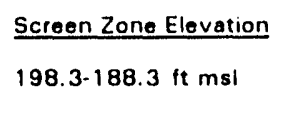

$01 / 07 / 92$
Top of Casing

$255.1 \mathrm{ft} \mathrm{msl}$

$04 / 15 / 92$
ANALYTICAL DATA

$\begin{array}{ll}\text { H. } & \text { Analyte } \\ \text { Aluminum } \\ \text { Antimony } \\ \text { Antimony-125 } \\ \text { Arsenic } \\ \text { Barium } \\ \text { Cadmium } \\ \text { Calcium } \\ \text { Corium-144 } \\ \text { Cesium-134 } \\ \text { Cesium-137 } \\ \text { Chloride } \\ \text { Chromium } \\ \text { Chromium-51 } \\ \text { Cobalt } \\ \text { Cobalt-57 } \\ \text { Cobatt-60 } \\ \text { Copper } \\ \text { Cyanide } \\ \text { Europium-152 } \\ \text { Europium-154 } \\ \text { Europium-155 } \\ \text { Fluoride } \\ \text { Gross alpho } \\ \text { lodine-131 } \\ \text { Iron } \\ \text { Lead } \\ \text { Magnesium } \\ \text { Manganese } \\ \text { Manganese-54 } \\ \text { Mercury } \\ \text { Nickel } \\ \text { Niobium-95 } \\ \text { Nitrate-nitrite as nitrogen } \\ \text { Nonvolatile beta } \\ \text { pH } \\ \text { Phenols } \\ \text { Potassium } \\ \text { Potassium-40 } \\ \text { Promethium-144 } \\ \text { Promethium-146 } \\ \text { Ruthenium-106 } \\ \text { Ruthenium-106 } \\ \end{array}$

2092
222.6
4.4
448
19.4
0
4.0

1092
222.0
4.8
437
19.8
0
4.0

4.0

$\begin{array}{ll}1092 & \underline{2092} \\ 126 & 136 \\ <2.0 & <2.0 \\ <2.0 & <2.0 \\ 18 & 19 \\ <2.0 & <2.0 \\ 1.620 & 1.730\end{array}$

$\begin{array}{ll}2.680 & 2.360 \\ <4.0 & <4.0 \\ 5.2 & 5.6\end{array}$

5.2

$<4.0$

$<5.0$

$<4.0$

$<5.0$

$<100$

4. $1 E+00$

191
$2.6 E+00$

$<4.0$
$<3.0$

1.090

4.6
$<3.0$

$<3.0$

1.170
202

$<0.20$

$<4.0$

48.800

$7.5 E+01$

$7.5 \mathrm{E}+$
4.7

4.7
$<5.0$

730

$\frac{\text { Casing }}{\text { 4" PVC }}$

$07 / 16 / 92$

$10 / 25 / 92$

\section{Formation}

Water table $\left(11 \mathrm{~B}_{2}\right)$ 
Well HSB112D continued

ANALYTICAL DATA

An $\quad$ Analyte
Selonium
Silica
Silica, total
Silvor
Sodium
Sodium-22
Specific conductance
Sulfato
Total activity
Total alpha-emitting radium
Total dissolved solids
Total organic carbon
Total organic halogens
Total phosphates las P)
Tributyl phosphate
Tritium
Vanadium
Yttrium-88
Zinc
Zinc-65
Zirconium-95

\begin{tabular}{lll}
\hline 1092 & $\underline{2092}$ & $\underline{3092}$ \\
$<2.0$ & $<2.0$ & $<2.0$ \\
8.800 & 8.950 & 8.040 \\
& & 8.570 \\
$<2.0$ & $<2.0$ & $<2.0$ \\
72.700 & 76.100 & 73.500 \\
340 & 420 & $<1.0 \mathrm{E}+01$ \\
10.500 & 8.170 & 362 \\
$1.9 \mathrm{E}+07$ & $1.6 \mathrm{E}+07$ & 8.490 \\
$2.1 \mathrm{E}+00$ & $8.6 \mathrm{E}+00$ & $6.4 \mathrm{E}+07$ \\
317.000 & 349.000 & 311.000 \\
$<1.000$ & $<1.000$ & $<1.000$ \\
71 & $<5.0$ & $<5.0$ \\
70 & 90 & $<50$ \\
& & $<10$ \\
$1.8 \mathrm{E}+04$ & $1.8 \mathrm{E}+04$ & $1.5 \mathrm{E}+04$ \\
$<8.0$ & $<8.0$ & $<8.0$ \\
& & $<6.0 \mathrm{E}+01$ \\
8.7 & 8.1 & 8.1 \\
& & $<2.0 \mathrm{E}+01$ \\
& & $<2.0 \mathrm{E}+01$
\end{tabular}

\begin{tabular}{|c|c|c|c|c|}
\hline 4092 & Mod & Unit & Lab & Flag \\
\hline$<2.0$ & & $\mu g / L$ & $\mathrm{GE}$ & 0 \\
\hline 7.140 & & $\mu g / L$ & $\mathrm{GE}$ & 0 \\
\hline 7.220 & & $\mu \mathrm{g} / \mathrm{L}$. & GE & 2 \\
\hline$<2.0$ & & $\mu \mathrm{g} / \mathrm{L}$ & GE & 0 \\
\hline 80.700 & J2 & $\mu g / L$ & $G E$ & 0 \\
\hline$<1.0 E+01$ & & $\mathrm{pCi} / \mathrm{L}$ & $G P$ & 0 \\
\hline 360 & & $\mu \mathrm{S} / \mathrm{cm}$ & $\mathrm{GE}$ & 1 \\
\hline $\begin{array}{l}8.980 \\
1.5 E+07\end{array}$ & J & $\begin{array}{l}\mu_{g} / \mathrm{L} \\
\mathrm{pCi} / \mathrm{L}\end{array}$ & $\begin{array}{l}\text { GE } \\
\text { EM }\end{array}$ & $\begin{array}{l}0 \\
0\end{array}$ \\
\hline $3.4 E+00$ & & $\mathrm{pCi} / \mathrm{L}$ & GE & 1 \\
\hline 296.000 & v & $\mu g / L$ & GE & 0 \\
\hline$<1.000$ & & $\mu_{\theta} / L$ & GE & 0 \\
\hline$<5.0$ & J & $\mu_{\theta} / \mathrm{L}$ & GE & 0 \\
\hline$<50$ & & $\mu g / L$. & GE & $\begin{array}{l}0 \\
0\end{array}$ \\
\hline $\begin{array}{l}<10 \\
1.5 E+04\end{array}$ & J1 & $\begin{array}{l}\mu \mathrm{g} / \mathrm{L} \\
\mathrm{pCi} / \mathrm{mL}\end{array}$ & $\begin{array}{l}\text { GE } \\
\text { GE }\end{array}$ & $\begin{array}{l}0 \\
2\end{array}$ \\
\hline$<8.0$ & & $\mu \mathrm{g} / \mathrm{L}$ & $\mathrm{GE}$ & 0 \\
\hline$<6.0 E+01$ & & $p \mathrm{Ci} / \mathrm{L}$ & GP & 0 \\
\hline 10 & & $\mu \mathrm{g} / \mathrm{L}$ & $\mathrm{GE}$ & 0 \\
\hline$<2.0 E+01$ & & $\begin{array}{l}\mathrm{pCi} / \mathrm{L} \\
\mathrm{pCi} / \mathrm{L}\end{array}$ & GP & 0 \\
\hline
\end{tabular}

\section{WELL HSB112E}

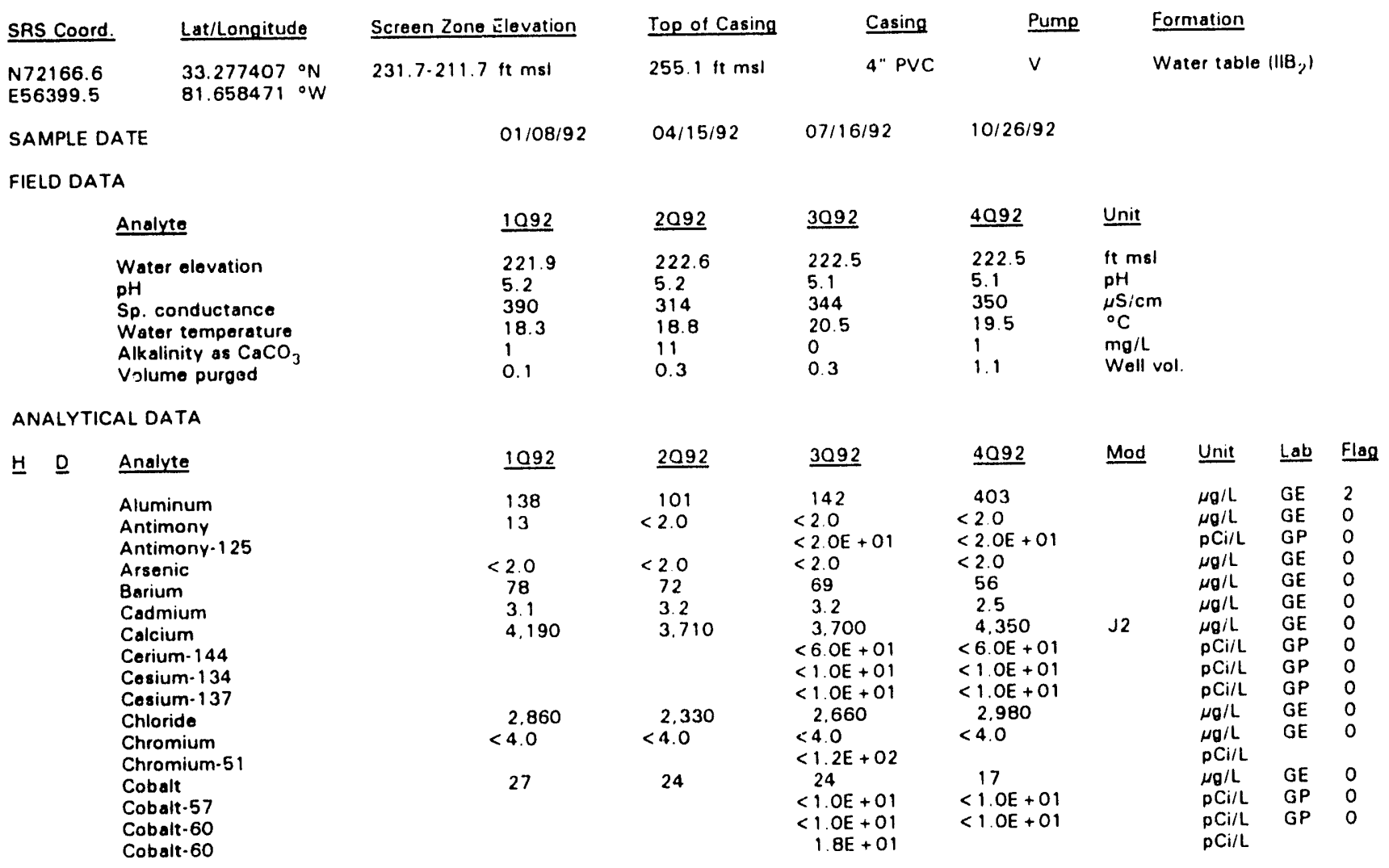

Note: Flagging levels, modifiers, and laboratories are for 4th quarter 1992 data only. See Appendix B for flagging criteria. - =xceeded holding time for 4th quarter 1992.

- exceeded final primary drinking water standard for 4th quarter 1992. 
WSRC-TR-93-059

Well HSB112E continued

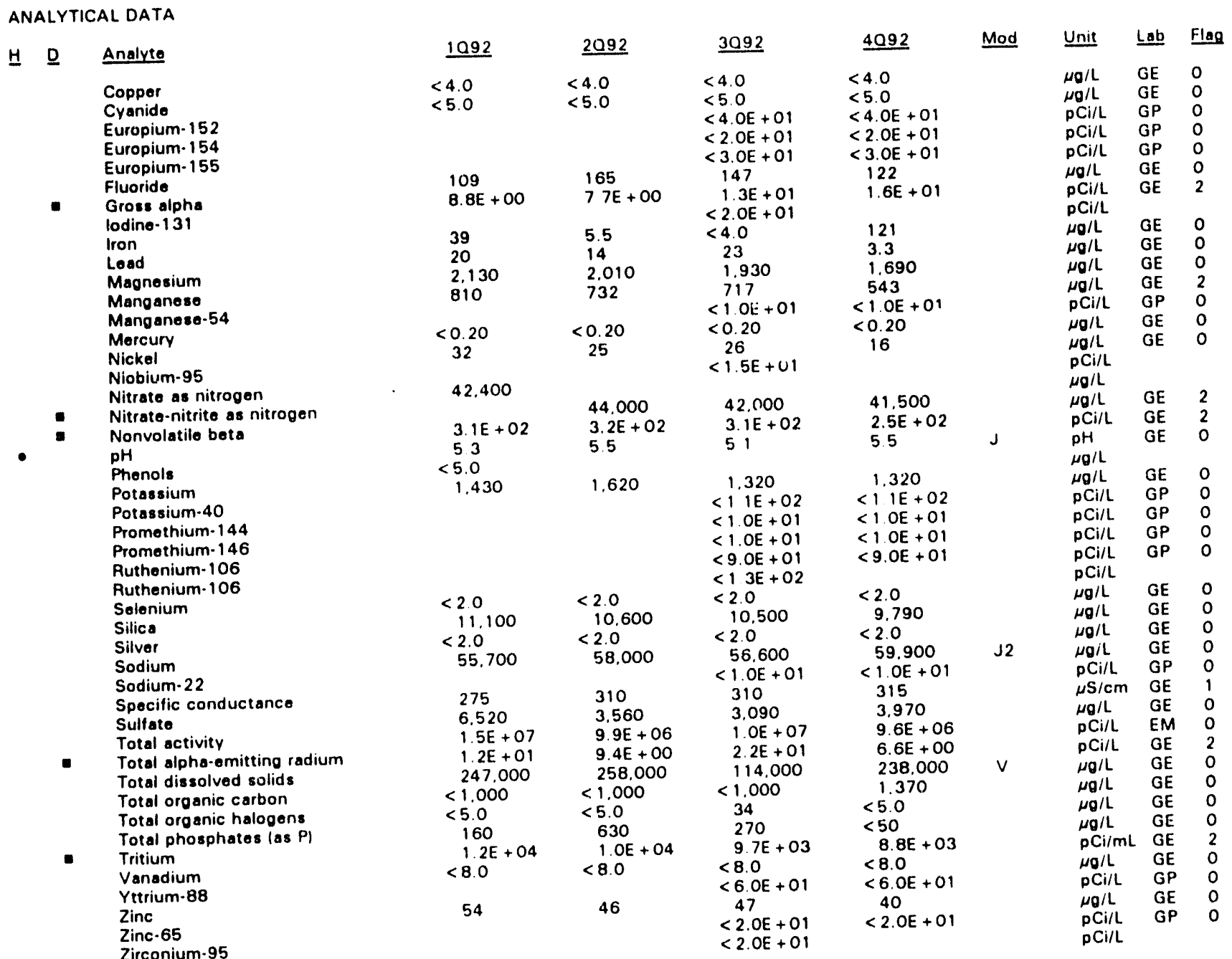

Note: Flagging levels, modifiers, and laboratories are for 4th quarter 1992 data only. See Appendix B for flagging criterla. - =xceeded holding tıme for 4th quarter 1992

- exceeded final primary drinking water standard for 4th quarter 1992. 


\section{WELL HSB113C}

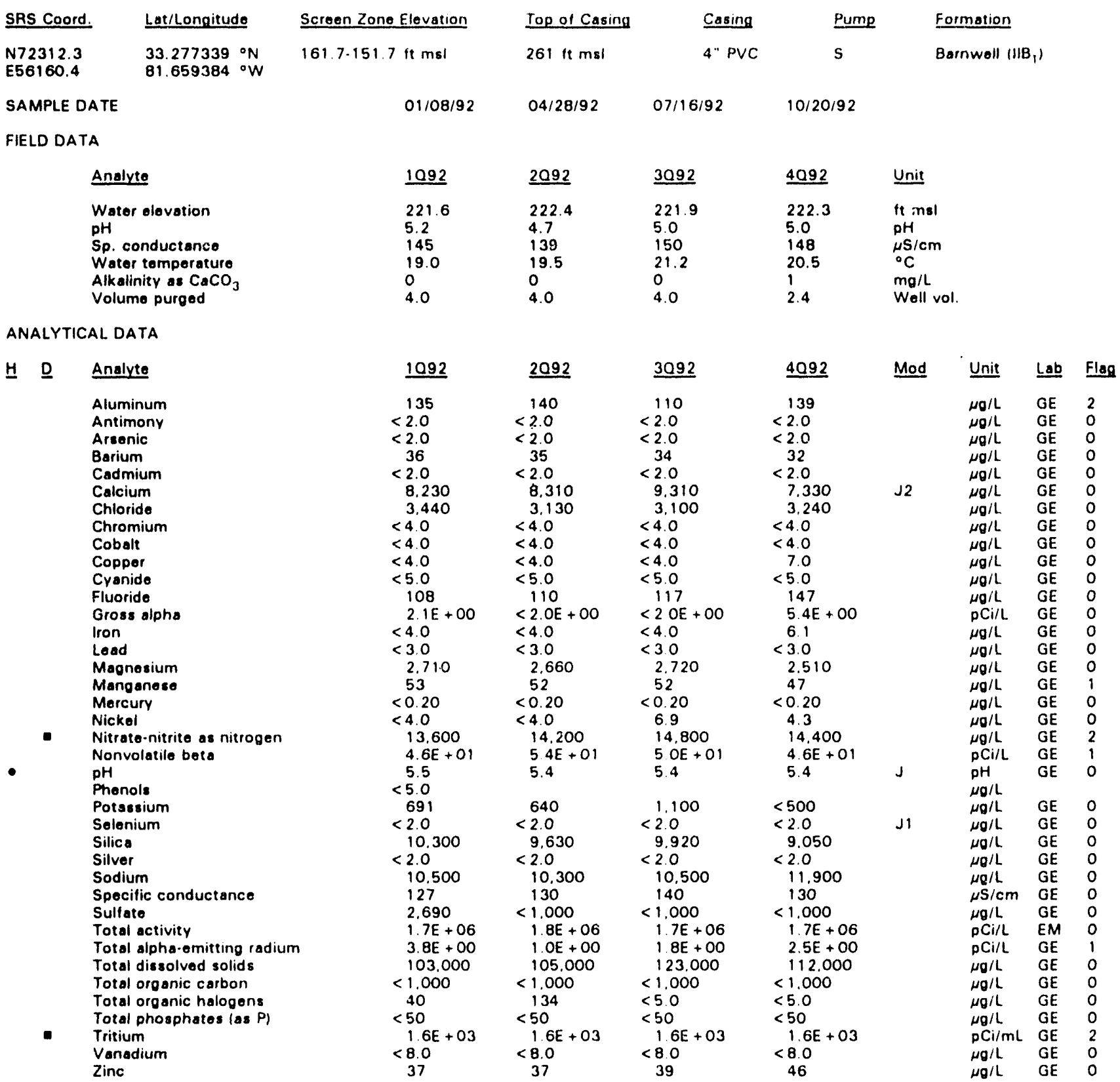

Note: Flagging levels, modifiers, and laboratories are for 4th quarter 1992 data only. See Appendix B for flagging criteria. - = exceeded holding time for 4th quarter 1992.

- exceeded final primary drinkıng water standard for 4th quarter 1992. 
WELL HSB1130

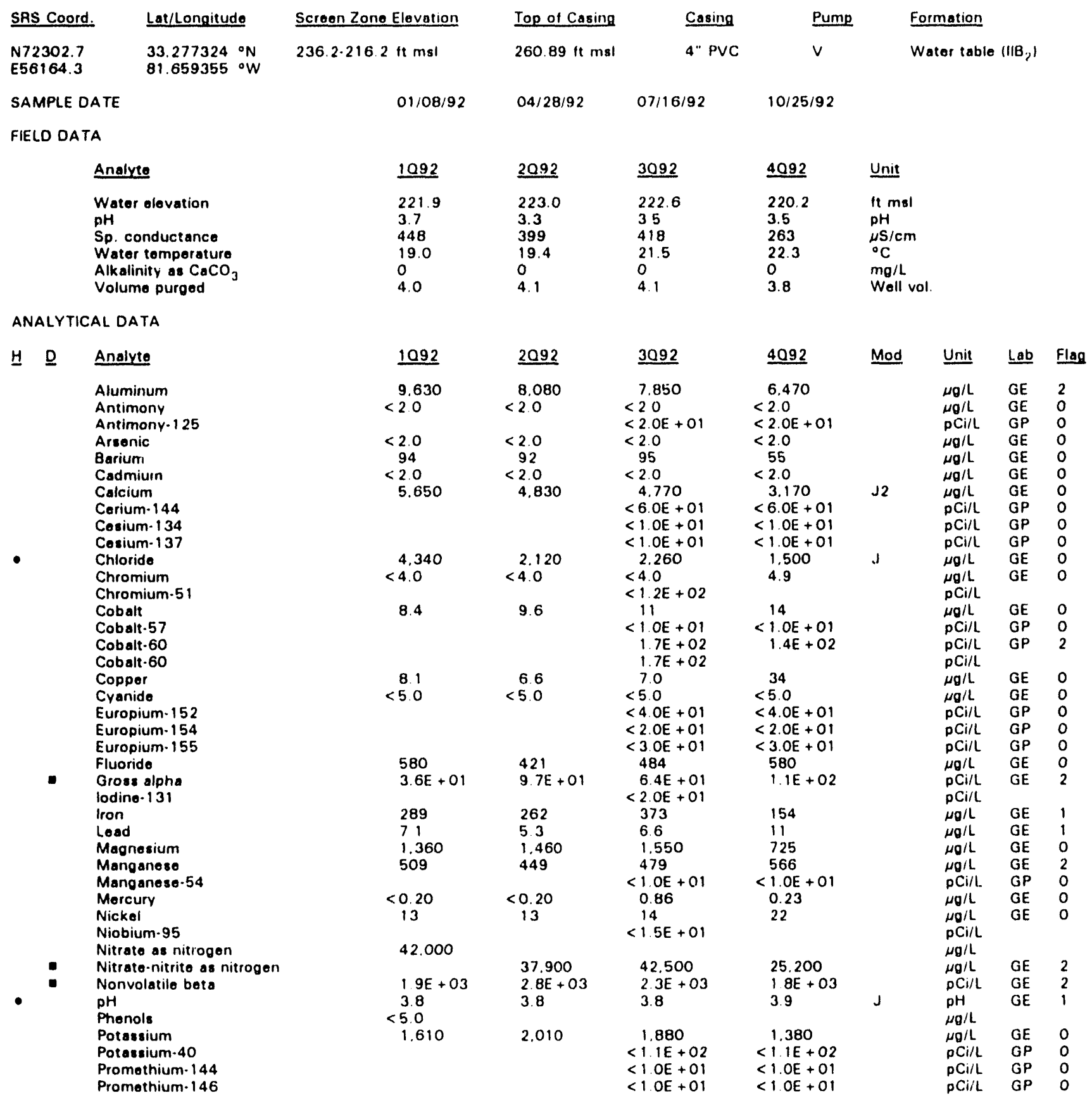

Note: Flagging levels, modifiers, and laboratories are for 4th quarter 1992 data only. See Appendix B for flagging criteria. - = exceeded holding lime for 4th quarter 1992.

- =xceeded final primary drinking water standard for 4th quarter 1992. 
ANALYTICAL DATA

H D Analyte
Ruthenium-106
Ruthonium-106
Solonium
Silica
Silver
Sodium
Sodium-22
Specific conductance
Sulfate
Total activity
Total alpha-emitting radium
Total dissolvod solids
Total organic carbon
Total organic halogens
Total phosphates las P)
Tritium
Vanadium
Yttrium-88
Zinc
Zinc-65
Zirconium-95

$\begin{array}{lll}1092 & \underline{2092} & \underline{3092} \\ & & <9.0 E+01 \\ & <1.3 \mathrm{E}+02 \\ <2.0 & <2.0 & <2.0 \\ 28.500 & 24.600 & 25.500 \\ <2.0 & <2.0 & <2.0 \\ 24.300 & 27.100 & 28.100 \\ 340 & 340 & <1.0 \mathrm{E}+01 \\ 5.840 & 1.810 & 350 \\ 1.1 \mathrm{E}+07 & 1.0 \mathrm{E}+07 & 1.500 \\ 5.7 \mathrm{E}+01 & 4.4 \mathrm{E}+01 & 3.0 \mathrm{BE}+07 \\ 247.000 & 221.000 & 211.000 \\ <1.000 & <1.000 & <1.000 \\ <5.0 & 6.9 & <5.0 \\ 70 & <50 & <50 \\ 1.1 \mathrm{E}+04 & 1.0 \mathrm{OE}+04 & 9.6 \mathrm{O}+03 \\ <8.0 & <8.0 & <8.0 \\ 83 & 74 & <6.0 \mathrm{O}+01 \\ & & 74 \\ & & <2.0 \mathrm{O}+01 \\ & & <2.0 \mathrm{O}+01\end{array}$

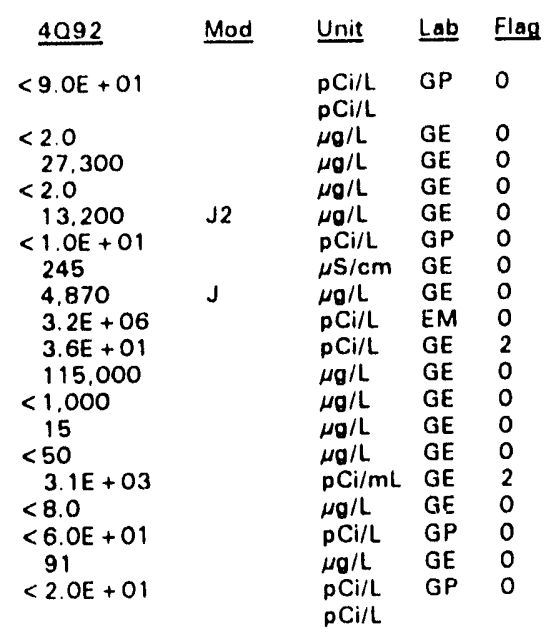

\section{WELL HSB 1 14C}

$\begin{array}{ll}\text { SARS Coord. } & \text { Lat/Longitude } \\ \text { N72464.6 } & 33.2775899^{\circ} \mathrm{N} \\ \text { E56107.0 } & 81.659820^{\circ} \mathrm{W} \\ \text { SAMPLE DATE } & \\ \text { FIELD DATA } & \end{array}$

$\frac{\text { Screen Zone Elevation }}{195.6 \cdot 185.6 \mathrm{ft} \mathrm{msl}}$

$\begin{array}{llll}\text { Top of Casing } & \text { Casing } & \text { Pump } & \text { Formation } \\ 263.8 \mathrm{ft} \mathrm{msl} & 4^{\prime \prime} P V C & \mathrm{~S} & \text { Barnwell (IIB,) }\end{array}$

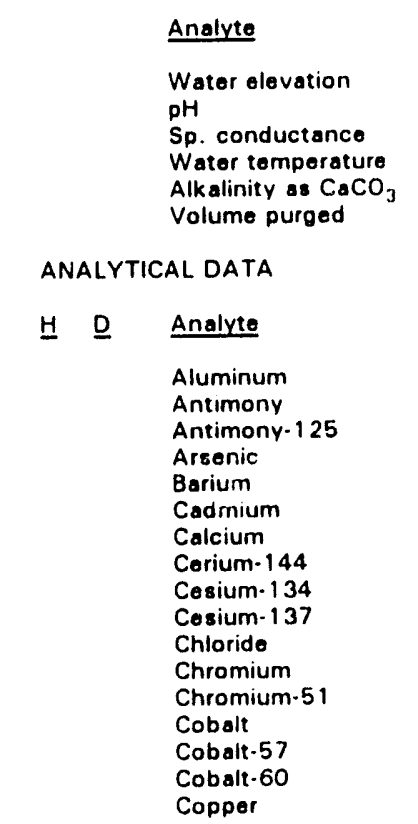

$01 / 08 / 92$

$04 / 28 / 92$

$07 / 16 / 92$

$10 / 25 / 92$

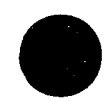

\begin{tabular}{|c|c|c|c|c|}
\hline 1092 & $\underline{2092}$ & 3092 & 4092 & Unit \\
\hline $\begin{array}{l}222.4 \\
4.5 \\
498 \\
19.6 \\
0 \\
4.0\end{array}$ & $\begin{array}{l}223.6 \\
4.1 \\
488 \\
20.0 \\
0 \\
4.0\end{array}$ & $\begin{array}{l}223.2 \\
4.4 \\
496 \\
21.4 \\
0 \\
4.0\end{array}$ & $\begin{array}{l}225.1 \\
4.7 \\
148 \\
20.8 \\
0 \\
2.7\end{array}$ & $\begin{array}{l}\text { ft msl } \\
\mathrm{pH} \\
\mu \mathrm{S} / \mathrm{cm} \\
{ }^{\circ} \mathrm{C} \\
\mathrm{mg} / \mathrm{L} \\
\text { Well vol. }\end{array}$ \\
\hline
\end{tabular}

H $\quad$ Analyte
Aluminum
Antimony
Antimony-125
Arsenic
Barium
Cadmium
Calcium
Corium-144
Cesium-134
Cosium-137
Chloride
Chromium
Chromium-51
Cobalt
Cobalt-57
Cobalt-60
Copper

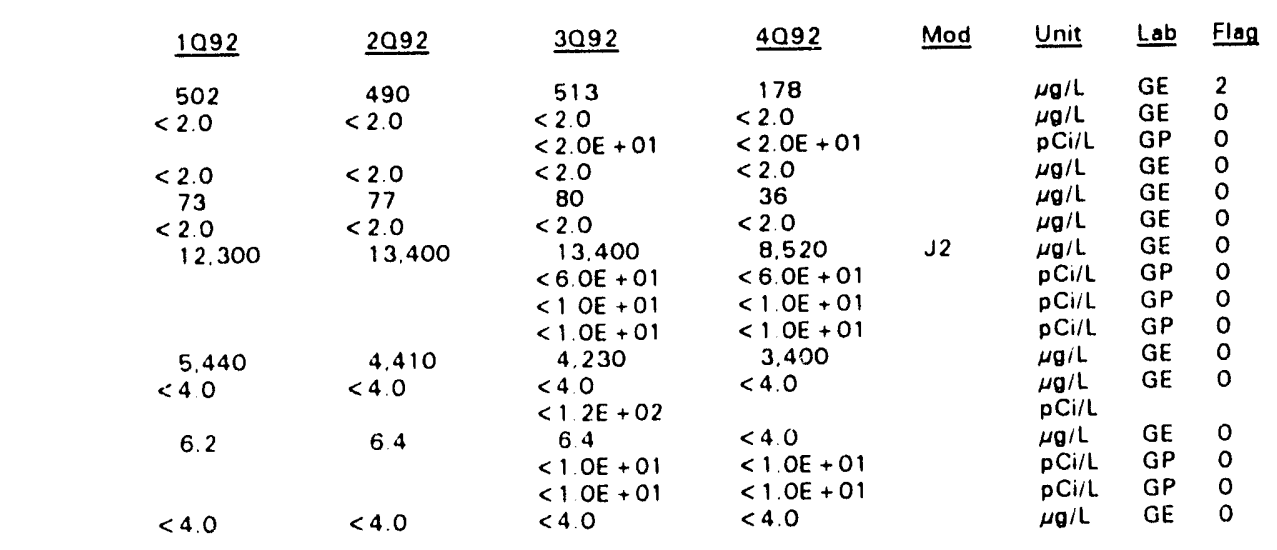

\footnotetext{
Note: Flagging levels, modifiers, and laboratories are for 4 th quarter 1992 data oniy. See Appendix $B$ for flagging criteria.

- = exceeded holding time for 4 th quarter 1992.

- =xceeded final primary drinking water standard for 4 th quarter 1992.
}

H-Area Seepage Basins 
WSRC-TR-93-059

Woll HSB114C continued

ANALYTICAL DATA

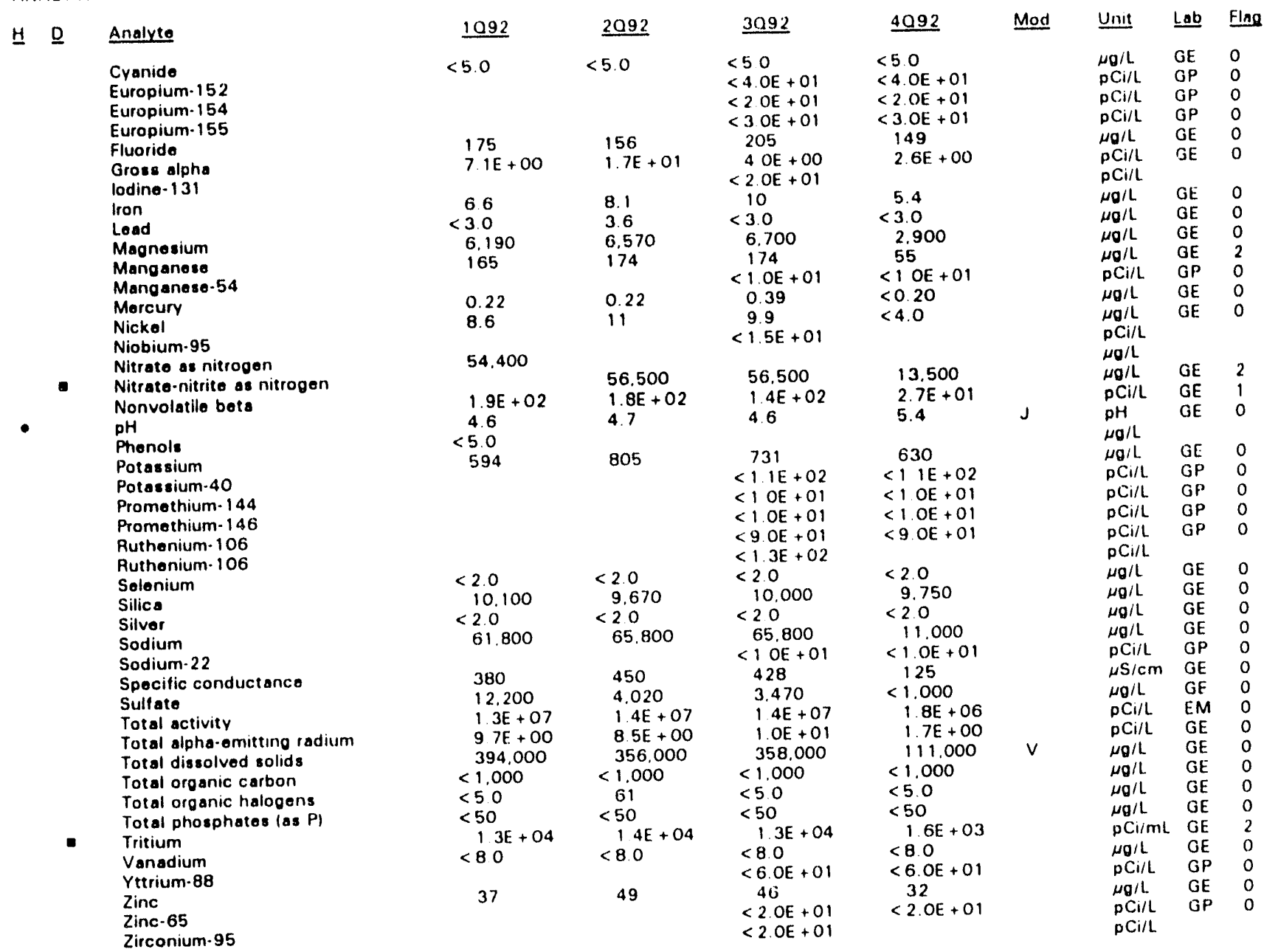

Note: Flagging levels, modifiers, and laboratories are for 4 th quarter 1992 data only. See Appendix B for flagging criteria.

- =xceeded holding tıme for 4th quarter 1992.

- exceeded final primary drinking water standard for 4ih quarter 1992.

H-Area Seepage Basins 


\section{WELL HSB114D}

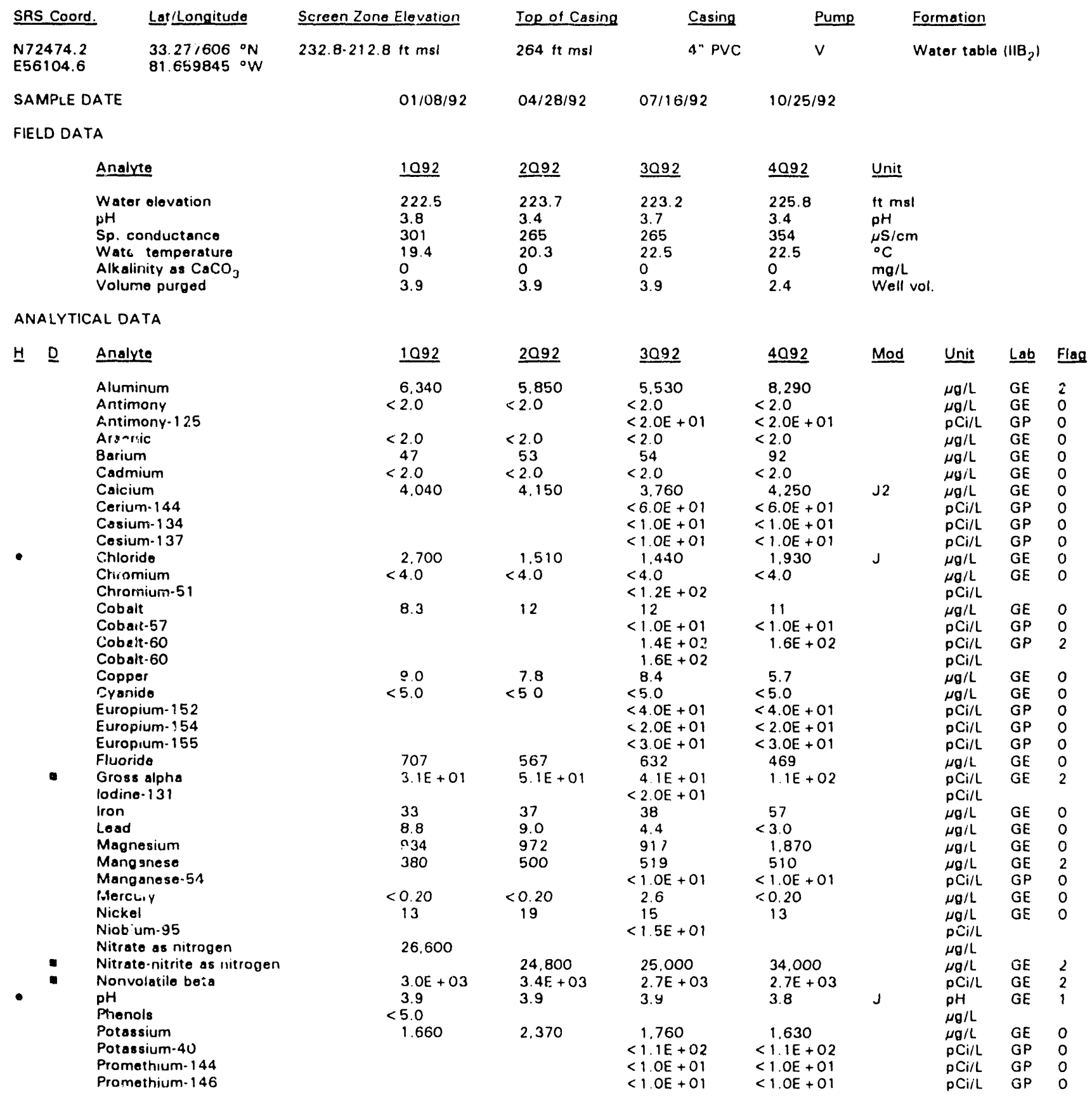

Note: Flagging levels, modifiers, and laboratories are for 4 th quarter 1992 data only. See Appendix B for flagging criteria. - = exceeded holding time for 4th quarter 1992.

- =xceeded final primary drinking water standard for 4th quarter 1992. 
Well HSB1140 continued

\begin{abstract}
ANALYTICAL DATA
\end{abstract}

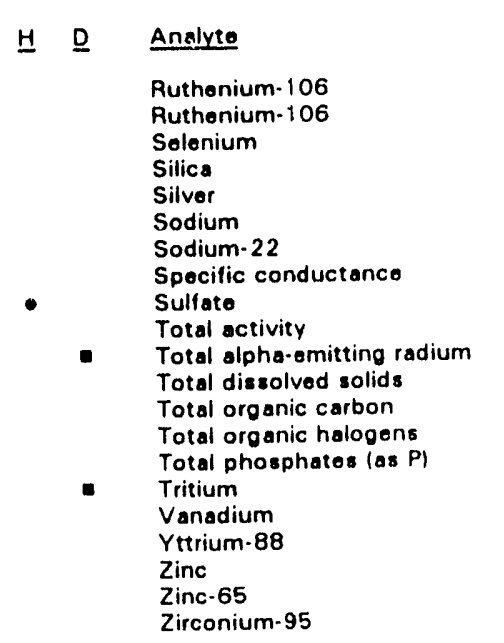

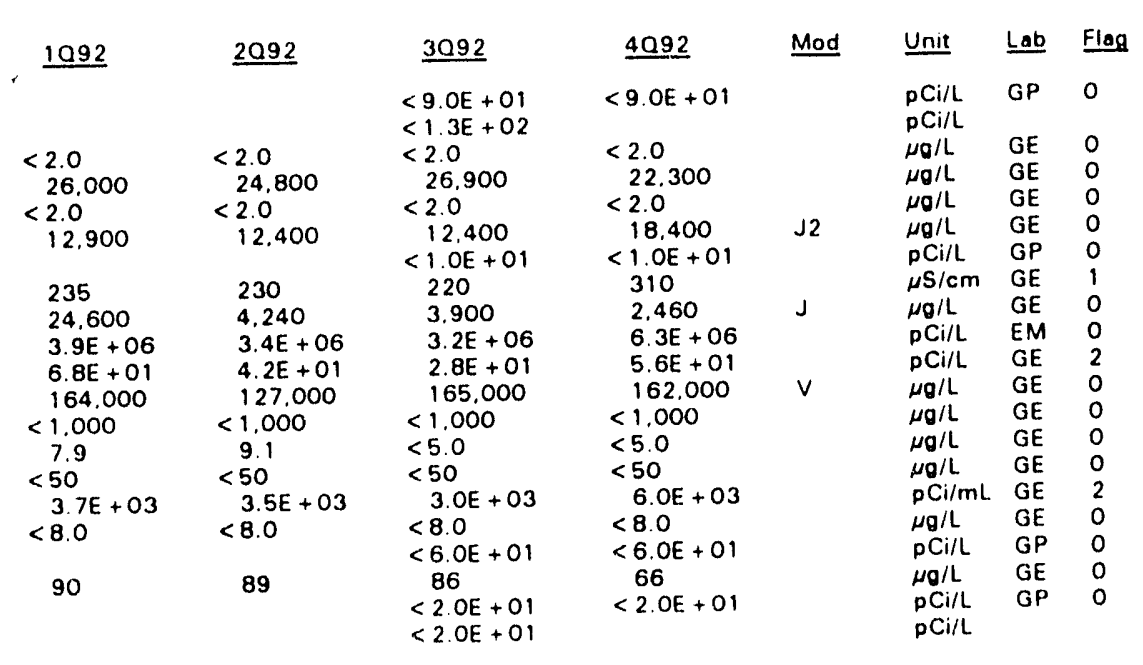

\title{
WELL HSB115C
}

$\begin{array}{ll}\text { SRS Coord. } & \text { Lat/Longitude } \\ \text { N72653.2 } & 33.277902{ }^{\circ} \mathrm{N} \\ \text { E56043.2 } & \mathbf{8 1 . 6 6 0 3 5 5 { } ^ { \circ } \mathrm { W }} \\ \text { SAMPLE DATE } & \\ \text { FIELD DATA } & \end{array}$

$\frac{\text { Screen Zone Elevation }}{192.8 \cdot 182.8 \mathrm{ft} \mathrm{msl}}$

\section{Top of Casing \\ $269.3 \mathrm{ft} \mathrm{mol}$}

$04 / 15 / 92$

$01 / 07 / 92$

1092
223.4
7.0
474
19.7
19
4.0

ANALYTICAL DATA

H D Analyte
Aluminum
Antimony
Antimony-125
Arsenic
Barium
Cadmium
Calcium
Corium-144
Cosium-134
Cosium-137
Chloride
Chromium
Chromium-51
Cobalt
Cobalt-57
Cobalt-60
Copper

Analyte

Water elevation

Sp. conductance

Water tamperature

Alkalinity as $\mathrm{CaCO}_{3}$

Alkalinity as Ca
Volume purged

4.0

$\begin{array}{cc}\frac{1092}{109} & \underline{2092} \\ 107 & <2.0 \\ <2.0 & <2.0 \\ <2.0 & 53 \\ 47 & <2.0 \\ <2.0 & 16.400 \\ 16.600 & \\ & \\ 2.400 & 2.400 \\ <4.0 & <4.0 \\ <40 & <4.0 \\ <4.0 & <4.0\end{array}$

2092
224.3
6.8
472
20.1
20
4.0

$<40$

$\begin{array}{lc}\text { Casing } & \text { Pump } \\ \text { 4" PVC } & \text { S } \\ & \\ 6 / 92 & 10 / 20 / 92\end{array}$

Formation

Barnwell (II8, ) 
ANALYTICAL DATA

$\begin{array}{ll}\text { D. } & \text { Analyte } \\ \text { Cyanide } \\ \text { Europium-152 } \\ \text { Europium-154 } \\ \text { Europium-155 } \\ \text { Fluoride } \\ \text { Gross alpha } \\ \text { lodine-131 } \\ \text { Iron } \\ \text { Lead } \\ \text { Magnesium } \\ \text { Manganese } \\ \text { Manganese-54 } \\ \text { Mercury } \\ \text { Nickel } \\ \text { Niobium-95 } \\ \text { Nitrate-nitrite as nitrogen } \\ \text { Nonvolatile beta } \\ \text { pH } \\ \text { Phenols } \\ \text { Potassium } \\ \text { Potassium-40 } \\ \text { Promethium-144 } \\ \text { Promethium-146 } \\ \text { Ruthenium-106 } \\ \text { Ruthenium-106 } \\ \text { Selenium } \\ \text { Silics } \\ \text { Silver } \\ \text { Sodium } \\ \text { Sodium-22 } \\ \text { Specific conductance } \\ \text { Sulfate } \\ \text { Total activity } \\ \text { Total aipha-emitting radium } \\ \text { Total dissolved solids } \\ \text { Total organic carbon } \\ \text { Total organic halogens } \\ \text { Total phosphates las P) } \\ \text { Tritium } \\ \text { Vanadium } \\ \text { Yttrium-88 } \\ \text { Zinc } \\ \text { Zinc-65 } \\ \text { Zirconium-95 } \\ \end{array}$

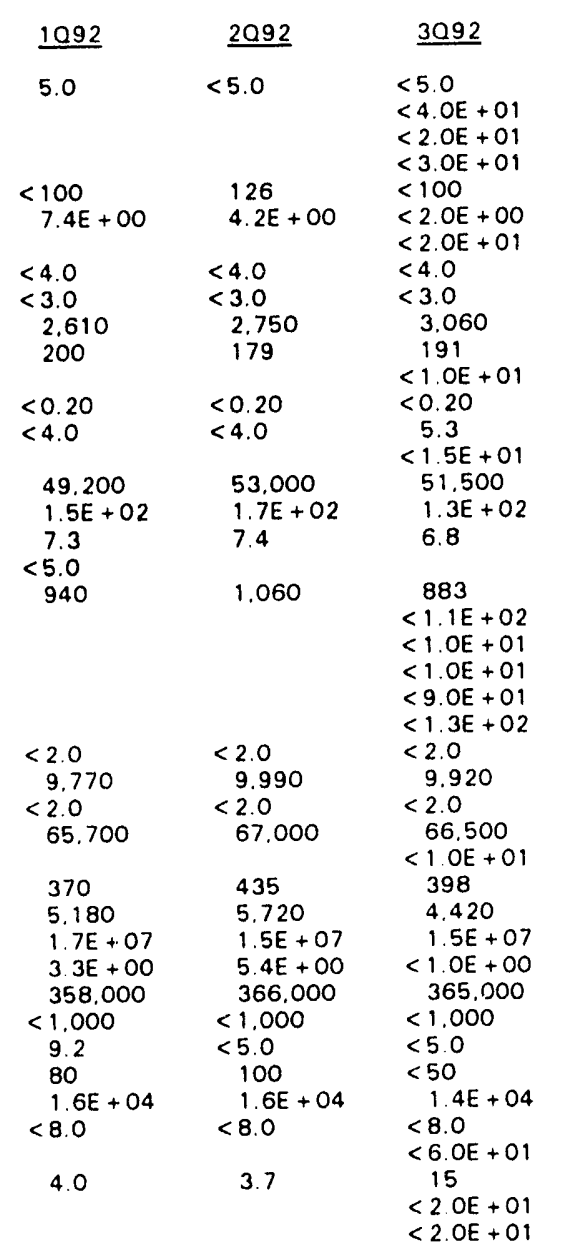

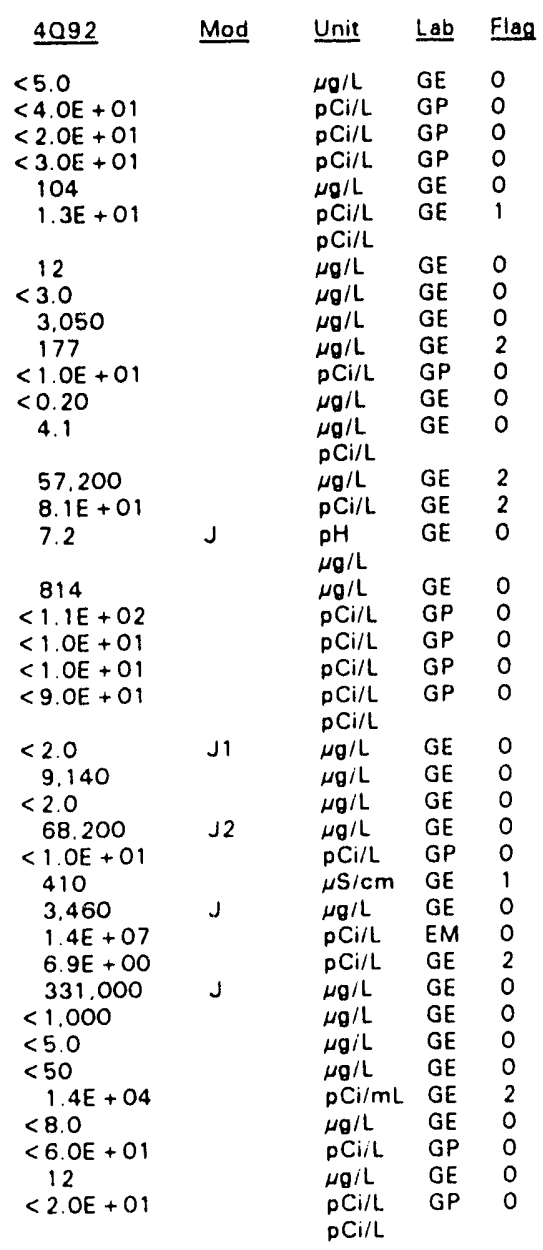

Note: Flagging levels, modifiers, and laboratories are for 4 th quarter 1992 data only. See Appendix $B$ for flagging criteria.

- =xceeded holding time for 4th quarter 1992 .

- exceeded final primary drinking water standard for 4th quarter 1992. 
WELL HSF'115D

$\begin{array}{lll}\text { SRS Coord. } & \text { Lat/Longitude } \\ \text { N72662.3 } & 33.277916^{\circ} \mathrm{N} \\ \text { E56039.8 } & 81.660381^{\circ} \mathrm{W} \\ \text { SAMPLE DATE } & \\ \text { FIELD DATA } & \end{array}$

\section{Analyte}

Water elevation

pH

$\mathrm{Sp}$. conductance

Water temperature

Alkalinity as $\mathrm{CaCO}_{3}$

Volume purged

ANALYTICAL DATA

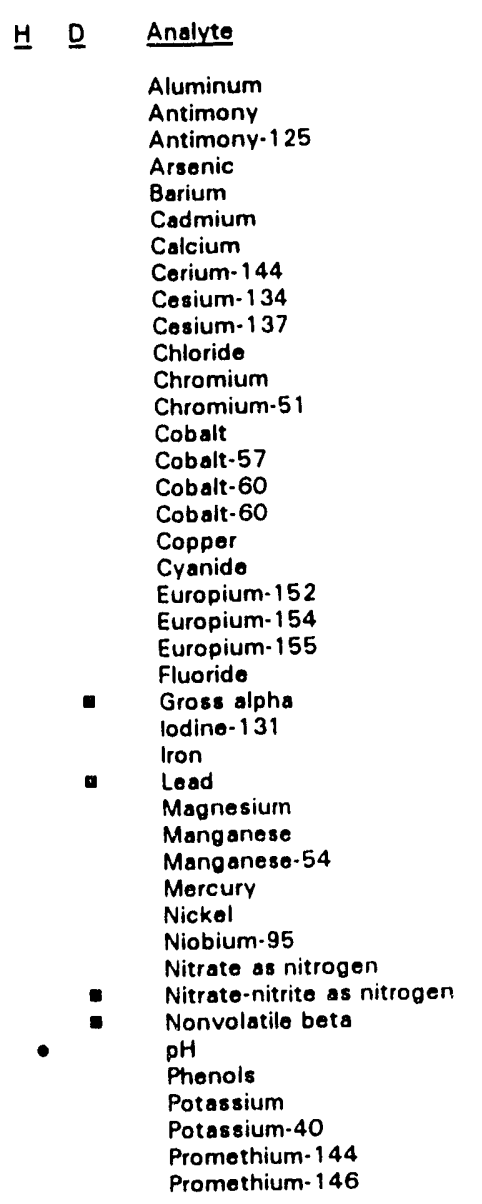

\begin{tabular}{|c|c|c|c|c|}
\hline Screen Zone Elevation & Top of Casing & Casing & Pump & Formation \\
\hline $233.9-213.9 \mathrm{ft} \mathrm{msl}$ & $269.1 \mathrm{ft} \mathrm{msl}$ & 4" PVC & $s$ & Water table $\left(11 B_{2}\right)$ \\
\hline $01 / 08 / 92$ & $04 / 15 / 92$ & $07 / 16 / 92$ & $10 / 21 / 92$ & \\
\hline 1092 & $\underline{2092}$ & $\underline{3092}$ & 4092 & $\underline{\text { Unit }}$ \\
\hline $\begin{array}{l}223.3 \\
4.3 \\
310 \\
18.4 \\
0 \\
1.1\end{array}$ & $\begin{array}{l}224.4 \\
3.8 \\
341 \\
19.2 \\
0 \\
0.4\end{array}$ & $\begin{array}{l}224.3 \\
3.9 \\
331 \\
21.4 \\
0 \\
0.6\end{array}$ & $\begin{array}{l}224.3 \\
3.5 \\
336 \\
18.2 \\
0 \\
0.4\end{array}$ & $\begin{array}{l}\mathrm{ft} \mathrm{msl} \\
\mathrm{pH} \\
\mu \mathrm{S} / \mathrm{cm} \\
{ }^{\circ} \mathrm{C} \\
\mathrm{mg} / \mathrm{L} \\
W \text { ll vol. }\end{array}$ \\
\hline
\end{tabular}

\begin{tabular}{|c|c|}
\hline 1092 & $\underline{2092}$ \\
\hline $\begin{array}{l}2.310 \\
9.8\end{array}$ & $\begin{aligned} & 3.450 \\
< & 2.0\end{aligned}$ \\
\hline $\begin{aligned} &< 2.0 \\
& 174 \\
&<2.0 \\
& 12.500\end{aligned}$ & $\begin{array}{l}<2.0 \\
202 \\
2.4 \\
12.500\end{array}$ \\
\hline
\end{tabular}

\section{$\underline{3092}$}

4092

Mod Unit Lab Flag

4.090

$<2.0$

$<2.0 E+01$

$<2.0$

212

3.5
11.200

11.200
$<6.0 E \cdots 01$

$<1.0 E+01$

$<1.0 E+01$

$\begin{array}{cc}2.500 & 1.570 \\ <4.0 & <4.0 \\ 36 & 46\end{array}$

1.630

$<4.0$

$<1.2 \mathrm{E}+02$

51

$<1.0 E+01$

$1.5 E+02$

$1.8 E+02$

$\begin{array}{rr}17 & 18 \\ <5.0 & <5.0\end{array}$

19
$<5.0$

$<4.0 E+01$

$<2.0 E+01$

$<3 . \mathrm{OE}+01$

640

$\begin{array}{ll}585 & 936 \\ 2.1 E+01 & 3.3 E+01\end{array}$

$2.5 \mathrm{E}+01$

$<2.0 E+01$

113

$\begin{array}{ll}148 & 127 \\ 54 & 71\end{array}$

54
3.100

1.060

71
2.460

2.460
1.560

$<0.20$

$<0.20$

60

2.850

1.710

$<1 . O E+01$

3.6

58
$<1.5 E+01$

31,600

$1.4 E+03$

$1.4 E+C$
4.2
$<5.0$

$<5.0$

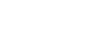

36.000

36.000
$2.4 E+03$

$2.4 \mathrm{E}$
4.1

$2.6 \mathrm{E}+03$

$2.6 \mathrm{E}$
4.0

1.710

$<1.1 E+02$

$<1.0 E+01$

$<1.0 E+01$
8.360

$<2.0$

$<2.0 \mathrm{E}+01$

$<2.0$

222

222
3.7

9.460

$<6.0 E+01$

$<1.0 E+01$

$<1.0 E+01$

1.690

$<4.0$

50

$<1.0 E+01$

$1.7 E+02$

20
$<5.0$

$<5.0$

$<4.0 E+0$

$<3.0 E+01$

585

$7.7 E+01$

1.690

66

2.650

1.730

$<1.0 E+01$

0.42

55

31.600

3. $\mathrm{OE}+03$

4.2

1,770

$<1.1 E+02$

$<1.0 E+01$

$<1.0 E+01$

\begin{tabular}{|c|c|c|}
\hline \multicolumn{3}{|l|}{$\mu_{\mathrm{g}} / \mathrm{L}$} \\
\hline $\begin{array}{l}\mu \mathrm{g} / \mathrm{L} \\
\mathrm{pCi} / \mathrm{L}\end{array}$ & $\begin{array}{l}G E \\
G P\end{array}$ & $\begin{array}{l}0 \\
0\end{array}$ \\
\hline$\mu \mathrm{g} / \mathrm{L}$ & GE & 0 \\
\hline$\mu \mathrm{g} / \mathrm{L}$ & $\mathrm{GE}$ & 0 \\
\hline$\mu g / L$ & GE & 1 \\
\hline $\begin{array}{l}\mu \mathrm{g} / \mathrm{L} \\
\mathrm{pCi/L}\end{array}$ & GE & 0 \\
\hline $\begin{array}{l}\text { pCi/L } \\
\text { pCi/L }\end{array}$ & & 0 \\
\hline $\mathrm{pCi} / \mathrm{L}$ & GP & 0 \\
\hline$\mu \boldsymbol{g} / \mathrm{L}$ & $\mathrm{GE}$ & 0 \\
\hline $\begin{array}{l}\mu g / L \\
p \mathrm{Ci} / \mathrm{L}\end{array}$ & GE & 0 \\
\hline$\mu \mathrm{g} / \mathrm{L}$ & GE & 2 \\
\hline $\mathrm{PC} / / \mathrm{L}$ & GP & 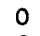 \\
\hline $\begin{array}{l}\text { pCi } / / L \\
{ }_{p} C_{i} / L\end{array}$ & GP & 2 \\
\hline$\mu \mathrm{g} / \mathrm{L}$ & GE & 0 \\
\hline$\mu \mathrm{g} / \mathrm{L}$ & $\mathrm{GE}$ & 0 \\
\hline $\mathrm{pCi} / \mathrm{L}$ & GP & 0 \\
\hline $\mathrm{pCi} / \mathrm{L}$ & GP & 0 \\
\hline $\mathrm{pCi} / \mathrm{L}$ & GP & 0 \\
\hline$\mu \mathrm{g} / \mathrm{L}$ & GE & 0 \\
\hline $\begin{array}{l}\mathrm{pCi} / \mathrm{L} \\
\mathrm{pCi} / \mathrm{L}\end{array}$ & & 2 \\
\hline$\mu \mathrm{g} / \mathrm{L}$ & GE & 2 \\
\hline$\mu \mathrm{g} / \mathrm{L}$ & $\mathrm{GE}$ & 2 \\
\hline$\mu \theta / L$ & GE & $\begin{array}{l}0 \\
2\end{array}$ \\
\hline $\begin{array}{l}\mu g / L \\
\mathrm{pCCi/L}\end{array}$ & $\begin{array}{l}\text { GE } \\
\text { GP }\end{array}$ & 0 \\
\hline$\mu_{\mathbf{g}} / \mathrm{L}$ & $\mathrm{GE}$ & 0 \\
\hline $\begin{array}{l}\mu \mathrm{g} / \mathrm{L} \\
\mathrm{pC \textrm {C } / \mathrm { L }}\end{array}$ & GE & 1 \\
\hline$\mu g / L$ & & \\
\hline$\mu \mathrm{g} / \mathrm{L}$ & $\mathrm{GE}$ & 2 \\
\hline $\begin{array}{l}\mathrm{PCC} / \mathrm{L} \\
\mathrm{pH}\end{array}$ & $\mathrm{GE}$ & 0 \\
\hline$\mu \mathrm{g} / \mathrm{L}$ & & \\
\hline $\begin{array}{l}\mu \mathrm{g} / \mathrm{L} \\
\mathrm{pCi} / \mathrm{L}\end{array}$ & $\begin{array}{l}G E \\
G P\end{array}$ & $\begin{array}{l}0 \\
0\end{array}$ \\
\hline $\mathrm{pCi} / \mathrm{L}$ & GP & 0 \\
\hline $\mathrm{pCi} / \mathrm{L}$ & $G P$ & 0 \\
\hline
\end{tabular}

Note: Flagging levels, modifiers, and laboratories are for 4th quarter 1992 data only. See Appendix B for flagging criteria. $-=$ exceeded holding time for 4 th quarter 1992.

- exceeded final primary drinking water standard for 4th quarter 1992. 
WSRC-TR.93.059

Well HSB1150 continued

ANALYTICAL DATA

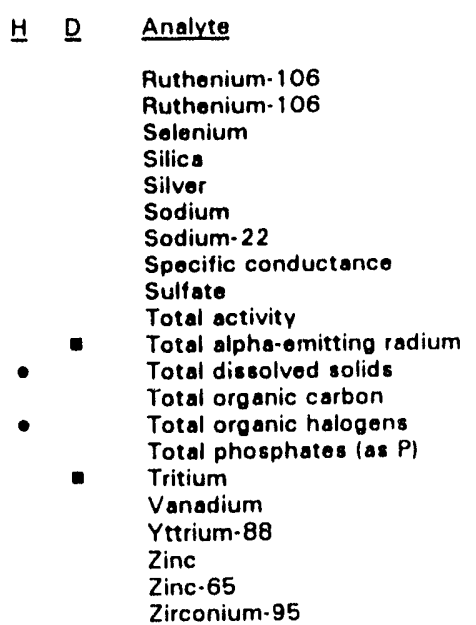

$\begin{array}{lll}1092 & \underline{2092} & \underline{3092} \\ & & <9.0 E+01 \\ & & <1.3 E+02 \\ <2.0 & <2.0 & <2.0 \\ 24.700 & 31.600 & 30.100 \\ <2.0 & <2.0 & <2.0 \\ 22.600 & 19.000 & 20.700 \\ 240 & 300 & <1.0 E+01 \\ 8.360 & 1.040 & 288 \\ 7.5 E+06 & 9.1 E+06 & 2.070 \\ 3.9 E+01 & 3.8 E+01 & 2.8 E+06 \\ 198.000 & 241.000 & 220.000 \\ <1.000 & <1.000 & <1.000 \\ <5.0 & <5.0 & <5.0 \\ 100 & 250 & 190 \\ 9.0 E+03 & 8.6 E+03 & 7.0 E+03 \\ <8.0 & <8.0 & <8.0 \\ & & <6.0 E+01 \\ 178 & 191 & 183 \\ & & <2.0 E+01 \\ & & <2.0 E+01\end{array}$

\begin{tabular}{|c|c|c|c|c|}
\hline 4092 & Mod & Unit & $\underline{L a b}$ & Flag \\
\hline$<9.0 E+01$ & & $\begin{array}{l}p \mathrm{Ci} / \mathrm{L} \\
p \mathrm{Ci} / \mathrm{L}\end{array}$ & GP & 0 \\
\hline$<2.0$ & & $\mu_{g} / \mathrm{L}$. & GE & 0 \\
\hline 37.500 & & $\mu g / L$ & GE & 0 \\
\hline$<2.0$ & & $\mu \mathrm{g} / \mathrm{L}$ & GE & 0 \\
\hline 21.100 & J2 & $\mu \sigma / L$ & GE & 0 \\
\hline$<1.0 E+01$ & & $\mathrm{pCi} / \mathrm{L}$ & GP & 0 \\
\hline 300 & & $\mu \mathrm{S} / \mathrm{cm}$ & $\begin{array}{l}\mathrm{GE} \\
\mathrm{GE}\end{array}$ & $\begin{array}{l}1 \\
0\end{array}$ \\
\hline $\begin{array}{l}1.240 \\
8.4 E+06\end{array}$ & & $\begin{array}{l}\mu g / L \\
p C i / L\end{array}$ & $E M$ & 0 \\
\hline $6.1 E+01$ & & $\mathrm{pCi} / \mathrm{L}$ & GE & 2 \\
\hline 187.000 & $J$ & $\mu g / L$ & $G E$ & 0 \\
\hline 1.800 & & $\mu \mathrm{g} / \mathrm{h}$ & GE & $\begin{array}{l}0 \\
0\end{array}$ \\
\hline $\begin{array}{l}<5.0 \\
<50\end{array}$ & $\mathrm{~J}$ & $\begin{array}{l}\mu g / L \\
\mu g / L\end{array}$ & $\begin{array}{l}\text { GE } \\
\mathrm{GE}\end{array}$ & 0 \\
\hline $7.6 E+03$ & & $\mathrm{pCi} / \mathrm{mL}$ & $\mathrm{GE}$ & 2 \\
\hline$<8.0$ & & $\mu \mathbf{g} / \mathrm{L}$ & $\mathrm{GE}$ & 0 \\
\hline$<6.0 E+01$ & & $\mathrm{pCi} / \mathrm{L}$ & $G P$ & 0 \\
\hline 174 & & $\mu \mathrm{g} / \mathrm{L}$ & $\mathrm{GE}$ & 0 \\
\hline$<2.0 E+01$ & & $\begin{array}{l}\mathrm{pCi} / \mathrm{L} \\
\mathrm{pCi} / \mathrm{L}\end{array}$ & GP & 0 \\
\hline
\end{tabular}

\section{WELL HSB116C}

$\begin{array}{ll}\text { SpS Coord. } & \text { Lat/Longitude } \\ \text { N72888.1 } & 33.278333^{\circ} \mathrm{N} \\ \text { E55989.1 } & 81.660953^{\circ} \mathrm{W}\end{array}$

$\frac{\text { Screen Zone Elevation }}{190.5 \cdot 180.5 \mathrm{ft} \mathrm{msl}}$

$\begin{array}{llll}\text { Top of Casing } & \text { Casing } & \text { Pump } & \text { Formation } \\ 257.5 \mathrm{ft} \mathrm{msl} & \text { 4" PVC } & V & \text { Barnwell (IIB,) }\end{array}$

SAMPLE DATE

$01 / 02 / 92$

$04 / 13 / 92$

$07 / 16 / 92$

$10 / 25 / 92$

FIELD DATA

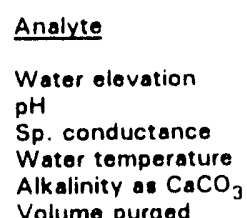

$\begin{array}{ll}\underline{1092} & \underline{2092} \\ 223.6 & 225.4 \\ 5.2 & 4.6 \\ 547 & 540 \\ 18.6 & 19.6 \\ 0 & 0 \\ 4.0 & 4.0\end{array}$

3092
225.4
4.7
530
20.8
0
4.0

4092
225.6
4.7
491
21.4
0
2.1

Unit
ft msl
$\mathrm{pH}$
$\mu \mathrm{S} / \mathrm{cm}$
${ }^{\circ} \mathrm{C}$
$\mathrm{mg} / \mathrm{L}$
Well vol

ANALYTICAL DATA

$\begin{array}{ll}\text { H D } \quad & \text { Anglyte } \\ & \text { Aluminum } \\ \text { Antimony } \\ \text { Antimony-125 } \\ \text { Arsenic } \\ \text { Barium } \\ \text { Cadmium } \\ \text { Calcium } \\ \text { Corium-144 } \\ \text { Cesium-134 } \\ \text { Cosium-137 } \\ \text { Chloride } \\ \text { Chromium } \\ \text { Chromium-51 } \\ \text { Cobalt } \\ \text { Cobalt-57 } \\ \text { Cobalt-60 } \\ \text { Copper }\end{array}$

$\begin{array}{cc}\frac{1092}{2092} \\ 146 & 161 \\ <2.0 & <2.0 \\ <2.0 & <2.0 \\ 81 & 84 \\ <2.0 & <2.0 \\ 4.070 & 4.310 \\ & \\ 4.480 & 4.470 \\ <4.0 & <4.0 \\ 67 & 70 \\ <4.0 & <4.0\end{array}$

$\begin{aligned} & 3092 \\ & 178 \\ < & 2.0 \\ < & 2.0 E+01 \\ < & 2.0 \\ & 84 \\ < & 2.0 \\ & 4.480 \\ < & 6.0 E+01 \\ < & 1.0 E+01 \\ < & 1.0 E+01 \\ & 4.010 \\ < & 4.0 \\ < & 1.2 E+02 \\ & 72 \\ < & 1.0 E+01 \\ < & 1.0 E+01 \\ < & 4.0\end{aligned}$

$\begin{aligned} & 4092 \\ & 198 \\ < & 2.0 \\ < & 2.0 E+01 \\ < & 2.0 \\ & 88 \\ < & 2.0 \\ & 4.870 \\ < & 6.0 E+01 \\ < & 1.0 E+01 \\ < & 1.0 E+01 \\ & 4.100 \\ < & 4.0 \\ & 70 \\ < & 1.0 E+01 \\ < & 1.0 E+01 \\ < & 4.0\end{aligned}$

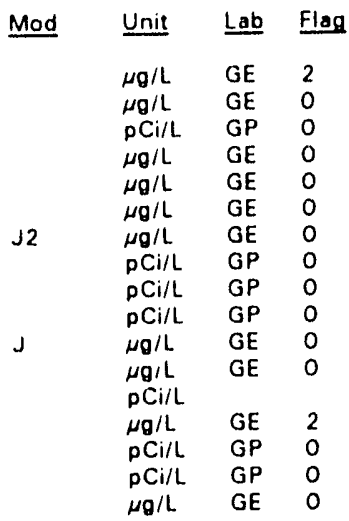

Note: Flagging levels, modifiers, and laboratories are for 4 th quarter 1992 data only. See Appendix B for flagging criteria.

- = exceeded holding time for 4 th quarter 1992.

- =xceeded final primary drinking water standard for 4th quarter 1992.

H-Area Seepage Basins 
WSRC.TR-93-059

Well HSB116C continued

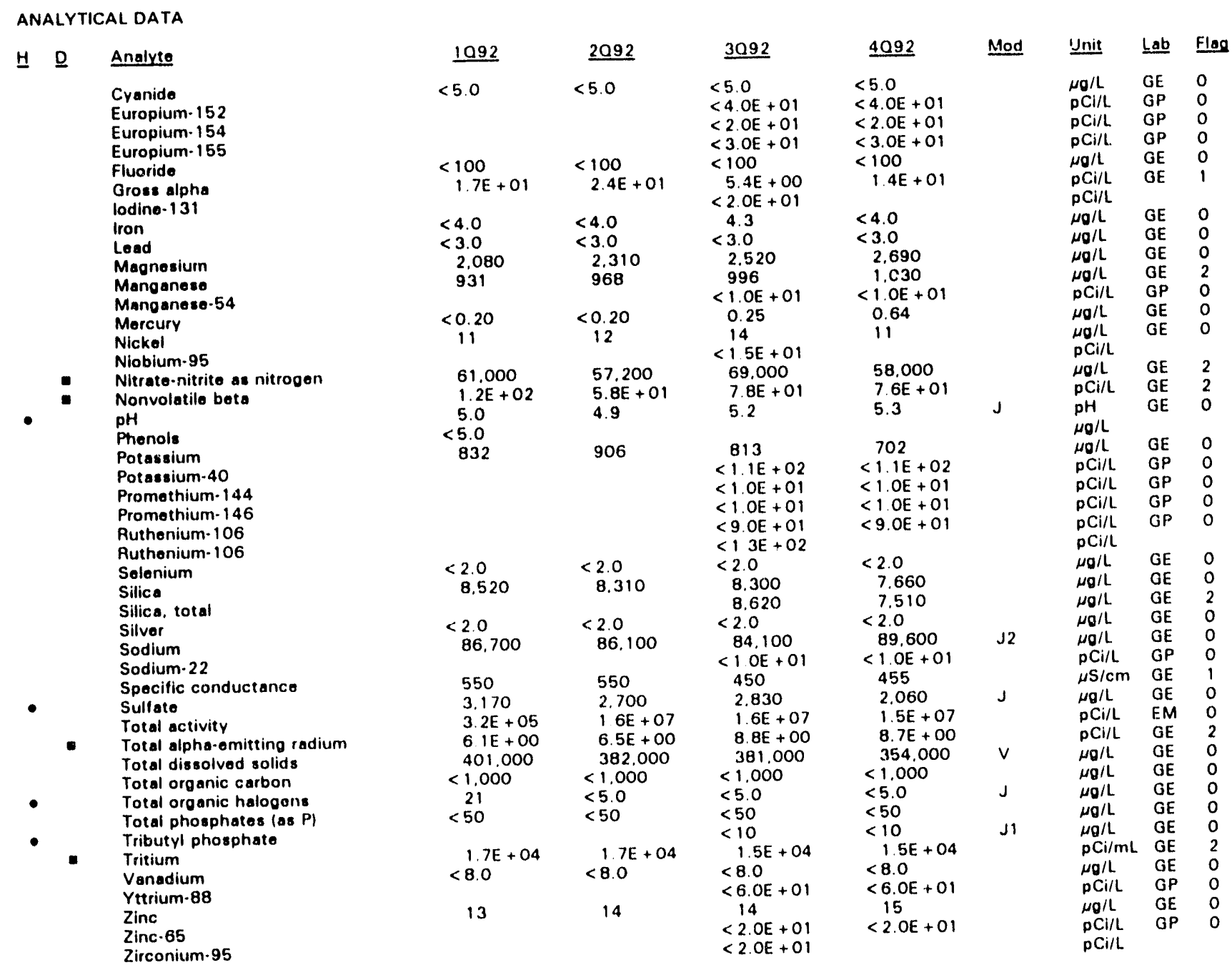

Note: Flegging levels, modifiers, and laboratories are for 4 ih quarter 1992 data only. See Appendix B for flagging criteria.

- = exceeded holding time for 4th quarter 1992.

- exceeded final primary drinkıng water standard for 4th quarter 1992. 
WELL HSB1 16D

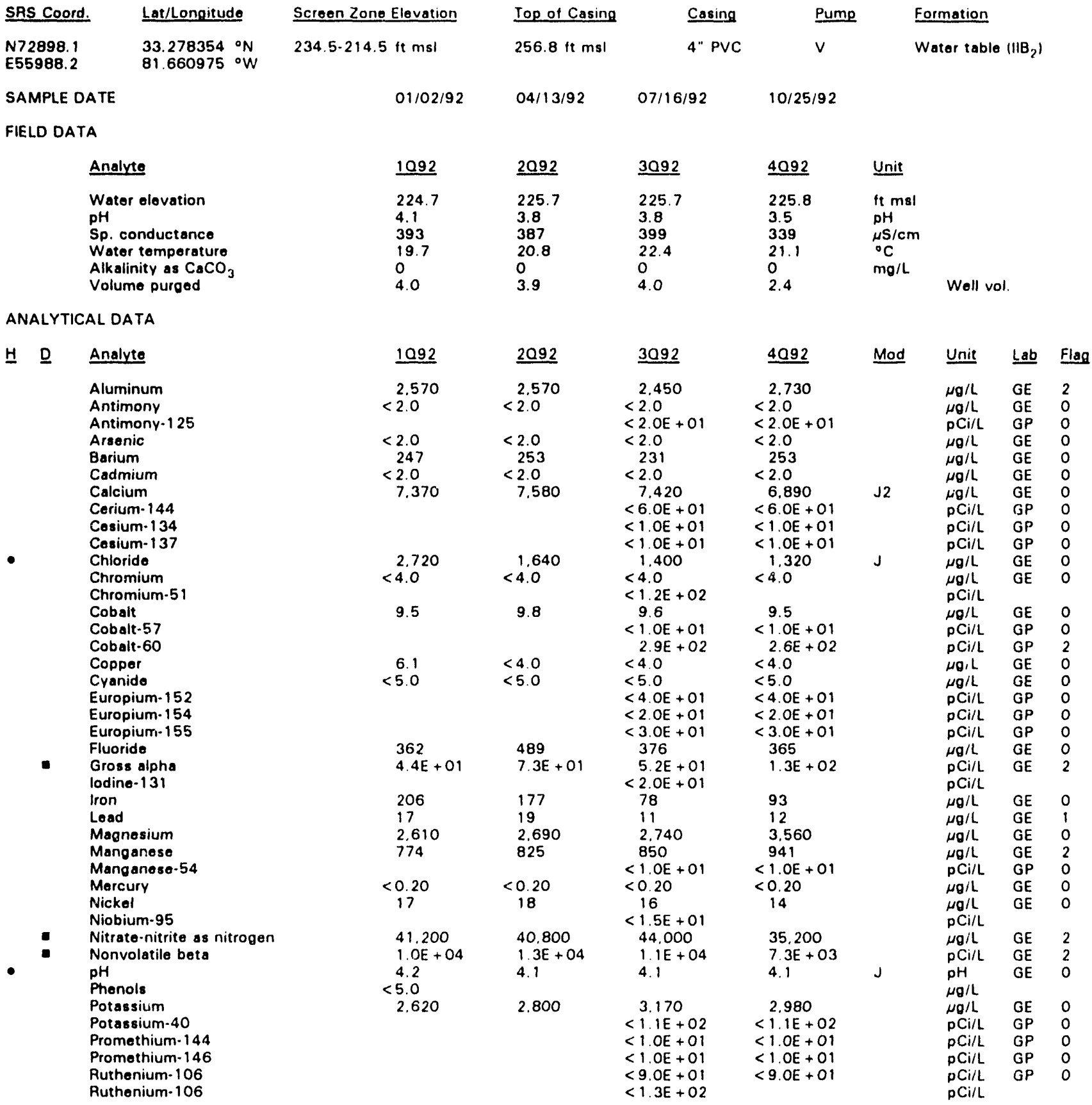

Note: Flagging levels, modifiers, and laboratories are for 4 th quarter 1992 data only. See Appendix 8 for tlagging criteria. - =xceeded holding time for 4 th quarter 1992.

- exceeded final primary drinking water standard for 4th quarter 1992. 
Woll HSB1160 continued

ANALYTICAL DATA

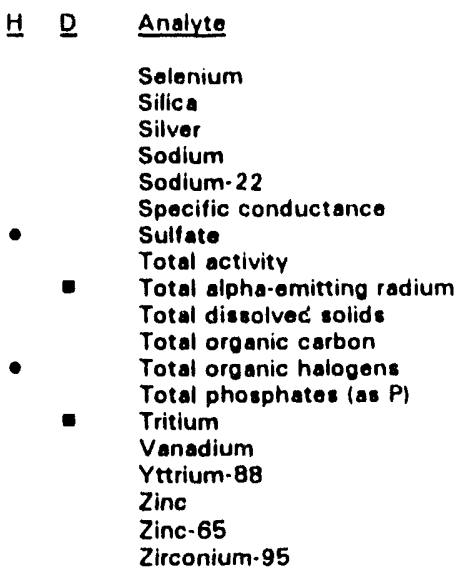

$\begin{array}{lll}1092 & \underline{2092} & \underline{3092} \\ <2.0 & <2.0 & <20 \\ 11.200 & 10.600 & 12.000 \\ <2.0 & <2.0 & <2.0 \\ 36.400 & 34.600 & 35.900 \\ & & <1.0 E+01 \\ 305 & 385 & 338 \\ 12.600 & 2.080 & 2.650 \\ 1.0 E+07 & 9.7 E+06 & 6.5 E+06 \\ 1.2 E+02 & 1.7 E+02 & 5.8 E+01 \\ 228.000 & 224.000 & 234.000 \\ <1.000 & <1.000 & <1.000 \\ 86 & <5.0 & <5.0 \\ <50 & <50 & <50 \\ 1.1 E+04 & 8.1 E+03 & 6.3 E+03 \\ <8.0 & <8.0 & <8.0 \\ & & <6.0 E+01 \\ 79 & 73 & 69 \\ & & <2.0 E+01 \\ & & <2.0 E+01\end{array}$

\begin{tabular}{|c|c|c|c|c|}
\hline 4092 & Mod & Unit & $\underline{\text { Lab }}$ & Flag \\
\hline$<2.0$ & & $\mu \mathrm{g} / \mathrm{L}$ & $\mathrm{GE}$ & 0 \\
\hline 9.400 & & $\mu \mathrm{g} / \mathrm{L}$ & $\mathrm{GE}$ & 0 \\
\hline$<2.0$ & & $\mu \mathrm{g} / \mathrm{L}$ & $\mathrm{GE}$ & 0 \\
\hline 26.300 & $\mathrm{~J} 2$ & $\mu \mathrm{g} / \mathrm{L}$ & $\mathrm{GE}$ & 0 \\
\hline$<1.0 E+01$ & & $\mathrm{pCi} / \mathrm{L}$ & $G P$ & 0 \\
\hline 280 & & $\mu \mathrm{S} / \mathrm{cm}$ & $\mathrm{GE}$ & 1 \\
\hline 3.250 & $J$ & $\mu_{0} / \mathrm{L}$ & GE & 0 \\
\hline $2.6 \mathrm{E}+06$ & & $\mathrm{PCi} / \mathrm{L}$ & EM & 0 \\
\hline $\begin{array}{l}8.9 E+01 \\
181,000\end{array}$ & $\mathrm{v}$ & $\begin{array}{l}\mathrm{PC} / \mathrm{L} \\
\mathrm{HO} / \mathrm{L}\end{array}$ & GE & 2 \\
\hline$<1,000$ & & $\mu \mathrm{g} / \mathrm{L}$ & GE & 0 \\
\hline$<5.0$ & $J$ & $\mu \mathrm{g} / \mathrm{h}$ & $\mathrm{GE}$ & 0 \\
\hline$<50$ & & $\mu \mathrm{g} / \mathrm{L}$ & $\mathrm{GE}$ & 0 \\
\hline 2. $7 E+03$ & & $\mathrm{pCi} / \mathrm{mL}$ & $G E$ & 2 \\
\hline$<8.0$ & & $\mu_{\mathbf{g}} / \mathrm{L}$ & $G E$ & 0 \\
\hline$<6.0 E+01$ & & $\mathrm{pCi} / \mathrm{L}$ & $G P$ & 0 \\
\hline 65 & & $\mu \mathrm{g} / \mathrm{h}$ & GE & 0 \\
\hline$<2.0 E+$ & & $\begin{array}{l}\mathrm{pCi} / \mathrm{L} \\
\mathrm{pCi} / \mathrm{L}\end{array}$ & $G P$ & 0 \\
\hline
\end{tabular}

\section{WELL HSB117A}

\begin{tabular}{|c|c|c|c|c|c|c|c|c|c|}
\hline \multirow{2}{*}{$\begin{array}{l}\text { SAS Coord. } \\
\text { N72733.6 } \\
\text { E55170.1 }\end{array}$} & Lat/Longitude & Screen Zone Elevation & Top of Casing & Casing & Pump & \multicolumn{2}{|c|}{ Formation } & & \\
\hline & $\begin{array}{l}33.2766555^{\circ} \mathrm{N} \\
81.662810^{\circ} \mathrm{W}\end{array}$ & $94.1 .84 .1 \mathrm{ft} \mathrm{msl}$ & $236.3 \mathrm{ft} \mathrm{msl}$ & & $\mathbf{s}$ & $M$ & Congaree & ||$|A|$ & \\
\hline \multicolumn{2}{|c|}{ SAMPLE DATE } & $01 / 14 / 92$ & $04 / 13 / 92$ & $07 / 22 / 92$ & $10 / 16 / 92$ & & & & \\
\hline \multicolumn{10}{|c|}{ FIELD DATA } \\
\hline \multicolumn{2}{|r|}{ Analyte } & 1092 & $\underline{2092}$ & $\underline{3092}$ & 4092 & \multicolumn{4}{|l|}{ Unit } \\
\hline & $\begin{array}{l}\text { Water olevation } \\
\text { pH } \\
\text { Sp. conductance } \\
\text { Water temperature } \\
\text { Alkalinity as } \mathrm{CaCO}_{3} \\
\text { Volume purged }\end{array}$ & $\begin{array}{l}166.9 \\
6.7 \\
142 \\
17.4 \\
47 \\
4.0\end{array}$ & $\begin{array}{l}166.7 \\
6.6 \\
151 \\
18.1 \\
53 \\
4.0\end{array}$ & $\begin{array}{l}166.5 \\
6.5 \\
150 \\
20.1 \\
55 \\
4.0\end{array}$ & $\begin{array}{l}166.6 \\
6.5 \\
143 \\
18.8 \\
46 \\
3.3\end{array}$ & $\begin{array}{l}\mathrm{ft} \mathrm{msl} \\
\mathrm{pH} \\
\mu \mathrm{S} / \mathrm{cm} \\
{ }^{\circ} \mathrm{C} \\
\mathrm{mg} / \mathrm{L} \\
\text { Well vol. }\end{array}$ & & & \\
\hline \multicolumn{10}{|c|}{ ANALYTICAL DATA } \\
\hline \multirow[t]{2}{*}{$\underline{\mathrm{H}} \quad \underline{\mathrm{D}}$} & Analyte & 1092 & $\underline{2092}$ & 3092 & 4092 & Mod & Unit & $\underline{L a b}$ & Flag \\
\hline & $\begin{array}{l}\text { Aluminum } \\
\text { Antimony } \\
\text { Arsenic } \\
\text { Barium } \\
\text { Cadmium } \\
\text { Calcium } \\
\text { Chloride } \\
\text { Chromium } \\
\text { Cobalt } \\
\text { Copper } \\
\text { Cyanide } \\
\text { Fluoride } \\
\text { Gross alpha } \\
\text { lron } \\
\text { Lead } \\
\text { Magnesium } \\
\text { Manganose } \\
\text { Mercury } \\
\text { Nickel }\end{array}$ & $\begin{aligned} &< 20 \\
&< 2.0 \\
&< 2.0 \\
& 31 \\
&< 2.0 \\
& 23.900 \\
& 4.800 \\
&< 4.0 \\
&< 4.0 \\
&< 4.0 \\
&< 5.0 \\
& 128 \\
&< 2.0 E+00 \\
&<4.0 \\
&<3.0 \\
& 814 \\
& 101 \\
&<0.20 \\
&<4.0\end{aligned}$ & $\begin{aligned} &< 20 \\
&< 2.0 \\
&< 2.0 \\
& 30 \\
&< 2.0 \\
& 23.600 \\
& 2.370 \\
&< 4.0 \\
&< 4.0 \\
&< 4.0 \\
&< 5.0 \\
& 185 \\
&<2.0 E+O 0 \\
&<4.0 \\
&<3.0 \\
& 792 \\
& 89 \\
&< 0.20 \\
&<4.0\end{aligned}$ & $\begin{aligned} &< 20 \\
&< 2.0 \\
&< 2.0 \\
& 29 \\
&< 2.0 \\
& 24.500 \\
& 2.340 \\
&< 4.0 \\
&< 4.0 \\
&< 4.0 \\
&<5.0 \\
&< 100 \\
&< 2.0 E+00 \\
&<4.0 \\
&<3.0 \\
& 765 \\
& 81 \\
&< 0.20 \\
&< 4.0\end{aligned}$ & $\begin{aligned} &< 20 \\
&< 2.0 \\
&< 2.0 \\
& 23 \\
&< 20 \\
& 24.800 \\
& 2.390 \\
&< 4.0 \\
&< 4.0 \\
&< 4.0 \\
&< 5.0 \\
& 131 \\
&< 2.0 E+00 \\
& 4.9 \\
&< 3.0 \\
& 803 \\
& 74 \\
&<0.20 \\
&<4.0\end{aligned}$ & $J 2$ & 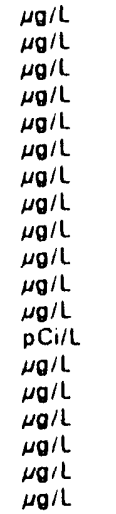 & $\begin{array}{l}G E \\
G E \\
G E \\
G E \\
G E \\
G E \\
G E \\
G E \\
G E \\
G E \\
G E \\
G E \\
G E \\
G E \\
G E \\
G E \\
G E \\
G E \\
G E\end{array}$ & $\begin{array}{l}0 \\
0 \\
0 \\
0 \\
0 \\
0 \\
0 \\
0 \\
0 \\
0 \\
0 \\
0 \\
0 \\
0 \\
0 \\
0 \\
2 \\
0 \\
0\end{array}$ \\
\hline
\end{tabular}

Note: Flagging levels, modifiers, and laboratories are for 4th quarter 1992 data only. See Appendix $B$ for flagging criteria.

- exceeded holding time for 4th quarter 1992.

- =xceeded final primary drinking water standard for 4th quarter 1992.

H-Area Seepage Basins

$D \cdot 118$
Fourth Quarter 1992 
WSRC.TR.93.059

Woll HSB117A continued

ANALYTICAL DATA

H D Analyte

Nitrate as nitrogen Nitrate-nitrite as nitrogen

Nonvolatile bete

pH

Phonols

Potasaium

Solonium

Silica

Silver

Sodium

Specific conductance

Sulfate

Total alpha-emitting radium

Total dissolved solids

Total organic carbon

Total organic halogens

Total phosphates las PI

Tritium

Vanadium

Zine

$\begin{array}{lll}1092 & \underline{2092} & \underline{3092} \\ <50 & & \\ & & \\ <2.0 E+00 & <2.0 E+00 & <50 \\ 6.8 & 6.9 & 2.3 E+00 \\ <5.0 & & 6.8 \\ 1.060 & 1.150 & \\ <2.0 & <2.0 & 1.380 \\ 29.300 & 27.900 & <2.0 \\ <2.0 & <2.0 & 28.500 \\ 1.770 & 1.810 & <2.0 \\ 148 & 115 & 1.820 \\ 5.740 & 6.300 & 132 \\ <1.0 E+00 & 1.2 E+00 & <1.0 E+00 \\ 97.000 & 98.000 & 103.000 \\ <1.000 & <1.000 & <1.000 \\ 11 & 52 & <5.0 \\ 70 & 150 & 170 \\ <7.0 E \cdot 01 & <7.0 E \cdot 01 & <7.0 E \cdot 01 \\ <8.0 & <8.0 & <8.0 \\ <2.0 & <2.0 & <2.0 \\ & & \end{array}$

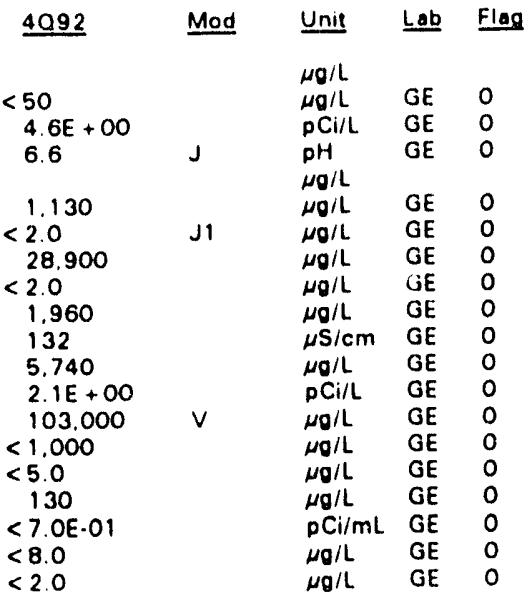

WELL HSB $117 \mathrm{C}$

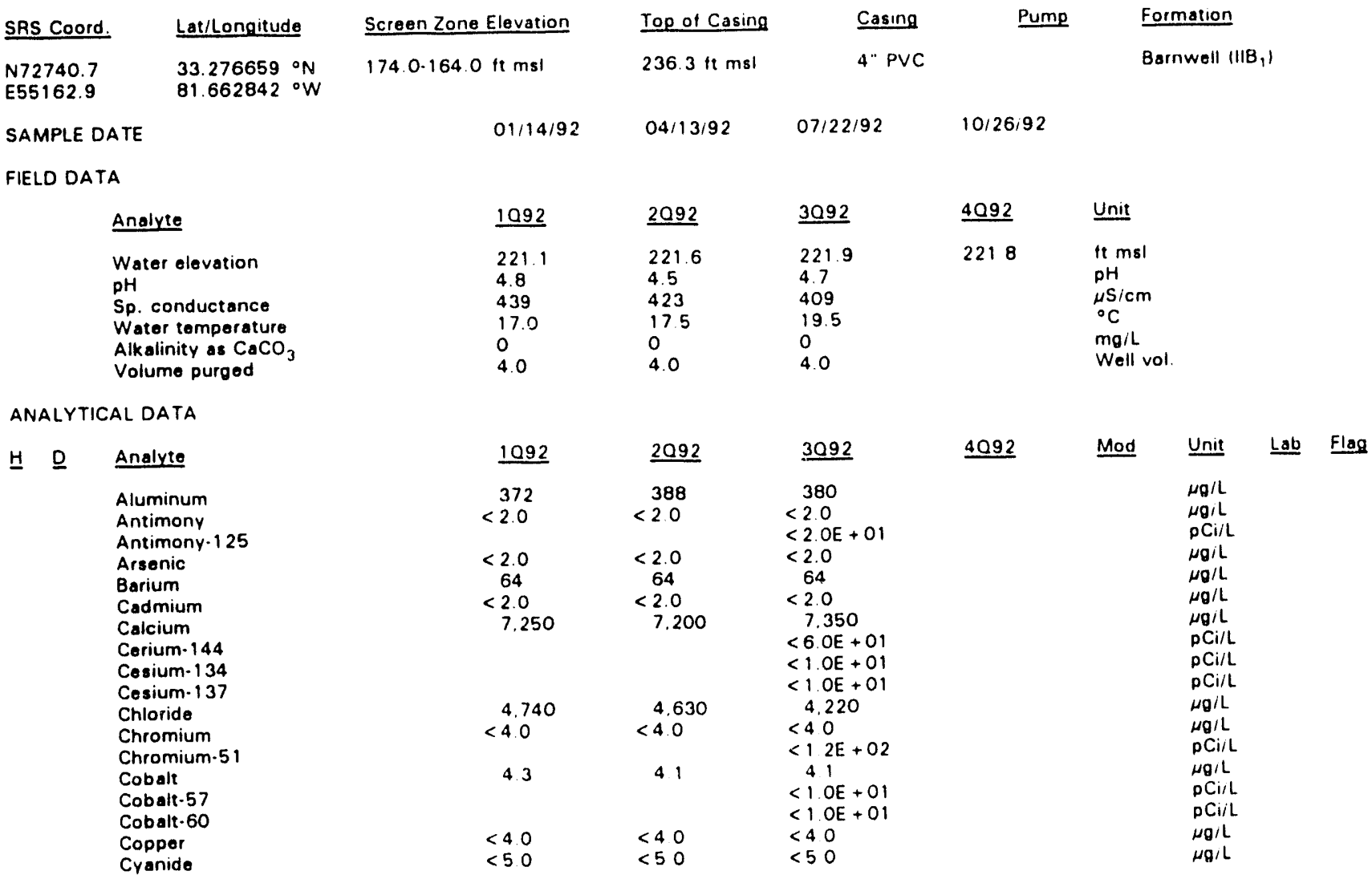

Note: Flagging levels, modifiers, and laboratories are for 4 th quarter 1992 data only. See Appendix B for flagging criteria.

- = exceeded holding ume for 4 th quarter 1992.

- =xceeded final primary drinking water standard for 4th quarter 1992. 
WSRC.TR-93.059

Woll HSB117C continued

\section{ANALYTICAL DATA}

브 D

\section{Anolvte}

Europium-152

Europium-154

Europium- 155

Fluoride

Grose alphe

lodine-131

Iron

Load

Magnesium

Manganese

Manganese. 54

Mercury

Nickol

Niobium-95

Nitrate-nitrite as nitrogen

Nonvolatile beta

pH

Phenols

Potassium

Potsssium-40

Promethium-144

Promethium-146

Ruthenium +106

Ruthenium.106

Solonium

Silica

Silver

Sodium

Sodium-22

Specific conductance

Sulfate

Total activity

Total alpha-omitting radium

Total dissolved solids

Total organic carbon

Total organic halogens

Total phosphates las PI

Tritium

Vanadium

Yttrium.88

Zinc

Zinc. 65

Zirconium-95

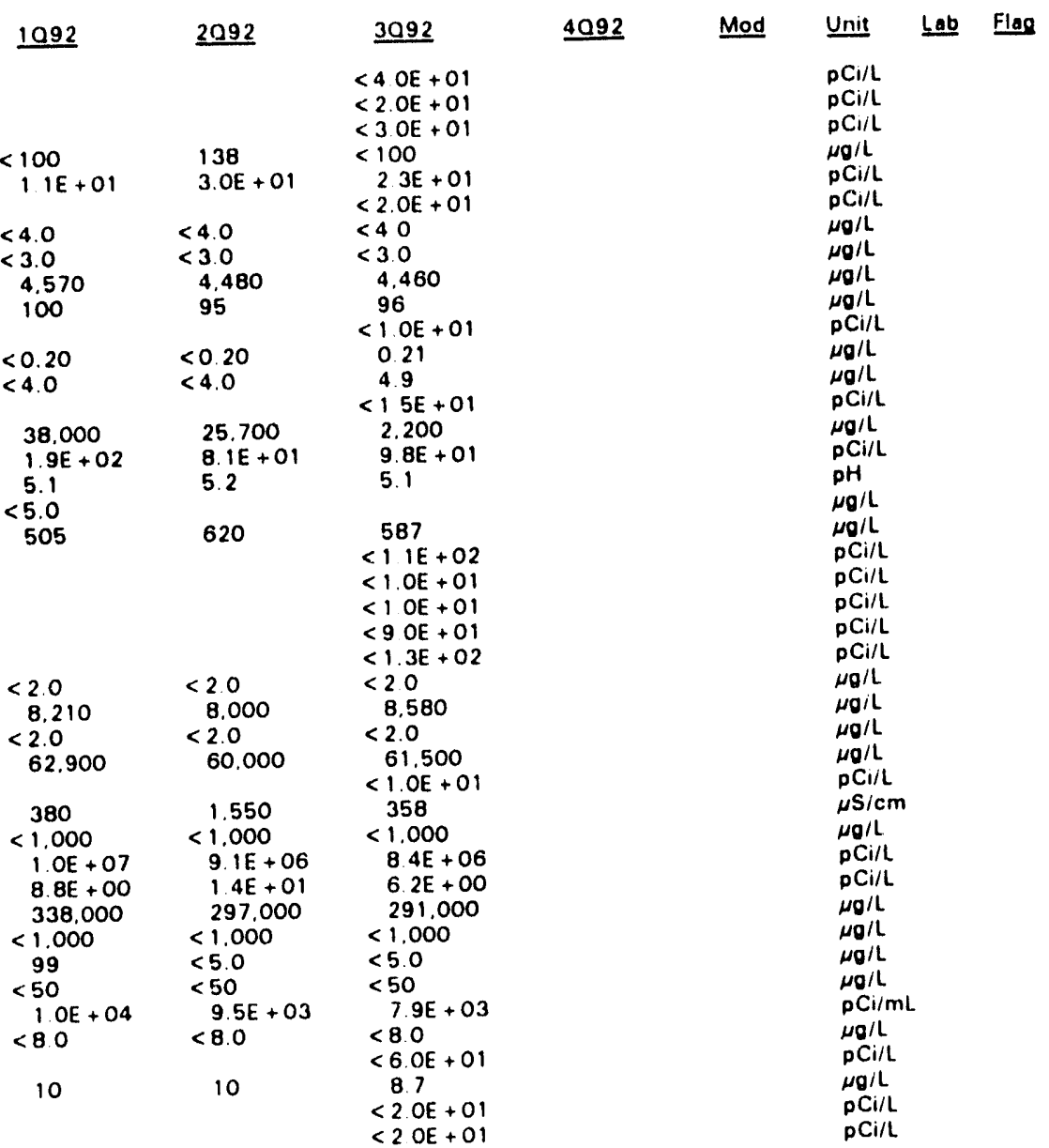

Note: Flagging levels, modifiers, and laboratories are for 4 th quarter 1992 data only. See Appendix B for flagging criteria.

- =xceecied holding time for 4th quarter 1992.

- exceeded final primary drinking water standard for 4th quarter 1992.

H-Area Seepage Basins

D.120 
WSRC.TR.93.059

WELL HSB117D

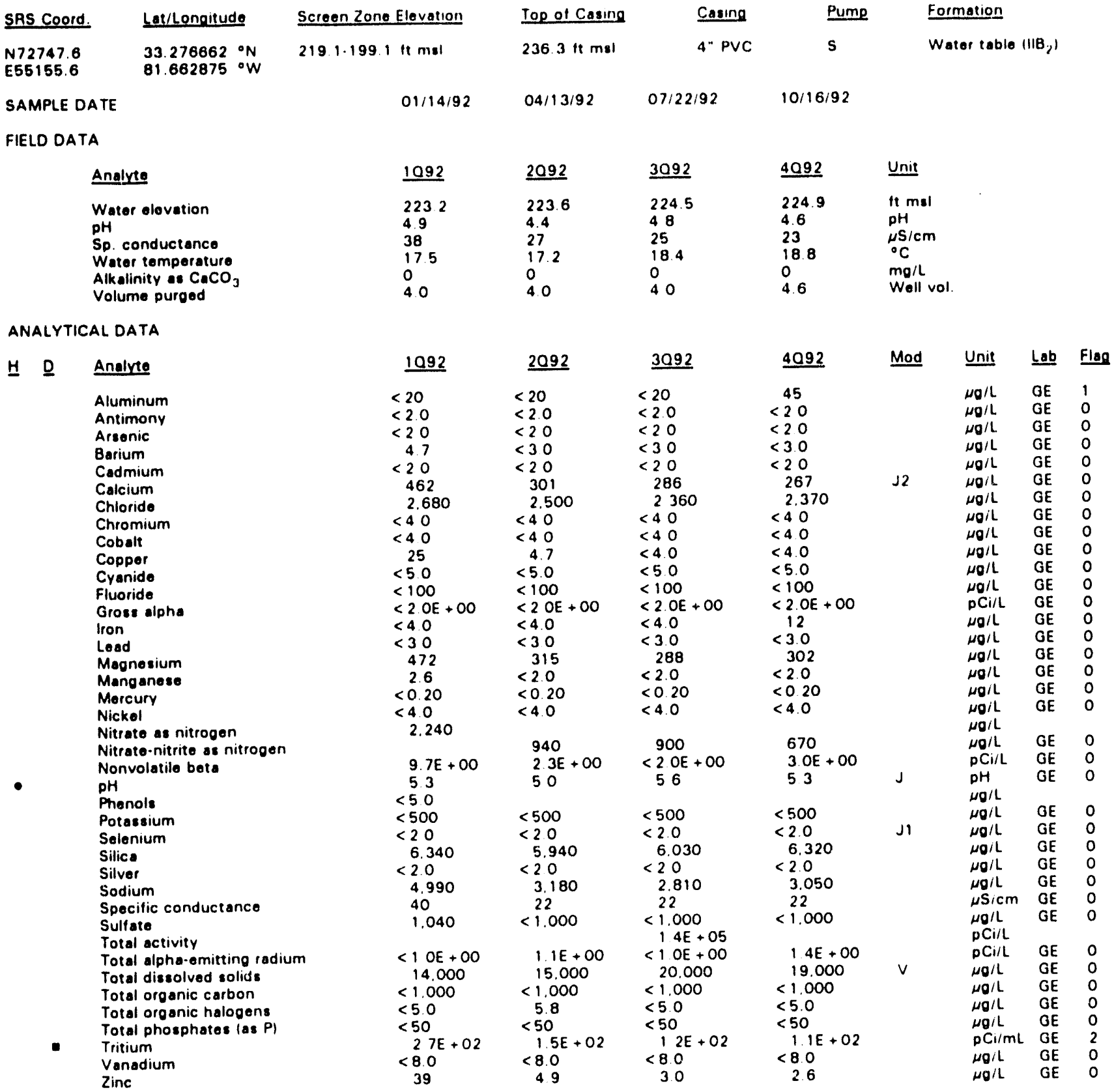

Note: Flagging levels, modifiers, and laboratories are for 4th quarter 1992 data only. See Appendix 8 for flagging criteria.

- =xceeded holding time for 4th quarter 1992.

- exceeded final primary drinking water standard for 4th quarter 1992.

H-Area Seepage Basins

D. 121

Fourth Quarter 1992 
WELL HSB1 18A

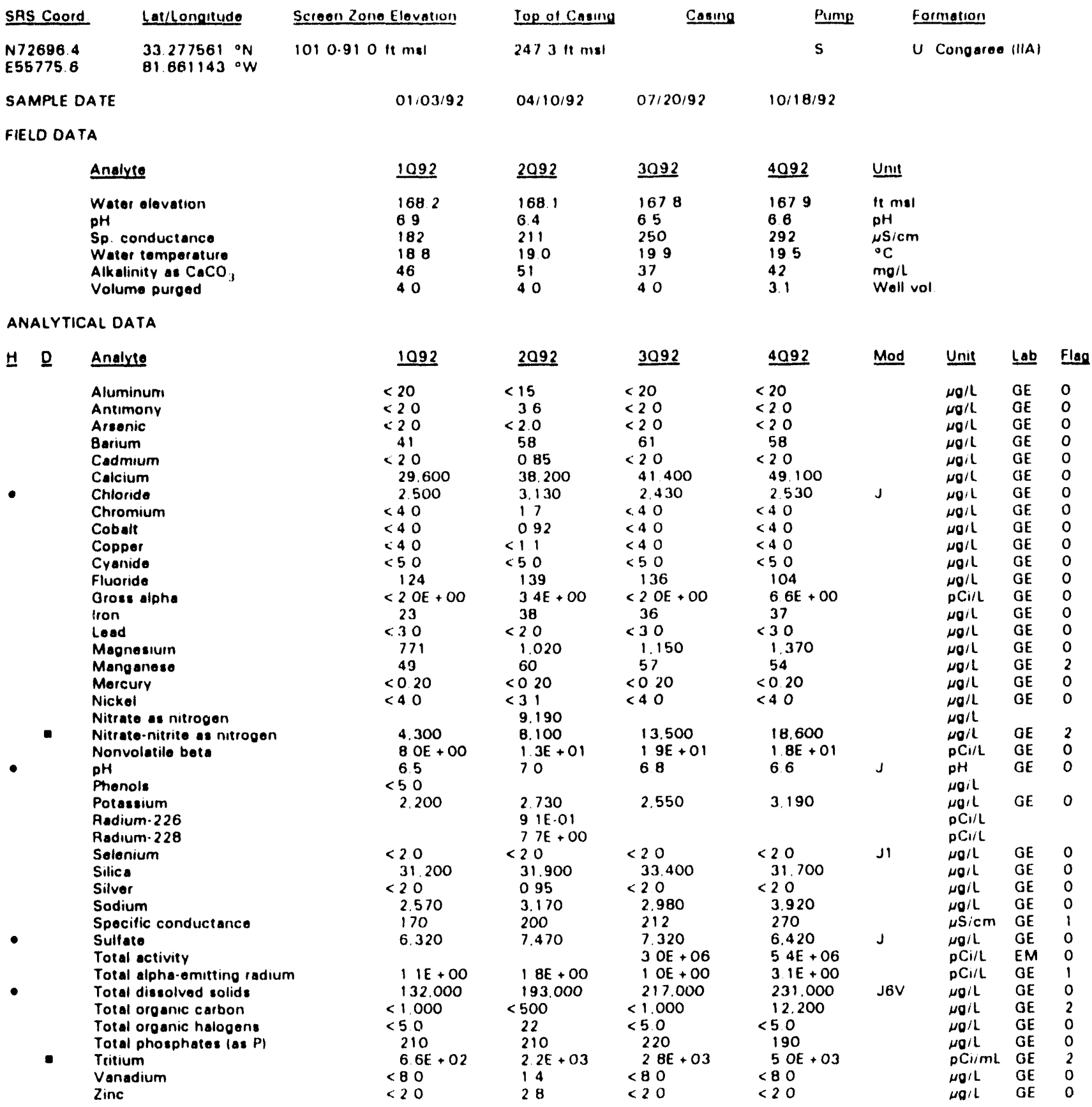

Note: Flagging levels, modifiers, and laboratories are for 4th quarter 1992 data only. See Appendix B for flagging criteria.

- excoeded holding lime for 4th quarter 1992 .

- exceeded final primary drinking water standard for 4th quarter 1992. 
WELL HSB119A

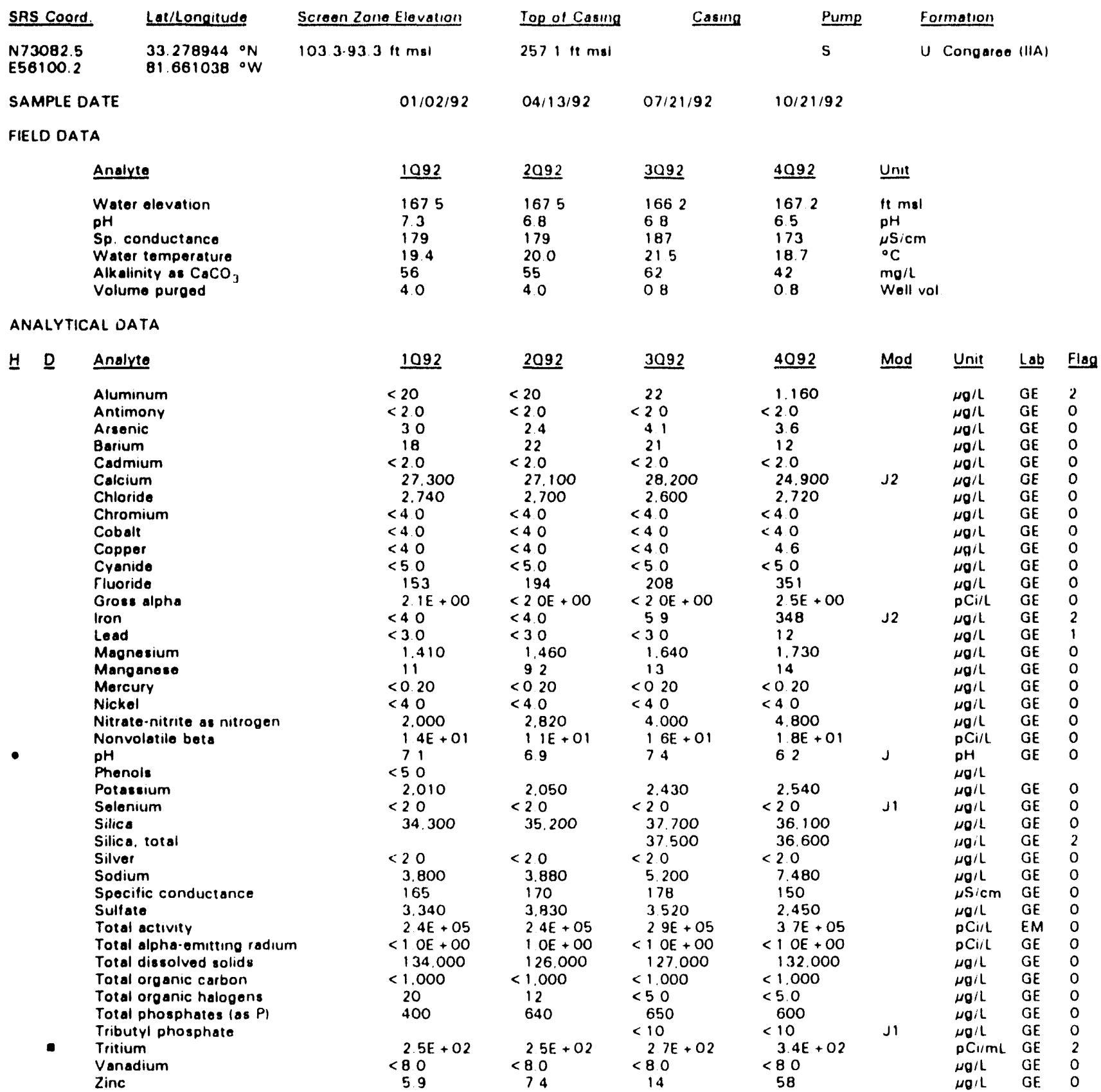

Note: Flagging levels, modifiers, and laboratories are for 4 th quarter 1992 data only. See Appendix B for flagging criteria. - =xceeded holding time for 4th quarter 1992.

- =xceeded final primary drinking water standard for 4th quarter 1992. 
WSRC.TR-93.059

\section{WELL HSB120A}

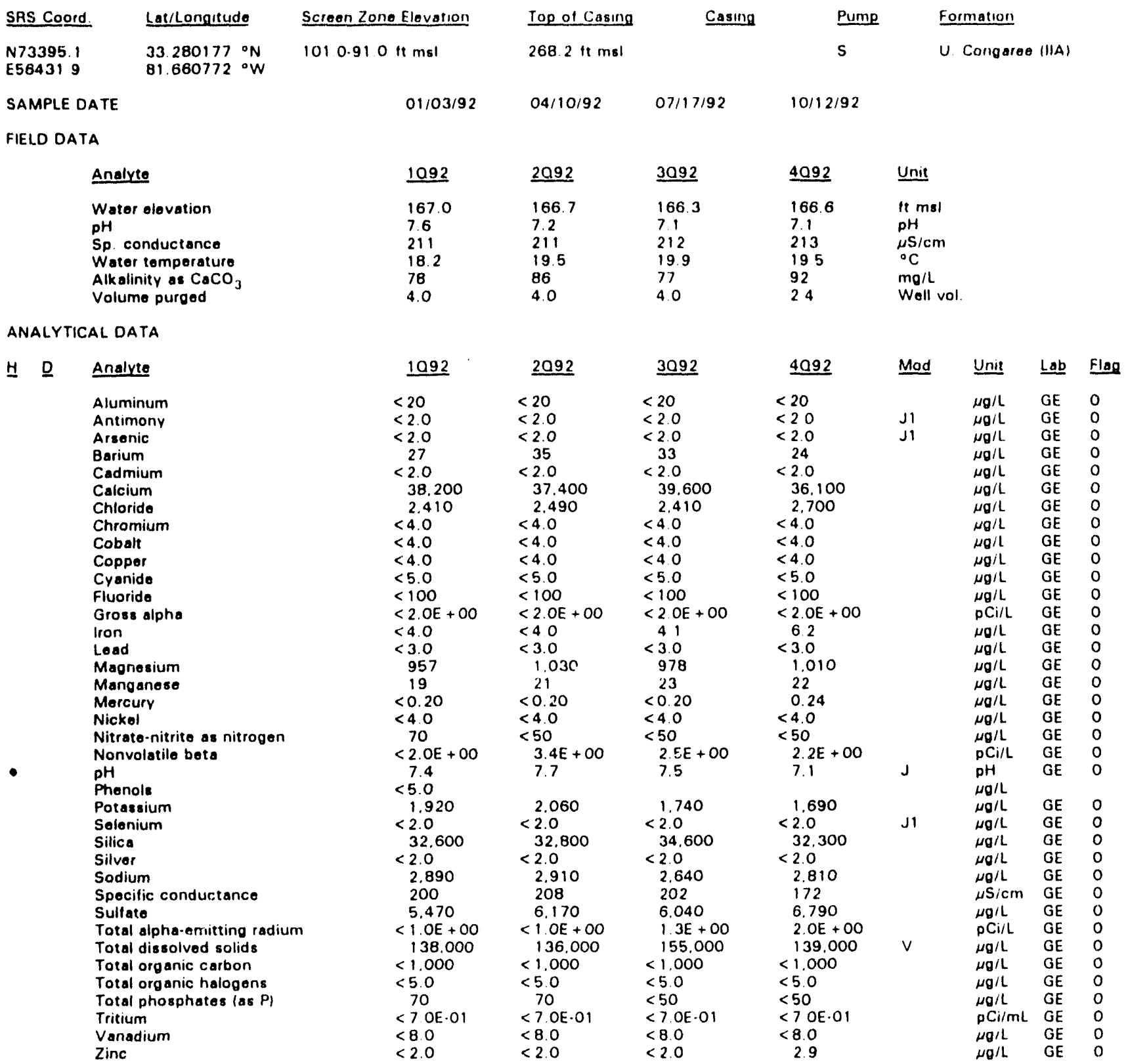

Note: Flagging levels, modifiers, and laboratories are for 4th quarter 1992 data only. See Appendix B for flagging criteria.

- = exceeded holding tıme for 4th quarter 1992.

- =xceeded final primary drinking water standard for 4th quarter 1992. 
WELL HISB121A

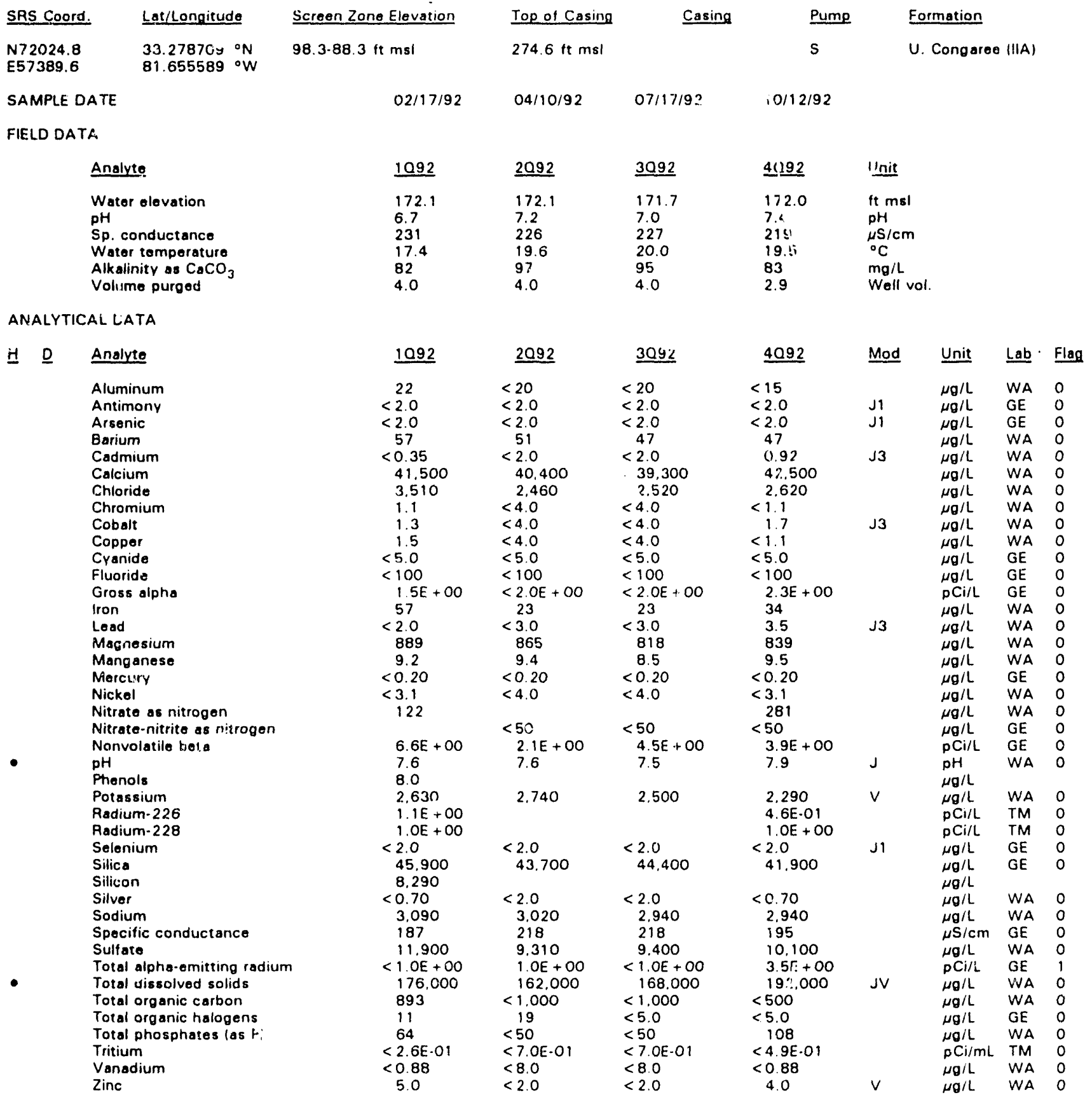

Note: Flagging levels, modifiers, and laboratories are for 4 th quarter 1992 data only. See Appendix B for flagging criteria.

- = exceeded hoiding time for 4th quarter 1992.

- exceeded tinal primary drinking water standard for 4th quarter 1992. 
WELL HSB122A

\begin{tabular}{|c|c|c|c|c|c|c|c|c|c|}
\hline SRS Coord & Lat/Longitude & Screen Zone Elevation & Top of Casing & Casing & Pump & For & imation & & \\
\hline $\begin{array}{l}N 72195.9 \\
E 57747.4\end{array}$ & $\begin{array}{l}33.279671^{\circ} \mathrm{N} \\
81.654979{ }^{\circ} \mathrm{W}\end{array}$ & $95.4-85.4 \mathrm{ft} \mathrm{msl}$ & $271.6 \mathrm{ft} \mathrm{msl}$ & & S & $U$ & Congaree & $(I I A)$ & \\
\hline SAMPLE D & ATE & $01 / 03 / 92$ & $04 / 10 / 92$ & $07 / 17 / 92$ & $10 / 12 / 92$ & & & & \\
\hline IELD OAT & & & & & & & & & \\
\hline & Analyte & 1092 & 2092 & 3092 & 4092 & Unit & & & \\
\hline & $\begin{array}{l}\text { Water elevation } \\
\text { pH } \\
\text { Sp. conductance } \\
\text { Water tomperature } \\
\text { Alkalinity as } \mathrm{CaCO}_{3} \\
\text { Volume purged }\end{array}$ & $\begin{array}{l}172.1 \\
7.2 \\
223 \\
17.8 \\
84 \\
4.0\end{array}$ & $\begin{array}{l}171.9 \\
6.9 \\
225 \\
19.6 \\
89 \\
4.0\end{array}$ & $\begin{array}{l}171.4 \\
6.9 \\
228 \\
19.9 \\
84 \\
4.0\end{array}$ & $\begin{array}{l}171.6 \\
7.1 \\
224 \\
19.5 \\
85 \\
2.6\end{array}$ & $\begin{array}{l}\text { ft msl } \\
\mathrm{pH} \\
\mu \mathrm{S} / \mathrm{cm} \\
{ }^{\circ} \mathrm{C} \\
\mathrm{mg} / \mathrm{L} \\
\text { Well vol }\end{array}$ & & & \\
\hline NALYTIC & AL DATA & & & & & & & & \\
\hline$\underline{D}$ & Analyte & 1092 & $\underline{2092}$ & 3092 & 4092 & Mod & Unit & $\underline{L a b}$ & Flag \\
\hline & 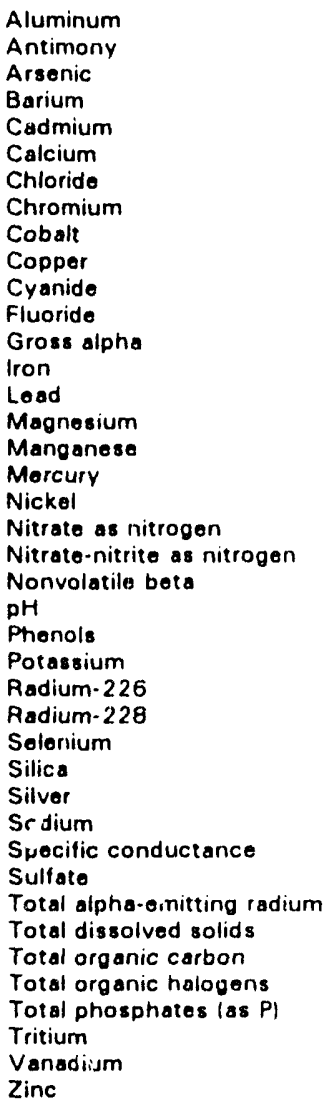 & $\begin{aligned}< & 20 \\
< & 2.0 \\
< & 2.0 \\
& 17 \\
< & 2.0 \\
& 43.100 \\
& 2.680 \\
< & 4.0 \\
< & 4.0 \\
< & 4.0 \\
< & 5.0 \\
< & 100 \\
< & 2.0 E+00 \\
& 18 \\
< & 3.0 \\
& 790 \\
& 5.1 \\
< & 0.20 \\
< & 4.0 \\
< & 50 \\
< & 2.0 E+00 \\
& 7.1 \\
< & 5.0 \\
& 738\end{aligned}$ & $\begin{aligned}< & 20 \\
< & 2.0 \\
< & 2.0 \\
& 24 \\
< & 2.0 \\
& 42.000 \\
& 2.540 \\
< & 4.0 \\
< & 4.0 \\
< & 4.0 \\
< & 5.0 \\
< & 100 \\
< & 2.0 E+00 \\
& 16 \\
< & 3.0 \\
& 840 \\
& 5.6 \\
< & 0.20 \\
< & 4.0 \\
< & 50 \\
< & 2.0 E+00 \\
& 73 \\
& 879\end{aligned}$ & 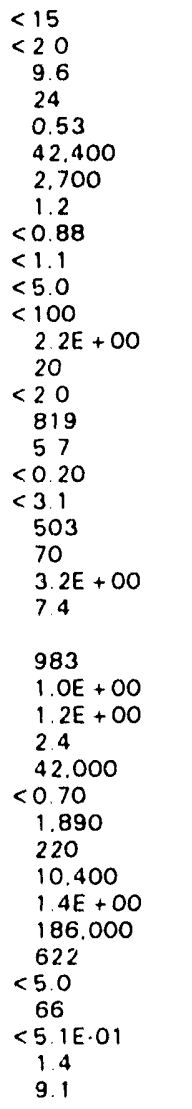 & $\begin{aligned}< & 20 \\
< & 2.0 \\
< & 2.0 \\
& 11 \\
< & 2.0 \\
& 39.700 \\
& 2.690 \\
< & 4.0 \\
< & 4.0 \\
< & 4.0 \\
< & 5.0 \\
< & 100 \\
< & 2.0 E+00 \\
& 17 \\
< & 3.0 \\
& 795 \\
& 3.6 \\
< & 0.20 \\
< & 4.0 \\
< & 50 \\
& 2.0 E+00 \\
& 6.8 \\
& 690\end{aligned}$ & $\begin{array}{l}J 1 \\
J 1\end{array}$ & 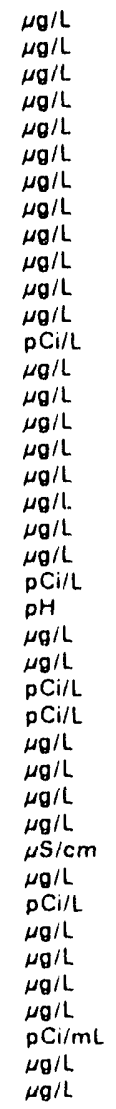 & $\begin{array}{l}\mathrm{GE} \\
\mathrm{GE} \\
\mathrm{GE} \\
\mathrm{GE} \\
\mathrm{GE} \\
\mathrm{GE} \\
\mathrm{GE} \\
\mathrm{GE} \\
\mathrm{GE} \\
\mathrm{GE} \\
\mathrm{GE} \\
\mathrm{GE} \\
\mathrm{GE} \\
\mathrm{GE} \\
\mathrm{GE} \\
\mathrm{GE} \\
\mathrm{GE} \\
\mathrm{GE} \\
\mathrm{GE} \\
\mathrm{GE} \\
\mathrm{GE} \\
\mathrm{GE} \\
\mathrm{GE}\end{array}$ & $\begin{array}{l}0 \\
0 \\
0 \\
0 \\
0 \\
0 \\
0 \\
0 \\
0 \\
0 \\
0 \\
0 \\
0 \\
0 \\
0 \\
0 \\
0 \\
0 \\
0 \\
0 \\
0 \\
0 \\
0 \\
0\end{array}$ \\
\hline
\end{tabular}

Note: Flagging levels, modifiers, and laboratories are for 4 th quarter 1992 data only. See Appendix B for flagging criteria.

- = exceeded holding time for 4th quarter 1992.

- =xceeded final primary drinking water standard for 4 th quarter 1992.

H-Area Seepage Basins

$D \cdot 126$
Fourin Quurter 1392 
WELL HSB1 23A

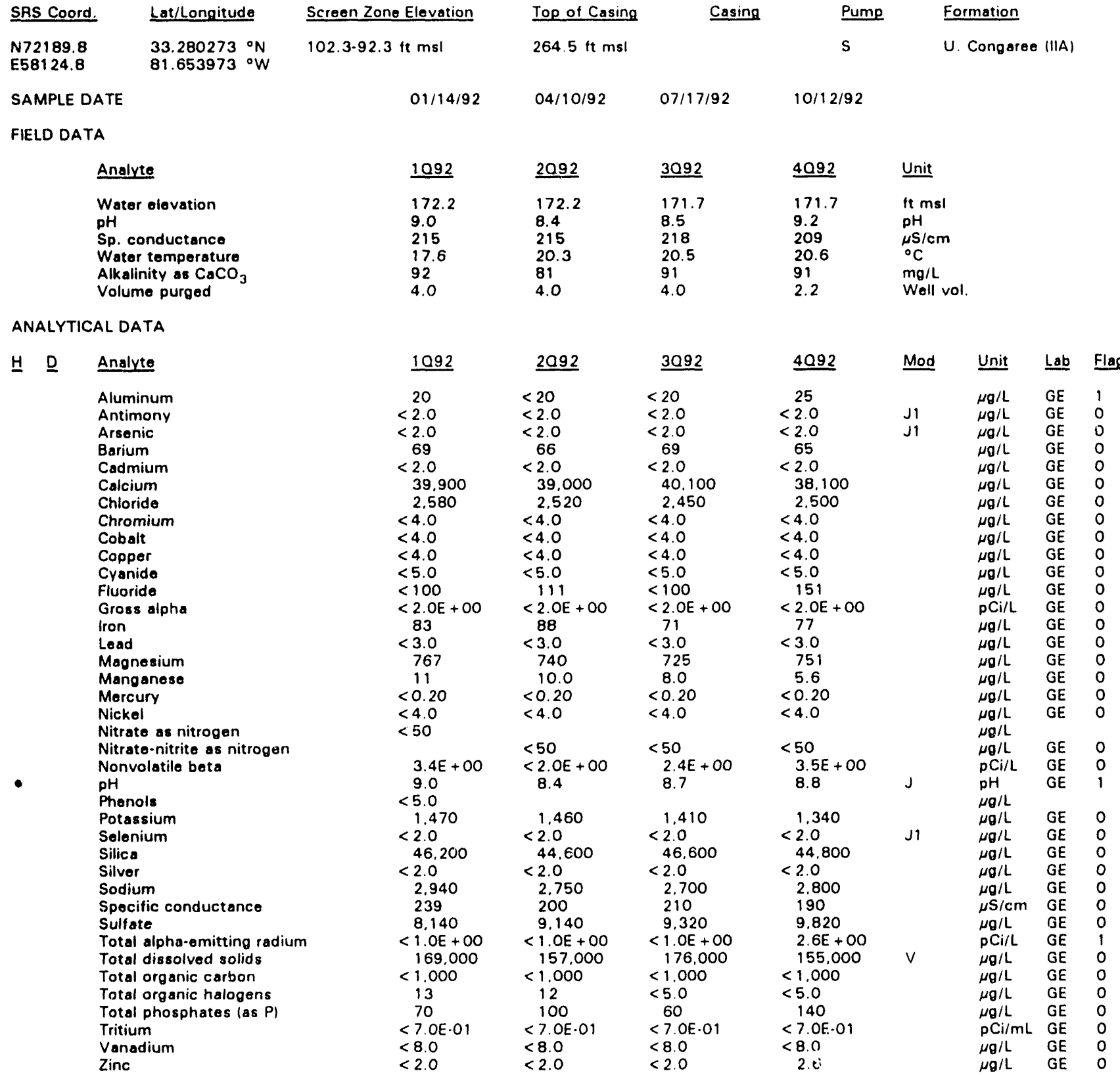

Note: Flagging levels, modifiers, and laboratories are for 4 th quarter 1992 data only. See Appendix $B$ for flagging criteria. - =xceeded holding time for 4th quarter 1992.

- =xceeded final primary drinking water standard for 4th quarter 1992. 


\section{WELL HSB124A}

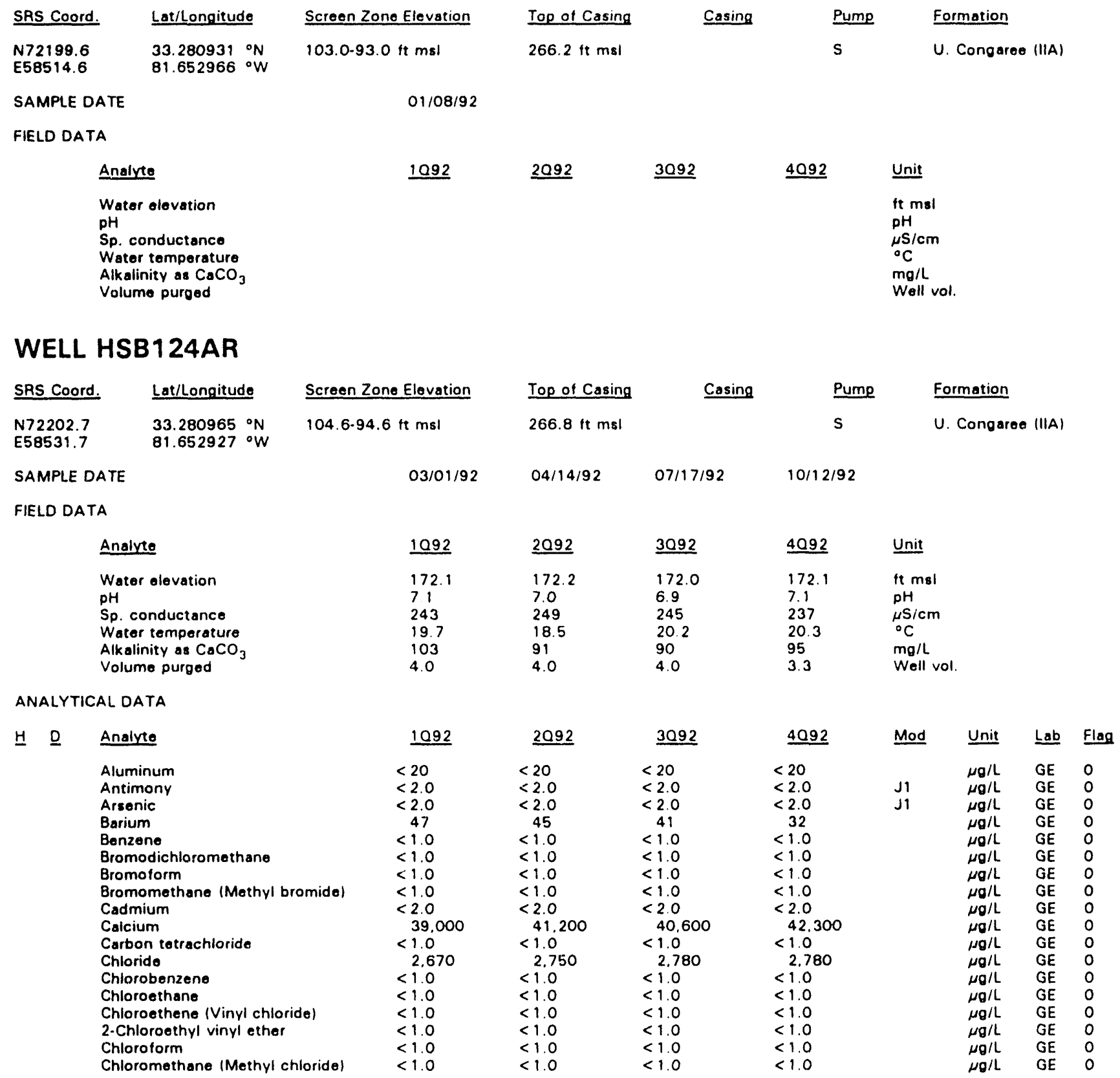

Note: Flagging levels, modifiers, and laboratories are for 4 th quarter 1992 data only. See Appendix B for flagging criteria.

- =xceeded holding time for 4 th quarter 1992.

- =xceeded final primary drinking water standard for 4th quarter 1992. 
WSRC-TR-93-059

Woll HSB1 24AR continued

ANALYTICAL DATA

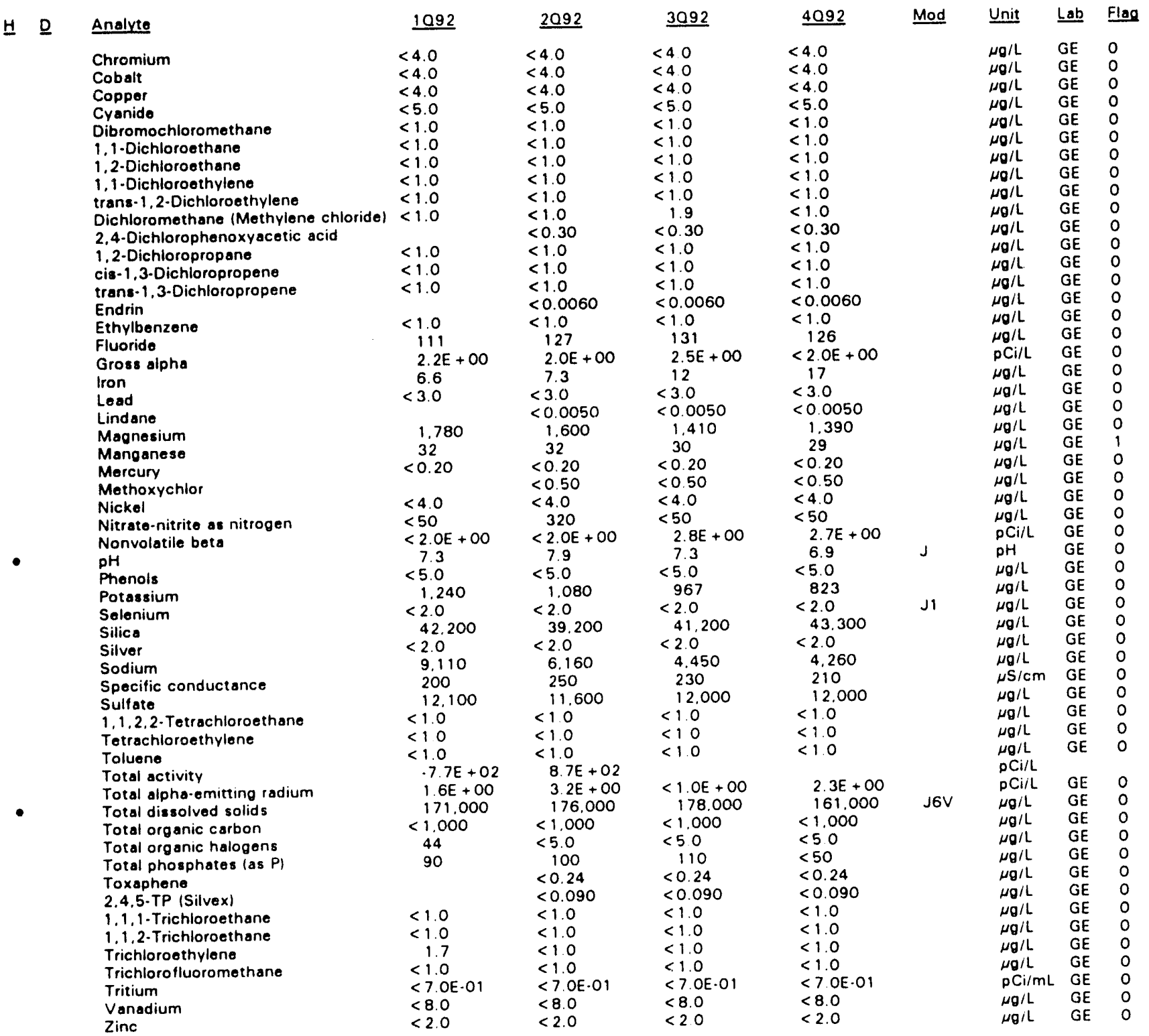

Note: Flagging levels, modifiers, and laboratories are for 4 th quarter 1992 data only. See Appendix 8 for flagging Criteria.

- =xceeded holding time for 4 th quarter 1992.

- exceeded final primary drinking water standard for 4 th quarter 1992.

H-Area Seepage Basins 
WELL HSB $125 \mathrm{C}$

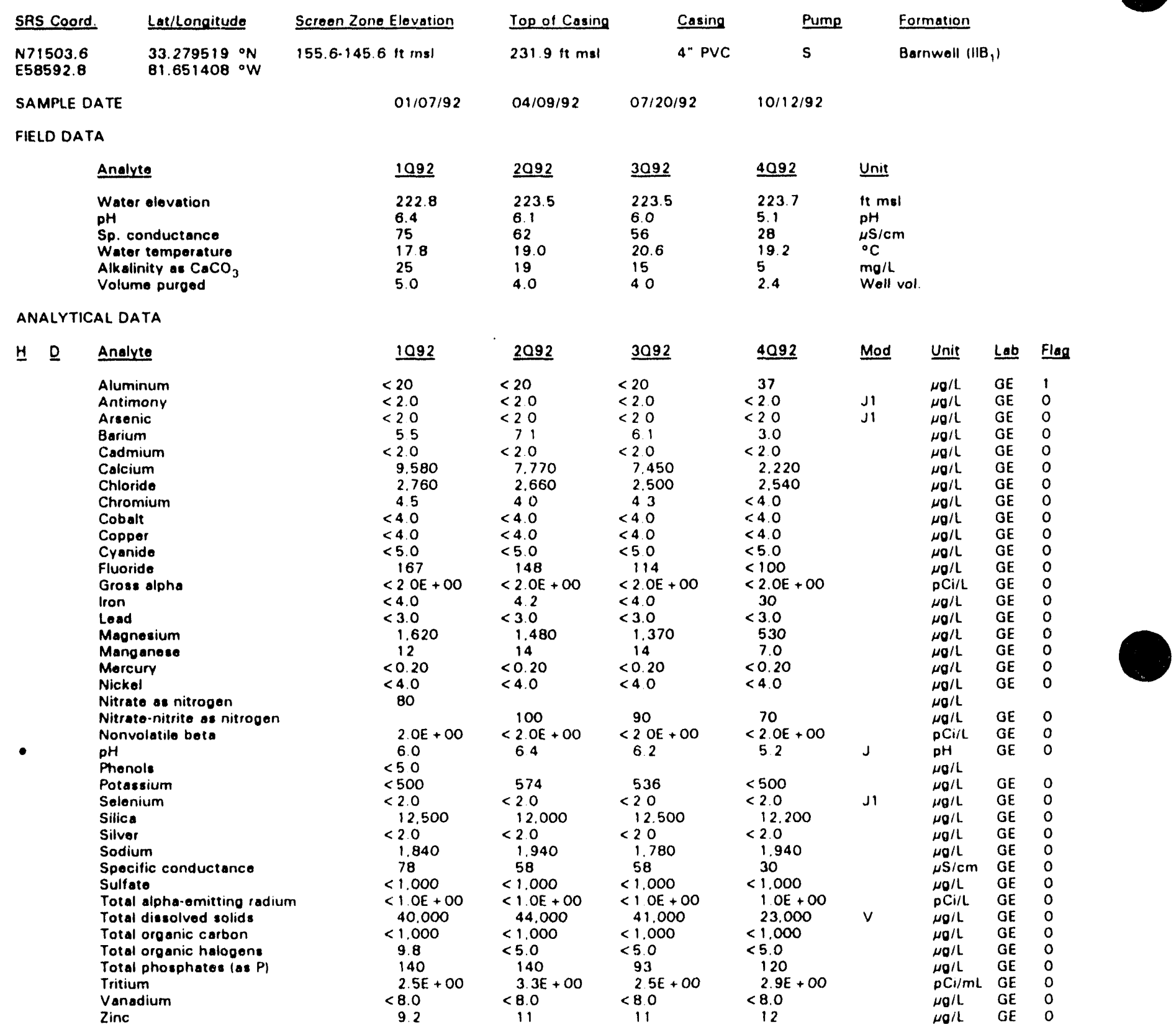

Note: Flagging levels, modifiers, and laboratories are for 4th quarter 1992 data only. See Appendix B for flagging criteria. - exceeded holding tıme for 4th quarter 1992.

- exceeded final primary drinking water standard for 4th quarter 1992. 
WELL HSB125D

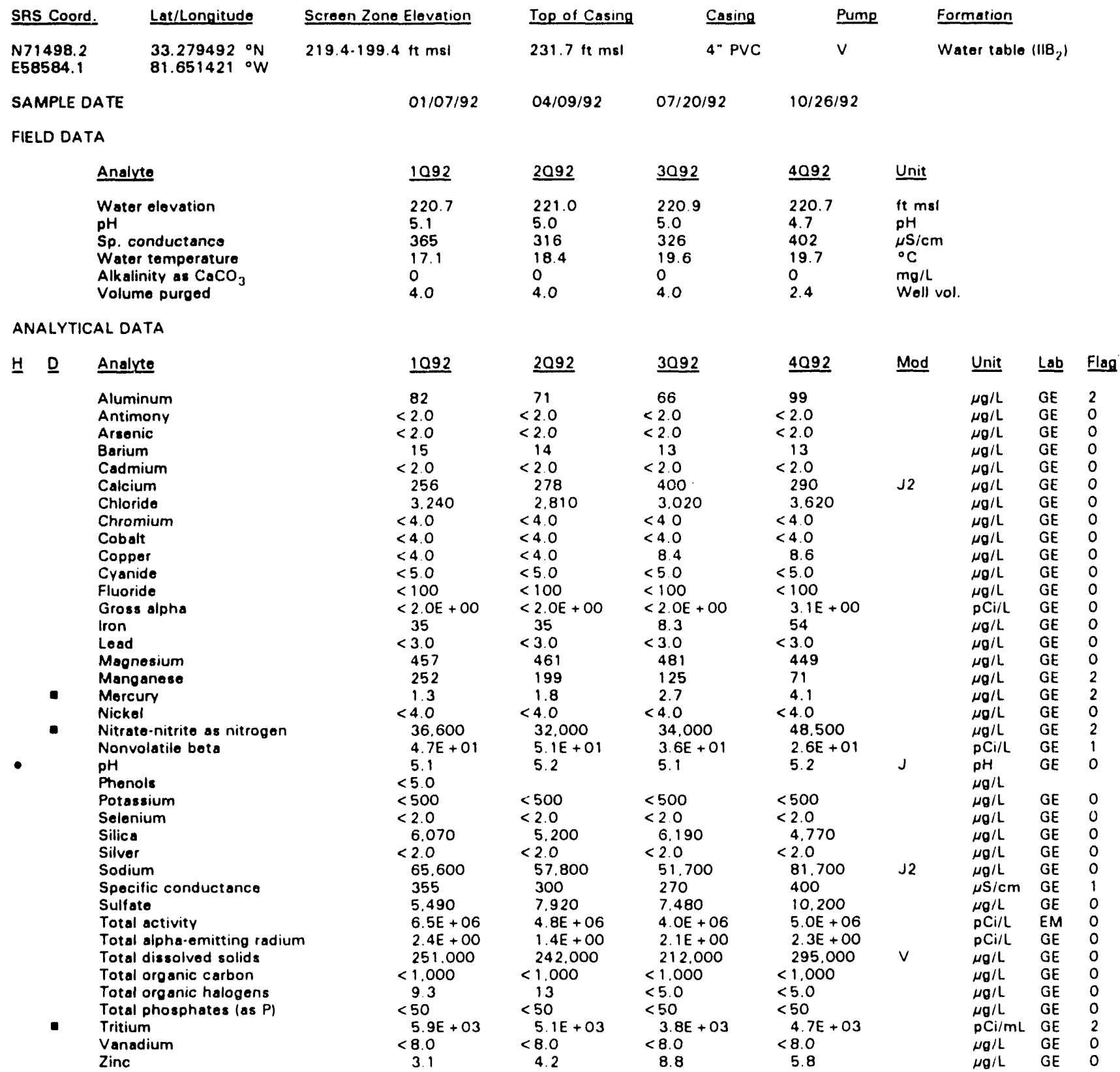

Note: Flagging levels, modifiers, and laboratories are for 4 th quarter 1992 data only. See Appendix B for flagging criteria.

- =xceeded holding time for 4 th quarter 1992.

- exceeded final primary drinking water standard for 4th quarter 1992. 
WSRC-TR-93-059

\section{WELL HSB126C}

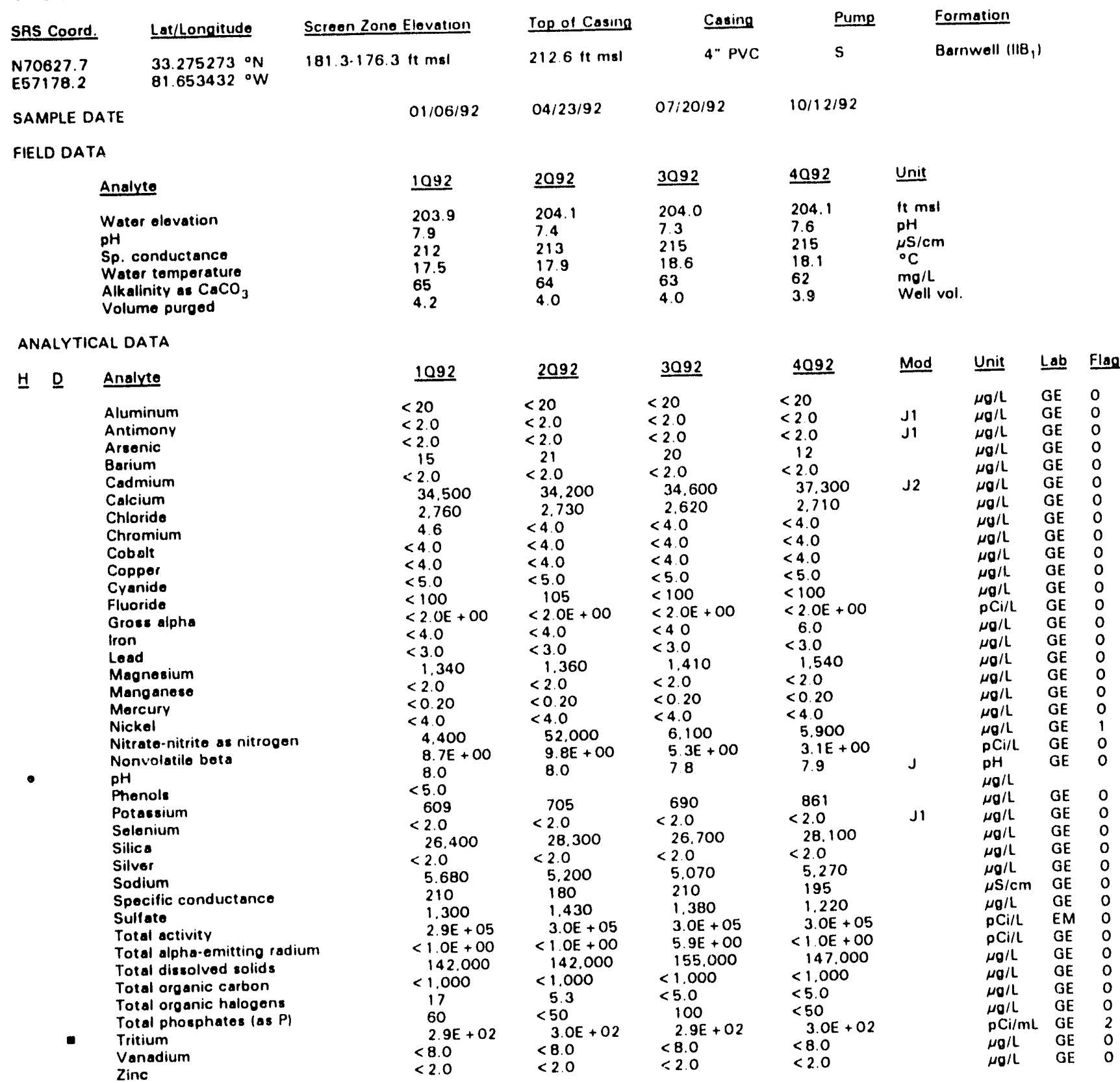

Note: Flagging levels, modifiers, and laboratories are for 4 th quarter 1992 data only. See Appendix B for flagging criteria. - =xceeded holding time for 4th quarter 1992

- exceeded final primary drinking water standard for 4th quarter 1992. 
WELL HSB 126D

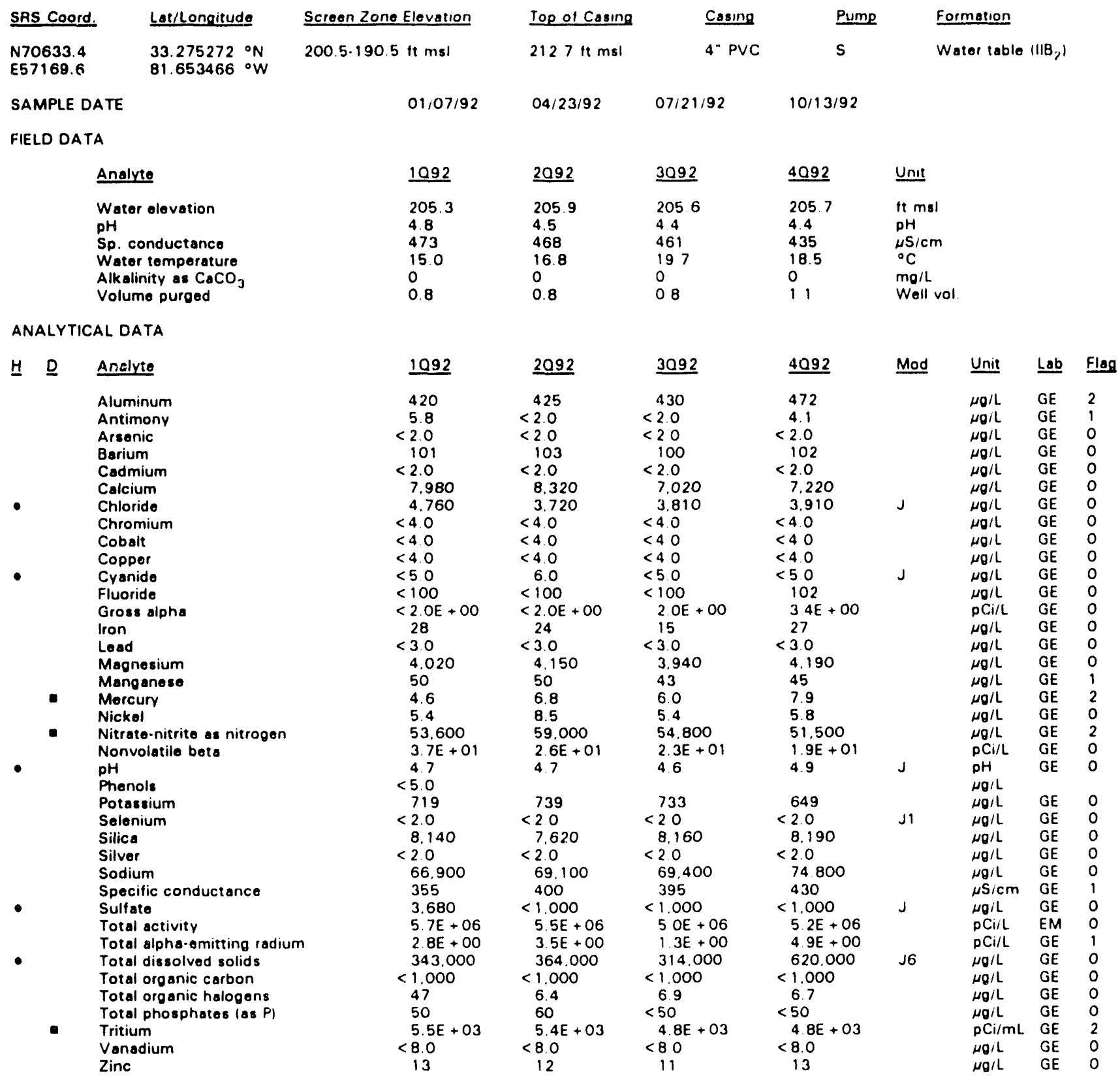

Note: Flagging levels, modifiers, and laboratories are for 4th quarter 1992 data only. See Appendix B for flagging criteria. - =xceeded holding time for 4ih quarter 1992.

- exceeded final primary drınkıng water standard for 4th quarter 1992. 
WSRC.TR.93.059

WELL HSB 127C

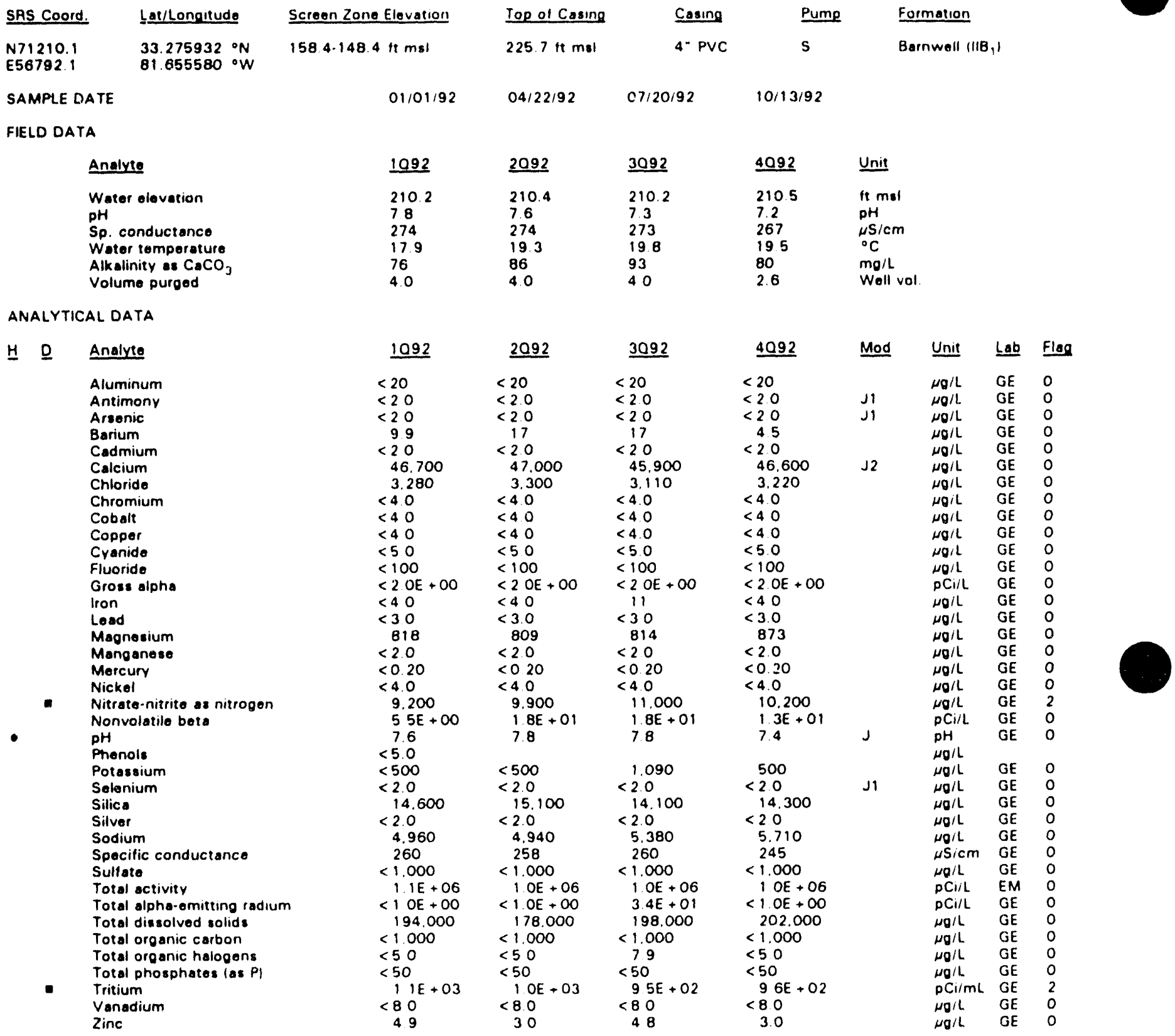

Note: Flagging levels, modifiers, and laboratories are for 4ih quarter 1992 data only. See Appendix 8 tor flagging criteria. - =xceeded holding ime for 4ih quarter 1992.

- exceeded final primary drinking water standard for 4in quarter 1992. 
WELL HSB127D

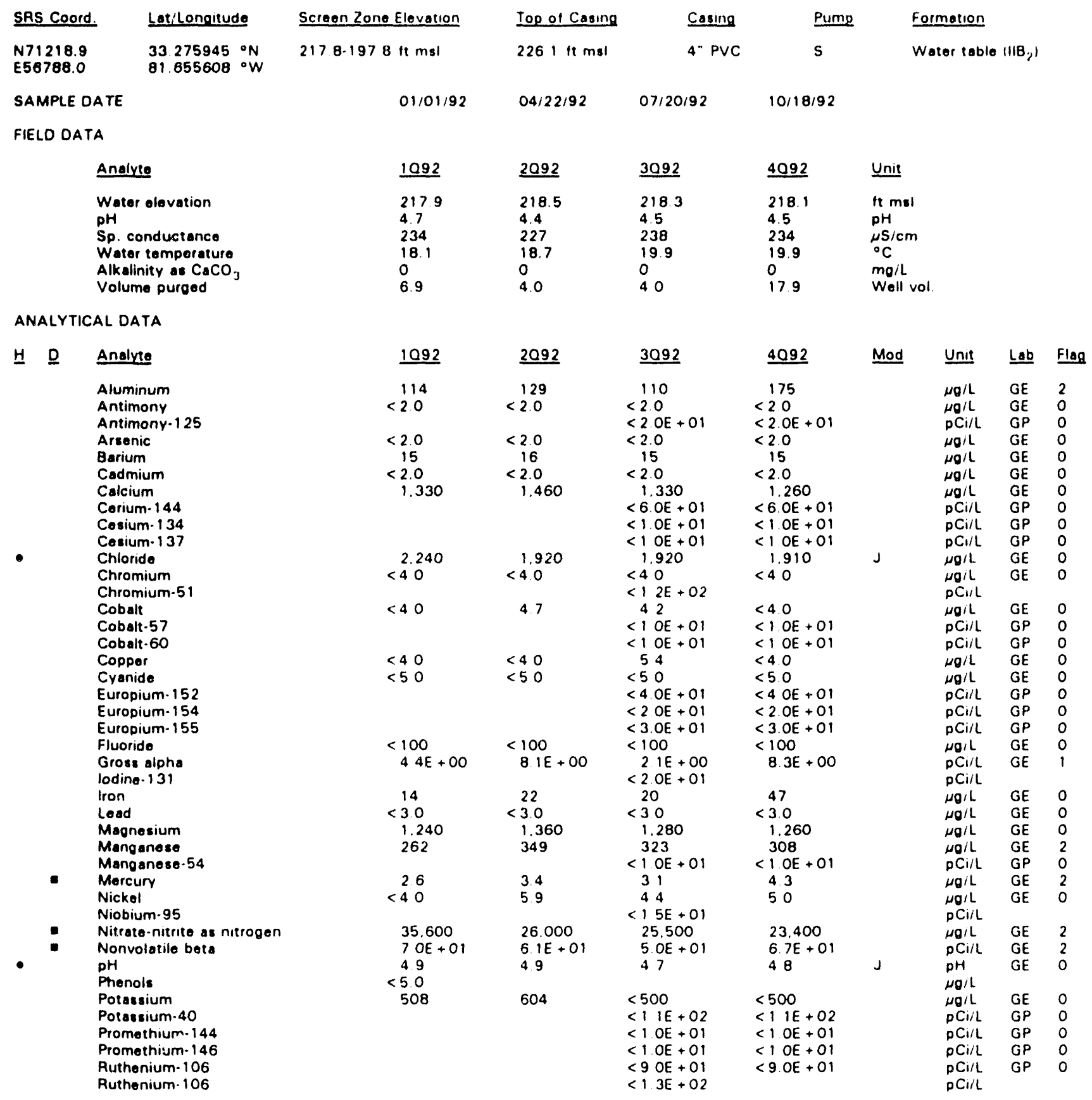

Note: Flagging levels, modifiers, and laboratories are for 4th quarter 1992 data only. See Appendix B for flagging criteria. - exceeded holding ume for 4ih quarter 1992 .

- =xceeded final primary drinkıng water standard for 4th quarter 1992 
WSRC-TR-93.059

Well HSB127D continued

ANALYTICAL DATA
H Q $\quad$ Analyte
Solenium
Silica
Silver
Sodium
Sodium-22
Specific conductance
Sulfate
Total activity
Total alpha-omittino radium
Total diseolved solids
Total organic carbon
Totel organic halogens
Total phosphates las PI
Tritium
Vonadium
Yttrium-88
Zinc
Zinc-65
Zirconium-95

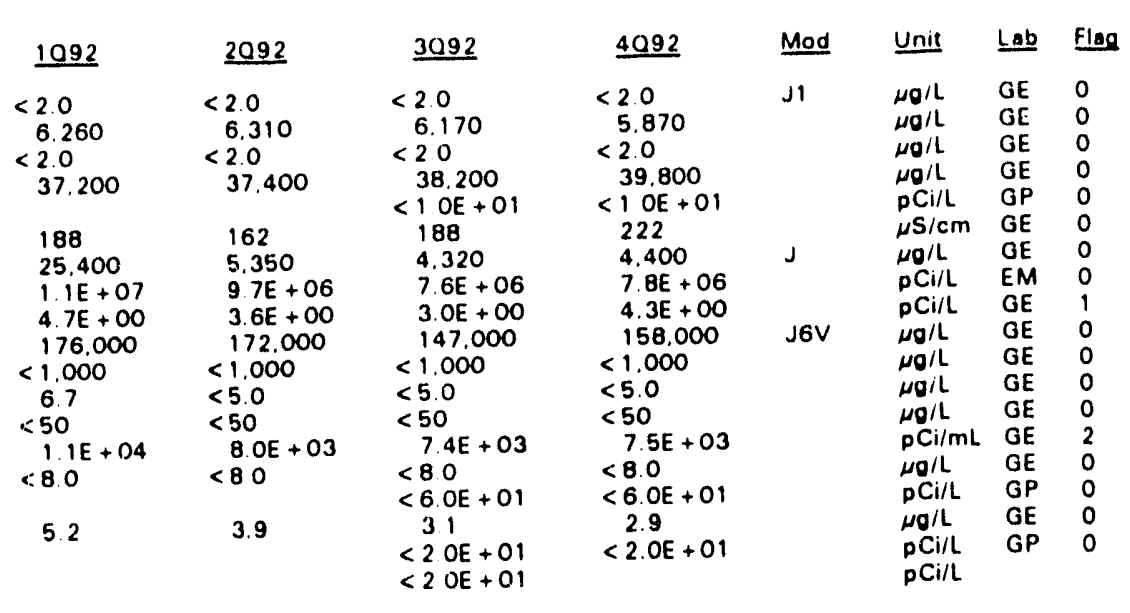

\section{WELL HSB129C}

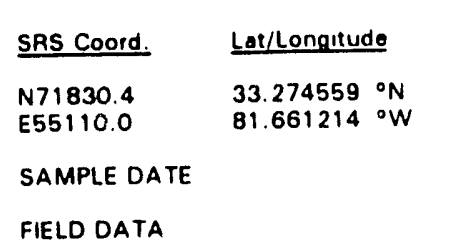

$\frac{\text { Screen Zone Elevation }}{157.8 .147 .8 \mathrm{it} \mathrm{msl}}$

\section{Top of Casing}

$215.1 \mathrm{ft} \mathrm{msl}$

$01 / 13 / 92$

$04 / 23 / 92$

$07 / 21 / 92$
Formation

Barnwell (IIB,
Analyte

Water elevation

pH

Sp. conductance

Water temperature

Alkalinity os $\mathrm{CaCO}_{3}$

Volume purged

ANALYTICAL DATA

H D Analyte

Aluminum

Antimony

Antimony-125

Arsenic

Barium

Cadmium

Calcium

Cerium-144

Cesium-134

Cesium-137

Chloride

Chromium

Chromium-51

Cobalt

Cobalt -57

Cobalt-60

Copper

Cyanide

Europium. 152

1092
205.8
5.2
209
17.6
17.0

2092
196.9
5.4
210
20.0
1
4.8

1092

93
$<2.0$

$<2.0$

57
$<2.0$

16.400

$\underline{2092}$

94
$<2.0$

$<2.0$

$<2.0$

$<20$

$<2.0$
$\quad 16.800$

$\begin{array}{ll}4.240 & 4.020 \\ <4.0 & <4.0\end{array}$

$<40$

$<4.0$

$<4.0$
$<5.0$

3092
206.3
5.6
178
19.6
5
0.9

3092

49

$<2.0$

$<2$ OE + 01

$<2.0$

47

$<2.0$

13.700

$<6 . O E+01$

$<1 O E+01$

$<1 . O E+01$

3.770

$<4.0$

$<1.2 \mathrm{E}+02$

$<4.0$

$<1 . O E+01$

$<1$ OE +01

$<4.0<4.0$

$<5.0<5.0$ asing

S

$10 / 21 / 92$ log

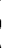

0

1

0

0

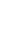


Well HSB129C continued

\section{ANALYTICAL DATA}

H D Analyte

Europium-154

Europium-155

Fluoride

Gross alpho

lodine-131

Iron

Lead

Magnesium

Manganese

Manganese-54

Mercury

Nickel

Niobium.95

Nitrate-nitrite as nitrogen

Nonvolatile beta

pH

Phonols

Potassium

Potassium.40

Promethium-144

Promethium - 146

Ruthenium-106

Ruthenium-106

Selenium

Silica

Silver

Sodium

Sodium- 22

Specific conductance

Sultate

Total activity

Total alpha-emitting radium

Total dissolved solids

Total organic carbon

Total organic halogens

Total phosphates las P)

Tritium

Vanadium

Yttrium.88

Zine

Zinc. 65

Zirconium-95

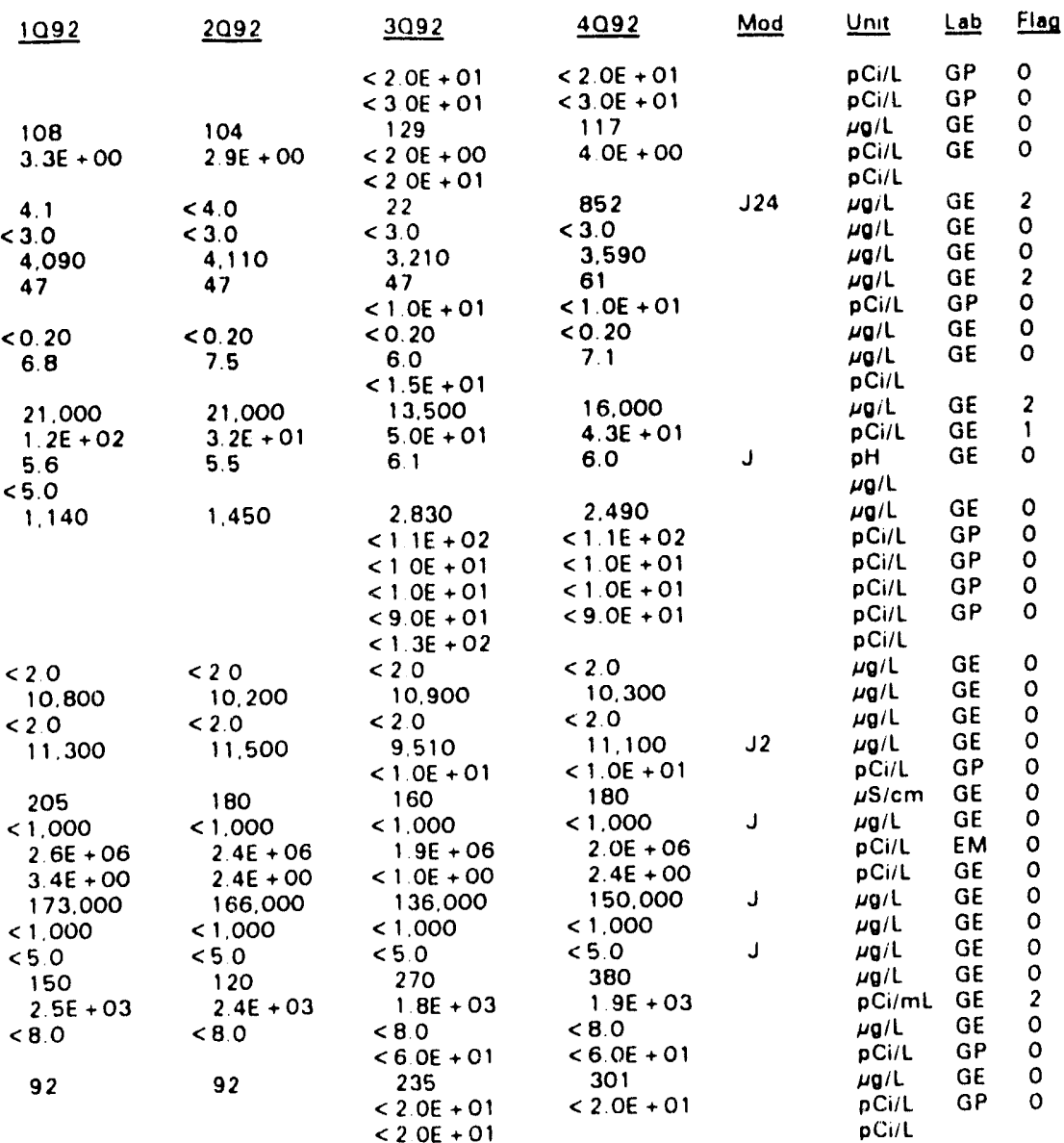

Note: Flagging levels, modifiers, and laboratortes are for 4 th quarter 1992 data only. See Appendix 8 for flaggıng criterla.

- exceeded holding time for 4th quarter 1992.

- exceeded final primary drinking water standard for 4th quarter 1992.

H-Area Seepage Basins 
WELL HSB129D

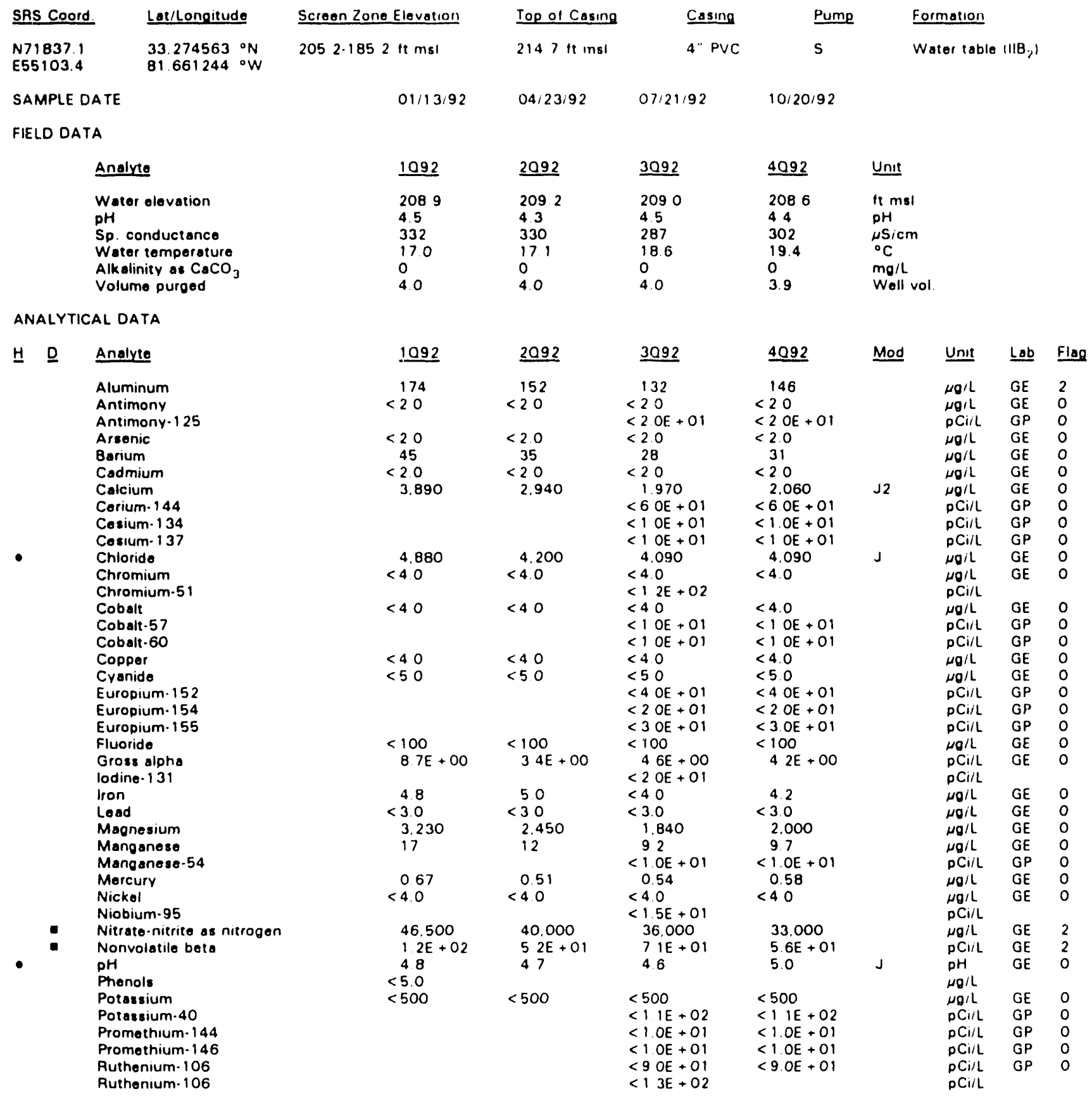

Note: Flagging levels, modifiers, and laboratories are for 4 th quarter 1992 data only. See Appendix B for flagging criteria.

- =xceeded holding time for 4 in quarter 1992.

- exceeded final primary drinking water standard for 4th quarter 1992. 
ANALYTICAL DATA

H D $\quad$ Analyte
Solenium
Silica
Silver
Sodium
Sodium-22
Specific conductance
Sulfate
Total activity
Total alpha-emitting radium
Trital dissolved solids
Total organic carbon
Total organic halogens
Total phosphates las P)
Tritium
Vanadium
Yttrium-88
Zinc
Zinc-65
Zircorium+95

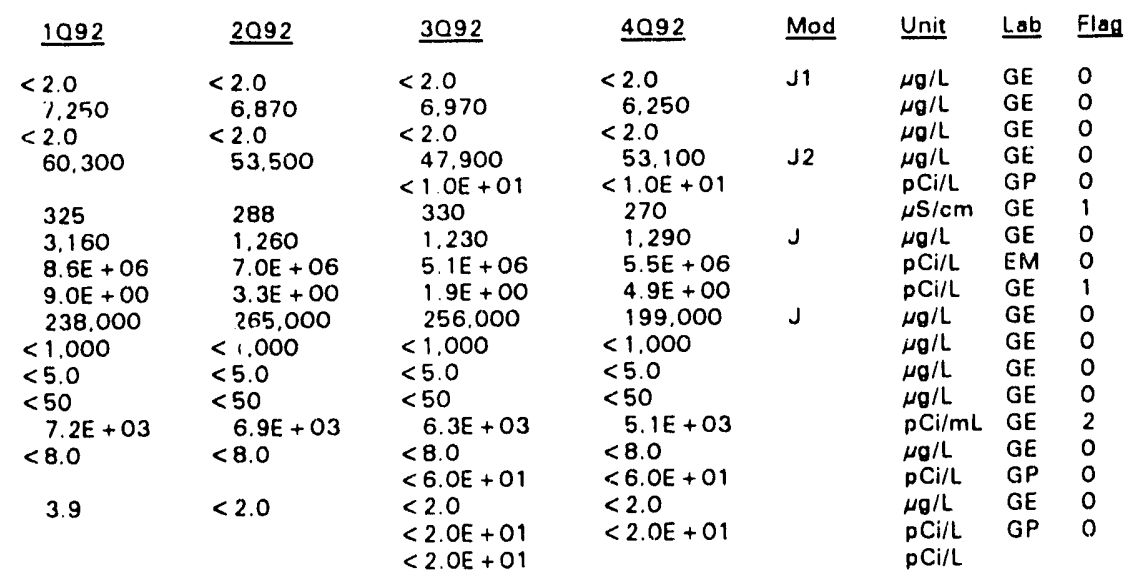

\section{WELL HSB130C}

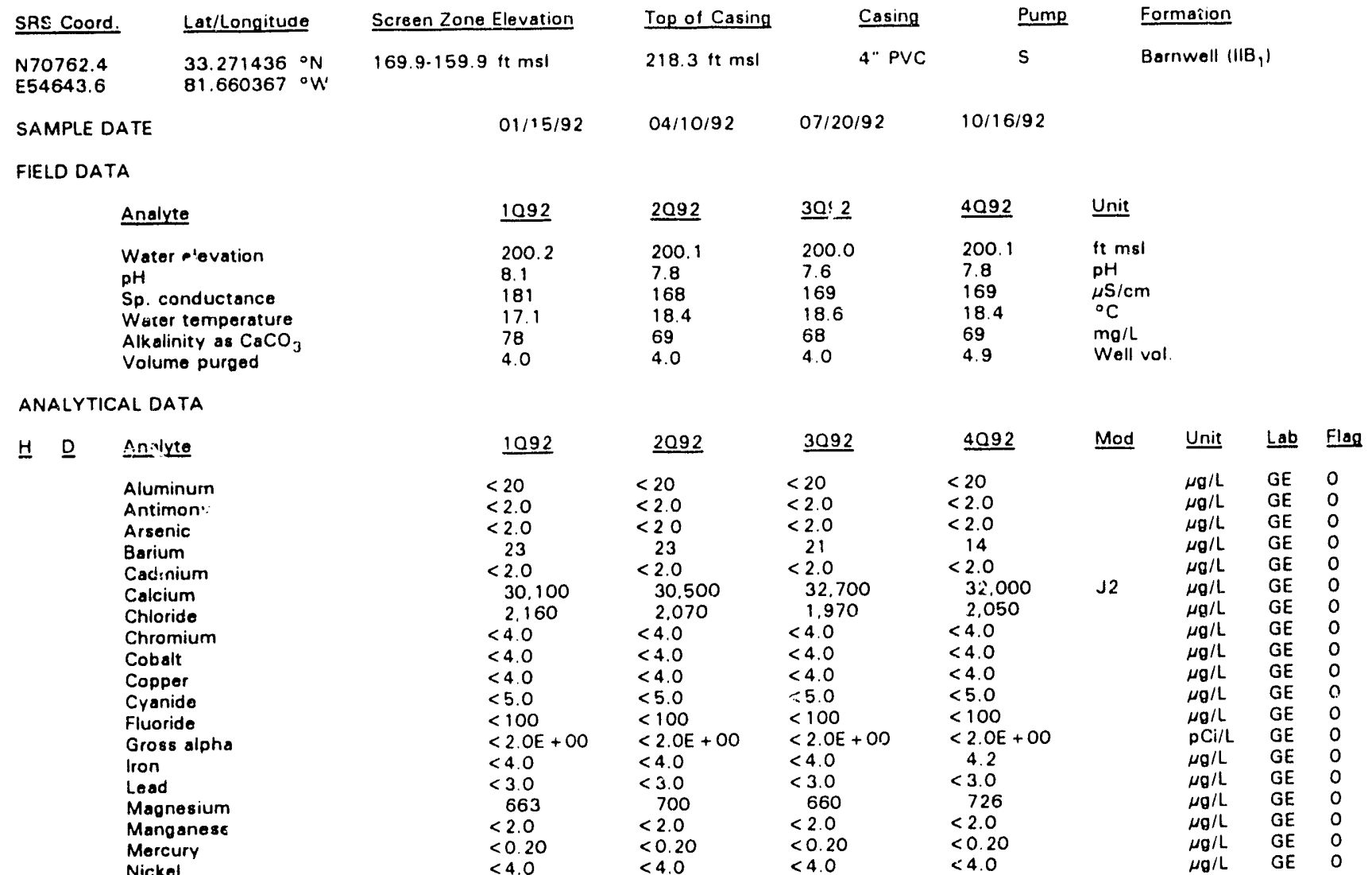

Note: Flagging levels, modifiers. and laboratories are for 4 th quarter 1992 data only. See Apperi \& 8 for flagging criteria. - = exceeded holding time for 4th quarter 1992.

- =xceeded final primary drinking water standard for 4th quarter 1992. 
WSRC.TR.93-059

Well HSB130C continued

ANALYTICAL DATA

$\begin{array}{ll}\text { H. } & \text { Analyte } \\ \text { Nitrate as nitrogen } \\ \text { Nitrate-nitrite as nitrogen } \\ \text { Nonvolatile beta } \\ \text { pH } \\ \text { Phenols } \\ \text { Potassium } \\ \text { Solenium } \\ \text { Silica } \\ \text { Silver } \\ \text { Sodium } \\ \text { Specific conductance } \\ \text { Sulfate } \\ \text { Total alpho-emitting radium } \\ \text { Total dissolved solids } \\ \text { Total organic carbon } \\ \text { Total organic halogens } \\ \text { Total phosphates las PI } \\ \text { Tritium } \\ \text { Vanadium } \\ \text { Zinc }\end{array}$

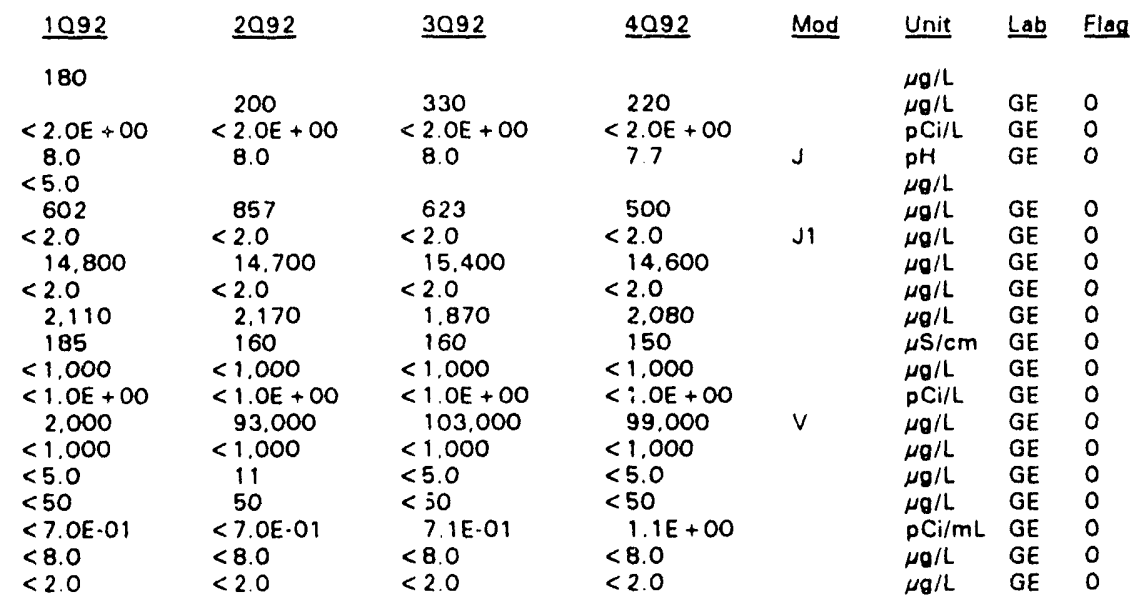

\section{WELL HSB130D}

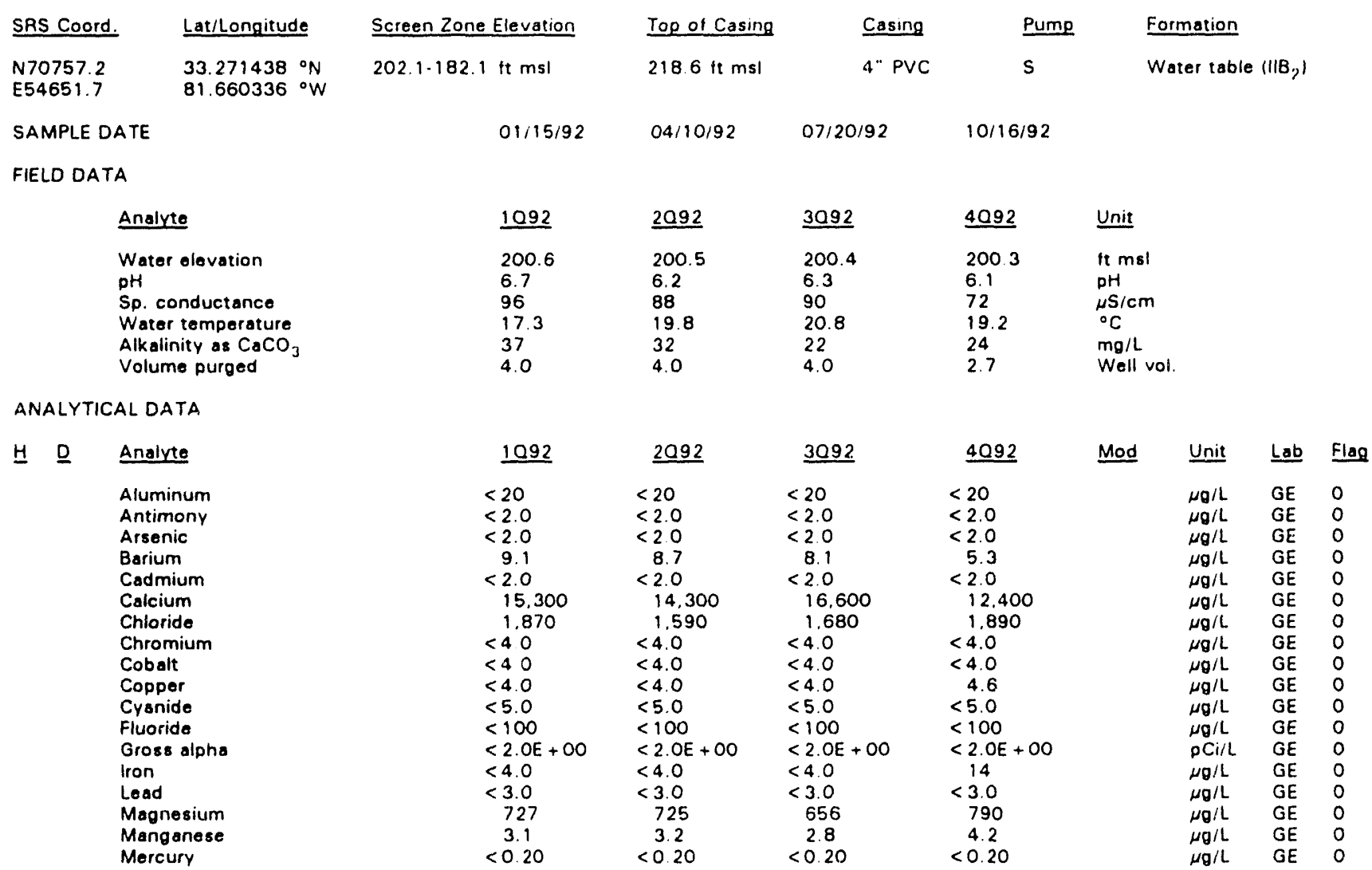

Note: Flagging levels, modifiers, and laboratories are for 4th quarter 1992 data only. See Appendix B for flagging criteria.

- = exceeded holding time for 4th quarter 1992.

- exceeded final primary drinking water standard for 4th quarter 1992. 
Woll HSB1300 continued

ANALYTICAL DATA

H. $\quad$ Analyte
Nickol
Nitrate as nitrogen
Nitrate-nitrite as nitrogen
Nonvolatile beta
pH
Phenols
Potassium
Selenium
Silica
Silver
Sodium
Specific conductance
Sulfate
Total alpha-emitting radium
Total dissolved solids
Total organic carbon
Total orgaric halogens
Total phosphates las PI
Tritium
Vanadium
Zinc

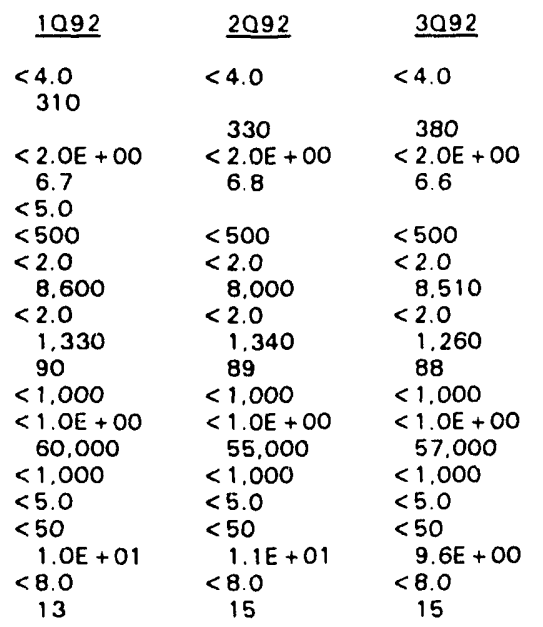

\begin{tabular}{|c|c|c|c|}
\hline 4092 & Mod & Unit & Lob \\
\hline$<4.0$ & & $\begin{array}{l}\mu \mathrm{g} / \mathrm{L} \\
\mu \mathrm{g} / \mathrm{L}\end{array}$ & $\mathrm{GE}$ \\
\hline $\begin{array}{l}740 \\
<2.0 E+00\end{array}$ & & $\begin{array}{l}\mu \mathrm{g} / \mathrm{L} \\
\mathrm{pCi} / \mathrm{L}\end{array}$ & $\begin{array}{l}\mathrm{GE} \\
\mathrm{GE}\end{array}$ \\
\hline 6.9 & $J$ & $\begin{array}{l}\rho H \\
\mu g / L\end{array}$ & GE \\
\hline$<500$ & & $\mu \mathrm{g} / \mathrm{L}$ & $\mathrm{GE}$ \\
\hline $\begin{array}{l}<2.0 \\
7.930\end{array}$ & J1 & $\begin{array}{l}\mu \mathrm{g} / \mathrm{L} \\
\mu \mathrm{g} / \mathrm{L}\end{array}$ & $\begin{array}{l}\mathrm{GE} \\
\mathrm{GE}\end{array}$ \\
\hline$<2.0$ & & $\mu g / L$ & $\mathrm{GE}$ \\
\hline $\begin{array}{l}1.390 \\
75\end{array}$ & & $\begin{array}{l}\mu \mathrm{g} / \mathrm{L} \\
\mu \mathrm{S} / \mathrm{cm}\end{array}$ & $\begin{array}{l}\mathrm{GE} \\
\mathrm{GE}\end{array}$ \\
\hline$<1,000$ & & $\mu \mathrm{g} / \mathrm{L}$ & $\mathrm{GE}$ \\
\hline$<1 . O E+O O$ & & $\mathrm{pCi} / \mathrm{L}$ & $\mathrm{GE}$ \\
\hline $\begin{aligned} & 47,000 \\
< & 1.000\end{aligned}$ & $V$ & $\begin{array}{c}\mu g / L \\
\mu g / L\end{array}$ & $\begin{array}{l}\mathrm{GE} \\
\mathrm{GE}\end{array}$ \\
\hline$<5.0$ & & $\mu \mathrm{g} / \mathrm{L}$ & G \\
\hline$<50$ & & $\mu g / L$ & G \\
\hline $9.3 E+00$ & & $\mathrm{pCi} / \mathrm{mL}$ & $\mathrm{GE}$ \\
\hline$<8.0$ & & $\mu g / L$ & GE \\
\hline 24 & & $\mu \mathrm{g} / \mathrm{L}$ & $\mathrm{GE}$ \\
\hline
\end{tabular}

\section{WELL HSB131C}

\begin{tabular}{|c|c|c|c|c|c|c|c|c|c|}
\hline SRS Coord. & Lat/Longitude & Screen Zone Elevation & Top of Casing & Casing & Pump & \multicolumn{3}{|c|}{ Formation } & \\
\hline $\begin{array}{l}\text { N70374.7 } \\
\text { E56894.9 }\end{array}$ & $\begin{array}{l}33.274252{ }^{\circ} \mathrm{N} \\
81.653687{ }^{\circ} \mathrm{W}\end{array}$ & $158.5 \cdot 148.5 \mathrm{ft} \mathrm{msl}$ & $211.7 \mathrm{ft} \mathrm{msl}$ & 4" PVC & s & \multicolumn{4}{|c|}{ Barnwell $\left(11 B_{1}\right)$} \\
\hline \multicolumn{2}{|c|}{ SAMPLE DATE } & $01 / 10 / 92$ & $04 / 03 / 92$ & $07 / 20 / 92$ & $10 / 16 / 92$ & & & & \\
\hline \multicolumn{10}{|l|}{ FIELD DATA } \\
\hline \multicolumn{2}{|r|}{ Analyte } & 1092 & 2092 & 3092 & 4092 & \multicolumn{4}{|l|}{ Unit } \\
\hline \multicolumn{2}{|r|}{$\begin{array}{l}\text { Water elevation } \\
\text { pH } \\
\text { Sp. conductance } \\
\text { Water temperature } \\
\text { Alkalinity as } \mathrm{CaCO}_{3} \\
\text { Volume purged }\end{array}$} & $\begin{array}{l}203.6 \\
8.0 \\
222 \\
17.2 \\
85 \\
4.0\end{array}$ & $\begin{array}{l}203.9 \\
7.8 \\
227 \\
17.4 \\
85 \\
4.0\end{array}$ & $\begin{array}{l}203.6 \\
7.5 \\
224 \\
18.6 \\
84 \\
4.0\end{array}$ & $\begin{array}{l}203.7 \\
7.6 \\
223 \\
18.2 \\
80 \\
4.2\end{array}$ & \multicolumn{3}{|c|}{$\begin{array}{l}\text { ft msl } \\
\mathrm{pH} \\
\mu \mathrm{S} / \mathrm{cm} \\
{ }^{\circ} \mathrm{C} \\
\mathrm{mg} / \mathrm{L} \\
\text { Well vol. }\end{array}$} & \\
\hline \multicolumn{10}{|c|}{ ANALYTICAL DATA } \\
\hline \multirow[b]{2}{*}{, } & Analyte & 1092 & $\underline{2092}$ & $\underline{3 Q 92}$ & 4092 & Mod & Unit & Lab & Flag \\
\hline & $\begin{array}{l}\text { Aluminum } \\
\text { Antimony } \\
\text { Arsenic } \\
\text { Barium } \\
\text { Cadmium } \\
\text { Calcium } \\
\text { Chloride } \\
\text { Chromium } \\
\text { Cobait } \\
\text { Copper } \\
\text { Cyanide } \\
\text { Fluoride } \\
\text { Gross alpha } \\
\text { Iron } \\
\text { Lead } \\
\text { Magnesium } \\
\text { Manganese }\end{array}$ & $\begin{aligned} &< 20 \\
&< 2.0 \\
&< 2.0 \\
& 22 \\
&< 2.0 \\
& 40.600 \\
& 2.730 \\
&< 4.0 \\
&<4.0 \\
&<4.0 \\
&<5.0 \\
&<100 \\
&<2.0 E+00 \\
&<4.0 \\
&<3.0 \\
& 696 \\
&< 2.0\end{aligned}$ & $\begin{aligned} &< 20 \\
&< 2.0 \\
&< 2.0 \\
& 22 \\
&< 2.0 \\
& 38.200 \\
& 2.620 \\
&< 4.0 \\
&< 4.0 \\
&< 4.0 \\
&<5.0 \\
&<100 \\
&<2.0 E+O 0 \\
&<4.0 \\
&<3.0 \\
& 697 \\
&< 2.0\end{aligned}$ & $\begin{aligned}< & 20 \\
< & 2.0 \\
< & 2.0 \\
& 21 \\
< & 2.0 \\
& 43.400 \\
& 2.480 \\
< & 4.0 \\
< & 4.0 \\
< & 4.0 \\
< & 5.0 \\
< & 100 \\
< & 2.0 E+00 \\
< & 4.0 \\
< & 3.0 \\
& 658 \\
< & 2.0\end{aligned}$ & $\begin{aligned}< & 20 \\
< & 2.0 \\
< & 2.0 \\
& 10 \\
< & 2.0 \\
& 42.600 \\
& 2.580 \\
< & 4.0 \\
< & 4.0 \\
< & 4.0 \\
< & 5.0 \\
< & 100 \\
< & 2.0 E+00 \\
< & 4.0 \\
& 3.2 \\
& 730 \\
< & 2.0\end{aligned}$ & & $\begin{array}{l}\mu g / L \\
\mu g / L \\
\mu g / L \\
\mu g / L \\
\mu g / L \\
\mu g / L \\
\mu g / L \\
\mu g / L \\
\mu g / L \\
\mu g / L \\
\mu g / L \\
\mu g / L \\
\rho C i / L \\
\mu g / L \\
\mu g / L \\
\mu g / L \\
\mu g / L\end{array}$ & $\begin{array}{l}\mathrm{GE} \\
\mathrm{GE} \\
\mathrm{GE} \\
\mathrm{GE} \\
\mathrm{GE} \\
\mathrm{GE} \\
\mathrm{GE} \\
\mathrm{GE} \\
\mathrm{GE} \\
\mathrm{GE} \\
\mathrm{GE} \\
\mathrm{GE} \\
\mathrm{GE} \\
\mathrm{GE} \\
\mathrm{GE} \\
\mathrm{GE} \\
\mathrm{GE}\end{array}$ & $\begin{array}{l}0 \\
0 \\
0 \\
0 \\
0 \\
0 \\
0 \\
0 \\
0 \\
0 \\
0 \\
0 \\
0 \\
0 \\
0 \\
0 \\
0\end{array}$ \\
\hline
\end{tabular}

Note: Flagging levels, modifiers, and laboratories are for 4 th quarter 1992 data only. See Appendix B for flagging criteria.

- =xceeded holding time for 4th quarter 1992.

- =xceeded final primary drinking water standard for 4th quarter 1992.

H-Area Seepage Basins

D. 141

Fourth Quarter 1992 
Woll HSB131C continued

ANALYTICAL DATA

H. $\quad$ Analyte
Mercury
Nickel
Nitrate-nitrite as nitrogen
Nonvolatile beta
pH
Phenols
Potassium
Selenium
Silica
Silver
Sodium
Specific conductance
Sulfate
Total alpha-emitting radium
Total dissolved solids
Total organic carbon
Total organic halogens
Total phosphates las PI
Tritium
Vanadium
Zinc

$\begin{array}{lll}1092 & \underline{2092} & \underline{3092} \\ <0.20 & <0.20 & <0.20 \\ <4.0 & <4.0 & <4.0 \\ 3.400 & 2.940 & 3.380 \\ 2.4 \mathrm{E}+00 & <2.0 \mathrm{E}+00 & <2.0 \mathrm{E}+00 \\ 8.0 & 8.0 & 7.9 \\ <5.0 & & \\ 654 & 632 & 818 \\ <2.0 & <2.0 & <2.0 \\ 16.400 & 16.400 & 16.900 \\ <2.0 & <2.0 & <2.0 \\ 3.480 & 3.510 & 3.230 \\ 202 & 188 & 218 \\ <1.000 & <1.000 & <1.000 \\ <1.0 \mathrm{E}+00 & <1.0 \mathrm{E}+00 & <1.0 \mathrm{E}+00 \\ 133.000 & 143.000 & 161.000 \\ <1.000 & <1.000 & <1.000 \\ <5.0 & <5.0 & <5.0 \\ <50 & 70 & <50 \\ 1.8 \mathrm{E}+02 & 1.8 \mathrm{E}+02 & 1.6 \mathrm{E}+02 \\ <8.0 & <8.0 & <8.0 \\ <2.0 & <2.0 & <2.0\end{array}$

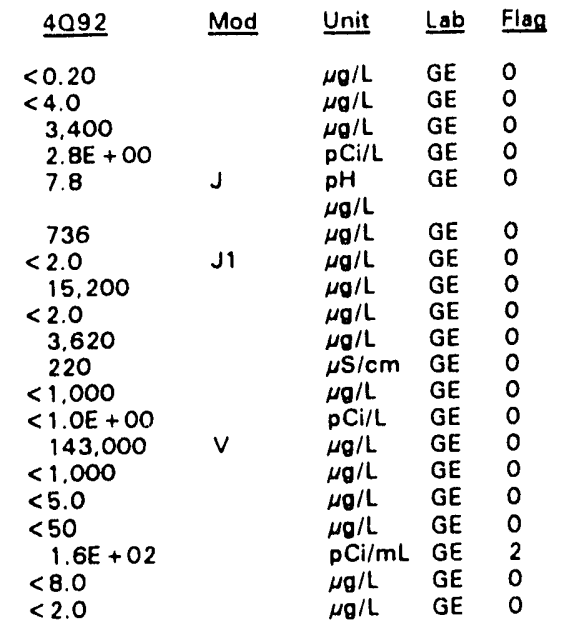

\section{WELL HSB131D}

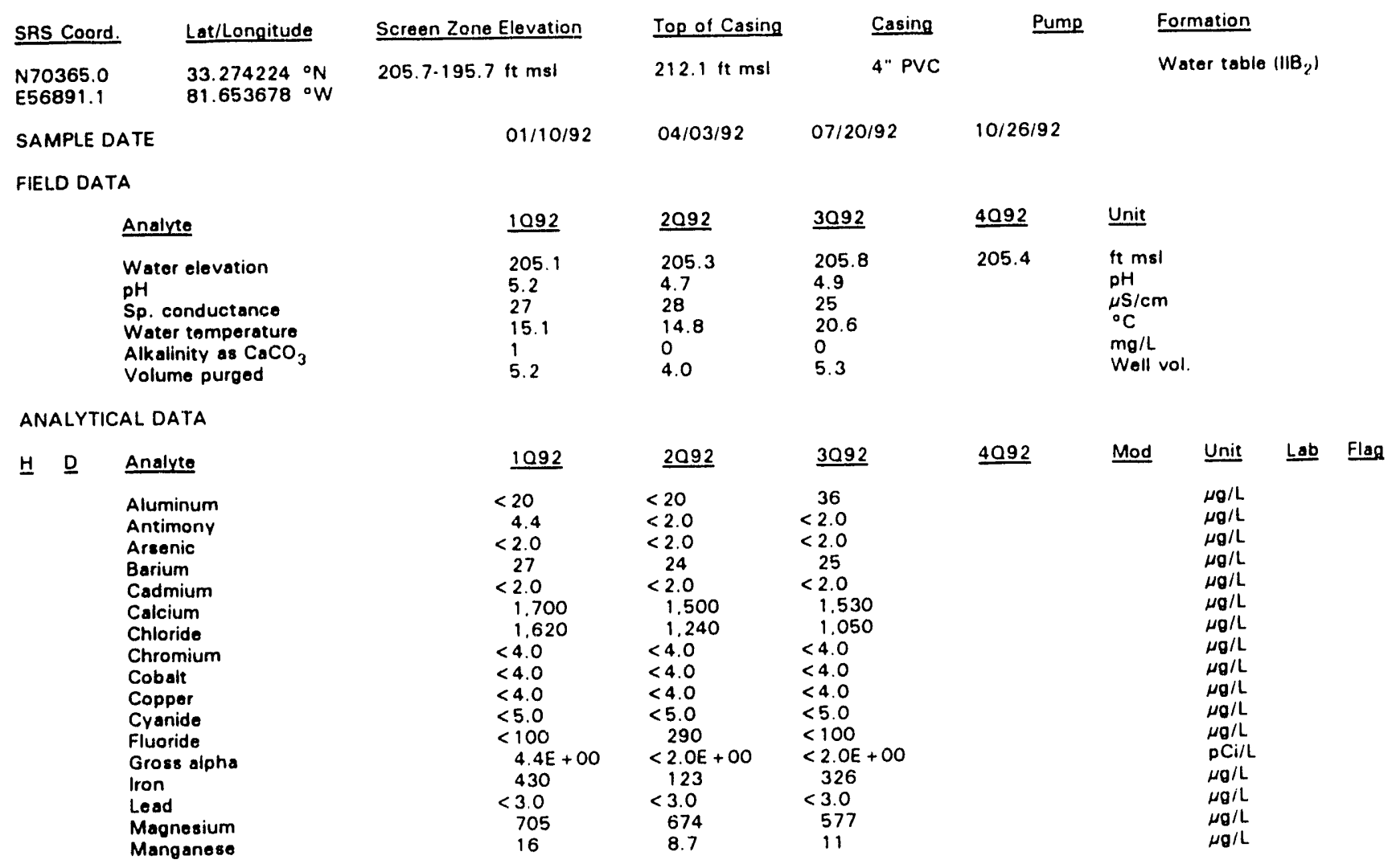

Note: Flagging levels, modifiers, and laboratories are for 4 th quarter 1992 data only. See Appendix B for flagging criteria.

- = exceeded holding time for 4th quarter 1992.

- =xceeded final primary drinking water standard for 4th quarter 1992 . 
WSRC-TR-93.059

Well HSB1310 continued

ANALYTICAL DATA

H $\quad \underline{D}$

Analyte
Mercury
Nickel
Nitrate-nitrite as nitrogen
Nonvolatile beta
pH
Phenols
Potassium
Solenium
Silica
Silver
Sodium
Specific conductance
Sulfate
Total alpha-emitting radium
Total dissolved solids
Total organic carbon
Total organic halogens
Total phosphotes las P)
Tritium
Vanadium
Zinc

$\begin{array}{lll}\frac{1092}{2092} & \underline{3092} \\ <0.20 & <0.20 & <0.20 \\ <4.0 & <4.0 & <4.0 \\ 520 & 290 & 290 \\ 7.7 \mathrm{E}+\mathrm{OO} & <2.0 \mathrm{E}+00 & <2.0 \mathrm{E}+00 \\ 5.4 & 5.4 & 5.2 \\ <5.0 & & \\ <500 & <500 & 597 \\ <2.0 & <2.0 & <2.0 \\ 5.620 & 5.190 & 5.990 \\ <2.0 & <2.0 & <2.0 \\ 774 & 804 & 690 \\ 25 & 22 & 25 \\ 2.630 & 2.620 & 2.040 \\ 2.8 \mathrm{E}+00 & <1.0 \mathrm{E}+00 & <1.0 \mathrm{E}+00 \\ 17.000 & 21.000 & 19.000 \\ <1.000 & <1.000 & <1.000 \\ <5.0 & <5.0 & <5.0 \\ <50 & <50 & <50 \\ 1.1 \mathrm{E}+01 & 8.6 \mathrm{E}+00 & 9.3 \mathrm{E}+00 \\ <8.0 & <8.0 & <8.0 \\ <2.0 & 2.6 & 2.5\end{array}$

4092

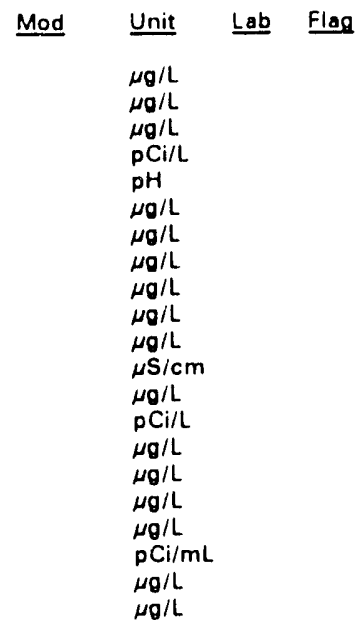

\section{WELL HSB132C}

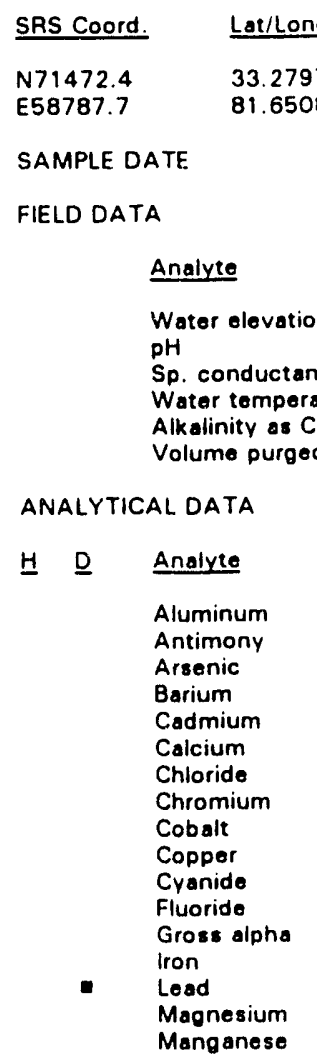

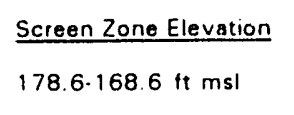

$01 / 10 / 92$

\section{Top of Casing \\ $240.5 \mathrm{ft} \mathrm{ms}$}

$04 / 03 / 92$

2092
221.5
4.4
34
17.2
1
4.0

3092
221.4
5.2
31
20.5
3
0.9

4092
221.5
4.9
29
18.0
2
0.9

Unit

tt msl

$\mathrm{pH}$

$\mu \mathrm{S} / \mathrm{cm}$

${ }^{\circ} \mathrm{C}$

$\mathrm{mo} / \mathrm{L}$

Well vol.
Formation

Barnwell (IIB,

$\begin{array}{lcc}1092 & \underline{2092} & \underline{3092} \\ 47 & 67 & 34 \\ 6.3 & <2.0 & <2.0 \\ <2.0 & <2.0 & <2.0 \\ 3.8 & 4.4 & 4.3 \\ <2.0 & <2.0 & <2.0 \\ 762 & 707 & 801 \\ 2.700 & 2.570 & 2.420 \\ <4.0 & <4.0 & <4.0 \\ <4.0 & <4.0 & <4.0 \\ <4.0 & <4.0 & 5.1 \\ <5.0 & <5.0 & <5.0 \\ <100 & <100 & <100 \\ <2.0 E+00 & <2.0 E+00 & <2.0 E+O O \\ 14 & 18 & 23 \\ <3.0 & <3.0 & <3.0 \\ 298 & 306 & 293 \\ 14 & 12 & 10\end{array}$

\begin{tabular}{|c|c|c|c|c|}
\hline 4092 & Mod & $\underline{\text { Unit }}$ & $\underline{\text { Lab }}$ & Flas \\
\hline 96 & & $\mu g / L$ & $G E$ & 2 \\
\hline$<2.0$ & & $\mu g / L$ & $\mathrm{GE}$ & 0 \\
\hline$<2.0$ & & $\mu g / L$ & $\mathrm{GE}$ & 0 \\
\hline 4.7 & & $\mu g / L$ & $\mathrm{GE}$ & 0 \\
\hline$<2.0$ & & $\mu g / \mathrm{L}$ & $\mathrm{GE}$ & 0 \\
\hline 736 & $J 2$ & $\mu g / L$ & $\mathrm{GE}$ & 0 \\
\hline 2.440 & & $\mu g / L$ & $\mathrm{GE}$ & 0 \\
\hline$<4.0$ & & $\mu \theta / L$ & $\mathrm{GE}$ & 0 \\
\hline$<4.0$ & & $\mu g / L$ & $\mathrm{GE}$ & 0 \\
\hline 11 & & $\mu g / L$ & $\mathrm{GE}$ & 0 \\
\hline$<5.0$ & & $\mu \mathrm{g} / \mathrm{L}$ & $\mathrm{GE}$ & 0 \\
\hline$<100$ & & $\mu g / L$ & GE & 0 \\
\hline$<2.0 E+00$ & & $p C i / L$ & $G E$ & 0 \\
\hline $\begin{array}{l}1.020 \\
16\end{array}$ & 4 & $\begin{array}{l}\mu \boldsymbol{g} / \mathrm{L} \\
\mu \boldsymbol{g} / \mathrm{L}\end{array}$ & $\begin{array}{l}\mathrm{GE} \\
\mathrm{GE}\end{array}$ & $\begin{array}{l}2 \\
2\end{array}$ \\
\hline 323 & & $\mu g / L$ & $\mathrm{GE}$ & 0 \\
\hline 15 & & /L & $\mathrm{GE}$ & 0 \\
\hline
\end{tabular}

Note: Flagging levels, modifiers, and laboratories are for 4th quarter 1992 data only. See Appendix B for flagging criteria.

- exceeded holding time for 4th quarter 1992.

- =xceeded final primary drinking water standard for 4th quarter 1992. 
WSRC-TR-93-059

Woll HSB132C continued

ANALYTICAL DATA

H D Analyte
Mercury
Nickel
Nitrate-nitrite as nitrogen
Nonvolatile beta
pH
Phenols
Potassium
Solenium
Silica
Silver
Sodium
Specific conductance
Sulfate
Total alpha-emitting radium
Total dissolved solids
Total organic carbon
Total organic halogens
Total phosphates las PI
Tritium
Vanadium
Zinc

\begin{tabular}{lll}
1092 & $\underline{2092}$ & $\underline{3092}$ \\
\hline$<0.20$ & $<0.20$ & $<0.20$ \\
$<4.0$ & $<4.0$ & $<4.0$ \\
90 & 110 & 150 \\
$<2.0 E+00$ & $<2.0 E+00$ & $<2.0 \mathrm{E}+00$ \\
5.6 & 5.8 & 6.0 \\
$<5.0$ & & \\
583 & 621 & 609 \\
$<2.0$ & $<2.0$ & $<2.0$ \\
9.620 & 9.610 & 9.730 \\
$<2.0$ & $<2.0$ & $<2.0$ \\
3.470 & 3.740 & 4.240 \\
29 & 25 & 30 \\
2.080 & 2.090 & 2.560 \\
$1.7 \mathrm{E}+00$ & $<1.0 \mathrm{E}+00$ & $<1.0 \mathrm{E}+00$ \\
22.000 & 22.000 & 26.000 \\
$<1.000$ & $<1.000$ & $<1.000$ \\
10 & $<5.0$ & $<5.0$ \\
120 & 210 & 330 \\
$<7.0 \mathrm{E}-01$ & $<7.0 \mathrm{E} \cdot 01$ & $<7.0 \mathrm{E} \cdot 01$ \\
$<8.0$ & $<8.0$ & $<8.0$ \\
10 & 12 & 12 \\
& &
\end{tabular}

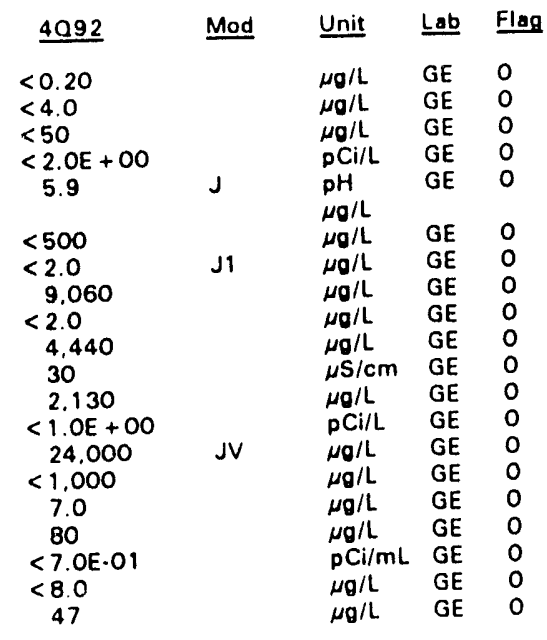

WELL HSB132D

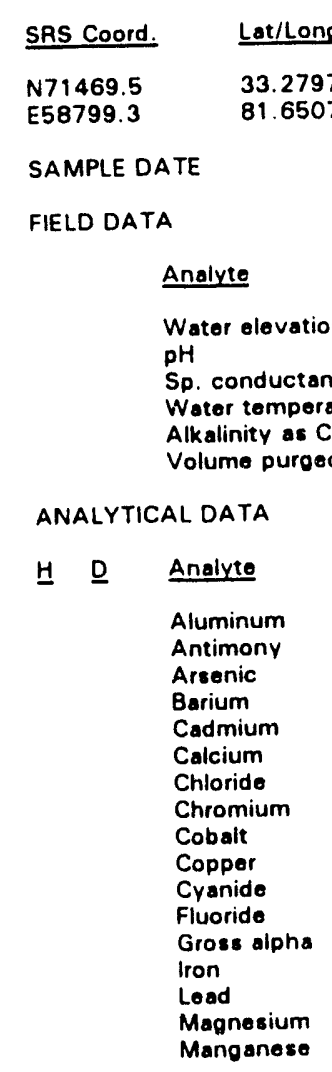

$\frac{\text { Screen Zone Elevation }}{226.5 \cdot 206.5 \mathrm{ft} \mathrm{msl}}$

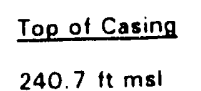

$01 / 10 / 92$

$04 / 03 / 92$

$\begin{array}{ll}1092 & \underline{2092} \\ 220.5 & 221.1 \\ 5.1 & 4.1 \\ 21 & 23 \\ 18.4 & 18.0 \\ 1 & 1 \\ 4.0 & 4.0\end{array}$

$\begin{aligned} & 1092 \\ &< 20 \\ & 5.8 \\ &<2.0 \\ & 5.7 \\ &<2.0 \\ & 562 \\ & 2.390 \\ &<4.0 \\ &<4.0 \\ & 4.7 \\ &<5.0 \\ &<100 \\ &<2.0 \mathrm{E}+00 \\ &<4.0 \\ &<3.0 \\ & 411 \\ & 11\end{aligned}$

$\begin{aligned} & 2092 \\ &< 20 \\ &< 2.0 \\ &< 2.0 \\ & 6.4 \\ &< 2.0 \\ & 524 \\ & 2.090 \\ &<4.0 \\ &<4.0 \\ &<4.0 \\ &<5.0 \\ &<100 \\ &<2.0 E+00 \\ &<4.0 \\ &<3.0 \\ & 378 \\ & 8.8\end{aligned}$

$\begin{aligned} & 3092 \\ &< 20 \\ &< 2.0 \\ &< 2.0 \\ & 7.9 \\ &< 2.0 \\ & 640 \\ & 1.830 \\ &< 4.0 \\ &< 4.0 \\ & 9.2 \\ &<5.0 \\ &< 100 \\ &< 2.0 E+00 \\ & 35 \\ &< 30 \\ & 425 \\ & 12\end{aligned}$

$\begin{aligned} & 4092 \\ & 685 \\ &< 2.0 \\ &< 2.0 \\ & 9.0 \\ &<2.0 \\ & 587 \\ & 1.960 \\ &<4.0 \\ &<4.0 \\ & 21 \\ &<5.0 \\ &<100 \\ &<2.0 E+00 \\ & 238 \\ & 5.0 \\ & 491 \\ & 13\end{aligned}$

\begin{tabular}{|c|c|c|c|}
\hline Mod & $\underline{\text { Unit }}$ & Lab & Flas \\
\hline \multirow{16}{*}{ J2 } & $\mu g / L$ & GE & 2 \\
\hline & $\mu \boldsymbol{g} / \mathrm{L}$ & GE & 0 \\
\hline & $\mu \mathrm{g} / \mathrm{L}$ & $\mathrm{GE}$ & 0 \\
\hline & $\mu g / L$ & GE & 0 \\
\hline & $\mu \mathrm{g} / \mathrm{L}$ & $\mathrm{GE}$ & 0 \\
\hline & $\mu \mathrm{g} / \mathrm{L}$ & GE & 0 \\
\hline & $\mu_{\theta} / \mathrm{L}$ & $\mathrm{GE}$ & 0 \\
\hline & $\mu \mathrm{g} / \mathrm{L}$ & $\mathrm{GE}$ & 0 \\
\hline & $\mu \boldsymbol{g} / \mathrm{L}$ & $\mathrm{GE}$ & 0 \\
\hline & $\begin{array}{l}\mu \mathrm{g} / \mathrm{L} \\
\mu \mathrm{g} / \mathrm{L}\end{array}$ & $\begin{array}{l}\mathrm{GE} \\
\mathrm{GE}\end{array}$ & $\begin{array}{l}0 \\
0\end{array}$ \\
\hline & $\mu g / L$ & $\mathrm{GE}$ & 0 \\
\hline & $\mathrm{pCi} / \mathrm{L}$ & $\mathrm{GE}$ & 0 \\
\hline & $\mu_{0} / \mathrm{L}$ & $G E$ & 1 \\
\hline & $\mu \boldsymbol{g} / \mathbf{L}$ & $G E$ & 0 \\
\hline & $\mu g / L$ & GE & 0 \\
\hline & $\mu g / L$ & Ge & \\
\hline
\end{tabular}

\section{Formation}

Water table (IIB.) ) 
Well HSB132D continued

ANALYTICAL DATA

H $\quad$ Analyte
Morcury
Nickel
Nitrate-nitrite as nitrogen
Nonvolatilo bota
pH
Phenols
Potassium
Solenium
Silica
Silver
Sodium
Specific conductance
Sulfate
Total alpho-emitting radium
Total dissolved solids
Total organic carbon
Total organic halogens
Total phosphates (as P)
Tritium
Vanadium
Zinc

$\begin{array}{lll}\frac{1092}{2} & \underline{2092} & \underline{3092} \\ <0.20 & <0.20 & <0.20 \\ <4.0 & <4.0 & 5.3 \\ 860 & 770 & 720 \\ <2.0 \mathrm{E}+00 & <2.0 \mathrm{E}+00 & <2.0 \mathrm{E}+00 \\ 5.4 & 5.4 & 5.4 \\ <5.0 & & \\ <500 & <500 & <500 \\ <2.0 & <2.0 & <2.0 \\ 6.830 & 6.880 & 6.860 \\ <2.0 & <2.0 & <2.0 \\ 1.930 & 1.810 & 1.570 \\ 20 & 20 & 20 \\ <1.000 & <1.000 & <1.000 \\ <1.0 \mathrm{OE}+00 & <1.0 \mathrm{O}+00 & <1.0 \mathrm{E}+00 \\ 11.000 & 20.000 & 18.000 \\ <1.000 & <1.000 & <1.000 \\ <5.0 & 35 & <5.0 \\ <50 & <50 & <50 \\ 1.6 \mathrm{E}+01 & 2.0 \mathrm{O}+01 & 1.7 \mathrm{E}+01 \\ <8.0 & <8.0 & <8.0 \\ 9.1 & 7.0 & 9.0 \\ & & \end{array}$

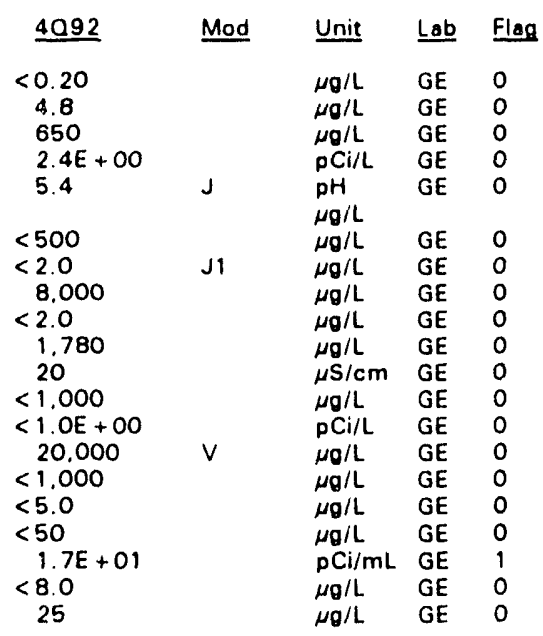

\section{WELL HSB133C}

\begin{tabular}{|c|c|}
\hline SRS Coord. & Lat/Longitude \\
\hline $\begin{array}{l}N 71949.5 \\
E 59110.3\end{array}$ & $\begin{array}{l}33.281349{ }^{\circ} \mathrm{N} \\
81.650912^{\circ} \mathrm{W}\end{array}$ \\
\hline
\end{tabular}

\section{Scroen Zone Elevation}

183.3.173.3 ft $\mathrm{msl}$

SAMPLE DATE

$01 / 03 / 92$

\section{Top of Casing}

$255.6 \mathrm{ft} \mathrm{ms}$

$04 / 03 / 92$

$\underline{2092}$
235.6
5.5
44
18.6
7
3.7

ㄴ D

Analyte
Aluminum
Antimony
Arsenic
Barium
Cadmium
Calcium
Chloride
Chromium
Cobalt
Copper
Cyonide
Fluoride
Gross alpho
Iron
Lead
Magnesium
Manganese

1092

1092
230.3
6.0
45
18.0
11
4.0

$\begin{aligned} & 1092 \\ &< 20 \\ &<2.0 \\ &<2.0 \\ & 5.0 \\ &<2.0 \\ & 4.150 \\ & 2.630 \\ &<4.0 \\ &<4.0 \\ &<4.0 \\ &<5.0 \\ & 120 \\ &<2.0 E+00 \\ &<4.0 \\ &<3.0 \\ & 467 \\ & 5.6\end{aligned}$

$\begin{aligned} & 2092 \\ &< 20 \\ &< 2.0 \\ &< 2.0 \\ & 5.5 \\ &< 2.0 \\ & 3.800 \\ & 2.690 \\ &< 4.0 \\ &< 4.0 \\ &< 4.0 \\ &<5.0 \\ & 106 \\ &< 2.0 \mathrm{E}+00 \\ &<4.0 \\ &<3.0 \\ & 495 \\ & 5.3\end{aligned}$

FIELD DATA

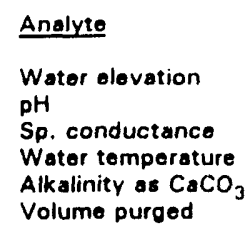

$\begin{aligned} & 3092 \\ < & 20 \\ < & 2.0 \\ < & 2.0 \\ & 5.4 \\ < & 2.0 \\ & 3.800 \\ & 2.500 \\ < & 4.0 \\ < & 4.0 \\ < & 4.0 \\ < & 5.0 \\ < & 100 \\ < & 2.0 E+00 \\ < & 4.0 \\ & 3.5 \\ & 446 \\ & 4.3\end{aligned}$

$\begin{aligned} & 4092 \\ & 22 \\ &< 2.0 \\ &< 2.0 \\ & 5.2 \\ &<2.0 \\ & 4.430 \\ & 2.640 \\ & 4.2 \\ &<4.0 \\ &<4.0 \\ &<5.0 \\ & 104 \\ &<2.0 \mathrm{E}+00 \\ & 6.5 \\ &<3.0 \\ & 532 \\ & 5.3\end{aligned}$

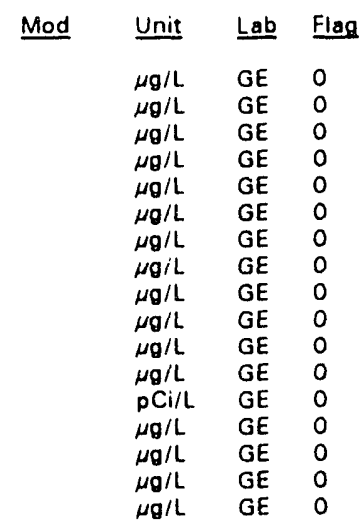

$\begin{array}{lll}3092 & \text { 4092 } & \text { Unit } \\ 230.4 & 231.0 & \mathrm{ft} \mathrm{msl} \\ 5.7 & 5.9 & \mathrm{pH} \\ 42 & 44 & \mu \mathrm{S} / \mathrm{cm} \\ 19.9 & 19.7 & { }^{\circ} \mathrm{C} \\ 10 & 10 & \mathrm{mg} / \mathrm{L} \\ 4.0 & 3.0 & \mathrm{Well} \text { vol. }\end{array}$

Formation

Barnwell (IIB, 
WSRC-TR-93-059

Well HSB133C continued

ANALYTICAL DATA

H. $\quad$ Analyte
Mercury
Nickel
Nitrate-nitrite as nitrogen
Nonvolatile beta
pH
Phonols
Potassium
Solenium
Silica
Silver
Sodium
Spocific conductance
Sulfate
Total alpha-emitting radium
Total dissolved solids
Total organic carbon
Total organic halogens
Total phosphates las PI
Tritium
Vanadium
Zinc

$\begin{array}{lll}1092 & \underline{2092} & \underline{3092} \\ <0.20 & <0.20 & <0.20 \\ <4.0 & <4.0 & <4.0 \\ 70 & <50 & <50 \\ <2.0 E+00 & <2.0 E+00 & <2.0 E+00 \\ 5.9 & 6.2 & 6.2 \\ <5.0 & & \\ 1.110 & 1.180 & 1.220 \\ <2.0 & <2.0 & <2.0 \\ 13.300 & 14.600 & 14.000 \\ <2.0 & <2.0 & <2.0 \\ 2.290 & 2.330 & 2.350 \\ 45 & 35 & 40 \\ <1.000 & <1.000 & 1.010 \\ <1.0 E+00 & <1.0 E+00 & <1.0 E+00 \\ 39.000 & 33.000 & 38.000 \\ <1.000 & <1.000 & <1.000 \\ <5.0 & <5.0 & <5.0 \\ 230 & 140 & 230 \\ <7.0 E-01 & <7.0 E-01 & <7.0 E-01 \\ <8.0 & <8.0 & <8.0 \\ 4.7 & 4.8 & 5.6\end{array}$

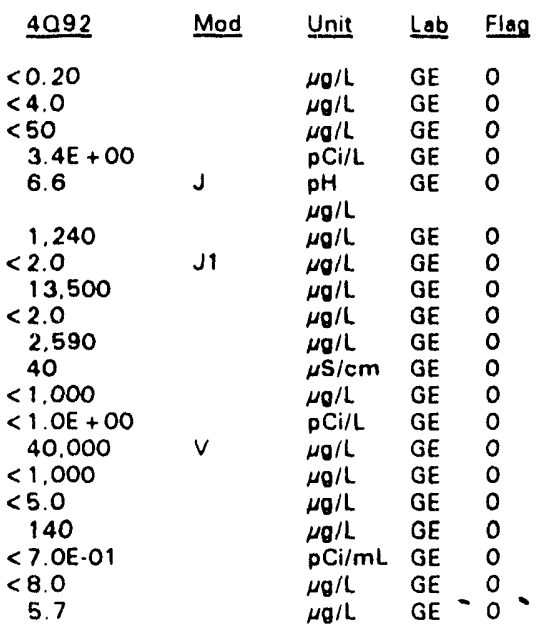

\section{WELL HSB133D}

\begin{tabular}{|c|c|c|c|c|c|c|c|c|c|}
\hline SRS Coord. & Lat/Longitude & Screen Zone Elevation & Top of Casing & Casing & Pump & \multicolumn{3}{|c|}{ Formation } & \\
\hline $\begin{array}{l}\text { N71943.5 } \\
E 59102.3\end{array}$ & $\begin{array}{l}33.281323{ }^{\circ} \mathrm{N} \\
81.650921^{\circ} \mathrm{W}\end{array}$ & $228.5-208.5 \mathrm{ft} \mathrm{msl}$ & $255.3 \mathrm{ft} \mathrm{msl}$ & 4" PVC & $\mathbf{S}$ & \multicolumn{4}{|c|}{ Water table $\left(I 1 B_{2}\right)$} \\
\hline \multicolumn{2}{|c|}{ SAMPLE DATE } & $01 / 03 / 92$ & $04 / 03 / 92$ & $07 / 17 / 92$ & $10 / 16 / 92$ & & & & \\
\hline \multicolumn{10}{|l|}{ FIELD DATA } \\
\hline \multicolumn{2}{|r|}{ Analyte } & 1092 & 2092 & 3092 & 4092 & \multicolumn{4}{|l|}{ Unit } \\
\hline & $\begin{array}{l}\text { Water elevation } \\
\text { pH } \\
\text { Sp. conductance } \\
\text { Water temperature } \\
\text { Alkalinity as } \mathrm{CaCO}_{3} \\
\text { Volume purged }\end{array}$ & $\begin{array}{l}234.8 \\
5.5 \\
72 \\
17.3 \\
5 \\
4.3\end{array}$ & $\begin{array}{l}235.4 \\
5.3 \\
72 \\
18.1 \\
6 \\
4.3\end{array}$ & $\begin{array}{l}235.2 \\
5.2 \\
69 \\
18.9 \\
5 \\
5.3\end{array}$ & $\begin{array}{l}230.5 \\
5.3 \\
68 \\
18.7 \\
5 \\
16.1\end{array}$ & \multicolumn{3}{|c|}{$\begin{array}{l}\mathrm{ft} \mathrm{msl} \\
\mathrm{pH} \\
\mu \mathrm{S} / \mathrm{cm} \\
{ }^{\circ} \mathrm{C} \\
\mathrm{mg} / \mathrm{h} \\
\text { Well vol. }\end{array}$} & \\
\hline \multicolumn{10}{|c|}{ ANALYTICAL DATA } \\
\hline$\underline{H} \quad \underline{D}$ & Analyte & 1092 & $\underline{2092}$ & 3092 & 4092 & Mod & Unit & Lab & Flag \\
\hline A & $\begin{array}{l}\text { Aluminum } \\
\text { Antimany } \\
\text { Arsenic } \\
\text { Barium } \\
\text { Cadmium } \\
\text { Calcium } \\
\text { Chloride } \\
\text { Chromium } \\
\text { Cobalt } \\
\text { Copper } \\
\text { Cyanide } \\
\text { Fluoride } \\
\text { Gross alpha } \\
\text { Iron } \\
\text { Lead } \\
\text { Magnesium } \\
\text { Manganese }\end{array}$ & $\begin{aligned} &< 20 \\
&<2.0 \\
&<2.0 \\
&<3.0 \\
&<2.0 \\
& 1.780 \\
& 6.100 \\
&<4.0 \\
&<4.0 \\
&<4.0 \\
&<5.0 \\
&<100 \\
&<2.0 E+00 \\
& 8.7 \\
&<3.0 \\
& 104 \\
& 3.1\end{aligned}$ & $\begin{aligned} &< 20 \\
&<2.0 \\
&<2.0 \\
&<3.0 \\
&<2.0 \\
& 1.610 \\
& 5.700 \\
&< 4.0 \\
&<4.0 \\
&<4.0 \\
&<5.0 \\
&<100 \\
&<2.0 E+00 \\
& 13 \\
&<3.0 \\
& 133 \\
& 4.0\end{aligned}$ & $\begin{aligned} &< 20 \\
&<2.0 \\
&<2.0 \\
&<3.0 \\
&<2.0 \\
& 1.440 \\
& 6.070 \\
&< 4.0 \\
&< 4.0 \\
&<4.0 \\
&<5.0 \\
&<100 \\
&<2.0 E+00 \\
& 4.7 \\
&<3.0 \\
& 109 \\
& 3.4\end{aligned}$ & $\begin{aligned} & 156 \\
&< 2.0 \\
&< 2.0 \\
&< 3.0 \\
&< 2.0 \\
& 1.260 \\
& 6.540 \\
&< 4.0 \\
&< 4.0 \\
&< 4.0 \\
&<5.0 \\
&<100 \\
&< 2.0 E+00 \\
& 64 \\
&< 3.0 \\
& 113 \\
& 3.7\end{aligned}$ & J6 & $\begin{array}{l}\mu g / L \\
\mu g / L \\
\mu g / L \\
\mu g / L \\
\mu g / L \\
\mu g / L \\
\mu g / L \\
\mu g / L \\
\mu g / L \\
\mu g / L \\
\mu g / L \\
\mu g / L \\
p C i / L \\
\mu g / L \\
\mu g / L \\
\mu g / L \\
\mu g / L\end{array}$ & $\begin{array}{l}\mathrm{GE} \\
\mathrm{GE} \\
\mathrm{GE} \\
\mathrm{GE} \\
\mathrm{JE} \\
\mathrm{GE} \\
\mathrm{GE} \\
\mathrm{GE} \\
\mathrm{GE} \\
\mathrm{GE} \\
\mathrm{GE} \\
\mathrm{GE} \\
\mathrm{GE} \\
\mathrm{GE} \\
\mathrm{GE} \\
\mathrm{GE} \\
\mathrm{GE}\end{array}$ & $\begin{array}{l}2 \\
0 \\
0 \\
0 \\
0 \\
0 \\
0 \\
0 \\
0 \\
0 \\
0 \\
0 \\
0 \\
0 \\
0 \\
0 \\
0\end{array}$ \\
\hline
\end{tabular}

Note: Flagging levels, modifiers, and laboratories are for 4 th quarter 1992 data only. See Appendix B for flagging criteria.

- exceeded holding time for 4th quarter 1992.

- =xceeded final primary drinking water standard for 4th quarter 1992. 
ANALYTICAL DATA

D D Analyte
Mercury
Nickel
Nitrate-nitrite as nitrogen
Nonvolatile beta
pH
Phenols
Potassium
Selenium
Silica
Silver
Sodium
Specific conductance
Sulfate
Total alpha-emitting radium
Total dissolved solids
Total organic carbon
Total organic hologens
Total phosphates las PI
Tritium
Vanadium
Zinc

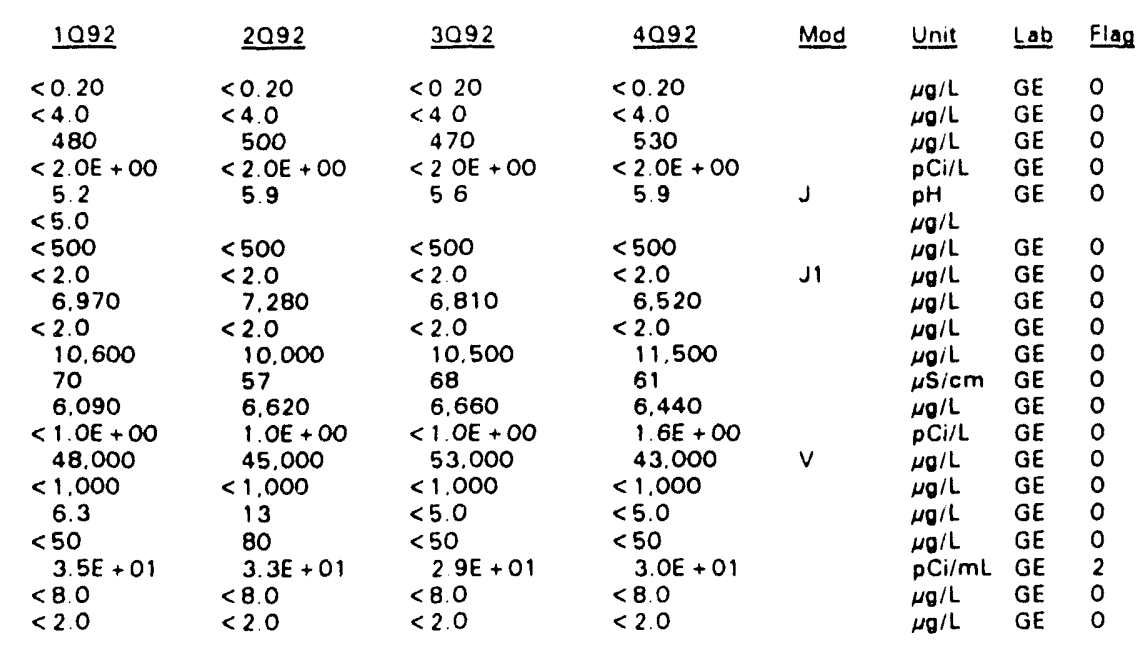

WELL HSB134C

\begin{tabular}{|c|c|c|c|c|c|c|c|c|c|}
\hline sRS Coord. & Lat/Longitude & Screen Zone Elevation & Top of Casing & Casing & Pump & \multicolumn{3}{|c|}{ Formation } & \\
\hline $\begin{array}{l}N 71210.3 \\
E 58289.9\end{array}$ & $\begin{array}{l}33.278376^{\circ} \mathrm{N} \\
81.651636^{\circ} \mathrm{W}\end{array}$ & $159.1 \cdot 149.1 \mathrm{ft} \mathrm{msl}$ & $238.4 \mathrm{tt} \mathrm{msl}$ & 4" PVC & s & \multicolumn{4}{|c|}{ Barnwell (IIB, ) } \\
\hline \multicolumn{2}{|c|}{ SAMPLE DATE } & $01 / 07 / 92$ & $04 / 09 / 92$ & $07 / 22 / 92$ & $10 / 16 / 92$ & & & & \\
\hline \multicolumn{10}{|c|}{ FIELD DATA } \\
\hline \multicolumn{2}{|r|}{ Analyte } & 1092 & $\underline{2092}$ & 3092 & 4092 & \multicolumn{4}{|l|}{$\underline{\text { Unit }}$} \\
\hline \multicolumn{2}{|r|}{$\begin{array}{l}\text { Water elevation } \\
\text { pH } \\
\text { Sp. conductance } \\
\text { Water temperature } \\
\text { Alkalinity as } \mathrm{CaCO}_{3} \\
\text { Volume purged }\end{array}$} & $\begin{array}{l}220.6 \\
5.8 \\
49 \\
18.0 \\
7 \\
4.0\end{array}$ & $\begin{array}{l}220.8 \\
55 \\
46 \\
19.1 \\
5 \\
4.0\end{array}$ & $\begin{array}{l}220.9 \\
5.4 \\
43 \\
19.4 \\
4 \\
4.0\end{array}$ & $\begin{array}{l}221.2 \\
5.2 \\
39 \\
19.3 \\
2 \\
2.5\end{array}$ & \multicolumn{3}{|c|}{$\begin{array}{l}\mathrm{ft} \mathrm{msl} \\
\mathrm{pH} \\
\mu \mathrm{S} / \mathrm{cm} \\
{ }^{\circ} \mathrm{C} \\
\mathrm{mg} / \mathrm{L} \\
\text { Well vol. }\end{array}$} & \\
\hline \multicolumn{10}{|c|}{ ANALYTICAL DATA } \\
\hline \multirow[t]{2}{*}{$\underline{H} \quad \underline{D}$} & Analyte & 1092 & $\underline{2092}$ & 3092 & 4092 & Mod & $\underline{\text { Unit }}$ & Lob & Flag \\
\hline & $\begin{array}{l}\text { Aluminum } \\
\text { Antimony } \\
\text { Arsenic } \\
\text { Barium } \\
\text { Cadmium } \\
\text { Calcium } \\
\text { Chloride } \\
\text { Chromium } \\
\text { Cobalt } \\
\text { Copper } \\
\text { Cyanide } \\
\text { Fluoride } \\
\text { Gross alpha } \\
\text { Iron } \\
\text { Lead } \\
\text { Magnesium } \\
\text { Manganese }\end{array}$ & $\begin{aligned} & 25 \\
< & 2.0 \\
< & 2.0 \\
& 6.6 \\
< & 2.0 \\
& 4.400 \\
& 3.020 \\
< & 4.0 \\
< & 4.0 \\
< & 4.0 \\
< & 5.0 \\
< & 100 \\
< & 2.0 E+00 \\
< & 4.0 \\
< & 3.0 \\
& 1.000 \\
& 18\end{aligned}$ & $\begin{aligned} & 26 \\
< & 2.0 \\
< & 2.0 \\
& 7.6 \\
< & 2.0 \\
& 3.440 \\
& 2.940 \\
< & 4.0 \\
< & 4.0 \\
< & 4.0 \\
< & 5.0 \\
< & 100 \\
< & 2.0 E+00 \\
< & 4.0 \\
< & 3.0 \\
& 1.090 \\
& 20\end{aligned}$ & $\begin{aligned} & 27 \\
&< 2.0 \\
&< 2.0 \\
& 6.9 \\
&< 2.0 \\
& 3.470 \\
& 2.720 \\
&< 4.0 \\
&< 4.0 \\
&< 4.0 \\
&< 5.0 \\
& 117<2 \\
&< 2 . \mathrm{OE}+00 \\
&<40 \\
&<3.0 \\
& 1.100 \\
& 21\end{aligned}$ & $\begin{aligned} & 37 \\
&< 2.0 \\
&< 2.0 \\
& 7.1 \\
&< 2.0 \\
& 2.960 \\
& 2.810 \\
&< 4.0 \\
&< 4.0 \\
&<4.0 \\
&<5.0 \\
&<100 \\
&<2.0 E+00 \\
& 8.5 \\
&<3.0 \\
& 1.330 \\
& 23\end{aligned}$ & & $\begin{array}{l}\mu g / L \\
\mu g / L \\
\mu g / L \\
\mu g / L \\
\mu g / L \\
\mu g / L \\
\mu g / L \\
\mu g / L \\
\mu g / L \\
\mu g / L \\
\mu g / L \\
\mu g / L \\
\rho C i / L \\
\mu g / L \\
\mu g / L \\
\mu g / L \\
\mu g / L\end{array}$ & $\begin{array}{l}\mathrm{GE} \\
\mathrm{GE} \\
\mathrm{GE} \\
\mathrm{GE} \\
\mathrm{GE} \\
\mathrm{GE} \\
\mathrm{GE} \\
\mathrm{GE} \\
\mathrm{GE} \\
\mathrm{GE} \\
\mathrm{GE} \\
\mathrm{GE} \\
\mathrm{GE} \\
\mathrm{GE} \\
\mathrm{GE} \\
\mathrm{GE} \\
\mathrm{GE}\end{array}$ & $\begin{array}{l}1 \\
0 \\
0 \\
0 \\
0 \\
0 \\
0 \\
0 \\
0 \\
0 \\
0 \\
0 \\
0 \\
0 \\
0 \\
0 \\
0\end{array}$ \\
\hline
\end{tabular}

\footnotetext{
Note: Flagging levels, modifiers, and laboratories are for 4 th quarter 1992 data only. See Appendix B for flagging criterta. - =xceeded holding time for 4th quarter 1992.

- =xceeded final primary drinking water standard for 4th quarter 1992.
} 
WSRC.TR.93.059

Well HSB134C continued

ANALYTICAL DATA

H $\quad$ Analyte
Mercury
Nickol
Nitrste as nitrogon
Nitrate-nitrite as nitrogen
Nonvolatile beta
pH
Phenols
Potassium
Solenium
Silica
Silver
Sodium
Specific conductance
Sulfate
Total alpha-emitting radium
Total dissolvod solid:
Total organic carbon
Total organic halogens
Total phosphates las P)
Tritium
Vanadium
Zinc

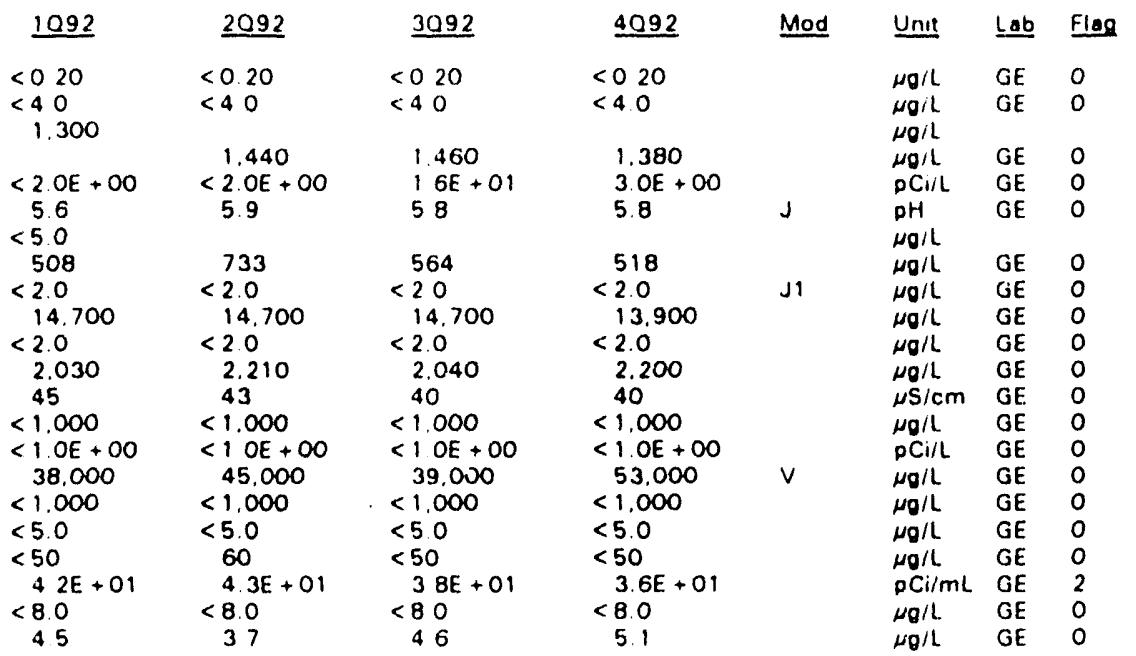

\section{WELL HSB 134D}

\begin{tabular}{|c|c|}
\hline SRS Coord. & Lat/Longitude \\
\hline $\begin{array}{l}N 71217.3 \\
E 58296.5\end{array}$ & $\begin{array}{l}33.278402{ }^{\circ} \mathrm{N} \\
81.651633^{\circ} \mathrm{W}\end{array}$ \\
\hline
\end{tabular}

$\frac{\text { Screen Zone Elevation }}{225.8 .205 .8 \mathrm{ft} \mathrm{msl}}$

$\frac{\text { Top of Casing }}{2381 \mathrm{ft} \mathrm{msi}}$

$\begin{array}{ll}\text { Casing } & \text { Pump } \\ \text { 4* PVC } & \text { S }\end{array}$

Formation

SAMPLE DATE

$01 / 07 / 92$

$04 / 09 / 92$

$07 / 22 / 92$

$10 / 18 / 92$

FIELO DATA

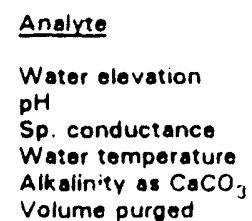

$\begin{array}{ll}1092 & \underline{2092} \\ 221.3 & 2224 \\ 44 & 44 \\ 114 & 144 \\ 178 & 182 \\ 0 & 0 \\ 40 & 40\end{array}$

$\mathbf{3 0 9 2}$
222.1
4.2
120
19.3
0
40

4092
222.0
4.2
141
19.4
0
32

Unit
tt msil
$\mathrm{oH}$
$\mu=1 \mathrm{~cm}$
${ }^{\circ} \mathrm{C}$
$\mathrm{mg} / \mathrm{L}$
Well vol

ANALYTICAL DATA

\begin{tabular}{|c|c|}
\hline$\underline{D}$ & Anolyte \\
\hline & $\begin{array}{l}\text { Aluminum } \\
\text { Antimony } \\
\text { Antimony-125 } \\
\text { Arsenic } \\
\text { Barium } \\
\text { Cadmium } \\
\text { Colcium } \\
\text { Cerium.144 } \\
\text { Cesium-134 } \\
\text { Cesium.137 } \\
\text { Chloride } \\
\text { Chromium } \\
\text { Chromium.51 } \\
\text { Cobait } \\
\text { Cobatt.57 } \\
\text { Cobatr. } 60\end{array}$ \\
\hline
\end{tabular}

$\begin{array}{ll}\frac{1092}{235} & \underline{2092} \\ <2.0 & <24 \\ <2.0 & <2.0 \\ 24 & 26 \\ <2.0 & <2.0 \\ 385 & 410 \\ & \\ 2.620 & 2.320 \\ <4.0 & <40 \\ <4.0 & <4.0\end{array}$

$\begin{aligned} & 3092 \\ & 299 \\ < & 2.0 \\ < & 2.0 E+01 \\ < & 2.0 \\ & 29 \\ < & 2.0 \\ & 585 \\ < & 6 . O E+01 \\ < & 1 . O E+01 \\ < & 1 O E+01 \\ & 1.830 \\ < & 40 \\ < & 12 E+02 \\ < & 4 O \\ < & 1 O E+01 \\ < & 1 O E+01\end{aligned}$

$\begin{aligned} & 4092 \\ & 473 \\ < & 2.0 \\ < & 2.0 E+01 \\ < & 2.0 \\ & 40 \\ < & 2.0 \\ & 645 \\ < & 6.0 E+01 \\ < & 1 O E+01 \\ < & 1.0 E+01 \\ & 1.910 \\ < & 40 \\ < & 40 \\ < & 1.0 E+01 \\ < & 1 O E+01\end{aligned}$

\begin{tabular}{|c|c|c|c|}
\hline Mod & $\underline{\text { Unit }}$ & $\underline{\text { Lab }}$ & Fla \\
\hline & $\mu g / L$ & $\mathrm{GE}$ & 2 \\
\hline & $\mu \mathrm{g} / \mathrm{L}$ & $\mathrm{GE}$ & 0 \\
\hline & $\mathrm{pCi} / \mathrm{L}$ & $G P$ & 0 \\
\hline & $\mu \mathrm{g} / \mathrm{L}$ & GE & 0 \\
\hline & $\mu \mathrm{g} / \mathrm{L}$ & $G E$ & 0 \\
\hline & $\mu \mathrm{g} / \mathrm{L}$ & $G E$ & 0 \\
\hline & $\mu_{\theta} / \mathrm{L}$ & $\mathrm{GE}$ & 0 \\
\hline & $\rho \mathrm{C} i / \mathrm{L}$ & $G P$ & 0 \\
\hline & $\mathrm{pCi} / \mathrm{L}$ & GP & 0 \\
\hline & $\mathrm{pCi} / \mathrm{L}$ & GP & 0 \\
\hline & $\mu g / L$ & $\mathrm{GE}$ & 0 \\
\hline & $\mu \mathrm{g} / \mathrm{L}$ & $\mathrm{GE}$ & 0 \\
\hline & $\mu \sigma / L$ & $\mathrm{GE}$ & 0 \\
\hline & $\rho C i / L$ & $G P$ & 0 \\
\hline & $\mathrm{pCi/L}$ & GP & 0 \\
\hline
\end{tabular}

Note: Flagging levels, modifiers, and laboratories are for 4 th quarter 1992 data only. See Appendix B for flagging criteria.

- =xceeded holding time for 4 th quarter 1992.

- exceeded final primary drinking watef standard for 4th quarter 1992. 
WSRC.TR-9.3.059

Well HSB1340 continued

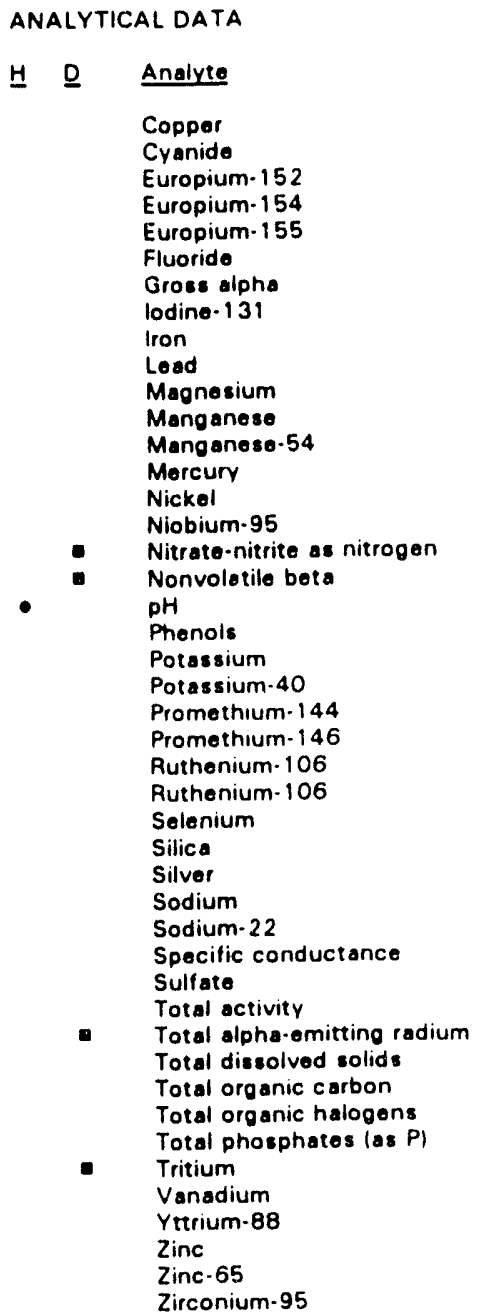

\begin{tabular}{|c|c|c|}
\hline 1092 & 2092 & 3092 \\
\hline $\begin{array}{l}<4.0 \\
<5.0\end{array}$ & $\begin{array}{l}<4.0 \\
<5.0\end{array}$ & $\begin{array}{l}<4.0 \\
<5.0 \\
<4.0 E+01 \\
<2.0 E+01 \\
<3.0 E+01\end{array}$ \\
\hline $\begin{array}{l}<100 \\
\quad 2.3 E+\infty 0\end{array}$ & $\begin{array}{l}<100 \\
\quad 3.3 E+00\end{array}$ & $\begin{array}{l}<100 \\
<2.0 E+00 \\
<2.0 E+01\end{array}$ \\
\hline $\begin{array}{r}8.3 \\
<3.0 \\
751 \\
66\end{array}$ & $\begin{array}{c}21 \\
<3.0 \\
789 \\
67\end{array}$ & $\begin{aligned} & 11 \\
&< 3.0 \\
& 961 \\
& 90 \\
&<1.0 E+01\end{aligned}$ \\
\hline $\begin{aligned} & 0.60 \\
< & 4.0\end{aligned}$ & $\begin{aligned} & 0.48 \\
< & 4.0\end{aligned}$ & $\begin{aligned} & 0.38 \\
< & 4.0 \\
< & 15 E+01\end{aligned}$ \\
\hline $\begin{array}{l}10.200 \\
2.6 \mathrm{E}+02 \\
4.6\end{array}$ & $\begin{array}{l}15.800 \\
3.2 E+02 \\
4.7\end{array}$ & $\begin{array}{l}12.000 \\
4.6 E+02 \\
4.5\end{array}$ \\
\hline $\begin{array}{l}<5.0 \\
<500\end{array}$ & $<500$ & $\begin{array}{l}<500 \\
<11 E+02 \\
<1 O E+01 \\
<1 O E+01 \\
<9 O E+01 \\
<13 E+02\end{array}$ \\
\hline $\begin{aligned}< & 2.0 \\
& 6.570 \\
< & 2.0 \\
& 13.500\end{aligned}$ & $\begin{array}{l}<2.0 \\
6.190 \\
<2.0 \\
22.200\end{array}$ & $\begin{aligned}< & 2.0 \\
& 6.630 \\
< & 2.0 \\
& 15.000 \\
< & 1.0 E+01\end{aligned}$ \\
\hline $\begin{array}{l}110 \\
1.810 \\
1.6 E+06 \\
3.3 E+00 \\
70.000\end{array}$ & $\begin{array}{l}132 \\
<1.000 \\
1.5 E+06 \\
1.1 E+01 \\
104.000\end{array}$ & $\begin{array}{l}110 \\
1.290 \\
8.9 E+05 \\
2.7 E+00 \\
79.000\end{array}$ \\
\hline $\begin{array}{l}<1.000 \\
39 \\
<50\end{array}$ & $\begin{array}{l}<1,000 \\
38 \\
<50\end{array}$ & $\begin{aligned}< & 1.000 \\
& 5.5 \\
< & 50\end{aligned}$ \\
\hline $\begin{array}{l}<50 \\
1.5 E+03 \\
<8.0\end{array}$ & $\begin{array}{l}<50 \\
1.6 E+03 \\
<8.0\end{array}$ & $\begin{aligned} & 8.6 E+02 \\
< & 8.0 \\
< & 6.0 E+01\end{aligned}$ \\
\hline 26 & $<2.0$ & $\begin{aligned} & 4.3 \\
< & 2.0 E+01 \\
< & 2.0 E+01\end{aligned}$ \\
\hline
\end{tabular}

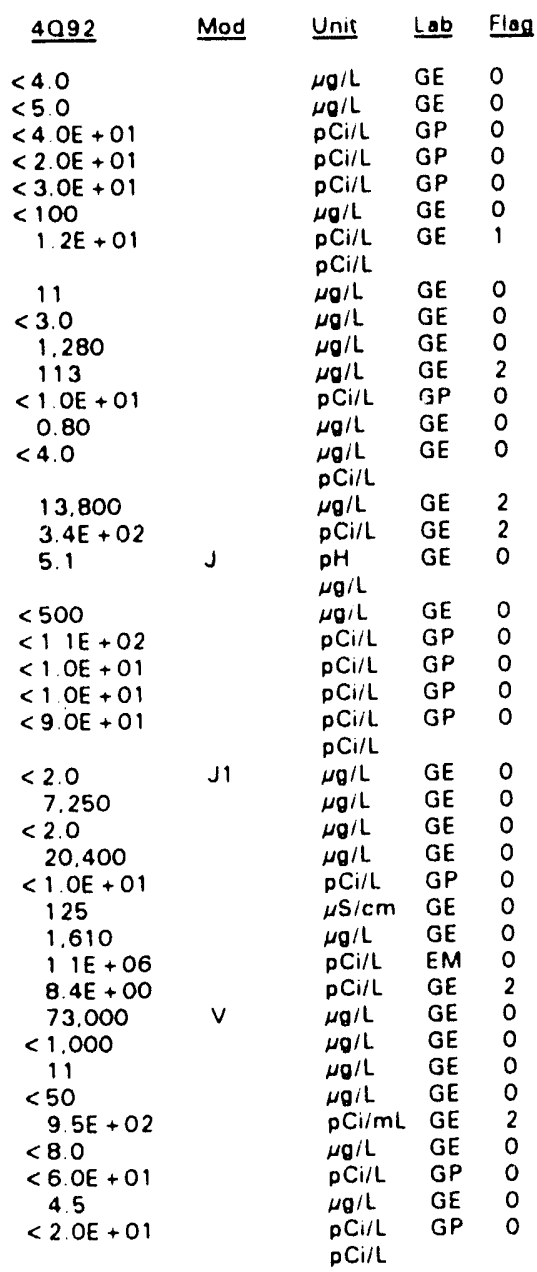

Note: Flagging levels, modifiers, and laboratories are for 4 th quarter 1992 data only. See Appendix B for flagging criteria

- =xceeded holding time for 4th quarter 1992.

- =xceeded final primary drinkıing water standard for 4th quarter 1992. 
WSRC.TR.93-059

WELL HSB135C

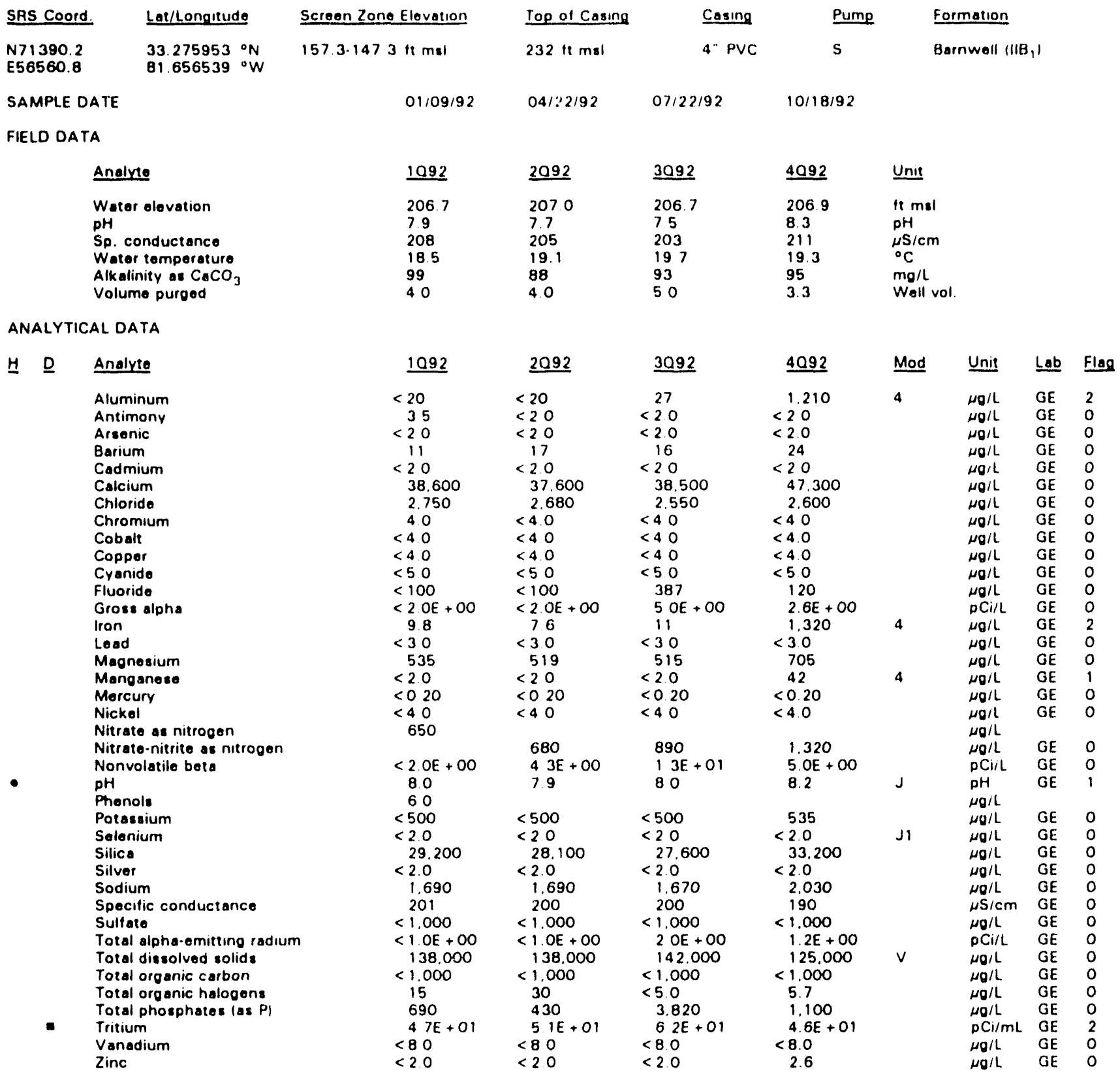

Note: Flagging levels, modifiers, and laboratories are for 4 th quarter 1992 data only. See Appendix $B$ for flagging criteria - =xceeded holding time for 4th quarter 1992.

- =xceeded final primary drinking water standard for 4th quarter 1992. 
WSRC-TR.93-059

WELL HSB 135D

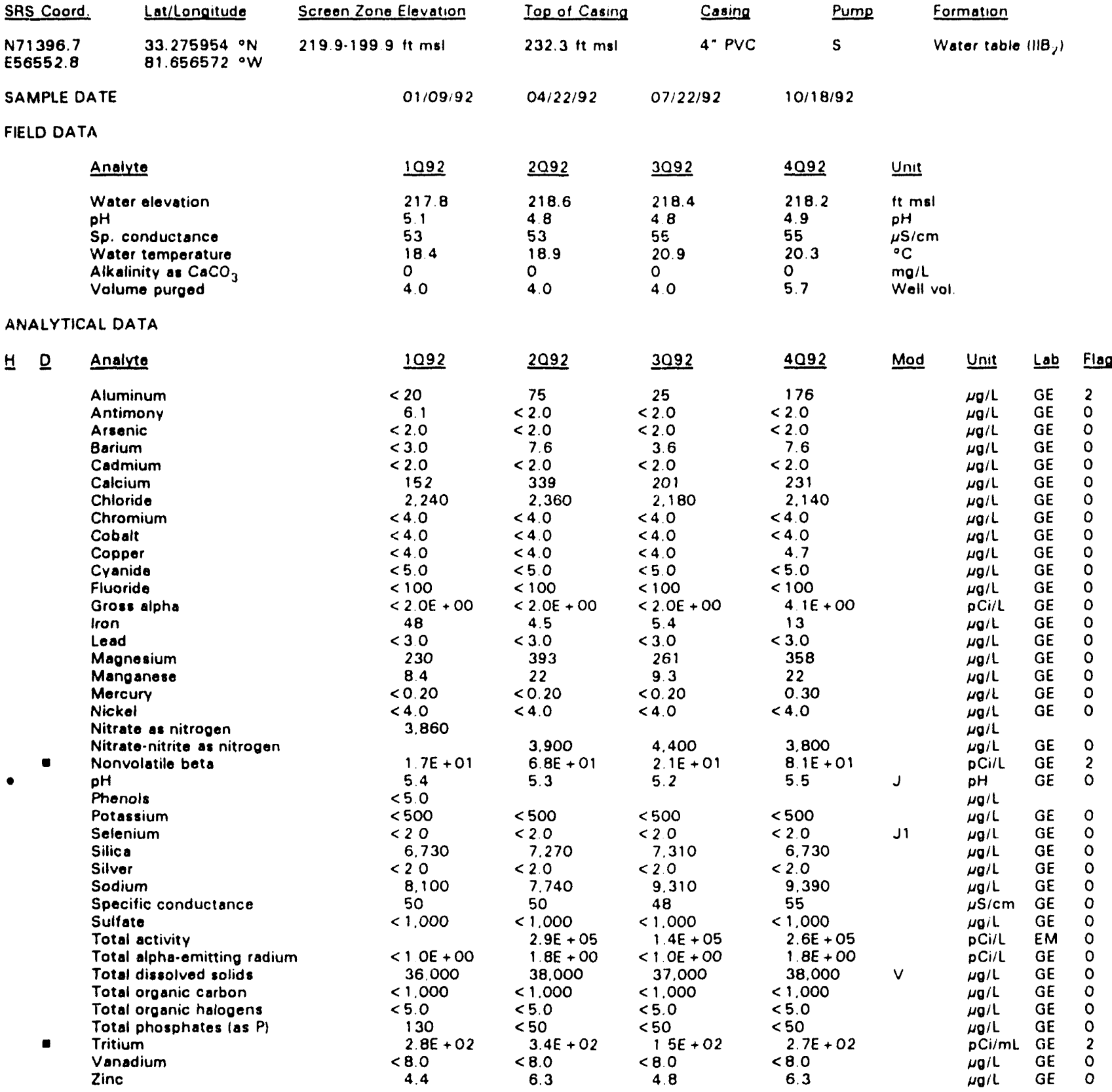

Note: Flagging levels, modifiers, and laboratories are for 4 th quarter 1992 data only. See Appendix B for flagging criteria. - =xceoded holding time for 4th quarter 1992.

- =xceeded final primary drinking water standard for 4th quarter 1992. 
WELL HSB 136C

\begin{tabular}{|c|c|c|c|c|c|c|}
\hline SAS Coord. & Lat/Longitude & Screen Zono Elovation & Top of Casing & Casing & Pump & Formation \\
\hline $\begin{array}{l}\text { N71900.3 } \\
\text { E55949.6 }\end{array}$ & $\begin{array}{l}33.276084{ }^{\circ} \mathrm{N} \\
81.659139{ }^{\circ} \mathrm{W}\end{array}$ & $170.5 \cdot 1605 \mathrm{ft} \mathrm{ms}$ & $227.9 \mathrm{ft} \mathrm{ms}$ & 4" PVC & S & Barnwell IIIB, \\
\hline SAMPLE D & & $01 / 13 / 92$ & $04 / 27 / 92$ & $07 / 21 / 92$ & $10 / 21 / 92$ & \\
\hline \multicolumn{7}{|l|}{ FIELD DATA } \\
\hline \multicolumn{2}{|c|}{ Analyte } & 1092 & $\underline{2092}$ & 3092 & 4092 & Unit \\
\hline \multicolumn{2}{|c|}{$\begin{array}{l}\text { Water elevation } \\
\text { pH } \\
\text { Sp. conductance } \\
\text { Water temperature } \\
\text { Alkalinity as } \mathrm{CaCO}_{3} \\
\text { Volume purged }\end{array}$} & $\begin{array}{l}217.1 \\
60 \\
425 \\
19.3 \\
11 \\
4.0\end{array}$ & $\begin{array}{l}2177 \\
5.7 \\
421 \\
19.1 \\
11 \\
41\end{array}$ & $\begin{array}{l}217.6 \\
96 \\
421 \\
211 \\
33 \\
0.8\end{array}$ & $\begin{array}{l}217.5 \\
8.9 \\
418 \\
18.6 \\
29 \\
0.7\end{array}$ & $\begin{array}{l}\text { ft mal } \\
\mathrm{pH} \\
\mu \mathrm{S} / \mathrm{cm} \\
{ }^{\circ} \mathrm{C} \\
\mathrm{mg} / \mathrm{L} \\
\text { Well vol. }\end{array}$ \\
\hline
\end{tabular}

ANALYTICAL DATA

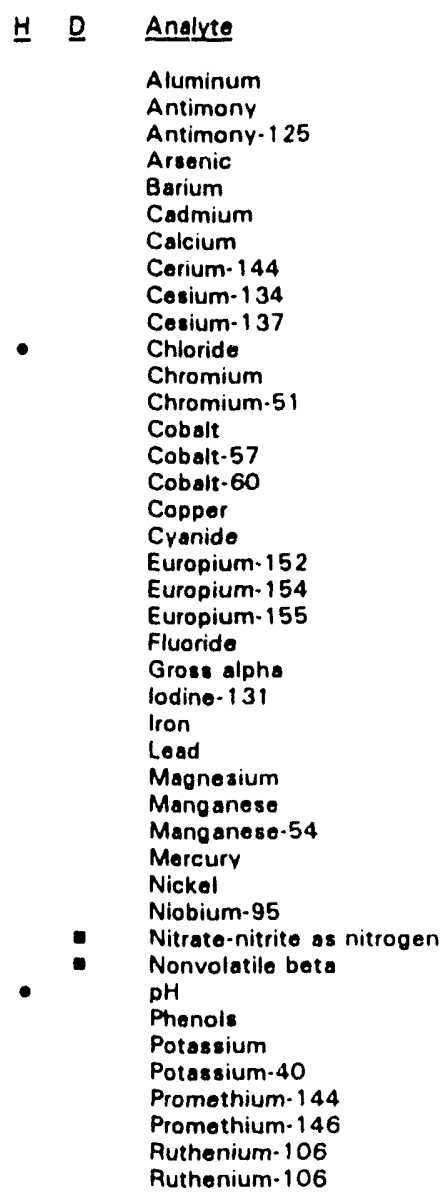

$\begin{array}{ll}\frac{1092}{50} & \underline{2092} \\ <2.0 & 57 \\ <2.0 & <2.0 \\ 75 & <2.0 \\ <2.0 & <5 \\ 13.500 & <2.0 \\ & 14.400\end{array}$

\section{2}

$\frac{4092}{241}$

86
$<20$

$<2.0 E+01$

$<2.0$

67

$<.0$
20.500

$<6.0 E+01$

$<1.0 E+01$

$<1.0 E+01$

$\begin{array}{ll}4.220 & 3.810 \\ <40 & <40 \\ <40 & <40\end{array}$

3.800

$<40$

$<12 E+02$

$<4.0$

$<1 . O E+01$

$<1.0 E+01$

$<4.0$

$<4.0$
$<50$

$<4.0$

$<5.0$

$<4.0 E+01$

$<2.0 E+01$

$<3 . \mathrm{OE}+0$

11

111
7. $3 E+00$

104

3. $1 E+00$

$<2.0 E+01$

$<4.0$

$<30$

4.450

$<$ (

$<2 . \mathrm{OE}+01$
$<40$

$<40$
$<30$

2.280

8.6

$<1.0 E+01$

$<0.20$
5.5

4. 380

$<0.20$

$<0.20$

$<4.0$

$<1.5 E+01$

$41.500 \quad 44.200 \quad 37.600$

$1.5 \mathrm{E}+02$ 8. $7 E+01$

6.2

5. $2 E+01$

10

7.230

$<1 . \mathrm{IE}+02$

$<1.0 E+01$

$<1 . O E+01$

$<1.3 E+02$

$<2.0$

$<2.0 E+01$

$<2.0$

69

$<20$

15.900

$<6.0 E+01$

$<1.0 E+01$

$<1 . O E+01$

4.130

$<40$

$<4.0$

$<1 O E+O 1$

$<1.0 E+01$

$<4.0$

$<5.0$

$<4.0 E+01$

$<2.0 E+01$

$<3.0 E+01$

118

1. $2 E+01$

160

$<30$

3.960

$<1$. OE + 01

$<0.20$

$<0.20$
$<4.0$

Ruthenium-1

38.400

1. $1 \mathrm{E}+02$

9.2

4.400

$<1.1 E+02$

$<1.0 E+01$

$<1 . O E+01$

Mod

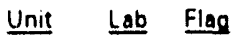

$\mu \mathrm{g} / \mathrm{L}$ GE 2

$\mu \mathrm{g} / \mathrm{L} \quad \mathrm{GE} \quad \mathrm{O}$

PCi/L GP 0

$\mu \mathrm{g} / \mathrm{L} \quad \mathrm{GE} \quad \mathrm{O}$

$\mu \mathrm{g} / \mathrm{L}$

$\begin{array}{lll}\mu \mathrm{g} / \mathrm{L} & \mathrm{GE} & 0 \\ \mu \mathrm{g} / \mathrm{L} & \mathrm{GE} & 0\end{array}$

$\begin{array}{lll}\mathrm{PC} i / \mathrm{L} & \mathrm{GP} & 0 \\ \mathrm{PC} & 0\end{array}$

$P C i L$ GP

PCIN GP 0

$\mu \mathrm{g} / \mathrm{L}$ GE

pCi/L

$\mu \mathrm{g} / \mathrm{L}$ GE 0

$\rho \mathrm{Ci} / \mathrm{L}$ GP 0

PCIL GP O

$\begin{array}{lll}\mu \mathrm{g} / \mathrm{L} & \mathrm{GE} & 0 \\ \mu_{\mathrm{g} / \mathrm{L}} & \mathrm{GE} & 0\end{array}$

OCIIL GP 0

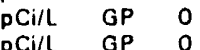

$\mu \mathrm{g} / \mathrm{L}$ GE $\quad 0$

pCi/L GE

PCi/l

$\mathrm{pCi} / \mathrm{L}$

$\mu \mathrm{G} / \mathrm{L} \quad \mathrm{GE} \quad 1$

$\mu \mathrm{g} / \mathrm{L} \quad \mathrm{GE} \quad \mathrm{O}$

$\mu g / L$ GE $\quad 0$

$\mathrm{PCi} / \mathrm{L}$ GP 0

$\mu g / L$ GE 0

$\mu \mathrm{g} / \mathrm{L} \quad \mathrm{GE} \quad 0$

pCi/L

$\mu_{0} / \mathrm{L} \quad \mathrm{GE} \quad 2$

$\mathrm{PCi} / \mathrm{G}$ GE 2

$\mathrm{pH}$

$\mu \mathrm{g} / \mathrm{L}$

$\mu g / L \quad G E-O$

pCi/L GP

$\mathrm{PCi} / \mathrm{L}$ GP $\mathrm{O}$

PCi/L GP 0

$\mathrm{pCi} / \mathrm{L}$

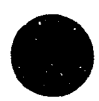

Note: Flagging levels, modifiers, and laboratories are for 4th quarter 1992 data only. See Appendix $B$ for flagging criteria - = exceeded holding time for 4th quarter 1992.

- =xceeded final primary drinkıng water standard for 4th quarter 1992. 
ANALYTICAL DATA

H D $\quad$ Analyte
Solenium
Silica
Silver
Sodium
Sodium- 22
Soecific co iductance
Sulfats
Total activity
Total alpha-emitting radium
Total dissolved solids
Total organic carbon
Total organic halogens
Total phosphates las P)
Tritium
Vanadium
Yttrium-88
Zinc
Zinc-65
Zirconium-95

$\begin{array}{ccc}1092 & \underline{2092} & \underline{3092} \\ <2.0 & <2.0 & <2.0 \\ 11.300 & 11.000 & 9.080 \\ <2.0 & <2.0 & <2.0 \\ 56.500 & 58.600 & 51.900 \\ & & <1.0 E+11 \\ 410 & 355 & 345 \\ <1.000 & <1.000 & <1.000 \\ 1.1 \mathrm{E}+07 & 7.4 \mathrm{E}+06 & 8.4 \mathrm{E}+06 \\ 2.9 \mathrm{E}+00 & 3.3 \mathrm{O}+00 & 2.3 \mathrm{E}+00 \\ 344.000 & 330.000 & 286.000 \\ <1.000 & <1.000 & 2.830 \\ 6.3 & 36 & <5.0 \\ <50 & <50 & 50 \\ 1.1 \mathrm{E}+04 & 1.0 \mathrm{O}+04 & 8.1 \mathrm{E}+03 \\ <8.0 & <8.0 & <8.0 \\ 18 & 19 & <6.0 \mathrm{O}+01 \\ & & <2.0 \\ & & <2.0 \mathrm{O}+01 \\ & & <2.0 \mathrm{O}+01\end{array}$

\begin{tabular}{|c|c|c|c|c|}
\hline 4092 & Mod & Unit & Lab & Flas \\
\hline$<2.0$ & & $\mu g / L$ & $\mathrm{GE}$ & 0 \\
\hline 8.330 & & $\mu \mathrm{g} / \mathrm{L}$ & $\mathrm{GE}$ & 0 \\
\hline$<2.0$ & & $\mu \mathrm{g} / \mathrm{L}$ & $\mathrm{GE}$ & 0 \\
\hline 58,900 & J2 & $\mu \mathrm{g} / \mathrm{L}$ & $\mathrm{GE}$ & 0 \\
\hline$<1.0 E+01$ & & $\mathrm{pCi} / \mathrm{L}$ & GP & 0 \\
\hline 380 & & $\mu \mathrm{S} / \mathrm{cm}$ & GE & 1 \\
\hline$<1,000$ & $J$ & $\mu g / L$ & GE & 0 \\
\hline $9.3 E+06$ & & $\mathrm{pCi} / \mathrm{L}$ & EM & 0 \\
\hline $4.1 E+00$ & & $\mathrm{pCi} / \mathrm{L}$ & $\mathrm{GE}$ & 1 \\
\hline 286,000 & J & $\mu \mathbf{g} / \mathrm{L}$ & GE & 0 \\
\hline 1,600 & & $\mu \mathrm{g} / \mathrm{L}$ & GE & 0 \\
\hline$<5.0$ & $J$ & $\mu_{g} / \mathrm{L}$ & $\mathrm{GE}$ & 0 \\
\hline$<50$ & & $\mu g / L$ & $\mathrm{GE}$ & 0 \\
\hline $9.0 E+03$ & & $\mathrm{pCi} / \mathrm{mL}$ & $\mathrm{GE}$ & 2 \\
\hline$<8.0$ & & $\mu g / L$ & $\mathrm{GE}$ & 0 \\
\hline$<6.0 E+01$ & & $\mathrm{pCI} / \mathrm{L}$ & GP & 0 \\
\hline 11 & & $\mu_{g} / L$ & $\mathrm{GE}$ & 0 \\
\hline$<2.0 \mathrm{E}$ & & $\begin{array}{l}\mathrm{pCi} / \mathrm{L} \\
\mathrm{pCi} / \mathrm{L}\end{array}$ & $G P$ & 0 \\
\hline
\end{tabular}

\section{WELL HSB136D}

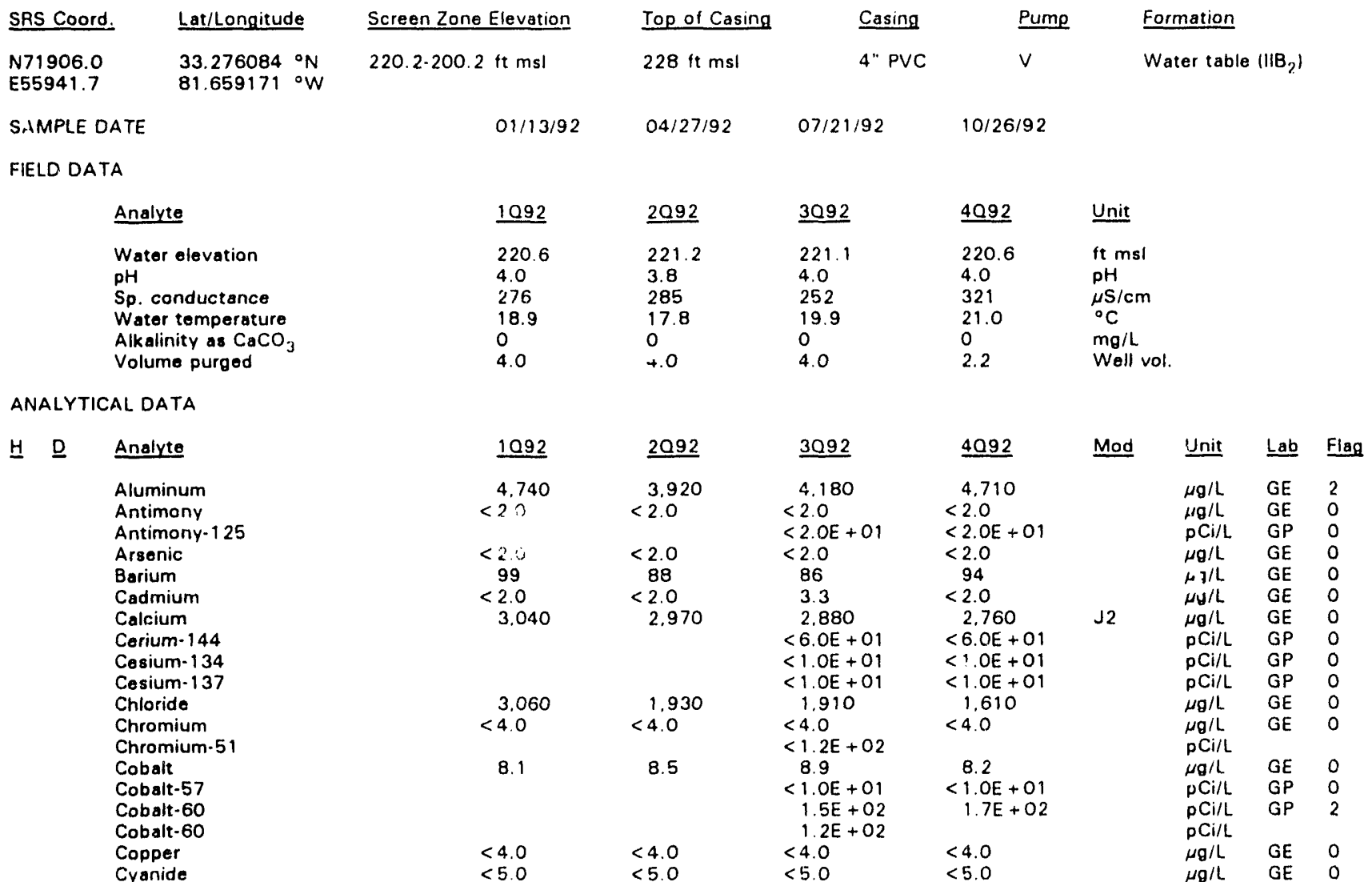

Note: Flagging levels, musifiers, and laboratories are for 4 th quarter 1992 data only. See Appendix B for flagging criteria. - =xceeded holding time for 4th quarter 1992.

- exceeded final primary drinking water standard for 4th quarter 1992. 


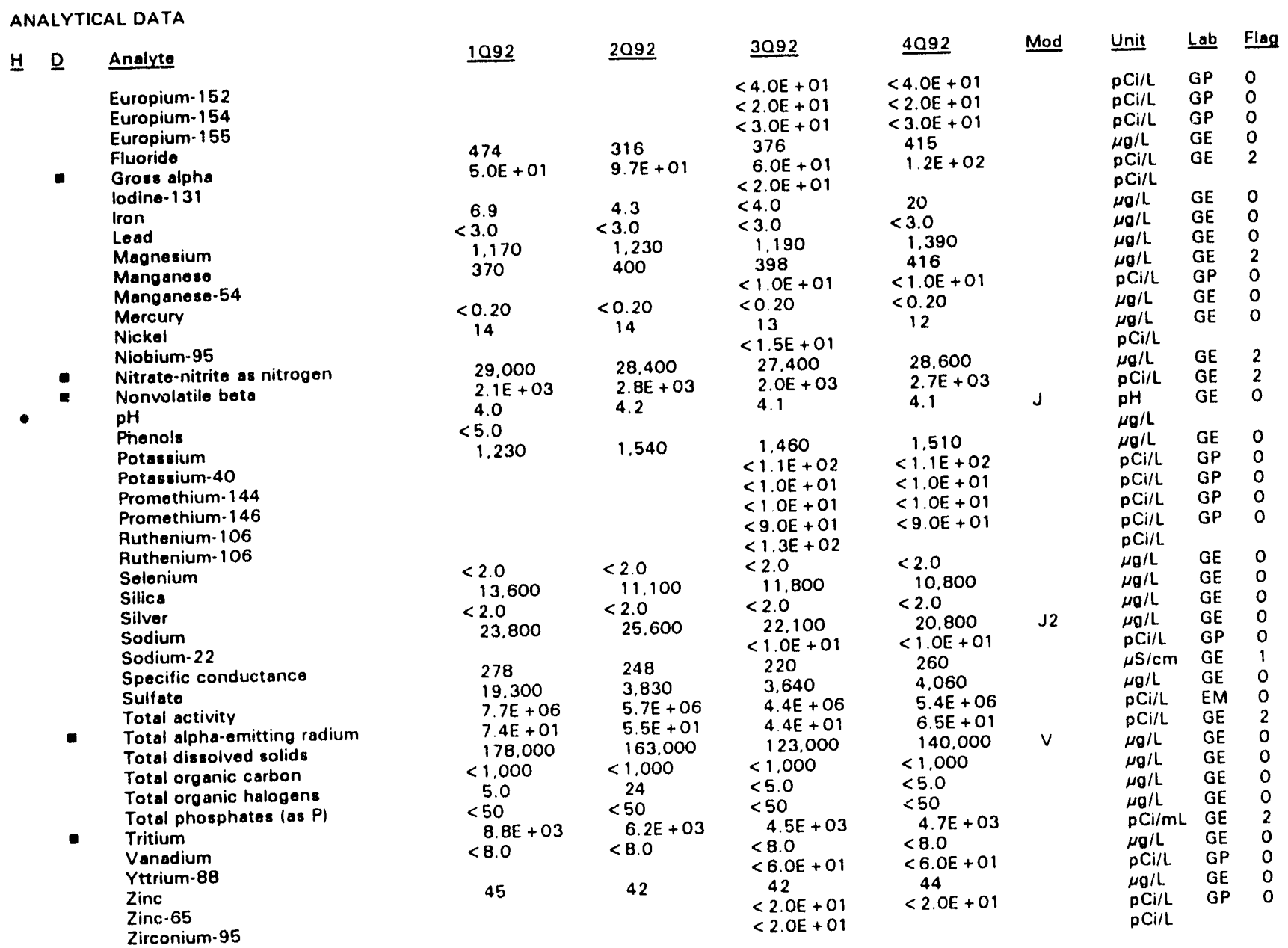

Note: Flagging levels, modifiers, and laboratories are for 4 th quarter 1992 data only. See Appendix B for flagging criteria. - exceeded holding time for 4th quarter 1992.

- =xceeded final primary drinking water standard for 4th quarter 1992. 
WSRC-TR-93-059

WELL HSB137C

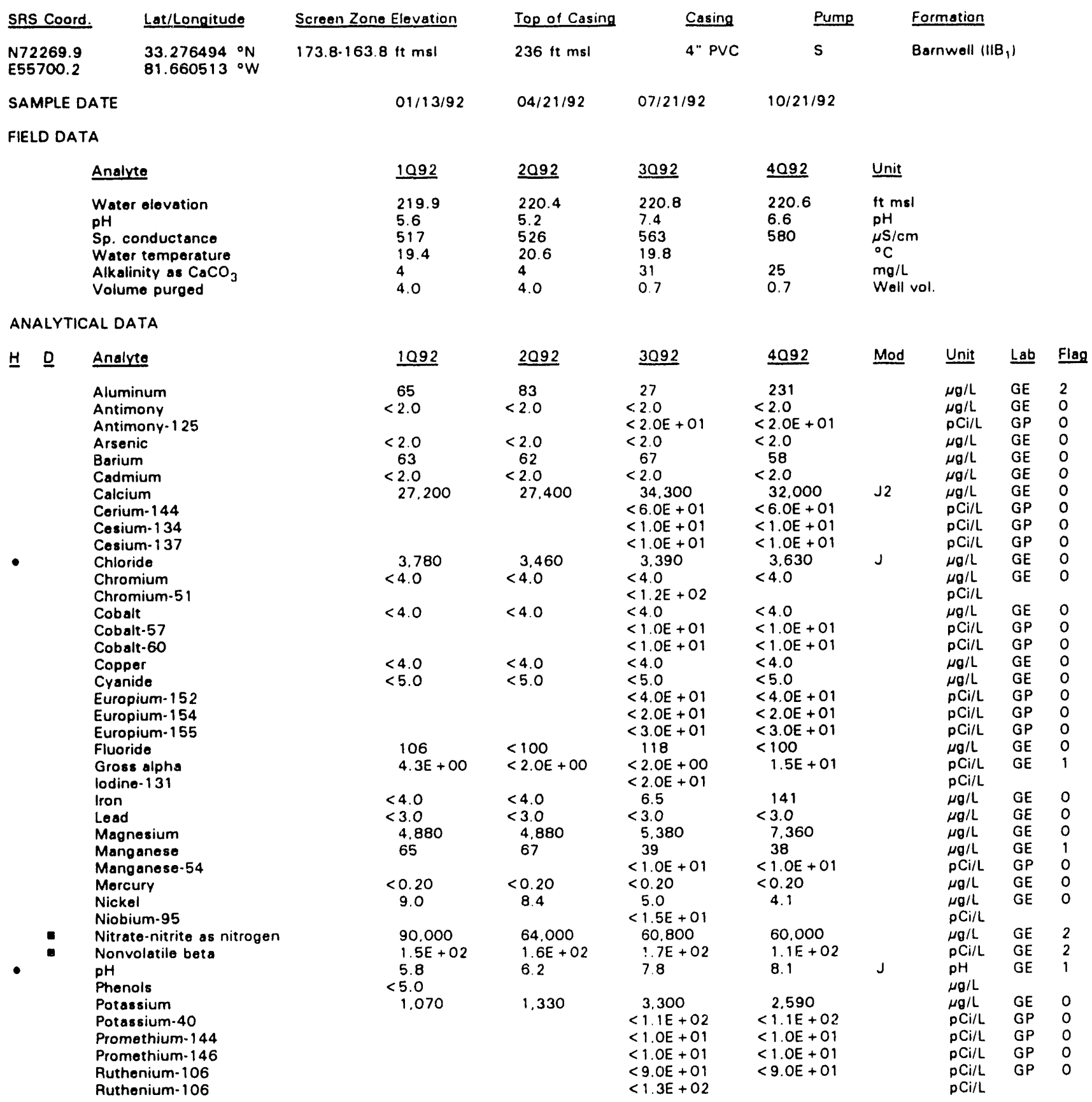

Note: Flagging levels, modifiers, and laboratories are for 4 th quarter 1992 data only. See Appendix B for flagging criteria. - = exceeded holding time for 4th quarter 1992.

- = exceeded final primary drinking water standard for 4 th quarter 1992. 
Woll HSB137C continued

ANALYTICAL DATA

H $\quad$ Analyte
Solenium
Silica
Silver
Sodium
Sodium-22
Specific conductance
Sulfate
Total activity
Total alpha-emitting radium
Total dissolved solids
Total organic carbon
Total organic halogens
Total phosphates las PI
Tritium
Vanadium
Yttrium-88
Zinc
Zinc-65
Zirconium-95

$\begin{array}{lll}1092 & \underline{2092} & \underline{3092} \\ <2.0 & <2.0 & <2.0 \\ 11.200 & 11.100 & 11.200 \\ <2.0 & <2.0 & <2.0 \\ 60.500 & 63.200 & 68.400 \\ & & <1.0 \mathrm{E}+01 \\ 490 & 420 & 480 \\ 5.680 & 4.030 & 4.760 \\ 1.3 \mathrm{E}+07 & 1.2 \mathrm{E}+07 & 1.2 \mathrm{E}+07 \\ 1.6 \mathrm{E}+00 & 2.0 \mathrm{E}+00 & 1.6 \mathrm{E}+00 \\ 421.000 & 450.000 & 412.000 \\ <1.000 & <1.000 & 5.270 \\ <5.0 & <5.0 & <5.0 \\ <50 & <50 & 100 \\ 1.2 \mathrm{E}+04 & 1.2 \mathrm{E}+04 & 1.2 \mathrm{E}+04 \\ <8.0 & <8.0 & <8.0 \\ & & <6.0 \mathrm{E}+01 \\ 39 & 43 & 18 \\ & & <2.0 \mathrm{E}+01 \\ & & <2.0 \mathrm{E}+01\end{array}$

\begin{tabular}{|c|c|c|c|c|}
\hline 4092 & Mod & Unit & $\underline{\text { Lab }}$ & Flag \\
\hline$<2.0$ & & $\mu g / L$ & GE & 0 \\
\hline 12,700 & & $\mu \mathrm{g} / \mathrm{L}$ & GE & 0 \\
\hline$<2.0$ & & $\mu \mathrm{g} / \mathrm{L}$ & $\mathrm{GE}$ & 0 \\
\hline 72.800 & $\sqrt{ } 2$ & $\mu \mathrm{g} / \mathrm{L}$ & $\mathrm{GE}$ & 0 \\
\hline$<1.0 E+01$ & & $\mathrm{pCi} / \mathrm{L}$ & GP & 0 \\
\hline 600 & & $\mu \mathrm{S} / \mathrm{cm}$ & GE & 2 \\
\hline 4,670 & $J$ & $\mu \mathrm{g} / \mathrm{L}$ & GE & 0 \\
\hline $1.2 E+07$ & & $\mathrm{pCi} / \mathrm{L}$ & $E M$ & 0 \\
\hline $1.4 E+00$ & & $\mathrm{pCi} / \mathrm{L}$ & $\mathrm{GE}$ & 0 \\
\hline 440,000 & $J$ & $\mu g / L$ & GE & 0 \\
\hline 1,590 & & $\mu \mathrm{g} / \mathrm{L}$ & $\overline{G E}$ & 0 \\
\hline$<5.0$ & $J$ & $\mu \mathrm{g} / \mathrm{L}$ & GE & 0 \\
\hline 70 & & $\mu \mathrm{g} / \mathrm{L}$ & $G E$ & 0 \\
\hline 1. $2 E+04$ & & $\mathrm{pCi} / \mathrm{mL}$ & G & 2 \\
\hline$<8.0$ & & $\mu g / h$ & G & 0 \\
\hline$<6.0 E+01$ & & $\mathrm{pCi} / \mathrm{L}$ & $\mathrm{G}$ & 0 \\
\hline 17 & & $\mu g / L$ & GE & 0 \\
\hline$<2.0 E+$ & & $\begin{array}{l}p \mathrm{Ci} / \mathrm{L} \\
\mathrm{pCi} / \mathrm{L}\end{array}$ & & 0 \\
\hline
\end{tabular}

WELL HSB137D

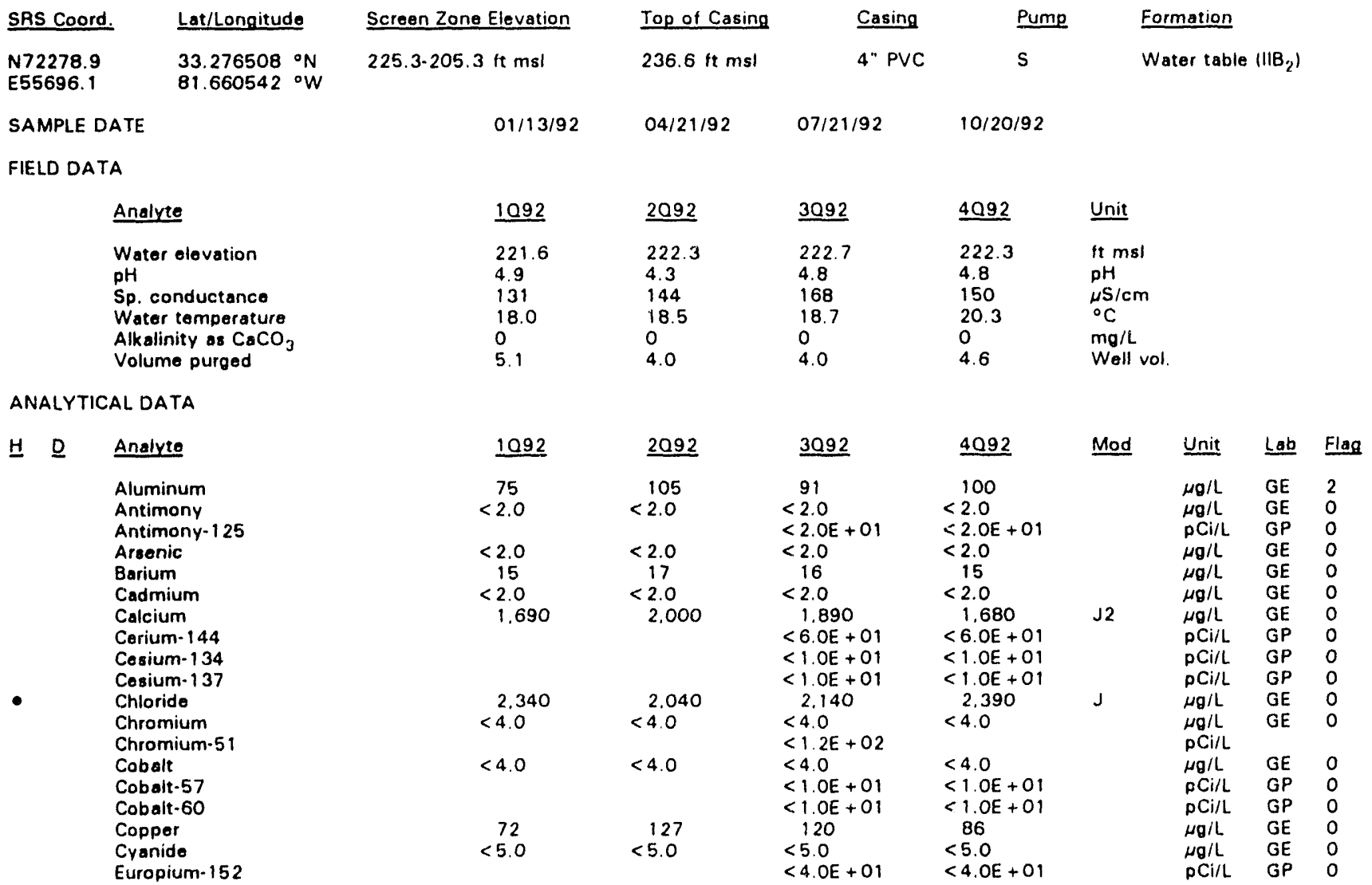

Note: Flagging levels, modifiers, and laboratories are for 4 th quarter 1992 data only. See Appendix B for flagging criteria.

- exceeded holding time for 4 in quarter 1992.

- exceeded final primary drinking water standard for 4th quarter 1992.

H-Area Seepage Basins

D.156
Fourth Quarter 1992 
ANALYTICAL DATA

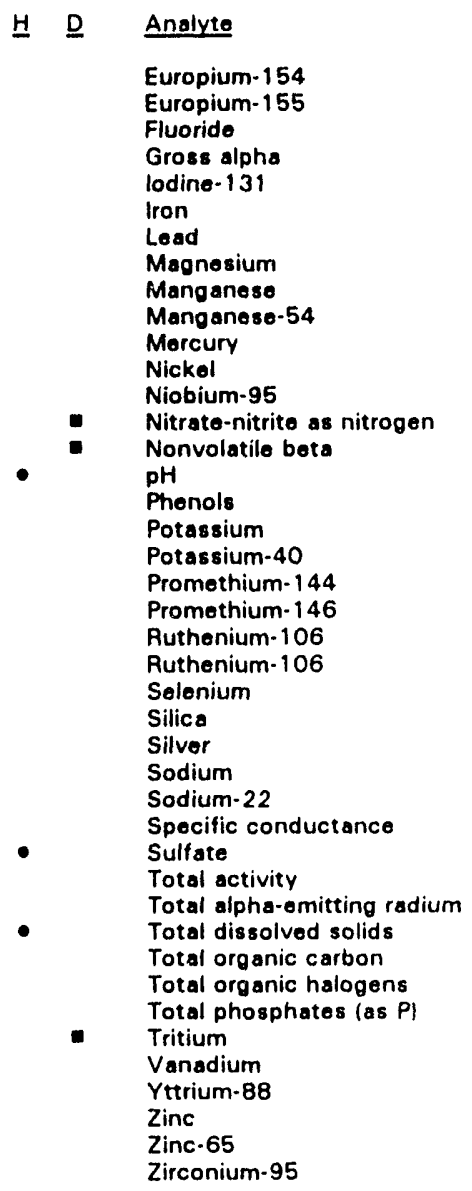

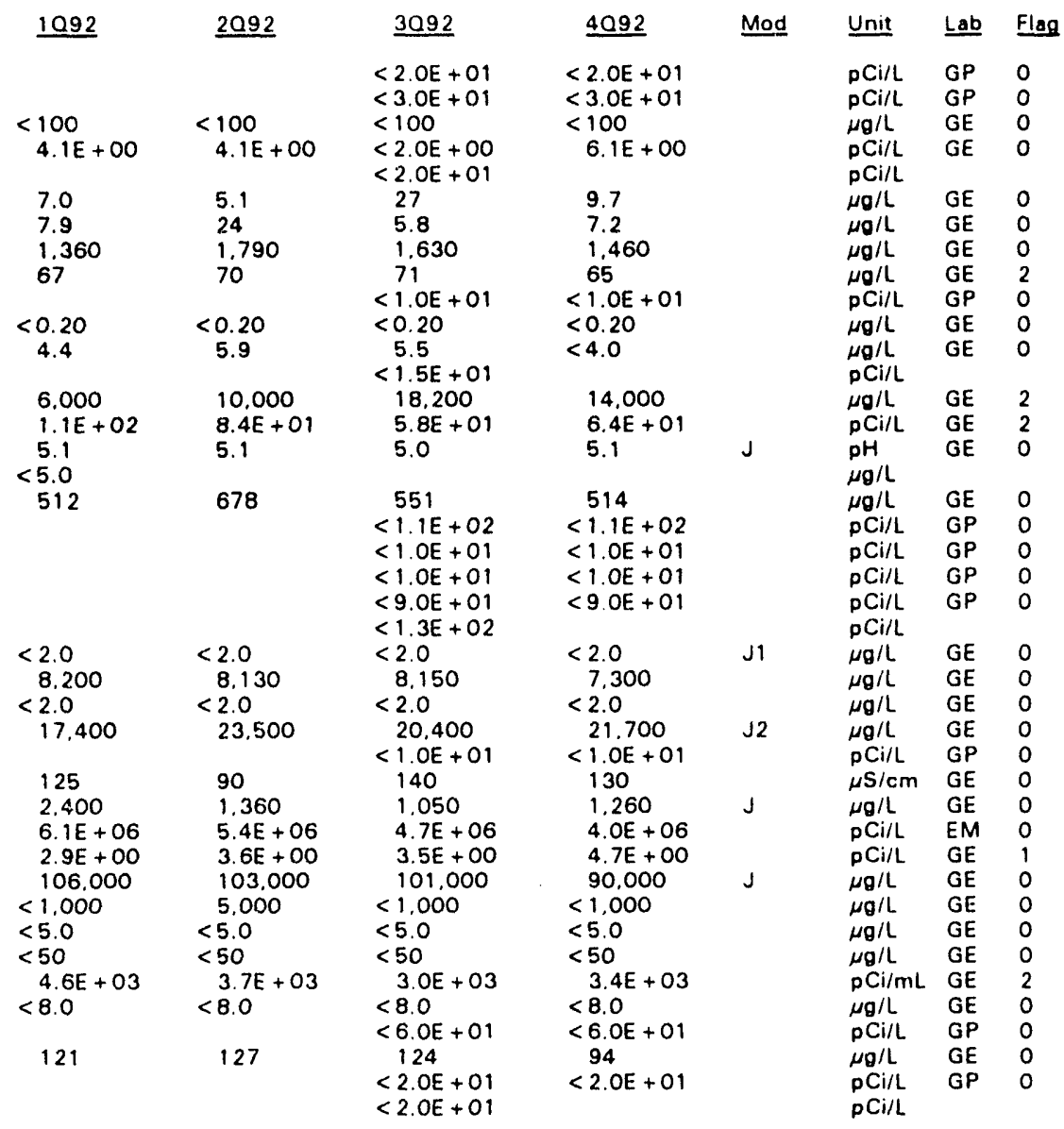

Note: Flagging levels, modifiers, and laboratories are for 4th quarter 1992 data only. See Appendix B for flagging criteria.

- = exceeded holding time for 4th quarter 1992.

- =xceeded final primary drinking water standard for 4th quarter 1992. 
WSRC-TR-93-059

WELL HSB138D

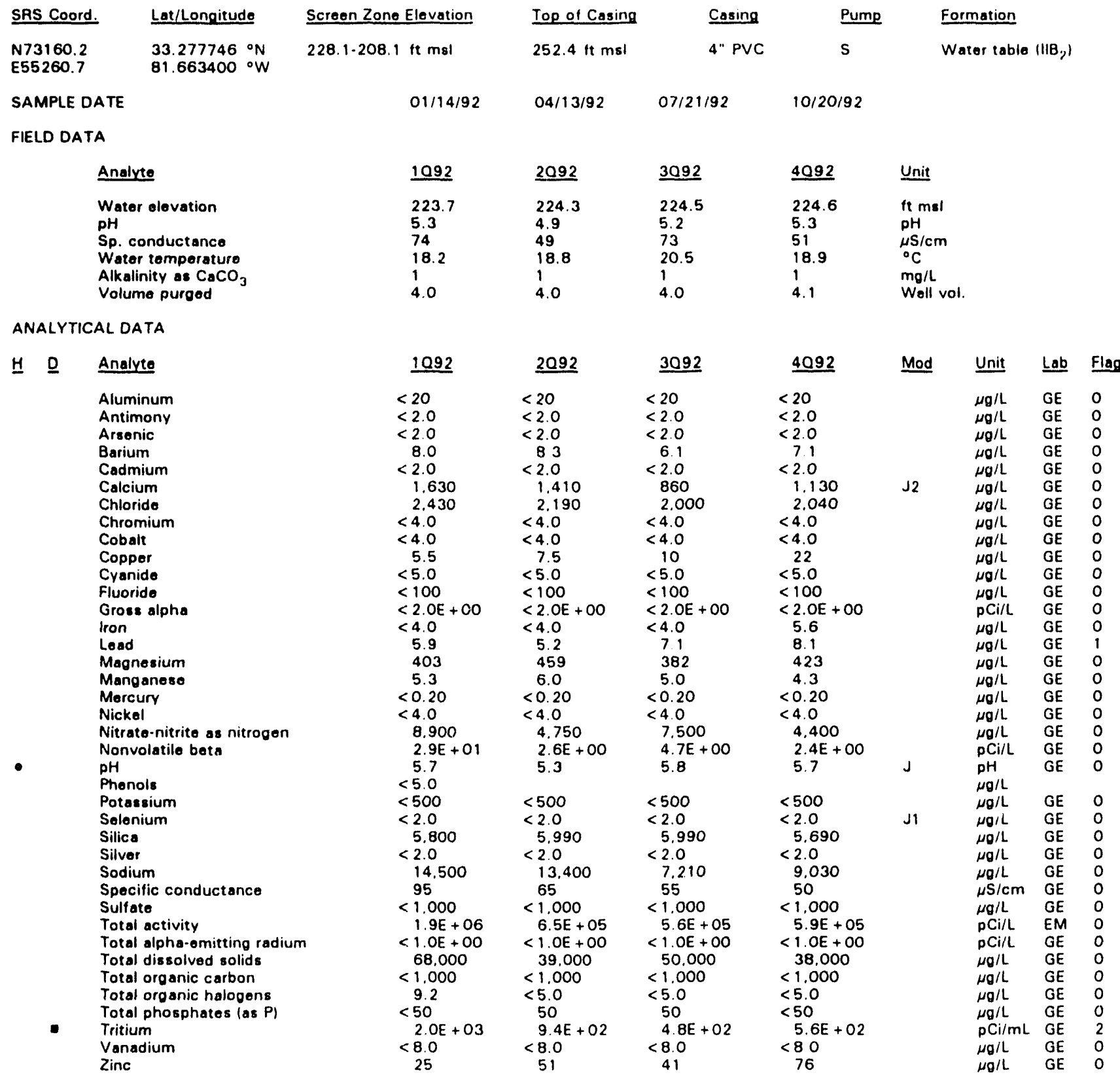

Note: Flagging levels, modifiers, and laboratories are for 4th quarter 1992 data only. See Appendix B for flagging criteria.

- =xceeded holding time for 4 th quarter 1992.

- =xceeded final primary drinking water standard for 4 th quarter 1992.

H-Area Seepage Basins

D. 158

Fourth Quarter 1992 
WSRC-TR-93-059

WELL HSB139A

\begin{tabular}{|c|c|c|c|c|c|c|}
\hline SRS Coord. & Lat/Longitude & Screen Zone Elovation & Top of Casing & Casing & Pump & Formation \\
\hline $\begin{array}{l}N 71127.4 \\
E 57365.4\end{array}$ & $\begin{array}{l}33.2766844^{\circ} \mathrm{N} \\
81.653910^{\circ} \mathrm{W}\end{array}$ & $97.6 \cdot 87.6 \mathrm{ft} \mathrm{msl}$ & $233.7 \mathrm{ft} \mathrm{msl}$ & & $\mathbf{s}$ & $U$. Congaree ||$|A|$ \\
\hline \multicolumn{2}{|c|}{ SAMPLE DATE } & $02 / 11 / 92$ & $04 / 23 / 92$ & $07 / 22 / 92$ & $10 / 18 / 92$ & \\
\hline \multicolumn{7}{|c|}{ FIELD DATA } \\
\hline \multicolumn{2}{|c|}{ Analyte } & 1092 & $\underline{2092}$ & 3092 & 4092 & $\underline{\text { Unit }}$ \\
\hline \multicolumn{2}{|c|}{$\begin{array}{l}\text { Water elevation } \\
\text { pH } \\
\text { Sp. conductance } \\
\text { Water temperature } \\
\text { Alkalinity as } \mathrm{CaCO}_{3} \\
\text { Volume purged }\end{array}$} & $\begin{array}{l}174.1 \\
8.0 \\
223 \\
17.4 \\
93 \\
4.0\end{array}$ & $\begin{array}{l}174.4 \\
7.6 \\
229 \\
19.0 \\
102 \\
4.0\end{array}$ & $\begin{array}{l}173.8 \\
7.4 \\
227 \\
19.3 \\
85 \\
4.0\end{array}$ & $\begin{array}{l}173.9 \\
7.6 \\
229 \\
19.0 \\
92 \\
3.0\end{array}$ & $\begin{array}{l}\text { ft msl } \\
\mathrm{pH} \\
\mu \mathrm{S} / \mathrm{cm} \\
{ }^{\circ} \mathrm{C} \\
\mathrm{mg} / \mathrm{h} \\
\text { Well vol. }\end{array}$ \\
\hline
\end{tabular}

ANALYTICAL DATA

H D Analyte

Aluminum

Antimony

Arsenic

Barium

Cadmium

Calcium

Chloride

Chromium

Cobalt

Copper

Cyanido

Fluoride

Gross alpha

Iron

Load

Magnesium

Manganese

Mercury

Nickol

Nitrate as nitrogen

Nitrate-nitrite as nitrogen

Nonvolatile beta

pH

Phenols

Potassium

Solenium

Silica

Silver

Sodium

Specific conductance

Sulfate

Total alpha-emitting radium

Total dissolved solids

Total organic carbon

Total organic halogen

Total phosphates (as P)

Tritium

Vanadium

Zinc

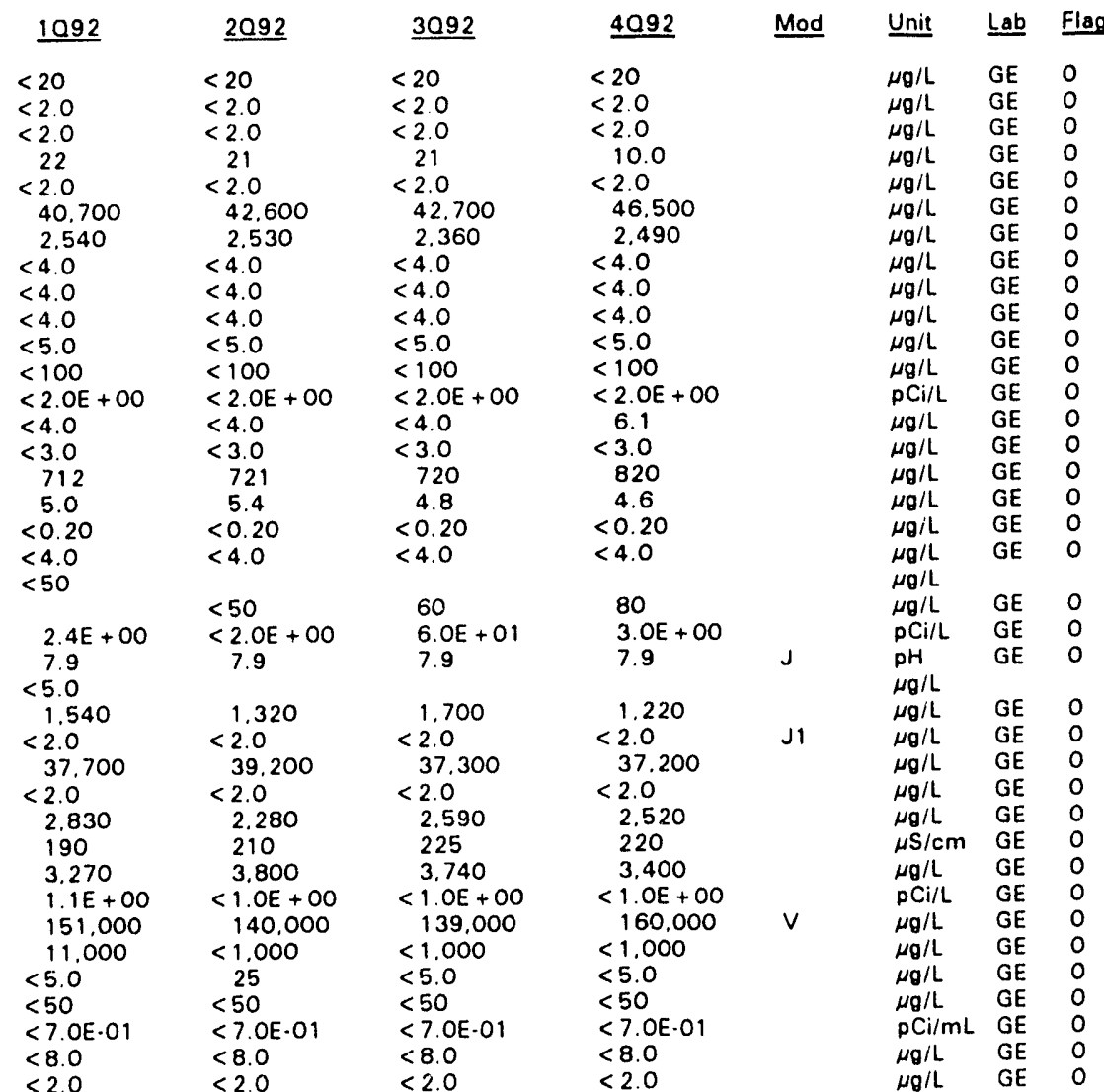

Note: Flagging levels, modifiers, and laboratories are for 4 th quarter 1992 data only. See Appendix B for flagging criteria.

- = exceeded holding time for 4th quarter 1992

- =xceeded final primary drinking water standard for 4th quarter 1992. 
WSRC-TR-93.059

WELL HSB139C

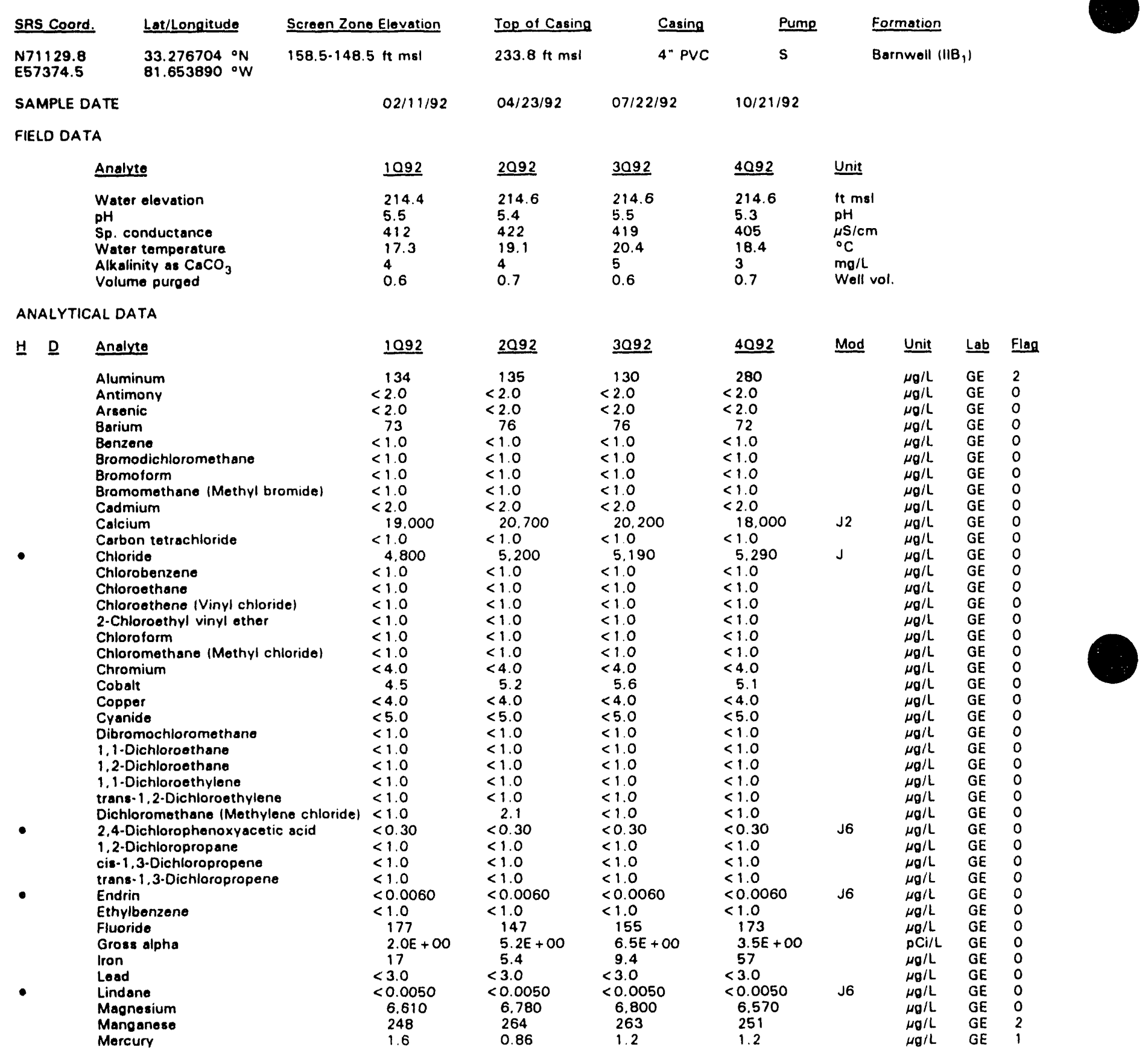

Note: Flagging levels, modifiers, and laboratories are for 4 th quarter 1992 data only. See Appendix 8 for flagging criteria.

- = exceeded holding time for 4th quarter 1992 .

- =xceeded final primary drinking water standard for 4th quarter 1992. 


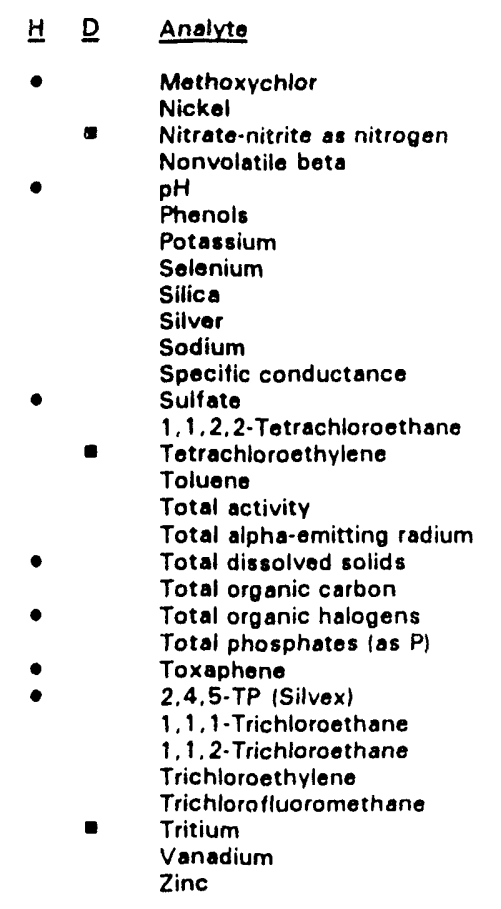

$\begin{array}{lll}1092 & \underline{2092} & \underline{3092} \\ <0.50 & <0.50 & <0.50 \\ 11 & 12 & 8.0 \\ 43.000 & 50.000 & 48.800 \\ 3.7 \mathrm{E}+01 & 4.0 \mathrm{E}+01 & 3.4 \mathrm{E}+01 \\ 5.5 & 5.6 & 5.6 \\ <5.0 & <5.0 & <5.0 \\ 1.490 & 1.780 & 1.570 \\ <2.0 & <2.0 & <2.0 \\ 10.800 & 10.900 & 11.100 \\ <2.0 & <2.0 & <2.0 \\ 43.700 & 44.500 & 44.400 \\ 335 & 340 & 360 \\ <1.000 & <1.000 & <1.000 \\ <1.0 & <1.0 & <1.0 \\ 9.9 & 8.8 & 8.8 \\ <1.0 & <1.0 & <1.0 \\ 3.5 \mathrm{E}+06 & 3.3 \mathrm{E}+06 & 3.1 \mathrm{E}+06 \\ 1.2 \mathrm{E}+00 & 1.3 \mathrm{E}+00 & 1.5 \mathrm{E}+00 \\ 316.000 & 348.000 & 302.000 \\ <1.000 & <1.000 & <1.000 \\ 15 & 5.8 & 8.5 \\ 150 & 200 & 160 \\ <0.24 & <0.24 & <0.24 \\ <0.090 & <0.090 & <0.090 \\ <1.0 & <1.0 & <1.0 \\ <1.0 & <1.0 & <1.0 \\ 1.7 & 1.4 & 1.6 \\ <1.0 & 2.4 & <1.0 \\ 3.4 \mathrm{E}+03 & 3.3 \mathrm{E}+03 & 3.0 \mathrm{E}+03 \\ <8.0 & <8.0 & <8.0 \\ 46 & 48 & 49 \\ & & \end{array}$

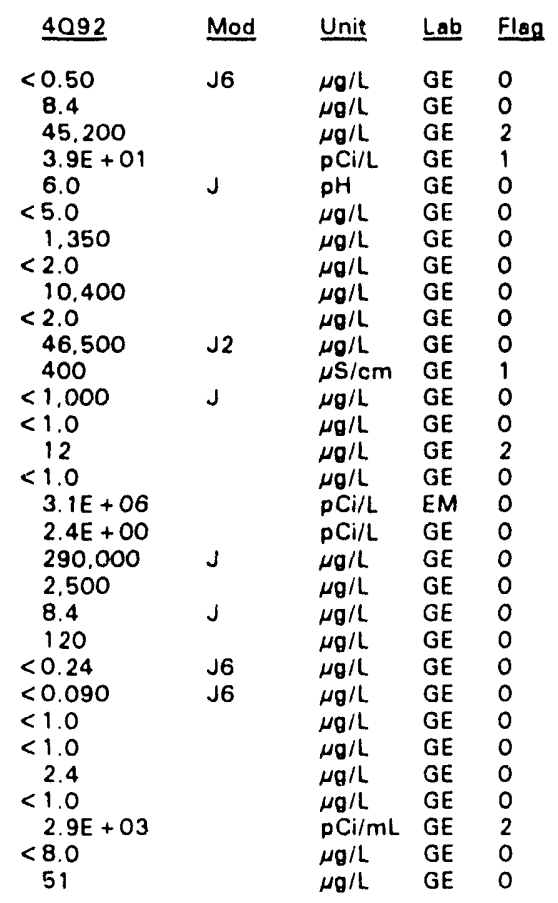

\section{WELL HSB139D}

$\begin{array}{ll}\text { SRS Coord. } & \text { Lat/Longitude } \\ \text { N71133.2 } & 33.276728 \circ \mathrm{N}\end{array}$ E57384.4

SAMPLE DATE $81.653871^{\circ} \mathrm{W}$

Screen Zone Elevation

226.7.206. $7 \mathrm{ft} \mathrm{msl}$

$02 / 11 / 92$
Top of Casing

$233.8 \mathrm{ft} \mathrm{msl}$

$04 / 23 / 92$

\begin{tabular}{|c|c|}
\hline 1092 & 2092 \\
\hline 222.6 & 224.5 \\
\hline $\begin{array}{l}4.9 \\
29\end{array}$ & $\begin{array}{l}4.8 \\
39\end{array}$ \\
\hline 17.6 & 17.7 \\
\hline 0 & 0 \\
\hline 4 & 4.0 \\
\hline
\end{tabular}

\section{2}

39
7.1
$<2.0$
9.7
0.40
1.150
3.720

2092
41
$<2.0$
$<2.0$
11
$<2.0$
1.550
3.050

\section{Casing \\ 4" PVC}

$07 / 22 / 92$

10/18/92

\section{Analvie}

Water elevation

pH

Sp. conductance

Water temperature

ANALYTICAL DATA

3092
223.8
4.7
32
19.7
0
4.0

4092
223.1
4.9
30
20.5
0
5.8

Unit
$\mathrm{tt} \mathrm{msl}$
$\mathrm{pH}$
$\mu \mathrm{S} / \mathrm{cm}$
${ }^{\circ} \mathrm{C}$
$\mathrm{mg} / \mathrm{L}$
Well vol.

Analyte
Aluminum
Antimony
Arsenic
Barium
Cadmium
Calcium
Chloride

H D

(2)

$\begin{aligned} & 4092 \\ & 51 \\ &< 2.0 \\ &<2.0 \\ & 11 \\ &<2.0 \\ & 1.260 \\ & 2.630\end{aligned}$

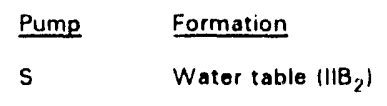

Water table $\left(11 \mathrm{~B}_{2}\right)$ 
WSRC.TR.9.3-059

Well HSB139D continued

ANALYTICAL DATA

\begin{tabular}{|c|c|}
\hline$\underline{\mathbf{D}}$ & Analyto \\
\hline - & 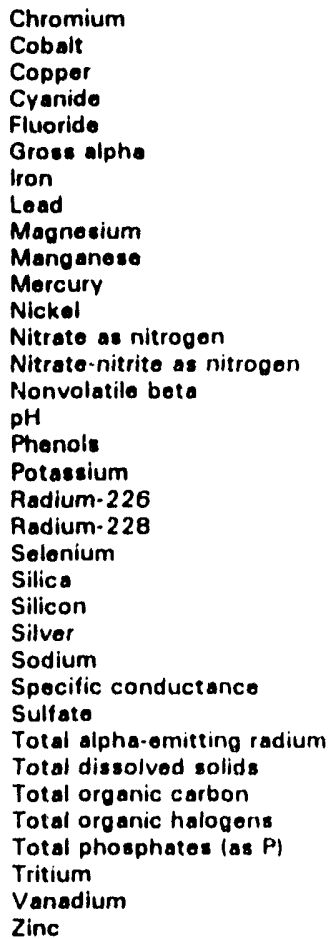 \\
\hline
\end{tabular}

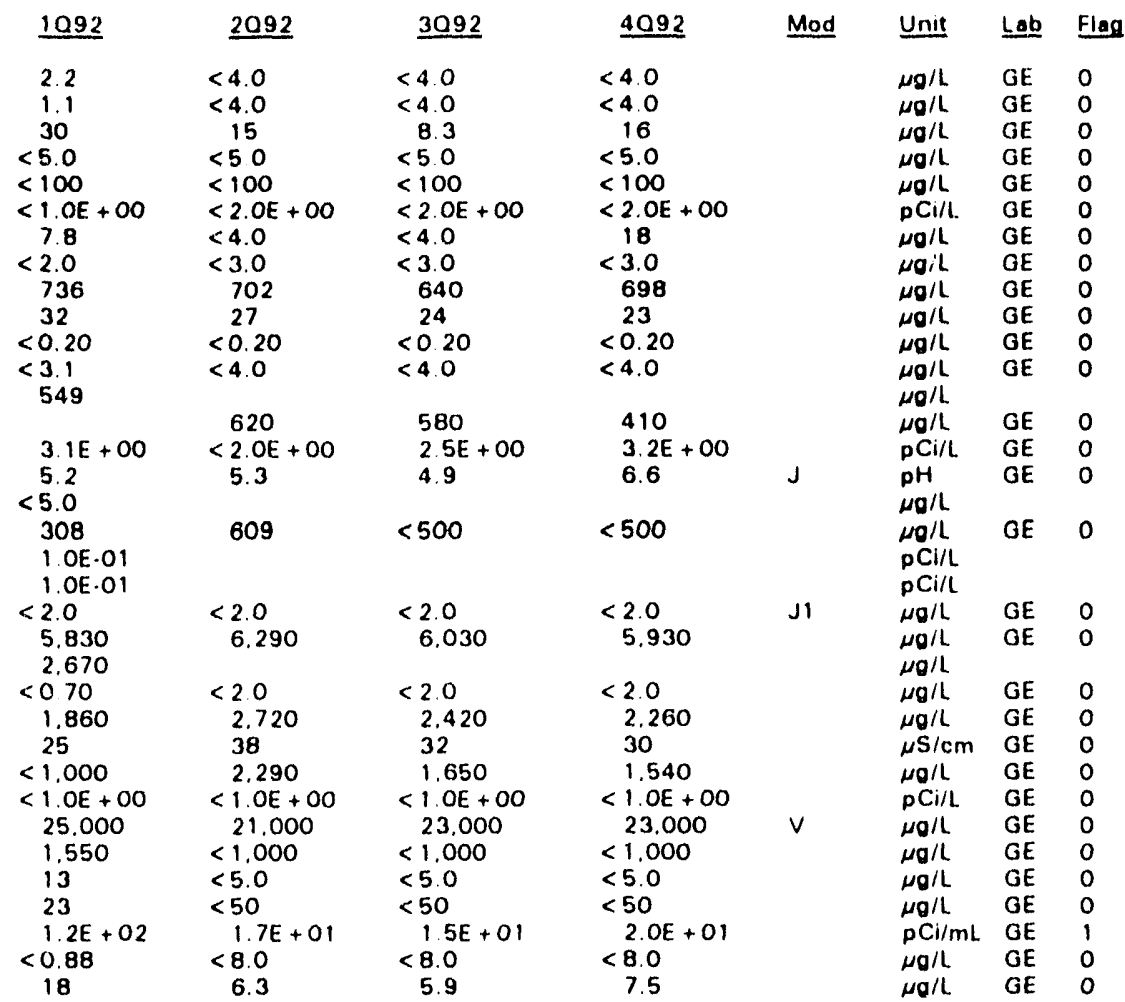

\section{WELL HSB140A}

\begin{tabular}{|c|c|c|c|c|c|c|c|c|c|}
\hline SRS Coord. & Lat/Longitude & Screen Zone Elevation & Top of Casing & Casing & Pump & & imation & & \\
\hline $\begin{array}{l}N 70050.3 \\
E 56535.4\end{array}$ & $\begin{array}{l}33.272948{ }^{\circ} \mathrm{N} \\
81.654003{ }^{\circ} \mathrm{W}\end{array}$ & $91.0 .81 .0 \mathrm{ft} \mathrm{msl}$ & $235.9 \mathrm{ft} \mathrm{msl}$ & 4" PVC & s & $u$. & Congaree & ||$|A|$ & \\
\hline \multicolumn{2}{|c|}{ SAMPLE DATE } & $01 / 15 / 92$ & $04 / 24 / 92$ & $07 / 22 / 92$ & $10 / 23 / 92$ & & & & \\
\hline \multicolumn{10}{|c|}{ FIELD DATA } \\
\hline \multicolumn{2}{|r|}{ Analyte } & 1092 & $\underline{2092}$ & 3092 & 4092 & Unit & & & \\
\hline \multicolumn{2}{|r|}{$\begin{array}{l}\text { Water elevation } \\
\text { pH } \\
\text { Sp. conductance } \\
\text { Water temperature } \\
\text { Alkalinity as } \mathrm{CaCO}_{3} \\
\text { Volume purged }\end{array}$} & $\begin{array}{l}176.1 \\
7.4 \\
176 \\
18.8 \\
61 \\
4.0\end{array}$ & $\begin{array}{l}176.2 \\
6.7 \\
160 \\
19.7 \\
61 \\
4.0\end{array}$ & $\begin{array}{l}175.6 \\
6.9 \\
162 \\
20.4 \\
57 \\
4.0\end{array}$ & $\begin{array}{l}175.5 \\
7.1 \\
161 \\
20.3 \\
59 \\
2.2\end{array}$ & $\begin{array}{l}\text { tt msl } \\
\mathrm{pH} \\
\mu \mathrm{S} / \mathrm{cm} \\
{ }^{\circ} \mathrm{C} \\
\mathrm{mg} / \mathrm{L} \\
\text { Well vol. }\end{array}$ & & & \\
\hline \multicolumn{10}{|c|}{ ANALYTICAL DATA } \\
\hline \multirow[t]{2}{*}{$\underline{H} \quad \underline{D}$} & Analyte & 1092 & $\underline{2092}$ & 3092 & 4092 & Mod & $\underline{\underline{\text { nit }}}$ & $\underline{L a b}$ & Flag \\
\hline & $\begin{array}{l}\text { Alkalinity las } \mathrm{CaCO}_{3} \text { ) } \\
\text { Aluminum } \\
\text { Antimony }\end{array}$ & $\begin{array}{r}42 \\
<20\end{array}$ & $\begin{aligned} & 34 \\
< & 2.0\end{aligned}$ & $\begin{array}{r}63 \\
26 \\
<20\end{array}$ & $\begin{array}{r}67 \\
33 \\
<2.0\end{array}$ & & $\begin{array}{l}\mathrm{mg}_{\mathrm{g}} / \mathrm{L} \\
\mu \mathrm{g} / \mathrm{L} \\
\mu \mathrm{g} / \mathrm{L}\end{array}$ & $\begin{array}{l}\mathrm{GE} \\
\mathrm{GE} \\
\mathrm{GE}\end{array}$ & $\begin{array}{l}0 \\
1 \\
0\end{array}$ \\
\hline
\end{tabular}

Note: Flagging levels, modifiers, and laboratories are for 4th quarter 1992 data only. See Appendix B for flagging criteria. - =xceeded holding time for 4th quarter 1992.

- exceeded final primary drinking water standard for 4th quarter 1992. 
Well HSB140A continued

ANALYTICAL DATA

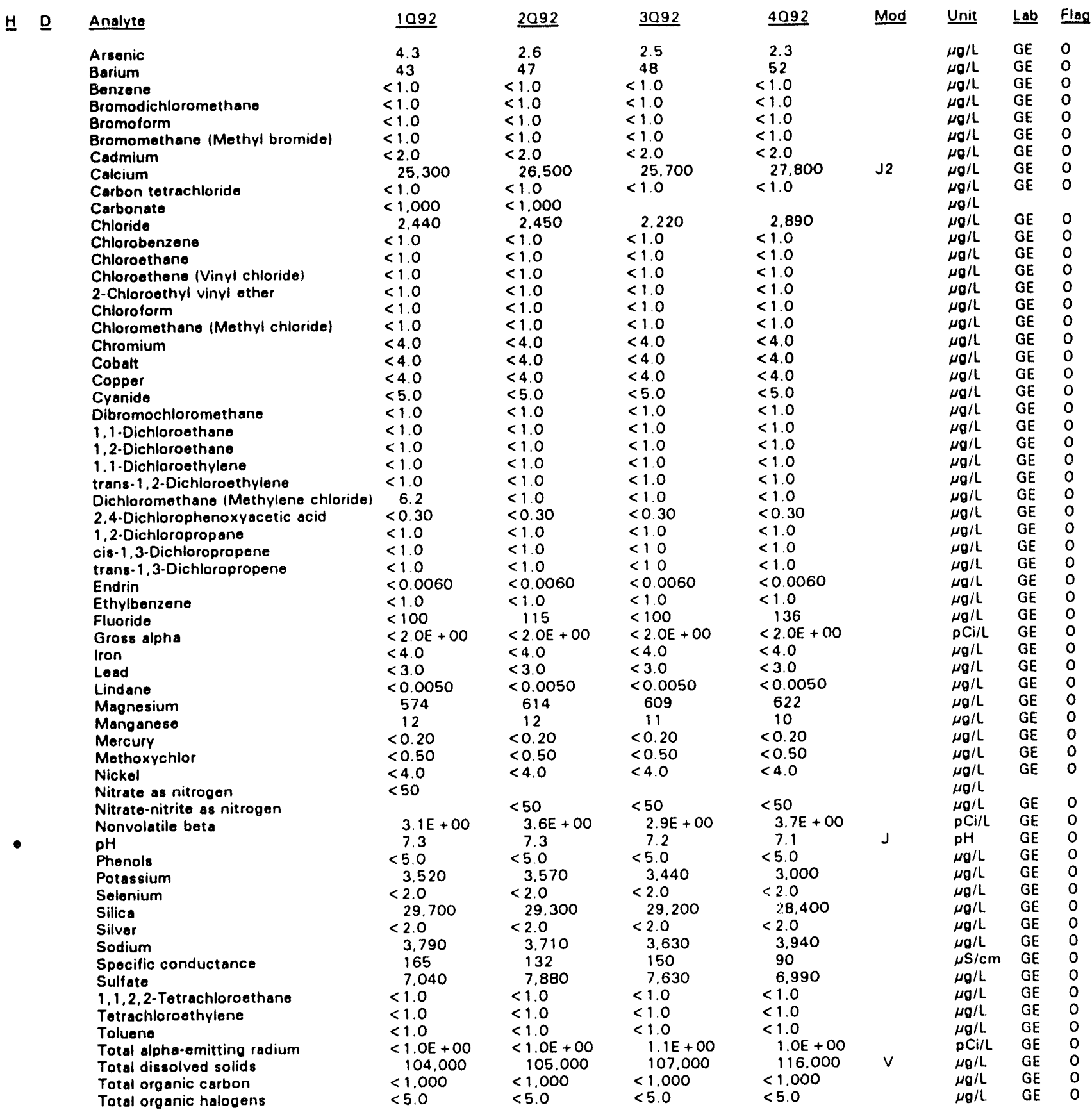

Note: Flagging levels, modifiers, and laboratories are for 4 th quarter 1992 data only. See Appendix B for flagging criteria. - = exceeded holding time for 4 th quarter 1992.

- =xceeded final primary drinking water standard for 4th quarter 1992. 
WSRC-TR-93-059

Well HSB140A continued

ANALYTICAL DATA

H D Analyte

Total phosphates (as P)

Toxaphene

2,4,5-TP (Silvex)

1,1,1-Trichloroethane

1.1,2-Trichloroethane

Trichloroethylane

Trichlorofluoromethane

Tritium

Uranium

Vanadium

Zinc

\begin{tabular}{|c|c|c|c|c|c|c|c|}
\hline 1092 & 2092 & 3092 & 40.92 & Mod & Unit & Lab & Flog \\
\hline $\begin{aligned} & 220 \\
< & 0.24 \\
< & 0.090 \\
< & 1.0 \\
< & 1.0 \\
< & 1.0 \\
< & 1.0 \\
< & 7.0 \mathrm{E} \cdot 01 \\
< & 8.0 \\
< & 2.0\end{aligned}$ & $\begin{aligned} & 190 \\
< & 0.24 \\
< & 0.090 \\
< & 1.0 \\
< & 1.0 \\
< & 1.0 \\
< & 1.0 \\
< & 7.0 \text { E. } 01 \\
& <8.0 \\
< & 2.0\end{aligned}$ & $\begin{aligned} & 300 \\
< & 0.24 \\
< & 0.090 \\
< & 1.0 \\
< & 1.0 \\
< & 1.0 \\
< & 1.0 \\
& 8.4 \mathrm{E} \cdot 01 \\
< & 20 \\
< & 8.0 \\
< & 2.0\end{aligned}$ & $\begin{aligned} & 120 \\
< & 0.24 \\
< & 0.090 \\
< & 1.0 \\
< & 1.0 \\
< & 1.0 \\
< & 1.0 \\
< & 7.0 \mathrm{E} \cdot 01 \\
< & 20 \\
< & 8.0 \\
< & 2.0\end{aligned}$ & & $\begin{array}{l}\mu g / \mathrm{L} \\
\mu g / \mathrm{L} \\
\mu g / \mathrm{L} \\
\mu g / \mathrm{L} \\
\mu g / \mathrm{L} \\
\mu g / \mathrm{L} \\
\mu g / \mathrm{L} \\
\mathrm{pCi} / \mathrm{mL} \\
\mu g / \mathrm{L} \\
\mu g / \mathrm{L} \\
\mu g / \mathrm{L}\end{array}$ & $\begin{array}{l}G E \\
G E \\
G E \\
G E \\
G E \\
G E \\
G E \\
G E \\
G E \\
G E \\
G E\end{array}$ & $\begin{array}{l}0 \\
0 \\
0 \\
0 \\
0 \\
0 \\
0 \\
0 \\
0 \\
0 \\
0\end{array}$ \\
\hline
\end{tabular}

\section{WELL HSB140C}

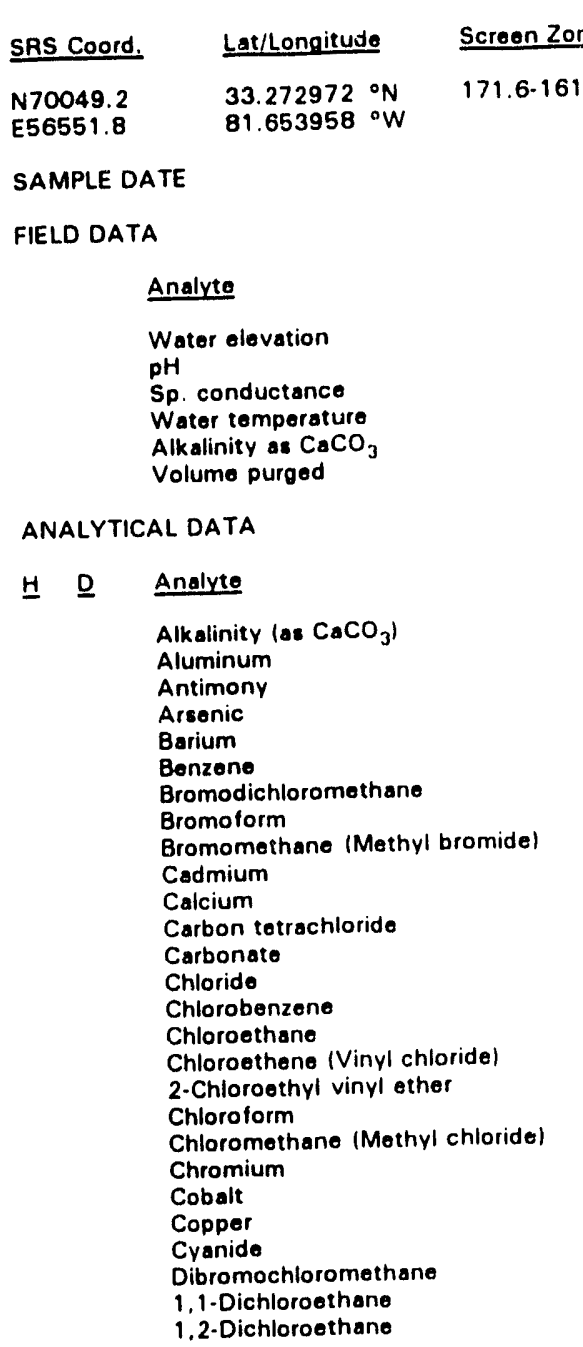

$01 / 15 / 92$

Top of Casing

$04 / 24 / 92 \quad 07 / 22 / 92 \quad 10 / 23 / 92$

$\begin{array}{lllll}1092 & \underline{2092} & \underline{3092} & \underline{4092} & \text { Unit } \\ 205.8 & 205.8 & 205.5 & 205.6 & \mathrm{ft} \mathrm{msi} \\ 5.6 & 4.9 & 5.3 & 5.3 & \mathrm{pH} \\ 26 & 24 & 25 & 24 & \mu \mathrm{S} / \mathrm{cm} \\ 17.5 & 19.1 & 20.4 & 19.4 & { }^{\circ} \mathrm{C} \\ 3 & 3 & 3 & 3 & \mathrm{mg} / \mathrm{L} \\ 4.0 & 4.0 & 4.0 & 2.8 & \text { Well vol. }\end{array}$

1092

$<20$

$<2.0$

$<.0$
7.6

$<1.0$

$<1.0$

$<1.0$

$<10$

$<2.0$

$<2.0$

$<1.0$

$<1.000$

2.030

$<1.0$

$<1.0$

$<1.0$

$<1.0$

$<10$

$<10$

$<4.0$

$<4.0$

$<4.0$
$<4.0$

$<5.0$

$<1.0$

$<1.0$

$<1.0$
2092

$<20$

$<2.0$

$<2.0$

8.5
$<1.0$

$<1.0$

$<1.0$

$<1.0$

$<1.0$

$<2.0$

1.760

$<1.0$

$<1,000$

1.980

$<1.0$

$<1.0$

$<1.0$

$<1.0$

$<1.0$

$<1.0$

$<4.0$

$<4.0$

$<4.0$

$<5.0$

$<1.0$

$<1.0$

$<1.0$
3092

5.5
$<15$

$<15$
4.3

4.3
$<2.0$

11

$<1.0$

$<1.0$

$<1.0$

$<1.0$

1.950

$<1.0$

1.930

$<1.0$

$<1.0$

$<1.0$

$<1.0$

$<1.0$

$<1.0$

3.9

$<0.8$

1.3
$<5.0$

$<5.0$
$<1.0$

$<1.0$

$<1.0$

$\begin{array}{lll}\text { Casing } & \text { Pump } & \text { Formation } \\ 4^{n} \text { PVC } & S & \text { Barnwell }\left(118_{1}\right)\end{array}$

1.1-Dichloroethane

$\begin{aligned} & 4092 \\ & 6.1 \\ &< 20 \\ &< 2.0 \\ &< 2.0 \\ & 7.3 \\ &< 1.0 \\ &< 1.0 \\ &< 1.0 \\ &< 1.0 \\ &< 2.0 \\ & 1.750 \\ &< 1.0 \\ & \\ & 1.680 \\ &< 1.0 \\ &< 1.0 \\ &<1.0 \\ &<1.0 \\ &<1.0 \\ &<1.0 \\ &<4.0 \\ &<4.0 \\ &<4.0 \\ &<5.0 \\ &<1.0 \\ &<1.0 \\ &<1.0\end{aligned}$

Mod

\begin{tabular}{|c|c|}
\hline Unit & Lav \\
\hline $\mathrm{mg} / \mathrm{l}$ & $\mathrm{GE}$ \\
\hline$\mu g / L$ & $\mathrm{GE}$ \\
\hline$\mu \mathrm{g} / \mathrm{L}$ & GE \\
\hline$\mu g / L$ & GE \\
\hline$\mu_{\theta} / L$ & GE \\
\hline$\mu \mathrm{g} / \mathrm{L}$ & GE \\
\hline$\mu g / L$ & GE \\
\hline$\mu \mathrm{g} / \mathrm{L}$ & GE \\
\hline$\mu g / L$ & GE \\
\hline$\mu \mathrm{g} / \mathrm{L}$ & GE \\
\hline $\begin{array}{c}\mu g / L \\
\mu g / L\end{array}$ & $\begin{array}{l}\mathrm{GE} \\
\mathrm{GE}\end{array}$ \\
\hline$\mu g / L$ & \\
\hline$\mu \mathrm{g} / \mathrm{L}$ & GE \\
\hline$\mu \boldsymbol{g} / L$ & GE \\
\hline$\mu \boldsymbol{\sigma} / \mathrm{L}$ & GE \\
\hline$\mu \mathrm{g} / \mathrm{L}$ & $\mathrm{GE}$ \\
\hline$\mu g / L$ & $\mathrm{GE}$ \\
\hline$\mu g / L$ & $\mathrm{GE}$ \\
\hline$\mu \mathrm{g} / \mathrm{L}$ & $\mathrm{GE}$ \\
\hline$\mu \mathrm{g} / \mathrm{L}$ & $\mathrm{GE}$ \\
\hline$\mu \mathrm{g} / \mathrm{L}$ & GE \\
\hline$\mu \mathrm{g} / \mathrm{L}$ & GE \\
\hline$\mu g / L$ & GE \\
\hline$\mu g / L$ & GE \\
\hline
\end{tabular}

Note: Flagging levels, modifiers, and laboratories are for 4 th quarter 1992 data only. See Appendix B for flagging criteria.

- =xceeded holding time for 4th quarter 1992.

= exceeded final primary drinking water standard for 4th quarter 1992.

H-Area Seepage Basins

D. 164

Fourth Quarter 1992 
Well HSB140C continued

ANALYTICAL DATA

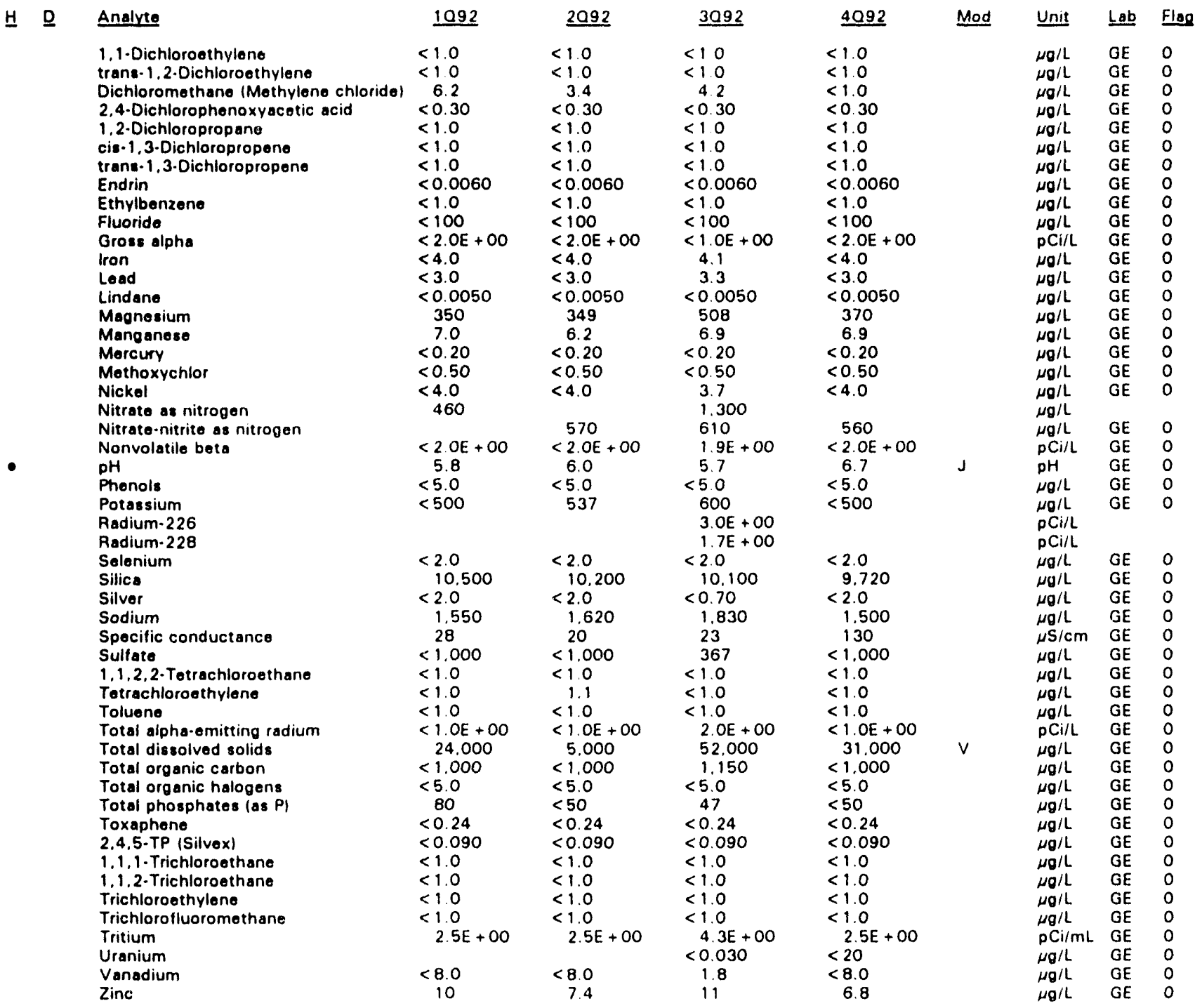

Note: Flagging levels, modifiers, and laboratories are for 4th quarter 1992 data only. See Appendix B for flagging criteria.

- =xceeded holding time for 4 th quarter 1992.

- exceeded final primary drinking water standard for 4th quarter 1992. 
WSRC.TR.93.059

WELL HSB140D

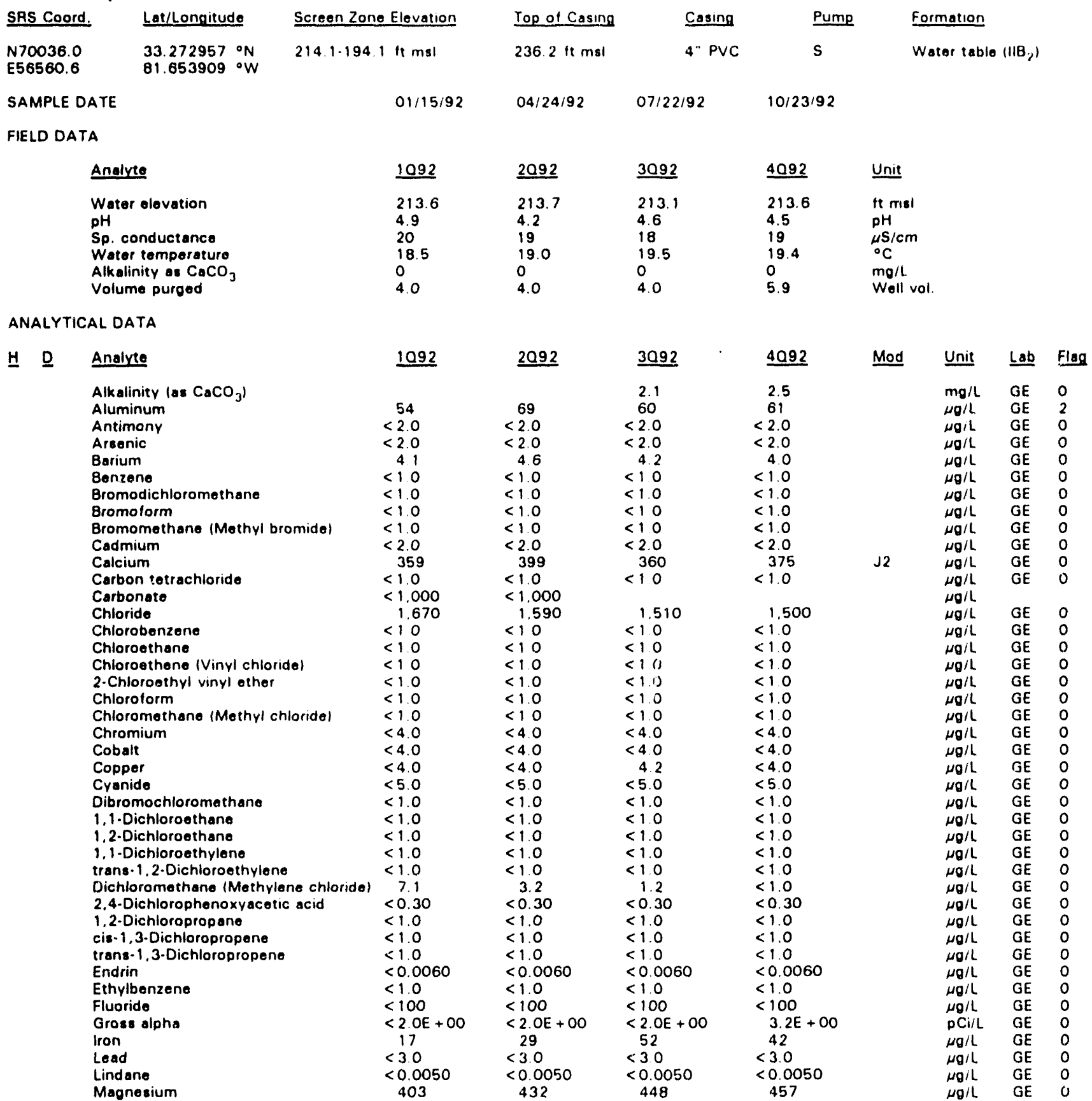

Note: Flagging levels, modifiers, and laboratories are for 4th quarter 1992 data only. See Appendix B for flagging criteria.

- = exceeded holding time for 4th quarter 1992.

- exceeded final primary drinking water standard for 4th quarter 1992.

H-Area Seepage Basins

D.166

Fourth Quarter 1992 
Well HSB140D continued

ANALYTICAL DATA

\begin{tabular}{|c|c|}
\hline$\underline{H} \underline{D}$ & Analyte \\
\hline • & $\begin{array}{l}\text { Manganese } \\
\text { Mercury } \\
\text { Methoxychlor } \\
\text { Nickel } \\
\text { Nitrate as nitrogen } \\
\text { Nitrate-nitrite as nitrogen } \\
\text { Nonvolatile beta } \\
\text { pH } \\
\text { Phenol:s } \\
\text { Potassium } \\
\text { Selenium } \\
\text { Silica } \\
\text { Silver } \\
\text { Sodium } \\
\text { Specific conductance } \\
\text { Sulfate } \\
\text { 1,1,2,2-Tetrachloroethane } \\
\text { Tetrachloroethylene } \\
\text { Toluene } \\
\text { Total alpha-emitting radium } \\
\text { Total dissolved solids } \\
\text { Total organic carbon } \\
\text { Total organic halogens } \\
\text { Total phosphates (as P) } \\
\text { Toxaphene } \\
2,4,5-\text { TP (Silvex) } \\
1,1,1-\text { Trichloroethane } \\
1,1,2-\text { Trichloroethane } \\
\text { Trichloroethylene } \\
\text { Trichlorofluoromethane } \\
\text { Tritium } \\
\text { Uranium } \\
\text { Vanadium } \\
\text { Zinc }\end{array}$ \\
\hline
\end{tabular}

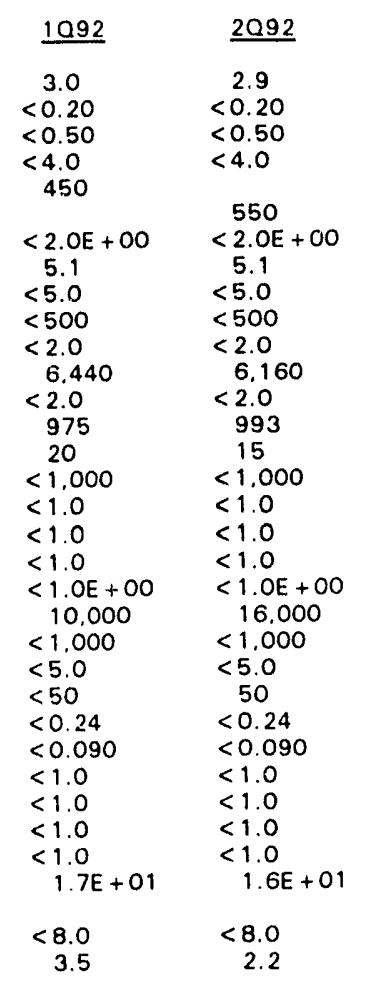

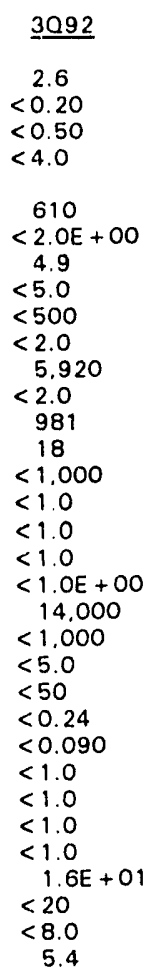

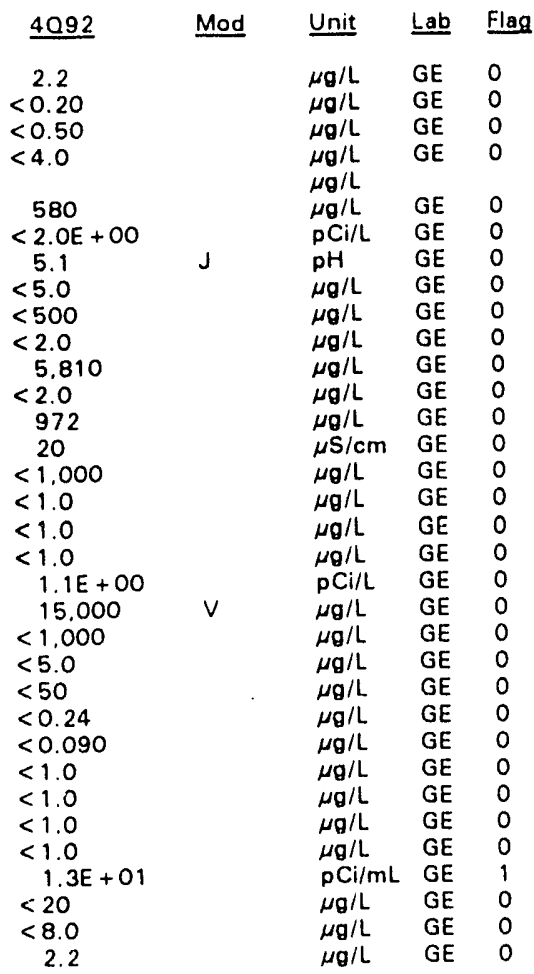

WELL HSB141A

\begin{tabular}{|c|c|c|c|c|c|c|c|c|c|}
\hline SRS Coord. & Lat/Longitude & Screen Zone Elevation & Top of Casing & Casing & Pump & \multicolumn{3}{|c|}{ Formation } & \\
\hline $\begin{array}{l}\text { N71213.6 } \\
\text { E59168.7 }\end{array}$ & $\begin{array}{l}33.279817{ }^{\circ} \mathrm{N} \\
81.649329 \circ \mathrm{W}\end{array}$ & $90.6-80.6 \mathrm{ft} \mathrm{msl}$ & $254.6 \mathrm{ft} \mathrm{msl}$ & 4" PVC & $\mathrm{s}$ & \multicolumn{4}{|c|}{$U$. Congaree (IIA) } \\
\hline \multicolumn{2}{|c|}{ SAMPLE DATE } & $01 / 10 / 92$ & $04 / 27 / 92$ & $07 / 23 / 92$ & $10 / 21 / 92$ & & & & \\
\hline \multicolumn{10}{|c|}{ FIELD DATA } \\
\hline \multicolumn{2}{|c|}{ Analyte } & $\underline{1092}$ & $\underline{2092}$ & $\underline{3092}$ & $\underline{4092}$ & \multicolumn{4}{|l|}{ Unit } \\
\hline \multicolumn{2}{|c|}{$\begin{array}{l}\text { Water elevation } \\
\mathrm{pH} \\
\text { Sp. conductance } \\
\text { Water temperature } \\
\text { Alkalinity as } \mathrm{CaCO}_{3} \\
\text { Volume purged }\end{array}$} & $\begin{array}{l}175.1 \\
11.2 \\
258 \\
18.8 \\
64 \\
12.0\end{array}$ & $\begin{array}{l}175.1 \\
11.4 \\
867 \\
19.0 \\
192 \\
4.0\end{array}$ & $\begin{array}{l}174.7 \\
11.3 \\
841 \\
20.7 \\
185 \\
4.0\end{array}$ & $\begin{array}{l}174.8 \\
11.4 \\
1212 \\
19.8 \\
268 \\
2.4\end{array}$ & \multicolumn{3}{|l|}{$\begin{array}{l}\text { it } \mathrm{msl} \\
\mathrm{pH} \\
\mu \mathrm{S} / \mathrm{cm} \\
{ }^{\circ} \mathrm{C} \\
\mathrm{mg} / \mathrm{L} \\
\text { Well vol. }\end{array}$} & \\
\hline \multicolumn{10}{|c|}{ ANALYTICAL DATA } \\
\hline \multirow[t]{2}{*}{$\underline{H} \quad \underline{D}$} & & 1092 & $\underline{2092}$ & $\underline{3092}$ & $\underline{4092}$ & Mod & $\underline{\text { Unit }}$ & $\underline{\text { Lab }}$ & Flag \\
\hline & $\begin{array}{l}\text { inity (as } \mathrm{CaCO}_{3} \text { ) } \\
\text { inum } \\
\text { nony } \\
\text { ic }\end{array}$ & $\begin{array}{l}1.440 \\
9.7 \\
5.1\end{array}$ & $\begin{aligned} & 2.730 \\
< & 2.0 \\
< & 2.0\end{aligned}$ & $\begin{aligned} & 180 \\
& 2.690 \\
< & 2.0 \\
& 2.0\end{aligned}$ & $\begin{aligned} & 12 \\
& 2.960 \\
< & 2.0 \\
< & 2.0\end{aligned}$ & & $\begin{array}{l}m g / L \\
\mu g / L \\
\mu g / L \\
\mu g / L\end{array}$ & $\begin{array}{l}\mathrm{GE} \\
\mathrm{GE} \\
\mathrm{GE} \\
\mathrm{GE}\end{array}$ & $\begin{array}{l}0 \\
2 \\
0 \\
0\end{array}$ \\
\hline
\end{tabular}

Note: Flagging levels, modifiers, and laboratories are for 4 th quarter 1992 data only. See Appendix $B$ for flagging criteria.

- = exceeded holding time for 4th quarter 1992.

- =xceeded final primary drinking water standard for 4th quarter 1992.

H-Area Seepage Basins

$D \cdot 167$

Fourth Quarter 1992 
WSRC-TR-93-059

Well HSB141A continued

ANALYTICAL DATA

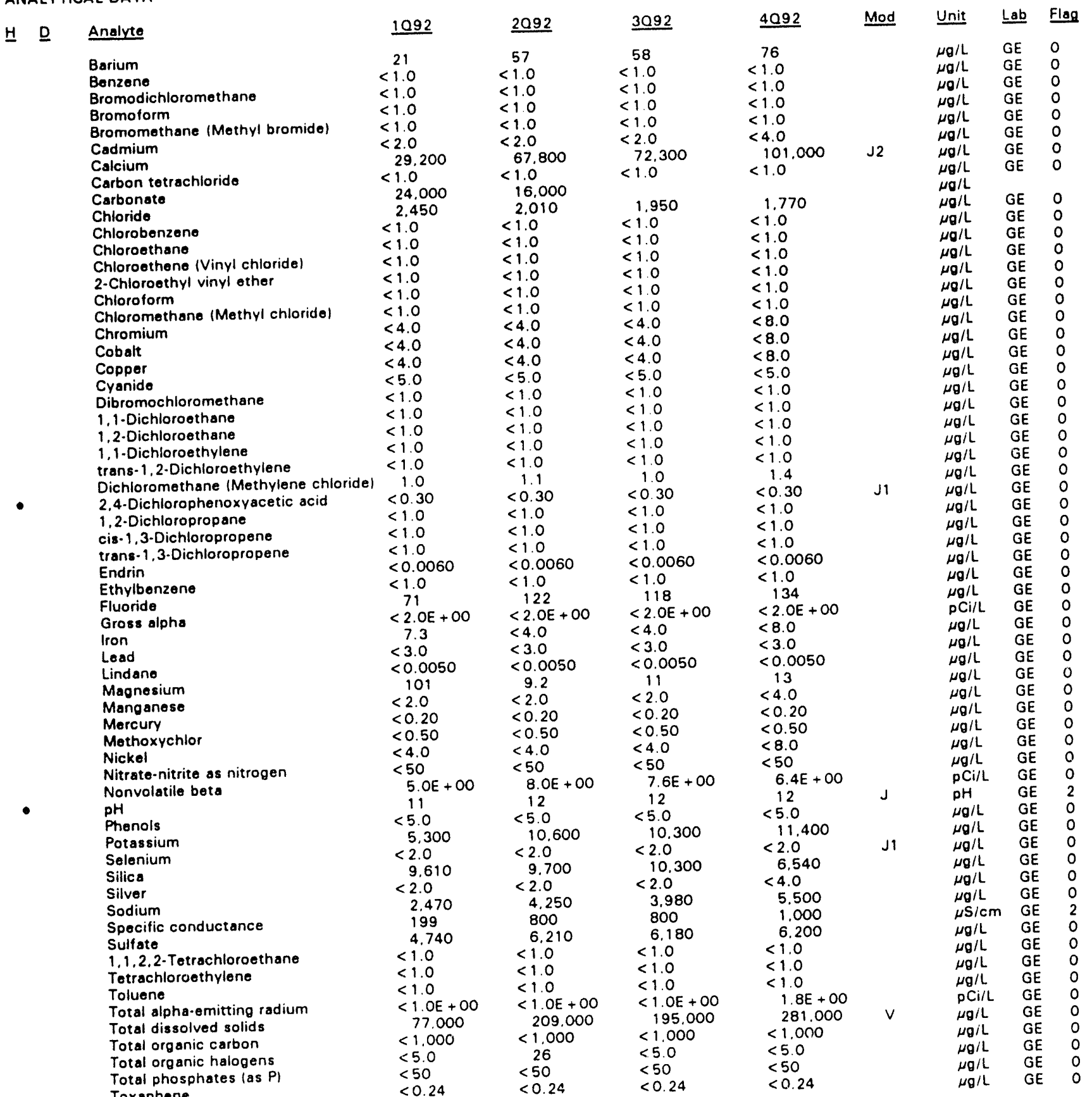

Note: Flagging levels, modifiers, and laboratories are for 4th quarter 1992 data only. See Appendix $B$ for flagging criteria.

- = exceeded ho!ding time for 4th quarter 1992.

$=$ = exceeded fina! primary drinking water standard for 4th quarter 1992.

H-Area Seepage Basins 
WSRC-TR-93-059

Well HSB141A continued

ANALYTICAL DATA

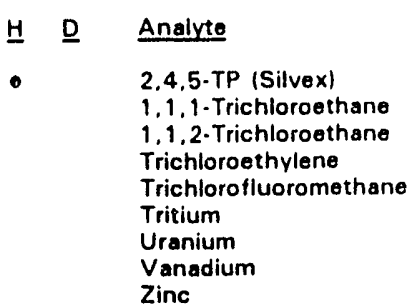

\begin{tabular}{|c|c|c|}
\hline 1092 & 2092 & $\underline{3092}$ \\
\hline$<0.090$ & $<0.090$ & $<0.090$ \\
\hline$<1.0$ & $<1.0$ & $<1.0$ \\
\hline$<1.0$ & $<1.0$ & $<1.0$ \\
\hline$<1.0$ & $<1.0$ & $<1.0$ \\
\hline$<1.0$ & $<1.0$ & $<1.0$ \\
\hline$<7.0 \mathrm{E} \cdot 01$ & $<7.0$ E.01 & $\begin{array}{l}<7.0 E \cdot 01 \\
<20\end{array}$ \\
\hline $\begin{array}{l}<8.0 \\
<2.0\end{array}$ & $\begin{array}{l}11 \\
3.8\end{array}$ & $\begin{array}{l}12 \\
2.4\end{array}$ \\
\hline
\end{tabular}

$\begin{aligned} & 4092 \\ < & 0.090 \\ < & 1.0 \\ < & 1.0 \\ < & 1.0 \\ < & 1.0 \\ & 9.5 E-01 \\ < & 40 \\ < & 16 \\ & 5.0\end{aligned}$

$\begin{array}{llll}\text { Mod } & \text { Unit } & \text { Lab } & \text { Flag } \\ & & & \\ & \mu g / L & G E & 0 \\ & \mu g / L & G E & 0 \\ & \mu g / L & G E & 0 \\ & \mu g / L & G E & 0 \\ & \mu g / L & G E & 0 \\ & \mathrm{pCi} / \mathrm{mL} & \text { GE } & 0 \\ \mu g / L & G E & 0 \\ & \mu g / L & G E & 0 \\ & \mu g / L & G E & 0\end{array}$

WELL HSB141C

\begin{tabular}{|c|c|}
\hline SRS Coord. & Lat/Longitude \\
\hline $\begin{array}{l}N 71196.7 \\
\text { E59170.2 }\end{array}$ & $\begin{array}{l}33.279782{ }^{\circ} \mathrm{N} \\
81.649292{ }^{\circ} \mathrm{W}\end{array}$ \\
\hline
\end{tabular}

$\frac{\text { Screen Zone Elevation }}{164.7-154.7 \mathrm{ft} \mathrm{msl}}$

Top of Casi
$254.7 \mathrm{ft} \mathrm{ms}$
$04 / 17 / 92$

2092
228.4
11.6
1176
18.5
252
0.8

\begin{tabular}{l}
$01 / 10 / 92$ \\
1092 \\
\hline 227.8 \\
11.7 \\
601 \\
17.9 \\
124 \\
0.9
\end{tabular}

\section{ANALYTICAL DATA}

$\mathrm{H} \quad$ Analyte
Alkalinity (as $\mathrm{CaCO}_{3}$ )
Aluminum
Antimony
Arsenic
Barium
Benzene
Bromodichloromethane
Bromoform
Bromomethane (Methyl bromide)
Cadmium
Calcium
Carbon tetrachloride
Carbonate
Chloride
Chlorobenzene
Chloroethane
Chloroethene (Vinyl chloride)
2 -Chloroethyl vinyl ether
Chloroform
Chloromethane (Methyl chloride)
Chromium
Cobalt
Copper
Cyanide
Dibromochloromethane
1 1-Dichloroethane
1 2-Dichloroethane
1 1-Dichloroethylene
trans-1,2-Dichloroethylene

\begin{tabular}{|c|c|}
\hline 1092 & 2092 \\
\hline $\begin{array}{l}2.010 \\
8.7\end{array}$ & 2.810 \\
\hline $\begin{array}{r}8.7 \\
<2.0\end{array}$ & $<2.0$ \\
\hline $\begin{array}{c}<2.0 \\
46\end{array}$ & $<2.0$ \\
\hline 46 & 88 \\
\hline$<1.0$ & $<1.0$ \\
\hline$<1.0$ & $<1.0$ \\
\hline$<1.0$ & $<1.0$ \\
\hline$<1.0$ & $<1.0$ \\
\hline$<2.0$ & $<2.0$ \\
\hline 14.300 & 34.100 \\
\hline$<1.0$ & $<1.0$ \\
\hline $\begin{array}{l}35,300 \\
2,100\end{array}$ & $\begin{array}{l}48,400 \\
1.850\end{array}$ \\
\hline$<1.0$ & $<1.0$ \\
\hline$<1.0$ & $<1.0$ \\
\hline$<1.0$ & $<1.0$ \\
\hline$<1.0$ & $<1.0$ \\
\hline$<1.0$ & $<1.0$ \\
\hline$<1.0$ & $<1.0$ \\
\hline$<4.0$ & $<4.0$ \\
\hline$<4.0$ & $<4.0$ \\
\hline$<4.0$ & $<4.0$ \\
\hline$<5.0$ & $<5.0$ \\
\hline$<1.0$ & $<1.0$ \\
\hline$<1.0$ & $<1.0$ \\
\hline$<1.0$ & $<1.0$ \\
\hline$<1.0$ & $<1.0$ \\
\hline$<1.0$ & $<1.0$ \\
\hline
\end{tabular}

$\begin{aligned} & 3092 \\ & 74 \\ & 1.260 \\ &< 2.0 \\ &< 2.0 \\ & 48 \\ &< 1.0 \\ &< 1.0 \\ &<1.0 \\ &<1.0 \\ &<2.0 \\ & 9.180 \\ &< 1.0 \\ & 2.010 \\ &< 1.0 \\ &<1.0 \\ &<1.0 \\ &<1.0 \\ &<1.0 \\ &<1.0 \\ & 4.5 \\ &<4.0 \\ &<4.0 \\ &<5.0 \\ &<1.0 \\ &<1.0 \\ &<1.0 \\ &<1.0 \\ &<1.0 \\ &<1.0\end{aligned}$

$\begin{array}{lll}\underline{3092} & \underline{4092} & \text { Unit } \\ 228.7 & 229.4 & \mathrm{ft} \mathrm{msl} \\ 11.2 & 11.3 & \mathrm{pH} \\ 469 & 1087 & \mu \mathrm{S} / \mathrm{cm} \\ 19.2 & 17.7 & { }^{\circ} \mathrm{C} \\ 111 & 224 & \mathrm{mg} / \mathrm{L} \\ 0.9 & 0.9 & \text { Well vol. }\end{array}$

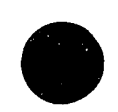

SAMPLE DATE

Anslyte

Water elevation

Sp. conductance

Water temperature

Alkalinity as $\mathrm{CaCO}_{3}$

Volume purged

0.9

$\begin{array}{lll}\text { Casing } & \text { Pumg } & \text { Formation } \\ \text { 4" PVC } & \text { S } & \left.\text { Barnwell (IIB } B_{1}\right)\end{array}$

FIELD DATA 
Well HSB141C continued

\begin{tabular}{|c|c|c|c|c|c|c|c|c|c|}
\hline$\underline{\text { H}} \quad \underline{\mathrm{D}}$ & Analyte & 1092 & $\underline{2092}$ & 3092 & 4092 & Mod & Unit & $\underline{\text { Lab }}$ & Flag \\
\hline
\end{tabular}

Note: Flagging levels, modifiers, and laboratories are for 4 th quarter 1992 data only. See Appendix B for flagging criteria. - =xceeded holding time for 4 th quarter 1992.

- =xceeded final primary drinking water standard for 4th quarter 1992. 
WELL HSB141D

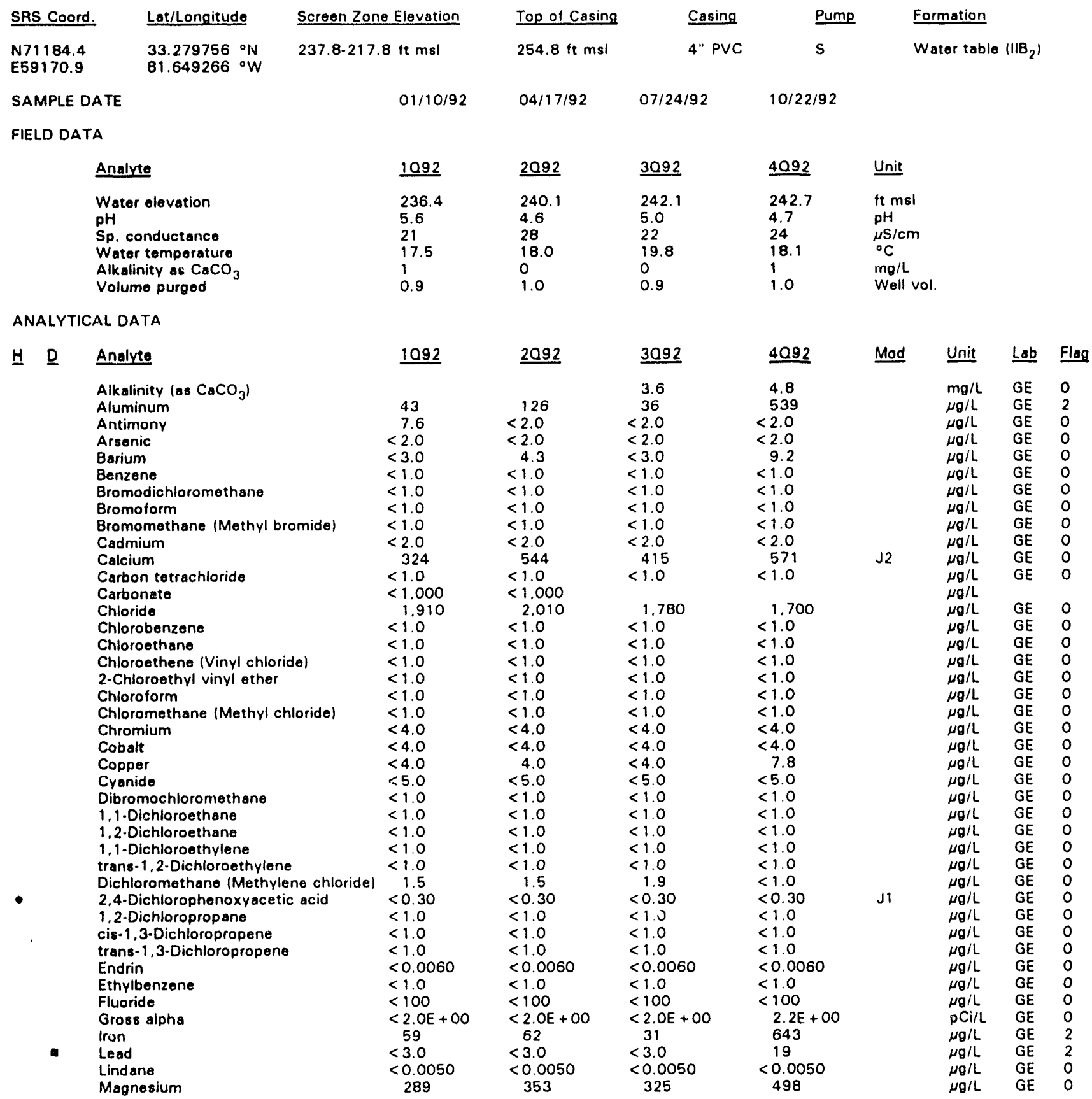

Note: Flagging levels, modifiers, and laboratories are for 4 th quarter 1992 data only. See Appendix B for flagging criteria. - = exceeded holding time for 4th quarter 1992.

- exceeded final primary drinking water standard for 4th quarter 1992. 
WSRC-TR-93-059

Woll HSB1410 continued

ANALYTICAL DATA

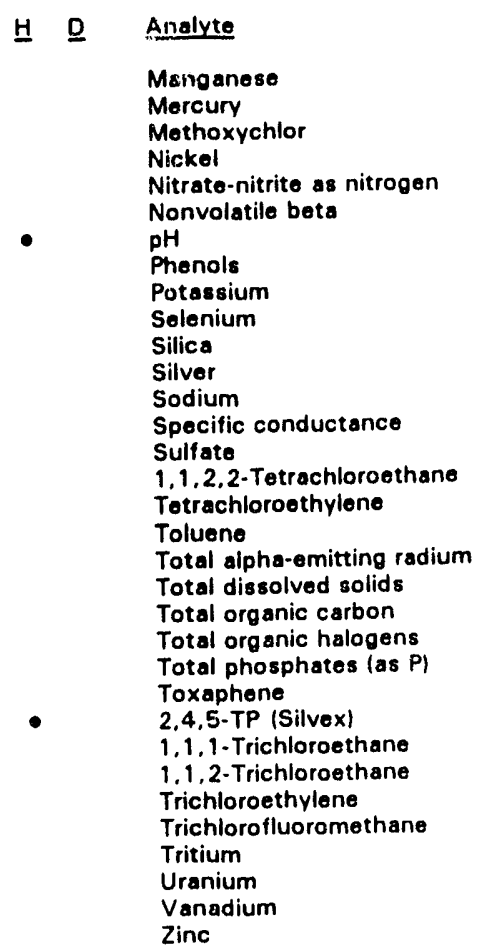

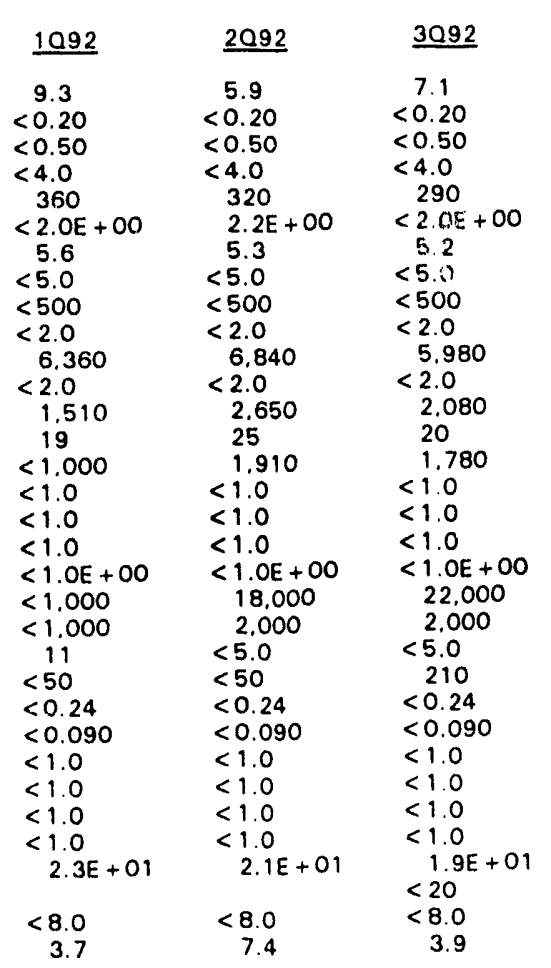

\begin{tabular}{|c|c|c|c|c|}
\hline 4092 & Mod & Unit & $\underline{\text { Lab }}$ & Flag \\
\hline 42 & & $\mu \boldsymbol{g} / \mathrm{L}$ & GE & 1 \\
\hline $\begin{array}{l}<0.20 \\
<0.50\end{array}$ & & $\mu \mathrm{g} / \mathrm{L}$ & GE & 0 \\
\hline $\begin{array}{l}<0.50 \\
<4.0\end{array}$ & & $\mu \sigma / L$ & GE & 0 \\
\hline $\begin{array}{r}<4.0 \\
250\end{array}$ & & $\mu g / L$ & GE & 0 \\
\hline $\begin{aligned} & 250 \\
< & 2.0 E+00\end{aligned}$ & & $\begin{array}{l}\mu \mathrm{g} / \mathrm{L} \\
\mathrm{pCi} / \mathrm{L}\end{array}$ & $\begin{array}{l}\mathrm{GE} \\
\mathrm{GE}\end{array}$ & $\begin{array}{l}0 \\
0\end{array}$ \\
\hline 6.0 & J & $\mathrm{pH}$ & GE & 0 \\
\hline$<5.0$ & & $\mu g / L$ & GE & 0 \\
\hline $\begin{array}{l}<500 \\
<2.0\end{array}$ & J1 & & $\begin{array}{l}\mathrm{GE} \\
\mathrm{GE}\end{array}$ & $\begin{array}{l}0 \\
0\end{array}$ \\
\hline $\begin{array}{r}<2.0 \\
8.300\end{array}$ & & $\begin{array}{l}\mu \mathrm{g} / \mathrm{L} \\
\mu \mathrm{g} / \mathrm{L}\end{array}$ & $\mathrm{GE}$ & 0 \\
\hline$<2.0$ & & $\mu \mathrm{g} / \mathrm{L}$ & $\mathrm{GE}$ & 0 \\
\hline $\begin{array}{l}2,490 \\
20\end{array}$ & & $\begin{array}{l}\mu \mathrm{g} / \mathrm{L} \\
\mu \mathrm{S} / \mathrm{cm}\end{array}$ & $\begin{array}{l}\mathrm{GE} \\
\mathrm{GE}\end{array}$ & $\begin{array}{l}0 \\
0\end{array}$ \\
\hline 1.090 & & $\mu \mathrm{g} / \mathrm{L}$ & GE & 0 \\
\hline $\begin{array}{l}<1.0 \\
<1.0\end{array}$ & & $\mu \mathrm{g} / \mathrm{L}$ & $\mathrm{GE}$ & $\begin{array}{l}0 \\
0\end{array}$ \\
\hline $\begin{array}{l}<1.0 \\
<1.0\end{array}$ & & $\begin{array}{l}\mu \mathrm{g} / \mathrm{L} \\
\mu \mathrm{g} / \mathrm{L}\end{array}$ & GE & 0 \\
\hline $\begin{aligned}< & 1.0 \\
& 1.5 E+00\end{aligned}$ & & $\begin{array}{l}\mu g / \mathrm{L} \\
\mathrm{pCi} / \mathrm{L}\end{array}$ & $\mathbf{G E}$ & 0 \\
\hline $\begin{aligned} & 19.000 \\
< & 1.000\end{aligned}$ & V & $\mu g / L$ & $\begin{array}{l}\mathrm{GE} \\
\mathrm{GE}\end{array}$ & $\begin{array}{l}0 \\
0\end{array}$ \\
\hline $\begin{array}{l}<1.000 \\
<5.0\end{array}$ & & $\begin{array}{l}\mu g / L \\
\mu g / L\end{array}$ & GE & 0 \\
\hline$<50$ & & $\mu \mathrm{g} / \mathrm{L}$ & GE & 0 \\
\hline$<0.24$ & & $\mu g / L$ & $\mathrm{GE}$ & 0 \\
\hline$<0.090$ & J1 & $\mu g / L$ & GE & 0 \\
\hline$<1.0$ & & $\mu \mathrm{g} / \mathrm{L}$ & $\mathrm{GE}$ & 0 \\
\hline$<1.0$ & & $\mu g / L$ & $\mathrm{GE}$ & 0 \\
\hline$<1.0$ & & $\mu \mathrm{g} / \mathrm{L}$ & GE & 0 \\
\hline$<1.0$ & & $\mu \mathrm{g} / \mathrm{L}$ & GE & 0 \\
\hline $1.7 E+01$ & & $\mathrm{pCi} / \mathrm{mL}$ & GE & 1 \\
\hline$<20$ & & $\mu_{g} / \mathrm{L}$ & GE & 0 \\
\hline$<8.0$ & & $\mu g / L$ & $\begin{array}{l}\mathrm{GE} \\
\mathrm{GE}\end{array}$ & $\begin{array}{l}0 \\
0\end{array}$ \\
\hline 22 & & $\mu_{g} / \mathrm{L}$ & & \\
\hline
\end{tabular}

\section{WELL HSB142C}

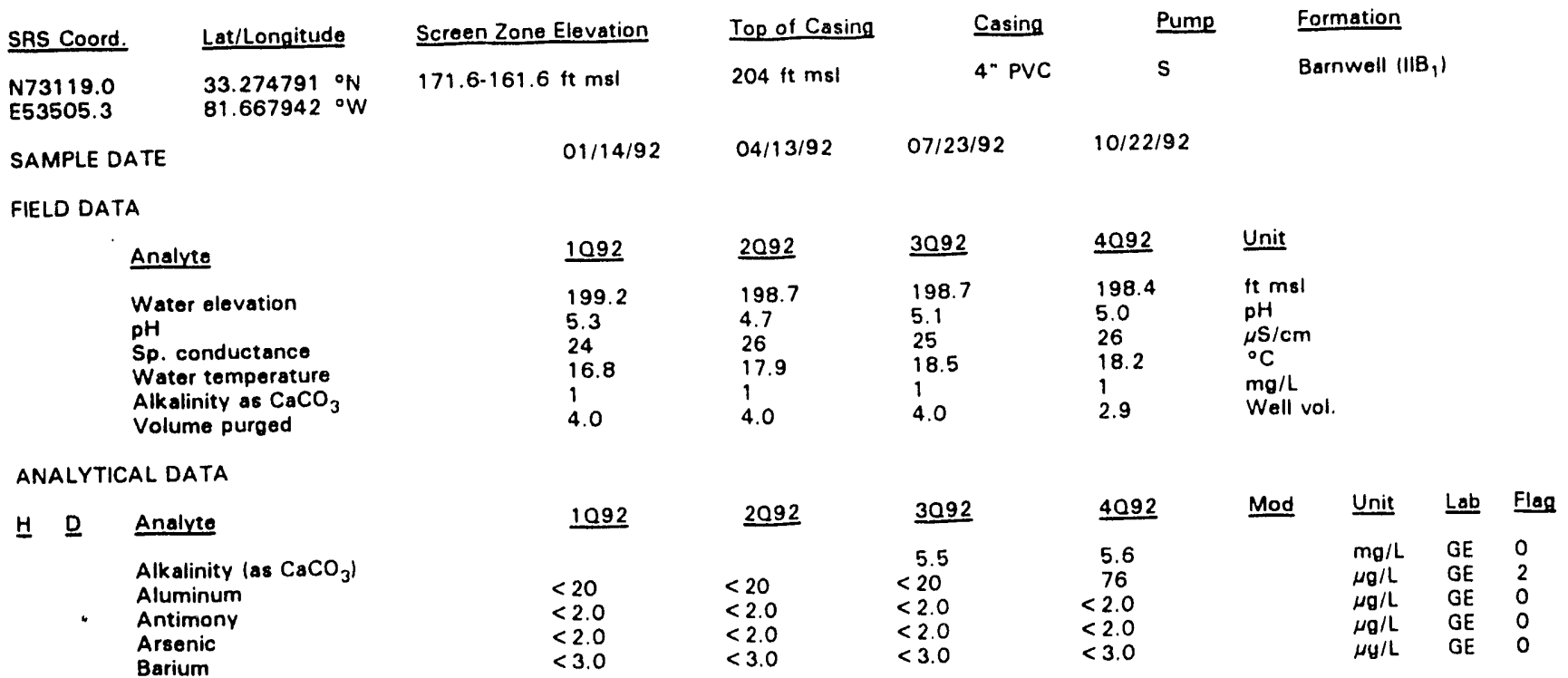

Note: Flagging levels, modifiers, and laboratories are for 4 th quarter 1992 data only. See Appendix B for flagging criteria.

- = exceeded holding time for 4 th quarter 1992.

= exceeded final primary drinking water standard for 4th quarter 1992.

H.Area Seppage Basins 


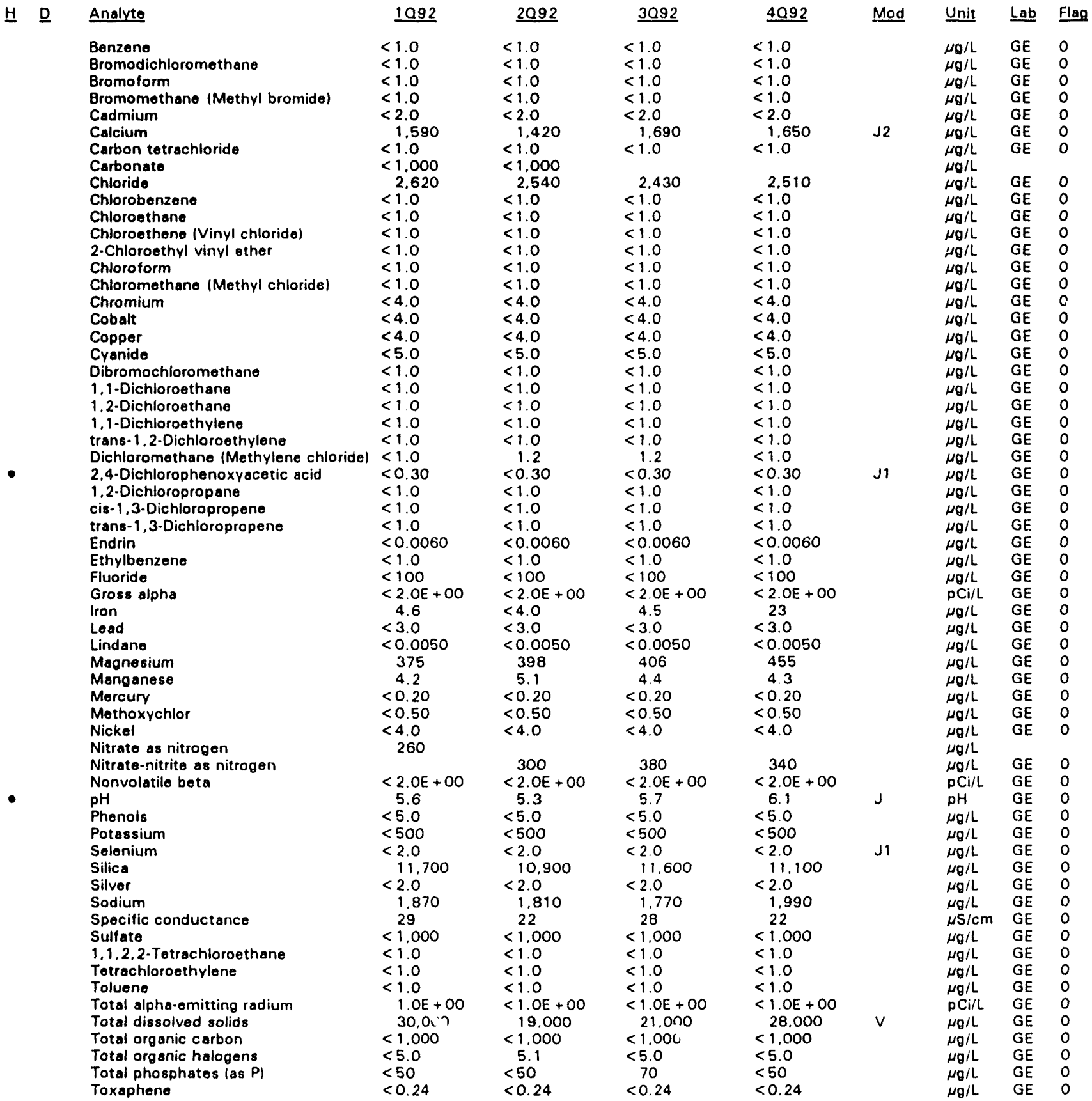

Note: Flagging levels, modifiers, and laboratories are for 4th quarter 1992 data only. See Appendix B for flagging criteria. - =xceeded holding time for 4th quarter 1992.

- =xceeded final primary drinking water standard for 4th quarter 1992. 
WSRC-TR-93-059

Well HSB142C continued

\section{ANALYTICAL DATA}

H D

\section{Analyte}

- $\quad 2,4,5-T P$ (Silvex)

1,1.1-Trichloroethane

1,1,2-Trichloroethane

Trichloroethylene

Trichlorofluoromethane

Tritium

Uranium

Vanadium

Zinc

$\begin{array}{ll}\underline{1092} & \underline{2092} \\ <0.090 & <0.090 \\ <1.0 & <1.0 \\ <1.0 & <1.0 \\ <1.0 & <1.0 \\ <1.0 & <1.0 \\ <7.0 \text { E-O } & <7.0 \mathrm{E}-01 \\ <8.0 & <8.0 \\ 7.3 & 5.2\end{array}$

$\begin{aligned} & 3092 \\ < & 0.090 \\ < & 1.0 \\ < & 1.0 \\ < & 1.0 \\ < & 1.0 \\ < & 7.0 \mathrm{E}-01 \\ < & 20 \\ < & 8.0 \\ & 4.5\end{aligned}$

$\begin{array}{llll}\text { Mod } & \text { Unit } & \text { Lab } & \text { Flag } \\ \text { J1 } & & & \\ & \mu g / L & G E & 0 \\ & \mu g / L & G E & 0 \\ & \mu g / L & G E & 0 \\ \mu g / L & G E & 0 \\ & \mu g / L & G E & 0 \\ & \mathrm{pC} / / m L & G E & 0 \\ & \mu g / L & G E & 0 \\ & \mu g / L & G E & 0 \\ & \mu \sigma / L & G E & 0\end{array}$

\section{WELL HSB142D}

\begin{tabular}{|c|c|}
\hline SRS Coord. & Lat/Longitude \\
\hline $\begin{array}{l}\text { N73113.0 } \\
\text { E53493.1 }\end{array}$ & $\begin{array}{l}33.274758{ }^{\circ} \mathrm{N} \\
81.6679622^{\circ} \mathrm{W}\end{array}$ \\
\hline
\end{tabular}

SAMPLE DATE

Screen Zone Elevation

199.7.189.7 ft msl

$01 / 14 / 92$

$\frac{1092}{102}$
199.5
4.8
54
15.2
5
4.0

\section{ANALYTICAL DATA}

브

Analyte
Alkalinity (as $\mathrm{CaCO}_{3}$ )
Aluminum
Antimony
Arsenic
Barium
Benzene
Bromodichloromethane
Bromoform
Bromomethane (Methyl bromide)
Cadmium
Calcium
Carbon tetrachloride
Carbonate
Chloride
Chlorobenzene
Chloroethane
Chloroethene (Vinyl chloride)
2 -Chloroethyl vinyl ether
Chloroform
Chloromethane (Methyl chloride)
Chromium
Cobalt
Copper
Cyanide
Dibromochloromethane
1 1-Dichloroethane
1,2 -Dichloroethane
1.1 -Dichloroethylene
trans-1,2-Dichloroethylene

Water elevation
pH
Sp. conductance
Water temperature
Alkalinity as $\mathrm{CaCO}_{3}$
Volume purged

trans-1,2-Dichloroethylene

\begin{tabular}{|c|c|}
\hline 1092 & $\underline{2092}$ \\
\hline $\begin{aligned} & 154 \\
&< 2.0 \\
&< 2.0 \\
& 1.0 \\
&<1.0 \\
&<1.0 \\
&<1.0 \\
&<1.0 \\
&<2.0 \\
& 1.060 \\
&<1.0 \\
&<1.000 \\
& 4.680 \\
&<1.0 \\
&<1.0 \\
&<1.0 \\
&<1.0 \\
&<1.0 \\
&<1.0 \\
&<4.0 \\
&<4.0 \\
& 8.9 \\
&<5.0 \\
&<1.0 \\
&<1.0 \\
&<1.0 \\
&<1.0 \\
&<1.0 \\
&<1.0\end{aligned}$ & $\begin{aligned} & 142 \\
&< 2.0 \\
&< 2.0 \\
& 24 \\
&< 1.0 \\
&< 1.0 \\
&< 1.0 \\
&< 1.0 \\
&< 2.0 \\
& 1.190 \\
&< 1.0 \\
&< 1.000 \\
& 4.290 \\
&< 1.0 \\
&< 1.0 \\
&< 1.0 \\
&< 1.0 \\
&<1.0 \\
&<1.0 \\
&<4.0 \\
&<4.0 \\
&<4.0 \\
&<5.0 \\
&<1.0 \\
&<1.0 \\
&<1.0 \\
&<1.0 \\
&<1.0\end{aligned}$ \\
\hline
\end{tabular}

\section{Top of Casing \\ 204.2 ft msl}

$04 / 13 / 92$

2092
198.1
4.4
48
15.7
0
0.5

0.5

3092
198.2
4.8
46
21.2
0
0.7

$\begin{array}{ll}\text { Casing } & \text { Pump } \\ \text { 4" PVC } & \mathrm{s}\end{array}$

\section{Formation}

Water table $\left(11 \mathrm{~B}_{2}\right)$

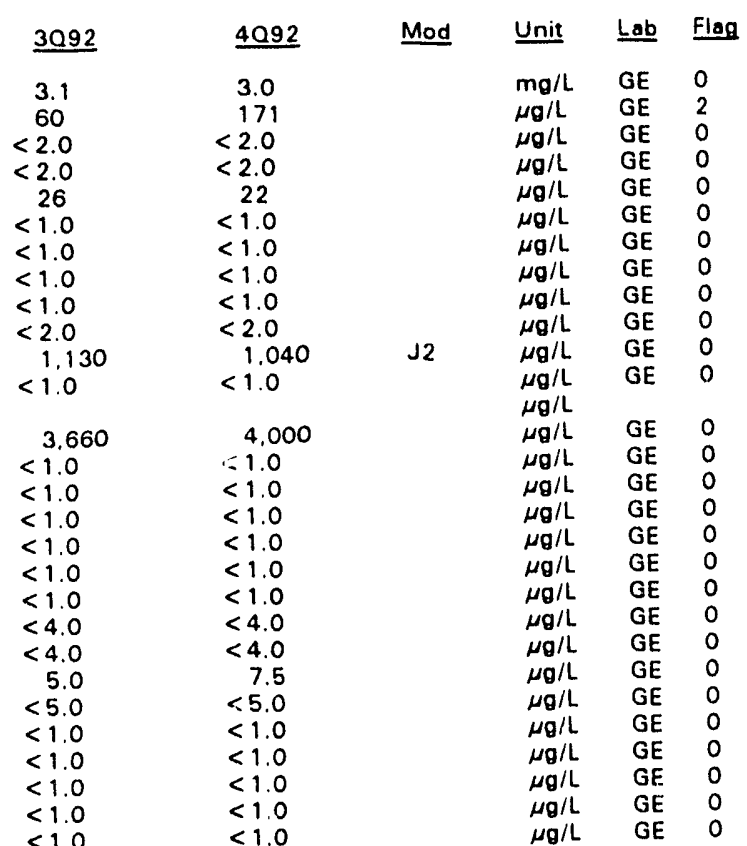




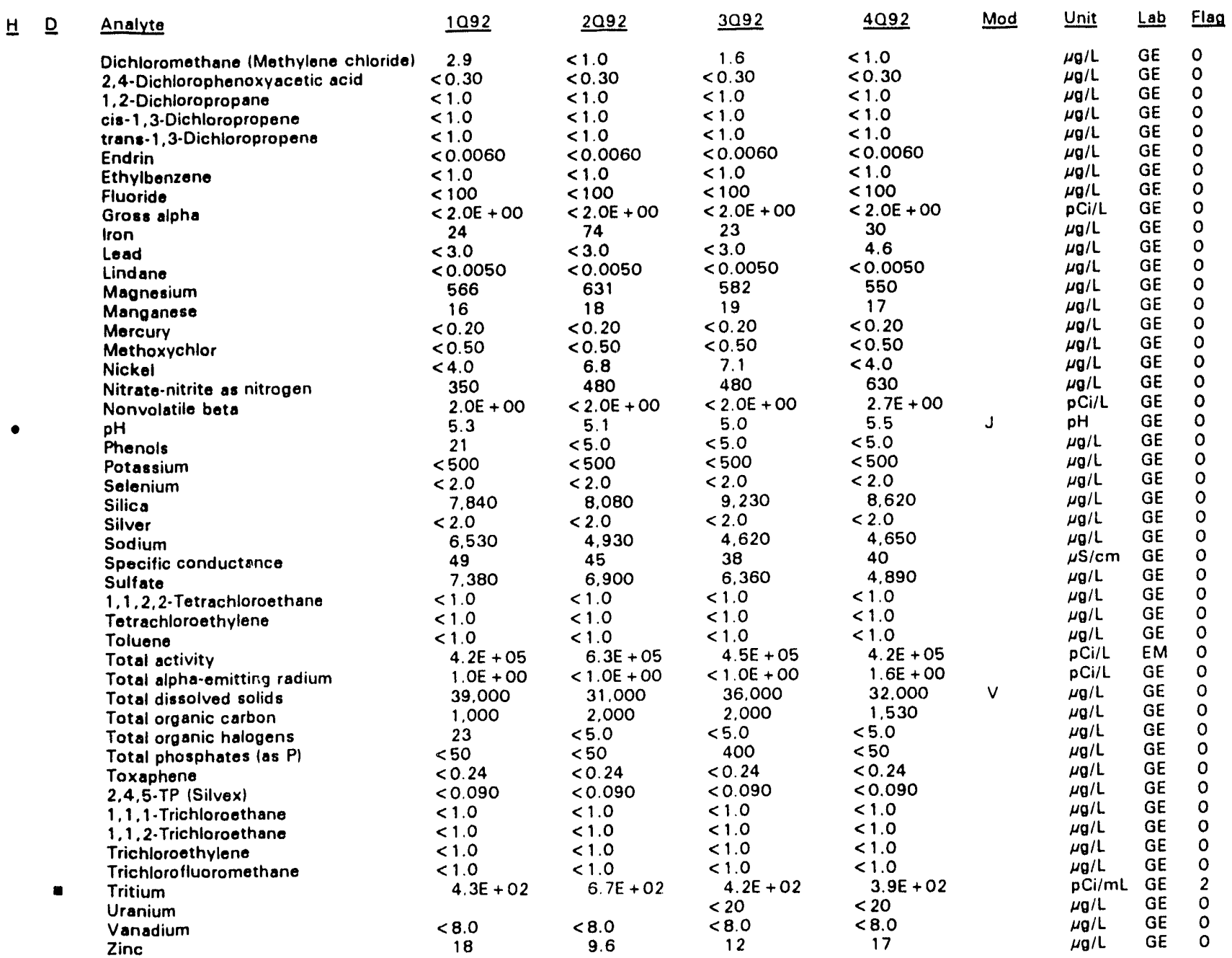

Noto: Flagging levels, modifiers, and laboratories are for 4th quarter 1992 data only. See Appendix $B$ for flagging criteria. - = exceeded holding time for 4th quarter 1992.

- =xceeded final primary drinking water standard for 4th quarter 1992. 
WSRC.TR.93-059

WELL HSB143C

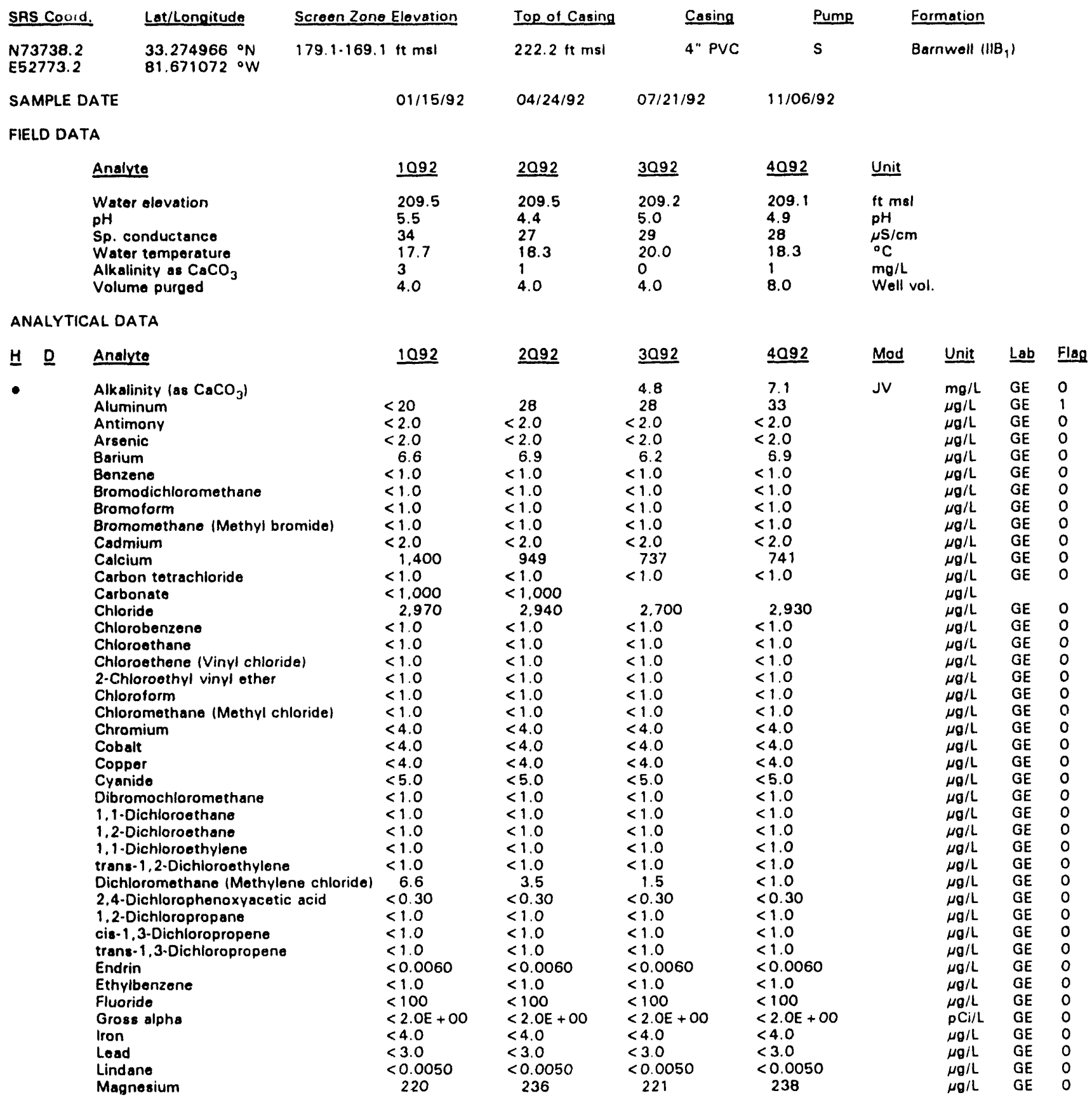

Note: Flagging levels, modifiers, and laboratories are for 4 th quarter 1992 data only. See Appendix B for flagging criteria.

- = exceeded holding time for 4th quarter 1992.

- =xceeded final primary drinking water standard for 4th quarter 1992.

H-Area Seepage Basins

D.176

Fourth Quarter 1992 
WSRC.TR.93.059

Well HSB143C continued

ANALYTICAL DATA

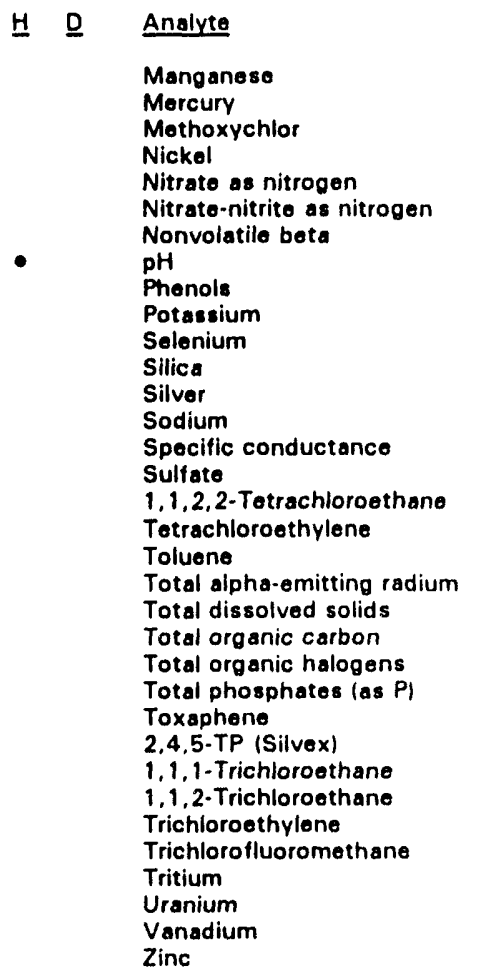

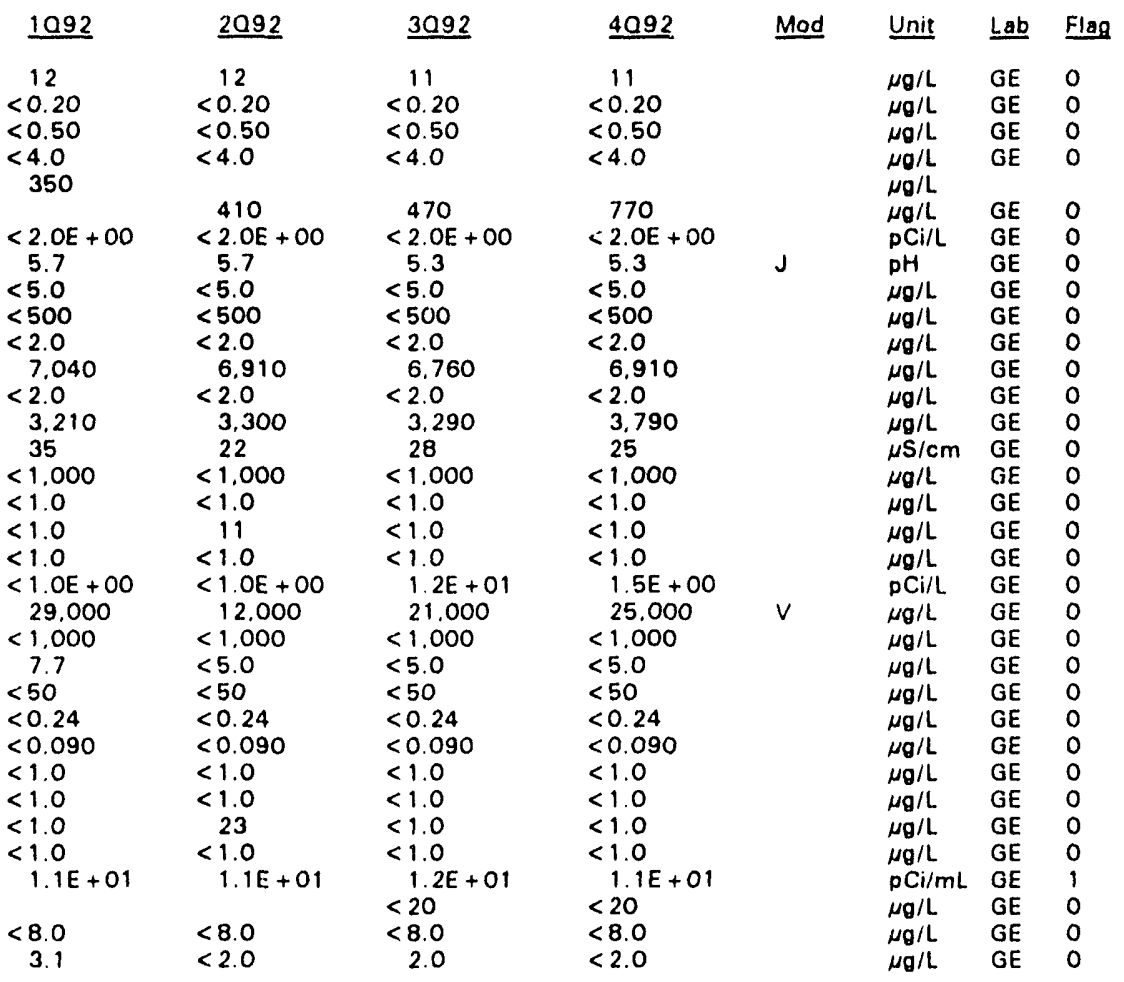

\section{WELL HSB143D}

\begin{tabular}{|c|c|c|c|c|c|c|c|c|c|}
\hline \multirow{2}{*}{$\begin{array}{l}\text { SRS Coord. } \\
\text { N73754.0 } \\
\text { E52774.5 }\end{array}$} & Lat/Longitude & Screen Zone Elevation & Top of Casing & Casing & Pump & \multicolumn{4}{|c|}{ Formation } \\
\hline & $\begin{array}{l}33.275003^{\circ} \mathrm{N} \\
81.671099^{\circ} \mathrm{W}\end{array}$ & $216.9 \cdot 196.9 \mathrm{ft} \mathrm{msl}$ & $222.9 \mathrm{ft} \mathrm{msl}$ & 4" PVC & s & & ater tab & $\left(11 \mathrm{~B}_{2}\right)$ & \\
\hline \multicolumn{2}{|c|}{ SAMPLE DATE } & $01 / 15 / 92$ & $04 / 24 / 92$ & $07 / 21 / 92$ & $11 / 06 / 92$ & & & & \\
\hline \multicolumn{10}{|l|}{ FIELD DATA } \\
\hline \multicolumn{2}{|c|}{ Analyte } & 1092 & $\underline{2092}$ & 3092 & 4092 & \multicolumn{4}{|l|}{ Unit } \\
\hline & $\begin{array}{l}\text { Water olevation } \\
\text { pH } \\
\text { Sp. conductance } \\
\text { Water temperature } \\
\text { Alkalinity as } \mathrm{CaCO}_{3} \\
\text { Volume purged }\end{array}$ & $\begin{array}{l}213.3 \\
4.8 \\
20 \\
17.8 \\
0 \\
4.0\end{array}$ & $\begin{array}{l}213.3 \\
4.0 \\
18 \\
18.1 \\
0 \\
4.0\end{array}$ & $\begin{array}{l}213.1 \\
4.5 \\
21 \\
19.0 \\
0 \\
4.0\end{array}$ & $\begin{array}{l}212.1 \\
4.5 \\
19 \\
19.0 \\
0 \\
8.8\end{array}$ & \multicolumn{3}{|c|}{$\begin{array}{l}\text { ft msl } \\
\mathrm{pH} \\
\mu \mathrm{S} / \mathrm{cm} \\
{ }^{\circ} \mathrm{C} \\
\mathrm{mg} / \mathrm{L} \\
\text { Well vol. }\end{array}$} & \\
\hline \multicolumn{10}{|c|}{ ANALYTICAL DATA } \\
\hline \multirow[t]{2}{*}{$\underline{H} \quad \underline{D}$} & Analyte & 1092 & 2092 & $\underline{3092}$ & 4092 & Mod & Unit & Lab & Flag \\
\hline & $\begin{array}{l}\text { ity (as } \mathrm{CaCO}_{3} \text { ) } \\
\text { num } \\
\text { ony } \\
\text { ic }\end{array}$ & $\begin{array}{r}\quad 43 \\
<2.0 \\
<2.0\end{array}$ & $\begin{aligned} & 56 \\
< & 2.0 \\
< & 2.0\end{aligned}$ & $\begin{aligned} & 2.2 \\
& 51 \\
< & 2.0 \\
< & 2.0\end{aligned}$ & $\begin{aligned} & 4.0 \\
& 56 \\
< & 2.0 \\
< & 2.0\end{aligned}$ & v & $\begin{array}{l}\mathrm{mg} / \mathrm{L} \\
\mu \mathrm{g} / \mathrm{L} \\
\mu \mathrm{g} / \mathrm{L} \\
\mu \mathrm{g} / \mathrm{L}\end{array}$ & $\begin{array}{l}\mathrm{GE} \\
\mathrm{GE} \\
\mathrm{GE} \\
\mathrm{GE}\end{array}$ & $\begin{array}{l}0 \\
2 \\
0 \\
0\end{array}$ \\
\hline
\end{tabular}

Note: Flagging levels, modifiers, and laboratories are for 4th quarter 1992 data only. See Appendix B for flagging criteria.

- = exceeded holding time for 4 th quarter 1992 .

- = exceeded final primary drinking water standard for 4th quarter 1992.

H-Area Seepage Basins 


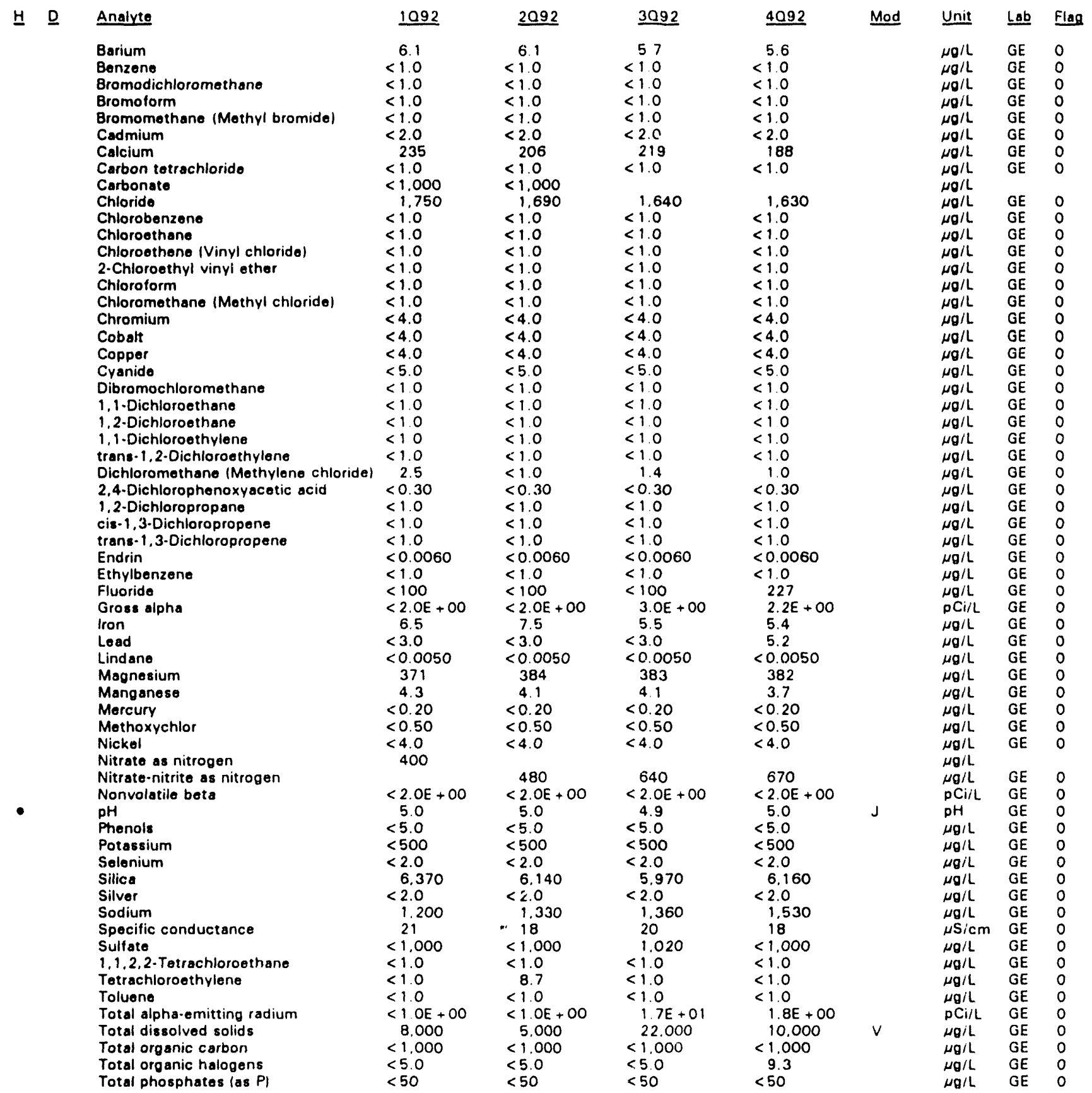

Note: Flagging levels, modifiers, and laboratories are for 4 th quarter 1992 data only. See Appendix B for flagging criteria.

- =xceeded holding time for 4th quarter 1992.

= exceeded final primary drinking water standard for 4th quarter 1992.

H-Area Seepage Basins

$D \cdot 178$
Fourth Quarter 1992 
WSRC.TR.93-059

Well HSB143D continued

ANALYTICAL DATA

H D Analyto

Toxaphene

2,4,5-TP (Silvex)

1,1,1-Trichloroetliane

1.1.2-Trichloroethan

Trichloroethylene

Trichlorofluoromethane

Tritium

Uranium

Vanadium

Zinc

$\begin{array}{lll}1092 & \underline{2092} & \underline{3092} \\ <0.24 & <0.24 & <0.24 \\ <0.090 & <0.090 & <0.090 \\ <1.0 & <1.0 & <1.0 \\ <1.0 & <1.0 & <1.0 \\ <1.0 & 1.3 & <1.0 \\ <1.0 & <1.0 & <1.0 \\ 1.3 E+01 & 1.3 E+01 & 1.1 E+01 \\ <8.0 & <8.0 & <20 \\ 2.3 & <2.0 & <2.0\end{array}$

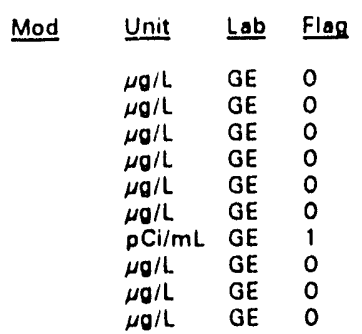

\section{WELL HSB144A}

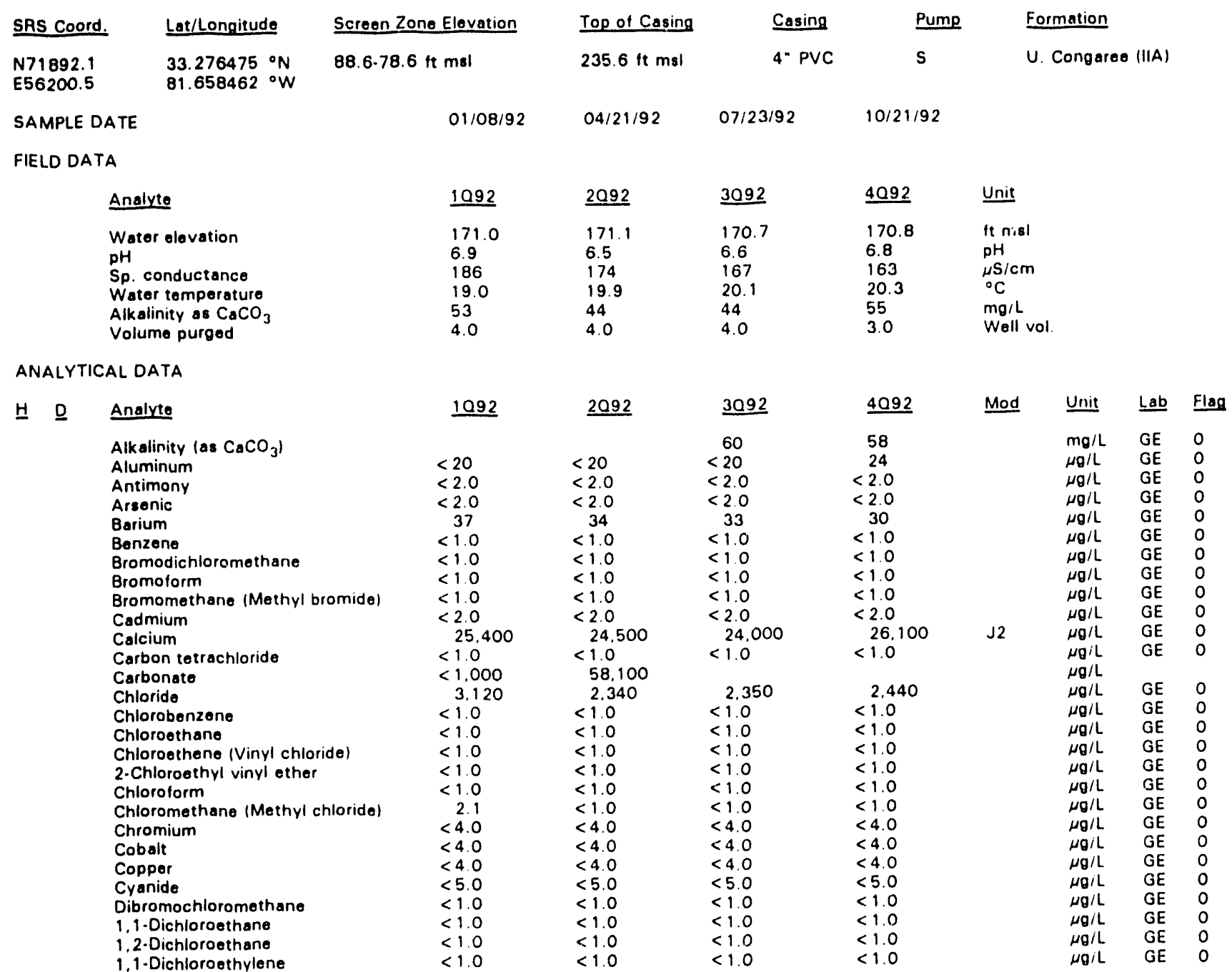

Note: Flagging levels, modifiers, and laboratories are for 4 th quarter 1992 data only. See Appendix 8 for flagging criteria. - =xceeded holding time for 4th quarter 1992.

- =xceeded final primary drinking water standard for 4 th quarter 1992 . 
WSRC-TR-93-059

Woll HSB144A continued

ANALYTICAL DATA

므 Analyte

1092

$\begin{aligned} & 1092 \\ &< 1.0 \\ &< 1.0 \\ &< 0.30 \\ &< 1.0 \\ &< 1.0 \\ &< 1.0 \\ &< 0.0060 \\ &< 1.0 \\ & 128 \\ & 3.2 \mathrm{E}+00 \\ &< 4.0 \\ &< 3.0 \\ &< 0.0050 \\ & 716 \\ & 52 \\ &<0.20 \\ &<0.50 \\ &<4.0\end{aligned}$

$\begin{aligned} & 2092 \\ < & 1.0 \\ < & 1.0 \\ < & 0.30 \\ < & 1.0 \\ < & 1.0 \\ < & 1.0 \\ < & 0.0060 \\ < & 1.0 \\ & 143 \\ < & 2.0 \mathrm{E}+00 \\ < & 4.0 \\ < & 3.0 \\ < & 0.0050 \\ & 696 \\ & 46 \\ < & 0.20 \\ < & 0.50 \\ < & 4.0\end{aligned}$

\section{2}

409․

$<10$

trane-1,2-Dichioronthylene

Dichloromethane (Mothylene chloride)

2.4-Dichlorophenoxyacetic acid

1,2-Dichloropropane

trans-1,3-Dichloropropene

Endrin

Ethylbenzene

Fluoride

Groses alpha

Iron

Lead

Lindane

Magnesium

Manganese

Mercury

Mothoxychlor

Nitrate as nitrogen

3.000

Nitrate-nitrite as nitrogen

Nonvolatile beta

$\mathrm{pH}$

Phenols

Potassium

Selonium

Silica

Silver

Sodium

Specific conductance

Sulfate

1,1,2,2-Totrachloroethane

Tetrachloroethylene

Toluene

Total activity

Total alpha-omitting radium

1. $5 E+01$

7.1
$<5.0$

2.700

$<2.0$

28.700

$<2.0$
6.260

6.260
138

138
6.120

$<1.0$

$<1.0$

$<1.0$

2. $0 E+06$

$<1 . O E+00$
138,000

Total dissolved solids

Total organic halogens

Total phosphates (as P)

$<1.000$

$<5.0$

290

$<0.24$

2,4,5-TP (Silvex)

1.1,1-Trichloroethane

1,1,2-Trichloroethane

Trichloroethylene

$<0.090$

$<1.0$

$<1.0$

$<1.0$

Trichlorofluoromethane

$<1.0$

1.180
$5.6 E+00$

$5.6 \mathrm{E}$
6.3
$<5.0$

$<5.0$

2.400

28.600

$<2.0$

$<2.0$
5.920

5.920
160

6.450

$<1.0$

$<1.0$

$<1.0$

$\begin{aligned} & 1.8 \\ < & 0.30\end{aligned}$

$<1.0$

$<10$

$<0.0060$

$<1.0$

131

$<2 . \mathrm{OE}+0 \mathrm{OO}$

$<4.0$

$<3.0$

$<0.0050$

660
42

$\begin{aligned} & 42 \\ < & 0.20\end{aligned}$

$<0.20$
$<0.50$

4.4

Tritium

Uranium

Vanadium

2. $1 E+03$

$1.5 E+06$

$<1 . \mathrm{OE}+\mathrm{OO}$

112,000

$<1.000$

15

330

$<0.24$

$<0.090$

$<1.0$
$<1.0$

$<1.0$

1.550

6. $7 E+00$

7.0
$<5.0$

2.350

$<2.0$

27.600

$<2.0$

5.590

310

6.470

$<1.0$

$<1.0$

1.1E+06

$1.4 E+00$

111,000

$<1.000$

7.4

$<50$

$<0.24$

$<0.090$

$<1.0$

$<1.0$

$<1.0$

$<1.0$

$1.5 E+03 \quad 1.1 E+03$

$<20$

$<8.0$
$<2.0$

$<8.0$

$<8.0$

$<2.0$

$<1.0$
1.6
$<0.30$
$<1.0$
$<1.0$
$<1.0$
$<0.0060$
$<1.0$
169
$<2.0 E+00$
$<4.0$
$<3.0$
$<0.0050$
723
43
$<0.20$
$<0.50$

Mod Unit Lab Flag

$\begin{array}{lll}\mu \mathrm{g} / \mathrm{L} & \text { GE } & 0 \\ \mu \mathrm{g} / \mathrm{L} & \text { GE } & 0\end{array}$

$\mu v / L \quad$ GE

$\mu / L$ GE O

$\mu / L$ GE O

$\mu \mathrm{g} / \mathrm{L} \quad \mathrm{GE}$

$\mu / L$ GE 0

$\mathrm{PCi} / \mathrm{L}$ GE

$\mu / L$ GE

$\mu \mathrm{g} / \mathrm{L}$ GE 0

$\mu d / L$ GE 0

$\mu / L$

$\mu \mathrm{g} / \mathrm{L}$

1.020

$\begin{array}{lllll}5.9 E+00 & & \mathrm{pCi} / \mathrm{L} & \mathrm{GE} & 0 \\ 6.9 & \mathrm{~J} & \mathrm{pH} & \mathrm{GE} & 0\end{array}$

$<5.0$

2.420

$<2.0$

30.300

$<2.0$

6.220

140

5,870

$<1.0$

$<1.0$

$<1.0$

8. $7 E+05$

$<1.0 E+00$

$113.000 \mathrm{JV}$

$<1,000$

$<5.0$

310

$<0.24$

$<0.090$

$<1.0$

$<1.0$

$<1.0$

$<1.0$

8. $O E+02$

$<20$

$<8.0$

$<2.0$

$\mathrm{J} 1$
4

$\mu \mathrm{g} / \mathrm{L}$

$\mu \mathrm{g} / \mathrm{L}$ GE 0

$\mu \mathrm{g} / \mathrm{L}$ GE 0

Zinc

$<2.0$ 
WELL HSB 145C

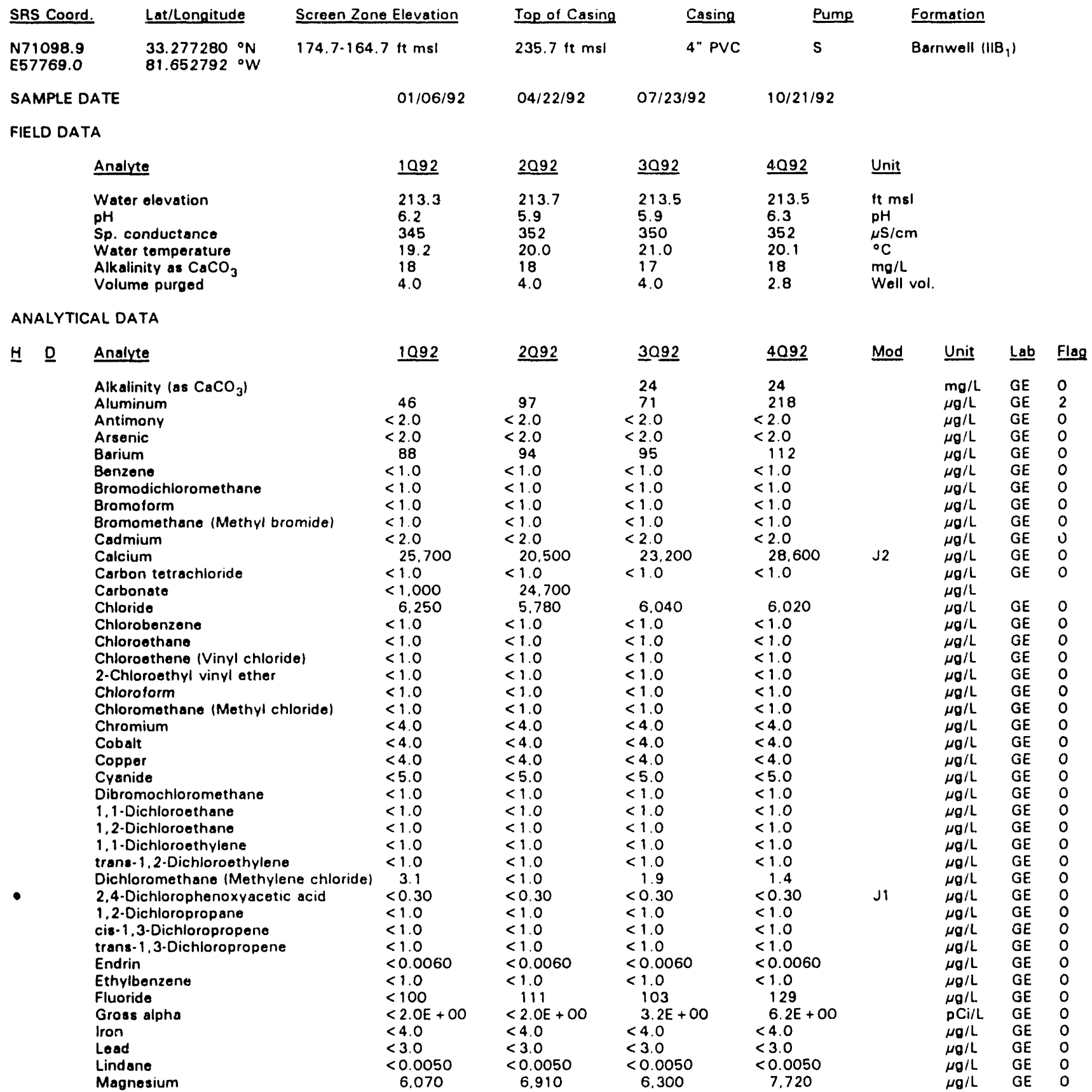

Note: Flagging levels, modifiers, and laboratories are for 4 th quarter 1992 data only. See Appendix B for flagging criteria. - exceeded holding time for 4th quarter 1992.

- =xceeded final primary drinking water standard for 4th quarter 1992.

H-Area Seepage Basins 


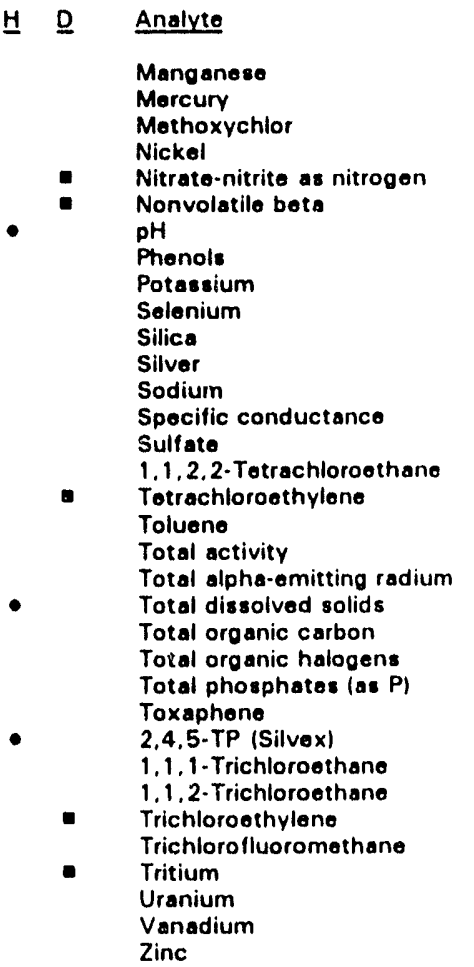

\section{WELL HSB 145D}

$\begin{array}{ll}\text { SRS Coord. } & \text { Lat/Longitude } \\ \text { N71088.0 } & 33.277231 \mathrm{n} N \\ \text { E57753.9 } & 81.652810^{\circ} \mathrm{W} \\ \text { SAMPLE DATE } & \\ \text { FIELD DATA } & \end{array}$

\begin{tabular}{|c|c|}
\hline 1092 & 2092 \\
\hline 88 & 120 \\
\hline$<0.20$ & 0.21 \\
\hline$<0.50$ & $<0.50$ \\
\hline 4.2 & $<4.0$ \\
\hline $\begin{array}{l}33,600 \\
4.1 E+01\end{array}$ & 33,200 \\
\hline $\begin{array}{l}4.1 E+01 \\
6.4\end{array}$ & $\begin{array}{l}4.7 E+01 \\
6.2\end{array}$ \\
\hline$<50$ & $<5.0$ \\
\hline 2.900 & 2.120 \\
\hline$<2.0$ & $<2.0$ \\
\hline $\begin{aligned} & 11.300 \\
< & 2.0\end{aligned}$ & 11.700 \\
\hline $\begin{array}{l}<2.0 \\
27.300\end{array}$ & $<2.0$ \\
\hline $\begin{array}{l}27,300 \\
375\end{array}$ & $\begin{array}{l}28,900 \\
330\end{array}$ \\
\hline$<1.000$ & $<1,000$ \\
\hline$<1.0$ & $<1.0$ \\
\hline 12 & 14 \\
\hline$<1.0$ & $<1.0$ \\
\hline $2.0 E+06$ & $1.8 E+06$ \\
\hline $1.4 E+00$ & $1.9 E+00$ \\
\hline 238,000 & 252,000 \\
\hline$<1,000$ & $<1,000$ \\
\hline 6.4 & 14 \\
\hline$<50$ & 50 \\
\hline$<0.24$ & $<0.24$ \\
\hline$<0.090$ & $<0.090$ \\
\hline$<1.0$ & $<1.0$ \\
\hline$<1.0$ & $<1.0$ \\
\hline 1.8 & 1.9 \\
\hline$<1.0$ & $<1.0$ \\
\hline $2.0 E+03$ & $1.9 E+03$ \\
\hline$<8.0$ & $\begin{array}{c}<8.0 \\
26\end{array}$ \\
\hline
\end{tabular}

\begin{tabular}{|c|c|c|c|c|}
\hline 4092 & Mod & Unit & $\underline{\text { Lab }}$ & Flo \\
\hline 127 & & $\mu \mathrm{g} / \mathrm{L}$ & GE & 2 \\
\hline 0.26 & & $\mu g / L$ & GE & 0 \\
\hline$<0.50$ & & $\mu \sigma / L$ & GE & 0 \\
\hline 4.6 & & $\mu \mathrm{g} / \mathrm{L}$ & GE & 0 \\
\hline 39.200 & & $\mu \sigma / L$ & $\mathrm{GE}$ & 2 \\
\hline $\begin{array}{l}5.2 \mathrm{E}+01 \\
6.4\end{array}$ & & $\mathrm{pCi} / \mathrm{L}$ & GE & 2 \\
\hline $\begin{array}{r}6.4 \\
<5.0\end{array}$ & $J$ & pH & GE & 0 \\
\hline $\begin{aligned}< & 5.0 \\
& 2.380\end{aligned}$ & & $\mu \sigma / L$ & GE & 0 \\
\hline 2.380 & & $\mu_{0} / L$ & GE & 0 \\
\hline $\begin{aligned}< & 2.0 \\
& 11.200\end{aligned}$ & J1 & $\mu \mathrm{g} / \mathrm{L}$ & GE & 0 \\
\hline 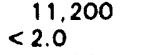 & & $\begin{array}{l}\mu_{g} / \mathrm{L} \\
\mu_{\mathrm{g}} / \mathrm{L}\end{array}$ & $\begin{array}{l}\text { GE } \\
\text { GE }\end{array}$ & $\begin{array}{l}0 \\
0\end{array}$ \\
\hline 34.200 & & $\mu \sigma / L$ & GE & 0 \\
\hline 310 & & $\mu \mathrm{S} / \mathrm{cm}$ & GE & 1 \\
\hline$<1.000$ & & $\mu \mathrm{g} / \mathrm{L}$ & GE & 0 \\
\hline$<1.0$ & & $\mu_{\sigma} / L$ & GE & 0 \\
\hline 32 & & $\mu g / L$ & GE & 2 \\
\hline$<1.0$ & & $\mu \sigma / L$ & GE & 0 \\
\hline $1.8 E+06$ & & $\mathrm{pCi} / \mathrm{L}$ & $E M$ & 0 \\
\hline $4.0 E+00$ & & $\mathrm{pCi} / \mathrm{L}$ & $\mathrm{GE}$ & 1 \\
\hline 253,000 & JV & $\mu \mathrm{g} / \mathrm{L}$ & GE & 0 \\
\hline$<1,000$ & & $\mu g / L$ & GE & 0 \\
\hline 7.1 & & $\mu \mathrm{g} / \mathrm{L}$ & $G E$ & 0 \\
\hline$<50$ & & $\mu g / L$ & $\mathrm{GE}$ & 0 \\
\hline$<0.24$ & & $\mu \mathrm{g} / \mathrm{L}$ & GE & 0 \\
\hline$<0.090$ & J1 & $\mu_{\mathrm{g}} / \mathrm{L}$ & GE & 0 \\
\hline$<1.0$ & & $\mu_{0} / \mathrm{L}$ & GE & 0 \\
\hline$<1.0$ & & $\mu g / \mathrm{L}$ & GE & 0 \\
\hline 35 & & $\mu \mathrm{g} / \mathrm{L}$ & GE & 2 \\
\hline$<1.0$ & & $\mu g / L$ & GE & 0 \\
\hline $1.7 E+03$ & & $\mathrm{pCi} / \mathrm{mL}$ & GE & 2 \\
\hline$<20$ & & $\mu \sigma / L$ & $\mathrm{GE}$ & 0 \\
\hline$<8.0$ & & $\mu_{0} / \mathrm{L}$ & $\mathrm{GE}$ & 0 \\
\hline & & $\mu g / L$ & GE & 0 \\
\hline
\end{tabular}

\section{Analyte}

Water elevation

pH

Sp. conductance

Water temperature

Alkalinity as $\mathrm{CaCO}_{3}$

Volume purged

\section{ANALYTICAL DATA}

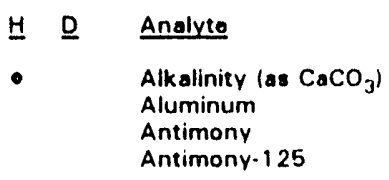

\section{Screen Zone Elovation \\ $194.2 \cdot 184.2 \mathrm{ft} \mathrm{ms}$}

$01 / 06 / 92$

\begin{tabular}{lrrrl} 
Top of Casing & Casing & $\frac{\text { Pump }}{\text { Formation }}$ & \\
\hline $236.2 \mathrm{tt} \mathrm{msl}$ & $4 "$ PVC & S & Wator table (II8.) \\
$04 / 22 / 92$ & $07 / 23 / 92$ & $10 / 21 / 92$ &
\end{tabular}

$\begin{array}{llll}\underline{2092} & \underline{3092} & \underline{4092} & \text { Unit } \\ 222.1 & 221.1 & 220.4 & \mathrm{tt} \mathrm{msl} \\ 5.5 & 5.5 & 5.4 & \mathrm{pH} \\ 361 & 359 & 359 & \mu \mathrm{H} / \mathrm{cm} \\ 19.8 & 20.1 & 20.0 & \mathrm{o} \\ 13 & 12 & 10 & \mathrm{mg} / \mathrm{L} \\ 4.0 & 4.0 & 2.5 & \text { Well vol. }\end{array}$

\begin{tabular}{|c|c|c|c|c|c|c|}
\hline 1092 & $\underline{2092}$ & $\underline{3092}$ & 4092 & Mod & $\underline{\text { Unit }}$ & Lab \\
\hline & & 18 & & JV & $\mathrm{mg} / \mathrm{L}$ & $\mathrm{GE}$ \\
\hline 306 & 411 & 478 & 1,140 & & $\mu g / \mathrm{l}$ & $G E$ \\
\hline & & $\begin{array}{l}<2.0 \\
<2 . O E+01\end{array}$ & $\begin{array}{l}<2.0 \\
<2.0 E+01\end{array}$ & & $\begin{array}{l}\mu \mathrm{g} / \mathrm{h} . \\
\mathrm{pC} / \mathrm{L}\end{array}$ & GP \\
\hline
\end{tabular}

Note: Flagging levels, modifiers, and laboratories are for 4 th quarter 1992 data only. See Appendix B for flagging criteria.

- = exceeded holding time for 4th quarter 1992.

m = exceeded final primary drinking water standard for 4th quarter 1992.

H-Area Seepage Basins

D.182
Fourth Quarter 1992 
WSRC-TR.93-059

Well HSB145D continued

ANALYTICAL DATA

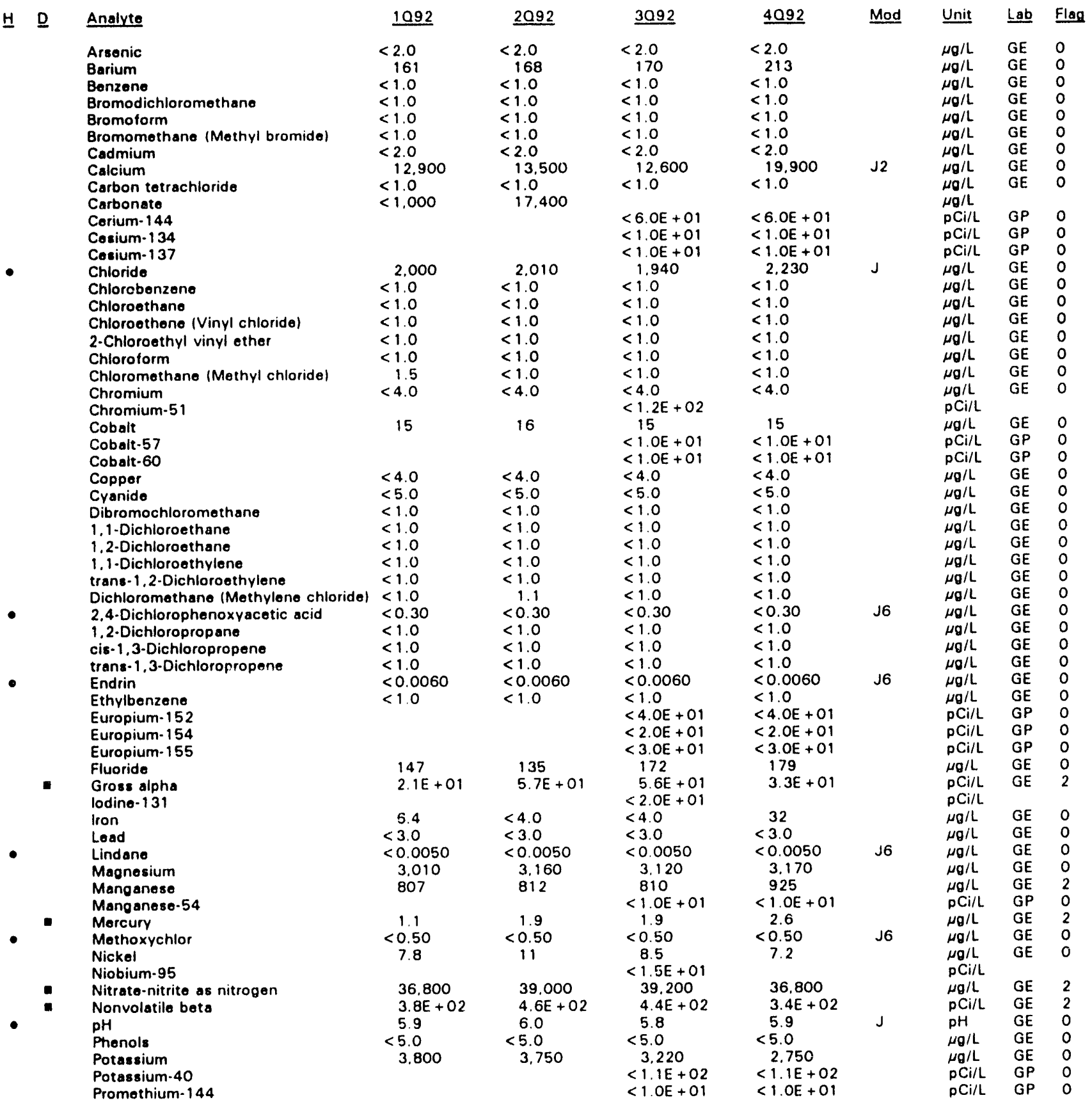

Note: Flagging levels, modifiers, and laboratories are for 4 th quarter 1992 data only. See Appendix B for flagging criteria. - =xceeded holding time for 4th quarter 1992.

- = exceeded final primary drinking water standard for 4th quarter 1992. 
WSRC-TR.93.059

Well HSB145D continued

ANALYTICAL DATA

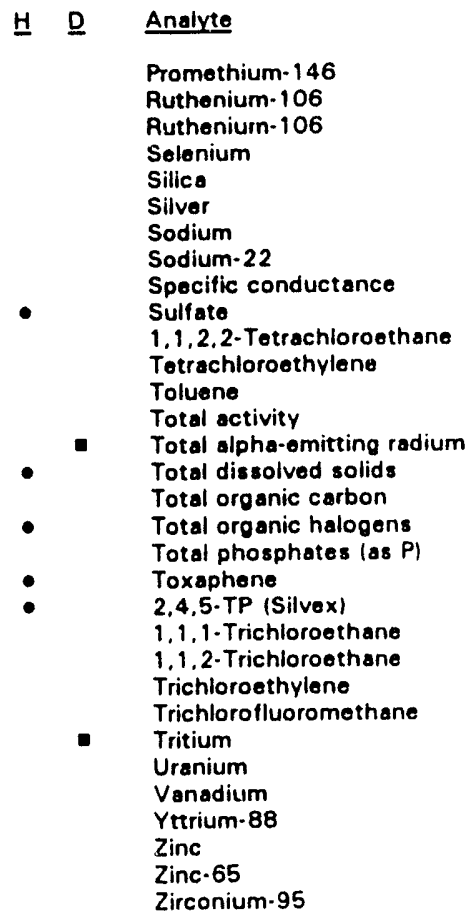

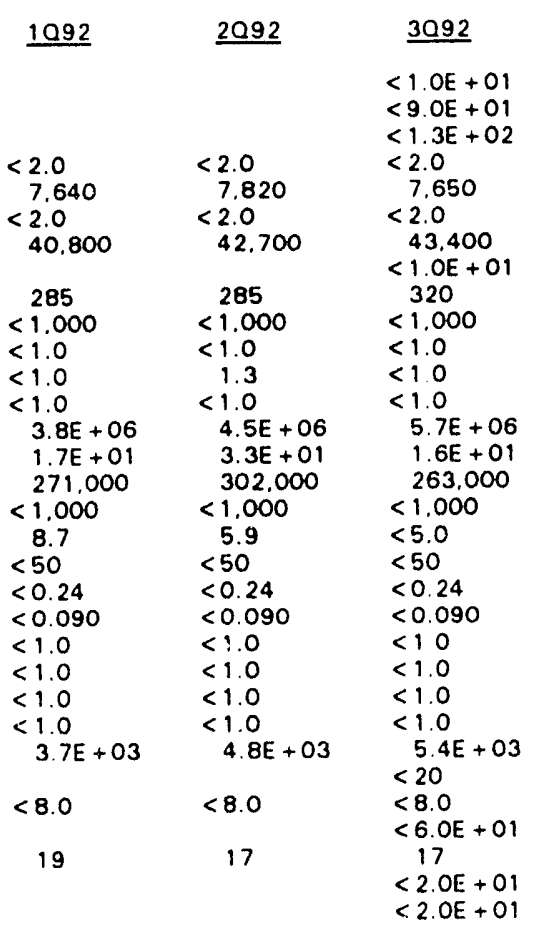

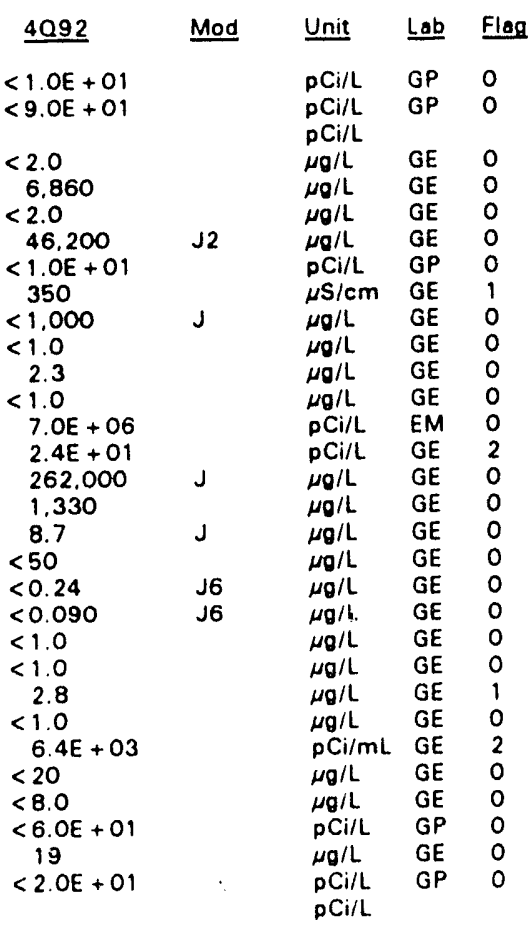

WELL HSB 146A

$\begin{array}{ll}\text { SRS Coord. } & \text { Lat/Longitude } \\ \text { N70478.9 } & 33.2770266^{\circ} \mathrm{N} \\ \text { E58454.0 } & 81.649784{ }^{\circ} \mathrm{W}\end{array}$

Screen Zone Elevation

Top of Casing

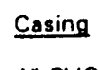

Pump

Formation

$251.6 \mathrm{ft} \mathrm{msl}$

4" PVC

S

U. Congaree (IIA)

SAMPLE DATE

$01 / 10 / 92$

$04 / 03 / 92$

07/23/92

10/23/92

FIELD DATA

Analyte
Water elevation
pH
$\mathrm{Sp}$. conductance
Water temperature
Alkalinity as $\mathrm{CaCO}_{3}$
Volume purged

ANALYTICAL DATA

H D Analyte

Alkalinity (as $\mathrm{CaCO}_{3}$ )

Aluminum

Antimony

Arsenic

Barium

Benzene

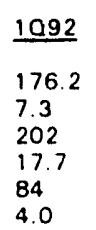

2092
176.1
7.3
209
18.7
83
4.0

$\underline{3092}$
175.8
7.0
202
20.5
94
40

4092
175.8
7.3
206
19.9
81
2.5

Unit

ft $\mathrm{msl}$

pH

${ }^{\mu \mathrm{S} / \mathrm{cm}}$

$\mathrm{mg} / \mathrm{L}$

Well vol.

\begin{tabular}{|c|c|c|c|c|c|c|c|}
\hline 1092 & 2092 & 3092 & 4092 & Mod & Unit & 느므 & Flag \\
\hline $\begin{array}{r}<20 \\
<2.0 \\
<2.0 \\
35 \\
<1.0\end{array}$ & $\begin{aligned}<20 \\
<2.0 \\
<2.0 \\
36 \\
<1.0\end{aligned}$ & $\begin{array}{r}88 \\
<20 \\
<2.0 \\
<2.0 \\
38 \\
<1.0\end{array}$ & $\begin{aligned} & 96 \\
& 119 \\
< & 2.0 \\
< & 2.0 \\
& 34 \\
< & 1.0\end{aligned}$ & & $\begin{array}{l}m g / L \\
\mu g / L \\
\mu g / L \\
\mu g / L \\
\mu g / L \\
\mu g / L\end{array}$ & $\begin{array}{l}G E \\
G E \\
G E \\
G E \\
G E \\
G E\end{array}$ & $\begin{array}{l}0 \\
2 \\
0 \\
0 \\
0 \\
0\end{array}$ \\
\hline
\end{tabular}

Note: Flagging levels, modifiers, and laboratories are for 4th quarter 1992 data only. See Appendix B for flagging criteria.

- exceeded holding time for 4th quarter 1992.

- exceeded final primary drinking water standard for 4th quarter 1992.

H-Area Seepage Basins

D.184 


\section{ANALYTICAL DATA}

\begin{tabular}{|c|c|c|c|c|c|c|c|c|c|}
\hline$\underline{H} \quad \underline{D}$ & Analyte & 1092 & 2092 & 3092 & 4092 & Mlod & Unit & $\underline{\text { Lab }}$ & Flas \\
\hline & 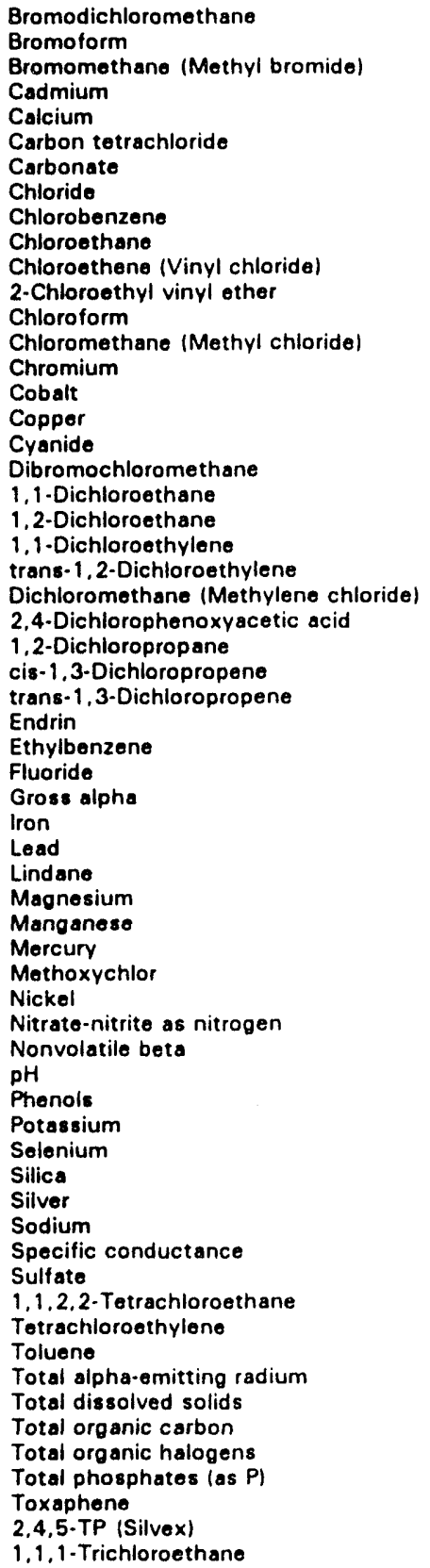 & $\begin{aligned}< & 1.0 \\
< & 1.0 \\
< & 1.0 \\
< & 2.0 \\
& 37.200 \\
< & 1.0 \\
< & 1.000 \\
& 2.750 \\
< & 1.0 \\
< & 1.0 \\
< & 1.0 \\
< & 1.0 \\
< & 1.0 \\
< & 1.0 \\
< & 4.0 \\
< & 4.0 \\
< & 4.0 \\
< & 5.0 \\
< & 1.0 \\
< & 1.0 \\
< & 1.0 \\
< & 1.0 \\
< & 1.0 \\
& 1.1 \\
< & <0.30 \\
< & 1.0 \\
< & 1.0 \\
& <1.0 \\
& <\end{aligned}$ & 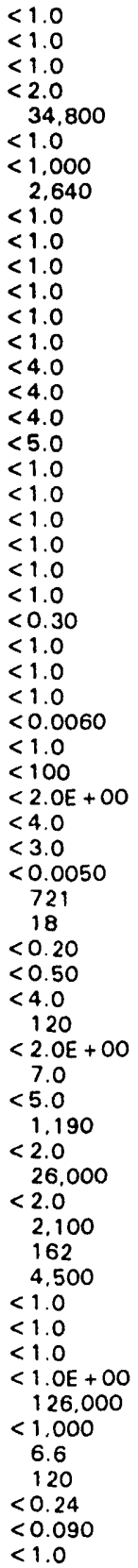 & 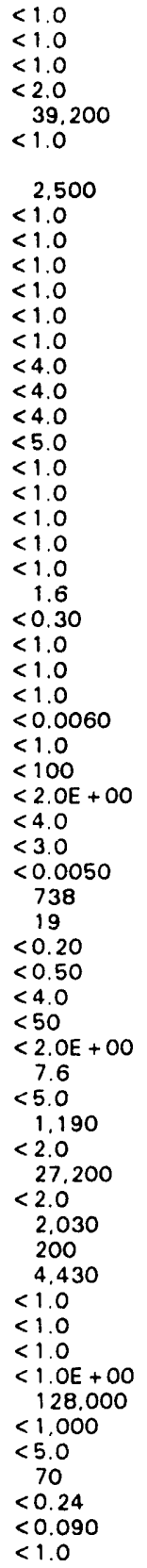 & 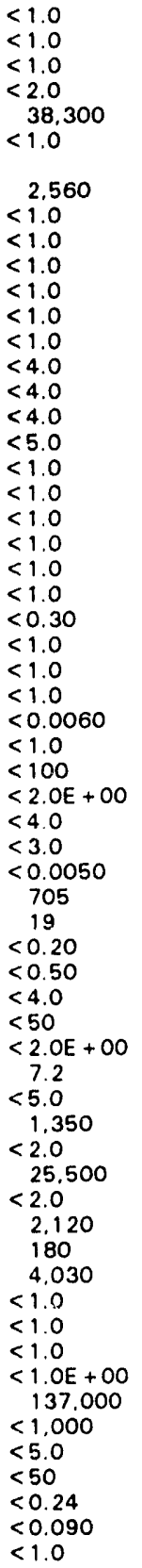 & J2 & 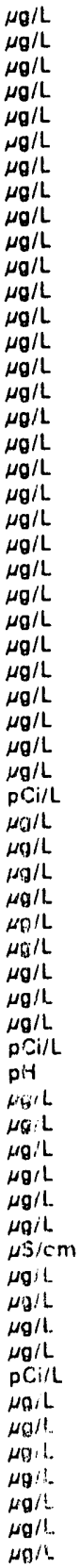 & $\begin{array}{l}\mathrm{GE} \\
\mathrm{GE} \\
\mathrm{GE} \\
\mathrm{GE} \\
\mathrm{GE} \\
\mathrm{GE} \\
\mathrm{GE} \\
\mathrm{GE} \\
\mathrm{GE} \\
\mathrm{GE} \\
\mathrm{GE} \\
\mathrm{GE} \\
\mathrm{GE} \\
\mathrm{GE} \\
\mathrm{GE} \\
\mathrm{GE} \\
\mathrm{GE} \\
\mathrm{GE} \\
\mathrm{GE} \\
\mathrm{GE} \\
\mathrm{GE} \\
\mathrm{GE} \\
\mathrm{GE} \\
\mathrm{GE} \\
\mathrm{GE} \\
\mathrm{GE} \\
\mathrm{GE} \\
\mathrm{GE} \\
\mathrm{GE} \\
\mathrm{GE} \\
\mathrm{GE} \\
\mathrm{GE} \\
\mathrm{GE} \\
\mathrm{GE} \\
\mathrm{GE} \\
\mathrm{GE} \\
\mathrm{GE} \\
\mathrm{GE} \\
\mathrm{GE} \\
\mathrm{GE} \\
\mathrm{GE} \\
\mathrm{GE} \\
\mathrm{GE} \\
\mathrm{GE} \\
\mathrm{GE} \\
\mathrm{GE} \\
\mathrm{GE} \\
\mathrm{GE} \\
\mathrm{GE} \\
\mathrm{GE} \\
\mathrm{GE} \\
\mathrm{GE} \\
\mathrm{GE} \\
\end{array}$ & 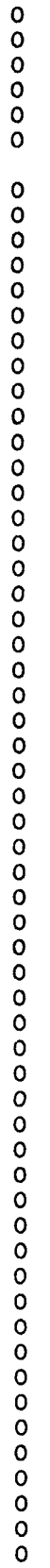 \\
\hline
\end{tabular}

Note: Flagging levels, modifiers, and laboratories are for 4 th quarter 1992 data only. See Appendix $B$ for flagging criteria. - = exceeded holding time for 4th quarter 1992.

- exceeded final primary drinking water standard for 4 th quarter 1992.

H-Area Seepage Basins

$D .185$
Fourth Quarter 1992 
WSRC-TR-93-059

Well HSB146A continised

ANALYTICAL DATA

H D Analyte

1,1,2-Trichloroethane

Trichloroethylene

Trichlorofluoromethane

Tritium

Uranium

Vanadium

Zinc

\begin{tabular}{|c|c|c|}
\hline 1092 & $\underline{2092}$ & 3092 \\
\hline $\begin{array}{l}<1.0 \\
<1.0 \\
<1.0 \\
<7.0 \text { E-O1 }\end{array}$ & $\begin{array}{l}<1.0 \\
<1.0 \\
<1.0 \\
<7 . \text { OE-01 }\end{array}$ & $\begin{array}{l}<1.0 \\
<1.0 \\
<1.0 \\
<7.0 E \cdot 01 \\
<20\end{array}$ \\
\hline $\begin{array}{l}<8.0 \\
<2.0\end{array}$ & $\begin{array}{l}<8.0 \\
<2.0\end{array}$ & $\begin{array}{l}<8.0 \\
<2.0\end{array}$ \\
\hline
\end{tabular}

4092

$<1.0$

$<1.0$

$<1.0$

$<7.0$ E.01

$<20$

$<8.0$

$<2.0$

$\begin{array}{llll}\text { Mod } & \text { Unit } & \text { Lab } & \text { Flag } \\ \mu g / L & G E & 0 \\ \mu g / L & G E & 0 \\ \mu g / L & G E & 0 \\ \mathrm{pCi} / \mathrm{mL} & \mathrm{GE} & 0 \\ \mu \mathrm{g} / \mathrm{L} & \mathrm{GE} & 0 \\ \mu \mathrm{g} / \mathrm{L} & \mathrm{GE} & 0 \\ \mu \mathrm{g} / \mathrm{L} & \mathrm{GE} & 0\end{array}$

\section{W'ELL HSB146C}

$\begin{array}{ll}\text { SAs; Coord. } & \text { Lat/Longitude } \\ \text { N70471.6 } & 33.277041^{\circ} \mathrm{N} \\ \text { E58473.1 } & 81.649719^{\circ} \mathrm{W}\end{array}$

SAMPLE DATE

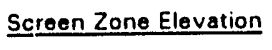

162.3-152.3 ft mst

$01 / 10 / 92$

$\begin{array}{ll}1092 & \underline{2092} \\ 209.8 & 209.9 \\ 7.2 & 9.0 \\ 103 & 84 \\ 17.7 & 19.0 \\ 29 & 22 \\ 4.0 & 5.0\end{array}$

\section{2}

H D Analyte

Alkalinity (as $\mathrm{CaCO}_{3}$ )

Aluminum

Antimony

Arsenic

Barium

Benzene

Bromodichloromethane

Bromoform

Bromomethane (Methyl bromide)

Cadmium

Calcium

Carbon tetrachloride

Carbonate

Chloride

Chlorobenzene

Chloroethane

Chloroethene (Vinyl chloride)

2-Chloroethyl vinyl ether

Chloroform

Chioromethane (Methyl chioride)

Chromium

Cobait

Copper

Cyanide

Dibromochloromethane

1,1-Dichloroethane

1,2-Dichloroethane

1.1-Dichloroethylene

Dichloromethane (Methylene chloride) 1.0

2,4-Dichlorophenoxyacetic acid

\section{2}

$<20$

2.4
$<2.0$

34
$<1.0$

$<1.0$

$<1.0$

$<1.0$

$<2.0$

8.630

$<1.0$

$<1.000$

2.330

$<1.0$

$<1.0$

$<1.0$

$<1.0$

$<1.0$

$<4.0$

$<4.0$

$<4.0$

$<5.0$

$<1.0$

$<1.0$

$<1.0$

$<1.0$

$<0.30$

3092
210.0
7.3
74
19.1
25
4.0

Casing
4" PVC

Pump

s

Formation

Barnwell (IIB,

$07 / 24 / 92$

$10 / 23 / 92$

3092

409

35
111

$<2.0$

2.6
37

37
$<1.0$

$<1.0$

$<10$

$<1.0$

$<0.35$

$\begin{aligned} & 6.560 \\ < & 1.0\end{aligned}$

2.220

$<1.0$

$<1.0$

$<1.0$

$<1.0$

$<1.0$

$<1.0$

2.3

$<0.88$

$<1.1$

$<5.0$

$<1.0$

$<1.0$

$<1.0$

$<1.0$

$<1.0$

2.2

$<0.30$

$\begin{array}{ll}4092 & \text { Unit } \\ 209.8 & \text { ft msl } \\ 7.9 & \mathrm{pH} \\ 76 & \mu \mathrm{S} / \mathrm{cm} \\ 19.3 & { }^{\circ} \mathrm{C} \\ 17 & \mathrm{mg} / \mathrm{L} \\ 3.2 & \text { Well vol. }\end{array}$

26
165
$<2.0$

$<2.0$

34

$<1.0$

$<1.0$

$<1.0$

$<1.0$

$<20$

5.890

$<1.0$

2.320

$<1.0$

$<1.0$

$<1.0$

$<1.0$

$<1.0$

$<1.0$

$<4.0$

$<4.0$

$<4.0$

$<5.0$

$<1.0$

$<1.0$

$<1.0$

$<1.0$

$<1.0$

$<1.0$

$<0.30$

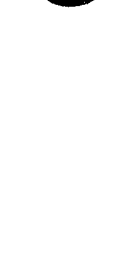


Well HSB146C continued

ANALYTICAL DATA

H D Analyte

1,2-Dichloropropane

cis-1,3-Dichloropropene

trans-1,3-Dichloropropene

Ethylbenzene

Fluoride

Gross alpha

Iron

Lead

Lind ane

Magnesium

Manganese

Mercury

Mathoxychior

Nickel

Nitrate as nitrogen

Nitrate-nitrite as nitrogen

Nonvolatile beta

pH

Phenols

Potassium

Radium-226

Radium-228

Solenium

Silica

Sodium

Specific conductance

Sulfate

1,1,2,2-Tetrachloroethane

Totrachloroethylene

Toluene

Total alpha-emitting radium

Total dissolved solids

Total organic carbon

Total organic halogens

Total phosphates (as P)

Toxaphene

2,4,5-TP (Silvex)

1,1,1-Trichloroethane

1,1,2-Trichloroethane

Trichloroethylene

Trichlorofluoromethane

Tritium

Uranium

Vanadium

Zinc
1092

$<1.0$

$<1.0$

$<1.0$

$<0.0060$

$<1.0$

$<100$

$<2.0 \mathrm{E}+\mathrm{OO}$

$<4.0$

$<3.0$

$<0.0050$

353

$<2.0$

$<0.20$

$<0.50$

$<4.0$

550
$7.1 \mathrm{E}+$

$7.1 E+00$

7.6
$<5.0$

6.020

$<2.0$

4.990

$<2.0$

8.410

102

1.190

$<1.0$

$<1.0$

$<1.0$

$<1.0 \mathrm{E}+00$

58.000

$<1.000$

9.6

$<50$

$<0.24$

$<0.090$

$<1.0$

$<1.0$

$<1.0$

1. $O E+01$

$<8.0$
$<2.0$
2092

$<1.0$

$<1.0$

$<1.0$

$<1.0$

110

$<2.0 E+00$

$<2.0$

$<3.0$

$<0.0050$

241

$<2.0$

$<0.20$

$<0.50$

$<4.0$

710

2. $4 \mathrm{E}+00$

7.6
$<5.0$

4.440

$<2.0$

6.110

$<2.0$

70

70
1.110

$<1.0$

$<1.0$

$<10$

$<1 . O E+00$

38.000

$<1.000$

$<.3$
80

80

$<0.24$

$<0.090$

$<1.0$
$<1.0$

$<1.0$

1. $1 E+01$

$<8.0$
$<2.0$
3092

$<1.0$

$<1.0$

$<1.0$
$<0.0060$

$<1.0$

105

1. $2 E+00$

7.9

$<2.0$

$<0.0050$

249

1.2

$<0.20$

$<0.50$

$<3.1$

581

$6.7 E+00$

8.9

$<5.0$

4.550

2. $7 E+00$

2. $7 E+0 O$

$<2.0$

6.100

$<0.70$

6.190

79

1.030

$<1.0$

$<1.0$

$<1.0$

$<1.0 E+O O$

46.000

$<500$

19

120

$<0.24$

$<0.090$

$<1.0$

$<1.0$

$<1.0$

$<10$

1. $2 E+01$

$<0.030$

3.7
7.2
4092 Mod Unit Lab Flag

$<1.0$

$<1.0$

$<1.0$
$<0.0060$

$<1.0$

$<100$

$<2.0 E+00$

16

$<3.0$

$<0.0050$

307

$<2.0$

$<0.20$

$<0.50$

$<4.0$

650

$<2.0 E+00$

7.4

$<5.0$

4.040

$<2.0$

5.120

$<2.0$

5,940

60

$<1.000$

$<1.0$

$<1.0$

$<1.0$

$<1.0 E+00$

45,000

$<1,000$

$<5.0$

$<50$

$<0.24$

$<0.090$

$<1.0$

$<1.0$

$<1.0$

$<10$

$9.9 E+00$

$<20$

$<8.0$

2.0 $\mu g / L \quad G E \quad 0$

$\mu g / L$ GE $\quad 0$

$\mu \mathrm{g} / \mathrm{L}$ GE 0

$\mu g / L \quad G E$

$\mu g / L$ GE 0

PCi/L GE

$\mu g / L$ GE

$\mu \mathrm{O} / \mathrm{L} \quad \mathrm{GE}$

$\mu \mathrm{g} / \mathrm{L} \quad \mathrm{GE}$

$\mu \mathrm{g} / \mathrm{L}$ GE

UE $O$

$\mu g / L$ GE 0

$\mu g / L \quad G E$

$\mu \mathrm{g} / \mathrm{L}$ GE 0

$\mu g / L$ GE 0

$\mathrm{pCi}$ GE

$\begin{array}{lll}\mathrm{pH} & \mathrm{GE} & 0 \\ \mu \mathrm{g} / \mathrm{L} & \mathrm{GE} & 0\end{array}$

$\mu g / L$ GE 0

pCill

$\mathrm{pCi} / \mathrm{L}$ GE 0

$\mu \mathrm{g} / \mathrm{L}$ GE 0

$\mu g / L$ GE 0

$\mu \mathrm{g} / \mathrm{L}$ GE 0

$\mu \mathrm{S} / \mathrm{cm}$ GE

NSICM GE

$\begin{array}{lll}\mu \mathrm{g} / \mathrm{L} & \mathrm{GE} & 0 \\ \mu \mathrm{g} / \mathrm{L} & \mathrm{GE} & 0\end{array}$

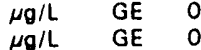

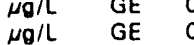

$\mathrm{pCi} / \mathrm{G}$ GE

$\mu g / L$ GE 0

$\mu \mathrm{g} / \mathrm{L}$ GE 0

$\mu g / L$ GE 0

$\mu / \mathrm{L}$ GE

$\mu g / L$ GE 0

$\mu \mathrm{g} / \mathrm{L} \quad \mathrm{GE} \quad 0$

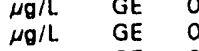

$\mu g / L$ GE 0

$\mu \mathrm{g} / \mathrm{L} \quad \mathrm{GE}$

$\mathrm{pCi} / \mathrm{mL}$ GE

$\begin{array}{lll}\mu g / L & G E & 0 \\ \mu g / L & G E & \end{array}$

$\begin{array}{lll}\mu g / L & G E & 0 \\ \mu g / L & G E & 0\end{array}$

Note: Flagging levels, modifiers, and laboratories are for 4 th quarter 1992 data only. See Appendix B fop flagging criteria. - = exceeded holding time for 4th quarter 1992.

- =xceeded final primary drinking water standard for 4th quarter 1992. 
WSRC-TR-93.059

WELL HSB146D

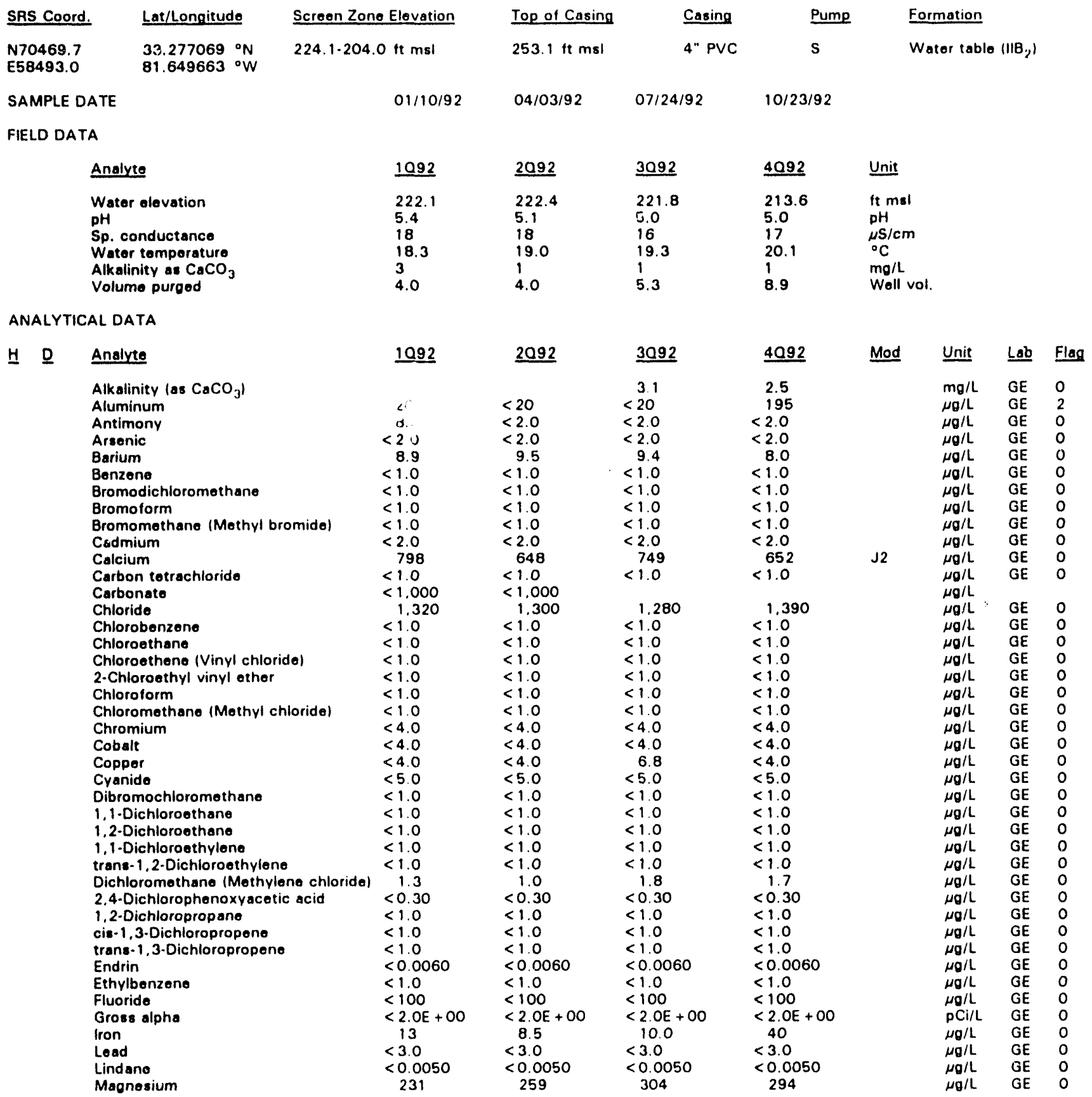

Note: Flagging levels, modifiers, and laboratories are for 4 th quarter 1992 data only. See Appendix $B$ for flagging criteria.

- =xceeded holding time for 4 th quarter 1992.

- exceeded final primary drinking water standard for 4 th quarter 1992.

H-Area Seepage Basins

D.188

Fourth Quarter 1992 
WSRC-TR-93.059

Well HSB 1460 continued

ANALYTICAL DATA

H

Andyte
Manganese
Mercury
Methoxychlor
Nickel
Nitrate-nitrite as nitrogen
Nonvolatile beta
pH
Phenols
Potassium
Selenium
Silica
Silver
Sodium
Specific conductance
Sulfate
1,1,2,2-Tetrachloroethane
Tetrachloroethylene
Toluene
Total alpha-emitting radium
Total dissolved solids
Total organic carbon
Total organic halogens
Total phosphates (as P)
Toxaphene
$2,4,5-$ TP (Silvex)
$1,1,1$-Trichloroethane
1,1,2-Trichloroethane
Trichloroethylene
Trichlorofluoromethane
Tritium
Uranium
Vanadium
Zinc

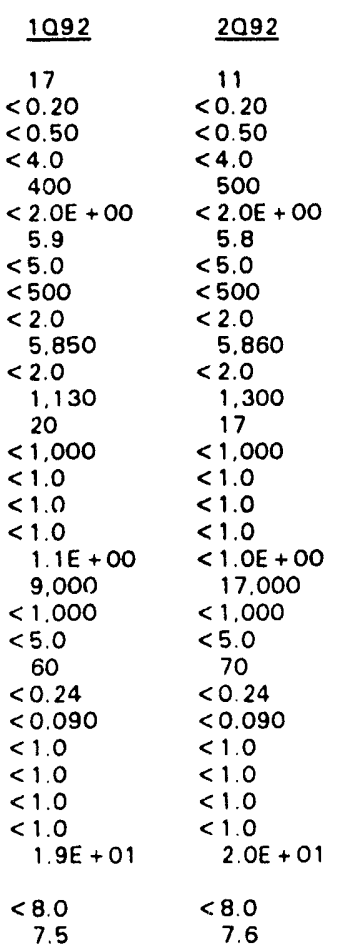

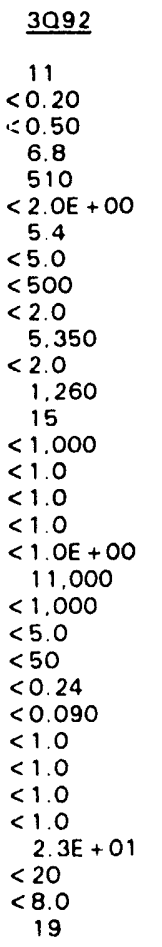

\begin{tabular}{|c|c|c|c|c|}
\hline 4092 & Mod & Unit & $\underline{\mathrm{Lab}}$ & Flas \\
\hline 7.8 & & $\mu g / \mathrm{L}$ & $\mathrm{GE}$ & 0 \\
\hline$<0.20$ & & $\mu g / \mathrm{L}$ & $\mathrm{GE}$ & 0 \\
\hline$<0.50$ & & $\mu_{\theta} / \mathrm{L}$ & $\mathrm{GE}$ & 0 \\
\hline$<4.0$ & & $\mu g / L$ & GE & 0 \\
\hline 510 & & $\mu g / L$ & GE & 0 \\
\hline $2.1 E+00$ & & $\mathrm{pCi} / \mathrm{L}$ & GE & 0 \\
\hline 5.8 & $J$ & $\mathrm{pH}$ & GE & 0 \\
\hline $\begin{array}{l}<5.0 \\
<500\end{array}$ & & $\mu g / L$ & GE & 0 \\
\hline $\begin{array}{l}<500 \\
<2.0\end{array}$ & & $\mu g / L$ & $\begin{array}{l}\mathrm{GE} \\
\mathrm{GE}\end{array}$ & $\begin{array}{l}0 \\
0\end{array}$ \\
\hline $\begin{array}{l}<2.0 \\
\quad 5.530\end{array}$ & & $\mu g / L$ & GE & 0 \\
\hline$<2.0$ & & $\mu \mathrm{g} / \mathrm{L}$ & $\mathrm{GE}$ & 0 \\
\hline 1.210 & & $\mu g / L$ & GE & 0 \\
\hline 18 & & $\mu \mathrm{S} / \mathrm{cm}$ & GE & 0 \\
\hline$<1,000$ & & $\mu_{\theta} / L$ & GE & 0 \\
\hline$<1.0$ & & $\mu g / L$ & GE & 0 \\
\hline$<1.0$ & & $\mu \mathrm{g} / \mathrm{L}$ & $\mathrm{GE}$ & 0 \\
\hline$<1.0$ & & $\mu g / L$ & $\mathrm{GE}$ & 0 \\
\hline$<1 . \mathrm{OE}+00$ & & $\mathrm{pCi} / \mathrm{L}$ & GE & 0 \\
\hline 17,000 & V & $\mu g / L$ & $\mathrm{GE}$ & 0 \\
\hline$<1,000$ & & $\mu g / L$ & $G E$ & 0 \\
\hline$<5.0$ & & $\mu \mathrm{g} / \mathrm{L}$ & GE & 0 \\
\hline $\begin{array}{l}<50 \\
<0.24\end{array}$ & & $\mu g / L$ & $\mathrm{GE}$ & 0 \\
\hline$<0.24$ & & $\mu g / L$ & $G E$ & 0 \\
\hline$<0.090$ & & $\mu g / L$ & $\mathrm{GE}$ & 0 \\
\hline$<1.0$ & & $\mu g / L$ & $\mathrm{GE}$ & 0 \\
\hline$<1.0$ & & $\mu_{g} / L$ & $\mathrm{GE}$ & 0 \\
\hline$<1.0$ & & $\mu g / L$ & $\mathrm{GE}$ & 0 \\
\hline$<1.0$ & & $\mu g / L$ & GE & 0 \\
\hline $2.0 E+01$ & & $\mathrm{pCi} / \mathrm{mL}$ & GE & 2 \\
\hline $\begin{array}{l}<20 \\
<8.0\end{array}$ & & $\mu g / L$ & $G E$ & $\begin{array}{l}0 \\
0\end{array}$ \\
\hline $\begin{array}{r}<8.0 \\
4.0\end{array}$ & & $\begin{array}{l}\mu \mathrm{g} / \mathrm{L} \\
\mu \mathrm{g} / \mathrm{L}\end{array}$ & $\begin{array}{l}\mathrm{GE} \\
\mathrm{GE}\end{array}$ & 0 \\
\hline 4.0 & & & & \\
\hline
\end{tabular}

WELL HSB147D

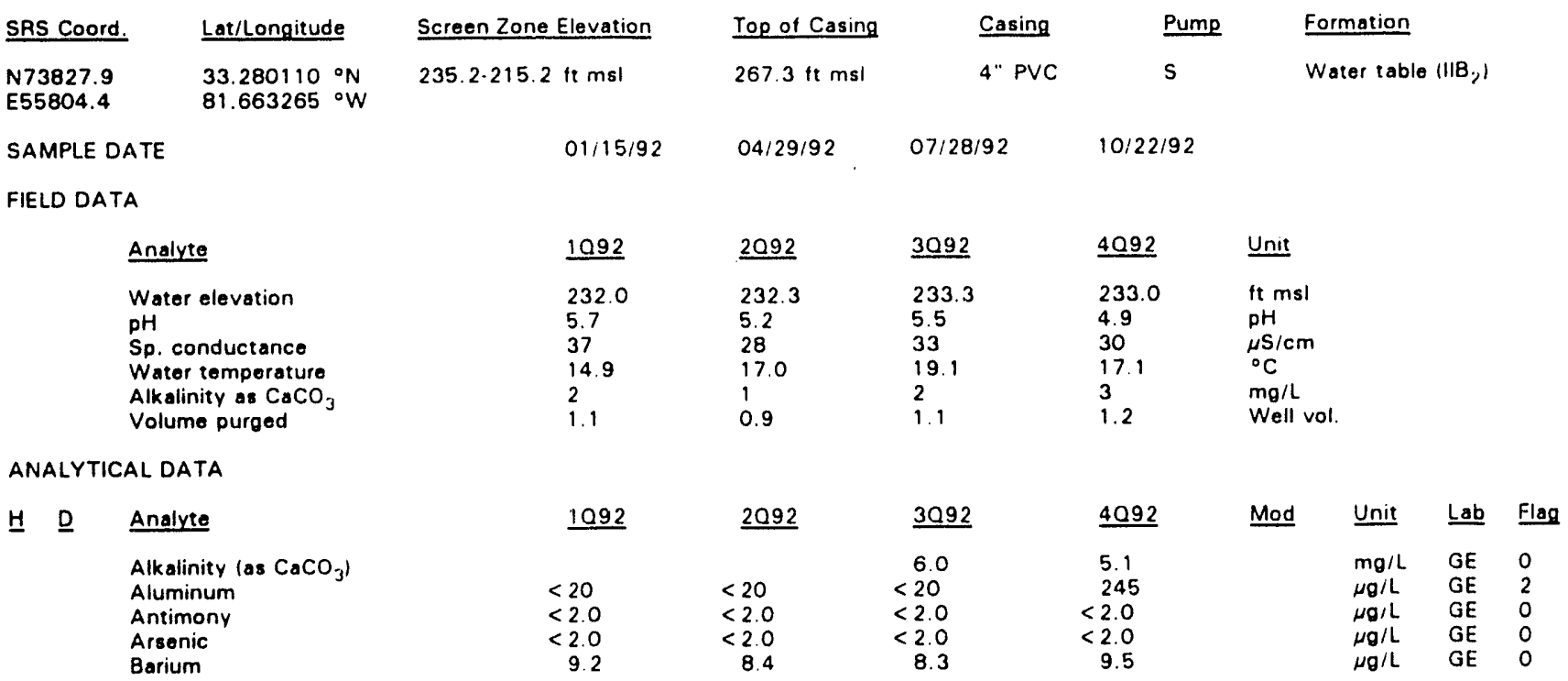

Note: Flagging levels, modifiers, and laboratories are for 4th quarter 1992 data only. See Appendix 8 for flagging criteria.

- =xceeded holding time for 4th quarter 1992.

- = exceeded final primary drinking water standard for 4th quarter 1992.

H-Area Seepage Basins

D.189

Fourth Quarter 1992 
WSRC-TR-93-059

Well HSB147D continued

ANALYTICAL DATA

H $\underline{D}$

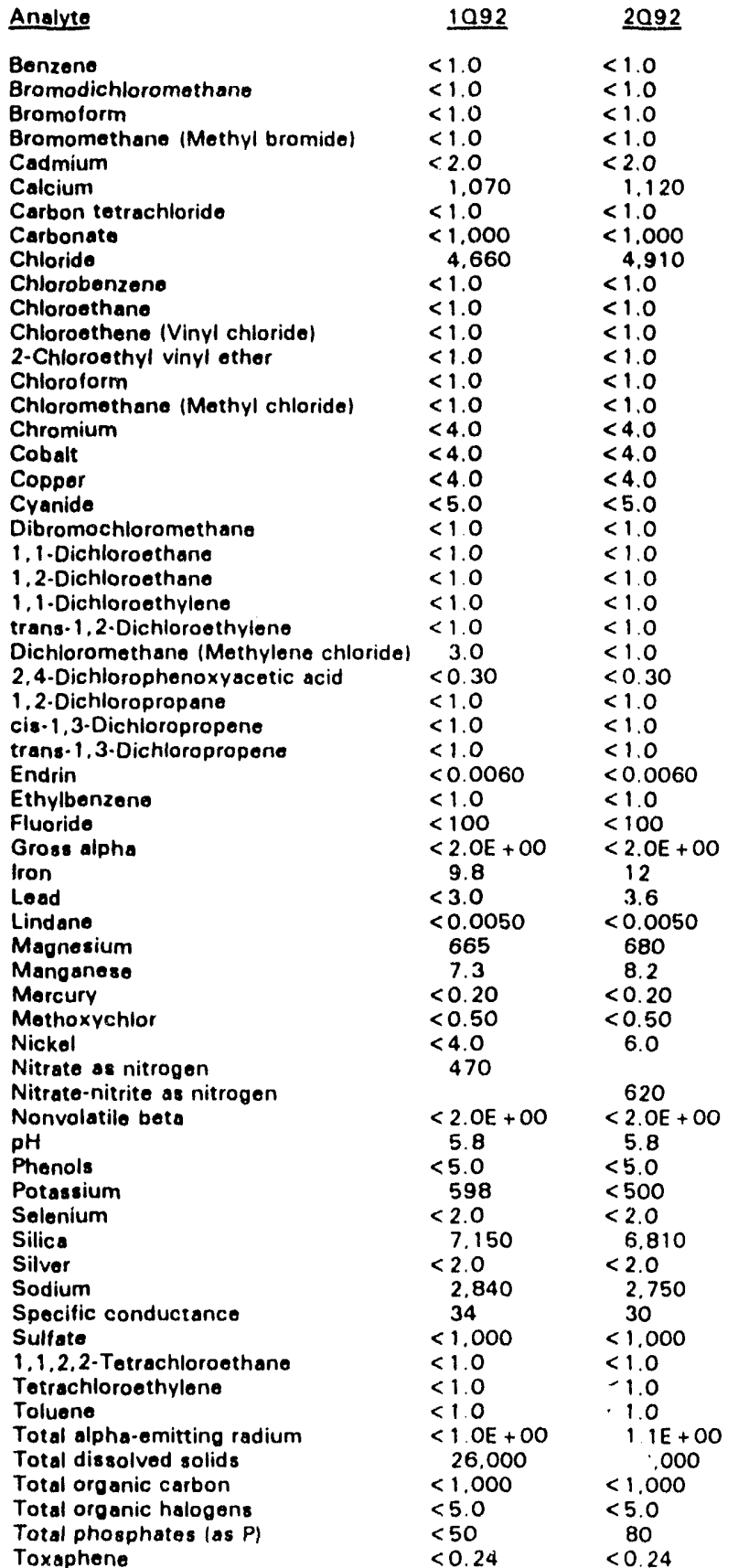

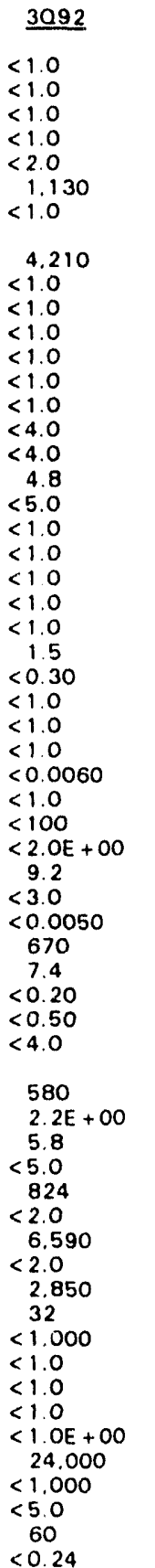

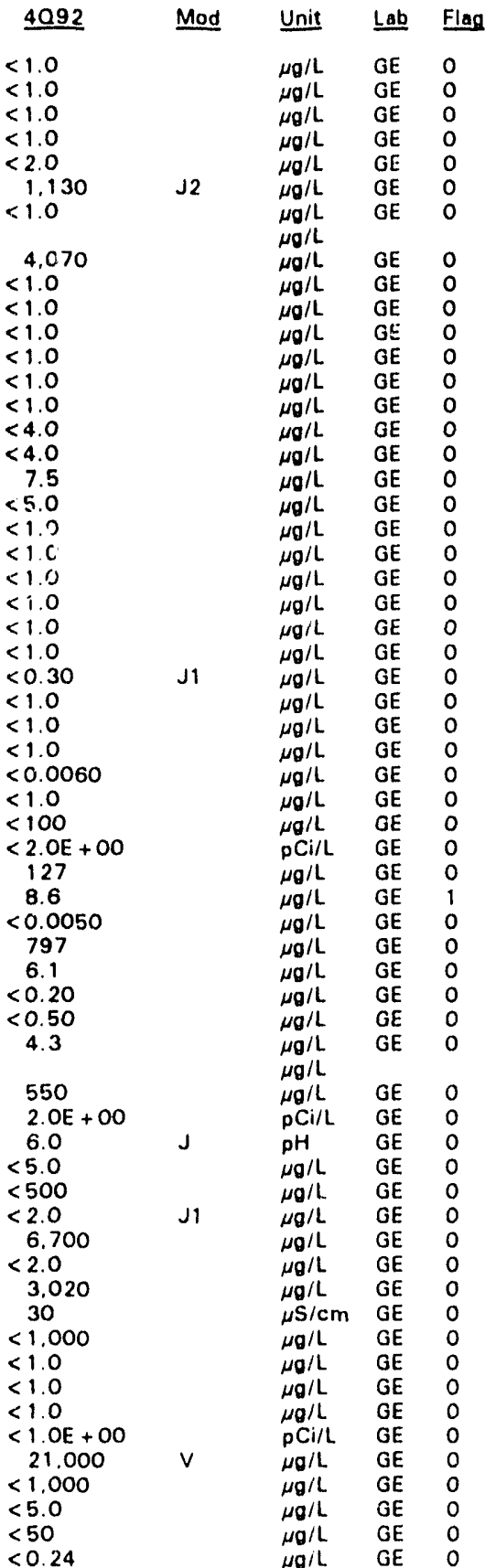

Note: Flagging levels, modifiers, and laboratories are for 4 th quarter 1992 data only. See Appendix 8 for flagging criteria. - =xceeded holding time for 4th quarter 1992.

- =xceeded final primary drinking water standard for 4th quarter 1992. 
WSRC.TR.93.059

Well HSB147D continued

ANALYTICAL DATA

H D $\quad$ Analyte
2.4.5-TP (Silvex)
1.1.1. Trichloroethane
1.1.2.Trichloroethane
Trichloroethylene
Trichlorofluoromethane
Tritium
Uranium
Vanadium
Zinc

$\begin{array}{lll}\frac{1092}{} & \underline{2092} & \underline{3092} \\ <0.090 & <0.090 & <0.090 \\ <1.0 & <1.0 & <1.0 \\ <1.0 & <1.0 & <1.0 \\ <1.0 & <1.0 & <1.0 \\ <1.0 & <1.0 & <1.0 \\ 2.4 \mathrm{E}+01 & 2.2 \mathrm{E}+01 & 2.1 \mathrm{E}+01 \\ <8.0 & <8.0 & <20 \\ 23 & 23 & <8.0 \\ & & 18\end{array}$

$\begin{aligned} & 4092 \\ < & 0.090 \\ < & 1.0 \\ < & 1.0 \\ < & 1.0 \\ < & 1.0 \\ & 1.8 E+01 \\ < & 20 \\ < & 8.0 \\ & 22\end{aligned}$

$\begin{array}{llll}\text { Mod } & \text { Unit } & \text { Lab } & \text { Flag } \\ \text { J1 } & & & \\ & \mu g / L & G E & 0 \\ & \mu g / L & G F & 0 \\ & \mu g / L & G E & 0 \\ & \mu g / L & G E & 0 \\ & \mu g / L & G E & 0 \\ & \mathrm{DCi} / \mathrm{mL} & \mathrm{GE} & 1 \\ \mu \mathrm{g} / \mathrm{L} & \mathrm{GE} & 0 \\ \mu g / L & \mathrm{GE} & 0 \\ & \mu g / \mathrm{L} & \mathrm{GE} & 0\end{array}$

\section{WELL HSB148C}

$\begin{array}{ll}\text { SAS Coord. } & \text { Lat/Longitude } \\ \text { N70151.5 } & 33.271228{ }^{\circ} \mathrm{N} \\ \text { E55344.2 } & 81.657336{ }^{\circ} \mathrm{W}\end{array}$

SAMPLE DATE

FIELD DATA

\section{Screen Zone Elevation \\ 168.9.158.9 ft msl}

$01 / 15 / 92$

\section{Top of Casing}

$250.9 \mathrm{ft} \mathrm{msl}$

$04 / 24 / 92$

2092
201.8
10.6
298
17.8
64
0.8

ANALYTICAL DATA

\section{H D Analyte}

Alkalinity (as $\mathrm{CaCO}_{3}$ )

Aluminum

Antimony

Arsenic

Barium

Benzene

Brómodichloromethane

Bromoform

Bromomethane (Methyl bromide)

Cadmium

Calcium

Carbon tetrachloride

Carbonate

Chloride

Chlorobenzene

Chloroethane

Chloroethene (Vinyl chloride)

2-Chloroethyl vinyl ether

Chloroform

Chloromethane (Methyl chloride)

Chromium

Cobalt

Copper

Cyanide

Dibromochloromethane

1.1-Dichloroethane

1.2-Dichloroethane

1.1-Dichloroethylene

trans-1.2-Dichloroethylene

1092
201.9
11.7
345
16.3
71
0.9

\section{2}

1.040

$<2.0$

$<2.0$
41

41
$<1.0$

$<1.0$

$<1.0$

$<1.0$

$<2.0$

23.100

$<1.0$

23.200

1.690

$<1.0$

$<1.0$

$<1.0$

$<1.0$

$<1.0$

$<1.0$

$<4.0$

$<4.0$

$<4.0$

5.7

$<1.0$

$<1.0$

$<1.0$

$<1.0$

$<1.0$

\section{2}

988

$<2.0$
$<2.0$

42

42
$<1.0$

$<1.0$

$<1.0$

$<1.0$

$<2.0$

24.200

$<1.0$

29.000

1.740

$<1.0$

$<1.0$

$<1.0$

$<1.0$

$<1.0$

$<1.0$

$<4.0$

$<4.0$

$<4.0$

$<5.0$

$<1.0$

$<1.0$

$<1.0$

$<1.0$
$<1.0$

92
8
0
0
0
0
0
0
200
0
.000
40
0
0
0
0
0
0
0
0
0
0
0
0
0

$\begin{array}{lll}\text { Casing } & \text { Pump } & \text { Formation } \\ \text { 4" PVC } & \text { S } & \text { Barnwoll (IIB, ) }\end{array}$

$07 / 24 / 92$

$10 / 23 / 92$

$\begin{array}{lll}\underline{3092} & \underline{4092} & \text { Unit } \\ 201.5 & 201.6 & \mathrm{ft} \mathrm{msl} \\ 10.8 & 11.1 & \mathrm{pH} \\ 314 & 290 & \mu \mathrm{S} / \mathrm{cm} \\ 19.3 & 17.1 & { }^{\circ} \mathrm{C} \\ 68 & 68 & \mathrm{mg} / \mathrm{L} \\ 0.9 & 0.8 & W e l l \mathrm{vol}\end{array}$

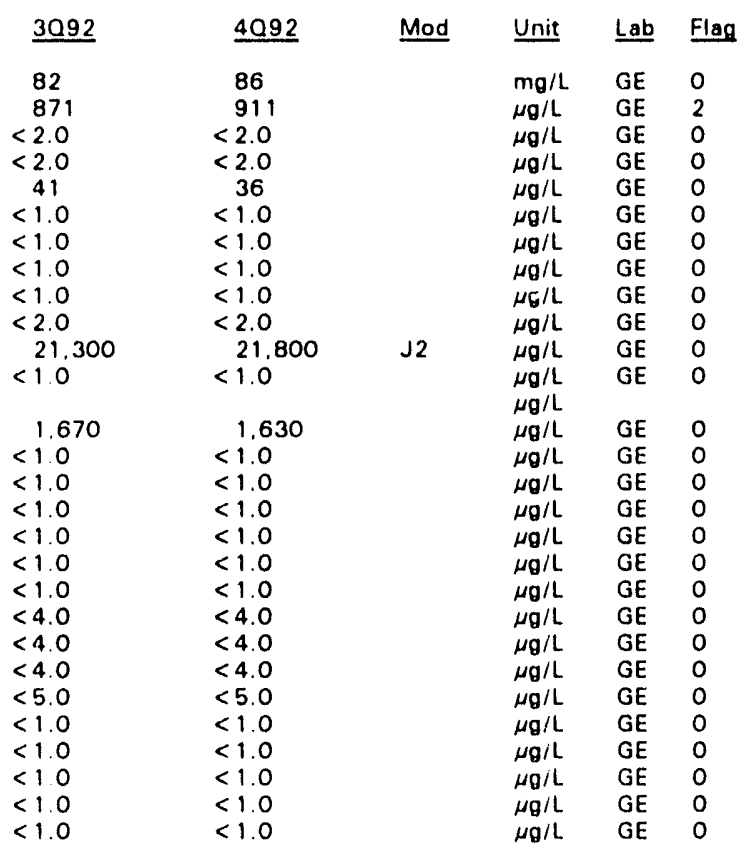

Note: Flagging levels, modifiers, and laboratories are for 4 th quarter 1992 data only. See Appendix $B$ for flagging criteria. - = exceeded holding time for 4th quarter 1992.

- =xceeded final primary dririking water standard for 4th quarter 1992.

H-Area Seepage Basins 
Well HSB148C continued

ANALYTICAL DATA

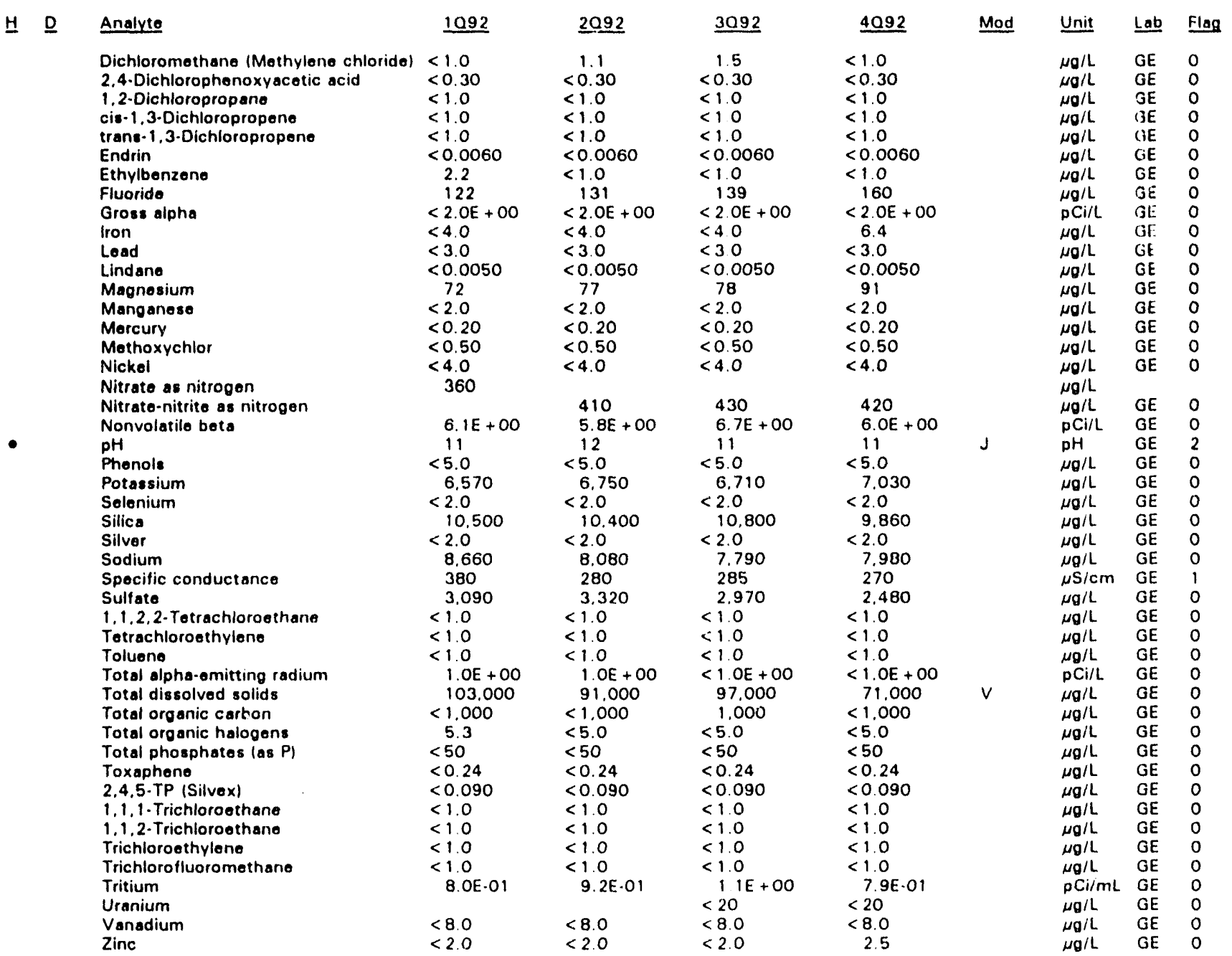

Note: Flagging levels, modifiers, and laboratories are for 4th quarter 1992 data only. See Appendix B for flagging criteria.

- =xceeded holding time for 4th quarter 1992.

- exceeded final primary drinking water standard for 4th quarter 1992.

H-Area Seepage Basins

D-192
Fourth Quarter 1992 
WELL HSB148D

\begin{tabular}{|c|c|c|c|c|c|c|}
\hline SRS Coord. & Lat/Longitude & Screen Zone Elevation & Top of Casing & Casing & Pump & Formation \\
\hline $\begin{array}{l}\text { N70160.9 } \\
\text { E55355.7 }\end{array}$ & $\begin{array}{l}33.271268{ }^{\circ} \mathrm{N} \\
81.657324{ }^{\circ} \mathrm{W}\end{array}$ & $218.1-198.1 \mathrm{ft} \mathrm{msl}$ & $251.1 \mathrm{ft} \mathrm{msl}$ & 4" PVC & s & Water table $\left(11 \mathrm{~B}_{2}\right)$ \\
\hline \multicolumn{2}{|c|}{ SAMPLE DATE } & $01 / 15 / 92$ & $04 / 24 / 92$ & $07 / 24 / 92$ & $10 / 23 / 92$ & \\
\hline \multicolumn{7}{|l|}{ FIELD DATA } \\
\hline \multicolumn{2}{|c|}{ Analyte } & 1092 & $\underline{2092}$ & 3092 & 4092 & Unit \\
\hline \multicolumn{2}{|c|}{$\begin{array}{l}\text { Water elevation } \\
\text { pH } \\
\text { Sp. conductance } \\
\text { Water temperature } \\
\text { Alkalinity as } \mathrm{CaCO}_{3} \\
\text { Volume purged }\end{array}$} & $\begin{array}{l}213.6 \\
11.3 \\
157 \\
16.4 \\
36 \\
0.6\end{array}$ & $\begin{array}{l}213.9 \\
9.6 \\
90 \\
17.8 \\
26 \\
0.6\end{array}$ & $\begin{array}{l}213.3 \\
9.6 \\
72 \\
19.3 \\
21 \\
0.7\end{array}$ & $\begin{array}{l}213.9 \\
9.7 \\
77 \\
16.8 \\
25 \\
0.6\end{array}$ & $\begin{array}{l}\text { ft msl } \\
\mathrm{pH} \\
\mu \mathrm{S} / \mathrm{cm} \\
{ }^{\circ} \mathrm{C} \\
\mathrm{mg} / \mathrm{L} \\
\text { Well voi }\end{array}$ \\
\hline
\end{tabular}

\section{ANALYTICAL DATA}

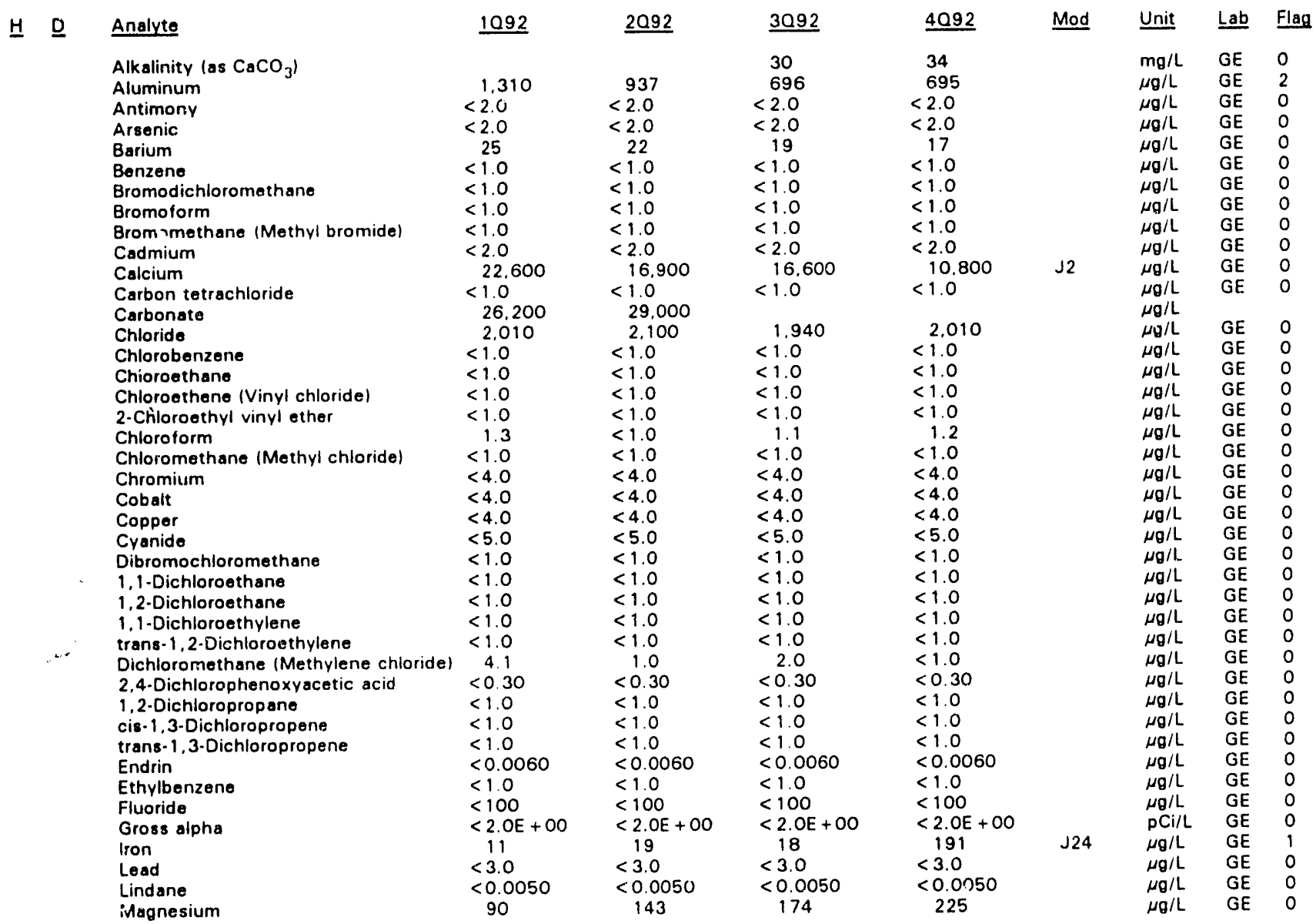

Note: Flagging levels, modifiers, and laboratories are for 4 th quarter 1992 data only. See Appendix B for flagging criteria. - = exceeded holding time for 4 th quarter 1992.

- exceeded final primary drinking water standard for 4th quarter 1992. 
WSRC-TR-93-059

Well HSB1480 continued

ANALYTICAL DATA

H D Analyte

\begin{tabular}{ll}
$\quad 1092$ & $\underline{2092}$ \\
$<2.0$ & $<2.0$ \\
$<0.20$ & $<0.20$ \\
$<0.50$ & $<0.50$ \\
$<4.0$ & $<4.0$ \\
$<50$ & \multicolumn{1}{c}{$<$} \\
& 50 \\
$<2.0 E+00$ & $<2.0 E+00$ \\
11.0 & $<0$ \\
$<5.0$ & $<5.0$ \\
1.760 & 1.570 \\
$<2.0$ & $<2.0$ \\
9.670 & 9.280 \\
$<2.0$ & $<2.0$ \\
1.970 & 2.240 \\
180 & 65 \\
3.000 & 3.540 \\
$<1.0$ & $<1.0$ \\
$<1.0$ & $<1.0$ \\
$<1.0$ & $<1.0$ \\
$<1.0 E+00$ & $1.0 E+00$ \\
57.000 & 37.000 \\
$<1.000$ & $<1.000$ \\
$<5.0$ & 18 \\
$<50$ & $<50$ \\
$<0.24$ & $<0.24$ \\
$<0.090$ & $<0.090$ \\
$<1.0$ & $<1.0$ \\
$<1.0$ & $<1.0$ \\
$<1.0$ & $<1.0$ \\
$<1.0$ & $<1.0$ \\
$1.5 E+01$ & $1.5 E+01$ \\
112 & 11 \\
$<2.0$ & $<2.0$ \\
$<2.0$ &
\end{tabular}

$\begin{aligned} & 3092 \\ &< 2.0 \\ &<0.20 \\ &<0.50 \\ &<4.0 \\ &<50 \\ & 2.4 E+00 \\ & 9.5 \\ &<5.0 \\ & 1.260 \\ &<2.0 \\ & 9.690 \\ &<2.0 \\ & 2.560 \\ & 65 \\ & 3.760 \\ &<1.0 \\ &<1.0 \\ &<1.0 \\ &<1.0 E+00 \\ & 52.000 \\ &<1.000 \\ &<5.0 \\ &<50 \\ &<0.24 \\ &<0.090 \\ &<1.0 \\ &<1.0 \\ &<1.0 \\ &<1.0 \\ &<1.4 E+01 \\ &<20 \\ & 11 \\ &<2.0\end{aligned}$

$4092 \quad$ Mod

Unit Lab Flag

Manganese
Mercury
Methoxychlor
Nickel
Nitrate as nitrogen
Nitrate-nitrite as nitrogen
Nonvolatile bets
pH
Phenols
Potassium
Solenium
Silica
Silver
Sodium
Specific conductance
Sulfate
1,1.2.2-Tetrachloroethane
Tetrachloroethylene
Toluene
Total aipha-emitting radium
Total dissolved solids
Total organic carbon
Total organic halogens
Total phosphates (as P)
Toxaphene
$2,4.5-T P$ (Silvex)
1.1 .1 -Trichloroethone
$1,1,2$-Trichloroethane
Trichloroethylene
Trichlorofluoromethane
Tritium
Uranium
Vanadium
Zinc

WELL HSB149D

$\begin{array}{ll}\text { SRS Coord. } & \text { Lat/Longitude } \\ \text { N71338.8 } & 33.277023^{\circ} \mathrm{N} \\ \text { E57286.3 } & 81.654529^{\circ} \mathrm{W} \\ \text { SAMPLE DATE } & \\ \text { FIELD DATA } & \end{array}$

Scroen Zone Elevation

$227.0 \cdot 207.0 \mathrm{ft} \mathrm{mst}$

$01 / 08 / 92$

Casing

Pump

Formation
$240 \mathrm{ft} \mathrm{msl} \quad 4$ " PVC S Water table (IIB.)

$240 \mathrm{ft} \mathrm{msl} \quad 4$ " PVC S Water table (IIB.)

$10 / 21 / 92$
Analyte

Water slevation

pH

Sp. conductance

Water temperature

Alkalinity as $\mathrm{CaCO}_{3}$

Volume purged

ANALYTICAL DATA

H D Analyte

Alkalinity las $\mathrm{CaCO}_{3}$ )

Aluminum

Antimony

Arsenic

$\begin{array}{lll}1092 & \underline{2092} & \underline{3092} \\ 222.5 & 224.1 & 224.6 \\ 4.7 & 4.1 & 4.5 \\ 27 & 20 & 21 \\ 19.2 & 18.8 & 21.4 \\ 0 & 0 & 0 \\ 4.0 & 4.0 & 4.0\end{array}$

1092

\section{2}

61

$<2.0$
$<0.20$

$<0.50$

$<4.0$

50

$2.5 \mathrm{E}+00$
9.8

9.8

1.200

$<2.0$

$<2.0$

2.430

80

3.320
$<1.0$

$<1.0$

$<10$

58.000

$<1.000$
$<5.0$

$<50$

$<0.24$

$<0.090$

$<1.0$

$<1.0$

$<1.0$

1. $2 E+01$

$<20$

9.0
4.8

$\begin{array}{lll}\mu \mathrm{g} / \mathrm{L} & \mathrm{GE} & 0 \\ \mu \mathrm{g} / \mathrm{L} & \mathrm{GE} & 0 \\ \mu \mathrm{g} / \mathrm{L} & \mathrm{GE} & 0 \\ \mu \mathrm{g} / \mathrm{L} & \mathrm{GE} & 0 \\ \mu \mathrm{g} / \mathrm{L} & & \\ \mu \mathrm{g} / \mathrm{L} & \mathrm{GE} & 0 \\ \mathrm{pCi} / \mathrm{L} & \mathrm{GE} & 0 \\ \mathrm{pH} & \mathrm{GE} & 1 \\ \mu \mathrm{g} / \mathrm{L} & \mathrm{GE} & 0 \\ \mu \mathrm{g} / \mathrm{L} & \mathrm{GE} & 0 \\ \mu \mathrm{g} / \mathrm{L} & \mathrm{GE} & 0 \\ \mu \mathrm{g} / \mathrm{L} & \mathrm{GE} & 0 \\ \mu \mathrm{g} / \mathrm{L} & \mathrm{GE} & 0 \\ \mu \mathrm{g} / \mathrm{L} & \mathrm{GE} & 0 \\ \mu \mathrm{S} / \mathrm{cm} & \mathrm{GE} & 0 \\ \mu \mathrm{g} / \mathrm{L} & \mathrm{GE} & 0 \\ / / \mathrm{L} & \mathrm{GE} & 0 \\ \mu \mathrm{g} / \mathrm{L} & \mathrm{GE} & 0 \\ \mu \mathrm{g} / \mathrm{L} & \mathrm{GE} & 0 \\ \mathrm{pCi} / \mathrm{L} & \mathrm{GE} & 0 \\ \mu \mathrm{g} / \mathrm{L} & \mathrm{GE} & 0 \\ \mu \mathrm{g} / \mathrm{L} & \mathrm{GE} & 0 \\ \mu \mathrm{g} / \mathrm{L} & \mathrm{GE} & 0 \\ \mu \mathrm{g} / \mathrm{L} & \mathrm{GE} & 0 \\ \mu \mathrm{g} / \mathrm{L} & \mathrm{GE} & 0 \\ \mu \mathrm{g} / \mathrm{L} & \mathrm{GE} & 0 \\ \mu \mathrm{g} / \mathrm{L} & \mathrm{GE} & 0 \\ \mu \mathrm{g} / \mathrm{L} & \mathrm{GE} & 0 \\ \mu \mathrm{g} / \mathrm{L} & \mathrm{GE} & 0 \\ \mu \mathrm{g} / \mathrm{L} & \mathrm{GE} & 0 \\ \mathrm{pCC} / \mathrm{mL} & \mathrm{GE} & 1 \\ \mu \mathrm{g} / \mathrm{L} & \mathrm{GE} & 0 \\ \mu \mathrm{g} / \mathrm{L} & \mathrm{GE} & 0 \\ \mu \mathrm{g} / \mathrm{L} & \mathrm{GE} & 0 \\ & & \end{array}$ 
WSRC-TR-93-059

Woll HSB149D continued

ANALYTICAL DATA

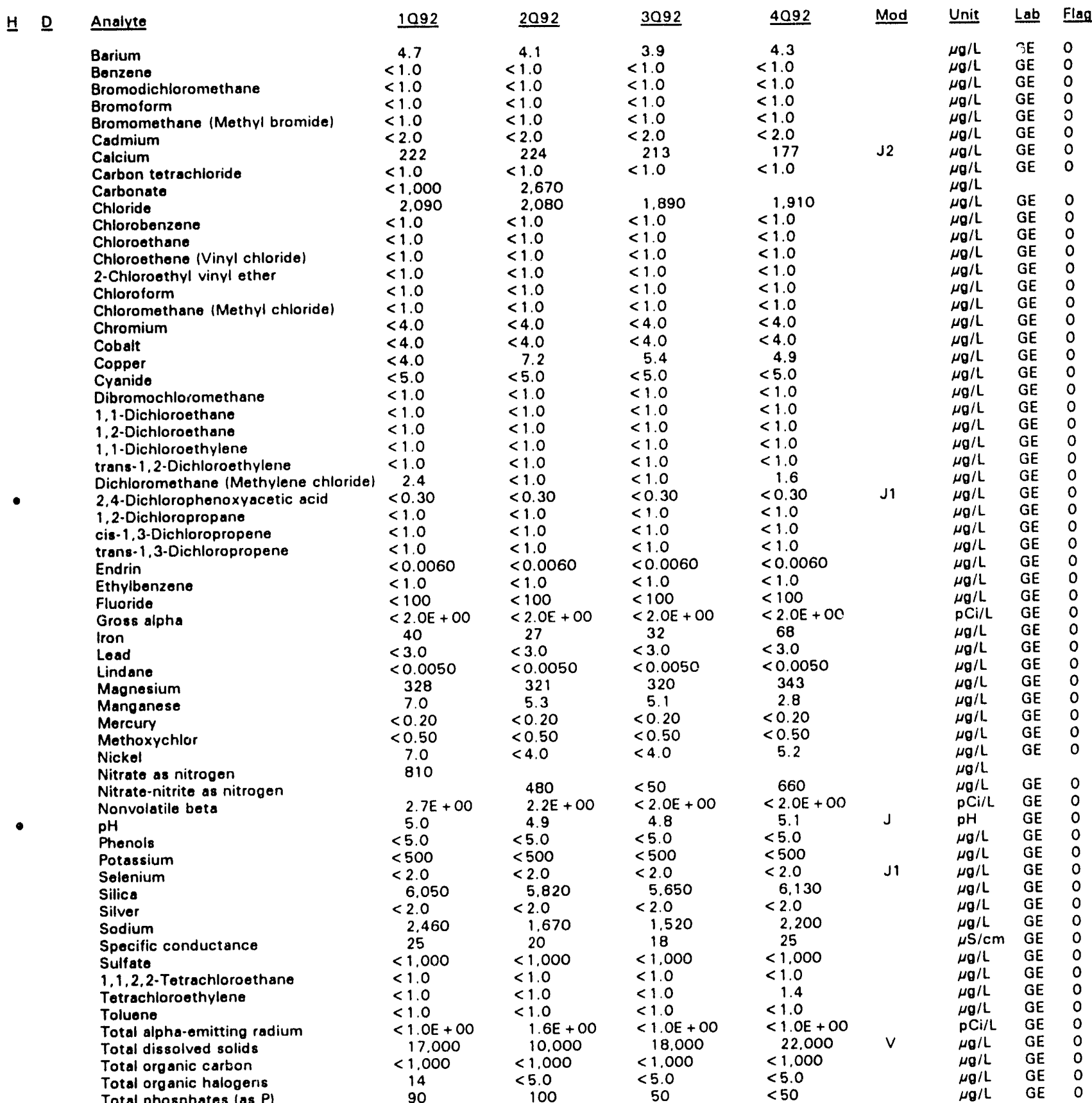

Note: Flagging levels, modifiers, and laboratories are for 4 th quarter 1992 data only. See Appendix 8 for flagging criteria. - =xceeded holding time for 4th quarter 1992.

- = exceeded final primary drinking water standard for 4th quarter 1992 . 
ANALYTICAL DATA

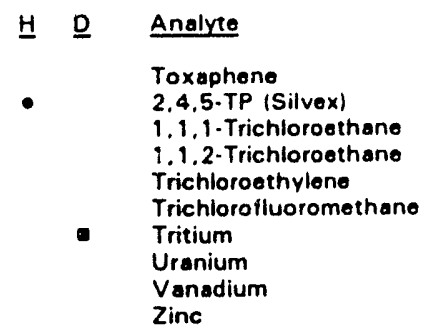

WELL HSB150D

$\begin{array}{ll}\text { SRS Coord. } & \text { Lat/Longitude } \\ \text { N71692.6 } & 33.280100^{\circ} \mathrm{N} \\ \text { E58692.8 } & 81.651512^{\circ} \mathrm{W} \\ \text { SAMPLE DATE } & \end{array}$

$\frac{\text { Scrnell Zone Elevation }}{226.9 \cdot 206.9 \mathrm{ft} \mathrm{msl}}$

Top of Casing

$01 / 07 / 92$

$\begin{aligned} & 2092 \\ < & 0.24 \\ < & 0.090 \\ < & 1.0 \\ < & 1.0 \\ < & 1.0 \\ < & 1.0 \\ & 3.1 E+01 \\ < & 8.0 \\ & 8.4\end{aligned}$

$04 / 27 / 92$

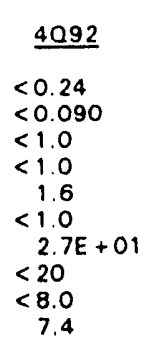

\begin{tabular}{clll} 
Mod & Unit & Lab & Flag \\
\cline { 2 - 3 }$J 1$ & $\mu g / L$ & GE & 0 \\
& $\mu g / L$ & $G E$ & 0 \\
& $\mu g / L$ & $G E$ & 0 \\
& $\mu g / L$ & $G E$ & 0 \\
& $\mu g / L$ & $G E$ & 0 \\
& $\mu g / L$ & $G E$ & 0 \\
& $p C i / m L$ & $G E$ & 2 \\
& $\mu g / L$ & $G E$ & 0 \\
& $\mu g / L$ & $G E$ & 0 \\
& $\mu g / L$ & $G E$ & 0
\end{tabular}

FIELD DATA

\section{Analyte \\ Water elevation \\ pH \\ Sp. conductance \\ Water temperature \\ Alkalinity as $\mathrm{CaCO}_{3}$ \\ Volume purged}

ANALYTICAL DATA

1092
226.6
5.4
37
17.1
1
4.0

\section{2}

Alkalinity las $\mathrm{CaCO}_{3}$ l

Aluminum

Antimony

Arsonic

Barium

Benzene

Bromodichloromethane

Bromoform

Bromomethane (Methyl bromide)

Cadmium

Calcium

Carbonato

Chloride

Chlorobenzene

Chloroethane

Chloroethene (Vinyl chloride)

2.Chloroethyl vinyl ethe

Chloroform

Chloromethane (Methyl chloride)

Chromium

Cobalt

Cranide

Dibromochloromethane

1 1-Dichloroethane

1.2-Dichloroethane

1.1-Dichloroethylene

\begin{tabular}{|c|c|}
\hline 1092 & 2092 \\
\hline$<20$ & 107 \\
\hline$<2.0$ & $<2.0$ \\
\hline$<2.0$ & $<2.0$ \\
\hline 3.3 & 5.1 \\
\hline$<1.0$ & $<1.0$ \\
\hline$<1.0$ & $<1.0$ \\
\hline$<1.0$ & $<1.0$ \\
\hline$<1.0$ & $<1.0$ \\
\hline$<2.0$ & $<0.35$ \\
\hline 482 & 835 \\
\hline$<1.0$ & $<1.0$ \\
\hline$<1.000$ & $<500$ \\
\hline 3.580 & 5.500 \\
\hline$<1.0$ & $<10$ \\
\hline$<1.0$ & $<1.0$ \\
\hline$<10$ & $<1.0$ \\
\hline$<10$ & $<1.0$ \\
\hline$<10$ & $<10$ \\
\hline$<10$ & $<1.0$ \\
\hline$<4.0$ & $<1.1$ \\
\hline$<4.0$ & $<0.88$ \\
\hline$<4.0$ & 4.3 \\
\hline$<5.0$ & $<5.0$ \\
\hline$<1.0$ & $<1.0$ \\
\hline$<10$ & $<1.0$ \\
\hline$<1.0$ & $<1.0$ \\
\hline$<1.0$ & $<1.0$ \\
\hline
\end{tabular}

$\frac{2092}{}$
2274
4.2
35
16.8
0
4.0

3092
229.4
4.9
32
21.0
0
4.0

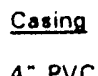

Pump

Formation

4" PVC

S

Water table $\left(11 \mathrm{~B}_{2}\right)$

$07 / 24 / 92$

10/23/92

$\begin{array}{ll}\text { 4092 } & \text { Unit } \\ 225.3 & \mathrm{ft} \mathrm{msl} \\ 4.4 & \mathrm{oH} \\ 35 & \mu \mathrm{S} / \mathrm{cm} \\ 18.5 & { }^{\circ} \mathrm{C} \\ 1 & \mathrm{mg} / \mathrm{L} \\ 1.2 & \text { Well vol. }\end{array}$

\begin{tabular}{|c|c|c|c|c|}
\hline 3092 & 4092 & Mod & Unit & $\underline{\text { Lab }}$ \\
\hline 6.4 & 6.6 & & $\mathrm{mg} / \mathrm{L}$ & $\mathrm{GE}$ \\
\hline 30 & 1.770 & & $\mu g / L$ & GE \\
\hline$<2.0$ & $<2.0$ & & $\mu_{\theta} / \mathrm{L}$ & GE \\
\hline$<2.0$ & $<2.0$ & & $\mu g / L$ & GE \\
\hline 4.4 & 7.0 & & $\mu \mathrm{g} / \mathrm{L}$ & $\mathrm{GE}$ \\
\hline$<1.0$ & $<1.0$ & & $\mu \mathrm{g} / \mathrm{L}$ & $\mathrm{GE}$ \\
\hline$<1.0$ & $<1.0$ & & $\mu \mathrm{g} / \mathrm{L}$ & GE \\
\hline$<1.0$ & $<1.0$ & & $\mu \mathrm{g} / \mathrm{L}$ & $G E$ \\
\hline$<1,0$ & $<1.0$ & & $\mu \mathrm{g} / \mathrm{L}$ & $\mathrm{GE}$ \\
\hline$<2.0$ & $<2.0$ & & $\mu g / L$ & GE \\
\hline 686 & 773 & J2 & $\mu_{\mathrm{g}} / \mathrm{L}$ & $\mathrm{GE}$ \\
\hline$<1.0$ & $<1.0$ & & $\begin{array}{l}\mu \mathrm{g} / \mathrm{L} \\
\mu \mathrm{g} / \mathrm{L}\end{array}$ & $\mathrm{GE}$ \\
\hline 2.760 & 3.980 & & $\mu \mathrm{g} / \mathrm{h}$ & $G E$ \\
\hline$<1.0$ & $<1,0$ & & $\mu \mathrm{g} / \mathrm{L}$ & $G E$ \\
\hline$<10$ & $<1.0$ & & $\mu g / L$ & GE. \\
\hline$<10$ & $<10$ & & $\mu \sigma / L$ & $\mathrm{GE}$ \\
\hline$<10$ & $<1.0$ & & $\mu_{\theta} / \mathrm{L}$ & $\mathrm{GE}$ \\
\hline$<10$ & $<10$ & & $\mu g / L$ & GE \\
\hline$<10$ & $<1.0$ & & $\mu_{g} / \mathrm{L}$ & $G E$ \\
\hline$<4.0$ & $<4.0$ & & $\mu \mathrm{g} / \mathrm{L}$ & $G E$ \\
\hline$<4.0$ & $<4.0$ & & $\mu \mathrm{g} / \mathrm{L}$ & $\mathrm{GE}$ \\
\hline 62 & 13 & & $\mu \mathrm{g} / \mathrm{L}$ & $G E$ \\
\hline$<5.0$ & $<5.0$ & & $\mu g / \mathrm{L}$ & $G E$ \\
\hline$<1.0$ & $<1.0$ & & $\mu g / L$ & $G E$ \\
\hline$<10$ & $<1.0$ & & $\mu g / L$ & GE \\
\hline$<1.0$ & $<1.0$ & & $\mu g / L$ & $\mathrm{GE}$ \\
\hline$<1.0$ & $<1.0$ & & $\mu g / L$ & $\mathrm{GE}$ \\
\hline
\end{tabular}

Note: Flagging levels, modifiers, and laboratories are for 4 th quarter 1992 data only. See Appendix $B$ for flagging criteria.

- =xceeded holding time for 4th quarter 1992.

- =xceeded final primary drinking water standard for 4ih quarter 1992.

H-Area Seepage Basins 


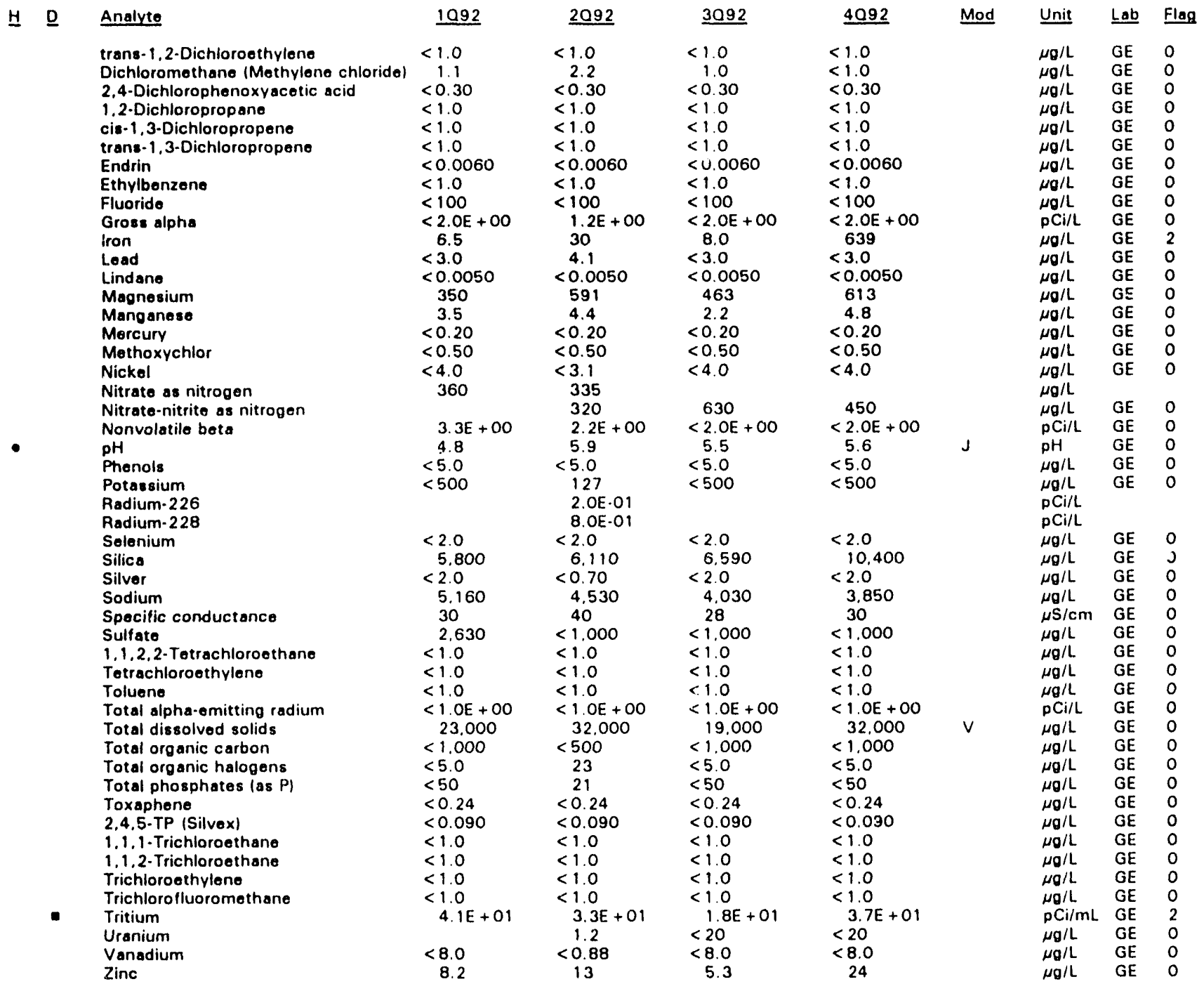

Note: Flagging levels, modifiers, and laboratories are for 4 th quarter 1992 data only. See Appendix $B$ for flagging criteria. - Exceeded holding time for 4th quarter 1992.

- =xceeded final primary drinking water standard for 4th quarter 1992. 
WSRC-TR-93-059

WELL HSB151C

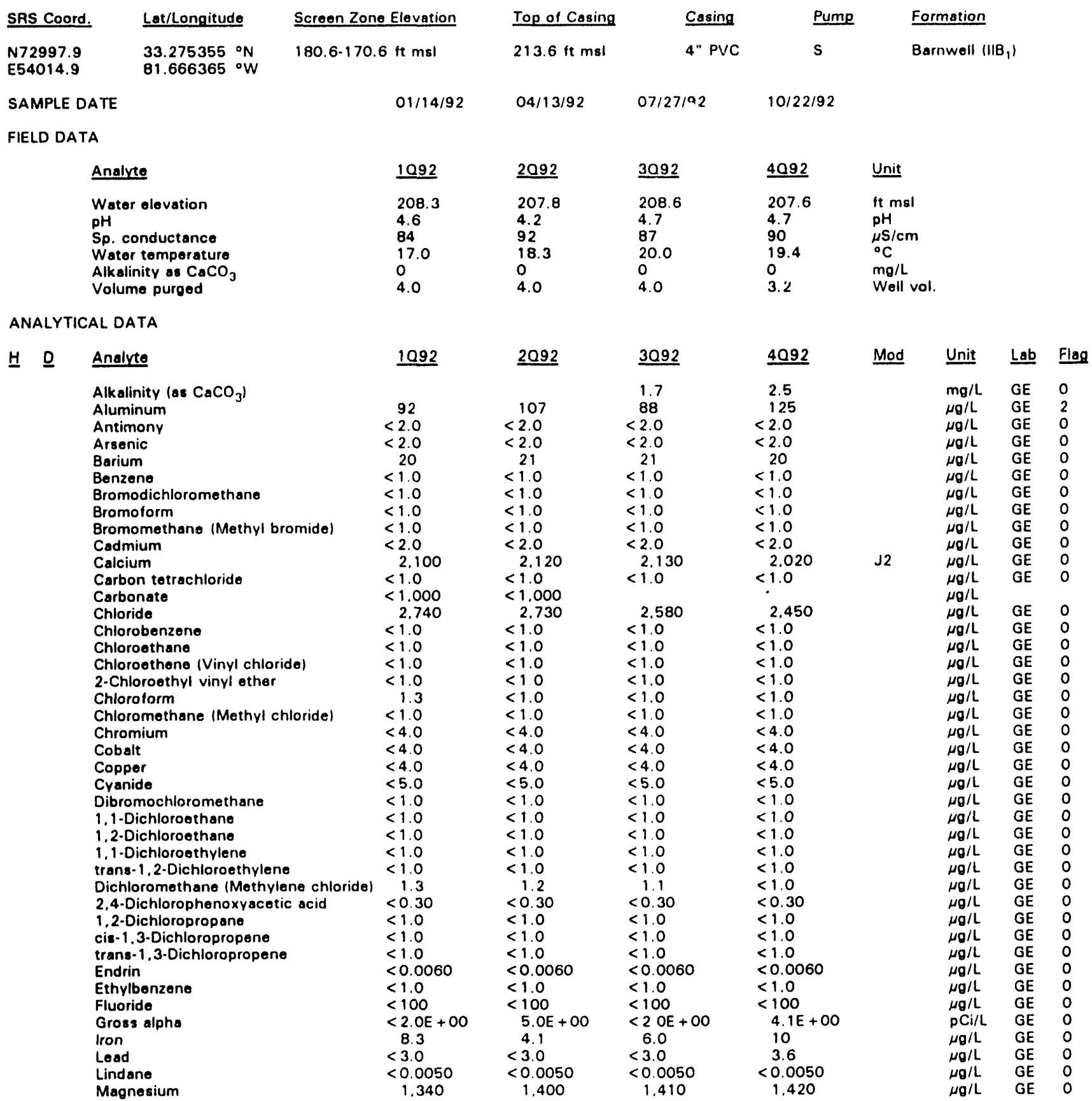

Note: Flagging levels, modifiers, and laboratories are for 4 th quarter 1992 data only. See Appendix $B$ for flagging criteria. - exceeded holding time for 4th quarter 1992 .

- exceeded final primary drinking water standard for 4th quarter 1992. 
Well HSB151C continued

ANALYTICAL DATA

H. $\quad$ Analyte
Manganese
Mercury
Methoxychlor
Nickel
Nitrate-nitrite as nitrogen
Nonvolatile beta
pH
Phenols
Potassium
Selenium
Silics
Silver
Sodium
Specific conductance
Sulfate
1.1.2.2-Totrachloroethane
Tetrachloroethylene
Toluene
Total activity
Total alpha-emitting radium
Total dissolved solids
Total organic carbon
Total organic halogens
Total phosphates las P)
Toxaphene
2.4.5-TP (Silvex)
$1,1.1-$ Trichloroethane
1.1.2-Trichloroethane
Trichloroethylene
Trichlorofluoromethane
Tritium
Uranium
Vanadium
Zinc

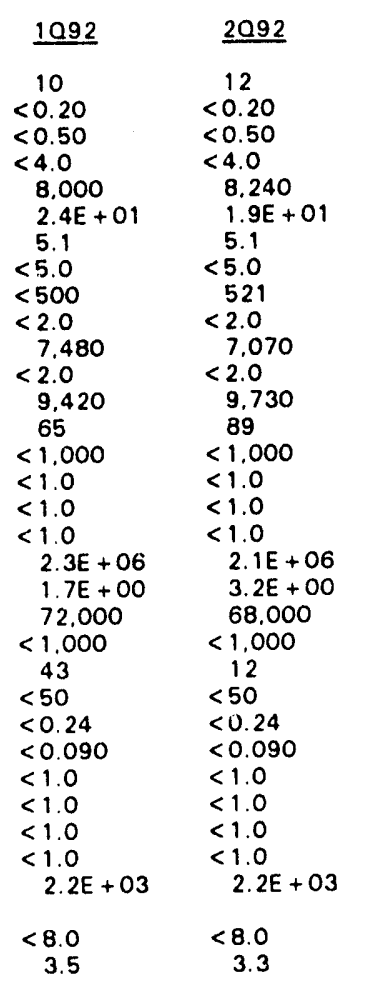

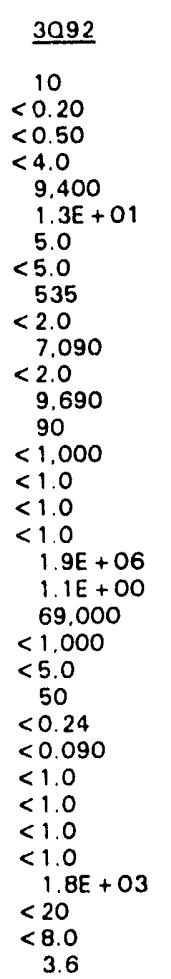

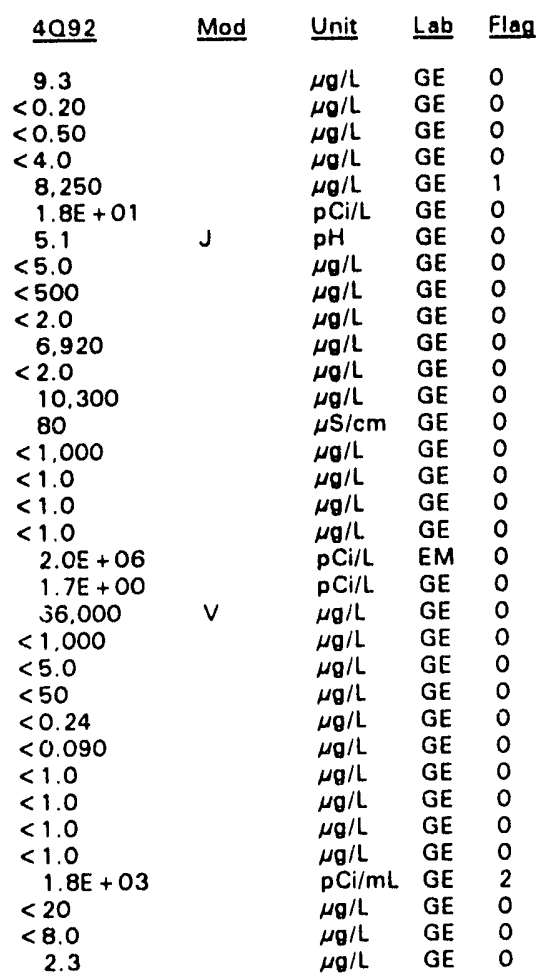

\section{WELL HSB151D}

$\begin{array}{ll}\text { SRS Coord. } & \text { Lat/Longitude } \\ \text { N72997.8 } & 33.275373{ }^{\circ} \mathrm{N} \\ \text { E54026.4 } & 81.666334{ }^{\circ} \mathrm{W}\end{array}$

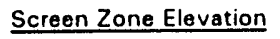

207.6-197.6 ft msl

SAMPLE DATE

FIELD DATA

Analyte

Water elevation

pH

Sp. conductance

Water temperoture

Alkalinity as $\mathrm{CaCO}_{3}$

Volume purged

ANALYTICAL DATA

H

Analyte
Alkalinity (as $\mathrm{CaCO}_{3}$ )
Aluminum
Antimony
Arsenic

$01 / 14 / 92$

\section{Top of Casing}

$213.6 \mathrm{ft} \mathrm{msl}$

$04 / 13 / 92$

1092
207.9
6.7
29
15.4
0
4.0

1092

$\underline{2092}$

2092
207.2
4.3
28
16.0
0
4.0

3092
208.2
4.7
24
22.4
0
4.0

Casing
4" PVC

$07 / 27 / 92$

$10 / 22 / 92$

$\begin{array}{rrr} & & \\ 27 & 33 & 2.6 \\ <2.0 & <2.0 & <20 \\ <2.0 & <2.0 & <2.0\end{array}$

$\begin{aligned} & 3092 \\ & 2.6 \\ < & 20 \\ < & 2.0 \\ < & 2.0\end{aligned}$

$\begin{array}{ll}4092 & \text { Unit } \\ 207.0 & \mathrm{ft} \mathrm{msl} \\ 4.8 & \mathrm{pH} \\ 25 & \mu \mathrm{S} / \mathrm{cm} \\ 21.1 & { }^{\circ} \mathrm{C} \\ 0 & \mathrm{mg} / \mathrm{L} \\ 8.3 & \text { Weil vol. }\end{array}$

Formation

Water table (IIB.,) 
WSRC-TR-93-059

Well HSB151D continued

ANALYTICAL DATA

H D Analyte

Barium

Benzene

Bromodichloromethane

Bromoform

Bromomethane (Mothyl bromide)

Cadmium

Calcium

Carbon tetrachloride

Carbonate

Chloride

Chlorobenzene

Chloroethane

Chloroethene (Vinyl chloride)

2.Chloroethyl vinyl other

Chloroform

Chloromethane (Methyl chloride)

Chromium

Cobait

Copper

Cyanide

Dibromochloromethane

1.1-Dichloroethane

1.2-Dichloroethane

1.1-Dichloroethylene

trans-1,2-Dichloroethylene

Dichloromethane (Methylene chloride)

2.4-Dichlorophenoxyacetic acid

1.2-Dichloropropane

cis-1.3-Dichloropropene

trans-1.3-Dichloropropene

Endrin

Ethylbenzene

Fluoride

Gross alpha

Iron

Lead

Lind ane

Magnesium

Manganese

Mercury

Methoxychlor

Nickol

Nitrato as nitrogen

Nitrato-nitrite as nitrogen

Nonvolatile bete

$\mathrm{pH}$

Phenols

Potassium

Solonium

Silica

Silver

Sodium

Specific conductance

Sulfate

1,1,2,2-Totrachloroethane

Tetrachloroethylene

Toluene

Total activity

Total alpha-omitting radium

Total dissolved solids

Total organic carbon

Total organic halogens

1092

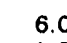

$<1.0$

$<1.0$

$<1.0$

$<1.0$

$<2.0$

512

$<1.0$

$<1,000$

1.780

$<1.0$

$<1.0$

$<1.0$

$<1.0$

$<1.0$

$<4.0$

$<4.0$

8. 3

$<5.0$

$<1.0$

$<1.0$

$<10$

$<1.0$

$<1.0$

$<1.0$

$<0.30$

$<1.0$

$<1.0$

$<1.0$

$<0.0060$

$<1.0$

$<100$

$<2$. OE + 00

7.2
$<3.0$

$<3.0$
$<0.0050$

563

$<0.20$

$<0.20$

$<0.50$
$<4.0$

1.860

$6.8 E+00$

5.2
8.5

8.5
$<500$

$<2.0$

5.780

$<2.0$

3.160

31

$<1.000$

$<1.0$

$<1.0$

$<1.0$

$<1 . \mathrm{OE}+00$

25.000

2.000

15
2092

5.2

$<1.0$

$<1.0$

$<1.0$

$<1.0$

$<2.0$

474

$<1.0$

$<1.000$

1.720
$<1.0$

$<1.0$

$<1.0$

$<1.0$

$<1.0$

$<1.0$

$<4.0$

$<4.0$

6.2

$<5.0$

$<1.0$

$<1.0$

$<1.0$

$<1.0$

$<1.0$

$<1.0$

$<0.30$

$<1.0$

$<1.0$

$<1.0$

$<1.0$

$<100$

$<2.0 E+00$

11

$<3.0$

$<0.0050$

537

$<0.20$

$<0.20$
$<0.50$

$<4.0$

1,600

$<2.0 E+00$

4.8

$<5.0$

$<500$

$<2.0$

5.330

$<2.0$

2.850

30
$<1.000$

$<1.0$

$<1.0$

$<1.0$

$<1.0$

$4.6 E+05$
$<1.0 E+00$

17.000

$<1,000$

14
3092

5.0

$<1.0$

$<1.0$

$<1.0$

$<2.0$

444

$<1.0$

1.400

$<1.0$

$<1.0$

$<1.0$

$<1.0$

$<1.0$

$<1.0$

$<4.0$

$<5.0$

$<1.0$

$<1.0$

$<1.0$

$<1,0$

$<1.0$

$<0.30$

$<1.0$

$<1.0$

$<0.0060$

$<1.0$

$<100$

$<2 . \mathrm{OE}+00$

12

$<3.0$

$<0.0050$

464

$<0.20$

$<0.20$

$<0.50$
$<4.0$

1.290

$<2.0 E+00$

5.2
$<5.0$

$<5.0$
$<500$

$<2.0$

6.030

$<2.0$

2.330

25

$<1.000$

$<1.0$

$<1.0$

$<1.0$

2. $6 E+05$

20.000

$<1,000$

12

$\begin{aligned} & 4092 \quad \text { Mod } \\ 4.8 & \\ <1.0 & \\ <1.0 & \\ <1.0 & \\ <1.0 & \\ <2.0 & \\ 403 & \text { J2 } \\ <1.0 & \\ & \\ & \\ <1.320 & \end{aligned}$

Unit Lab Flag

$\mu \mathrm{g} / \mathrm{L}$ GE 0

$\mu \sigma / L$ GE $\quad 0$

wo/

$\mu \mathrm{ol}$

$\mu \mathrm{g} / \mathrm{L}$

$\mu \mathrm{g} / \mathrm{L}$

$\mu \mathrm{g} / \mathrm{L}$

$\mu \sigma / L$

$<1.0$

$<1.0$

$<1.0$

$<1.0$

$<1.0$

$<1.0$

$<4.0$

$<4.0$

$<4.0$

$<5.0$

$<1.0$

$<1.0$

$<1.0$

$<10$

$<1.0$

$<1.0$

$<0.30$

$<1.0$

$<1.0$

$<1.0$

$<0.0060$

$<1.0$

$<2.0 \mathrm{E}+00$

28

$<3.0$

$<0.0050$

482

$<0.20$

$<0.20$

$<0.50$
$<4.0$

1.290

2. $7 \mathrm{E}+00$

$\begin{array}{r}5.6 \\ <5.0 \\ \hline\end{array}$

$<5.0$
$<500$

$<2.0$

5.710

$<2.0$

2.470

25

$<1.000$

$<1.0$

$<1.0$

$<1.0$

2.8E + 05

$<1.0 E+O 0$ 
WSRC-TR-93-059

Well HSB151D continued

ANALYTICAL DATA

H D Analyte

Total phosphates (as P) Toxaphene

2.4,5-TP (Silvex)

1,1,1-Trichloroethane

1.1.2. Trichloroethane

Trichloroethylene

Trichlorofluoromethane

- Tritium

Uranium

Vanadium

Zinc

$\begin{aligned} & 1092 \\ < & 50 \\ < & 0.24 \\ < & 0.090 \\ < & 1.0 \\ < & 1.0 \\ < & 1.0 \\ < & 1.0 \\ & 6.9 \mathrm{E}+02 \\ < & 8.0 \\ & 13\end{aligned}$

$\begin{aligned} & 2092 \\ < & 50 \\ < & 0.24 \\ < & 0.090 \\ < & 1.0 \\ < & 1.0 \\ < & 1.0 \\ < & 1.0 \\ & 4.8 \mathrm{E}+02 \\ < & 8.0 \\ & 7.4\end{aligned}$

$\begin{aligned} & 4092 \\ < & 50 \\ < & 0.24 \\ < & 0.090 \\ < & 1.0 \\ < & 1.0 \\ < & 1.0 \\ < & 1.0 \\ & 2.6 E+02 \\ < & 20 \\ < & 8.0 \\ & 5.6\end{aligned}$

Mod

$\begin{array}{lll}\text { Unit } & \text { Lab } & \text { Flag } \\ \mu \mathrm{g} / \mathrm{L} & \mathrm{GE} & 0 \\ \mu \mathrm{g} / \mathrm{L} & \mathrm{GE} & 0 \\ \mu \mathrm{g} / \mathrm{L} & \mathrm{GE} & 0 \\ \mu \mathrm{g} / \mathrm{L} & \mathrm{GE} & 0 \\ \mu \mathrm{g} / \mathrm{L} & \mathrm{GE} & 0 \\ \mu \mathrm{g} / \mathrm{L} & \mathrm{GE} & 0 \\ \mu \mathrm{g} / \mathrm{L} & \mathrm{GE} & 0 \\ \mathrm{pCi} / \mathrm{mL} & \mathrm{GE} & 2 \\ \mu \mathrm{g} / \mathrm{L} & \mathrm{GE} & 0 \\ \mu \mathrm{g} / \mathrm{L} & \mathrm{GE} & 0 \\ \mu \mathrm{g} / \mathrm{L} & \mathrm{GE} & 0\end{array}$

\section{WELL HSB152C}

$\begin{array}{ll}\text { SAS Coord. } & \text { Lat/Longitude } \\ \text { N72012.0 } & 33.273716^{\circ} \mathrm{N} \\ \text { E54346.7 } & 81.663576{ }^{\circ} \mathrm{W}\end{array}$

\section{Screen Zone Elevation \\ 183.1-173.1 it msl}

$01 / 14 / 92$

$04 / 13 / 92$

$10 / 22 / 92$

FIELD DATA
Analyte

Water elevation

$\mathrm{pH}$

Sp. conductance

Water temperature

Alkalinity as $\mathrm{CaCO}_{3}$

Volume purged

ANALYTICAL DATA

H

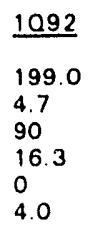

1092

Alkalinity (as $\mathrm{CaCO}_{3}$ )

Aluminum

Antimony

Arsonic

Barium

Benzene

Bromodichloromethane

Bromoform

Bromomethane (Methyl bromide)

Cadmium

Carbon tetrachlorid

Carbonate

Chloride

Chlorobenzene

Chloroethane

2.Chloroethyl vinyl ether

Chlorotorm

Chloromethane (Methyl chloride)

Chromium

Cobalt

Copper

Cyanide

Dibromochloromethane

1,1-Dichloroethane

1,2-Dichloroethane
Chloroethene (Vinyl chloride)

$\begin{array}{lll} & & \\ 85 & 84 & 3.3 \\ <2.0 & <2.0 & 57 \\ <2.0 & <2.0 & <2.0 \\ 29 & 31 & <2.0 \\ <1.0 & <1.0 & 33 \\ <1.0 & <1.0 & <1.0 \\ <1.0 & <1.0 & <1.0 \\ <1.0 & <1.0 & <1.0 \\ <2.0 & <2.0 & <1.0 \\ 3.590 & 4.090 & <2.0 \\ <1.0 & <1.0 & 4.530 \\ <1.000 & <1.000 & <1.0 \\ 3.360 & 3.170 & \\ <1.0 & <1.0 & 3.230 \\ <1.0 & <1.0 & <1.0 \\ <1.0 & <1.0 & <1.0 \\ <1.0 & <1.0 & <1.0 \\ <1.0 & <1.0 & <1.0 \\ <1.0 & <1.0 & <1.0 \\ <4.0 & <4.0 & <4.0 \\ <4.0 & <4.0 & <4.0 \\ <4.0 & <4.0 & <4.0 \\ <5.0 & <5.0 & <5.0 \\ <1.0 & <1.0 & <1.0 \\ <1.0 & <1.0 & <1.0 \\ <1.0 & <1.0 & <1.0\end{array}$

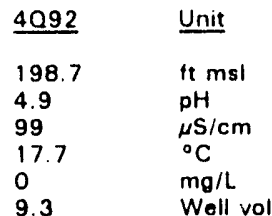

\section{Formation}

Barnwell (IIB, )

2092
198.8
4.4
100
17.2
0
4.0

2092

$<1.0$

3092
199.0
4.8
105
18.7
0
4.0

$\underline{3092}$

$\begin{array}{rl} & 4092 \\ & 5.1 \\ 87 & 87 \\ < & 2.0 \\ < & 2.0 \\ & 32 \\ < & 1.0 \\ < & 1.0 \\ < & 1.0 \\ < & 1.0 \\ < & 2.0 \\ & 4.340 \\ < & 1.0 \\ & \\ & 3.320 \\ < & 1.0 \\ <1.0 \\ <1.0 \\ <1.0 \\ <1.0 \\ <1.0 \\ <4.0 \\ <4.0 \\ <4.0 \\ <5.0 \\ <1.0 \\ <1.0 \\ <1.0 \\ <1.0\end{array}$

Mod

$\begin{array}{lll}\text { Unit } & \text { Lab } & \text { Flag } \\ \mathrm{mg} / \mathrm{L} & \mathrm{GE} & 0 \\ \mu \mathrm{g} / \mathrm{L} & \mathrm{GE} & 2 \\ \mu \mathrm{g} / \mathrm{L} & \mathrm{GE} & 0 \\ \mu \mathrm{g} / \mathrm{L} & \mathrm{GE} & 0 \\ \mu \mathrm{g} / \mathrm{L} & \mathrm{GE} & 0 \\ \mu \mathrm{g} / \mathrm{L} & \mathrm{GE} & 0 \\ \mu \mathrm{g} / \mathrm{L} & \mathrm{GE} & 0 \\ \mu \mathrm{g} / \mathrm{L} & \mathrm{GE} & 0 \\ \mu \mathrm{g} / \mathrm{L} & \mathrm{GE} & 0 \\ \mu \mathrm{g} / \mathrm{L} & \mathrm{GE} & 0 \\ \mu \mathrm{g} / \mathrm{L} & \mathrm{GE} & 0 \\ \mu \mathrm{g} / \mathrm{L} & \mathrm{GE} & 0 \\ \mu \mathrm{g} / \mathrm{L} & & \\ \mu \mathrm{g} / \mathrm{L} & \mathrm{GE} & 0 \\ \mu \mathrm{g} / \mathrm{L} & \mathrm{GE} & 0 \\ \mu \mathrm{g} / \mathrm{L} & \mathrm{GE} & 0 \\ \mu \mathrm{g} / \mathrm{L} & \mathrm{GE} & 0 \\ \mu \mathrm{g} / \mathrm{L} & \mathrm{GE} & 0 \\ \mu \mathrm{g} / \mathrm{L} & \mathrm{GE} & 0 \\ \mu \mathrm{g} / \mathrm{L} & \mathrm{GE} & 0 \\ \mu \mathrm{g} / \mathrm{L} & \mathrm{GE} & 0 \\ \mu \mathrm{g} / \mathrm{L} & \mathrm{GE} & 0 \\ \mu \mathrm{g} / \mathrm{L} & \mathrm{GE} & 0 \\ \mu \mathrm{g} / \mathrm{L} & \mathrm{GE} & 0 \\ \mu \mathrm{g} / \mathrm{L} & \mathrm{GE} & 0 \\ \mu \mathrm{g} / \mathrm{L} & \mathrm{GE} & 0 \\ \mu \mathrm{g} / \mathrm{L} & \mathrm{GE} & 0 \\ & & \end{array}$

Note: Flagging levels, modifiers, and laboratories are for 4 th quarter 1992 data only. See Appendix B for flagging criteria. - =xceeded holding time for 4th quarter 1992.

- exceeded final primary drinking water standard for 4th quarter 1992. 


\begin{abstract}
ANALYTICAL DATA
\end{abstract}

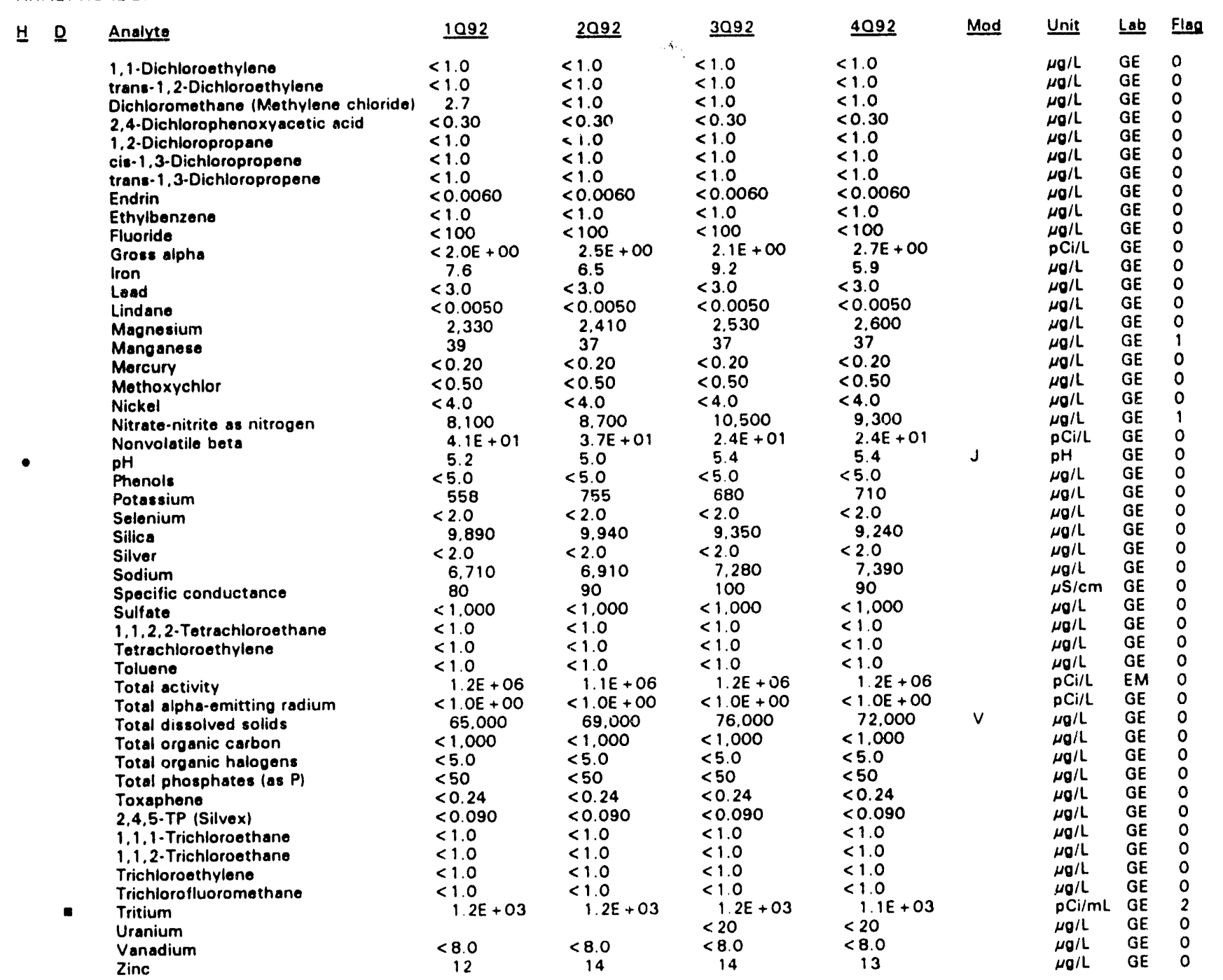

Note: Flagging levels, modifiers, and laboratories are for 4 th quarter 1992 data oniy. See Appendix $B$ for flagging criteria. - = exceeded holding time for 4th quarter 1992.

- =xceeded final primary drinking water standard for 4th quarter 1992. 
WELL HSB152D

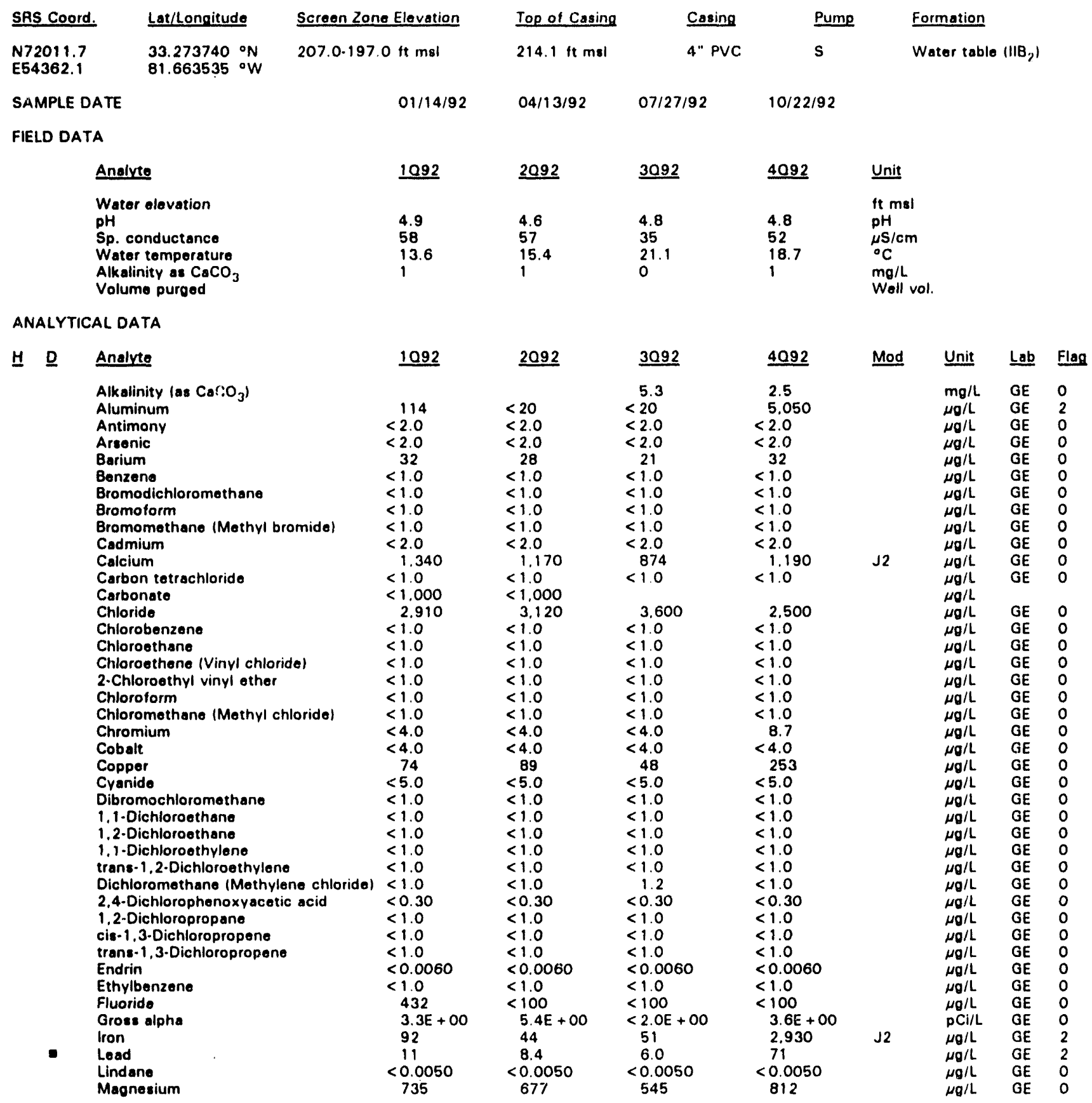

Note: Flagging levels, modifiers, and laboratories are for 4 th quarter 1992 data only. See Appendix B for flagging criteria. - = exceeded holding time for 4th quarter 1992.

- exceeded final primary drinking water standard for 4th quarter 1992. 
H D Analyte

Manganeso

Mercury

Mothoxychlor

Nickol

Nitrate as nitrogen

Nitrate-nitrite as nitrogon

Nonvolatilo bote

pH

Phonole

Potastium

Solonium

Silica

Silver

Sodium

Specific conductance

Sulfate

1,1,2,2-Tetrachloroethane

Totrachloroethylene

Toluene

Total activity

Total alpha-emitting rodium

Total dissolved solids

Total organic carbon

Total organic halogens

Total phosphates (as P

Toxaphone

2.4.5.TP (Silvex)

1.1.1.Trichloroethene

1.1.2. Trichloroethane

Trichloroethylono

Trichlorofluoromeshane

- Tritium

Uranium

Vanadium

Zinc
1092

35

$<0.20$

11

3,460

$1.6 E+01$

5.3

$<5.0$

$<500$

$<2.0$

6.800

$<2.0$

6.650

60

2.970

$<1.0$

$<1.0$

$<1.0$

$2.0 E+00$

46.000

2.000

13

340

$<0.24$

$<0.090$

3.1

$<1.0$

$<1.0$

$<1.0$

5. $6 E+02$

$<8.0$

128 $\underline{2092}$

33

$<0.50$

4.4

3.260

1. $4 \mathrm{E}+01$

5.1

$<5.0$

$<500$

$<2.0$

$<2.0$

6.200

50

1.510

$<1.0$

$<1.0$

$<1.0$

4. $8 E+05$

1. $2 E+\infty$

37.000

2.000

41

1.180
$<0.24$

$<0.090$

3.0
$<1.0$

$<1.0$

$<1.0$

4. $7 E+02$

$<8.0$

107
3092

4092

Mod Unir Lab Flag

$<24$

$<0.20$

$<0.50$
$<4.0$

1.170

4. $7 E+\infty$

5.5

$<5.0$

$<500$

$<2.0$

5.960

$<2.0$

4.530

40

1.150

$<1.0$

$<1.0$

$<1.0$

$1.1 E+05$

$<1.0 E+\infty 0$

33,000

1.600

25

630

$<0.24$

$<0.090$

17

$<1.0$

$<1.0$

$<1.0$

$2.0 E+02$

$<20$

$<8.0$

68
36

$<0.20$

$<.50$
8.8

3.200

1. $4 \mathrm{E}+01$

5.3

$<5.0$

$<500$

$<2.0$

16.000

$<2.0$

5.960

50

1.190

$<1.0$

$<1.0$

$<1.0$

4. $2 E+05$

$1.6 E+00$

44.000

1.480

14

300

$<0.24$

$<0.090$

2.1

$<1.0$

$<1.0$

$<1.0$

3. $9 \mathrm{E}+02$

$<20$

$<8.0$
194 $\mu \mathrm{O} / \mathrm{L}$ GE 1

$\mu \mu_{\mu \sigma / L}$ GE 0

$\mu O / L$ GE $O$

$\mu \mathrm{OL}$ GE $O$

PCi/L GE O

PH $O E$ O

$\mu_{\mathrm{O} / \mathrm{L}} \mathrm{GE} O$

$\mu \mathrm{g} / \mathrm{L}$ GE 0

$\mu g / L$ GE

HOL OE

$\mu g / L$ GE 0

$\mu \mathrm{S} / \mathrm{cm}$ GE 0

$\mu \mathrm{g} / \mathrm{L}$ GE

$\mu \mathrm{g} / \mathrm{L}$ GE 0

$\mu \mathrm{O} / \mathrm{L}$ GE 0

$\begin{array}{lll}\mu \mathrm{O} / \mathrm{L} & \mathrm{GE} & 0 \\ \mathrm{pC} / \mathrm{L} & \mathrm{EM} & 0\end{array}$

$\begin{array}{lll}p C \mathrm{C} i / \mathrm{L} & \mathrm{EM} & 0 \\ \mathrm{pCi} / \mathrm{L} & \mathrm{GE} & 0\end{array}$

$\mu \mathrm{g} / \mathrm{L}$ GE $\mathrm{O}$

$\mu \mathrm{g} / \mathrm{L}$ GE

$\mu g / L$ GE

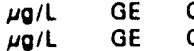

$\mu \sigma / L$ GE

$\mu g / L$ GE

$\mu \mathrm{g} / \mathrm{L}$ GE 0

$\mu \mathrm{g} / \mathrm{L}$ GE

$\mu g / L$ GE 0

$\mathrm{pCi} / \mathrm{mL}$ GE 2

$\mu g / \mathrm{L}$ GE 0

$\begin{array}{lll}\mu_{0} / \mathrm{L} & \mathrm{GE} & 0 \\ \mu_{\mathrm{g}} / \mathrm{L} & \mathrm{GE} & 0\end{array}$ $\mu g / L$ GE 0

Note: Flagging levels, modifiers, and laboratories are for 4th quarter 1992 data only. See Appendix 8 for flagging criteria.

- = exceeded holding time for 4th quarter 1992.

- =xceeded final primary drinkıng water standard for 4th quarter 1992. 
WSRC-TR-93-059

\section{Appendix E - Data Quality/Useability Assessment}




\section{Data Quality/Useability Assessment}

Quality assurance/quality control (QA/QC) procedures relating to accuracy and precision of analyses performed on groundwater samples are followed in the field and laboratory and are reviewed prior to publication of results. The Environmental Protection Department/ Environmental Monitoring Section's (EPD/EMS) review of the volume of analytical data acquired each quarter and presented in various reports is an ongoing process; its review of the QA/QC data cannot be completed in time to meet the deadlines for the reports required by the Resource Conservation and Recovery Act and associated regulations. Other site and regulatory personnel can obtain further information on the data quality and useability in a variety of ways, including those described below.

\section{Data Qualification}

The contract laboratories continually assess their own accuracy and precision according to U.S. Environmental Protection Agency (EPA) guidelines. They submit sample- or batchspecific $Q A / Q C$ information either at the same time as analytical results or in a quarterly summary. Properly defined and used result modifiers (also referred to as qualifiers) can be a key component in assessing data useability. Result modifiers designed by EPD/EMS and provided to the primary laboratories are presented in Appendix D.

\section{Assessment of Accuracy of the Data}

Accuracy, or the nearness of the reported result to the true concentration of a constituent in a sample, can be assessed in several ways.

A laboratory's general accuracy can be judged by analysis of results obtained from known samples. The non-radionuclide contract laboratories analyze commercial reference samples every quarter at EPD/EMS' request. The results of these analyses are presented in the EPD/EMS quarterly report, The Savannah River Site's Groundwater Monitoring Program. The primary laboratories also seek or maintain state certification by participating periodically in performance studies; reference samples and analysis of results are provided by EPA. Results of these studies also are published in the EPD/EMS quarterly reports.

Analysis of blanks provides a tool for assessing the accuracy of both sampling and laboratory analysis. Results for all field blanks for the quarter can be found in the EPD/EMS quarterly reports. Any field or laboratory blanks that exceeded established minimums are identified in the same reports, in tables associating them with groundwater samples analyzed in the same batches.

Surrogates, organic compounds similar in chemical behavior to the compounds of interest but not normally found in environmental samples, are used to monitor the effect of the matrix on the accuracy of analyses for organic parameters. For example, for analyses of volatile organics by EPA Method 8240 , three surrogate compounds are added to all samples 
and blanks in each analytical batch. In analyses of semivolatile organics, three to four acid compounds and three to four baseineutral compounds are used. Other surrogates are used in pesticides analyses. Percent recoveries for surrogate analyses are calculated by laboratory personnel, reported to EPD/EMS, reviewed, and entered into the database, but they are not published. If recoveries are rot within specified limits, the laboratory is expected to re-run the samples or attach result qualifiers to the data identifying the anomalous results.

Sample-specific accuracy for both oryanic and inorganic parameters can be assessed by examination of matrix spilke/matrix spike duplicate results. A sample is analyzed unspiked to determine a baseline set of ves. A second portion of sample is spiked with known concentrations of compounds appropriate to the analyses being performed, typically 5 volatile organic compounus for volatile organics analyses, 11 semivolatile compounds for semivolatiles, 6 pesticide compounds for pesticides, all metals for metals analyses, and a known quantity of cyanide for cyanide analysis. The percentage of the spike compound that is recovered (i.e., measured in excess of the valie obtained for the unspiked sample) is a direct measure of analytical accuracy. EPA requires matrix spike/matrix spike duplicates to be run at least once per 20 samples of similar matrix.

Matrix spike/matrix spik.e duplicate results are reported to EPD/EMS but are not published. For organic compounds, according to EPA guidelines, no action is taken on the basis of matrix spike/matrix spike duplicate tata alone (i.e., no result modifiers are assigned solely on the basis of matrix spike results); however, the results can indicate if a lab is having a systematic problem in the analysis of one or more analytes.

In the case of inorganic compounds, such as metals, the matrix spike sample analysis provides information about the effect of each sample matrix on the digestion and measurement methodology. Data qualifiers can be assigned on the basis of the percentage of spike recovery and are reported in the pubinshed results tables.

\section{Assessment of Precision}

Precision of the analyses, or agreement of a set of replicate results among themselves, is assessed through the use of duplicates (laboratory-initiated, and blind replicates (provided by EPD/EMS). The results of duplicate and replicate analyses are presented in the results tables of the first, second, and third quarter reports as multiple entries for an analyte under a single well heading. The results of replicate analyses are presented in the results tables in first, second, and third quarter reports as two separate sets of results for the saine well. Duplicate and replicate results are not presented in fourth quarter reports; the results tables present instead only the highest result for each analyte for each quarter of the year.

The laboratories assess precision by calculating the relative percent difference, or RPD, for each pair of laboratory-initiated duplicate results. During 1992, at le.st one of the contract laboratories used a data qualifier (J3) to modify metais analyses when the RPD for laboratory duplicates was greater than $20 \%$. 
Additional statistical comparisons of laboratory duplicate and blind replicate results, both intra- and interlabcratory, are presented in the EPD/EMS quarterly reports. The calculation used for these reports is the MRD, or mean relative difference, which is similar to EPA's RPD except that the MRD provides a single value for all of the analyses of a particular compound, either inter- or intralaboratory, during one quarter. Because detection limits may vary among samples, the MRD requires calculation of a reference detection limit, which is the detection limit at the 90 th percentile of the array of limits in the population of all replicate and duplicate analyses for a given analyte during a particular quarter. The MRD is not method-specific.

\section{Method-Specific Accuracy and Precision}

The contract laboratories' EPA-approved laboratory procedures include QA/QC requirements as an integral part of the methods. Thus, knowledge of the method used in obtaining data is an important component of determining data useability. EPA has conducted extensive research and development on the methods approved for the analysis of water and waste water; information on the accuracy and precision of the method is available from EPA publications, as is full information on required QA/QC procedures. A listing of the methods used by the primary laboratories during first quarter 1992 is given below along with the source for the method description. Many, if not all, of these sources include presentations of representative accuracy and precision results.

\begin{tabular}{ll} 
Method & Used to Analyze \\
EPA120.1 & Specific conductance \\
EPA150.1 & pH \\
EPA160.1 & Filterable residue (total dissolved solids) \\
EPA160.2 & Nonfilterable residue \\
EPA180.1 & Turbidity \\
EPA200.7 & Trace elements \\
EPA206.2 & Arsenic \\
EPA208.2 & Barium \\
EPA239.2 & Lead \\
EPA245.1 & Mercury \\
EPA270.2 & Selenium \\
EPA279.2 & Thallium \\
EPA300.0 & Inorganics, non-metallics \\
EPA310.1 & Alkalinity \\
EPA325.2 & Chloride \\
EPA335.3 & Cyanide \\
EPA340.2 & Fluoride \\
EPA353.1 & Nitrogen, nitrate-nitrite \\
EPA353.2 & Nitrogen, nitrate, nitrite, or combined \\
EPA353.3 & Nitrogen, nitrate-nitrite, or nitrite only \\
EPA354.1 & Nitrogen, nitrite \\
EPA365.1 & Phosphorus, all forms (reported as total phosphates) \\
EPA365.2 & Phosphorus, all forms (reported as total phosphates) \\
EPA375.4 & Sulfate, turbidimetric \\
EPA376.2 & Sulfide \\
APHA403 & Alkalinity \\
EPA413.1 & Oil \& grease \\
APHA415A & lodine \\
\hline
\end{tabular}

Source

EPA EMSL 1983 EPA EMSL 1983 EPA EMSL 1983 EPA EMSL 1983 EPA EMSL 1983 EPA EMSL 1983 EPA EMSL 1983 EPA EMSL 1983 EPA EMSL 1983 EPA EMSL 1983 EPA EMSL 1983 EPA EMSL 1983 EPA EMSL 1991 EPA EMSL 1983 EPA EMSL 1983 EPA EMSL 1983 EPA EMSL 1983 EPA EMSL 1983 EPA EMSL 1983 EPA EMSL 1983 EPA EMSL 1983 EPA EMSL 1983 EPA EMSL 1983 EPA EMSL 1983 EPA EMSL 1983 APHA 1985 EPA EMSL 1983 APHA 1985 
Method

EPA415.1
EPA418.1
EPA420.1
EPA420.2
APHA705
ASTMD3869C
APHA5320
EPA6010
EPA7041
EPA7060
EPA7421
EPA7470
EPA7740
EPA7841
EPA8010
EPA8020
EPA8080
EPA8140
EPA8150
EPA8240
EPA8270
EPA8280
EPA9012
EPA9020
EPA9030

Used to Analyze

Total organic carbon
Petroleum hydrocarbons
Phenolics
Phenolics
Total alpha-emitting radium
lodide
Dissolved organic halogen
Metals
Antimony
Arsenic
Lead
Mercury
Selenium
Thallium
Halogenated volatile organics
Aromatic volatile organics
Organochlorine pesticides and PCBs
Organophosphorus pesticides
Chlorinated herbicides
GCMS VOA
GCMS semivolatiles
Dioxins and furans
Total cyanide
Total organic halides
Sulfides

Source

EPA EMSL 1983
EPA EMSL 1983
EPA EMSL 1983
EPA EMSL 1983
APHA 1985
ASTM 1992
APHA 1989
EPA 1986
EPA 1986
EPA 1986
EPA 1986
EPA 1986
EPA 1986
EPA 1986
EPA 1986
EPA 1986
EPA 1986
EPA 1986
EPA 1986
EPA 1986
EPA 1986
EPA 1986
EPA 1986
EPA 1986
EPA 1986

An example of the available method-specific QA/QC information is that for the analysis of metals by EPA Method 6010/200.7 (EPA, 1986/EPA EMSL, 1983). The primary laboratories, General Engineering Laboratories (GE) and Roy F. Weston, Inc. (Weston), use this inductively coupled plasma (ICP) atomic emission spectrometric method.

The following precision and accuracy data are based on the experience of seven laboratories that applied the ICP technique to acid-distilled water matrices that had been dosed with various metal concentrates. (Note: not all seven laboratories analyzed all 14 elements.) The references give results for samples having three concentration ranges; the results here are for samples having the lowest values, similar to actual groundwater results for SRS.

ICP Precision and Accuracy Data

\section{Element}

Beryllium

Manganese

Vanadium

Arsenic

Chromium

Copper

Iron

Aluminum

\section{True value $(\mu \mathrm{g} / \mathrm{L})$}

20

15

70

22

10

11

20

60
Mean reported

value $(\mu \mathrm{g} / \mathrm{L})$

20

15

69

19

10

11

19

62
Mean percent

$\underline{\mathrm{RSD}}^{\mathrm{a}}$

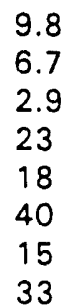




\begin{tabular}{llll} 
Element & True value $(\mu \mathrm{g} / \mathrm{L})$ & $\begin{array}{l}\text { Mean reported } \\
\text { value }(\mu \mathrm{g} / \mathrm{L})\end{array}$ & $\begin{array}{l}\text { Mea } \\
\text { RSD }\end{array}$ \\
\cline { 2 - 3 } & 2.5 & 2.9 & 16 \\
Cobalt & 20 & 20 & 4.1 \\
Nickel & 30 & 28 & 11 \\
Lead & 24 & 30 & 32 \\
Zinc & 16 & 19 & 45 \\
Selenium & 6 & 8.5 & 42
\end{tabular}

Note: In EPA (1986), the column heading is Mean Standard Deviation (\%).

a Relative standard deviation.

As another example, EPA Method 601/8010 (CFR, 1991/EPA, 1986) is used by both GE and Weston for analyses of halogenated volatile organics. In the presentation of the method in both references, the following table gives method-specific accuracy and precision as functions of concentration. Contract laboratories are expected to achieve or at least approach these limits.

Accuracy and Precision as Functions of Concentration for EPA Method 601/8010

\section{Parameter}

Bromodichloromethane

Bromoform

Bromomethane

Carbon tetrachloride

Chlorobenzene

Chloroethane

2.-Chloroethyl vinyl ether ${ }^{f}$

Chloroform

Chloromethane

Dibromochloromethane

1,2-Dichlorobenzene

1,3-Dichlorobenzene

1,4-Dichlorobenzene

1,1-Dichloroethane

1,2-Dichloroethane

1,1-Dichloroethene trans-1,2-Dichloroethene 1,2-Dichloropropane ${ }^{f}$ cis-1,3-Dichloropropene ${ }^{f}$ trans-1,3-Dichloropropene ${ }^{f}$ Methylene chloride

1,1,2,2-Tetrachloretharie

Tetrachloroethylene

$1,1,1$-Trichloroethane

1,1,2-Trichloroethane

Trichloroethylene
Accuracy as recovery, $x^{\prime a}(\mu \mathrm{g} / L)$

$1.12 C-1.02^{d}$

$0.96 C-2.05$

$0.76 C-1.27$

$0.98 C-1.04$

$1.00 C-1.23$

$0.99 C-1.53$

$1.00 \mathrm{C}$

$0.93 C-0.39$

$0.77 C+0.18$

$0.94 C+2.72$

$0.93 C+1.70$

$0.95 C+0.43$

$0.93 C-0.09$

$0.95 C-1.08$

$1.04 C-1.06$

$0.98 C-0.87$

$0.97 C-0.16$

$1.00 \mathrm{C}$

$1.00 \mathrm{C}$

$1.00 \mathrm{C}$

$0.91 C-0.93$

$0.95 C+0.19$

$0.94 C+0.06$

$0.90 C-0.16$

$0.86 C+0.30$

$0.87 C+0.48$
Single analyst precision $(\mu \mathrm{g} / \mathrm{L})^{b}$

$0.11 \bar{x}+0.04^{e}$

$0.12 \bar{x}+0.58$

$0.28 \bar{x}+0.27$

$0.15 \bar{x}+0.38$

$0.15 \bar{x}-0.02$

$0.14 \bar{X}-0.13$

$0.20 \bar{x}$

$0.13 \bar{x}+0.15$

$0.28 \bar{x}-0.31$

$0.11 \bar{x}+1.10$

$0.20 \bar{x}+0.97$

$0.14 \bar{X}+2.33$

$0.15 \bar{X}+0.29$

$0.09 \bar{x}+0.17$

$0.11 \bar{x}+0.70$

$0.21 \bar{x}-0.23$

$0.11 \bar{x}+1.46$

$0.13 \bar{x}$

$0.18 \bar{X}$

$0.18 \bar{X}$

$0.11 \bar{x}+0.33$

$0.14 \bar{x}+2.41$

$0.14 \bar{X}+0.38$

$0.15 \bar{x}+0.04$

$0.13 \bar{x}-0.14$

$0.13 \bar{x}-0.03$
Overall precision $\left(\mu \mathrm{g} / \mathrm{Ll}^{\mathrm{C}}\right.$

$0.20 \bar{X}+1.00$

$0.21 \bar{x}+2.41$

$0.36 \bar{x}+0.94$

$0.20 \bar{x}+0.39$

$0.18 \bar{x}+1.21$

$0.17 \bar{x}+0.63$

$0.35 \bar{x}$

$0.19 \bar{x}-0.02$

$0.52 \bar{x}+1.31$

$0.24 \bar{x}+1.68$

$0.13 \bar{x}+6.13$

$0.26 \bar{x}+2.34$

$0.20 \bar{x}+0.41$

$0.14 \bar{X}+0.94$

$0.15 \bar{x}+0.94$

$0.29 \bar{x}-0.40$

$0.17 \bar{X}+1.46$

$0.23 \bar{x}$

$0.32 \bar{x}$

$0.32 \bar{X}$

$0.21 \bar{x}+1.43$

$0.23 \bar{x}+2.79$

$0.18 \bar{X}+2.21$

$0.20 \bar{X}+0.37$

$0.19 \bar{X}+0.67$

$0.23 \bar{x}+0.30$ 
Parameter

Trichlorofluoromethane

Vinyl chloride

\begin{tabular}{|c|c|c|}
\hline $\begin{array}{l}\text { Accuracy as } \\
\text { recovery, } x^{\prime a}(\mu \mathrm{g} / \mathrm{L})\end{array}$ & $\begin{array}{l}\text { Single analyst } \\
\text { precision }(\mu \mathrm{g} / \mathrm{L})^{b}\end{array}$ & $\begin{array}{l}\text { Overall } \\
\text { precision }(\mu \mathrm{g} / \mathrm{L})^{\mathrm{c}}\end{array}$ \\
\hline $\begin{array}{l}0.89 C-0.07 \\
0.97 C-0.36\end{array}$ & $\begin{array}{l}0.15 \bar{x}+0.67 \\
0.13 \bar{x}+0.65\end{array}$ & $\begin{array}{l}0.26 \bar{x}+0.91 \\
0.27 \bar{x}+0.40\end{array}$ \\
\hline
\end{tabular}

a $X^{\prime}=$ expected recovery for one or more measurements of a sample containing a concentration of $C$, in $\mu g / L$.

b Expected single analyst standaro deviation of measurements.

c Expected interlaboratory standard deviation of measurements.

d $C=$ true value for the concentration, in $\mu \mathrm{g} / \mathrm{L}$.

$\bar{X}=$ average recovery found for measurements of samples containing a concentration of $C$, in $\mu \mathrm{g} / \mathrm{L}$.

$f$ Estimates based on performance in a single laboratory.

\section{References}

APHA (American Public Health Association), 1985. Standard Methods for the Examination of Water and Wastewater, 16th edition. Washington, DC.

APHA (American Public Health Association), 1989. Standard Methods for the Examination of Water and Wastewater, 17th edition. Washington, DC.

ASTM (American Society for Testing and Materials), 1992. 1992 Annual Book of ASTM Standards, Volume 11.02, Water (II). Philadelphia, PA.

CFR (Code of Federal Regulations), 1991. Guidelines Establishing Test Procedures for the Analysis of Groundwater, Title 40, Part 136, Appendix A. Revised July 1, 1991. Washington, DC.

EPA (U.S. Environmental Protection Agency), 1986. Test Methods for Evaluating Solid Waste (SW-846), Volumes IA-IC. Washington, DC.

EPA (U.S. Environmental Protection Agency), 1987. Data Quality Objectives for Remedial Response Activities. PB88-131870; EPA/540/G-87/003. Washington, DC.

EPA (U.S. Environmental Protection Agency), 1988a. Contract Laboratory Program Statement of Work for Inorganics Analysis, Multi-Media, Multi-Concentration. SOW No. 788. Washington, DC.

EPA (U.S. Environmental Protection Agency), 1988b. Contract Laboratory Program Statement of Work for Organics Analysis Multi-Media, Multi-Concentration. SOW No. 288. Washington, DC.

EPA (U.S. Environmental Protection Agency), 1990. Guidance for Data Useability in Risk Assessment. Interim Final. EPA/540/G-90/008. Washington, DC.

EPA EMSL (U.S. Environmental Protection Agency, Environmental Monitoring and Systems Laboratory), 1979. Handbook for Analytical Quality Control in Water Wastewater Laboratories. PB-297 451; EPA-600/4-79-019. Cincinnati, OH. 
EPA EMSL (U.S. Environmental Protection Agency, Environmental Monitoring and Systems Laboratory), 1983. Methods for Chemical Analysis of Water and Wastes. Revised March 1983. Cincinnati, $\mathrm{OH}$.

EPA EMSL (U.S. Environmental Protection Agency, Environmental Monitoring and Systems Laboratory), 1991. Test Method, The Determination of Inorganic Anions in Water by Ion Chromatography-Method 300.0. Revised August 1991. Cincinnati, OH. 
WSRC-TR-93.059

\section{Appendix F - Time Series Plots}




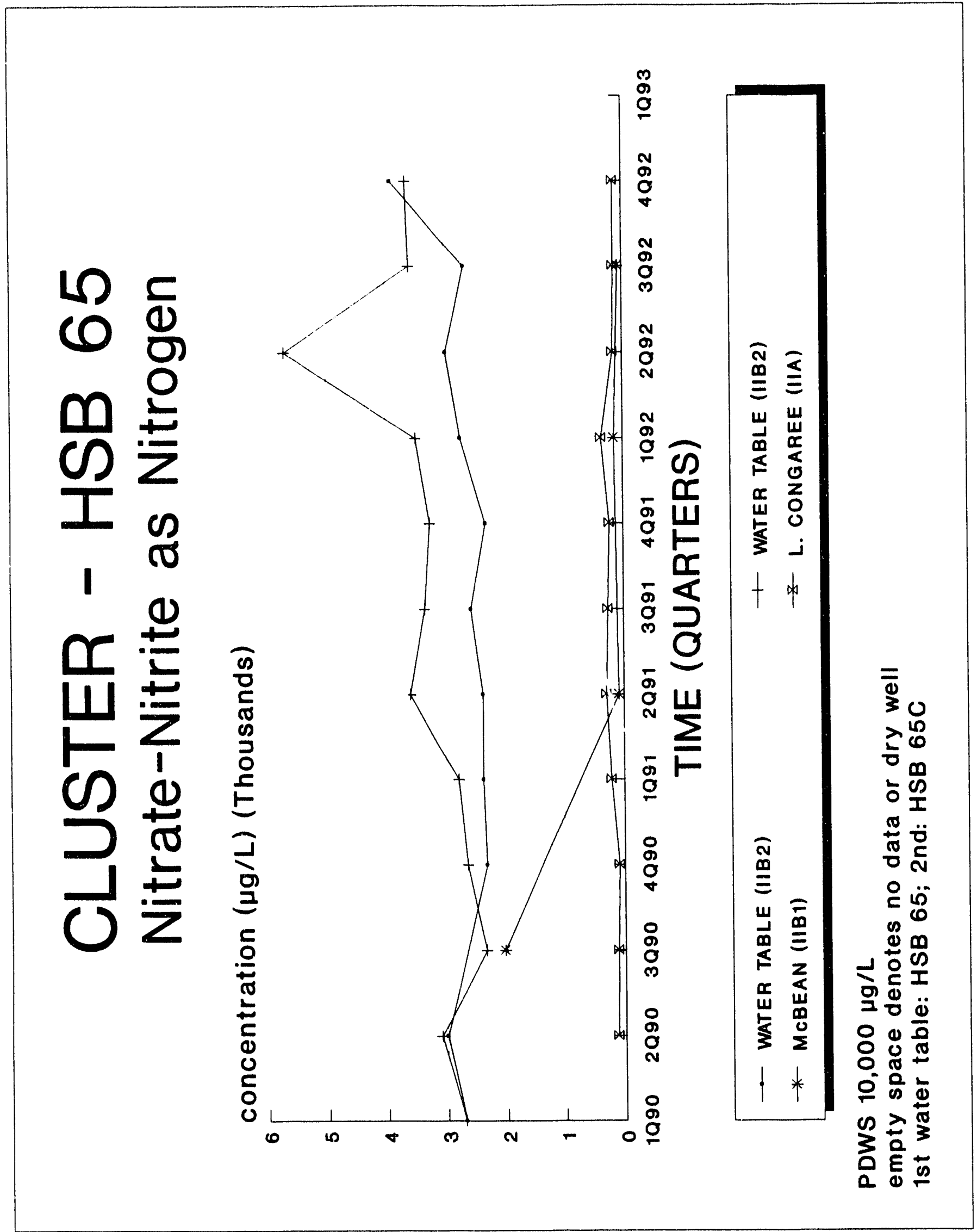




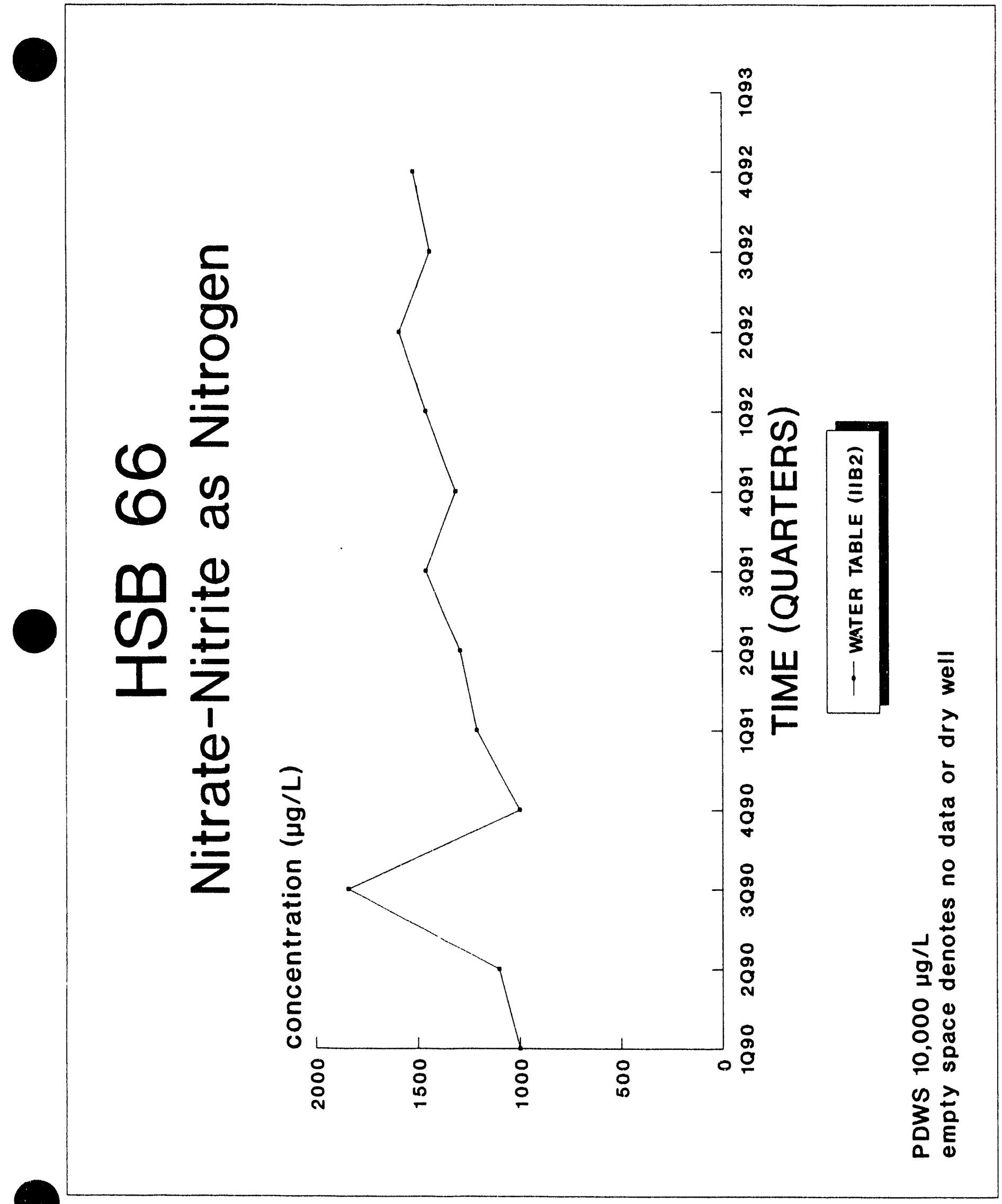




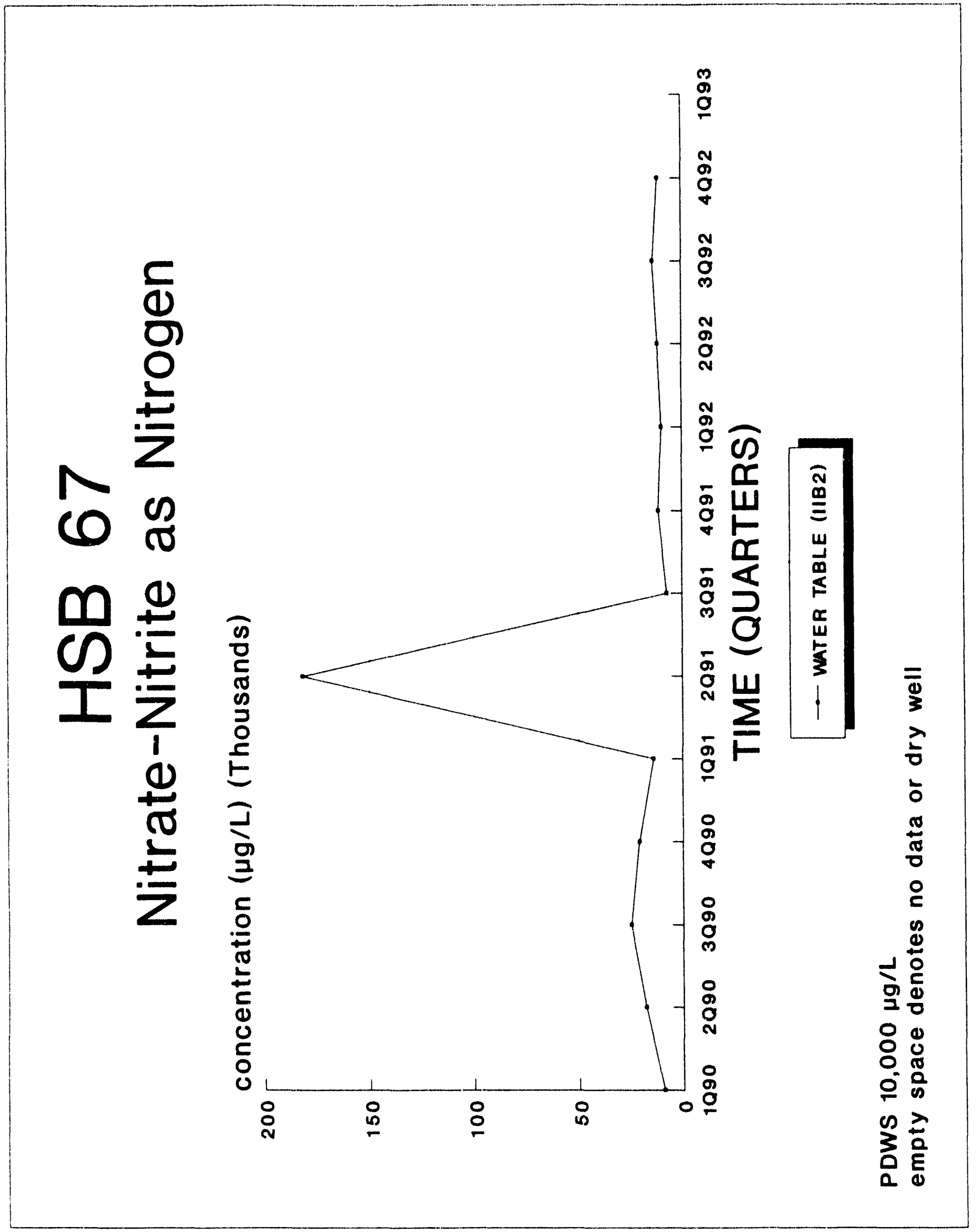




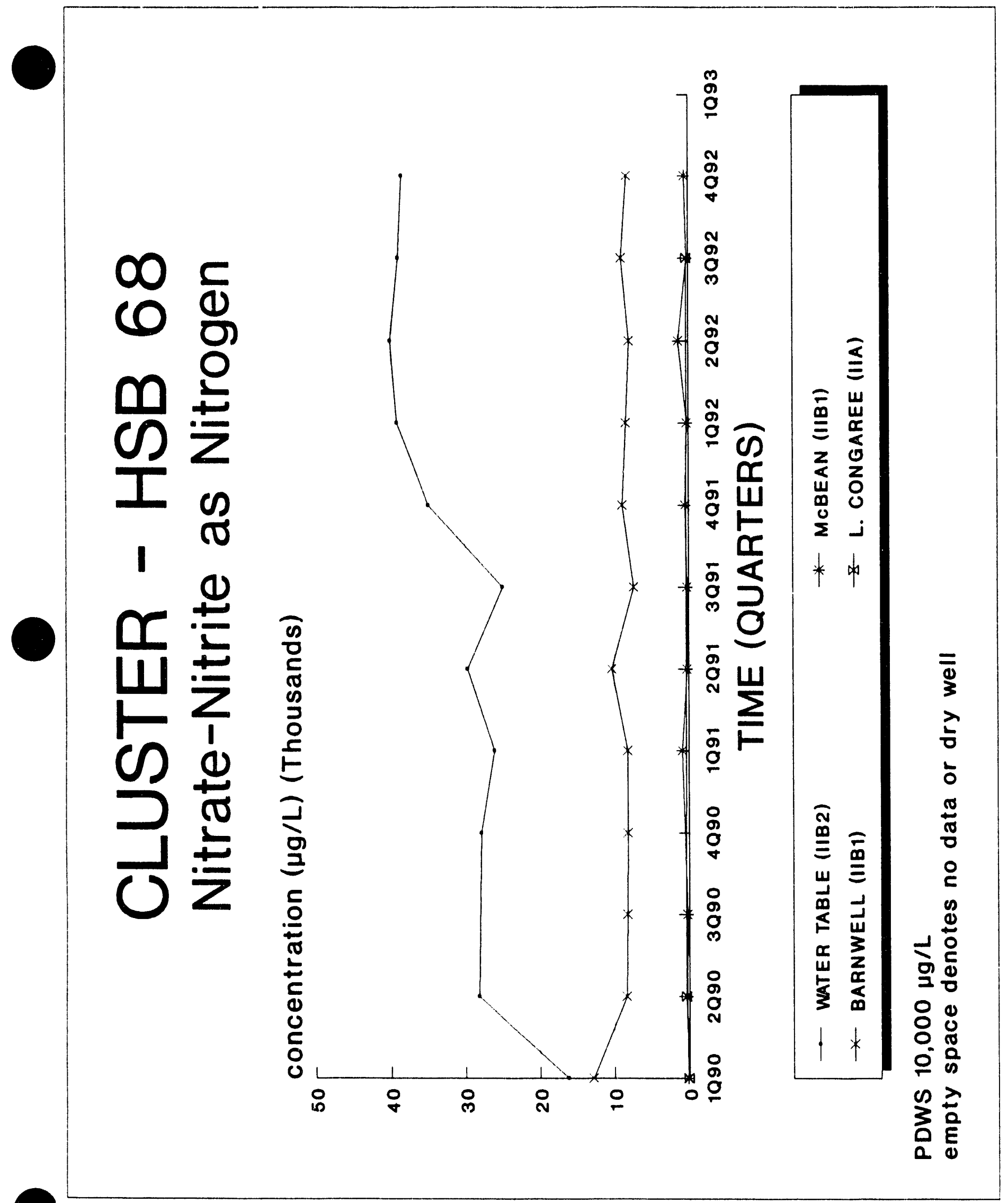




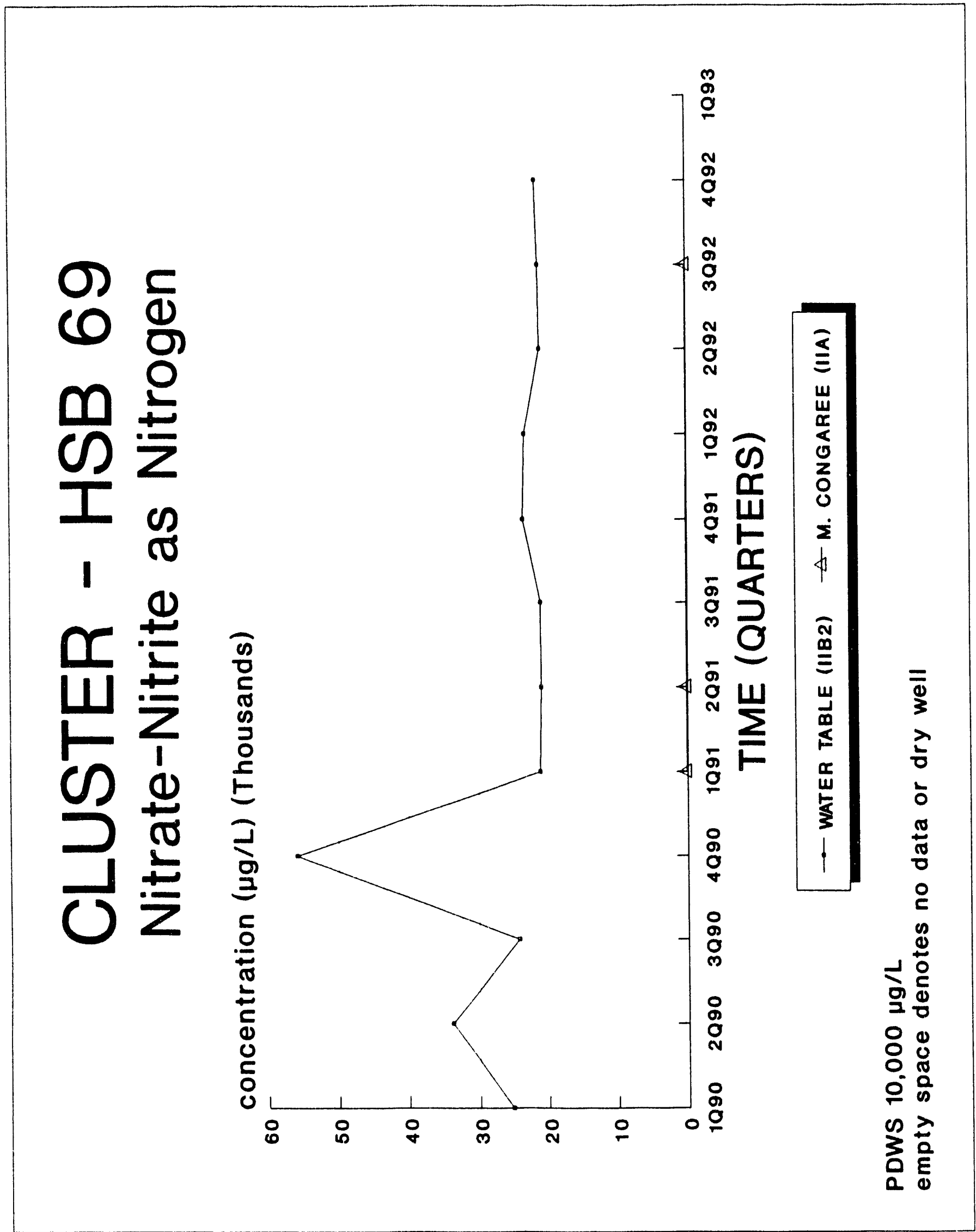




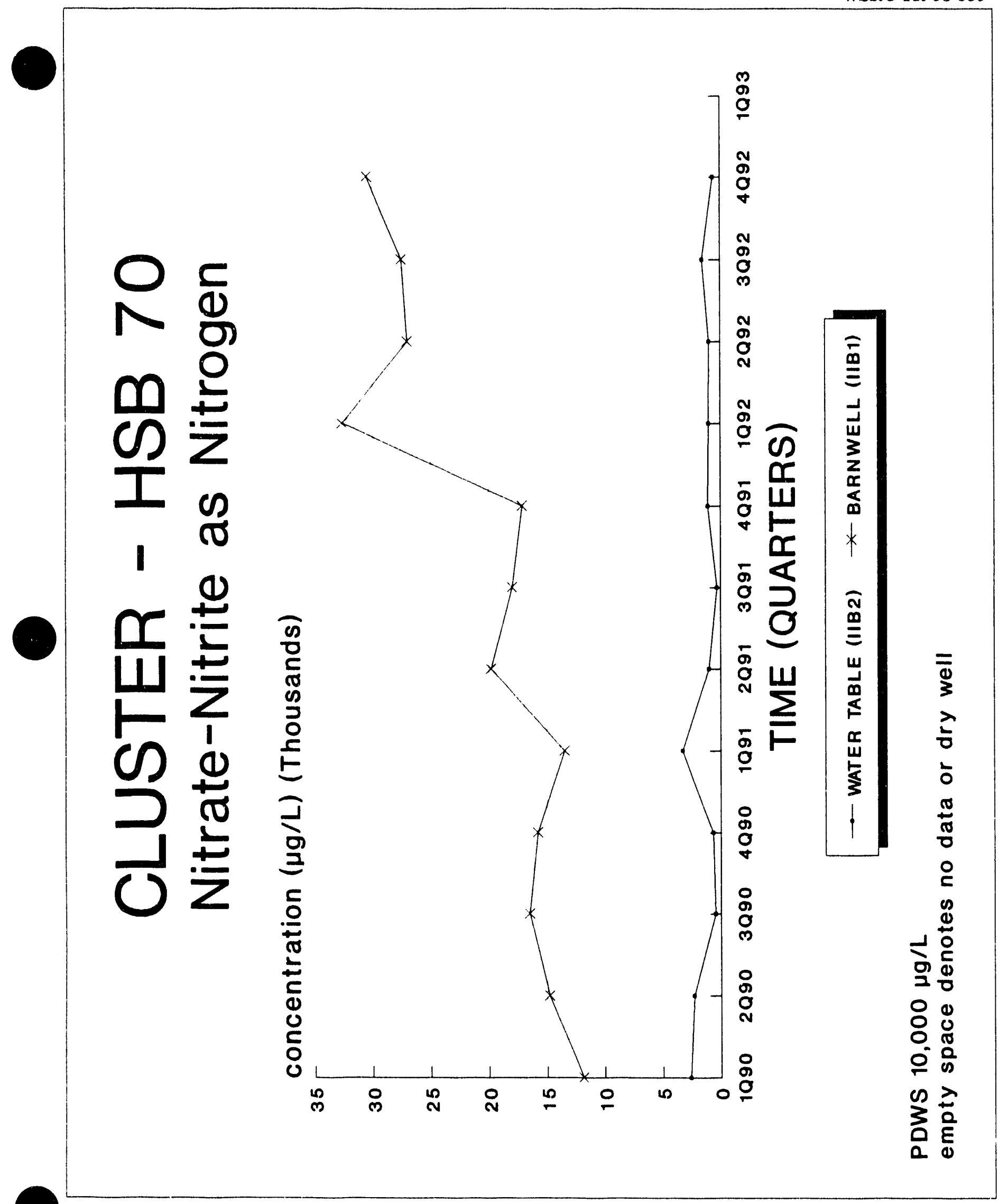




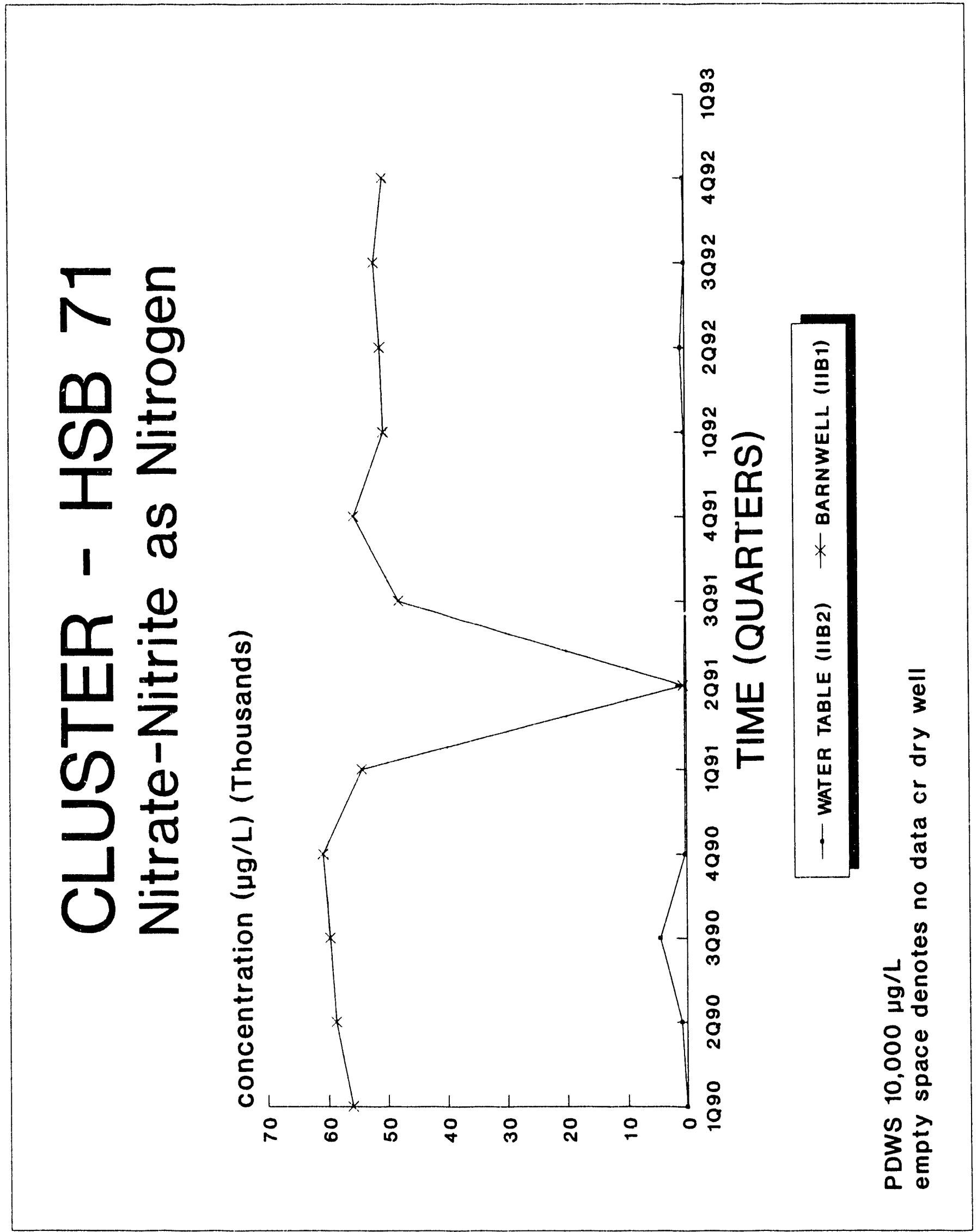




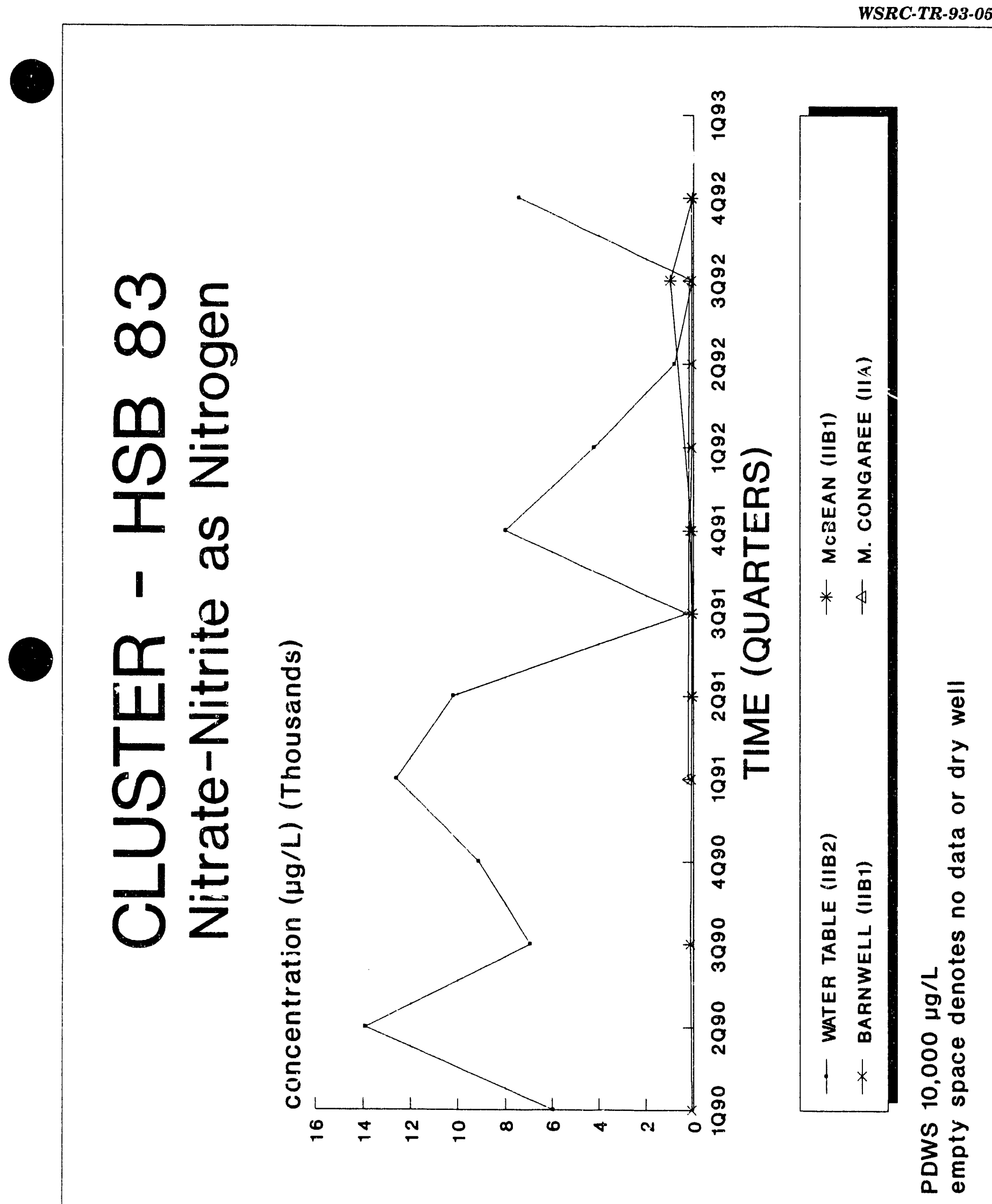




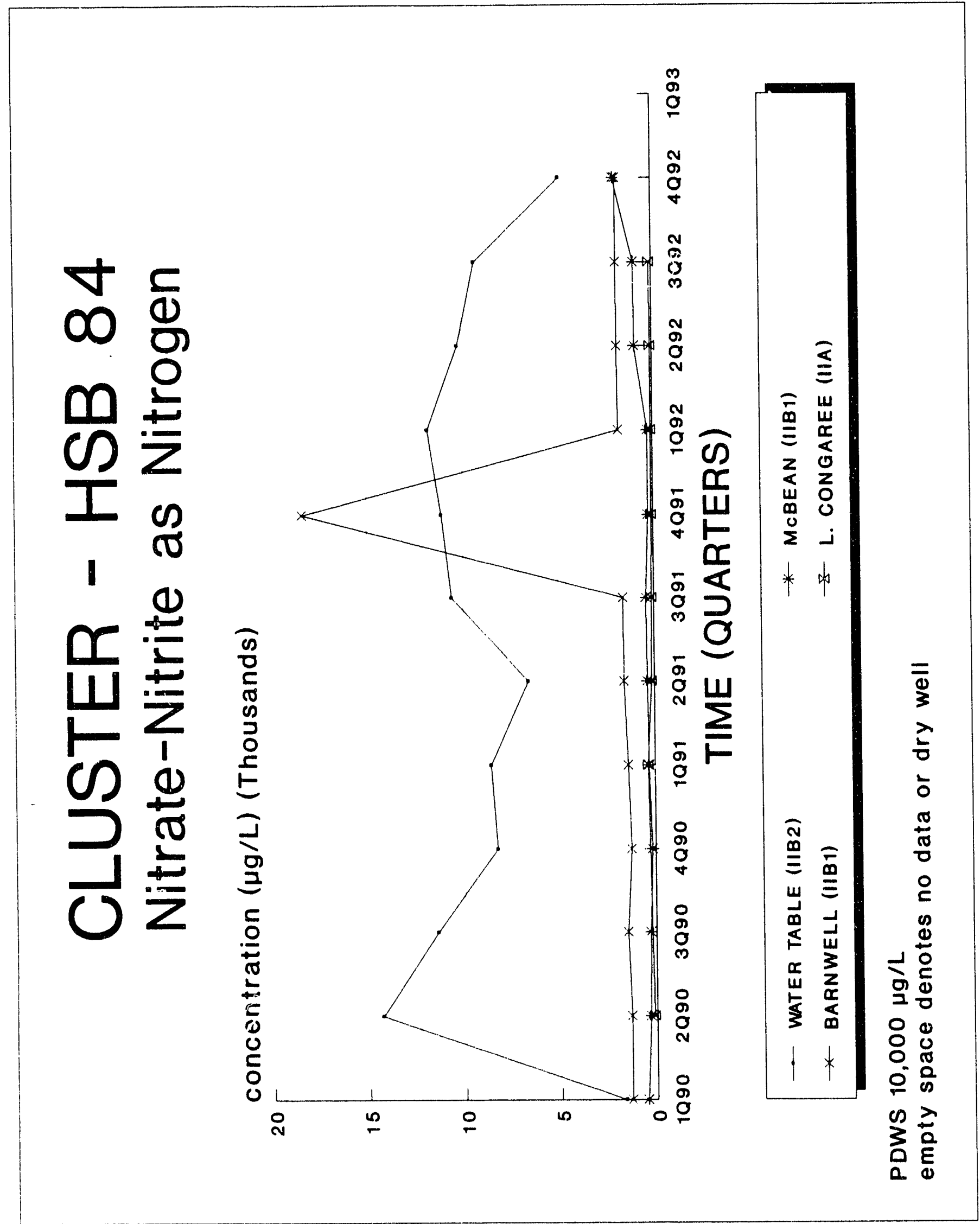




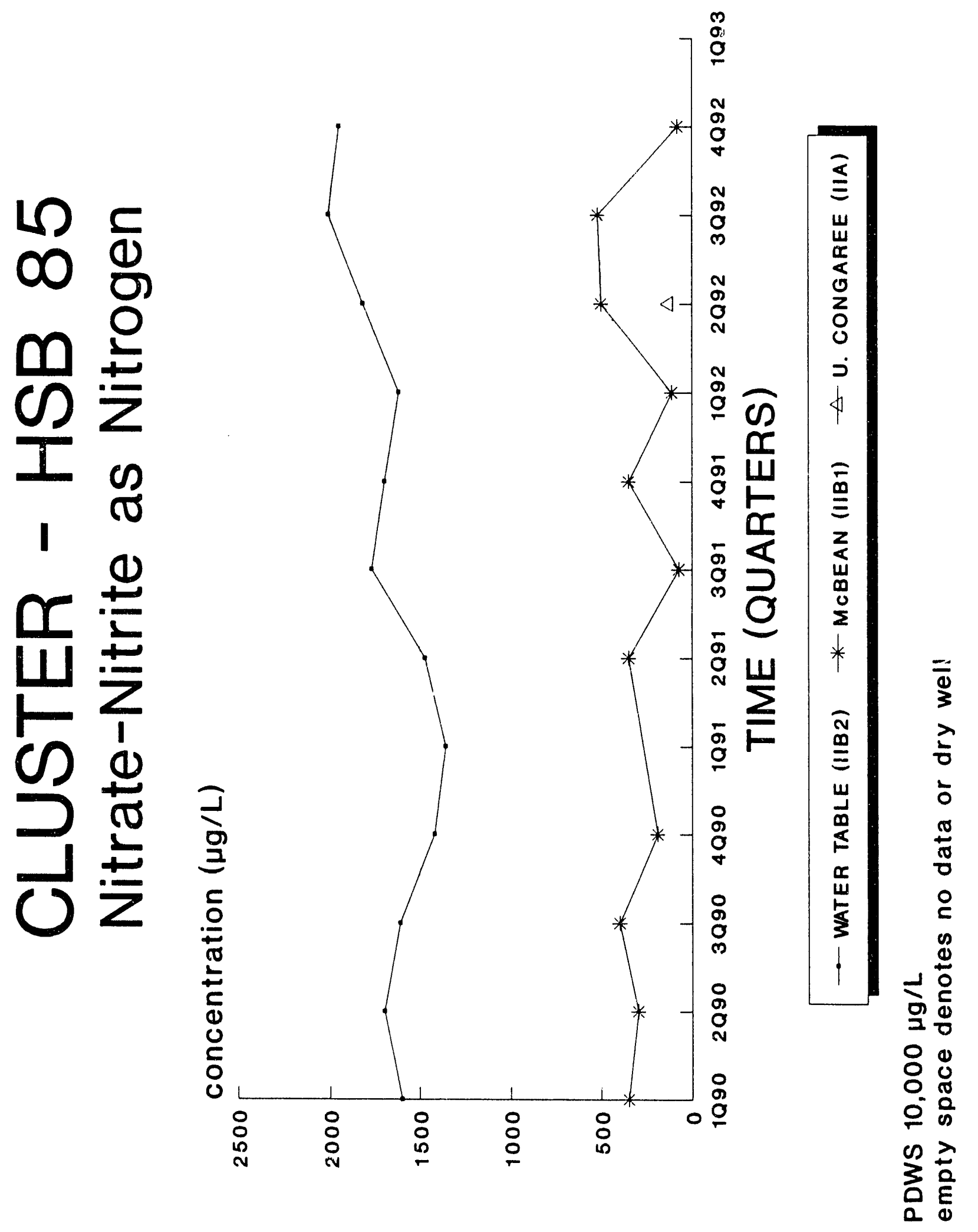




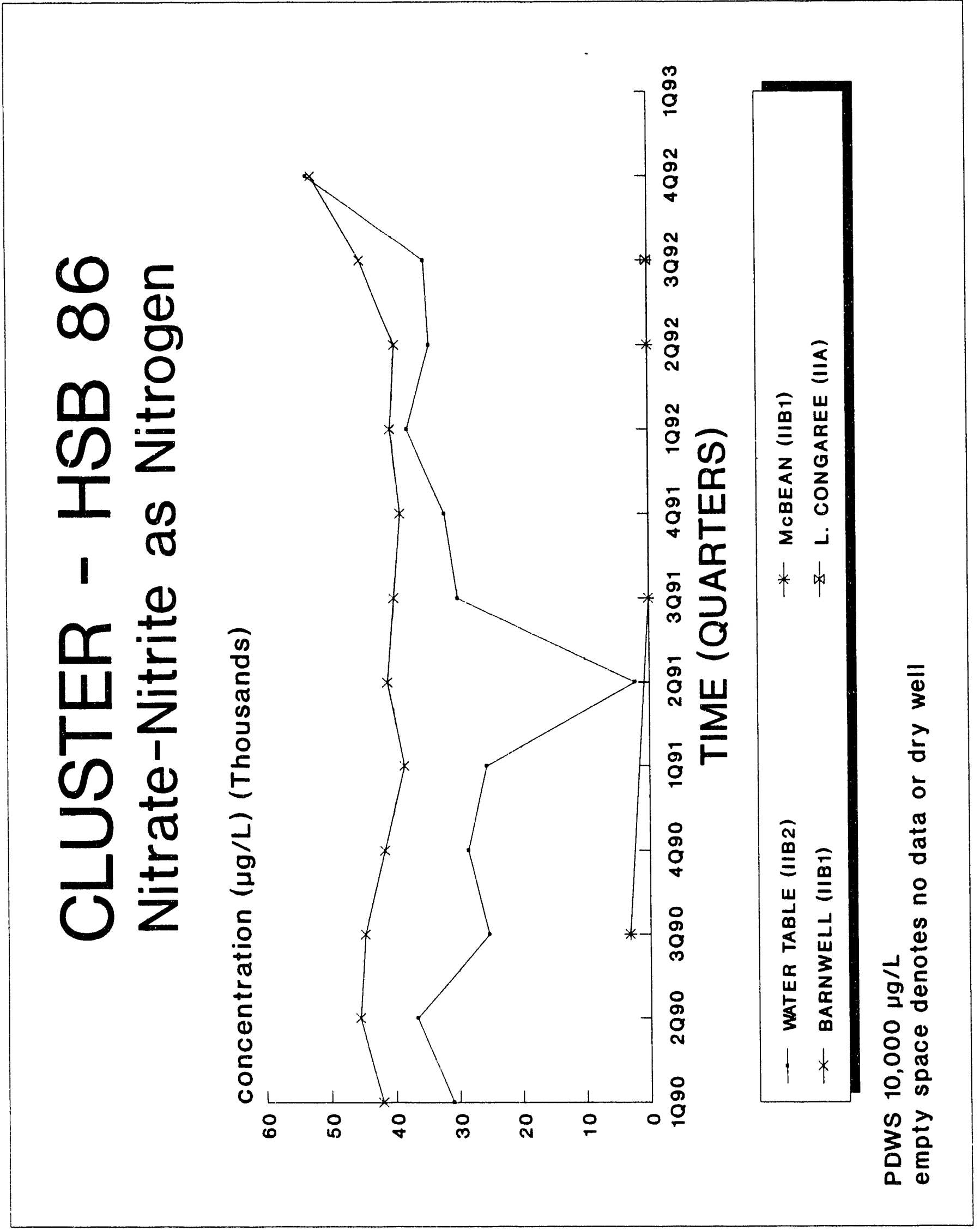




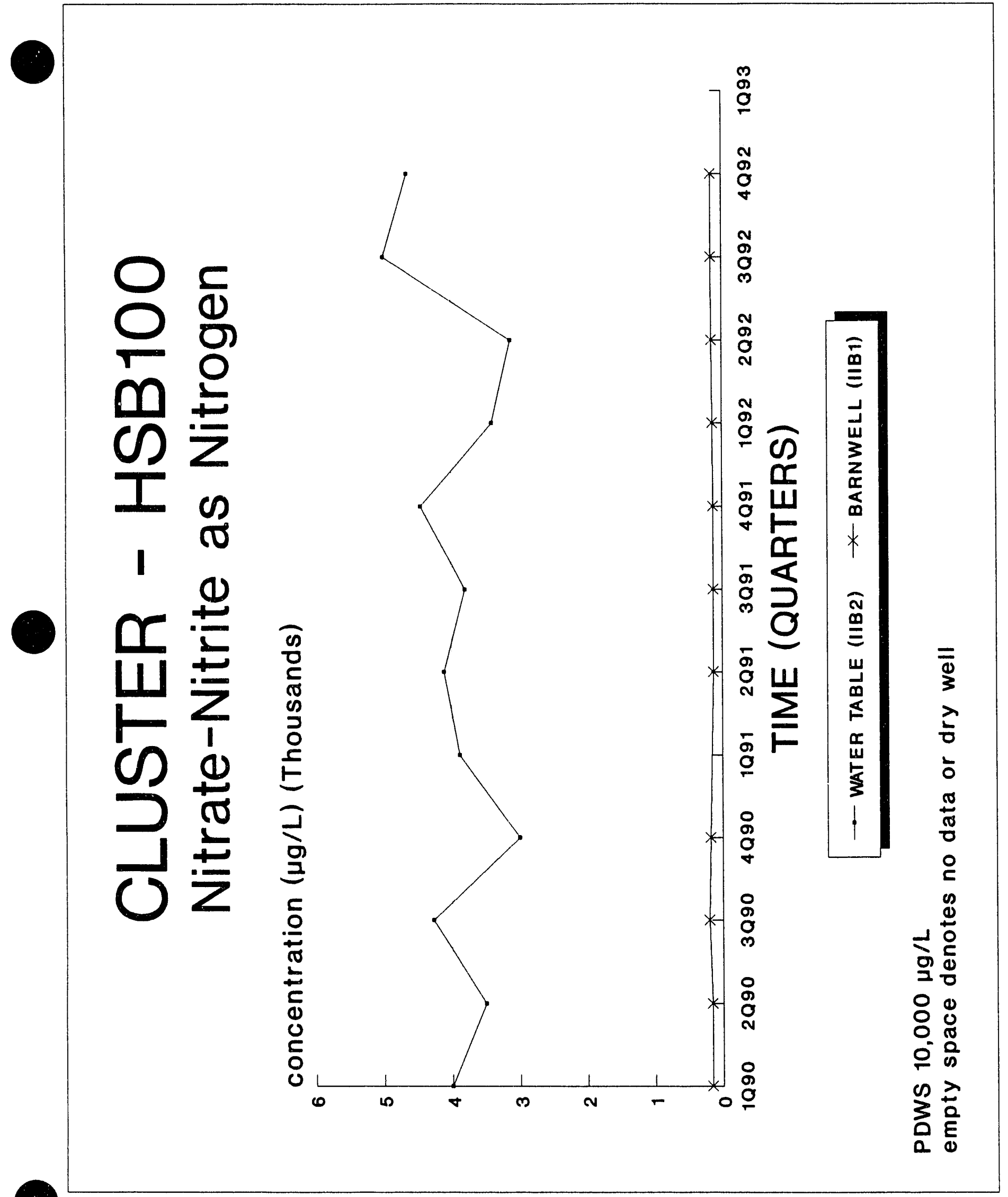




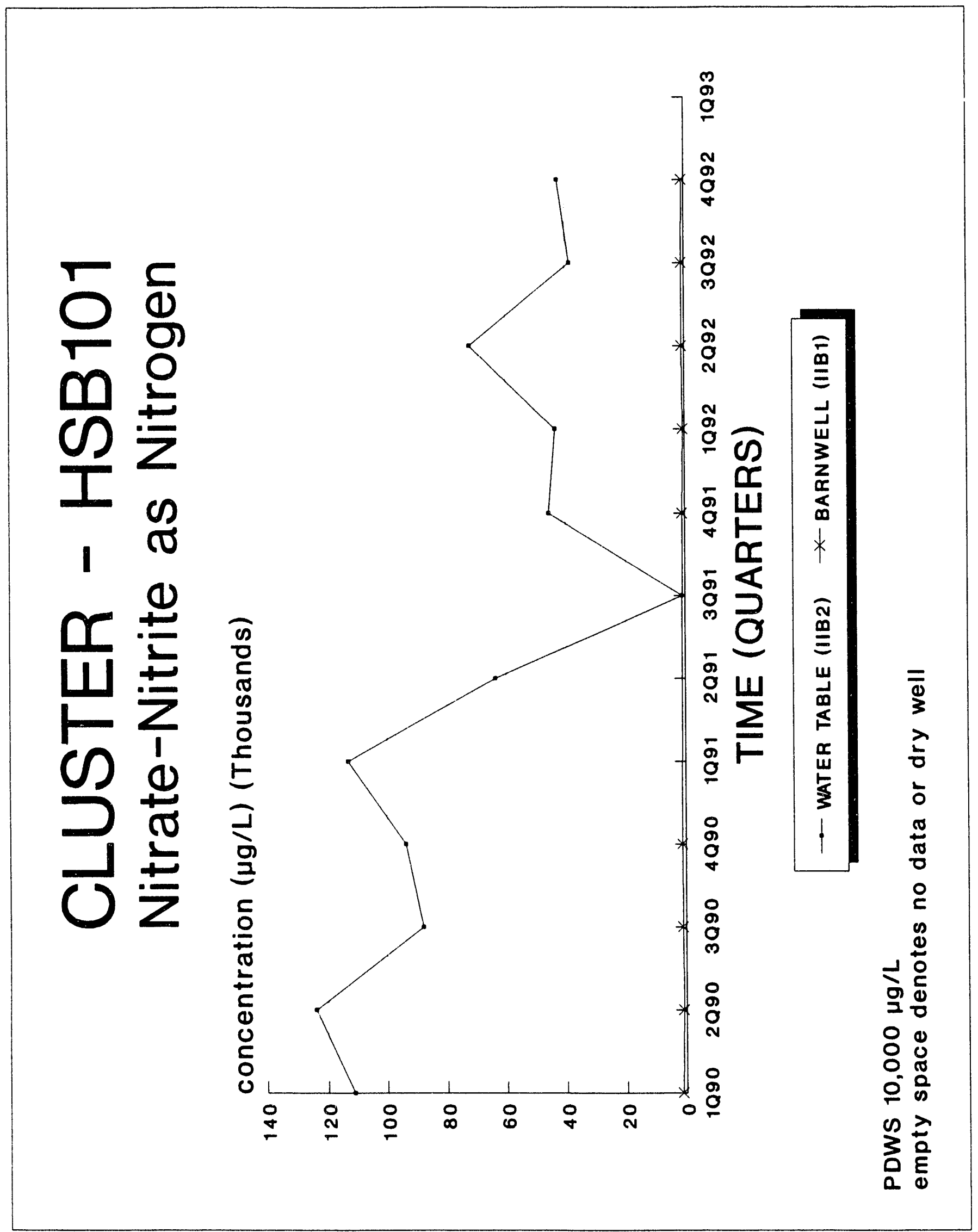




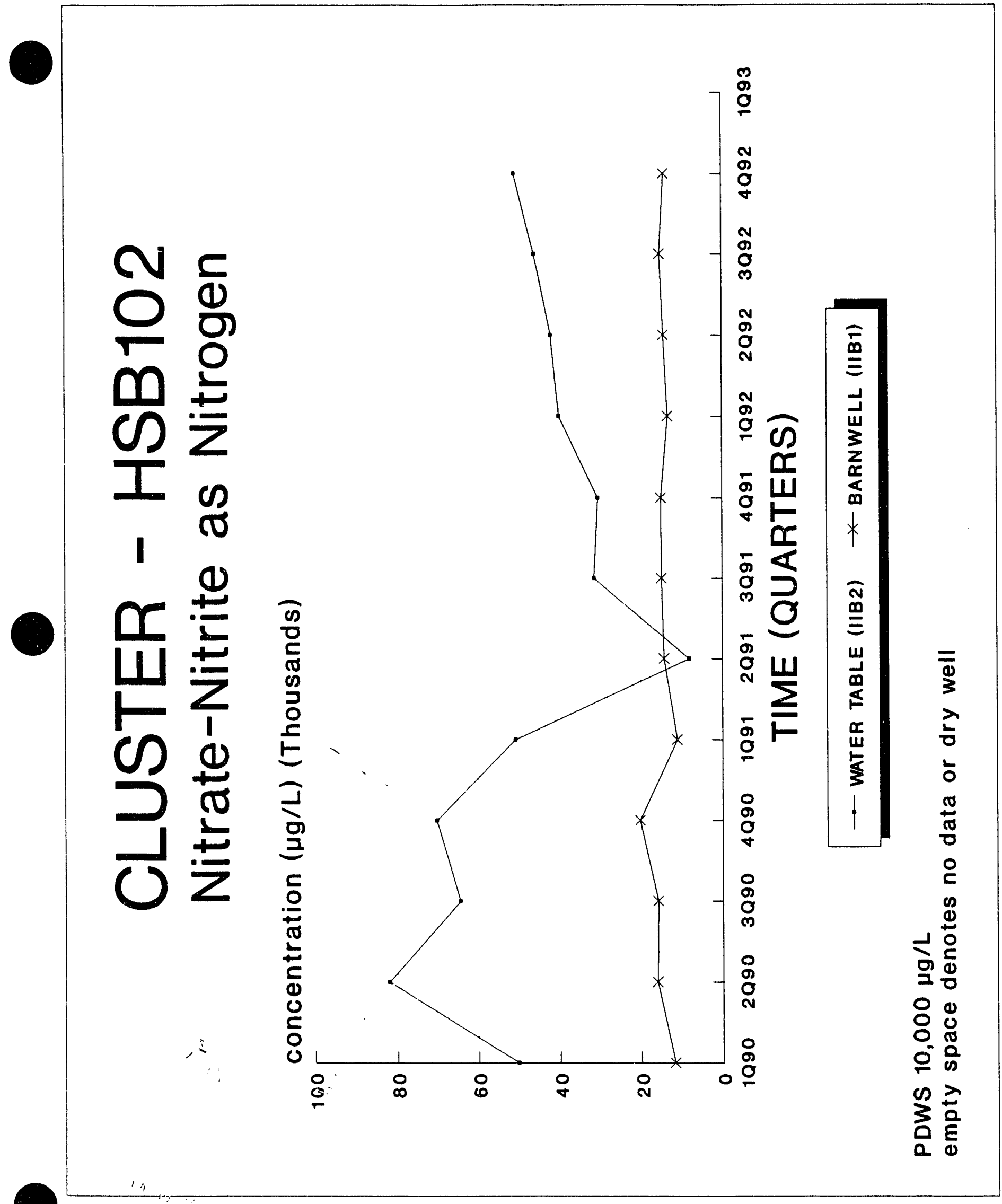




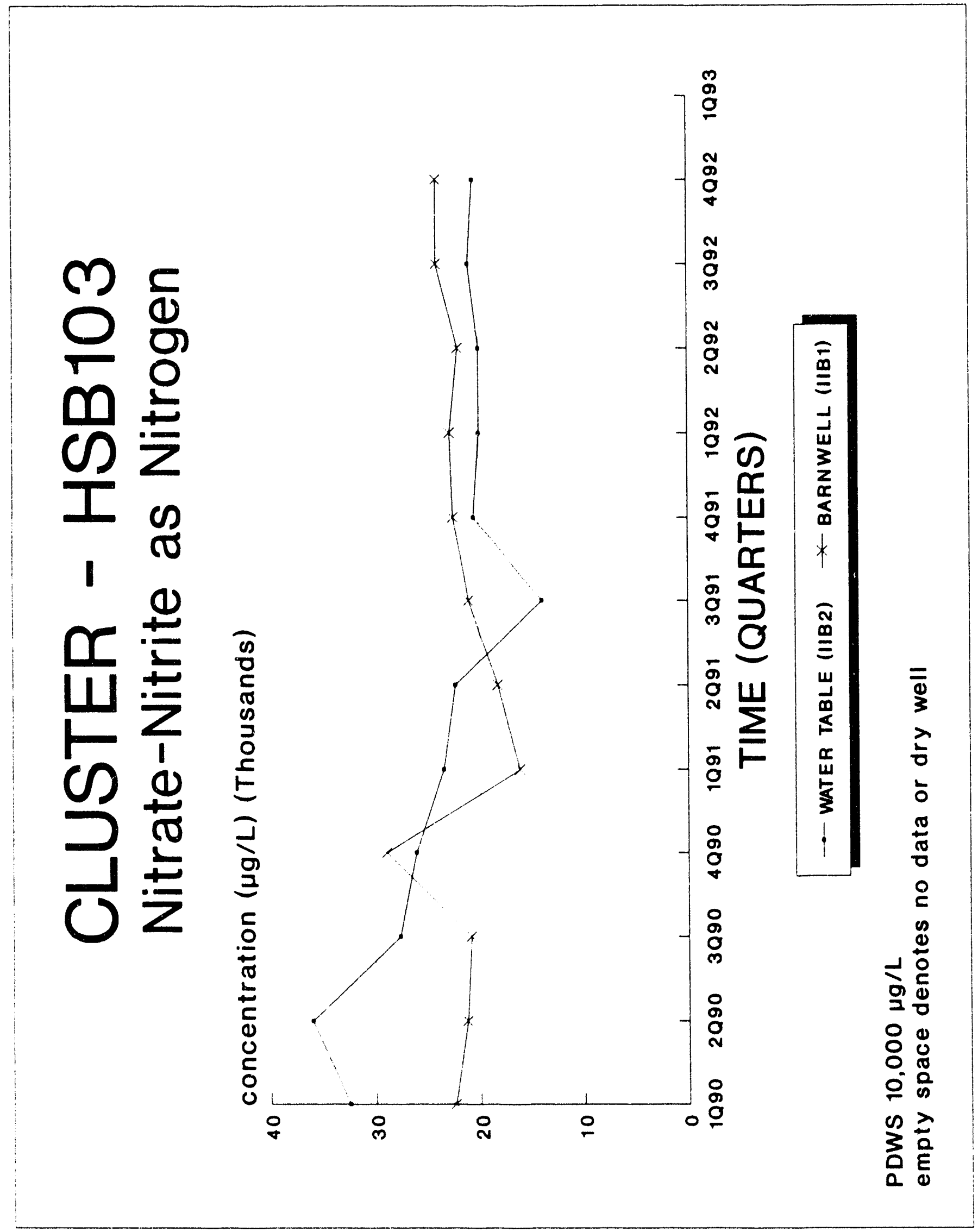




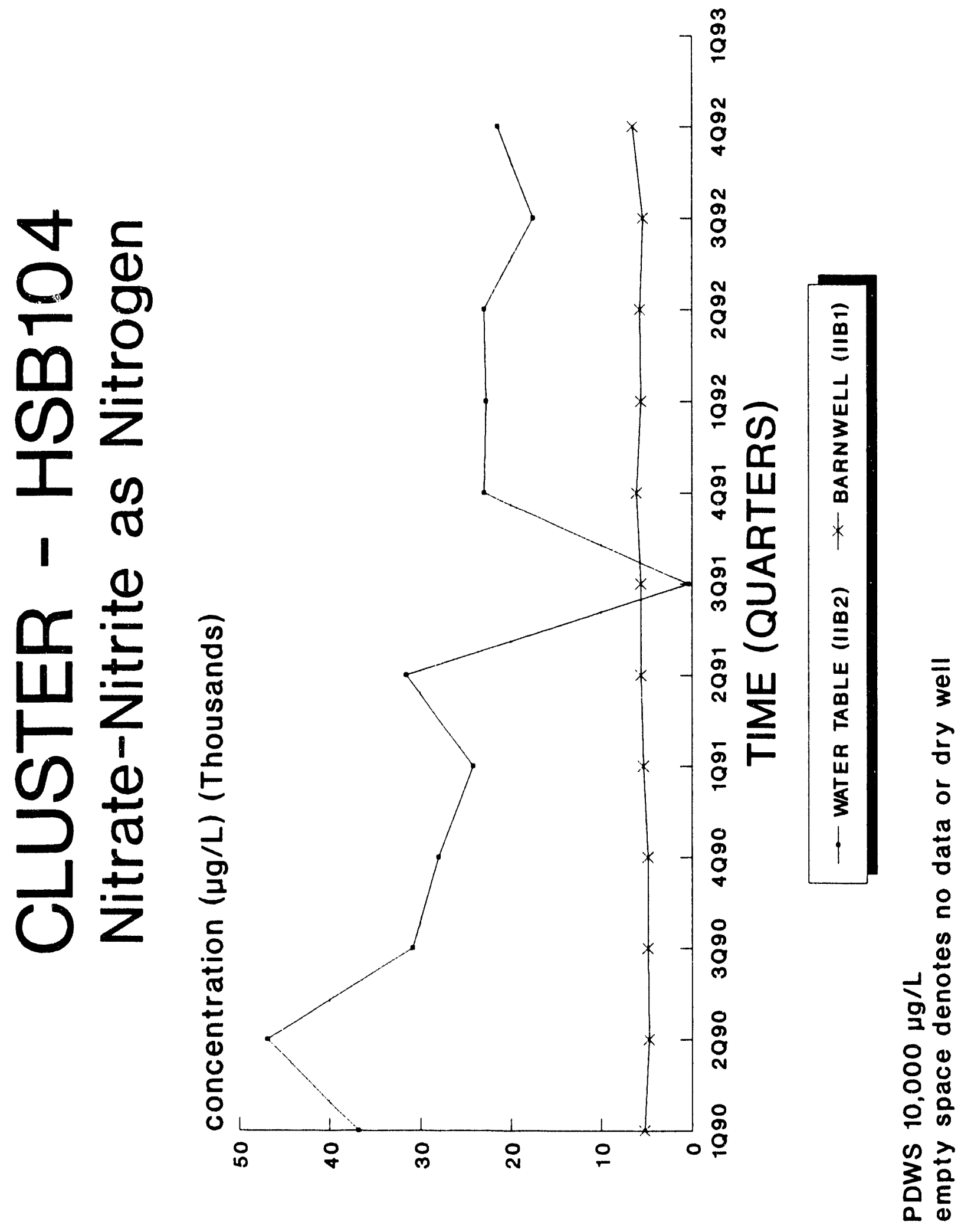




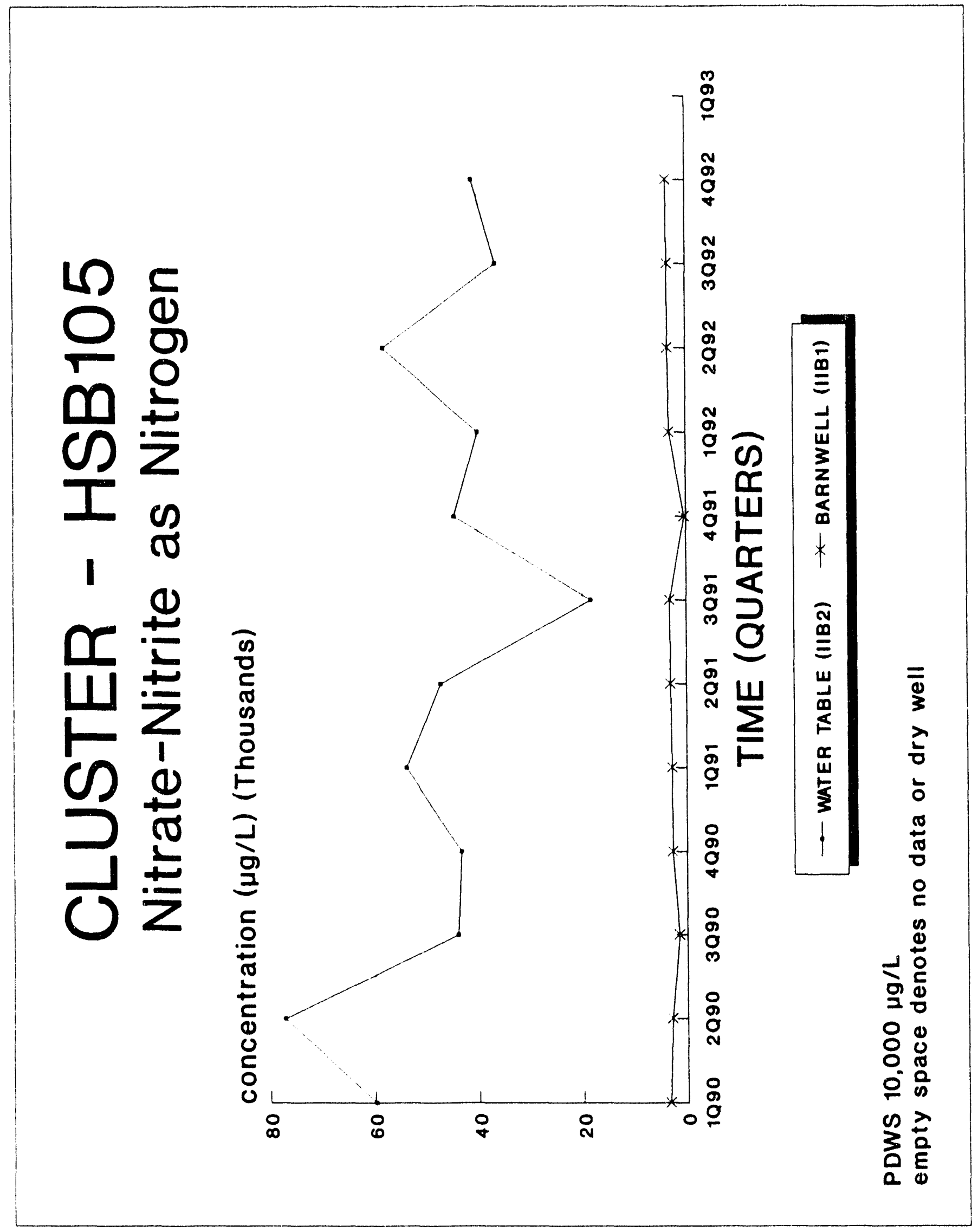




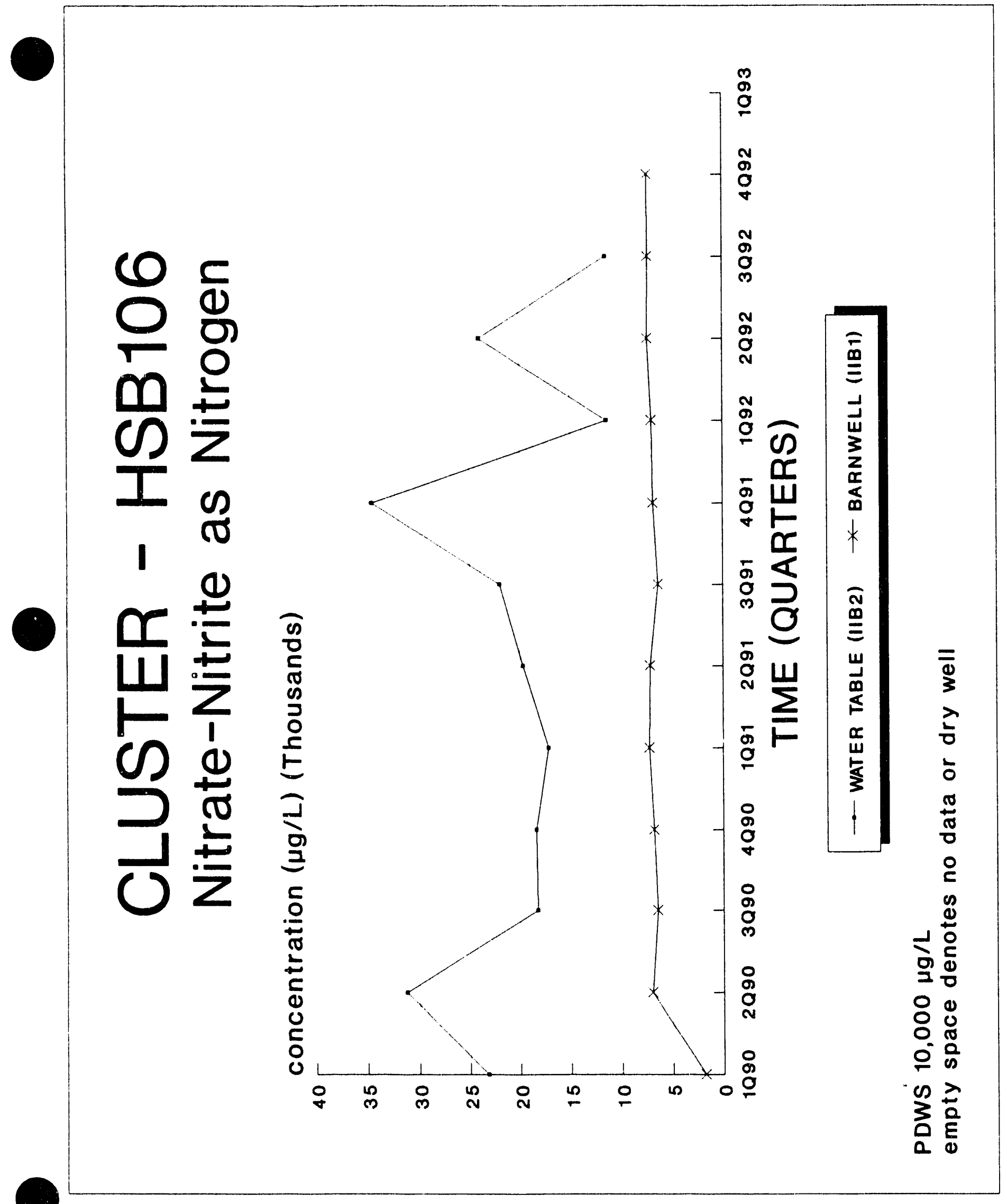




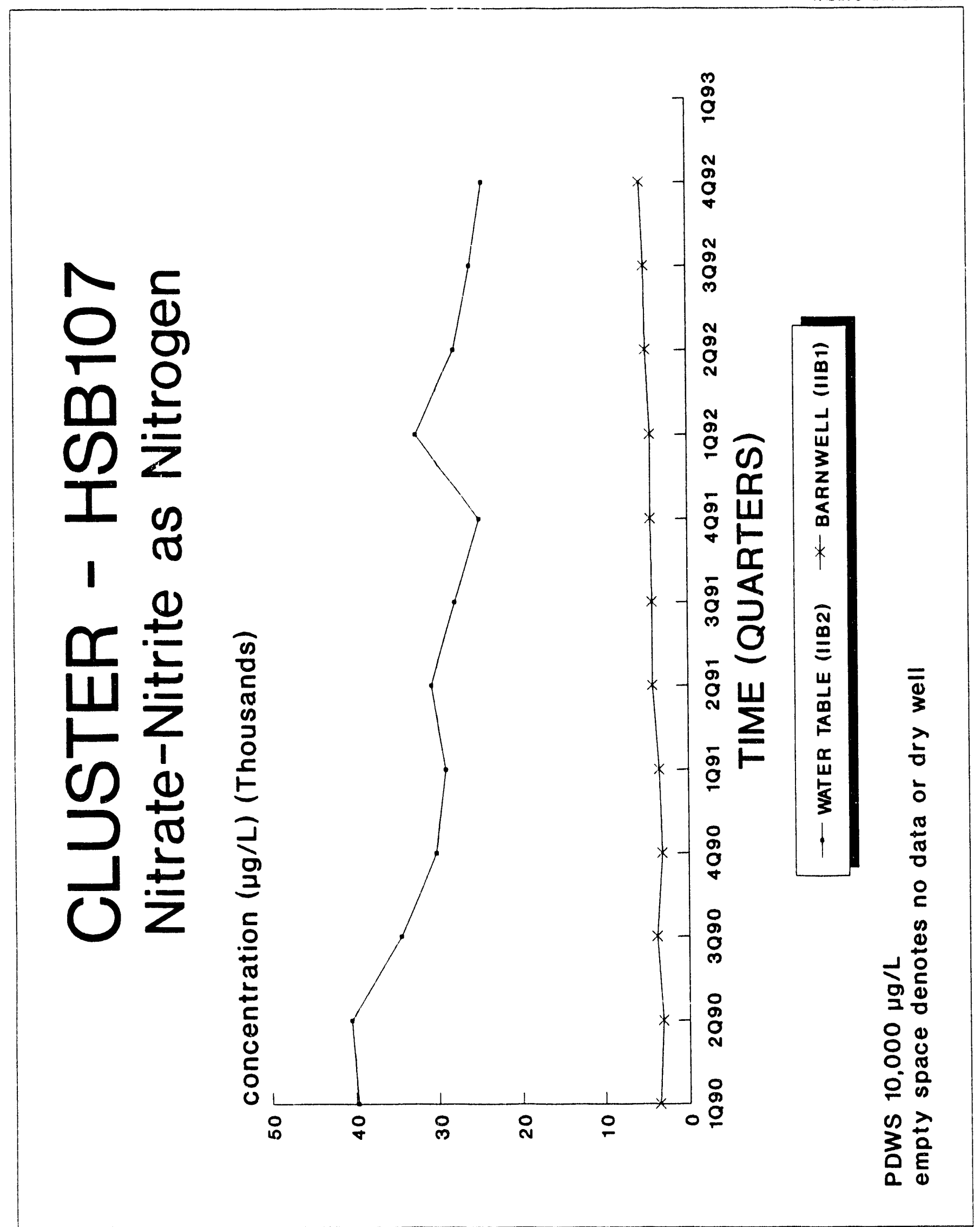




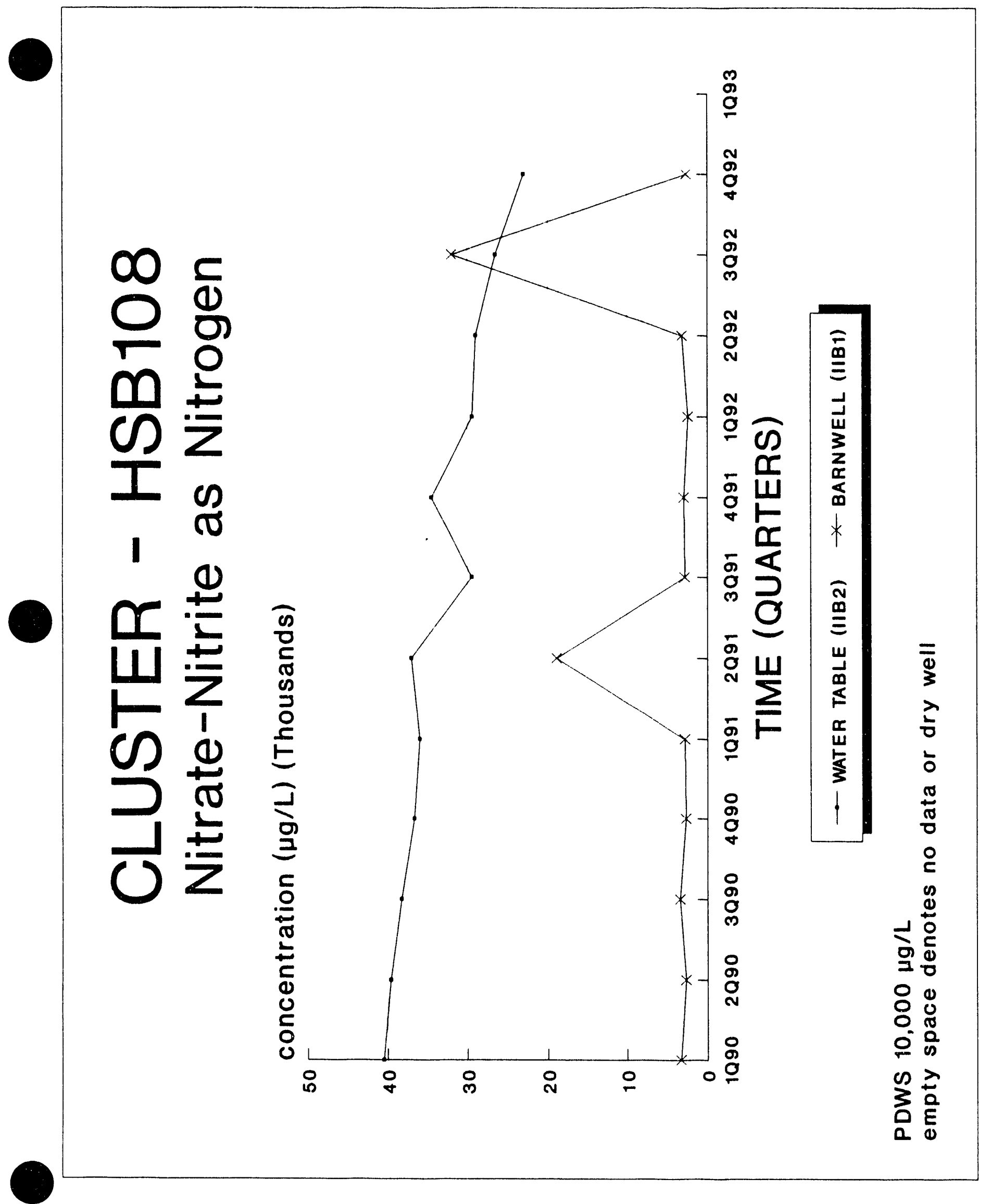




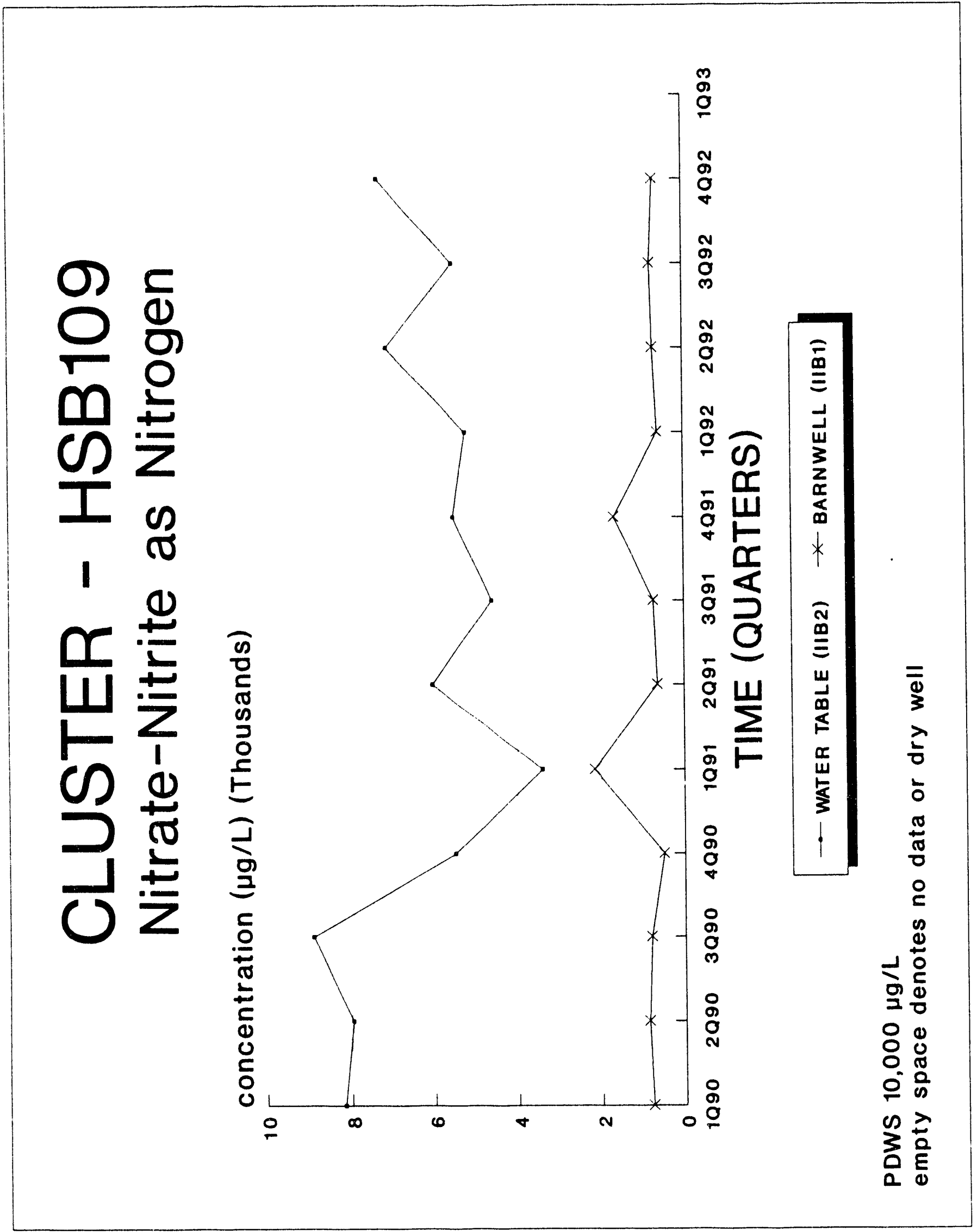




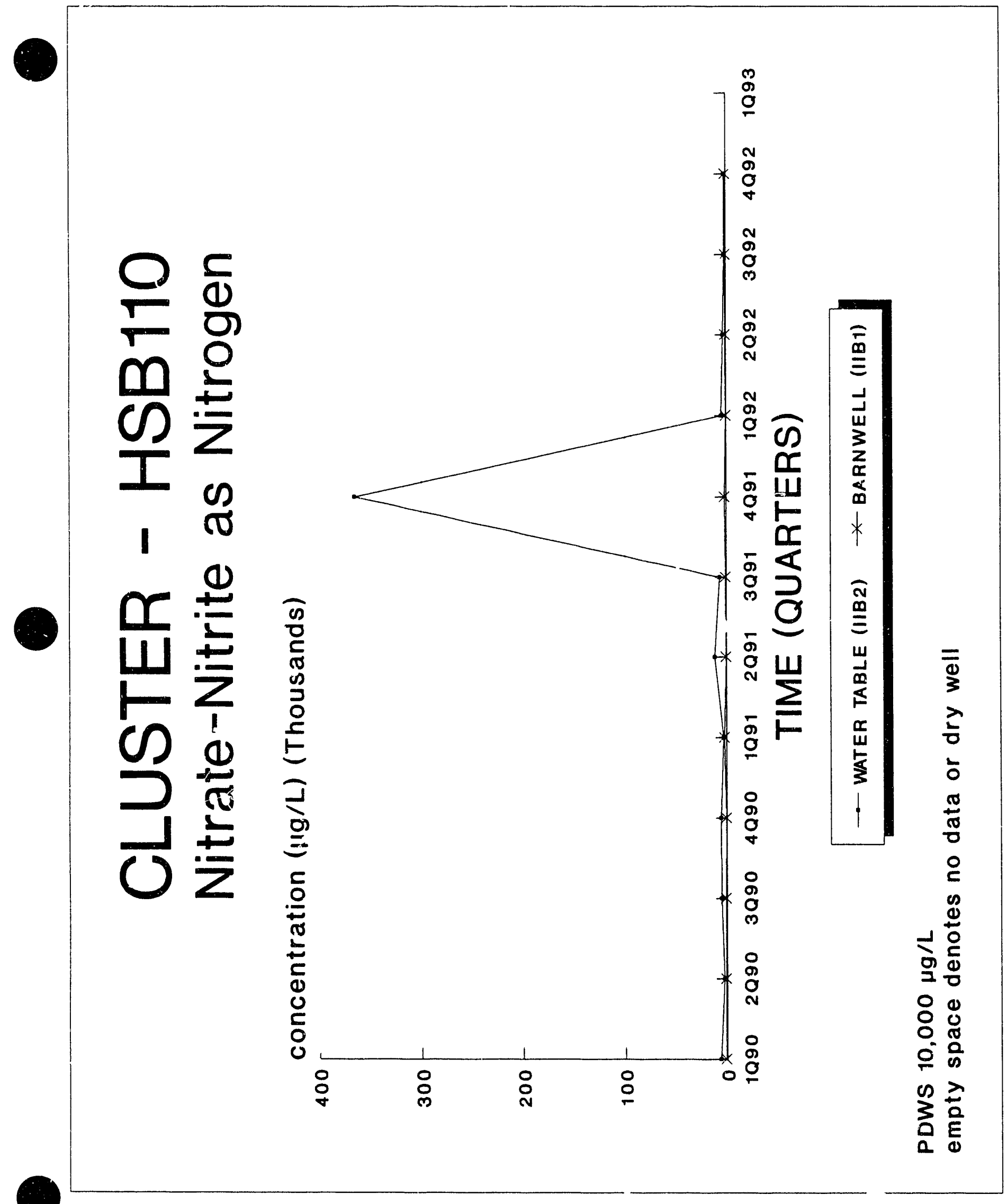




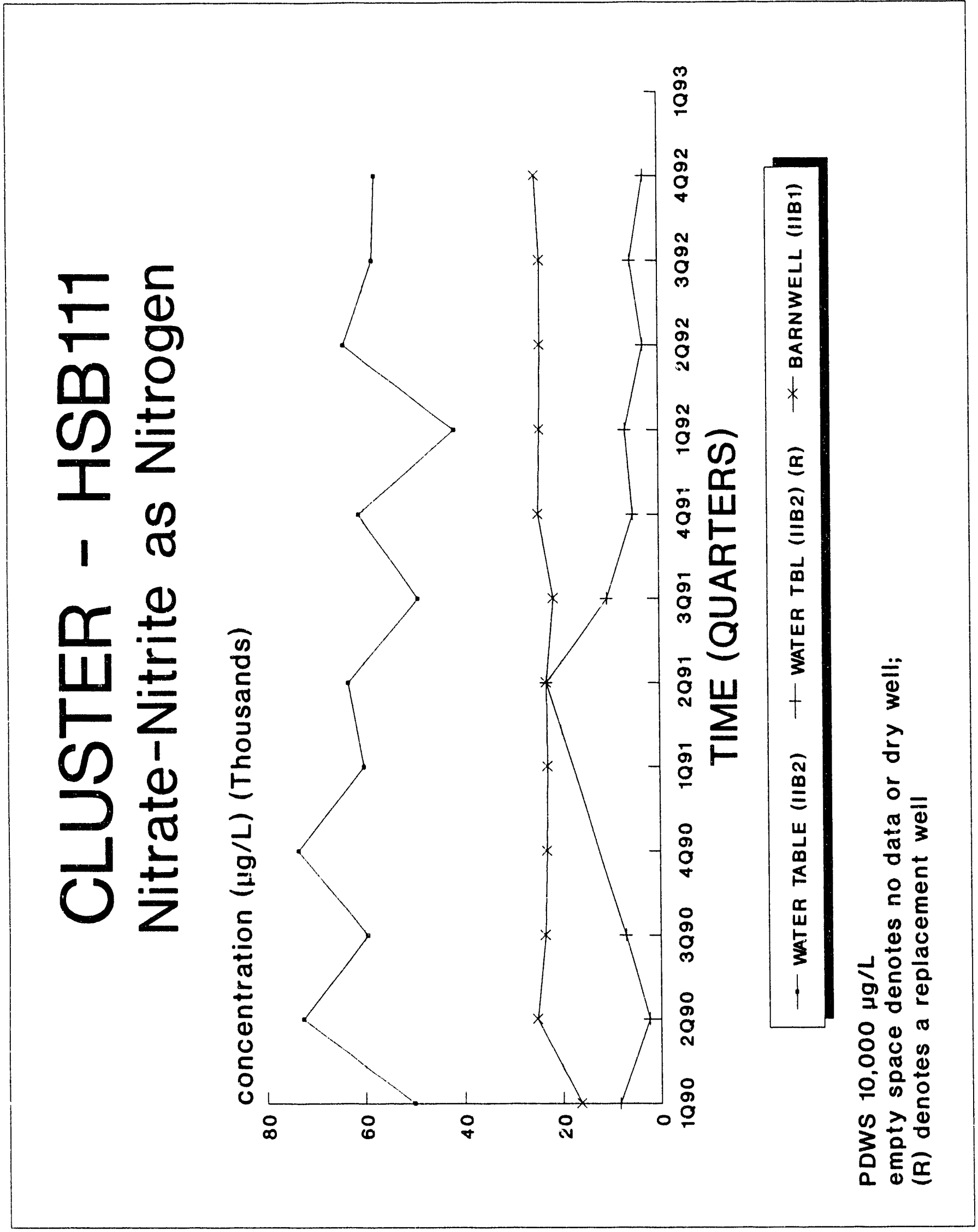




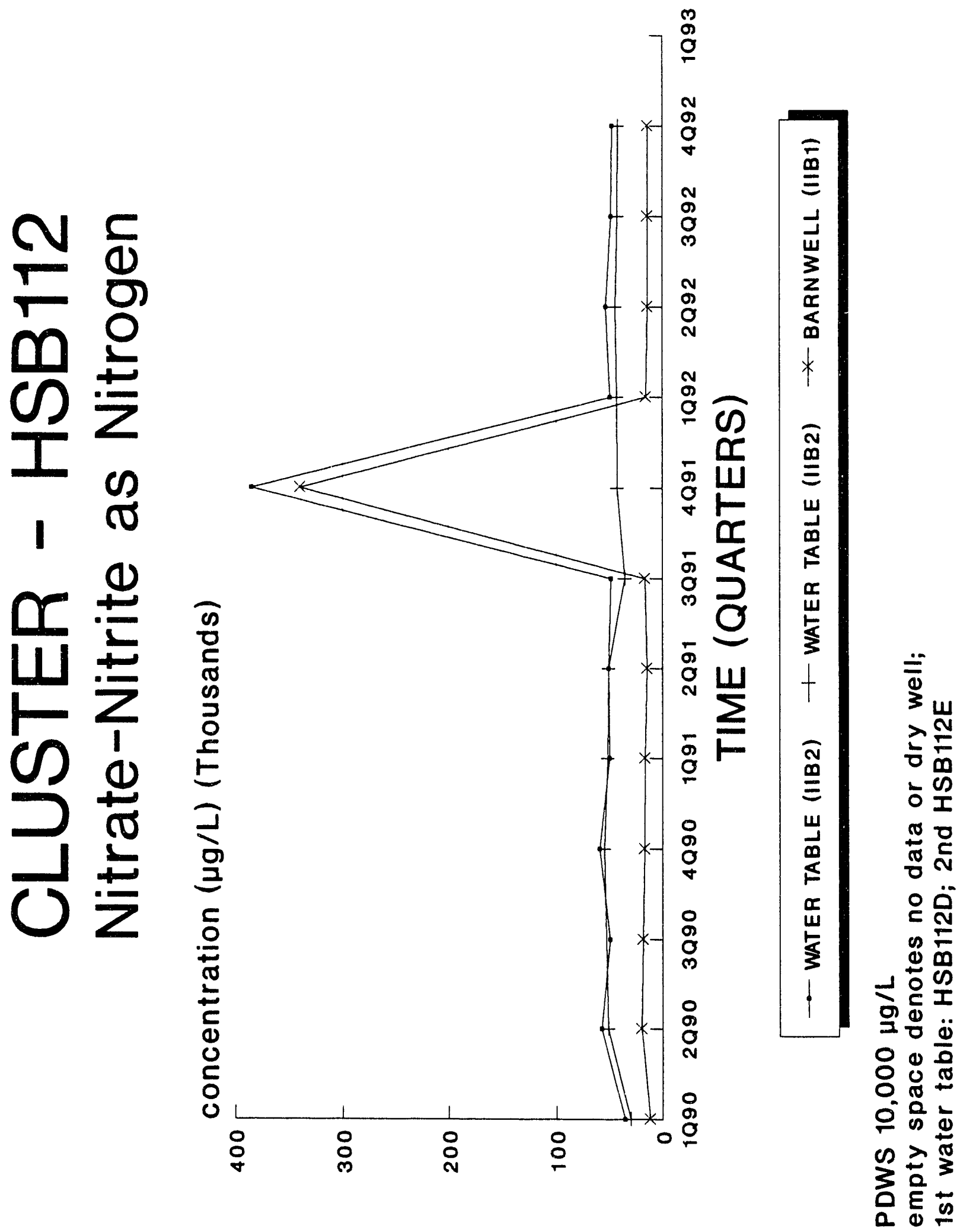




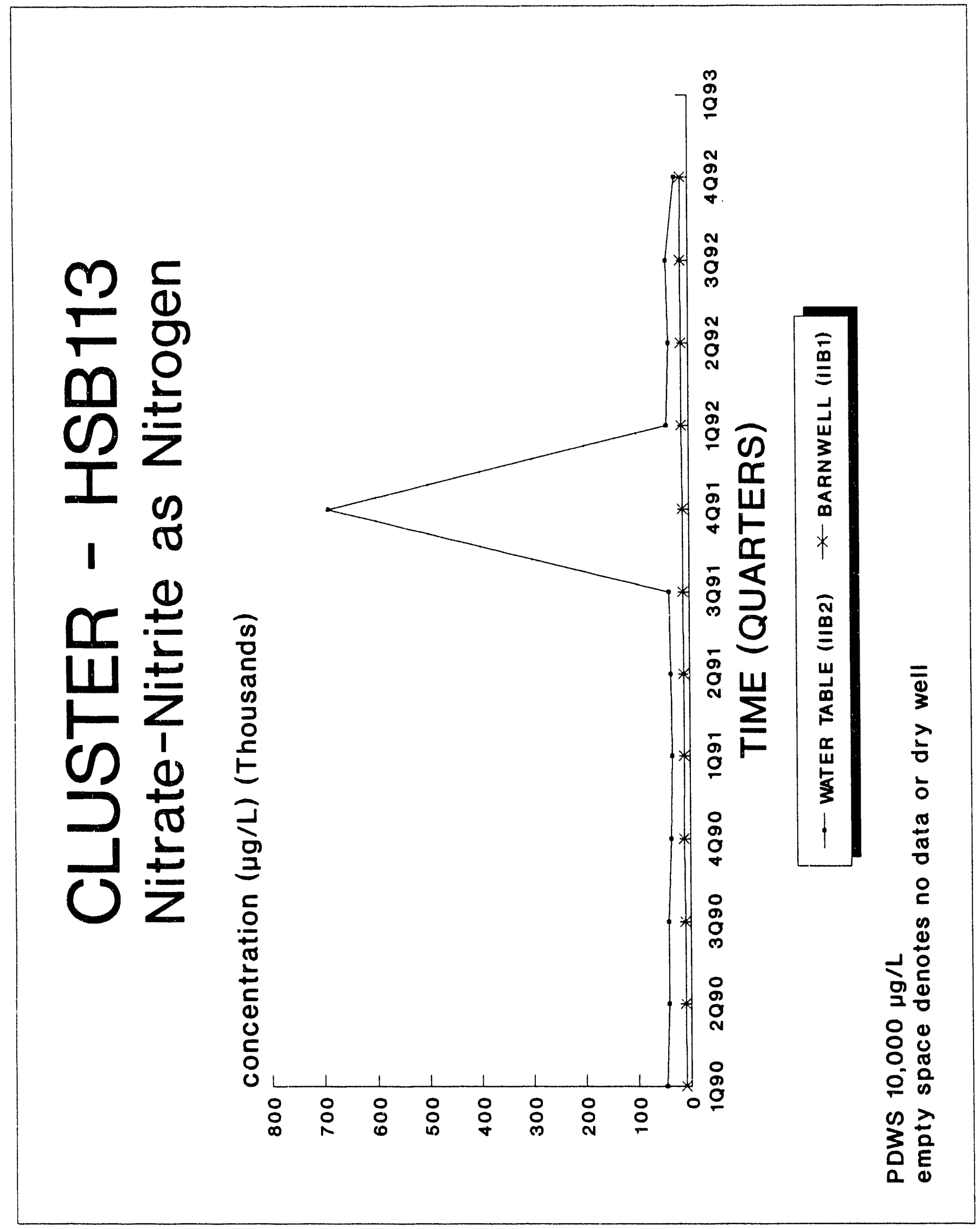




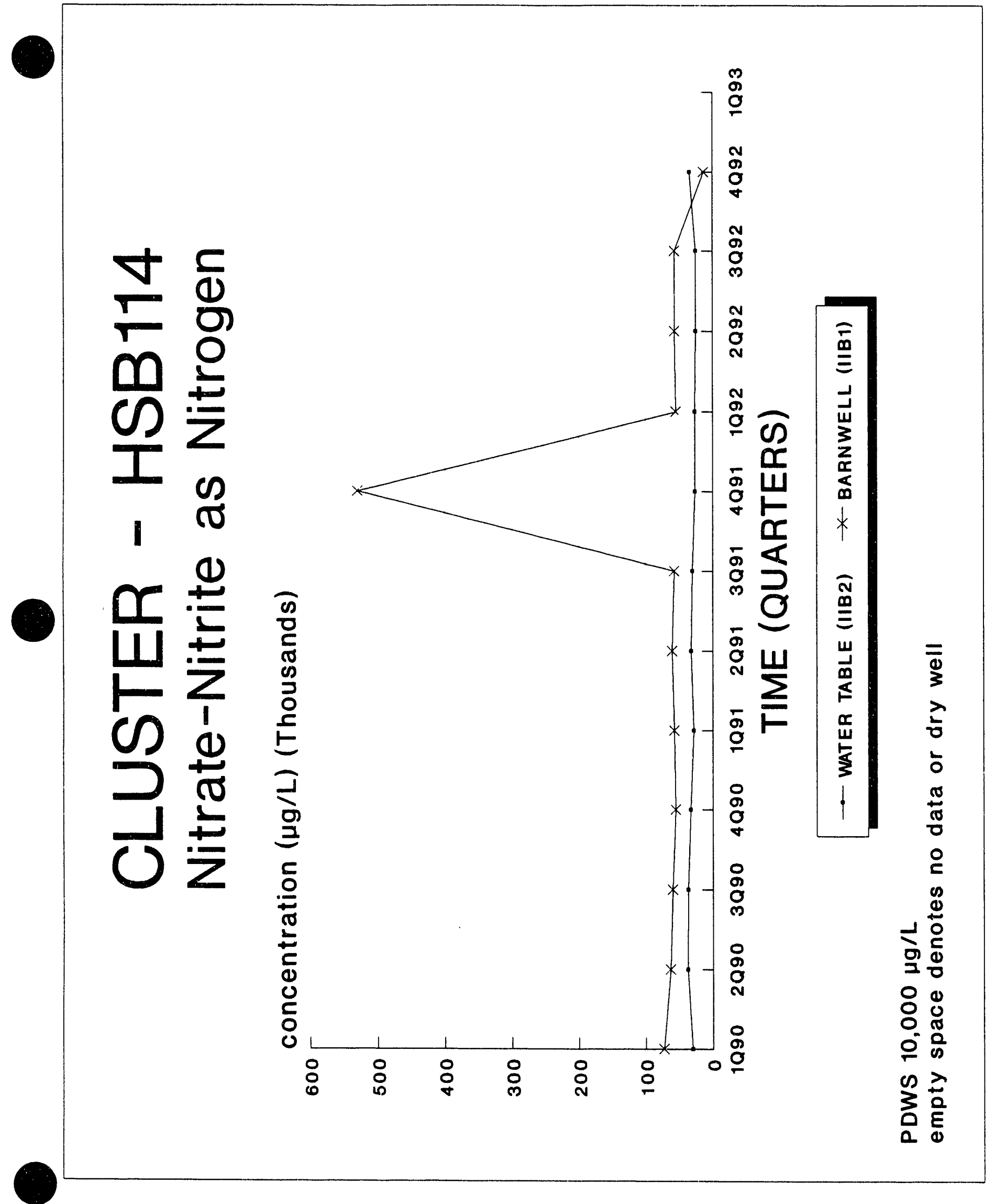




\section{WSRC-TR-93-059}

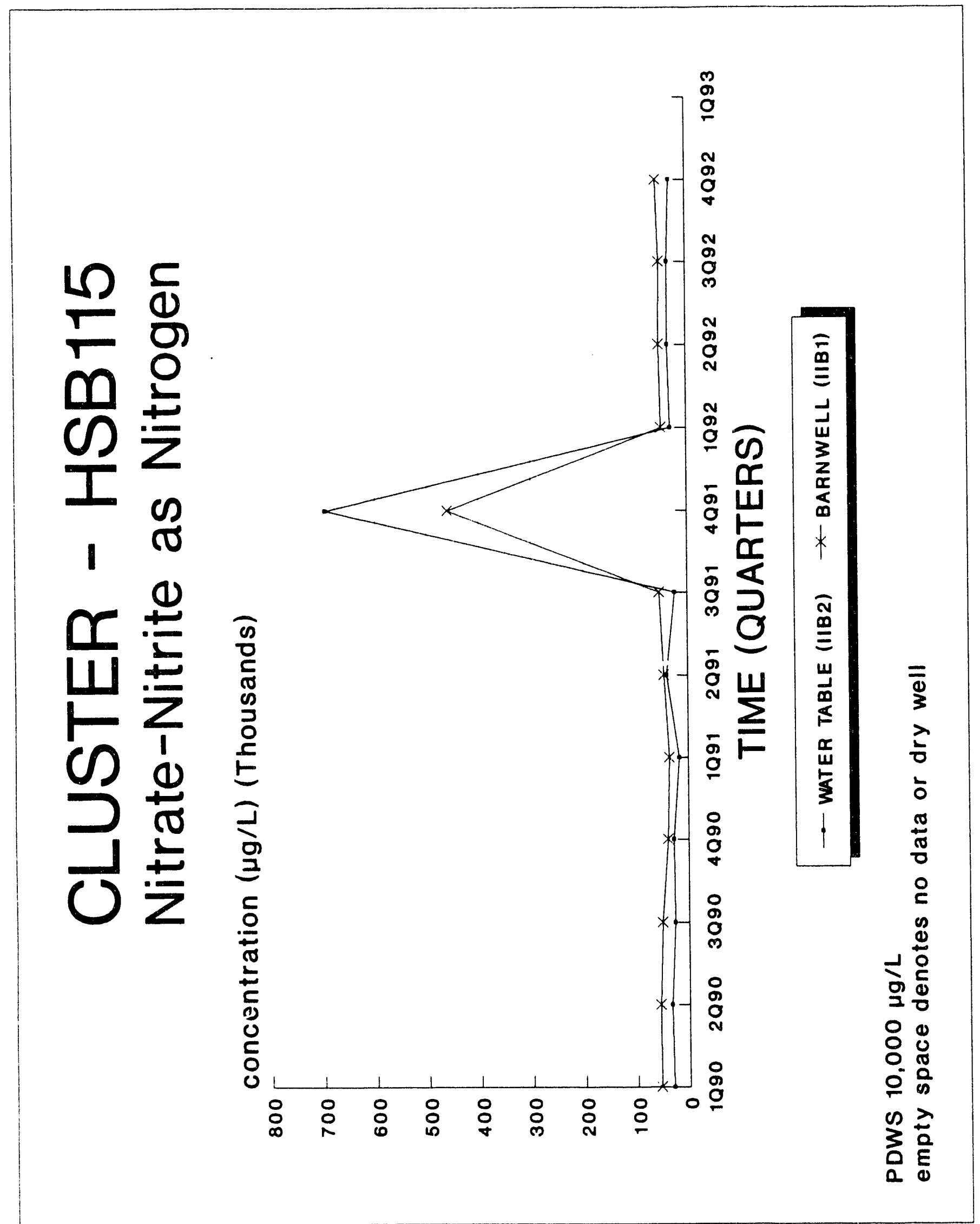



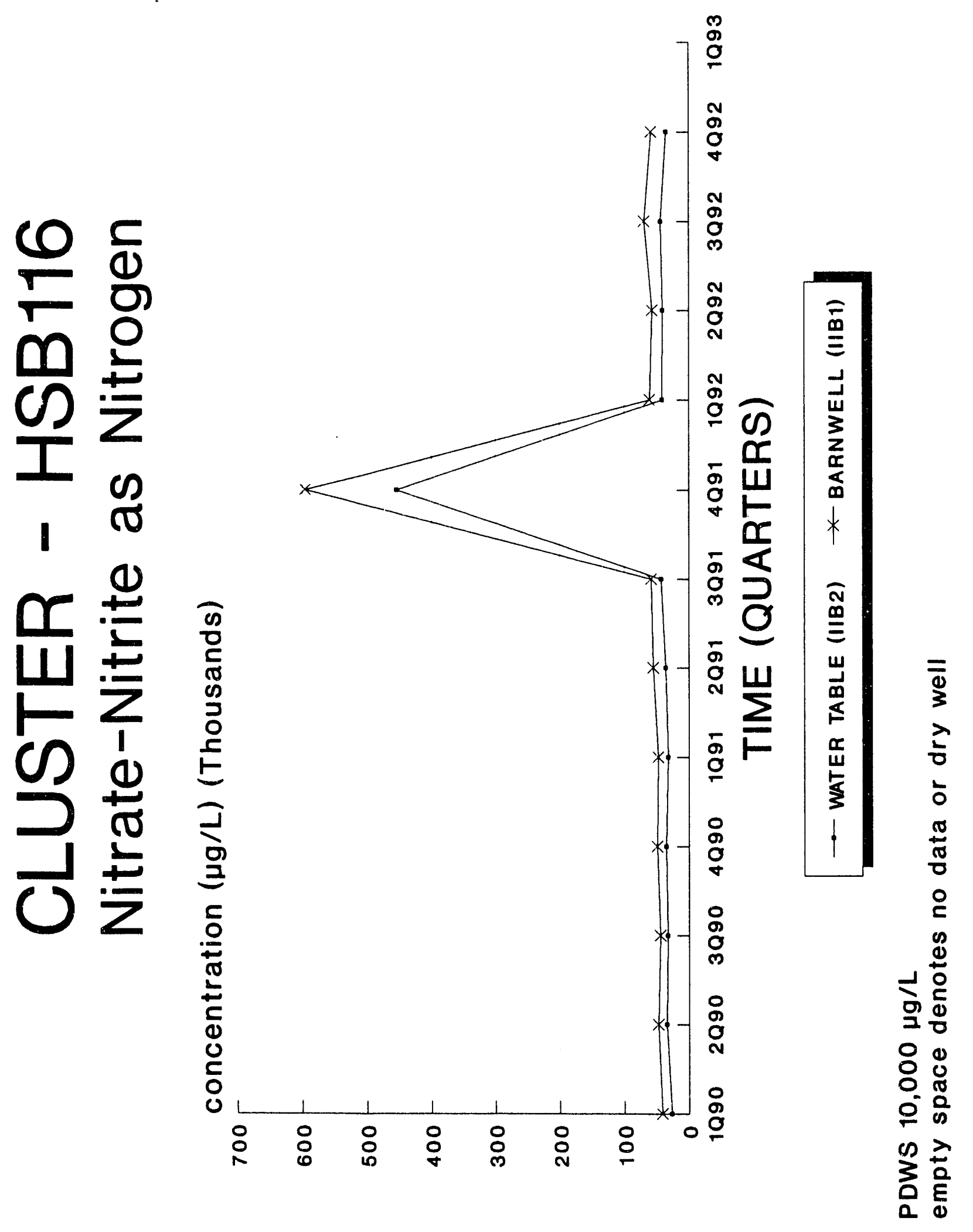


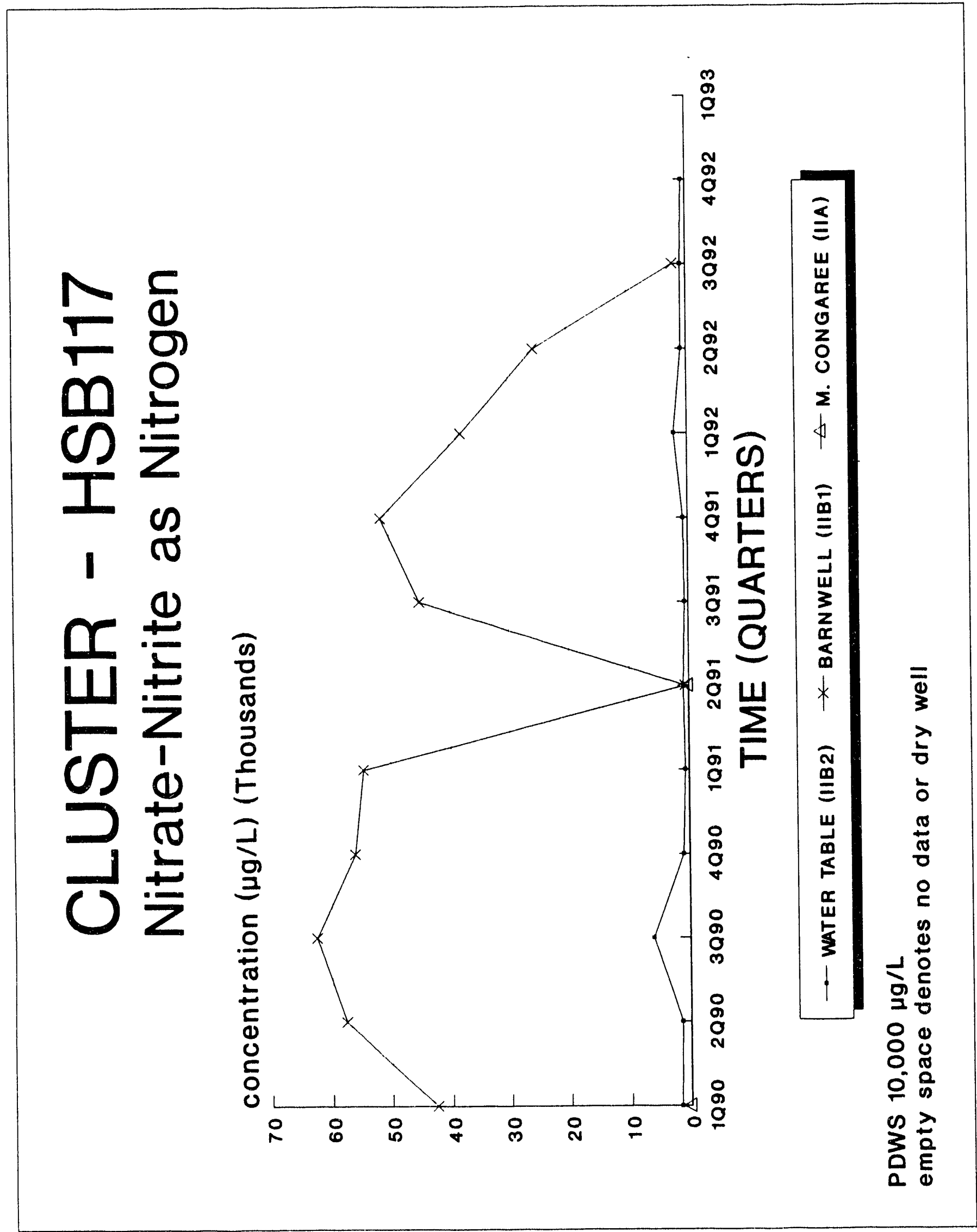




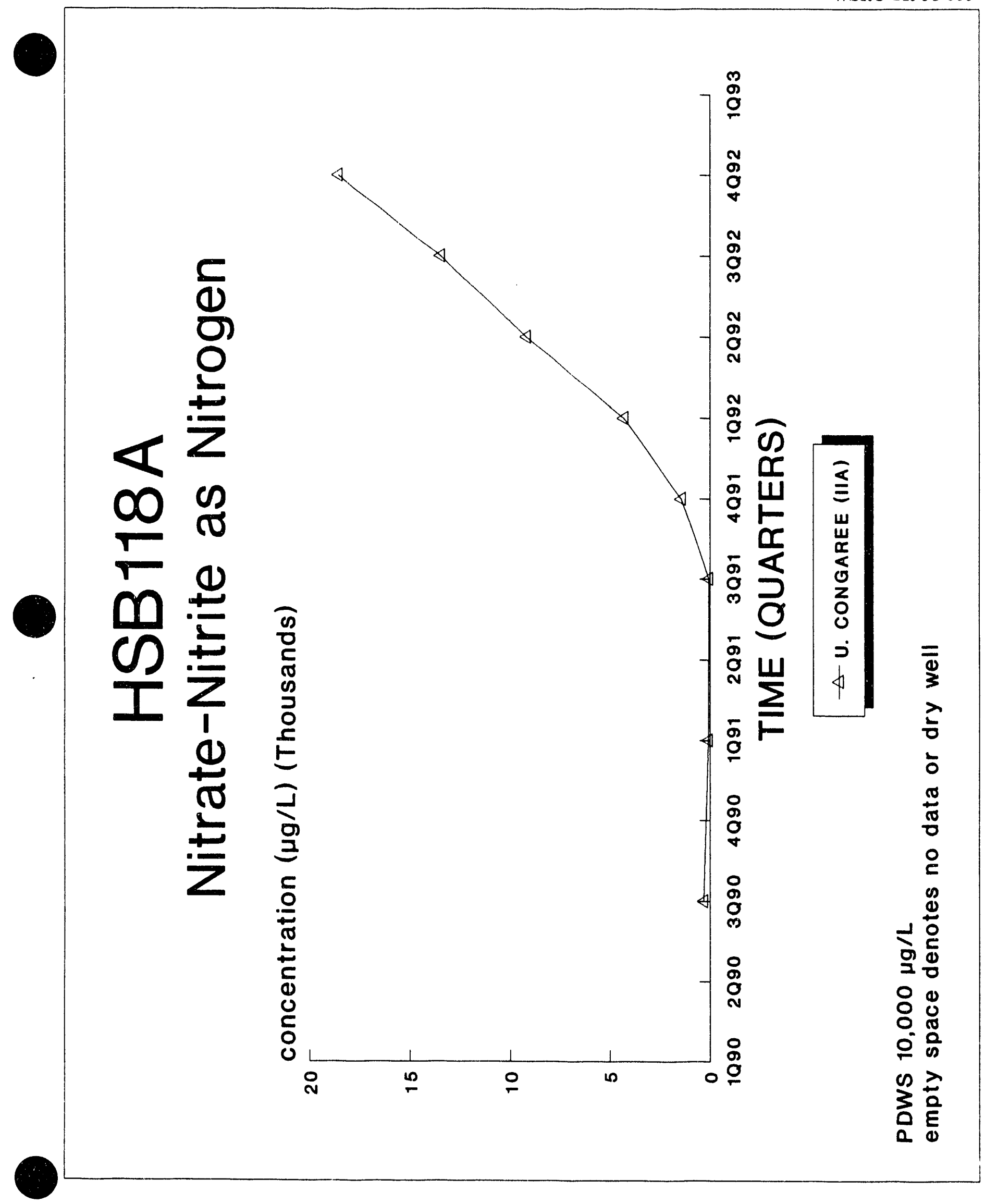




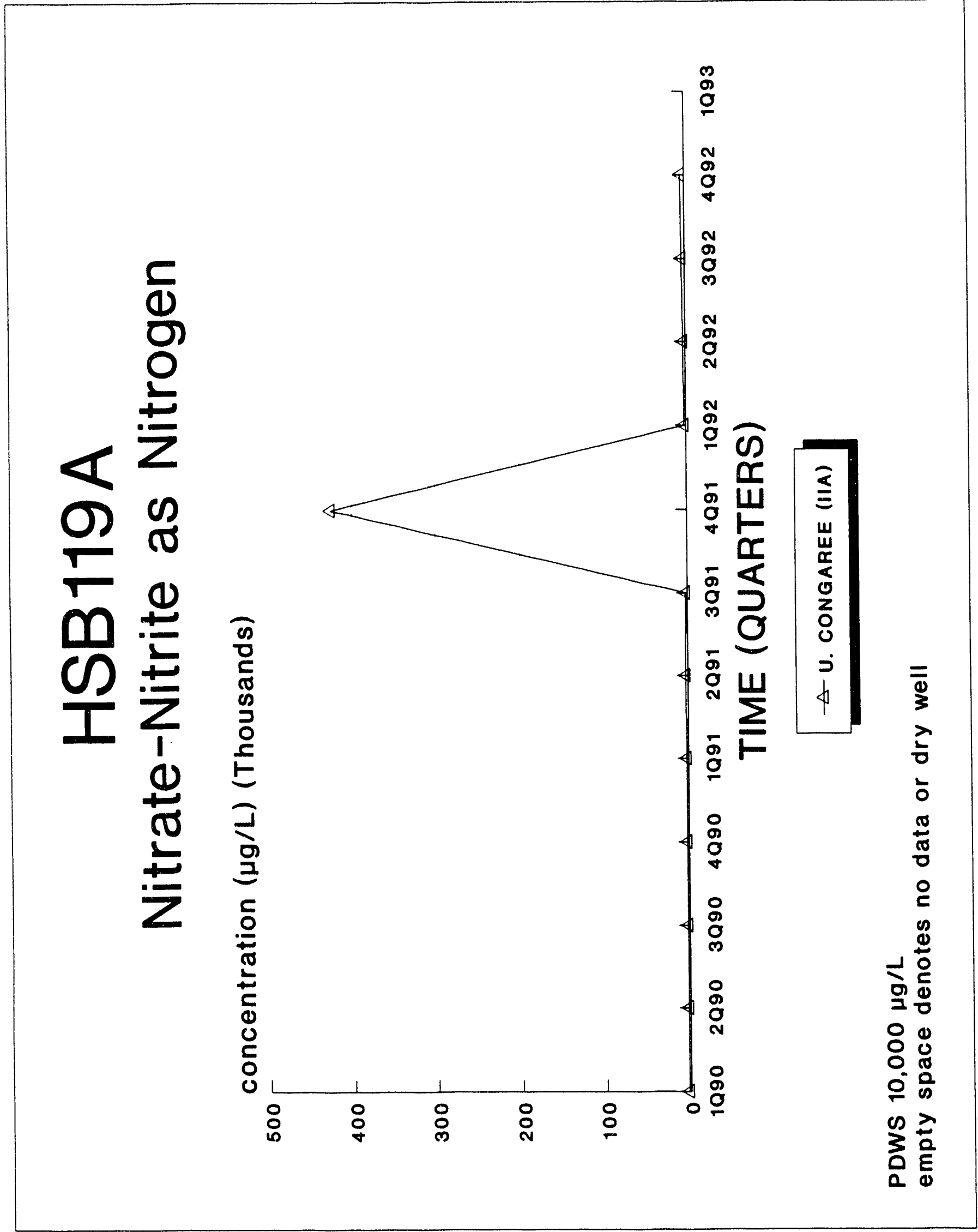




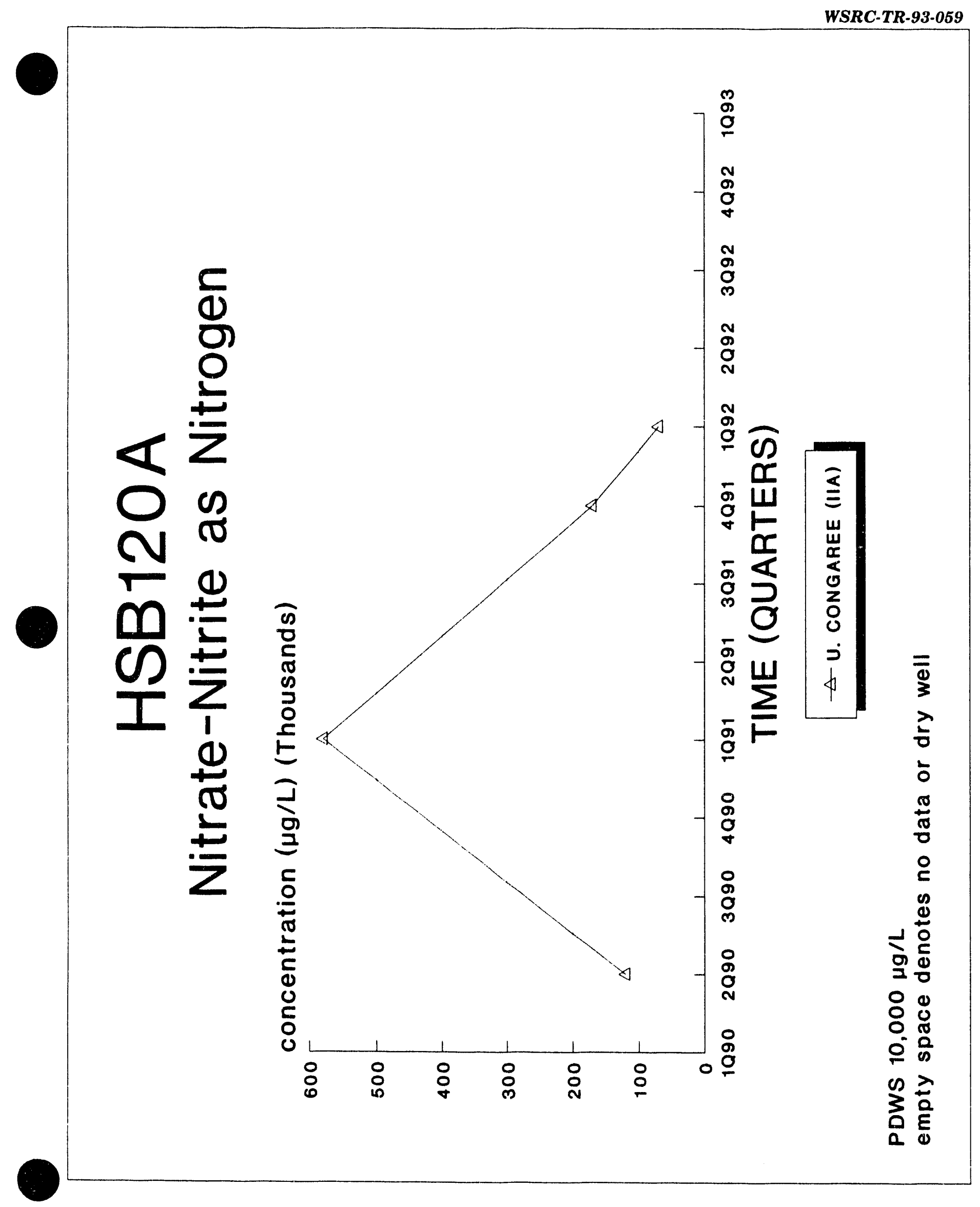



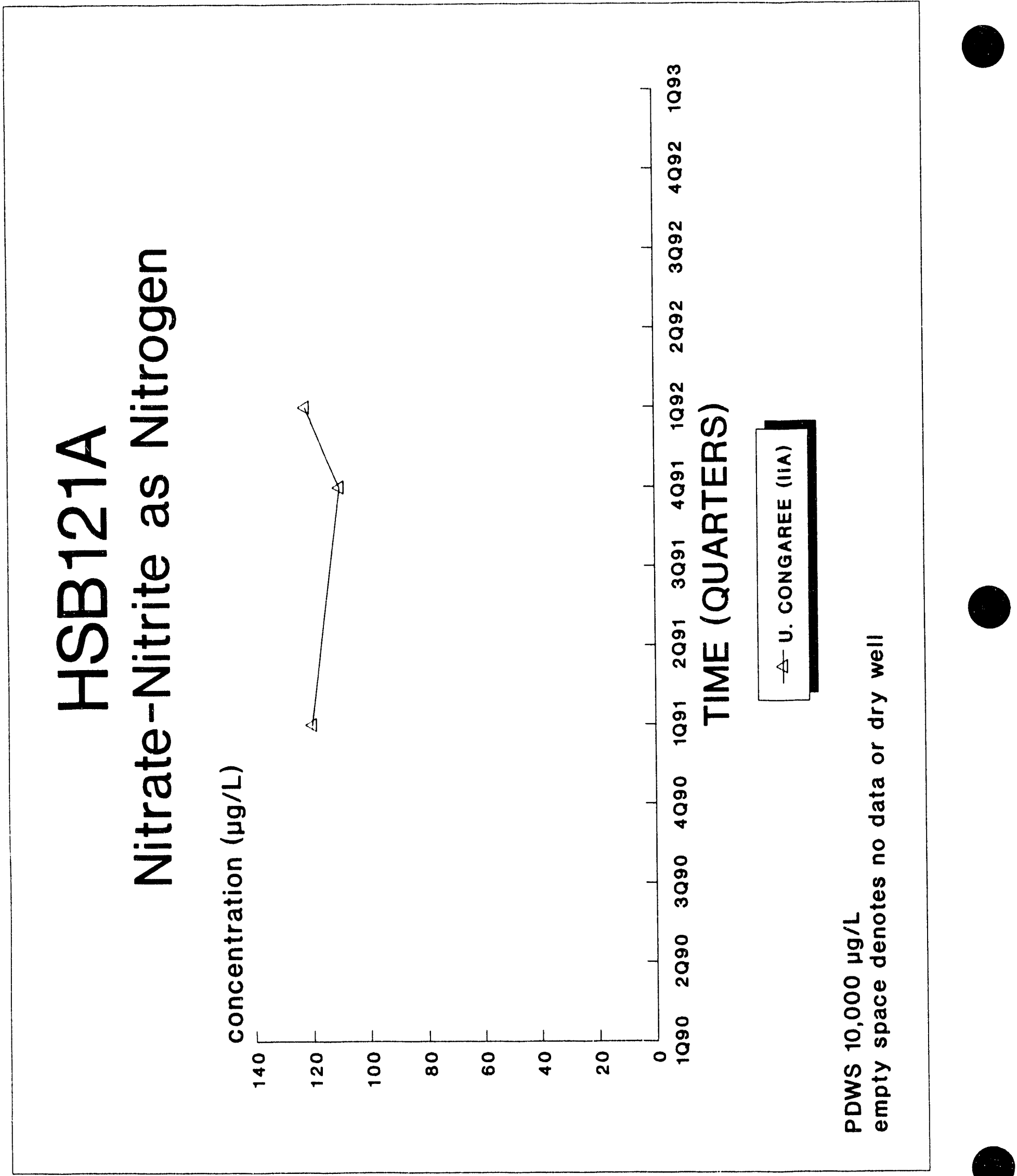


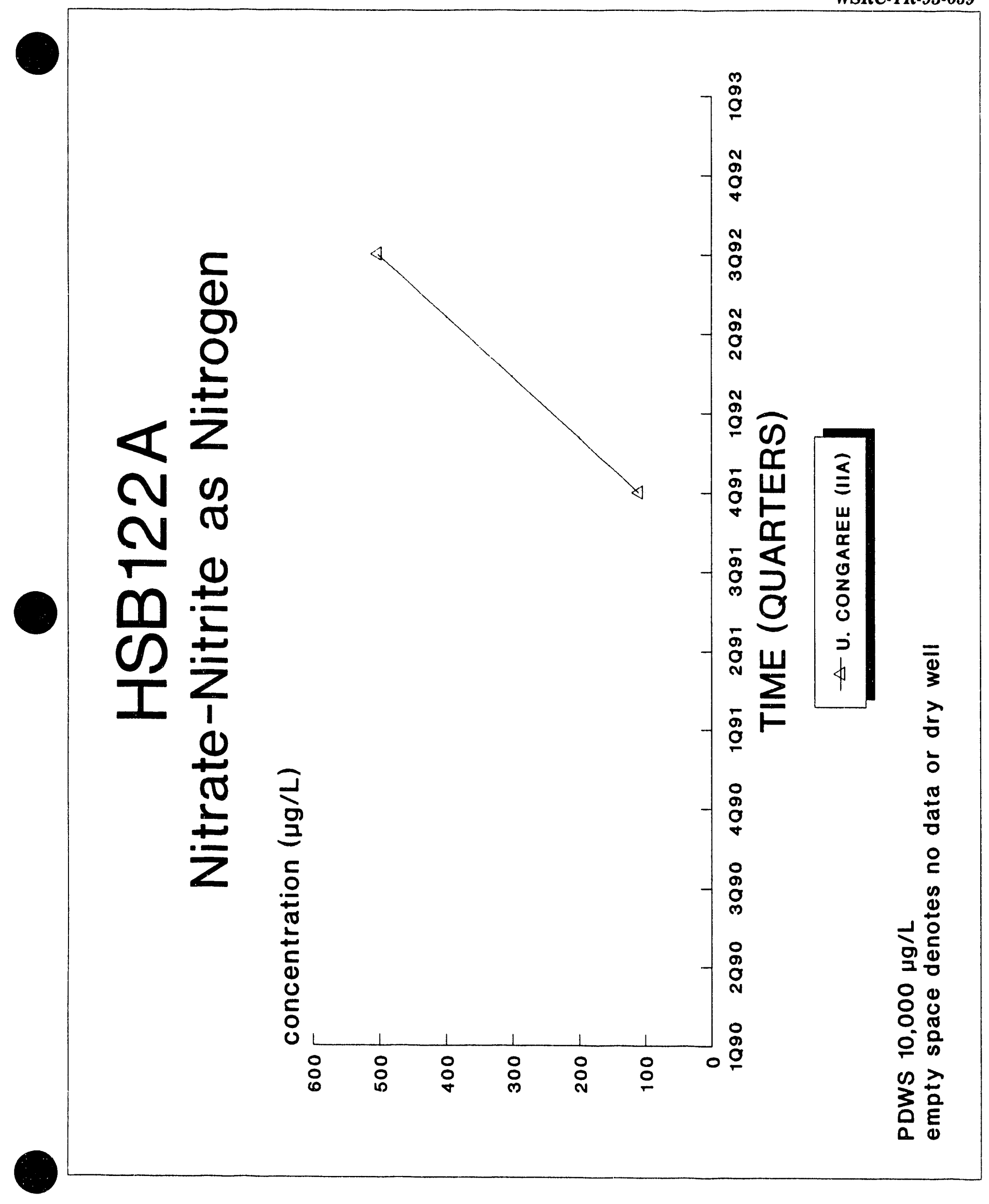



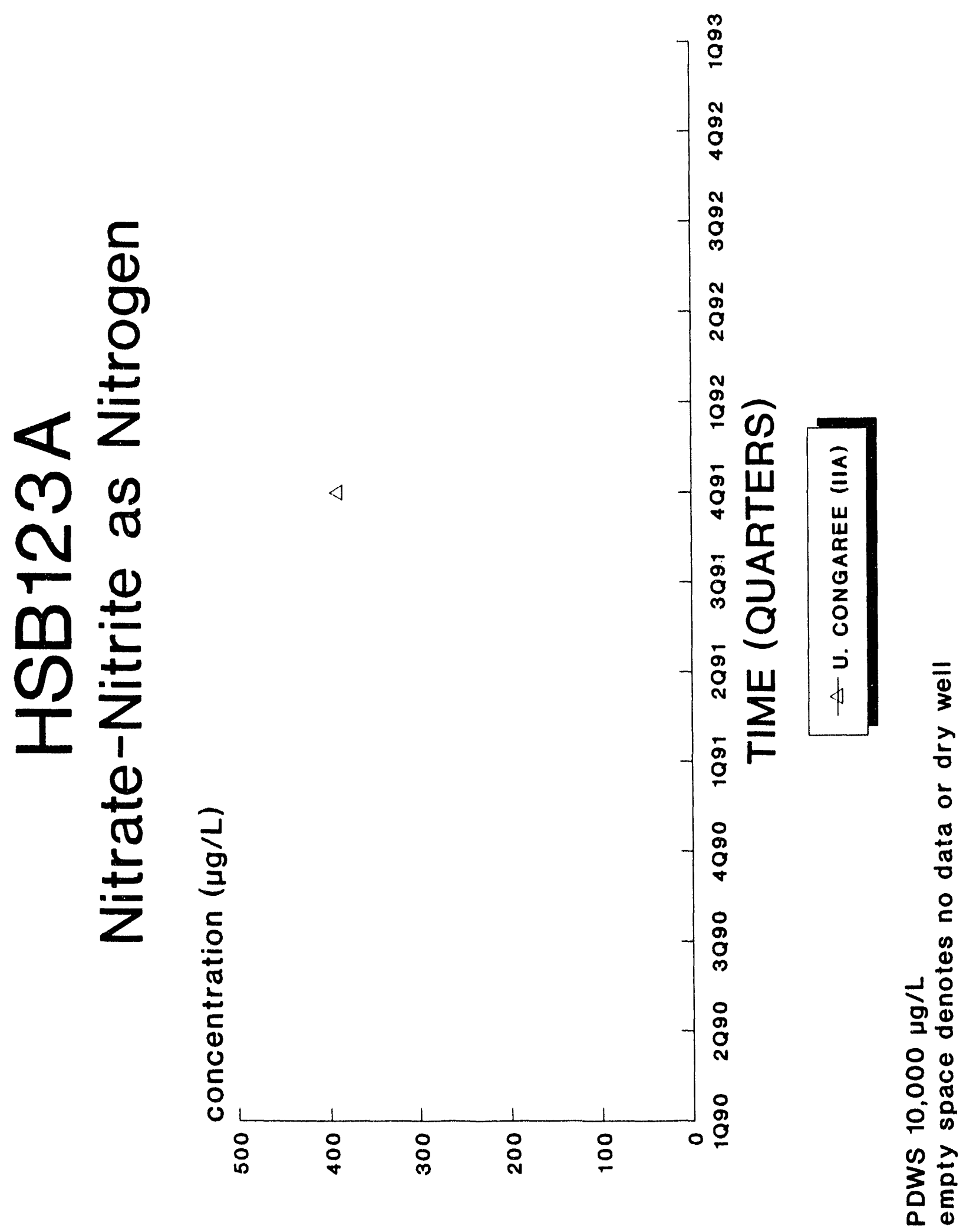


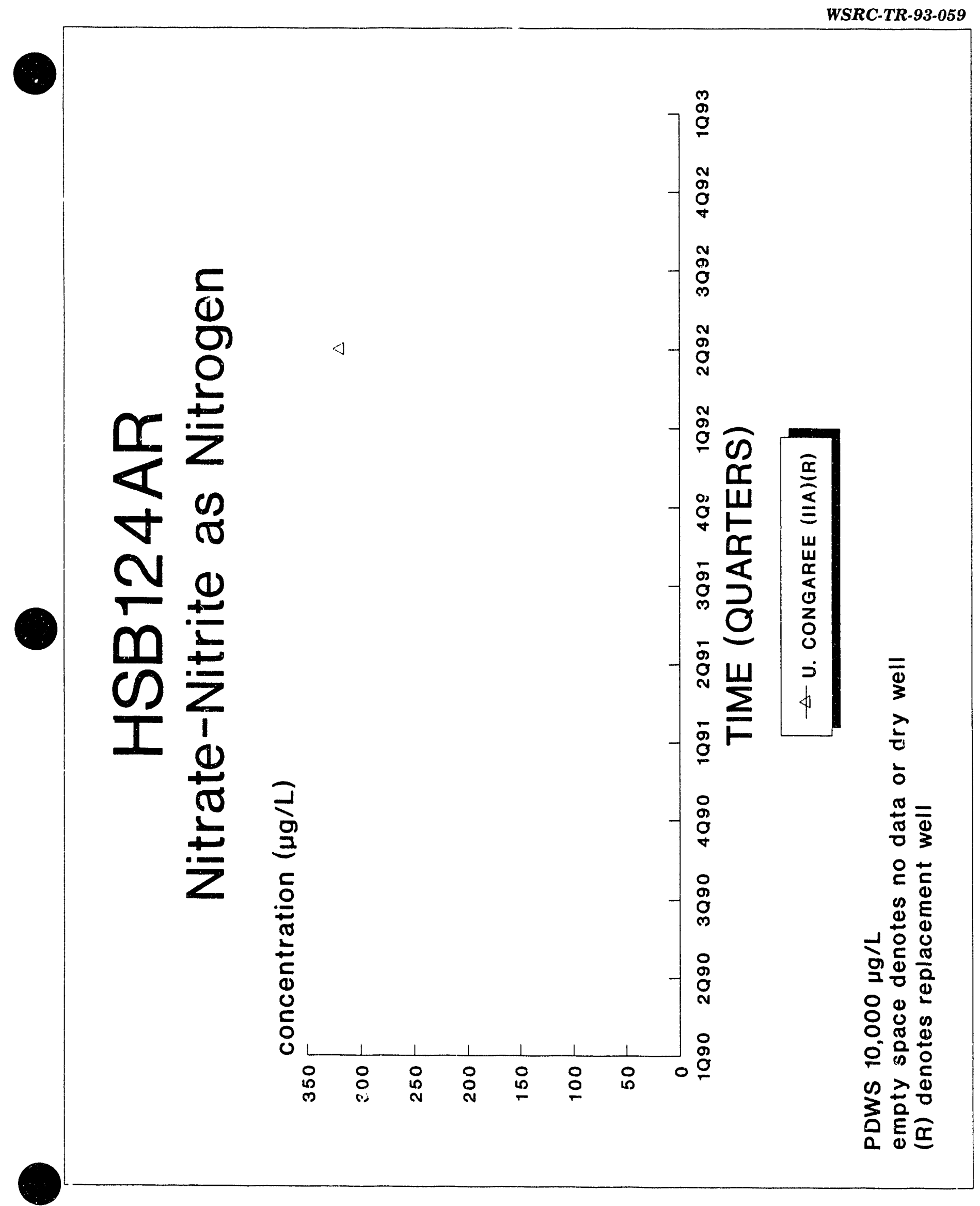




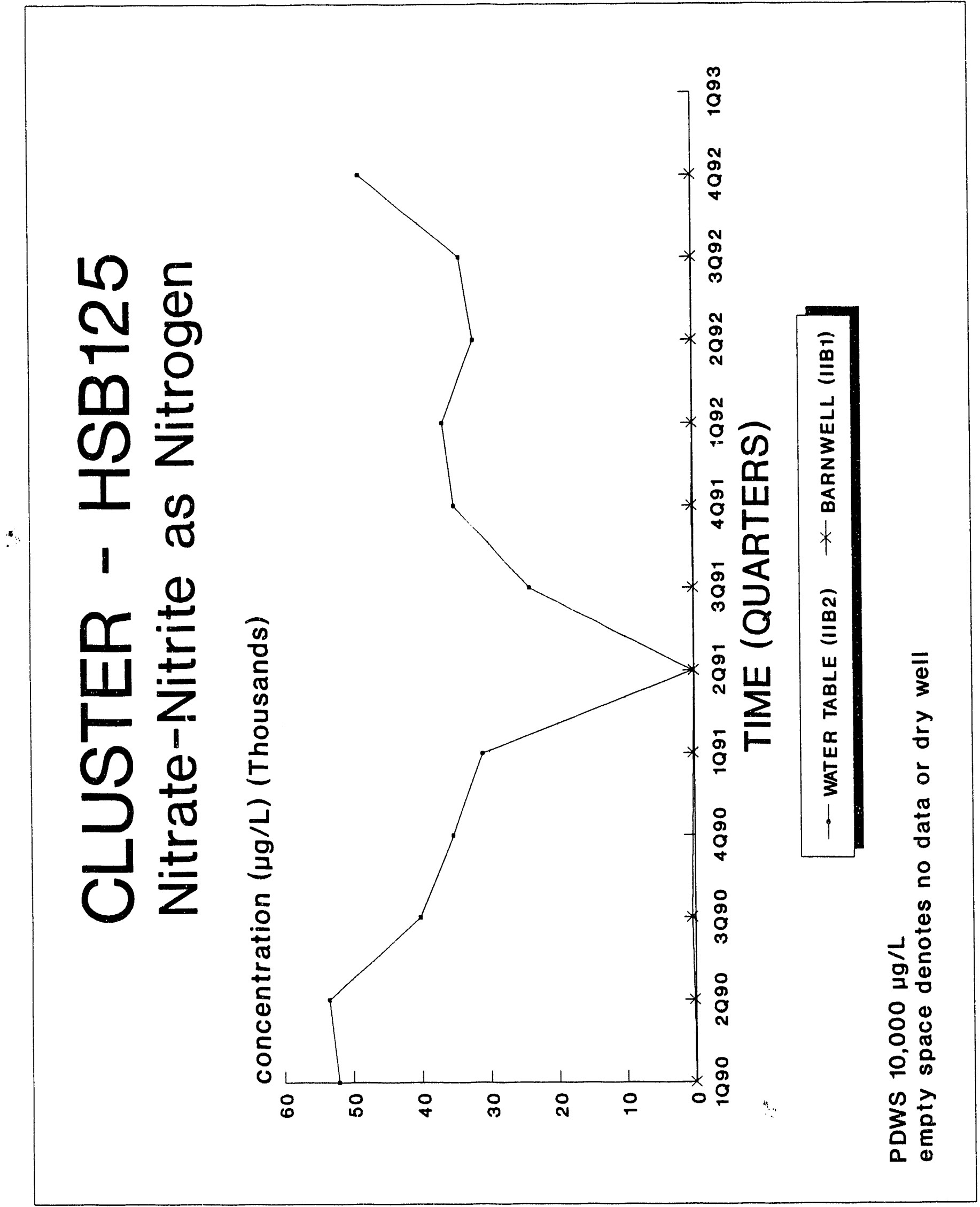




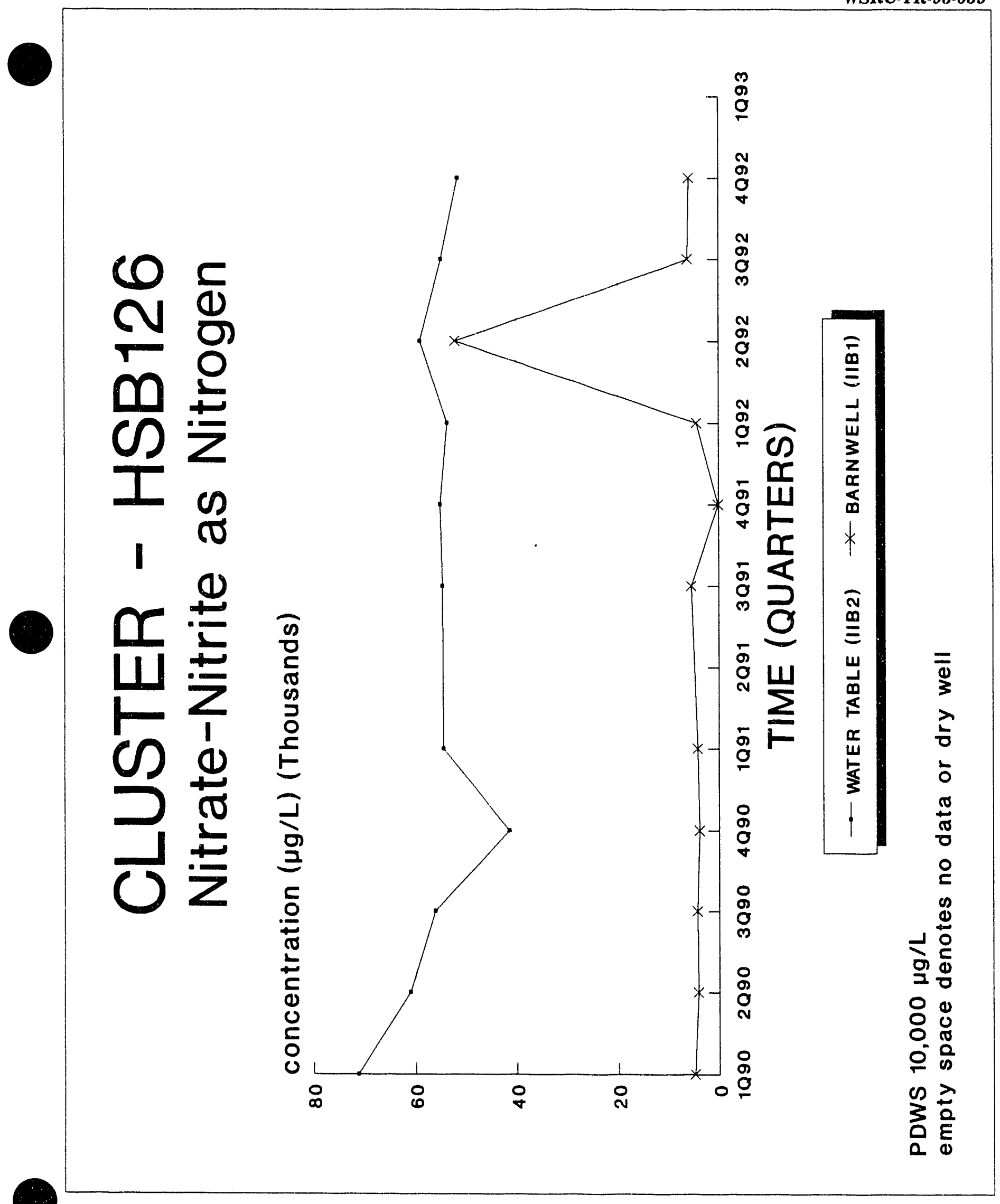




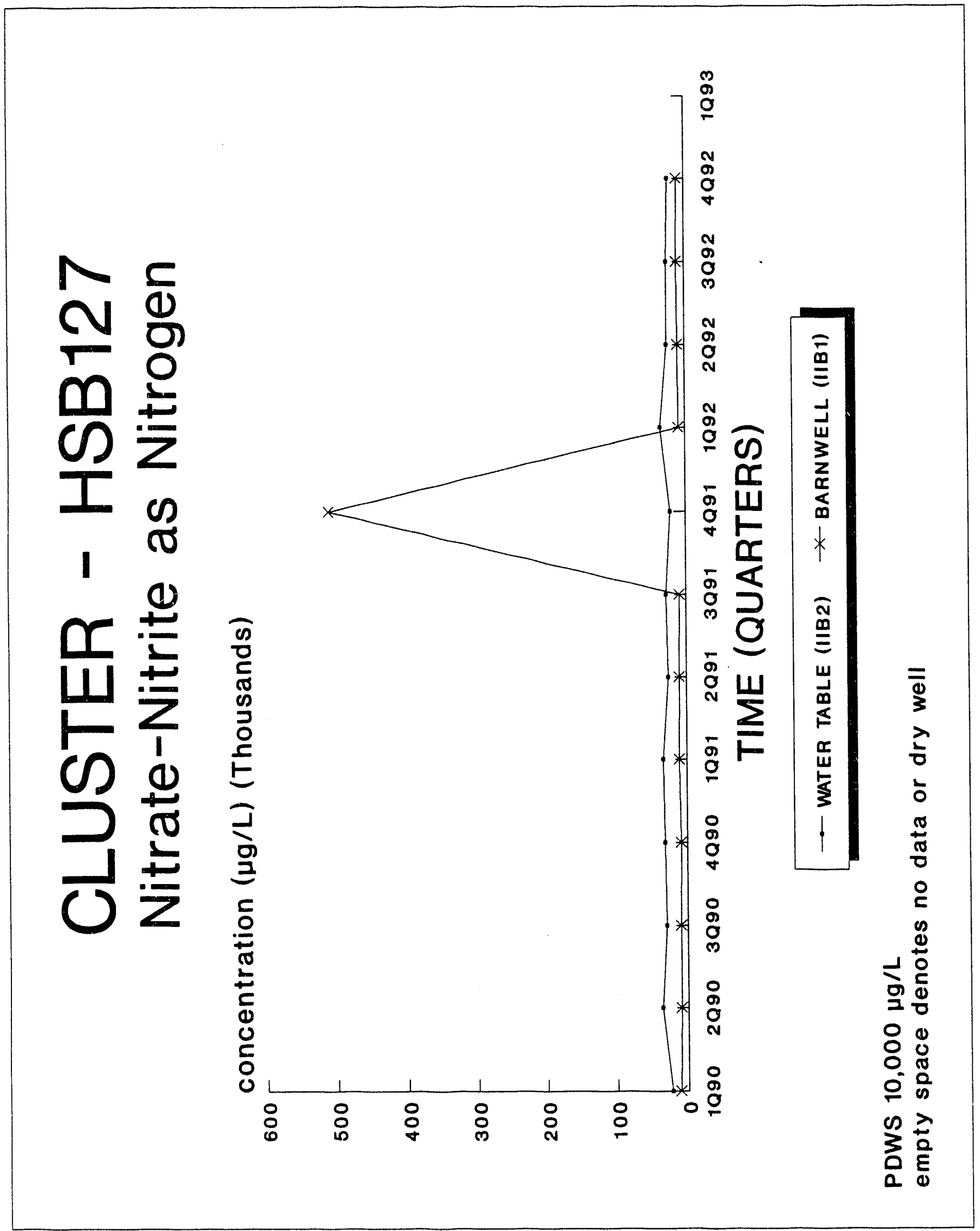




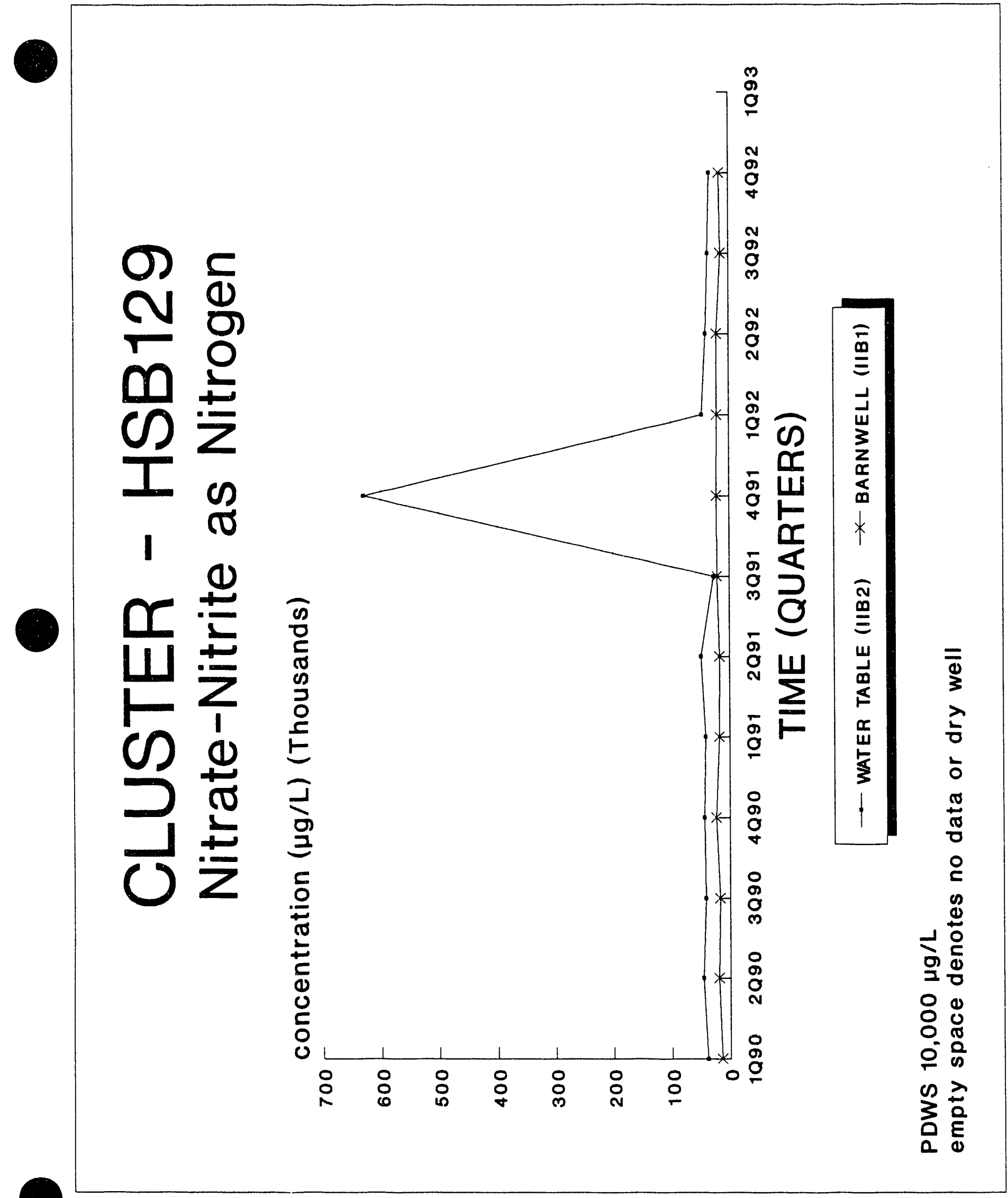




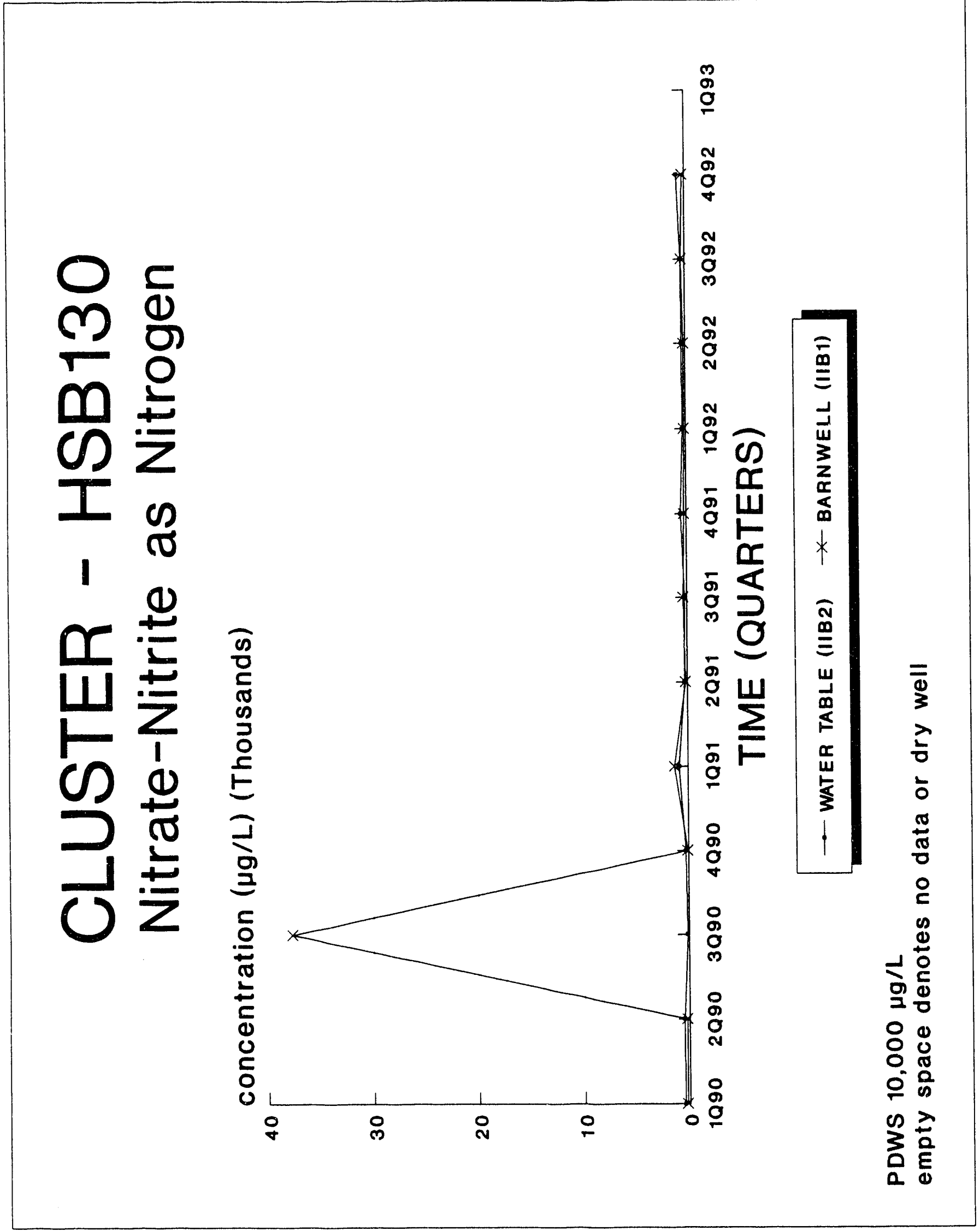




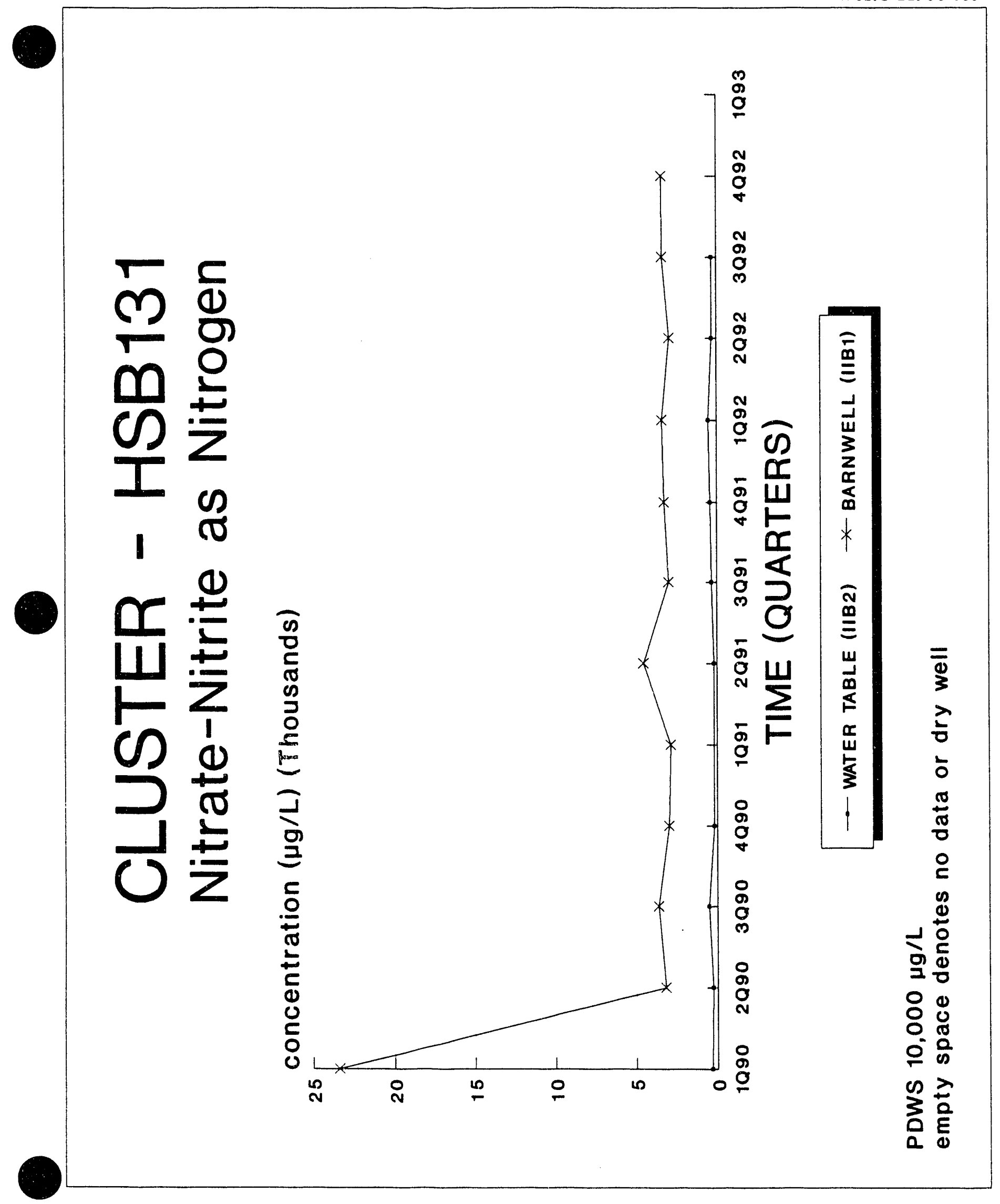




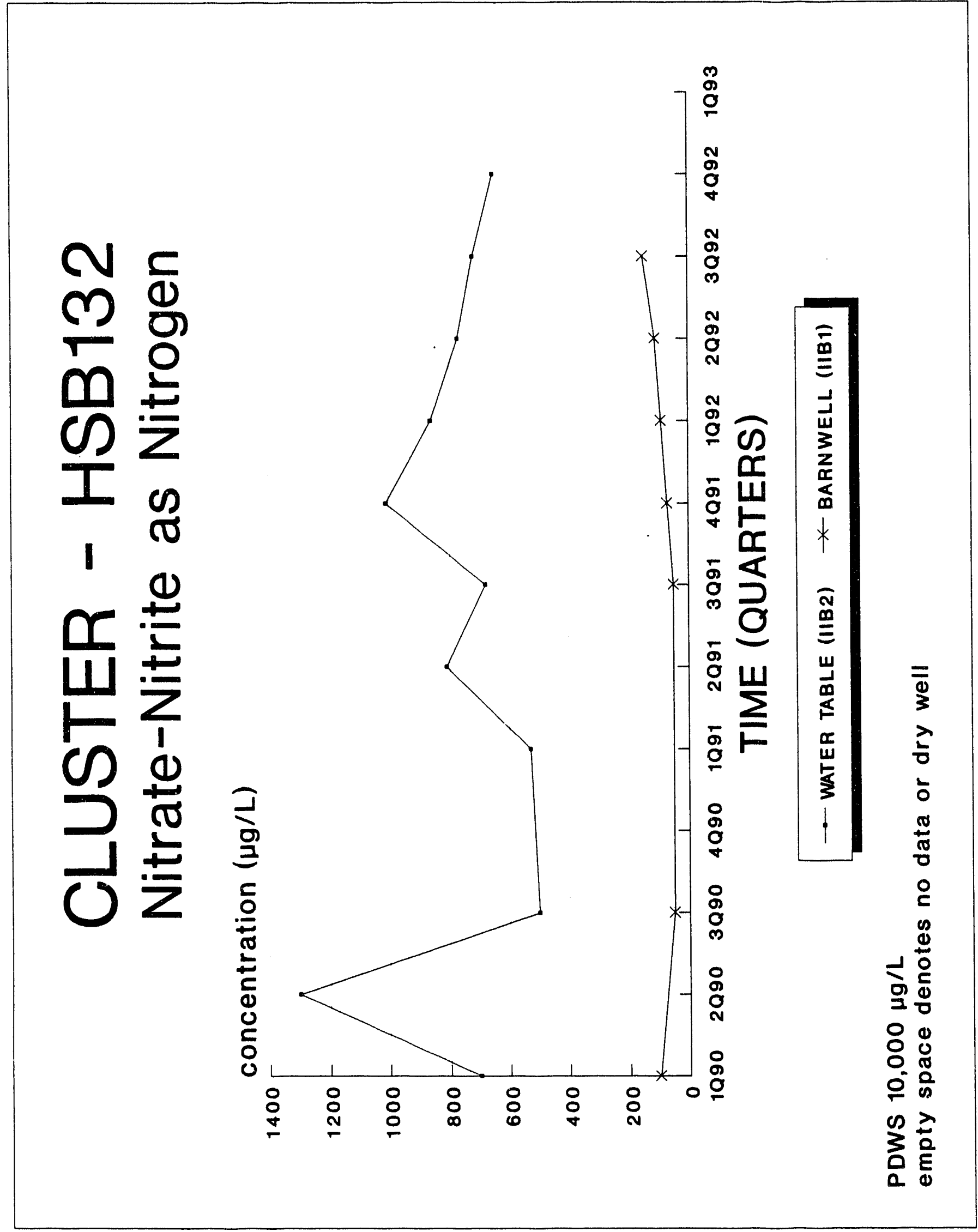




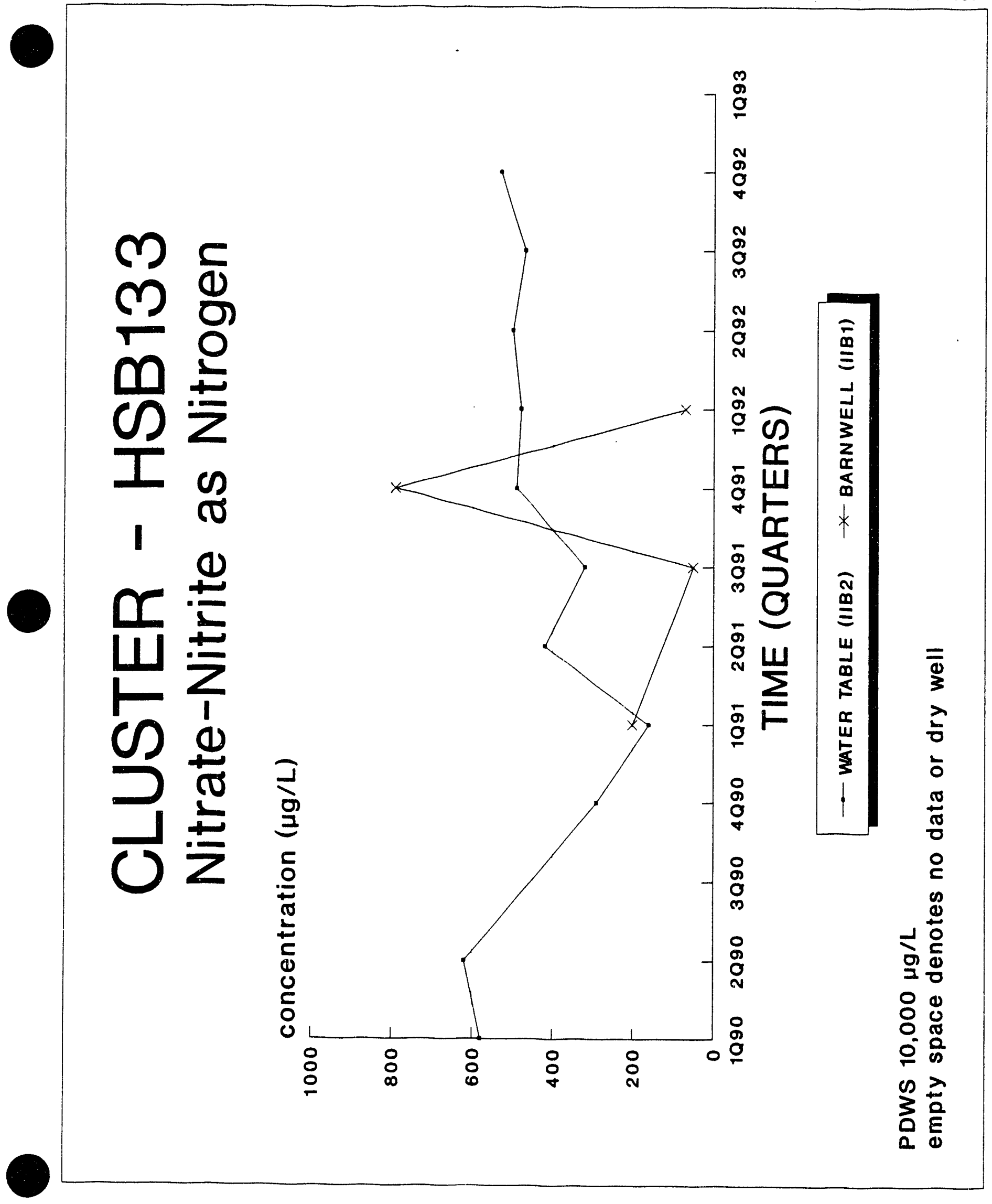




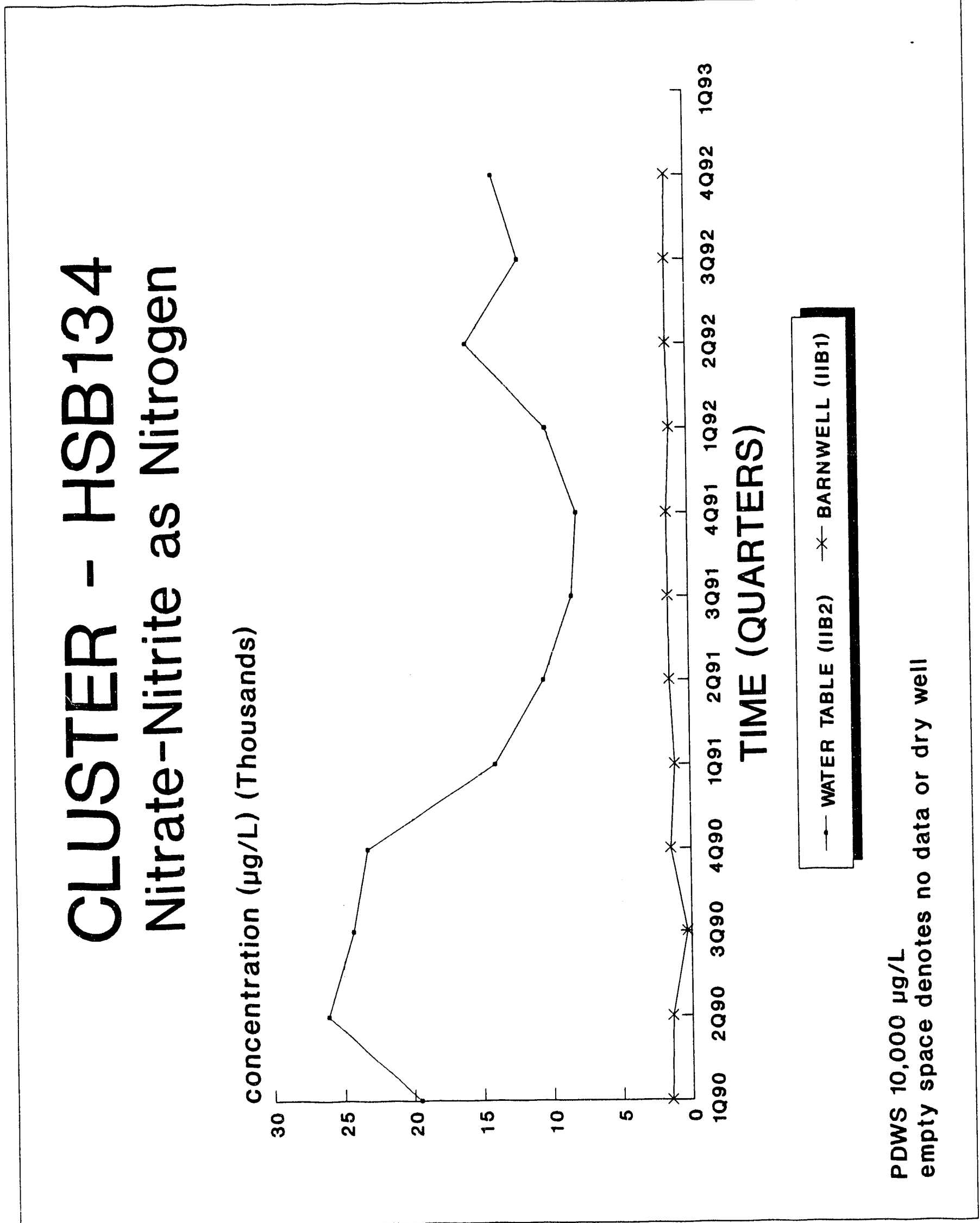




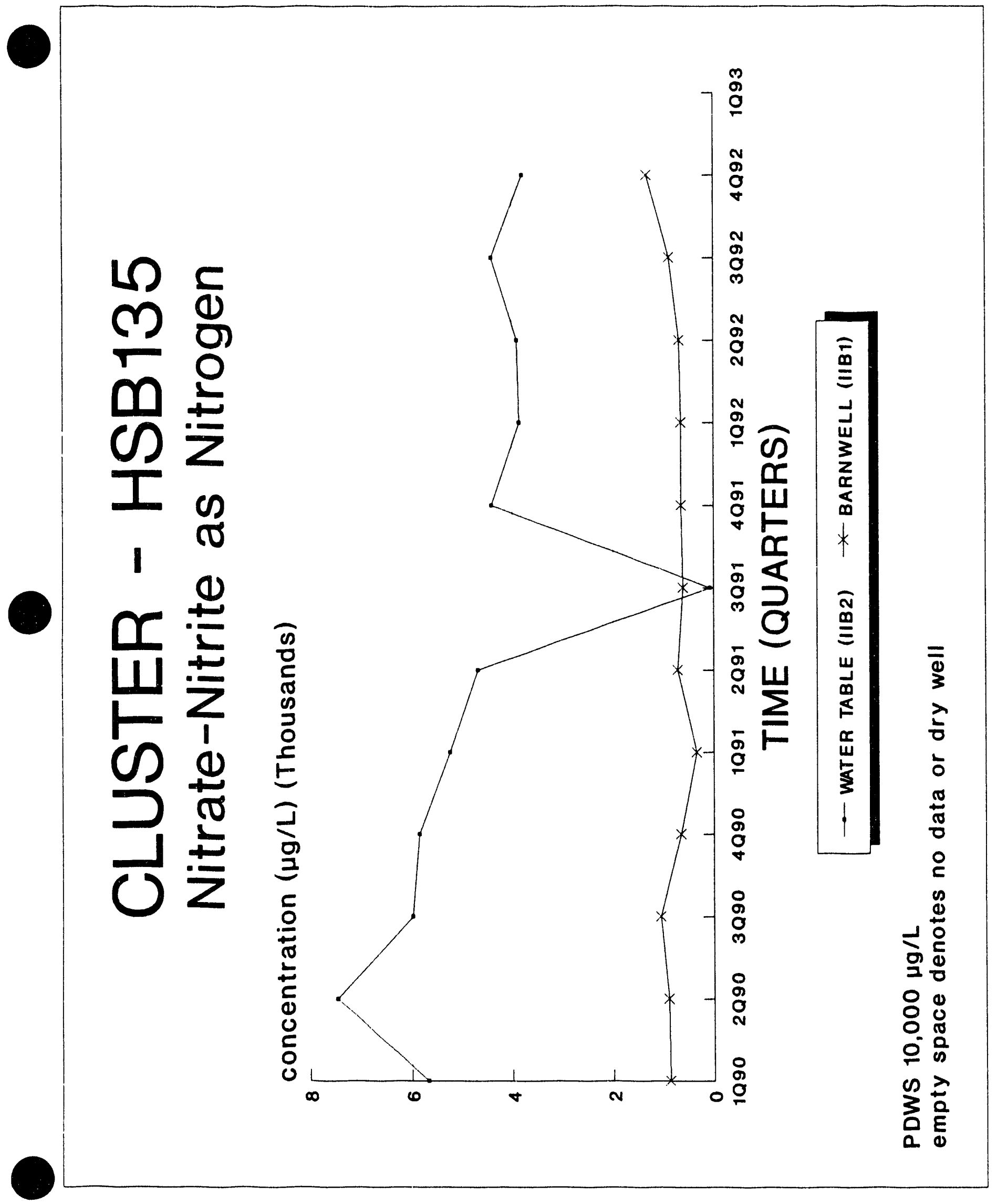




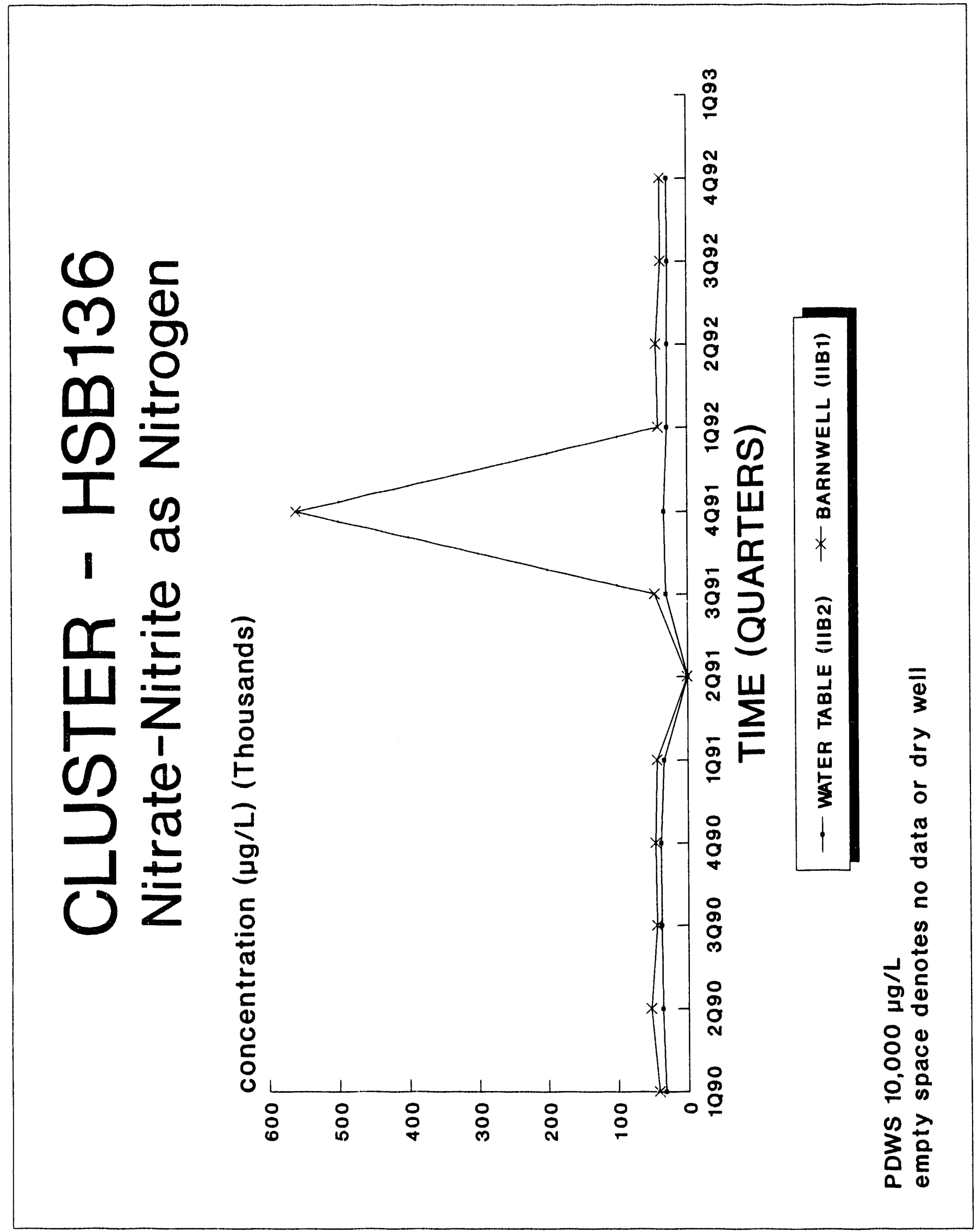




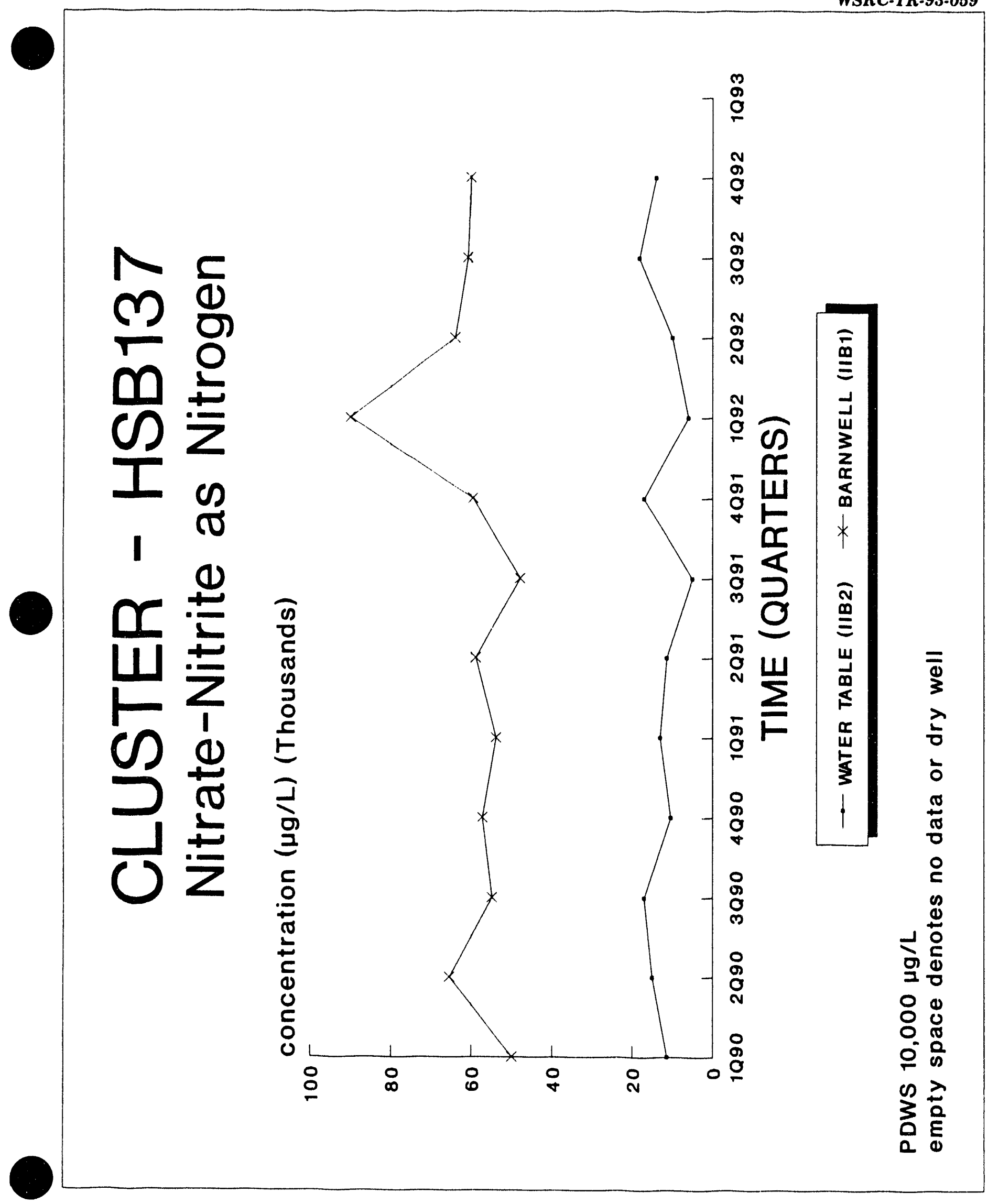




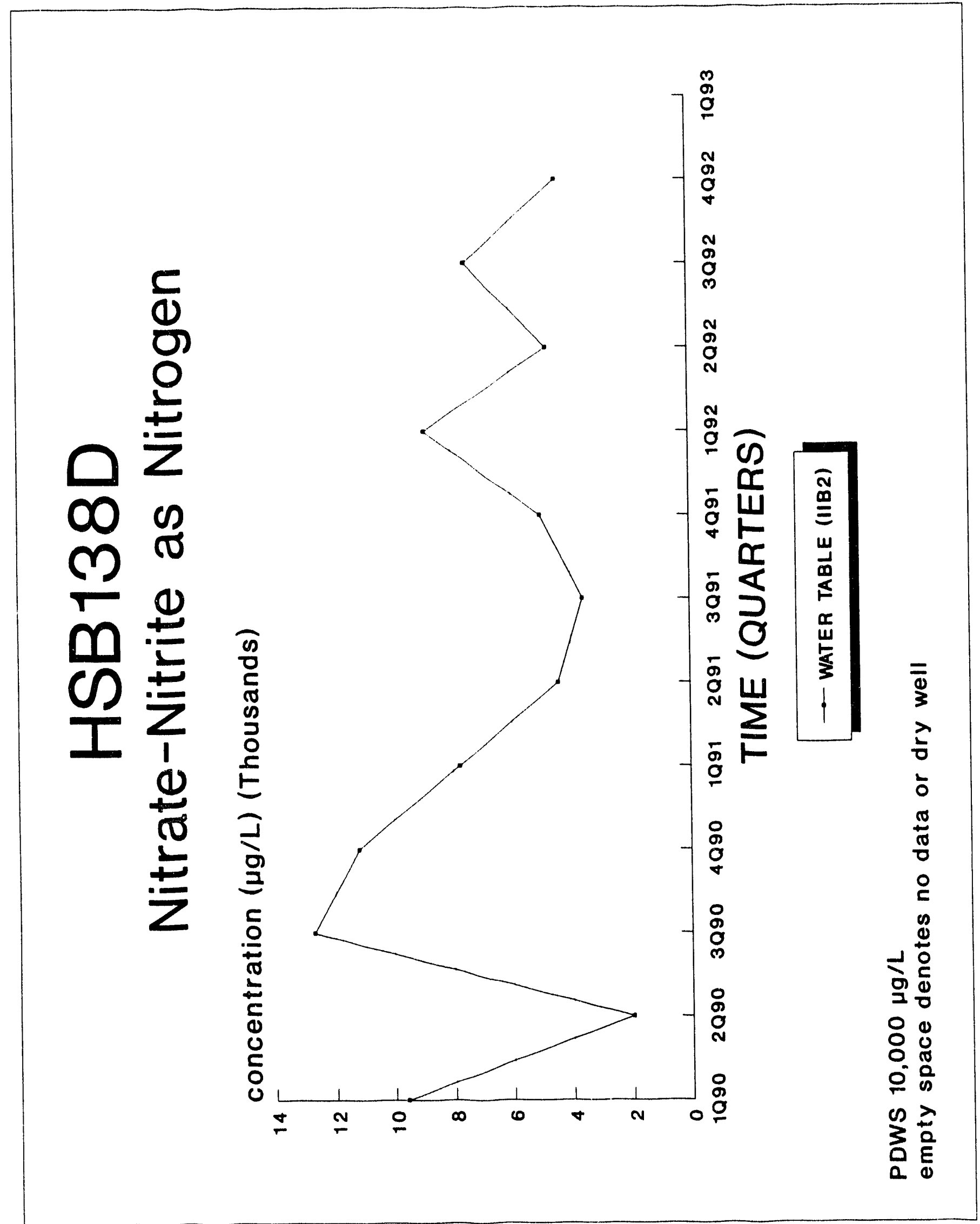




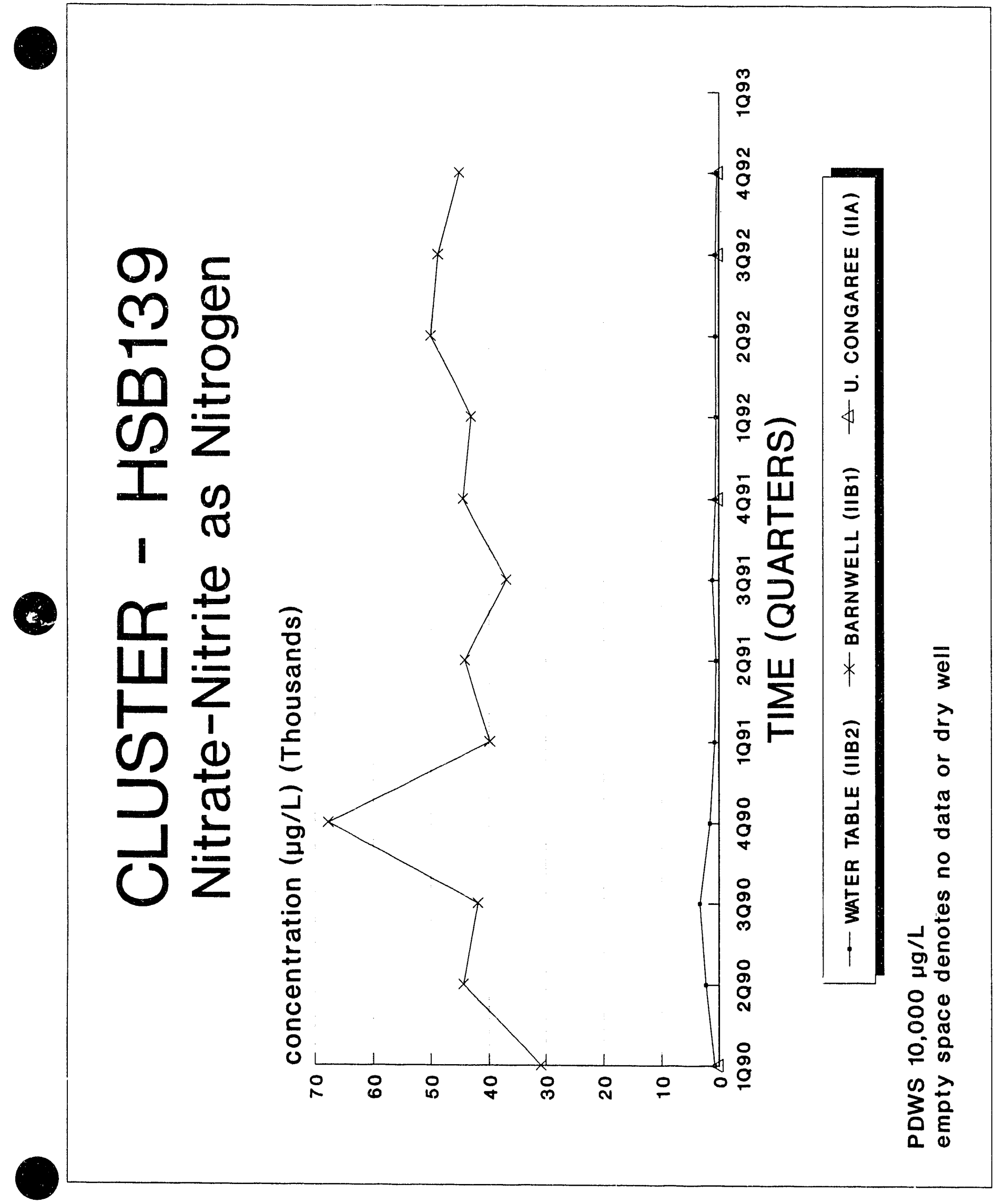




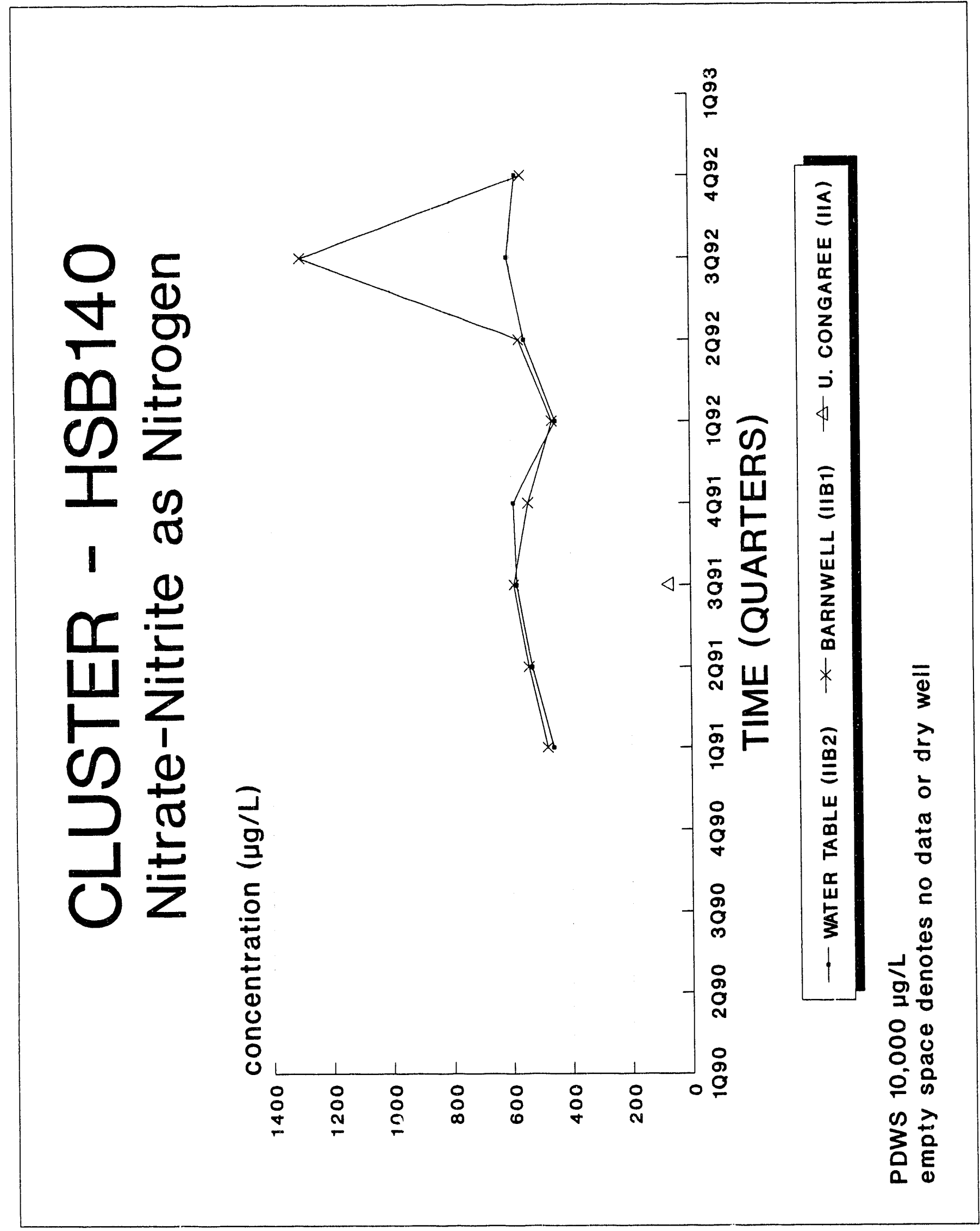




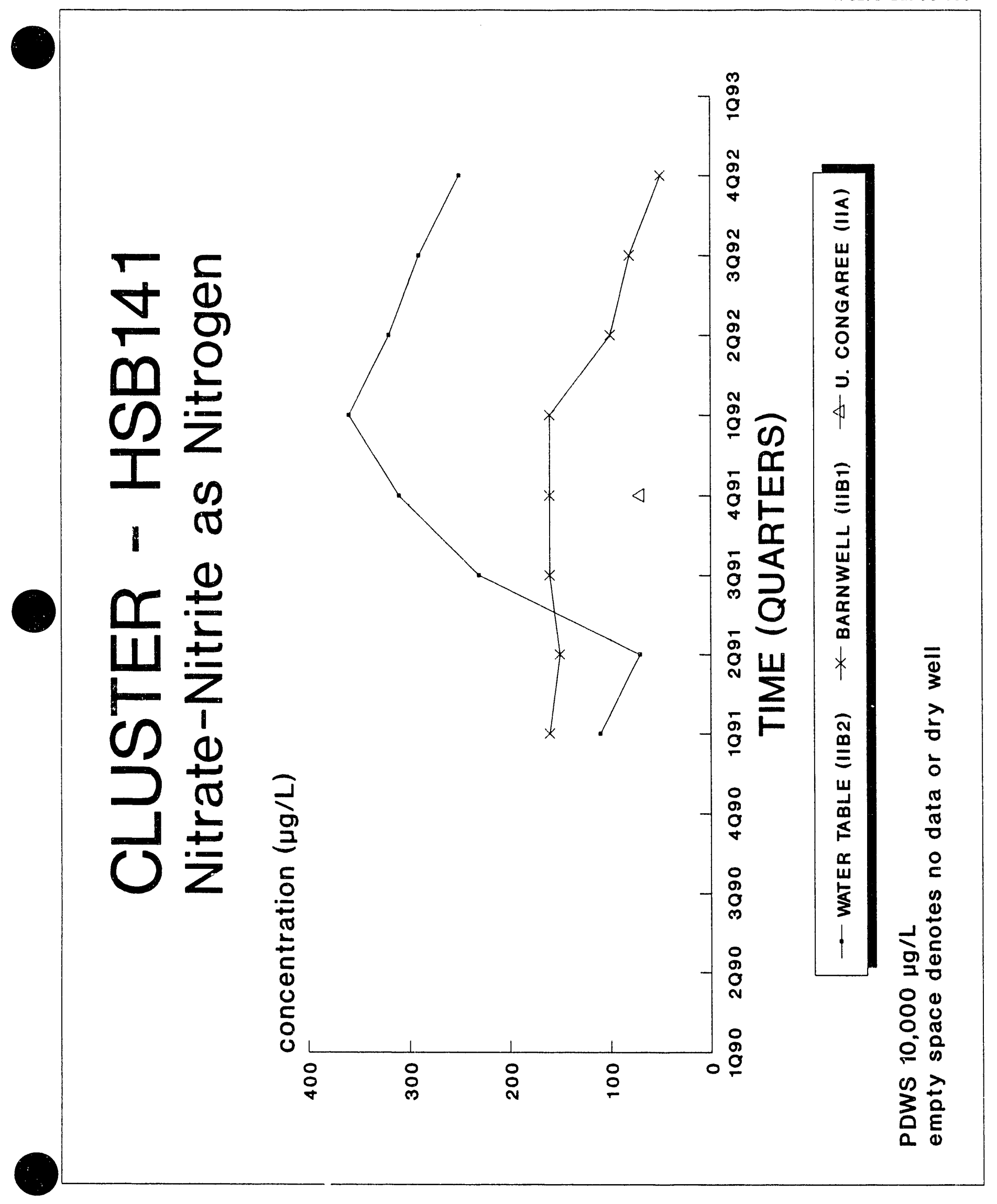




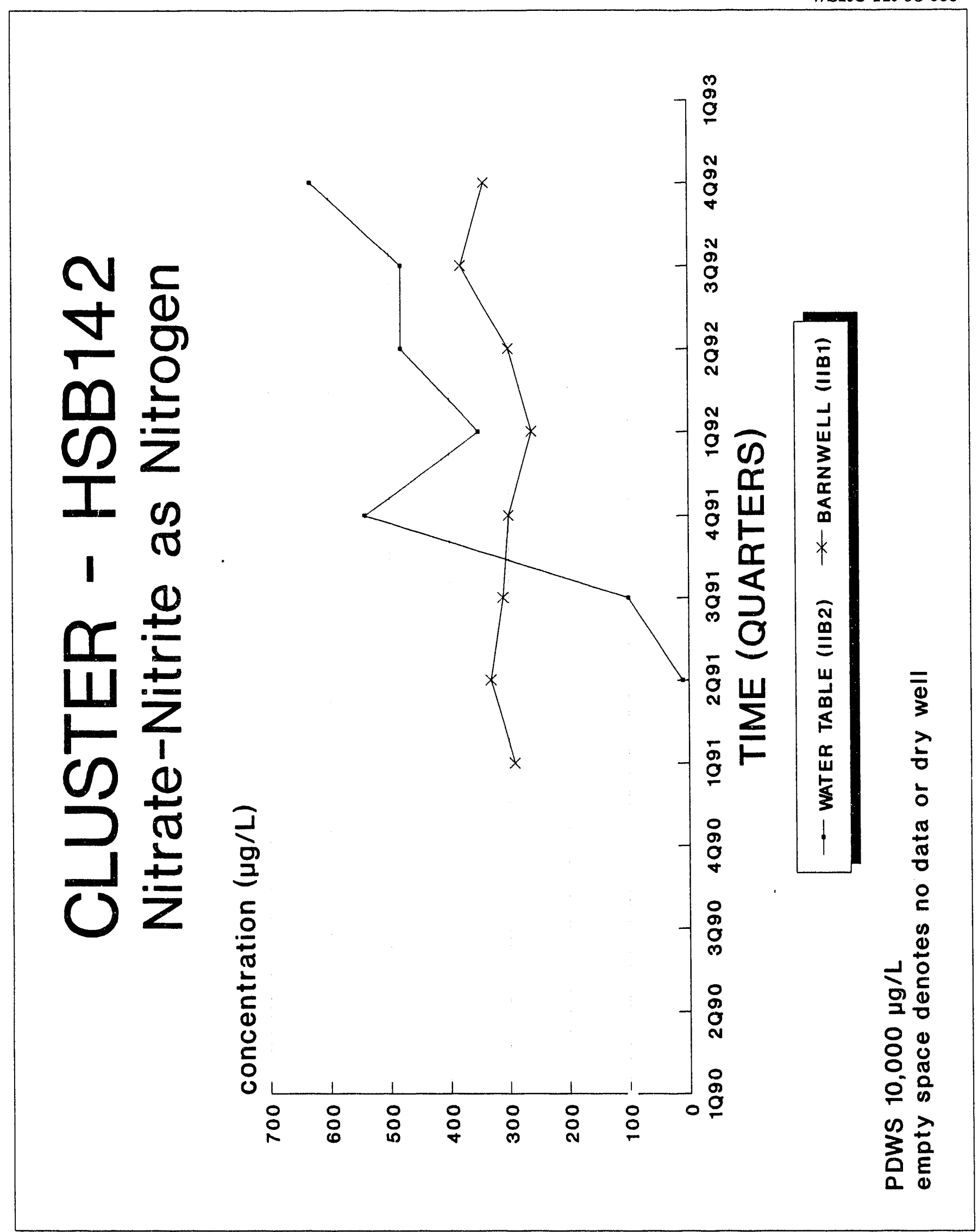




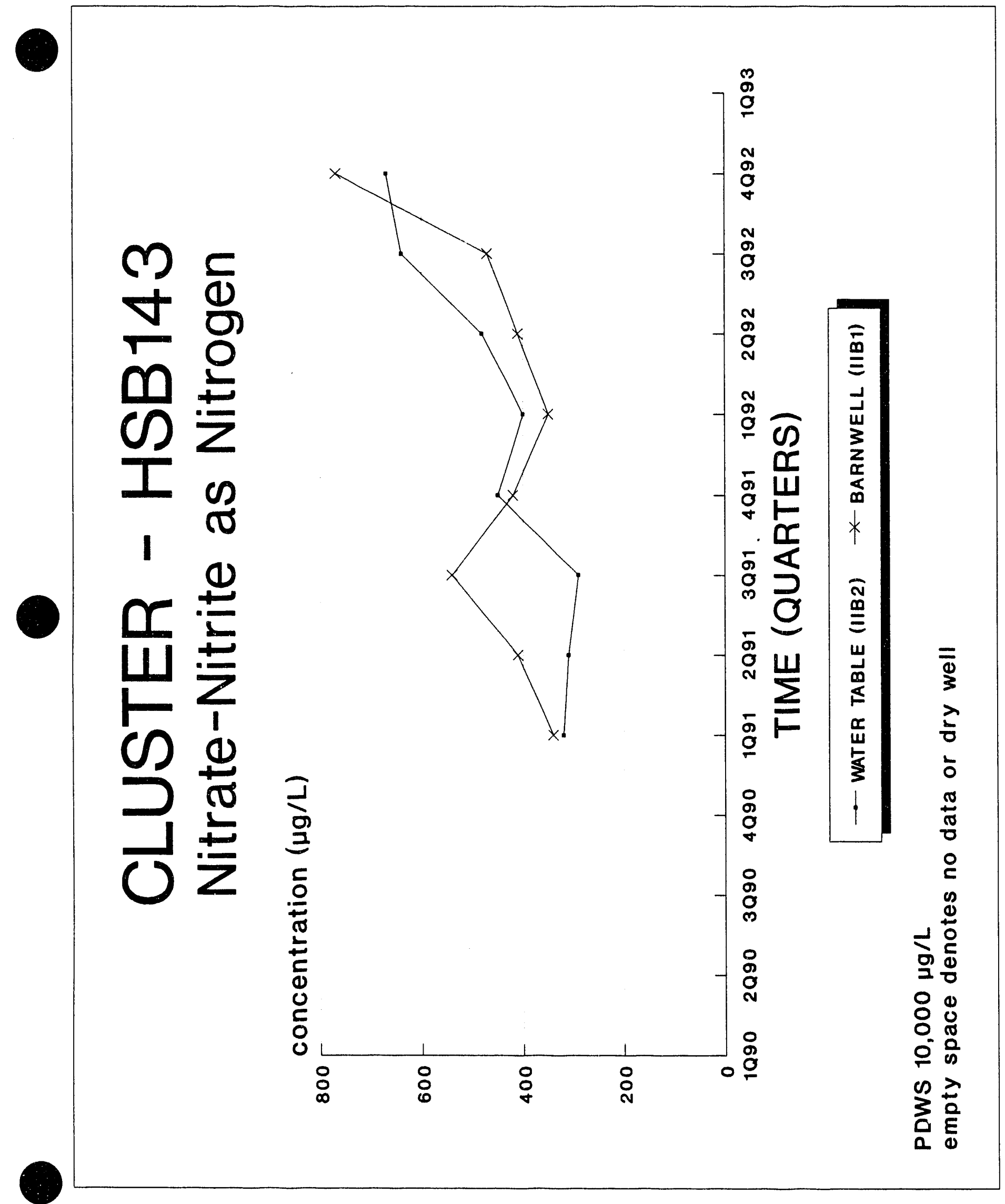




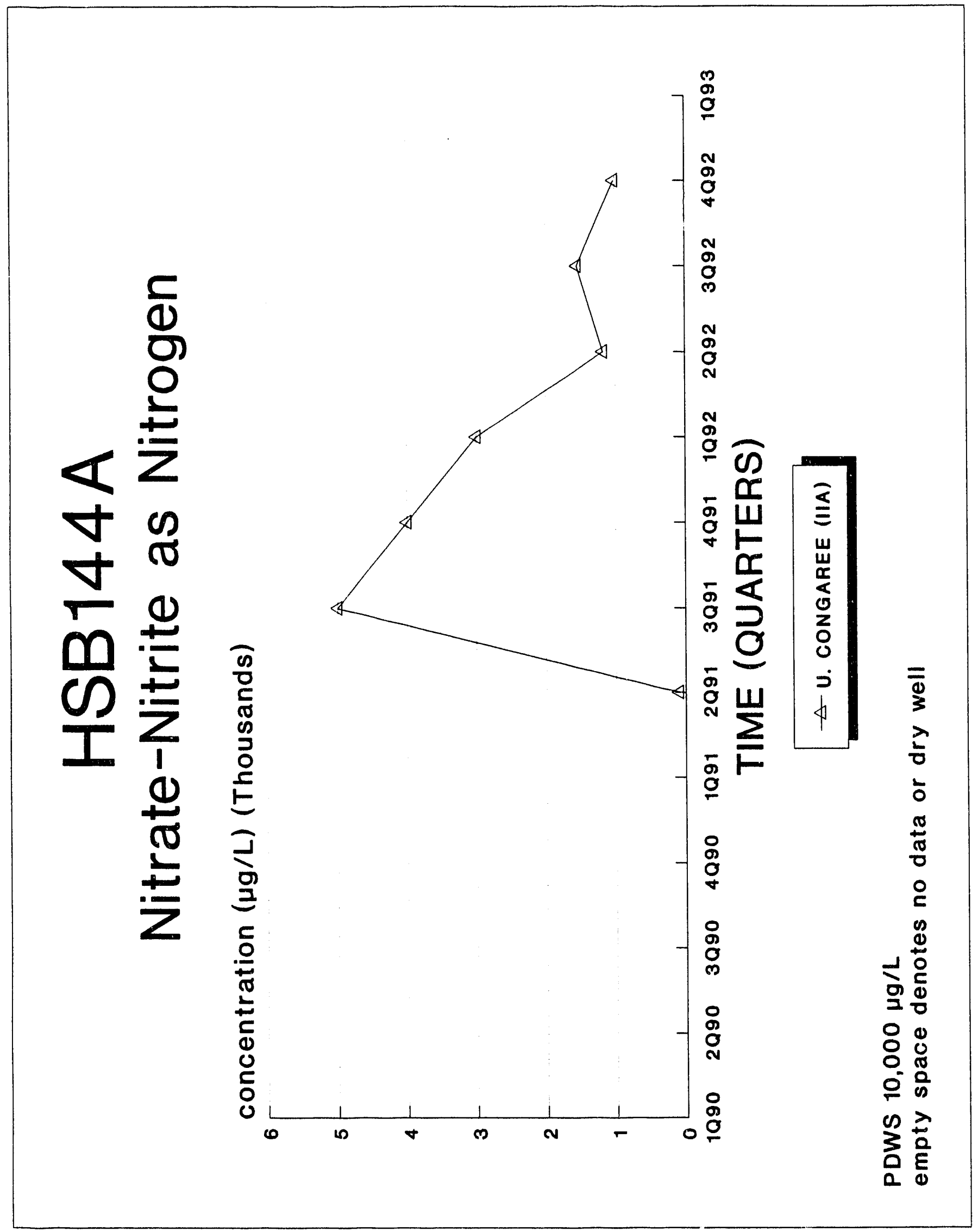




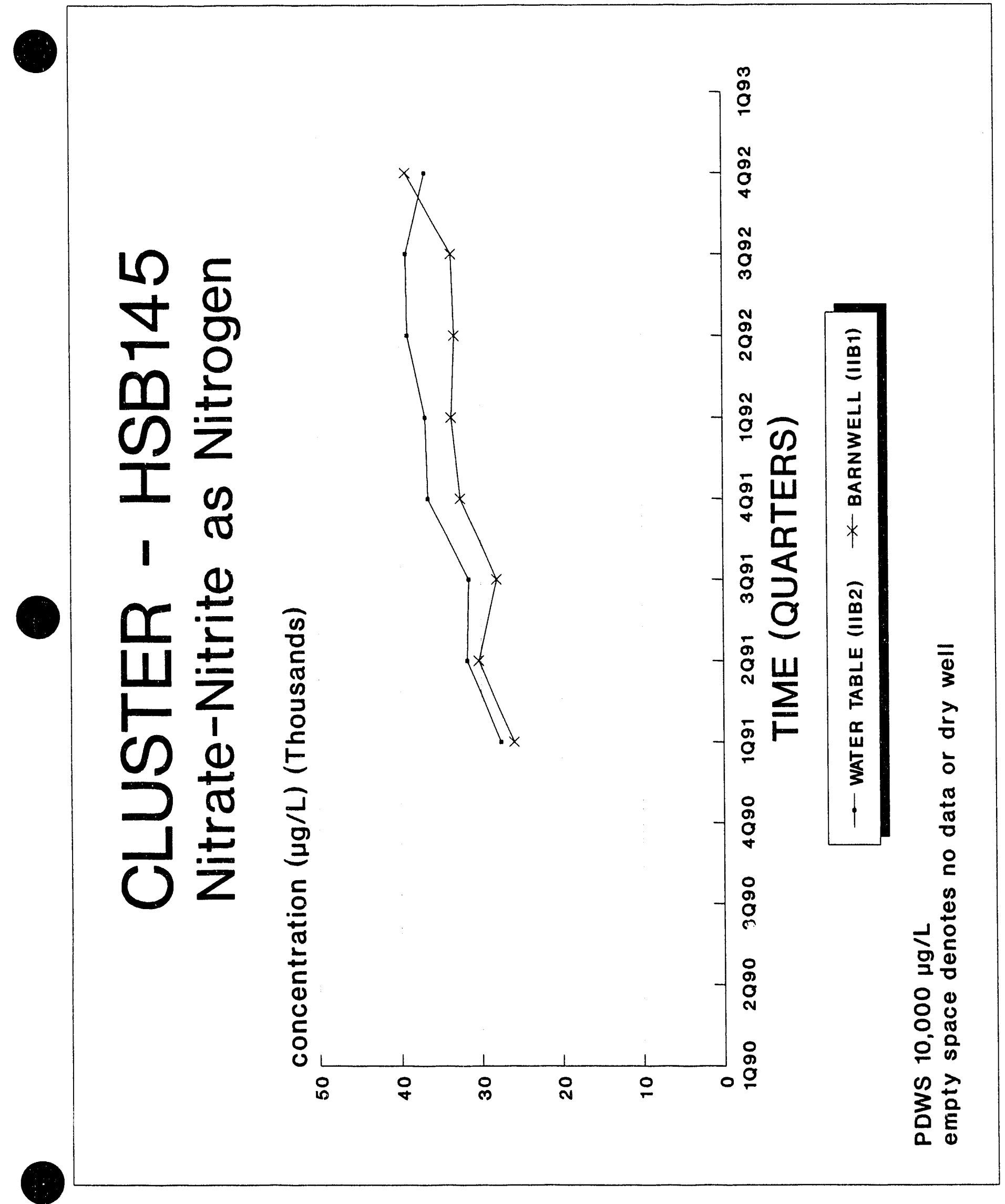




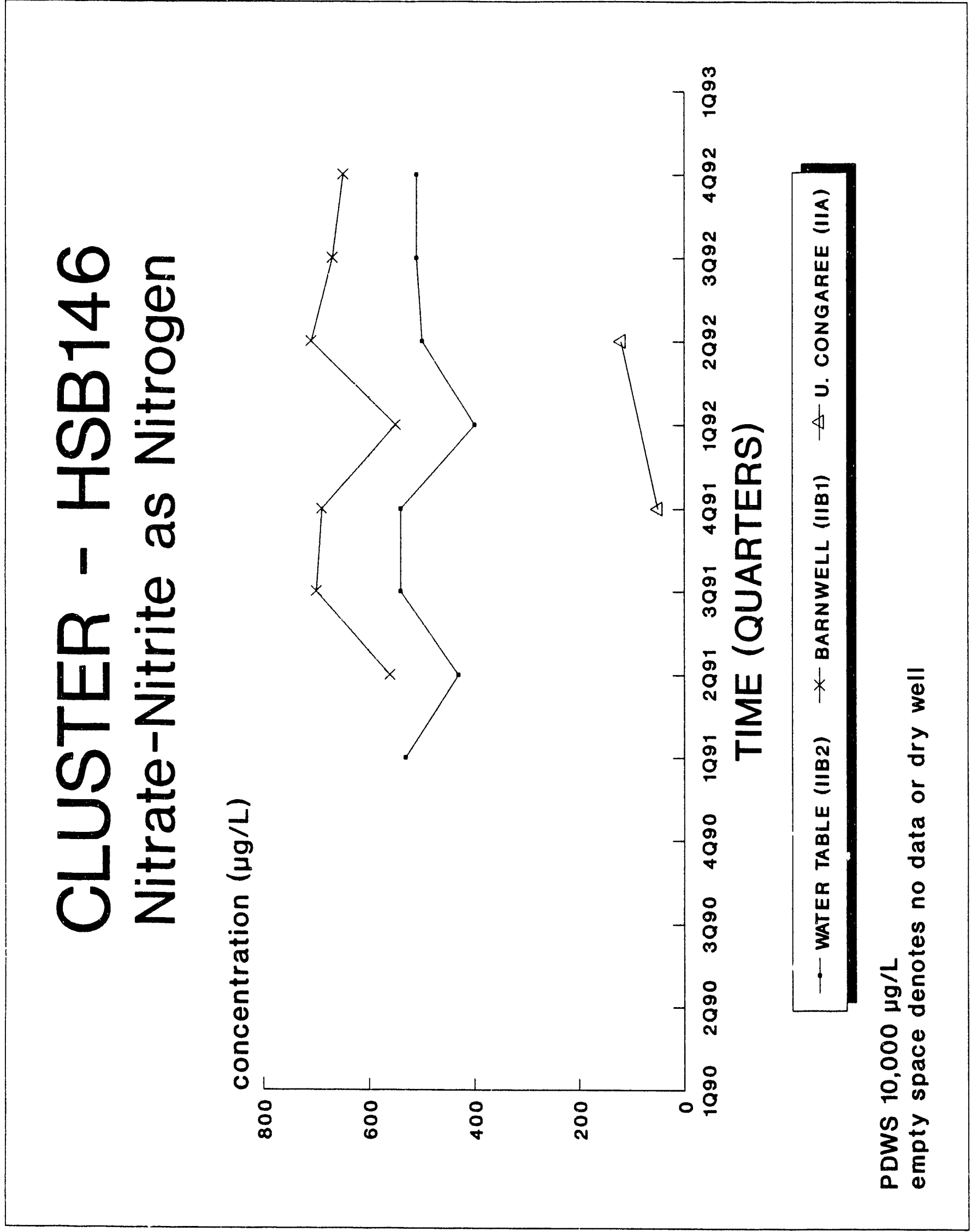




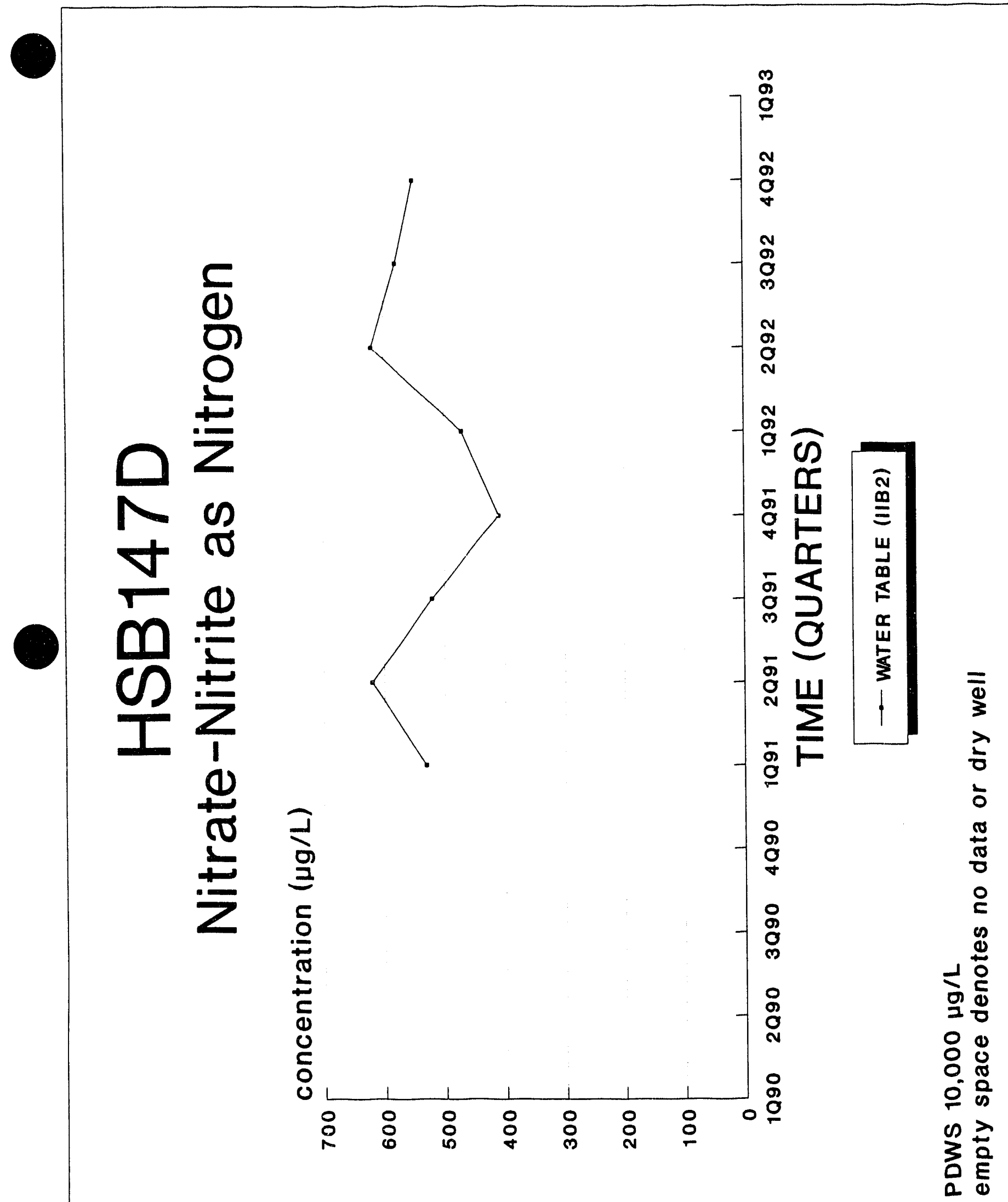




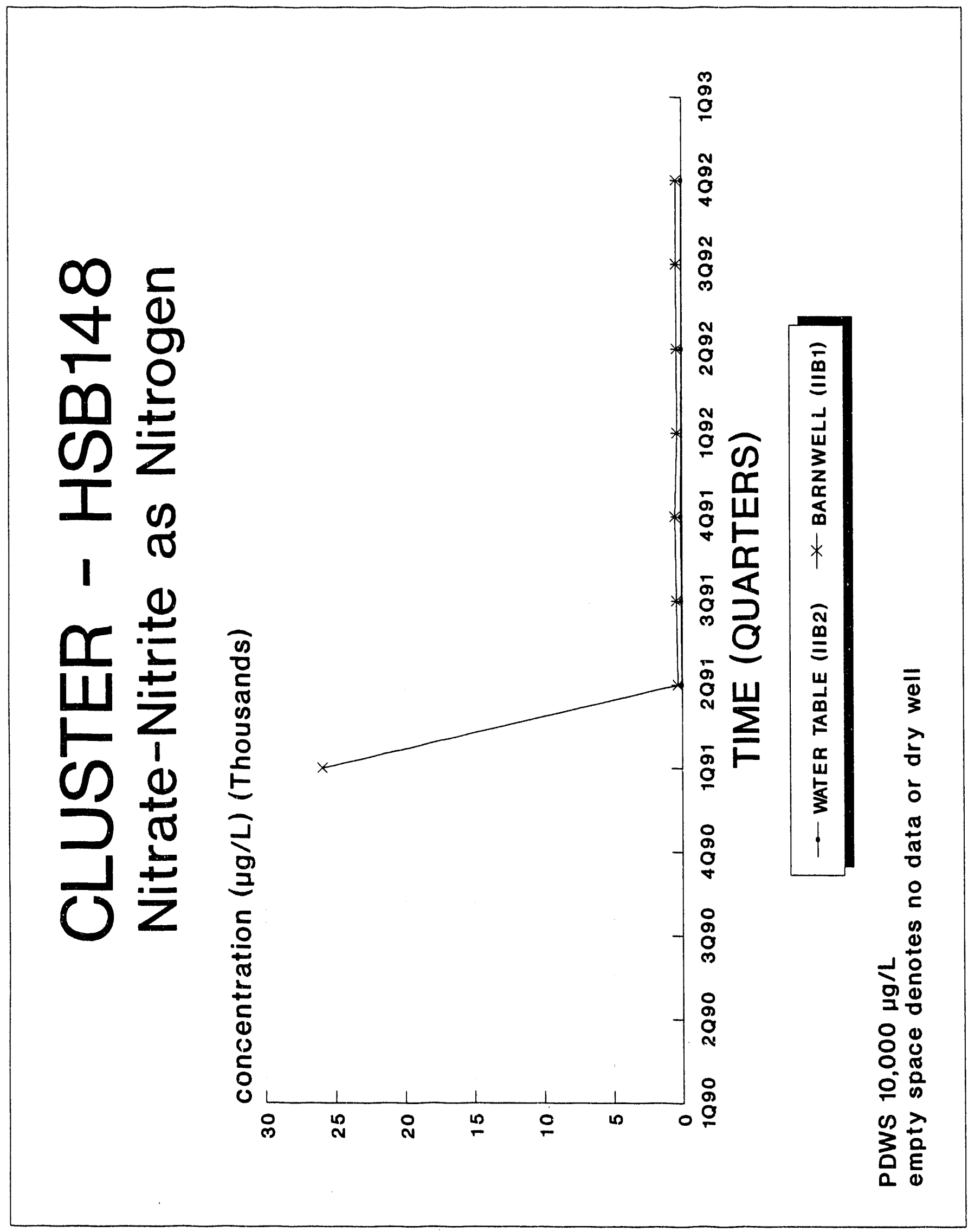




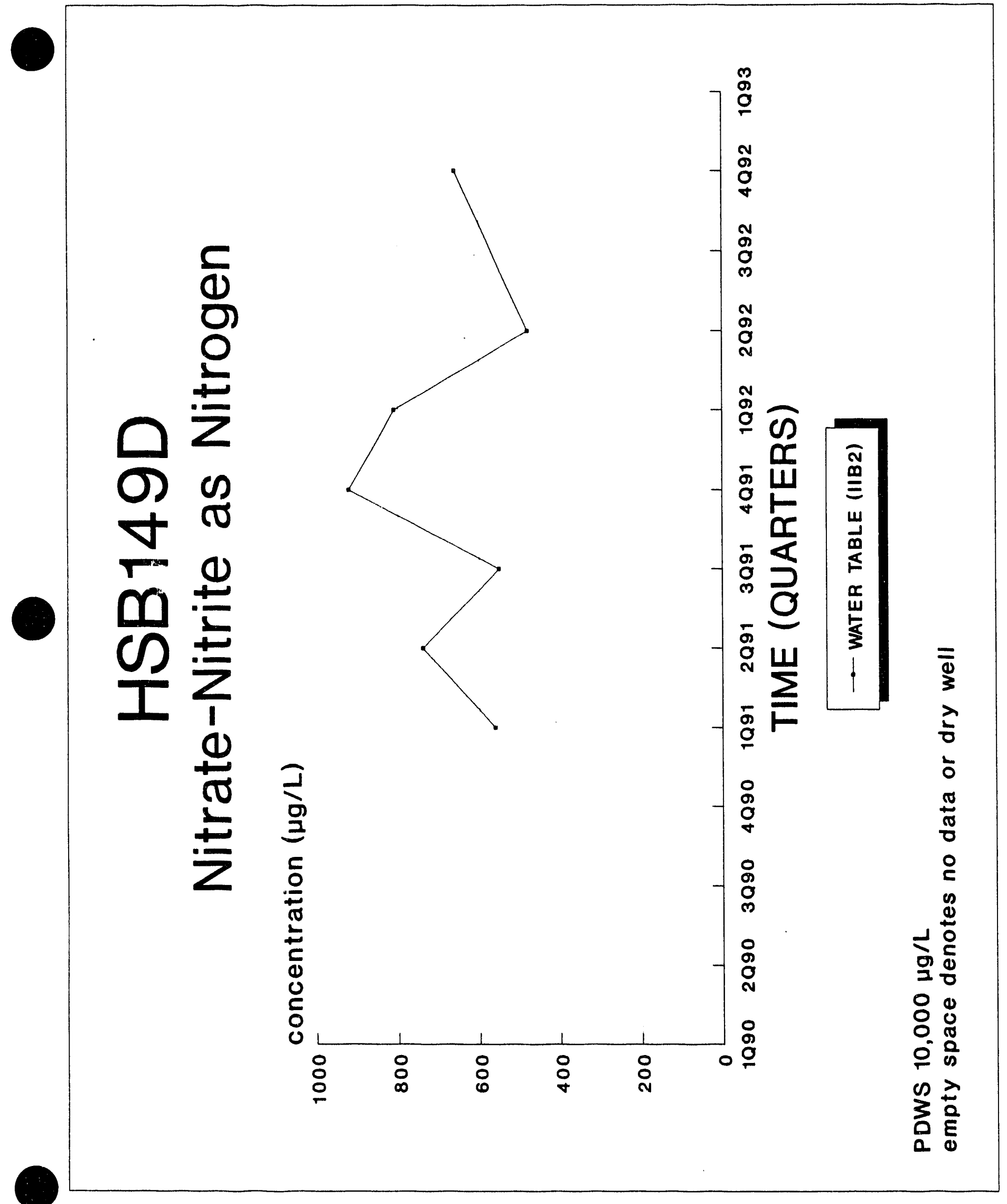




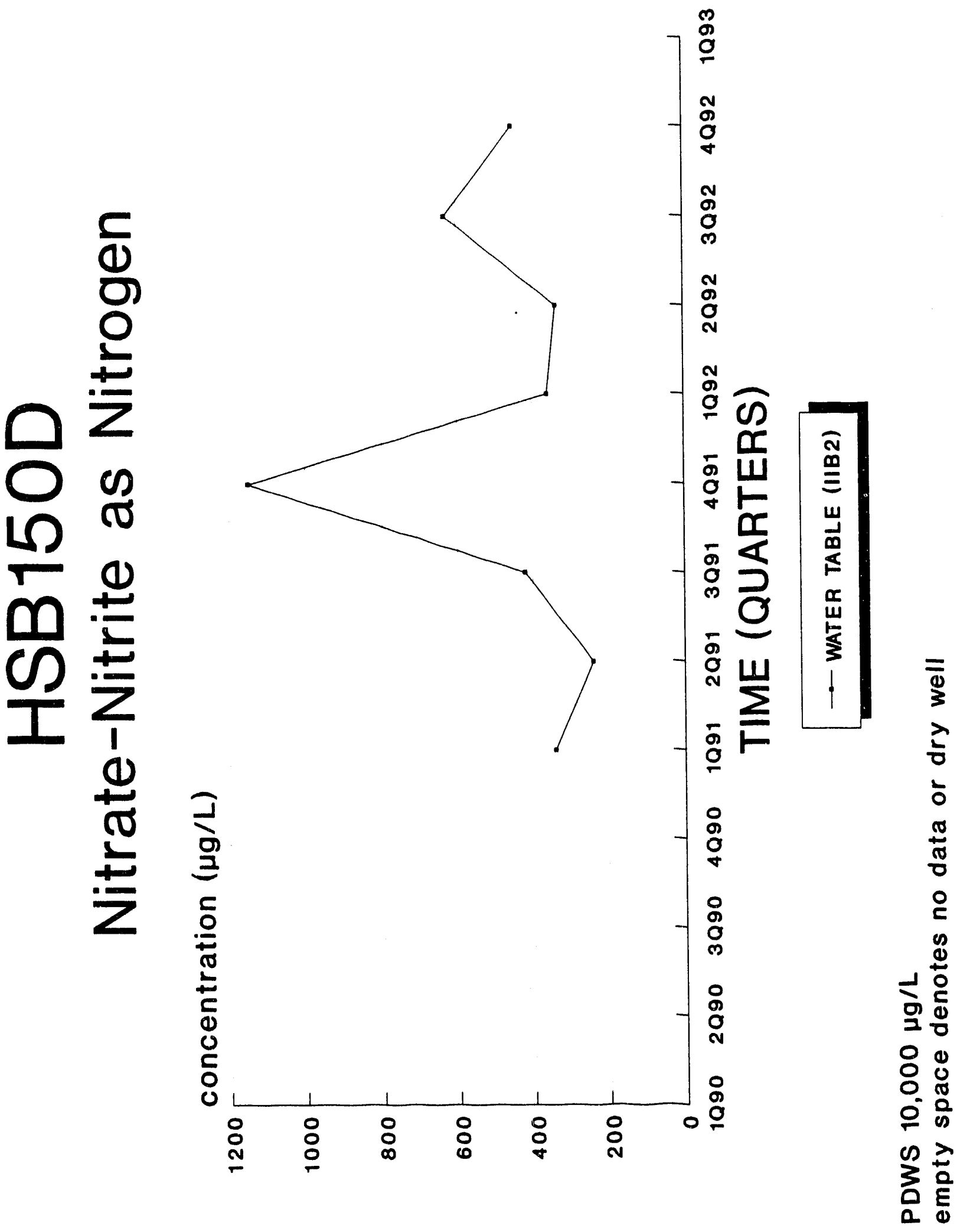




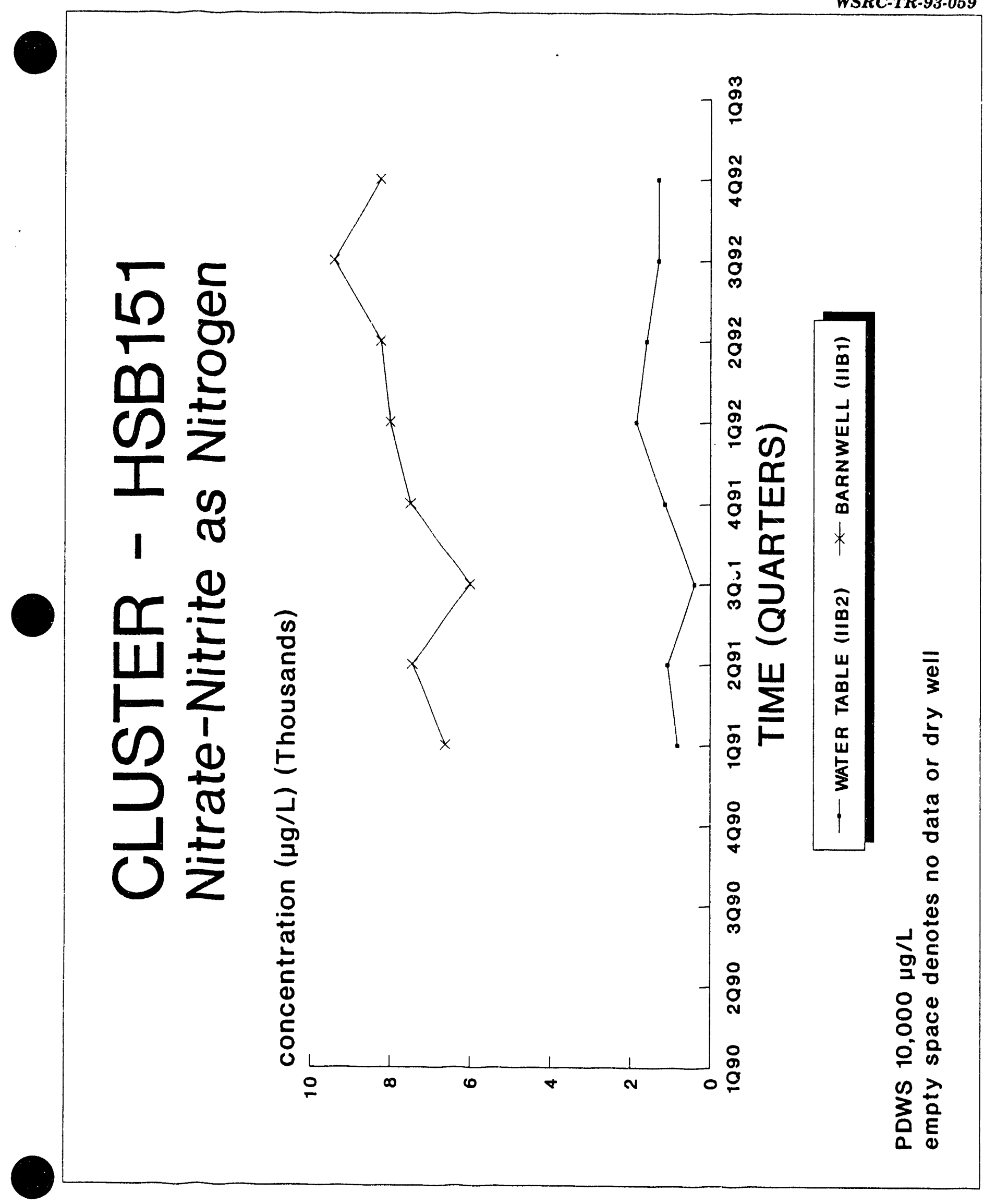




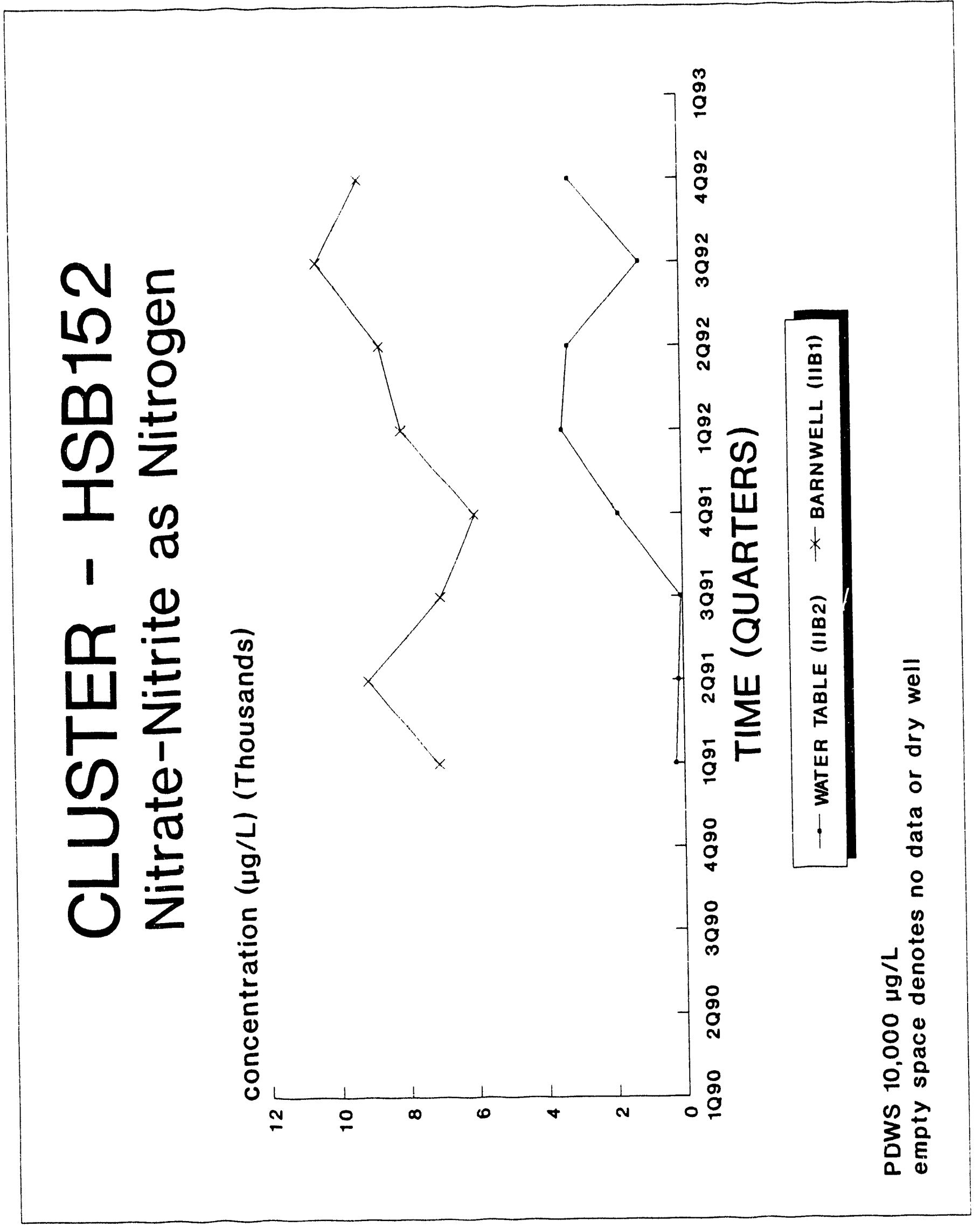




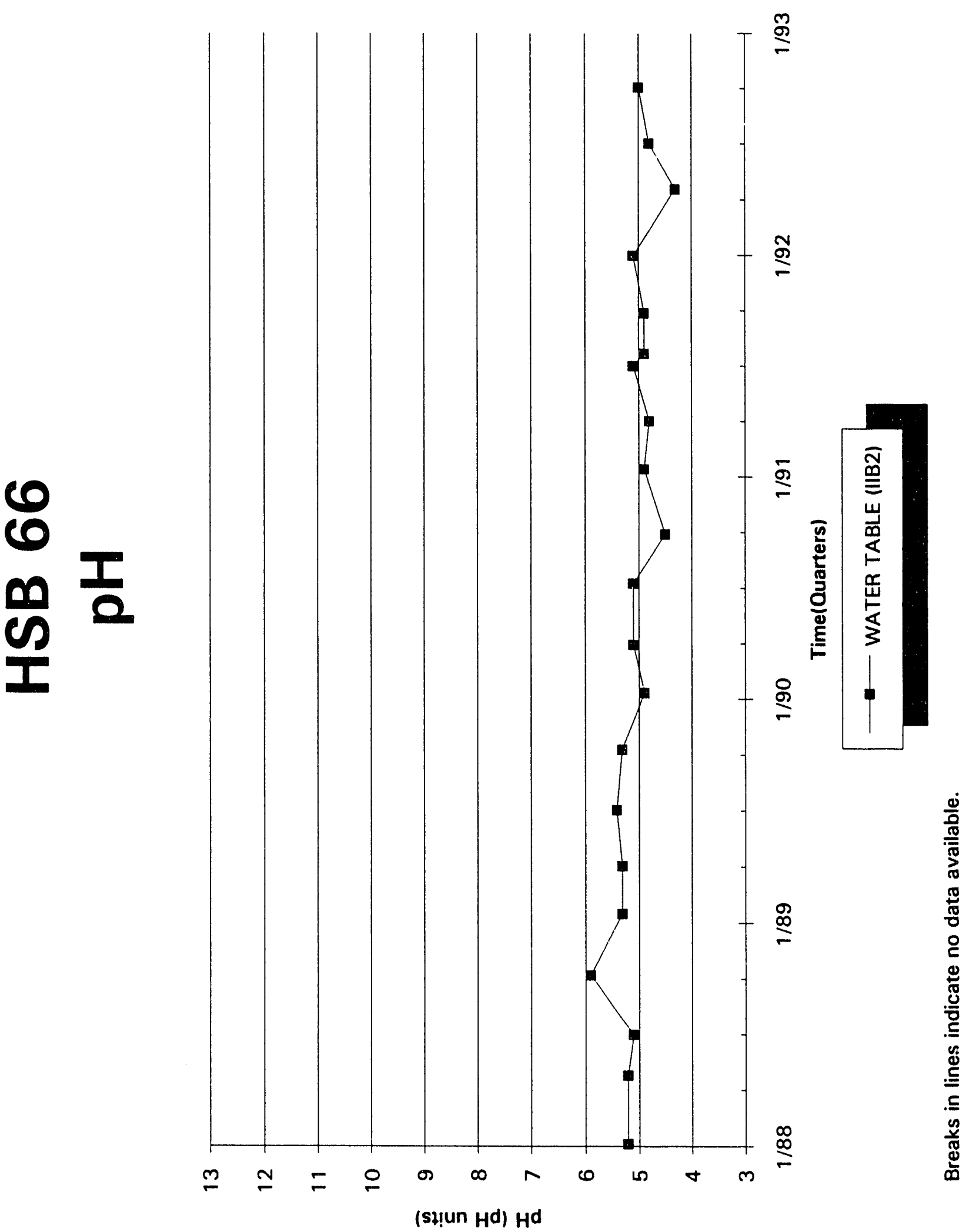




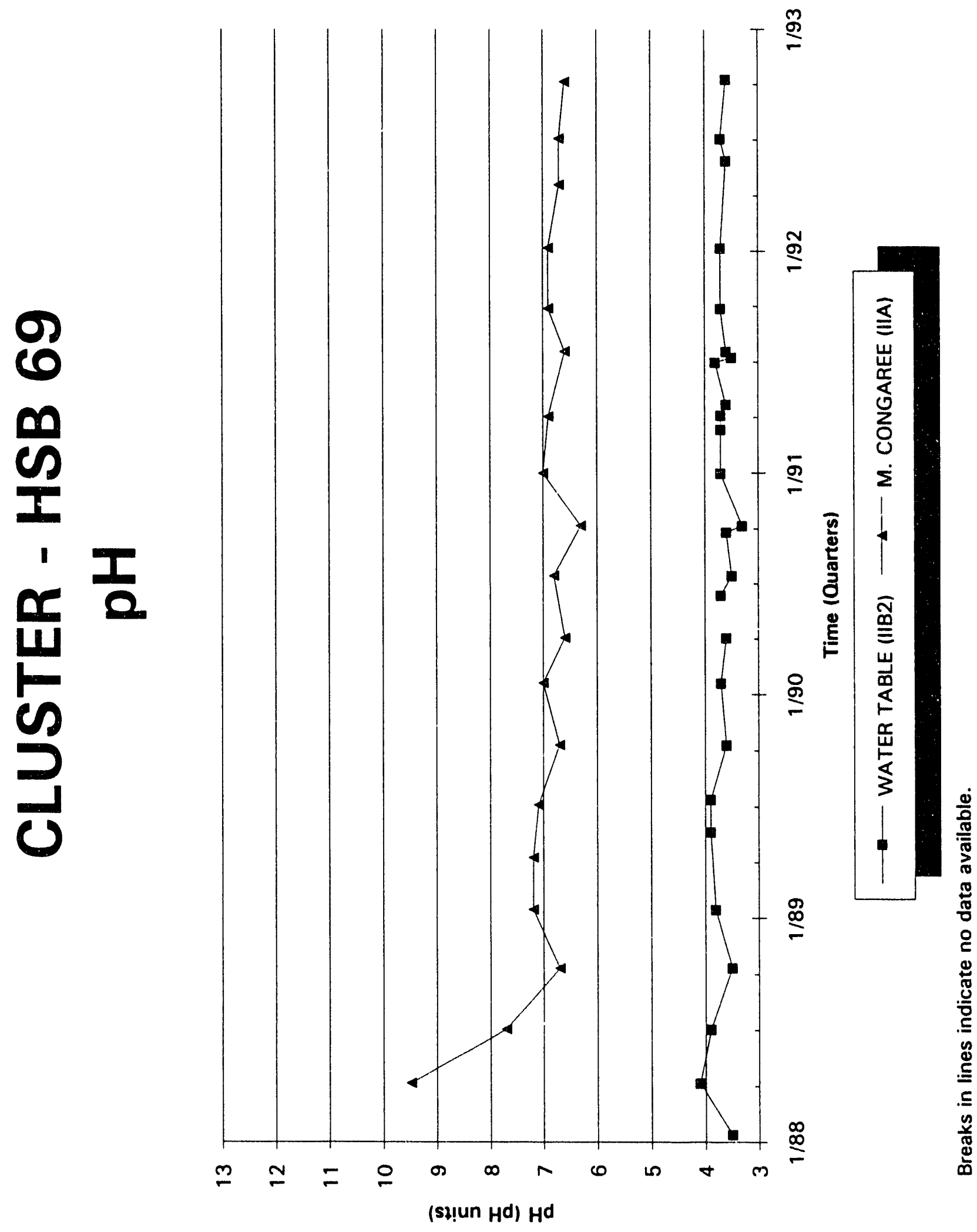


WSRC-TR-93-059

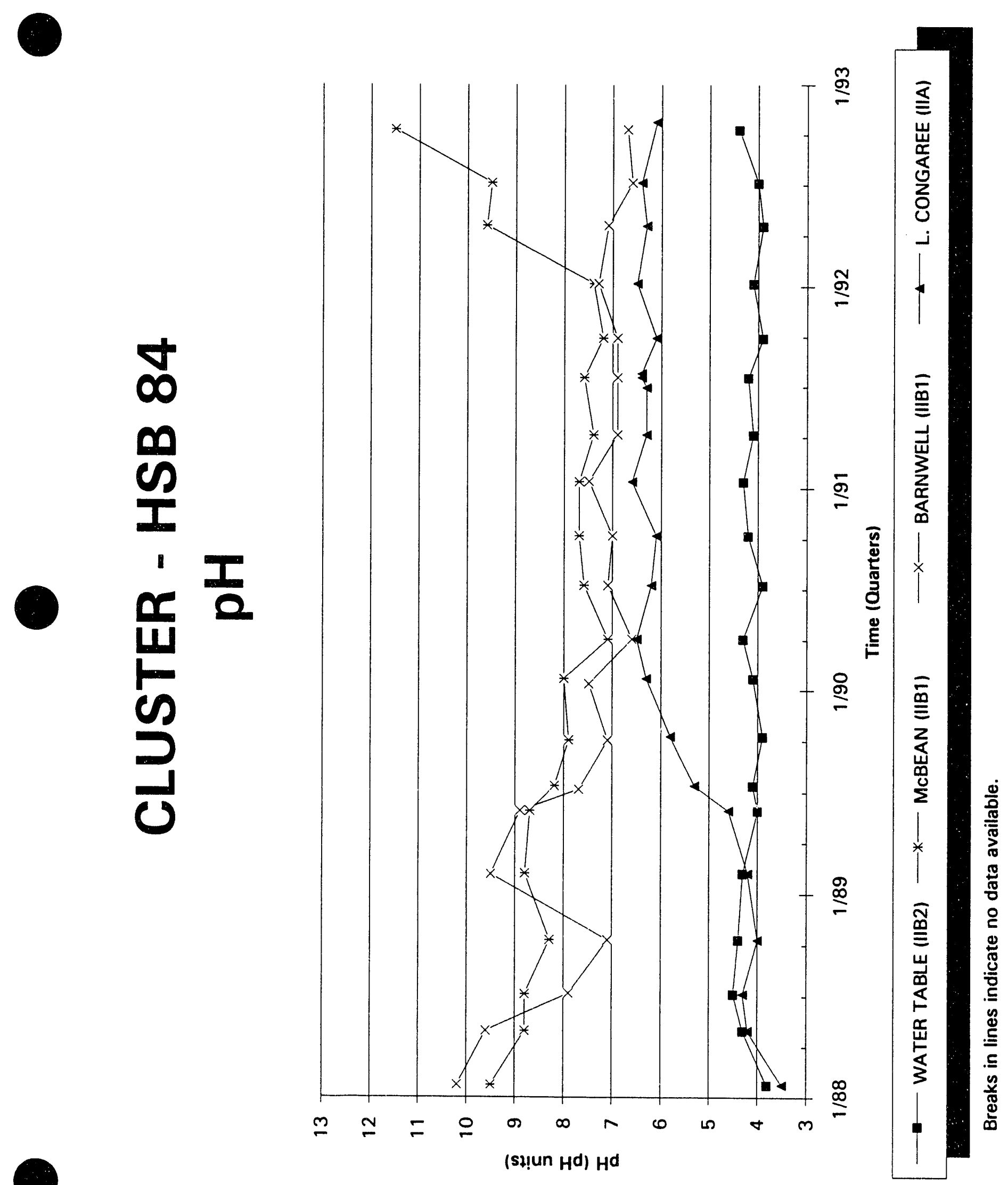




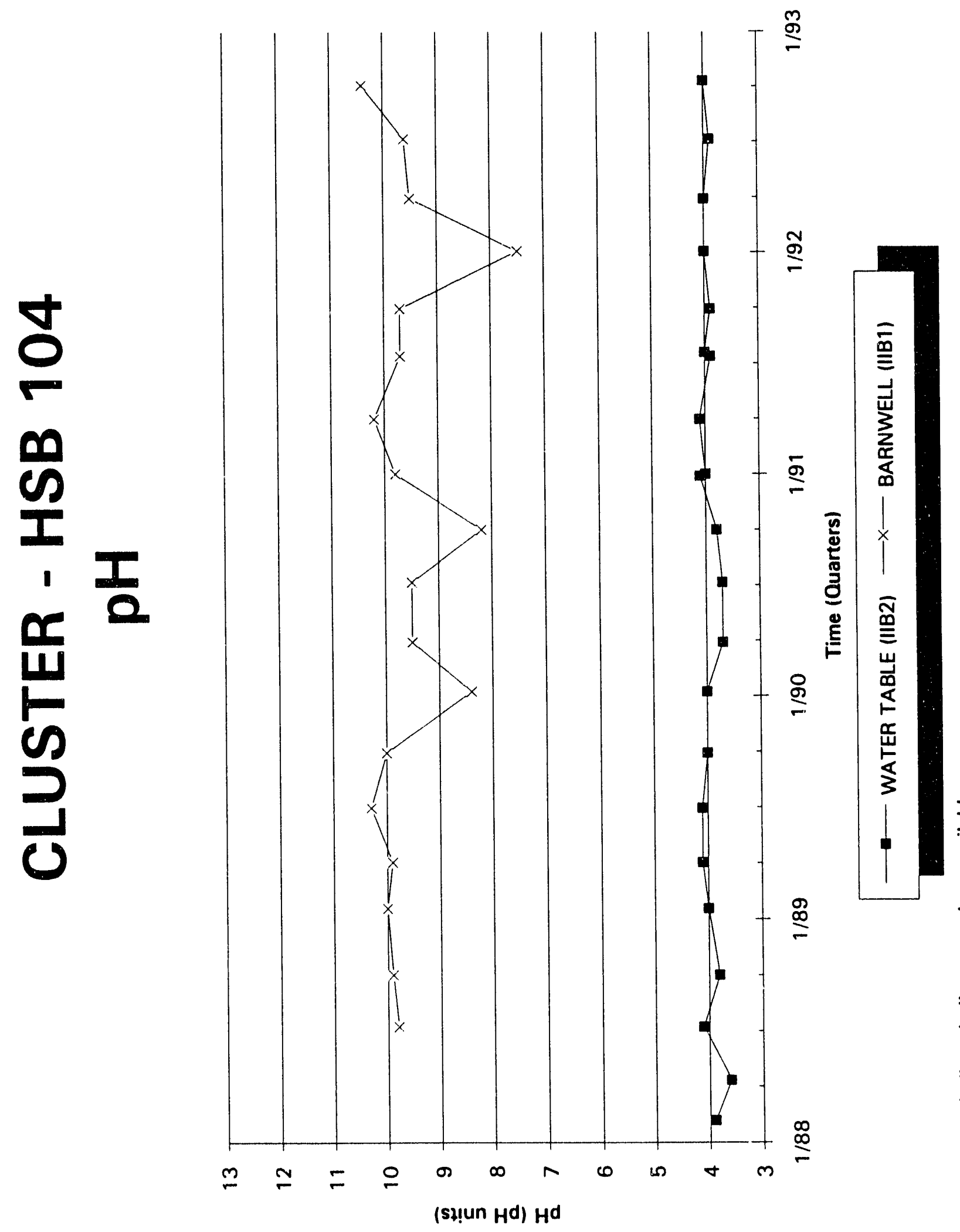

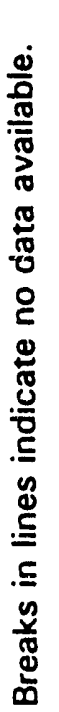




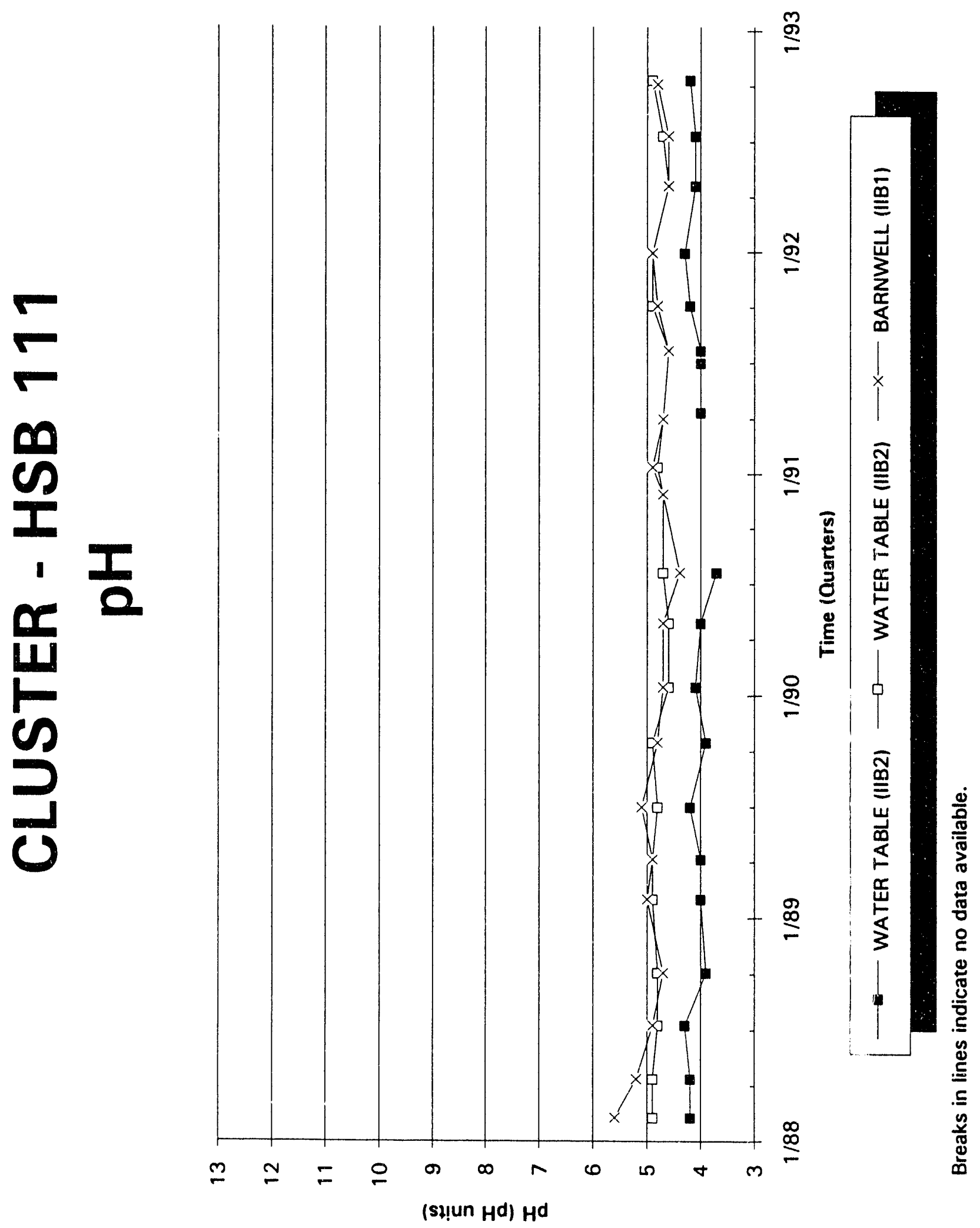




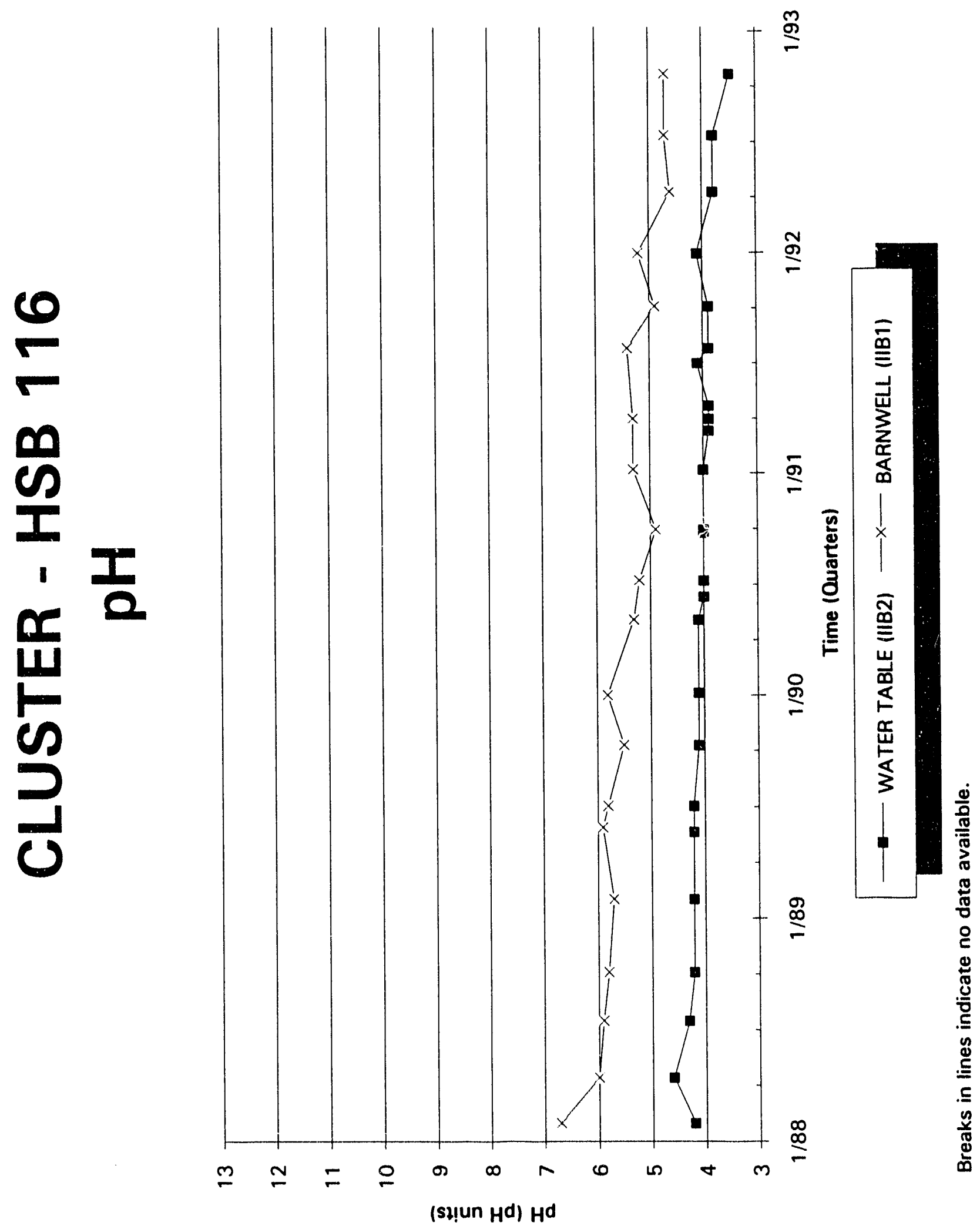




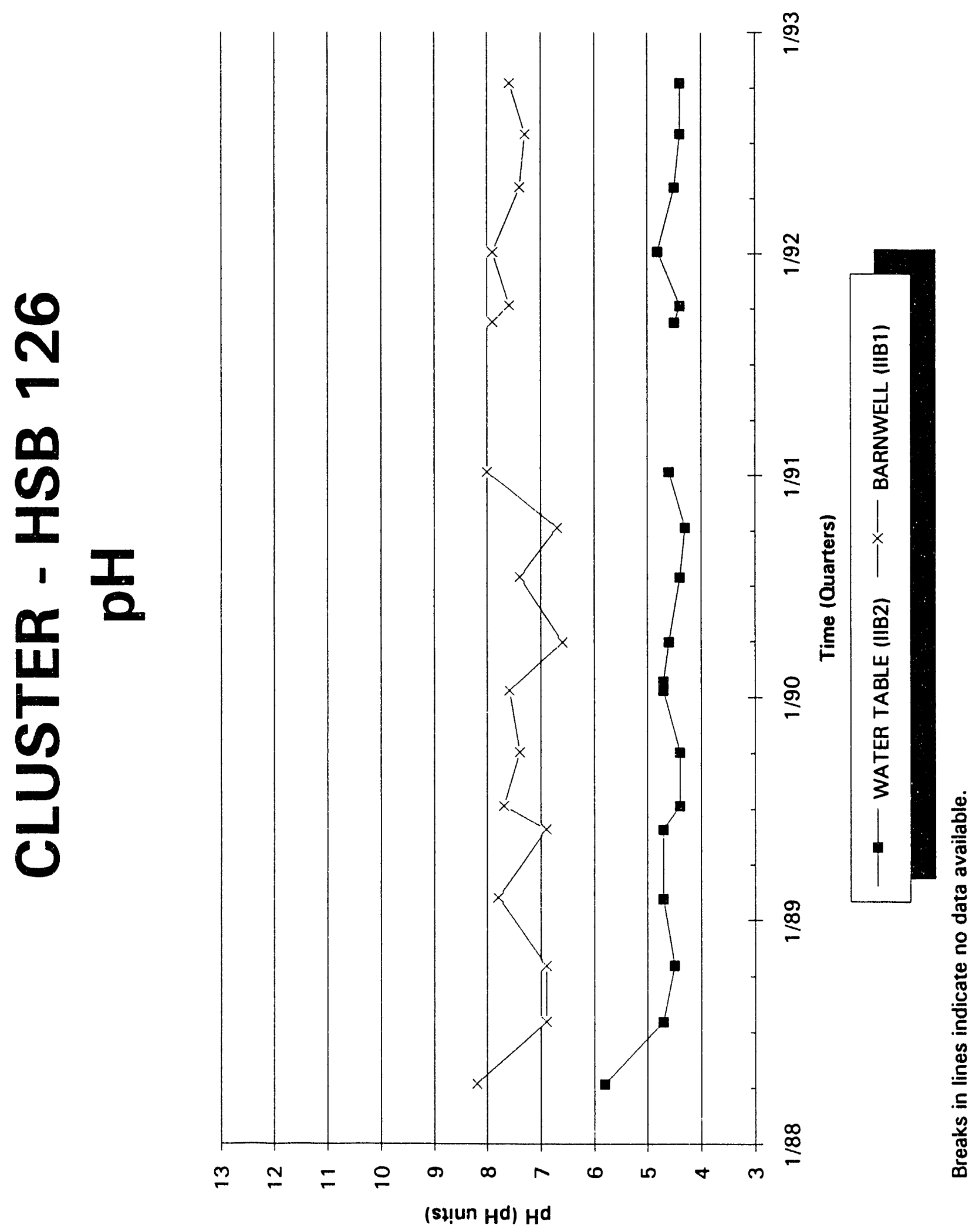




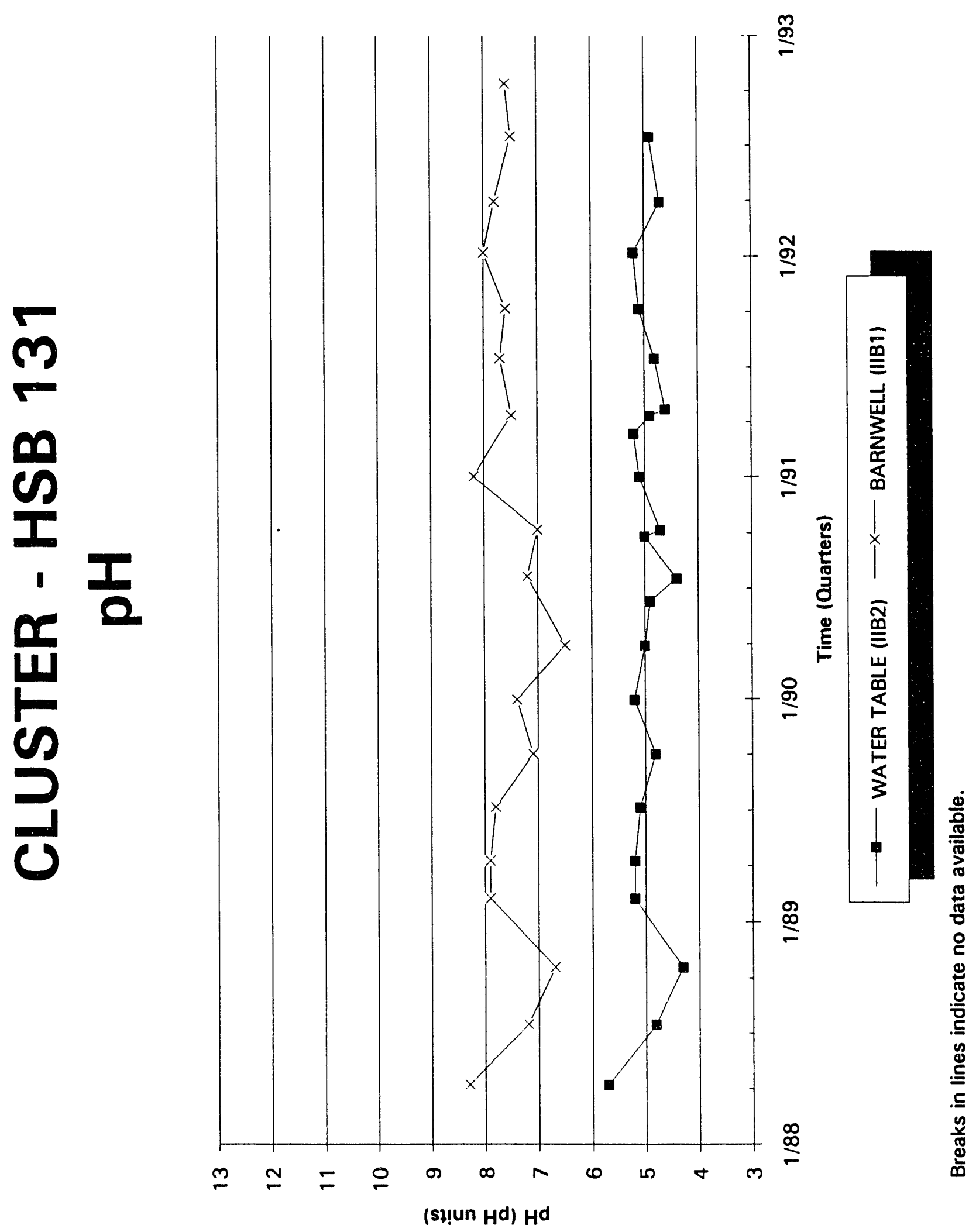




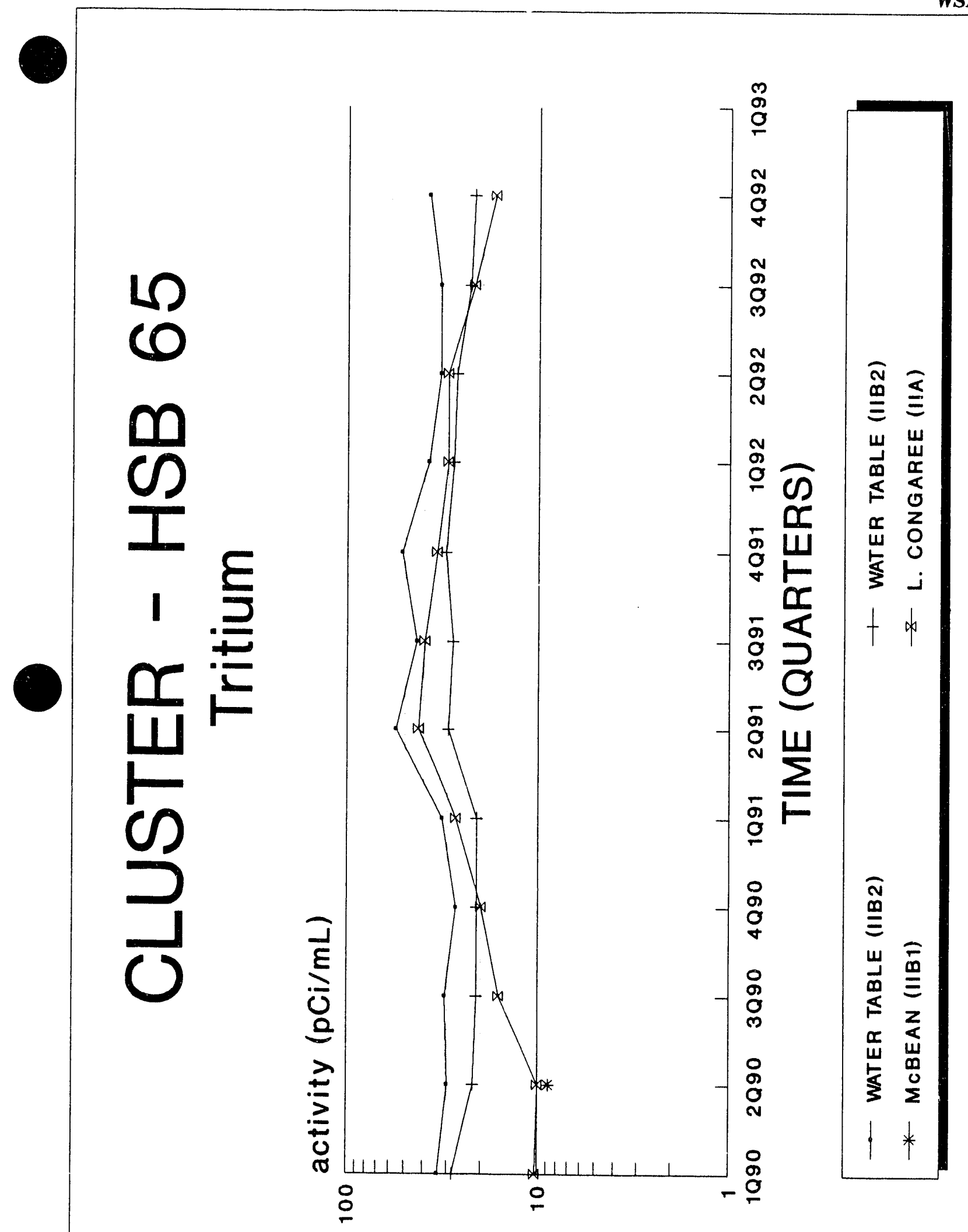

$\overline{\bar{\Phi}}$

30
260

눙

○感

$\infty$

๘্

$2 \mathrm{c}$

ำ

$\infty 0$

$\stackrel{0}{\infty} \infty$

$+\frac{1}{1} I$

E

ऽ。

Q 0

은 은

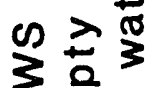

О है 


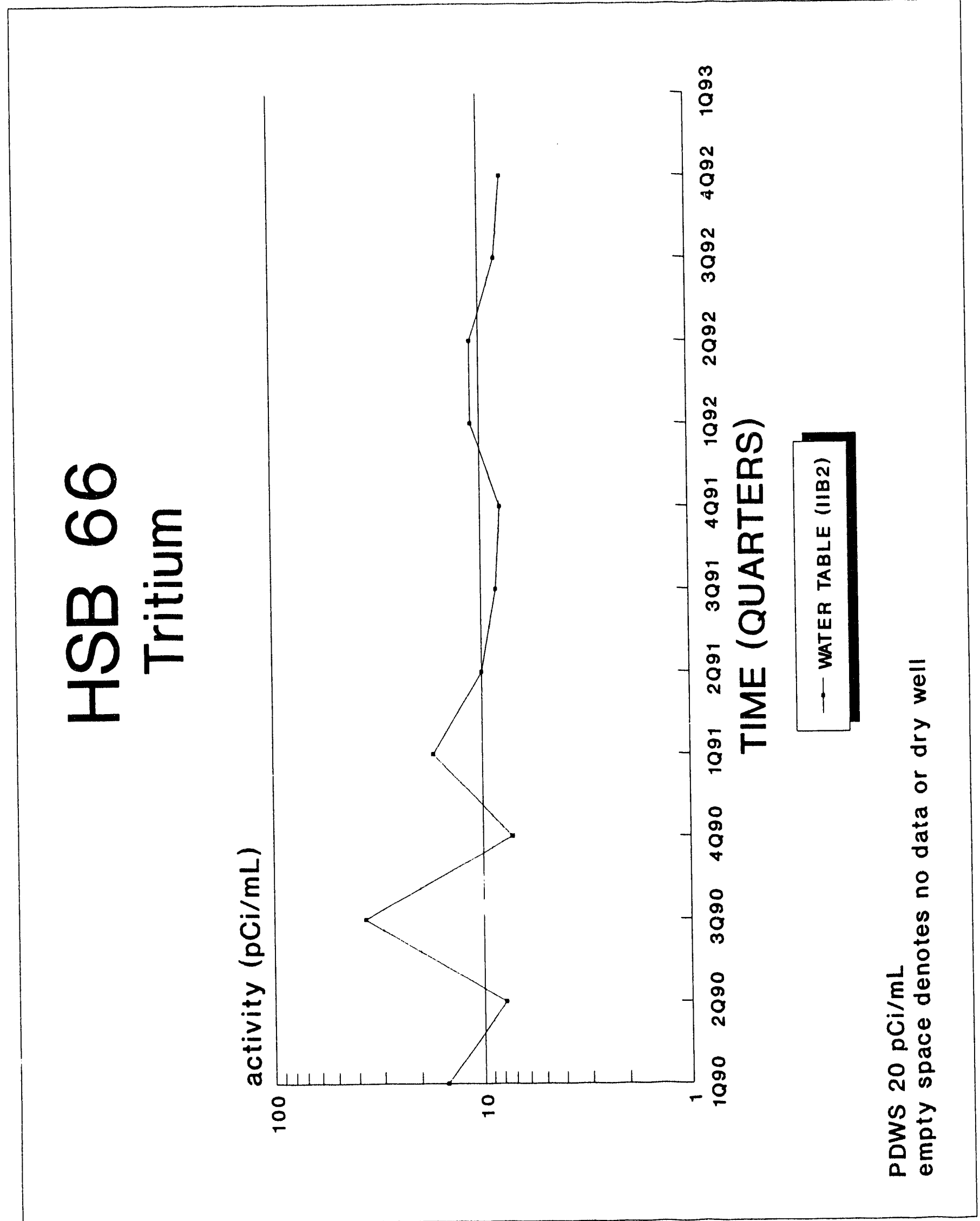




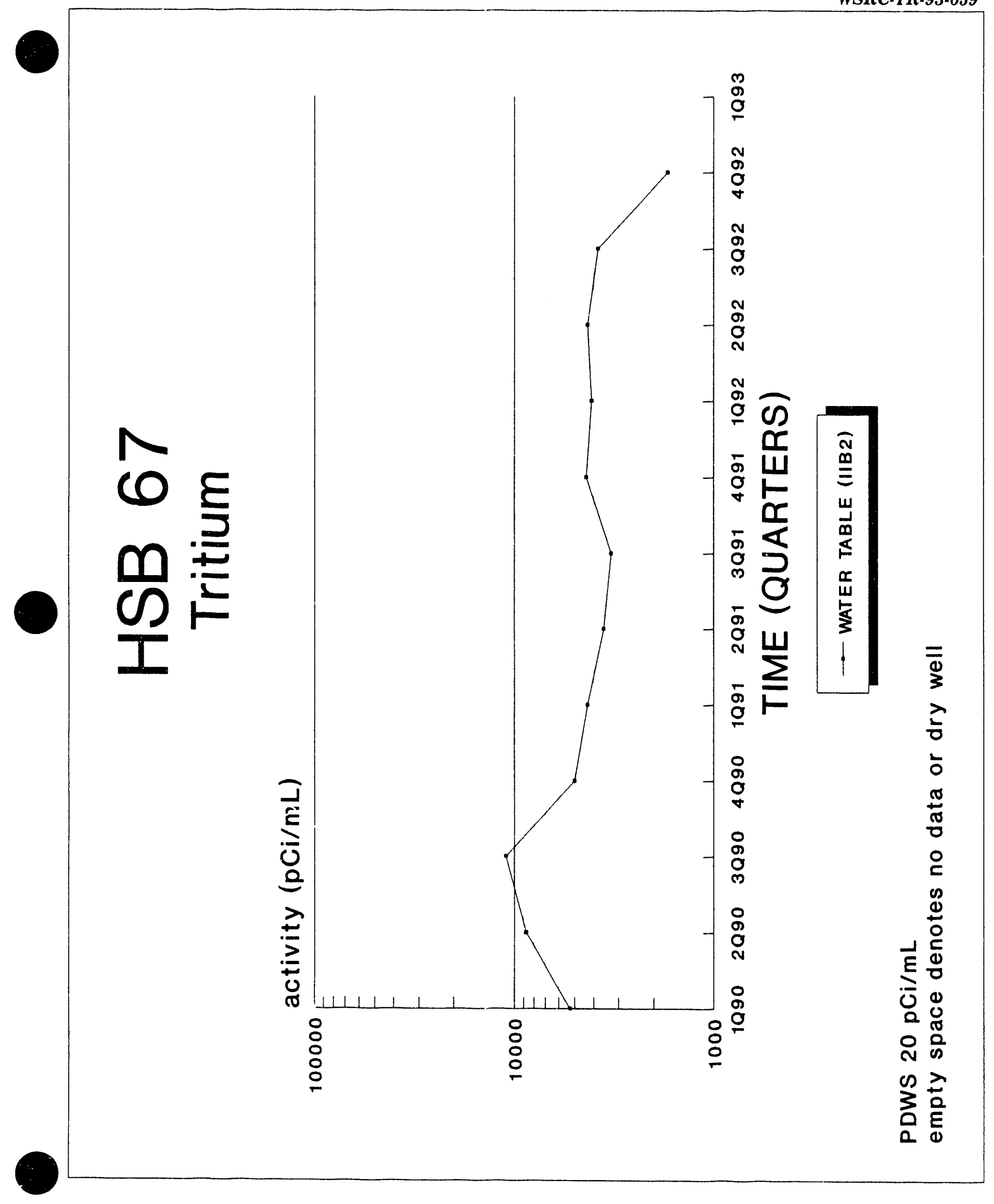




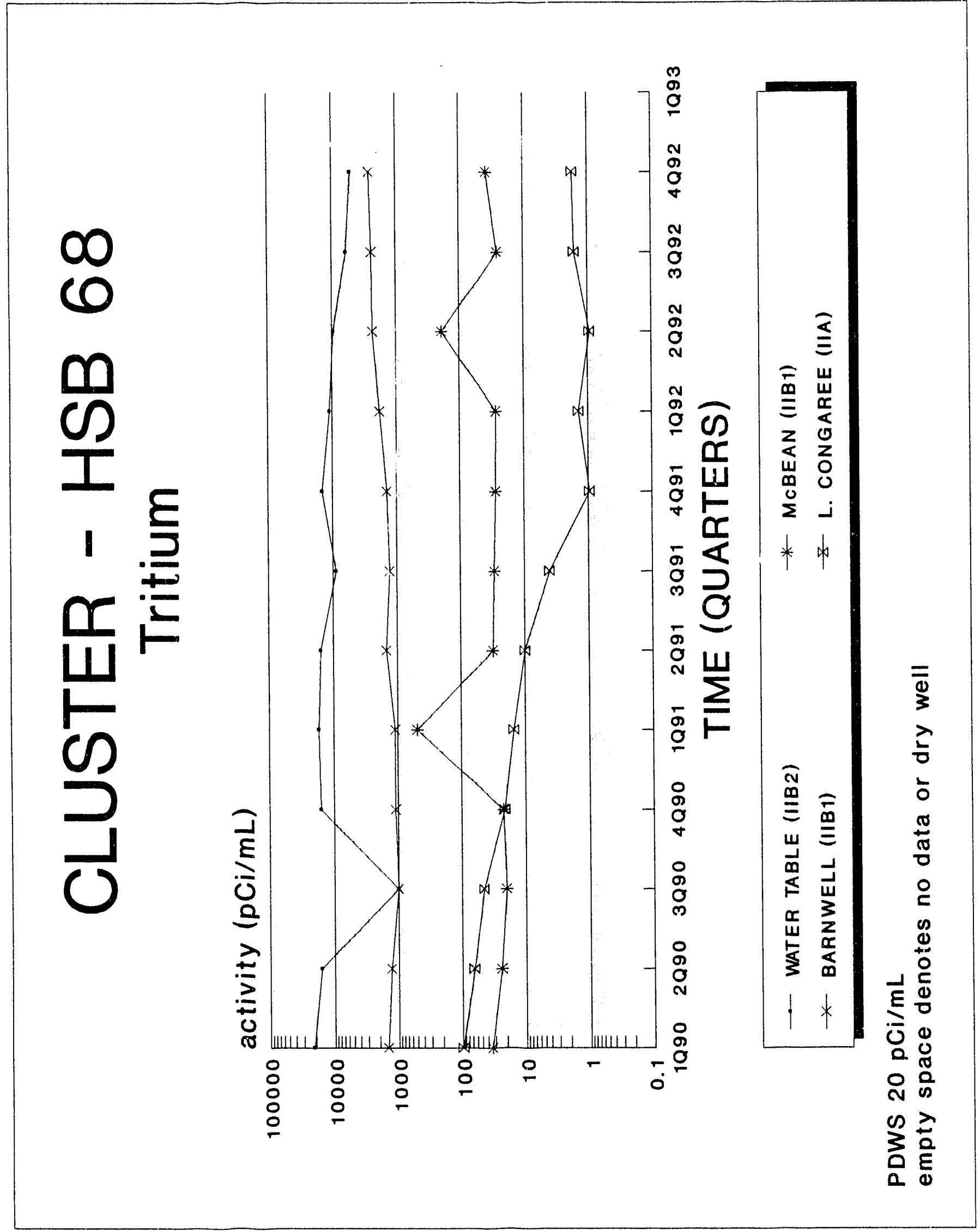




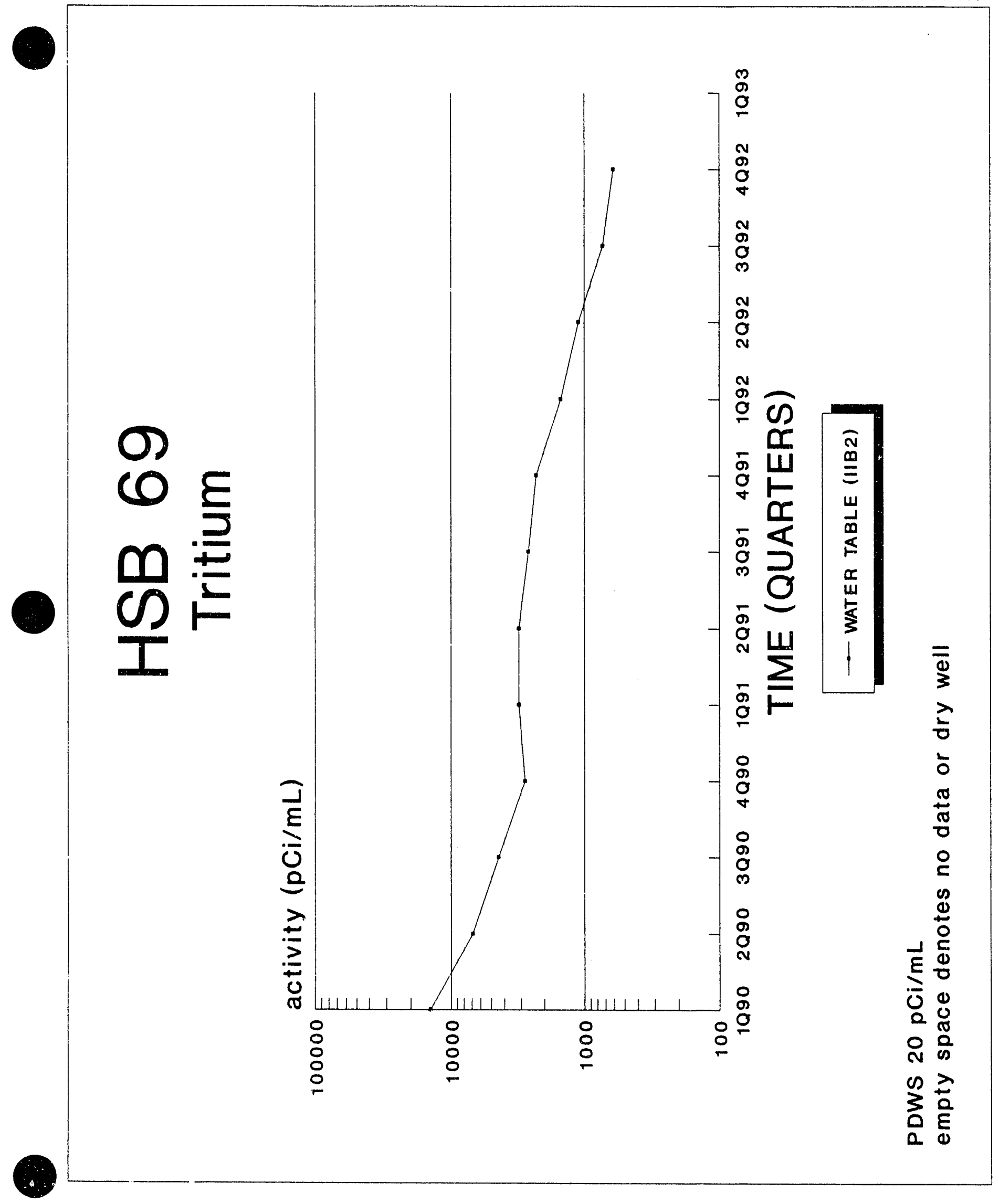




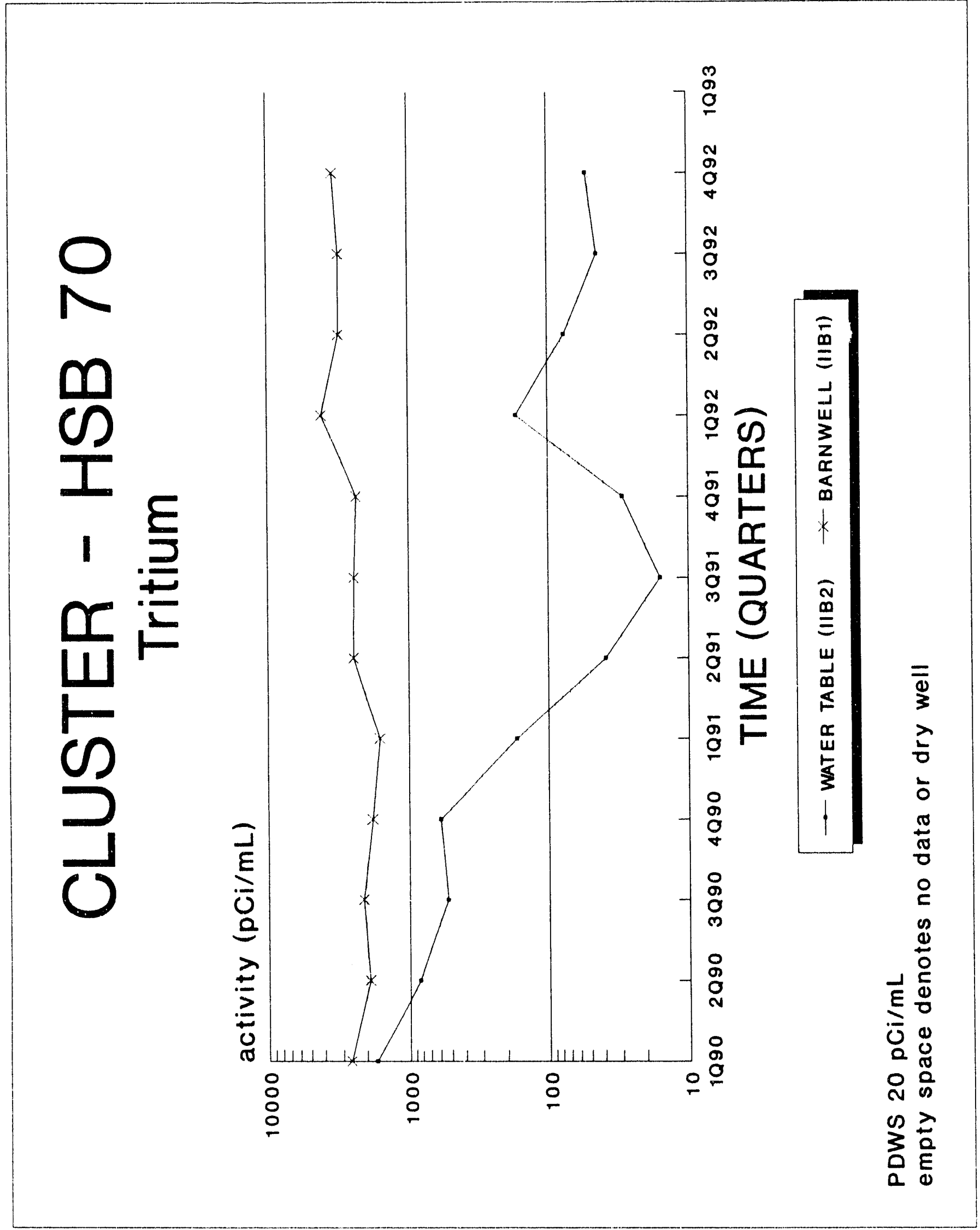




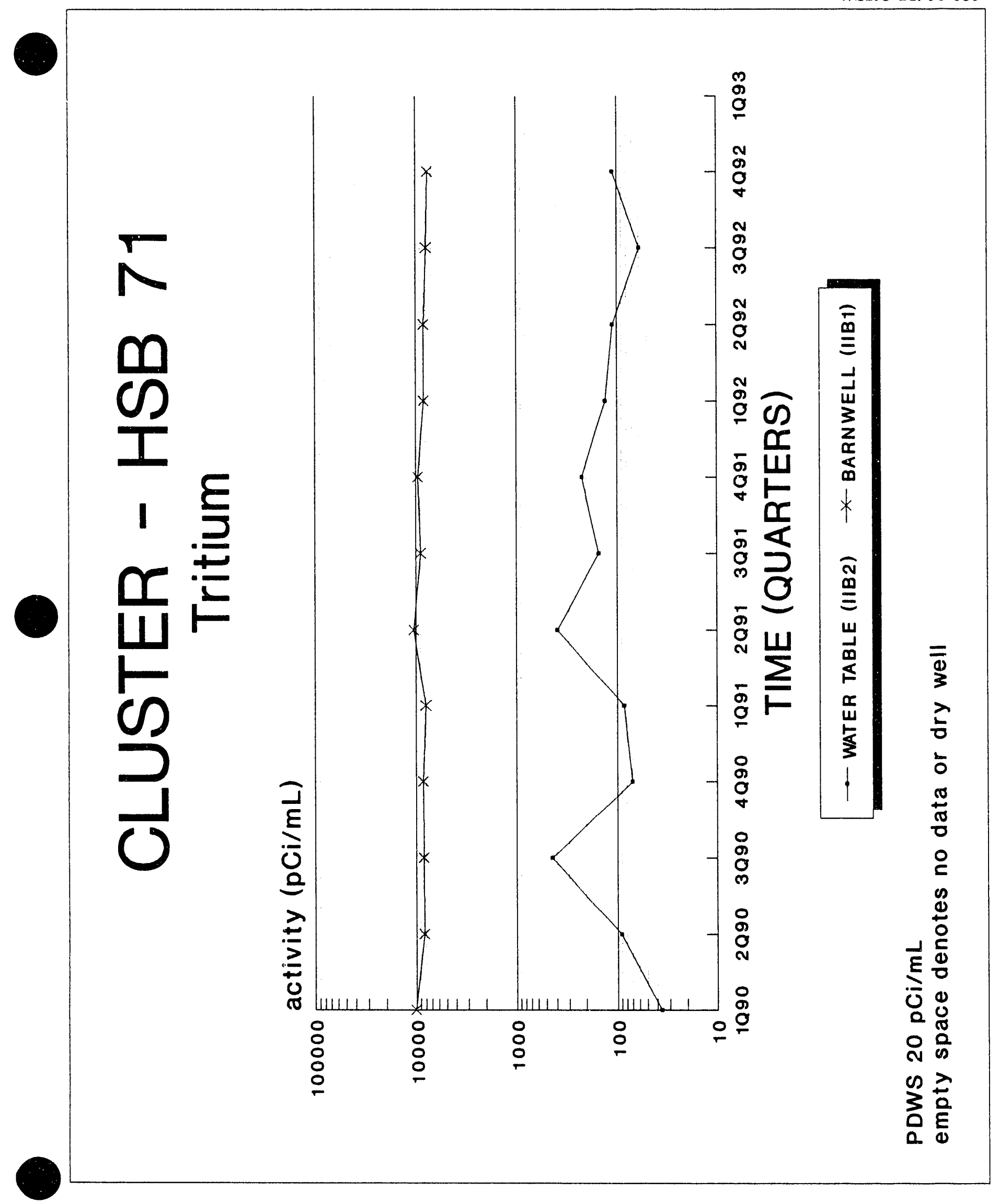




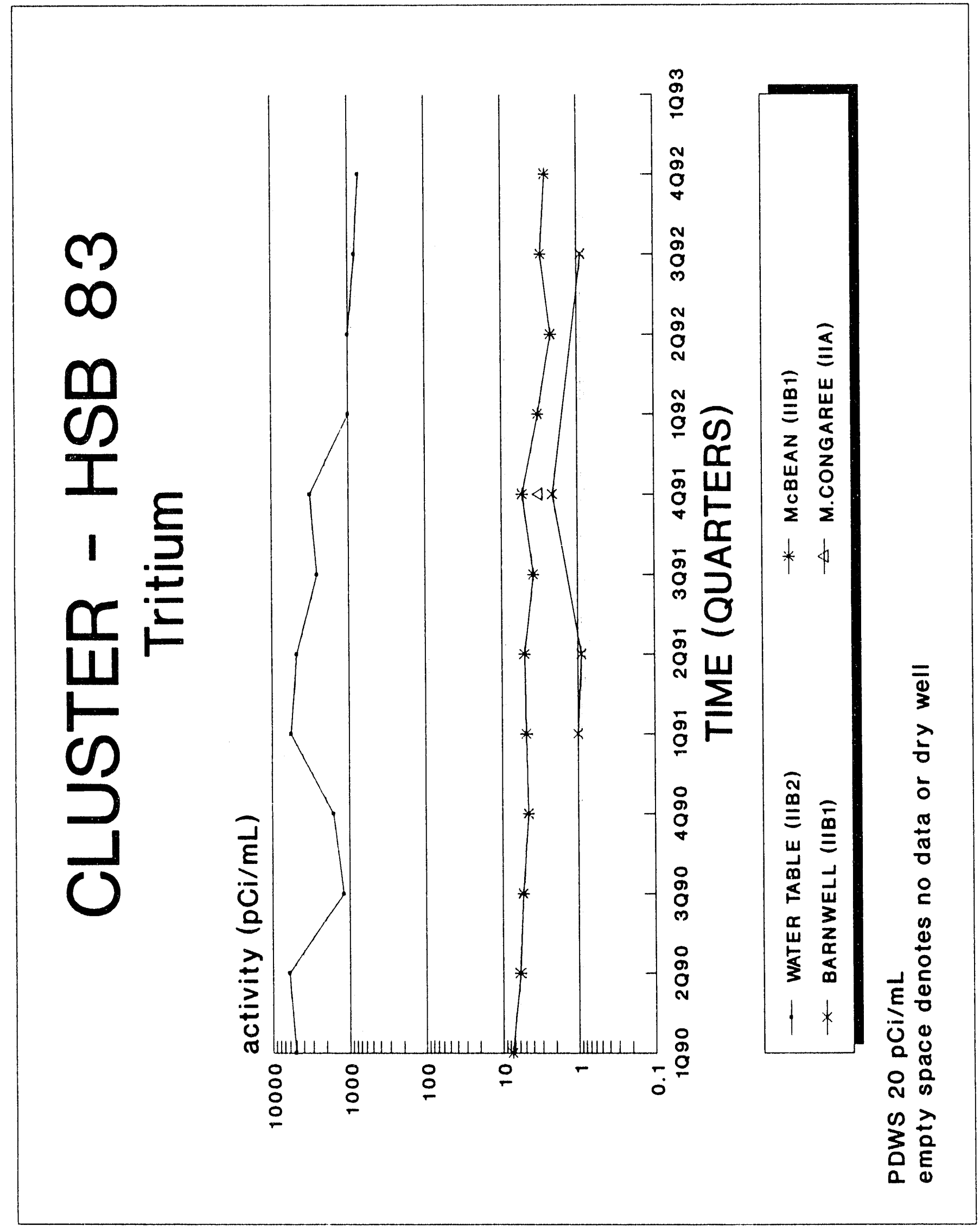




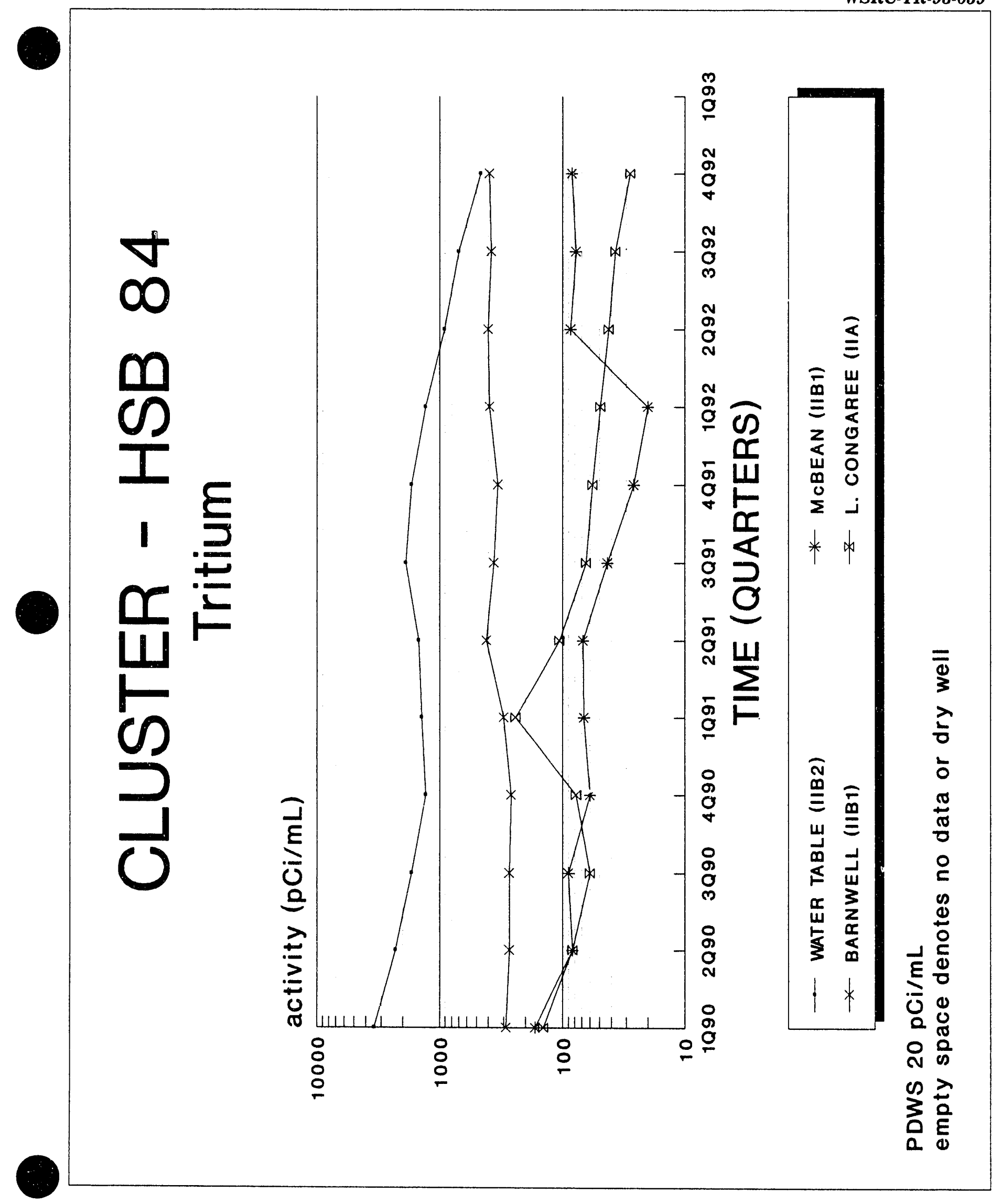



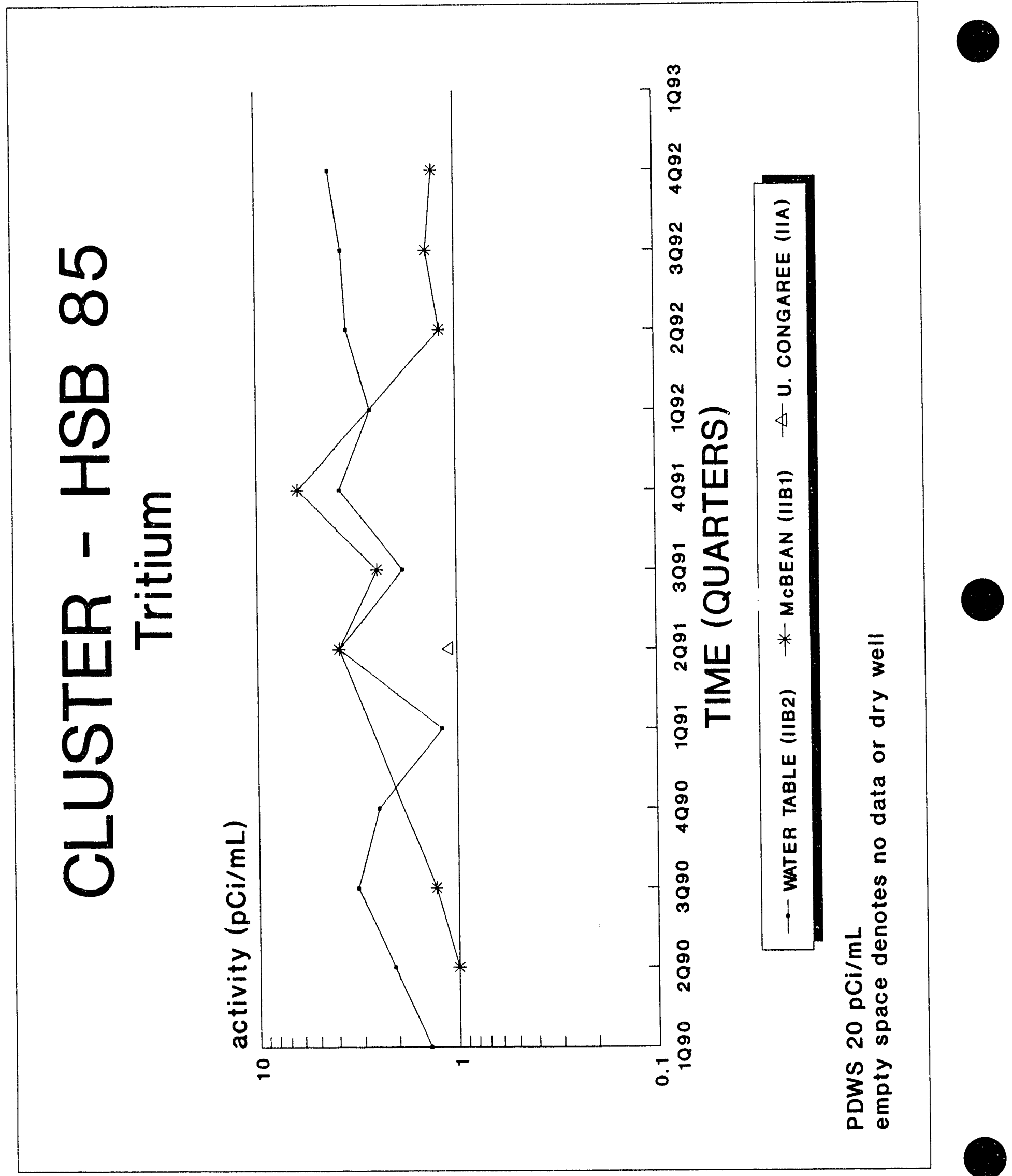


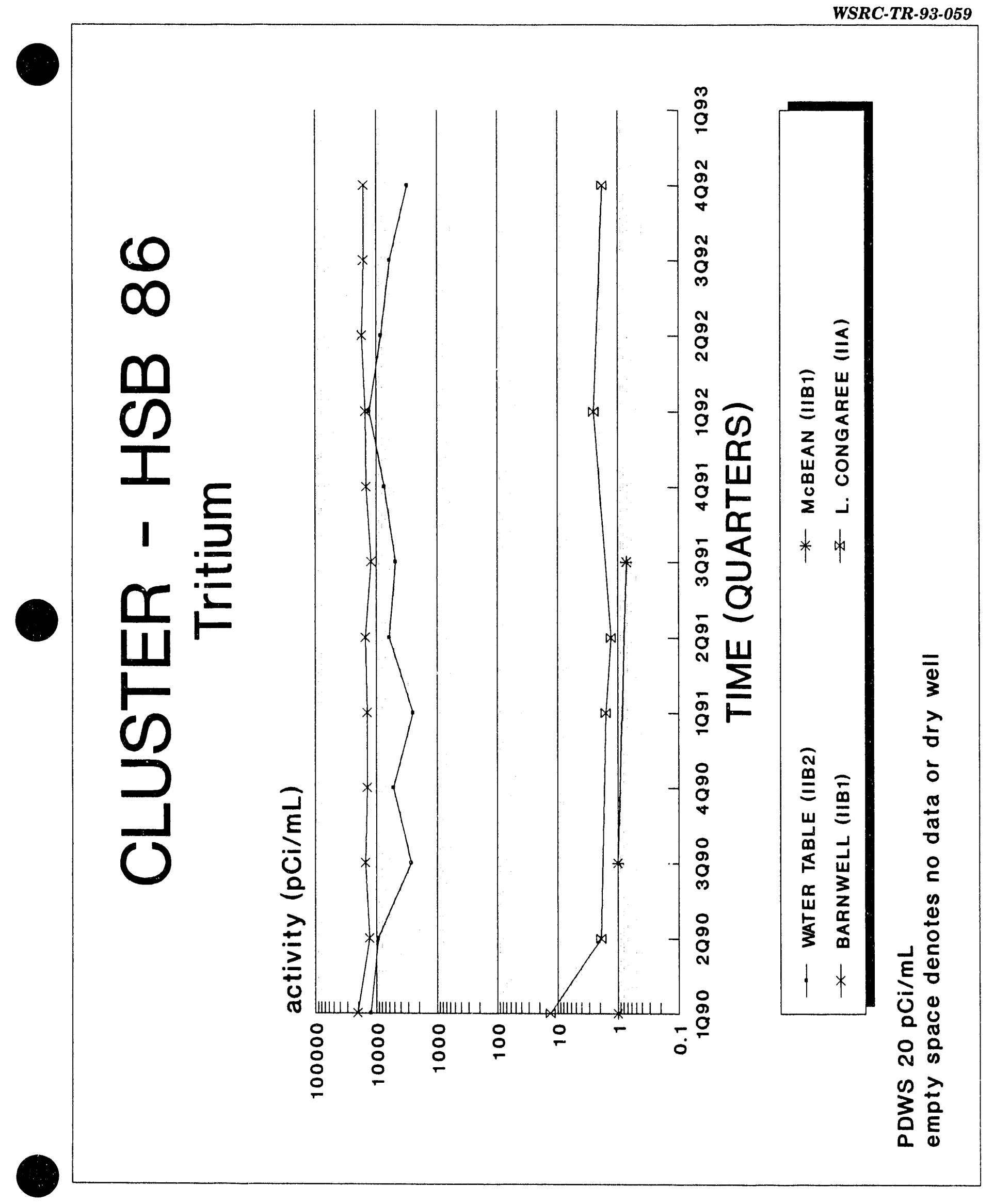




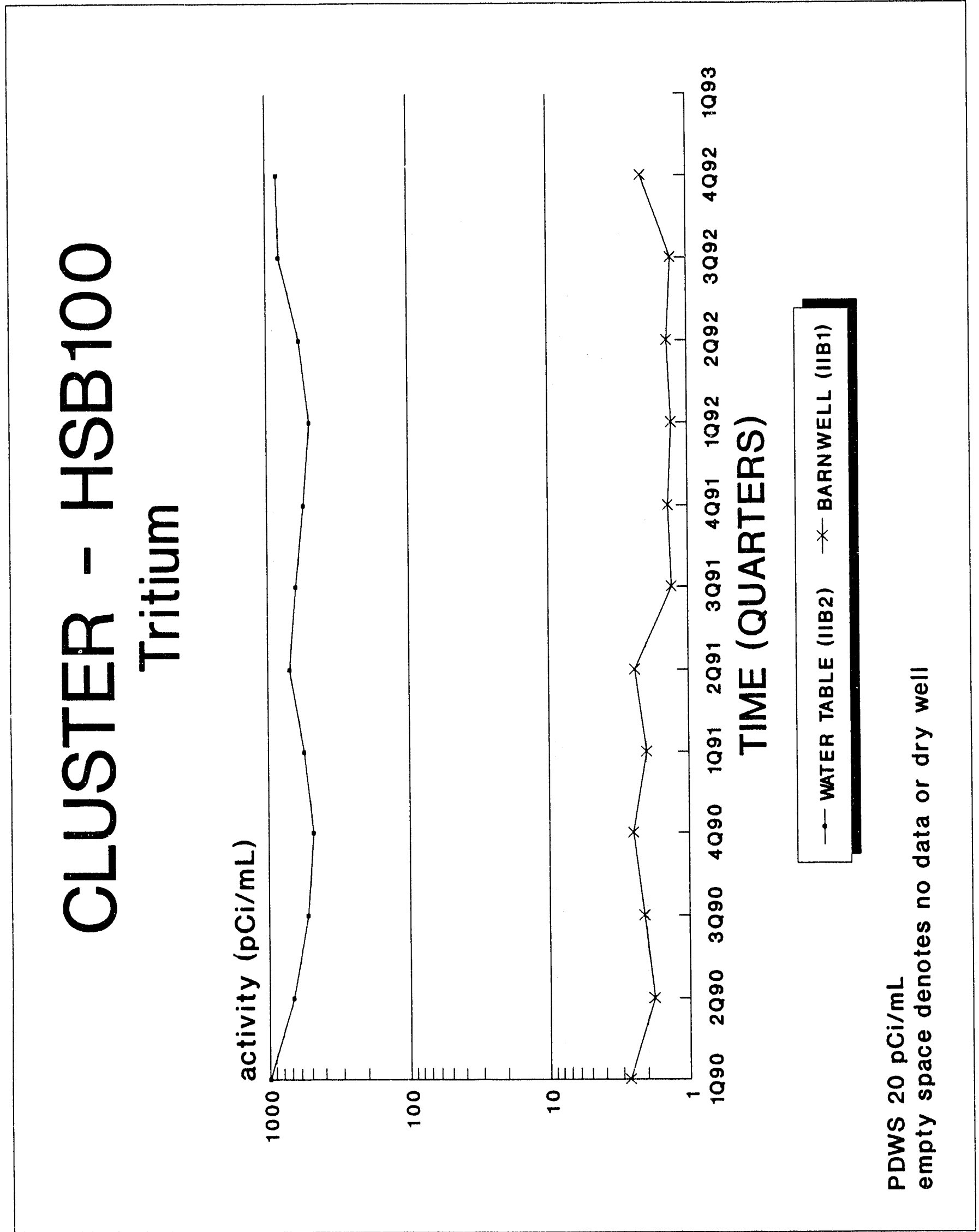




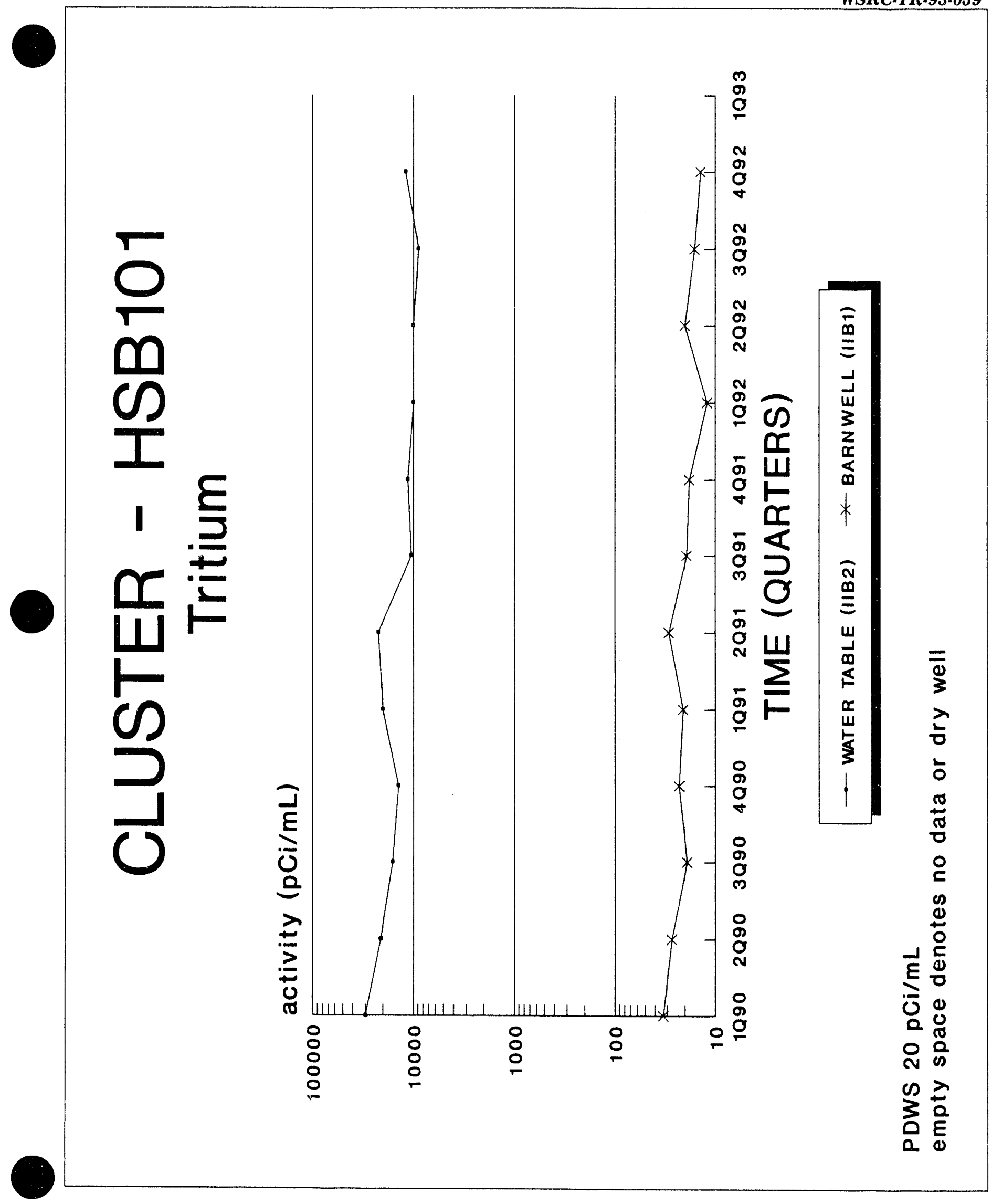




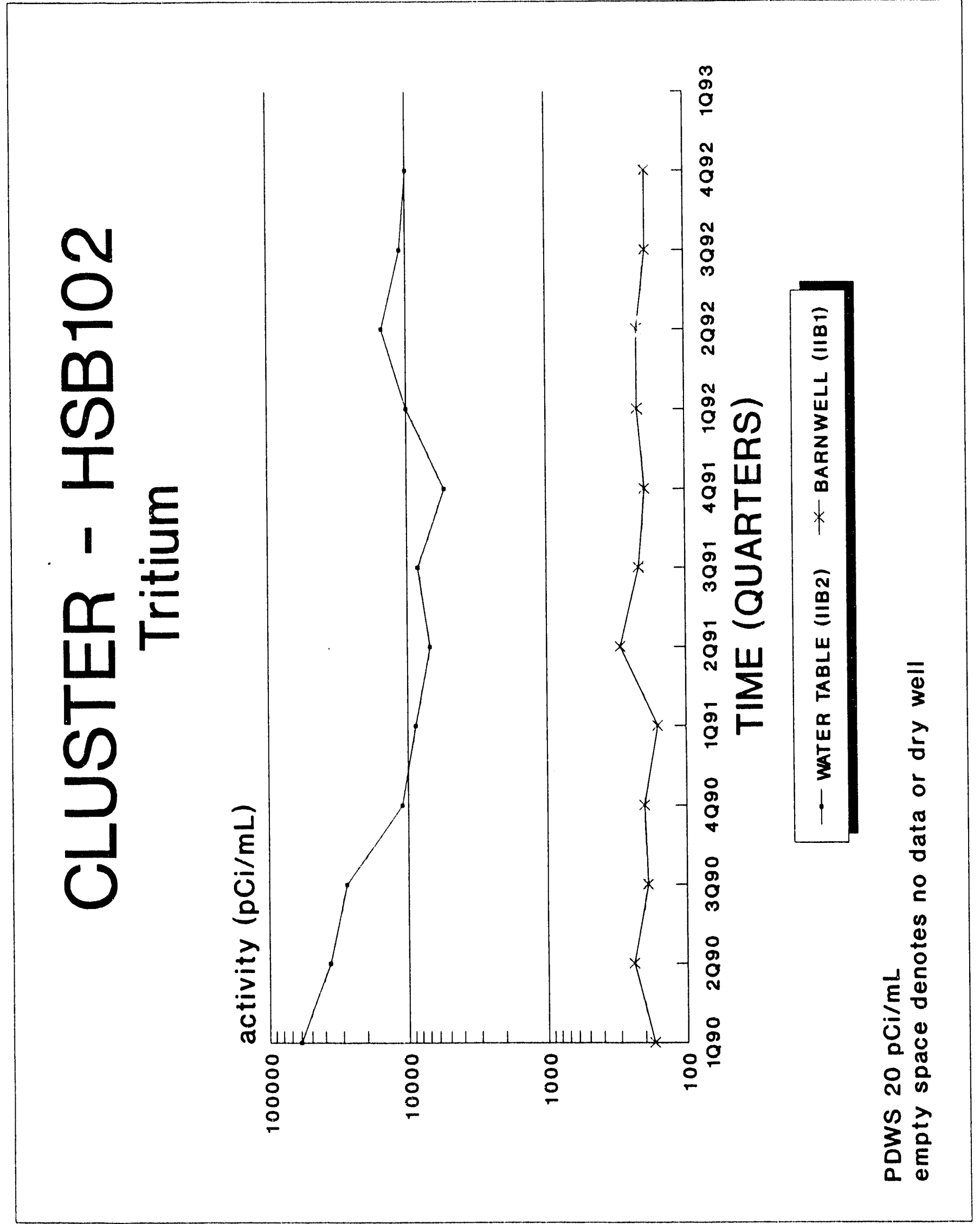




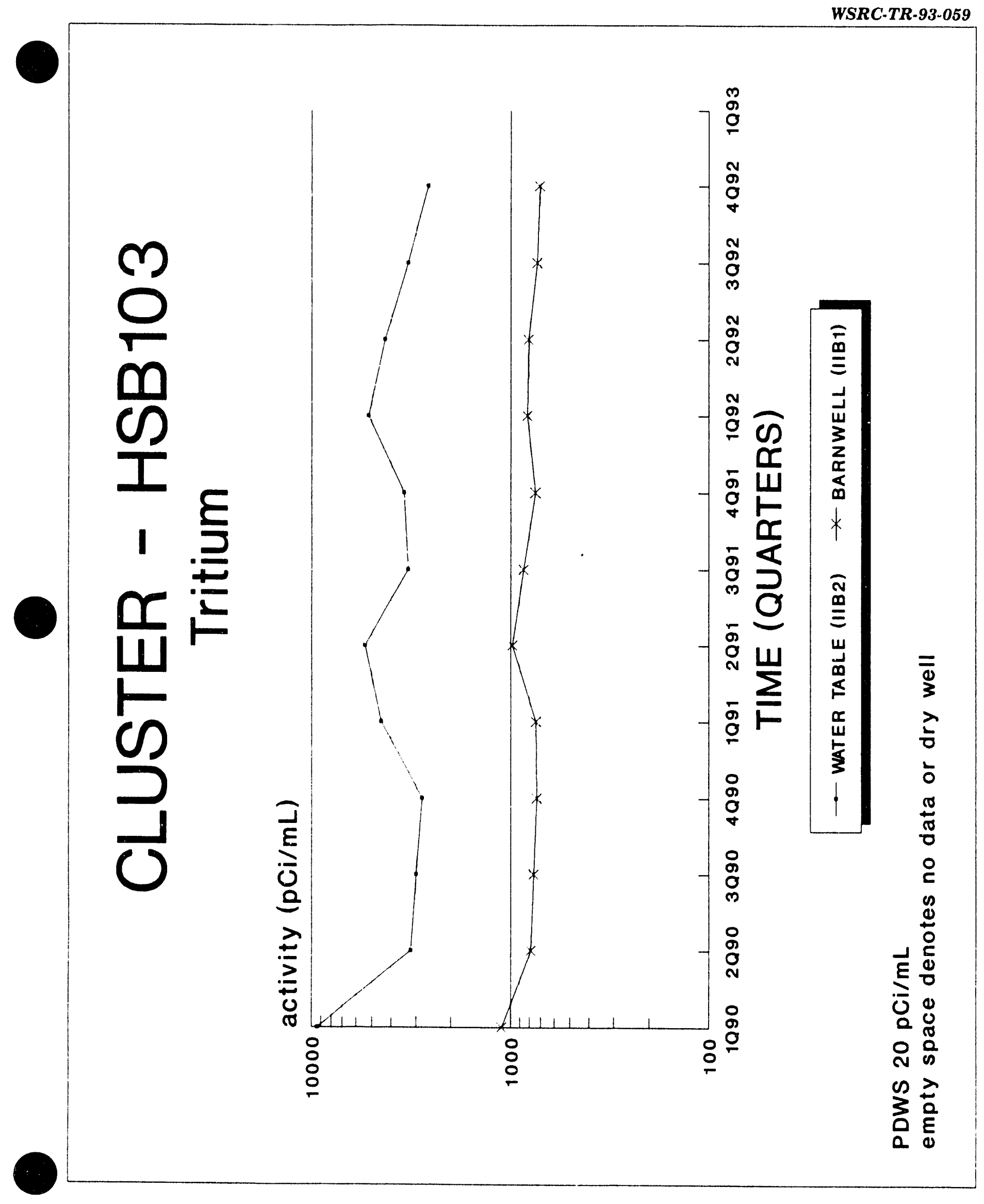




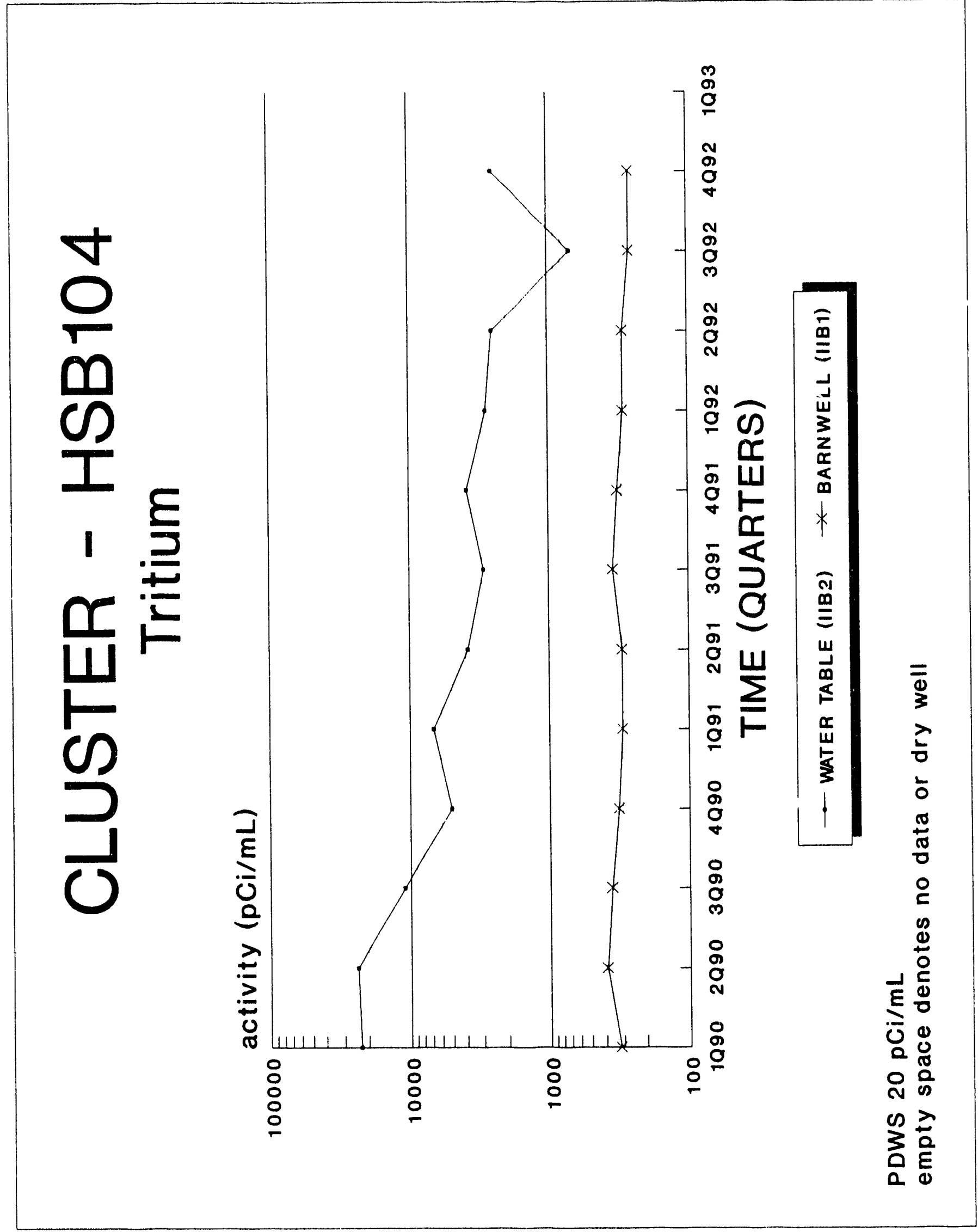




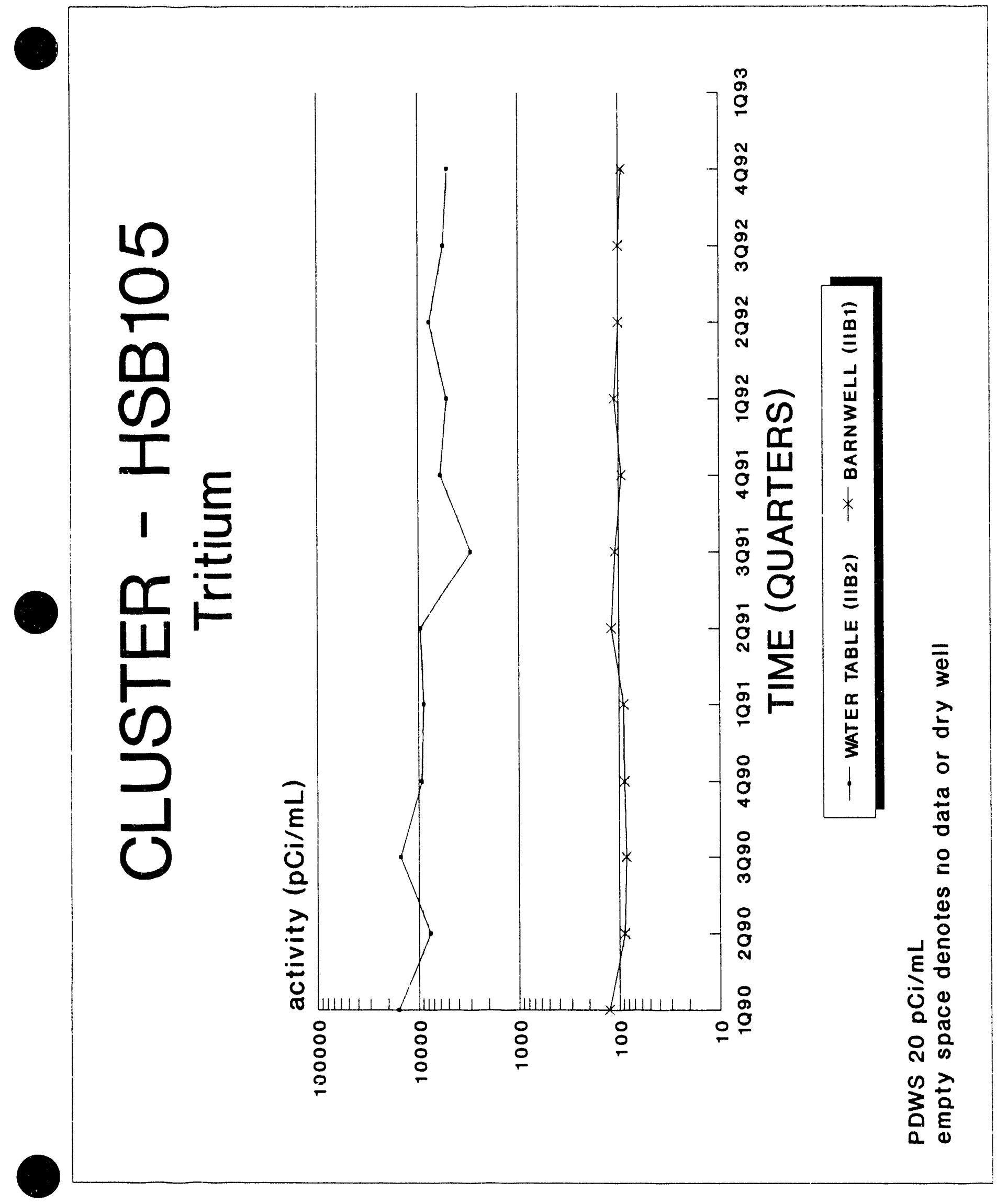




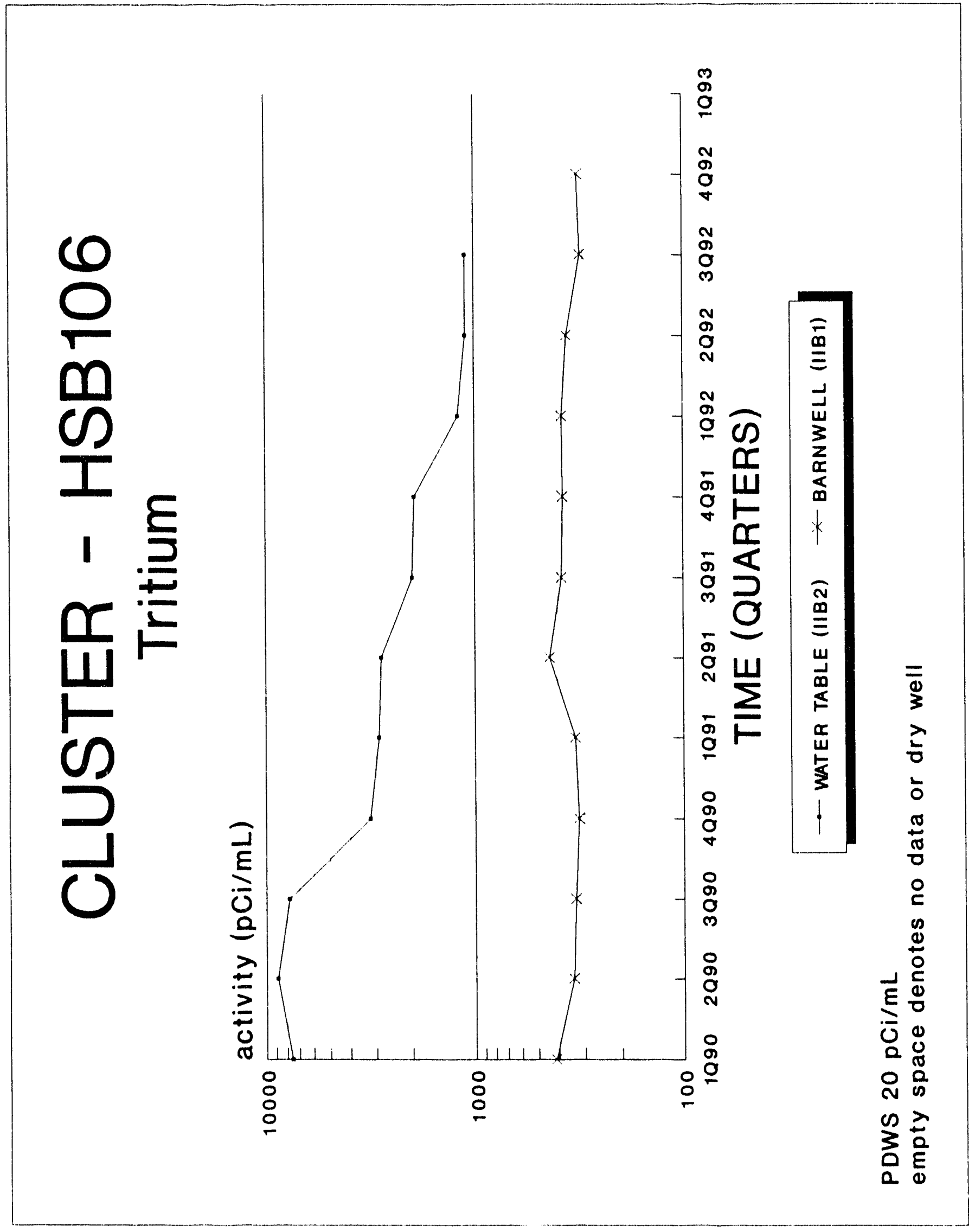




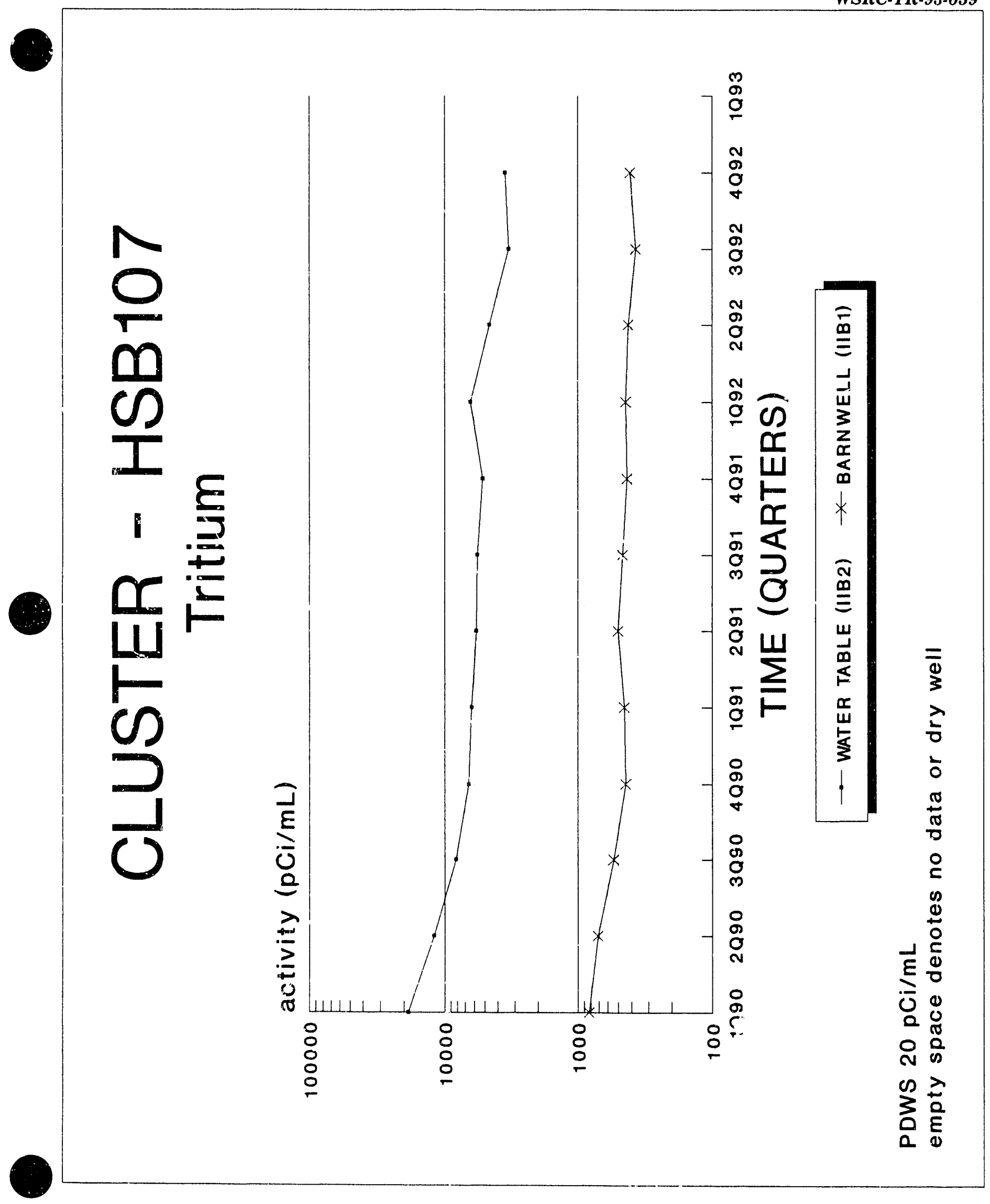




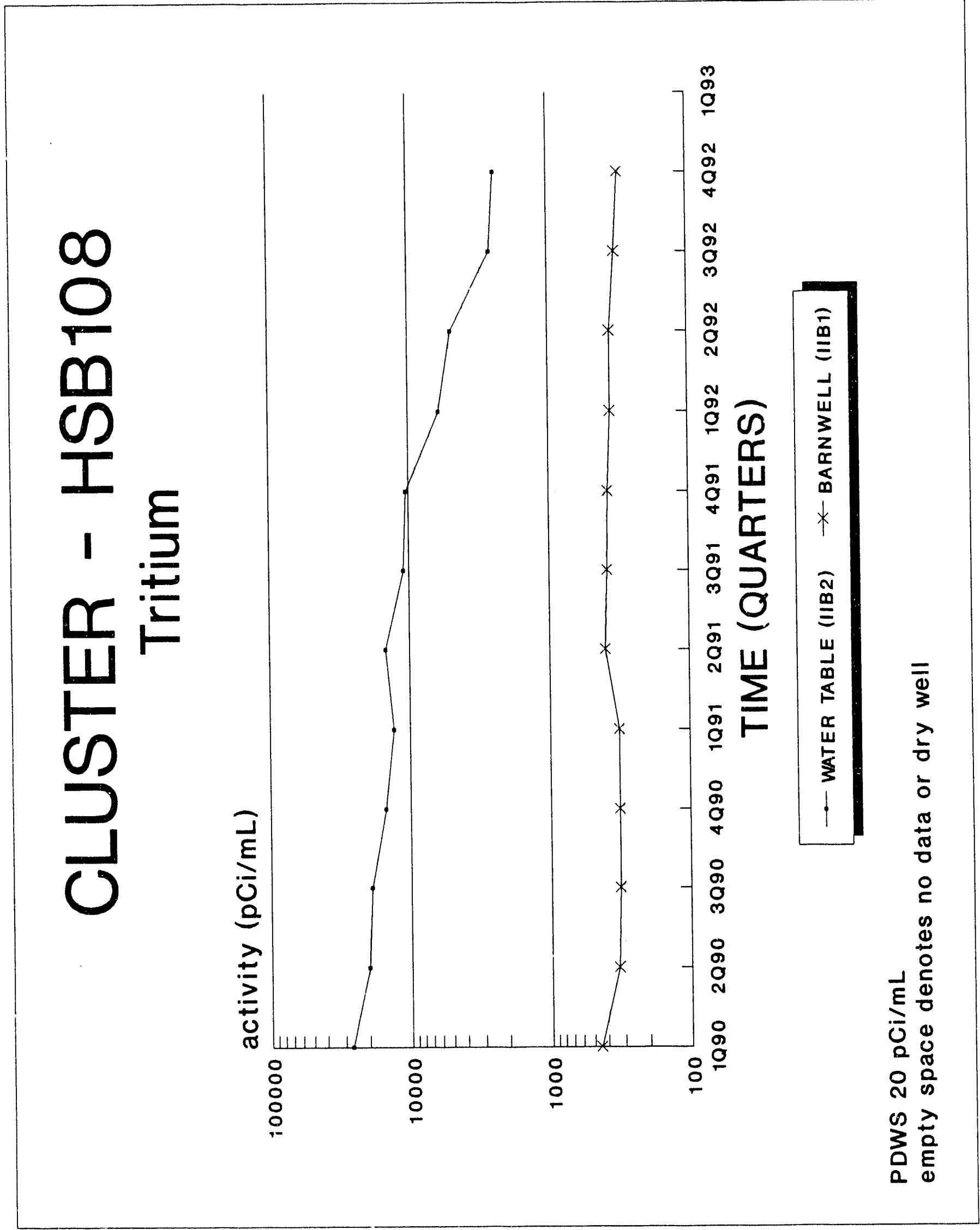




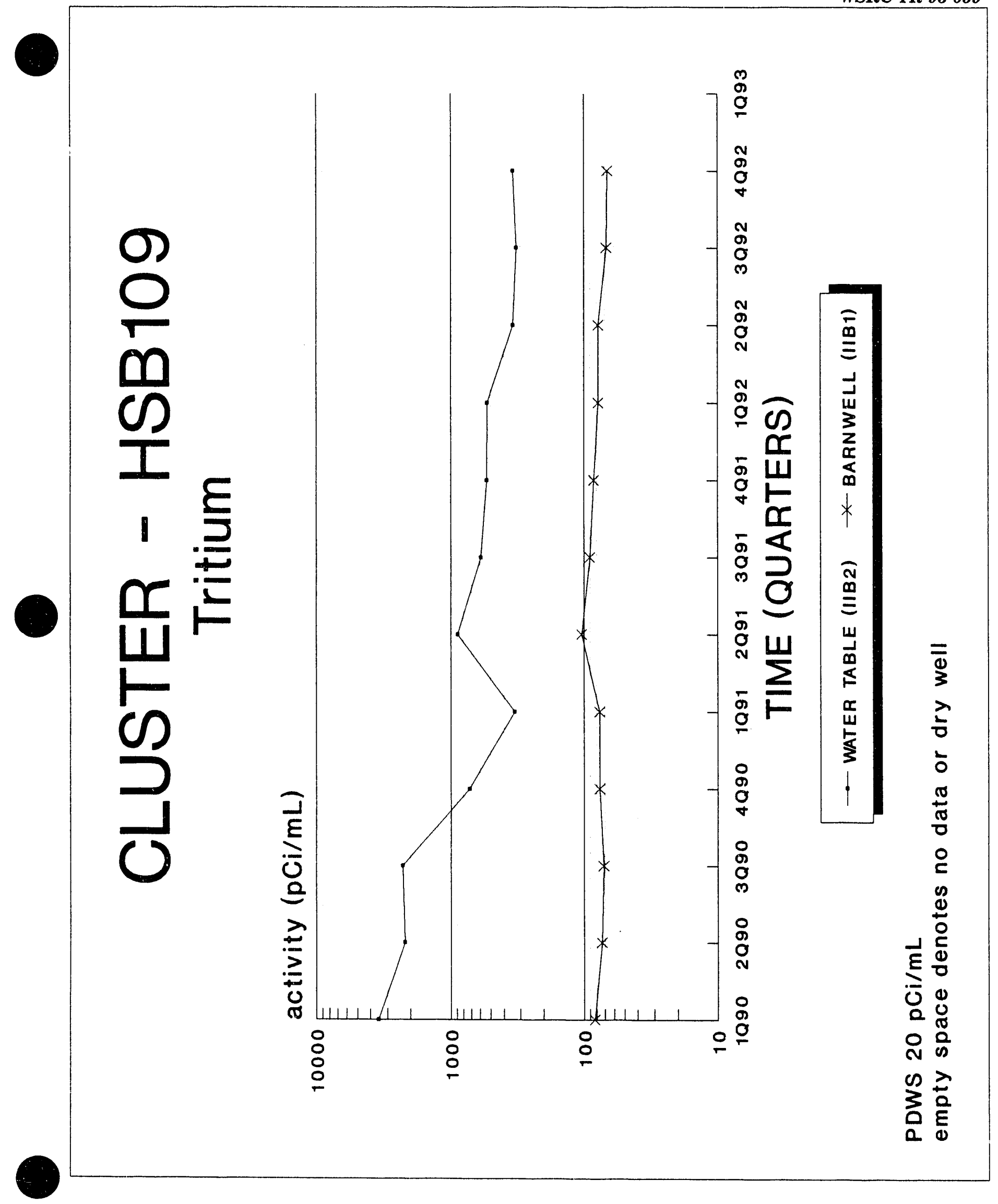




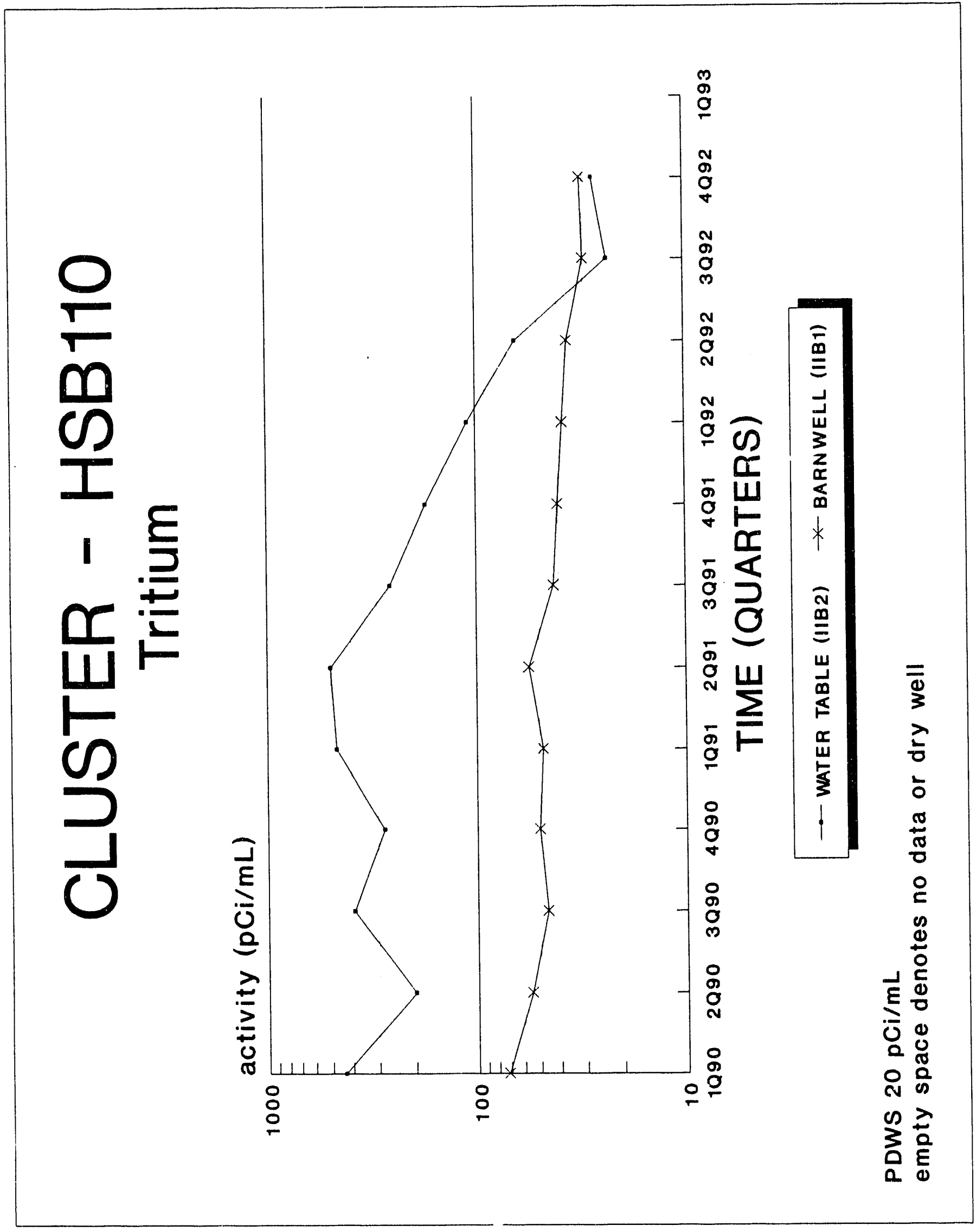




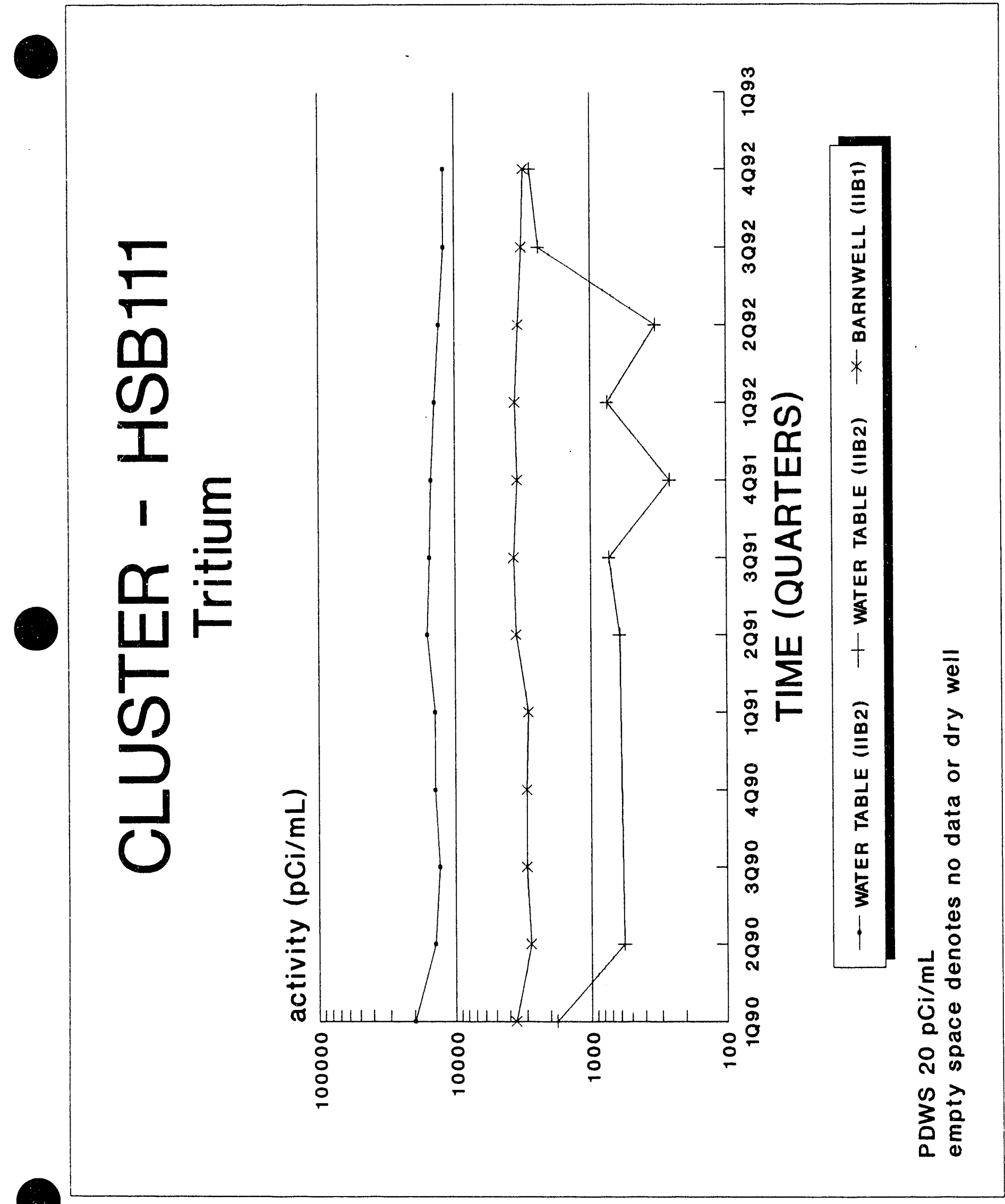




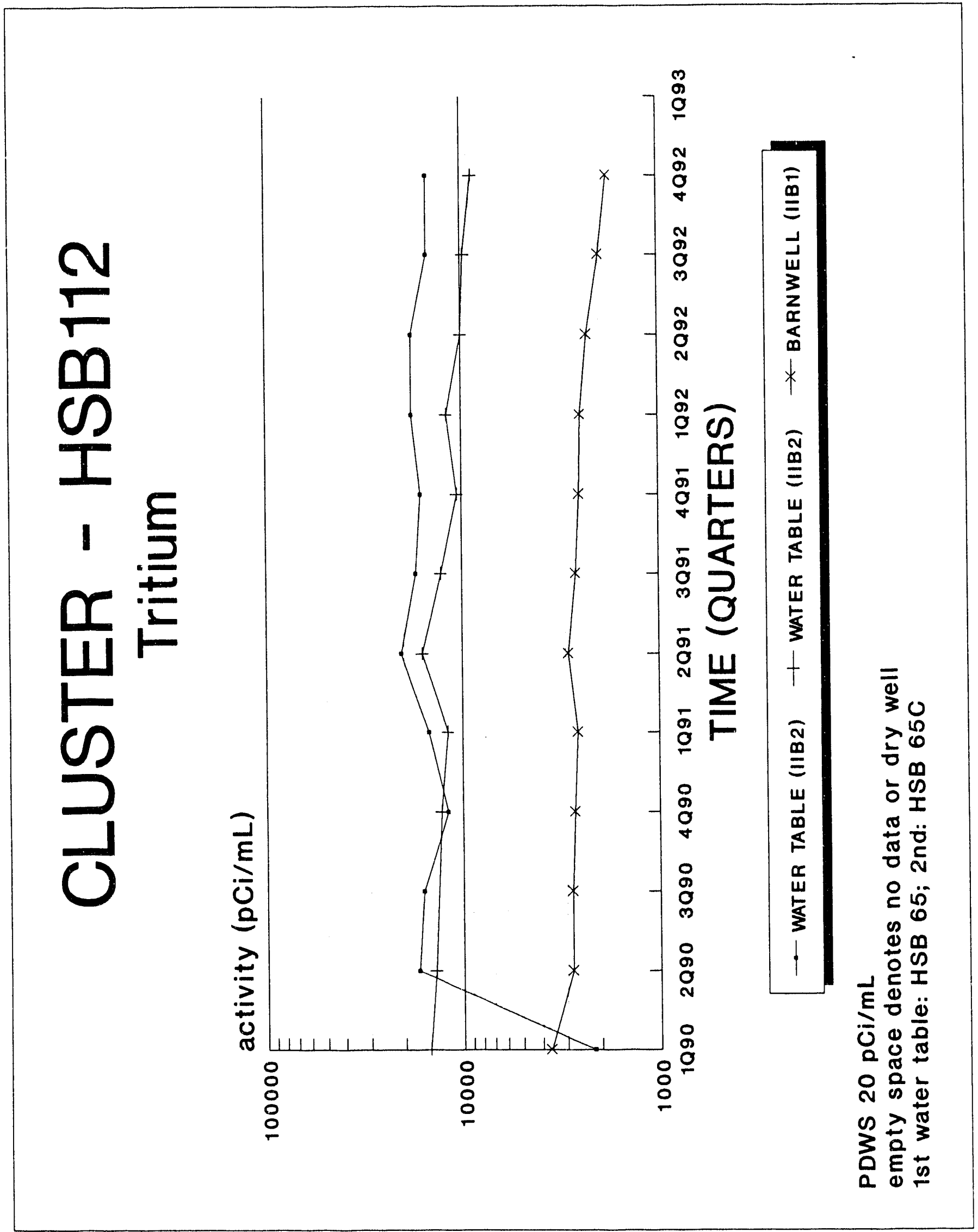




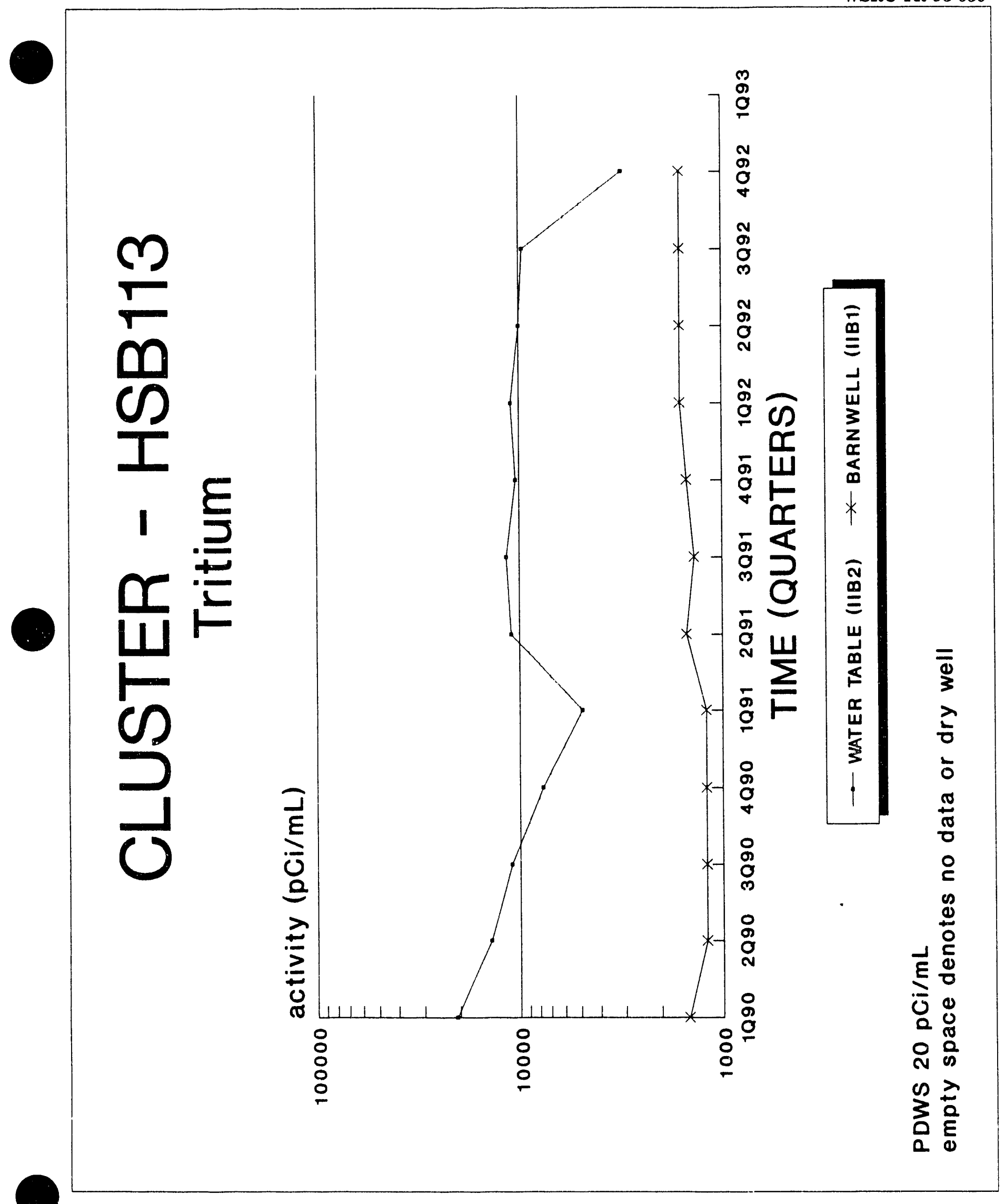




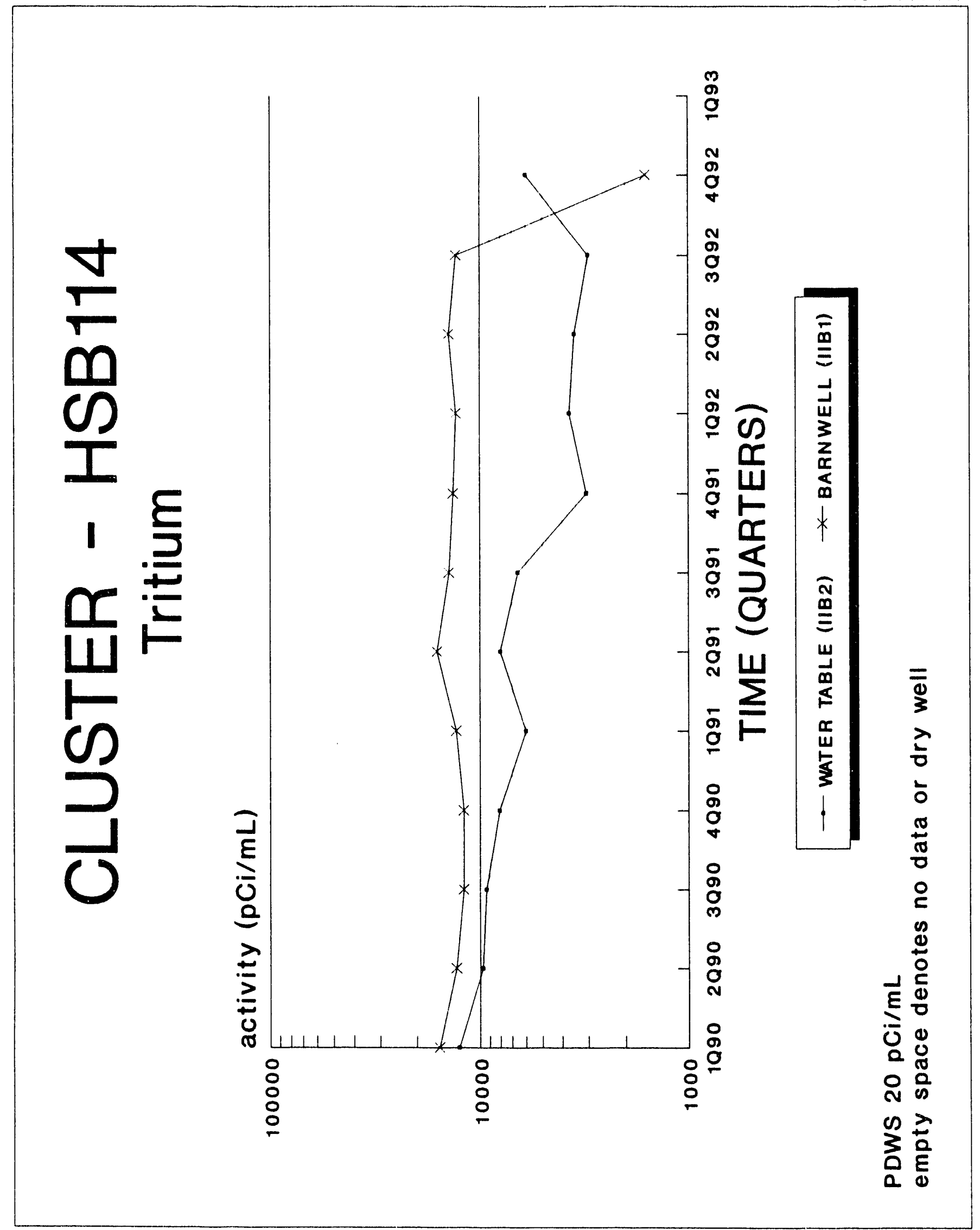




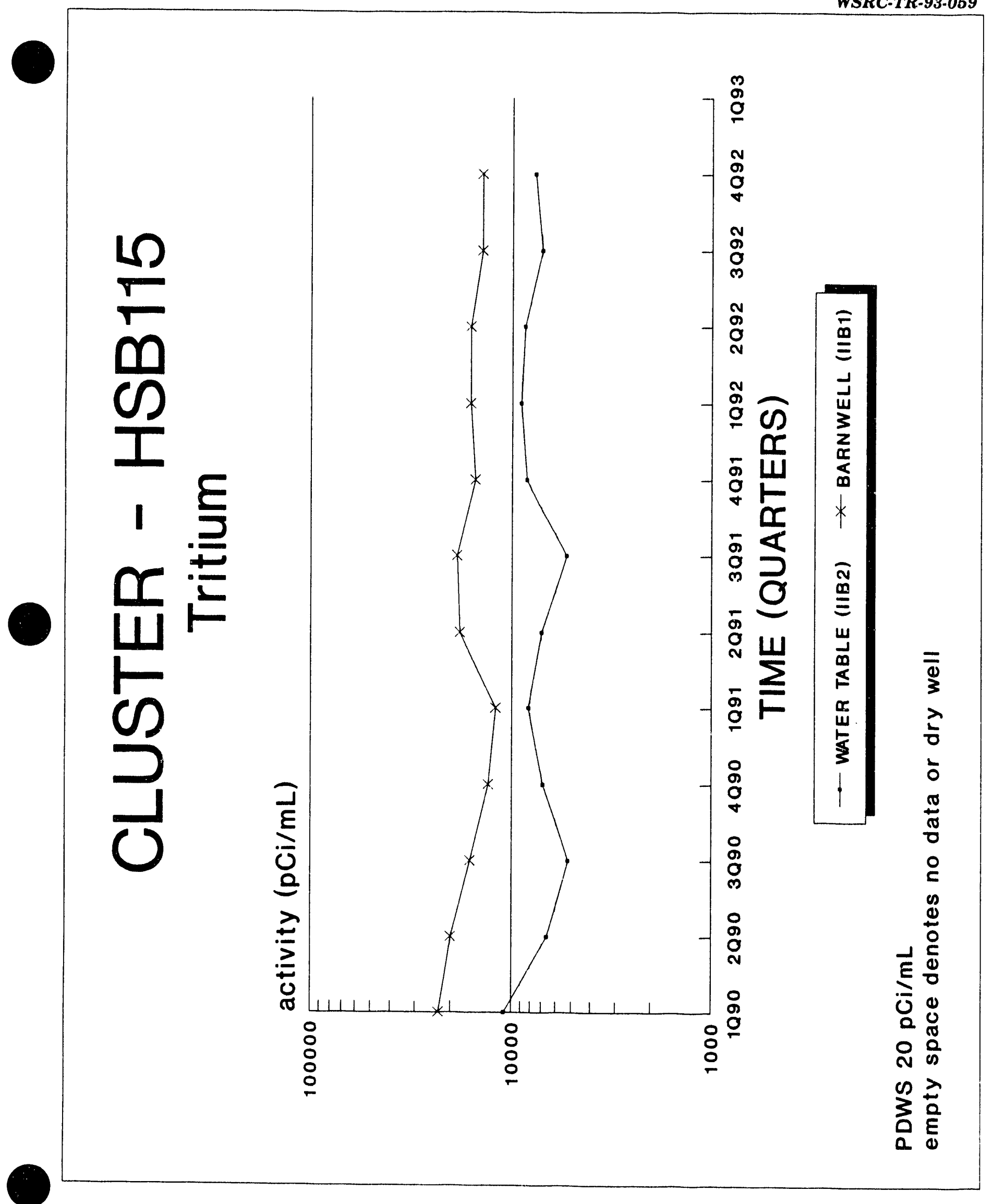




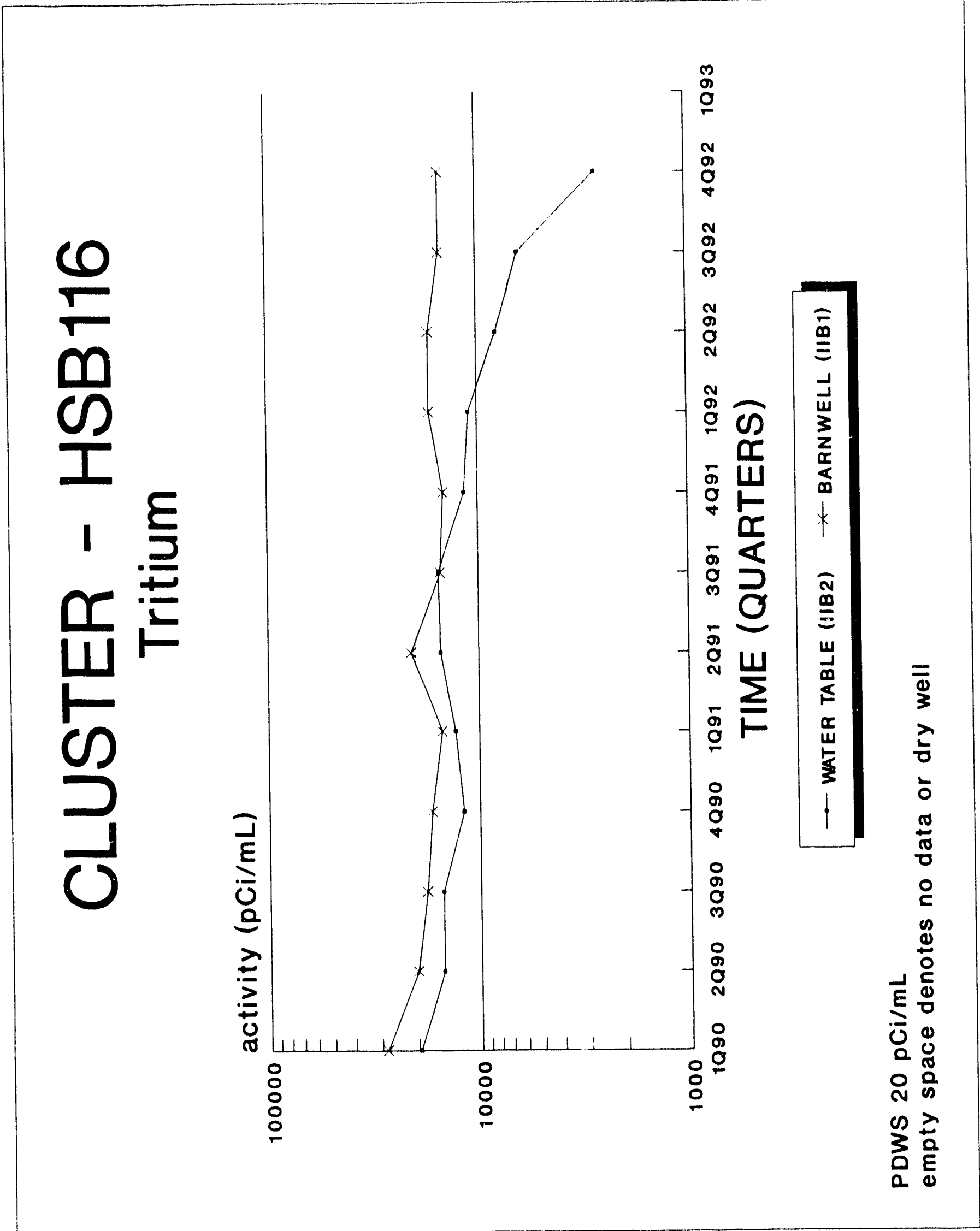




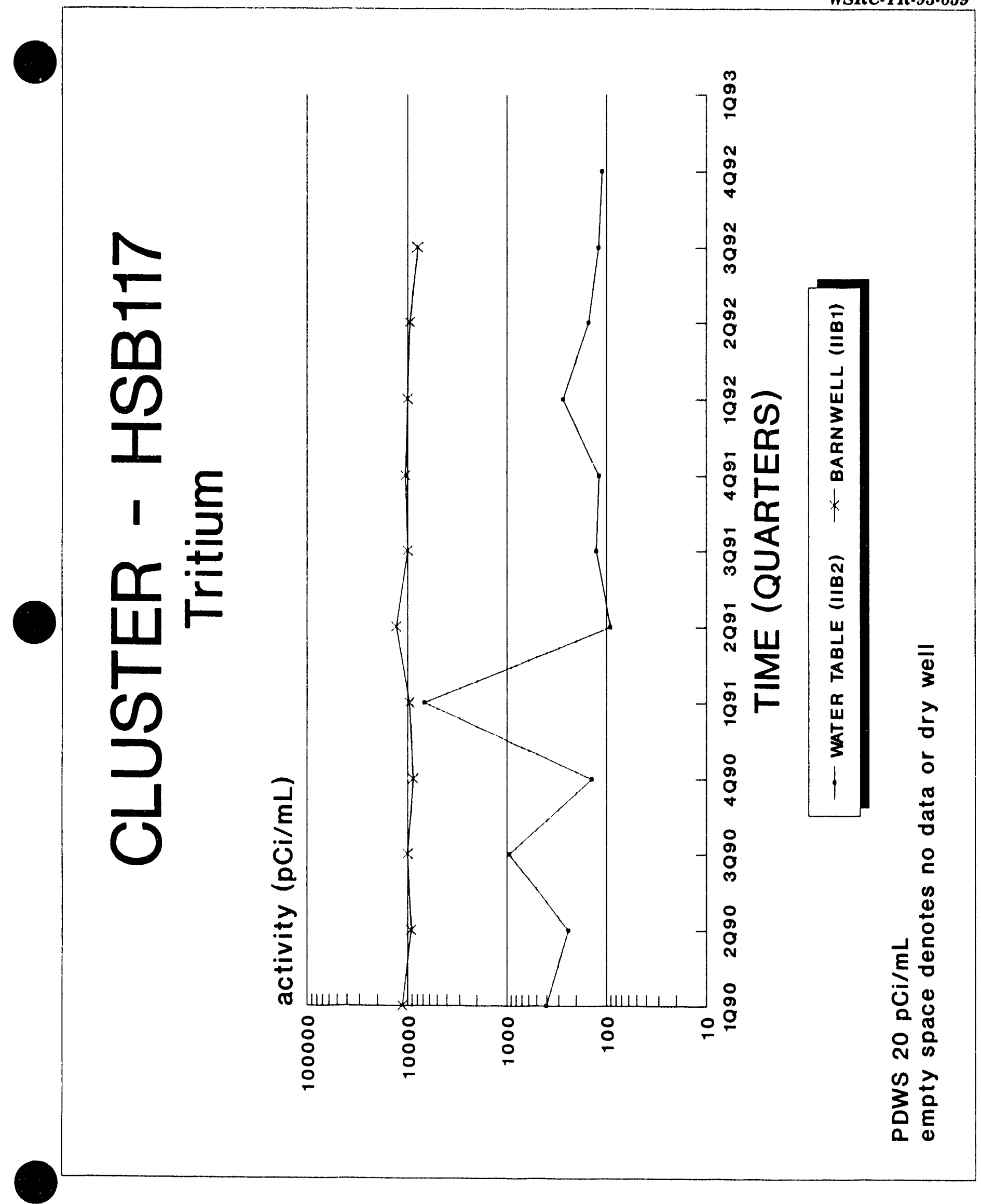




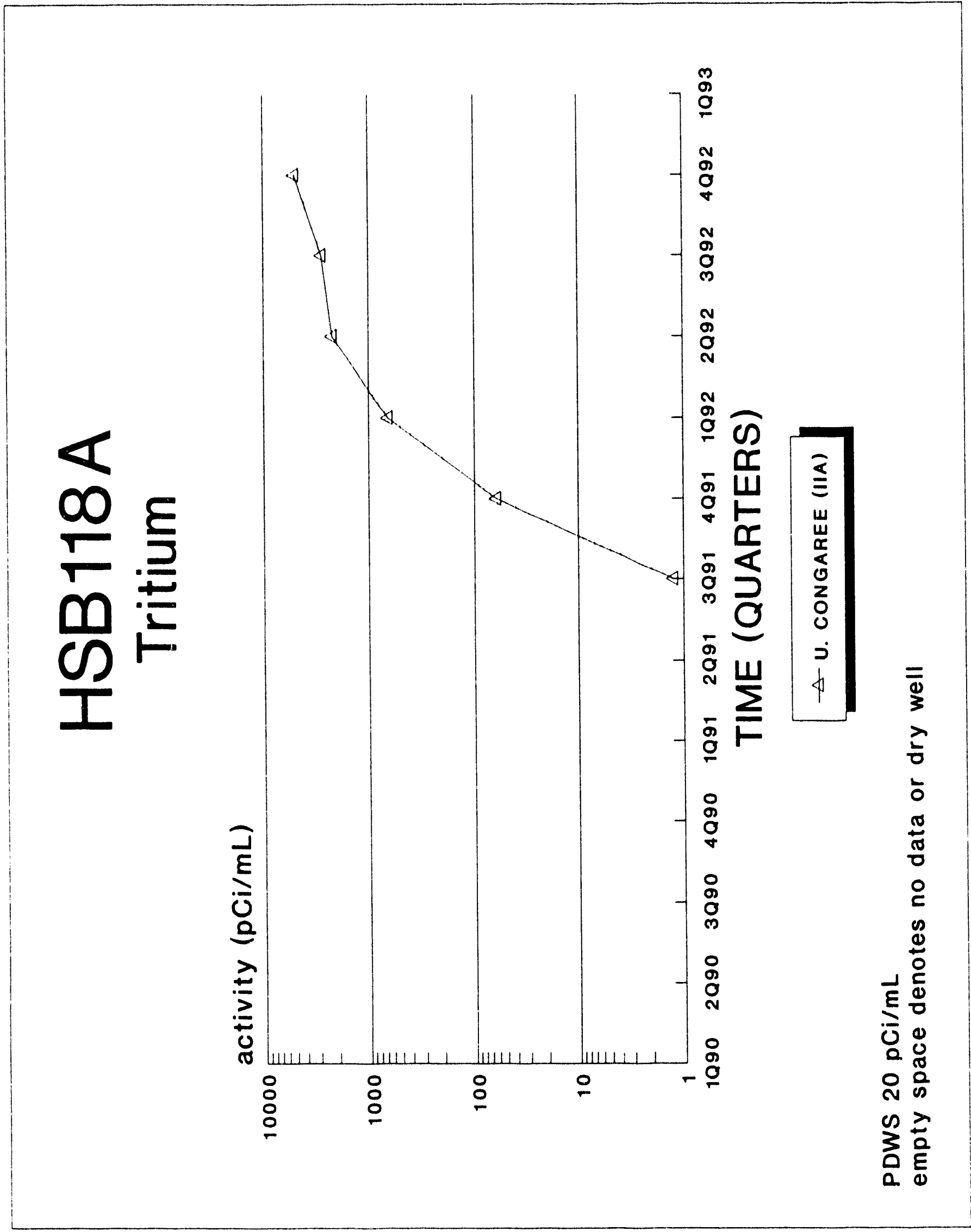




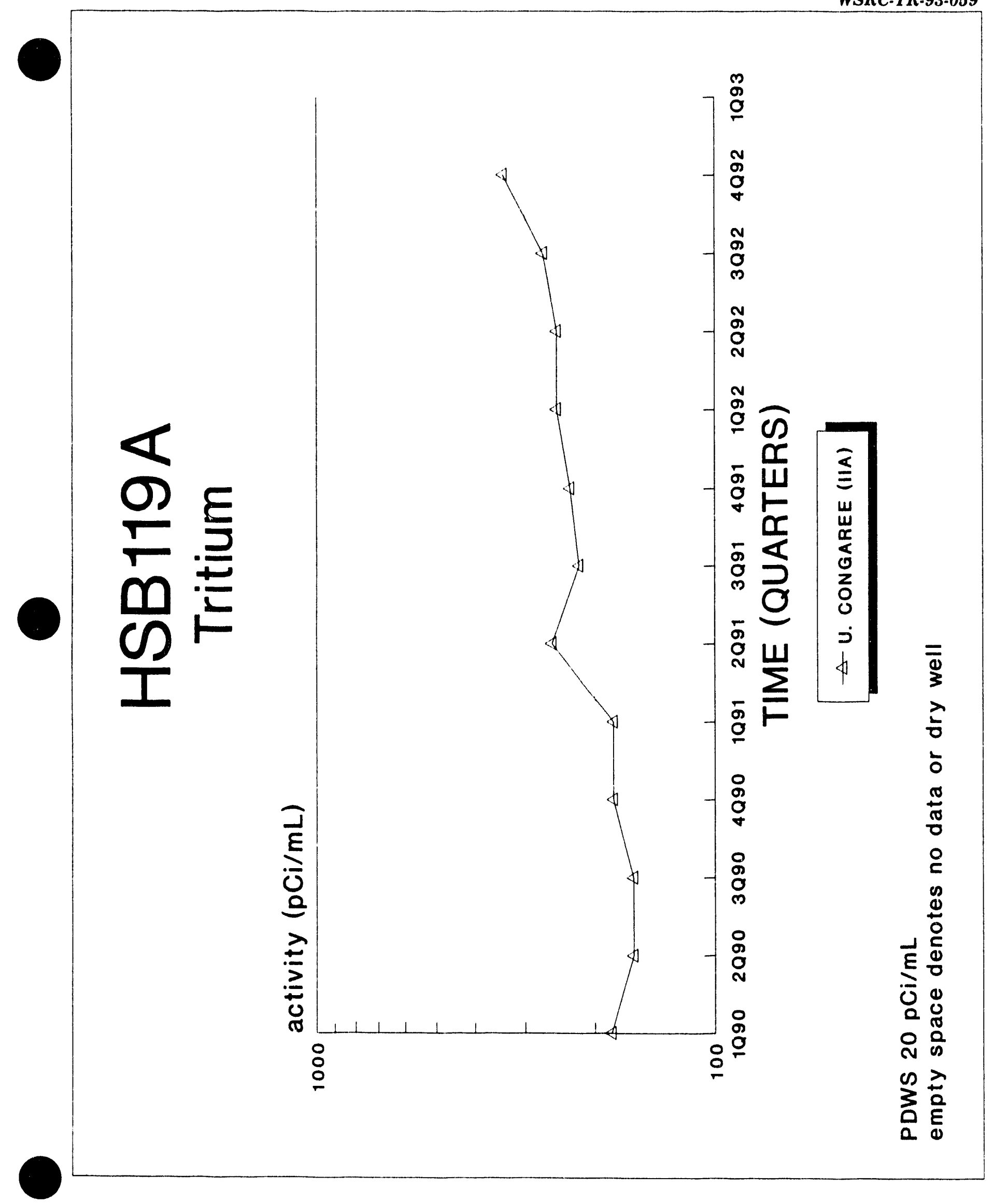




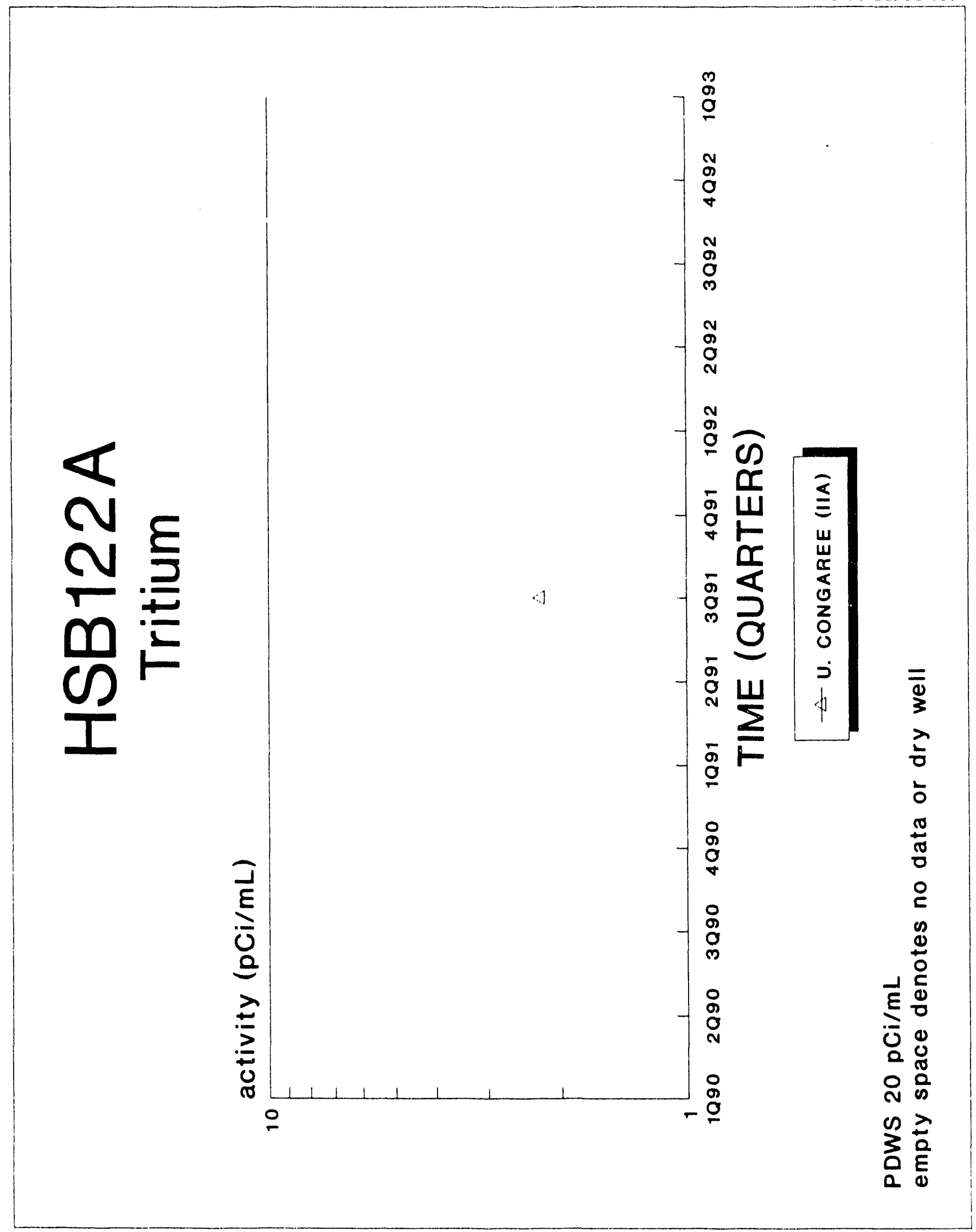




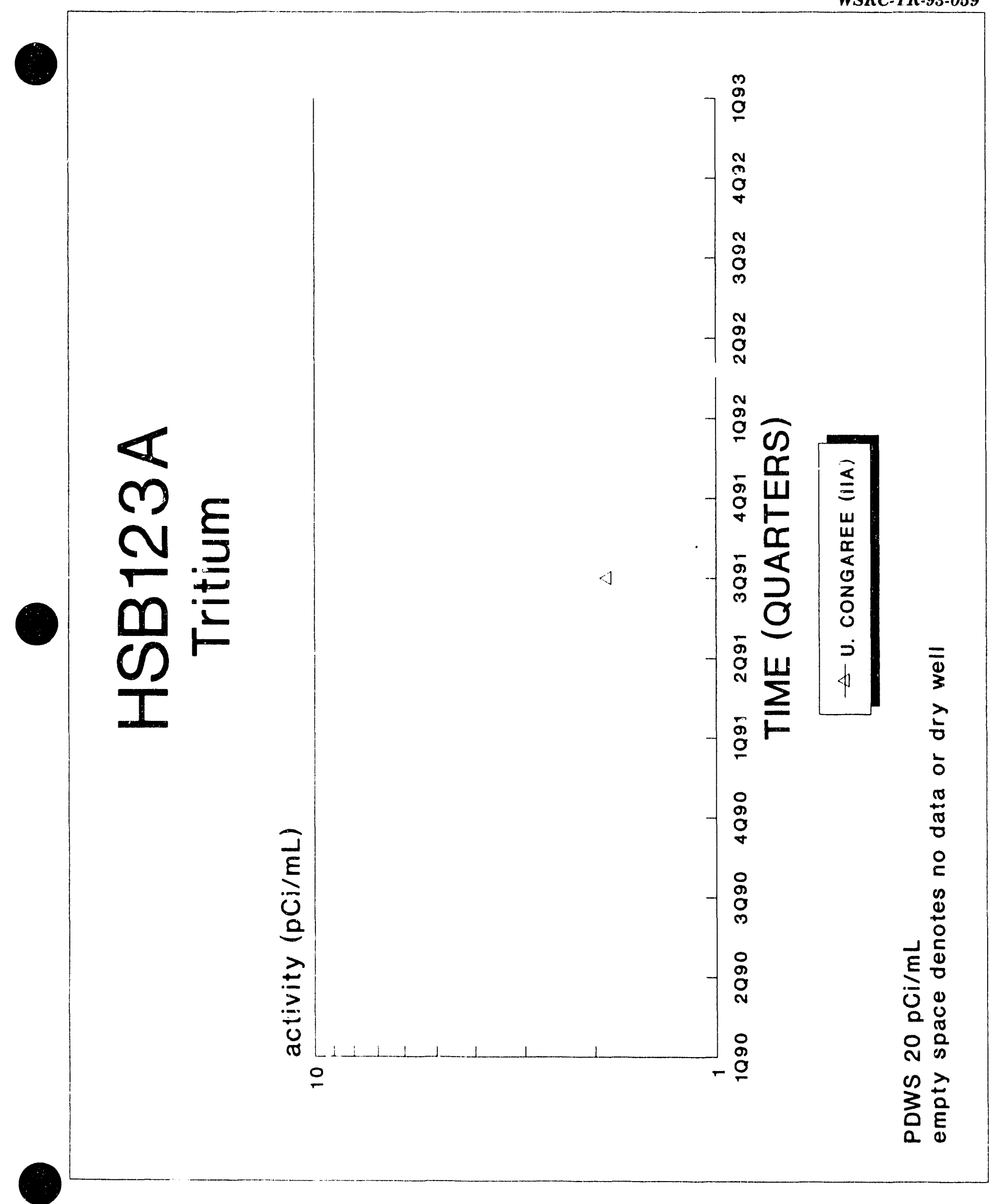




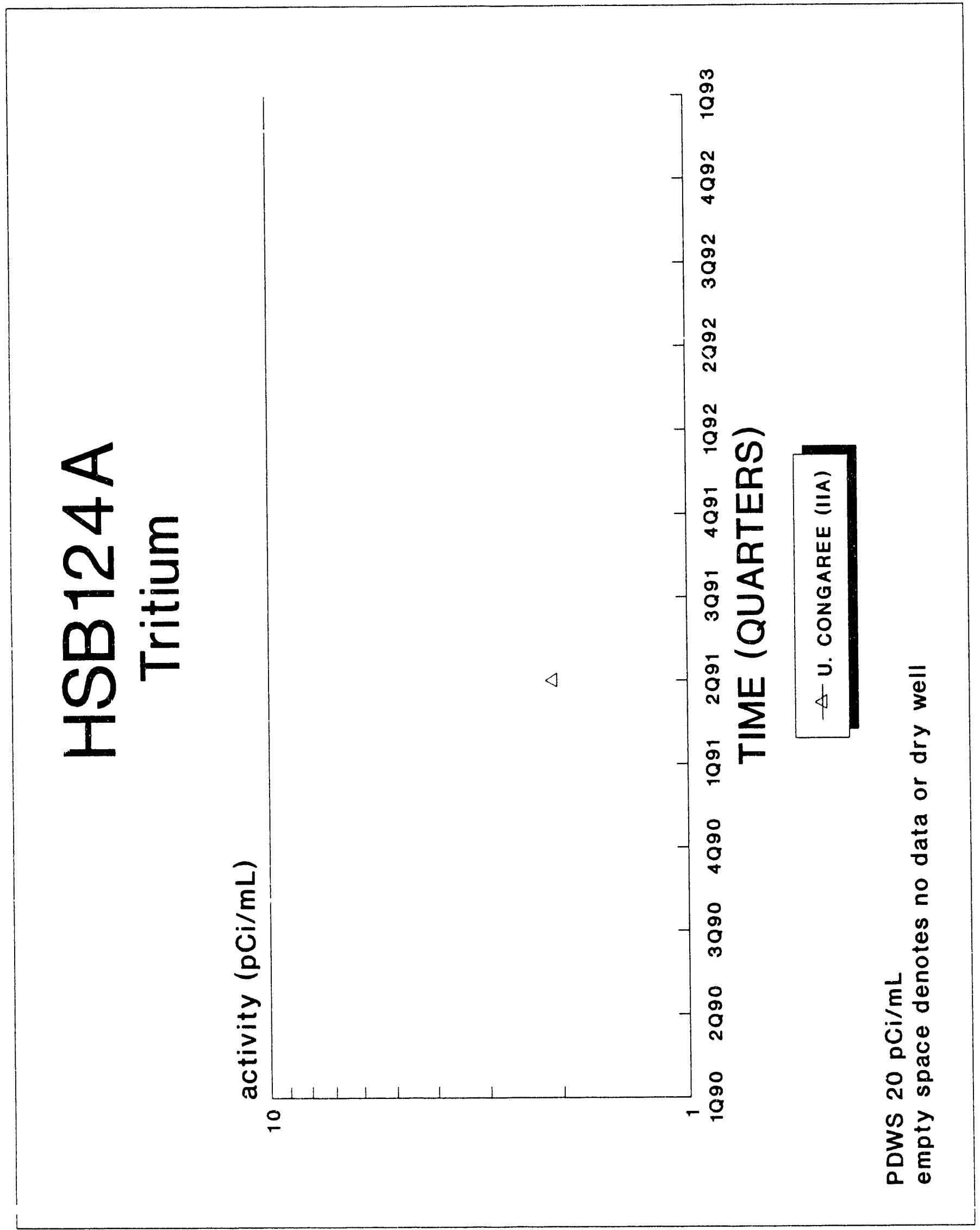




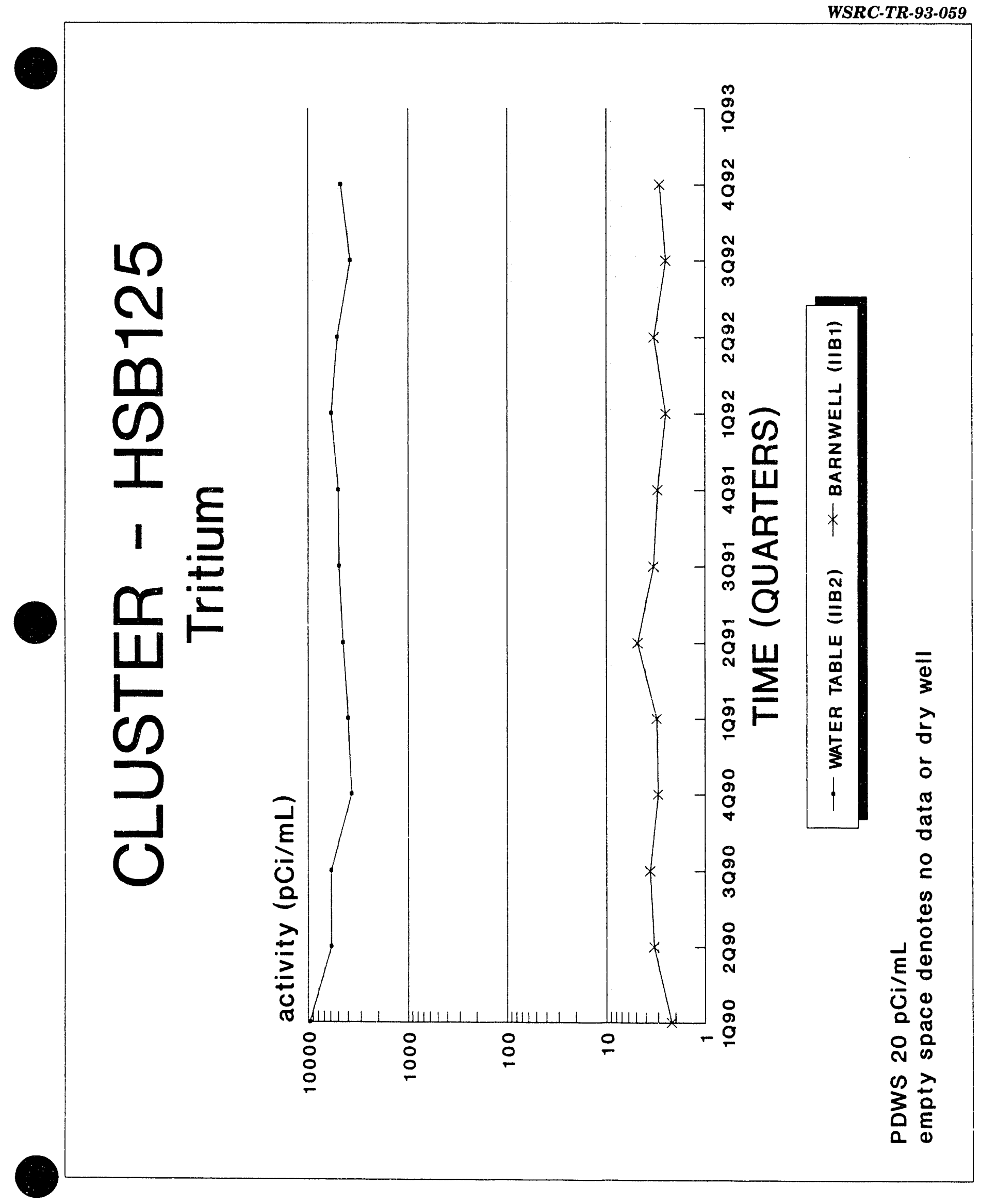




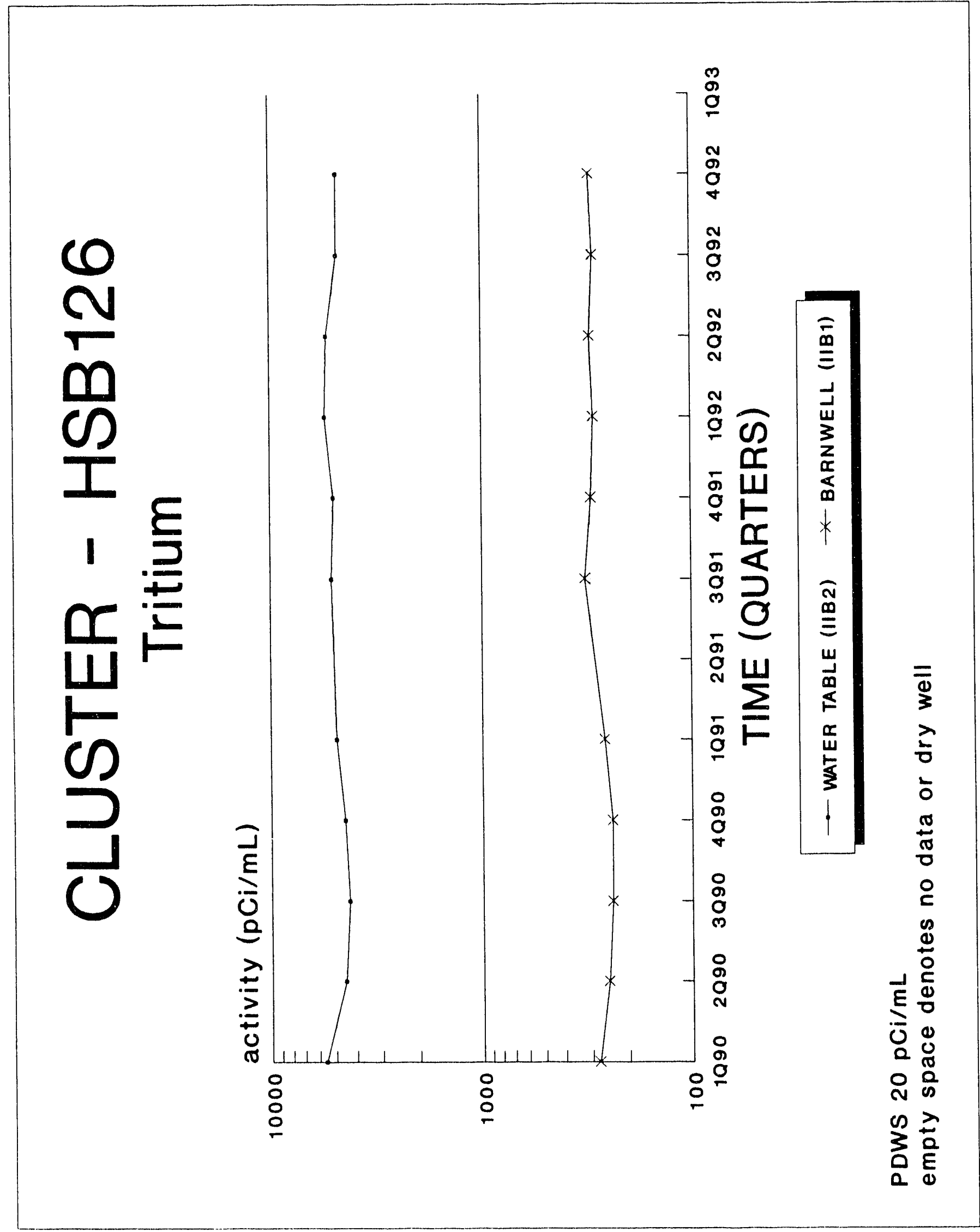




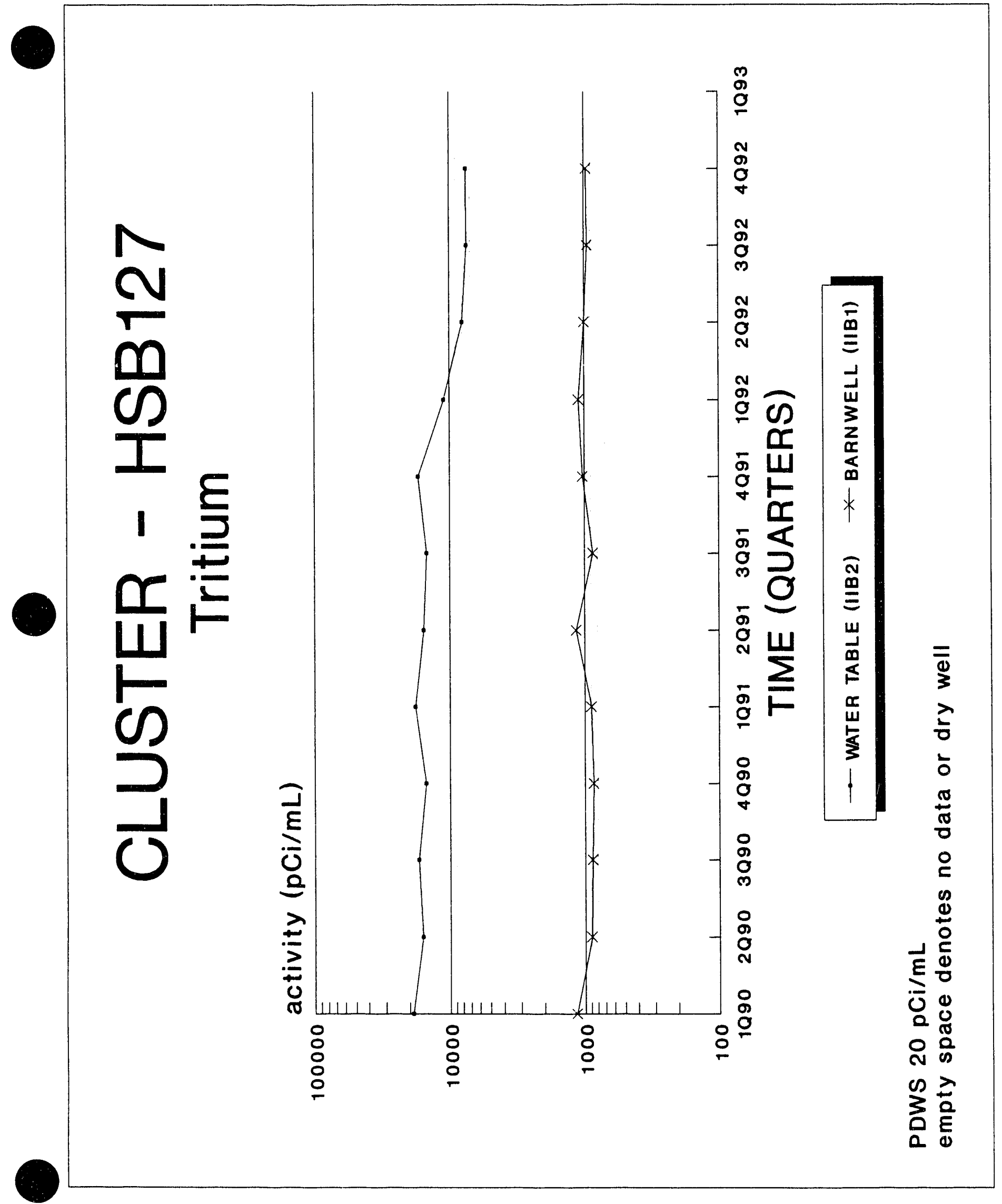




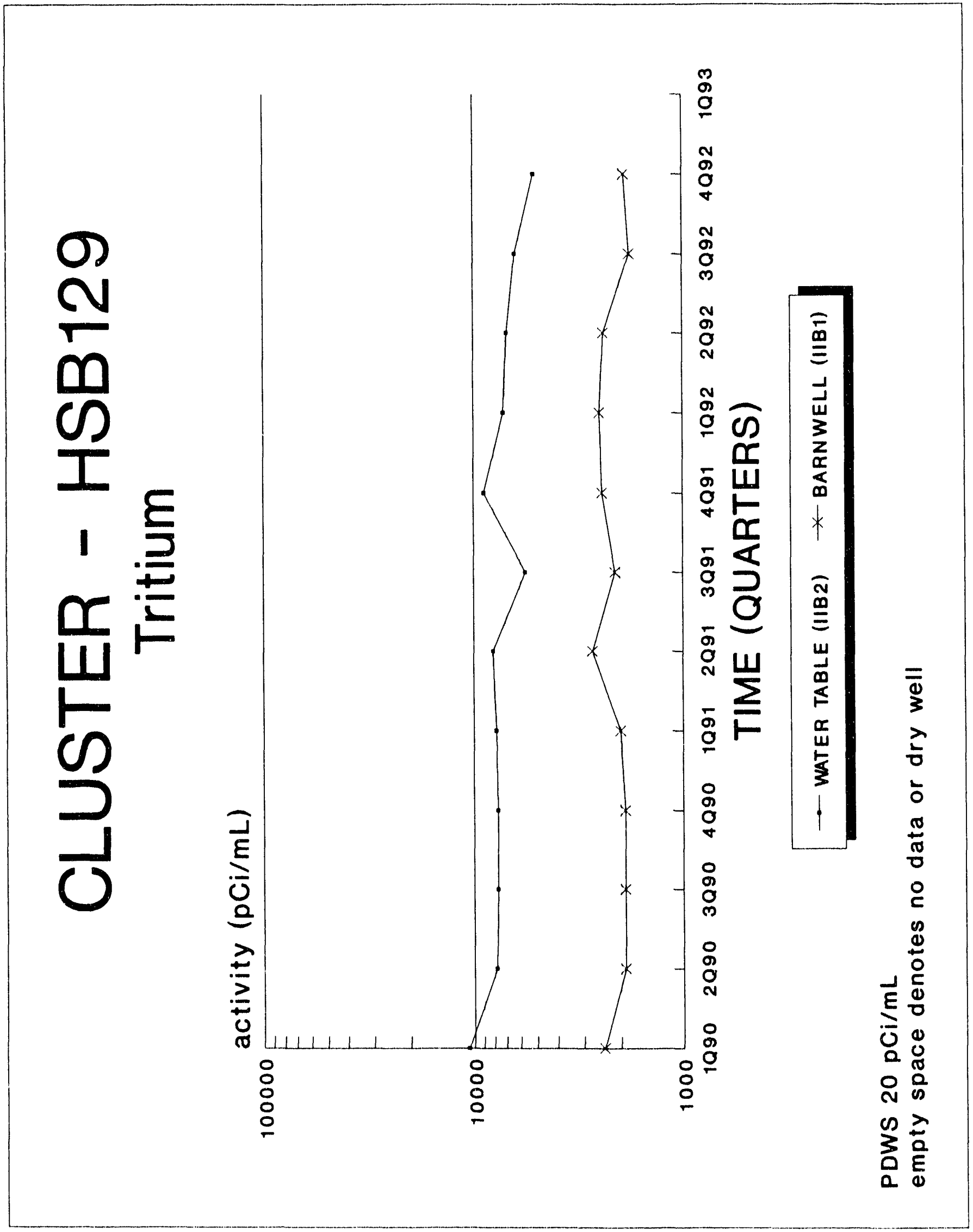




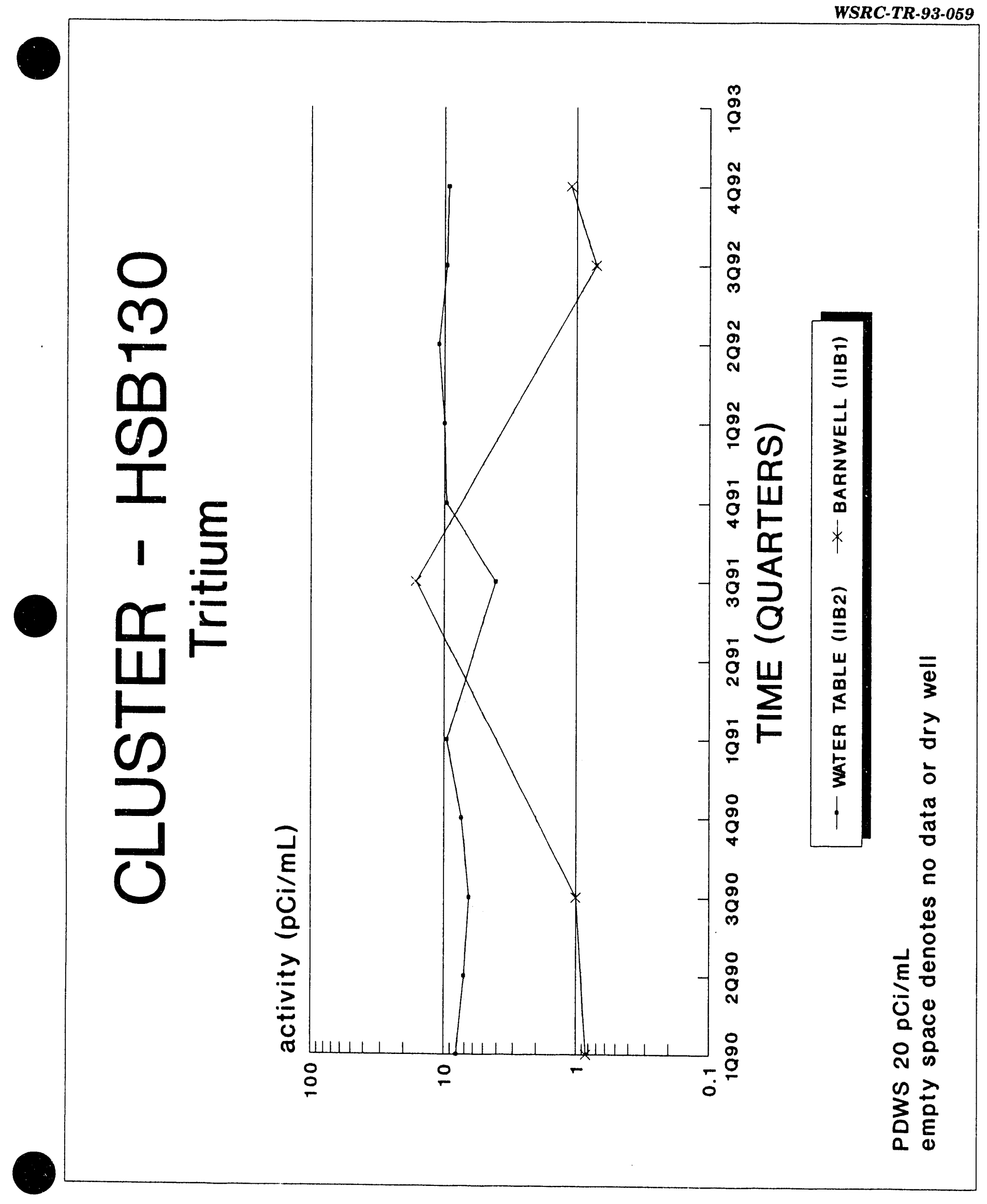




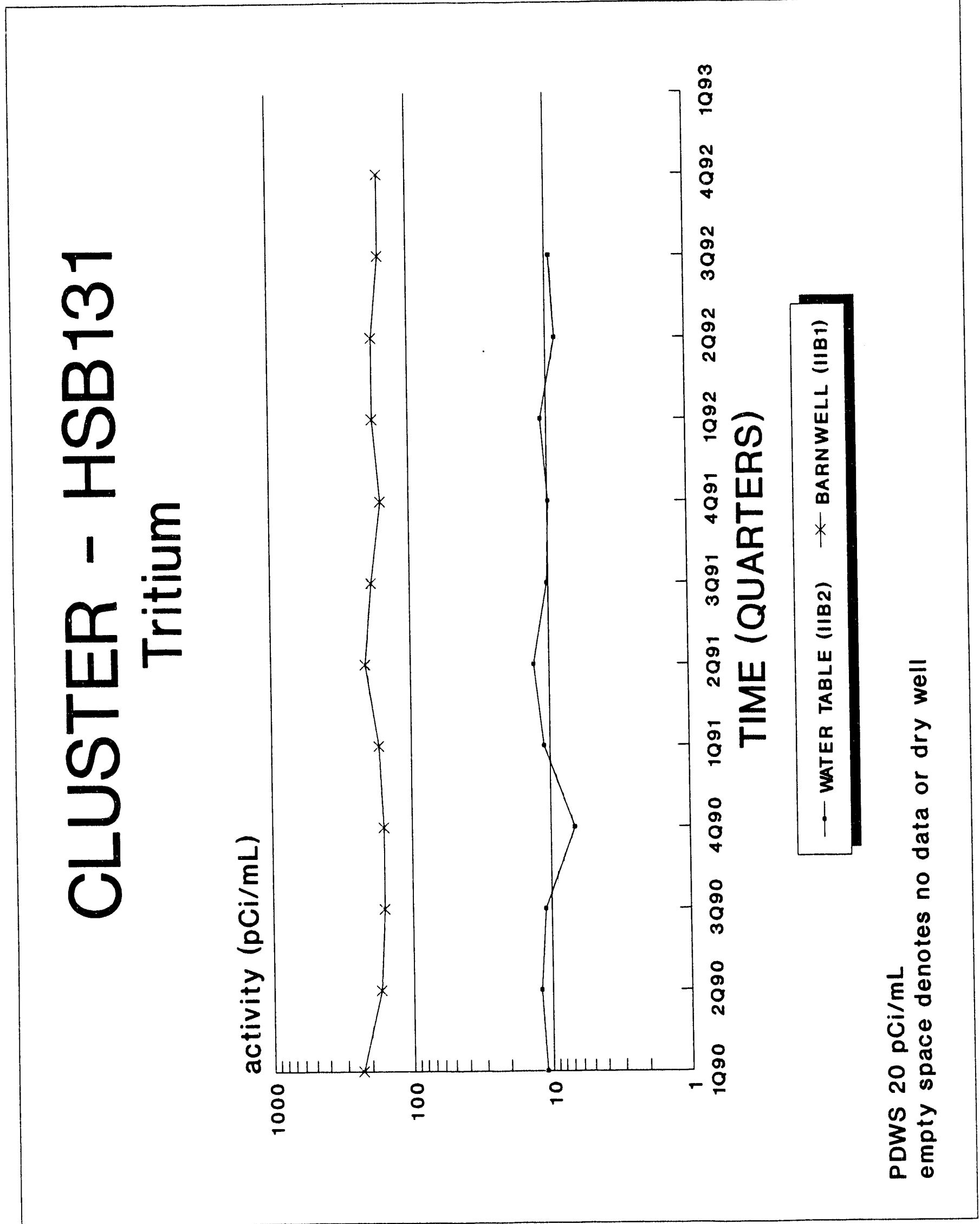




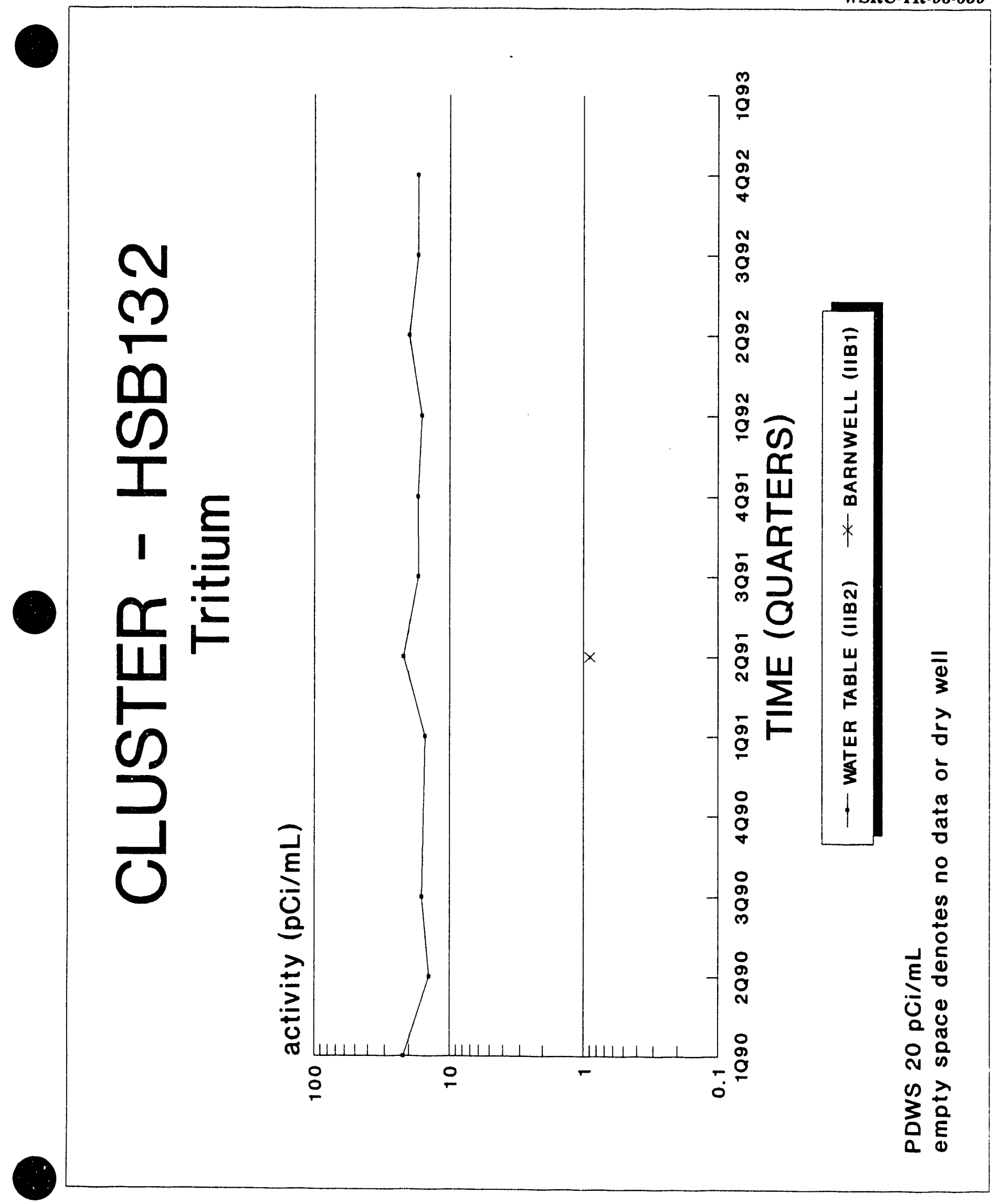



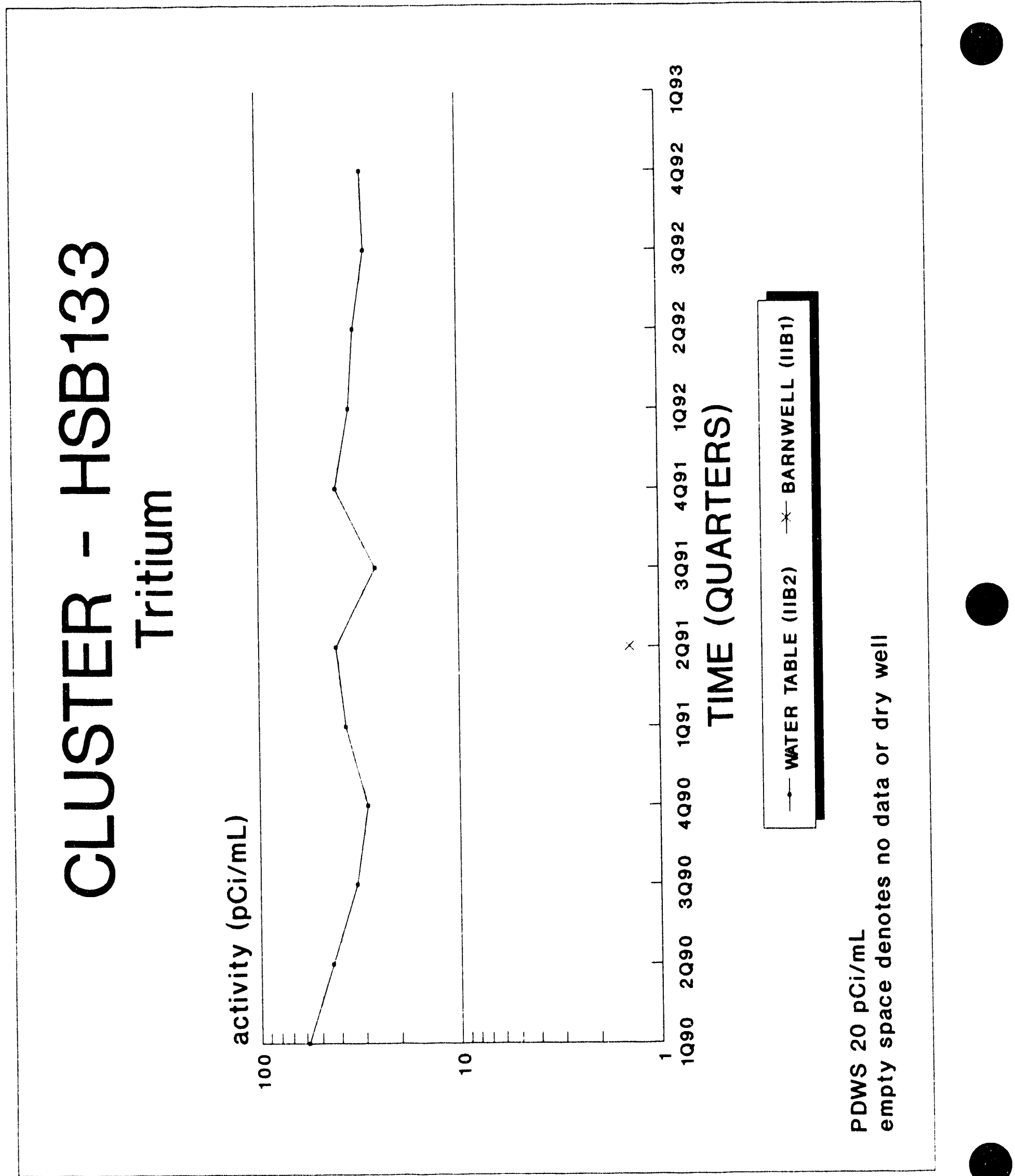


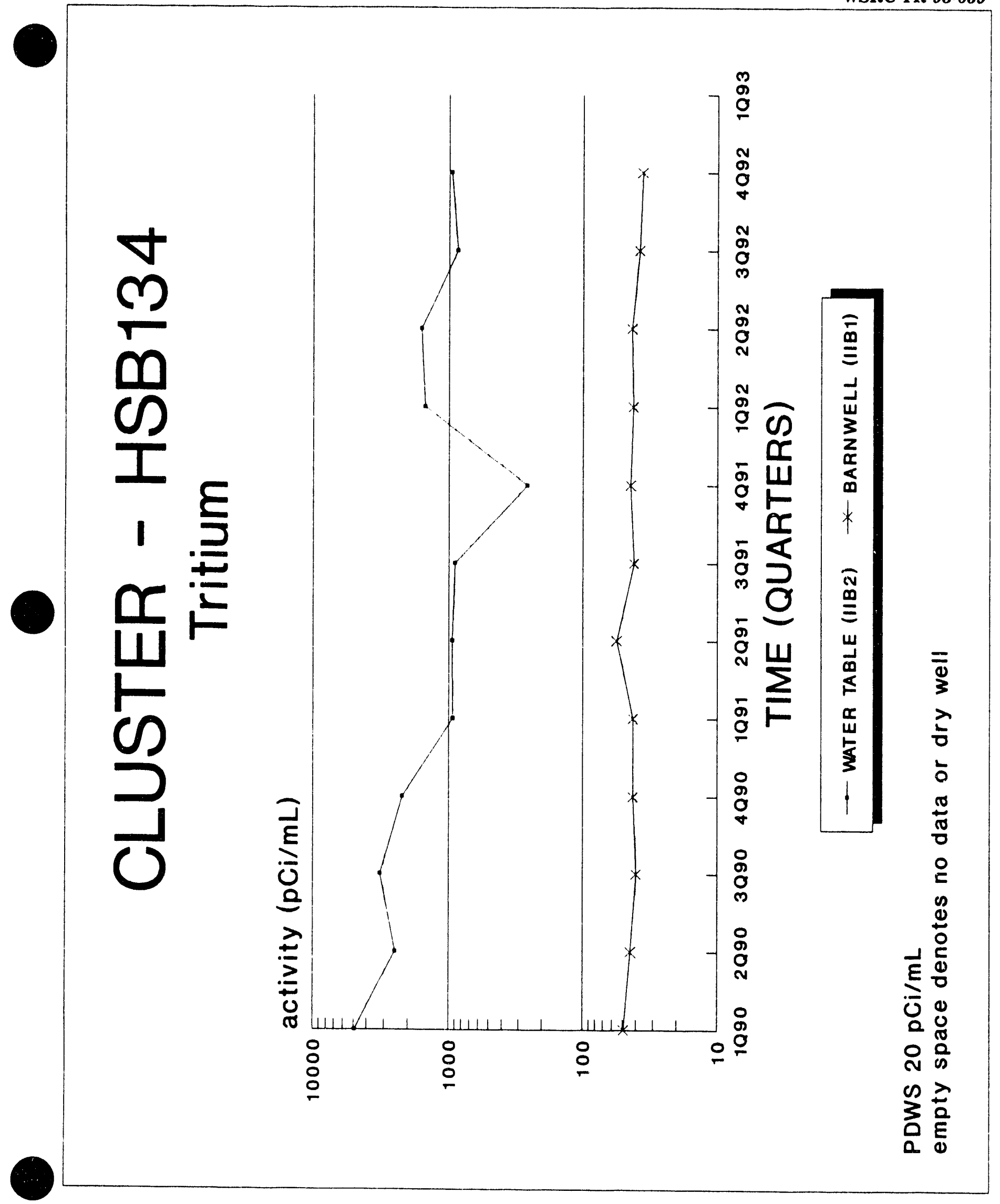




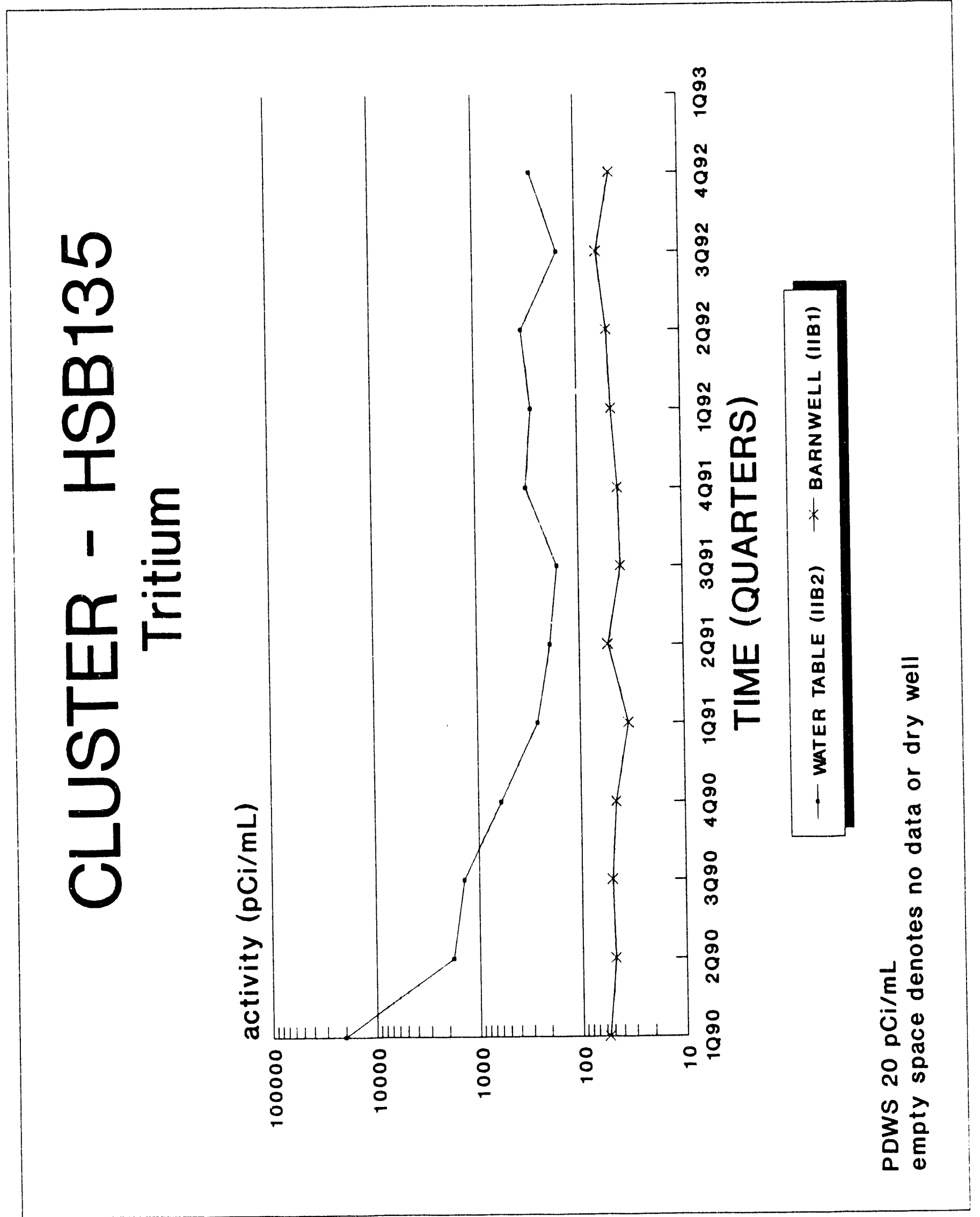




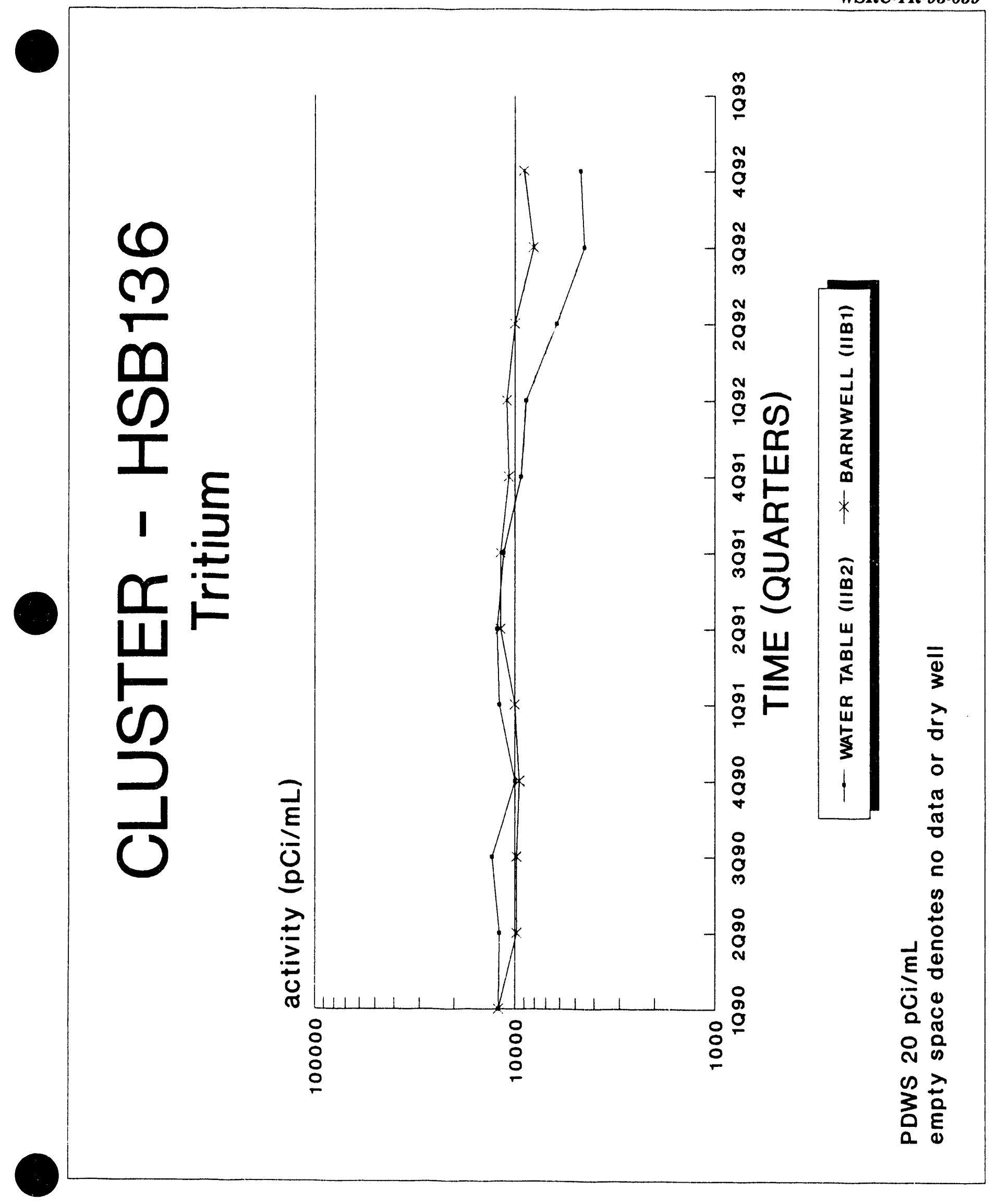




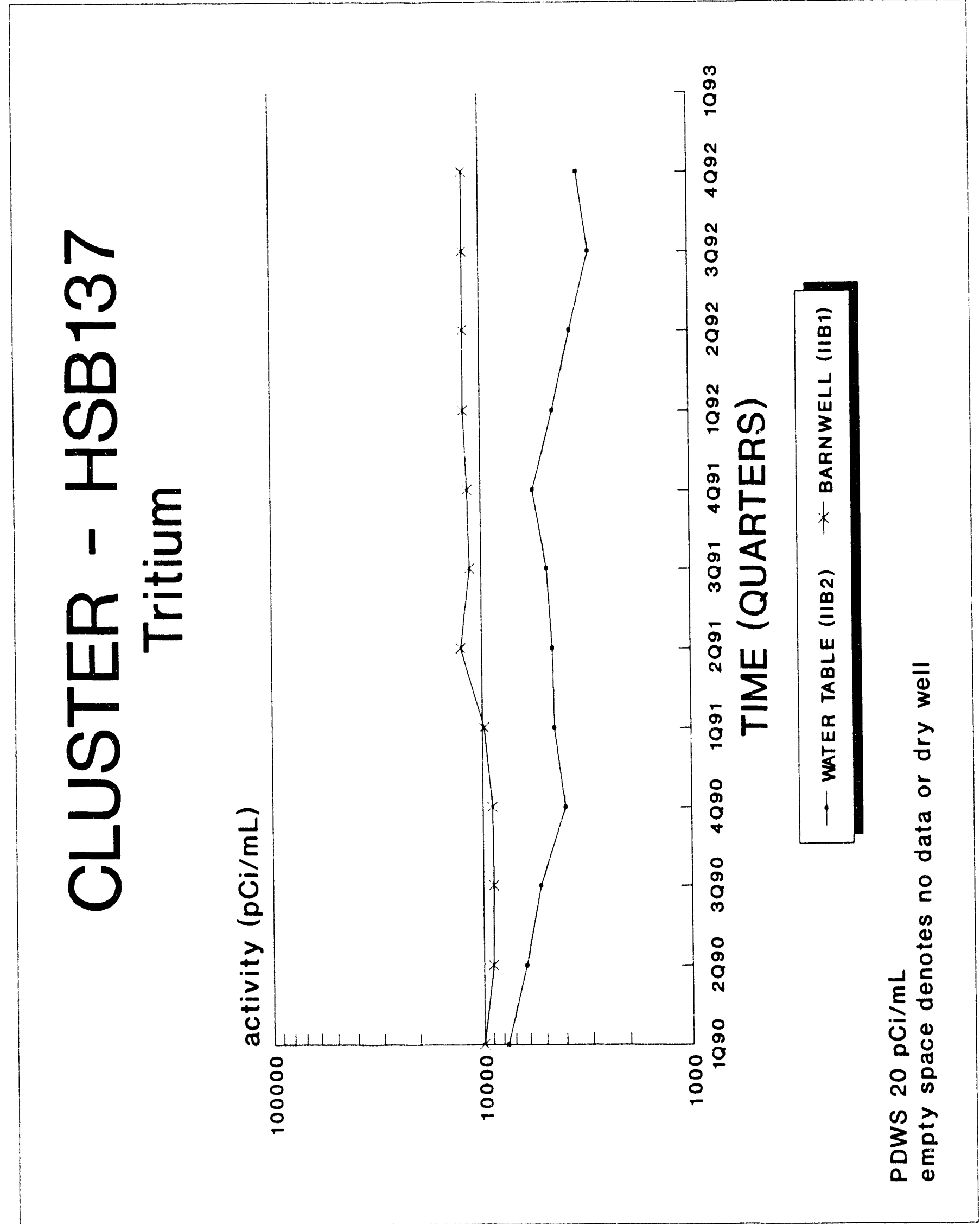




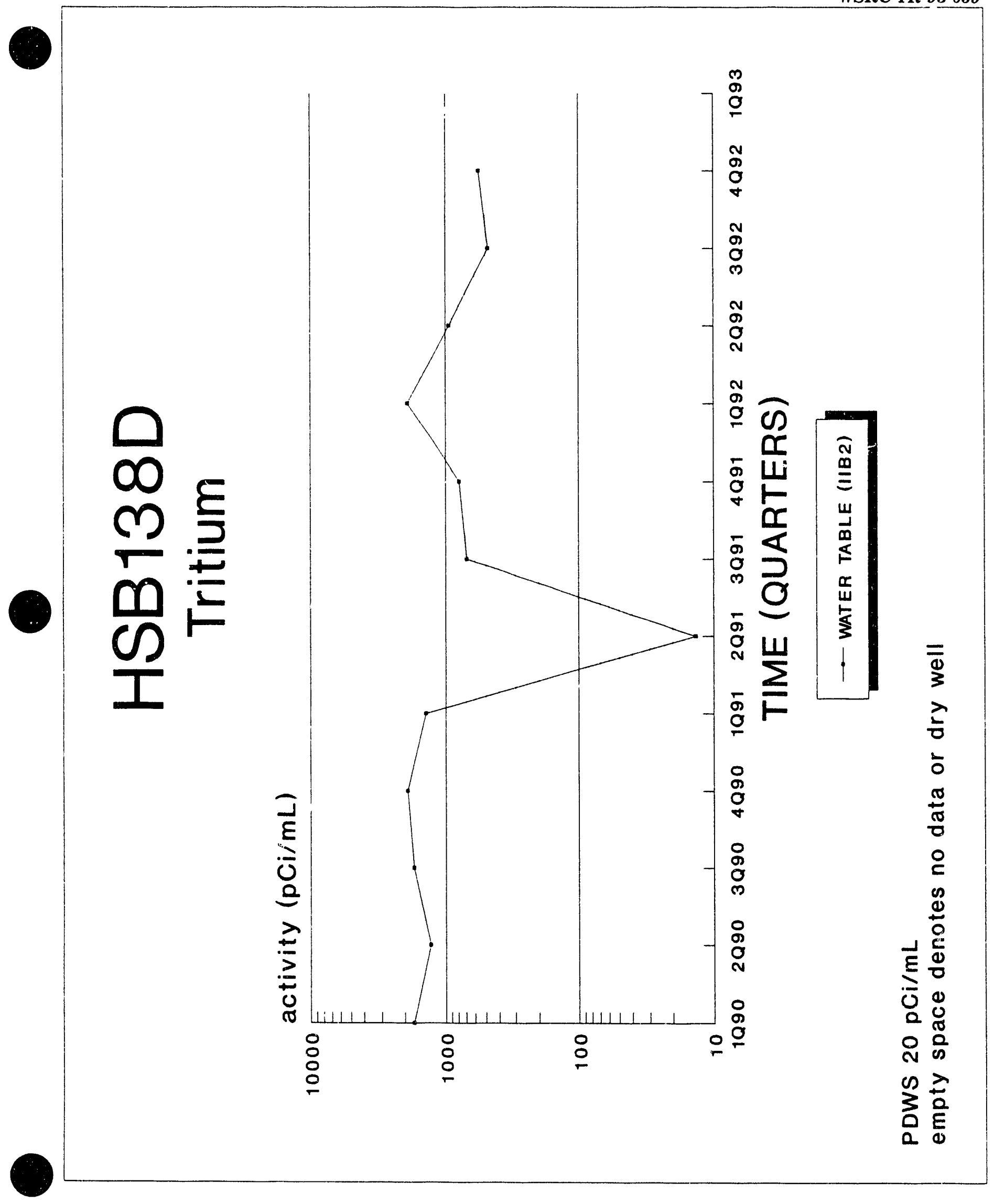




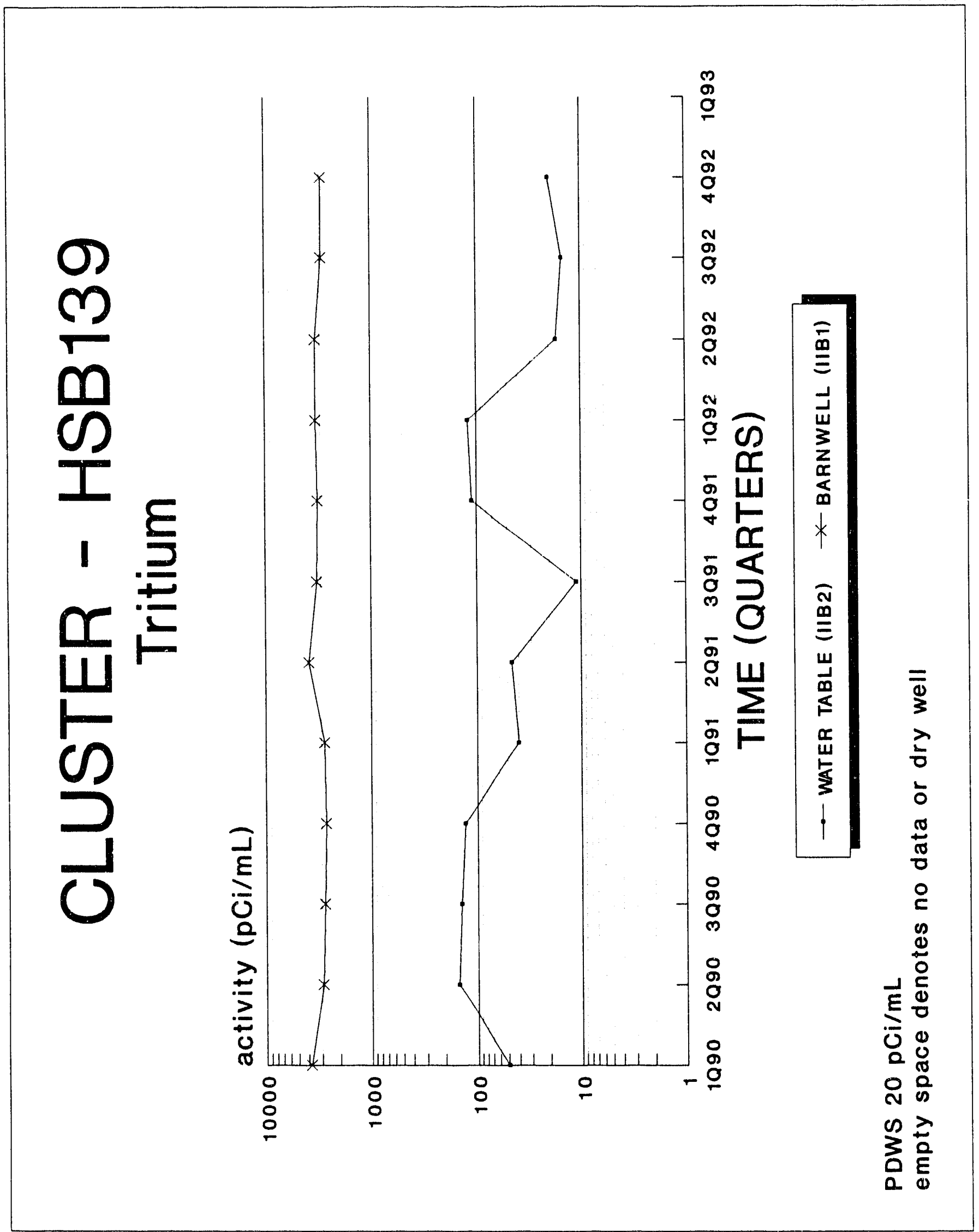




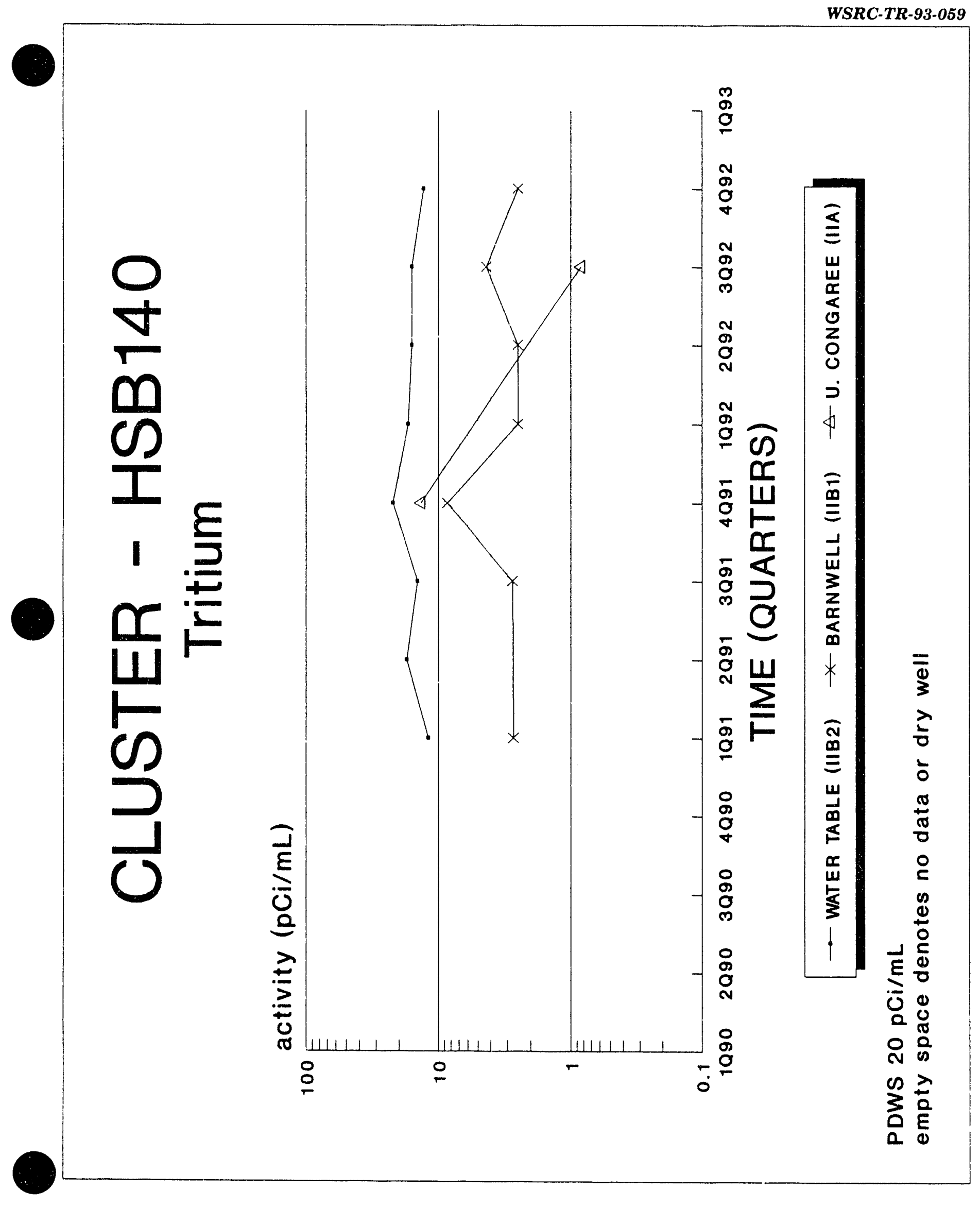




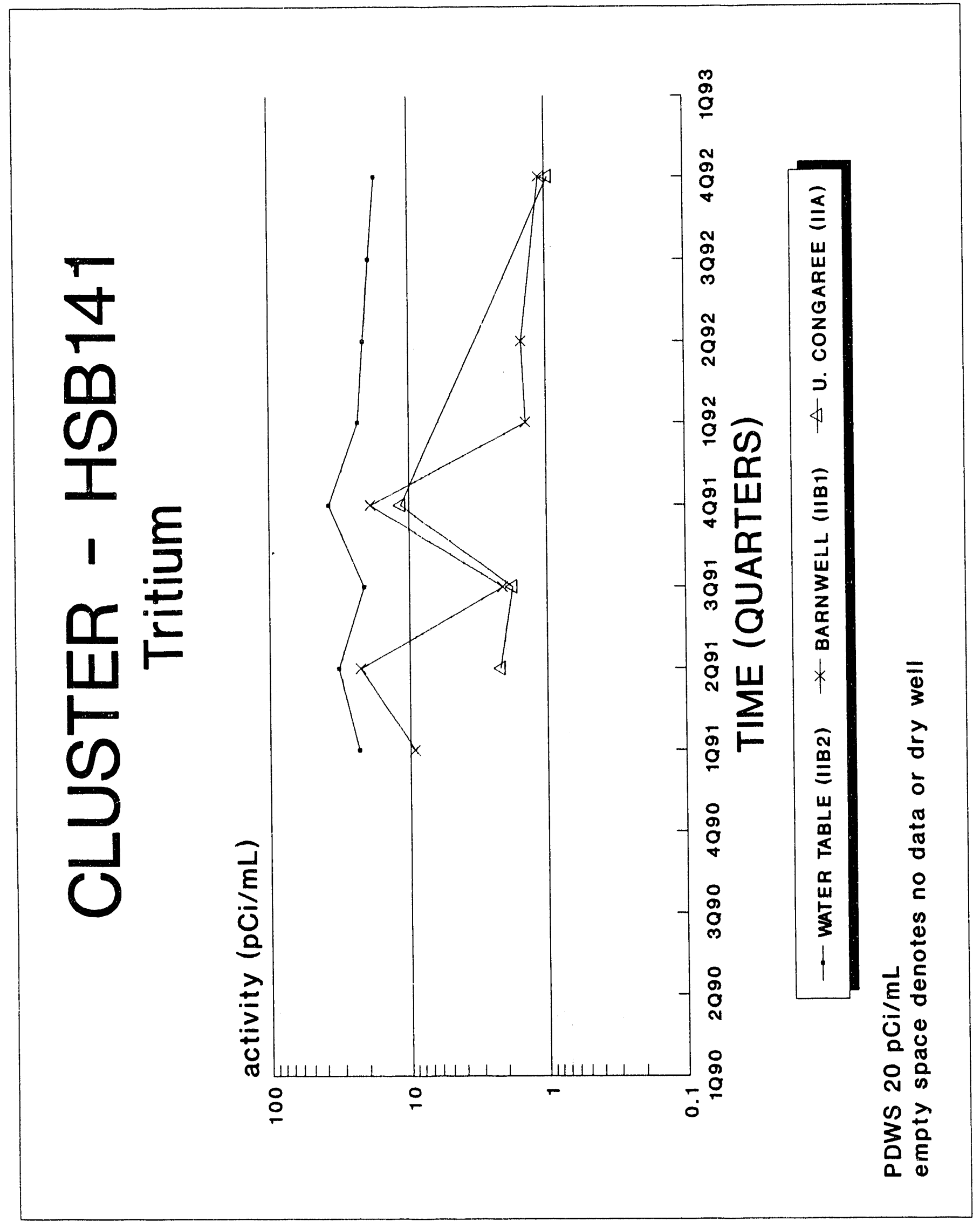




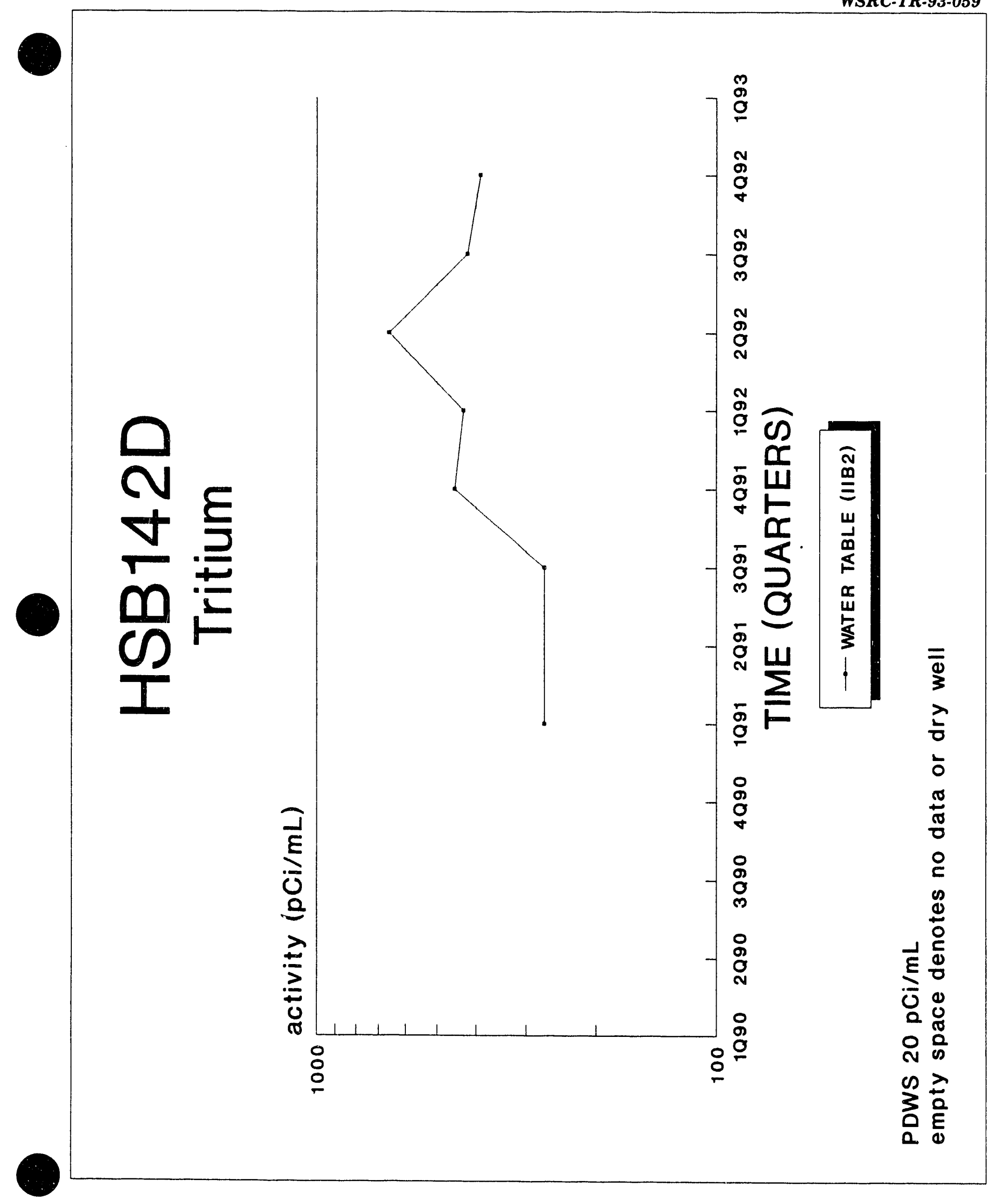




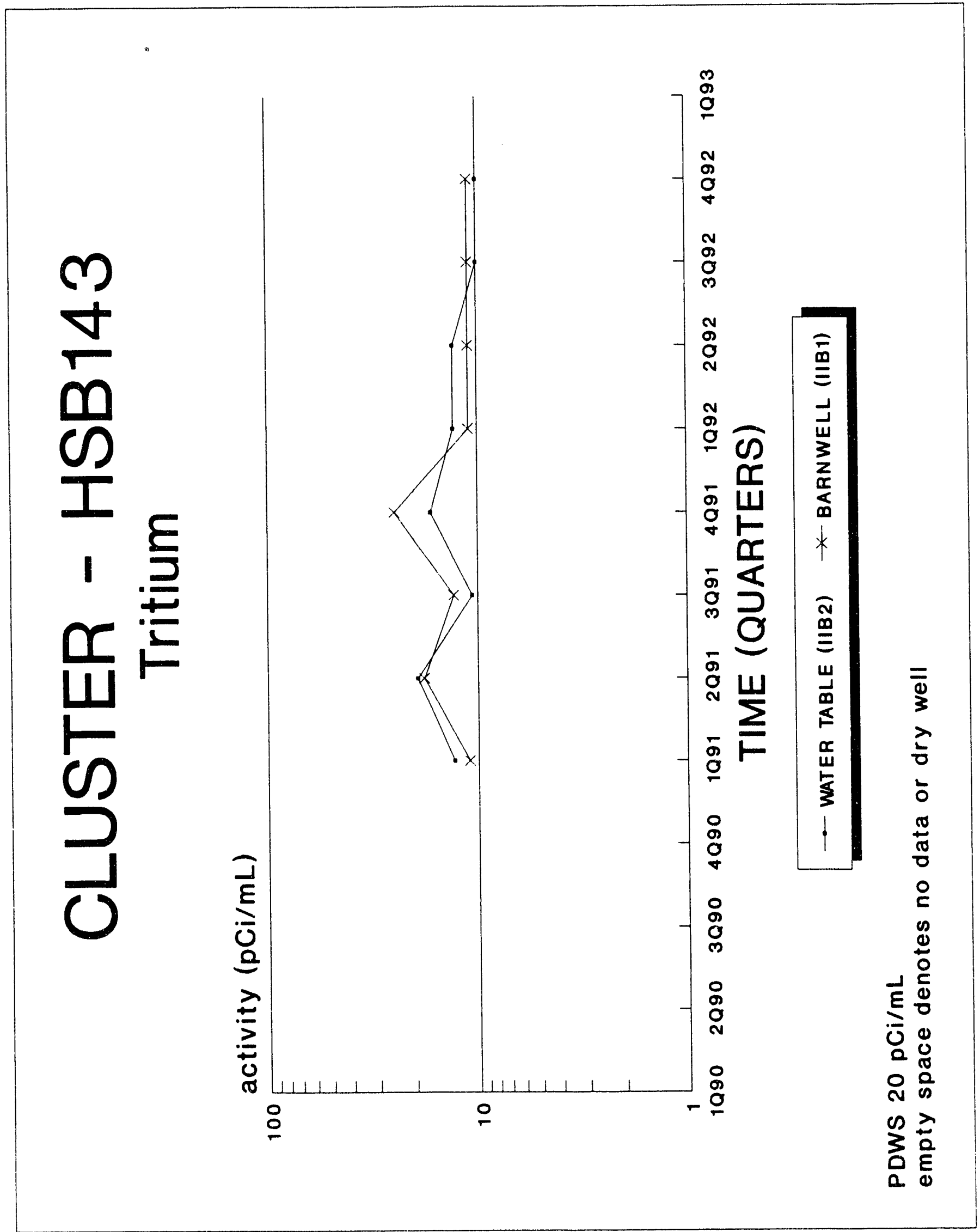




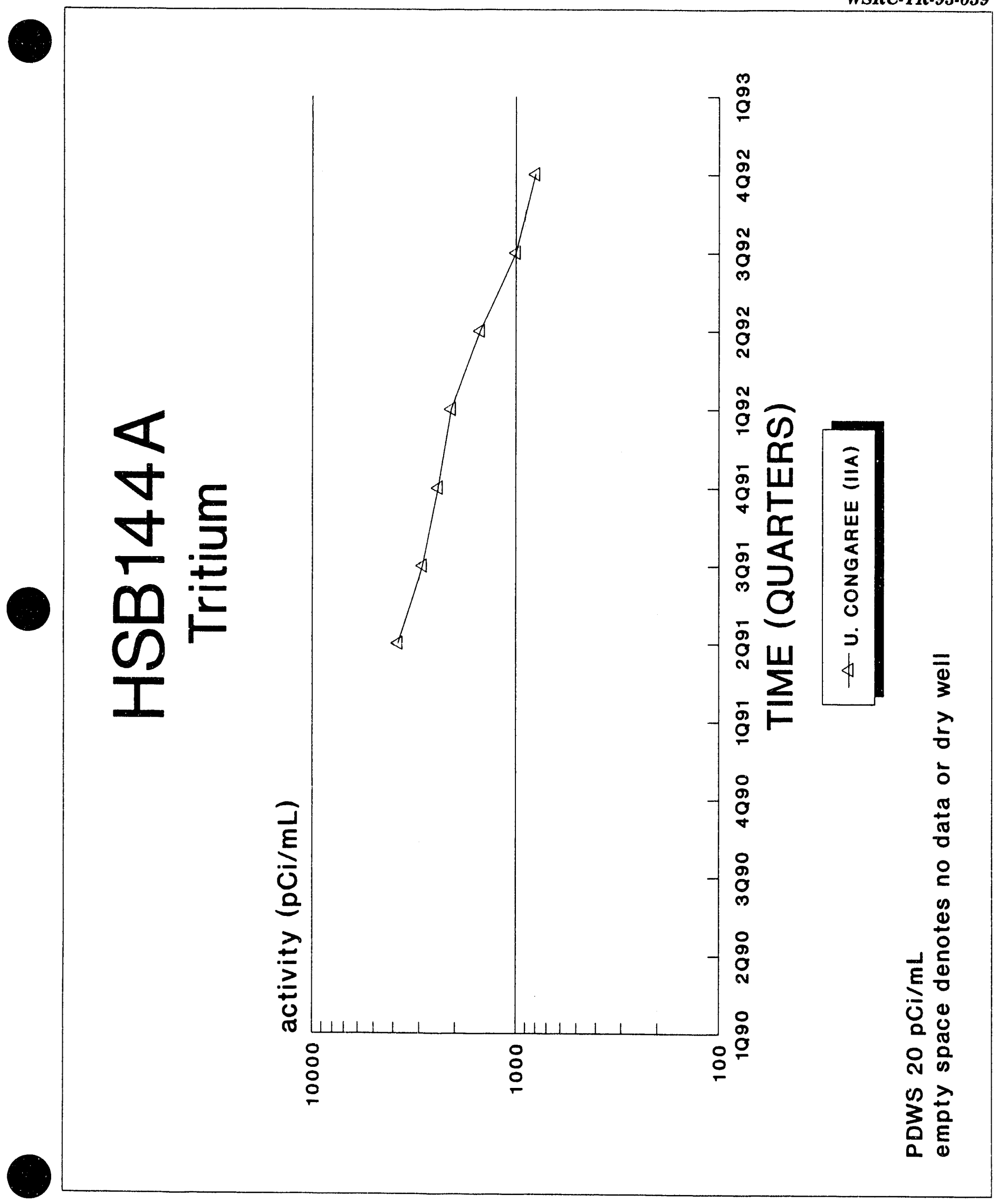




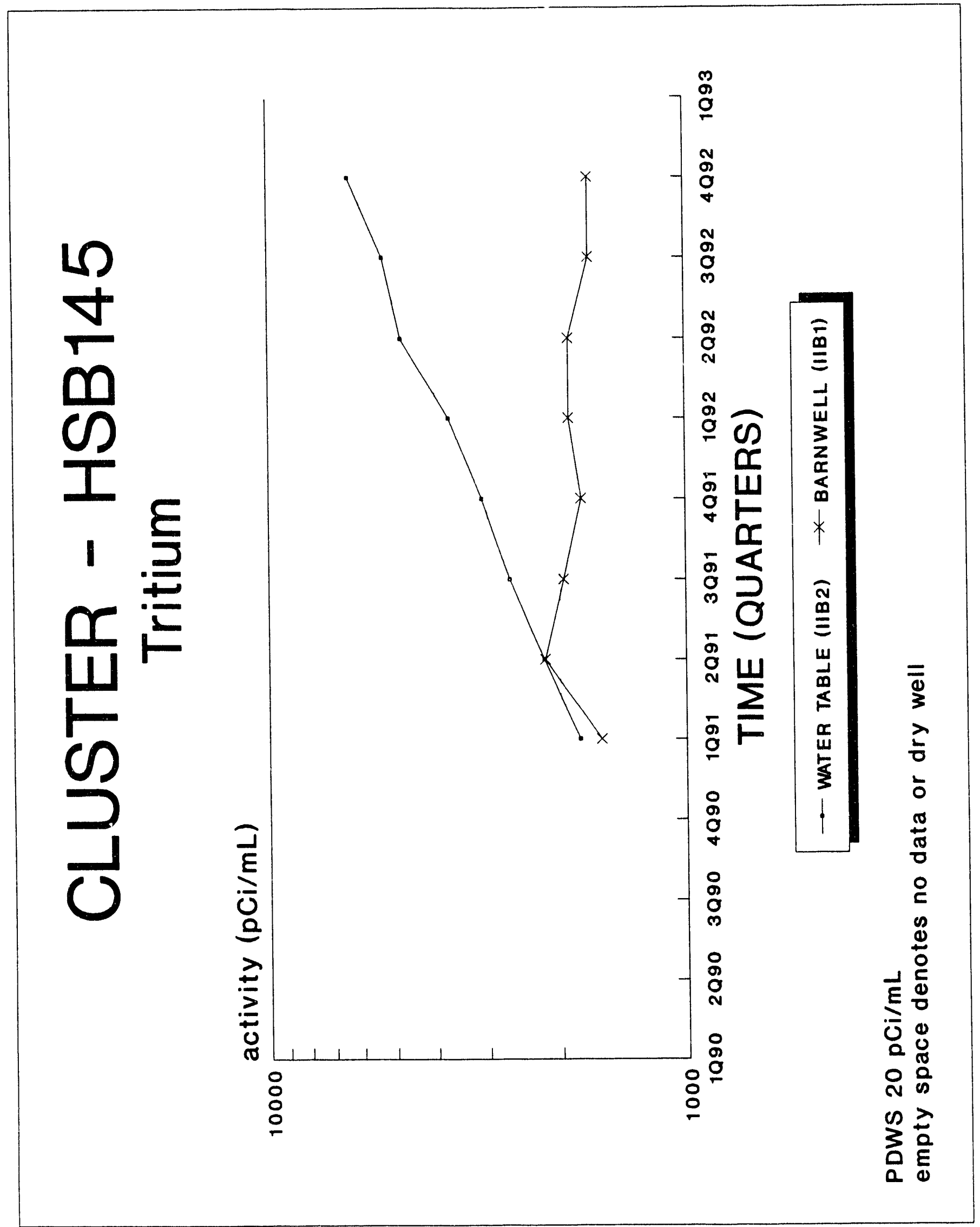




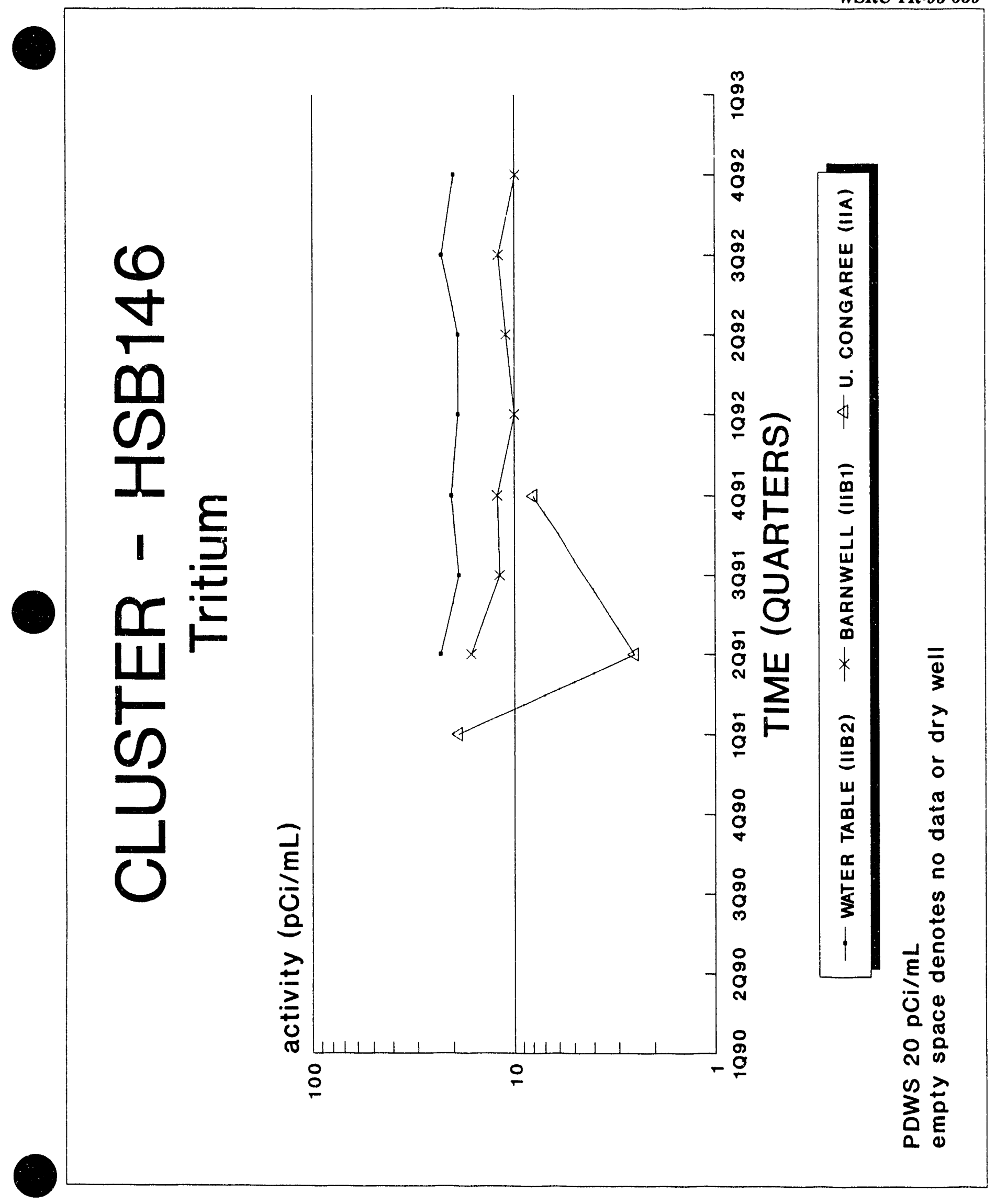




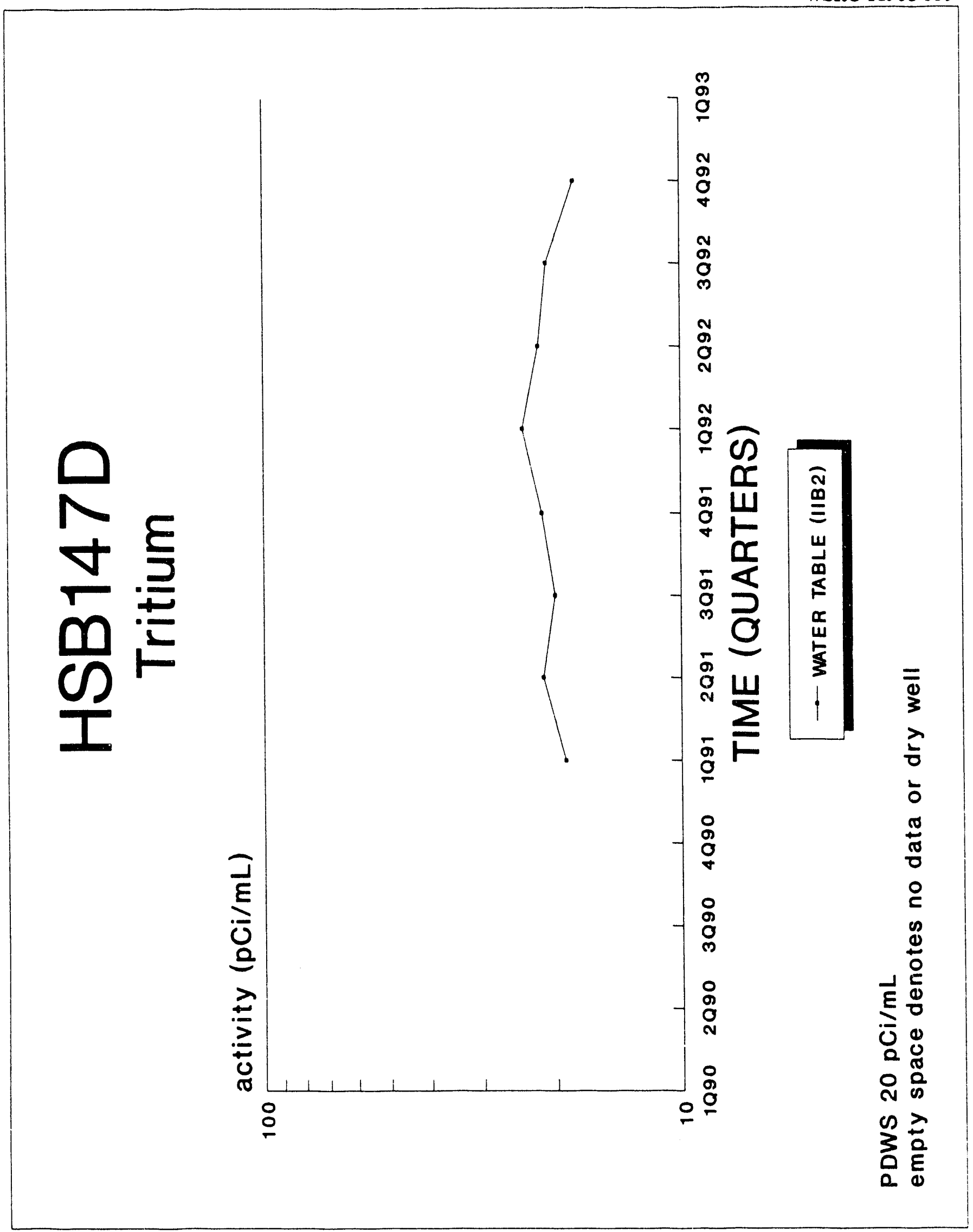




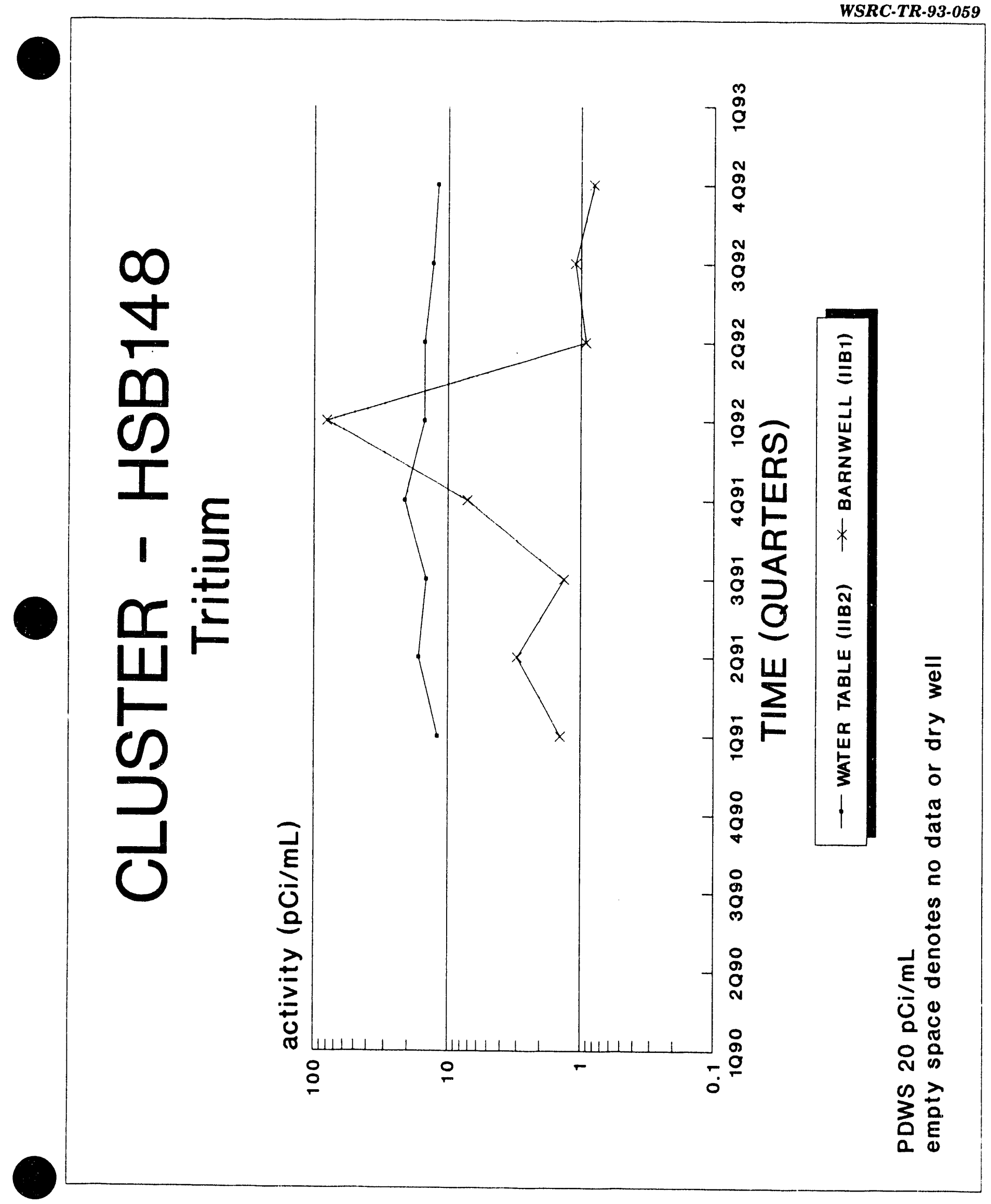



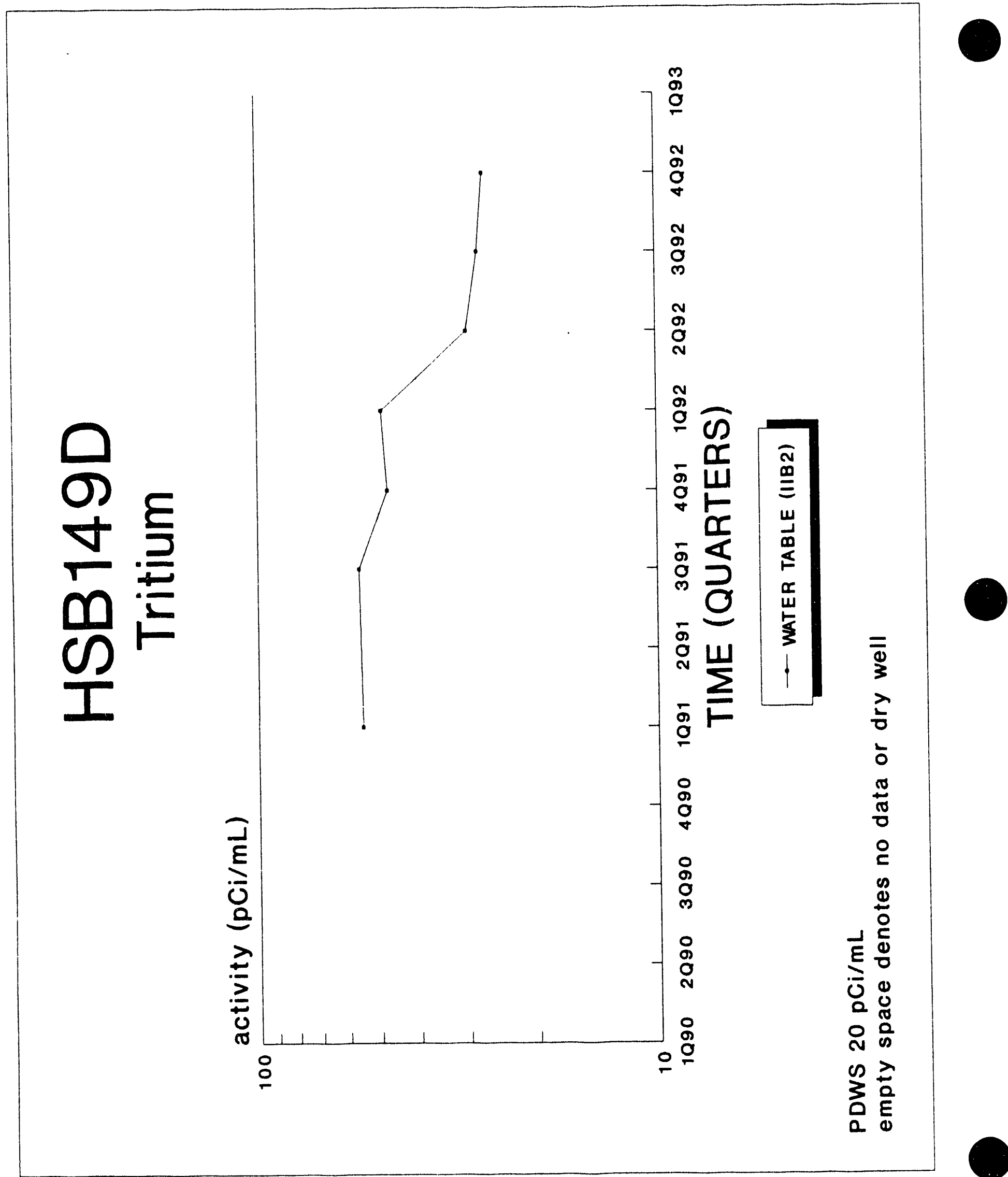


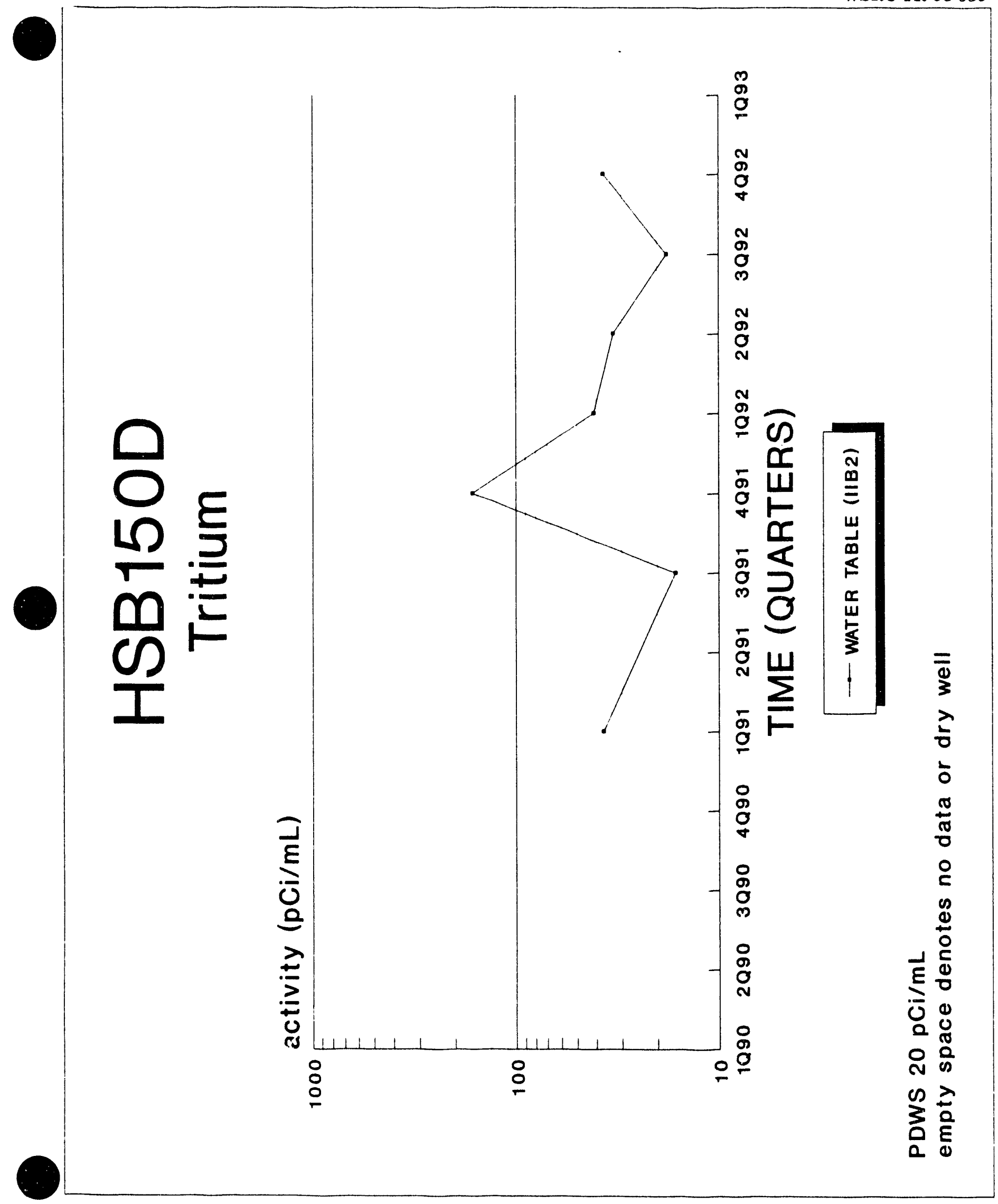




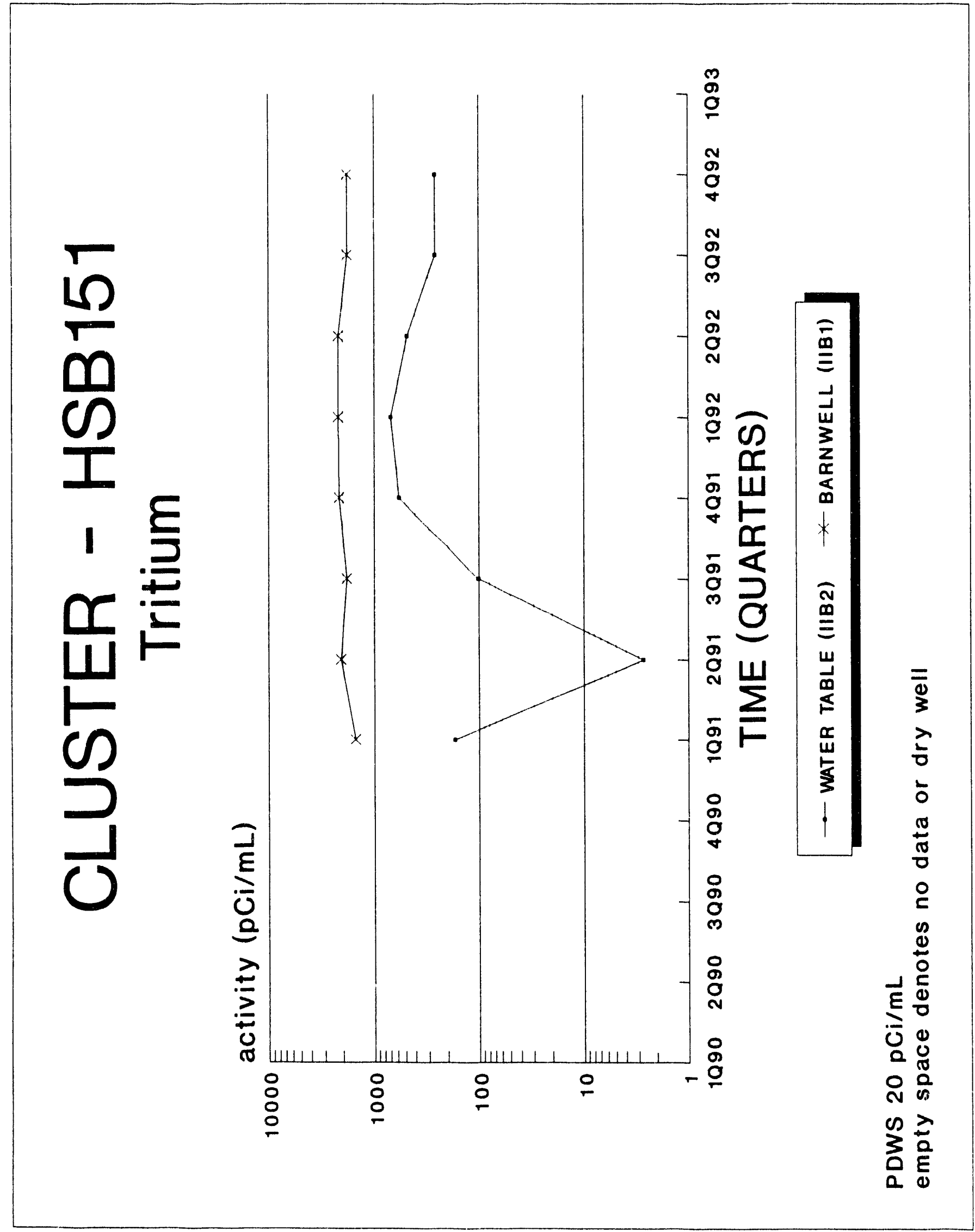




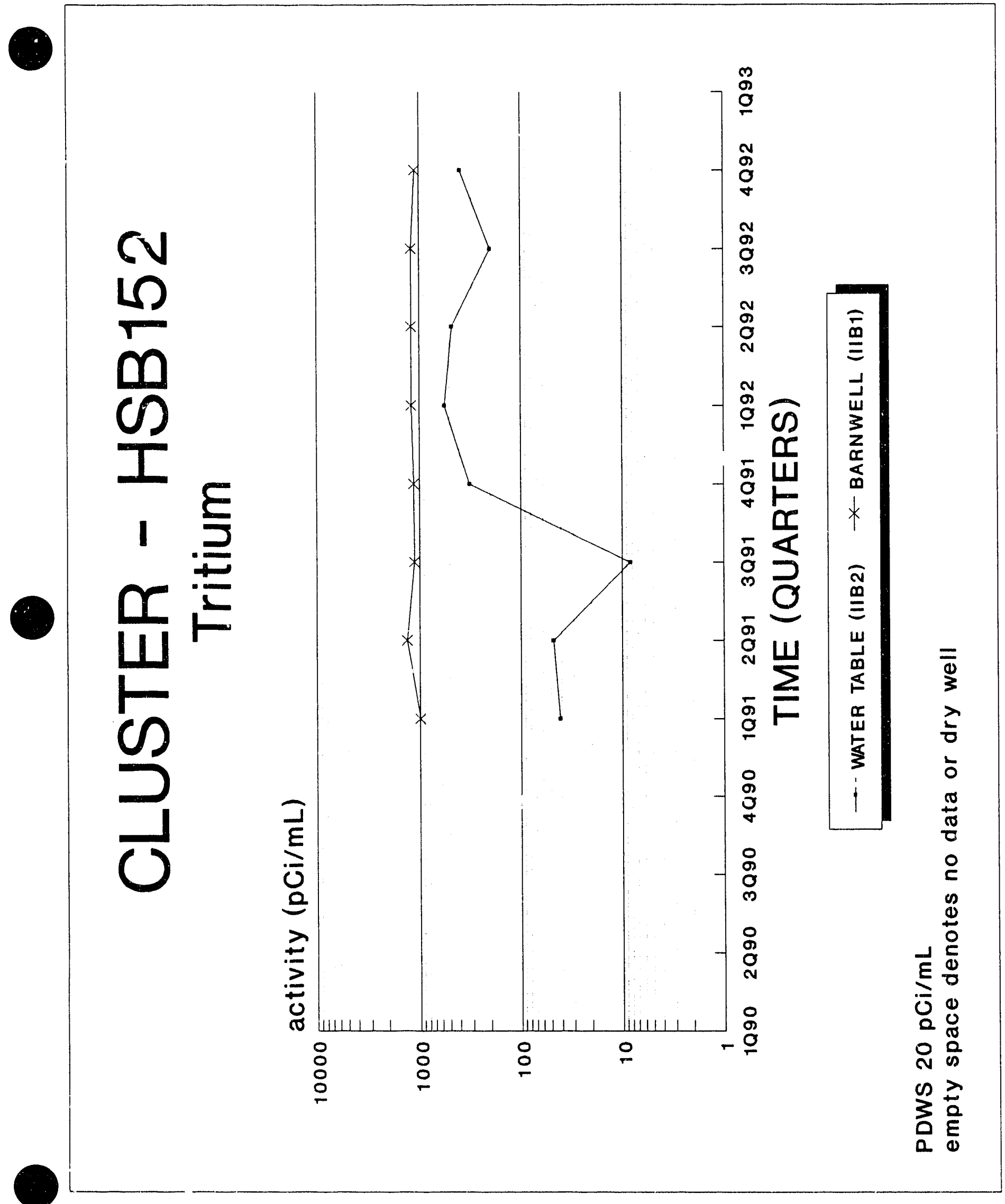


WSRC-TR-93-059 


\section{Appendix G - Hydrographs}




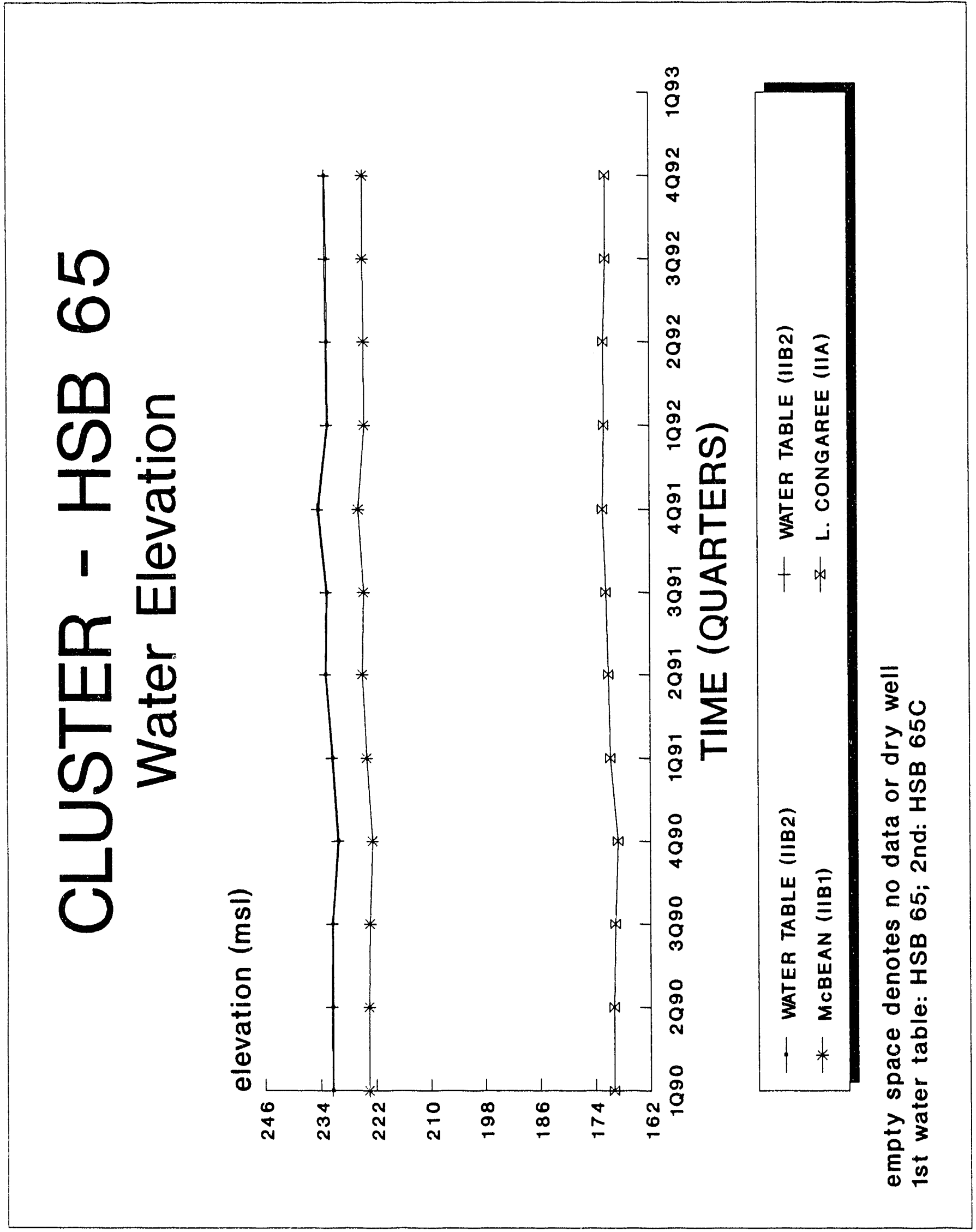




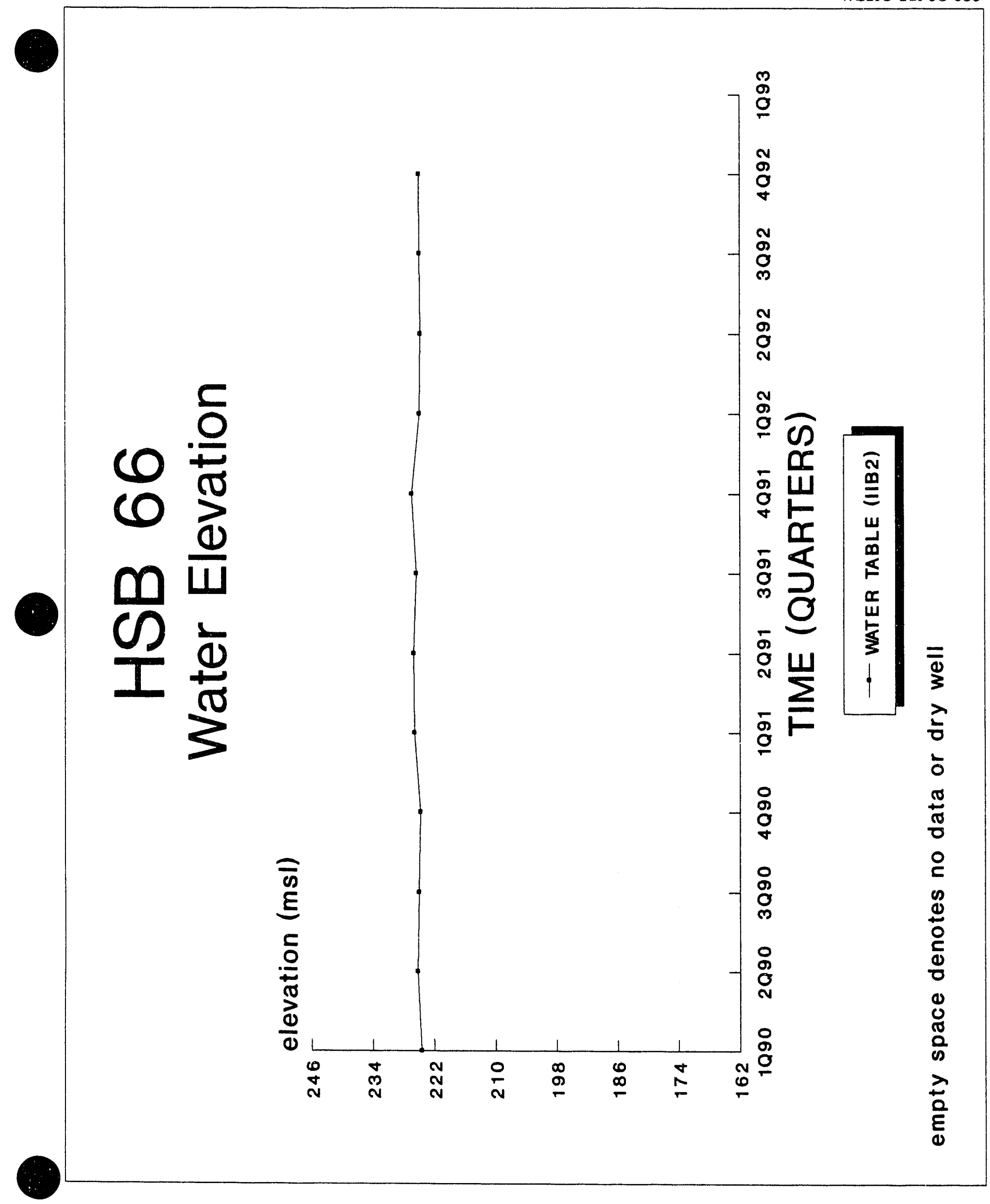




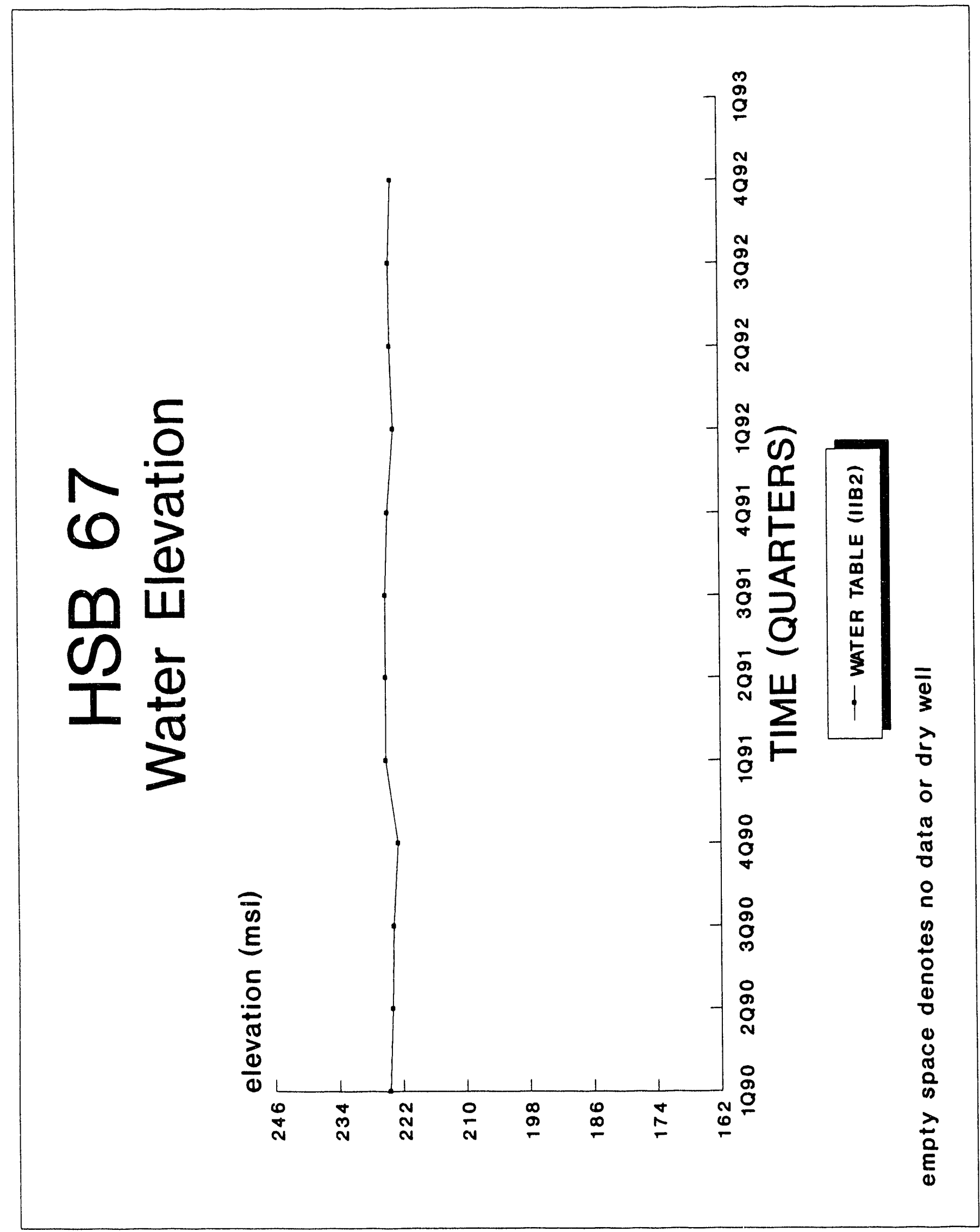




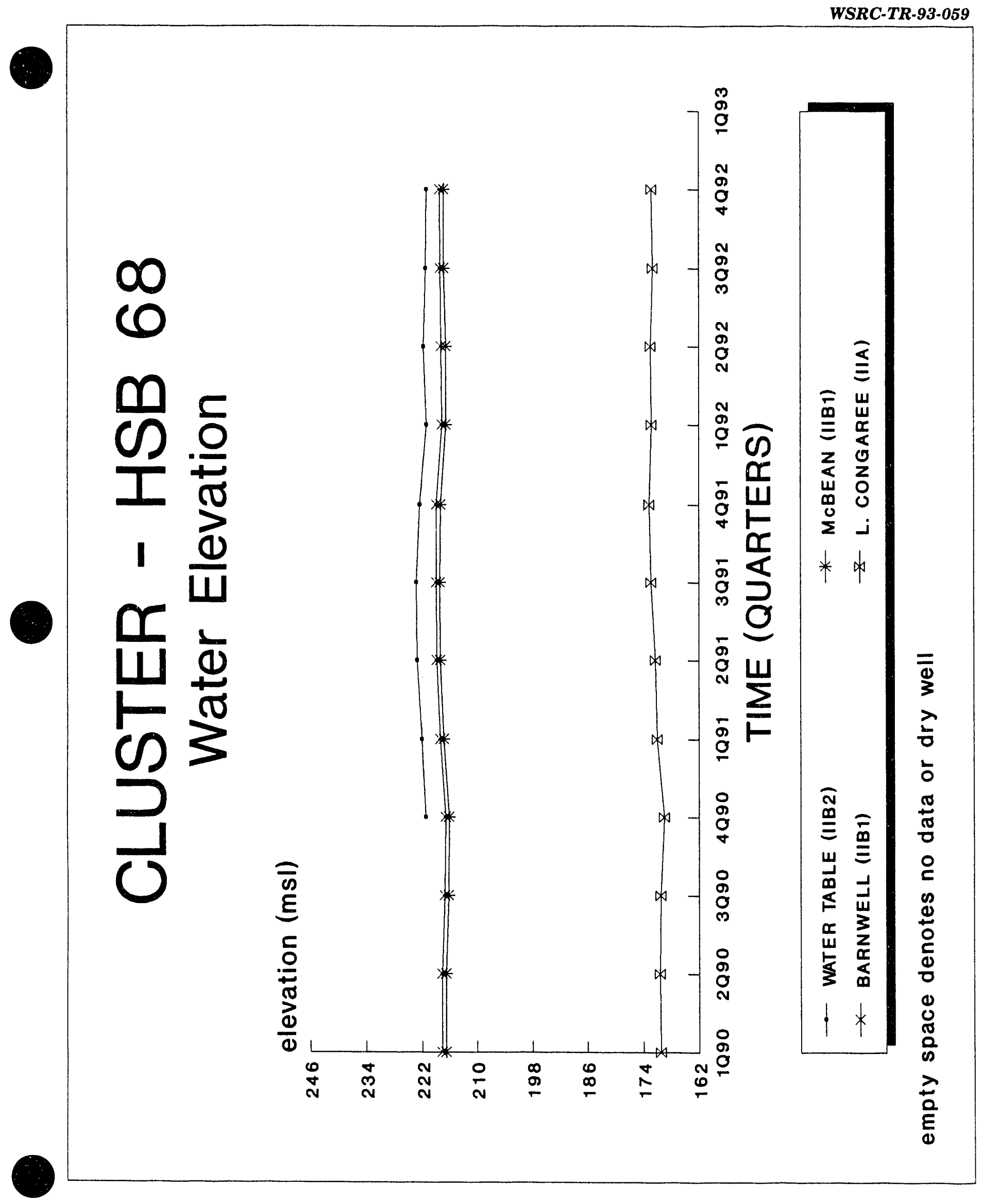




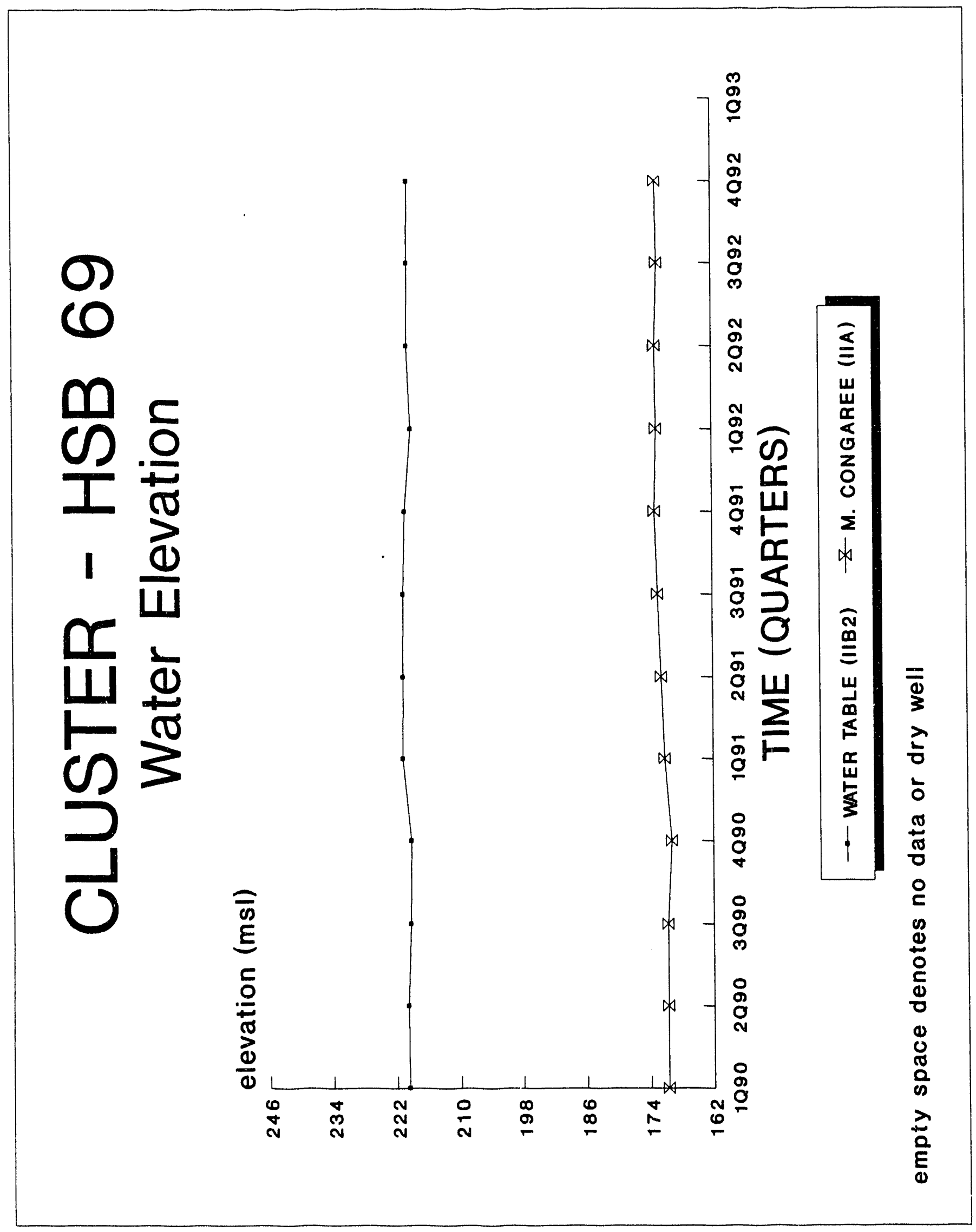




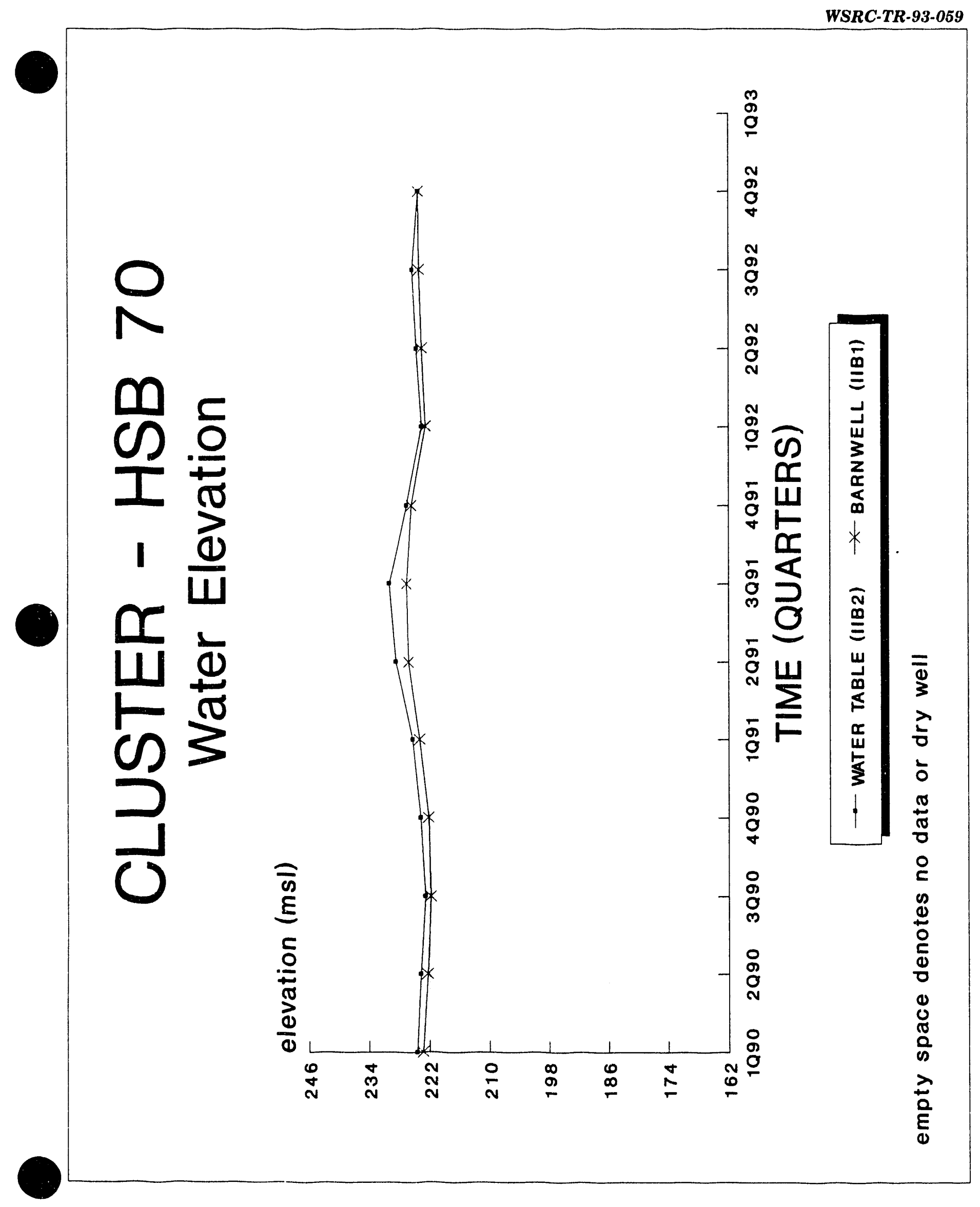




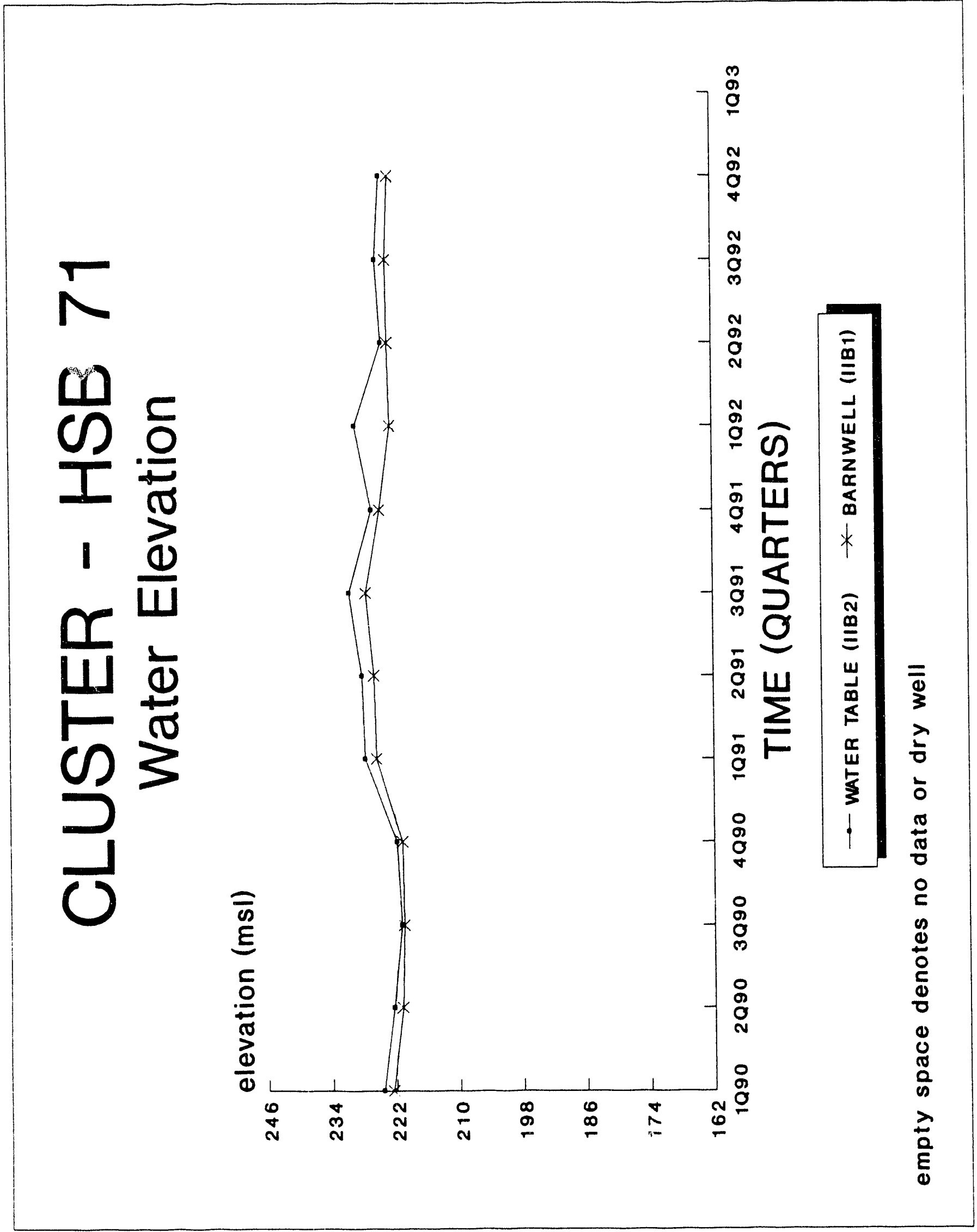




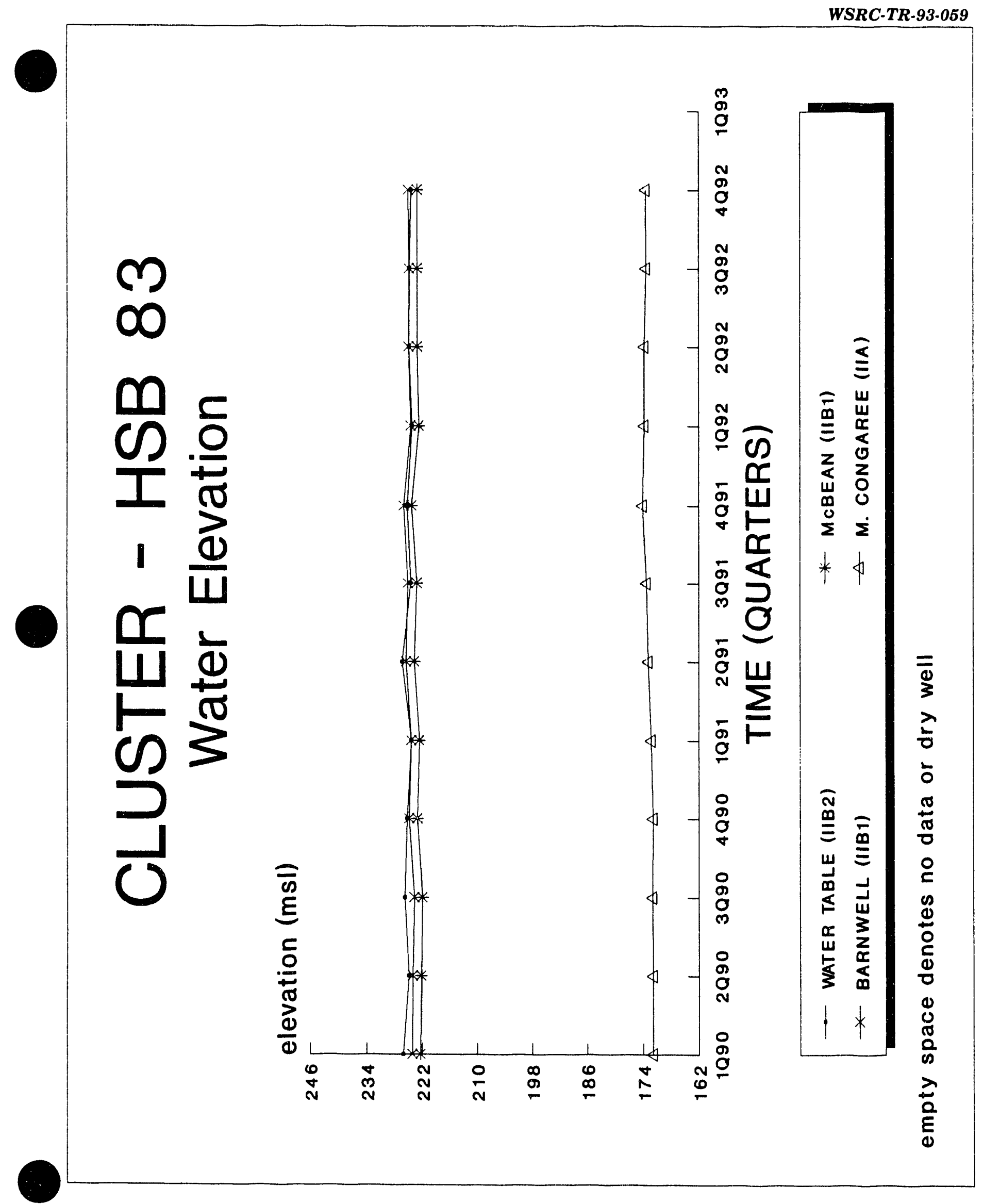




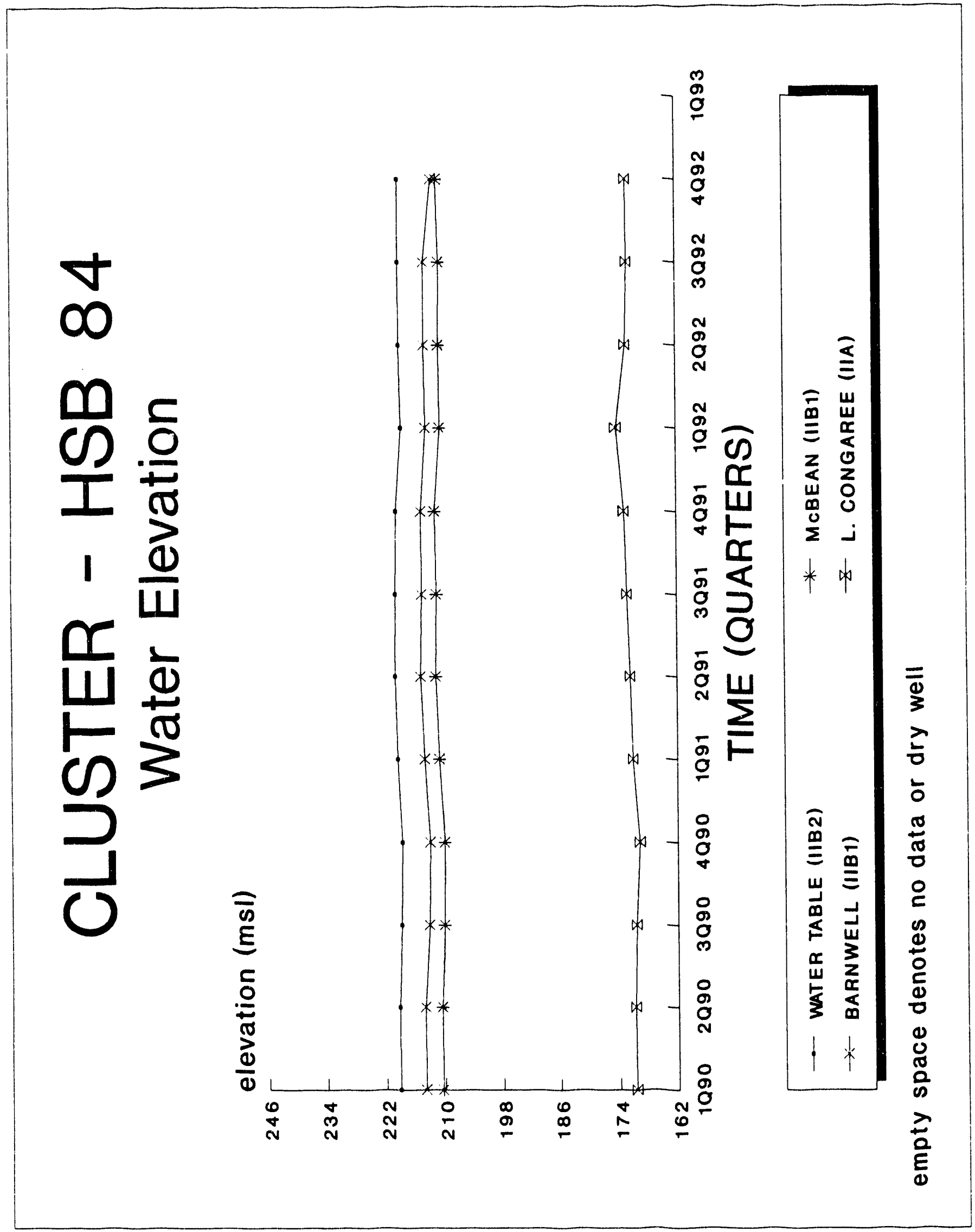




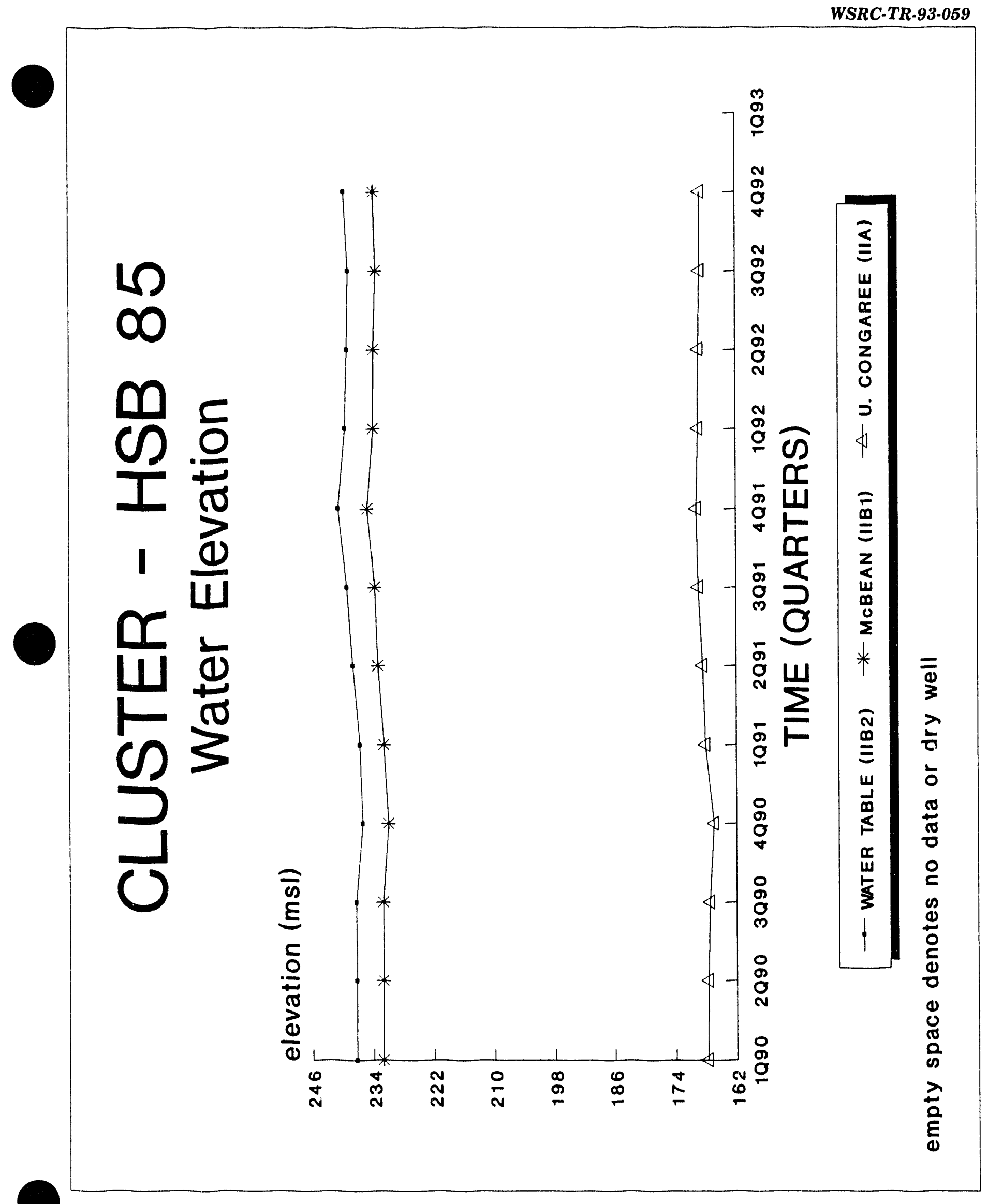




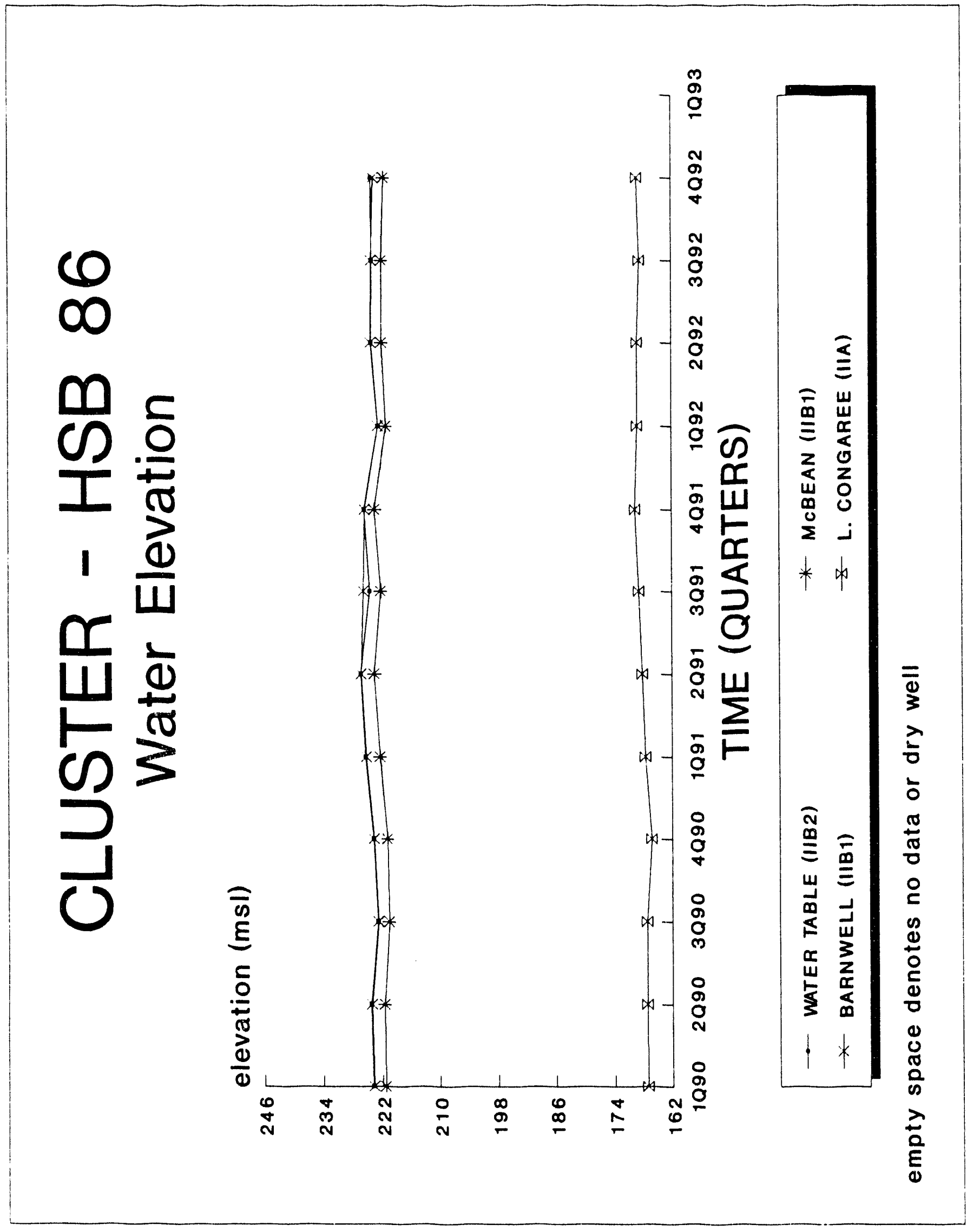




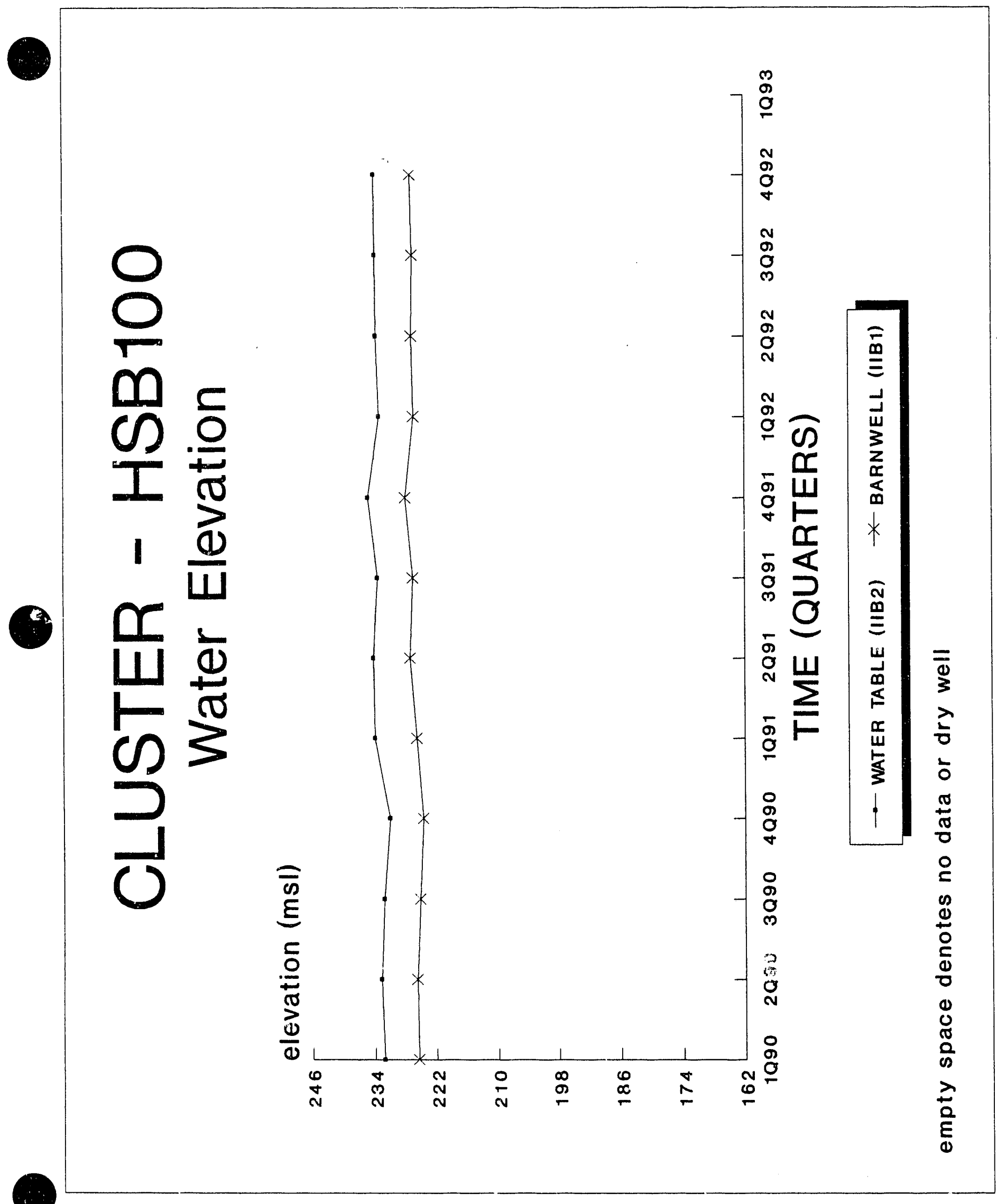




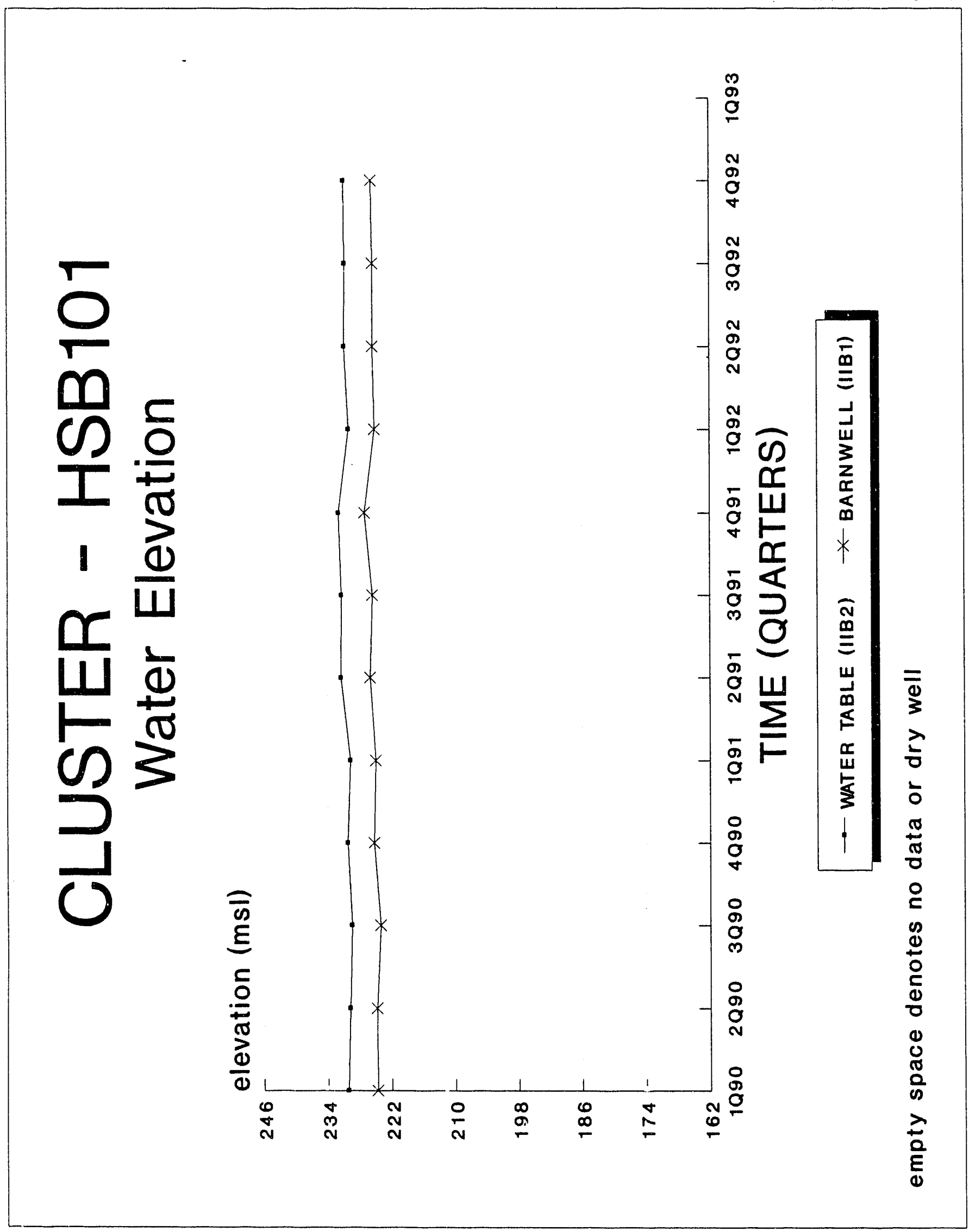




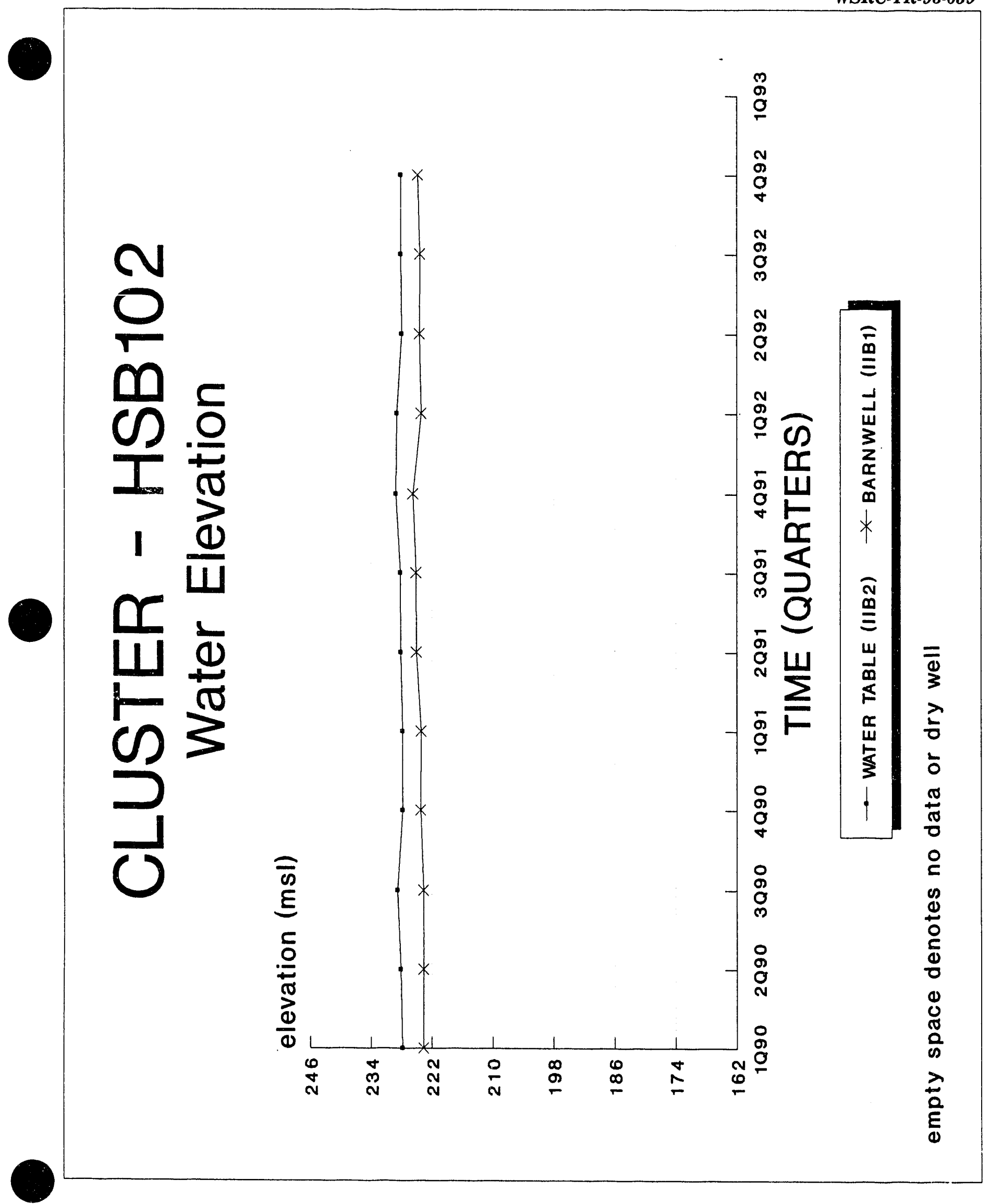




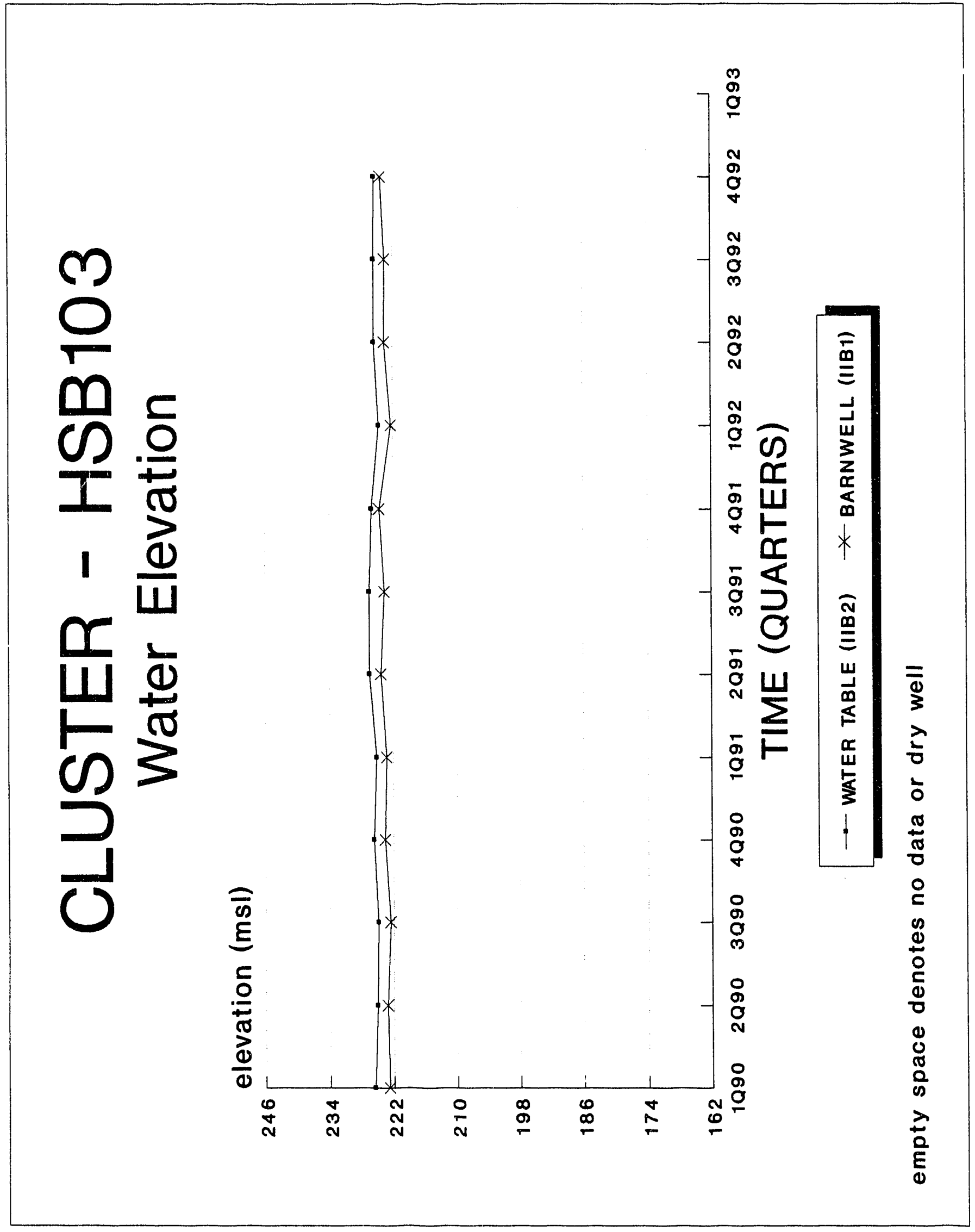




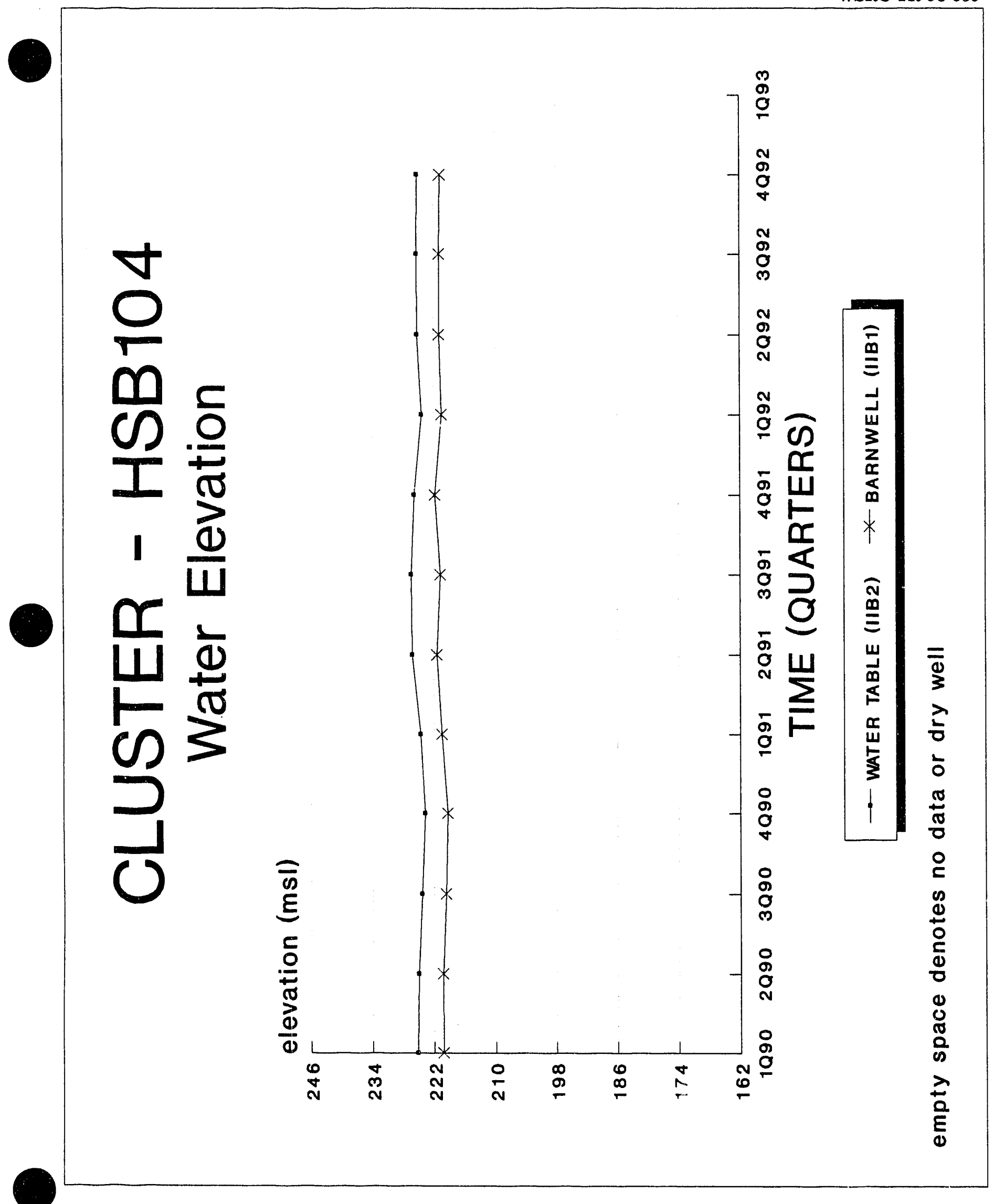




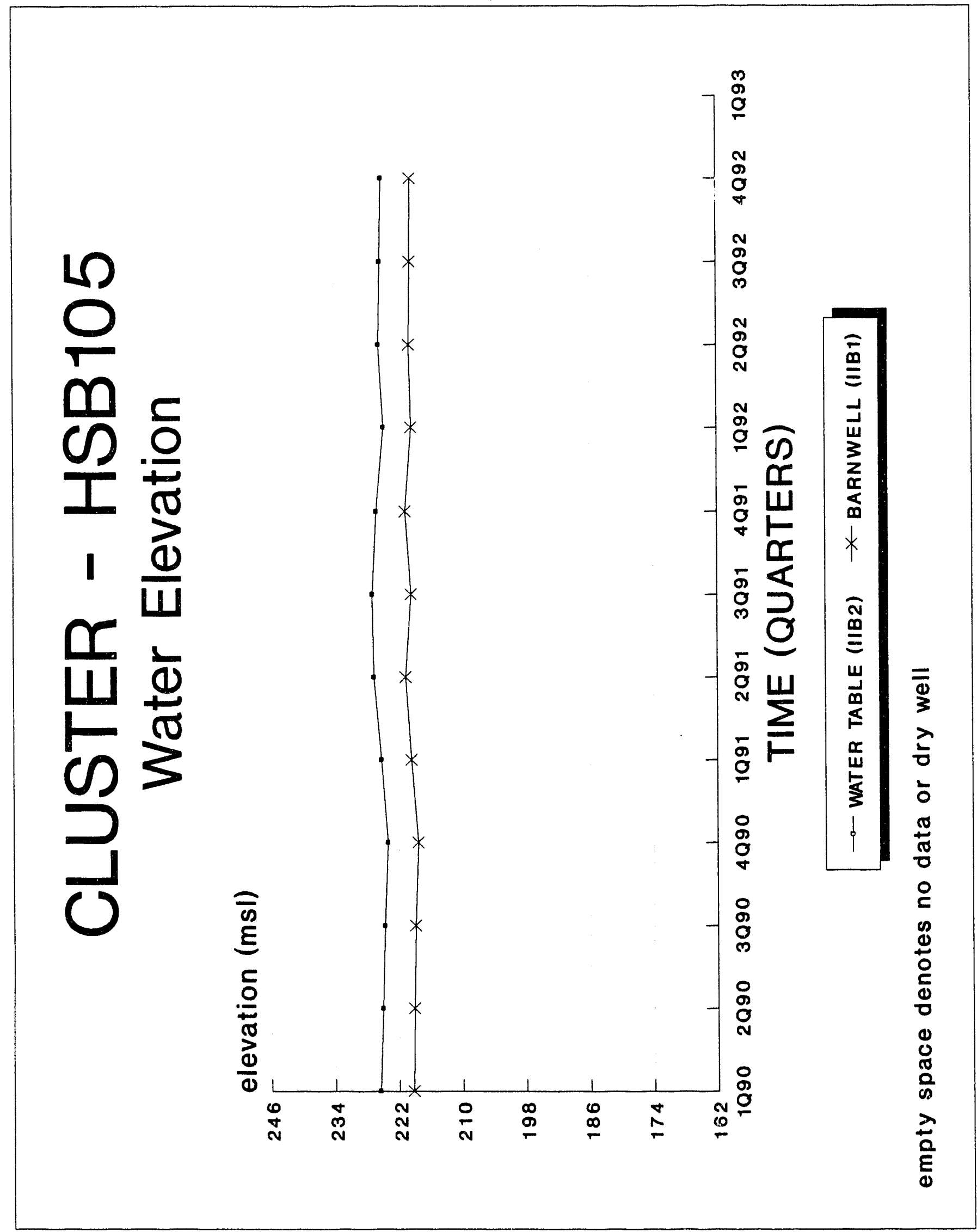




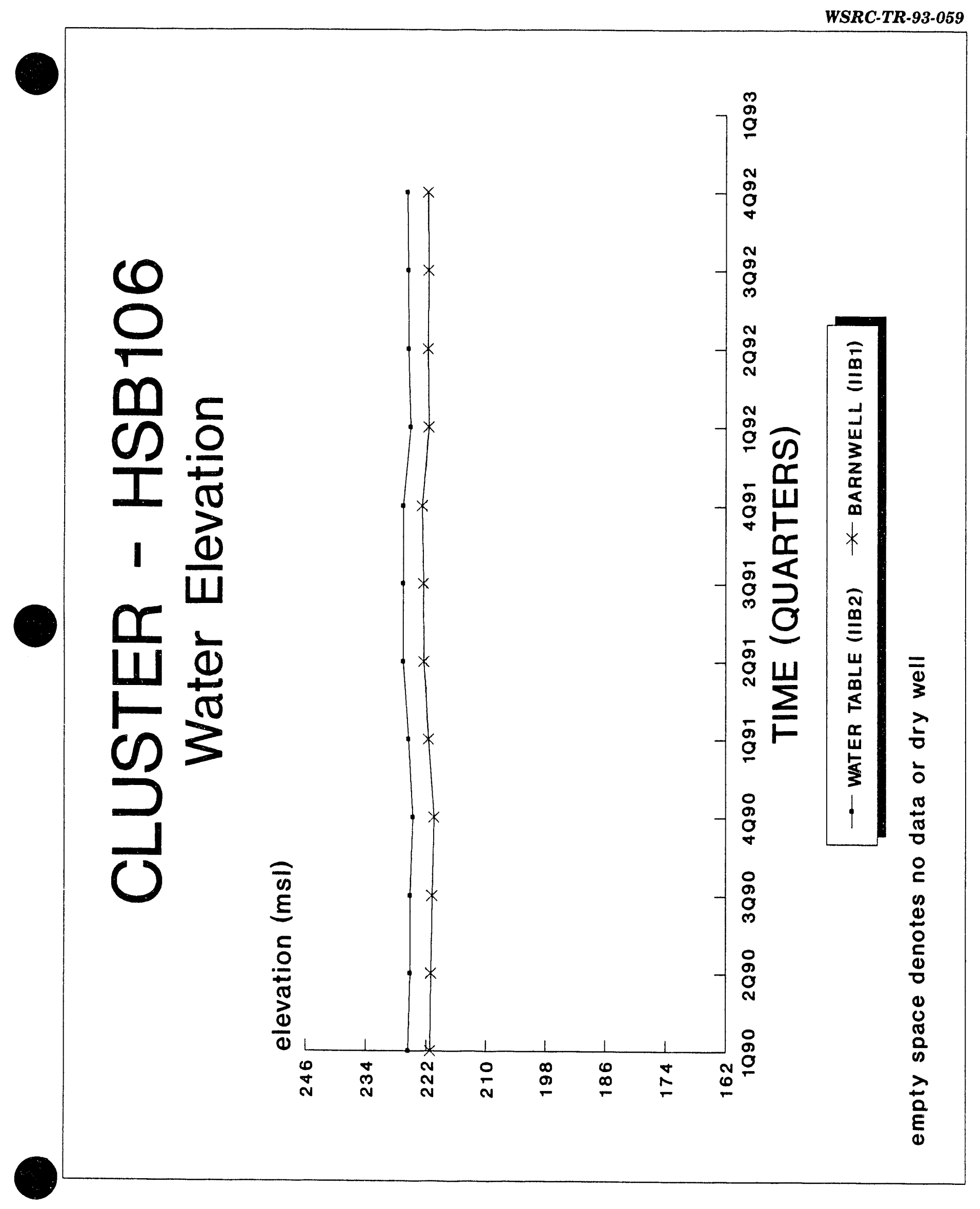




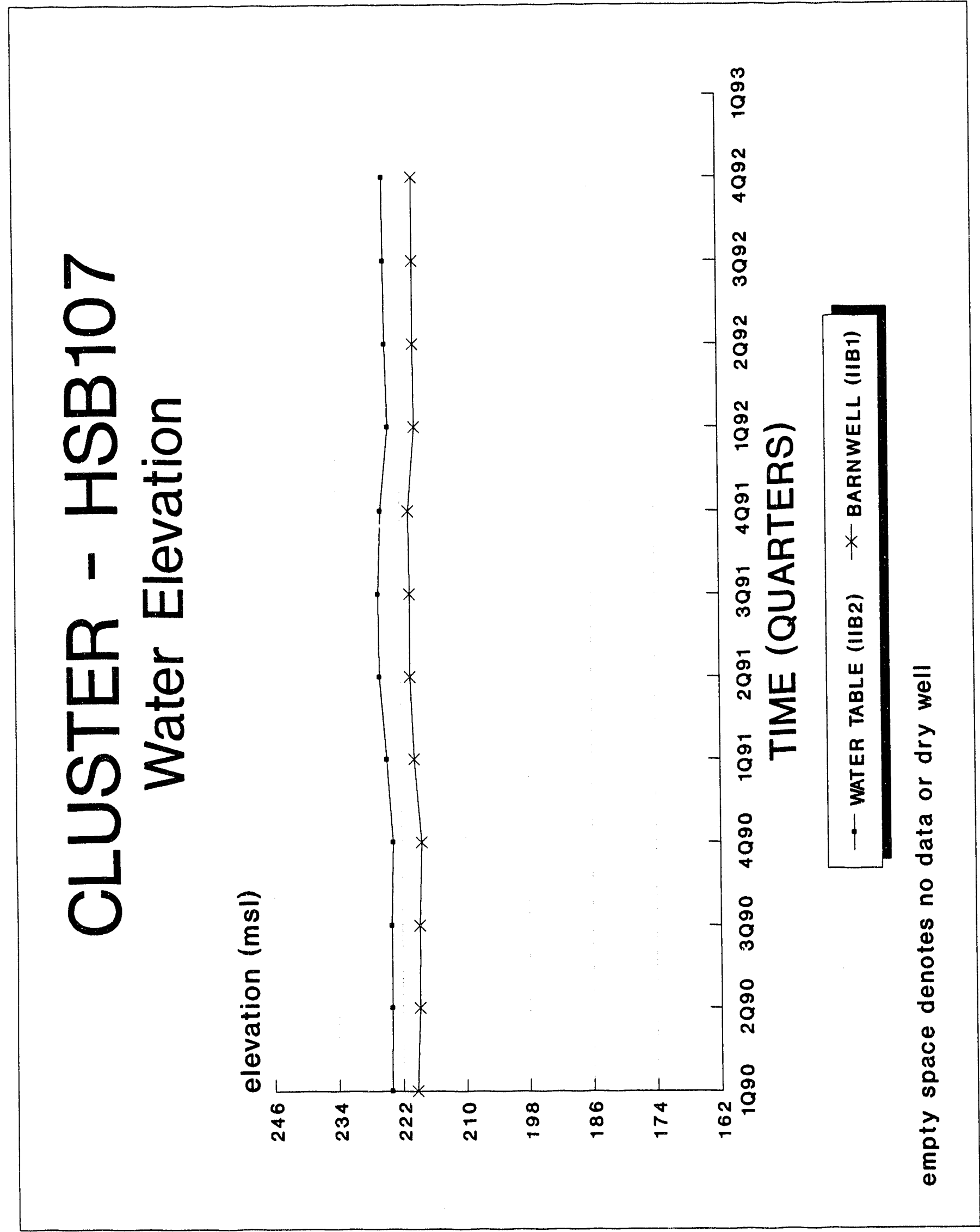




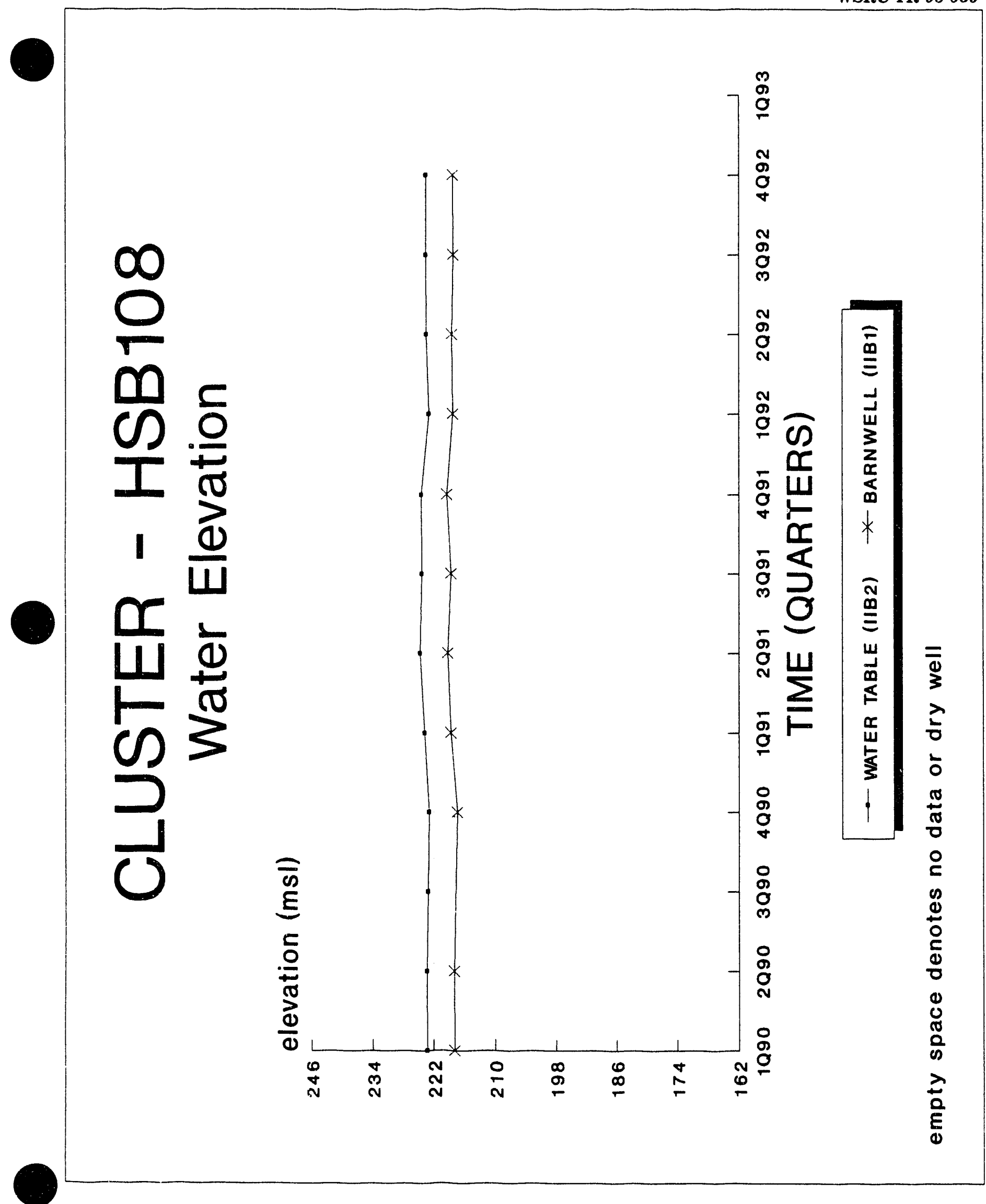




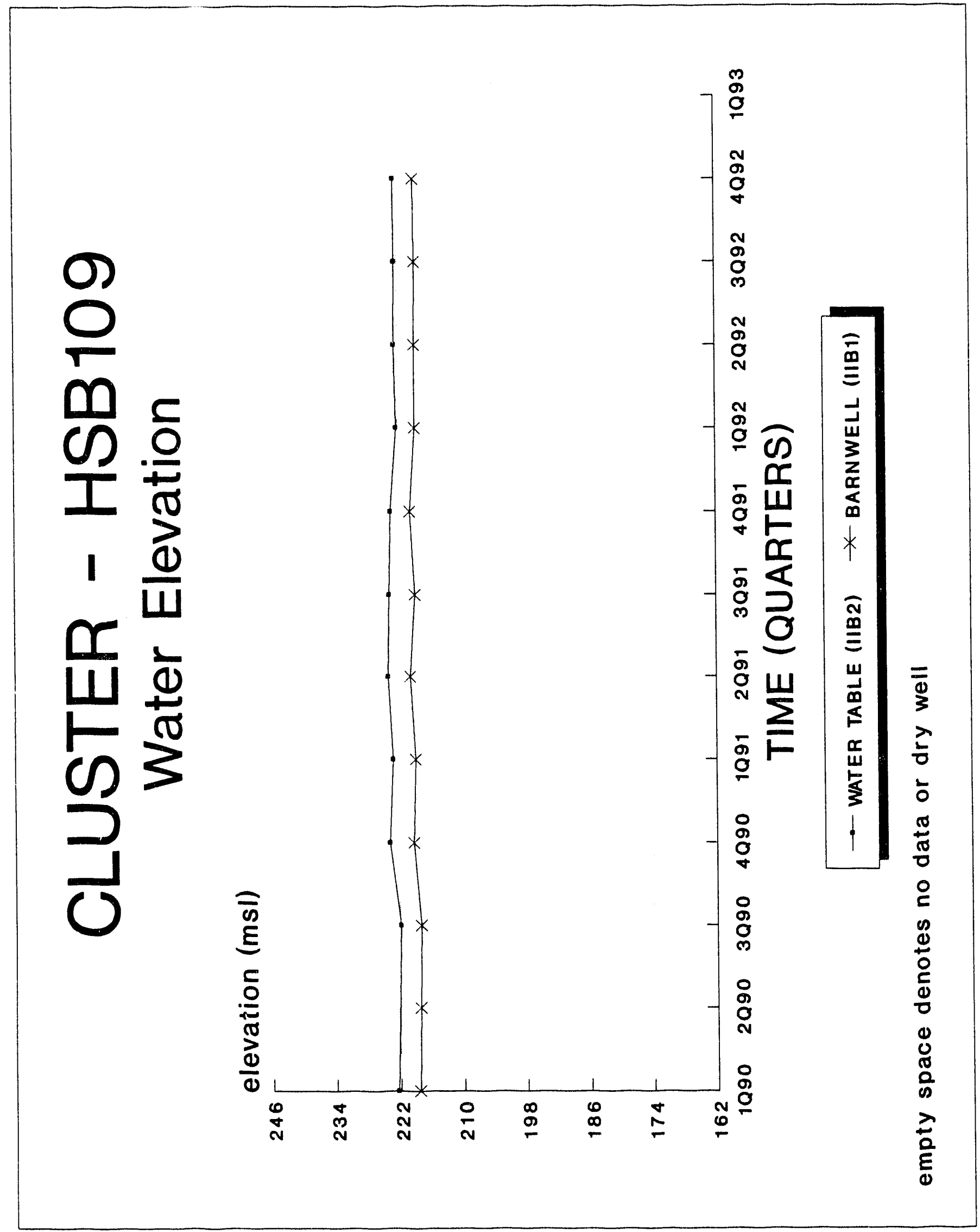




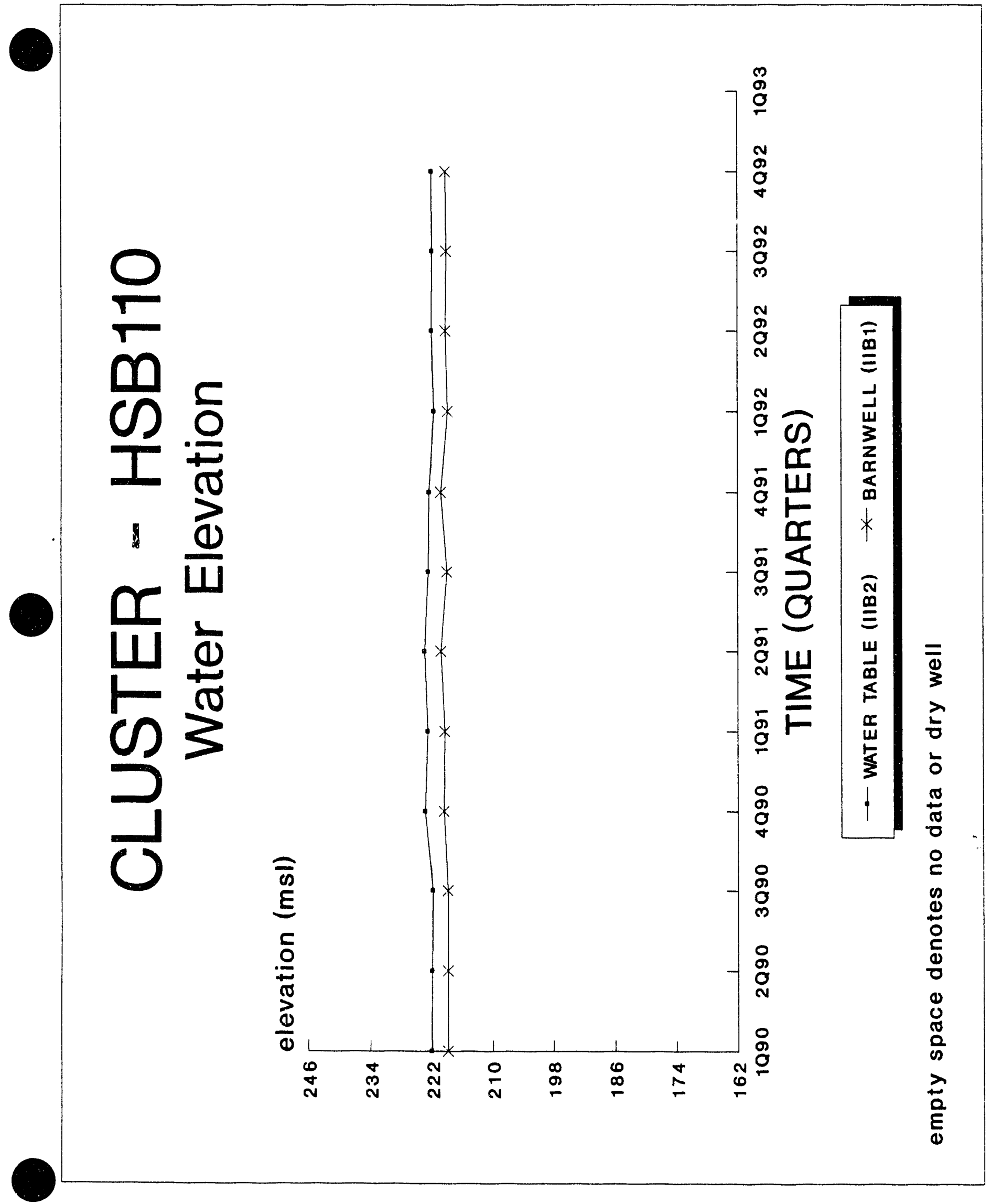




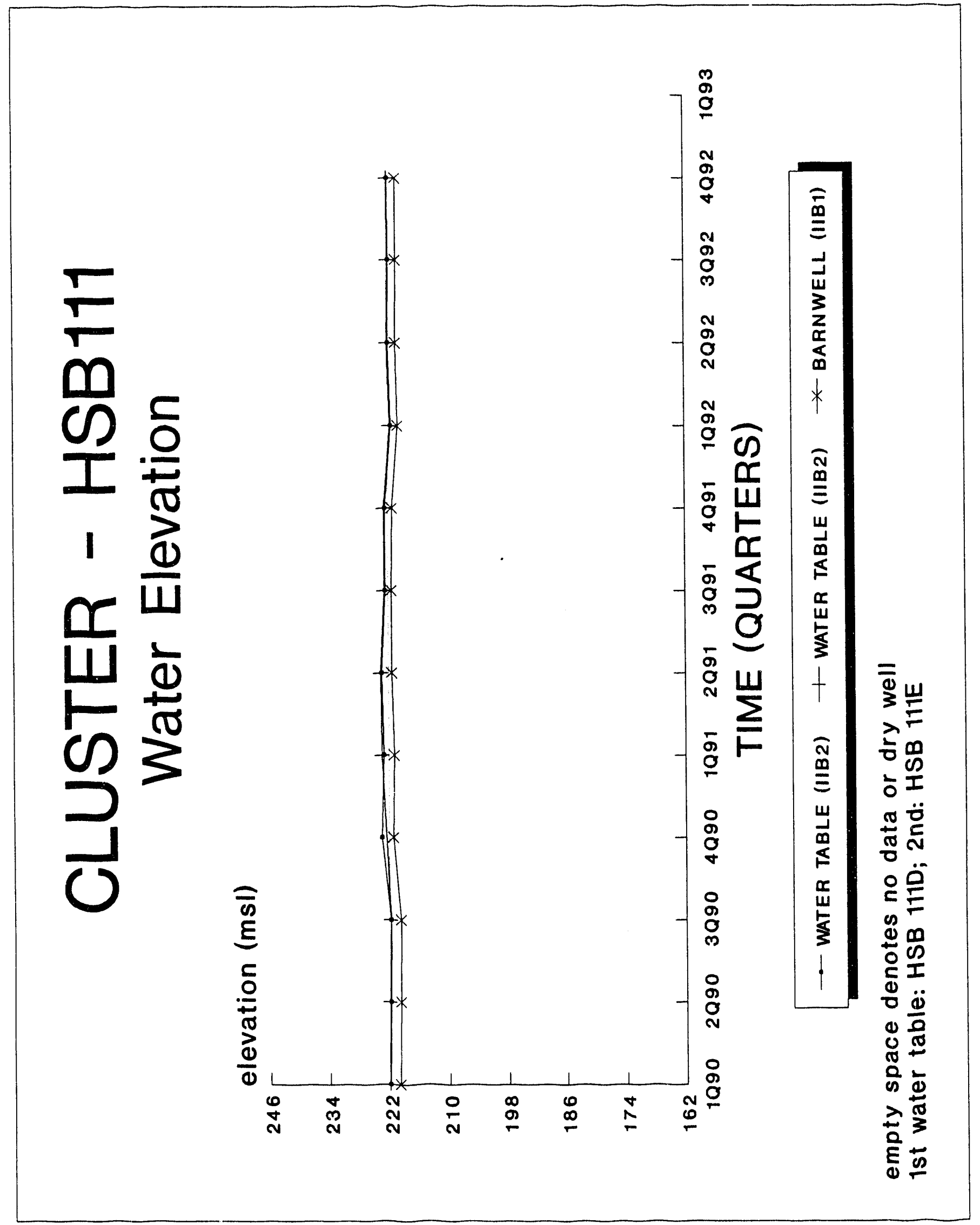




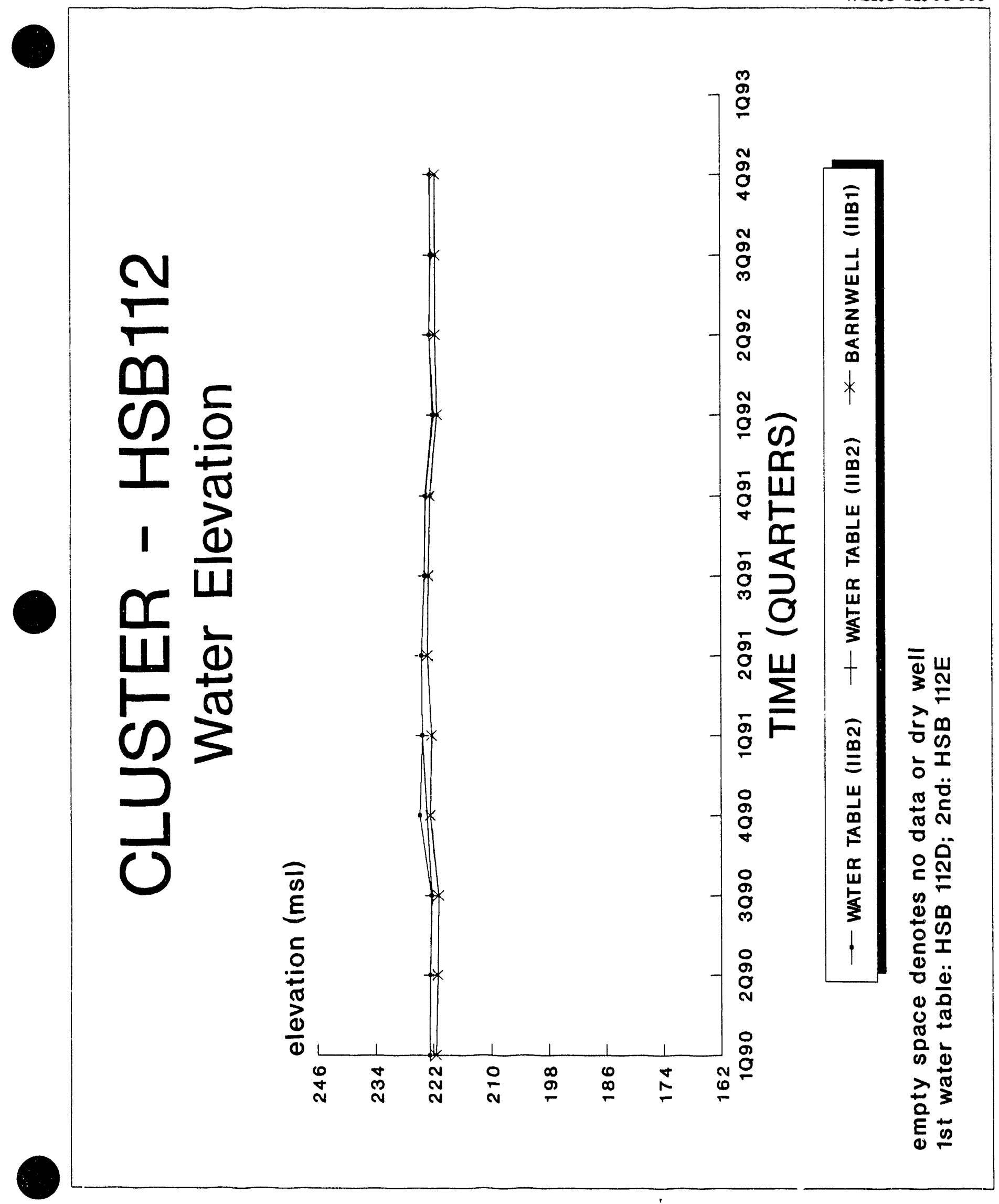




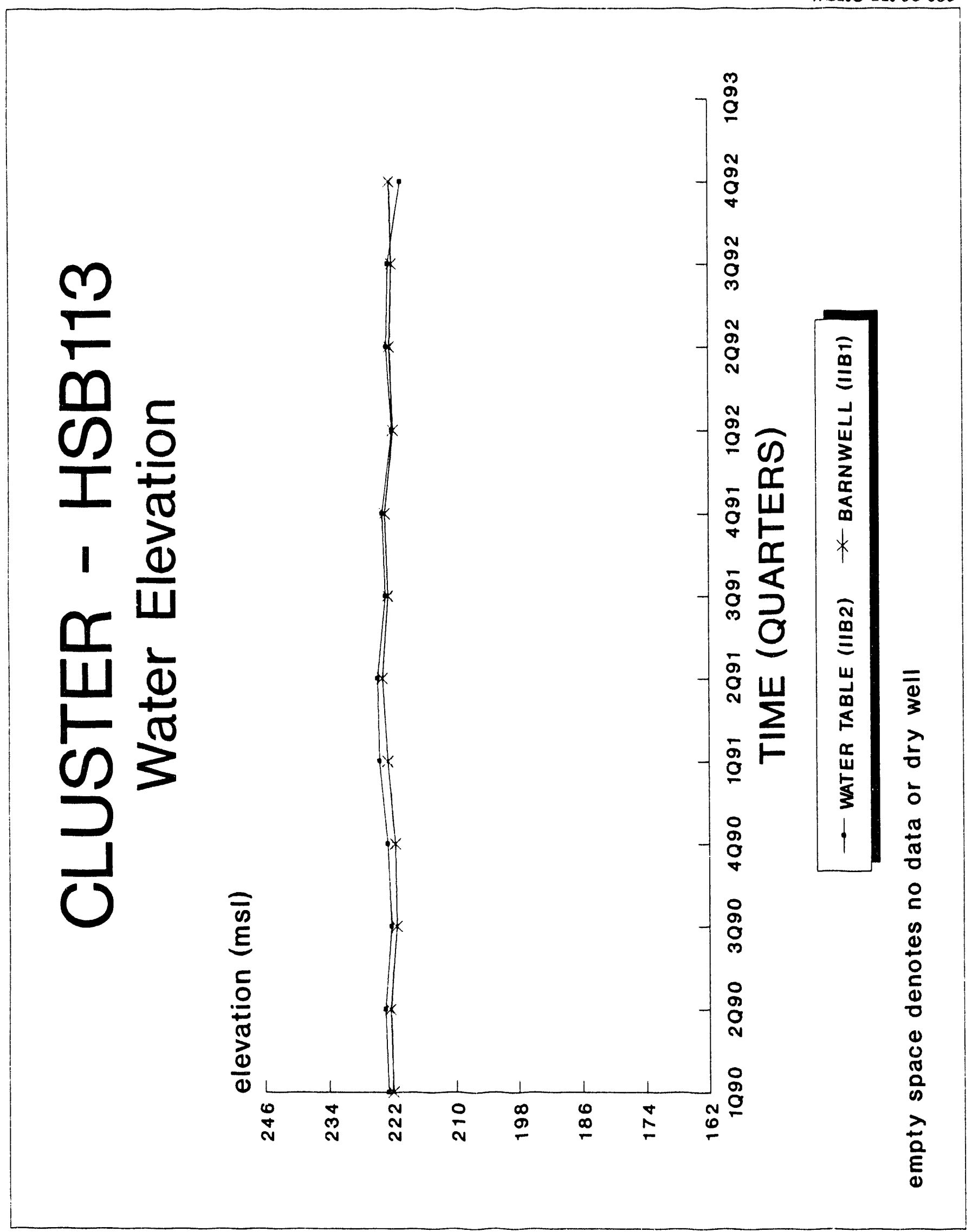




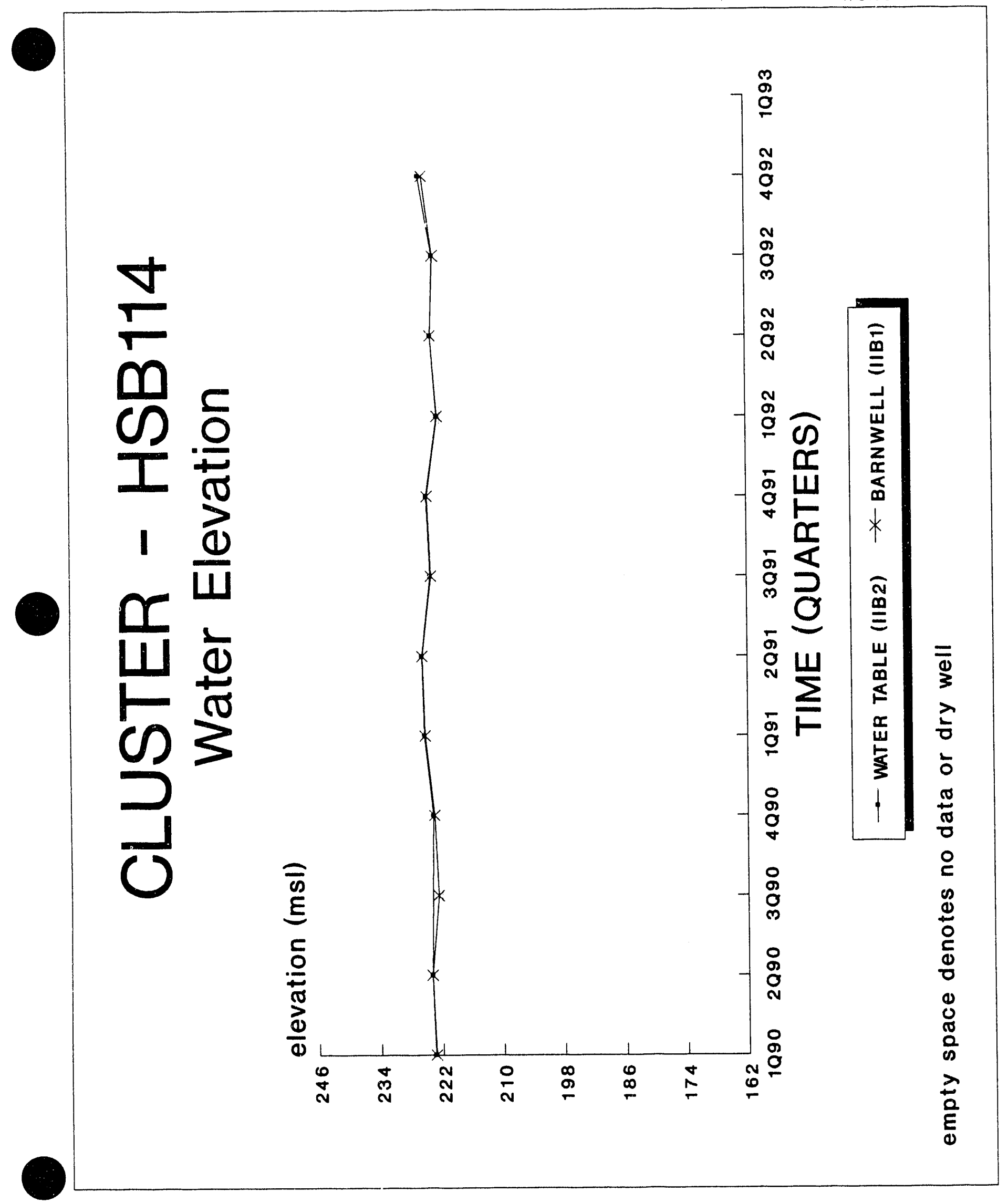




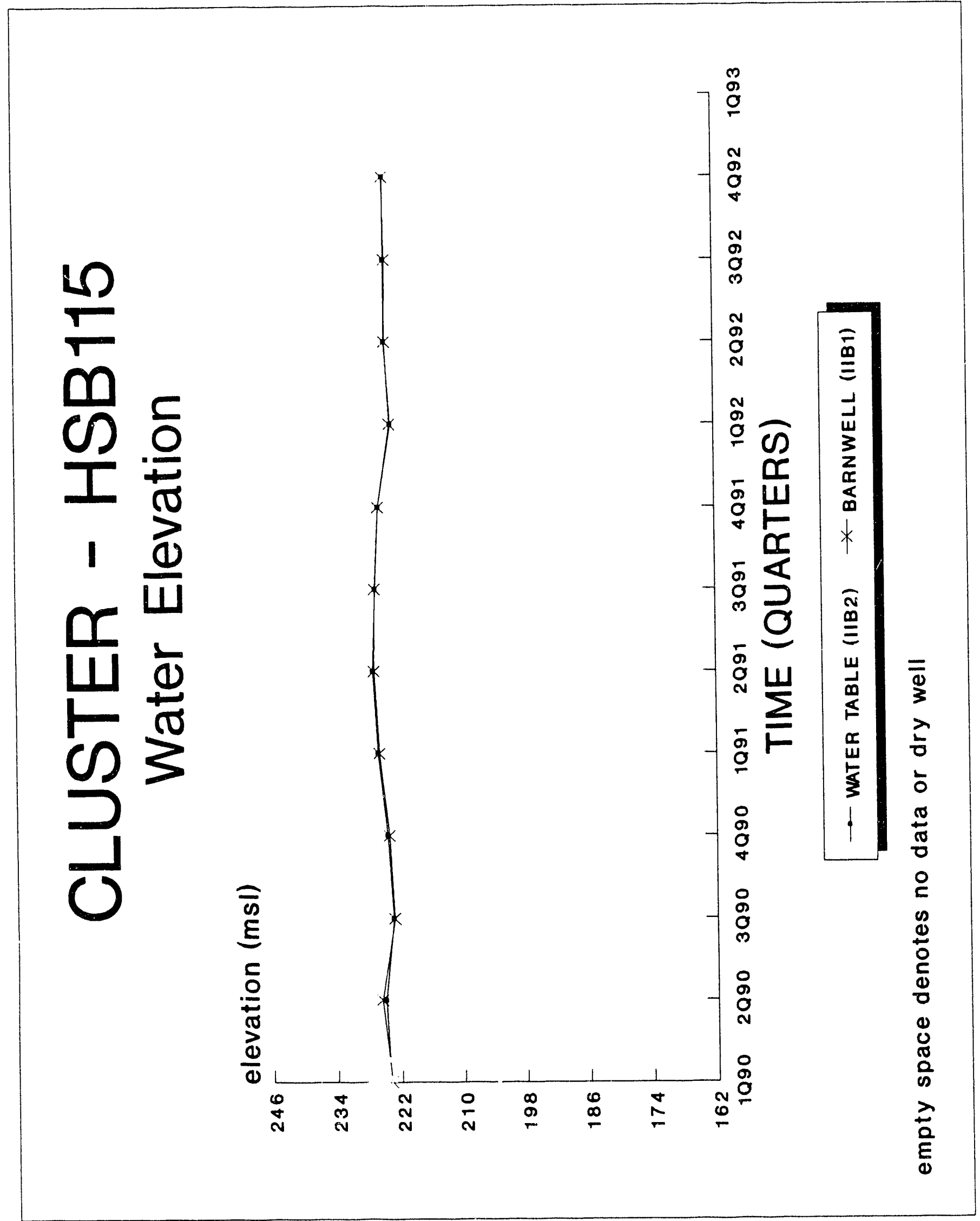




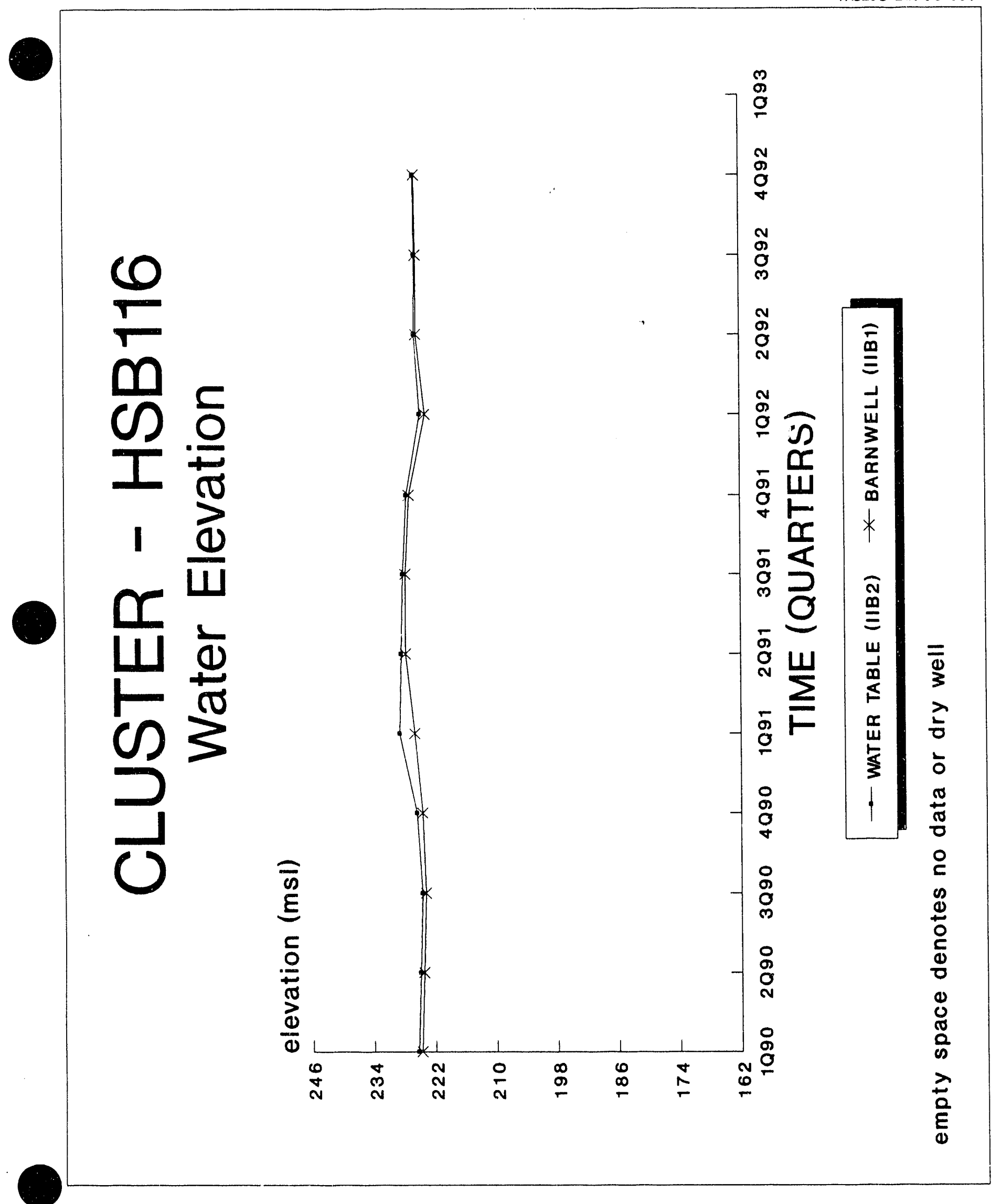




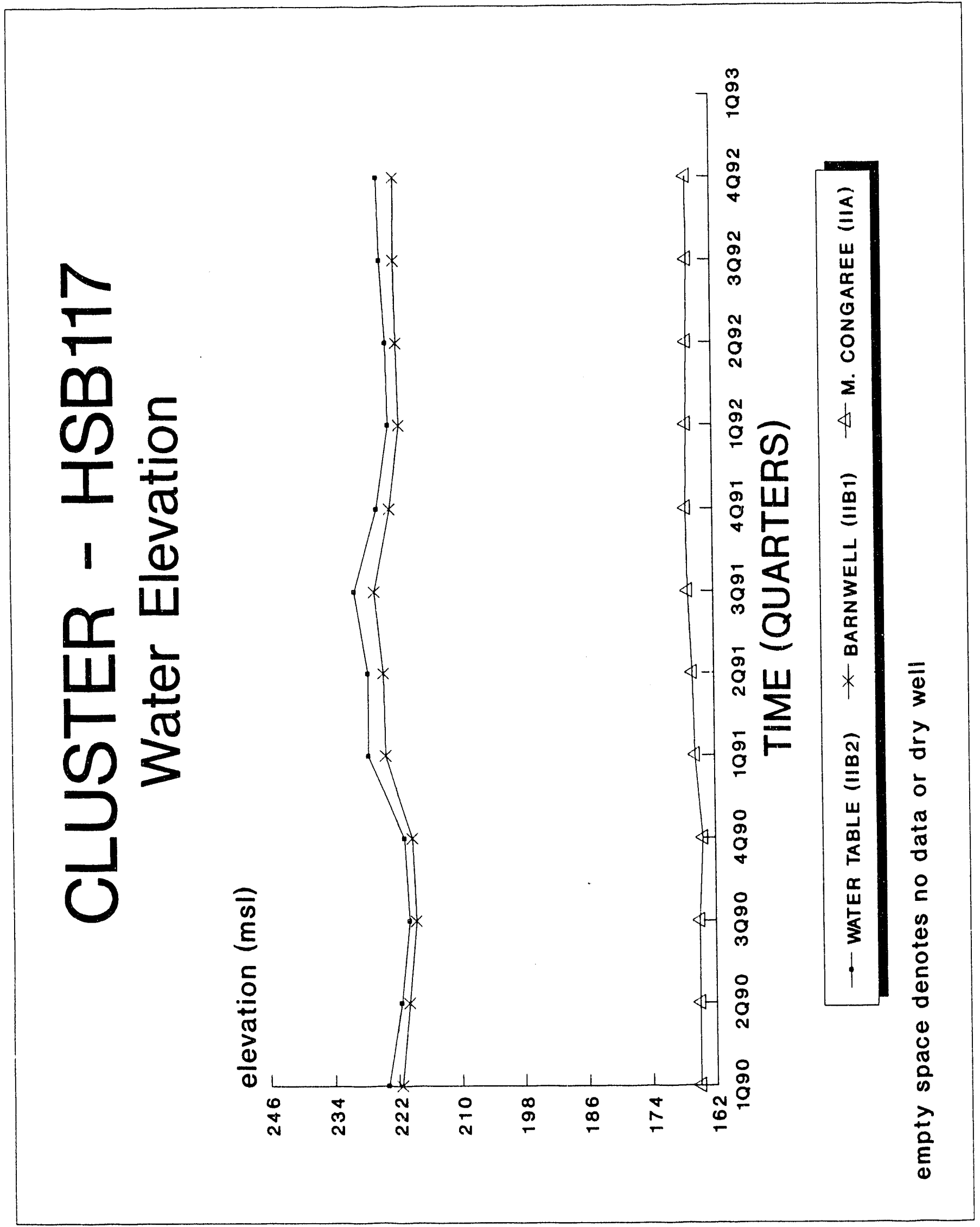




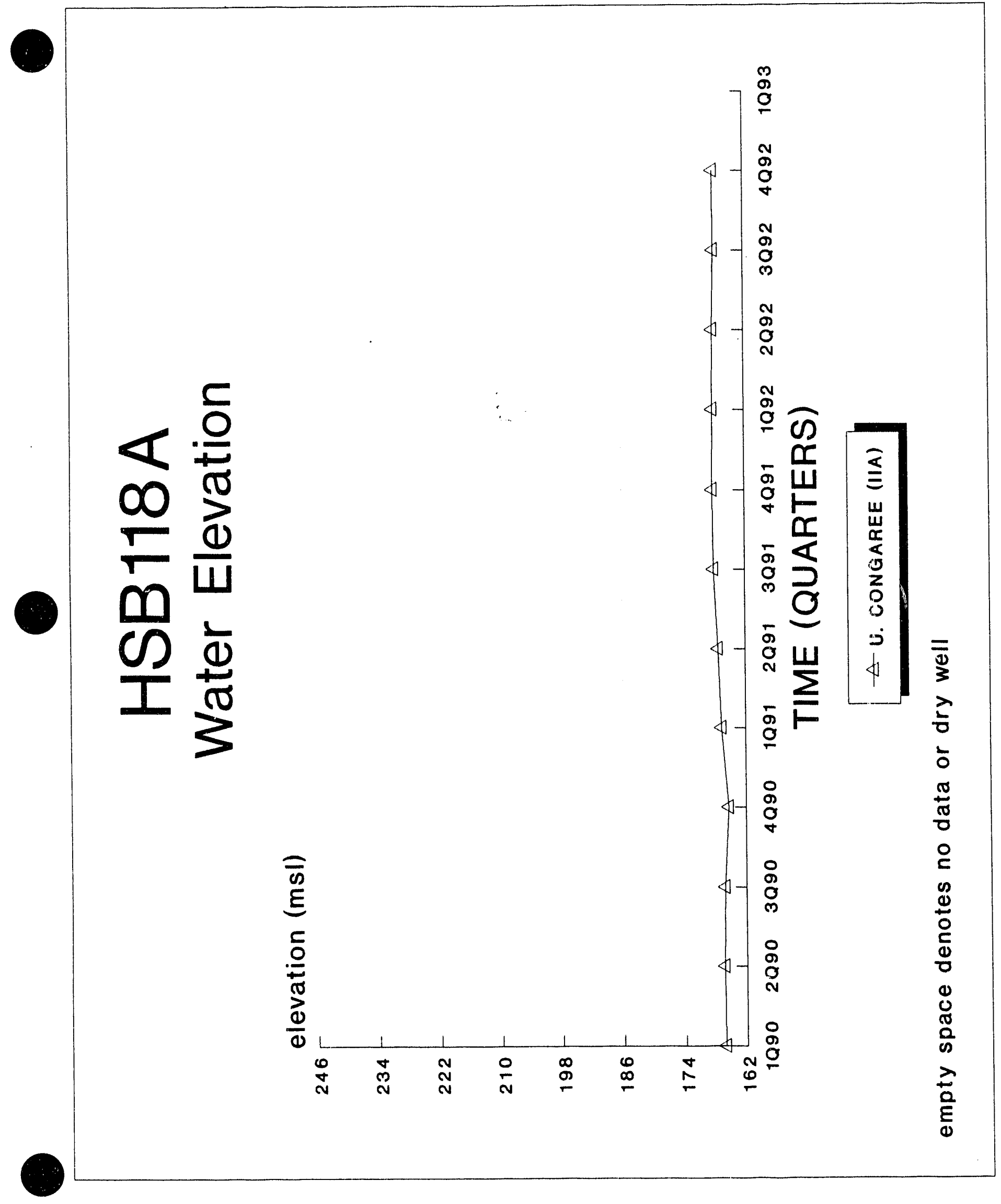




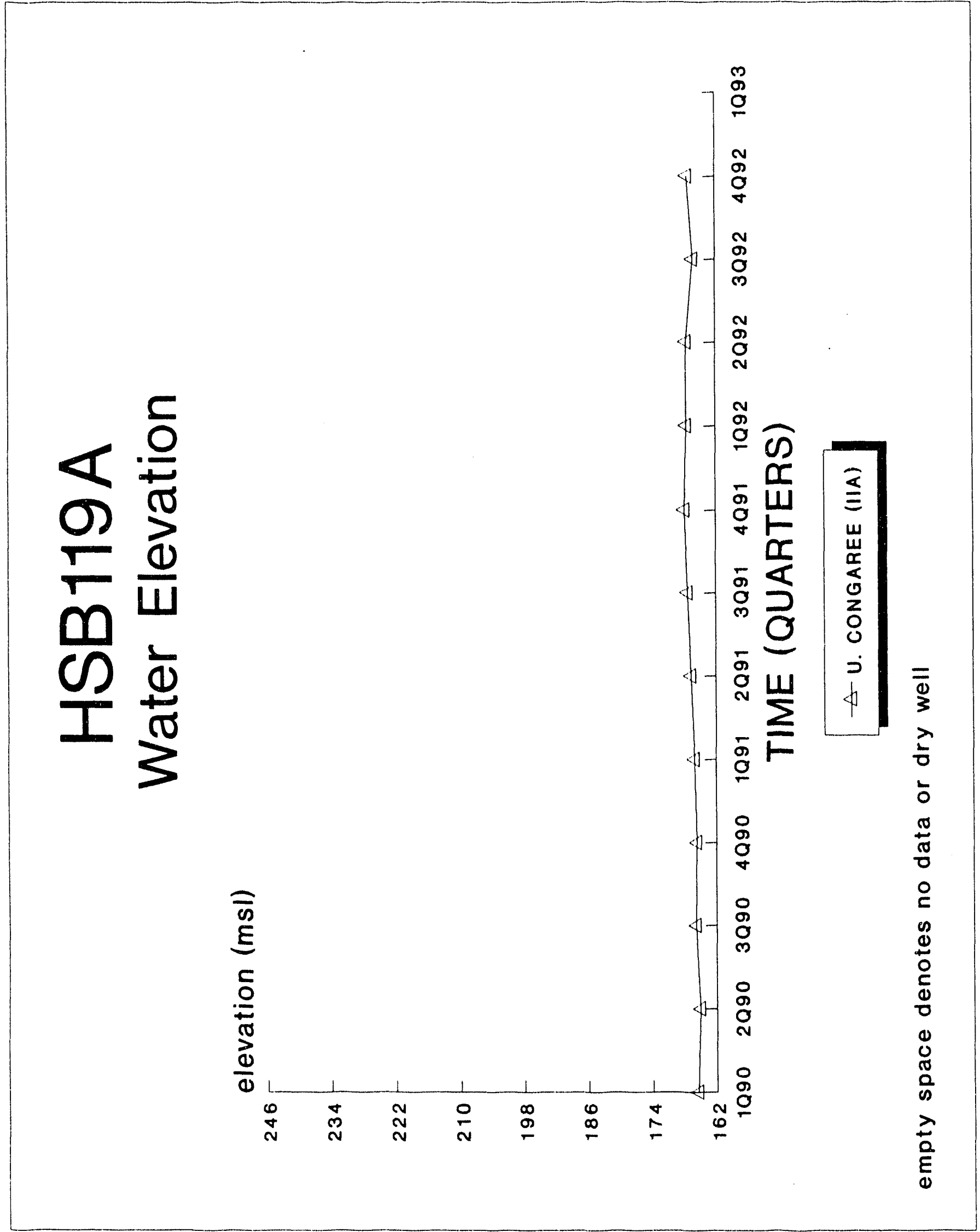




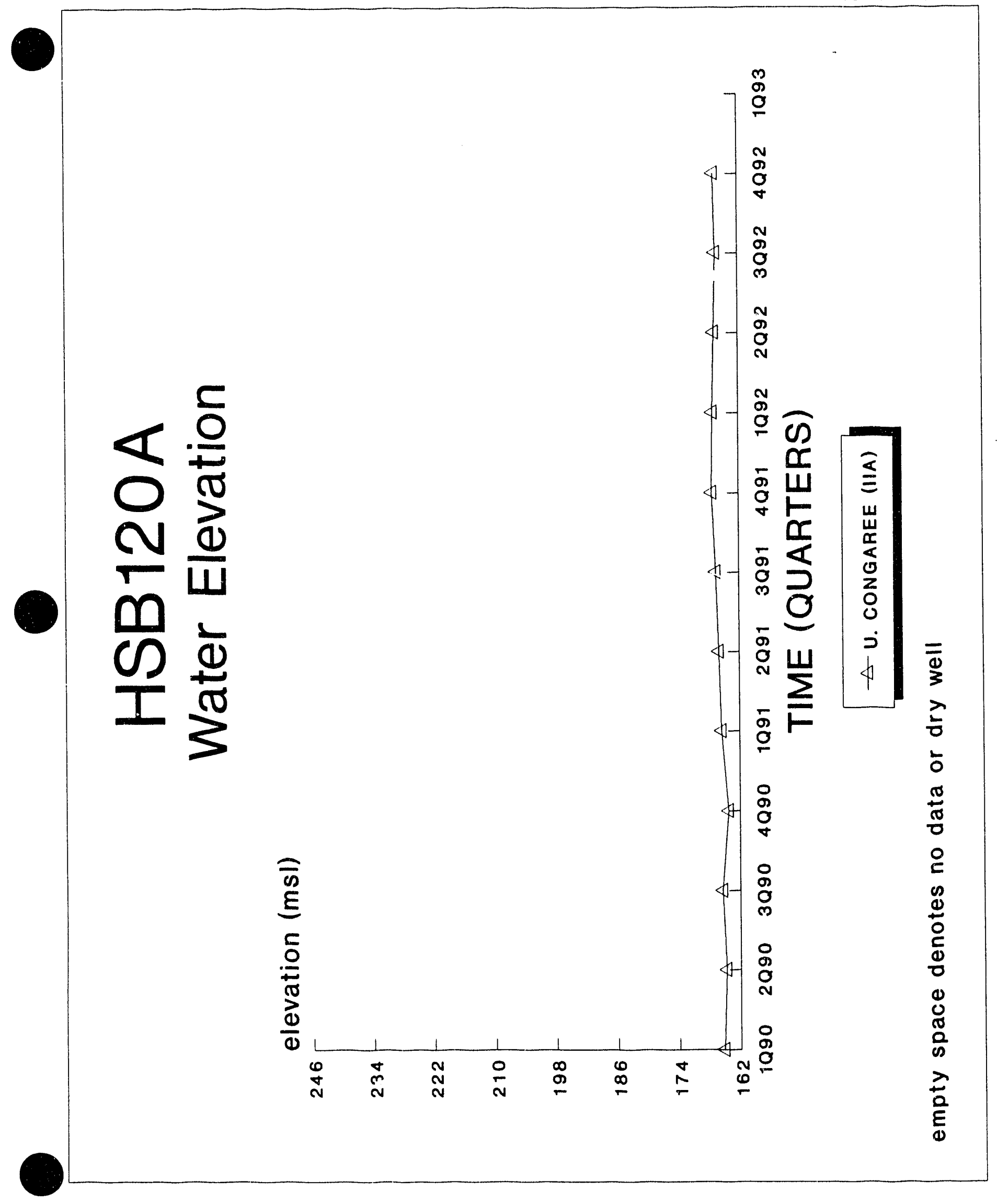




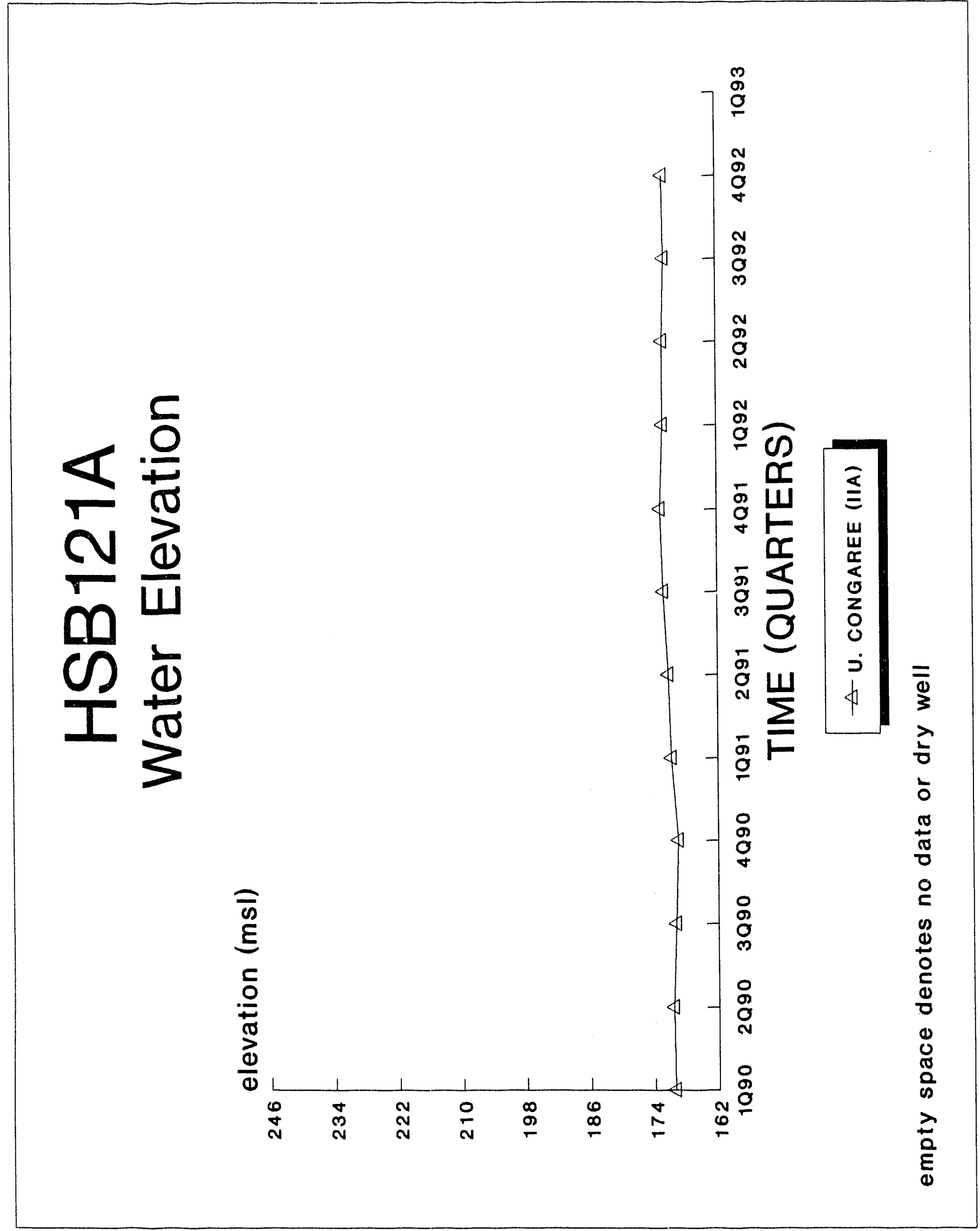




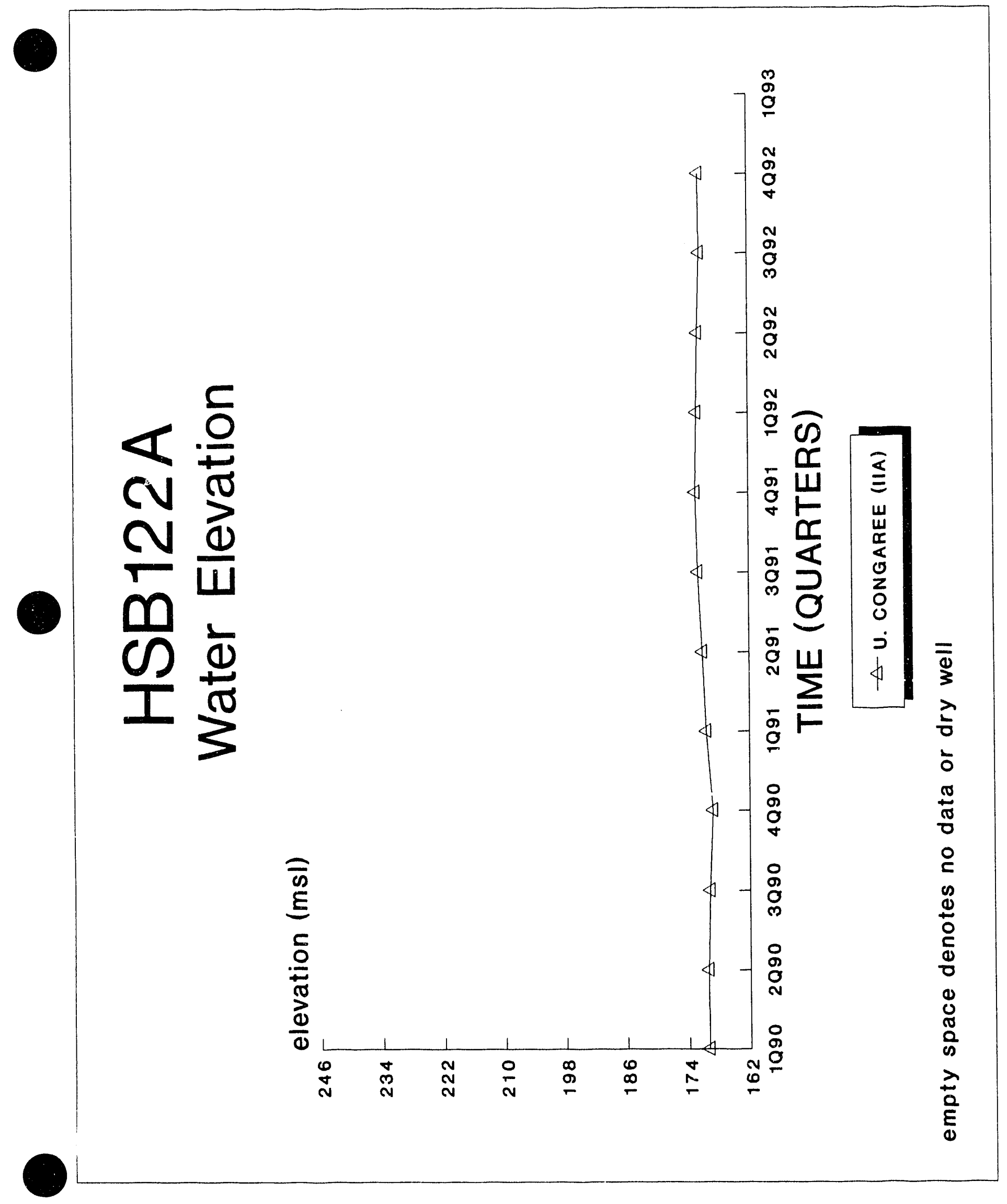




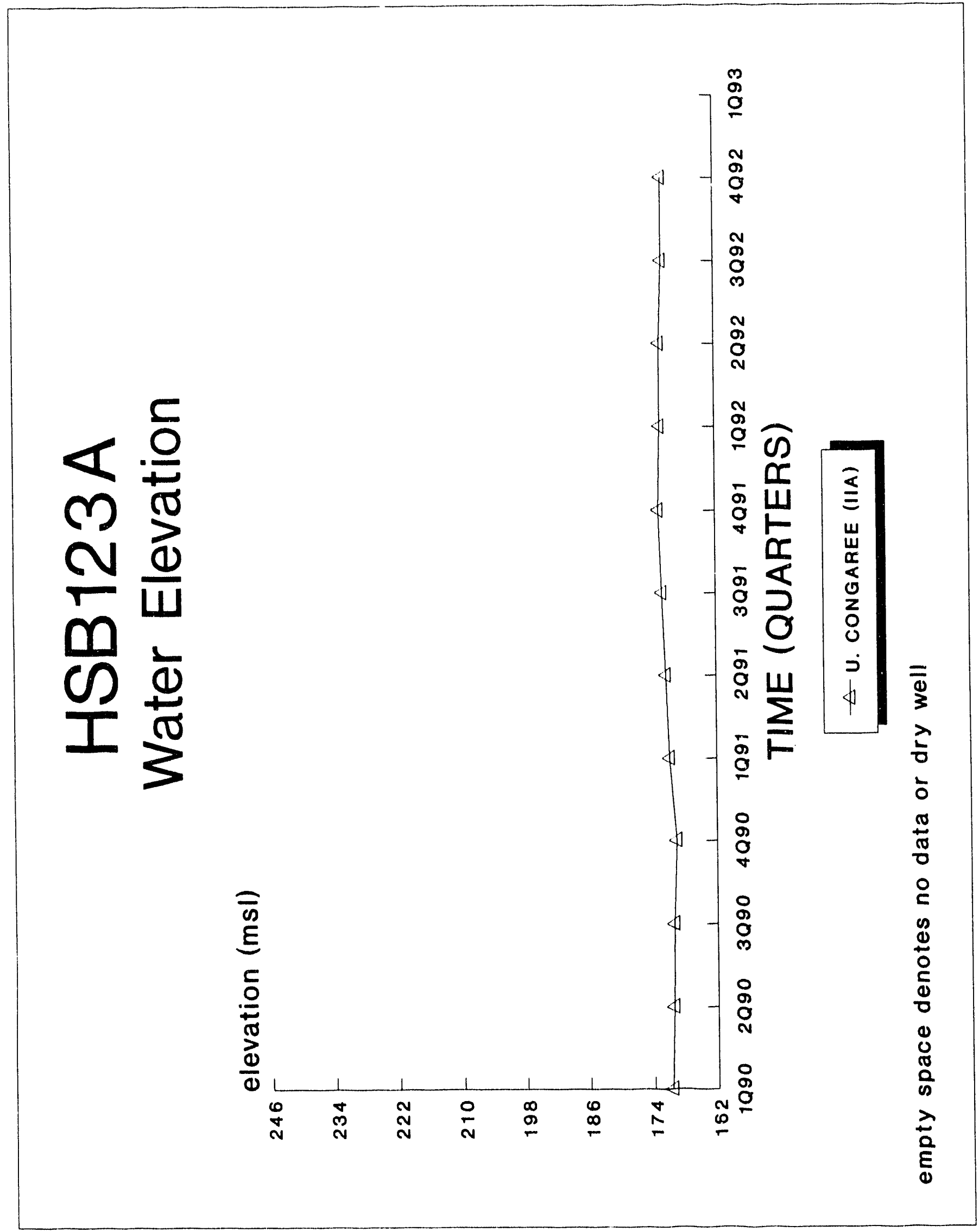




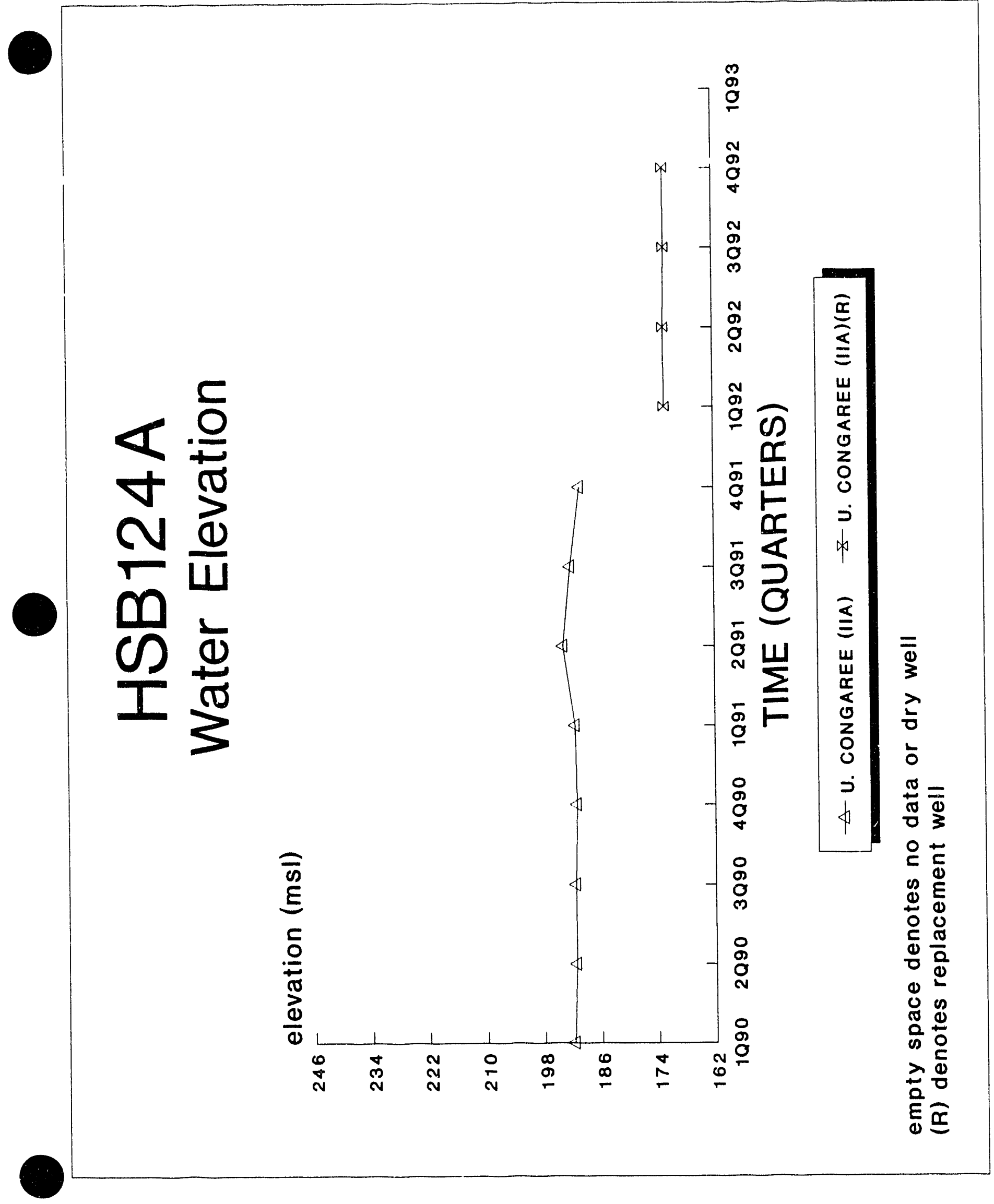




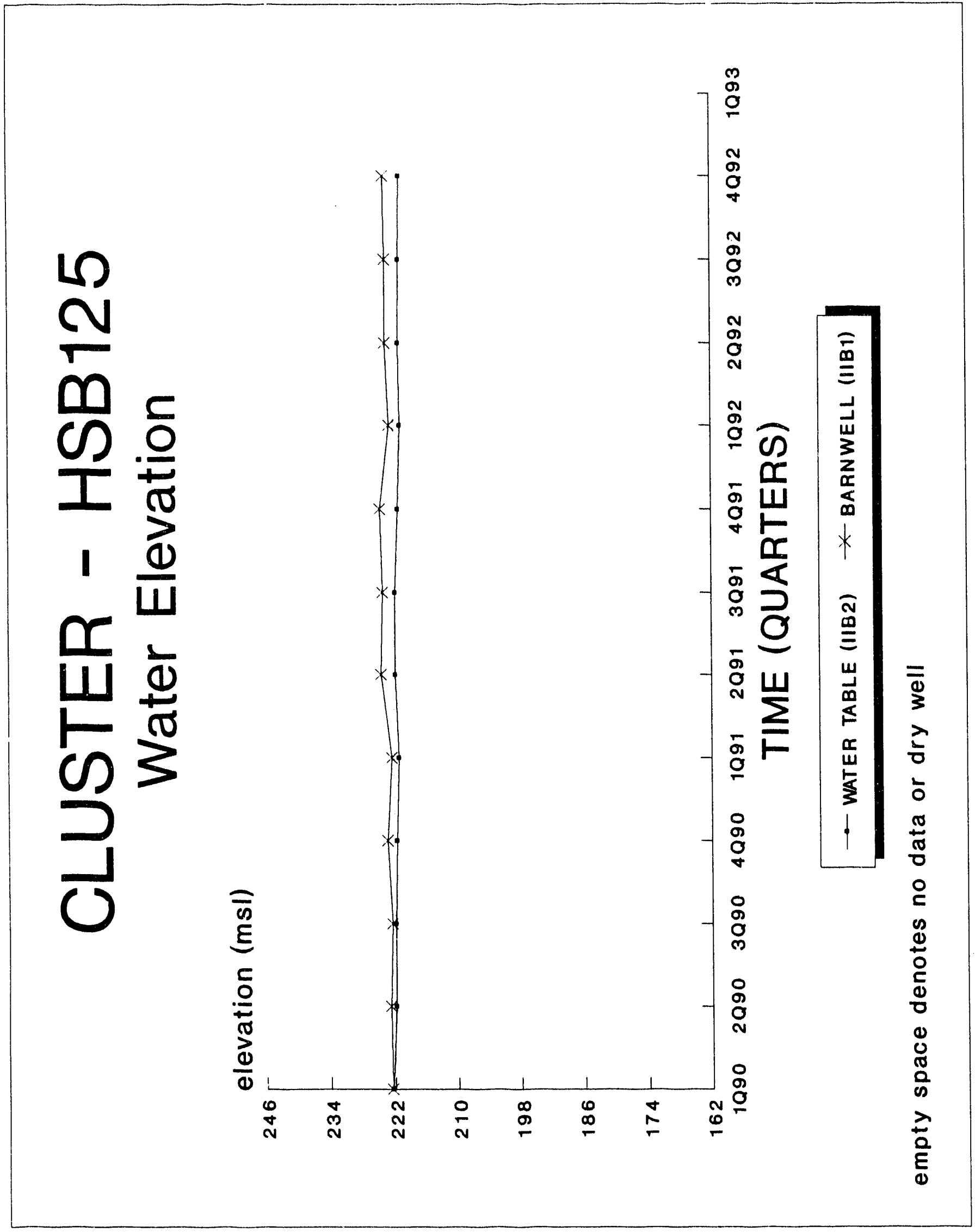




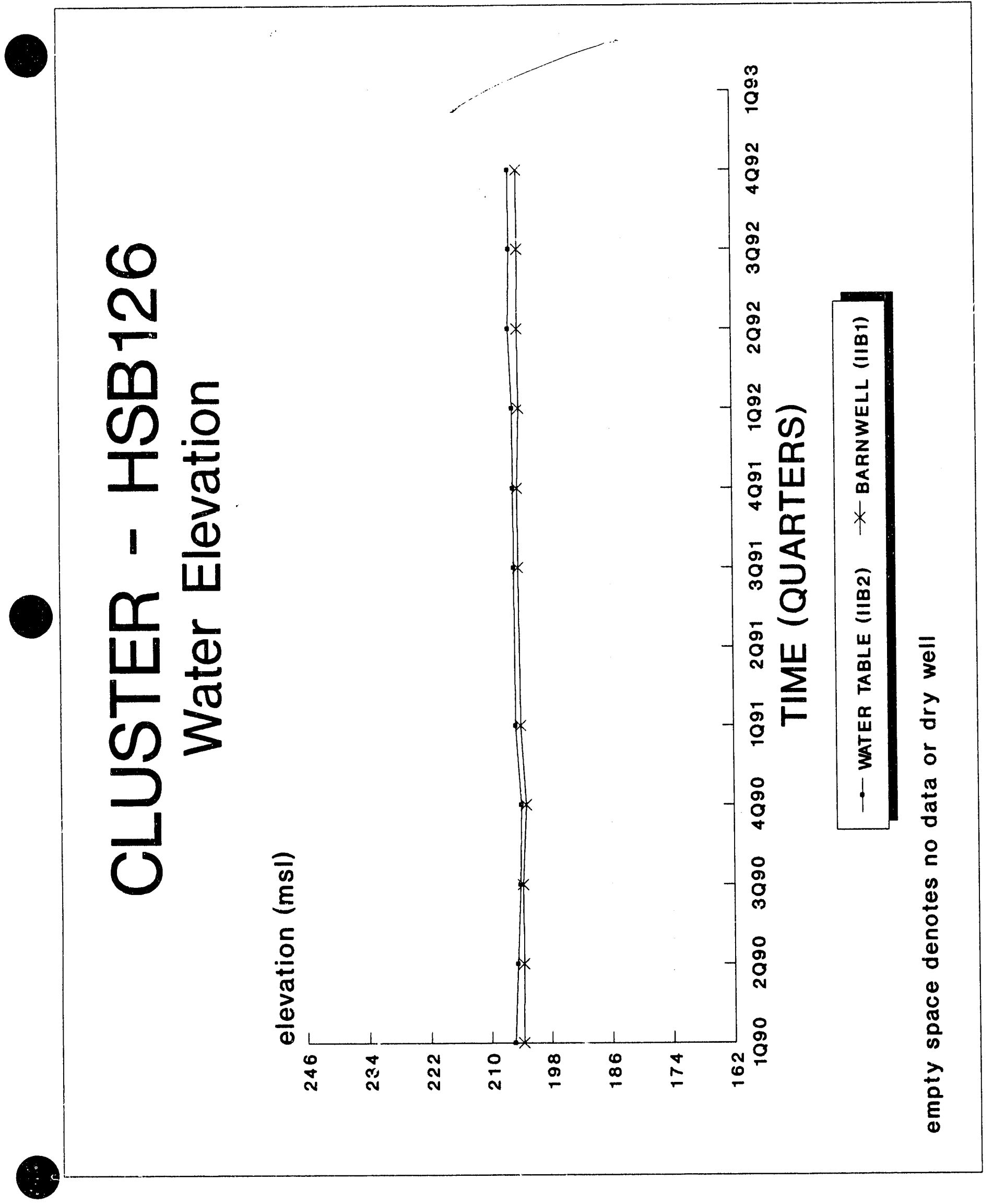



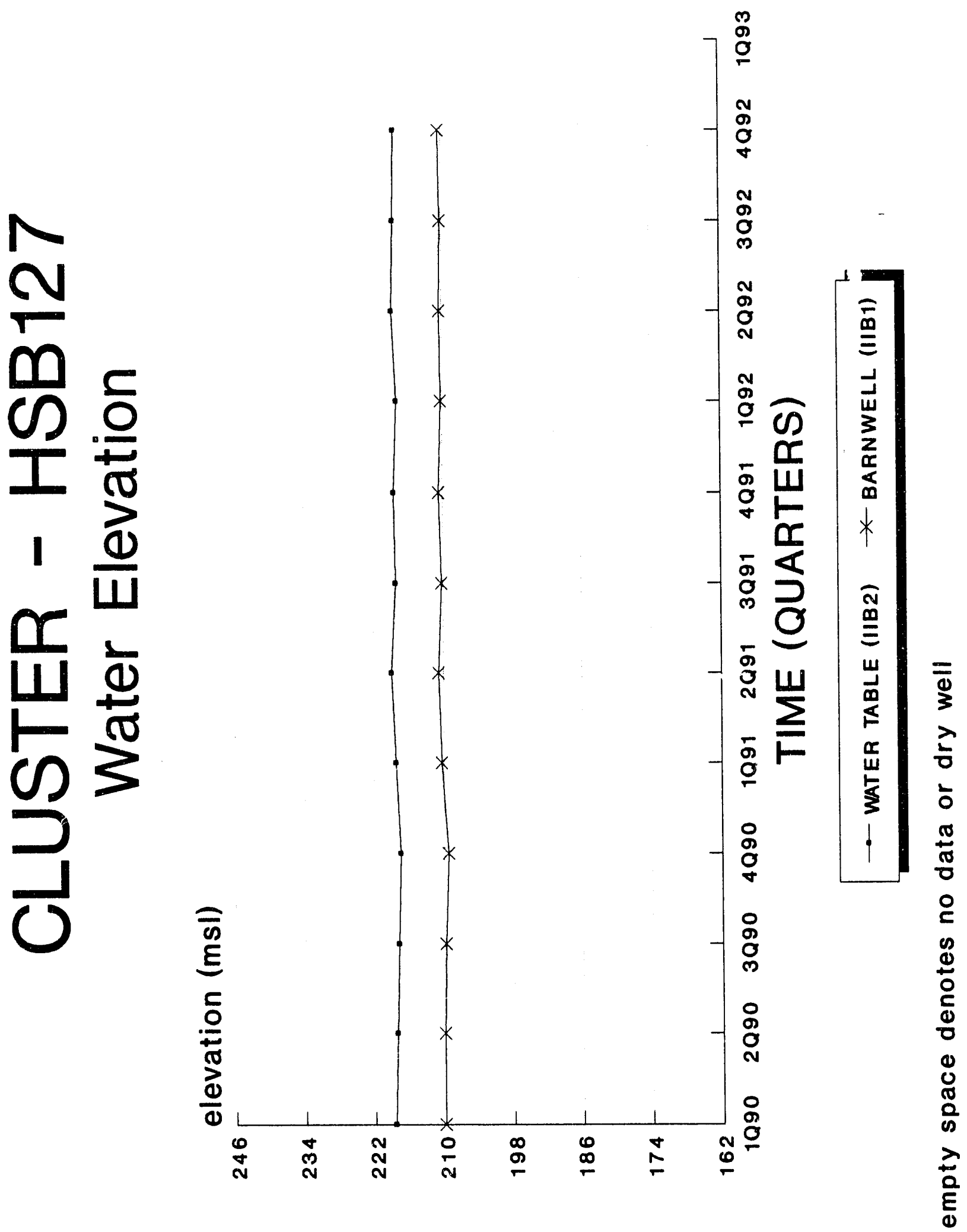


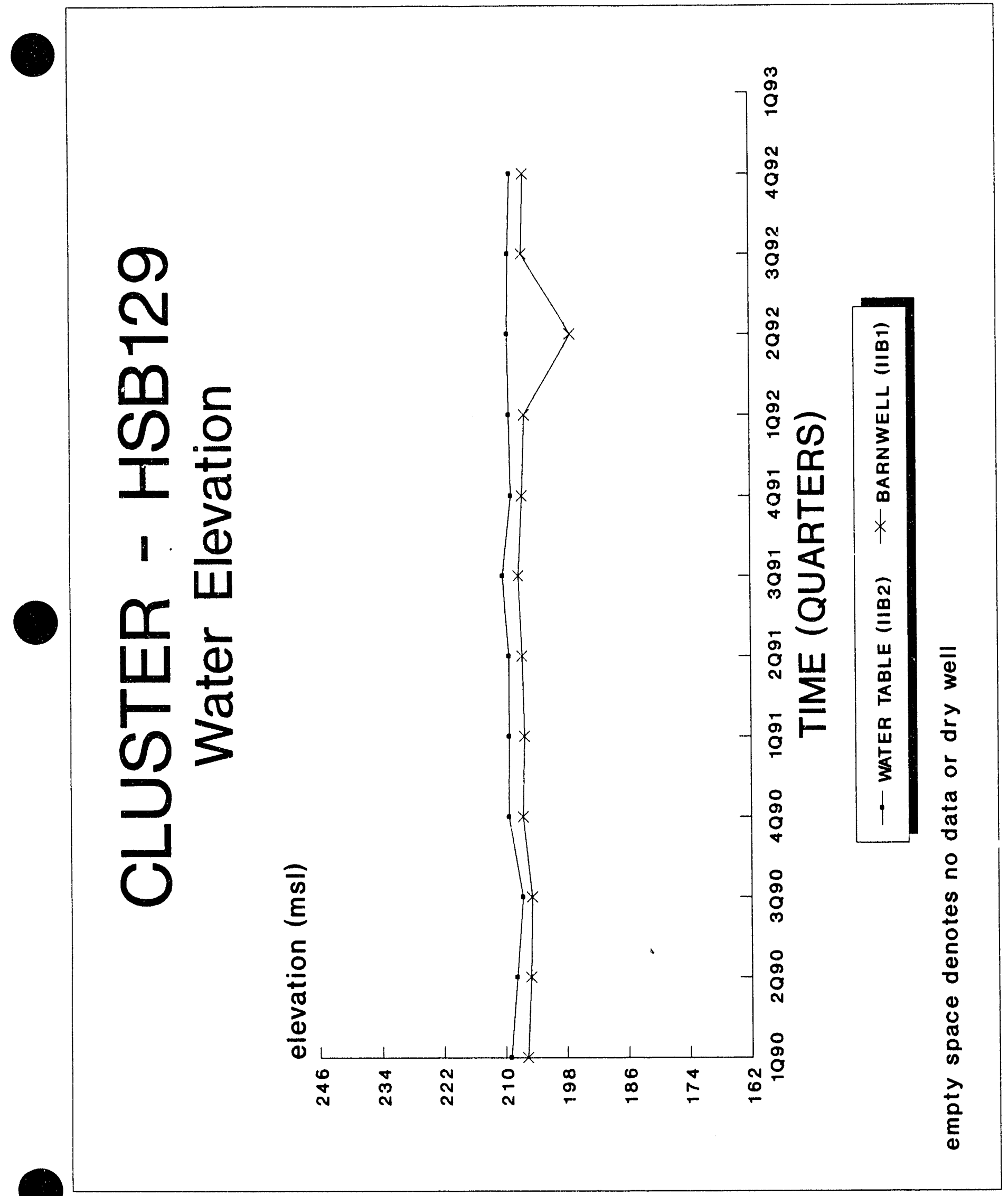




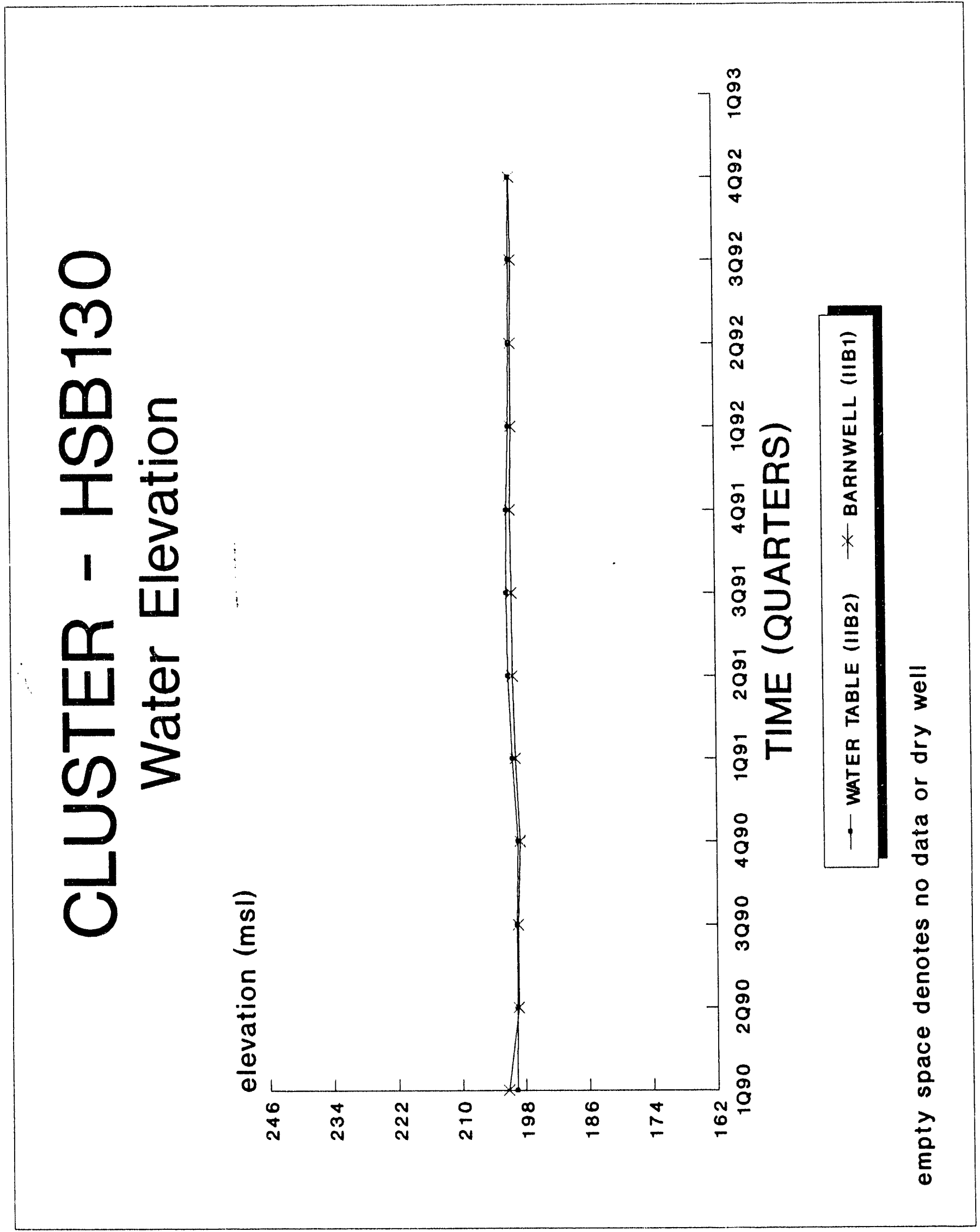




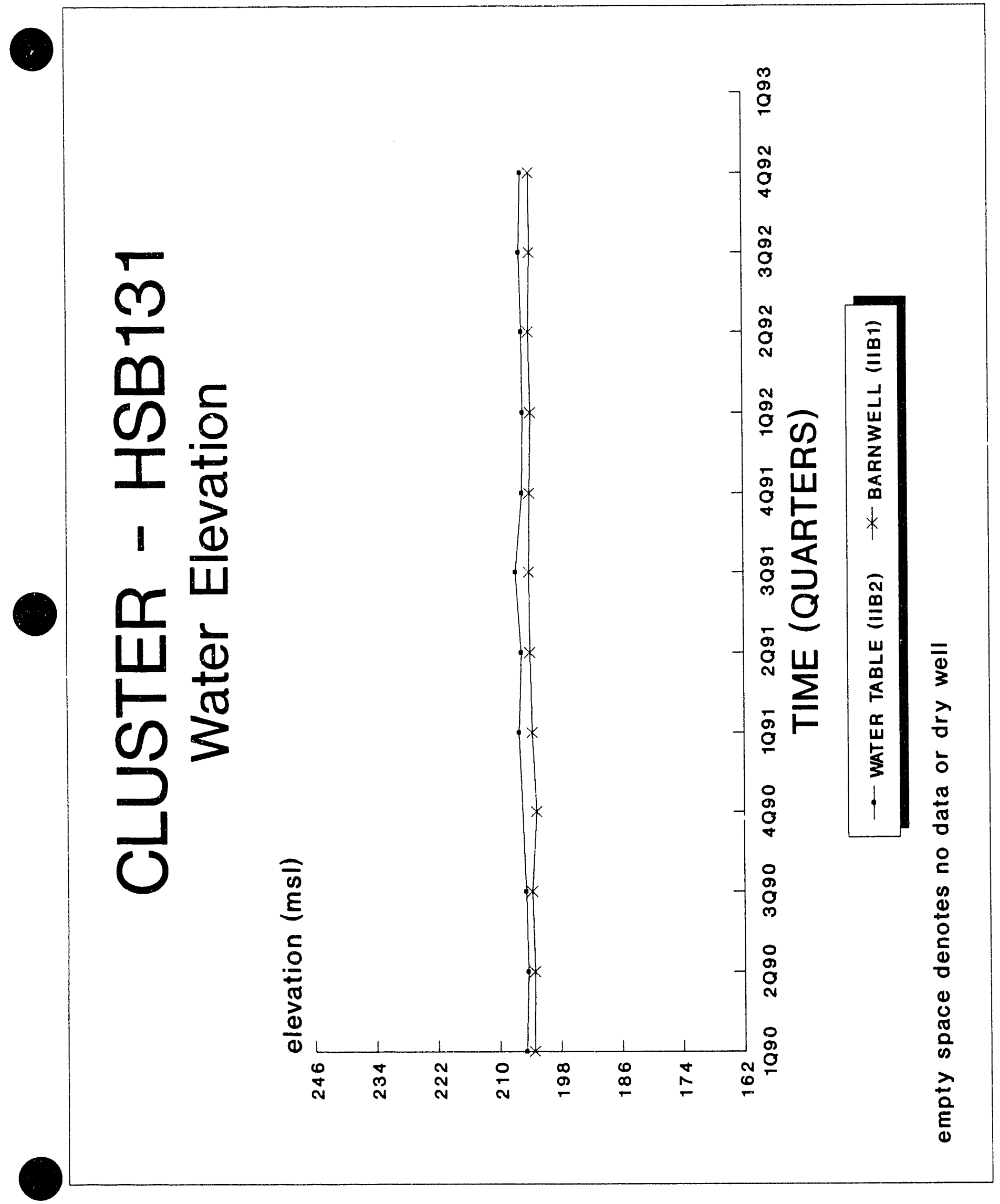




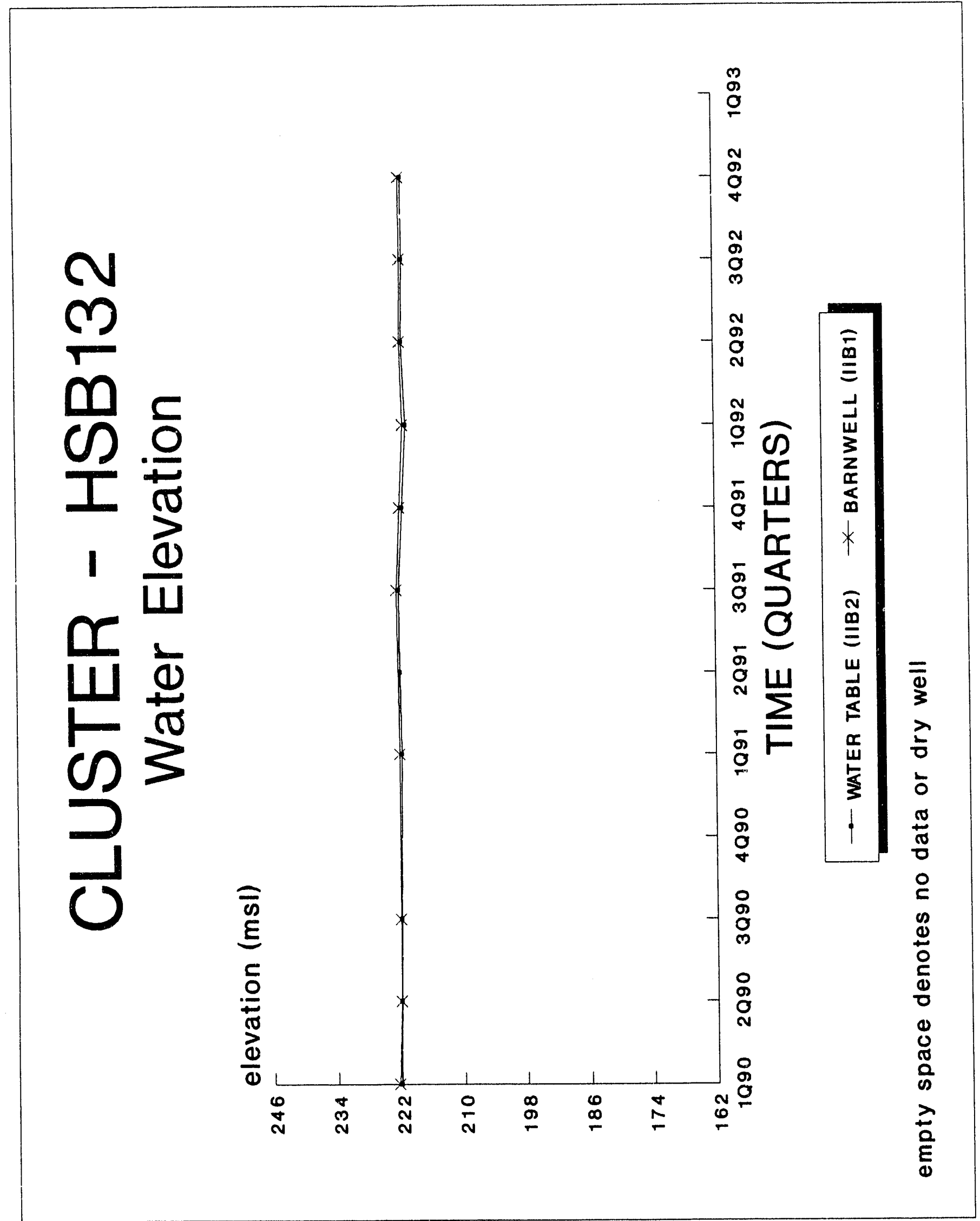




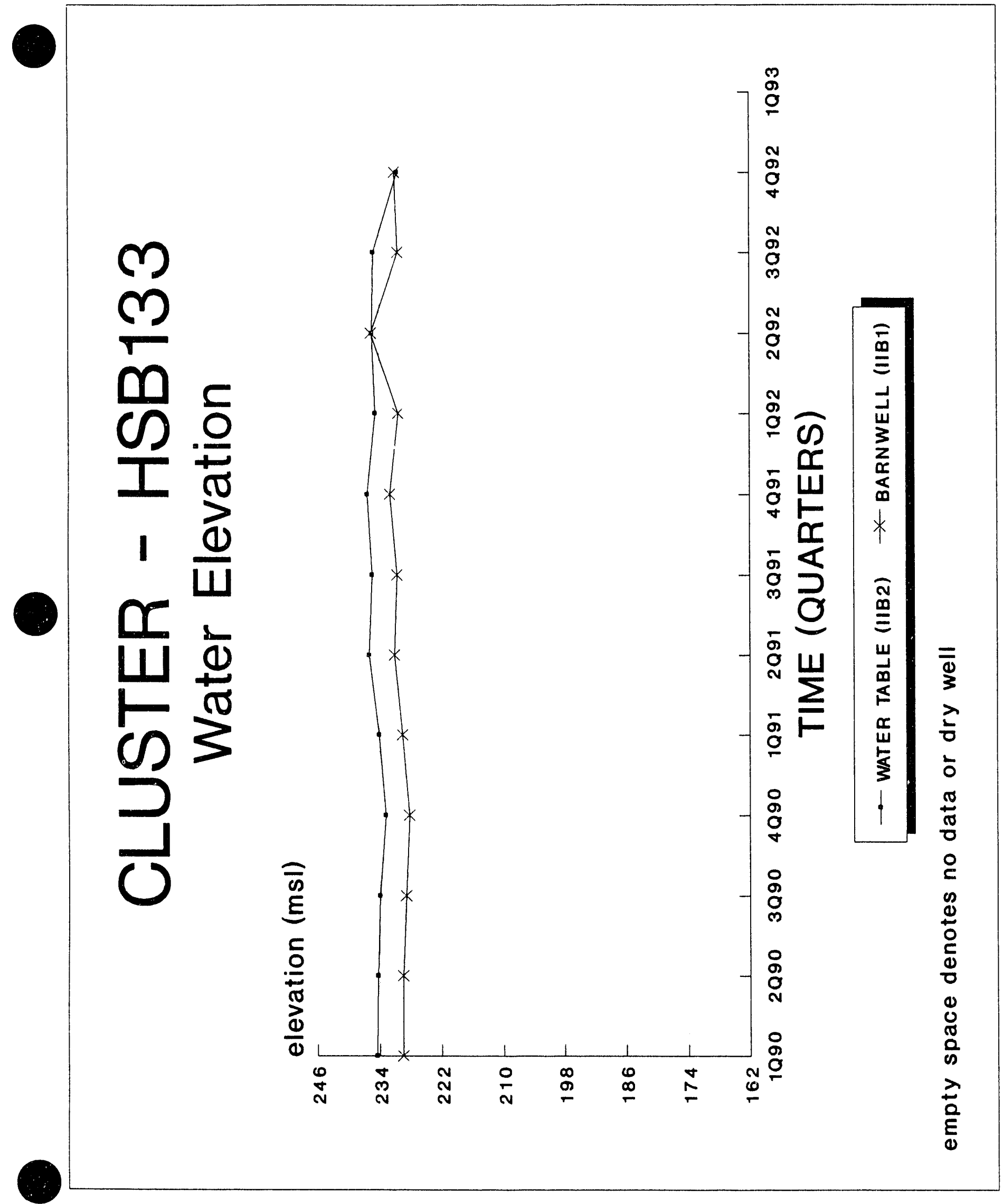




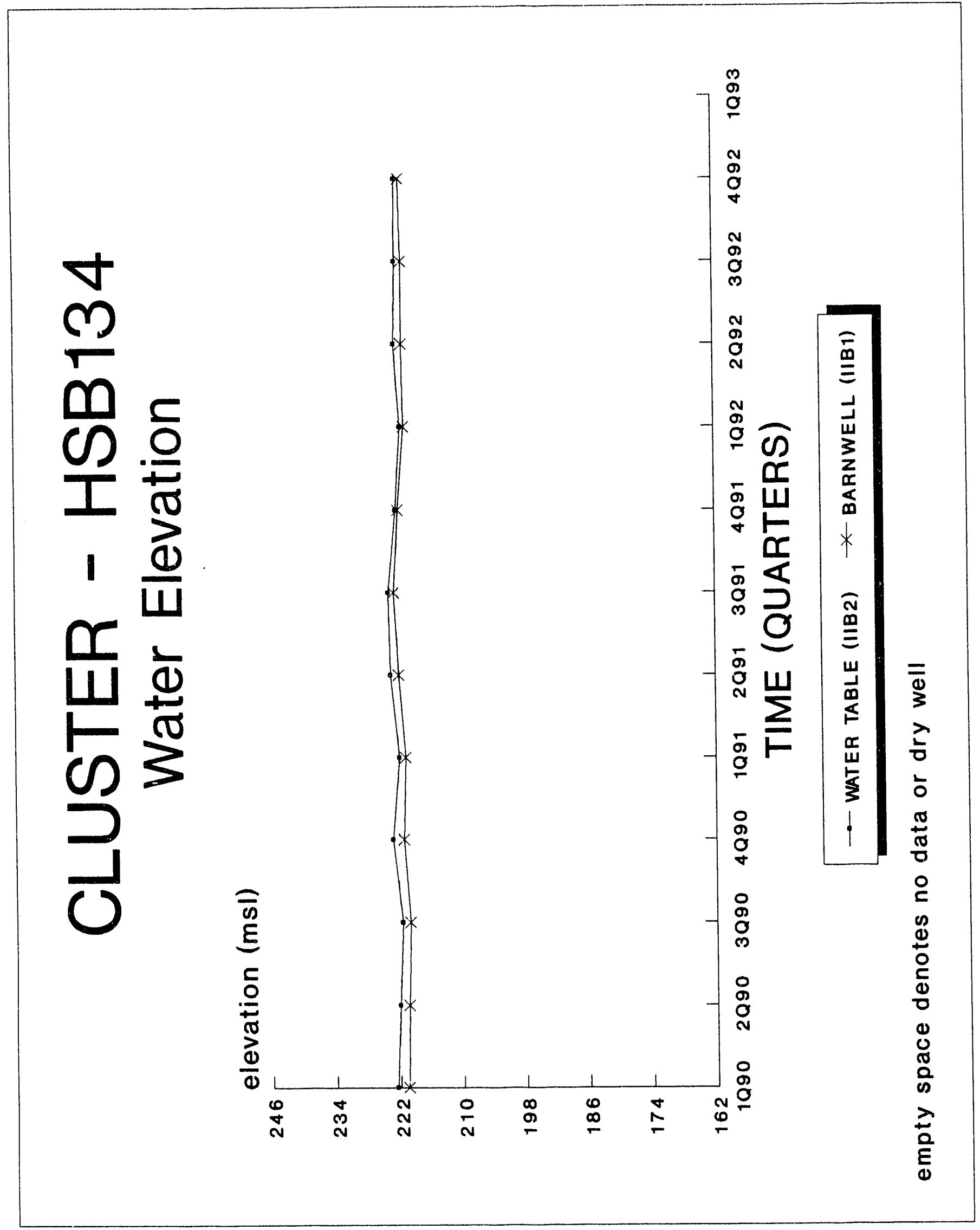




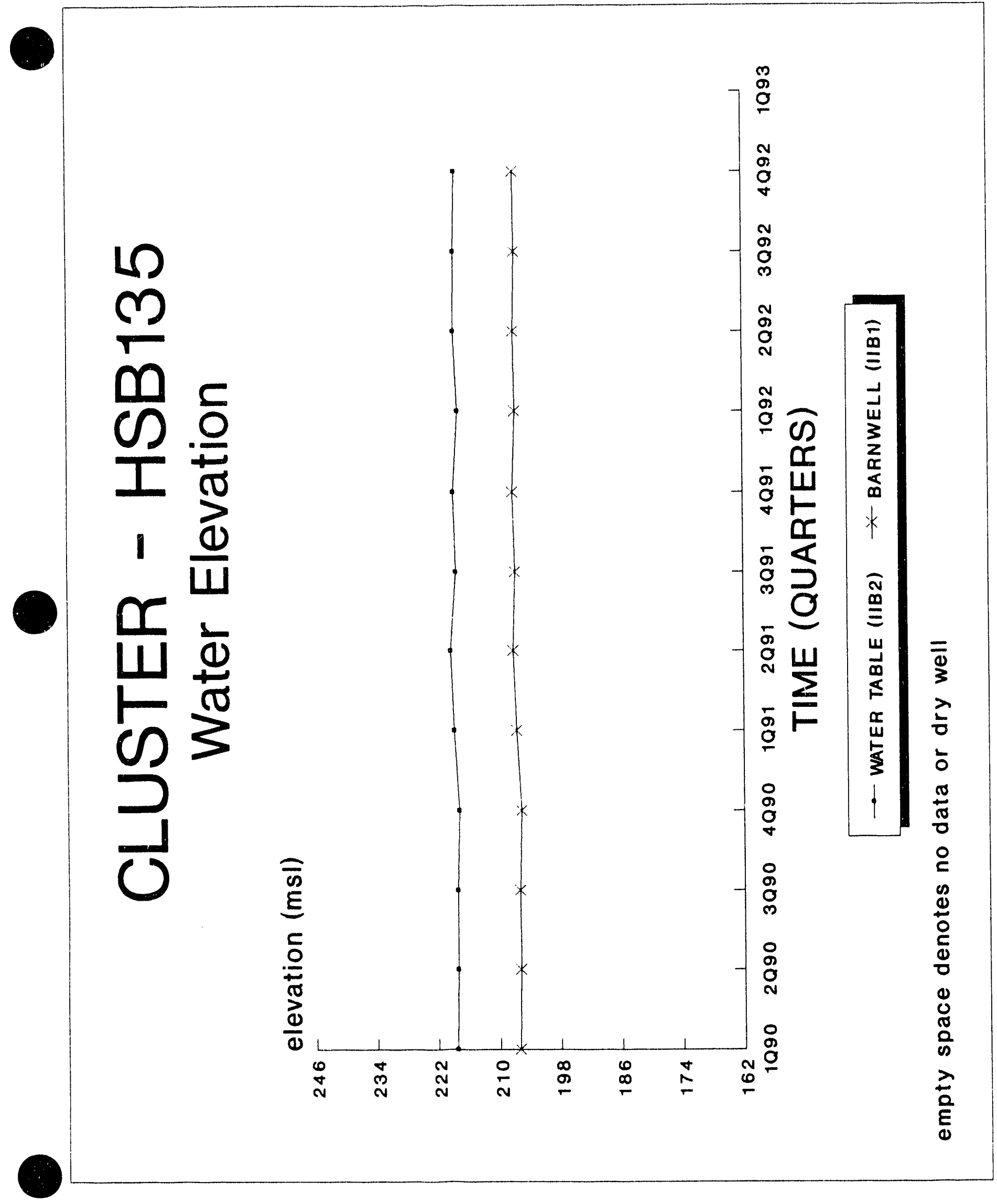




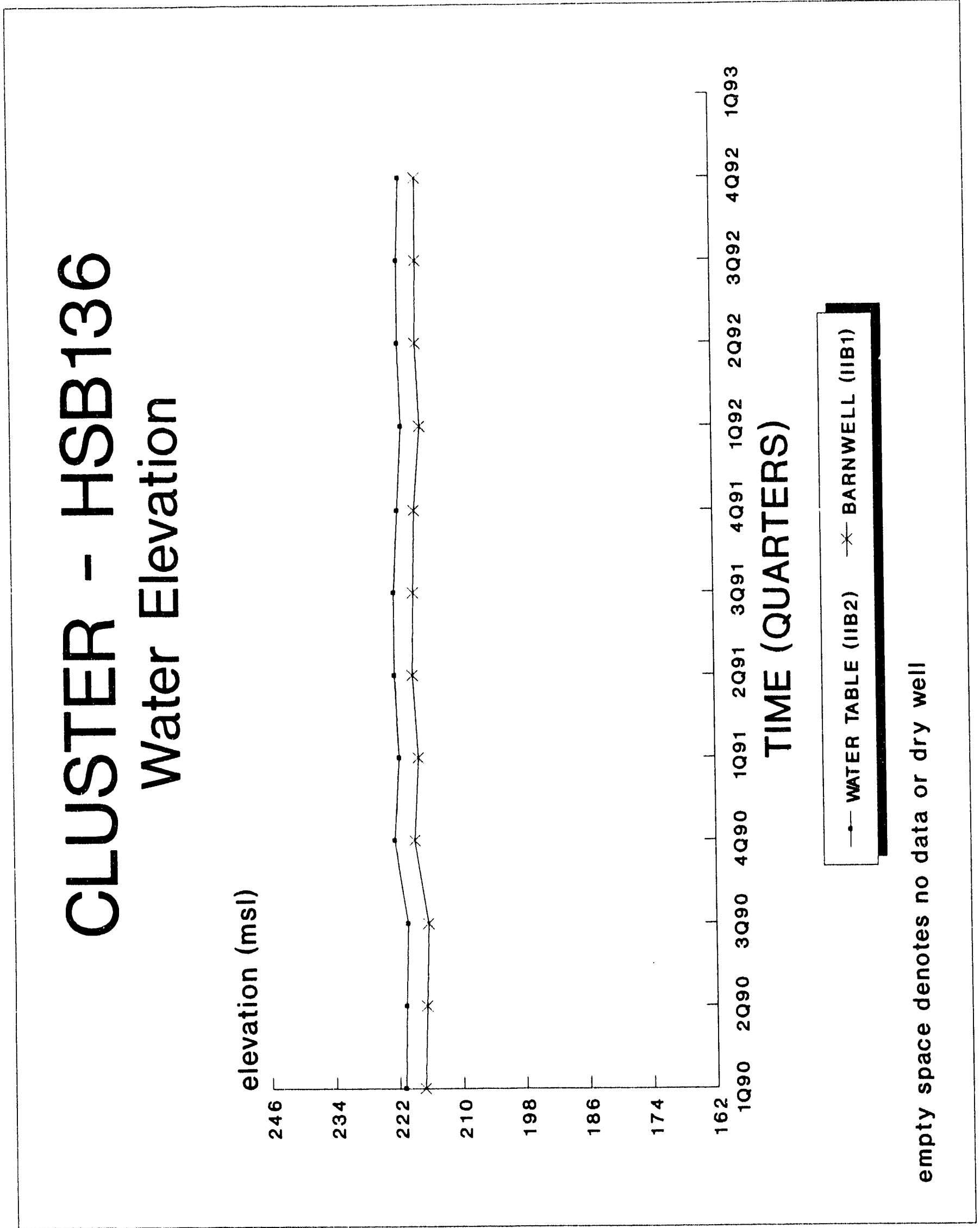




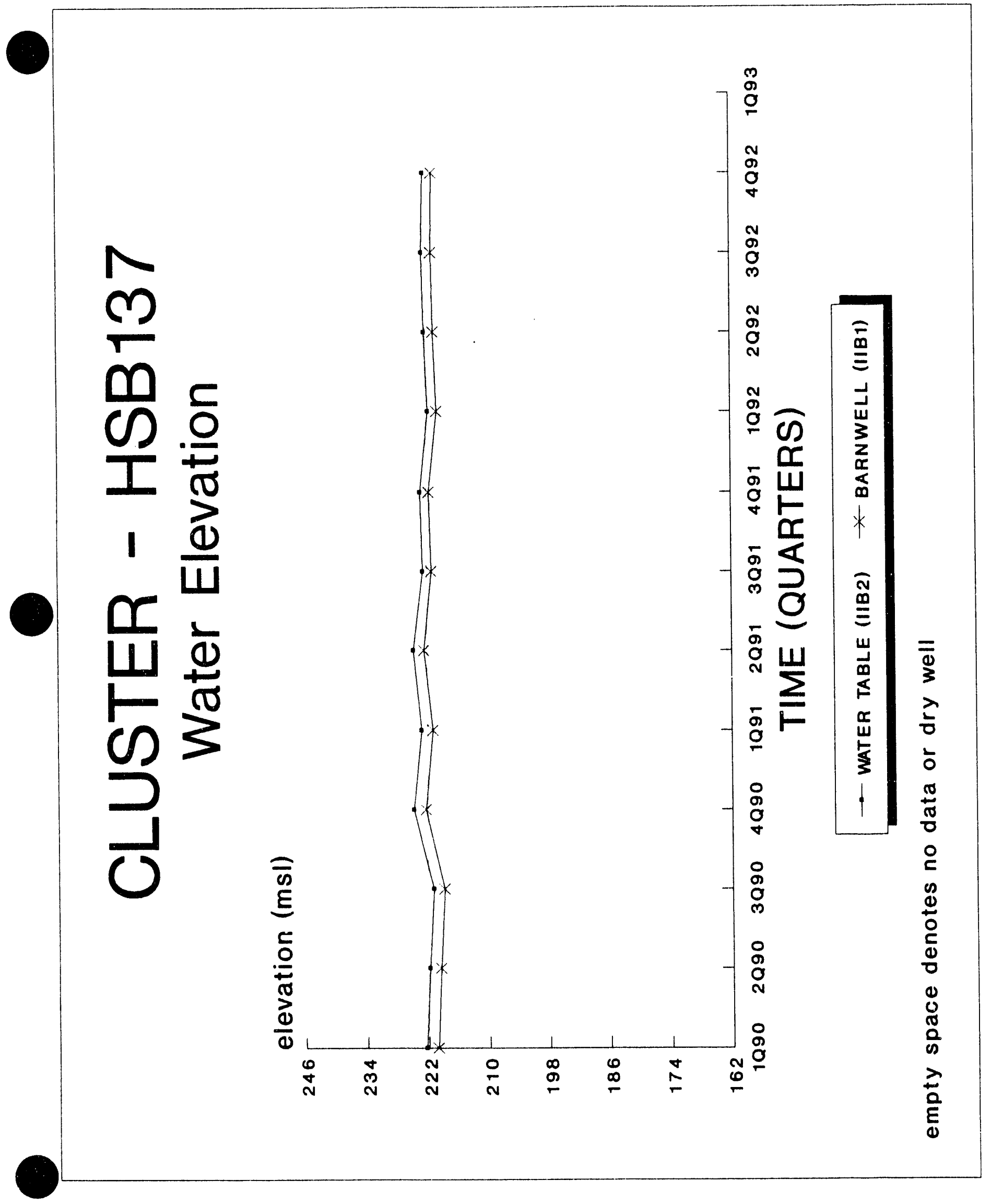




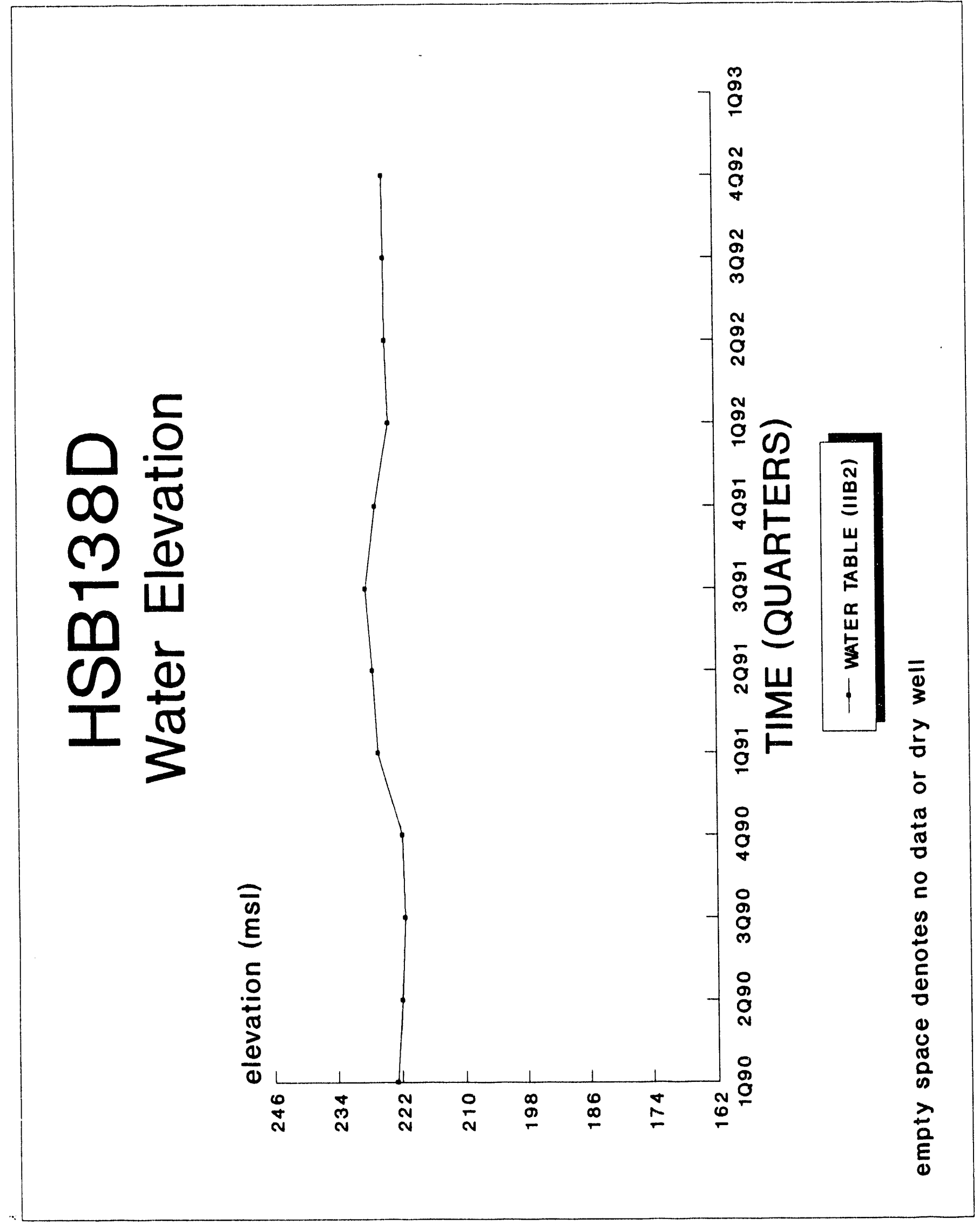




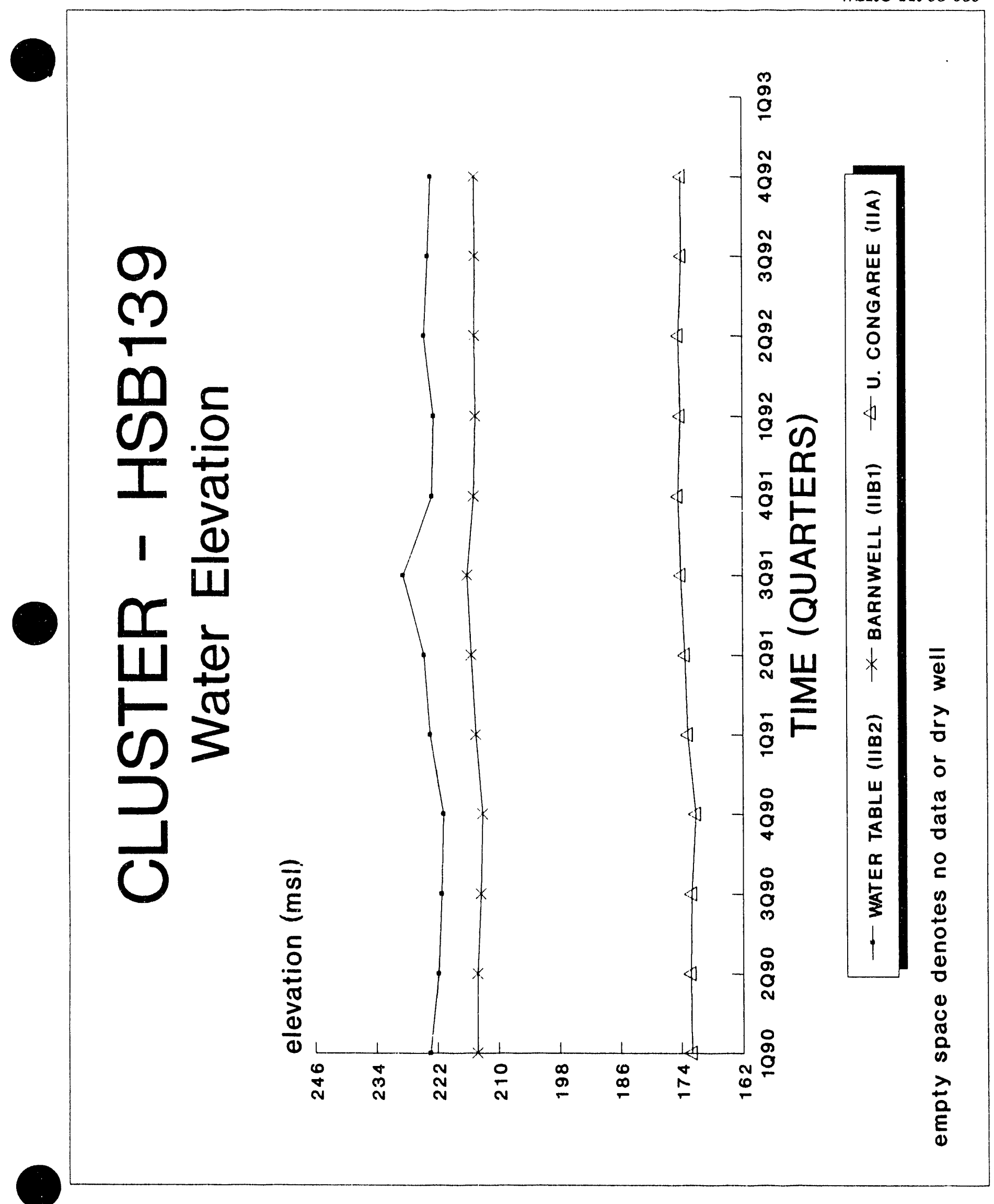



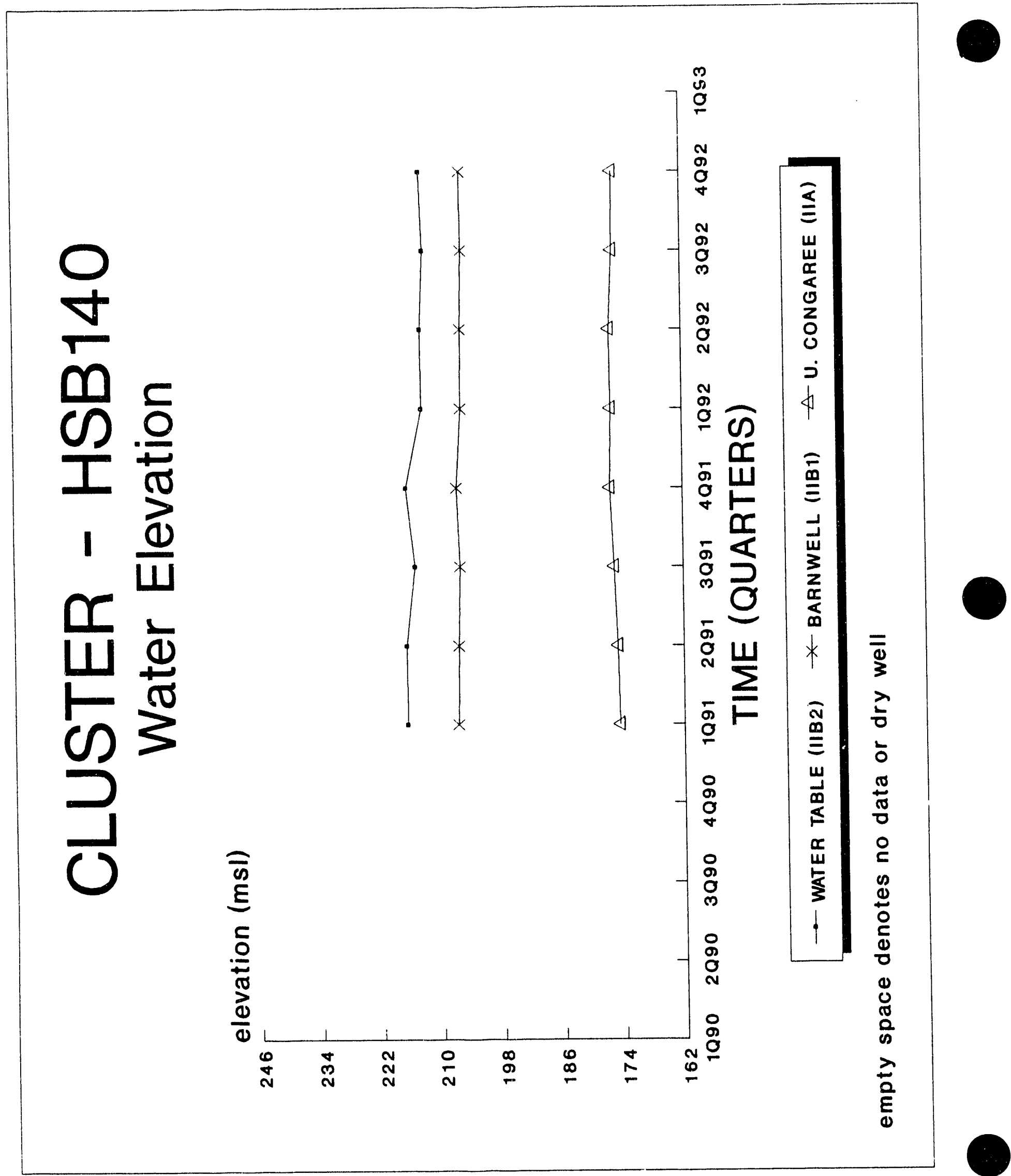


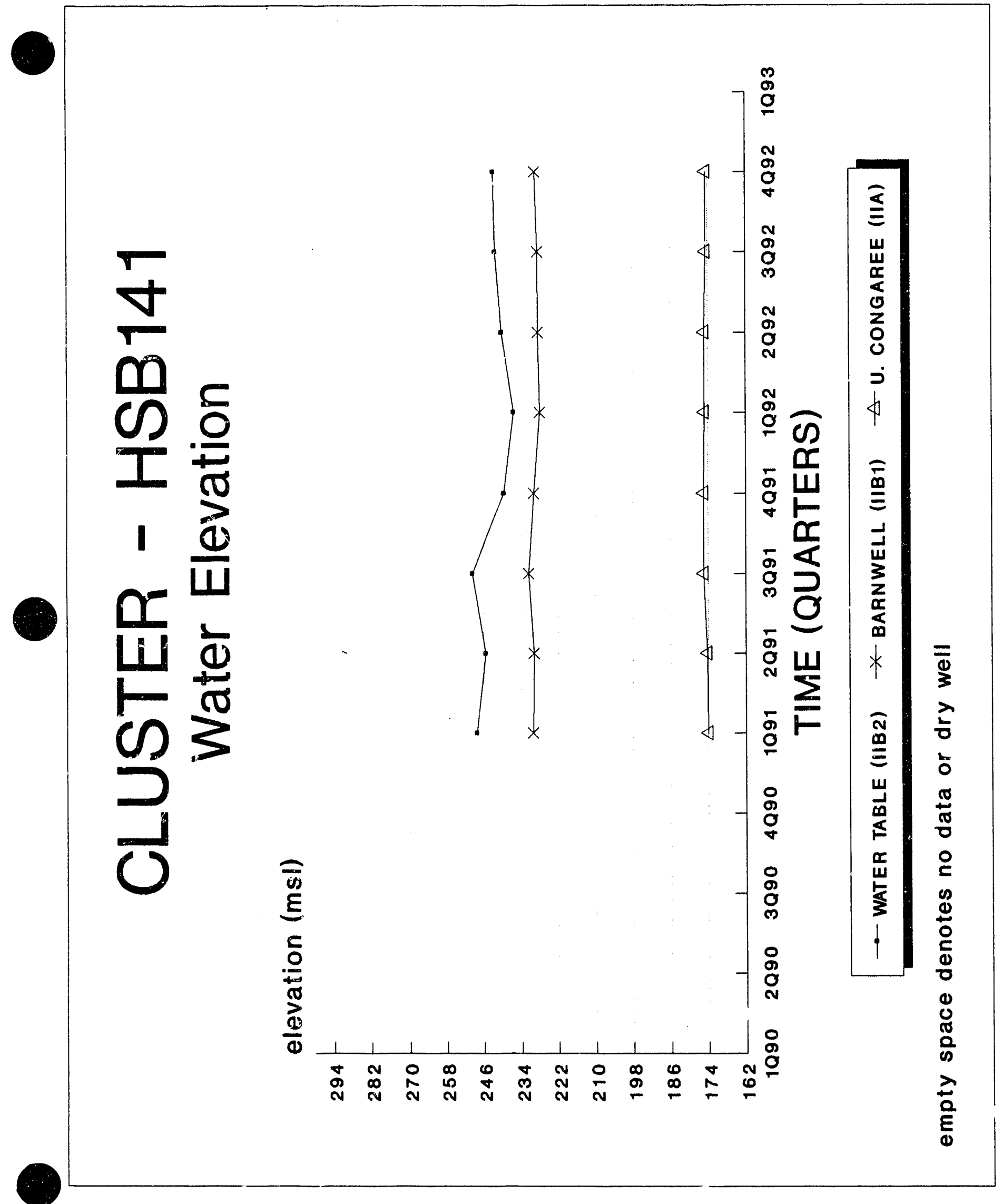




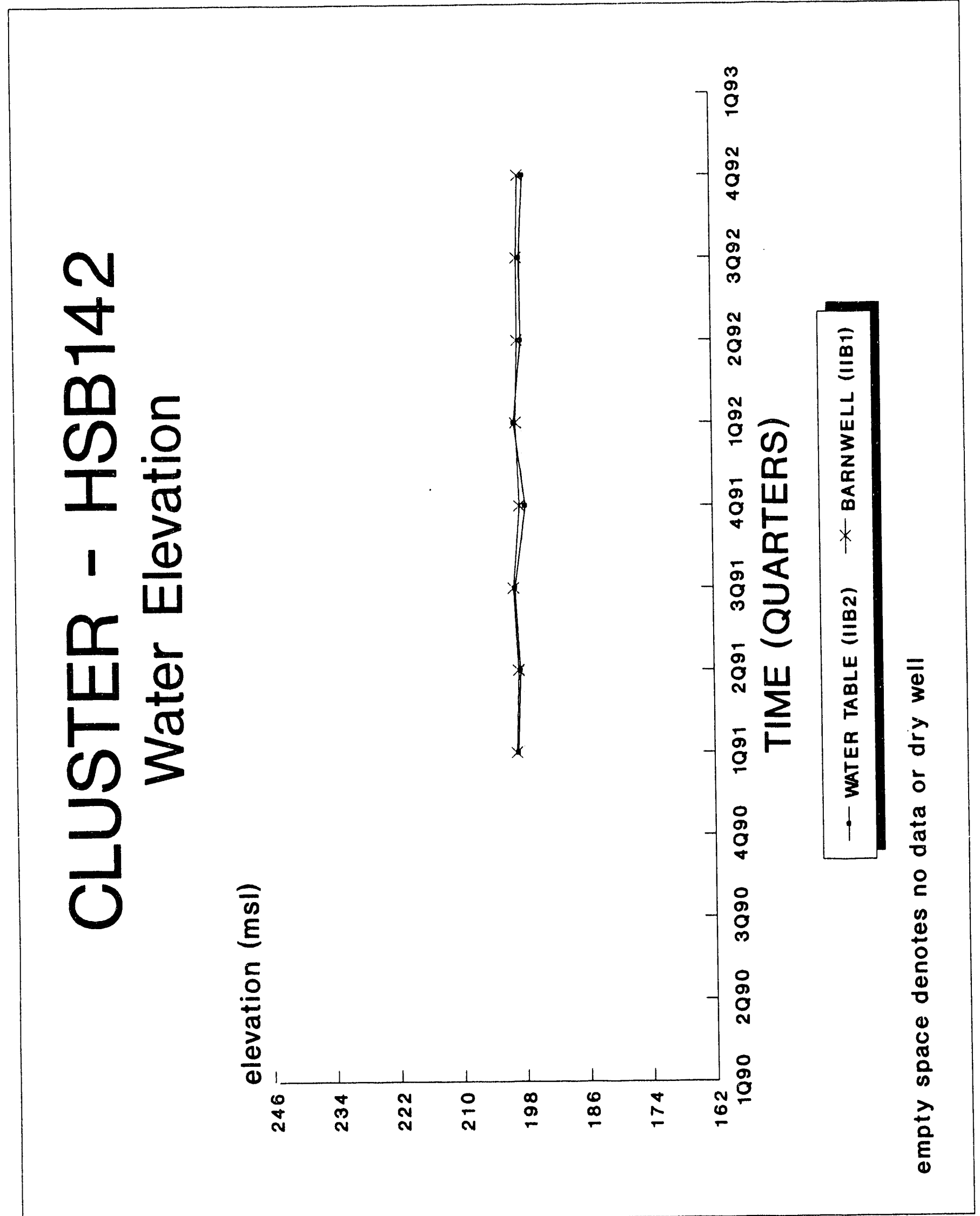




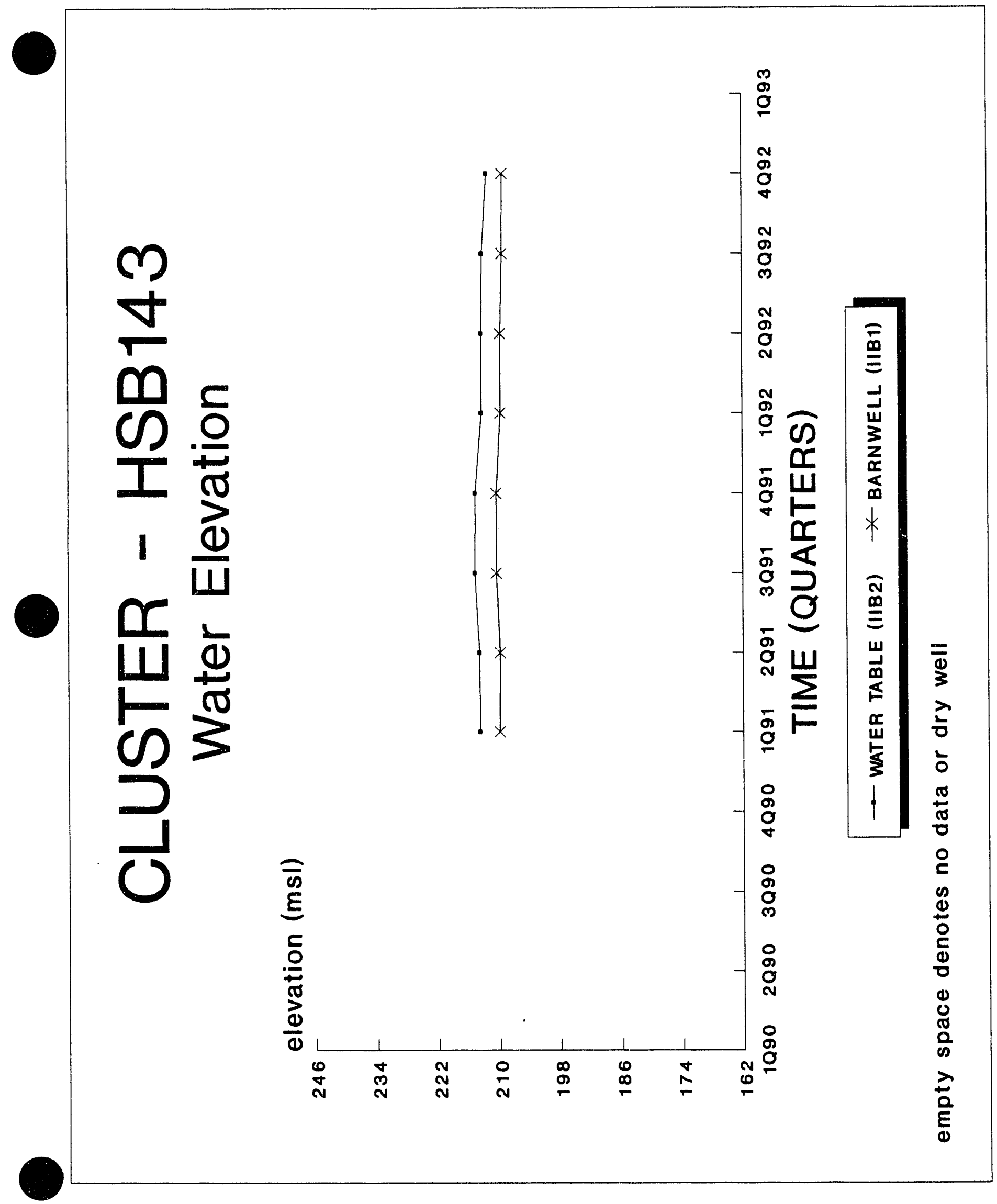




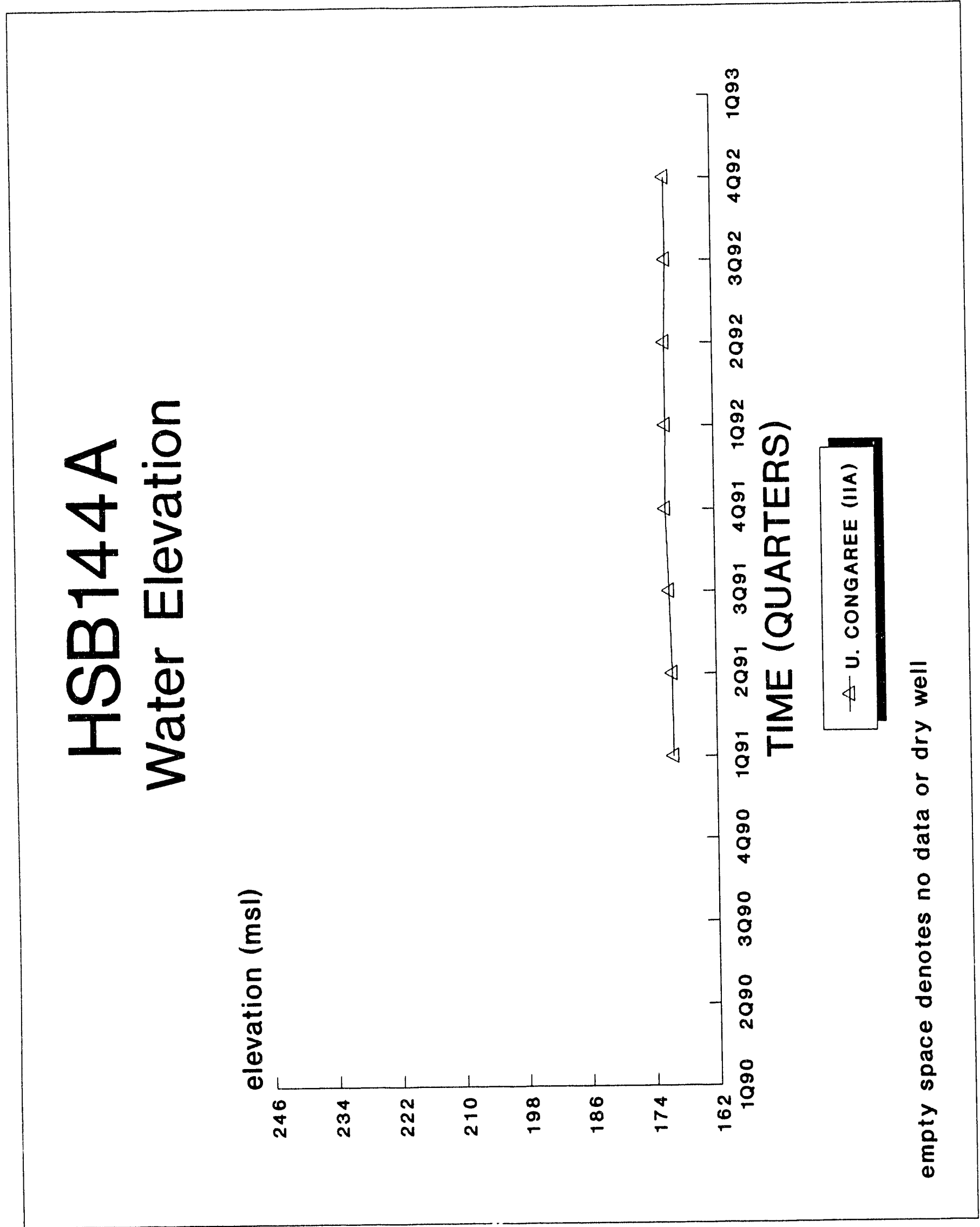




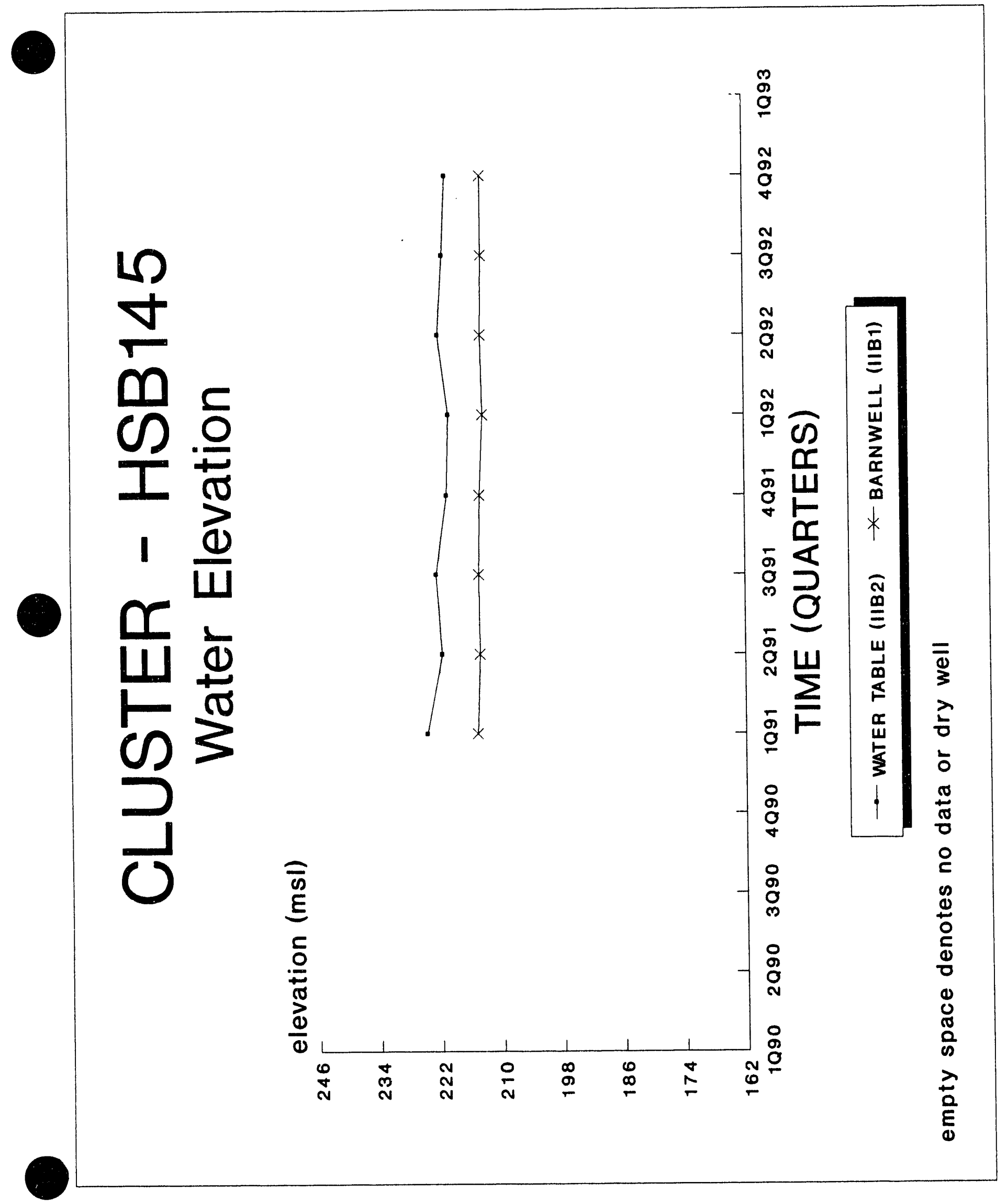




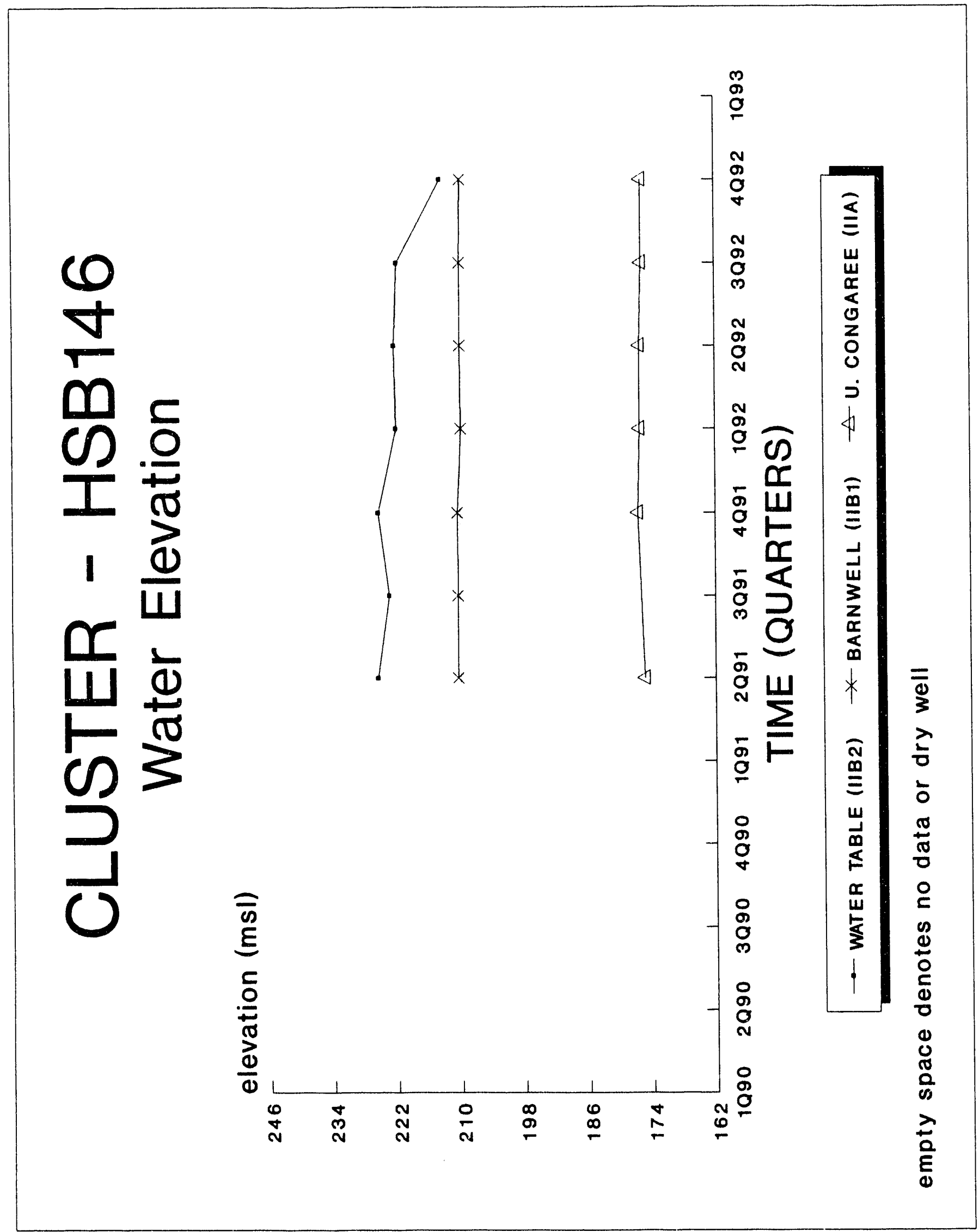




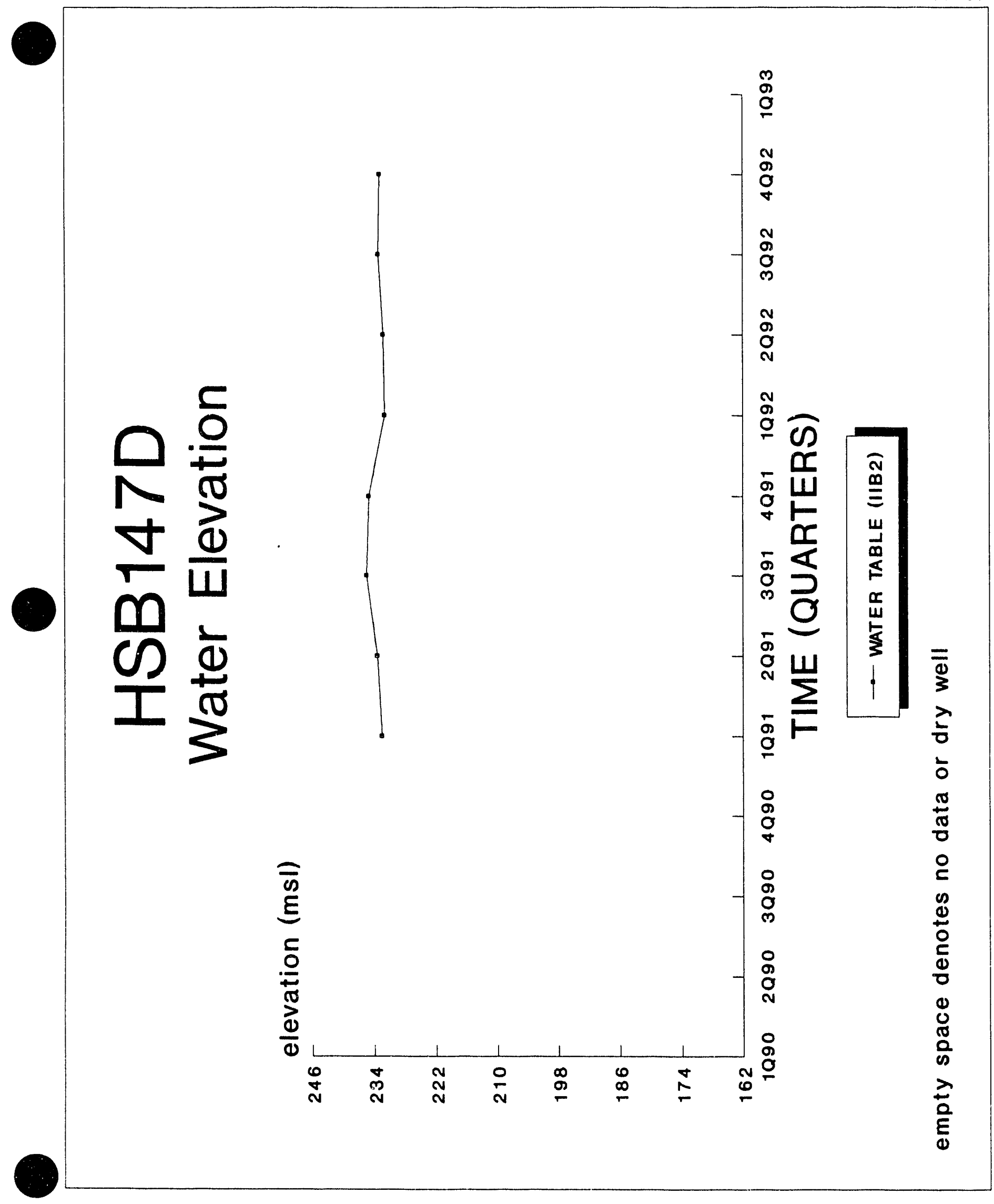



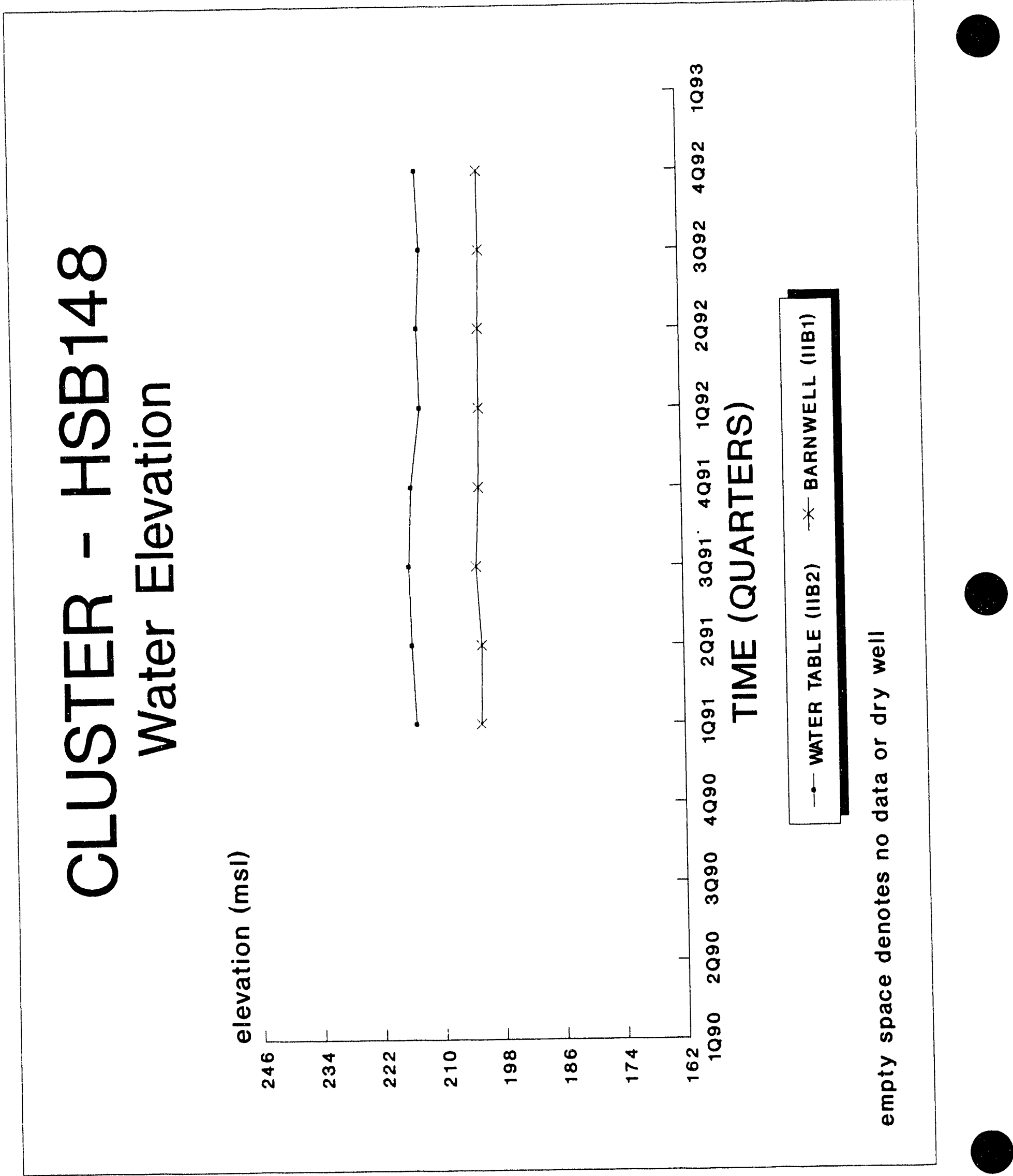


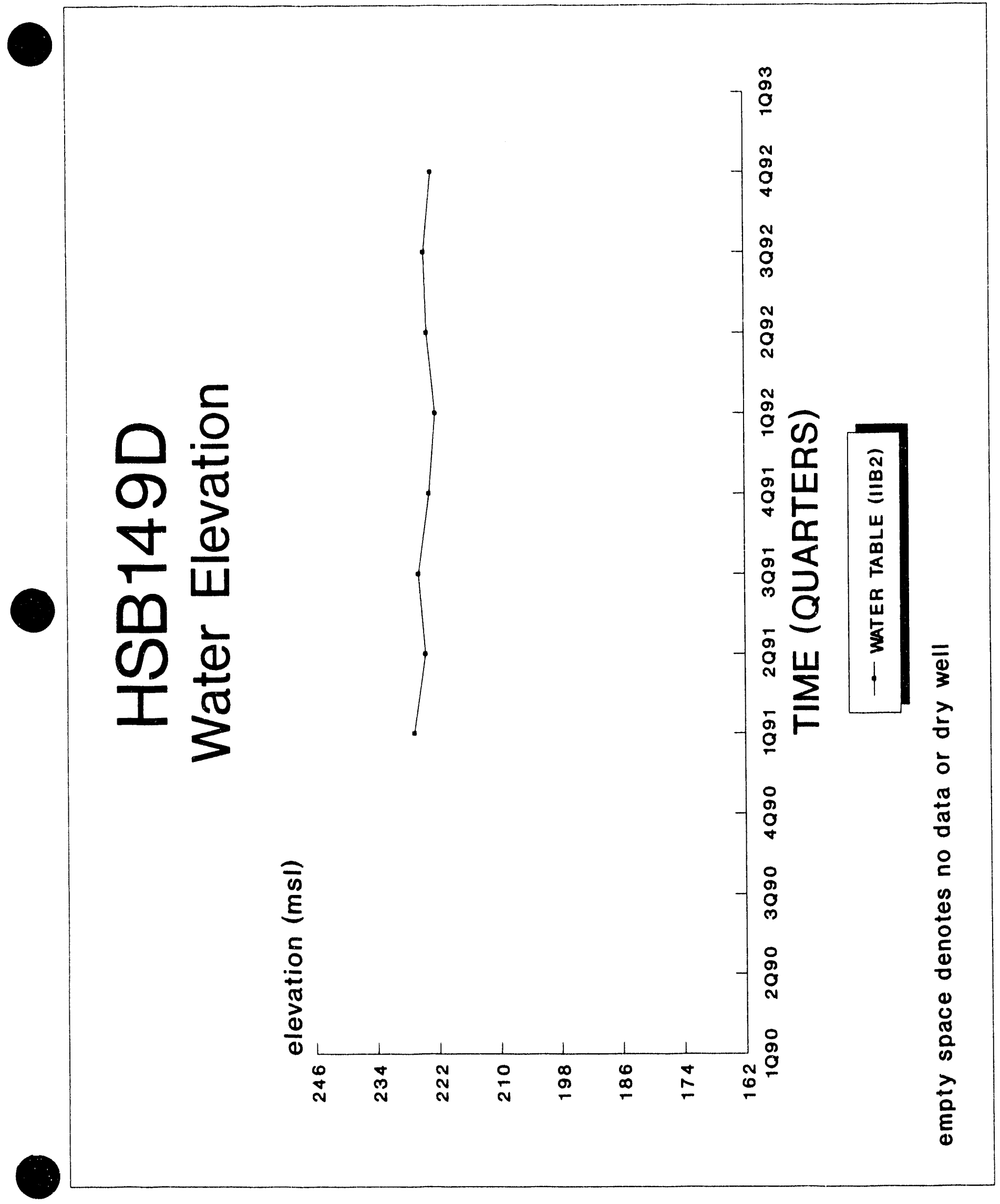


WSRC-TR-93.059

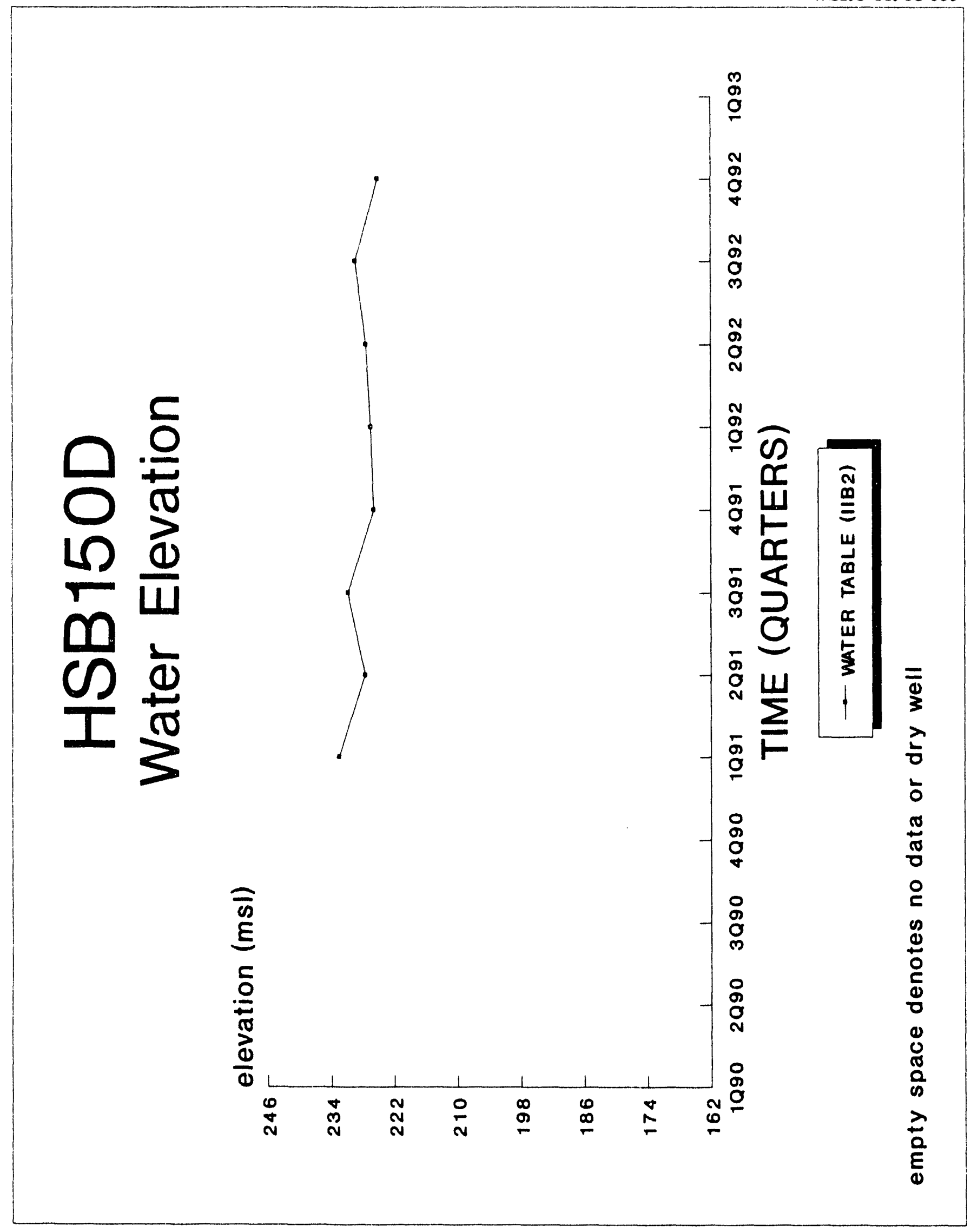




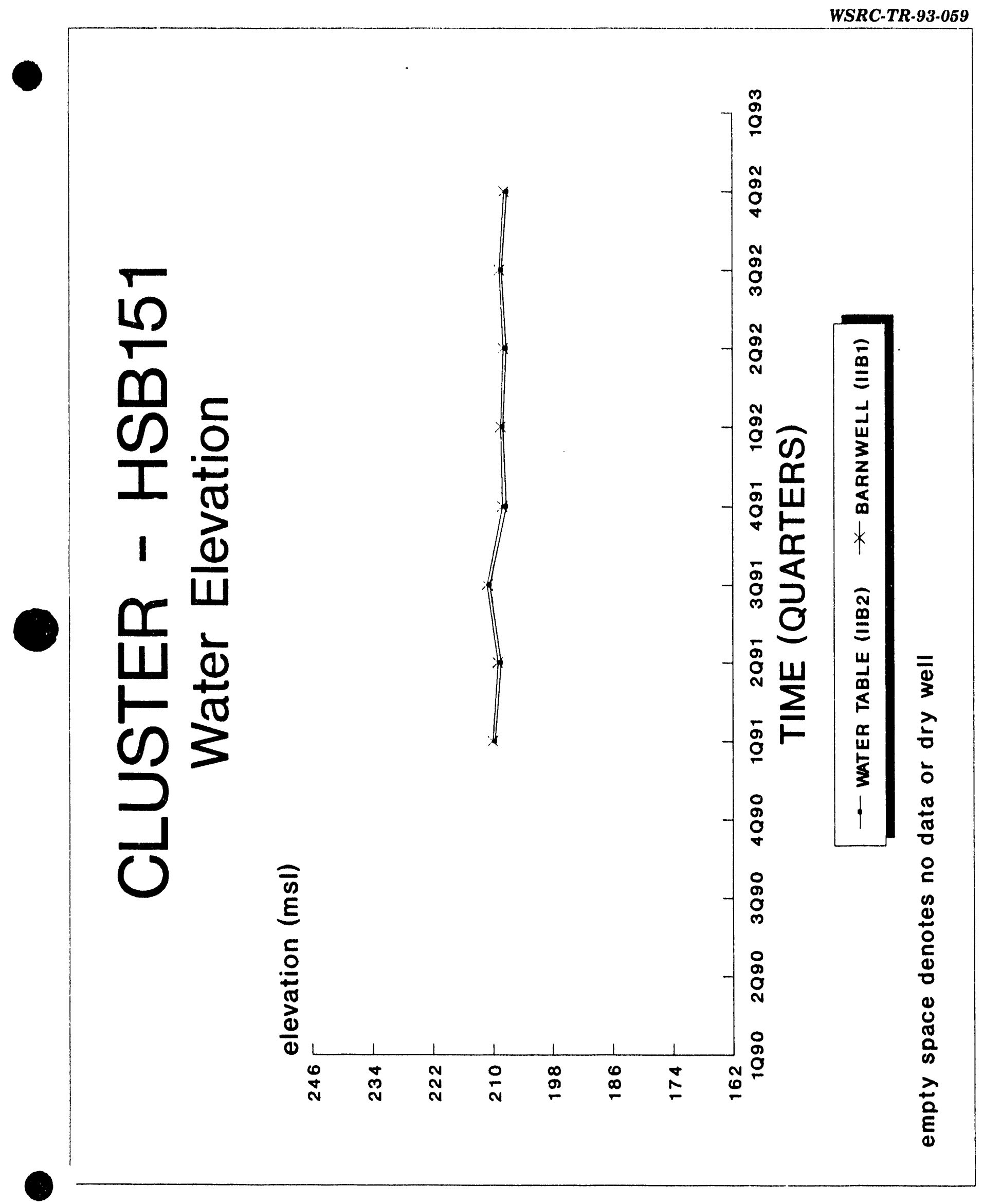




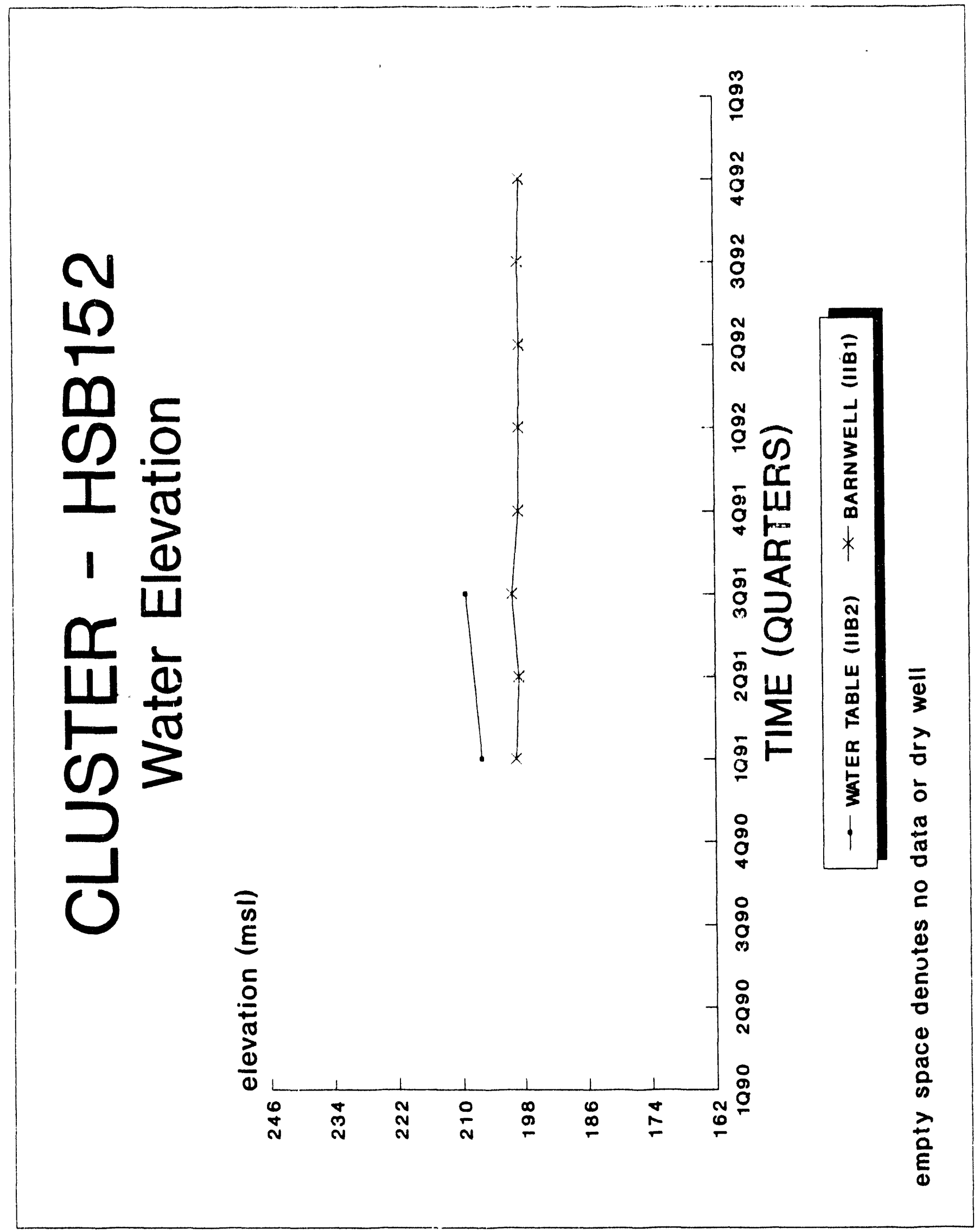


